Supporting Information

\title{
Catalytic Enantioselective House-Meinwald Rearrangement: Efficient Construction of All-Carbon Quaternary Stereocenters
}

\author{
Dengke $\mathrm{Ma}^{\dagger}{ }^{\dagger}$ Chun-Bao Miao, ${ }^{\ddagger}$ and Jianwei Sun ${ }^{\dagger *}$
}

${ }^{+}$Department of Chemistry and Shenzhen Research Institute, the Hong Kong University of Science and Technology, Clear Water Bay, Kowloon, Hong Kong SAR, China; ¥ Jiangsu Key Laboratory of Advanced Catalytic Materials and Technology, School of Petrochemical Engineering, Changzhou University, Changzhou, Jiangsu, China

\section{Table of Contents}

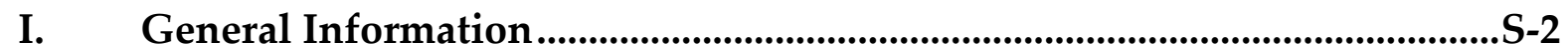

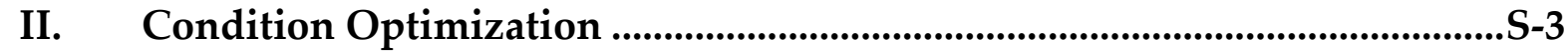

III. Substrate Preparation .................................................................................................

IV. Organocatalytic Asymmetric Rearrangement of Epoxides ........................S-48

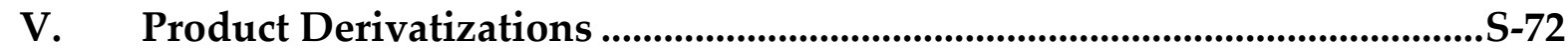

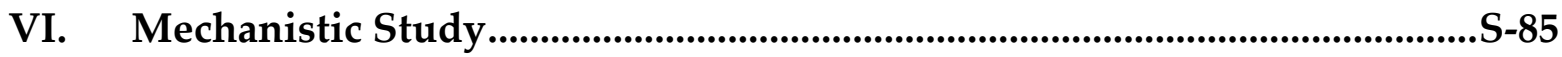

VII. Determination of the Stereochemistry ..............................................................S-88

NMR Spectra and HPLC Traces 


\section{General Information}

Flash column chromatography was performed over silica gel (200-300 mesh) purchased from Qindao Puke Co., China. All air or moisture sensitive reactions were conducted in oven-dried glassware under nitrogen atmosphere using anhydrous solvents. Anhydrous dichloromethane, toluene, diethyl ether, and tetrahydrofuran were purified by the Innovative ${ }^{\circledR}$ solvent purification system. Reagents were purchased at the highest commercial quality and used without further purification, unless otherwise stated. ${ }^{1} \mathrm{H},{ }^{13} \mathrm{C},{ }^{19} \mathrm{~F}$, and ${ }^{31} \mathrm{P}$ NMR spectra were collected on a Bruker AV $400 \mathrm{MHz}$ NMR spectrometer using residue solvent peaks as an internal standard ( ${ }^{1} \mathrm{H}$ NMR: $\mathrm{CDCl}_{3}$ at $7.26 \mathrm{ppm}, \mathrm{DMSO}-d_{6}$ at $2.54 \mathrm{ppm}$, Acetone- $d_{6}$ at 2.09 ppm; ${ }^{13} \mathrm{C}$ NMR: $\mathrm{CDCl}_{3}$ at $77.0 \mathrm{ppm}$, DMSO- $d_{6}$ at $39.52 \mathrm{ppm}$, Acetone- $d_{6}$ at $29.84 \mathrm{ppm}$ ). Mass spectra were collected on an Agilent GC/MS 5975C system, a MALDI Micro MX mass spectrometer, or an API QSTAR XL System. IR spectra were recorded on Bruker TENSOR 27 spectrometer and reported in terms of frequency of absorption $\left(\mathrm{cm}^{-1}\right)$. Optical rotations were measured on JASCO P-2000 polarimeter with $[\alpha]_{\mathrm{D}}$ values reported in degrees; concentration (c) is in $10 \mathrm{mg} / \mathrm{mL}$. The enantiomeric excess values were determined by chiral HPLC using an Agilent 1200 LC instrument with Daicel CHIRALPAK ${ }^{\circledR}$ AD-H, IC, AS-H, or CHIRALCEL ${ }^{\circledR}$ OD-H columns. 


\section{Condition Optimization}

Table S1. Reaction Optimization ${ }^{a}$

\begin{tabular}{|c|c|c|c|c|c|}
\hline entry & Cat. & Solvent & additive & Conv. $(\%)^{b}$ & ee $(\%)^{b}$ \\
\hline 1 & $(R)-\mathbf{A} 1$ & DCM & - & 100 & -27 \\
\hline 2 & $(R)-\mathbf{A} 2$ & $"$ & - & 100 & -24 \\
\hline 3 & $(S)-\mathbf{A} 3$ & $"$ & - & 100 & 70 \\
\hline 4 & (S)-A4 & $"$ & - & 100 & 79 \\
\hline 5 & $(R)-\mathrm{B} 1$ & $"$ & - & 100 & -58 \\
\hline 6 & $(R)-\mathrm{B} 2$ & $"$ & - & 100 & -64 \\
\hline 7 & $(R)-\mathrm{B} 3$ & $"$ & - & 100 & -43 \\
\hline 8 & $(R)-\mathbf{C} 1$ & $"$ & - & 100 & 89 \\
\hline 9 & $(R)-\mathbf{C 2}$ & $"$ & - & 100 & 59 \\
\hline 10 & $(R)-\mathbf{C} 3$ & $"$ & - & 100 & 42 \\
\hline 11 & $(R)-\mathrm{C} 4$ & $"$ & - & 100 & 51 \\
\hline 12 & $(R)-\mathrm{C5}$ & $"$ & - & 100 & 34 \\
\hline 13 & $(R)-\mathrm{C} 6$ & $"$ & - & 100 & 53 \\
\hline 14 & $(R)-\mathrm{C} 7$ & $"$ & - & 100 & 75 \\
\hline 15 & $(R)-\mathbf{C 8}$ & $"$ & - & 100 & 39 \\
\hline 16 & $(R)-\mathbf{C} 1$ & $\mathrm{CHCl}_{3}$ & - & 31 & 79 \\
\hline 17 & $(R)-\mathbf{C} 1$ & $\mathrm{CCl}_{4}$ & - & 100 & 76 \\
\hline 18 & $(R)-\mathbf{C 1}$ & DCE & - & 100 & 71 \\
\hline 19 & $(R)-\mathbf{C} 1$ & toluene & - & 100 & 88 \\
\hline 20 & $(R)-\mathbf{C 1}$ & DCM & $5 \AA \mathrm{MS}$ & 100 & 90 \\
\hline $21^{c}$ & $(R)-\mathbf{C} 1$ & DCM & $5 \AA \mathrm{MS}$ & 100 & 90 \\
\hline $22^{c, d}$ & $(R)-\mathbf{C} 1$ & $\mathrm{DCM}$ & $5 \AA \mathrm{MS}$ & 100 & 90 \\
\hline $23^{c, d, e}$ & $(R)-\mathbf{C} 1$ & $\mathrm{PhCl} / \mathrm{DCM}$ & $5 \AA \mathrm{MS}$ & 100 & 94 \\
\hline $24^{c, e, f}$ & $(R)-\mathbf{C} 1$ & $\mathrm{PhCl} / \mathrm{DCM}$ & $5 \AA \mathrm{MS}$ & 100 & 94 \\
\hline $25^{c, e, g}$ & $(R)-\mathbf{C 1}$ & $\mathrm{PhCl} / \mathrm{DCM}$ & $5 \AA \mathrm{MS}$ & 100 & 86 \\
\hline \multicolumn{2}{|c|}{$\begin{array}{l}(R)-A 1, R=2,4,6-i \operatorname{Pr}_{3} \mathrm{C}_{6} \\
(R)-\mathrm{A} 2, \mathrm{R}=9-\text {-Anthranyl } \\
\text { (S)-A3, R = 1-Napthyl } \\
\text { (S)-A4, R = 1-Pyrenyl }\end{array}$} & $\begin{array}{l}\mathrm{J}_{2} \\
(R)-\mathrm{B} 1, \mathrm{R}= \\
(R)-\mathrm{B} 2, \mathrm{R}= \\
(R)-\mathrm{B} 3, \mathrm{R}=\end{array}$ & $\begin{array}{l}\text { renyl } \\
\text { pthyl } \\
-i \mathrm{Pr}_{3} \mathrm{C}_{6} \mathrm{H}_{2}\end{array}$ & \multicolumn{2}{|c|}{$\begin{array}{l}(R)-\mathrm{C} 1, \mathrm{R}=1-\text {-Pyrenyl } \\
(R)-\mathrm{C} 2, \mathrm{R}=\mathrm{Mes} \\
(R)-\mathrm{C} 3, \mathrm{R}=4-\mathrm{ClC}_{6} \mathrm{H}_{4} \\
(R)-\mathrm{C} 4, \mathrm{R}=4-\mathrm{NO}_{2} \mathrm{C}_{6} \mathrm{H}_{4} \\
(R)-\mathrm{C} 5, \mathrm{R}=3,5-\text { bisClC }_{6} \mathrm{H}_{3} \\
(R)-\mathrm{C} 6, \mathrm{R}=3,5-\text { bisCF }_{3} \mathrm{C}_{6} \mathrm{H}_{3} \\
(R)-\mathrm{C} 7, \mathrm{R}=3,5-\text { bisPhC }_{6} \mathrm{H}_{3} \\
(R)-\mathrm{C} 8, \mathrm{R}=\text { Biphenyl }\end{array}$} \\
\hline
\end{tabular}

${ }^{a}$ Reaction conditions: $1 \mathrm{a}(0.05 \mathrm{mmol})$, cat. $(10 \mathrm{~mol} \%)$, additive $(20 \mathrm{mg})$, and solvent $(1 \mathrm{~mL})$ at $0{ }^{\circ} \mathrm{C}$. ${ }^{b}$ The conversion was determined by NMR using $\mathrm{CH}_{2} \mathrm{Br}_{2}$ as an internal standard. The ee value was determined by chiral HPLC with a chiral stationary phase. ${ }^{c} \mathrm{C}=0.025 \mathrm{M}$. ${ }^{d}$ Run with $5 \mathrm{~mol} \%$ of cat. ${ }^{e} \mathrm{PhCl} / \mathrm{DCM}=7: 1 .{ }^{f}$ Run with $1 \mathrm{~mol} \%$ of catalyst, $12 \mathrm{~h} .{ }^{8}$ Run with $0.1 \mathrm{~mol} \%$ of cat., $23 \mathrm{~h}$. 
Table S2. Solvent Screening ${ }^{a}$

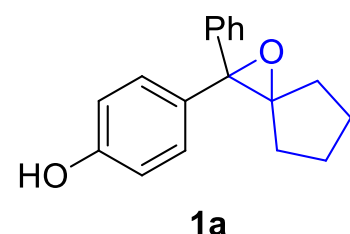

$1 \mathrm{a}$

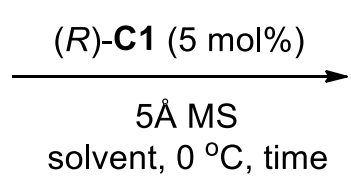

solvent, $0^{\circ} \mathrm{C}$, time<smiles>O=C1CCCC[C@@]1(c1ccccc1)c1ccc(O)cc1</smiles>

$2 a$

\begin{tabular}{|c|c|c|c|c|}
\hline entry & solvent & time $(\mathrm{h})$ & Conv. $(\%)^{b}$ & ee $(\%)^{b}$ \\
\hline 1 & DCM & 3 & 100 & 90 \\
\hline 2 & $\mathrm{PhCl} / \mathrm{DCM}=7 / 1$ & 10 & 100 & 94 \\
\hline 3 & $\mathrm{PhCF}_{3} / \mathrm{DCM}=7 / 1$ & 10 & 100 & 90 \\
\hline 4 & $o$-Xylene/DCM = 7/1 & 10 & 100 & 94 \\
\hline 5 & $m$-Xylene/DCM = 7/1 & 10 & 100 & 94 \\
\hline 6 & $p$-Xylene/DCM = 7/1 & 10 & 100 & 94 \\
\hline 7 & $\mathrm{PhF} / \mathrm{DCM}=7 / 1$ & 12 & 100 & 94 \\
\hline 8 & $\mathrm{PhBr} / \mathrm{DCM}=7 / 1$ & 12 & 100 & 94 \\
\hline 9 & Mesitylene/DCM = 7/1 & 12 & 100 & 92 \\
\hline 10 & n-hexane & 10 & 100 & 42 \\
\hline 11 & EA & 23 & NR & - \\
\hline 12 & THF & 23 & NR & - \\
\hline 13 & $\mathrm{Et}_{2} \mathrm{O}$ & 23 & NR & - \\
\hline 14 & $\mathrm{CH}_{3} \mathrm{NO}_{2}$ & 23 & NR & - \\
\hline 15 & Dioxane & 23 & NR & - \\
\hline 16 & MTBE & 23 & NR & - \\
\hline 17 & DME & 23 & NR & - \\
\hline
\end{tabular}

${ }^{a}$ Reaction condition: 1a $(0.05 \mathrm{mmol}),(R)-\mathrm{C1}(5 \mathrm{~mol} \%), 5 \AA \mathrm{MS}(20 \mathrm{mg})$, and solvent $(2 \mathrm{~mL})$ at $0{ }^{\circ} \mathrm{C}$. ${ }^{b}$ Determined by NMR using $\mathrm{CH}_{2} \mathrm{Br}_{2}$ as internal standard. The ee value was determined by chiral HPLC with a chiral stationary phase.

Note: The tetrasubstituted epoxide substrate was found to be unstable at room temperature in its pure form due to spontaneous background reaction to form racemic product. However, it is stable when stored in DCM at $-20^{\circ} \mathrm{C}$. This explains why DCM is a co-solvent for many entries in the above Table. 
Table S3. Reaction Optimization for $3 \mathbf{a}^{a}$

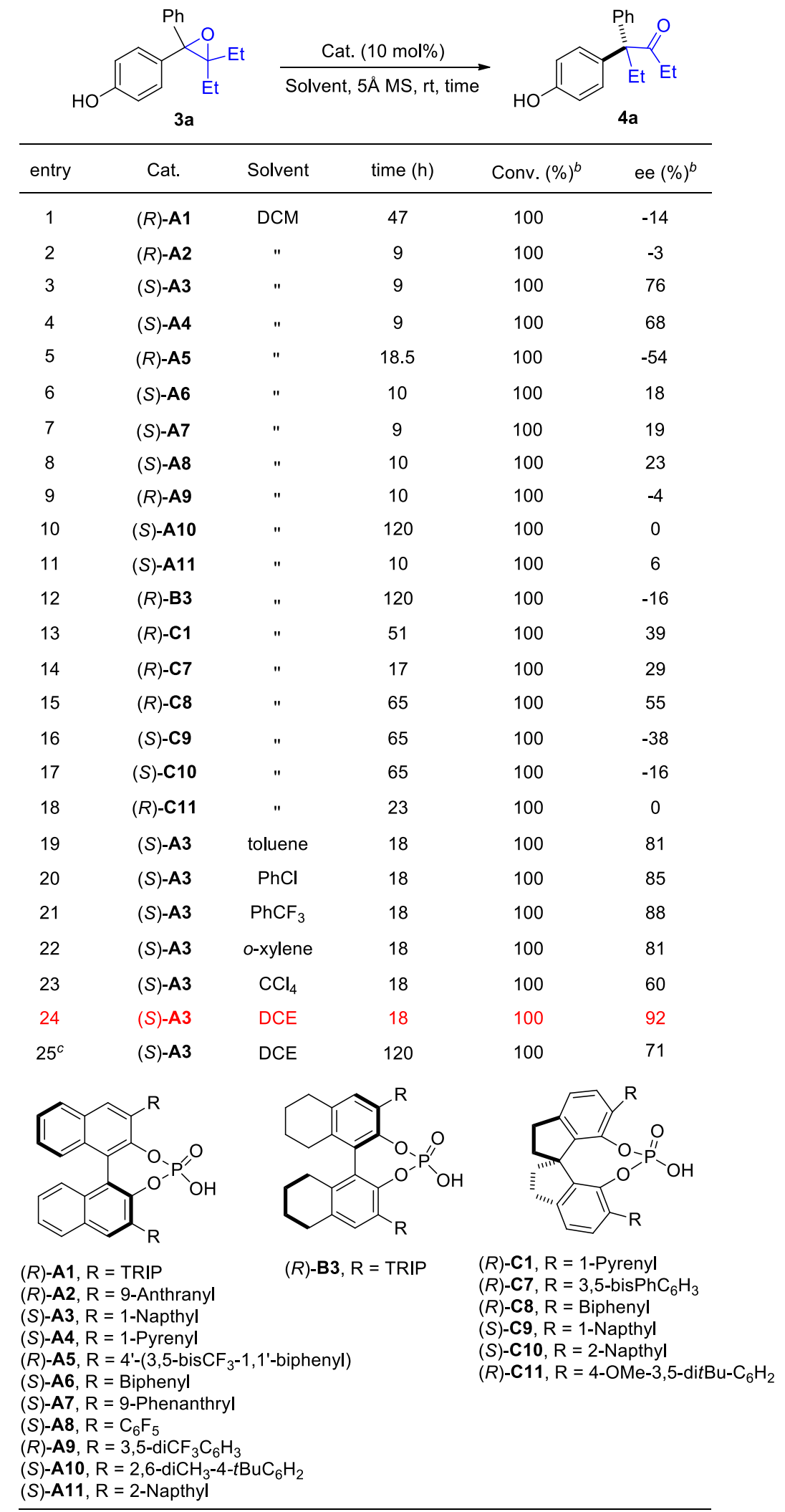

a Reaction condition: 3a (0.05 mmol), cat. (10 mol\%), $5 \AA$ MS $(20 \mathrm{mg})$, and solvent $(1 \mathrm{~mL})$ at rt. ${ }^{b}$ The conversion was determined by NMR using $\mathrm{CH}_{2} \mathrm{Br}_{2}$ as an internal standard. The ee value was determined by chiral HPLC with a chiral stationary phase. ${ }^{c}$ Run with $5 \%$ mol of cat. 


\section{Substrate Preparation}

\section{General Procedure A: General Scheme.}

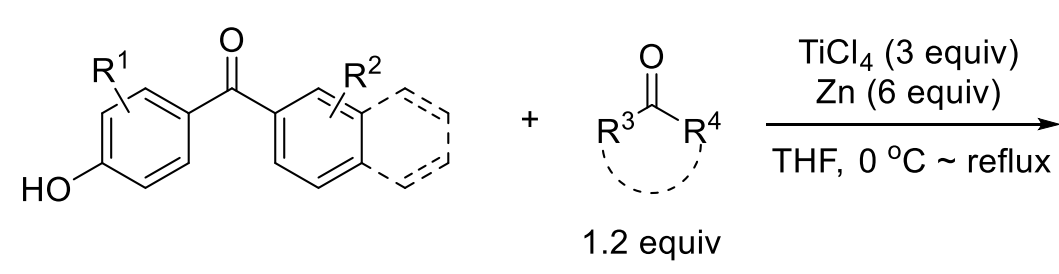<smiles>[R][R]1cc(O)ccc1C(=C1[R]=CCCN1)c1ccc2ccccc2c1</smiles>

S1

The alkenes were prepared using the McMurry reaction according to the literature procedure. ${ }^{1}$ The following general procedure is described with S1a as an example.

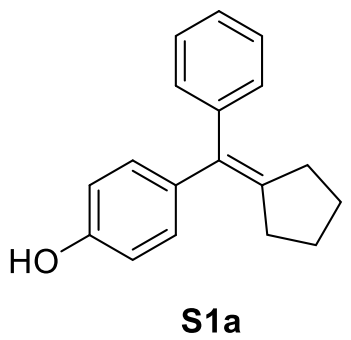

4-(Cyclopentylidene(phenyl)methyl)phenol (S1a). Under $\mathrm{N}_{2}$ at $0{ }^{\circ} \mathrm{C}$, to a mixture of zinc powder (7.85 g, $120 \mathrm{mmol}, 6$ equiv) in anhydrous THF (200 mL) was slowly added $\mathrm{TiCl}_{4}(11.38 \mathrm{~g}, 60 \mathrm{mmol}, 3$ equiv). After addition, the mixture was heated to reflux at $75{ }^{\circ} \mathrm{C}$ for $1 \mathrm{~h}$ before it was cooled to room temperature. Next, a solution of 4-hydroxybenzophenone (3.96 g, $20 \mathrm{mmol})$ and cyclopentanone ( $2.02 \mathrm{~g}, 24 \mathrm{mmol}, 1.2$ equiv) in anhydrous THF (40 mL) was added. The mixture was then heated to reflux at $75{ }^{\circ} \mathrm{C}$ again. The reaction progress was monitored by TLC. Upon completion $(\sim 2 \mathrm{~h})$, the reaction mixture was cooled to room temperature. Next, an aqueous solution of $\mathrm{K}_{2} \mathrm{CO}_{3}(10 \%)$ was slowly added to quench the reaction. The layers were separated, and the aqueous layer was extracted with ethyl acetate $(100 \mathrm{~mL} \times 3)$. The combined organic layers were washed with brine $(40 \mathrm{~mL})$, dried over $\mathrm{Na}_{2} \mathrm{SO}_{4}$, and concentrated. 
The residue was purified by silica gel flash column chromatography (eluent: hexanes/ethyl acetate $=15: 1)$ to afford S1a as a white solid ( $4.79 \mathrm{~g}$, 96\% yield).

${ }^{1} \mathrm{H}$ NMR $\left(400 \mathrm{MHz}, \mathrm{CDCl}_{3}\right) \delta$ 7.29-7.21 (m, 2H), 7.20-7.10 (m, 3H), $7.03(\mathrm{~d}, J=8.5 \mathrm{~Hz}$, $2 \mathrm{H}), 6.71(\mathrm{~d}, J=8.5 \mathrm{~Hz}, 2 \mathrm{H}), 5.74(\mathrm{~s}, 1 \mathrm{H}), 2.48-2.23(\mathrm{~m}, 4 \mathrm{H}), 1.73-1.54(\mathrm{~m}, 4 \mathrm{H}) \mathrm{ppm}$.

${ }^{13} \mathrm{C}$ NMR (100 MHz, $\left.\mathrm{CDCl}_{3}\right) \delta 153.2,143.7,142.8,136.2,132.2,130.4,129.1,127.9,125.9$, $114.8,33.17,33.15,26.83,26.81 \mathrm{ppm}$.

IR (ATR) 3345, 2944, 2868, 2821, 1604, 1590, 1507, 1492, 1449, 1440, 1427, 1275, 1259, $1226,1169,835 \mathrm{~cm}^{-1}$.

HRMS (CI+) Calcd for $\mathrm{C}_{18} \mathrm{H}_{18} \mathrm{O}\left[\mathrm{M}^{+}\right]$: 250.1358, Found: 250.1353.

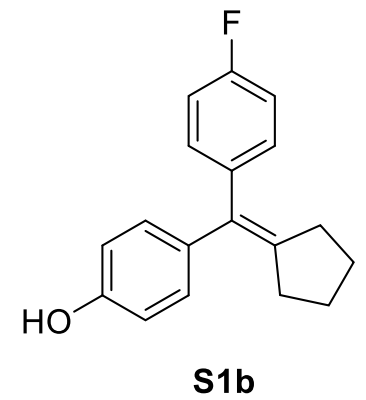

4-(Cyclopentylidene(4-fluorophenyl)methyl)phenol (S1b) was prepared as a white

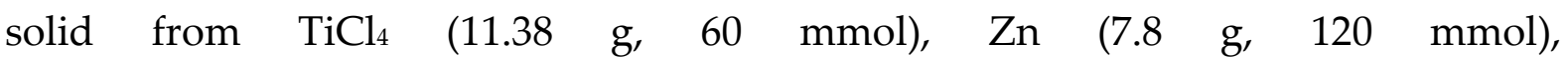
(4-fluorophenyl)(4-hydroxyphenyl)methanone $\quad\left(\begin{array}{llll}4.32 & \mathrm{~g}, & 20 & \mathrm{mmol}), \quad \text { and }\end{array}\right.$ cyclopentanone $(1.85 \mathrm{~g}, 22 \mathrm{mmol})$ in THF $(120 \mathrm{~mL})$ according to the General Procedure A $(2 \mathrm{~h}$, purified by flash column chromatography: hexanes/ethyl acetate $=$ $20: 1,4.39 \mathrm{~g}, 82 \%$ yield).

${ }^{1} \mathrm{H}$ NMR $\left(400 \mathrm{MHz}, \mathrm{CDCl}_{3}\right) \delta$ 7.21-7.11 (m, 2H), $7.07(\mathrm{~d}, J=8.6 \mathrm{~Hz}, 2 \mathrm{H}), 7.03-6.94(\mathrm{~m}$, $2 \mathrm{H}), 6.79(\mathrm{~d}, J=8.6 \mathrm{~Hz}, 2 \mathrm{H}), 5.10(\mathrm{brs}, 1 \mathrm{H}), 2.48-2.30(\mathrm{~m}, 4 \mathrm{H}), 1.79-1.61(\mathrm{~m}, 4 \mathrm{H}) \mathrm{ppm}$. ${ }^{13} \mathrm{C}$ NMR (100 MHz, $\left.\mathrm{CDCl}_{3}\right) \delta 161.1(\mathrm{~d}, J=243.3 \mathrm{~Hz}), 153.5,143.1,139.6(\mathrm{~d}, J=3.5 \mathrm{~Hz})$, 136.1, 131.3, 130.7 (d, J = 7.8 Hz), 130.4, 114.8, 114.7 (d, J = 20.8 Hz), $33.2(2 \mathrm{C}), 26.9$, $26.8 \mathrm{ppm}$.

${ }^{19}$ F NMR $\left(376 \mathrm{MHz}, \mathrm{CDCl}_{3}\right) \delta-116.8 \mathrm{ppm}$. 
IR (ATR) 3337, 2944, 2868, 1601, 1589, 1504, 1449, 1429, 1223, 1156, 1099, 1013, 835 $\mathrm{cm}^{-1}$.

HRMS (CI+) Calcd for $\mathrm{C}_{18} \mathrm{H}_{17} \mathrm{FO}\left[\mathrm{M}^{+}\right]$: 268.1263, Found: 268.1256.

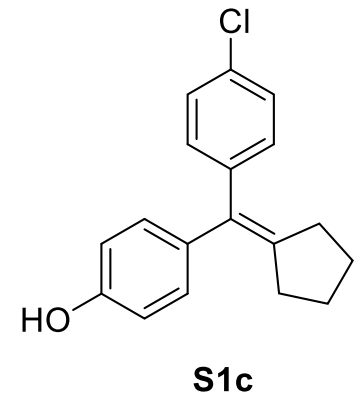

4-((4-Chlorophenyl)(cyclopentylidene)methyl)phenol (S1c) was prepared as a white solid from $\mathrm{TiCl}_{4}(11.38 \mathrm{~g}, 60 \mathrm{mmol}), \mathrm{Zn} \quad(7.8 \mathrm{~g}$, $120 \mathrm{mmol})$, (4-chlorophenyl)(4-hydroxyphenyl)methanone $\quad\left(\begin{array}{llll}4.64 & \mathrm{~g}, & 20 \mathrm{mmol}) & \text { and }\end{array}\right.$ cyclopentanone $(1.85 \mathrm{~g}, 22 \mathrm{mmol})$ in THF $(120 \mathrm{~mL})$ according to the General Procedure A ( $2 \mathrm{~h}$, purified by flash column chromatography: hexanes/ethyl acetate = $20: 1,4.21 \mathrm{~g}, 74 \%$ yield).

${ }^{1} \mathrm{H}$ NMR $\left(400 \mathrm{MHz}, \mathrm{CDCl}_{3}\right) \delta 7.27(\mathrm{~d}, J=8.4 \mathrm{~Hz}, 2 \mathrm{H}), 7.13(\mathrm{~d}, J=8.4 \mathrm{~Hz}, 2 \mathrm{H}), 7.06(\mathrm{~d}, J$ $=8.5 \mathrm{~Hz}, 2 \mathrm{H}), 6.79(\mathrm{~d}, J=8.6 \mathrm{~Hz}, 2 \mathrm{H}), 5.45(\mathrm{~s}, 1 \mathrm{H}), 2.47-2.31(\mathrm{~m}, 4 \mathrm{H}), 1.76-1.62(\mathrm{~m}, 4 \mathrm{H})$ ppm.

${ }^{13} \mathrm{C}$ NMR $\left(100 \mathrm{MHz} \mathrm{CDCl}_{3}\right) \delta 153.6,143.6,142.0,135.7,131.5,131.1,130.5,130.4,128.0$, $114.9,33.3,33.2,26.84,26.81 \mathrm{ppm}$.

IR (ATR) 3326, 2955, 2939, 2865, 1604, 1589, 1507, 1487, 1448, 1427, 1226, 1168, 1091, $1012,832 \mathrm{~cm}^{-1}$.

HRMS (CI+) Calcd for $\mathrm{C}_{18} \mathrm{H}_{17} \mathrm{ClO}\left[\mathrm{M}^{+}\right]$: 284.0968, Found: 284.0964 . 
<smiles>Oc1ccc(C(=C2CCCC2)c2ccc(Br)cc2)cc1</smiles>

4-((4-Bromophenyl)(cyclopentylidene)methyl)phenol (S1d) was prepared as a white solid from $\mathrm{TiCl}_{4}(11.38 \mathrm{~g}$, $60 \mathrm{mmol})$, $\mathrm{Zn}(7.85 \mathrm{~g}, 120 \mathrm{mmol})$, (4-bromophenyl)(4-hydroxyphenyl)methanone $\quad(5.54 \quad \mathrm{~g}, \quad 20 \mathrm{mmol})$ and cyclopentanone $(2.02 \mathrm{~g}, 24 \mathrm{mmol})$ in THF $(240 \mathrm{~mL})$ according to the General Procedure A ( $1 \mathrm{~h}$, purified by flash column chromatography: hexanes/ethyl acetate = $15: 1,4.72 \mathrm{~g}, 72 \%$ yield).

${ }^{1}$ H NMR (400 MHz, $\left.\mathrm{CDCl}_{3}\right) \delta$ 7.44-7.34 (m, 2H), 7.08-6.99 (m, 4H), 6.80-6.70 (m, 2H), $4.89(\mathrm{~s}, 1 \mathrm{H}), 2.46-2.24(\mathrm{~m}, 4 \mathrm{H}), 1.71-1.62(\mathrm{~m}, 4 \mathrm{H}) \mathrm{ppm}$.

${ }^{13} \mathrm{C}$ NMR $\left(100 \mathrm{MHz}, \mathrm{CDCl}_{3}\right) \delta 153.8,143.6,142.6,135.6,131.2,131.0,130.9,130.4,119.8$, $114.9,33.3,33.2,26.9,26.8$ ppm.

IR (ATR) 3277, 2952, 2865, 1609, 1588, 1510, 1483, 1448, 1254, 1227, 1045, 1009, 829 $\mathrm{cm}^{-1}$.

HRMS (CI+) Calcd for $\mathrm{C}_{18} \mathrm{H}_{17} \mathrm{BrO}\left[\mathrm{M}^{+}\right]$: 328.0463, Found: 328.0462 .<smiles>Oc1ccc(C(=C2CCCC2)c2ccc(-c3ccccc3)cc2)cc1</smiles>

4-([1,1'-Biphenyl]-4-yl(cyclopentylidene)methyl)phenol (S1e) was prepared as a white solid from $\mathrm{TiCl}_{4}(2.28 \mathrm{~g}, 12 \mathrm{mmol}), \mathrm{Zn} \quad(1.57 \mathrm{~g}$, $24 \mathrm{mmol})$, [1,1'-biphenyl]-4-yl(4-hydroxyphenyl)methanone $\quad(1.10 \quad \mathrm{~g}, \quad 4 \quad \mathrm{mmol})$ and cyclopentanone $(0.40 \mathrm{~g}, 4.8 \mathrm{mmol})$ in THF $(60 \mathrm{~mL})$ according to the General 
Procedure A (13 h, purified by flash column chromatography: hexanes/ethyl acetate $=15: 1,0.87 \mathrm{~g}, 66 \%$ yield $)$.

${ }^{1} \mathrm{H}$ NMR $\left(400 \mathrm{MHz}, \mathrm{CDCl}_{3}\right) \delta$ 7.67-7.55 (m, 2H), $7.51(\mathrm{~d}, J=7.1 \mathrm{~Hz}, 2 \mathrm{H}), 7.45-7.35(\mathrm{~m}$, 2H), 7.35-7.27 (m, 1H), $7.24(\mathrm{~d}, J=7.3 \mathrm{~Hz}, 2 \mathrm{H}), 7.08(\mathrm{~d}, J=7.3 \mathrm{~Hz}, 2 \mathrm{H}), 6.76(\mathrm{~d}, J=7.4$ $\mathrm{Hz}, 2 \mathrm{H}), 4.92$ (brs, 1H), 2.54-2.26 (m, 4H), 1.80-1.48 (m, 4H) ppm.

${ }^{13} \mathrm{C}$ NMR $\left(100 \mathrm{MHz}, \mathrm{CDCl}_{3}\right) \delta 153.6,143.2,142.7,140.9,138.5,136.2,131.9,130.5,129.6$, $128.7,127.0,126.9,126.5,114.8,33.4,33.3,27.0,26.8$ ppm.

IR (ATR) 3270, 2945, 2863, 2829, 1609, 1509, 1486, 1431, 1401, 1227, 1171, 1045, 836 $\mathrm{cm}^{-1}$.

HRMS (CI+) Calcd for $\mathrm{C}_{24} \mathrm{H}_{22} \mathrm{O}\left[\mathrm{M}^{+}\right]$: 326.1671, Found: 326.1673.<smiles>Oc1ccc(C(=C2CCCC2)c2ccc(O)cc2)cc1</smiles>

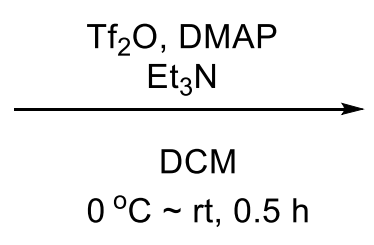

\section{4-(Cyclopentylidene(4-hydroxyphenyl)methyl)phenyl trifluoromethanesulfonate}

(S1f). Under $\mathrm{N}_{2}$ at $0 \quad{ }^{\circ} \mathrm{C}$, to an oven-dried flask charged with 4,4'-(cyclopentylidenemethylene)diphenol ${ }^{1}$ (1.07 g, $4 \mathrm{mmol}$ ), DMAP (24.3 mg, 0.2 $\mathrm{mmol})$, and anhydrous DCM (30 mL) were sequentially added $\mathrm{Et}_{3} \mathrm{~N}(0.40 \mathrm{~g}, 4 \mathrm{mmol})$ and $\mathrm{Tf}_{2} \mathrm{O}(1.15 \mathrm{~g}, 4 \mathrm{mmol})$. The reaction was then stirred at room temperature, and the reaction progress was monitored by TLC. Upon completion $(\sim 0.5 \mathrm{~h}), \mathrm{H}_{2} \mathrm{O}(20 \mathrm{~mL})$ was slowly added to quench the reaction. The layers were separated, and the aqueous layer was extracted with DCM $(20 \mathrm{~mL} \times 3)$. The combined organic layers were washed with brine $(20 \mathrm{~mL})$, dried over $\mathrm{Na}_{2} \mathrm{SO}_{4}$, and concentrated. The residue was purified by silica gel flash column chromatography (eluent: hexanes/ethyl acetate $=20: 1$ to $10: 1)$ to afford S1f $(0.44 \mathrm{~g}, 28 \%$ yield $)$ as a colorless oil. 
${ }^{1} \mathrm{H}$ NMR $\left(400 \mathrm{MHz}, \mathrm{CDCl}_{3}\right) \delta 7.24(\mathrm{~d}, J=8.7 \mathrm{~Hz}, 2 \mathrm{H}), 7.17(\mathrm{~d}, J=8.8 \mathrm{~Hz}, 2 \mathrm{H}), 7.02(\mathrm{~d}, J$ $=8.6 \mathrm{~Hz}, 2 \mathrm{H}), 6.77(\mathrm{~d}, J=8.6 \mathrm{~Hz}, 2 \mathrm{H}), 5.11$ (brs, $1 \mathrm{H}), 2.46-2.25(\mathrm{~m}, 4 \mathrm{H}), 1.76-1.60(\mathrm{~m}$, 4H) ppm.

${ }^{13} \mathrm{C}$ NMR $\left(100 \mathrm{MHz} \mathrm{CDCl}_{3}\right) \delta 153.9,147.5,144.6,144.0,135.3,130.9,130.7,130.4,120.7$, $118.7(\mathrm{q}, J=318.9 \mathrm{~Hz}), 115.0,33.4,33.3,26.9,26.7 \mathrm{ppm}$.

${ }^{19}$ F NMR (376 $\left.\mathrm{MHz}_{2} \mathrm{CDCl}_{3}\right) \delta-72.9 \mathrm{ppm}$.

IR (thin film) 3378, 2956, 2869, 1611, 1591, 1511, 1497, 1424, 1250, 1214, 1140, 890, 839 $\mathrm{cm}^{-1}$.

HRMS (CI+) Calcd for $\mathrm{C}_{19} \mathrm{H}_{17} \mathrm{~F}_{3} \mathrm{O}_{4} \mathrm{~S}$ [M+1: 398.0800, Found: 398.0812 .<smiles>Oc1ccc(C(=C2CCCC2)c2ccc(O)cc2)cc1</smiles>
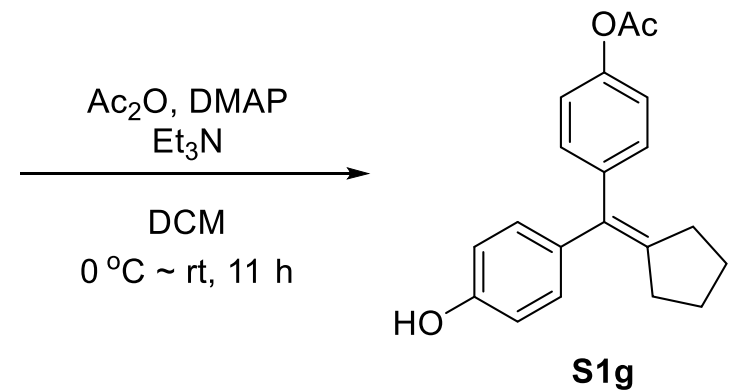

4-(Cyclopentylidene(4-hydroxyphenyl)methyl)phenyl acetate (S1g). Under $\mathrm{N}_{2}$ at 0 ${ }^{\circ} \mathrm{C}$, to an oven-dried flask were added 4,4'-(cyclopentylidenemethylene)diphenol ${ }^{1}$ (1.07 g, $4 \mathrm{mmol})$, DMAP (24.4 mg, $0.2 \mathrm{mmol})$, and anhydrous DCM (30 mL) atmosphere. Then the reaction mixture was cooled to $0{ }^{\circ} \mathrm{C}$ using an ice water bath, after which $\mathrm{Et}_{3} \mathrm{~N}(0.40 \mathrm{~g}, 4 \mathrm{mmol})$ and $\mathrm{Ac}_{2} \mathrm{O}(0.41 \mathrm{~g}, 4 \mathrm{mmol})$ were added sequentially. After that, the reaction was warmed up to $\mathrm{rt}$. The reaction progress was monitored by TLC. Upon completion $(11 \mathrm{~h}), \mathrm{H}_{2} \mathrm{O}(20 \mathrm{~mL})$ was added to quench the reaction slowly, the layers were separated, and the aqueous layer was extracted with DCM (20 mL×3). The combined organic layers were washed with brine $(20 \mathrm{~mL})$, dried over $\mathrm{Na}_{2} \mathrm{SO}_{4}$, and concentrated. The residue was purified by silica gel flash column chromatography (eluent: hexanes/ethyl acetate $=15: 1$ to 8:1) to afford S1g (0.37 g, $30 \%$ yield) as a white oil.

${ }^{1} \mathbf{H}$ NMR (400 MHz, $\left.\mathrm{CDCl}_{3}\right) \delta$ 7.20-7.12 (m, 2H), 7.06-6.94 (m, 4H), 6.76-6.68 (m, 2H), $5.17(\mathrm{~s}, 1 \mathrm{H}), 2.44-2.33(\mathrm{~m}, 4 \mathrm{H}), 2.29(\mathrm{~s}, 3 \mathrm{H}), 1.71-1.60(\mathrm{~m}, 4 \mathrm{H}) \mathrm{ppm}$. 
${ }^{13} \mathrm{C}$ NMR $\left(100 \mathrm{MHz}, \mathrm{CDCl}_{3}\right) \delta 169.8,153.8,148.6,143.3,141.3,135.8,131.5,130.4,130.1$, $120.8,114.8,33.3,33.2,26.9,26.8,21.2 \mathrm{ppm}$.

IR (thin film) 3429, 2953, 2867, 1738, 1611, 1511, 1432, 1370, 1231, 1198, 1165, 1017, 913 , $836 \mathrm{~cm}^{-1}$.

HRMS (CI+) Calcd for $\mathrm{C}_{20} \mathrm{H}_{20} \mathrm{O}_{3}\left[\mathrm{M}^{+}\right]$: 308.1412, Found: 308.1415.

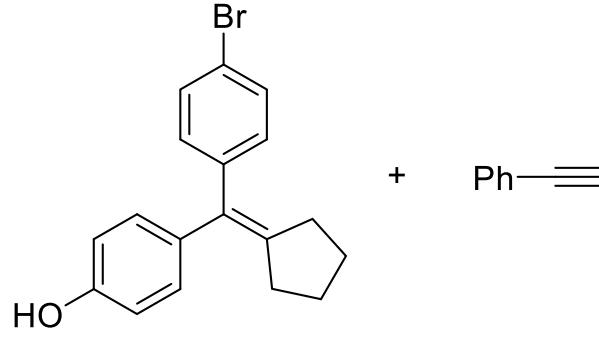

S1d

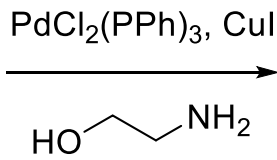

THF, $60^{\circ} \mathrm{C}, 27 \mathrm{~h}$

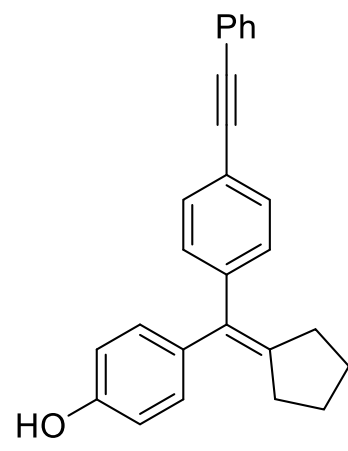

S1h

4-(Cyclopentylidene(4-(phenylethynyl)phenyl)methyl)phenol (S1h) ${ }^{2}$ Under $\mathrm{N}_{2}$, to an oven-dried flask charged with S1d $(0.99 \mathrm{~g}, 3 \mathrm{mmol}), \mathrm{PdCl}_{2}\left(\mathrm{PPh}_{3}\right)_{2}(63.0 \mathrm{mg}, 90$ $\mu \mathrm{mol})$, CuI (11.5 mg, $60 \mu \mathrm{mol})$, and THF $(20 \mathrm{~mL})$ were sequentially added phenylacetylene $(0.46 \mathrm{~g}, 4.5 \mathrm{mmol})$ and a solution of 2 -aminoethanol $(0.37 \mathrm{~g}, 6 \mathrm{mmol})$ in $\mathrm{H}_{2} \mathrm{O}$. The reaction mixture was stirred at $60{ }^{\circ} \mathrm{C}$, and the reaction progress was monitored by TLC. Upon completion $(\sim 27 \mathrm{~h})$, the mixture was extracted with ethyl acetate $(20 \mathrm{~mL} \times 3)$. The combined organic layers were washed with brine $(20 \mathrm{~mL})$, dried over $\mathrm{Na}_{2} \mathrm{SO}_{4}$, and concentrated. The residue was purified by silica gel flash column chromatography (eluent: hexanes/ethyl acetate $=20: 1$ to $10: 1$ ) to afford S1h (0.50 $\mathrm{g}, 47 \%$ yield) as a yellow solid.

${ }^{1} \mathbf{H}$ NMR $\left(400 \mathrm{MHz}, \mathrm{CDCl}_{3}\right) \delta$ 7.56-7.48 (m, 2H), $7.44(\mathrm{~d}, J=8.2 \mathrm{~Hz}, 2 \mathrm{H}), 7.36-7.26$ (m, $3 \mathrm{H}), 7.15(\mathrm{~d}, J=8.2 \mathrm{~Hz}, 2 \mathrm{H}), 7.04(\mathrm{~d}, J=8.6 \mathrm{~Hz}, 2 \mathrm{H}), 6.75(\mathrm{~d}, J=8.5 \mathrm{~Hz}, 2 \mathrm{H}), 5.06(\mathrm{~s}$, 1H), 2.48-2.26 (m, 4H), 1.74-1.56 (m, 4H) ppm.

${ }^{13} \mathrm{C}$ NMR $\left(100 \mathrm{MHz} \mathrm{CDCl}_{3}\right) \delta 153.7,143.78,143.77,135.8,131.8,131.5,131.2,130.5$, $129.2,128.3,128.1,123.4,120.6,114.9,89.6,89.2,33.4,33.3,26.9,26.8$ ppm. 
IR (thin film) 3321, 2975, 2953, 2867, 2216, 1611, 1596, 1511, 1442, 1264, 1226, 1170, $1101,836 \mathrm{~cm}^{-1}$.

HRMS (CI+) Calcd for $\mathrm{C}_{26} \mathrm{H}_{22} \mathrm{O}\left[\mathrm{M}^{+}\right]$: 350.1671, Found: 350.1682 .

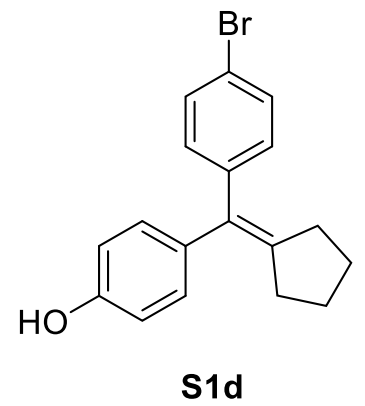

(1) $n$ BuLi (2.1 equiv), THF, $-78^{\circ} \mathrm{C}$

(2) DMF (5 equiv), $-78^{\circ} \mathrm{C} \sim \mathrm{rt}, 5 \mathrm{~h}$

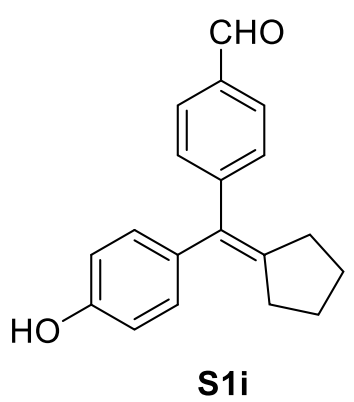

4-(Cyclopentylidene(4-hydroxyphenyl)methyl)benzaldehyde (S1i). At $-78^{\circ} \mathrm{C}$, to an oven-dried flask charged with S1d $(0.99 \mathrm{~g}, 3 \mathrm{mmol})$ and anhydrous THF (12 mL) was slowly added $n$-BuLi (6.3 mmol, 2.6 mL, 2.4 $\mathrm{M}$ in $n$-hexane) over $5 \mathrm{~min}$. The mixture was then stirred at $-78{ }^{\circ} \mathrm{C}$ for $30 \mathrm{~min}$ before anhydrous DMF $(1.10 \mathrm{~g}, 15 \mathrm{mmol})$ was added. Next, the reaction was stirred at room temperature. The reaction progress was monitored by TLC. Upon completion $(\sim 5 \mathrm{~h})$, a saturated aqueous solution of $\mathrm{NH}_{4} \mathrm{Cl}(10 \mathrm{~mL})$ was added slowly. The layers were separated, and the aqueous layer was extracted with ethyl acetate $(20 \mathrm{~mL} \times 3)$. The combined organic layers were washed with brine $(20 \mathrm{~mL})$, dried over $\mathrm{Na}_{2} \mathrm{SO}_{4}$, and concentrated. The residue was purified by silica gel flash column chromatography (eluent: hexanes/ethyl acetate = $15: 1$ to $8: 1)$ to afford $\mathrm{S} 1 \mathrm{i}(0.39 \mathrm{~g}, 46 \%$ yield) as a white solid.

${ }^{1} \mathrm{H}$ NMR (400 MHz, $\left.\mathrm{CDCl}_{3}\right) \delta 9.95(\mathrm{~s}, 1 \mathrm{H}), 7.80(\mathrm{~d}, J=8.3 \mathrm{~Hz}, 2 \mathrm{H}), 7.34(\mathrm{~d}, J=8.2 \mathrm{~Hz}$, 2H), 7.08-6.96 (m, 2H), 6.86-7.76 (m, 2H), $6.10(\mathrm{~s}, 1 \mathrm{H}), 2.46-2.30(\mathrm{~m}, 4 \mathrm{H}), 1.75-1.62(\mathrm{~m}$, 4H) ppm.

${ }^{13} \mathrm{C}$ NMR $\left(100 \mathrm{MHz}, \mathrm{CDCl}_{3}\right) \delta 192.6,154.3,150.5,145.4,134.9,133.9,131.6,130.5,129.8$, $129.6,115.1,33.5,33.3,26.9,26.6 \mathrm{ppm}$.

IR (ATR) 3345, 2951, 2865, 2832, 1686, 1597, 1562, 1509, 1263, 1211, 1168, $831 \mathrm{~cm}^{-1}$. HRMS (CI+) Calcd for $\mathrm{C}_{19} \mathrm{H}_{18} \mathrm{O}_{2}\left[\mathrm{M}^{+}\right]$: 278.1307, Found: 278.1315. 
<smiles>Oc1ccc(C(=C2CCCC2)c2ccccc2F)cc1</smiles>

S1j

4-(Cyclopentylidene(2-fluorophenyl)methyl)phenol (S1j) was prepared as a colorless oil from $\mathrm{TiCl}_{4}(1.42 \mathrm{~g}, 7.5 \mathrm{mmol}), \mathrm{Zn} \quad(0.98 \mathrm{~g}$, $15 \mathrm{mmol})$, (2-fluorophenyl)(4-hydroxyphenyl)methanone $\quad(0.54 \quad \mathrm{~g}, \quad 2.5 \mathrm{mmol})$ and cyclopentanone $(0.25 \mathrm{~g}, 3 \mathrm{mmol})$ in THF $(35 \mathrm{~mL})$ according to the General Procedure A ( $2 \mathrm{~h}$, purified by flash column chromatography: hexanes/ethyl acetate $=15: 1,0.67 \mathrm{~g}$, $99 \%$ yield).

${ }^{1} \mathbf{H}$ NMR $\left(400 \mathrm{MHz} \mathrm{CDCl}_{3}\right) \delta$ 7.24-7.17 (m, 1H), 7.17-7.11 (m, 1H), 7.11-7.00 (m, 4H), 6.77-6.69 (m, 2H), 4.89 (brs, 1H), $2.48(\mathrm{t}, J=6.9 \mathrm{~Hz}, 2 \mathrm{H}), 2.18(\mathrm{t}, J=7.0 \mathrm{~Hz}, 2 \mathrm{H})$, 1.77-1.57 (m, 4H) ppm.

${ }^{13} \mathrm{C}$ NMR $\left(100 \mathrm{MHz}, \mathrm{CDCl}_{3}\right) \delta 159.6(\mathrm{~d}, J=243.5 \mathrm{~Hz}), 153.6,145.2,135.0,131.5(\mathrm{~d}, J=$ $4.1 \mathrm{~Hz}), 131.1(\mathrm{~d}, J=17.4 \mathrm{~Hz}), 129.9,128.2(\mathrm{~d}, J=8.0 \mathrm{~Hz}), 125.9,123.9(\mathrm{~d}, J=3.3 \mathrm{~Hz})$, $115.7(\mathrm{~d}, J=22.6 \mathrm{~Hz}), 114.7,32.9(\mathrm{~d}, J=1.7 \mathrm{~Hz}), 32.8,27.1,26.3 \mathrm{ppm}$.

${ }^{19} \mathbf{F}$ NMR $\left(376 \mathrm{MHz}, \mathrm{CDCl}_{3}\right) \delta-115.0 \mathrm{ppm}$.

IR (thin film) 3355, 2954, 2867, 1610, 1510, 1487, 1449, 1257, 1220, 1273, 1101, 835, 757 $\mathrm{cm}^{-1}$.

HRMS (CI+) Calcd for $\mathrm{C}_{18} \mathrm{H}_{17} \mathrm{FO}\left[\mathrm{M}^{+}\right]$: 268.1263, Found: 268.1271 .

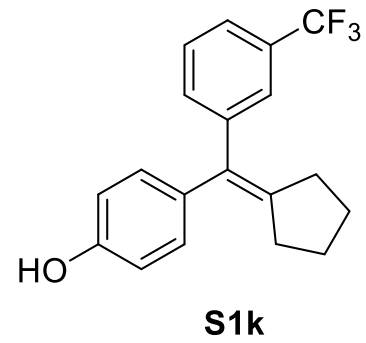

4-(Cyclopentylidene(3-(trifluoromethyl)phenyl)methyl)phenol (S1k) was prepared as a light yellow oil from $\mathrm{TiCl}_{4}(1.31 \mathrm{~g}, 6.9 \mathrm{mmol}), \mathrm{Zn}(0.90 \mathrm{~g}, 13.8 \mathrm{mmol})$, (4-hydroxyphenyl)(3-(trifluoromethyl)phenyl)methanone (0.61 g, $2.3 \mathrm{mmol}$ ) and 
cyclopentanone $(0.23 \mathrm{~g}, 2.76 \mathrm{mmol})$ in THF $(30 \mathrm{~mL})$ according to the General Procedure A (11 h, purified by flash column chromatography: hexanes/ethyl acetate $=15: 1,0.70 \mathrm{~g}, 96 \%$ yield $)$.

${ }^{1}$ H NMR (400 MHz, $\left.\mathrm{CDCl}_{3}\right) \delta$ 7.50-7.41 (m, 2H), 7.41-7.28 (m, 2H), 7.09-6.99 (m, 2H), 6.82-6.72 (m, 2H), 5.18 (brs, $1 \mathrm{H}), 2.45-2.38(\mathrm{~m}, 2 \mathrm{H}), 2.38-2.29(\mathrm{~m}, 2 \mathrm{H}), 1.75-1.61(\mathrm{~m}, 4 \mathrm{H})$ ppm.

${ }^{13} \mathrm{C}$ NMR $\left(100 \mathrm{MHz}, \mathrm{CDCl}_{3}\right) \delta 153.8,144.5,144.4,135.4,132.5,131.2,130.5,130.3(\mathrm{q}, J=$ 31.7 Hz), 128.4, $125.8(q, J=3.7 \mathrm{~Hz}), 124.3(\mathrm{q}, J=270.7 \mathrm{~Hz}), 122.7(\mathrm{q}, J=3.8 \mathrm{~Hz}), 115.0$, $33.3,33.2,26.9,26.8 \mathrm{ppm}$.

${ }^{19}$ F NMR (376 MHz, $\left.\mathrm{CDCl}_{3}\right) \delta-62.4 \mathrm{ppm}$.

IR (thin film) 3422, 2957, 2869, 1610, 1510, 1432, 1337, 1310, 1262, 1165, 1125, 1073, 704 $\mathrm{cm}^{-1}$.

HRMS (CI+) Calcd for $\mathrm{C}_{19} \mathrm{H}_{17} \mathrm{~F}_{3} \mathrm{O}\left[\mathrm{M}^{+}\right]$: 318.1231, Found: 318.1234.

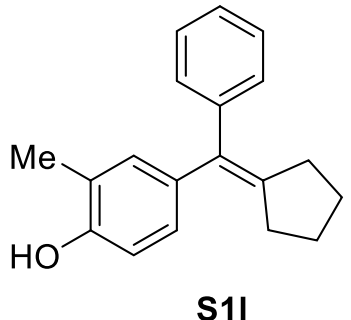

4-(Cyclopentylidene(phenyl)methyl)-2-methylphenol (S11) was prepared as a yellow oil from $\mathrm{TiCl}_{4}(1.25 \mathrm{~g}, 6.6 \mathrm{mmol}), \mathrm{Zn} \quad(0.86 \mathrm{~g}, 13.2 \mathrm{mmol})$, (4-hydroxy-3-methylphenyl)(phenyl)methanone $\quad\left(\begin{array}{lllll}0.47 & \mathrm{~g}, & 2.2 \mathrm{mmol}) & \text { and }\end{array}\right.$ cyclopentanone $(0.22 \mathrm{~g}, 2.64 \mathrm{mmol})$ in THF $(30 \mathrm{~mL})$ according to the General Procedure A $(1 \mathrm{~h}$, purified by flash column chromatography: hexanes/ethyl acetate = $15: 1,0.57 \mathrm{~g}, 97 \%$ yield).

${ }^{1} \mathbf{H}$ NMR $\left(400 \mathrm{MHz}, \mathrm{CDCl}_{3}\right) \delta$ 7.32-7.24 (m, 2H), 7.21-1.12 (m, 3H), 6.96-6.92 (m, 1H), $6.90\left(\mathrm{dd}, J_{1}=8.2 \mathrm{~Hz}, J_{2}=2.0 \mathrm{~Hz}, 1 \mathrm{H}\right), 6.67(\mathrm{~d}, J=8.1 \mathrm{~Hz}, 1 \mathrm{H}), 4.77(\mathrm{~s}, 1 \mathrm{H}), 2.45-2.28(\mathrm{~m}$, $4 \mathrm{H}), 2.18(\mathrm{~s}, 3 \mathrm{H}), 1.76-1.57(\mathrm{~m}, 4 \mathrm{H}) \mathrm{ppm}$. 
${ }^{13} \mathrm{C}$ NMR $\left(100 \mathrm{MHz}, \mathrm{CDCl}_{3}\right) \delta 151.9,143.8,142.6,136.1,132.5,131.7,129.1,127.91$, $127.86,125.8,123.0,114.4,33.2,33.1,26.9,26.8,15.8$ ppm.

IR (thin film) 3441, 2592, 2834, 1637, 1609, 1503, 1245, 1113, $701 \mathrm{~cm}^{-1}$.

HRMS (CI+) Calcd for $\mathrm{C}_{19} \mathrm{H}_{20} \mathrm{O}\left[\mathrm{M}^{+}\right]$: 264.1514, Found: 264.1510.

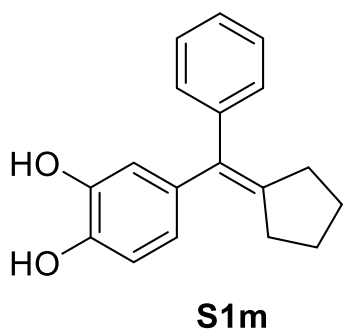

4-(Cyclopentylidene(phenyl)methyl)benzene-1,2-diol (S1m) was prepared as a white solid from $\mathrm{TiCl}_{4}(11.38 \mathrm{~g}, 60 \mathrm{mmol}), \mathrm{Zn} \quad(7.8 \mathrm{~g}, 120 \mathrm{mmol})$, (3,4-dihydroxyphenyl)(phenyl)methanone (4.28 g, $20 \mathrm{mmol})$ and cyclopentanone (2.02 $\mathrm{g}, 24 \mathrm{mmol})$ in THF $(150 \mathrm{~mL})$ according to the General Procedure A (2 $\mathrm{h}$, purified by flash column chromatography: hexanes/ethyl acetate $=10: 1,3.30 \mathrm{~g}$, 62\% yield).

${ }^{1} \mathrm{H}$ NMR (400 MHz, $\left.\mathrm{CDCl}_{3}\right) \delta$ 7.34-7.25 (m, 2H), 7.24-1.14 (m, 3H), $6.78(\mathrm{~d}, J=8.2 \mathrm{~Hz}$, $1 \mathrm{H}), 6.71(\mathrm{~d}, J=1.7 \mathrm{~Hz}, 1 \mathrm{H}), 6.67\left(\mathrm{dd}, J_{1}=8.1 \mathrm{~Hz}, J_{2}=1.8 \mathrm{~Hz}, 1 \mathrm{H}\right), 5.48$ (brs, 2H), 2.47-2.30 (m, 4H), 1.74-1.60 (m, 4H) ppm.

${ }^{13} \mathrm{C}$ NMR $\left(100 \mathrm{MHz} \mathrm{CDCl}_{3}\right) \delta 143.6,143.1,142.7,141.5,136.8,132.1,129.1,127.9,125.9$, $122.2,116.5,114.9,33.2(2 \mathrm{C}), 26.9,26.8 \mathrm{ppm}$.

IR (ATR) 3343, 2950, 2865, 1597, 1510, 1429, 1295, 1262, 1189, $1105 \mathrm{~cm}^{-1}$.

HRMS (CI+) Calcd for $\mathrm{C}_{18} \mathrm{H}_{18} \mathrm{O}_{2}\left[\mathrm{M}^{+}\right]$: 266.1307, Found: 266.1313 . 


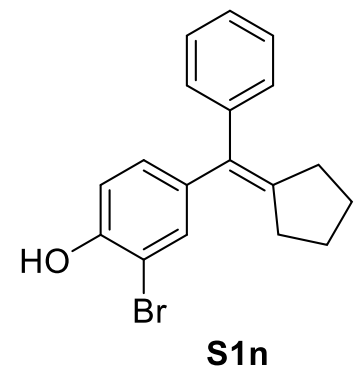

2-Bromo-4-(cyclopentylidene(phenyl)methyl)phenol (S1n) was prepared as a light yellow oil from $\mathrm{TiCl}_{4}(3.41 \mathrm{~g}, 18 \mathrm{mmol}), \mathrm{Zn} \quad(2.35 \mathrm{~g}, 36 \mathrm{mmol})$, (3-bromo-4-hydroxyphenyl)(phenyl)methanone $\left(\begin{array}{llll}1.66 & \mathrm{~g}\end{array} \quad 6 \mathrm{mmol}\right)$ and cyclopentanone $(0.61 \mathrm{~g}, 7.2 \mathrm{mmol})$ in THF $(80 \mathrm{~mL})$ according to the General Procedure A (11 h, purified by flash column chromatography: hexanes/ethyl acetate $=15: 1,1.67 \mathrm{~g}, 68 \%$ yield $)$.

${ }^{1} \mathbf{H}$ NMR $\left(400 \mathrm{MHz}, \mathrm{CDCl}_{3}\right) \delta$ 7.31-7.23 (m, 3H), 7.21-1.12 (m, 3H), $7.03\left(\mathrm{dd}, J_{1}=8.4 \mathrm{~Hz}\right.$, $\left.J_{2}=2.0 \mathrm{~Hz}, 1 \mathrm{H}\right), 6.92(\mathrm{~d}, J=8.4 \mathrm{~Hz}, 1 \mathrm{H}), 5.56(\mathrm{~s}, 1 \mathrm{H}), 2.45-2.26(\mathrm{~m}, 4 \mathrm{H}), 1.74-1.57(\mathrm{~m}$, 4H) ppm.

${ }^{13} \mathrm{C}$ NMR $\left(100 \mathrm{MHz}, \mathrm{CDCl}_{3}\right) \delta 150.3,144.0,143.0,137.4,132.4,131.2,130.0,129.1,128.0$, $126.1,115.4,109.7,33.21,33.18,26.82,26.81$ ppm.

IR (thin film) 3510, 2953, 2866, 1600, 1567, 1491, 1319, 1273, 1194, 1178, $1038 \mathrm{~cm}^{-1}$. HRMS (LD+) Calcd for $\mathrm{C}_{18} \mathrm{H}_{18} \mathrm{BrO}\left[\mathrm{M}^{+}+\mathrm{H}\right]: 329.0541$, Found: 329.0568 .

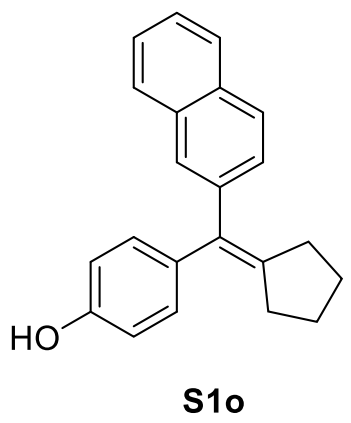

4-(Cyclopentylidene(naphthalen-2-yl)methyl)phenol (S1o) was prepared as a yellow oil from $\mathrm{TiCl}_{4}(3.41 \mathrm{~g}, 18 \mathrm{mmol}), \mathrm{Zn}(2.35 \mathrm{~g}, 36 \mathrm{mmol})$, (4-hydroxyphenyl)(naphthalen-2-yl)methanone $(1.49 \mathrm{~g}, 6 \mathrm{mmol})$ and cyclopentanone $(0.61 \mathrm{~g}, 7.2 \mathrm{mmol})$ in THF $(80 \mathrm{~mL})$ according to the General Procedure A $(2 \mathrm{~h}$, 
purified by flash column chromatography: hexanes/ethyl acetate $=15: 1,1.14 \mathrm{~g}, 64 \%$ yield).

${ }^{1} \mathrm{H}$ NMR $\left(400 \mathrm{MHz}, \mathrm{CDCl}_{3}\right) \delta$ 7.82-7.69 (m, 3H), $7.65(\mathrm{~s}, 1 \mathrm{H}), 7.48-7.35(\mathrm{~m}, 2 \mathrm{H}), 7.27$ $\left(\mathrm{dd}, J_{1}=8.4 \mathrm{~Hz}, J_{2}=1.6 \mathrm{~Hz}, 1 \mathrm{H}\right), 7.13-7.02(\mathrm{~m}, 2 \mathrm{H}), 6.78-6.67(\mathrm{~m}, 2 \mathrm{H}), 5.01(\mathrm{~s}, 1 \mathrm{H})$, 2.52-2.34 (m, 4H), 1.78-1.56 (m, 4H) ppm.

${ }^{13} \mathrm{C}$ NMR (100 MHz, $\left.\mathrm{CDCl}_{3}\right) \delta 153.6,143.4,141.3,136.0,133.3,132.2,131.9,130.6,127.9$, $127.8,127.7,127.5,127.4,125.8,125.4,114.8,33.3$ (2C), 27.0, 26.9 ppm.

IR (thin film) 3406, 2952, 2865, 1609, 1509, 1431, 1257, 1235, 1170, $746 \mathrm{~cm}^{-1}$.

HRMS (LD+) Calcd for $\mathrm{C}_{22} \mathrm{H}_{20} \mathrm{O}\left[\mathrm{M}^{+}\right]$: 300.1514, Found: 300.1517 .

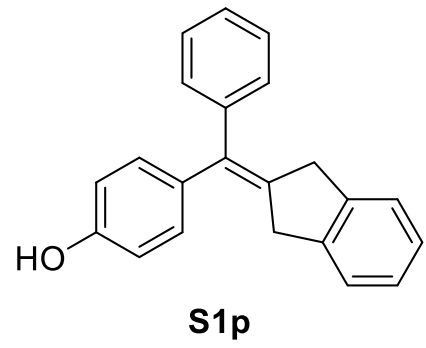

4-((1,3-Dihydro-2H-inden-2-ylidene)(phenyl)methyl)phenol (S1p) was prepared as a light yellow solid from $\mathrm{TiCl}_{4}(11.38 \mathrm{~g}, 60 \mathrm{mmol}), \mathrm{Zn}(7.8 \mathrm{~g}, 120 \mathrm{mmol})$, 4-hydroxybenzophenone (3.96 g, $20 \mathrm{mmol})$ and 1,3-dihydro-2H-inden-2-one (2.64 g, $20 \mathrm{mmol})$ in THF $(120 \mathrm{~mL})$ according to the General Procedure A $(2 \mathrm{~h}$, purified by flash column chromatography: hexanes/ethyl acetate $=20: 1,4.16 \mathrm{~g}$, $70 \%$ yield). ${ }^{1} \mathrm{H}$ NMR $\left(400 \mathrm{MHz}, \mathrm{CDCl}_{3}\right) \delta$ 7.33-7.23 (m, 2H), 7.23-7.15 (m, 3H), 7.15-7.03 (m, 6H), $6.73(\mathrm{~d}, J=8.6 \mathrm{~Hz}, 2 \mathrm{H}), 5.30$ (brs, $1 \mathrm{H}), 3.75(\mathrm{~s}, 2 \mathrm{H}), 3.71(\mathrm{~s}, 2 \mathrm{H}) \mathrm{ppm}$.

${ }^{13} \mathrm{C}$ NMR (100 MHz, $\left.\mathrm{CDCl}_{3}\right) \delta 153.8,142.9,141.9,141.8,138.1,135.3,135.2,130.2,128.9$, $128.3,126.4,126.3(2 \mathrm{C}), 124.3(2 \mathrm{C}), 115.1,39.0$ (2C) ppm.

IR (ATR) 3299, 3021, 2973, 1609, 1509, 1485, 1440, 1263, 1212, 1170, 1043, $830 \mathrm{~cm}^{-1}$.

HRMS (CI+) Calcd for $\mathrm{C}_{22} \mathrm{H}_{18} \mathrm{O}\left[\mathrm{M}^{+}\right]$: 298.1358, Found: 298.1356. 


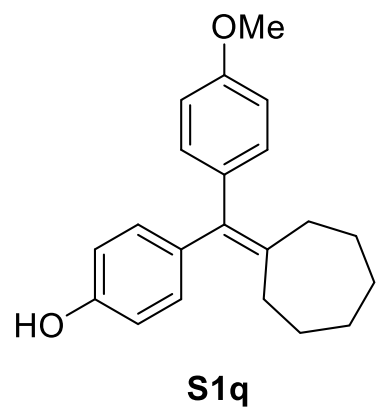

4-(Cycloheptylidene(4-methoxyphenyl)methyl)phenol (S1q) was prepared as a

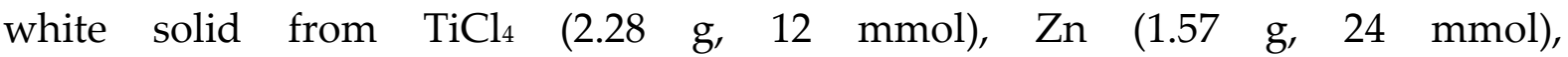
(4-hydroxyphenyl)(4-methoxyphenyl)methanone $\quad\left(\begin{array}{lllll}0.91 & \mathrm{~g}, & 4 & \mathrm{mmol})\end{array}\right.$ and cycloheptanone $(0.54 \mathrm{~g}, 4.8 \mathrm{mmol})$ in THF $(50 \mathrm{~mL})$ according to the General Procedure A $(2 \mathrm{~h}$, purified by flash column chromatography: hexanes/ethyl acetate $=$ 15:1 to $10: 1,0.93 \mathrm{~g}$, $76 \%$ yield).

${ }^{1} \mathrm{H}$ NMR $\left(400 \mathrm{MHz}, \mathrm{CDCl}_{3}\right)$ 8 7.09-7.03 (m, 2H), 7.03-6.96 (m, 2H), 6.84-6.77 (m, 2H), 6.75-6.68 (m, 2H), $4.97(\mathrm{~s}, 1 \mathrm{H}), 3.77(\mathrm{~s}, 3 \mathrm{H}), 2.38-2.22(\mathrm{~m}, 4 \mathrm{H}), 1.64-1.48(\mathrm{~m}, 8 \mathrm{H}) \mathrm{ppm}$.

${ }^{13} \mathrm{C}$ NMR (100 MHz, $\left.\mathrm{CDCl}_{3}\right) \delta 157.6,153.6,139.6,136.5,136.4,136.1,130.5,130.3,114.7$, $113.3,55.2,33.42,33.41,29.4(2 \mathrm{C}), 28.1(2 \mathrm{C}) \mathrm{ppm}$.

IR (thin film) 3423, 2922, 2851, 1606, 1508, 1242, 1173, 1105, 1035, $830 \mathrm{~cm}^{-1}$.

HRMS (CI+) Calcd for $\mathrm{C}_{21} \mathrm{H}_{24} \mathrm{O}_{2}\left[\mathrm{M}^{+}\right]$: 308.1776, Found: 308.1779.

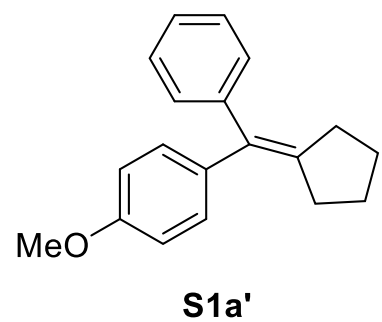

1-(cyclopentylidene(phenyl)methyl)-4-methoxybenzene (S1a') was prepared as a colorless oil from $\mathrm{TiCl}_{4}(11.38 \mathrm{~g}, 60 \mathrm{mmol}), \mathrm{Zn} \quad(7.85 \mathrm{~g}, 120 \mathrm{mmol})$, 4-methoxybezophenone ( $3.19 \mathrm{~g}, 15 \mathrm{mmol})$ and cyclopentanone $(1.51 \mathrm{~g}, 18 \mathrm{mmol})$ in THF $(120 \mathrm{~mL})$ according to the General Procedure A $(11 \mathrm{~h}$, purified by flash column chromatography: hexanes/ethyl acetate $=80: 1,3.20 \mathrm{~g}, 81 \%$ yield) . 
${ }^{1} \mathbf{H}$ NMR $\left(400 \mathrm{MHz} \mathrm{CDCl}_{3}\right) \delta$ 7.30-7.21 (m, 2H), 7.20-7.13 (m, 3H), 7.13-7.07 (m, 2H), 6.84-6.77 (m, 2H), 3.74 (s, 3H), 2.46-2.30 (m, 4H), 1.72-1.58 (m, 4H) ppm.

${ }^{13} \mathrm{C}$ NMR (100 MHz, $\left.\mathrm{CDCl}_{3}\right) \delta 157.6,143.7,142.6,135.9,132.4,130.2,129.1,127.8,125.8$, 113.2, 55.0, 33.19, 33.16, 26.9, 26.8 ppm.

This is a known compound. The characterization data match the literature data. ${ }^{3}$<smiles>CCC(CC)=C(c1ccccc1)c1ccc(O)cc1</smiles>

S3a

4-(2-Ethyl-1-phenylbut-1-en-1-yl)phenol (S3a) was prepared as a white solid from $\mathrm{TiCl}_{4}(15.17 \mathrm{~g}$, $80 \mathrm{mmol}), \mathrm{Zn}(10.46 \mathrm{~g}, 160 \mathrm{mmol})$, 4-hydroxybenzophenone (3.96 g, 20 $\mathrm{mmol})$ and 3-pentanone $(5.17 \mathrm{~g}, 60 \mathrm{mmol})$ in THF $(140 \mathrm{~mL})$ according to the General Procedure A (14.5 h, purified by flash column chromatography: hexanes/ethyl acetate $=20: 1,3.76 \mathrm{~g}, 75 \%$ yield $)$.

${ }^{1} \mathrm{H}$ NMR $\left(400 \mathrm{MHz} \mathrm{CDCl}_{3}\right) \delta$ 7.30-7.21 (m, 2H), 7.21-7.08 (m, 3H), $7.00(\mathrm{~d}, J=8.5 \mathrm{~Hz}$, $2 \mathrm{H}), 6.70(\mathrm{~d}, J=8.4 \mathrm{~Hz}, 2 \mathrm{H}), 5.27(\mathrm{~s}, 1 \mathrm{H}), 2.20-2.06(\mathrm{~m}, 4 \mathrm{H}), 1.00(\mathrm{t}, J=7.4 \mathrm{~Hz}, 3 \mathrm{H}), 0.99$ $(\mathrm{t}, J=7.5 \mathrm{~Hz}, 3 \mathrm{H}) \mathrm{ppm}$.

${ }^{13} \mathrm{C}$ NMR $\left(100 \mathrm{MHz}_{2} \mathrm{CDCl}_{3}\right) \delta 153.4,143.7,141.9,136.4,136.2,130.5,129.2,127.9,125.9$, $114.9,24.35,24.26,13.3(2 \mathrm{C}) \mathrm{ppm}$.

This is a known compound. The characterization data match the literature data. ${ }^{4}$<smiles>CC(C)=C(c1ccccc1)c1ccc(O)cc1</smiles>

4-(2-Methyl-1-phenylprop-1-en-1-yl)phenol (S3b) was prepared as a white solid from $\mathrm{TiCl}_{4}(11.38 \mathrm{~g}, 60 \mathrm{mmol}), \mathrm{Zn}(7.85 \mathrm{~g}, 120 \mathrm{mmol})$, 4-hydroxybenzophenone (3.96 $\mathrm{g}$, $20 \mathrm{mmol})$ and acetone $(1.63 \mathrm{~g}, 28 \mathrm{mmol})$ in THF $(140 \mathrm{~mL})$ according to the General 
Procedure A (14.5 h, purified by flash column chromatography: hexanes/ethyl acetate $=20: 1,3.60 \mathrm{~g}, 80 \%$ yield).

${ }^{1} \mathrm{H}$ NMR $\left(400 \mathrm{MHz}, \mathrm{CDCl}_{3}\right) \delta$ 7.31-7.21 (m, 2H), 7.21-7.06 (m, 3H), 6.99 (d, J = 8.6 Hz, 2H), $6.72(\mathrm{~d}, \mathrm{~J}=8.6 \mathrm{~Hz}, 2 \mathrm{H}), 5.08(\mathrm{~s}, 1 \mathrm{H}), 1.80(\mathrm{~s}, 3 \mathrm{H}), 1.78(\mathrm{~s}, 3 \mathrm{H}) \mathrm{ppm}$.

${ }^{13} \mathrm{C}$ NMR (100 MHz, $\left.\mathrm{CDCl}_{3}\right) \delta 153.6,143.6,136.4,136.0,131.1,130.6,129.8,127.8,125.9$, 114.7, 22.5 (2C) ppm.

IR (ATR) 3386, 2986, 2910, 2855, 1609, 1509, 1440, 1264, 1170, 831, $734 \mathrm{~cm}^{-1}$.

HRMS (CI+) Calcd for $\mathrm{C}_{16} \mathrm{H}_{16} \mathrm{O}\left[\mathrm{M}^{+}\right]$: 224.1201, Found: 224.1200.<smiles>CCC(CC)=C(c1ccc(O)cc1)c1ccc(Cl)cc1</smiles>

S3c

4-(1-(4-Chlorophenyl)-2-ethylbut-1-en-1-yl)phenol (S3c) was prepared as a white solid from $\mathrm{TiCl}_{4}(11.38 \mathrm{~g}, 60 \mathrm{mmol}), \mathrm{Zn} \quad(7.85 \mathrm{~g}, 120 \mathrm{mmol})$, (4-chlorophenyl)(4-hydroxyphenyl)methanone $(4.67 \mathrm{~g}, 20 \mathrm{mmol})$ and 3-pentanone $(2.07 \mathrm{~g}, 24 \mathrm{mmol})$ in THF $(160 \mathrm{~mL})$ according to the General Procedure A $(12 \mathrm{~h}$, purified by flash column chromatography: hexanes/ethyl acetate $=15: 1,4.96 \mathrm{~g}$, 86\% yield).

${ }^{1}$ H NMR $\left(400 \mathrm{MHz}, \mathrm{CDCl}_{3}\right) \delta 7.22(\mathrm{~d}, J=8.2 \mathrm{~Hz}, 2 \mathrm{H}), 7.05(\mathrm{~d}, J=8.2 \mathrm{~Hz}, 2 \mathrm{H}), 6.97(\mathrm{~d}, J$ $=8.4 \mathrm{~Hz}, 2 \mathrm{H}), 6.73(\mathrm{~d}, J=8.4 \mathrm{~Hz}, 2 \mathrm{H}), 5.23(\mathrm{~s}, 1 \mathrm{H}), 2.21-2.04(\mathrm{~m}, 4 \mathrm{H}), 0.994(\mathrm{t}, J=7.3$ $\mathrm{Hz}, 3 \mathrm{H}), 0.988(\mathrm{t}, J=7.3 \mathrm{~Hz}, 3 \mathrm{H}) \mathrm{ppm}$.

${ }^{13} \mathrm{C}$ NMR $\left(100 \mathrm{MHz} \mathrm{CDCl}_{3}\right) \delta 153.8,142.7,142.1,135.6,135.3,131.7,130.6,130.5,128.1$, $114.9,24.38,24.35,13.3(2 \mathrm{C}) \mathrm{ppm}$.

IR (ATR) 3352, 2966, 2934, 2873, 1607, 1590, 1508, 1485, 1262, 1251, 1223, 1169, 1091, $1014,822 \mathrm{~cm}^{-1}$.

HRMS (CI+) Calcd for $\mathrm{C}_{18} \mathrm{H}_{19} \mathrm{ClO}\left[\mathrm{M}^{+}\right]$: 286.1124, Found: 286.1126. 
<smiles>CCC(CC)=C(c1ccccc1)c1ccc(O)c(Br)c1</smiles>

S3d

2-Bromo-4-(2-ethyl-1-phenylbut-1-en-1-yl)phenol (S3d) was prepared as a light yellow oil from $\mathrm{TiCl}_{4}(3.41 \mathrm{~g}, 18 \mathrm{mmol}), \mathrm{Zn}(2.35 \mathrm{~g}, 36 \mathrm{mmol})$, (3-bromo-4-hydroxyphenyl)(phenyl)methanone (1.66 g, $6 \mathrm{mmol}$ ) and 3-pentanone (0.62 $\mathrm{g}, 7.2 \mathrm{mmol})$ in THF $(80 \mathrm{~mL})$ according to the General Procedure A $(2 \mathrm{~h}$, purified by flash column chromatography: hexanes/ethyl acetate $=15: 1,1.42 \mathrm{~g}, 57 \%$ yield).

${ }^{1} \mathbf{H}$ NMR (400 MHz, $\left.\mathrm{CDCl}_{3}\right) \delta$ 7.31-7.25 (m, 2H), $7.24(\mathrm{~d}, J=2.0 \mathrm{~Hz}, 1 \mathrm{H}), 7.22-7.15(\mathrm{~m}$, 1H), 7.14-7.08 (m, 2H), $7.01\left(\mathrm{dd}, J_{1}=8.3 \mathrm{~Hz}, J_{2}=2.0 \mathrm{~Hz}, 1 \mathrm{H}\right), 6.92(\mathrm{~d}, J=8.3 \mathrm{~Hz}, 1 \mathrm{H})$, $5.41(\mathrm{~s}, 1 \mathrm{H}), 2.14(\mathrm{q}, J=7.5 \mathrm{~Hz}, 2 \mathrm{H}), 2.11(\mathrm{q}, J=7.5 \mathrm{~Hz}, 2 \mathrm{H}), 1.01(\mathrm{t}, J=8.0 \mathrm{~Hz}, 3 \mathrm{H}), 0.99$ $(\mathrm{t}, J=8.0 \mathrm{~Hz}, 3 \mathrm{H}) \mathrm{ppm}$.

${ }^{13} \mathrm{C}$ NMR (100 MHz, $\left.\mathrm{CDCl}_{3}\right) \delta 150.4,143.0,137.4,135.4,132.4,130.1,129.1$ (2C), 128.1, $126.2,115.5,109.8,24.39,24.36,13.3$ (2C) ppm.

IR (thin film) 3512, 2967, 2934, 2873, 1600, 1490, 1273, 1177, 1039, $821 \mathrm{~cm}^{-1}$.

HRMS (LD+) Calcd for $\mathrm{C}_{18} \mathrm{H}_{19} \mathrm{BrO}\left[\mathrm{M}^{+}\right]$: 330.0619, Found: 330.0615.<smiles>CCC(CC)=C(c1ccc(O)cc1)c1ccccc1F</smiles>

4-(2-Ethyl-1-(2-fluorophenyl)but-1-en-1-yl)phenol (S3e) was prepared as a yellow

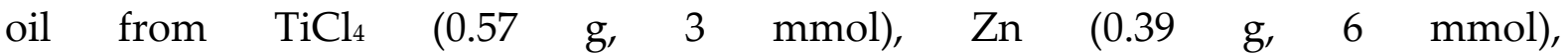
(2-fluorophenyl)(4-hydroxyphenyl)methanone $(0.22 \mathrm{~g}, 1 \mathrm{mmol}$ ) and 3-pentanone 
(0.10 g, $1.2 \mathrm{mmol})$ in THF $(12 \mathrm{~mL})$ according to the General Procedure A $(1 \mathrm{~h}$, purified by flash column chromatography: hexanes/ethyl acetate $=15: 1,0.25 \mathrm{~g}$, 92\% yield).

${ }^{1}$ H NMR (400 MHz, $\left.\mathrm{CDCl}_{3}\right) \delta$ 7.22-7.10 (m, 2H), 7.10-7.02 (m, 3H), 7.02-6.94 (m, 1H), 6.77-6.68 (m, 2H), 4.73 (brs, 1H), 2.17 (q, J = 7.5 Hz, 2H), $2.02(\mathrm{q}, J=7.5 \mathrm{~Hz}, 2 \mathrm{H}), 1.01$ (t, $J=7.5 \mathrm{~Hz}, 3 \mathrm{H}), 0.96(\mathrm{t}, J=7.5 \mathrm{~Hz}, 3 \mathrm{H}) \mathrm{ppm}$.

${ }^{13} \mathrm{C}$ NMR $\left(100 \mathrm{MHz}, \mathrm{CDCl}_{3}\right) \delta 159.5(\mathrm{~d}, J=242.3 \mathrm{~Hz}), 153.8,144.4,135.0,131.3$ (d, $J=$ $3.8 \mathrm{~Hz}), 131.1(\mathrm{~d}, J=17.5 \mathrm{~Hz}), 130.3,129.6,128.0(\mathrm{~d}, J=7.9 \mathrm{~Hz}), 123.8(\mathrm{~d}, J=3.5 \mathrm{~Hz})$, $115.6(\mathrm{~d}, J=22.6 \mathrm{~Hz}), 114.8,24.9,24.0,13.2,12.7 \mathrm{ppm}$.

${ }^{19} \mathrm{~F}$ NMR $\left(376 \mathrm{MHz}, \mathrm{CDCl}_{3}\right) \delta-114.7 \mathrm{ppm}$.

IR (ATR) 3361, 2969, 1609, 1509, 1486, 1447, 1264, 1218, 1170, 1104, $832 \mathrm{~cm}^{-1}$.

HRMS (CI+) Calcd for $\mathrm{C}_{18} \mathrm{H}_{19} \mathrm{FO}\left[\mathrm{M}^{+}\right]$: 270.1420, Found: 270.1414.

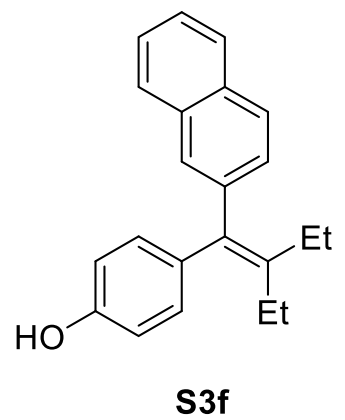

4-(2-Ethyl-1-(naphthalen-2-yl)but-1-en-1-yl)phenol (S3f) was prepared as a yellow oil from $\mathrm{TiCl}_{4}(1.14 \mathrm{~g}, 6 \mathrm{mmol}), \mathrm{Zn} \quad(0.78 \mathrm{~g}, 12 \mathrm{mmol})$, (4-hydroxyphenyl)(naphthalen-2-yl)methanone $(0.50 \mathrm{~g}, 2 \mathrm{mmol})$ and 3-pentanone $(0.21 \mathrm{~g}, 2.4 \mathrm{mmol})$ in THF $(25 \mathrm{~mL})$ according to the General Procedure A $(2 \mathrm{~h}$, purified by flash column chromatography: hexanes/ethyl acetate $=15: 1,0.42 \mathrm{~g}$, 69\% yield).

${ }^{1} \mathrm{H}$ NMR $\left(400 \mathrm{MHz}^{\mathrm{CDCl}}\right) \delta 7.76(\mathrm{~d}, J=8.9 \mathrm{~Hz}, 2 \mathrm{H}), 7.71(\mathrm{~d}, J=8.4 \mathrm{~Hz}, 1 \mathrm{H}), 7.61(\mathrm{~s}$, $1 \mathrm{H}), 7.47-7.34(\mathrm{~m}, 2 \mathrm{H}), 7.23\left(\mathrm{dd}, J_{1}=8.4 \mathrm{~Hz}, J_{2}=1.1 \mathrm{~Hz}, 1 \mathrm{H}\right), 7.04(\mathrm{~d}, J=8.4 \mathrm{~Hz}, 2 \mathrm{H})$, $6.80(\mathrm{~d}, J=8.4 \mathrm{~Hz}, 2 \mathrm{H}), 5.18(\mathrm{~s}, 1 \mathrm{H}), 2.21(\mathrm{q}, J=8.0 \mathrm{~Hz}, 2 \mathrm{H}), 2.17(\mathrm{q}, J=8.0 \mathrm{~Hz}, 2 \mathrm{H})$, $1.04(\mathrm{t}, J=7.5 \mathrm{~Hz}, 3 \mathrm{H}), 1.01(\mathrm{t}, J=7.5 \mathrm{~Hz}, 3 \mathrm{H}) \mathrm{ppm}$. 
${ }^{13} \mathrm{C}$ NMR $\left(100 \mathrm{MHz}, \mathrm{CDCl}_{3}\right) \delta$ 153.7, 142.4, 141.3, 136.4, 135.9, 133.3, 131.9, 130.7, 128.1, $127.8,127.5,127.4(2 \mathrm{C}), 125.8,125.4,114.8,24.5,24.4,13.37,13.36$ ppm.

IR (ATR) 3353, 2966, 2933, 2872, 1607, 1508, 1461, 1431, 1263, 1169, $735 \mathrm{~cm}^{-1}$.

HRMS (CI+) Calcd for $\mathrm{C}_{22} \mathrm{H}_{22} \mathrm{O}\left[\mathrm{M}^{+}\right]$: 302.1671, Found: 302.1667.

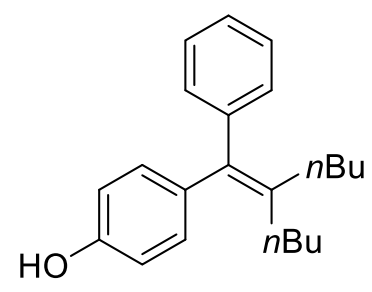

S3g

4-(2-Butyl-1-phenylhex-1-en-1-yl)phenol (S3g) was prepared as a light yellow oil from $\mathrm{TiCl}_{4}(5.69 \mathrm{~g}, 30 \mathrm{mmol}), \mathrm{Zn}(3.92 \mathrm{~g}$, $60 \mathrm{mmol})$, 4-hydroxybezophenone (1.98 g, $10 \mathrm{mmol})$ and 5-nonanone $(1.71 \mathrm{~g}, 12 \mathrm{mmol})$ in THF $(120 \mathrm{~mL})$ according to the General Procedure A (2 h, purified by flash column chromatography: hexanes/ethyl acetate $=15: 1,3.06 \mathrm{~g}, 99 \%$ yield).

${ }^{1} \mathrm{H}$ NMR $\left(400 \mathrm{MHz} \mathrm{CDCl}_{3}\right) \delta$ 7.28-7.21 (m, 2H), 7.19-7.07 (m, 3H), 7.02-6.93 (m, 2H), 6.74-6.66 (m, 2H), $5.16(\mathrm{~s}, 1 \mathrm{H}), 2.17-2.04(\mathrm{~m}, 4 \mathrm{H}), 1.45-1.33(\mathrm{~m}, 4 \mathrm{H}), 1.29-1.12(\mathrm{~m}, 4 \mathrm{H})$, $0.82(\mathrm{t}, J=7.5 \mathrm{~Hz}, 3 \mathrm{H}), 0.80(\mathrm{t}, J=7.5 \mathrm{~Hz}, 3 \mathrm{H}) \mathrm{ppm}$.

${ }^{13} \mathrm{C}$ NMR $\left(100 \mathrm{MHz}, \mathrm{CDCl}_{3}\right) \delta 153.5,143.9,139.4,137.3,136.3,130.6,129.3,127.9,125.8$, $114.8,31.4,31.3,30.7(2 \mathrm{C}), 22.7,22.6,14.0,13.9$ ppm.

IR (thin film) 3346, 2956, 2928, 2859, 1609, 1509, 1465, 1442, 1252, 1222, 1170, 1101, 829, $701 \mathrm{~cm}^{-1}$.

HRMS (CI+) Calcd for $\mathrm{C}_{22} \mathrm{H}_{28} \mathrm{O}\left[\mathrm{M}^{+}\right]$: 308.2140, Found: 308.2133. 
<smiles>CC(C)CCC(CCC(C)C)=C(c1ccccc1)c1ccc(O)cc1</smiles>

4-(2-Isopentyl-5-methyl-1-phenylhex-1-en-1-yl)phenol (S3h) was prepared as a yellow oil from $\mathrm{TiCl}_{4}(5.69 \mathrm{~g}, 30 \mathrm{mmol}), \mathrm{Zn}(3.92 \mathrm{~g}, 60 \mathrm{mmol})$, 4-hydroxybenzophenone (1.98 g, $10 \mathrm{mmol})$ and 2,8-dimethylnonan-5-one (2.04 g, 12 $\mathrm{mmol})$ in THF $(120 \mathrm{~mL})$ according to the General Procedure A $(10 \mathrm{~h}$, purified by flash column chromatography: hexanes/ethyl acetate $=15: 1,3.27 \mathrm{~g}$, 97\% yield).

${ }^{1}$ H NMR $\left(400 \mathrm{MHz} \mathrm{CDCl}_{3}\right) \delta$ 7.30-7.19 (m, 2H), 7.18-7.06 (m, 3H), 7.04-6.93 (m, 2H), 6.76-6.66 (m, 2H), 5.09 (s, 1H), 2.18-1.99 (m, 4H), 1.53-1.35 (m, 2H), 1.35-1.23 (m, 4H), $0.79(\mathrm{~d}, J=6.6 \mathrm{~Hz}, 6 \mathrm{H}), 0.76(\mathrm{~d}, J=6.6 \mathrm{~Hz}, 6 \mathrm{H}) \mathrm{ppm}$.

${ }^{13} \mathrm{C}$ NMR (100 MHz, $\left.\mathrm{CDCl}_{3}\right) \delta 153.6,143.8,139.9,137.1,136.2,130.6,129.3,127.8,125.9$, $114.8,37.9$ (2C), 29.73, 29.68, 28.13, 28.10, 22.51, 22.46 ppm.

IR (ATR) 3357, 2953, 2926, 2868, 1608, 1508, 1466, 1441, 1263, 1218, $1169 \mathrm{~cm}^{-1}$. HRMS (CI+) Calcd for $\mathrm{C}_{24} \mathrm{H}_{32} \mathrm{O}\left[\mathrm{M}^{+}\right]$: 336.2453, Found: 336.2449.

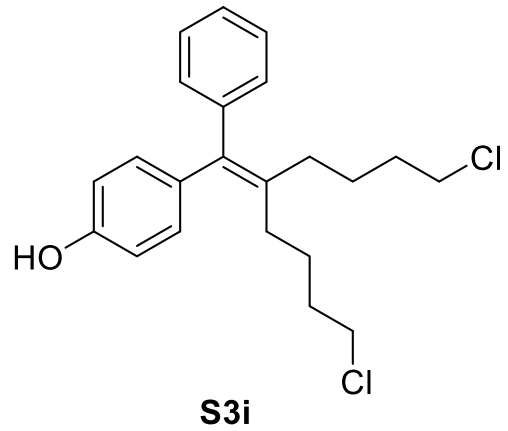

4-(6-Chloro-2-(4-chlorobutyl)-1-phenylhex-1-en-1-yl)phenol (S3i) was prepared as a colorless oil from $\mathrm{TiCl}_{4}(1.71 \mathrm{~g}, 9 \mathrm{mmol}), \mathrm{Zn}(1.18 \mathrm{~g}, 18 \mathrm{mmol})$, 4-hydroxybezophenone $(0.59 \mathrm{~g}, 3 \mathrm{mmol})$ and 1,9-dichlorononan-5-one (0.70 g, 3.3 $\mathrm{mmol})$ in THF (50 mL) according to the General Procedure A ( $1 \mathrm{~h}$, purified by flash column chromatography: hexanes/ethyl acetate $=12: 1,0.84 \mathrm{~g}, 74 \%$ yield). 
${ }^{1} \mathbf{H}$ NMR $\left(400 \mathrm{MHz} \mathrm{CDCl}_{3}\right) \delta$ 7.31-7.21 (m, 2H), 7.21-7.14 (m, 1H), 7.14-7.06 (m, 2H), 7.03-6.93 (m, 2H), 6.77-6.67 (m, 2H), $4.95(\mathrm{~s}, 1 \mathrm{H}), 3.43(\mathrm{t}, J=6.6 \mathrm{~Hz}, 2 \mathrm{H}), 3.41(\mathrm{t}, J=6.6$ $\mathrm{Hz}, 2 \mathrm{H}), 2.24-2.06(\mathrm{~m}, 4 \mathrm{H}), 1.74-1.62(\mathrm{~m}, 4 \mathrm{H}), 1.62-1.48(\mathrm{~m}, 4 \mathrm{H}) \mathrm{ppm}$.

${ }^{13} \mathrm{C}$ NMR $\left(100 \mathrm{MHz} \mathrm{CDCl}_{3}\right) \delta 153.8,143.3,139.0,137.4,135.7,130.4,129.1,128.1,126.2$, 115.0, 44.8 (2C), 32.2, 32.1, 30.5, 30.4, 25.4 (2C) ppm.

IR (thin film) 3407, 2954, 2936, 2865, 1609, 1509, 1442, 1260, 1226, 1170, 1099, $832 \mathrm{~cm}^{-1}$. HRMS (CI+) Calcd for $\mathrm{C}_{22} \mathrm{H}_{26} \mathrm{Cl}_{2} \mathrm{O}\left[\mathrm{M}^{+}\right]$: 376.1361, Found: 376.1348.

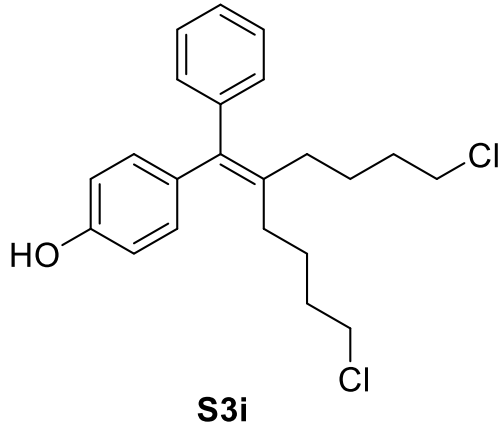

S3i

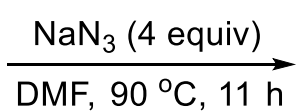

4-(6-Azido-2-(4-azidobutyl)-1-phenylhex-1-en-1-yl)phenol (S3j). ${ }^{5}$ Under $\mathrm{N}_{2}$, to an oven-dried Schlenk tube charged with $\mathrm{NaN}_{3}(52.0 \mathrm{mg}, 0.8 \mathrm{mmol})$ were added S3i (75.2 $\mathrm{mg}, 0.2 \mathrm{mmol})$ and anhydrous DMF (2 mL). The mixture was heated at $90{ }^{\circ} \mathrm{C}$ and monitored by TLC. Upon completion $(11 \mathrm{~h})$, the reaction mixture was cooled to room temperature and quenched with water $(4 \mathrm{~mL})$. The aqueous layer was extracted with ethyl acetate $(10 \mathrm{~mL} \times 3)$. The combined organic layers were washed with brine $(10 \mathrm{~mL})$, dried over $\mathrm{Na}_{2} \mathrm{SO}_{4}$, and concentrated. The residue was purified by silica gel flash column chromatography (eluent: hexanes/ethyl acetate $=15: 1$ ) to afford S3j as a yellow oil (58.4 $\mathrm{mg}, 75 \%$ yield).

${ }^{1}$ H NMR (400 MHz, acetone- $\left.d_{6}\right) \delta 8.32(\mathrm{~s}, 1 \mathrm{H}), 7.37-7.29(\mathrm{~m}, 2 \mathrm{H}), 7.26-7.17(\mathrm{~m}, 3 \mathrm{H})$, 7.07-7.00 (m, 2H), 6.86-6.78 (m, 2H), $3.28(\mathrm{t}, J=6.0 \mathrm{~Hz}, 2 \mathrm{H}), 3.25(\mathrm{t}, J=6.4 \mathrm{~Hz}, 2 \mathrm{H})$, 2.33-2.24 (m, 2H), 2.24-2.15 (m, 2H), 1.66-1.47 (m, 8H) ppm.

${ }^{13} \mathrm{C}$ NMR (100 MHz, acetone- $\left.d_{6}\right) \delta 156.7,144.7,140.2,138.1,135.3,131.1,130.0,128.9$, $126.9,115.74,115.65,51.68,51.65,31.5,31.4,29.19,29.16,26.1$ ppm.

IR (ATR) 3378, 2935, 2863, 2091, 1608, 1508, 1457, 1441, 1263, $1169 \mathrm{~cm}^{-1}$. 
HRMS (CI+) Calcd for $\mathrm{C}_{22} \mathrm{H}_{27} \mathrm{~N}_{6} \mathrm{O}\left[\mathrm{M}^{+}+\mathrm{H}\right]$ : 391.2246, Found: 391.2256.<smiles>CCC(CC)=C(c1ccccc1)c1ccc(O)cc1</smiles>

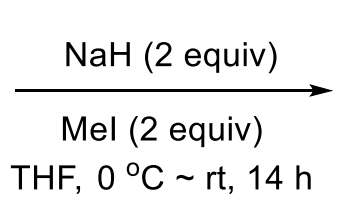

THF, $0{ }^{\circ} \mathrm{C} \sim \mathrm{rt}, 14 \mathrm{~h}$<smiles>CCC(=C(c1ccccc1)c1ccc(OC)cc1)c1ccccc1</smiles>

S3a'

1-(2-Ethyl-1-phenylbut-1-en-1-yl)-4-methoxybenzene (S3a'). Under $\mathrm{N}_{2}$ at $0{ }^{\circ} \mathrm{C}$, to an oven-dried Schlenk tube charged with $\mathrm{NaH}(0.24 \mathrm{~g}, 6 \mathrm{mmol}, 60 \mathrm{wt} \%$ in mineral oil) and anhydrous THF (8 mL) was added a solution of S3a $(0.76 \mathrm{~g}, 3 \mathrm{mmol})$ in anhydrous THF $(4 \mathrm{~mL})$ followed by MeI $(0.85 \mathrm{~g}, 6 \mathrm{mmol})$. Next, the mixture was warmed naturally to room temperature, and the reaction progress was monitored by TLC. Upon completion $(14 \mathrm{~h})$, the reaction mixture was at cooled to $0{ }^{\circ} \mathrm{C}$ and quenched with water $(10 \mathrm{~mL})$. The aqueous layer was extracted with ethyl ether (30 $\mathrm{mL} \times 3)$. The combined organic layers were washed with brine $(20 \mathrm{~mL})$, dried over $\mathrm{Na}_{2} \mathrm{SO}_{4}$, and concentrated. The residue was purified by silica gel flash column chromatography (eluent: hexanes/ethyl ether $=25: 1$ ) to afford S3a' as a colorless oil (0.77 g, 97\% yield).

${ }^{1} \mathrm{H}$ NMR $\left(400 \mathrm{MHz} \mathrm{CDCl}_{3}\right) \delta$ 7.29-7.21 (m, 2H), 7.19-7.10 (m, 3H), 7.09-7.02 (m, 2H), 6.83-6.77 (m, 2H), $3.75(\mathrm{~s}, 3 \mathrm{H}), 2.16(\mathrm{q}, J=7.4 \mathrm{~Hz}, 2 \mathrm{H}), 2.12(\mathrm{q}, J=7.3 \mathrm{~Hz}, 2 \mathrm{H}), 1.01(\mathrm{t}, J$ $=7.5 \mathrm{~Hz}, 3 \mathrm{H}), 1.00(\mathrm{t}, J=7.5 \mathrm{~Hz}, 3 \mathrm{H}) \mathrm{ppm}$.

${ }^{13} \mathrm{C}$ NMR $\left(100 \mathrm{MHz}, \mathrm{CDCl}_{3}\right) \delta 157.8,143.8,141.8,136.6,135.9,130.3,129.2,127.9,125.9$, 113.3, 55.1, 24.4, 24.3, 13.3 (2C) ppm.

IR (ATR) 2965, 2934, 1606, 1507, 1462, 1441, 1265, 1241, 1173, 1035, $825 \mathrm{~cm}^{-1}$. HRMS (CI+) Calcd for $\mathrm{C}_{19} \mathrm{H}_{22} \mathrm{O}\left[\mathrm{M}^{+}\right]$: 266.1671, Found: 266.1676 . 


\section{General Procedure B: Preparation of the epoxides.}<smiles></smiles>

S1 or 53
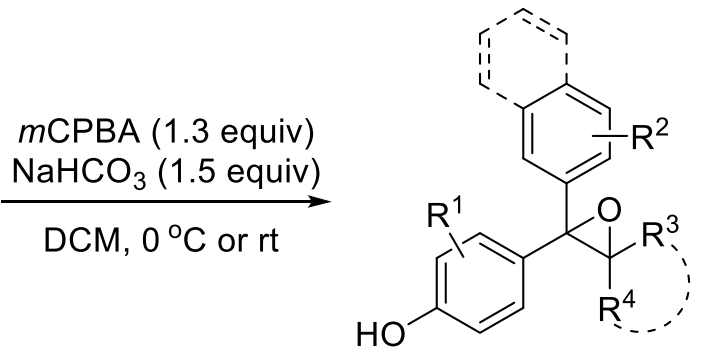

1 or 3

The general procedure is described using 1a as an example.

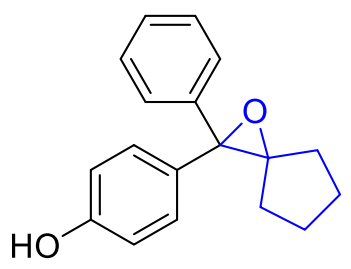

$1 \mathbf{a}$

4-(2-Phenyl-1-oxaspiro[2.4]heptan-2-yl)phenol (1a). At $0{ }^{\circ} \mathrm{C}$ under $\mathrm{N}_{2}$, to an oven-dried flask were added mCPBA (2.07 g, 85\%, $10.2 \mathrm{mmol}, 1.3$ equiv), $\mathrm{NaHCO}_{3}$ (1.00 g, $12 \mathrm{mmol}, 1.5$ equiv), and anhydrous DCM (60 mL) was added a solution of S1a $(2.00 \mathrm{~g}, 8 \mathrm{mmol})$ in anhydrous DCM $(20 \mathrm{~mL})$. The reaction was stirred at $0{ }^{\circ} \mathrm{C}$ and monitored by TLC. Upon completion ( 11.5 h), DCM (40 mL) was added to dilute the reaction mixture followed by saturated aqueous solutions of $\mathrm{Na}_{2} \mathrm{SO}_{3}(40$ $\mathrm{mL}$ ) and $\mathrm{NaHCO}_{3}$ (aq., $40 \mathrm{~mL}$ ). The layers were separated, and the aqueous layer was extracted with DCM $(50 \mathrm{~mL} \times 3)$. The combined organic layers were washed with brine $(20 \mathrm{~mL})$, dried over $\mathrm{Na}_{2} \mathrm{SO}_{4}$, and concentrated (water bath $<30{ }^{\circ} \mathrm{C}$ ). The residue was purified by silica gel (pre-treated with a solution of $0.5 \%$ of $\mathrm{Et}_{3} \mathrm{~N}$ in hexanes) flash column chromatography (eluent: hexanes/ethyl acetate $=8: 1)$ to afford 1a $(1.22$ g, 57\% yield) as a light yellow oil. Due to easy decomposition, the product was stored as a solution in anhydrous DCM $(0.2 \mathrm{M})$ at $-20^{\circ} \mathrm{C}$.

${ }^{1}$ H NMR (400 MHz, acetone- $\left.d_{6}\right) \delta 8.40(\mathrm{~s}, 1 \mathrm{H}), 7.54-7.46(\mathrm{~m}, 2 \mathrm{H}), 7.40-7.32(\mathrm{~m}, 4 \mathrm{H})$, 7.30-7.23 (m, 1H), 6.90-6.83 (m, 2H), 1.89-1.58 (m, 6H), 1.49-1.32 (m, 2H) ppm. 
${ }^{13} \mathrm{C}$ NMR (100 MHz, acetone- $\left.d_{6}\right) \delta$ 157.3, 142.1, 132.4, 129.1, 128.8, 127.71, 127.69, 115.6, 77.1, 69.3, 32.2 (2C), $25.6(2 \mathrm{C}) \mathrm{ppm}$.

IR (ATR) 3373, 2959, 2868, 1613, 1595, 1512, 1495, 1446, 1263, 1219, 1168, 1103, 827 $\mathrm{cm}^{-1}$.

HRMS (CI+) Calcd for $\mathrm{C}_{18} \mathrm{H}_{18} \mathrm{O}_{2}\left[\mathrm{M}^{+}\right]$: 266.1307, Found: 266.1301.

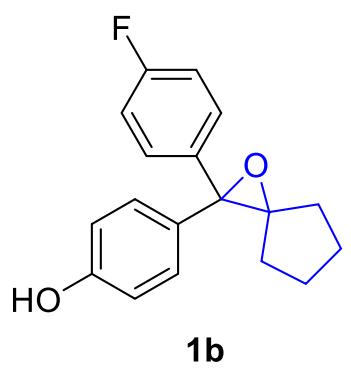

4-(2-(4-Fluorophenyl)-1-oxaspiro[2.4]heptan-2-yl)phenol (1b) was prepared as a yellow oil from mCPBA $(85 \%, 0.90 \mathrm{~g}, 4.4 \mathrm{mmol}), \mathrm{NaHCO}_{3}(0.44 \mathrm{~g}, 5.2 \mathrm{mmol})$, and S1b $(1.07 \mathrm{~g}, 4 \mathrm{mmol})$ in DCM $(40 \mathrm{~mL})$ according to the General Procedure B $(17 \mathrm{~h}$, purified by flash column chromatography: hexanes/ethyl acetate $=12: 1$ to $8: 1,0.68 \mathrm{~g}$, $59 \%$ yield).

${ }^{1}$ H NMR (400 MHz, acetone- $\left.d_{6}\right) \delta 8.47(\mathrm{~s}, 1 \mathrm{H})$, 7.57-7.47 $(\mathrm{m}, 2 \mathrm{H}), 7.38-7.29(\mathrm{~m}, 2 \mathrm{H})$, 7.18-7.08 (m, 2H), 6.91-6.83 (m, 2H), 1.88-1.58 (m, 6H), 1.47-1.34 (m, 2H) ppm.

${ }^{13} \mathrm{C}$ NMR (100 MHz, acetone- $\left.d_{6}\right) \delta 162.5(\mathrm{~d}, J=242.2 \mathrm{~Hz}), 157.4,138.3(\mathrm{~d}, J=3.2 \mathrm{~Hz})$, 132.1, $129.7(\mathrm{~d}, J=8.1 \mathrm{~Hz}), 129.0,115.7,115.5(\mathrm{~d}, J=21.5 \mathrm{~Hz}), 77.3,68.8,32.2,32.1$, 25.67, 25.65 ppm.

${ }^{19}$ F NMR (376 MHz, acetone- $\left.d_{6}\right) \delta-117.0 \mathrm{ppm}$.

IR (thin film) 3378, 2962, 2871, 1613, 1607, 1510, 1438, 1261, 1223, 1170, 1157, $833 \mathrm{~cm}^{-1}$. HRMS (CI+) Calcd for $\mathrm{C}_{18} \mathrm{H}_{17} \mathrm{FO}_{2}\left[\mathrm{M}^{+}\right]$: 284.1213, Found: 284.1220. 


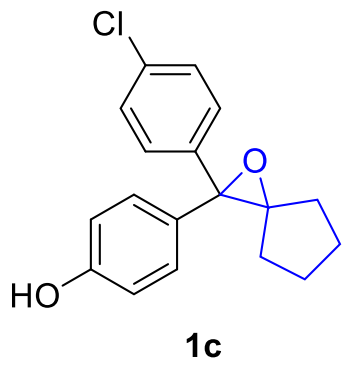

4-(2-(4-Chlorophenyl)-1-oxaspiro[2.4]heptan-2-yl)phenol (1c) was prepared as a yellow oil from $m$ CPBA $(85 \%, 0.90 \mathrm{~g}, 4.4 \mathrm{mmol}), \mathrm{NaHCO}_{3}(0.44 \mathrm{~g}, 5.2 \mathrm{mmol})$, and S1c (1.14 g, $4 \mathrm{mmol})$ in DCM (40 mL) according to the General Procedure B (5 h, purified by flash column chromatography: hexanes/ethyl acetate $=10: 1$ to $6: 1,0.76 \mathrm{~g}, 63 \%$ yield).

${ }^{1}$ H NMR (400 MHz, acetone- $\left.d_{6}\right) \delta 8.45(\mathrm{~s}, 1 \mathrm{H}), 7.54-7.46(\mathrm{~m}, 2 \mathrm{H}), 7.42-7.37(\mathrm{~m}, 2 \mathrm{H})$, 7.37-7.30 (m, 2H), $6.87(\mathrm{~d}, J=8.4 \mathrm{~Hz}, 2 \mathrm{H}), 1.88-1.60(\mathrm{~m}, 6 \mathrm{H}), 1.47-1.34(\mathrm{~m}, 2 \mathrm{H}) \mathrm{ppm}$.

${ }^{13} \mathrm{C}$ NMR (100 MHz, acetone- $\left.d_{6}\right) \delta 157.5,141.1,133.1,131.9,129.5,129.1,128.9,115.7$, $77.3,68.8,32.3,32.0,25.68,25.65$ ppm.

IR (thin film) 3385, 2962, 2871, 1614, 1596, 1515, 1489, 1437, 1264, 1226, 1169, 1091, $1014,829 \mathrm{~cm}^{-1}$.

HRMS (CI+) Calcd for $\mathrm{C}_{18} \mathrm{H}_{17} \mathrm{ClO}_{2}\left[\mathrm{M}^{+}\right]$: 300.0917, Found: 300.0906 .

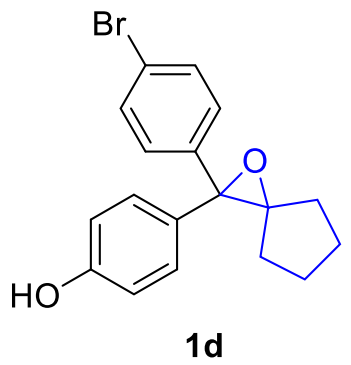

4-(2-(4-Bromophenyl)-1-oxaspiro[2.4]heptan-2-yl)phenol (1d) was prepared as a colorless oil from $m \mathrm{CPBA}(85 \%, 0.31 \mathrm{~g}, 1.5 \mathrm{mmol}), \mathrm{NaHCO}_{3}(0.15 \mathrm{~g}, 1.8 \mathrm{mmol})$, and S1d $(0.40 \mathrm{~g}, 1.2 \mathrm{mmol})$ in DCM $(12 \mathrm{~mL})$ according to the General Procedure B (5 h, purified by flash column chromatography: hexanes/ethyl acetate $=12: 1$ to $8: 1,0.34 \mathrm{~g}$, $81 \%$ yield). 
${ }^{1}$ H NMR (400 MHz, acetone- $\left.d_{6}\right) \delta 8.46(\mathrm{~s}, 1 \mathrm{H}), 7.58-7.51(\mathrm{~m}, 2 \mathrm{H}), 7.48-7.40(\mathrm{~m}, 2 \mathrm{H})$, 7.37-7.29 (m, 2H), 6.89-6.81 (m, 2H), 1.89-1.58 (m, 6H), 1.46-1.34 (m, 2H) ppm.

${ }^{13}$ C NMR (100 MHz, acetone- $\left.d_{6}\right) \delta 157.5,141.5,131.9,131.8,129.9,129.1,121.2,115.7$, 77.3, 68.9, 32.3, 32.1, 25.7, 25.6 ppm.

IR (thin film) 3387, 2960, 1614, 1594, 1515, 1487, 1437, 1384, 1264, 1227, 1169, 1070, $1011,828 \mathrm{~cm}^{-1}$.

HRMS (CI+) Calcd for $\mathrm{C}_{18} \mathrm{H}_{17} \mathrm{BrO}_{2}\left[\mathrm{M}^{+}\right]$: 344.0412, Found: 344.0407.

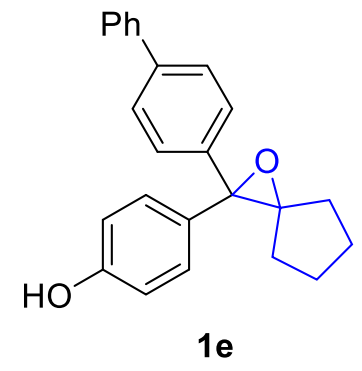

4-(2-([1,1'-Biphenyl]-4-yl)-1-oxaspiro[2.4]heptan-2-yl)phenol (1e) was prepared as a yellow oil from $m$ CPBA $(85 \%, 0.52 \mathrm{~g}$, $2.6 \mathrm{mmol}), \mathrm{NaHCO}_{3}(0.26 \mathrm{~g}, 3 \mathrm{mmol})$, and S1e $(0.65 \mathrm{~g}, 2 \mathrm{mmol})$ in DCM $(20 \mathrm{~mL})$ according to the General Procedure B (4 h, purified by flash column chromatography: hexanes/ethyl acetate $=8: 1$ to $6: 1,0.54 \mathrm{~g}, 79 \%$ yield).

${ }^{1}$ H NMR (400 MHz, acetone- $\left.d_{6}\right) \delta 8.43(\mathrm{~s}, 1 \mathrm{H}), 7.71-7.62(\mathrm{~m}, 4 \mathrm{H}), 7.61-7.55(\mathrm{~m}, 2 \mathrm{H})$, 7.52-7.43 (m, 2H), 7.42-7.34 (m, 3H), 6.92-6.83 (m, 2H), 1.90-1.62 (m, 6H), 1.52-1.38 (m, 2H) ppm.

${ }^{13} \mathrm{C}$ NMR (100 MHz, acetone- $\left.d_{6}\right) \delta 157.4,141.4,141.3,140.4,132.4,129.7,129.1,128.3$, $128.1,127.6,127.3,115.7,77.2,69.3,32.3,32.2,25.71,25.69$ ppm.

IR (thin film) 3384, 2969, 2870, 1614, 1598, 1515, 1487, 1439, 1384, 1268, 1225, 1169, $1101,831,764 \mathrm{~cm}^{-1}$.

HRMS (CI+) Calcd for $\mathrm{C}_{24} \mathrm{H}_{22} \mathrm{O}_{2}\left[\mathrm{M}^{+}\right]$: 342.1620, Found: 342.1623 . 


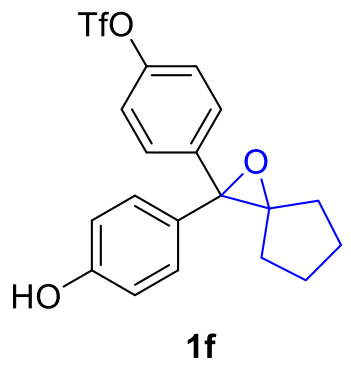

4-(2-(4-Hydroxyphenyl)-1-oxaspiro[2.4]heptan-2-yl)phenyl trifluoromethanesulfonate (1f) was prepared as a light yellow oil from $m$ CPBA $(85 \%, 0.28 \mathrm{~g}, 1.4 \mathrm{mmol})$, $\mathrm{NaHCO}_{3}(0.14 \mathrm{~g}, 1.65 \mathrm{mmol})$, and S1f $(0.44 \mathrm{~g}, 1.1 \mathrm{mmol})$ in DCM (11 mL) according to the General Procedure B (11 h, purified by flash column chromatography: hexanes/ethyl acetate $=8: 1$ to $6: 1,0.29 \mathrm{~g}, 63 \%$ yield).

${ }^{1} \mathrm{H}$ NMR (400 MHz, acetone- $\left.d_{6}\right) \delta 8.52(\mathrm{~s}, 1 \mathrm{H}), 7.75-7.65(\mathrm{~m}, 2 \mathrm{H}), 7.51-7.42(\mathrm{~m}, 2 \mathrm{H})$, 7.41-7.32 (m, 2H), 6.93-6.83 (m, 2H), 1.92-1.62 (m, 6H), 1.48-1.34 (m, 2H) ppm.

${ }^{13}$ C NMR (100 MHz, acetone- $\left.d_{6}\right) \delta$ 157.7, 149.4, 143.1, 131.5, 130.0, 129.2, 121.9, 119.6 $(q, J=318.0 \mathrm{~Hz}), 115.9,77.5,68.8,32.4,32.0,25.7,25.6 \mathrm{ppm}$.

${ }^{19}$ F NMR (376 MHz, acetone- $\left.d_{6}\right) \delta-74.2 \mathrm{ppm}$.

IR (thin film) 3406, 2964, 2873, 1615, 1598, 1516, 1500, 1425, 1250, 1215, 1141, 890, 835 $\mathrm{cm}^{-1}$.

HRMS (CI+) Calcd for $\mathrm{C}_{19} \mathrm{H}_{17} \mathrm{~F}_{3} \mathrm{O}_{5} \mathrm{~S}\left[\mathrm{M}^{+}\right]$: 414.0749, Found: 414.0740.

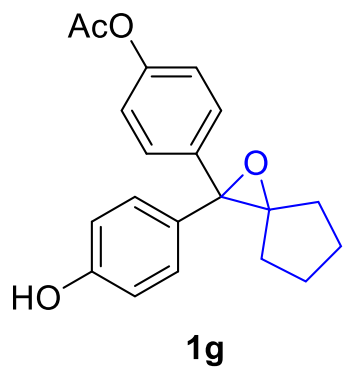

4-(2-(4-Hydroxyphenyl)-1-oxaspiro[2.4]heptan-2-yl)phenyl acetate (1g) was prepared as a light yellow oil from $m$ CPBA $(85 \%, 0.31 \mathrm{~g}, 1.5 \mathrm{mmol}), \mathrm{NaHCO}_{3}(0.15 \mathrm{~g}$, $1.8 \mathrm{mmol})$, and S1g $(0.37 \mathrm{~g}, 1.2 \mathrm{mmol})$ in DCM $(12 \mathrm{~mL})$ according to the General Procedure B (4.5 h, purified by flash column chromatography: hexanes/ethyl acetate $=6: 1 \rightarrow 4: 1,0.27 \mathrm{~g}, 70 \%$ yield $)$. 
${ }^{1}$ H NMR (400 MHz, acetone- $\left.d_{6}\right) \delta 8.42$ (brs, $\left.1 \mathrm{H}\right)$, 7.56-7.47 (m, 2H), 7.38-7.30 (m, 2H), 7.16-7.08 (m, 2H), 6.89-6.81 (m, 2H), $2.27(\mathrm{~s}, 3 \mathrm{H}), 1.88-1.60(\mathrm{~m}, 6 \mathrm{H}), 1.48-1.35(\mathrm{~m}, 2 \mathrm{H})$ ppm.

${ }^{13} \mathrm{C}$ NMR (100 MHz, acetone- $\left.d_{6}\right) \delta 169.6,157.4,150.8,139.5,132.2,129.1,128.7,122.1$, 115.7, 77.2, 69.0, 32.2, 32.1, 25.7, 25.6, 20.9 ppm.

IR (thin film) 3412, 2962, 2871, 1753, 1693, 1614, 1515, 1437, 1371, 1199, 1165, 1017, 913, $832 \mathrm{~cm}^{-1}$.

HRMS (CI+) Calcd for $\mathrm{C}_{20} \mathrm{H}_{20} \mathrm{O}_{4}\left[\mathrm{M}^{+}\right]$: 324.1362, Found: 324.1363 .

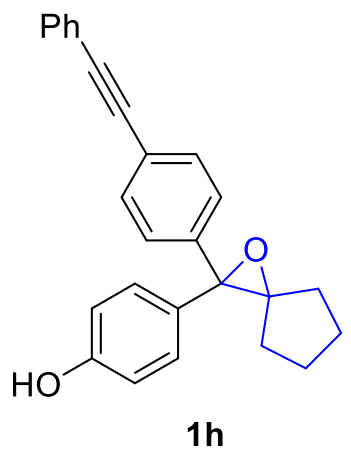

4-(2-(4-(Phenylethynyl)phenyl)-1-oxaspiro[2.4]heptan-2-yl)phenol (1h) was prepared as a yellow oil from $m$ CPBA $(85 \%, 0.31 \mathrm{~g}, 1.5 \mathrm{mmol}), \mathrm{NaHCO}_{3}(0.15 \mathrm{~g}, 1.8$ $\mathrm{mmol})$, and S1h $(0.42 \mathrm{~g}, 1.2 \mathrm{mmol})$ in DCM (12 mL) according to the General Procedure B (11 h, purified by flash column chromatography: hexanes/ethyl acetate = 6:1, $0.18 \mathrm{~g}, 40 \%$ yield).

${ }^{1} \mathrm{H}$ NMR (400 MHz, acetone- $\left.d_{6}\right) \delta 8.43(\mathrm{~s}, 1 \mathrm{H}), 7.63-7.50(\mathrm{~m}, 6 \mathrm{H}), 7.48-7.41(\mathrm{~m}, 3 \mathrm{H})$, 7.40-7.32 (m, 2H), 6.91-6.83 (m, 2H), 1.92-1.59 (m, 6H), 1.49-1.36 (m, 2H) ppm.

${ }^{13} \mathrm{C}$ NMR (100 MHz, acetone- $\left.d_{6}\right) \delta 157.5,142.7,132.3,132.0,131.9,129.4,129.3,129.2$, $128.1,124.0,122.6,115.8,90.1,89.9,77.4,69.2,32.3,32.0,25.71,25.66$ ppm.

IR (thin film) 3367, 2972, 2870, 2218, 1614, 1596, 1515, 1442, 1267, 1225, 1169, 1107, 829 $\mathrm{cm}^{-1}$.

HRMS (CI+) Calcd for $\mathrm{C}_{26} \mathrm{H}_{22} \mathrm{O}_{2}\left[\mathrm{M}^{+}\right]$: 366.1620, Found: 366.1609. 


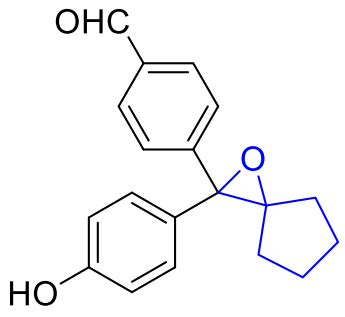

$1 \mathrm{i}$

4-(2-(4-Hydroxyphenyl)-1-oxaspiro[2.4]heptan-2-yl)benzaldehyde

(1i) was prepared as a colorless oil from $m \mathrm{CPBA}(85 \%, 0.31 \mathrm{~g}, 1.5 \mathrm{mmol}), \mathrm{NaHCO}_{3}(0.15 \mathrm{~g}, 1.8$ mmol), and S1i $(0.33 \mathrm{~g}, 1.2 \mathrm{mmol})$ in DCM $(12 \mathrm{~mL})$ according to the General Procedure B (11 h, purified by flash column chromatography: hexanes/ethyl acetate = $8: 1 \rightarrow 6: 1,0.22 \mathrm{~g}, 63 \%$ yield).

${ }^{1}$ H NMR (400 MHz, acetone- $\left.d_{6}\right) \delta 10.06$ (s, 1H), 8.47 (s, 1H), 7.98-7.89 (m, 2H), 7.76-7.69 (m, 2H), 7.43-7.36 (m, 2H), 6.91-6.85 (m, 2H), 1.93-1.83 (m, 1H), 1.82-1.65 (m, $5 \mathrm{H}), 1.49-1.36(\mathrm{~m}, 2 \mathrm{H}) \mathrm{ppm}$.

${ }^{13} \mathrm{C}$ NMR (100 MHz, acetone- $\left.d_{6}\right) \delta 192.5,157.6,148.7,136.5,131.4,130.1,129.2,128.4$, $115.8,77.5,69.2,32.3,32.0,25.7,25.6 \mathrm{ppm}$.

IR (thin film) 3381, 2962, 2870, 1698, 1607, 1516, 1438, 1385, 1265, 1211, 1170, $825 \mathrm{~cm}^{-1}$. HRMS (CI+) Calcd for $\mathrm{C}_{19} \mathrm{H}_{18} \mathrm{O}_{3}\left[\mathrm{M}^{+}\right]$: 294.1256, Found: 294.1254.

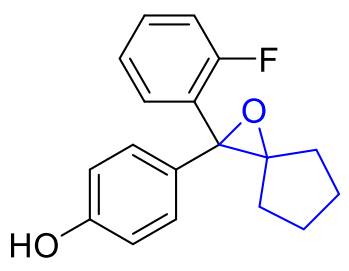

1j

4-(2-(2-Fluorophenyl)-1-oxaspiro[2.4]heptan-2-yl)phenol (1j) was prepared as a colorless oil from $m$ CPBA $(85 \%, 0.52 \mathrm{~g}, 2.5 \mathrm{mmol}), \mathrm{NaHCO}_{3}(0.25 \mathrm{~g}, 3 \mathrm{mmol})$, and S1j (0.54 g, $2 \mathrm{mmol})$ in DCM (20 mL) according to the General Procedure B (4 h, purified by flash column chromatography: hexanes/ethyl acetate $=10: 1 \rightarrow 8: 1,0.42 \mathrm{~g}, 73 \%$ yield). 
${ }^{1}$ H NMR (400 MHz, acetone- $\left.d_{6}\right) \delta 8.44(\mathrm{~s}, 1 \mathrm{H}), 7.50\left(\mathrm{td}, J_{1}=7.5 \mathrm{~Hz}, J_{2}=1.8 \mathrm{~Hz}, 1 \mathrm{H}\right)$, 7.44-7.34 (m, 1H), 7.34-7.27 (m, 2H), 7.27-7.14 (m, 2H), 6.88-6.76 (m,2H), 1.86-1.63 (m, $6 \mathrm{H}), 1.59-1.48$ (m, 1H), 1.41-1.29 (m, 1H) ppm.

${ }^{13} \mathrm{C}$ NMR (100 MHz, acetone- $\left.d_{6}\right) \delta 160.5$ (d, $\left.J=242.1 \mathrm{~Hz}\right), 157.6,131.4,130.2(\mathrm{~d}, J=8.0$ Hz), $130.1(\mathrm{~d}, J=4.9 \mathrm{~Hz}), 129.9(\mathrm{~d}, J=16.1 \mathrm{~Hz}), 128.6(\mathrm{~d}, J=2.7 \mathrm{~Hz}), 125.4(\mathrm{~d}, J=3.1$ $\mathrm{Hz}), 116.1(\mathrm{~d}, J=21.6 \mathrm{~Hz}), 115.7,76.8,66.6,32.8,31.1,25.8,25.6 \mathrm{ppm}$.

${ }^{19}$ F NMR (376 MHz, acetone- $\left.d_{6}\right) \delta-116.5 \mathrm{ppm}$.

IR (thin film) 3393, 2962, 2871, 1632, 1615, 1515, 1491, 1453, 1259, 1215, 1171, 1105, 832, $758 \mathrm{~cm}^{-1}$.

HRMS (CI+) Calcd for $\mathrm{C}_{18} \mathrm{H}_{17} \mathrm{FO}_{2}\left[\mathrm{M}^{+}\right]$: 284.1213, Found: 284.1212.

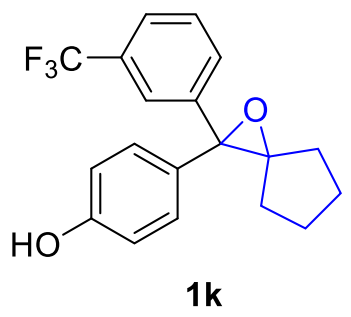

4-(2-(3-(Trifluoromethyl)phenyl)-1-oxaspiro[2.4]heptan-2-yl)phenol (1k) was prepared as a yellow oil from $m$ CPBA $(85 \%, 0.52 \mathrm{~g}, 2.5 \mathrm{mmol}), \mathrm{NaHCO}_{3}(0.25 \mathrm{~g}, 3$ $\mathrm{mmol})$, and S1k (0.63 $\mathrm{g}, 2 \mathrm{mmol})$ in DCM (20 mL) according to the General Procedure B (4 h, purified by flash column chromatography: hexanes/ethyl acetate = $10: 1 \rightarrow 8: 1,0.49 \mathrm{~g}, 74 \%$ yield).

${ }^{1}$ H NMR (400 MHz, acetone- $\left.d_{6}\right) \delta 8.50(\mathrm{~s}, 1 \mathrm{H}), 7.89-7.77(\mathrm{~m}, 2 \mathrm{H}), 7.70-7.58(\mathrm{~m}, 2 \mathrm{H})$, 7.46-7.33 (m, 2H), 6.98-6.82 (m, 2H), 1.94-1.64 (m, 6H), 1.49-1.30 (m, 2H) ppm.

${ }^{13} \mathrm{C}$ NMR (100 MHz, acetone- $\left.d_{6}\right) \delta 157.7,143.6,131.8,131.5,130.7(\mathrm{q}, J=31.8 \mathrm{~Hz})$, 129.9, 129.2, $125.3(\mathrm{q}, J=270.0 \mathrm{~Hz}), 124.6(\mathrm{q}, J=3.9 \mathrm{~Hz}), 124.2(\mathrm{q}, J=3.9 \mathrm{~Hz}), 115.9$, $77.6,68.9,32.4,32.0,25.71,25.65$ ppm.

${ }^{19}$ F NMR (376 MHz, acetone- $\left.d_{6}\right) \delta-63.0 \mathrm{ppm}$.

IR (thin film) 3396, 2964, 2873, 1614, 1516, 1440, 1335, 1264, 1228, 1167, 1126, 1073 $\mathrm{cm}^{-1}$.

HRMS (CI+) Calcd for $\mathrm{C}_{19} \mathrm{H}_{17} \mathrm{~F}_{3} \mathrm{O}_{2}\left[\mathrm{M}^{+}\right]$: 334.1181, Found: 334.1184 . 


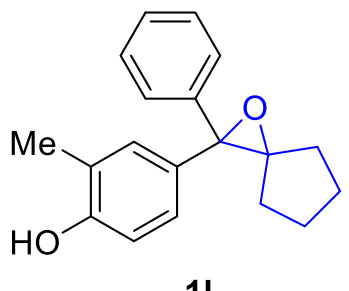

11

2-Methyl-4-(2-phenyl-1-oxaspiro[2.4]heptan-2-yl)phenol (11) was prepared as a colorless oil from $m$ CPBA $(85 \%, 0.39 \mathrm{~g}, 1.9 \mathrm{mmol}), \mathrm{NaHCO}_{3}(0.19 \mathrm{~g}, 2.25 \mathrm{mmol})$, and S11 (0.40 g, $1.5 \mathrm{mmol})$ in DCM (15 mL) according to the General Procedure B (3 h, purified by flash column chromatography: hexanes/ethyl acetate $=10: 1,0.33 \mathrm{~g}, 78 \%$ yield).

${ }^{1}$ H NMR (400 MHz, acetone- $\left.d_{6}\right) \delta 8.27(\mathrm{~s}, 1 \mathrm{H}), 7.52-7.45(\mathrm{~m}, 2 \mathrm{H}), 7.40-7.32(\mathrm{~m}, 2 \mathrm{H})$, 7.30-7.22 (m, 2H), $7.16\left(\mathrm{dd}, J_{1}=8.2 \mathrm{~Hz}, J_{2}=2.1 \mathrm{~Hz}, 1 \mathrm{H}\right), 6.82(\mathrm{~d}, J=8.2 \mathrm{~Hz}, 1 \mathrm{H}), 2.21(\mathrm{~s}$, $3 \mathrm{H}), 1.89-1.59(\mathrm{~m}, 6 \mathrm{H}), 1.47-1.30(\mathrm{~m}, 2 \mathrm{H}) \mathrm{ppm}$.

${ }^{13} \mathrm{C}$ NMR (100 MHz, acetone- $\left.d_{6}\right) \delta 155.3,142.3,132.4,130.3,128.7,127.75,127.66,126.3$, 124.6, 115.0, 77.0, 69.4, 32.3, 32.2, 25.68, 25.67, 16.3 ppm.

IR (thin film) 3407, 2960, 2870, 1630, 1612, 1509, 1275, 1114, 752, $701 \mathrm{~cm}^{-1}$.

HRMS (CI+) Calcd for $\mathrm{C}_{19} \mathrm{H}_{20} \mathrm{O}_{2}\left[\mathrm{M}^{+}\right]: 280.1463$, Found: 280.1461 .

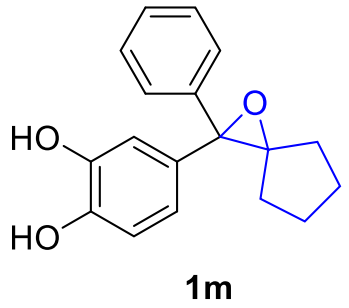

4-(2-Phenyl-1-oxaspiro[2.4]heptan-2-yl)benzene-1,2-diol $\mathbf{( 1 \mathrm { m } )}$ was prepared as a yellow oil from mCPBA $(85 \%, 0.78 \mathrm{~g}, 3.8 \mathrm{mmol}), \mathrm{NaHCO}_{3}(0.38 \mathrm{~g}, 4.5 \mathrm{mmol})$, and S1m $(0.80 \mathrm{~g}, 3 \mathrm{mmol})$ in DCM $(30 \mathrm{~mL})$ according to the General Procedure B $(3 \mathrm{~h}$, purified by flash column chromatography: hexanes/ethyl acetate $=8: 1 \rightarrow 5: 1,0.60 \mathrm{~g}$, $71 \%$ yield). The compound was too unstable to be characterized and thus used directly for the next step. 


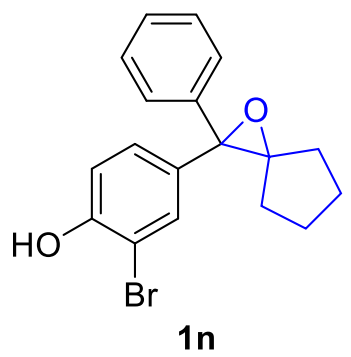

2-Bromo-4-(2-phenyl-1-oxaspiro[2.4]heptan-2-yl)phenol (1n) was prepared as a yellow oil from mCPBA $(85 \%, 0.78 \mathrm{~g}, 3.8 \mathrm{mmol}), \mathrm{NaHCO}_{3}(0.38 \mathrm{~g}, 4.5 \mathrm{mmol})$, and S1n $(0.99 \mathrm{~g}, 3 \mathrm{mmol})$ in DCM $(30 \mathrm{~mL})$ according to the General Procedure B (13 h, purified by flash column chromatography: hexanes/ethyl acetate $=8: 1,0.74 \mathrm{~g}, 71 \%$ yield).

${ }^{1}$ H NMR (400 MHz, acetone- $\left.d_{6}\right) \delta 8.93(\mathrm{~s}, 1 \mathrm{H}), 7.64(\mathrm{~d}, J=2.1 \mathrm{~Hz}, 1 \mathrm{H})$, 7.54-7.50 (m, $2 \mathrm{H}), 7.42-7.34(\mathrm{~m}, 3 \mathrm{H}), 7.33-7.27(\mathrm{~m}, 1 \mathrm{H}), 7.02(\mathrm{~d}, J=8.4 \mathrm{~Hz}, 1 \mathrm{H}), 1.87-1.65(\mathrm{~m}, 6 \mathrm{H})$, 1.49-1.33 (m, 2H) ppm.

${ }^{13} \mathrm{C}$ NMR (100 MHz, acetone- $\left.d_{6}\right) \delta 153.9,141.4,134.5,132.3,128.9,128.3,128.0,127.7$, $116.8,109.9,77.4,68.7,32.3,32.0,25.7$ (2C) ppm.

IR (thin film) 3441, 2961, 2870, 1605, 1495, 1447, 1407, 1384, 1286, 1179, 1042, $820 \mathrm{~cm}^{-1}$. HRMS (LD+) Calcd for $\mathrm{C}_{18} \mathrm{H}_{18} \mathrm{BrO}_{2}\left[\mathrm{M}^{+}+\mathrm{H}\right]: 345.0490$, Found: 345.0480 .

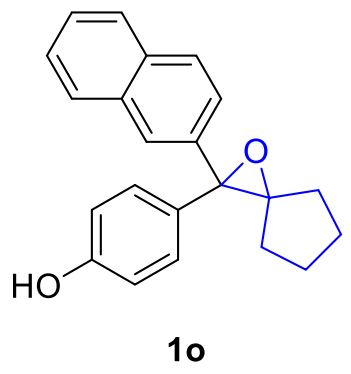

4-(2-(Naphthalen-2-yl)-1-oxaspiro[2.4]heptan-2-yl)phenol (10) was prepared as a yellow oil from $m$ CPBA $(85 \%, 0.65 \mathrm{~g}, 3.2 \mathrm{mmol}), \mathrm{NaHCO}_{3}(0.32 \mathrm{~g}, 3.75 \mathrm{mmol})$, and S1o $(0.75 \mathrm{~g}, 2.5 \mathrm{mmol})$ in DCM $(25 \mathrm{~mL})$ according to the General Procedure B (4 h, purified by flash column chromatography: hexanes/ethyl acetate $=8: 1,0.59 \mathrm{~g}, 74 \%$ yield). 
${ }^{1}$ H NMR (400 MHz, acetone- $\left.d_{6}\right) \delta 8.42(\mathrm{~s}, 1 \mathrm{H}), 8.04(\mathrm{~s}, 1 \mathrm{H})$, 7.98-7.85 (m, 3H), 7.66 (dd, $\left.J_{1}=8.5 \mathrm{~Hz}, J_{2}=1.7 \mathrm{~Hz}, 1 \mathrm{H}\right), 7.58-7.47(\mathrm{~m}, 2 \mathrm{H}), 7.47-7.39(\mathrm{~m}, 2 \mathrm{H}), 6.93-6.83(\mathrm{~m}, 2 \mathrm{H})$, 1.95-1.63 (m, 6H), 1.56-1.37 (m, 2H) ppm.

${ }^{13}$ C NMR (100 MHz, acetone- $\left.d_{6}\right) \delta$ 157.4, 139.7, 134.1, 133.5, 132.4, 129.2, 128.7, 128.45, $128.43,127.0,126.7,126.3,126.1,115.7,77.4,69.5,32.3,32.2,25.71,25.68$ ppm.

IR (thin film) 3381, 2961, 2870, 1613, 1597, 1514, 1436, 1265, 1228, 1170, $818 \mathrm{~cm}^{-1}$. HRMS (LD+) Calcd for $\mathrm{C}_{22} \mathrm{H}_{20} \mathrm{O}_{2}\left[\mathrm{M}^{+}\right]$: 316.1463, Found: 316.1471 .

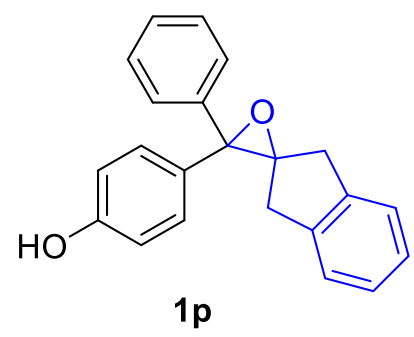

4-(3'-Phenyl-1,3-dihydrospiro[indene-2,2'-oxiran]-3'-yl)phenol (1p) was prepared as a yellow oil from $m$ CPBA $(85 \%, 0.90 \mathrm{~g}, 4.4 \mathrm{mmol}), \mathrm{NaHCO}_{3}(0.44 \mathrm{~g}, 5.2 \mathrm{mmol})$, and S1p $(1.19 \mathrm{~g}, 4 \mathrm{mmol})$ in DCM $(40 \mathrm{~mL})$ according to the General Procedure B $(24 \mathrm{~h}$, purified by flash column chromatography: hexanes/ethyl acetate $=10: 1 \rightarrow 8: 1,0.87 \mathrm{~g}$, $69 \%$ yield).

${ }^{1} \mathbf{H}$ NMR $\left(400 \mathrm{MHz} \mathrm{CDCl}_{3}\right) \delta$ 7.49-7.41 (m, 2H), 7.38-7.23 (m, 5H), 7.18-7.07 (m, 4H), 6.82-6.75 (m, 2H), $5.68(\mathrm{~s}, 1 \mathrm{H}), 3.13\left(\mathrm{dd}, J_{1}=20.9 \mathrm{~Hz}, J_{2}=18.4 \mathrm{~Hz}, 2 \mathrm{H}\right), 2.88\left(\mathrm{dd}, J_{1}=\right.$ $\left.18.3 \mathrm{~Hz}, J_{2}=10.6 \mathrm{~Hz}, 2 \mathrm{H}\right)$ ppm.

${ }^{13} \mathrm{C}$ NMR $\left(100 \mathrm{MHz}, \mathrm{CDCl}_{3}\right) \delta$ 155.0, 140.09, 140.07, 139.5, 131.4, 128.5, 128.2 (2C), $127.4,127.0,126.7$ (2C), 124.5, 115.1, 75.1, 69.4, 38.1, 38.0 ppm.

IR (thin film) 3396, 3059, 3027, 1613, 1597, 1514, 1446, 1265, 1224, 1171, 830, $738 \mathrm{~cm}^{-1}$. HRMS (CI+) Calcd for $\mathrm{C}_{22} \mathrm{H}_{18} \mathrm{O}_{2}\left[\mathrm{M}^{+}\right]$: 314.1307, Found: 314.1311. 


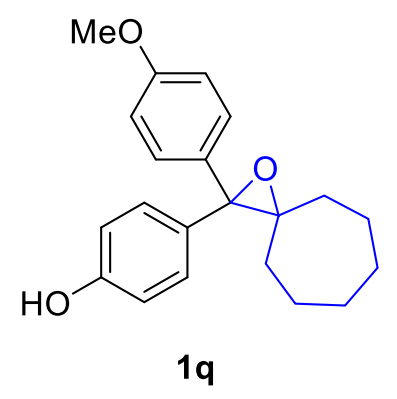

4-(2-(4-Methoxyphenyl)-1-oxaspiro[2.6]nonan-2-yl)phenol (1q) was prepared as a colorless oil from $m \mathrm{CPBA}(85 \%, 0.39 \mathrm{~g}, 1.9 \mathrm{mmol}), \mathrm{NaHCO}_{3}(0.19 \mathrm{~g}, 2.25 \mathrm{mmol})$, and S1q $(0.46 \mathrm{~g}, 1.5 \mathrm{mmol})$ in DCM $(15 \mathrm{~mL})$ according to the General Procedure B $(1 \mathrm{~h}$, purified by flash column chromatography: hexanes/ethyl acetate $=8: 1 \rightarrow 6: 1,0.34 \mathrm{~g}$, $70 \%$ yield).

${ }^{1}$ H NMR (400 MHz, acetone- $\left.d_{6}\right) \delta 8.36(\mathrm{~s}, 1 \mathrm{H}), 7.47-7.39(\mathrm{~m}, 2 \mathrm{H}), 7.38-7.31(\mathrm{~m}, 2 \mathrm{H})$, 6.94-6.86 (m, 2H), 6.85-6.78 (m, 2H), $3.79(\mathrm{~s}, 3 \mathrm{H}), 1.79-1.48(\mathrm{~m}, 10 \mathrm{H}), 1.48-1.36(\mathrm{~m}, 2 \mathrm{H})$ ppm.

${ }^{13} \mathrm{C}$ NMR (100 MHz, acetone- $\left.d_{6}\right) \delta 160.2,157.8,135.4,134.0,129.73,129.66,116.3,114.9$, $72.6,72.0,56.2,35.0(2 \mathrm{C}), 34.9(2 \mathrm{C}), 25.6(2 \mathrm{C}) \mathrm{ppm}$.

IR (thin film) 3405, 2928, 2855, 1612, 1513, 1443, 1247, 1170, 1111, 1034, $829 \mathrm{~cm}^{-1}$.

HRMS (CI+) Calcd for $\mathrm{C}_{21} \mathrm{H}_{25} \mathrm{O}_{3}\left[\mathrm{M}^{+}+\mathrm{H}\right]$ : 325.1804, Found: 325.1806.

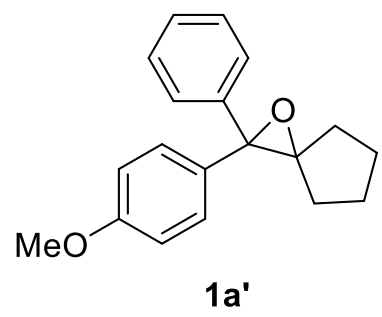

2-(4-methoxyphenyl)-2-phenyl-1-oxaspiro[2.4]heptane (1a') was prepared as a colorless oil from $m$ CPBA $(85 \%, 1.06 \mathrm{~g}, 5.2 \mathrm{mmol}), \mathrm{NaHCO}_{3}(0.50 \mathrm{~g}, 6 \mathrm{mmol})$, and S1a' (1.06 g, $4 \mathrm{mmol})$ in DCM (40 mL) according to the General Procedure B (14 h, purified by flash column chromatography: hexanes/ethyl acetate $=20: 1,1.05 \mathrm{~g}, 94 \%$ yield). 
${ }^{1}$ H NMR (400 MHz, acetone- $\left.d_{6}\right) \delta$ 7.55-7.48 (m, 2H), 7.48-7.41 (m, 2H), 7.41-7.33 (m, 2H), 7.32-7.24 (m, 1H), 6.98-6.90 (m, 2H), $3.80(\mathrm{~s}, 3 \mathrm{H}), 1.88-1.58(\mathrm{~m}, 6 \mathrm{H}), 1.49-1.33$ (m, 2H) ppm.

${ }^{13} \mathrm{C}$ NMR (100 MHz, acetone- $\left.d_{6}\right) \delta 159.7,141.9,133.6,129.0,128.8,127.8,127.7,114.2$, $77.1,69.2,55.4,32.19,32.17,25.67,25.66$ ppm.

IR (thin film) 2958, 2869, 1610, 1510, 1446, 1245, 1171, 1034, $825 \mathrm{~cm}^{-1}$.

HRMS (CI+) Calcd for $\mathrm{C}_{19} \mathrm{H}_{20} \mathrm{O}_{2}\left[\mathrm{M}^{+}\right]$: 280.1463, Found: 280.1454.

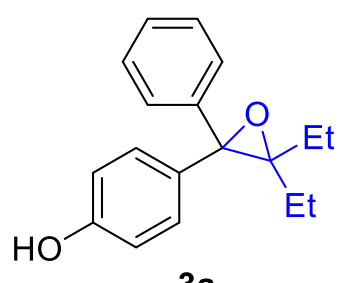

$3 \mathbf{a}$

4-(3,3-Diethyl-2-phenyloxiran-2-yl)phenol (3a) was prepared as a yellow oil from $m$ CPBA $(85 \%, 0.90 \mathrm{~g}, 4.4 \mathrm{mmol}), \mathrm{NaHCO}_{3}(0.44 \mathrm{~g}, 5.2 \mathrm{mmol})$, and S3a $(1.01 \mathrm{~g}, 4 \mathrm{mmol})$ in DCM $(40 \mathrm{~mL})$ according to the General Procedure B at room temperature $(3 \mathrm{~h}$, purified by flash column chromatography: hexanes/ethyl acetate $=15: 1,0.76 \mathrm{~g}, 70 \%$ yield).

${ }^{1} \mathrm{H}$ NMR (400 MHz, $\left.\mathrm{CDCl}_{3}\right) \delta$ 7.46-7.39 (m, 2H), 7.35-7.25 (m, 4H), 7.23-7.17 (m, 1H), 6.82-6.73 (m, 2H), $5.72(\mathrm{~s}, 1 \mathrm{H}), 1.53-1.35(\mathrm{~m}, 4 \mathrm{H}), 0.92(\mathrm{t}, J=7.5 \mathrm{~Hz}, 3 \mathrm{H}), 0.90(\mathrm{t}, J=7.5$ $\mathrm{Hz}, 3 \mathrm{H}) \mathrm{ppm}$.

${ }^{13} \mathrm{C}$ NMR $\left(100 \mathrm{MHz} \mathrm{CDCl}_{3}\right) \delta 154.7,140.6,132.4,128.3,128.1,127.0,126.8,115.0,72.6$, 72.4, 23.6, 23.4, 9.11, 9.08 ppm.

IR (thin film) 3387, 2974, 2940, 2880, 1614, 1597, 1515, 1446, 1266, 1224, 1169, 909, 830 $\mathrm{cm}^{-1}$.

HRMS (CI+) Calcd for $\mathrm{C}_{18} \mathrm{H}_{20} \mathrm{O}_{2}\left[\mathrm{M}^{+}\right]$: 268.1463, Found: 268.1468. 


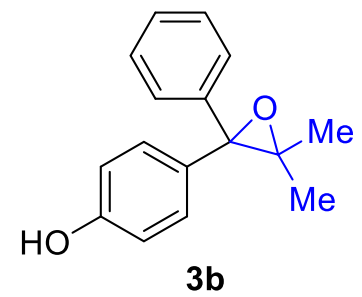

4-(3,3-Dimethyl-2-phenyloxiran-2-yl)phenol (3b) was prepared as a yellow oil from $m$ CPBA (1.04 g, 85\%, $5.1 \mathrm{mmol}), \mathrm{NaHCO}_{3}(0.51 \mathrm{~g}, 6 \mathrm{mmol})$, and S3b (0.90 g, $\left.4 \mathrm{mmol}\right)$ in DCM (40 mL) according to the General Procedure B at room temperature (13 h, purified by flash column chromatography: hexanes/ethyl acetate $=10: 1 \rightarrow 8: 1,0.76 \mathrm{~g}$, $79 \%$ yield).

${ }^{1} \mathrm{H}$ NMR (400 MHz, acetone- $\left.d_{6}\right) \delta 8.39(\mathrm{~s}, 1 \mathrm{H}), 7.56-7.48(\mathrm{~m}, 2 \mathrm{H}), 7.41-7.31(\mathrm{~m}, 4 \mathrm{H})$, 7.28-7.22 (m, 1H), 6.88-6.80 (m, 2H), $1.22(\mathrm{~s}, 3 \mathrm{H}), 1.18(\mathrm{~s}, 3 \mathrm{H}) \mathrm{ppm}$.

${ }^{13}$ C NMR (100 MHz, acetone- $\left.d_{6}\right) \delta$ 158.0, $143.2,133.5,129.8,129.5,128.5,128.4,116.4$, 72.1, 66.2, 22.8, $22.7 \mathrm{ppm}$.

IR (thin film) 3381, 3029, 2999, 2962, 2924, 2876, 1614, 1596, 1515, 1447, 1384, 1263, $1229,1196,1170,1103,1056,830 \mathrm{~cm}^{-1}$.

HRMS (CI+) Calcd for $\mathrm{C}_{16} \mathrm{H}_{16} \mathrm{O}_{2}\left[\mathrm{M}^{+}\right]$: 240.1150, Found: 240.1147 .

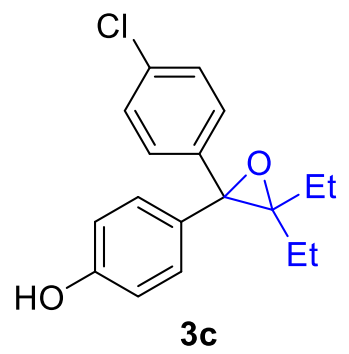

4-(2-(4-Chlorophenyl)-3,3-diethyloxiran-2-yl)phenol (3c) was prepared as a yellow oil from $m$ CPBA $(85 \%, 0.78 \mathrm{~g}, 3.8 \mathrm{mmol}), \mathrm{NaHCO}_{3}(0.38 \mathrm{~g}, 4.5 \mathrm{mmol})$, and $\mathrm{S} 3 \mathrm{c}(0.86 \mathrm{~g}$, $3 \mathrm{mmol})$ in DCM (30 mL) according to the General Procedure B at room temperature (16.5 $\mathrm{h}$, purified by flash column chromatography: hexanes/ethyl acetate $=8: 1 \rightarrow 6: 1$, $0.90 \mathrm{~g}, 99 \%$ yield). 
${ }^{1}$ H NMR (400 MHz, acetone- $\left.d_{6}\right) \delta 8.45(\mathrm{~s}, 1 \mathrm{H}), 7.60-7.49(\mathrm{~m}, 2 \mathrm{H}), 7.44-7.32(\mathrm{~m}, 4 \mathrm{H})$, 6.89-6.80 (m, 2H), 1.58-1.36 (m, 4H), $0.94(\mathrm{t}, J=7.5 \mathrm{~Hz}, 3 \mathrm{H}), 0.93(\mathrm{t}, J=7.5 \mathrm{~Hz}, 3 \mathrm{H})$ ppm.

${ }^{13} \mathrm{C}$ NMR (100 MHz, acetone- $\left.d_{6}\right) \delta 157.4,141.3,133.0,132.0,129.5,129.0,128.9,115.8$, 72.4, 72.1, 24.2, 24.1, $9.3(2 \mathrm{C}) \mathrm{ppm}$.

IR (thin film) 3386, 2974, 2940, 2880, 1614, 1596, 1515, 1489, 1459, 1440, 1264, 1227, $1169,1092,1015,914,831 \mathrm{~cm}^{-1}$.

HRMS (CI+) Calcd for $\mathrm{C}_{18} \mathrm{H}_{19} \mathrm{ClO}_{2}\left[\mathrm{M}^{+}\right]$: 302.1074, Found: 302.1063.

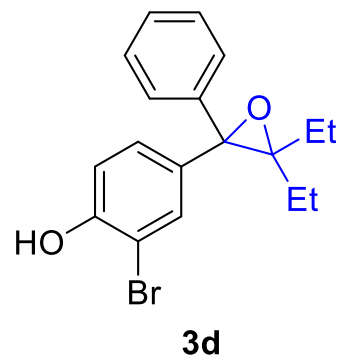

2-Bromo-4-(3,3-diethyl-2-phenyloxiran-2-yl)phenol (3d) was prepared as a yellow oil from $m$ CPBA $(85 \%, 0.78 \mathrm{~g}, 3.8 \mathrm{mmol}), \mathrm{NaHCO}_{3}(0.38 \mathrm{~g}, 4.5 \mathrm{mmol})$, and S3d $(0.99 \mathrm{~g}$, $3 \mathrm{mmol})$ in DCM (30 mL) according to the General Procedure B at room temperature (17 h, purified by flash column chromatography: hexanes/ethyl acetate $=8: 1,0.85 \mathrm{~g}$, $82 \%$ yield).

${ }^{1}$ H NMR (400 MHz, acetone- $\left.d_{6}\right) \delta 8.94(\mathrm{~s}, 1 \mathrm{H}), 7.67(\mathrm{~d}, J=2.1 \mathrm{~Hz}, 1 \mathrm{H})$, 7.59-7.50 (m, 2H), 7.45-7.33 (m, 3H), 7.31-7.24 (m, 1H), $7.01(\mathrm{~d}, J=8.4 \mathrm{~Hz}, 1 \mathrm{H}), 1.54-1.34(\mathrm{~m}, 4 \mathrm{H})$, $0.96(\mathrm{t}, J=7.5 \mathrm{~Hz}, 3 \mathrm{H}), 0.93(\mathrm{t}, J=7.5 \mathrm{~Hz}, 3 \mathrm{H}) \mathrm{ppm}$.

${ }^{13} \mathrm{C}$ NMR (100 MHz, acetone- $\left.d_{6}\right) \delta 153.9,141.6,134.6,132.3,129.0,128.3,127.9,127.7$, $116.9,109.9,72.4,72.0,24.3,24.0,9.33,9.29$ ppm.

IR (thin film) 3434, 2973, 2939, 1605, 1495, 1446, 1406, 1384, 1331, 1287, 1172, 1042, 928, $822 \mathrm{~cm}^{-1}$.

HRMS (LD+) Calcd for $\mathrm{C}_{18} \mathrm{H}_{19} \mathrm{BrO}_{2}\left[\mathrm{M}^{+}\right]$: 346.0568, Found: 346.0573. 


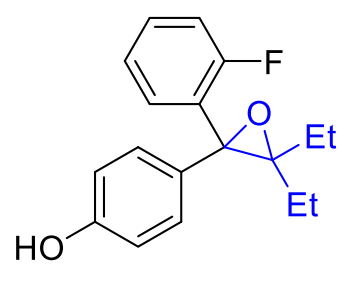

$3 e$

4-(3,3-Diethyl-2-(2-fluorophenyl)oxiran-2-yl)phenol (3e) was prepared as a yellow oil from $m$ CPBA $(85 \%, 0.18 \mathrm{~g}, 0.91 \mathrm{mmol}), \mathrm{NaHCO}_{3}(88.6 \mathrm{mg}, 1.05 \mathrm{mmol})$, and S3e $(0.19 \mathrm{~g}, 0.7 \mathrm{mmol})$ in DCM $(10 \mathrm{~mL})$ according to the General Procedure B at room temperature $(10 \mathrm{~h}$, purified by flash column chromatography: hexanes/ethyl acetate $=8: 1,0.18 \mathrm{~g}, 91 \%$ yield $)$.

${ }^{1}$ H NMR (400 MHz, acetone- $\left.d_{6}\right) \delta 8.42(\mathrm{~s}, 1 \mathrm{H}), 7.56-7.43(\mathrm{~m}, 1 \mathrm{H}), 7.43-7.36(\mathrm{~m}, 2 \mathrm{H})$, 7.36-7.29 (m, 1H), 7.25-7.10 (m, 2H), 6.88-6.78 (m, 2H), 1.84-1.58 (m, 2H), 1.42-1.30 (m, $1 \mathrm{H}), 1.30-1.18(\mathrm{~m}, 1 \mathrm{H}), 0.97(\mathrm{t}, J=7.5 \mathrm{~Hz}, 3 \mathrm{H}), 0.93(\mathrm{t}, J=7.6 \mathrm{~Hz}, 3 \mathrm{H}) \mathrm{ppm}$.

${ }^{13} \mathrm{C}$ NMR (100 MHz, acetone- $\left.d_{6}\right) \delta 159.8(\mathrm{~d}, J=242.6 \mathrm{~Hz}), 157.5,131.6,130.5(\mathrm{~d}, J=4.4$ Hz), 130.1 (d, J = 19.6 Hz), 130.0 (d, J = 8.0 Hz), 128.7 (d, J = 2.9 Hz), 125.4, 116.1 (d, J = 21.3 Hz), 115.7, 72.1, 69.3, 24.5, 23.4, 9.23, 9.22 ppm.

${ }^{19}$ F NMR (376 MHz, acetone- $\left.d_{6}\right) \delta-115.5 \mathrm{ppm}$.

IR (ATR) 3387, 2973, 2941, 2881, 1614, 1514, 1489, 1452, 1259, 1219, 1168, 1100, 1081, $916 \mathrm{~cm}^{-1}$.

HRMS (CI+) Calcd for $\mathrm{C}_{18} \mathrm{H}_{20} \mathrm{FO}_{2}\left[\mathrm{M}^{+}+\mathrm{H}\right]:$ 287.1447, Found: 287.1443.

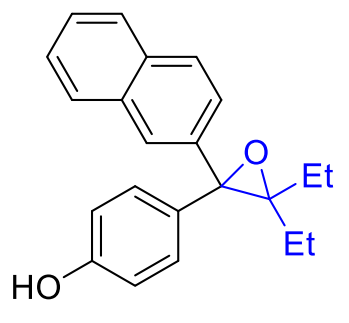

$3 f$

4-(3,3-Diethyl-2-(naphthalen-2-yl)oxiran-2-yl)phenol (3f) was prepared as a yellow solid from $m$ CPBA $(85 \%, 0.26 \mathrm{~g}, 1.3 \mathrm{mmol}), \mathrm{NaHCO}_{3}(0.13 \mathrm{~g}, 1.5 \mathrm{mmol})$, and S3f $(0.30$ g, $1 \mathrm{mmol})$ in DCM $(10 \mathrm{~mL})$ according to the General Procedure B at room temperature $(14 \mathrm{~h}$, purified by flash column chromatography: hexanes/ethyl acetate 
$=8: 1 \rightarrow 4: 1,0.19 \mathrm{~g}, 60 \%$ yield $)$.

${ }^{1} \mathrm{H}$ NMR $\left(400 \mathrm{MHz}\right.$, acetone- $\left.d_{6}\right) \delta 8.41(\mathrm{~s}, 1 \mathrm{H}), 8.06(\mathrm{~s}, 1 \mathrm{H}), 7.99-7.92(\mathrm{~m}, 1 \mathrm{H}), 7.89(\mathrm{~d}, J$ $=8.4 \mathrm{~Hz}, 2 \mathrm{H}), 7.70\left(\mathrm{dd}, J_{1}=8.5 \mathrm{~Hz}, J_{2}=1.7 \mathrm{~Hz}, 1 \mathrm{H}\right), 7.57-7.42(\mathrm{~m}, 4 \mathrm{H}), 6.91-6.83(\mathrm{~m}$, 2H), 1.62-1.38 (m, 4H), $0.99(\mathrm{t}, J=7.5 \mathrm{~Hz}, 3 \mathrm{H}), 0.94(\mathrm{t}, J=7.5 \mathrm{~Hz}, 3 \mathrm{H}) \mathrm{ppm}$.

${ }^{13} \mathrm{C}$ NMR (100 MHz, acetone- $\left.d_{6}\right) \delta 157.3,139.9,134.1,133.4,132.5,129.2,128.7,128.5$, $128.4,127.0,126.6,126.3,126.2,115.7,72.8,72.4,24.3,24.2,9.4$ (2C) ppm.

IR (ATR) 3372, 2972, 2938, 2879, 1613, 1595, 1514, 1459, 1437, 1263, 1223, 1168, 818 $\mathrm{cm}^{-1}$.

HRMS (CI+) Calcd for $\mathrm{C}_{22} \mathrm{H}_{22} \mathrm{O}_{2}\left[\mathrm{M}^{+}\right]$: 318.1620, Found: 318.1623.

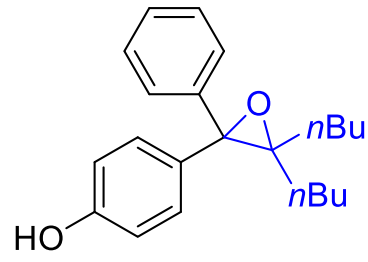

$3 \mathbf{g}$

4-(3,3-Dibutyl-2-phenyloxiran-2-yl)phenol (3g) was prepared as a yellow oil from $m$ CPBA (85\%, $0.78 \mathrm{~g}, 3.8 \mathrm{mmol}), \mathrm{NaHCO}_{3}(0.38 \mathrm{~g}, 4.5 \mathrm{mmol})$, and S3g $(0.93 \mathrm{~g}, 3 \mathrm{mmol})$ in DCM (30 mL) according to the General Procedure B at room temperature $(17.5 \mathrm{~h}$, purified by flash column chromatography: hexanes/ethyl acetate $=8: 1,0.91 \mathrm{~g}, 94 \%$ yield).

${ }^{1} \mathbf{H}$ NMR $\left(400 \mathrm{MHz}\right.$, acetone- $\left.d_{6}\right) \delta 8.36(\mathrm{~s}, 1 \mathrm{H}), 7.57-7.49(\mathrm{~m}, 2 \mathrm{H}), 7.42-7.29(\mathrm{~m}, 4 \mathrm{H})$, $7.25\left(\mathrm{tt}, J_{1}=7.4 \mathrm{~Hz}, J_{2}=1.5 \mathrm{~Hz}, 1 \mathrm{H}\right), 6.88-6.80(\mathrm{~m}, 2 \mathrm{H}), 1.54-1.31(\mathrm{~m}, 8 \mathrm{H}), 1.28-1.11(\mathrm{~m}$, $4 \mathrm{H}), 0.82(\mathrm{t}, J=7.1 \mathrm{~Hz}, 3 \mathrm{H}), 0.79(\mathrm{t}, J=7.2 \mathrm{~Hz}, 3 \mathrm{H}) \mathrm{ppm}$.

${ }^{13} \mathrm{C}$ NMR (100 MHz, acetone- $\left.d_{6}\right) \delta 157.2,142.3,132.6,129.1,128.7,127.8,127.6,115.6$, $71.9,71.0,31.6,31.5,27.9,27.8,23.55,23.50,14.13,14.07$ ppm.

IR (thin film) 3396, 2957, 2930, 2871, 1614, 1596, 1515, 1447, 1267, 1216, 1168, $830 \mathrm{~cm}^{-1}$. HRMS (CI+) Calcd for $\mathrm{C}_{22} \mathrm{H}_{28} \mathrm{O}_{2}\left[\mathrm{M}^{+}\right]$: 324.2089, Found: 324.2093. 


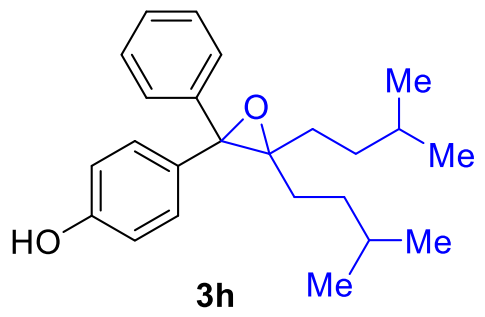

4-(3,3-Diisopentyl-2-phenyloxiran-2-yl)phenol (3h) was prepared as a yellow oil from $m$ CPBA $(85 \%, 0.79 \mathrm{~g}, 3.9 \mathrm{mmol}), \mathrm{NaHCO}_{3}(0.38 \mathrm{~g}, 4.5 \mathrm{mmol})$, and S3h (1.01 g, 3 $\mathrm{mmol})$ in DCM (30 $\mathrm{mL})$ according to the General Procedure B at room temperature ( $3 \mathrm{~h}$, purified by flash column chromatography: hexanes/ethyl acetate $=8: 1,0.72 \mathrm{~g}$, $68 \%$ yield).

${ }^{1}$ H NMR (400 MHz, acetone- $\left.d_{6}\right) \delta 8.36(\mathrm{~s}, 1 \mathrm{H}), 7.58-7.49(\mathrm{~m}, 2 \mathrm{H}), 7.42-7.31(\mathrm{~m}, 4 \mathrm{H})$, $7.25\left(\mathrm{tt}, J_{1}=7.4 \mathrm{~Hz}, J_{2}=1.5 \mathrm{~Hz}, 1 \mathrm{H}\right), 6.88-6.80(\mathrm{~m}, 2 \mathrm{H}), 1.56-1.24(\mathrm{~m}, 10 \mathrm{H}), 0.80(\mathrm{~d}, J=$ $6.2 \mathrm{~Hz}, 3 \mathrm{H}), 0.77(\mathrm{~d}, J=6.1 \mathrm{~Hz}, 3 \mathrm{H}), 0.76(\mathrm{~d}, J=6.3 \mathrm{~Hz}, 3 \mathrm{H}), 0.72(\mathrm{~d}, J=6.1 \mathrm{~Hz}, 3 \mathrm{H})$ ppm.

${ }^{13} \mathrm{C}$ NMR (100 MHz, acetone- $\left.d_{6}\right) \delta 157.3,142.3,132.5,129.1,128.7,127.9,127.6,115.6$, 72.1, 71.2, 34.7, 34.6, 29.7, 29.6, 28.9, 28.8, 22.93, 22.89, 22.4, 22.3 ppm.

IR (ATR) 3377, 2955, 2928, 2869, 1614, 1595, 1514, 1467, 1447, 1385, 1367, 1264, 1215, $1168,826 \mathrm{~cm}^{-1}$.

HRMS (CI+) Calcd for $\mathrm{C}_{24} \mathrm{H}_{33} \mathrm{O}_{2}\left[\mathrm{M}^{+}+\mathrm{H}\right]$ : 353.2481, Found: 353.2475.

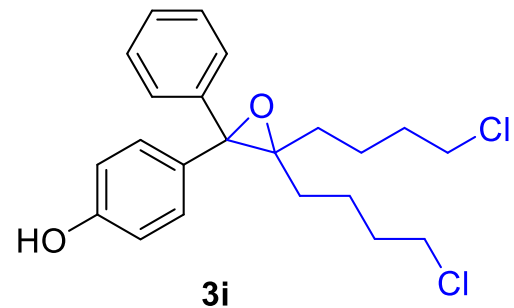

4-(3,3-Bis(4-chlorobutyl)-2-phenyloxiran-2-yl)phenol (3i) was prepared as a colorless oil from $m \mathrm{CPBA}(85 \%, 0.39 \mathrm{~g}, 1.9 \mathrm{mmol}), \mathrm{NaHCO}_{3}(0.19 \mathrm{~g}, 2.25 \mathrm{mmol})$, and S3i $(0.57 \mathrm{~g}, 1.5 \mathrm{mmol})$ in DCM $(15 \mathrm{~mL})$ according to the General Procedure B at room temperature $(11.5 \mathrm{~h}$, purified by flash column chromatography: hexanes/ethyl acetate $=8: 1,0.46 \mathrm{~g}, 78 \%$ yield). 
${ }^{1}$ H NMR (400 MHz, acetone- $\left.d_{6}\right) \delta 8.39(\mathrm{~s}, 1 \mathrm{H}), 7.58-7.49(\mathrm{~m}, 2 \mathrm{H})$, 7.44-7.32 (m, 4H), $7.26\left(\mathrm{tt}, J_{1}=7.4 \mathrm{~Hz}, J_{2}=1.5 \mathrm{~Hz}, 1 \mathrm{H}\right), 6.89-6.81(\mathrm{~m}, 2 \mathrm{H}), 3.60-3.40(\mathrm{~m}, 4 \mathrm{H}), 1.76-1.56(\mathrm{~m}$, $8 \mathrm{H}), 1.56-1.38(\mathrm{~m}, 4 \mathrm{H}) \mathrm{ppm}$.

${ }^{13} \mathrm{C}$ NMR (100 MHz, acetone- $\left.d_{6}\right) \delta 157.3,142.0,132.3,129.1,128.8,127.8,127.7,115.7$, $71.9,70.5,45.4,45.3,33.4,33.3,31.0,30.9,22.98,22.96$ ppm.

IR (thin film) 3395, 2957, 2868, 1614, 1596, 1514, 1446, 1265, 1225, 1168, 1103, $831 \mathrm{~cm}^{-1}$. HRMS (CI+) Calcd for $\mathrm{C}_{22} \mathrm{H}_{26} \mathrm{Cl}_{2} \mathrm{O}_{2}\left[\mathrm{M}^{+}\right]$: 392.1310, Found: 392.1318.

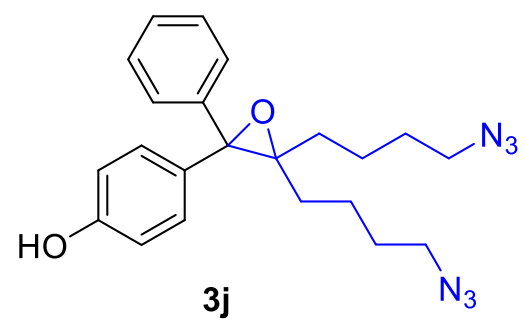

4-(3,3-Bis(4-azidobutyl)-2-phenyloxiran-2-yl)phenol (3j) was prepared as a colorless oil from $m$ CPBA (85\%, $39.8 \mathrm{mg}, 0.2 \mathrm{mmol}), \mathrm{NaHCO}_{3}(19.0 \mathrm{mg}, 0.225 \mathrm{mmol})$, and S3j (58.2 $\mathrm{mg}, 0.15 \mathrm{mmol})$ in DCM (2 mL) according to the General Procedure B at room temperature $(10 \mathrm{~h}$, purified by flash column chromatography: hexanes/ethyl acetate $=8: 1 \rightarrow 5: 1,43.9 \mathrm{mg}, 72 \%$ yield $)$.

${ }^{1}$ H NMR (400 MHz, acetone- $\left.d_{6}\right) \delta 8.41(\mathrm{~s}, 1 \mathrm{H}), 7.58-7.48(\mathrm{~m}, 2 \mathrm{H}), 7.44-7.31(\mathrm{~m}, 4 \mathrm{H})$, $7.26\left(\mathrm{tt}, J_{1}=7.4 \mathrm{~Hz}, J_{2}=1.5 \mathrm{~Hz}, 1 \mathrm{H}\right), 6.89-6.79(\mathrm{~m}, 2 \mathrm{H}), 3.26(\mathrm{t}, J=6.8 \mathrm{~Hz}, 2 \mathrm{H}), 3.23(\mathrm{t}, J$ $=6.9 \mathrm{~Hz}, 2 \mathrm{H}), 1.66-1.35(\mathrm{~m}, 12 \mathrm{H}) \mathrm{ppm}$.

${ }^{13} \mathrm{C}$ NMR (100 MHz, acetone- $\left.d_{6}\right) \delta 157.3,142.0,132.3,129.1,128.9,127.8,127.7,115.7$, $71.9,70.6,51.63,51.57,31.3,31.2,29.6,29.5,22.88,22.85$ ppm.

IR (ATR) 3382, 2937, 2867, 2093, 1613, 1595, 1514, 1447, 1264, 1169, $830 \mathrm{~cm}^{-1}$. HRMS (CI+) Calcd for $\mathrm{C}_{22} \mathrm{H}_{27} \mathrm{~N}_{6} \mathrm{O}_{2}\left[\mathrm{M}^{+}+\mathrm{H}\right]:$ 407.2195, Found: 407.2191. 


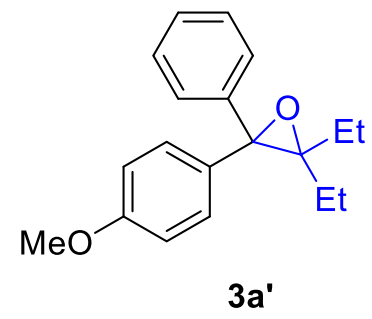

2,2-Diethyl-3-(4-methoxyphenyl)-3-phenyloxirane (3 $\left.\mathbf{a}^{\prime}\right)$ was prepared as a yellow oil from $m$ CPBA ( $85 \%, 0.39 \mathrm{~g}, 1.9 \mathrm{mmol}), \mathrm{NaHCO}_{3}(0.19 \mathrm{~g}, 2.25 \mathrm{mmol})$, and S3a' (0.40 $\mathrm{g}, 1.5 \mathrm{mmol})$ in DCM $(15 \mathrm{~mL})$ according to the General Procedure B at room temperature $(4 \mathrm{~h}$, purified by flash column chromatography: hexanes/ethyl acetate $=$ $30: 1,0.42 \mathrm{~g}, 98 \%$ yield).

${ }^{1}$ H NMR (400 MHz, acetone- $\left.d_{6}\right) \delta$ 7.58-7.52 $(\mathrm{m}, 2 \mathrm{H})$, 7.52-7.44 $(\mathrm{m}, 2 \mathrm{H})$, 7.39-7.31 (m, 2H), $7.26\left(\mathrm{tt}, J_{1}=7.4 \mathrm{~Hz}, J_{2}=1.5 \mathrm{~Hz}, 1 \mathrm{H}\right), 6.96-6.88(\mathrm{~m}, 2 \mathrm{H}), 3.79(\mathrm{~s}, 3 \mathrm{H}), 1.54-1.36(\mathrm{~m}$, $4 \mathrm{H}), 0.95(\mathrm{t}, J=7.5 \mathrm{~Hz}, 3 \mathrm{H}), 0.94(\mathrm{t}, J=7.5 \mathrm{~Hz}, 3 \mathrm{H}) \mathrm{ppm}$.

${ }^{13}$ C NMR (100 MHz, acetone- $\left.d_{6}\right) \delta 159.6,142.1,133.7,129.0,128.8,127.75,127.66,114.2$, 72.5, 72.2, 55.4, 24.3, 24.1, 9.4, $9.3 \mathrm{ppm}$.

IR (ATR) 2971, 2938, 1611, 1580, 1511, 1461, 1445, 1244, 1170, 1033, $827 \mathrm{~cm}^{-1}$.

HRMS (CI+) Calcd for $\mathrm{C}_{19} \mathrm{H}_{22} \mathrm{O}_{2}$ [M+]: 282.1620, Found: 282.1622. 


\section{Organocatalytic Asymmetric Rearrangement of Epoxides}

\section{General Procedure C: Formation of Cyclic Ketones}<smiles>[R]c1ccccc1C1(c2ccc(O)cc2)OC1([R])C1([R])CCCC1</smiles>

1

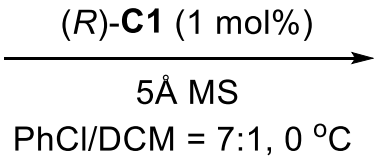

$\mathrm{PhCl} / \mathrm{DCM}=7: 1,0^{\circ} \mathrm{C}$

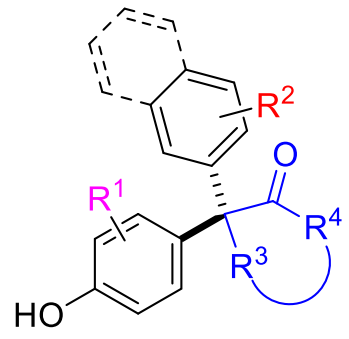

2

At $0{ }^{\circ} \mathrm{C}$, to an oven-dried vial charged with $(R)-\mathrm{C1}^{6}(2.9 \mathrm{mg}, 4 \mu \mathrm{mol}), 5 \AA \mathrm{MS}(0.16 \mathrm{~g})$, and anhydrous $\mathrm{PhCl}(14 \mathrm{~mL})$ was added a solution of the epoxide $\mathbf{1}(0.4 \mathrm{mmol})$ in anhydrous DCM (2 mL). Next, the reaction mixture was stirred at the same temperature, and the progress was monitored by TLC. Upon completion $(11.5 \sim 59 \mathrm{~h})$, the reaction mixture was filtered through a plug of silica gel (eluent: ethyl acetate). The filtrate was evaporated, and the residue was subjected to silica gel column chromatography to afford the pure product.

\section{General Procedure D: Formation of Acyclic Ketones}

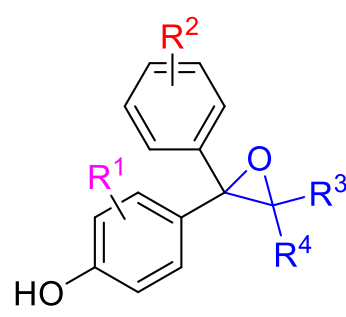

3

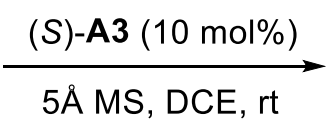<smiles>[R][R]C([R])(C([R])=O)c1ccc(O)cc1</smiles>

4

At room temperature, to an oven-dried vial charged with $(S)-\mathbf{A 3}^{7}$ (40 $\mu$ mol, 10 mol\%), $5 \AA \mathrm{MS}(0.16 \mathrm{~g})$, and anhydrous DCE $(6 \mathrm{~mL})$ was added a solution of the epoxide $3(0.4 \mathrm{mmol})$ in anhydrous DCE $(2 \mathrm{~mL})$. Next, the reaction was stirred at the 
same temperature, and the progress was monitored by TLC. Upon completion (11 24 h), the reaction mixture was filtered through a plug of silica gel (eluent: ethyl acetate). The filtrate was evaporated, and the residue was subjected to silica gel column chromatography to afford the pure product.

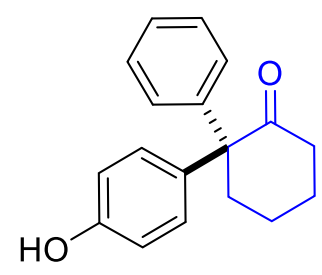

2a

(R)-2-(4-Hydroxyphenyl)-2-phenylcyclohexan-1-one (2a) was prepared as a colorless oil from $(R)-C 1(3.0 \mathrm{mg}, 4 \mu \mathrm{mol}), 5 \AA$ MS (0.16 g), and 1a $(106.5 \mathrm{mg}, 0.4$ $\mathrm{mmol})$ in $\mathrm{PhCl}(14 \mathrm{~mL})$ and DCM $(2 \mathrm{~mL})$ according to the General Procedure C (11.5 h, purified by flash column chromatography: hexanes/ethyl acetate $=8: 1 \rightarrow 6: 1,98.2$ $\mathrm{mg}$, 92\% yield, 92\% ee). A gram-scale reaction was also carried out with $(R)$-C1 (33.0 $\mathrm{mg}, 46 \mu \mathrm{mol}), 5 \AA \mathrm{MS}(1.84 \mathrm{~g})$, and $1 \mathrm{a}(1.22 \mathrm{~g}, 4.6 \mathrm{mmol})$ in $\mathrm{PhCl}(184 \mathrm{~mL})$ to form the product in $83 \%$ yield $(1.01 \mathrm{~g}, 92 \%$ ee).

$[\alpha]^{25}:-13.3\left(c=1.0, \mathrm{CHCl}_{3}\right)$. HPLC analysis of the product: Daicel CHIRALPAK AD-H column; $10 \%$ - $\mathrm{PrOH}$ in hexanes; $1.0 \mathrm{~mL} / \mathrm{min}$; retention times: $12.5 \mathrm{~min}$ (major), $14.3 \mathrm{~min}$ (minor).

${ }^{1} \mathrm{H}$ NMR $\left(400 \mathrm{MHz}, \mathrm{CDCl}_{3}\right) \delta$ 7.33-7.26 (m, 2H), 7.25-7.19 (m, 1H), 7.07-6.98 (m, 2H), 6.91-6.82 (m, 2H), 6.77-6.67 (m, 2H), $6.20(\mathrm{~s}, 1 \mathrm{H}), 2.61-2.43(\mathrm{~m}, 4 \mathrm{H}), 1.99-1.87(\mathrm{~m}, 2 \mathrm{H})$, $1.86-1.74(\mathrm{~m}, 2 \mathrm{H}) \mathrm{ppm}$.

${ }^{13} \mathrm{C}$ NMR (100 MHz, $\left.\mathrm{CDCl}_{3}\right) \delta 213.1,154.6,142.6,133.6,129.6,128.4,128.3,126.8,115.3$, $63.3,40.5,39.3,27.8,22.0 \mathrm{ppm}$.

IR (thin film) 3393, 2944, 2867, 1699, 1613, 1514, 1447, 1270, 1233, 1181, 1120, 910, 732 $\mathrm{cm}^{-1}$.

HRMS (CI+) Calcd for $\mathrm{C}_{18} \mathrm{H}_{18} \mathrm{O}_{2}\left[\mathrm{M}^{+}\right]$: 266.1307, Found: 266.1302 . 


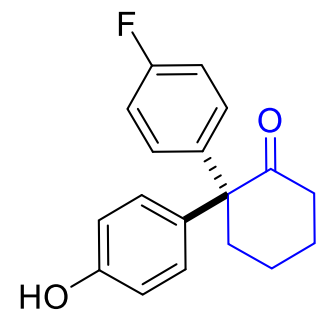

2b

(S)-2-(4-Fluorophenyl)-2-(4-hydroxyphenyl)cyclohexan-1-one (2b) was prepared as a white oil from $(R)-\mathbf{C} 1(3.0 \mathrm{mg}, 4 \mu \mathrm{mol}), 5 \AA \mathrm{MS}(0.16 \mathrm{~g})$, and $\mathbf{1 b}(113.9 \mathrm{mg}, 0.4 \mathrm{mmol})$ in $\mathrm{PhCl}(14 \mathrm{~mL})$ and DCM $(2 \mathrm{~mL})$ according to the General Procedure C $(17.5 \mathrm{~h}$, purified by flash column chromatography: hexanes/ethyl acetate $=8: 1 \rightarrow 6: 1,106.4 \mathrm{mg}$, 93\% yield, $92 \%$ ee).

$[\alpha]^{25}:-51.3\left(c=1.0, \mathrm{CHCl}_{3}\right)$. HPLC analysis of the product: Daicel CHIRALPAK AD-H column; $10 \%$ i-PrOH in hexanes; $1.0 \mathrm{~mL} / \mathrm{min}$; retention times: $11.4 \mathrm{~min}$ (major), 13.4 min (minor).

${ }^{1} \mathrm{H}$ NMR $\left(400 \mathrm{MHz}, \mathrm{CDCl}_{3}\right) \delta 6.95(\mathrm{~d}, J=6.9 \mathrm{~Hz}, 4 \mathrm{H}), 6.91(\mathrm{~d}, J=8.7 \mathrm{~Hz}, 2 \mathrm{H}), 6.76(\mathrm{~d}, J$ $=8.7 \mathrm{~Hz}, 2 \mathrm{H}), 6.23(\mathrm{brs}, 1 \mathrm{H}), 2.68-2.36(\mathrm{~m}, 4 \mathrm{H}), 2.04-1.72(\mathrm{~m}, 4 \mathrm{H}) \mathrm{ppm}$.

${ }^{13} \mathrm{C}$ NMR $\left(100 \mathrm{MHz}, \mathrm{CDCl}_{3}\right) \delta 213.0,161.4(\mathrm{~d}, J=244.5 \mathrm{~Hz}), 154.8,138.8(\mathrm{~d}, J=2.8 \mathrm{~Hz})$, 133.0, $130.1(\mathrm{~d}, J=7.8 \mathrm{~Hz}), 129.5,115.6,114.9$ (d, $J=21.1 \mathrm{~Hz}), 62.7,40.4,39.5,27.7,22.0$ ppm.

${ }^{19} \mathrm{~F}$ NMR $\left(376 \mathrm{MHz}, \mathrm{CDCl}_{3}\right) \delta-116.1 \mathrm{ppm}$.

IR (thin film) 3393, 2945, 2868, 1698, 1613, 1597, 1510, 1449, 1269, 1231, 1182, 1164, $1119,827,736 \mathrm{~cm}^{-1}$.

HRMS (CI+) Calcd for $\mathrm{C}_{18} \mathrm{H}_{17} \mathrm{FO}_{2}\left[\mathrm{M}^{+}\right]$: 284.1213, Found: 284.1213.

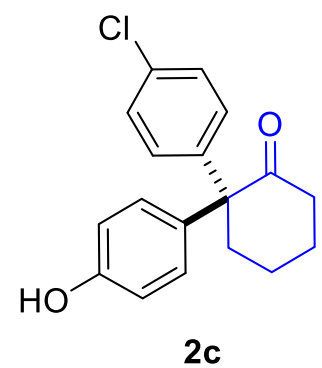

(S)-2-(4-Chlorophenyl)-2-(4-hydroxyphenyl)cyclohexan-1-one (2c) was prepared as 
a white oil from $(R)-C 1(3.0 \mathrm{mg}, 4 \mu \mathrm{mol}), 5 \AA \mathrm{MS}(0.16 \mathrm{~g})$, and 1c (120.0 mg, $0.4 \mathrm{mmol})$ in $\mathrm{PhCl}(14 \mathrm{~mL})$ and $\mathrm{DCM}(2 \mathrm{~mL})$ according to the General Procedure $\mathrm{C}(14 \mathrm{~h}$, purified by flash column chromatography: hexanes/ethyl acetate $=8: 1 \rightarrow 6: 1,114.7 \mathrm{mg}$, $96 \%$ yield, $92 \%$ ee).

Gram scale reaction: 2c was prepared as a yellow oil from $(R)-\mathbf{C 1}(50.5 \mathrm{mg}, 70 \mu \mathrm{mol})$, $5 \AA$ MS $(2.8 \mathrm{~g})$, and $1 \mathrm{c}(2.11 \mathrm{~g}, 7 \mathrm{mmol})$ in $\mathrm{PhCl}(280 \mathrm{~mL})$ according to the General Procedure C (17.5 h, purified by flash column chromatography: hexanes/ethyl acetate $=8: 1$ to $6: 1,2.08 \mathrm{~g}, 98 \%$ yield, $91 \%$ ee).

$[\alpha]^{25}:-49.4\left(c=1.0, \mathrm{CHCl}_{3}\right)$. HPLC analysis of the product: Daicel CHIRALPAK AD-H column; $10 \%$ - $\mathrm{PrOH}$ in hexanes; $1.0 \mathrm{~mL} / \mathrm{min}$; retention times: $11.8 \mathrm{~min}$ (major), $14.7 \mathrm{~min}$ (minor).

${ }^{1} \mathrm{H}$ NMR $\left(400 \mathrm{MHz}, \mathrm{CDCl}_{3}\right) \delta$ 7.29-7.18 (m, 2H), $6.92(\mathrm{~d}, J=8.5 \mathrm{~Hz}, 4 \mathrm{H}), 6.81-6.71(\mathrm{~m}$, 2H), 5.96 (brs, 1H), 2.70-2.34 (m, 4H), 2.05-1.70 (m, 4H) ppm.

${ }^{13} \mathrm{C}$ NMR $\left(100 \mathrm{MHz}, \mathrm{CDCl}_{3}\right) \delta 212.5,154.8,141.8,132.7,132.6,129.9,129.5,128.3,115.6$, 62.8, 40.4, 39.3, 27.7, $22.0 \mathrm{ppm}$.

IR (thin film) 3394, 2945, 2867, 1699, 1613, 1593, 1514, 1492, 1267, 1232, 1182, 1120, $1096,1014,824 \mathrm{~cm}^{-1}$.

HRMS (CI+) Calcd for $\mathrm{C}_{18} \mathrm{H}_{17} \mathrm{ClO}_{2}\left[\mathrm{M}^{+}\right]$: 300.0917, Found: 300.0912.

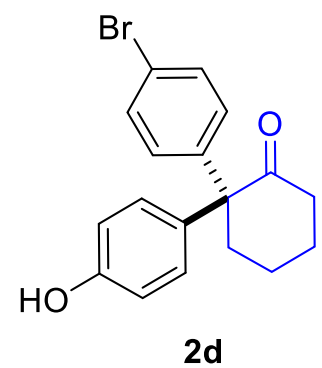

(S)-2-(4-Bromophenyl)-2-(4-hydroxyphenyl)cyclohexan-1-one (2d) was prepared as a colorless oil from $(R)-C 1(3.0 \mathrm{mg}, 4 \mu \mathrm{mol}), 5 \AA \mathrm{MS}(0.16 \mathrm{~g})$, and 1d (138.0 mg, 0.4 $\mathrm{mmol})$ in $\mathrm{PhCl}(14 \mathrm{~mL})$ and DCM (2 mL) according to the General Procedure $\mathrm{C}(18 \mathrm{~h}$, purified by flash column chromatography: hexanes/ethyl acetate $=8: 1 \rightarrow 6: 1,128.8 \mathrm{mg}$, 93\% yield, 93\% ee). 
$[\alpha]_{\mathrm{D}^{25}}:-20.9\left(c=1.0, \mathrm{CHCl}_{3}\right)$. HPLC analysis of the product: Daicel CHIRALPAK AD-H column; $10 \%$ - $\mathrm{PrOH}$ in hexanes; $1.0 \mathrm{~mL} / \mathrm{min}$; retention times: $12.6 \mathrm{~min}$ (major), 16.0 min (minor).

${ }^{1} \mathrm{H}$ NMR $\left(400 \mathrm{MHz}, \mathrm{CDCl}_{3}\right)$ d 7.43-7.34 (m, 2H), 6.96-6.88 (m, 2H), 6.88-6.81 (m, 2H), 6.80-6.73 (m, 2H), 6.02 (brs, 1H), 2.68-2.36 (m, 4H), 2.04-1.72 (m, 4H) ppm.

${ }^{13} \mathrm{C}$ NMR $\left(100 \mathrm{MHz}, \mathrm{CDCl}_{3}\right) \delta 212.5,154.8,142.3,132.5,131.2,130.3,129.5,120.8,115.6$, 62.9, 40.4, 39.2, 27.7, 21.9 ppm.

IR (thin film) 3399, 2944, 2867, 1699, 1613, 1593, 1514, 1489, 1268, 1233, 1182, 1120, $1010,909,822 \mathrm{~cm}^{-1}$.

HRMS (CI+) Calcd for $\mathrm{C}_{18} \mathrm{H}_{17} \mathrm{BrO}_{2}\left[\mathrm{M}^{+}\right]$]: 344.0412, Found: 344.0413 .

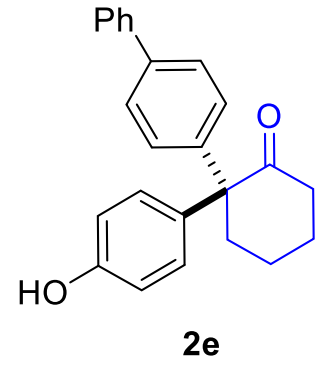

(R)-2-([1,1'-biphenyl]-4-yl)-2-(4-hydroxyphenyl)cyclohexan-1-one (2e) was prepared as a white solid from (R)-C1 (2.9 mg, $4 \mu \mathrm{mol}), 5 \AA \mathrm{MS}(0.16 \mathrm{~g})$, and 1e (137.2 $\mathrm{mg}, 0.4 \mathrm{mmol})$ in $\mathrm{PhCl}(14 \mathrm{~mL})$ and DCM $(2 \mathrm{~mL})$ according to the General Procedure $\mathrm{C}(16.5 \mathrm{~h}$, purified by flash column chromatography: hexanes/ethyl acetate $=8: 1 \rightarrow 6: 1$, $117.5 \mathrm{mg}$, 86\% yield, $90 \%$ ee).

$[\alpha]^{25}:-14.5\left(c=1.0, \mathrm{CHCl}_{3}\right)$. HPLC analysis of the product: Daicel CHIRALPAK AD-H column; 10\% $i$-PrOH in hexanes; $1.0 \mathrm{~mL} / \mathrm{min}$; retention times: 20.9 min (major), 32.1 min (minor).

${ }^{1} \mathrm{H}$ NMR (400 MHz, acetone- $\left.d_{6}\right) \delta 8.46(\mathrm{~s}, 1 \mathrm{H}), 7.73-7.65(\mathrm{~m}, 2 \mathrm{H}), 7.61(\mathrm{~d}, J=8.4 \mathrm{~Hz}$, 2H), 7.53-7.43 (m, 2H), 7.42-7.33 (m, 1H), $7.14(\mathrm{~d}, J=8.4 \mathrm{~Hz}, 2 \mathrm{H}), 7.03(\mathrm{~d}, J=8.7 \mathrm{~Hz}$, $2 \mathrm{H}), 6.93-6.85(\mathrm{~m}, 2 \mathrm{H}), 2.76-2.66(\mathrm{~m}, 1 \mathrm{H}), 2.66-2.56(\mathrm{~m}, 1 \mathrm{H}), 2.56-2.38(\mathrm{~m}, 2 \mathrm{H})$, 2.05-1.94 (m, 2H), 1.94-1.82 (m, 2H) ppm. 
${ }^{13} \mathrm{C}$ NMR (100 MHz, acetone- $\left.d_{6}\right) \delta$ 210.7, 157.1, 144.4, 141.4, 139.8, 133.5, 130.5, 130.1, $129.7,128.1,127.6,127.2,116.1,63.7,41.2,39.7,28.2,23.0$ ppm.

IR (ATR) 3242, 2960, 2936, 1680, 1616, 1597, 1511, 1484, 1451, 1266, 1121, $818 \mathrm{~cm}^{-1}$.

HRMS (CI+) Calcd for $\mathrm{C}_{24} \mathrm{H}_{22} \mathrm{O}_{2}\left[\mathrm{M}^{+}\right]$: 342.1620, Found: 342.1624.

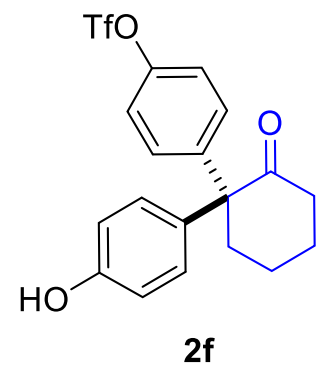

(S)-4-(1-(4-hydroxyphenyl)-2-oxocyclohexyl)phenyl trifluoromethanesulfonate (2f) was prepared as a white oil from $(R)-\mathbf{C 1}(2.9 \mathrm{mg}, 4 \mu \mathrm{mol}), 5 \AA \mathrm{MS}(0.16 \mathrm{~g})$, and $\mathbf{1 f}$ (165.6 mg, $0.4 \mathrm{mmol})$ in $\mathrm{PhCl}(14 \mathrm{~mL})$ and DCM $(2 \mathrm{~mL})$ according to the General Procedure C (26 h, purified by flash column chromatography: hexanes/ethyl acetate $=6: 1,138.6 \mathrm{mg}, 84 \%$ yield, $90 \%$ ee).

$[\alpha]^{25}:-56.5\left(c=1.0, \mathrm{CHCl}_{3}\right)$. HPLC analysis of the product: Daicel CHIRALPAK AD-H column; $10 \%$ - $\mathrm{PrOH}$ in hexanes; $1.0 \mathrm{~mL} / \mathrm{min}$; retention times: $11.8 \mathrm{~min}$ (major), $19.8 \mathrm{~min}$ (minor).

${ }^{1} \mathrm{H}$ NMR $\left(400 \mathrm{MHz}, \mathrm{CDCl}_{3}\right) \delta 7.14(\mathrm{~d}, J=8.8 \mathrm{~Hz}, 2 \mathrm{H}), 7.07-6.93(\mathrm{~m}, 4 \mathrm{H}), 6.82(\mathrm{~d}, J=8.7$ $\mathrm{Hz}, 2 \mathrm{H}), 6.19(\mathrm{~s}, 1 \mathrm{H}), 2.76-2.62(\mathrm{~m}, 1 \mathrm{H}), 2.62-2.30(\mathrm{~m}, 3 \mathrm{H}), 2.06-1.73(\mathrm{~m}, 4 \mathrm{H}) \mathrm{ppm}$.

${ }^{13} \mathrm{C}$ NMR $\left(100 \mathrm{MHz}, \mathrm{CDCl}_{3}\right) \delta 212.2,155.1,148.0,144.5,131.5,130.5,129.5,120.6,118.6$ $(\mathrm{q}, J=318.7 \mathrm{~Hz}), 115.9,62.9,40.4,39.4,27.7,21.9$ ppm.

${ }^{19}$ F NMR (376 MHz, $\left.\mathrm{CDCl}_{3}\right) \delta-72.9$ ppm.

IR (thin film) 3374, 2947, 2873, 1698, 1614, 1594, 1515, 1501, 1423, 1250, 1216, 1141, $1016,891,833 \mathrm{~cm}^{-1}$.

HRMS (CI+) Calcd for $\mathrm{C}_{19} \mathrm{H}_{17} \mathrm{~F}_{3} \mathrm{O}_{5} \mathrm{~S}\left[\mathrm{M}^{+}\right]$: 414.0749, Found: 414.0740. 


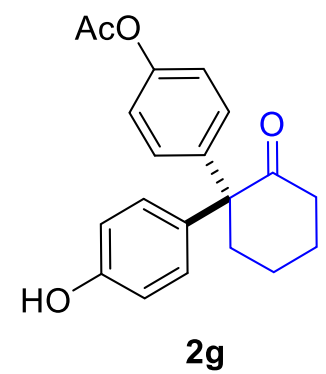

(S)-4-(1-(4-Hydroxyphenyl)-2-oxocyclohexyl)phenyl acetate (2g) was prepared as a white oil from $(R)-C 1(3.0 \mathrm{mg}, 4 \mu \mathrm{mol}), 5 \AA \mathrm{MS}(0.16 \mathrm{~g})$, and $1 \mathrm{~g}(129.8 \mathrm{mg}, 0.4 \mathrm{mmol})$ in $\mathrm{PhCl}(14 \mathrm{~mL})$ and $\mathrm{DCM}(2 \mathrm{~mL})$ according to the General Procedure $\mathrm{C}(16 \mathrm{~h}$, purified by flash column chromatography: hexanes/ethyl acetate $=2: 1,113.1 \mathrm{mg}, 87 \%$ yield, $82 \%$ ee).

$[\alpha]^{25}:-34.0\left(c=1.0, \mathrm{CHCl}_{3}\right)$. HPLC analysis of the product: Daicel CHIRALPAK AD-H column; $10 \%$ - $\mathrm{PrOH}$ in hexanes; $1.0 \mathrm{~mL} / \mathrm{min}$; retention times: $20.6 \mathrm{~min}$ (major), $44.8 \min$ (minor).

${ }^{1} \mathrm{H}$ NMR $\left(400 \mathrm{MHz}, \mathrm{CDCl}_{3}\right) \delta$ 7.09-6.95 (m, 4H), $6.88(\mathrm{~d}, J=8.7 \mathrm{~Hz}, 2 \mathrm{H}), 6.71(\mathrm{~d}, J=8.7$ $\mathrm{Hz}, 2 \mathrm{H}), 6.26(\mathrm{~s}, 1 \mathrm{H}), 2.65-2.40(\mathrm{~m}, 4 \mathrm{H}), 2.28(\mathrm{~s}, 3 \mathrm{H}), 2.00-1.72(\mathrm{~m}, 4 \mathrm{H}) \mathrm{ppm}$.

${ }^{13} \mathrm{C}$ NMR $\left(100 \mathrm{MHz} \mathrm{CDCl}_{3}\right) \delta 212.5,168.9,154.9,149.1,140.7,132.9,129.6,129.5,121.1$, $115.4,62.9,40.5,39.4,27.7,22.0,21.1 \mathrm{ppm}$.

IR (thin film) 3435, 2944, 2868, 1754, 1704, 1613, 1592, 1514, 1448, 1371, 1270, 1205, $1171,1120,1018,913,734 \mathrm{~cm}^{-1}$.

HRMS (CI+) Calcd for $\mathrm{C}_{20} \mathrm{H}_{20} \mathrm{O}_{4}\left[\mathrm{M}^{+}\right]$: 324.1362, Found: 324.1365.

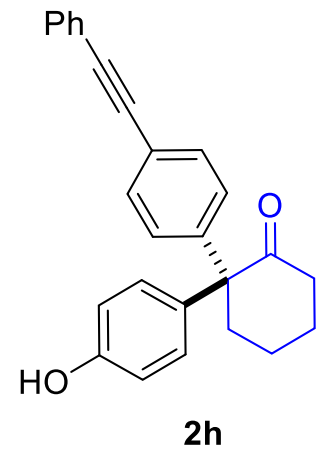

(R)-2-(4-Hydroxyphenyl)-2-(4-(phenylethynyl)phenyl)cyclohexan-1-one (2h) was prepared as a yellow solid from (R)-C1 (3.0 mg, $4 \mu \mathrm{mol}), 5 \AA \mathrm{MS}(0.16 \mathrm{~g})$, and $\mathbf{1 h}$ 
(146.5 mg, $0.4 \mathrm{mmol})$ in $\mathrm{PhCl}(14 \mathrm{~mL})$ and DCM $(2 \mathrm{~mL})$ according to the General Procedure C (23 h, purified by flash column chromatography: hexanes/ethyl acetate $=6: 1,105.3 \mathrm{mg}, 72 \%$ yield, $92 \%$ ee).

$[\alpha]^{25}:-4.3\left(c=1.0, \mathrm{CHCl}_{3}\right)$. HPLC analysis of the product: Daicel CHIRALPAK AD-H column; 10\% $i$-PrOH in hexanes; $1.0 \mathrm{~mL} / \mathrm{min}$; retention times: $19.7 \mathrm{~min}$ (major), $24.8 \mathrm{~min}$ (minor).

${ }^{1}$ H NMR (400 MHz, acetone- $\left.d_{6}\right) \delta 8.48(\mathrm{~s}, 1 \mathrm{H}), 7.61-7.53(\mathrm{~m}, 2 \mathrm{H}), 7.52-7.47(\mathrm{~m}, 2 \mathrm{H})$, 7.47-7.38 (m, 3H), 7.11-7.05 (m, 2H), 7.05-6.98 (m, 2H), 6.95-6.87 (m, 2H), 2.76-2.62 (m, $1 \mathrm{H}), 2.60-2.46(\mathrm{~m}, 2 \mathrm{H}), 2.46-2.34(\mathrm{~m}, 1 \mathrm{H}), 2.05-1.76(\mathrm{~m}, 4 \mathrm{H}) \mathrm{ppm}$.

${ }^{13} \mathrm{C}$ NMR (100 MHz, acetone- $\left.d_{6}\right) \delta$ 210.4, 157.2, 146.0, 132.8, 132.2, 131.7, 130.4, 129.8, $129.4,129.3,124.1,121.8,116.2,90.0,89.9,63.9,41.1,39.5,28.1,22.8$ ppm.

IR (ATR) 3256, 2941, 2865, 1686, 1611, 1592, 1511, 1440, 1265, 1229, 1180, 1117, 826, $806 \mathrm{~cm}^{-1}$.

HRMS (CI+) Calcd for $\mathrm{C}_{26} \mathrm{H}_{22} \mathrm{O}_{2}\left[\mathrm{M}^{+}\right]$: 366.1620, Found: 366.1606.

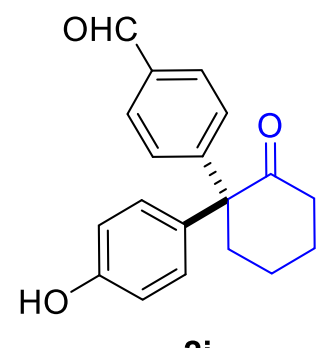

(R)-4-(1-(4-Hydroxyphenyl)-2-oxocyclohexyl)benzaldehyde (2i) was prepared as a colorless oil from $(R)-C 1(2.9 \mathrm{mg}, 4 \mu \mathrm{mol}), 5 \AA \mathrm{MS}(0.16 \mathrm{~g})$, and $1 \mathbf{i}(117.5 \mathrm{mg}, 0.4 \mathrm{mmol})$ in $\mathrm{PhCl}(14 \mathrm{~mL})$ and $\mathrm{DCM}(2 \mathrm{~mL})$ according to the General Procedure $\mathrm{C}(23 \mathrm{~h}$, purified by flash column chromatography: hexanes/ethyl acetate $=6: 1 \rightarrow 4: 1,113.1 \mathrm{mg}$, $96 \%$ yield, $87 \%$ ee).

$[\alpha]^{25}:-76.6\left(c=1.0, \mathrm{CHCl}_{3}\right)$. HPLC analysis of the product: Daicel CHIRALPAK AD-H column; 15\% $i$-PrOH in hexanes; $1.0 \mathrm{~mL} / \mathrm{min}$; retention times: $15.7 \mathrm{~min}$ (major), $19.1 \mathrm{~min}$ (minor). 
${ }^{1} \mathbf{H}$ NMR (400 MHz, $\left.\mathrm{CDCl}_{3}\right) \delta 9.95(\mathrm{~s}, 1 \mathrm{H}), 7.78(\mathrm{~d}, J=8.4 \mathrm{~Hz}, 2 \mathrm{H}), 7.14(\mathrm{~d}, J=8.4 \mathrm{~Hz}$, 2H), 7.01-6.95 (m, 2H), 6.87-6.82 (m, 2H), $6.46(\mathrm{~s}, 1 \mathrm{H}), 2.74-2.63(\mathrm{~m}, 1 \mathrm{H}), 2.62-2.42(\mathrm{~m}$, $3 \mathrm{H}), 2.05-1.77(\mathrm{~m}, 4 \mathrm{H}) \mathrm{ppm}$.

${ }^{13} \mathrm{C}$ NMR $\left(100 \mathrm{MHz}, \mathrm{CDCl}_{3}\right) \delta 211.6,192.4,155.2,151.2,134.5,131.5,129.5,129.44$, 129.36, 115.9, 63.5, 40.4, 39.1, 27.6, 21.9 ppm.

IR (thin film) 3392, 2945, 2867, 1698, 1605, 1573, 1515, 1449, 1271, 1220, 1181, 1120, 824, $803 \mathrm{~cm}^{-1}$.

HRMS (CI+) Calcd for $\mathrm{C}_{19} \mathrm{H}_{18} \mathrm{O}_{3}\left[\mathrm{M}^{+}\right]$: 294.1256, Found: 294.1257.

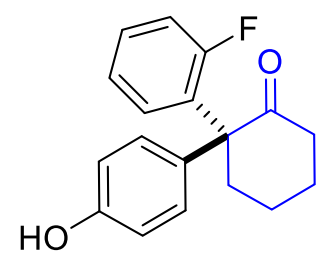

2j

(S)-2-(2-Fluorophenyl)-2-(4-hydroxyphenyl)cyclohexan-1-one (2j) was prepared as a white oil from $(R)-C 1(3.0 \mathrm{mg}, 4 \mu \mathrm{mol}), 5 \AA \mathrm{MS}(0.16 \mathrm{~g})$, and $\mathbf{1 j}(114.5 \mathrm{mg}, 0.4 \mathrm{mmol})$ in $\mathrm{PhCl}(14 \mathrm{~mL})$ and DCM $(2 \mathrm{~mL})$ according to the General Procedure $\mathrm{C}(17.5 \mathrm{~h}$, purified by flash column chromatography: hexanes/ethyl acetate $=8: 1 \rightarrow 6: 1,111.3 \mathrm{mg}$, 97\% yield, 94\% ee).

$[\alpha]^{25}:-142.1\left(c=1.0, \mathrm{CHCl}_{3}\right)$. HPLC analysis of the product: Daicel CHIRALPAK AD-H column; 10\% $i$-PrOH in hexanes; $1.0 \mathrm{~mL} / \mathrm{min}$; retention times: $11.4 \mathrm{~min}$ (major), $13.8 \mathrm{~min}$ (minor).

${ }^{1} \mathrm{H}$ NMR $\left(400 \mathrm{MHz}, \mathrm{CDCl}_{3}\right) \delta$ 7.24-7.11 (m, 3H), $7.04\left(\mathrm{ddd}, J_{1}=11.7 \mathrm{~Hz}, J_{2}=8.1 \mathrm{~Hz}, J_{3}=\right.$ $1.2 \mathrm{~Hz}, 1 \mathrm{H}), 6.95-6.82(\mathrm{~m}, 3 \mathrm{H}), 6.37\left(\mathrm{td}, J_{1}=7.8 \mathrm{~Hz}, J_{2}=1.7 \mathrm{~Hz}, 1 \mathrm{H}\right), 5.74(\mathrm{brs}, 1 \mathrm{H})$, 2.70-2.60 (m, 1H), 2.60-2.48 (m, 2H), 2.45-2.33 (m, 1H), 2.03-1.76 (m, 4H) ppm.

${ }^{13} \mathrm{C}$ NMR $\left(100 \mathrm{MHz}, \mathrm{CDCl}_{3}\right) \delta 210.9,160.1(\mathrm{~d}, J=245.6 \mathrm{~Hz}), 155.4,133.1(\mathrm{~d}, J=11.8$ $\mathrm{Hz}), 130.1(\mathrm{~d}, J=4.2 \mathrm{~Hz}), 129.6,128.8,128.6$ (d, $J=8.7 \mathrm{~Hz}), 123.7$ (d, J = 3.0 Hz), 116.0, $115.7(\mathrm{~d}, J=23.4 \mathrm{~Hz}), 60.7(\mathrm{~d}, J=3.2 \mathrm{~Hz}), 39.8,36.1$ (d, J=3.5 Hz), 26.7, 22.0 ppm.

${ }^{19} \mathrm{~F}$ NMR $\left(376 \mathrm{MHz}, \mathrm{CDCl}_{3}\right) \delta-106.0 \mathrm{ppm}$. 
IR (thin film) 3391, 2949, 2868, 1701, 1613, 1593, 1515, 1486, 1449, 1268, 1235, 1219, $1184,1123 \mathrm{~cm}^{-1}$.

HRMS (CI+) Calcd for $\mathrm{C}_{18} \mathrm{H}_{17} \mathrm{FO}_{2}\left[\mathrm{M}^{+}\right]$: 284.1213, Found: 284.1204.

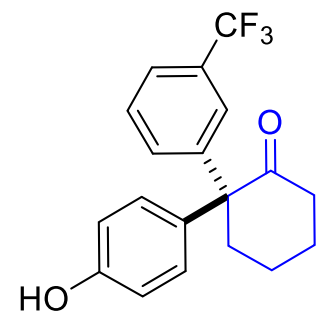

2k

(S)-2-(4-Hydroxyphenyl)-2-(3-(trifluoromethyl)phenyl)cyclohexan-1-one (2k) was prepared as a white solid from $(R)-\mathrm{C} 1(3.0 \mathrm{mg}, 4 \mu \mathrm{mol}), 5 \AA \mathrm{MS}(0.16 \mathrm{~g})$, and 1k (133.6 $\mathrm{mg}, 0.4 \mathrm{mmol})$ in $\mathrm{PhCl}(14 \mathrm{~mL})$ and DCM (2 mL) according to the General Procedure $\mathrm{C}(17 \mathrm{~h}$, purified by flash column chromatography: hexanes/ethyl acetate $=8: 1,112.0$ mg, $84 \%$ yield, $94 \%$ ee).

$[\alpha]^{25}:-74.2\left(c=1.0, \mathrm{CHCl}_{3}\right)$. HPLC analysis of the product: Daicel CHIRALPAK AD-H column; 10\% $i$-PrOH in hexanes; $1.0 \mathrm{~mL} / \mathrm{min}$; retention times: $8.2 \mathrm{~min}$ (major), $10.0 \min$ (minor).

${ }^{1}$ H NMR (400 MHz, acetone- $\left.d_{6}\right) \delta 8.63(\mathrm{~s}, 1 \mathrm{H}), 7.56(\mathrm{~d}, J=7.6 \mathrm{~Hz}, 1 \mathrm{H}), 7.49(\mathrm{t}, J=7.8$ $\mathrm{Hz}, 1 \mathrm{H}), 7.31(\mathrm{~s}, 1 \mathrm{H}), 7.27(\mathrm{~d}, J=7.8 \mathrm{~Hz}, 1 \mathrm{H}), 7.10(\mathrm{~d}, J=8.6 \mathrm{~Hz}, 2 \mathrm{H}), 6.97(\mathrm{~d}, J=8.6 \mathrm{~Hz}$, 2H), 2.92-2.71 (m, 1H), 2.64-2.46 (m, 2H), 2.46-2.31 (m, 1H), 2.07-1.75 (m, 4H) ppm.

${ }^{13} \mathrm{C}$ NMR (100 MHz, acetone- $\left.d_{6}\right) \delta 210.3,157.5,147.4,133.6,131.5,130.3,130.1$ (q, $J=$ $31.4 \mathrm{~Hz}), 129.2,126.1(\mathrm{q}, J=3.9 \mathrm{~Hz}), 125.4(\mathrm{q}, J=270.0 \mathrm{~Hz}), 123.8(\mathrm{q}, J=3.8 \mathrm{~Hz}), 116.6$, 63.8, 41.0, 39.5, 28.1, $22.8 \mathrm{ppm}$.

${ }^{19}$ F NMR (376 MHz, acetone- $\left.d_{6}\right) \delta-62.8 \mathrm{ppm}$.

IR (thin film) 3434, 2949, 2875, 1698, 1613, 1593, 1514, 1453, 1435, 1331, 1271, 1122 $\mathrm{cm}^{-1}$.

HRMS (CI+) Calcd for $\mathrm{C}_{19} \mathrm{H}_{17} \mathrm{~F}_{3} \mathrm{O}_{2}\left[\mathrm{M}^{+}\right]$: 334.1181, Found: 334.1186 . 


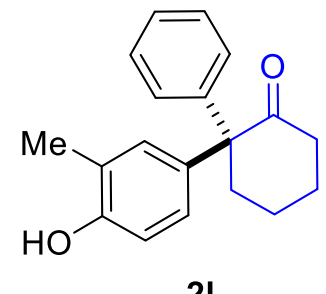

21

(R)-2-(4-Hydroxy-3-methylphenyl)-2-phenylcyclohexan-1-one (21) was prepared as a white solid from $(R)-C 1(3.0 \mathrm{mg}, 4 \mu \mathrm{mol}), 5 \AA \mathrm{MS}(0.16 \mathrm{~g})$, and 11 (112.0 mg, 0.4 $\mathrm{mmol})$ in $\mathrm{PhCl}(14 \mathrm{~mL})$ and DCM (2 mL) according to the General Procedure $\mathrm{C}(22 \mathrm{~h}$, purified by flash column chromatography: hexanes/ethyl acetate $=8: 1,98.0 \mathrm{mg}, 88 \%$ yield, $82 \%$ ee).

$[\alpha]^{25}:-31.7\left(c=1.0, \mathrm{CHCl}_{3}\right)$. HPLC analysis of the product: Daicel CHIRALPAK AD-H column; $10 \%$-PrOH in hexanes; $1.0 \mathrm{~mL} / \mathrm{min}$; retention times: $10.5 \mathrm{~min}$ (major), $11.6 \mathrm{~min}$ (minor).

${ }^{1} \mathbf{H}$ NMR $\left(400 \mathrm{MHz}\right.$, acetone- $\left.d_{6}\right) \delta 8.31(\mathrm{~s}, 1 \mathrm{H}), 7.33-7.25(\mathrm{~m}, 2 \mathrm{H}), 7.25-7.17(\mathrm{~m}, 1 \mathrm{H})$, 7.07-7.00 (m, 2H), $6.92(\mathrm{~d}, J=2.1 \mathrm{~Hz}, 1 \mathrm{H}), 6.85(\mathrm{~d}, J=8.4 \mathrm{~Hz}, 1 \mathrm{H}), 6.78\left(\mathrm{dd}, J_{1}=8.4 \mathrm{~Hz}\right.$, $\left.J_{2}=2.3 \mathrm{~Hz}, 1 \mathrm{H}\right), 2.72-2.61(\mathrm{~m}, 1 \mathrm{H}), 2.58-2.44(\mathrm{~m}, 2 \mathrm{H}), 2.44-2.35(\mathrm{~m}, 1 \mathrm{H}), 2.22(\mathrm{~s}, 3 \mathrm{H})$, 2.02-1.75 (m, 4H) ppm.

${ }^{13} \mathrm{C}$ NMR $\left(100 \mathrm{MHz}\right.$, acetone- $\left.d_{6}\right) \delta 210.7,155.0,145.3,133.3,131.6,129.5,128.6,127.6$, $127.0,124.9,115.3,63.9,41.2,39.7,28.2,22.9,16.5$ ppm.

IR (thin film) 3451, 2950, 2870, 1697, 1607, 1510, 1494, 1447, 1421, 1269, 1126, 1115 $\mathrm{cm}^{-1}$.

HRMS (CI+) Calcd for $\mathrm{C}_{19} \mathrm{H}_{20} \mathrm{O}_{2}\left[\mathrm{M}^{+}\right]$: 280.1463, Found: 280.1458 .

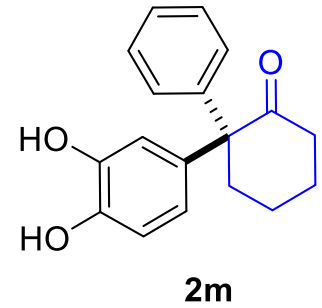

(R)-2-(3,4-Dihydroxyphenyl)-2-phenylcyclohexan-1-one $(2 \mathrm{~m})$ was prepared as a yellow oil from $(R)-C 1(3.0 \mathrm{mg}, 4 \mu \mathrm{mol}), 5 \AA \mathrm{MS}(0.16 \mathrm{~g})$, and $1 \mathrm{~m}(112.9 \mathrm{mg}, 0.4 \mathrm{mmol})$ 
in $\mathrm{PhCl}(14 \mathrm{~mL})$ and $\mathrm{DCM}(2 \mathrm{~mL})$ according to the General Procedure $\mathrm{C}(17 \mathrm{~h}$, purified by flash column chromatography: hexanes/ethyl acetate $=6: 1,91.0 \mathrm{mg}$, 81\% yield, $89 \%$ ee).

$[\alpha]^{25}:-41.9\left(c=1.0, \mathrm{CHCl}_{3}\right)$. HPLC analysis of the product: Daicel CHIRALPAK AD-H column; 10\% $i$-PrOH in hexanes; $1.0 \mathrm{~mL} / \mathrm{min}$; retention times: $19.1 \mathrm{~min}$ (major), $21.2 \mathrm{~min}$ (minor).

${ }^{1} \mathrm{H}$ NMR $\left(400 \mathrm{MHz}, \mathrm{CDCl}_{3}\right) \delta$ 7.34-7.14 (m, 3H), 7.03-6.92 (m, 2H), 6.84-6.74 (m, 1H), 6.56-6.45 (m, 2H), 5.40 (brs, 2H), 2.61-2.39 (m, 4H), 1.99-1.70 (m, 4H) ppm.

${ }^{13} \mathrm{C}$ NMR $\left(100 \mathrm{MHz} \mathrm{CDCl}_{3}\right) \delta 214.8,143.7,143.0,142.6,133.5,128.4,128.2,126.8,120.4$, $115.7,115.1,63.4,40.5,39.2,27.7,21.9$ ppm.

IR (thin film) 3382, 2945, 2867, 1698, 1605, 1520, 1446, 1286, 1266, 1200, 1118, $735 \mathrm{~cm}^{-1}$. HRMS (CI+) Calcd for $\mathrm{C}_{18} \mathrm{H}_{18} \mathrm{O}_{3}\left[\mathrm{M}^{+}\right]$: 282.1256, Found: 282.1247.

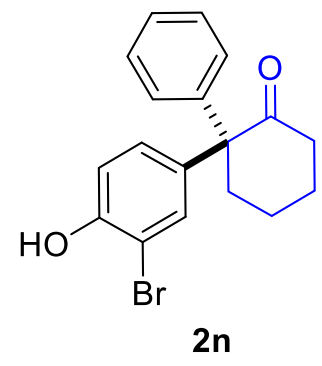

(R)-2-(3-Bromo-4-hydroxyphenyl)-2-phenylcyclohexan-1-one (2n) was prepared as a colorless oil from $(R)-C 1(3.0 \mathrm{mg}, 4 \mu \mathrm{mol}), 5 \AA \mathrm{MS}(0.16 \mathrm{~g})$, and 1n (138.0 mg, 0.4 $\mathrm{mmol})$ in $\mathrm{PhCl}(14 \mathrm{~mL})$ and DCM $(2 \mathrm{~mL})$ according to the General Procedure C (22.5 h, purified by flash column chromatography: hexanes/ethyl acetate $=8: 1,125.7 \mathrm{mg}$, 91\% yield, 93\% ee).

$[\alpha]^{25}:+15.9\left(c=1.0, \mathrm{CHCl}_{3}\right)$. HPLC analysis of the product: Daicel CHIRALPAK AD-H column; $10 \%$-PrOH in hexanes; $1.0 \mathrm{~mL} / \mathrm{min}$; retention times: $10.9 \mathrm{~min}$ (major), $12.2 \mathrm{~min}$ (minor).

${ }^{1} \mathbf{H}$ NMR $\left(400 \mathrm{MHz} \mathrm{CDCl}_{3}\right) \delta$ 7.38-7.31 (m, 2H), 7.30-7.22 (m, 1H), 7.13-7.04 (m, 3H), $6.90(\mathrm{~d}, J=8.6 \mathrm{~Hz}, 1 \mathrm{H}), 6.84\left(\mathrm{dd}, J_{1}=8.6 \mathrm{~Hz}, J_{2}=2.2 \mathrm{~Hz}, 1 \mathrm{H}\right), 5.76(\mathrm{~s}, 1 \mathrm{H}), 2.70-2.58(\mathrm{~m}$, $1 \mathrm{H}), 2.56-2.36(\mathrm{~m}, 3 \mathrm{H}), 2.00-1.88(\mathrm{~m}, 2 \mathrm{H}), 1.88-1.75(\mathrm{~m}, 2 \mathrm{H}) \mathrm{ppm}$. 
${ }^{13} \mathrm{C}$ NMR $\left(100 \mathrm{MHz}, \mathrm{CDCl}_{3}\right) \delta 211.4,151.0,141.3,136.4,131.9,129.4,128.6,128.3,127.1$, 115.6, 110.1, 63.0, 40.5, 39.2, 27.7, 22.0 ppm.

IR (thin film) 3412, 2944, 2867, 1703, 1604, 1577, 1496, 1448, 1407, 1292, 1265, 1176, $1119,1044,814,736 \mathrm{~cm}^{-1}$.

HRMS (CI+) Calcd for $\mathrm{C}_{18} \mathrm{H}_{17} \mathrm{BrO}_{2}\left[\mathrm{M}^{+}\right]$: 344.0412, Found: 344.0403.

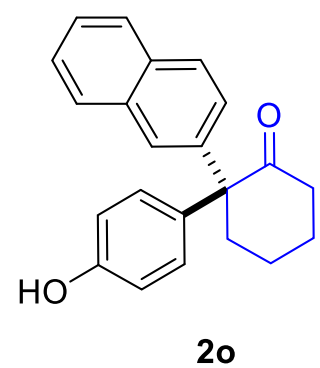

(S)-2-(4-Hydroxyphenyl)-2-(naphthalen-2-yl)cyclohexan-1-one (2o) was prepared as a white solid from $(R)-C 1(3.0 \mathrm{mg}, 4 \mu \mathrm{mol}), 5 \AA \mathrm{MS}(0.16 \mathrm{~g})$, and 10 (126.7 mg, 0.4 $\mathrm{mmol})$ in $\mathrm{PhCl}(14 \mathrm{~mL})$ and DCM $(2 \mathrm{~mL})$ according to the General Procedure C (16.5 h, purified by flash column chromatography: hexanes/ethyl acetate $=8: 1 \rightarrow 6: 1,123.9$ mg, $98 \%$ yield, $92 \%$ ee).

$[\alpha]^{25}:-0.9\left(c=1.0, \mathrm{CHCl}_{3}\right)$. HPLC analysis of the product: Daicel CHIRALPAK AD-H column; $10 \%$ i-PrOH in hexanes; $1.0 \mathrm{~mL} / \mathrm{min}$; retention times: $23.6 \mathrm{~min}$ (minor), $26.7 \mathrm{~min}$ (major).

${ }^{1} \mathrm{H}$ NMR $\left(400 \mathrm{MHz} \mathrm{CDCl}_{3}\right) \delta 7.79(\mathrm{~d}, J=8.3 \mathrm{~Hz}, 2 \mathrm{H}), 7.73-7.66(\mathrm{~m}, 1 \mathrm{H}), 7.49-7.40(\mathrm{~m}$, $2 \mathrm{H}), 7.38(\mathrm{~d}, J=1.2 \mathrm{~Hz}, 1 \mathrm{H}), 7.27\left(\mathrm{dd}, J_{1}=8.7 \mathrm{~Hz}, J_{2}=1.9 \mathrm{~Hz}, 1 \mathrm{H}\right), 6.96-6.88(\mathrm{~m}, 2 \mathrm{H})$, 6.79-6.70 (m, 2H), $5.38(\mathrm{~s}, 1 \mathrm{H}), 2.76-2.65(\mathrm{~m}, 1 \mathrm{H}), 2.65-2.46(\mathrm{~m}, 3 \mathrm{H}), 2.01-1.91(\mathrm{~m}, 2 \mathrm{H})$, 1.91-1.79 (m, 2H) ppm.

${ }^{13} \mathrm{C}$ NMR $\left(100 \mathrm{MHz}_{2} \mathrm{CDCl}_{3}\right) \delta 212.4,154.5,140.2,134.0,133.2,132.2,129.8,128.1,127.8$, 127.4, 127.3, 126.7, 126.04, 126.01, 115.3, 63.4, 40.7, 39.2, 27.8, $22.1 \mathrm{ppm}$.

IR (ATR) 3381, 2943, 2865, 1698, 1612, 1594, 1513, 1265, 1228, 1181, $1116 \mathrm{~cm}^{-1}$. HRMS (CI+) Calcd for $\mathrm{C}_{22} \mathrm{H}_{20} \mathrm{O}_{2}\left[\mathrm{M}^{+}\right]$: 316.1463, Found: 316.1451 . 


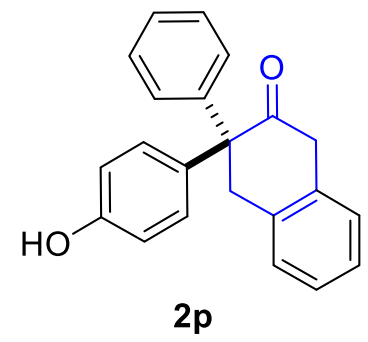

(R)-3-(4-Hydroxyphenyl)-3-phenyl-3,4-dihydronaphthalen-2(1H)-one (2p) was prepared as a white solid from $(R)-\mathbf{C 1}(28.6 \mathrm{mg}, 40 \mu \mathrm{mol}), 5 \AA \mathrm{MS}(0.16 \mathrm{~g})$, and 1p (125.8 $\mathrm{mg}, 0.4 \mathrm{mmol})$ in $\mathrm{PhCl}(14 \mathrm{~mL})$ and $\mathrm{DCM}(2 \mathrm{~mL})$ according to the General Procedure C (11.5 h, purified by flash column chromatography: hexanes/ethyl acetate $=8: 1,94.6 \mathrm{mg}, 75 \%$ yield, $80 \%$ ee).

$[\alpha]_{\mathrm{D}^{25}}:+17.9\left(c=1.0, \mathrm{CHCl}_{3}\right)$. HPLC analysis of the product: Daicel CHIRALPAK AD-H column; $10 \%$ i-PrOH in hexanes; $1.0 \mathrm{~mL} / \mathrm{min}$; retention times: $26.2 \mathrm{~min}$ (minor), $30.4 \mathrm{~min}$ (major).

${ }^{1} \mathrm{H}$ NMR (400 MHz, acetone- $\left.d_{6}\right) \delta 8.40(\mathrm{~s}, 1 \mathrm{H}), 7.34(\mathrm{~d}, J=7.3 \mathrm{~Hz}, 1 \mathrm{H}), 7.30-7.23$ (m, 2H), 7.23-7.10 (m, 5H), 7.10-6.98 (m, 3H), 6.80-6.72 (m, 2H), 3.90-3.75 (m, 4H) ppm.

${ }^{13}$ C NMR (100 MHz, acetone- $\left.d_{6}\right) \delta 210.2,157.7,145.0,137.6,134.9,133.7,131.4,130.2$, $129.6,129.4,128.9,128.1,128.02,128.00,116.4,61.7,45.8,43.2 \mathrm{ppm}$.

IR (ATR) 3404, 3054, 1708, 1686, 1611, 1598, 1512, 1495, 1444, 1264, 1180, $828 \mathrm{~cm}^{-1}$.

HRMS (CI+) Calcd for $\mathrm{C}_{22} \mathrm{H}_{18} \mathrm{O}_{2}\left[\mathrm{M}^{+}\right]$: 314.1307, Found: 314.1304.

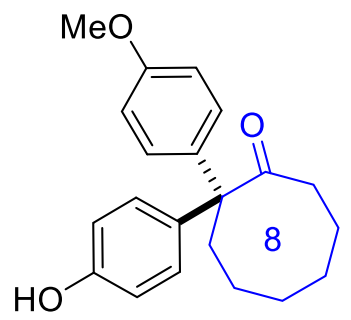

2q

(S)-2-(4-Hydroxyphenyl)-2-(4-methoxyphenyl)cyclooctan-1-one (2q) was prepared as a colorless oil from $(R)-C 1(28.7 \mathrm{mg}, 40 \mu \mathrm{mol}), 5 \AA \mathrm{AS}(0.16 \mathrm{~g})$, and 1q (129.9 mg, $0.4 \mathrm{mmol})$ in $\mathrm{PhCl}(14 \mathrm{~mL})$ and $\mathrm{DCM}(2 \mathrm{~mL})$ according to the General Procedure C (18 h at $0{ }^{\circ} \mathrm{C}$ and $41 \mathrm{~h}$ at rt, purified by flash column chromatography: hexanes/ethyl 
acetate $=8: 1 \rightarrow 6: 1,120.4 \mathrm{mg}, 93 \%$ yield, $75 \%$ ee).

$[\alpha]^{25}:-3.6\left(c=1.0, \mathrm{CHCl}_{3}\right)$. HPLC analysis of the product: Daicel CHIRALPAK AD-H column; 10\% $i$-PrOH in hexanes; $1.0 \mathrm{~mL} / \mathrm{min}$; retention times: $17.2 \mathrm{~min}$ (major), $19.5 \min$ (minor).

${ }^{1} \mathrm{H}$ NMR $\left(400 \mathrm{MHz}, \mathrm{CDCl}_{3}\right) \delta 7.15(\mathrm{~d}, J=8.9 \mathrm{~Hz}, 2 \mathrm{H}), 7.07(\mathrm{~d}, J=8.7 \mathrm{~Hz}, 2 \mathrm{H}), 6.85-6.77$ (m, 2H), 6.77-6.68 (m, 2H), 6.37 (brs, 1H), $3.77(\mathrm{~s}, 3 \mathrm{H}), 2.67-2.42(\mathrm{~m}, 2 \mathrm{H}), 2.42-2.20(\mathrm{~m}$, 2H), $1.92-1.72(\mathrm{~m}, 2 \mathrm{H}), 1.70-1.30(\mathrm{~m}, 6 \mathrm{H}) \mathrm{ppm}$.

${ }^{13} \mathrm{C}$ NMR $\left(100 \mathrm{MHz}, \mathrm{CDCl}_{3}\right) \delta 215.0,158.0,154.5,134.4,133.9,130.5,130.4,114.9,113.2$, $62.4,55.1,38.8,34.1,30.5,25.5,25.0,24.6 \mathrm{ppm}$.

IR (thin film) 3390, 2932, 2858, 1682, 1610, 1510, 1465, 1445, 1252, 1180, 1035, $827 \mathrm{~cm}^{-1}$. HRMS (CI+) Calcd for $\mathrm{C}_{21} \mathrm{H}_{24} \mathrm{O}_{3}\left[\mathrm{M}^{+}\right]$: 324.1725, Found: 324.1722.

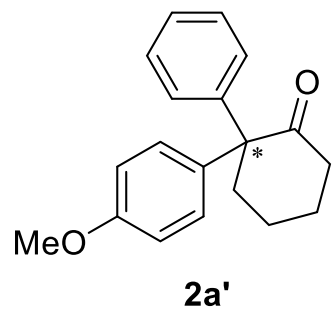

2-(4-methoxyphenyl)-2-phenylcyclohexan-1-one $\left(2 a^{\prime}\right)$ At $-78{ }^{\circ} \mathrm{C}$, to an oven-dried vial charged with $(R)-\mathrm{D}^{8}(26.8 \mathrm{mg}, 30 \mu \mathrm{mol}), 5 \AA \mathrm{MS}(0.12 \mathrm{~g})$, and anhydrous toluene $(10 \mathrm{~mL})$ was added a solution of the epoxide 1a' $(84.0 \mathrm{mg}, 0.3 \mathrm{mmol})$ in anhydrous toluene $(2 \mathrm{~mL})$. Next, the reaction mixture was stirred at the same temperature, and the progress was monitored by TLC. Upon completion $(5.5 \mathrm{~h})$, the reaction mixture was filtered through a plug of silica gel (eluent: ethyl acetate). The filtrate was evaporated, and the residue was subjected to silica gel column chromatography (eluent: hexanes/ethyl acetate $=15: 1)$ to afford $2 \mathbf{a}^{\prime}(75.9 \mathrm{mg}, 90 \%$ yield, $79 \%$ ee) as a colorless oil.

$[\alpha]^{25}:+10.3\left(c=1.0, \mathrm{CHCl}_{3}\right)$. HPLC analysis of the product: Daicel CHIRALPAK AD-H column; 5\% $i$-PrOH in hexanes; $1.0 \mathrm{~mL} / \mathrm{min}$; retention times: $9.6 \mathrm{~min}$ (minor), $10.2 \mathrm{~min}$ (major). 
${ }^{1} \mathrm{H}$ NMR $\left(400 \mathrm{MHz} \mathrm{CDCl}_{3}\right) \delta$ 7.34-7.26 (m, 2H), 7.26-7.19 (m, 1H), 7.08-7.01 (m, 2H), 7.01-6.93 (m, 2H), 6.89-6.80 (m, 2H), $3.77(\mathrm{~s}, 3 \mathrm{H}), 2.62-2.53(\mathrm{~m}, 2 \mathrm{H}), 2.53-2.44(\mathrm{~m}, 2 \mathrm{H})$, 1.99-1.87 (m, 2H), 1.87-1.75 (m, 2H) ppm.

${ }^{13} \mathrm{C}$ NMR $\left(100 \mathrm{MHz}_{2} \mathrm{CDCl}_{3}\right) \delta 211.5,158.2,142.7,134.1,129.5,128.4,128.2,126.7,113.6$, $63.1,55.1,40.5,39.2,27.7,22.0 \mathrm{ppm}$.

IR (thin film) 2941, 2865, 1705, 1607, 1510, 1250, 1184, 1119, 1033, $733 \mathrm{~cm}^{-1}$.

HRMS (CI+) Calcd for $\mathrm{C}_{19} \mathrm{H}_{20} \mathrm{O}_{2}\left[\mathrm{M}^{+}\right]$: 280.1463, Found: 280.1461.

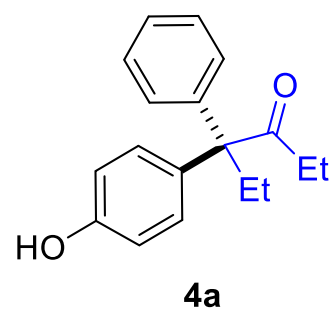

(R)-4-(4-Hydroxyphenyl)-4-phenylhexan-3-one (4a) was prepared as a white solid from $(S)-\mathbf{A} 3(24.2 \mathrm{mg}, 40 \mu \mathrm{mol}), 5 \AA \mathrm{MS}(0.16 \mathrm{~g})$, and 3a (107.0 mg, $0.4 \mathrm{mmol})$ in DCE (8 $\mathrm{mL})$ according to the General Procedure D (22 h, purified by flash column chromatography: hexanes/ethyl acetate $=6: 1,93.9 \mathrm{mg}, 88 \%$ yield, $91 \%$ ee).

$[\alpha]_{D^{25}}:+19.6\left(c=1.0, \mathrm{CHCl}_{3}\right)$. HPLC analysis of the product: Daicel CHIRALPAK AS-H column; $5 \%$ i-PrOH in hexanes; $1.0 \mathrm{~mL} / \mathrm{min}$; retention times: $13.1 \mathrm{~min}$ (minor), 15.4 min (major).

${ }^{1} \mathbf{H}$ NMR $\left(400 \mathrm{MHz}, \mathrm{CDCl}_{3}\right) \delta$ 7.38-7.29 (m, 2H), 7.29-7.18 (m, 3H), $7.13(\mathrm{~d}, J=8.6 \mathrm{~Hz}$, $2 \mathrm{H}), 6.80(\mathrm{~d}, J=8.6 \mathrm{~Hz}, 2 \mathrm{H}), 5.10(\mathrm{~s}, 1 \mathrm{H}), 2.46-2.20(\mathrm{~m}, 4 \mathrm{H}), 0.88(\mathrm{t}, J=7.3 \mathrm{~Hz}, 3 \mathrm{H}), 0.67$ $(\mathrm{t}, J=7.3 \mathrm{~Hz}, 3 \mathrm{H}) \mathrm{ppm}$.

${ }^{13} \mathrm{C}$ NMR (100 MHz, $\left.\mathrm{CDCl}_{3}\right) \delta 212.3,154.5,141.7,133.2,130.7,129.3,128.1,126.7,115.0$, 66.0, 32.3, 30.1, 9.4, $9.1 \mathrm{ppm}$.

IR (thin film) 3338, 2973, 2940, 2882, 1688, 1610, 1589, 1515, 1435, 1383, 1277, 1214, $1178 \mathrm{~cm}^{-1}$.

HRMS (CI+) Calcd for $\mathrm{C}_{18} \mathrm{H}_{21} \mathrm{O}_{2}\left[\mathrm{M}^{+}+\mathrm{H}\right]: 269.1542$, Found: 269.1540. 


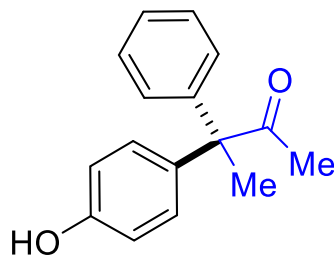

4b

(R)-3-(4-Hydroxyphenyl)-3-phenylbutan-2-one (4b) was prepared as a yellow oil from (S)-A3 (24.0 mg, $40 \mu \mathrm{mol}), 5 \AA \mathrm{MS}(0.16 \mathrm{~g})$, and 3b (96.3 mg, $0.4 \mathrm{mmol})$ in DCE (8 $\mathrm{mL})$ according to the General Procedure D (23 h, purified by flash column chromatography: hexanes/ethyl acetate $=10: 1 \rightarrow 8: 1,90.9 \mathrm{mg}, 94 \%$ yield, $86 \%$ ee). $[\alpha]_{D^{25}}:+13.2\left(c=1.0, \mathrm{CHCl}_{3}\right)$. HPLC analysis of the product: Daicel CHIRALPAK AS-H column; $10 \%$ i-PrOH in hexanes; $1.0 \mathrm{~mL} / \mathrm{min}$; retention times: $14.3 \mathrm{~min}$ (major), $16.3 \mathrm{~min}$ (minor).

${ }^{1} \mathrm{H}$ NMR $\left(400 \mathrm{MHz}^{\mathrm{CDCl}}\right)$ $\delta$ 7.37-7.29 (m, 2H), 7.28-7.21 (m, 1H), 7.21-7.13 (m, 2H), 7.08-6.98 (m, 2H), 6.84-6.72 (m, 2H), 6.26 (brs, 1H), $2.12(\mathrm{~s}, 3 \mathrm{H}), 1.84(\mathrm{~s}, 3 \mathrm{H}) \mathrm{ppm}$.

${ }^{13} \mathrm{C}$ NMR $\left(100 \mathrm{MHz}, \mathrm{CDCl}_{3}\right) \delta 211.0,154.8,143.8,134.7,129.6,128.3,128.2,126.8,115.3$, 61.6, 27.5, $26.4 \mathrm{ppm}$.

IR (thin film) 3391, 3059, 3026, 2985, 1696, 1613, 1595, 1514, 1494, 1445, 1354, 1271, $1228,1180,811 \mathrm{~cm}^{-1}$.

HRMS (CI+) Calcd for $\mathrm{C}_{16} \mathrm{H}_{17} \mathrm{O}_{2}\left[\mathrm{M}^{+}+\mathrm{H}\right]:$ 241.1229, Found: 241.1234 .

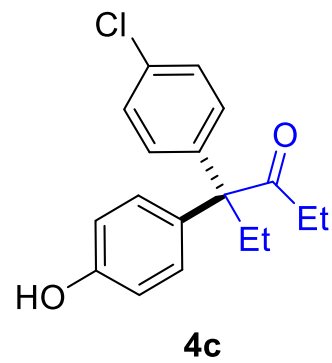

(S)-4-(4-Chlorophenyl)-4-(4-hydroxyphenyl)hexan-3-one (4c) was prepared as a

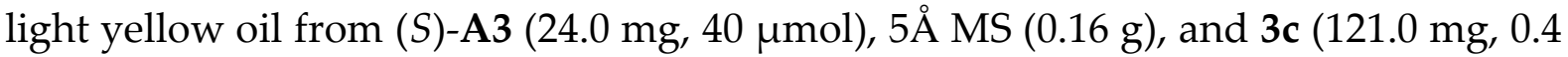
$\mathrm{mmol})$ in DCE (8 $\mathrm{mL})$ according to the General Procedure D $(24 \mathrm{~h}$, purified by flash column chromatography: hexanes/ethyl acetate $=3: 1,111.8 \mathrm{mg}, 92 \%$ yield, $92 \%$ ee). 
$[\alpha]^{25}:-4.9\left(c=1.0, \mathrm{CHCl}_{3}\right)$. HPLC analysis of the product: Daicel CHIRALPAK AS-H column; 5\% $i$-PrOH in hexanes; $1.0 \mathrm{~mL} / \mathrm{min}$; retention times: $12.8 \mathrm{~min}$ (minor), 14.2 $\min ($ major).

${ }^{1}$ H NMR (400 MHz, $\left.\mathrm{CDCl}_{3}\right) \delta$ 7.33-7.24 (m, 2H), 7.19-7.12 (m, 2H), 7.12-7.04 (m, 2H), 6.87-6.77 (m, 2H), $6.17(\mathrm{~s}, 1 \mathrm{H}), 2.42-2.22(\mathrm{~m}, 4 \mathrm{H}), 0.89(\mathrm{t}, J=7.3 \mathrm{~Hz}, 3 \mathrm{H}), 0.68(\mathrm{t}, J=7.3$ $\mathrm{Hz}, 3 \mathrm{H}) \mathrm{ppm}$.

${ }^{13} \mathrm{C}$ NMR $\left(100 \mathrm{MHz}, \mathrm{CDCl}_{3}\right) \delta 212.4,154.8,140.3,132.6,132.5,130.8,130.4,128.2,115.3$, $65.5,32.2,29.9,9.4,9.0$ ppm.

IR (thin film) 3397, 2976, 2939, 2880, 1695, 1613, 1593, 1513, 1491, 1457, 1438, 1266, $1226,1180,1097,1013,821 \mathrm{~cm}^{-1}$.

HRMS (CI+) Calcd for $\mathrm{C}_{18} \mathrm{H}_{20} \mathrm{ClO}_{2}\left[\mathrm{M}^{+}+\mathrm{H}\right]: 303.1152$, Found: 303.1146 .

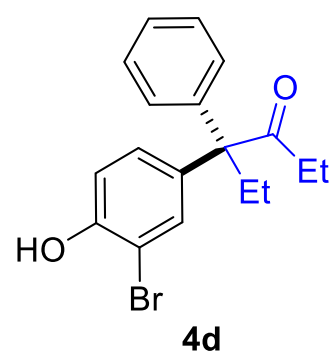

(R)-4-(3-Bromo-4-hydroxyphenyl)-4-phenylhexan-3-one (4d) was prepared as a yellow oil from $(S)-\mathbf{A} 3(24.1 \mathrm{mg}, 40 \mu \mathrm{mol}), 5 \AA \mathrm{MS}(0.16 \mathrm{~g})$, and 3d (138.9 mg, 0.4 $\mathrm{mmol})$ in DCE (8 $\mathrm{mL})$ according to the General Procedure D $(20 \mathrm{~h}$, purified by flash column chromatography: hexanes/ethyl acetate $=8: 1,119.1 \mathrm{mg}, 86 \%$ yield, $92 \%$ ee). $[\alpha]^{25}:+24.4\left(c=1.0, \mathrm{CHCl}_{3}\right)$. HPLC analysis of the product: Daicel CHIRALPAK AS-H column; 10\% $i$-PrOH in hexanes; $1.0 \mathrm{~mL} / \mathrm{min}$; retention times: $6.9 \mathrm{~min}$ (minor), 7.6 min (major).

${ }^{1}$ H NMR (400 MHz, $\left.\mathrm{CDCl}_{3}\right) \delta$ 7.40-7.31 (m, 3H), 7.31-7.25 (m, 1H), 7.24-7.18 (m, 2H), $7.08\left(\mathrm{dd}, J_{1}=8.6 \mathrm{~Hz}, J_{2}=2.3 \mathrm{~Hz}, 1 \mathrm{H}\right), 6.97(\mathrm{~d}, J=8.6 \mathrm{~Hz}, 1 \mathrm{H}), 5.68(\mathrm{~s}, 1 \mathrm{H}), 2.44-2.22(\mathrm{~m}$, $4 \mathrm{H}), 0.90(\mathrm{t}, J=7.3 \mathrm{~Hz}, 3 \mathrm{H}), 0.69(\mathrm{t}, J=7.3 \mathrm{~Hz}, 3 \mathrm{H}) \mathrm{ppm}$.

${ }^{13} \mathrm{C}$ NMR $\left(100 \mathrm{MHz}, \mathrm{CDCl}_{3}\right) \delta$ 211.1, 151.1, 141.2, 135.3, 132.7, 130.4, 129.1, 128.3, 127.0, $115.6,109.9,65.6,32.2,30.0,9.4,9.0$ ppm. 
IR (thin film) 3425, 2975, 2938, 2879, 1702, 1603, 1573, 1494, 1459, 1447, 1406, 1382, $1338,1291,1180,1043,822 \mathrm{~cm}^{-1}$.

HRMS (CI+) Calcd for $\mathrm{C}_{18} \mathrm{H}_{20} \mathrm{BrO}_{2}\left[\mathrm{M}^{+}+\mathrm{H}\right]$ : 347.0647, Found: 347.0641.

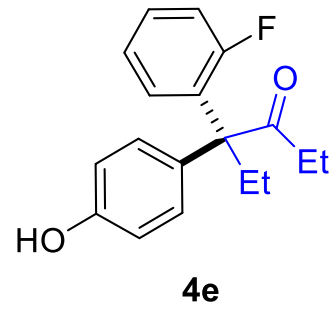

(S)-4-(2-Fluorophenyl)-4-(4-hydroxyphenyl)hexan-3-one (4e) was prepared as a yellow oil from $(S)$-A3 (18.2 mg, $30 \mu \mathrm{mol}), 5 \AA \mathrm{MS}(0.12 \mathrm{~g})$, and $3 \mathbf{e}(85.9 \mathrm{mg}, 0.3 \mathrm{mmol})$ in DCE $(6 \mathrm{~mL})$ according to the General Procedure D $(14 \mathrm{~h}$, purified by flash column chromatography: hexanes/ethyl acetate $=8: 1,66.6 \mathrm{mg}, 78 \%$ yield, 95\% ee).

$[\alpha]_{D^{25}}:-18.9\left(c=1.0, \mathrm{CHCl}_{3}\right)$. HPLC analysis of the product: Daicel CHIRALPAK OD-H column; $5 \%$-PrOH in hexanes; $1.0 \mathrm{~mL} / \mathrm{min}$; retention times: $11.6 \mathrm{~min}$ (minor), $13.6 \mathrm{~min}$ (major).

${ }^{1}$ H NMR (400 MHz, acetone- $\left.d_{6}\right) \delta 8.54(\mathrm{~s}, 1 \mathrm{H}), 7.43-7.33(\mathrm{~m}, 1 \mathrm{H}), 7.32-7.25(\mathrm{~m}, 2 \mathrm{H})$, 7.22-7.08 (m, 3H), 6.94-6.86 (m, 2H), 2.64-2.51 (m, 1H), 2.51-2.32 (m, 3H), $0.89(\mathrm{t}, J=7.3$ $\mathrm{Hz}, 3 \mathrm{H}), 0.69(\mathrm{t}, J=7.3 \mathrm{~Hz}, 3 \mathrm{H}) \mathrm{ppm}$.

${ }^{13} \mathrm{C}$ NMR (100 MHz, acetone- $\left.d_{6}\right) \delta 209.0,161.9(\mathrm{~d}, J=244.0 \mathrm{~Hz}), 157.4,132.3(\mathrm{~d}, J=4.6$ Hz), $131.6(\mathrm{~d}, J=12.6 \mathrm{~Hz}), 131.2,130.1,129.7$ (d, $J=8.9 \mathrm{~Hz}), 124.4(\mathrm{~d}, J=2.9 \mathrm{~Hz}), 116.5$ $(\mathrm{d}, J=23.4 \mathrm{~Hz}), 116.0,64.1(\mathrm{~d}, J=2.5 \mathrm{~Hz}), 31.5(\mathrm{~d}, J=2.6 \mathrm{~Hz}), 27.6,10.1,9.1 \mathrm{ppm}$.

${ }^{19} \mathrm{~F}$ NMR (376 MHz, acetone- $\left.d_{6}\right) \delta-109.1 \mathrm{ppm}$.

IR (ATR) 3383, 2975, 2939, 2881, 1693, 1612, 1592, 1511, 1486, 1449, 1265, 1220, 1180, $830 \mathrm{~cm}^{-1}$.

HRMS (CI+) Calcd for $\mathrm{C}_{18} \mathrm{H}_{20} \mathrm{FO}_{2}\left[\mathrm{M}^{+}+\mathrm{H}\right]$ : 287.1447, Found: 287.1448. 


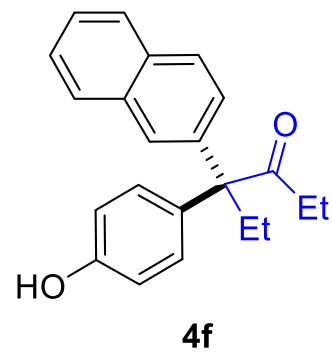

(S)-4-(4-Hydroxyphenyl)-4-(naphthalen-2-yl)hexan-3-one (4f) was prepared as a yellow oil from $(S)$-A3 (18.0 mg, $30 \mu \mathrm{mol}), 5 \AA \mathrm{MS}(0.12 \mathrm{~g})$, and $3 \mathbf{f}(95.5 \mathrm{mg}, 0.3 \mathrm{mmol})$ in DCE $(6 \mathrm{~mL})$ according to the General Procedure D $(21 \mathrm{~h}$, purified by flash column chromatography: hexanes/ethyl acetate $=8: 1,80.1 \mathrm{mg}, 84 \%$ yield, 92\% ee).

$[\alpha]^{25}:+1.3\left(c=1.0, \mathrm{CHCl}_{3}\right)$. HPLC analysis of the product: Daicel CHIRALPAK IC column; $5 \%$ i-PrOH in hexanes; $1.0 \mathrm{~mL} / \mathrm{min}$; retention times: $9.8 \mathrm{~min}$ (minor), 12.3 $\min$ (major).

${ }^{1}$ H NMR (400 MHz, acetone- $\left.d_{6}\right) \delta 8.50(\mathrm{~s}, 1 \mathrm{H})$, 7.96-7.86 (m, 3H), $7.84(\mathrm{~d}, J=1.8 \mathrm{~Hz}$, $1 \mathrm{H}), 7.58-7.46(\mathrm{~m}, 2 \mathrm{H}), 7.38\left(\mathrm{dd}, J_{1}=8.7 \mathrm{~Hz}, J_{2}=2.0 \mathrm{~Hz}, 1 \mathrm{H}\right), 7.25-7.15(\mathrm{~m}, 2 \mathrm{H})$, 6.95-6.86 (m, 2H), $2.48(\mathrm{q}, J=7.4 \mathrm{~Hz}, 2 \mathrm{H}), 2.43(\mathrm{q}, J=7.4 \mathrm{~Hz}, 2 \mathrm{H}), 0.89(\mathrm{t}, J=7.3 \mathrm{~Hz}$, $3 \mathrm{H}), 0.73(\mathrm{t}, J=7.3 \mathrm{~Hz}, 3 \mathrm{H}) \mathrm{ppm}$.

${ }^{13}$ C NMR (100 MHz, acetone- $\left.d_{6}\right) \delta$ 211.1, 157.1, 140.8, 134.1, 133.1, 133.0, 131.4, 128.9, $128.8,128.5,128.2$ (2C), 126.90, 126.86, 115.9, 66.6, 32.7, 30.6, 10.1, 9.4 ppm.

IR (ATR) 3383, 2975, 2937, 1689, 1612, 1594, 1510, 1457, 1435, 1264, 1221, 1180, 817 $\mathrm{cm}^{-1}$.

HRMS (CI+) Calcd for $\mathrm{C}_{22} \mathrm{H}_{23} \mathrm{O}_{2}\left[\mathrm{M}^{+}+\mathrm{H}\right]: 319.1698$, Found: 319.1700 .

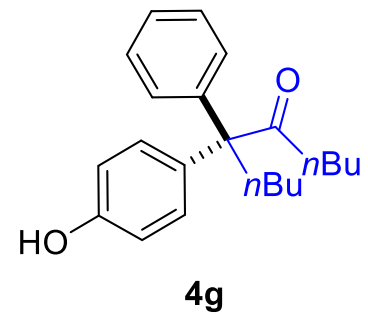

(S)-6-(4-Hydroxyphenyl)-6-phenyldecan-5-one (4g) was prepared as a yellow oil from $(R)-\mathrm{A}^{9}(36.9 \mathrm{mg}, 40 \mu \mathrm{mol}), 5 \AA \mathrm{MS}(0.16 \mathrm{~g})$, and $3 \mathrm{~g}(129.8 \mathrm{mg}, 0.4 \mathrm{mmol})$ in DCE $(8 \mathrm{~mL})$ according to the General Procedure D $\left(0{ }^{\circ} \mathrm{C}, 11 \mathrm{~h}\right.$, purified by flash 
column chromatography: hexanes/ethyl acetate $=8: 1,102.6 \mathrm{mg}, 79 \%$ yield, $89 \%$ ee). $[\alpha]^{25}:-15.1\left(c=1.0, \mathrm{CHCl}_{3}\right)$. HPLC analysis of the product: Daicel CHIRALPAK AS-H column; 5\% $i$-PrOH in hexanes; $1.0 \mathrm{~mL} / \mathrm{min}$; retention times: $9.9 \mathrm{~min}$ (major), $11.2 \mathrm{~min}$ (minor).

${ }^{1} \mathrm{H}$ NMR $\left(400 \mathrm{MHz}, \mathrm{CDCl}_{3}\right) \delta$ 7.35-7.28 (m, 2H), 7.28-7.19 (m, 3H), $7.12(\mathrm{~d}, J=8.7 \mathrm{~Hz}$, $2 \mathrm{H}), 6.82(\mathrm{~d}, J=8.7 \mathrm{~Hz}, 2 \mathrm{H}), 6.35(\mathrm{~s}, 1 \mathrm{H}), 2.43-2.30(\mathrm{~m}, 2 \mathrm{H}), 2.30-2.18(\mathrm{~m}, 2 \mathrm{H})$, 1.37-1.20 (m, 4H), 1.14-1.00 (m, 2H), 1.00-0.87 (m, 2H), $0.79(\mathrm{t}, J=7.3 \mathrm{~Hz}, 3 \mathrm{H}), 0.71(\mathrm{t}, J$ $=7.3 \mathrm{~Hz}, 3 \mathrm{H}) \mathrm{ppm}$.

${ }^{13} \mathrm{C}$ NMR $\left(100 \mathrm{MHz}, \mathrm{CDCl}_{3}\right) \delta$ 212.2, 154.7, 141.8, 132.9, 130.6, 129.3, 128.0, 126.7, 115.1, $65.7,38.8,37.1,27.0(2 \mathrm{C}), 23.2,22.1,13.9,13.6 \mathrm{ppm}$.

IR (thin film) 3401, 2958, 2933, 2872, 1692, 1613, 1594, 1513, 1466, 1447, 1267, 1224, $1180,831 \mathrm{~cm}^{-1}$.

HRMS (CI+) Calcd for $\mathrm{C}_{22} \mathrm{H}_{29} \mathrm{O}_{2}\left[\mathrm{M}^{+}+\mathrm{H}\right]: 325.2168$, Found: 325.2160 .

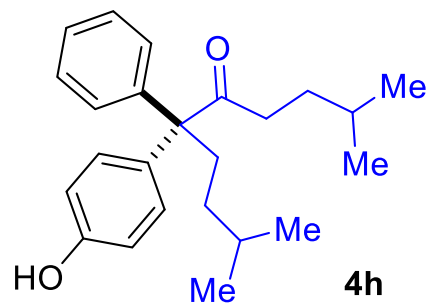

(S)-6-(4-Hydroxyphenyl)-2,9-dimethyl-6-phenyldecan-5-one (4h) was prepared as a

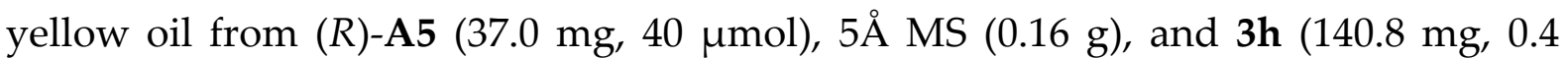
$\mathrm{mmol})$ in DCE $(8 \mathrm{~mL})$ according to the General Procedure $\mathrm{D}\left(0{ }^{\circ} \mathrm{C}, 15 \mathrm{~h}\right.$, purified by flash column chromatography: hexanes/ethyl acetate $=6: 1,114.2 \mathrm{mg}, 81 \%$ yield, $88 \%$ ee).

$[\alpha]^{25}:-10.3\left(c=1.0, \mathrm{CHCl}_{3}\right)$. HPLC analysis of the product: Daicel CHIRALPAK IC column; 5\% $i$-PrOH in hexanes; $1.0 \mathrm{~mL} / \mathrm{min}$; retention times: $5.6 \mathrm{~min}$ (major), $6.2 \mathrm{~min}$ (minor).

${ }^{1} \mathrm{H}$ NMR $\left(400 \mathrm{MHz} \mathrm{CDCl}_{3}\right) \delta$ 7.36-7.28 (m, 2H), 7.28-7.19 (m, 3H), 7.16-1.08 (m, 2H), 6.86-6.78 (m, 2H), $6.20(\mathrm{~s}, 1 \mathrm{H}), 2.40-2.31(\mathrm{~m}, 2 \mathrm{H}), 2.31-2.20(\mathrm{~m}, 2 \mathrm{H}), 1.52-1.40(\mathrm{~m}, 1 \mathrm{H})$, 
$1.35-1.25(\mathrm{~m}, 1 \mathrm{H}), 1.24-1.14(\mathrm{~m}, 2 \mathrm{H}), 0.91-0.83(\mathrm{~m}, 2 \mathrm{H}), 0.80(\mathrm{~d}, J=6.6 \mathrm{~Hz}, 6 \mathrm{H}), 0.68(\mathrm{~d}$, $J=6.5 \mathrm{~Hz}, 6 \mathrm{H}) \mathrm{ppm}$.

${ }^{13} \mathrm{C}$ NMR $\left(100 \mathrm{MHz}, \mathrm{CDCl}_{3}\right) \delta 212.4,154.7,141.7,132.9,130.6,129.3,128.1,126.7,115.1$, $65.8,37.1,35.2,34.0,33.7,28.6,27.5,22.5(2 \mathrm{C}), 22.2,22.1 \mathrm{ppm}$.

IR (ATR) 3401, 2955, 2869, 1690, 1613, 1593, 1511, 1263, 1219, 1179, $737 \mathrm{~cm}^{-1}$.

HRMS (CI+) Calcd for $\mathrm{C}_{24} \mathrm{H}_{33} \mathrm{O}_{2}\left[\mathrm{M}^{+}+\mathrm{H}\right]$ : 353.2481, Found: 353.2482.

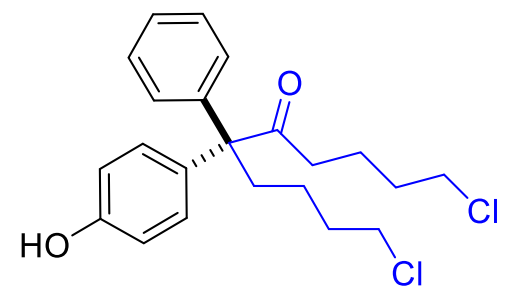

$4 \mathbf{i}$

(S)-1,10-Dichloro-6-(4-hydroxyphenyl)-6-phenyldecan-5-one (4i) was prepared as a yellow oil from $(R)$-A5 $(27.8 \mathrm{mg}, 30 \mu \mathrm{mol}), 5 \AA$ MS $(0.12 \mathrm{~g})$, and $3 \mathbf{i}(118.2 \mathrm{mg}, 0.3$ $\mathrm{mmol})$ in DCE $(6 \mathrm{~mL})$ according to the General Procedure $\mathrm{D}\left(0{ }^{\circ} \mathrm{C}, 23 \mathrm{~h}\right.$, purified by flash column chromatography: hexanes/ethyl acetate $=8: 1,103.6 \mathrm{mg}, 88 \%$ yield, $97 \%$ ee).

$[\alpha]^{25}:-13.2\left(c=1.0, \mathrm{CHCl}_{3}\right)$. HPLC analysis of the product: Daicel CHIRALPAK AS-H column; $10 \%$ i-PrOH in hexanes; $1.0 \mathrm{~mL} / \mathrm{min}$; retention times: $13.4 \mathrm{~min}$ (minor), $21.3 \mathrm{~min}$ (major).

${ }^{1} \mathrm{H}$ NMR $\left(400 \mathrm{MHz}, \mathrm{CDCl}_{3}\right) \delta$ 7.39-7.31 (m, 2H), 7.31-7.19 (m, 3H), $7.12(\mathrm{~d}, J=8.7 \mathrm{~Hz}$, 2H), $6.83(\mathrm{~d}, J=8.7 \mathrm{~Hz}, 2 \mathrm{H}), 5.87(\mathrm{brs}, 1 \mathrm{H}), 3.42(\mathrm{t}, J=6.8 \mathrm{~Hz}, 2 \mathrm{H}), 3.35-3.24(\mathrm{~m}, 2 \mathrm{H})$, 2.46-2.31 (m, 2H), 2.30-2.17 (m, 2H), 1.77-1.62 (m, 2H), 1.56-1.39 (m, 4H), 1.17-1.01 (m, 2H) ppm.

${ }^{13} \mathrm{C}$ NMR (100 MHz, $\left.\mathrm{CDCl}_{3}\right) \delta 210.9,154.8,141.1,132.4,130.5,129.2,128.3,127.0,115.3$, 65.7, 44.6, 44.4, 38.0, 36.6, 32.9, 31.6, 22.2, $22.1 \mathrm{ppm}$.

IR (thin film) 3401, 2956, 2871, 1695, 1613, 1594, 1513, 1446, 1268, 1223, 1180, 1116, 833 $\mathrm{cm}^{-1}$.

HRMS (CI+) Calcd for $\mathrm{C}_{22} \mathrm{H}_{27} \mathrm{Cl}_{2} \mathrm{O}_{2}\left[\mathrm{M}^{+}+\mathrm{H}\right]$ : 393.1388, Found: 393.1378. 


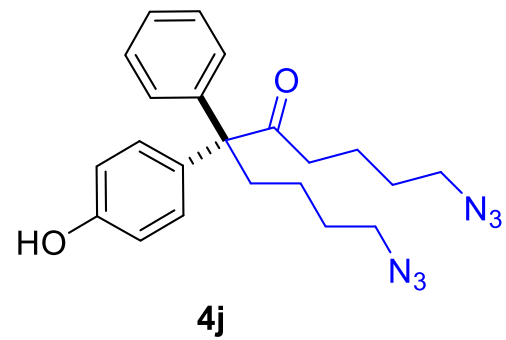

(S)-1,10-Diazido-6-(4-hydroxyphenyl)-6-phenyldecan-5-one (4j) was prepared as a

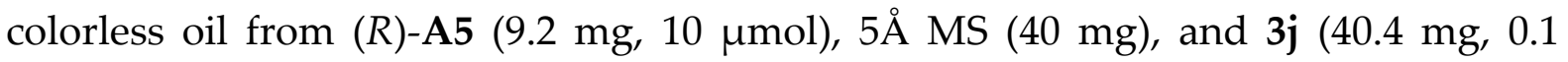
$\mathrm{mmol})$ in DCE $(2 \mathrm{~mL})$ according to the General Procedure D $\left(0{ }^{\circ} \mathrm{C}, 24 \mathrm{~h}\right.$, purified by flash column chromatography: hexanes/ethyl acetate $=5: 1,34.2 \mathrm{mg}$, 85\% yield, 97\% ee).

$[\alpha]^{25}:-17.1\left(c=1.0, \mathrm{CHCl}_{3}\right)$. HPLC analysis of the product: Daicel CHIRALPAK AS-H column; 10\% i-PrOH in hexanes; $1.0 \mathrm{~mL} / \mathrm{min}$; retention times: $20.2 \mathrm{~min}$ (major), $25.0 \mathrm{~min}$ (minor).

${ }^{1}$ H NMR (400 MHz, acetone- $\left.d_{6}\right) \delta 8.50(\mathrm{~s}, 1 \mathrm{H}), 7.43-7.36(\mathrm{~m}, 2 \mathrm{H}), 7.35-7.26(\mathrm{~m}, 3 \mathrm{H})$, 7.21-7.12 (m, 2H), 6.92-6.84 (m, 2H), $3.29(\mathrm{t}, J=6.9 \mathrm{~Hz}, 2 \mathrm{H}), 3.18(\mathrm{t}, J=6.7 \mathrm{~Hz}, 2 \mathrm{H})$, $2.47(\mathrm{t}, J=7.2 \mathrm{~Hz}, 2 \mathrm{H}), 2.41-2.31(\mathrm{~m}, 2 \mathrm{H}), 1.66-1.54(\mathrm{~m}, 2 \mathrm{H}), 1.51-1.42(\mathrm{~m}, 2 \mathrm{H})$, 1.42-1.29 (m, 2H), 1.17-1.02 (m, 2H) ppm.

${ }^{13}$ C NMR (100 MHz, acetone- $\left.d_{6}\right) \delta 209.8,157.2,143.1,132.7,131.3,130.2,128.9,127.5$, $115.9,66.2,51.7,51.6,38.6,37.6,29.9,28.7,23.1,22.7$ ppm.

IR (ATR) 3396, 2945, 2871, 2092, 1699, 1612, 1593, 1512, 1265, $832 \mathrm{~cm}^{-1}$.

HRMS (CI+) Calcd for $\mathrm{C}_{22} \mathrm{H}_{27} \mathrm{~N}_{6} \mathrm{O}_{2}\left[\mathrm{M}^{+}+\mathrm{H}\right]: 407.2195$, Found: 407.2189.

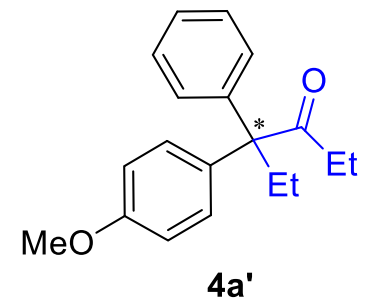

(4-(4-Methoxyphenyl)-4-phenylhexan-3-one (4a'). At $0{ }^{\circ} \mathrm{C}$, to an oven-dried vial charged with $(R)-E^{10}(25.4 \mathrm{mg}, 30 \mu \mathrm{mol}), 5 \AA \mathrm{MS}(0.12 \mathrm{~g})$, and anhydrous toluene (2 $\mathrm{mL})$ was added a solution of the epoxide $3 \mathbf{a}^{\prime}(84.6 \mathrm{mg}, 0.3 \mathrm{mmol})$ in anhydrous 
toluene $(1 \mathrm{~mL})$. Next, the reaction mixture was stirred at the same temperature, and the progress was monitored by TLC. Upon completion (48 h), the reaction mixture was filtered through a plug of silica gel (eluent: ethyl acetate). The filtrate was evaporated, and the residue was subjected to silica gel column chromatography (eluent: hexanes/ethyl acetate $=80: 1)$ to afford $4 a^{\prime}(69.4 \mathrm{mg}, 82 \%$ yield, $72 \%$ ee) as a light yellow oil.

$[\alpha]^{25}:+17.6\left(c=1.0, \mathrm{CHCl}_{3}\right)$. HPLC analysis of the product: Daicel CHIRALPAK $\mathrm{AD}-\mathrm{H}$ column; $1 \% \mathrm{i}-\mathrm{PrOH}$ in hexanes; $1.0 \mathrm{~mL} / \mathrm{min}$; retention times: $6.4 \mathrm{~min}$ (major), $6.7 \mathrm{~min}$ (minor).

${ }^{1} \mathrm{H}$ NMR (400 MHz, $\left.\mathrm{CDCl}_{3}\right) \delta$ 7.36-7.29 (m, 2H), 7.29-7.21 (m, 3H), 7.21-7.14 (m, 2H), 6.90-6.83 (m, 2H), $3.81(\mathrm{~s}, 3 \mathrm{H}), 2.39-2.26(\mathrm{~m}, 4 \mathrm{H}), 0.88(\mathrm{t}, J=7.3 \mathrm{~Hz}, 3 \mathrm{H}), 0.67(\mathrm{t}, J=7.4$ $\mathrm{Hz}, 3 \mathrm{H}) \mathrm{ppm}$.

${ }^{13} \mathrm{C}$ NMR $\left(100 \mathrm{MHz}, \mathrm{CDCl}_{3}\right) \delta 211.6,158.2,141.8,133.2,130.5,129.3,128.0,126.6,113.5$, $65.9,55.2,32.3,30.1,9.4,9.1 \mathrm{ppm}$.

IR (ATR) 2972, 2937, 1703, 1608, 1580, 1510, 1461, 1445, 1250, 1184, 1033, $734 \mathrm{~cm}^{-1}$. HRMS (CI+) Calcd for $\mathrm{C}_{19} \mathrm{H}_{23} \mathrm{O}_{2}\left[\mathrm{M}^{+}+\mathrm{H}\right]: 283.1698$, Found: 283.1692. 


\section{Product Derivatizations}

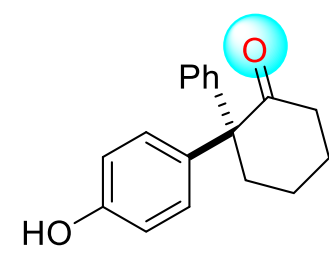

$2 a(92 \%$ ee $)$

$$
\begin{gathered}
\underset{\mathrm{HONH}_{2} \cdot \mathrm{HCl}(2 \text { equiv })}{\longrightarrow} \\
\mathrm{MaHCO}_{3}(2 \text { equiv }) \\
\mathrm{MeOH}, \text { reflux }, 52 \mathrm{~h}
\end{gathered}
$$

$95 \%$

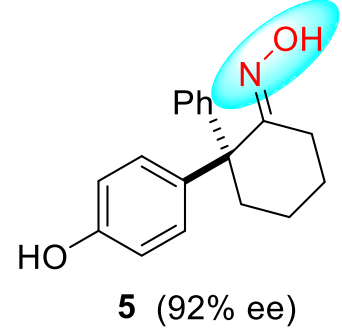

$5(92 \%$ ee $)$

(R)-2-(4-Hydroxyphenyl)-2-phenylcyclohexan-1-one oxime (5). Under $\mathrm{N}_{2}$, to an oven-dried Schlenk tube were sequentially added 2a (106.3 mg, $0.4 \mathrm{mmol}$ ), hydroxylamine hydrochloride $(55.6 \mathrm{mg}, 0.8 \mathrm{mmol}), \mathrm{NaHCO}_{3}(67.3 \mathrm{mg}, 0.8 \mathrm{mmol})$, and anhydrous $\mathrm{MeOH}(4 \mathrm{~mL})$. The reaction mixture was heated to reflux $\left(70{ }^{\circ} \mathrm{C}\right)$, and the progress was monitored by TLC. Upon completion $(\sim 52 \mathrm{~h})$, the reaction was concentrated cooled to room temperature. $\mathrm{H}_{2} \mathrm{O}(10 \mathrm{~mL})$ was added, and the layers were separated. The aqueous layer was extracted with DCM (10 $\mathrm{mL} \times 3)$. The combined organic layers were washed with brine $(10 \mathrm{~mL})$, dried over $\mathrm{Na}_{2} \mathrm{SO}_{4}$, and concentrated. The residue was dried under vacuum without further purification to afford pure oxime 5 (107.2 $\mathrm{mg}, 95 \%$ yield, 92\% ee) as a white solid.

$[\alpha]^{25}:-5.7\left(c=1.0, \mathrm{CHCl}_{3}\right)$. HPLC analysis of the product: Daicel CHIRALPAK AD-H column; $15 \%$ i-PrOH in hexanes; $1.0 \mathrm{~mL} / \mathrm{min}$; retention times: $14.7 \mathrm{~min}$ (major), $25.1 \mathrm{~min}$ (minor).

${ }^{1}$ H NMR (400 MHz, acetone- $\left.d_{6}\right) \delta 9.57(\mathrm{~s}, 1 \mathrm{H}), 8.25(\mathrm{~s}, 1 \mathrm{H}), 7.31-7.22(\mathrm{~m}, 2 \mathrm{H})$, 7.22-7.16 $(\mathrm{m}, 1 \mathrm{H}), 7.16-7.08(\mathrm{~m}, 2 \mathrm{H}), 7.05-6.95(\mathrm{~m}, 2 \mathrm{H}), 6.86-6.77(\mathrm{~m}, 2 \mathrm{H}), 2.82-2.64(\mathrm{~m}, 1 \mathrm{H})$, 2.60-2.41 (m, 3H), 1.76-1.56 (m, 4H) ppm.

${ }^{13}$ C NMR (100 MHz, acetone- $\left.d_{6}\right) \delta 162.8,156.4,146.7,136.0,130.6,129.7,128.3,126.5$, $115.5,55.4,38.8,26.4,23.0(2 \mathrm{C}) \mathrm{ppm}$.

IR (thin film) 3300, 2943, 2863, 1693, 1614, 1596, 1514, 1493, 1446, 1259, $1181 \mathrm{~cm}^{-1}$. HRMS (CI+) Calcd for $\mathrm{C}_{18} \mathrm{H}_{19} \mathrm{NO}_{2}\left[\mathrm{M}^{+}\right]$: 281.1416, Found: 281.1408 . 

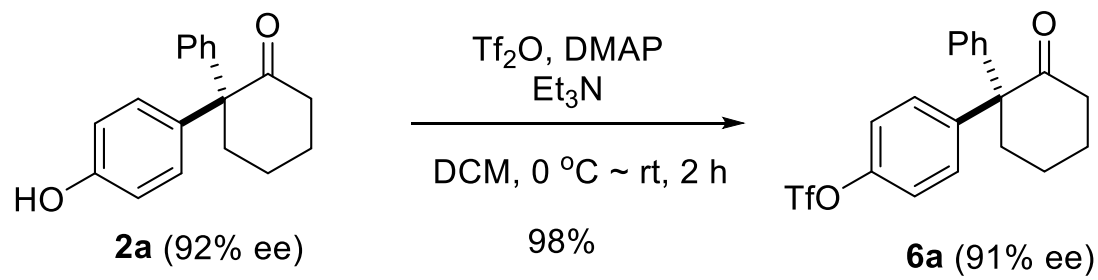

(R)-4-(2-Oxo-1-phenylcyclohexyl)phenyl trifluoromethanesulfonate (6a). At $0{ }^{\circ} \mathrm{C}$, to an oven-dried Schlenk tube were added 2a (532.8 mg, 2 mmol), DMAP (12.2 mg, $0.1 \mathrm{mmol})$, and anhydrous DCM $(10 \mathrm{~mL})$ were sequentially added anhydrous $\mathrm{Et}_{3} \mathrm{~N}$ $(404.8 \mathrm{mg}, 4 \mathrm{mmol})$ and $\mathrm{Tf}_{2} \mathrm{O}(846.4 \mathrm{mg}, 3 \mathrm{mmol})$. Then, the reaction mixture was allowed to warm to room temperature and stirred at the same temperature. The reaction progress was monitored by TLC. Upon completion $(\sim 2 \mathrm{~h})$, a saturated aqueous solution of $\mathrm{NaHCO}_{3}(20 \mathrm{~mL})$ was added. The layers were separated, and the aqueous layer was extracted with DCM $(20 \mathrm{~mL} \times 3)$. The combined organic layers were washed with brine $(20 \mathrm{~mL})$, dried over $\mathrm{Na}_{2} \mathrm{SO}_{4}$, and concentrated. The residue was purified by silica gel flash column chromatography (eluent: hexanes/ethyl acetate $=20: 1)$ to afford $\mathbf{6 a}(783.1 \mathrm{mg}, 98 \%$ yield, $91 \%$ ee $)$ as a yellow oil.

$[\alpha]^{25}:+137.3\left(c=1.0, \mathrm{CHCl}_{3}\right)$. HPLC analysis of the product: Daicel CHIRALPAK $\mathrm{AD}-\mathrm{H}$ column; 3\% $i$-PrOH in hexanes; $1.0 \mathrm{~mL} / \mathrm{min}$; retention times: $8.9 \mathrm{~min}$ (minor), $9.7 \min$ (major).

${ }^{1} \mathrm{H}$ NMR $\left(400 \mathrm{MHz} \mathrm{CDCl}_{3}\right) \delta$ 7.44-7.36 (m, 2H), 7.36-7.28 (m, 1H), $7.15(\mathrm{~d}, J=8.7 \mathrm{~Hz}$, $4 \mathrm{H}), 7.02(\mathrm{~d}, J=8.9 \mathrm{~Hz}, 2 \mathrm{H}), 2.81-2.68(\mathrm{~m}, 1 \mathrm{H}), 2.61-2.31(\mathrm{~m}, 3 \mathrm{H}), 2.10-1.75(\mathrm{~m}, 4 \mathrm{H})$ ppm.

${ }^{13} \mathrm{C}$ NMR $\left(100 \mathrm{MHz}, \mathrm{CDCl}_{3}\right) \delta 210.5,148.0,144.2,140.1,130.6,129.0,128.2,127.5,120.6$, $118.7(\mathrm{q}, J=318.8 \mathrm{~Hz}), 63.5,40.5,39.2,27.7,22.0 \mathrm{ppm}$.

${ }^{19}$ F NMR (376 MHz, $\left.\mathrm{CDCl}_{3}\right) \delta-72.9$ ppm.

IR (thin film) 2945, 2869, 1712, 1501, 1423, 1250, 1214, 1142, 1120, 1017, $890 \mathrm{~cm}^{-1}$. HRMS (CI+) Calcd for $\mathrm{C}_{19} \mathrm{H}_{17} \mathrm{~F}_{3} \mathrm{O}_{4} \mathrm{~S}\left[\mathrm{M}^{+}\right]$: 398.0800, Found: 398.0808 . 

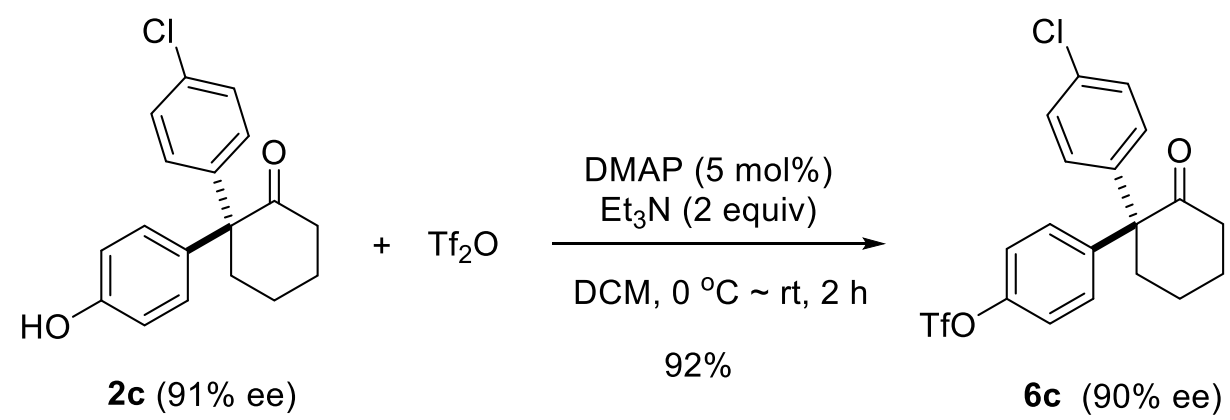

(S)-4-(1-(4-Chlorophenyl)-2-oxocyclohexyl)phenyl trifluoromethanesulfonate (6c).

Under $\mathrm{N}_{2}$ at $0{ }^{\circ} \mathrm{C}$, to an oven-dried Schlenk tube charged with $2 \mathrm{c}(1.96 \mathrm{~g}, 6.5 \mathrm{mmol})$, DMAP (40.0 mg, $0.33 \mathrm{mmol})$, and anhydrous DCM $(60 \mathrm{~mL})$ were sequentially added anhydrous $\mathrm{Et}_{3} \mathrm{~N}(1.32 \mathrm{~g}, 13 \mathrm{mmol})$ and $\mathrm{Tf}_{2} \mathrm{O}(2.75 \mathrm{~g}, 9.75 \mathrm{mmol})$. The reaction mixture was stirred room temperature, and the reaction progress was monitored by TLC. Upon completion $(2 \mathrm{~h})$, a saturated aqueous solution of $\mathrm{NaHCO}_{3}(40 \mathrm{~mL})$ was added to quench the reaction. The layers were separated, and the aqueous layer was extracted with DCM $(40 \mathrm{~mL} \times 3)$. The combined organic layers were washed with brine $(20 \mathrm{~mL})$, dried over $\mathrm{Na}_{2} \mathrm{SO}_{4}$, and concentrated. The residue was purified by silica gel flash column chromatography (eluent: hexanes/ethyl acetate $=20: 1$ ) to afford $6 \mathrm{c}(2.59 \mathrm{~g}, 92 \%$ yield, $90 \%$ ee) as a yellow oil.

$[\alpha]^{25}:+28.9\left(c=1.0, \mathrm{CHCl}_{3}\right)$. HPLC analysis of the product: Daicel CHIRALPAK IC column; $1 \%$ i-PrOH in hexanes; $1.0 \mathrm{~mL} / \mathrm{min}$; retention times: $7.7 \mathrm{~min}$ (minor), $9.2 \mathrm{~min}$ (major).

${ }^{1} \mathrm{H}$ NMR $\left(400 \mathrm{MHz}, \mathrm{CDCl}_{3}\right) \delta 7.35(\mathrm{~d}, J=8.6 \mathrm{~Hz}, 2 \mathrm{H}), 7.23-7.15(\mathrm{~m}, 2 \mathrm{H}), 7.11-6.99(\mathrm{~m}$, $4 \mathrm{H}), 2.71-2.58(\mathrm{~m}, 1 \mathrm{H}), 2.57-2.39(\mathrm{~m}, 3 \mathrm{H}), 2.06-1.75(\mathrm{~m}, 4 \mathrm{H}) \mathrm{ppm}$.

${ }^{13} \mathrm{C}$ NMR (100 MHz, $\left.\mathrm{CDCl}_{3}\right) \delta 210.0,148.2,143.3,139.2,133.4,130.4,129.7,129.0,120.9$, $118.6(\mathrm{q}, J=318.9 \mathrm{~Hz}), 62.9,40.4,39.2,27.5,21.9 \mathrm{ppm}$.

${ }^{19} \mathbf{F}$ NMR $\left(376 \mathrm{MHz}, \mathrm{CDCl}_{3}\right) \delta-72.9 \mathrm{ppm}$.

IR (ATR) 2944, 2868, 1710, 1493, 1421, 1249, 1206, 1136, 1118, 1095, 1014, 883, 827, 809 $\mathrm{cm}^{-1}$.

HRMS (CI+) Calcd for $\mathrm{C}_{19} \mathrm{H}_{16} \mathrm{ClF}_{3} \mathrm{O}_{4} \mathrm{~S}$ [M+]: 432.0410, Found: 432.0412. 


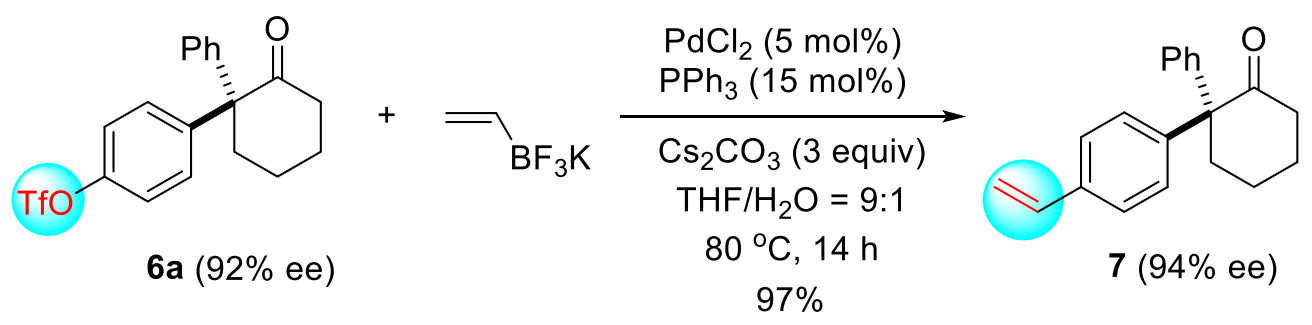

(R)-2-Phenyl-2-(4-vinylphenyl)cyclohexan-1-one (7). ${ }^{11}$ Under $\mathrm{N}_{2}$ at $\mathrm{rt}$, to an oven-dried Schlenk tube were sequentially added $\mathrm{PdCl}_{2}(3.6 \mathrm{mg}, 20 \mu \mathrm{mol}), \mathrm{PPh}_{3}(15.6$ $\mathrm{mg}, 60 \mu \mathrm{mol}), \mathrm{Cs}_{2} \mathrm{CO}_{3}(391.1 \mathrm{mg}, 1.2 \mathrm{mmol})$, potassium vinyltrifluoroborate (107.3 $\mathrm{mg}, 0.8 \mathrm{mmol})$, a solution of $6 \mathbf{6}(159.6 \mathrm{mg}, 0.4 \mathrm{mmol})$ in THF $(3.6 \mathrm{~mL})$, and $\mathrm{H}_{2} \mathrm{O}(0.4$ $\mathrm{mL}$ ). The mixture was stirred at $80{ }^{\circ} \mathrm{C}$, and the progress was monitored by TLC. Upon completion $(\sim 14 \mathrm{~h}), \mathrm{Na}_{2} \mathrm{SO}_{4}$ was added to remove water. The mixture was filtrated through a short pad of silica gel, which was washed with ethyl acetate (20 $\mathrm{mL} \times 3)$. The filtrate was concentrated. The residue was purified by silica gel flash column chromatography (eluent: hexanes/ethyl acetate $=30: 1 \rightarrow 20: 1$ ) to afford 7 (107.4 mg, 97\% yield, 92\% ee) as a colorless oil.

$[\alpha]^{25}:-9.7\left(c=1.0, \mathrm{CHCl}_{3}\right)$. HPLC analysis of the product: Daicel CHIRALPAK AD-H column; $1 \%$ i-PrOH in hexanes; $1.0 \mathrm{~mL} / \mathrm{min}$; retention times: $11.8 \mathrm{~min}$ (major), $13.1 \mathrm{~min}$ (minor).

${ }^{1} \mathrm{H}$ NMR $\left(400 \mathrm{MHz}, \mathrm{CDCl}_{3}\right) \delta$ 7.44-7.27 (m, 4H), 7.26-7.18 (m, 1H), $7.06(\mathrm{~d}, J=7.6 \mathrm{~Hz}$, 2H), $7.01(\mathrm{~d}, J=8.2 \mathrm{~Hz}, 2 \mathrm{H}), 6.68\left(\mathrm{dd}, J_{1}=17.6 \mathrm{~Hz}, J_{2}=10.9 \mathrm{~Hz}, 1 \mathrm{H}\right), 5.72(\mathrm{~d}, J=17.6$ $\mathrm{Hz}, 1 \mathrm{H}), 5.22(\mathrm{~d}, J=10.8 \mathrm{~Hz}, 1 \mathrm{H}), 2.58(\mathrm{t}, J=5.5 \mathrm{~Hz}, 2 \mathrm{H}), 2.49(\mathrm{t}, J=6.6 \mathrm{~Hz}, 2 \mathrm{H})$, 1.99-1.87 (m, 2H), 1.87-1.73 (m, 2H) ppm.

${ }^{13} \mathrm{C}$ NMR $\left(100 \mathrm{MHz}, \mathrm{CDCl}_{3}\right) \delta 211.1,142.2,141.9,136.2,136.0,128.6,128.4,128.3,126.8$, $126.1,113.9,63.6,40.6,39.0,27.7,22.0$ ppm.

IR (thin film) 2941, 2865, 1709, 1630, 1600, 1510, 1495, 1463, 1447, 1423, 1403, 1117, 992 $\mathrm{cm}^{-1}$.

HRMS (CI+) Calcd for $\mathrm{C}_{20} \mathrm{H}_{20} \mathrm{O}\left[\mathrm{M}^{+}\right]$: 276.1514, Found: 276.1526. 


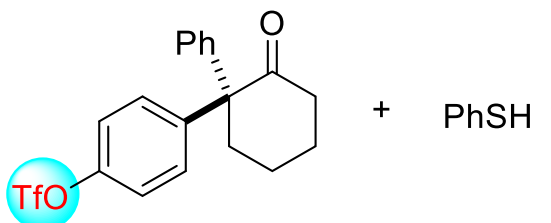

$6 \mathbf{a}(92 \%$ ee $)$

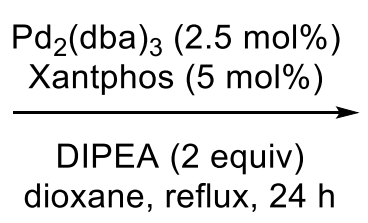

$63 \%$

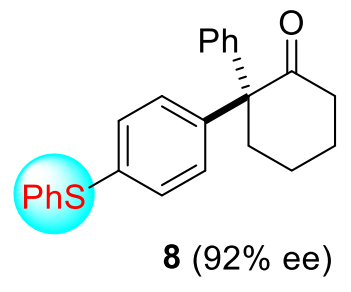

(R)-2-Phenyl-2-(4-(phenylthio)phenyl)cyclohexan-1-one (8). ${ }^{12}$ Under $\mathrm{N}_{2}$, to an oven-dried Schlenk tube were sequentially added $\operatorname{Pd}_{2}(\mathrm{dba})_{3}(9.3 \mathrm{mg}, 10 \mu \mathrm{mol})$, Xantphos $(11.6 \mathrm{mg}, 20 \mu \mathrm{mol})$, a solution of $6 \mathrm{a}(159.3 \mathrm{mg}, 0.4 \mathrm{mmol})$ in anhydrous dioxane (6 mL), PhSH (132.2 mg, $1.2 \mathrm{mmol})$, DIPEA (103.4 mg, $0.8 \mathrm{mmol})$, and anhydrous dioxane $(2 \mathrm{~mL})$. The reaction was heated to reflux $\left(110{ }^{\circ} \mathrm{C}\right)$, and the reaction progress was monitored by TLC. Upon completion $(\sim 24 \mathrm{~h})$, the reaction was filtered through a short pad of silica gel, which was washed with ethyl acetate (20 $\mathrm{mL} \times 3)$. The filtrate was concentrated. The residue was purified by silica gel flash column chromatography (eluent: hexanes/ethyl acetate $=2: 1 \rightarrow 1: 1)$ to afford 8 (90.6 mg, 63\% yield, 92\% ee) as a colorless oil.

$[\alpha]_{D^{25}}:+7.7\left(c=1.0, \mathrm{CHCl}_{3}\right)$. HPLC analysis of the product: Daicel CHIRALPAK AD-H column; $1 \%$ - $\mathrm{PrOH}$ in hexanes; $1.0 \mathrm{~mL} / \mathrm{min}$; retention times: $14.8 \mathrm{~min}$ (minor), $18.7 \mathrm{~min}$ (major).

${ }^{1} \mathbf{H}$ NMR (400 MHz, $\left.\mathrm{CDCl}_{3}\right) \delta$ 7.41-7.35 (m, 2H), 7.35-7.31 (m, 2H), 7.31-7.27 (m, 2H), 7.27-7.24 (m, 2H), 7.24-7.18 (m, 2H), $7.09(\mathrm{~d}, J=7.4 \mathrm{~Hz}, 2 \mathrm{H}), 6.93(\mathrm{~d}, J=8.4 \mathrm{~Hz}, 2 \mathrm{H})$, 2.70-2.58 (m, 1H), 2.56-2.43 (m, 3H), 2.01-1.89 (m, 2H), 1.87-1.77 (m, 2H) ppm.

${ }^{13} \mathrm{C}$ NMR (100 MHz, $\left.\mathrm{CDCl}_{3}\right) \delta 211.1,141.55,141.47,134.9,134.5,131.6,129.9,129.4$, $129.2,128.5,128.4,127.3,127.0,63.6,40.6,39.1,27.7,22.0$ ppm.

IR (thin film) 3058, 2941, 2864, 1710, 1599, 1493, 1477, 1447, 1119, 1085, 1071, 1016, 808 $\mathrm{cm}^{-1}$.

HRMS (CI+) Calcd for $\mathrm{C}_{24} \mathrm{H}_{22} \mathrm{OS}\left[\mathrm{M}^{+}\right]$: 358.1391, Found: 358.1389 . 


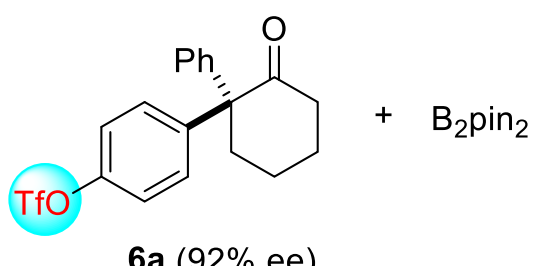

6a $(92 \%$ ee $)$

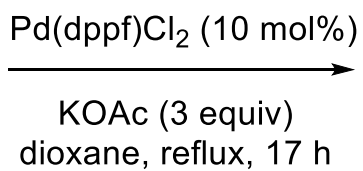

$79 \%$

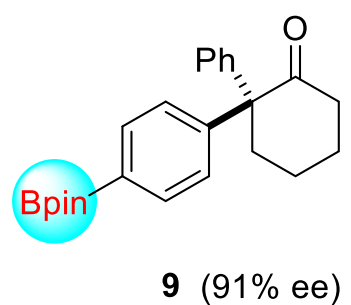

$9(91 \%$ ee $)$

\section{(R)-2-Phenyl-2-(4-(4,4,5,5-tetramethyl-1,3,2-dioxaborolan-2-yl)phenyl)cyclohexan-1}

-one (9). ${ }^{13}$ Under $\mathrm{N}_{2}$, to an oven-dried Schlenk tube were sequentially added bis(pinacolato)diboron (101.5 mg, $0.4 \mathrm{mmol}), \mathrm{Pd}(\mathrm{dppf}) \mathrm{Cl}_{2}(28.4 \mathrm{mg}, 40 \mu \mathrm{mol}), \mathrm{KOAc}$ $(117.5 \mathrm{mg}, 1.2 \mathrm{mmol})$, and a solution of $6 \mathrm{a}(159.5 \mathrm{mg}, 0.4 \mathrm{mmol})$ in anhydrous dioxane $(4 \mathrm{~mL})$. The mixture was heated to reflux $\left(110^{\circ} \mathrm{C}\right)$, and the reaction progress was monitored by TLC. Upon completion $(\sim 17 \mathrm{~h})$, the reaction was filtered through a short pad of silica gel, which was washed with ethyl acetate $(20 \mathrm{~mL} \times 3)$. The filtrate was concentrated, and the residue was purified by silica gel flash column chromatography (eluent: hexanes/ethyl acetate $=30: 1)$ to afford $9(118.8 \mathrm{mg}, 79 \%$ yield, $91 \%$ ee) as a colorless oil.

$[\alpha]^{25}:-33.4\left(c=1.0, \mathrm{CHCl}_{3}\right)$. HPLC analysis of the product: Daicel CHIRALPAK $\mathrm{AD}-\mathrm{H}$ column; $1 \% \mathrm{i}-\mathrm{PrOH}$ in hexanes; $1.0 \mathrm{~mL} / \mathrm{min}$; retention times: $7.4 \mathrm{~min}$ (minor), 9.9 min (major).

${ }^{1} \mathrm{H}$ NMR $\left(400 \mathrm{MHz}, \mathrm{CDCl}_{3}\right) \delta 7.78(\mathrm{~d}, J=8.0 \mathrm{~Hz}, 2 \mathrm{H}), 7.33-7.26(\mathrm{~m}, 2 \mathrm{H})$, 7.26-7.18 (m, $1 \mathrm{H}), 7.11(\mathrm{~d}, J=8.1 \mathrm{~Hz}, 2 \mathrm{H}), 7.01(\mathrm{~d}, J=7.4 \mathrm{~Hz}, 2 \mathrm{H}), 2.72-2.60(\mathrm{~m}, 1 \mathrm{H}), 2.60-2.42(\mathrm{~m}$, $3 \mathrm{H}), 2.01-1.88(\mathrm{~m}, 2 \mathrm{H}), 1.88-1.74(\mathrm{~m}, 2 \mathrm{H}), 1.33(\mathrm{~s}, 12 \mathrm{H}) \mathrm{ppm}$.

${ }^{13} \mathrm{C}$ NMR (100 MHz, $\left.\mathrm{CDCl}_{3}\right) \delta 211.0,145.1,142.4,134.8$ (2C), 128.5, 128.2, 127.9, 126.8, $83.7,64.0,40.7,38.9,27.7,24.8,22.0$ ppm.

IR (thin film) 2978, 2941, 2867, 1712, 1611, 1448, 1400, 1363, 1325, 1273, 1145, 1093, 859 $\mathrm{cm}^{-1}$.

HRMS (CI+) Calcd for $\mathrm{C}_{24} \mathrm{H}_{29} \mathrm{BO}_{3}\left[\mathrm{M}^{+}\right]$: 376.2210, Found: 376.2216 . 


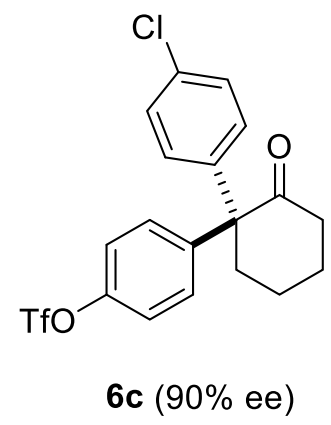

$$
\begin{gathered}
\underset{\mathrm{Pd}(\mathrm{OAc})_{2}(2 \mathrm{~mol} \%)}{\mathrm{PPh}_{3}(5 \mathrm{~mol} \%)} \\
\underset{\mathrm{Et}_{3} \mathrm{~N}(3 \text { equiv })}{\longrightarrow} \\
\mathrm{HCO}_{2} \mathrm{H}(2 \text { equiv }) \\
\mathrm{DMF}, 65^{\circ} \mathrm{C}, 17 \mathrm{~h}
\end{gathered}
$$

$87 \%$

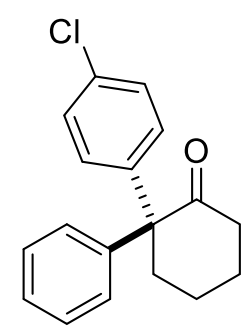

$10(90 \%$ ee $)$

(S)-2-(4-Chlorophenyl)-2-phenylcyclohexan-1-one (10). ${ }^{14}$ Under $\mathrm{N}_{2}$, to an oven-dried Schlenk flask were sequentially added $\mathrm{Pd}(\mathrm{OAc}) 2(25.7 \mathrm{mg}, 0.11 \mathrm{mmol})$, $\mathrm{PPh}_{3}(73.9 \mathrm{mg}, 0.28 \mathrm{mmol})$, anhydrous DMF (25 mL), anhydrous $\mathrm{Et}_{3} \mathrm{~N}(1.70 \mathrm{~g}, 16.8$ $\mathrm{mmol})$, and $\mathrm{HCO}_{2} \mathrm{H}(0.52 \mathrm{~g}, 11.2 \mathrm{mmol})$. Next, a solution of $6 \mathrm{c}(2.40 \mathrm{~g}, 5.6 \mathrm{mmol})$ in anhydrous DMF (5 mL) was added. The reaction mixture was stirred at $65{ }^{\circ} \mathrm{C}$, and the progress was monitored by TLC. Upon completion $(17 \mathrm{~h})$, the reaction was cooled to rt. A saturated aqueous solution of $\mathrm{NH}_{4} \mathrm{Cl}(30 \mathrm{~mL})$ was added to quench the reaction. The layers were separated, and the aqueous layer was extracted with ethyl acetate $(30 \mathrm{~mL} \times 3)$. The combined organic layers were washed with brine (20 $\mathrm{mL}$ ), dried over $\mathrm{Na}_{2} \mathrm{SO}_{4}$, and concentrated. The residue was purified by silica gel flash column chromatography (eluent: hexanes/ethyl acetate $=30: 1)$ to afford 10 (1.38 g, 87\% yield, $90 \%$ ee) as a colorless oil.

$[\alpha]^{25}:-22.6\left(c=1.0, \mathrm{CHCl}_{3}\right)$. HPLC analysis of the product: Daicel CHIRALPAK $\mathrm{AD}-\mathrm{H}$ column; $1 \% \mathrm{i}-\mathrm{PrOH}$ in hexanes; $1.0 \mathrm{~mL} / \mathrm{min}$; retention times: $9.3 \mathrm{~min}$ (major), $10.3 \mathrm{~min}$ (minor).

${ }^{1}$ H NMR (400 MHz, $\left.\mathrm{CDCl}_{3}\right) \delta$ 7.39-7.30 (m, 2H), 7.30-7.20 (m, 3H), 7.09 (d, J = 7.7 Hz, 2H), $6.93(\mathrm{~d}, J=8.6 \mathrm{~Hz}, 2 \mathrm{H}), 2.72-2.60(\mathrm{~m}, 1 \mathrm{H}), 2.57-2.40(\mathrm{~m}, 3 \mathrm{H}), 2.03-1.73(\mathrm{~m}, 4 \mathrm{H})$ ppm.

${ }^{13} \mathrm{C}$ NMR $\left(100 \mathrm{MHz} \mathrm{CDCl}_{3}\right) \delta 210.8,141.5,141.1,132.5,129.9,128.6,128.25,128.18$, 127.1, 63.3, 40.5, 39.0, 27.6, 21.9 ppm.

IR (ATR) 2941, 2865, 1707, 1492, 1447, 1265, 1118, 1095, 1014, $808 \mathrm{~cm}^{-1}$.

HRMS (CI+) Calcd for $\mathrm{C}_{18} \mathrm{H}_{17} \mathrm{ClO}\left[\mathrm{M}^{+}\right]$: 284.0968, Found: 284.0959. 

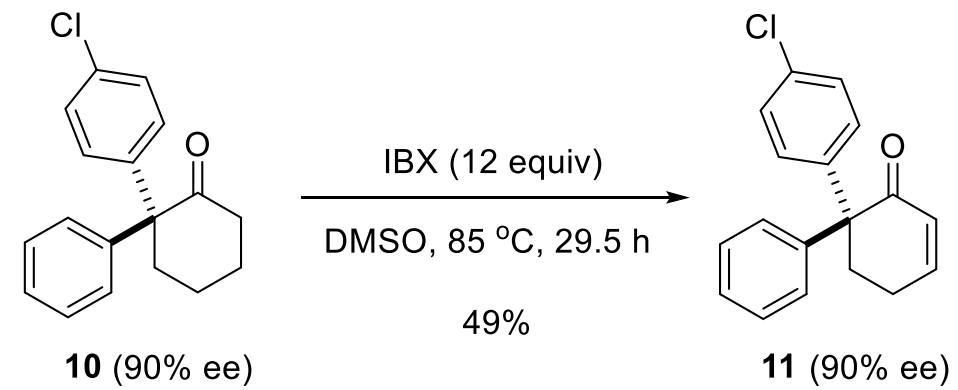

(S)-4-Chloro-5',6'-dihydro-2'H-[1,1':1',1"-terphenyl]-2'-one (11). ${ }^{15}$ Under $\mathrm{N}_{2}$, to an oven-dried Schlenk tube were sequentially added 2-iodoxybenzoic acid (560.1 mg, 2 $\mathrm{mmol})$, anhydrous DMSO (2 mL), and a solution of $10(113.6 \mathrm{mg}, 0.4 \mathrm{mmol})$ in anhydrous DMSO (2 mL). The reaction was stirred at $85{ }^{\circ} \mathrm{C}$ for $11 \mathrm{~h}$. An additional portion of 2-iodoxybenzoic acid (559.4 mg, $2 \mathrm{mmol}$ ) was added. After another $11 \mathrm{~h}$, another portion of 2-iodoxybenzoic acid $(223.9 \mathrm{mg}, 0.4 \mathrm{mmol})$ was added. The reaction progress was monitored by TLC. Upon completion (totally $29.5 \mathrm{~h}$ ), a saturated aqueous solution of $\mathrm{Na}_{2} \mathrm{CO}_{3}$ was added to quench the reaction. The layers were separated, and the aqueous layer was extracted with ethyl acetate $(10 \mathrm{~mL} \times 3)$. The combined organic layers were washed with brine $(10 \mathrm{~mL})$, dried over $\mathrm{Na}_{2} \mathrm{SO}_{4}$, and concentrated. The residue was purified by silica gel flash column chromatography (eluent: hexanes/ethyl acetate $=20: 1)$ to afford 11 (55.3 $\mathrm{mg}, 49 \%$ yield, $91 \%$ ee) as a yellow oil.

$[\alpha]^{25}:-42.5\left(c=1.0, \mathrm{CHCl}_{3}\right)$. HPLC analysis of the product: Daicel CHIRALPAK IC column; $1 \%$ i-PrOH in hexanes; $1.0 \mathrm{~mL} / \mathrm{min}$; retention times: $17.3 \mathrm{~min}$ (minor), 18.9 $\min ($ major).

${ }^{1}$ H NMR (400 MHz, $\left.\mathrm{CDCl}_{3}\right) \delta$ 7.37-7.19 (m, 5H), 7.14-7.06 (m, 2H), 7.05-6.97 (m, 2H), $6.83\left(\mathrm{dt}, J_{1}=9.9 \mathrm{~Hz}, J_{2}=4.0 \mathrm{~Hz}, 1 \mathrm{H}\right), 6.20\left(\mathrm{dt}, J_{1}=10.0 \mathrm{~Hz}, J_{2}=1.8 \mathrm{~Hz}, 1 \mathrm{H}\right), 2.81-2.61(\mathrm{~m}$, $2 \mathrm{H}), 2.40-2.17(\mathrm{~m}, 2 \mathrm{H}) \mathrm{ppm}$.

${ }^{13} \mathrm{C}$ NMR $\left(100 \mathrm{MHz}, \mathrm{CDCl}_{3}\right) \delta 199.7,149.3,140.8,140.7,132.7,130.0,129.8,128.24$, $128.17,128.1,127.1,59.0,34.8,24.3$ ppm.

IR (ATR) 3057, 2932, 1671, 1491, 1446, 1421, 1400, 1385, 1264, 1216, 1094, 1013, 733 $\mathrm{cm}^{-1}$. 
HRMS (CI+) $\mathrm{C}_{18} \mathrm{H}_{15} \mathrm{ClO}\left[\mathrm{M}^{+}\right]$: 282.0811, Found: 282.0819 .
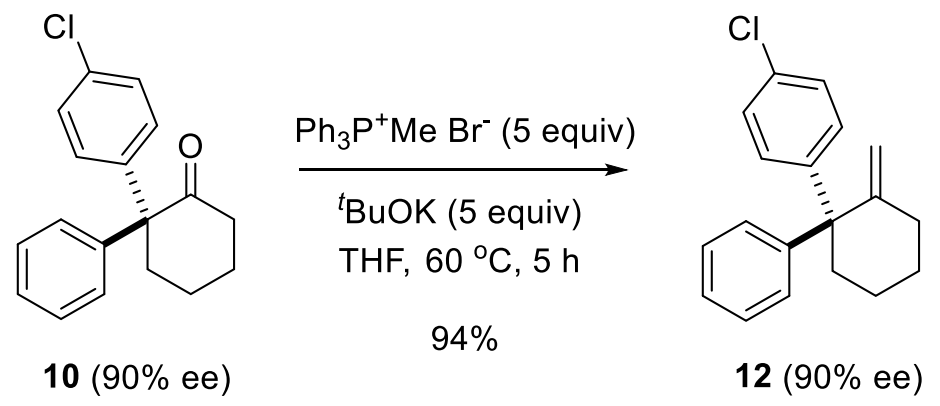

(S)-1-Chloro-4-(2-methylene-1-phenylcyclohexyl)benzene (12). Under $\mathrm{N}_{2}$, to an oven-dried Schlenk tube charged with methyltriphenylphosphonium bromide $(714.0$ $\mathrm{mg}, 2 \mathrm{mmol}),{ }^{t} \mathrm{BuOK}(224.3 \mathrm{mg}, 2 \mathrm{mmol})$, and anhydrous THF (2 mL) was added a solution of $10(114.3 \mathrm{mg}, 0.4 \mathrm{mmol})$ in anhydrous THF (2 mL). Next, the reaction mixture was stirred at $60{ }^{\circ} \mathrm{C}$, and the progress was monitored by TLC. Upon completion $(5 \mathrm{~h})$, the reaction mixture was cooled to room temperature, filtrated through a short pad of silica gel (eluent: ethyl acetate), and concentrated. The residue was purified by silica gel flash column chromatography (eluent: hexanes) to afford 12 (106.9 mg, 94\% yield, 90\% ee) as a colorless oil.

$[\alpha]^{25}:+0.6\left(c=1.0, \mathrm{CHCl}_{3}\right)$. HPLC analysis of the product: Daicel CHIRALPAK OD-H column; $0 \%$ - $\mathrm{PrOH}$ in hexanes; $0.5 \mathrm{~mL} / \mathrm{min}$; retention times: $11.5 \mathrm{~min}$ (major), $12.8 \mathrm{~min}$ (minor).

${ }^{1} \mathbf{H}$ NMR $\left(400 \mathrm{MHz} \mathrm{CDCl}_{3}\right) \delta$ 7.32-7.11 (m, 7H), 7.07 (d, J=8.6 Hz, 2H), $5.03(\mathrm{~s}, 1 \mathrm{H})$, $4.24(\mathrm{~d}, J=1.6 \mathrm{~Hz}, 1 \mathrm{H}), 2.54-2.34(\mathrm{~m}, 2 \mathrm{H}), 2.34-2.19(\mathrm{~m}, 2 \mathrm{H}), 1.70-1.50(\mathrm{~m}, 4 \mathrm{H}) \mathrm{ppm}$.

${ }^{13} \mathrm{C}$ NMR $\left(100 \mathrm{MHz}, \mathrm{CDCl}_{3}\right) \delta 153.5,145.1,144.5,131.7,130.3,128.8,128.1,128.0,126.1$, 113.8, 55.2, 37.7, 34.5, 28.5, 22.7 ppm.

IR (ATR) 2935, 2858, 1636, 1599, 1491, 1446, 1398, 1264, 1095, 1013, $908 \mathrm{~cm}^{-1}$.

HRMS (CI+) Calcd for $\mathrm{C}_{19} \mathrm{H}_{19} \mathrm{Cl}\left[\mathrm{M}^{+}\right]$: 282.1175, Found: 282.1165. 

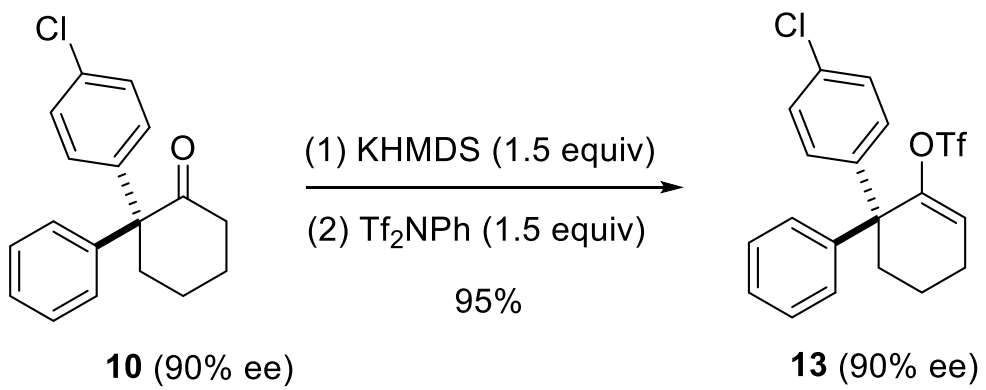

(S)-4-Chloro-3',4'-dihydro-2'H-[1,1':1',1"-terphenyl]-6'-yl trifluoromethanesulfonate (13). ${ }^{16}$ At $-78{ }^{\circ} \mathrm{C}$, to an oven-dried Schlenk tube charged with a solution of $\mathbf{1 0}$ (114.0 $\mathrm{mg}, 0.4 \mathrm{mmol})$ in anhydrous THF $(2 \mathrm{ml})$ was added a solution of KHMDS (0.6 mmol, $0.6 \mathrm{~mL}, 1 \mathrm{M}$ ) in THF over $5 \mathrm{~min}$. The mixture was stirred at $-78^{\circ} \mathrm{C}$ for $30 \mathrm{~min}$ and then a solution of $\mathrm{N}$-phenylbis(trifluoromethanesulfonimide) $(214.6 \mathrm{mg}, 0.6 \mathrm{mmol})$ in anhydrous THF (2 mL) was added. The reaction mixture was warmed to room temperature and stirred at the same temperature. The reaction progress was monitored by TLC. Upon completion $(0.5 \mathrm{~h})$, a saturated aqueous solution of $\mathrm{NH}_{4} \mathrm{Cl}$ $(10 \mathrm{~mL})$ was added to quench the reaction. The layers were separated, and the aqueous layer was extracted with ethyl acetate $(10 \mathrm{~mL} \times 3)$. The combined organic layers were washed with brine $(10 \mathrm{~mL})$, dried over $\mathrm{Na}_{2} \mathrm{SO}_{4}$, and concentrated. The residue was purified by silica gel flash column chromatography (eluent: hexanes/ethyl acetate $=80: 1)$ to afford $13(159.2 \mathrm{mg}, 95 \%$ yield, $90 \%$ ee $)$ as a colorless oil.

$[\alpha]_{D^{25}}:-5.8\left(c=1.0, \mathrm{CHCl}_{3}\right)$. HPLC analysis of the product: Daicel CHIRALPAK AD-H column; $0.5 \%$ i-PrOH in hexanes; $0.5 \mathrm{~mL} / \mathrm{min}$; retention times: $9.3 \mathrm{~min}$ (minor), 9.7 min (major).

${ }^{1} \mathrm{H}$ NMR $\left(400 \mathrm{MHz} \mathrm{CDCl}_{3}\right) \delta$ 7.38-7.27 (m, 5H), 7.27-7.17 (m, 4H), $6.06(\mathrm{t}, J=4.1 \mathrm{~Hz}$, $1 \mathrm{H}), 2.59-2.42(\mathrm{~m}, 2 \mathrm{H}), 2.36\left(\mathrm{td}, J_{1}=6.2 \mathrm{~Hz}, J_{2}=4.2 \mathrm{~Hz}, 2 \mathrm{H}\right), 1.53-1.43(\mathrm{~m}, 2 \mathrm{H}) \mathrm{ppm}$.

${ }^{13} \mathrm{C}$ NMR $\left(100 \mathrm{MHz} \mathrm{CDCl}_{3}\right) \delta 150.6,142.7,141.8,133.0,130.5,128.9,128.22,128.20$, $127.2,119.1,118.0(q, J=317.8 \mathrm{~Hz}), 53.4,39.2,24.7,17.9$ ppm.

${ }^{19}$ F NMR (376 MHz, $\left.\mathrm{CDCl}_{3}\right) \delta-75.2 \mathrm{ppm}$.

IR (ATR) 2942, 1671, 1491, 1415, 1399, 1205, 1139, 1096, 1027, $1014 \mathrm{~cm}^{-1}$. 
HRMS (CI+) Calcd for $\mathrm{C}_{19} \mathrm{H}_{16} \mathrm{ClF}_{3} \mathrm{O}_{3} \mathrm{~S}\left[\mathrm{M}^{+}\right]$: 416.0461, Found: 416.0451 .

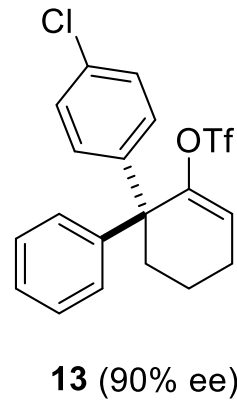

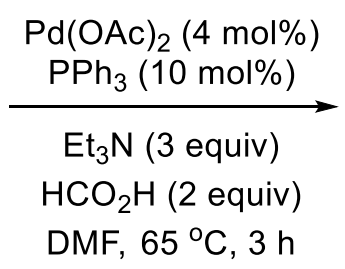

$76 \%$

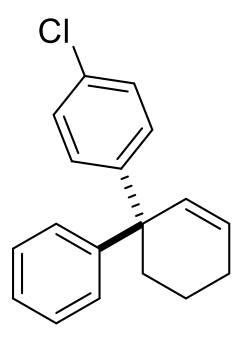

$14(88 \%$ ee $)$

(S)-4-Chloro-3',4'-dihydro-2'H-1,1':1',1'-terphenyl (14). ${ }^{14}$ Under $\mathrm{N}_{2}$, to an oven-dried Schlenk tube were sequentially added Pd(OAc)2 (1.9 mg, $8 \mu \mathrm{mol}), \mathrm{PPh}_{3}(5.3 \mathrm{mg}, 20$ $\mu \mathrm{mol})$, anhydrous DMF (1 mL), Et $3 \mathrm{~N}(60.7 \mathrm{mg}, 0.6 \mathrm{mmol}), \mathrm{HCO}_{2} \mathrm{H}(18.4 \mathrm{mg}, 0.4$ $\mathrm{mmol})$, and a solution of $13(83.6 \mathrm{mg}, 0.2 \mathrm{mmol})$ in anhydrous DMF $(1 \mathrm{~mL})$. The reaction was stirred at $65{ }^{\circ} \mathrm{C}$ and monitored by TLC. Upon completion $(3 \mathrm{~h})$, a saturated aqueous solution of $\mathrm{NH}_{4} \mathrm{Cl}(4 \mathrm{~mL})$ was added to quench the reaction. The layers were separated, and the aqueous layer was extracted with ethyl acetate (10 $\mathrm{mL} \times 3)$. The combined organic layers were washed with brine $(10 \mathrm{~mL})$, dried over $\mathrm{Na}_{2} \mathrm{SO}_{4}$, and concentrated. The residue was purified by silica gel flash column chromatography (eluent: hexanes/ethyl acetate $=100: 1)$ to afford $14(41.2 \mathrm{mg}, 76 \%$ yield, $88 \%$ ee) as a colorless oil.

$[\alpha]^{25}:-9.5\left(c=1.0, \mathrm{CHCl}_{3}\right)$. HPLC analysis of the product: Daicel CHIRALPAK OJ-H column; 2\% $i$-PrOH in hexanes; $0.9 \mathrm{~mL} / \mathrm{min}$; retention times: $7.0 \mathrm{~min}$ (major), $7.5 \mathrm{~min}$ (minor).

${ }^{1}$ H NMR $\left(400 \mathrm{MHz}, \mathrm{CDCl}_{3}\right) \delta$ 7.30-7.19 (m, 6H), 7.19-7.12 (m, 3H), $6.02(\mathrm{~d}, J=10.2 \mathrm{~Hz}$, $1 \mathrm{H}), 5.94\left(\mathrm{dt}, J_{1}=10.1 \mathrm{~Hz}, J_{2}=3.5 \mathrm{~Hz}, 1 \mathrm{H}\right), 2.36-2.16(\mathrm{~m}, 2 \mathrm{H}), 2.14-2.00(\mathrm{~m}, 2 \mathrm{H})$, 1.67-1.45 (m, 2H) ppm.

${ }^{13} \mathrm{C}$ NMR $\left(100 \mathrm{MHz} \mathrm{CDCl}_{3}\right) \delta 148.7,147.9,134.0,131.4,129.3,128.12,128.08,128.0$, $127.7,125.9,48.0,36.0,25.0,18.9$ ppm.

IR (ATR) 3023, 2937, 2866, 2834, 1598, 1489, 1446, 1264, 1075, 1013, $916 \mathrm{~cm}^{-1}$.

HRMS (CI+) Calcd for $\mathrm{C}_{18} \mathrm{H}_{17} \mathrm{Cl}\left[\mathrm{M}^{+}\right]$: 268.1019, Found: 268.1024. 


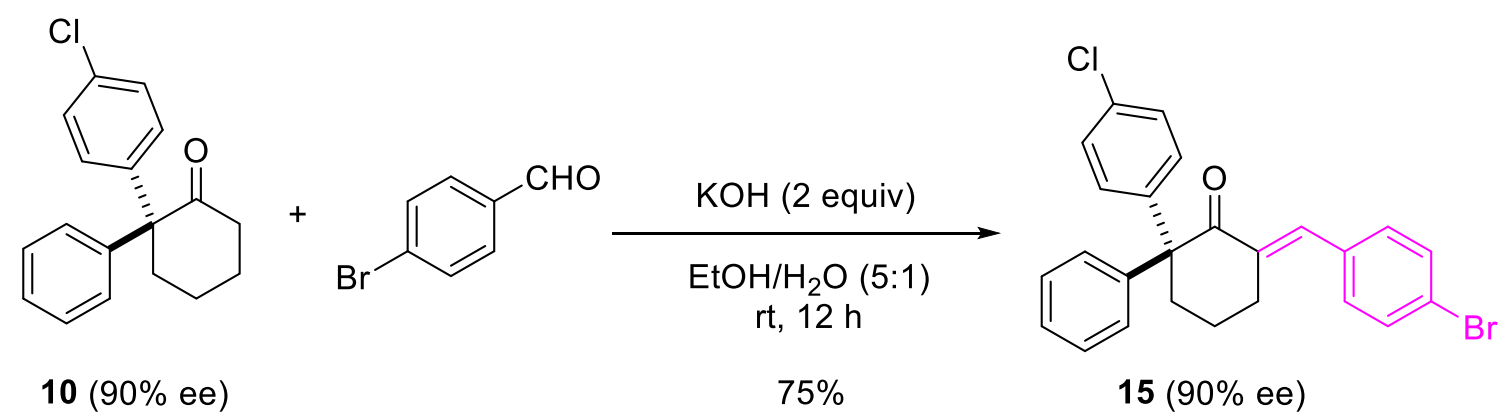

(S)-6-(4-Bromobenzylidene)-2-(4-chlorophenyl)-2-phenylcyclohexan-1-one (15). To a 10-mL vial were sequentially added 10 (114.0 mg, $0.4 \mathrm{mmol}), \mathrm{EtOH}(4 \mathrm{~mL}), \mathrm{KOH}$ $(85 \%, 53.0 \mathrm{mg}, 0.8 \mathrm{mmol})$, water $(0.8 \mathrm{~mL})$, and 4-bromobenzaldehyde $(74.2 \mathrm{mg}, 0.4$ $\mathrm{mmol})$. The mixture was stirred at room temperature, and the progress was monitored by TLC. Upon completion $(12 \mathrm{~h})$, ethyl acetate $(20 \mathrm{~mL})$ and water $(15 \mathrm{~mL})$ were added. The layers were separated, and the aqueous layer was extracted with ethyl acetate $(15 \mathrm{~mL} \times 3)$. The combined organic layers were washed with brine (10 $\mathrm{mL}$ ), dried over $\mathrm{Na}_{2} \mathrm{SO}_{4}$, and concentrated. The residue was purified by silica gel flash column chromatography (eluent: hexanes/ethyl acetate $=30: 1$ ) to afford 15 (136.3 mg, 75\% yield, 91\% ee) as a light yellow oil.

$[\alpha]^{25}:-53.8\left(c=1.0, \mathrm{CHCl}_{3}\right)$. HPLC analysis of the product: Daicel CHIRALPAK IC column; $1 \%$ i-PrOH in hexanes; $1.0 \mathrm{~mL} / \mathrm{min}$; retention times: $14.0 \mathrm{~min}$ (minor), 16.8 $\min ($ major).

${ }^{1} \mathbf{H}$ NMR $\left(400 \mathrm{MHz} \mathrm{CDCl}_{3}\right) \delta$ 7.52-7.43 (m, 3H), 7.36-7.29 (m, 2H), 7.29-7.18 (m, 5H), 7.15-7.08 (m, 2H), 7.07-7.00 (m, 2H), $2.80(\mathrm{t}, J=6.7 \mathrm{~Hz}, 2 \mathrm{H}), 2.75-2.56(\mathrm{~m}, 2 \mathrm{H})$, $1.84-1.68(\mathrm{~m}, 2 \mathrm{H}) \mathrm{ppm}$.

${ }^{13} \mathrm{C}$ NMR $\left(100 \mathrm{MHz}, \mathrm{CDCl}_{3}\right) \delta$ 202.2, 142.2, 142.0, 137.8, 136.4, 134.5, 132.6, 131.7, 131.5, 130.0, 128.4 (2C), 128.2, 127.0, 122.8, 61.7, 35.6, 28.4, 19.5 ppm.

IR (ATR) 3057, 3029, 2943, 2871, 1680, 1592, 1487, 1399, 1258, 1153, 1140, 1094, 1073, $1010 \mathrm{~cm}^{-1}$.

HRMS (CI+) $\mathrm{C}_{25} \mathrm{H}_{20} \mathrm{BrClO}\left[\mathrm{M}^{+}\right]$: 450.0386, Found: 450.0370 . 

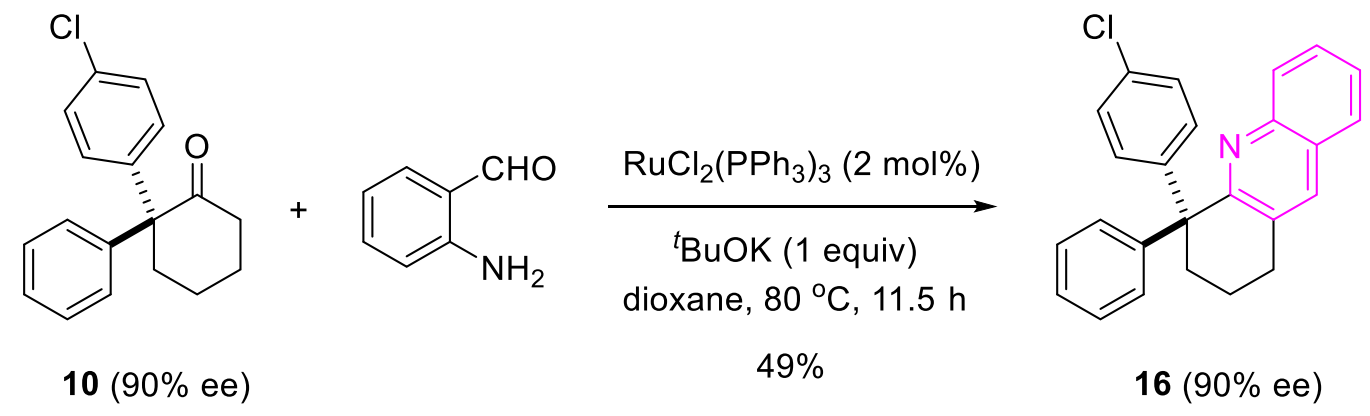

(S)-4-(4-Chlorophenyl)-4-phenyl-1,2,3,4-tetrahydroacridine (16). ${ }^{17}$ Under $\mathrm{N}_{2}$, to an oven-dried Schlenk tube were added $\mathrm{RuCl}_{2}\left(\mathrm{PPh}_{3}\right)_{3}(7.9 \mathrm{mg}, 8 \mu \mathrm{mol})$, ${ }^{t} \mathrm{BuOK}(45.2 \mathrm{mg}$, $0.4 \mathrm{mmol}), 2$-aminobenzaldehyde $(97.2 \mathrm{mg}, 0.8 \mathrm{mmol})$, and a solution of $\mathbf{1 0}(114.1 \mathrm{mg}$, $0.4 \mathrm{mmol})$ in anhydrous dioxane $(2 \mathrm{~mL})$. The mixture was stirred at $80{ }^{\circ} \mathrm{C}$ and the reaction progress was monitored by TLC. Upon completion (11.5 h), the reaction was cooled to room temperature, filtrated through a short pad of silica gel (eluent: ethyl acetate), and concentrated. The residue was purified by silica gel flash column chromatography (eluent: hexanes/ethyl acetate $=50: 1)$ to afford $16(72.0 \mathrm{mg}, 49 \%$ yield, $90 \%$ ee) as a colorless oil.

$[\alpha]^{25}:-14.7\left(c=1.0, \mathrm{CHCl}_{3}\right)$. HPLC analysis of the product: Daicel CHIRALPAK AD-H column; $1 \%$ i-PrOH in hexanes; $1.0 \mathrm{~mL} / \mathrm{min}$; retention times: 5.3 min (major), 5.7 min (minor).

${ }^{1} \mathrm{H}$ NMR $\left(400 \mathrm{MHz}, \mathrm{CDCl}_{3}\right) \delta$ 7.91-7.80 (m, 2H), $7.70(\mathrm{~d}, J=8.0 \mathrm{~Hz}, 1 \mathrm{H}), 7.52(\mathrm{t}, J=7.1$ $\mathrm{Hz}, 1 \mathrm{H}), 7.43(\mathrm{t}, J=7.4 \mathrm{~Hz}, 1 \mathrm{H}), 7.27-7.14(\mathrm{~m}, 5 \mathrm{H}), 7.14-7.02(\mathrm{~m}, 4 \mathrm{H}), 3.10-2.93(\mathrm{~m}, 2 \mathrm{H})$, 2.79-2.59 (m, 2H), 1.94-1.75 (m, 2H) ppm.

${ }^{13} \mathrm{C}$ NMR (100 MHz, $\left.\mathrm{CDCl}_{3}\right) \delta 162.1,147.3,146.5,146.3,135.4,131.6,131.2,130.9,129.6$, $129.2,128.2,127.7,127.5,127.0,126.5,126.2,126.0,55.6,37.9,29.2,19.0$ ppm.

IR (ATR) 3056, 3030, 2942, 2872, 1489, 1445, 1415, 1398, 1380, 1093, 1013, 960, 753 $\mathrm{cm}^{-1}$.

HRMS (CI+) Calcd for $\mathrm{C}_{25} \mathrm{H}_{20} \mathrm{ClN}$ [M+]: 369.1284, Found: 369.1282 . 


\section{Mechanistic Study}

(1) Study of non-linear effects for acyclic substrate 3a<smiles>CCC1(CC)OC1(c1ccccc1)c1ccc(O)cc1</smiles>

3a
(S)-A3 (10 mol\%) $5 \AA$ MS, DCE, rt, $19 \mathrm{~h}$<smiles>CCC(=O)[C@@](CC)(c1ccccc1)c1ccc(O)cc1</smiles>

$4 a$

\begin{tabular}{cccc}
\hline entry & ee of $(S)-\mathbf{A 3}(\%)$ & Conv. $(\%)$ & ee of $\mathbf{4 a}(\%)$ \\
\hline 1 & $\mathbf{0}$ & $100 \%$ & $\mathbf{0}$ \\
2 & $\mathbf{2 0}$ & $100 \%$ & $\mathbf{2 9}$ \\
3 & $\mathbf{4 0}$ & $100 \%$ & $\mathbf{5 8}$ \\
4 & $\mathbf{6 0}$ & $100 \%$ & $\mathbf{7 4}$ \\
5 & $\mathbf{8 0}$ & $100 \%$ & $\mathbf{8 7}$ \\
6 & $>99$ & $100 \%$ & 90 \\
\hline
\end{tabular}

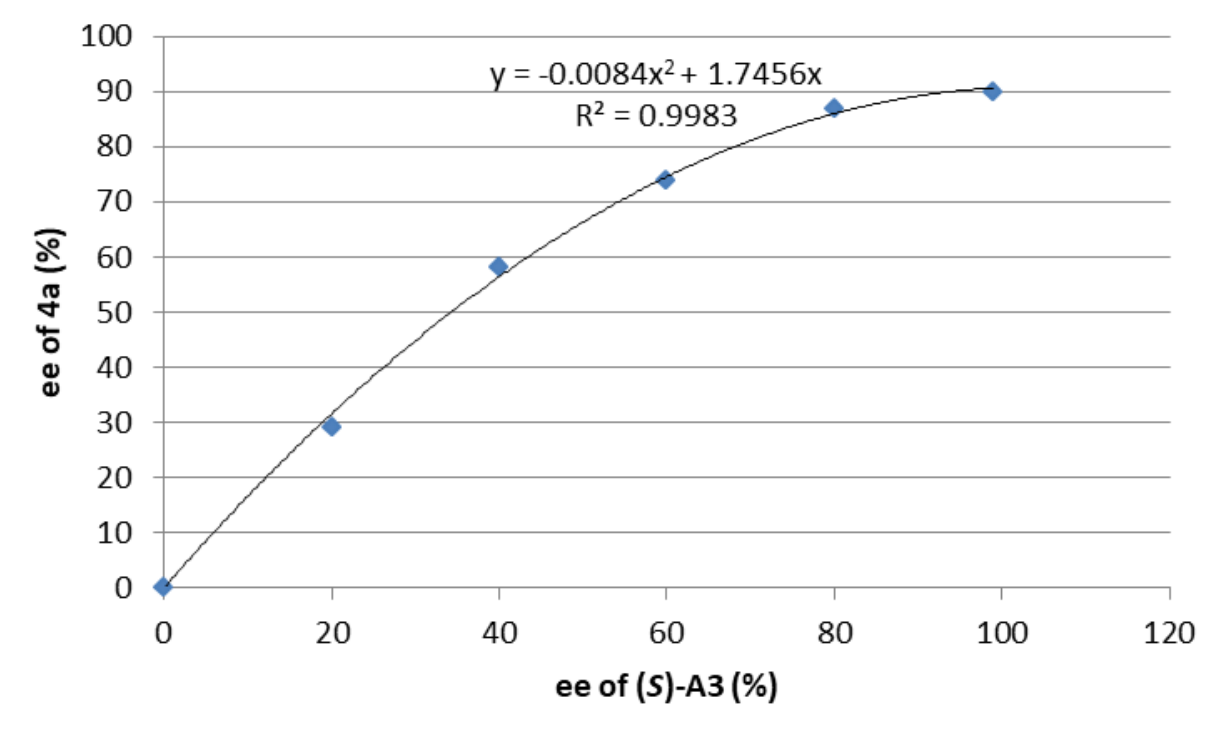


(2) Study of non-linear effects for cyclic substrate 1a<smiles>Oc1ccc(C2(c3ccccc3)OC23CCCC3)cc1</smiles>

$1 a$

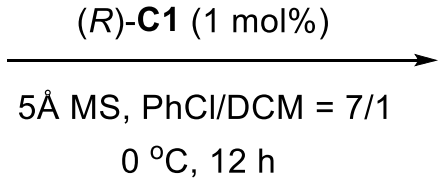

$0{ }^{\circ} \mathrm{C}, 12 \mathrm{~h}$

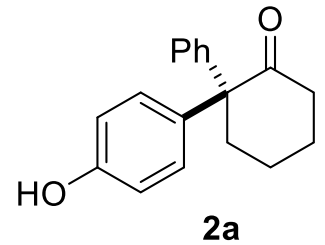

$2 a$

\begin{tabular}{cccc}
\hline entry & ee of $(R)-\mathbf{C 1}(\%)$ & Conv. $(\%)$ & ee of $\mathbf{2 a}(\%)$ \\
\hline 1 & 0 & $100 \%$ & 0 \\
2 & 20 & $100 \%$ & 19 \\
3 & 40 & $100 \%$ & 40 \\
4 & 60 & $100 \%$ & 59 \\
5 & 80 & $100 \%$ & 76 \\
6 & $>99$ & $100 \%$ & 91 \\
\hline
\end{tabular}

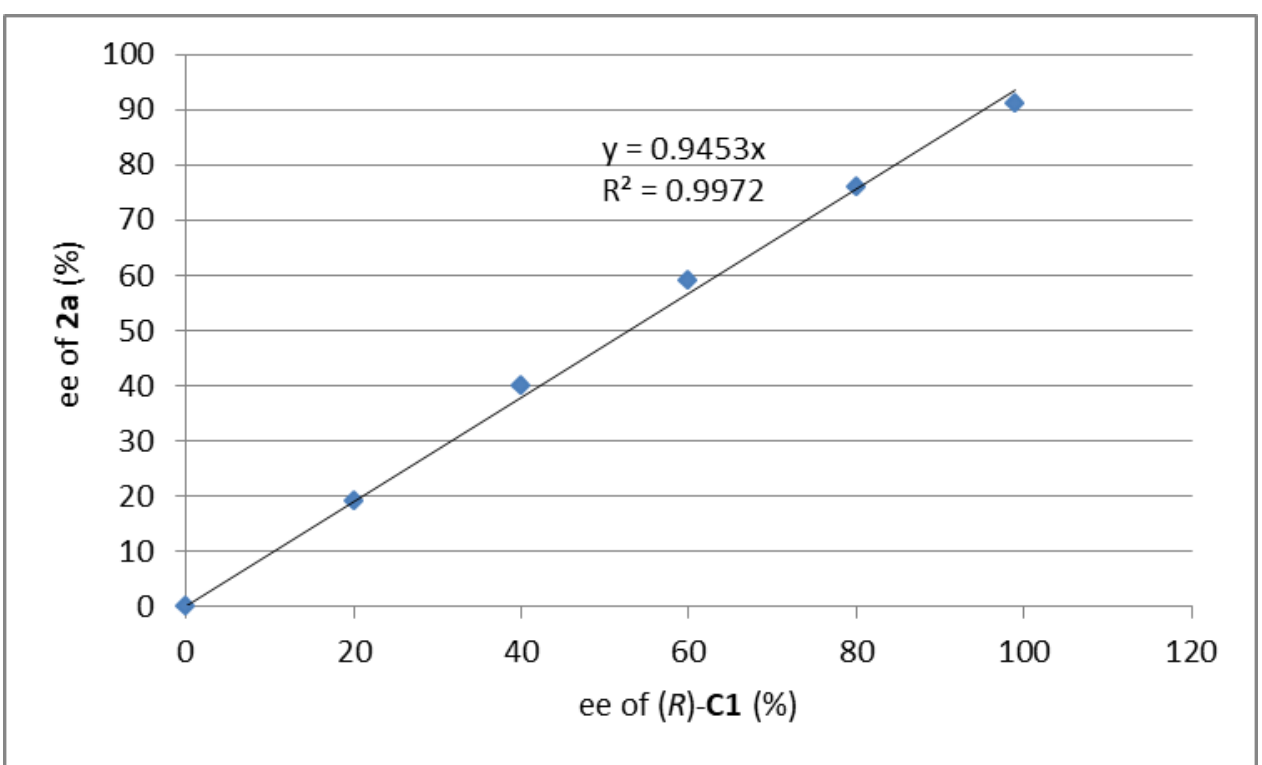

\section{(3) Kinetic study}<smiles>CCC1(CC)OC1(c1ccccc1)c1ccc(O)cc1</smiles>

$3 a$

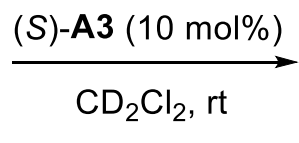

$\mathrm{HO}$<smiles>CCC(=O)C(CC)(CC)c1ccc(O)cc1</smiles>

$4 a$

At room temperature, to an oven-dried 4-mL vial charged with 3a (13.4 mg, $50 \mu \mathrm{mol})$ 
in $\mathrm{CD}_{2} \mathrm{Cl}_{2}(1-\mathrm{x} \mathrm{mL})$ was added $\mathrm{CH}_{2} \mathrm{Br}_{2}(7 \mu \mathrm{L}$, internal standard) and a solution of (S)-A3 in $\mathrm{CD}_{2} \mathrm{Cl}_{2}(\mathrm{x} \mathrm{mL})\left(1^{\text {st }}\right.$ run: $1.5 \mathrm{mg}$ in $300 \mu \mathrm{L}$ of $\mathrm{CD}_{2} \mathrm{Cl}_{2}, 2.5 \mu \mathrm{mol} ; 2^{\text {nd }}$ run: $2.3 \mathrm{mg}$ in $450 \mu \mathrm{L}$ of $\mathrm{CD}_{2} \mathrm{Cl}_{2}, 3.75 \mu \mathrm{mol}$; $3^{\text {rd }}$ run: $3.0 \mathrm{mg}$ in $600 \mu \mathrm{L}$ of $\mathrm{CD}_{2} \mathrm{Cl}_{2}, 5 \mu \mathrm{mol} ; 4^{\text {th }}$ run: $3.8 \mathrm{mg}$ in $750 \mu \mathrm{L}$ of $\mathrm{CD}_{2} \mathrm{Cl}_{2}, 6.25 \mu \mathrm{mol} ; 5^{\text {th }}$ run: $4.5 \mathrm{mg}$ in $900 \mu \mathrm{L}$ of $\left.\mathrm{CD}_{2} \mathrm{Cl}_{2}, 7.5 \mu \mathrm{mol}\right)$. The reaction progress was monitored with ${ }^{1} \mathrm{H}$ NMR analysis. $\left(1^{\text {st }}\right.$ and $2^{\text {nd }}$ runs: every $10 \mathrm{~min} ; 3^{\text {rd }}$ and $4^{\text {th }}$ runs: every $5 \mathrm{~min}$; $5^{\text {th }}$ run: every $2.5 \mathrm{~min}$ ). As shown below, a non-linear relationship between the initial rate and the concentration of the catalyst (S)-A3 was observed, indicating $1.6^{\text {th }}$-order kinetics in (S)-A3.

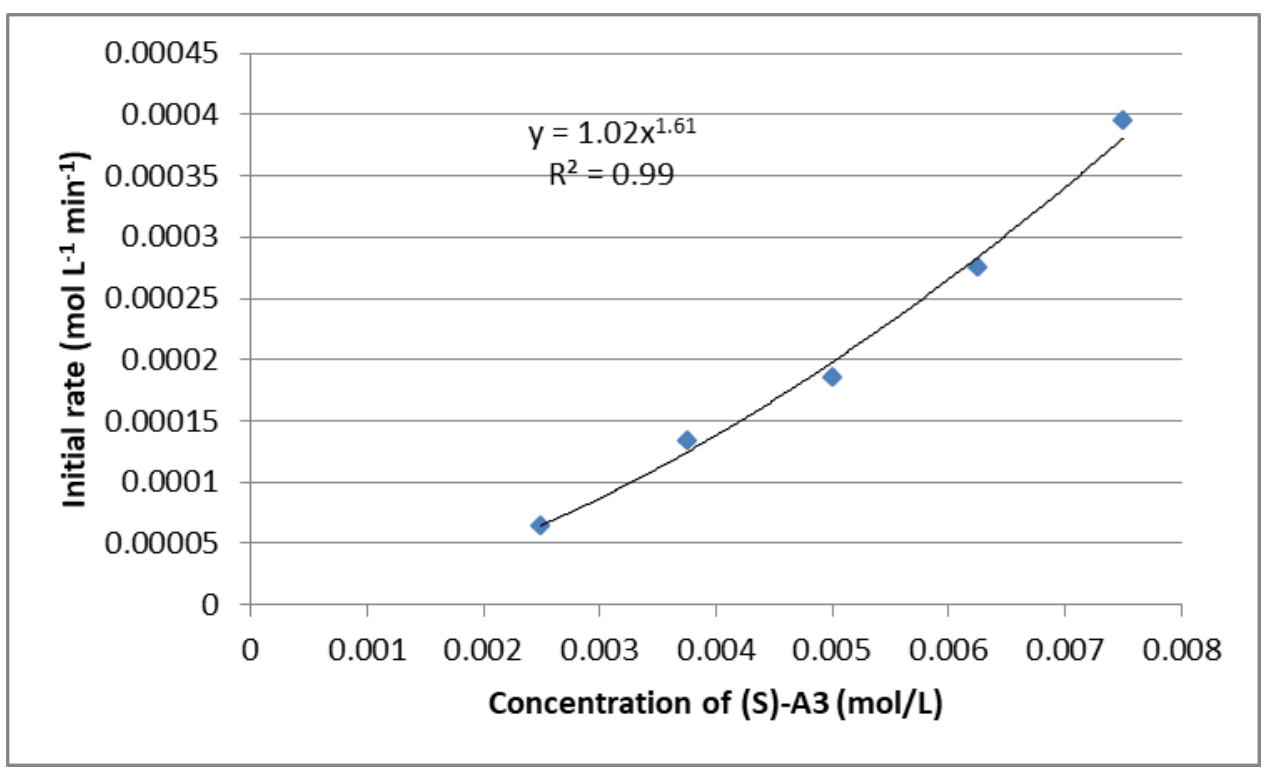




\section{Determination of the Stereochemistry}

The absolute stereochemistry of products $\mathbf{2 k}$ and $\mathbf{4 a}$ was determined by X-ray diffraction (shown below). The X-ray data have been deposited at the Cambridge Crystallographic Data Center (CCDC 1940153 for 2k and CCDC 1940150 for 4a). The stereochemistry of other products was assumed by analogy.<smiles>C#CC1CCC[C@@](c2ccc(O)cc2)(c2cccc(C(F)(F)F)c2)C1=O</smiles>

$2 k$<smiles>C#CCC(=O)[C@](CC)(c1ccccc1)c1ccc(O)cc1</smiles>

$4 a$
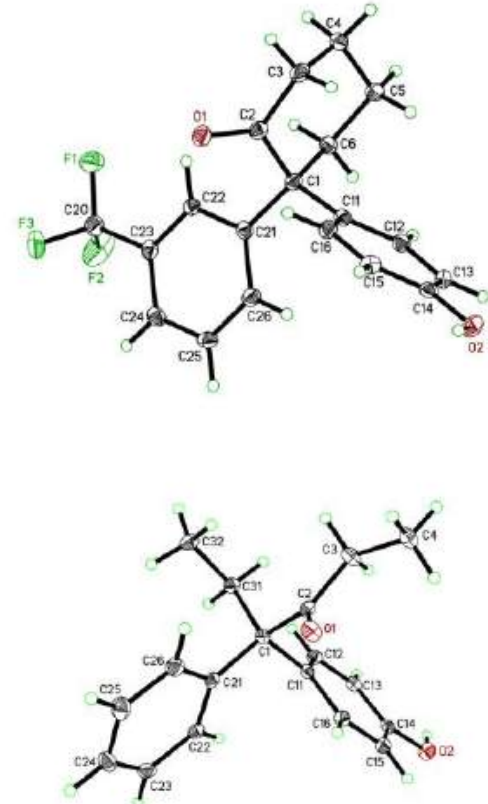

Table S4. Crystal data and structure refinement for $2 \mathrm{k}$.

Identification code

Empirical formula

Formula weight

Temperature/K

Crystal system

Space group

$\mathrm{a} / \AA$

$\mathrm{b} / \AA$

c/Å

$\alpha /{ }^{\circ}$

$\beta /{ }^{\circ}$

$\gamma /{ }^{\circ}$

Volume $/ \AA^{3}$

$\mathrm{Z}$ 2k

$\mathrm{C}_{19} \mathrm{H}_{17} \mathrm{~F}_{3} \mathrm{O}_{2}$

334.32

100.2(4)

orthorhombic

$\mathrm{P} 2{ }_{1} 22_{1}$

9.81945(10)

10.42071(11)

15.41623(15)

90

90

90

1577.48(3)

4 


$\begin{array}{ll}\rho_{\text {calcg/cm }}{ }^{3} & 1.408 \\ \mu / \mathrm{mm}^{-1} & 0.969 \\ \mathrm{~F}(000) & 696.0 \\ \text { Crystal size/mm }{ }^{3} & 0.25 \times 0.2 \times 0.15 \\ \text { Radiation } & \mathrm{CuK} \alpha(\lambda=1.54184) \\ 2 \Theta \text { range for data collection/ } & 10.246 \text { to } 134.978 \\ \text { Index ranges } & -11 \leq \mathrm{h} \leq 11,-12 \leq \mathrm{k} \leq 10,-18 \leq 1 \leq 18 \\ \text { Reflections collected } & 8586 \\ \text { Independent reflections } & 2818\left[\mathrm{R}_{\text {int }}=0.0133, \mathrm{R}_{\text {sigma }}=0.0132\right] \\ \text { Data/restraints/parameters } & 2818 / 0 / 218 \\ \text { Goodness-of-fit on } \mathrm{F}^{2} & 1.019 \\ \text { Final R indexes [I }>=2 \sigma(\mathrm{I})] & \mathrm{R}_{1}=0.0219, \mathrm{wR}_{2}=0.0572 \\ \text { Final R indexes [all data] } & \mathrm{R}_{1}=0.0220, \mathrm{wR}_{2}=0.0573 \\ \text { Largest diff. peak/hole / e } \AA^{-3} 0.16 /-0.15 \\ \text { Flack parameter } & 0.00(3)\end{array}$

Table S5. Fractional Atomic Coordinates $\left(\times 10^{4}\right)$ and Equivalent Isotropic Displacement Parameters $\left(\AA^{2} \times 10^{3}\right)$ for $2 \mathrm{k}$. Ueq is defined as $1 / 3$ of the trace of the orthogonalised UIJ tensor.

\begin{tabular}{lllrl} 
Atom & \multicolumn{1}{c}{$\boldsymbol{x}$} & $\boldsymbol{y}$ & \multicolumn{1}{l}{$\boldsymbol{z}$} & $\mathbf{U}(\mathbf{e q})$ \\
$\mathrm{F} 1$ & $1936.5(13)$ & $-313.1(9)$ & $4704.7(6)$ & $36.2(3)$ \\
F2 & $1101.0(14)$ & $-1320.5(11)$ & $3617.7(9)$ & $51.6(3)$ \\
F3 & $3249.1(13)$ & $-1322.9(11)$ & $3840.5(8)$ & $45.3(3)$ \\
O1 & $4108.6(11)$ & $3744.7(11)$ & $4404.9(7)$ & $22.8(3)$ \\
O2 & $3249.3(12)$ & $7908.0(11)$ & $863.5(7)$ & $24.1(2)$ \\
C1 & $2141.6(14)$ & $4236.8(14)$ & $3520.3(9)$ & $15.8(3)$ \\
C2 & $3153.6(15)$ & $4455.8(14)$ & $4273.4(9)$ & $17.6(3)$ \\
C3 & $2922.6(17)$ & $5637.7(15)$ & $4819.6(10)$ & $22.9(3)$ \\
C4 & $1438.7(18)$ & $5799.2(15)$ & $5118.8(10)$ & $24.8(4)$ \\
C5 & $438.7(16)$ & $5634.7(15)$ & $4372.2(10)$ & $21.1(3)$ \\
C6 & $686.8(15)$ & $4362.3(15)$ & $3909(1)$ & $17.9(3)$ \\
C11 & $2434.2(15)$ & $5277.8(13)$ & $2836.3(9)$ & $15.9(3)$ \\
C12 & $1411.2(15)$ & $5767.7(14)$ & $2306.9(10)$ & $17.9(3)$ \\
C13 & $1702.2(16)$ & $6634.1(14)$ & $1651.2(10)$ & $18.9(3)$ \\
C14 & $3027.3(15)$ & $7047.0(13)$ & $1519.6(9)$ & $17.9(3)$ \\
C15 & $4064.8(16)$ & $6577.5(16)$ & $2041.1(10)$ & $20.4(3)$ \\
C16 & $3763.8(15)$ & $5691.4(15)$ & $2687.9(10)$ & $19.2(3)$ \\
C20 & $2141.3(18)$ & $-578.6(15)$ & $3870.6(11)$ & $24.5(3)$ \\
C21 & $2297.2(14)$ & $2891.2(14)$ & $3128.5(10)$ & $16.4(3)$ \\
C22 & $2191.9(14)$ & $1806.9(14)$ & $3665.4(10)$ & $16.9(3)$
\end{tabular}




$\begin{array}{rrrrr}\mathrm{C} 23 & 2277.3(15) & 589.9(14) & 3310(1) & 18.3(3) \\ \mathrm{C} 24 & 2479.6(15) & 403.2(14) & 2424.3(10) & 20.0(3) \\ \mathrm{C} 25 & 2587.3(15) & 1467.2(16) & 1894.4(10) & 21.1(3) \\ \mathrm{C} 26 & 2493.0(16) & 2697.9(14) & 2242.5(10) & 19.7(3)\end{array}$

Table S6. Anisotropic Displacement Parameters $\left(\AA^{2} \times 10^{3}\right)$ for $2 k$. The Anisotropic displacement factor exponent takes the form: $-2 \pi^{2}\left[h^{2} a^{* 2} U_{11}+2 h k a * b * U_{12}+\ldots\right]$.

\begin{tabular}{|c|c|c|c|c|c|c|}
\hline Atom & $\mathbf{U}_{11}$ & $\mathbf{U}_{22}$ & $\mathbf{U}_{33}$ & $\mathbf{U}_{23}$ & $\mathbf{U}_{13}$ & $\mathbf{U}_{12}$ \\
\hline $\mathrm{F} 1$ & $64.5(7)$ & $20.9(5)$ & $23.3(5)$ & $4.5(4)$ & $6.0(5)$ & $1.2(5)$ \\
\hline $\mathrm{F} 2$ & $69.8(8)$ & $30.8(6)$ & $54.1(7)$ & $16.3(6)$ & $-24.4(6)$ & $-28.5(6)$ \\
\hline $\mathrm{F} 3$ & $61.0(7)$ & $30.9(5)$ & $44.0(6)$ & $11.1(5)$ & $4.3(6)$ & $23.8(6)$ \\
\hline $\mathrm{O} 1$ & $22.3(5)$ & $22.6(6)$ & $23.5(6)$ & $0.9(5)$ & $-4.5(4)$ & $1.9(4)$ \\
\hline $\mathrm{O} 2$ & $28.5(6)$ & $23.8(5)$ & $19.9(5)$ & $7.2(4)$ & $1.0(5)$ & $-4.9(5)$ \\
\hline $\mathrm{C} 1$ & $18.6(7)$ & $15.2(6)$ & $13.6(6)$ & $1.1(5)$ & $0.2(6)$ & $-0.7(5)$ \\
\hline $\mathrm{C} 2$ & $20.7(7)$ & $15.8(7)$ & $16.2(7)$ & $3.3(6)$ & $0.5(6)$ & $-3.0(6)$ \\
\hline $\mathrm{C} 3$ & $31.9(9)$ & $18.2(7)$ & $18.7(7)$ & $-0.7(6)$ & $-6.1(6)$ & $-0.7(7)$ \\
\hline $\mathrm{C} 4$ & $38.9(10)$ & $18.6(7)$ & $16.9(7)$ & $-1.6(6)$ & $0.8(6)$ & $5.9(7)$ \\
\hline $\mathrm{C} 5$ & $25.8(7)$ & $18.5(7)$ & $18.9(7)$ & $1.5(6)$ & $3.5(6)$ & $2.6(6)$ \\
\hline C6 & $20.0(7)$ & $15.8(7)$ & $17.9(7)$ & $1.1(6)$ & $1.9(6)$ & $-0.4(6)$ \\
\hline $\mathrm{C} 11$ & $19.9(7)$ & $12.4(7)$ & $15.5(7)$ & $-1.6(6)$ & $0.5(5)$ & $0.5(5)$ \\
\hline $\mathrm{C} 12$ & $18.4(7)$ & $17.1(7)$ & $18.2(7)$ & $0.1(6)$ & $1.6(6)$ & $0.3(6)$ \\
\hline C13 & $21.3(7)$ & $17.3(7)$ & $18.0(7)$ & $1.3(6)$ & $-1.0(6)$ & $3.8(6)$ \\
\hline C14 & $26.8(8)$ & $12.9(6)$ & $14.0(7)$ & $-1.4(5)$ & $2.6(6)$ & $-0.2(6)$ \\
\hline $\mathrm{C} 15$ & $20.5(7)$ & $21.9(8)$ & $18.7(7)$ & $-0.5(6)$ & $0.3(6)$ & $-4.6(6)$ \\
\hline $\mathrm{C} 16$ & $19.2(7)$ & $19.9(7)$ & $18.4(7)$ & $0.1(6)$ & $-2.6(6)$ & $0.3(6)$ \\
\hline $\mathrm{C} 20$ & $33.6(9)$ & $14.5(7)$ & $25.4(8)$ & $-1.4(6)$ & $-4.1(6)$ & $0.2(7)$ \\
\hline $\mathrm{C} 21$ & $14.2(6)$ & $15.4(7)$ & $19.5(7)$ & $-0.9(6)$ & $-0.9(6)$ & $0.3(6)$ \\
\hline $\mathrm{C} 22$ & $17.9(7)$ & $17.1(7)$ & $15.8(7)$ & $-0.4(6)$ & $-2.2(6)$ & $0.2(5)$ \\
\hline $\mathrm{C} 23$ & $16.8(7)$ & $16.6(7)$ & $21.5(7)$ & $0.9(6)$ & $-3.0(6)$ & $0.5(6)$ \\
\hline $\mathrm{C} 24$ & $19.2(7)$ & $17.3(7)$ & $23.4(8)$ & $-5.4(6)$ & $-2.9(6)$ & $0.3(6)$ \\
\hline $\mathrm{C} 25$ & $23.3(7)$ & $23.9(8)$ & $16.1(7)$ & $-3.7(6)$ & $-1.8(6)$ & $-0.8(6)$ \\
\hline $\mathrm{C} 26$ & $22.9(8)$ & $18.9(7)$ & $17.4(7)$ & $1.6(6)$ & $-0.5(6)$ & $-0.3(6)$ \\
\hline
\end{tabular}

Table S7. Bond Lengths for $2 \mathrm{k}$.

Atom Atom Length/Å Atom Atom Length/Å

$\begin{array}{llllll}\text { F1 } & \text { C20 } & 1.331(2) & \text { C11 } & \text { C12 } & 1.391(2) \\ \text { F2 } & \text { C20 } & 1.339(2) & \text { C11 } & \text { C16 } & 1.394(2)\end{array}$




$\begin{array}{llrlll}\mathrm{F} 3 & \mathrm{C} 20 & 1.337(2) & \mathrm{C} 12 & \mathrm{C} 13 & 1.385(2) \\ \mathrm{O} 1 & \mathrm{C} 2 & 1.2123(19) & \mathrm{C} 13 & \mathrm{C} 14 & 1.385(2) \\ \mathrm{O} 2 & \mathrm{C} 14 & 1.3695(18) & \mathrm{C} 14 & \mathrm{C} 15 & 1.387(2) \\ \mathrm{C} 1 & \mathrm{C} 2 & 1.5451(19) & \mathrm{C} 15 & \mathrm{C} 16 & 1.391(2) \\ \mathrm{C} 1 & \mathrm{C} 6 & 1.5546(19) & \mathrm{C} 20 & \mathrm{C} 23 & 1.499(2) \\ \mathrm{C} 1 & \mathrm{C} 11 & 1.5400(19) & \mathrm{C} 21 & \mathrm{C} 22 & 1.404(2) \\ \mathrm{C} 1 & \mathrm{C} 21 & 1.534(2) & \mathrm{C} 21 & \mathrm{C} 26 & 1.394(2) \\ \mathrm{C} 2 & \mathrm{C} 3 & 1.509(2) & \mathrm{C} 22 & \mathrm{C} 23 & 1.384(2) \\ \mathrm{C} 3 & \mathrm{C} 4 & 1.538(2) & \mathrm{C} 23 & \mathrm{C} 24 & 1.394(2) \\ \mathrm{C} 4 & \mathrm{C} 5 & 1.523(2) & \mathrm{C} 24 & \mathrm{C} 25 & 1.381(2) \\ \mathrm{C} 5 & \mathrm{C} 6 & 1.526(2) & \mathrm{C} 25 & \mathrm{C} 26 & 1.393(2)\end{array}$

Table S8. Bond Angles for 2k.

\begin{tabular}{|c|c|c|c|c|c|c|c|}
\hline \multicolumn{3}{|c|}{ Atom Atom Atom } & \multirow{2}{*}{$\begin{array}{l}\text { Angle } /^{\circ} \\
106.80(11)\end{array}$} & \multicolumn{3}{|c|}{ Atom Atom Atom } & \multirow{2}{*}{$\begin{array}{l}\text { Angle } /^{\circ} \\
119.70(13)\end{array}$} \\
\hline $\mathrm{C} 2$ & $\mathrm{C} 1$ & C6 & & $\mathrm{C} 13$ & $\mathrm{C} 14$ & C15 & \\
\hline C11 & $\mathrm{C} 1$ & $\mathrm{C} 2$ & $106.89(11)$ & $\mathrm{C} 14$ & $\mathrm{C} 15$ & C16 & $119.58(14)$ \\
\hline C11 & $\mathrm{C} 1$ & C6 & $112.09(12)$ & $\mathrm{C} 15$ & $\mathrm{C} 16$ & $\mathrm{C} 11$ & $121.47(14)$ \\
\hline $\mathrm{C} 21$ & $\mathrm{C} 1$ & $\mathrm{C} 2$ & $111.52(12)$ & $\mathrm{F} 1$ & $\mathrm{C} 20$ & $\mathrm{~F} 2$ & $106.63(15)$ \\
\hline $\mathrm{C} 21$ & $\mathrm{C} 1$ & C6 & $108.67(11)$ & $\mathrm{F} 1$ & $\mathrm{C} 20$ & F3 & $106.09(13)$ \\
\hline $\mathrm{C} 21$ & $\mathrm{C} 1$ & $\mathrm{C} 11$ & $110.83(11)$ & $\mathrm{F} 1$ & $\mathrm{C} 20$ & $\mathrm{C} 23$ & $113.68(13)$ \\
\hline $\mathrm{O} 1$ & $\mathrm{C} 2$ & $\mathrm{C} 1$ & $122.19(13)$ & $\mathrm{F} 2$ & $\mathrm{C} 20$ & $\mathrm{C} 23$ & $111.65(13)$ \\
\hline $\mathrm{O} 1$ & $\mathrm{C} 2$ & C3 & $121.46(14)$ & $\mathrm{F} 3$ & $\mathrm{C} 20$ & $\mathrm{~F} 2$ & $106.00(13)$ \\
\hline $\mathrm{C} 3$ & $\mathrm{C} 2$ & $\mathrm{C} 1$ & $116.30(12)$ & $\mathrm{F} 3$ & $\mathrm{C} 20$ & $\mathrm{C} 23$ & $112.26(14)$ \\
\hline $\mathrm{C} 2$ & $\mathrm{C} 3$ & $\mathrm{C} 4$ & $113.52(13)$ & $\mathrm{C} 22$ & $\mathrm{C} 21$ & $\mathrm{C} 1$ & $119.72(13)$ \\
\hline $\mathrm{C} 5$ & $\mathrm{C} 4$ & $\mathrm{C} 3$ & $111.84(12)$ & $\mathrm{C} 26$ & $\mathrm{C} 21$ & $\mathrm{C} 1$ & $122.11(13)$ \\
\hline $\mathrm{C} 4$ & $\mathrm{C} 5$ & C6 & $110.41(13)$ & $\mathrm{C} 26$ & $\mathrm{C} 21$ & $\mathrm{C} 22$ & $118.12(14)$ \\
\hline C5 & C6 & $\mathrm{C} 1$ & $113.59(12)$ & $\mathrm{C} 23$ & $\mathrm{C} 22$ & $\mathrm{C} 21$ & $119.96(14)$ \\
\hline $\mathrm{C} 12$ & $\mathrm{C} 11$ & $\mathrm{C} 1$ & $121.70(13)$ & $\mathrm{C} 22$ & $\mathrm{C} 23$ & $\mathrm{C} 20$ & $120.71(13)$ \\
\hline $\mathrm{C} 12$ & $\mathrm{C} 11$ & $\mathrm{C} 16$ & $117.81(13)$ & $\mathrm{C} 22$ & $\mathrm{C} 23$ & $\mathrm{C} 24$ & $121.63(14)$ \\
\hline C16 & $\mathrm{C} 11$ & $\mathrm{C} 1$ & $120.33(13)$ & $\mathrm{C} 24$ & $\mathrm{C} 23$ & $\mathrm{C} 20$ & $117.66(13)$ \\
\hline C13 & $\mathrm{C} 12$ & $\mathrm{C} 11$ & $121.22(14)$ & $\mathrm{C} 25$ & $\mathrm{C} 24$ & $\mathrm{C} 23$ & $118.58(14)$ \\
\hline $\mathrm{C} 12$ & $\mathrm{C} 13$ & $\mathrm{C} 14$ & $120.20(14)$ & $\mathrm{C} 24$ & $\mathrm{C} 25$ & $\mathrm{C} 26$ & $120.40(14)$ \\
\hline $\mathrm{O} 2$ & $\mathrm{C} 14$ & $\mathrm{C} 13$ & $117.46(13)$ & $\mathrm{C} 25$ & $\mathrm{C} 26$ & $\mathrm{C} 21$ & $121.30(14)$ \\
\hline $\mathrm{O} 2$ & $\mathrm{C} 14$ & $\mathrm{C} 15$ & $122.83(14)$ & & & & \\
\hline
\end{tabular}


Table S9. Hydrogen Bonds for $2 \mathrm{k}$.
D H A d(D-H)/Å

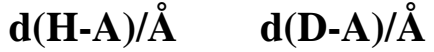
D-H-A/ ${ }^{\circ}$
$\mathrm{O} 2 \mathrm{H} 2 \mathrm{O} 1^{1}$
0.84
$1.942 .7680(16)$
168.1

${ }^{1} 1-X, 1 / 2+Y, 1 / 2-Z$

Table S10. Torsion Angles for 2k.

\begin{tabular}{|c|c|c|c|}
\hline C D & Angle ${ }^{\circ}$ & $\mathbf{A}$ & Angle ${ }^{\circ}$ \\
\hline $\mathrm{F} 1 \mathrm{C} 20 \mathrm{C} 23 \mathrm{C} 22$ & $0.1(2)$ & C6 C1 C21 C26 & $115.57(15)$ \\
\hline $\mathrm{F} 1 \mathrm{C} 20 \mathrm{C} 23 \mathrm{C} 24$ & $-179.32(14)$ & $\mathrm{C} 11 \mathrm{C} 1 \mathrm{C} 2 \mathrm{O} 1$ & $-107.68(15)$ \\
\hline $\mathrm{F} 2 \mathrm{C} 20 \mathrm{C} 23 \mathrm{C} 22$ & $120.84(16)$ & $\mathrm{C} 11 \mathrm{C} 1 \mathrm{C} 2 \mathrm{C} 3$ & $69.99(15)$ \\
\hline $\mathrm{F} 2 \mathrm{C} 20 \mathrm{C} 23 \mathrm{C} 24$ & $-58.6(2)$ & C11C1 C6 C5 & $-60.94(15)$ \\
\hline F3 C 20 C 23 C 22 & $-120.28(16)$ & $\mathrm{C} 11 \mathrm{C} 1 \mathrm{C} 21 \mathrm{C} 22$ & $174.38(13)$ \\
\hline F3 C20C23C24 & $60.24(19)$ & $\mathrm{C} 11 \mathrm{C} 1 \mathrm{C} 21 \mathrm{C} 26$ & $-8.02(19)$ \\
\hline $\mathrm{O} 1 \mathrm{C} 2 \mathrm{C} 3 \mathrm{C} 4$ & $-133.82(15)$ & C11 C12C13C14 & $-1.1(2)$ \\
\hline $\mathrm{O} 2 \mathrm{C} 14 \mathrm{C} 15 \mathrm{C} 16$ & $-179.15(14)$ & C12C11C16C15 & $0.9(2)$ \\
\hline $\mathrm{C} 1 \mathrm{C} 2 \mathrm{C} 3 \mathrm{C} 4$ & $48.49(18)$ & $\mathrm{C} 12 \mathrm{C} 13 \mathrm{C} 14 \mathrm{O} 2$ & $-179.74(13)$ \\
\hline $\mathrm{C} 1 \mathrm{C} 11 \mathrm{C} 12 \mathrm{C} 13$ & $-175.19(13)$ & C12C13C14C15 & $0.8(2)$ \\
\hline $\mathrm{C} 1 \mathrm{C} 11 \mathrm{C} 16 \mathrm{C} 15$ & $176.37(14)$ & C13C14C15C16 & $0.3(2)$ \\
\hline $\mathrm{C} 1 \mathrm{C} 21 \mathrm{C} 22 \mathrm{C} 23$ & $177.43(13)$ & C14C15C16C11 & $-1.2(2)$ \\
\hline $\mathrm{C} 1 \mathrm{C} 21 \mathrm{C} 26 \mathrm{C} 25$ & $-177.85(14)$ & C16C11 C12C13 & $0.3(2)$ \\
\hline C2C1 C6 C5 & $55.80(15)$ & C20C23 C24 C25 & $179.06(14)$ \\
\hline $\mathrm{C} 2 \mathrm{C} 1 \mathrm{C} 11 \mathrm{C} 12$ & $-149.06(14)$ & $\mathrm{C} 21 \mathrm{C} 1 \mathrm{C} 2 \mathrm{O} 1$ & $13.59(19)$ \\
\hline $\mathrm{C} 2 \mathrm{C} 1 \mathrm{C} 11 \mathrm{C} 16$ & $35.61(18)$ & $\mathrm{C} 21 \mathrm{C} 1 \quad \mathrm{C} 2 \mathrm{C} 3$ & $-168.74(12)$ \\
\hline $\mathrm{C} 2 \mathrm{C} 1 \mathrm{C} 21 \mathrm{C} 22$ & $55.42(17)$ & C21 C1 C6 C5 & $176.23(12)$ \\
\hline $\mathrm{C} 2 \mathrm{C} 1 \mathrm{C} 21 \mathrm{C} 26$ & $-126.97(14)$ & $\mathrm{C} 21 \mathrm{C} 1 \mathrm{C} 11 \mathrm{C} 12$ & $89.24(16)$ \\
\hline $\mathrm{C} 2 \mathrm{C} 3 \mathrm{C} 4 \mathrm{C} 5$ & $-48.39(18)$ & $\mathrm{C} 21 \mathrm{C} 1 \mathrm{C} 11 \mathrm{C} 16$ & $-86.10(16)$ \\
\hline $\mathrm{C} 3 \mathrm{C} 4 \mathrm{C} 5 \mathrm{C} 6$ & $53.58(17)$ & C21 C22 C23 C20 & $-178.86(14)$ \\
\hline C4 C5 C6 C1 & $-59.85(16)$ & C21 C22 C23 C24 & $0.6(2)$ \\
\hline C6C1 C2 O1 & $132.17(14)$ & C22 C21 C26 C25 & $-0.2(2)$ \\
\hline $\mathrm{C} 6 \mathrm{C} 1 \mathrm{C} 2 \mathrm{C} 3$ & $-50.16(16)$ & C22C23 C24 C25 & $-0.4(2)$ \\
\hline $\mathrm{C} 6 \mathrm{C} 1 \mathrm{C} 11 \mathrm{C} 12$ & $-32.37(18)$ & C23 C24C25 C26 & $-0.1(2)$ \\
\hline C6C1 C11C16 & $152.30(13)$ & C24C25 C26 C21 & $0.4(2)$ \\
\hline $\mathrm{C} 6 \mathrm{C} 1 \mathrm{C} 21 \mathrm{C} 22$ & $-62.03(16)$ & C26C21 C22 C23 & $-0.3(2)$ \\
\hline
\end{tabular}


Table S11. Hydrogen Atom Coordinates $\left(\AA \times 10^{4}\right)$ and Isotropic Displacement Parameters $\left(\AA^{2} \times 10^{3}\right)$ for $2 \mathbf{k}$.

$\begin{array}{lrrrr}\text { Atom } & \boldsymbol{x} & \boldsymbol{y} & \boldsymbol{z} & \mathbf{U}(\mathbf{e q}) \\ \text { H2 } & 4082 & 8088 & 840 & 36 \\ \text { H3A } & 3192 & 6404 & 4481 & 27 \\ \text { H3B } & 3518 & 5594 & 5337 & 27 \\ \text { H4A } & 1235 & 5157 & 5574 & 30 \\ \text { H4B } & 1321 & 6663 & 5376 & 30 \\ \text { H5A } & 548 & 6351 & 3956 & 25 \\ \text { H5B } & -505 & 5658 & 4598 & 25 \\ \text { H6A } & 12 & 4269 & 3436 & 22 \\ \text { H6B } & 537 & 3652 & 4325 & 23 \\ \text { H12 } & 496 & 5503 & 2397 & 24 \\ \text { H13 } & 991 & 6946 & 1291 & 23 \\ \text { H15 } & 4975 & 6859 & 1957 & 20 \\ \text { H16 } & 448 & 5361 & 3036 & 24 \\ \text { H22 } & 2062 & 1909 & 4272 & 25 \\ \text { H24 } & 2542 & -438 & 2190 & 24 \\ \text { H25 } & 2727 & 1359 & 1289 & 1869 \\ \text { H26 } & 2564 & 3420 & & \end{array}$

Table S12. Crystal data and structure refinement for $4 a$.

Identification code

$4 \mathrm{a}$

Empirical formula

$\mathrm{C}_{18} \mathrm{H}_{20} \mathrm{O}_{2}$

Formula weight

268.34

Temperature/K

$100.00(10)$

Crystal system

orthorhombic

Space group

$\mathrm{P} 2{ }_{1} 2{ }_{1}{ }_{1}$

$\mathrm{a} / \AA$

$7.43535(11)$

$\mathrm{b} / \AA$

12.70493(17)

c/Å

$15.2334(2)$

$\alpha /{ }^{\circ}$

90

$\beta /{ }^{\circ}$

90

$\gamma /{ }^{\circ}$

90

Volume/ $\AA^{3}$

1439.04(3)

Z

4

$\rho_{\text {calc }} \mathrm{g} / \mathrm{cm}^{3}$

1.239

$\mu / \mathrm{mm}^{-1}$

0.622 
$\mathrm{F}(000)$

Crystal size $/ \mathrm{mm}^{3}$

Radiation
576.0

$0.2 \times 0.18 \times 0.15$

$\mathrm{CuK} \alpha(\lambda=1.54184)$

$2 \Theta$ range for data collection/ ${ }^{\circ} 9.064$ to 134.976

Index ranges

Reflections collected $-6 \leq \mathrm{h} \leq 8,-15 \leq \mathrm{k} \leq 15,-14 \leq 1 \leq 18$

Independent reflections

Data/restraints/parameters

Goodness-of-fit on $\mathrm{F}^{2}$

8090

$2555\left[\mathrm{R}_{\text {int }}=0.0171, \mathrm{R}_{\text {sigma }}=0.0165\right]$

$2555 / 0 / 184$

1.049
Final $\mathrm{R}$ indexes $[\mathrm{I}>=2 \sigma(\mathrm{I})] \quad \mathrm{R}_{1}=0.0243, \mathrm{wR}_{2}=0.0614$

Final $\mathrm{R}$ indexes [all data] $\quad \mathrm{R}_{1}=0.0247, \mathrm{wR}_{2}=0.0617$

Largest diff. peak/hole / e $\AA^{-3} 0.18 /-0.14$

Flack parameter

$0.04(8)$

Table S13. Fractional Atomic Coordinates $\left(\times 10^{4}\right)$ and Equivalent Isotropic Displacement Parameters $\left(\AA^{2} \times 10^{3}\right)$ for $4 a$. $U_{\text {eq }}$ is defined as $1 / 3$ of of the trace of the orthogonalised UIJ tensor.

\begin{tabular}{|c|c|c|c|c|}
\hline Atom & $x$ & $y$ & $z$ & $\mathbf{U}(\mathbf{e q})$ \\
\hline $\mathrm{O} 1$ & $4738.8(15)$ & $4602.3(8)$ & $259.2(6)$ & $19.2(2)$ \\
\hline $\mathrm{O} 2$ & $9703.6(15)$ & $6249.3(8)$ & $3635.9(7)$ & $19.9(2)$ \\
\hline $\mathrm{C} 1$ & $3497(2)$ & $5035.7(10)$ & $1677.0(9)$ & $13.9(3)$ \\
\hline $\mathrm{C} 2$ & $4453(2)$ & $4311.0(11)$ & $1006.1(9)$ & $14.0(3)$ \\
\hline $\mathrm{C} 3$ & $5133(2)$ & $3260.2(11)$ & $1332.5(10)$ & $19.7(3)$ \\
\hline $\mathrm{C} 4$ & $6395(2)$ & $2707.9(13)$ & $703.4(11)$ & $21.5(3)$ \\
\hline $\mathrm{C} 11$ & $5074(2)$ & $5408.4(10)$ & $2255.7(9)$ & $13.7(3)$ \\
\hline $\mathrm{C} 12$ & $5204(2)$ & $5194.8(11)$ & $3153.5(9)$ & $15.8(3)$ \\
\hline C13 & $6722(2)$ & $5481.4(11)$ & $3632.0(9)$ & $16.2(3)$ \\
\hline C14 & $8148(2)$ & $5985.9(11)$ & $3219.3(10)$ & $15.2(3)$ \\
\hline C15 & $8014(2)$ & $6239.5(12)$ & $2329.8(10)$ & $16.6(3)$ \\
\hline C16 & $6501(2)$ & $5950.3(11)$ & $1860.9(10)$ & $15.7(3)$ \\
\hline $\mathrm{C} 21$ & $2560.0(19)$ & $5974.1(11)$ & $1233.1(9)$ & $13.9(3)$ \\
\hline $\mathrm{C} 22$ & $2567(2)$ & $6957.4(11)$ & $1636.9(9)$ & $15.5(3)$ \\
\hline $\mathrm{C} 23$ & $1630(2)$ & $7804.1(11)$ & $1278.9(11)$ & $20.1(3)$ \\
\hline $\mathrm{C} 24$ & $656(2)$ & $7683.5(13)$ & $511(1)$ & $22.3(3)$ \\
\hline $\mathrm{C} 25$ & $626(2)$ & $6708.3(13)$ & $105.4(10)$ & $22.8(3)$ \\
\hline $\mathrm{C} 26$ & $1568(2)$ & $5864.2(12)$ & $456.2(10)$ & $18.5(3)$ \\
\hline C31 & $2089(2)$ & $4397.5(11)$ & $2212.6(9)$ & $15.9(3)$ \\
\hline $\mathrm{C} 32$ & $590(2)$ & $3912.5(12)$ & $1662.2(10)$ & $20.1(3)$ \\
\hline
\end{tabular}


Table S14. Anisotropic Displacement Parameters $\left(\AA^{2} \times 10^{3}\right)$ for 4a. The Anisotropic displacement factor exponent takes the form: $-2 \pi^{2}\left[h^{2} a^{* 2} U_{11}+2 h k a * b * U_{12}+\ldots.\right]$.

\begin{tabular}{lllllrr} 
Atom & U11 & $\mathbf{U}_{22}$ & $\mathbf{U} 33$ & $\mathbf{U}_{23}$ & $\mathbf{U}_{13}$ & \multicolumn{1}{c}{$\mathbf{U}_{12}$} \\
O1 & $23.9(6)$ & $19.7(5)$ & $14.1(5)$ & $0.5(4)$ & \multicolumn{1}{c}{$2.9(4)$} & $3.5(4)$ \\
O2 & $19.0(5)$ & $24.3(5)$ & $16.5(5)$ & $1.0(4)$ & $-5.0(4)$ & $-1.5(4)$ \\
C1 & $14.8(7)$ & $13.7(6)$ & $13.2(6)$ & $-0.1(5)$ & $0.3(6)$ & $-0.5(6)$ \\
C2 & $12.3(7)$ & $15.2(7)$ & $14.4(6)$ & $-0.8(5)$ & $-1.3(6)$ & $-2.1(6)$ \\
C3 & $25.5(8)$ & $15.6(7)$ & $18.1(7)$ & $0.8(6)$ & $2.7(6)$ & $3.9(6)$ \\
C4 & $22.3(8)$ & $19.2(7)$ & $23.1(8)$ & $-0.2(6)$ & $1.8(7)$ & $6.5(7)$ \\
C11 & $14.5(7)$ & $12.5(6)$ & $14.2(6)$ & $-0.9(5)$ & $-0.2(5)$ & $2.1(6)$ \\
C12 & $17.8(7)$ & $14.8(6)$ & $14.8(6)$ & $0.5(5)$ & $1.2(6)$ & $-0.1(6)$ \\
C13 & $21.6(7)$ & $15.5(6)$ & $11.4(6)$ & $0.2(5)$ & $-0.2(6)$ & $2.8(6)$ \\
C14 & $15.3(7)$ & $12.6(6)$ & $17.6(7)$ & $-2.0(5)$ & $-2.6(6)$ & $3.3(5)$ \\
C15 & $15.0(7)$ & $17.0(7)$ & $17.8(7)$ & $1.2(6)$ & $1.1(5)$ & $0.1(6)$ \\
C16 & $17.0(7)$ & $17.1(6)$ & $13.0(6)$ & $2.0(5)$ & $0.0(6)$ & $0.7(6)$ \\
C21 & $11.0(6)$ & $16.3(7)$ & $14.5(6)$ & $1.7(5)$ & $3.2(5)$ & $-0.6(5)$ \\
C22 & $14.2(7)$ & $17.8(7)$ & $14.5(6)$ & $0.0(5)$ & $3.7(5)$ & $-2.6(6)$ \\
C23 & $19.2(7)$ & $13.9(6)$ & $27.3(8)$ & $2.0(6)$ & $8.1(7)$ & $-1.2(6)$ \\
C24 & $19.4(8)$ & $22.8(8)$ & $24.7(8)$ & $9.6(6)$ & $4.8(7)$ & $5.5(7)$ \\
C25 & $19.5(7)$ & $29.8(8)$ & $19.2(7)$ & $3.1(6)$ & $-2.7(6)$ & $2.5(7)$ \\
C26 & $17.4(7)$ & $19.5(7)$ & $18.5(7)$ & $-1.2(6)$ & $-0.6(6)$ & $-0.1(6)$ \\
C31 & $16.7(8)$ & $15.7(7)$ & $15.3(7)$ & $-1.0(5)$ & $1.7(6)$ & $-1.5(6)$ \\
C32 & $18.9(7)$ & $20.4(7)$ & $21.2(7)$ & $-1.4(6)$ & $2.9(6)$ & $-5.5(6)$
\end{tabular}

Table S15. Bond Lengths for 4a.

\begin{tabular}{|c|c|c|c|c|c|}
\hline \multicolumn{2}{|c|}{ Atom Atom } & Length/Å & Atom & Atom & Length/Å \\
\hline $\mathrm{O} 1$ & $\mathrm{C} 2$ & $1.2151(17)$ & C13 & $\mathrm{C} 14$ & $1.389(2)$ \\
\hline $\mathrm{O} 2$ & $\mathrm{C} 14$ & $1.3613(18)$ & $\mathrm{C} 14$ & $\mathrm{C} 15$ & $1.396(2)$ \\
\hline $\mathrm{C} 1$ & $\mathrm{C} 2$ & $1.5483(19)$ & $\mathrm{C} 15$ & $\mathrm{C} 16$ & $1.382(2)$ \\
\hline $\mathrm{C} 1$ & $\mathrm{C} 11$ & $1.541(2)$ & $\mathrm{C} 21$ & $\mathrm{C} 22$ & $1.392(2)$ \\
\hline $\mathrm{C} 1$ & $\mathrm{C} 21$ & $1.5378(19)$ & $\mathrm{C} 21$ & $\mathrm{C} 26$ & $1.401(2)$ \\
\hline $\mathrm{C} 1$ & C31 & $1.5556(19)$ & $\mathrm{C} 22$ & $\mathrm{C} 23$ & $1.393(2)$ \\
\hline $\mathrm{C} 2$ & $\mathrm{C} 3$ & $1.5116(19)$ & $\mathrm{C} 23$ & $\mathrm{C} 24$ & $1.385(2)$ \\
\hline $\mathrm{C} 3$ & $\mathrm{C} 4$ & $1.514(2)$ & $\mathrm{C} 24$ & $\mathrm{C} 25$ & $1.385(2)$ \\
\hline C11 & $\mathrm{C} 12$ & $1.398(2)$ & $\mathrm{C} 25$ & $\mathrm{C} 26$ & $1.388(2)$ \\
\hline C11 & $\mathrm{C} 16$ & $1.401(2)$ & C31 & C32 & $1.524(2)$ \\
\hline $\mathrm{C} 12$ & $\mathrm{C} 13$ & $1.392(2)$ & & & \\
\hline
\end{tabular}


Table S16. Bond Angles for 4a.

\begin{tabular}{|c|c|c|c|c|c|c|c|}
\hline \multicolumn{2}{|c|}{ Atom Atom } & \multirow{2}{*}{$\begin{array}{l}\text { Atom } \\
\text { C31 }\end{array}$} & \multirow{2}{*}{$\begin{array}{c}\text { Angle/ }{ }^{\circ} \\
110.20(11)\end{array}$} & \multirow{2}{*}{\multicolumn{2}{|c|}{$\begin{array}{l}\text { Atom Atom } \\
\mathrm{O} 2 \mathrm{C} 14\end{array}$}} & \multirow{2}{*}{$\begin{array}{l}\text { Atom } \\
\text { C13 }\end{array}$} & \multirow{2}{*}{$\begin{array}{l}\text { Angle }{ }^{\circ} \\
123.43(13)\end{array}$} \\
\hline $\mathrm{C} 2$ & $\mathrm{C} 1$ & & & & & & \\
\hline $\mathrm{C} 11$ & $\mathrm{C} 1$ & $\mathrm{C} 2$ & $102.19(11)$ & $\mathrm{O} 2$ & C14 & $\mathrm{C} 15$ & $117.15(13)$ \\
\hline $\mathrm{C} 11$ & $\mathrm{C} 1$ & C31 & $111.86(11)$ & $\mathrm{C} 13$ & $\mathrm{C} 14$ & $\mathrm{C} 15$ & $119.42(13)$ \\
\hline $\mathrm{C} 21$ & $\mathrm{C} 1$ & $\mathrm{C} 2$ & $112.26(11)$ & $\mathrm{C} 16$ & $\mathrm{C} 15$ & $\mathrm{C} 14$ & $119.89(14)$ \\
\hline $\mathrm{C} 21$ & $\mathrm{C} 1$ & $\mathrm{C} 11$ & $110.98(11)$ & $\mathrm{C} 15$ & $\mathrm{C} 16$ & $\mathrm{C} 11$ & $121.69(13)$ \\
\hline $\mathrm{C} 21$ & $\mathrm{C} 1$ & $\mathrm{C} 31$ & $109.25(12)$ & $\mathrm{C} 22$ & $\mathrm{C} 21$ & $\mathrm{C} 1$ & $119.97(13)$ \\
\hline $\mathrm{O} 1$ & $\mathrm{C} 2$ & $\mathrm{C} 1$ & $121.14(12)$ & $\mathrm{C} 22$ & $\mathrm{C} 21$ & $\mathrm{C} 26$ & $117.68(13)$ \\
\hline $\mathrm{O} 1$ & $\mathrm{C} 2$ & $\mathrm{C} 3$ & $121.23(13)$ & $\mathrm{C} 26$ & $\mathrm{C} 21$ & $\mathrm{C} 1$ & $122.18(13)$ \\
\hline $\mathrm{C} 3$ & $\mathrm{C} 2$ & $\mathrm{C} 1$ & $117.49(11)$ & $\mathrm{C} 21$ & $\mathrm{C} 22$ & $\mathrm{C} 23$ & $121.20(14)$ \\
\hline $\mathrm{C} 2$ & $\mathrm{C} 3$ & $\mathrm{C} 4$ & $114.11(12)$ & $\mathrm{C} 24$ & $\mathrm{C} 23$ & $\mathrm{C} 22$ & $120.47(14)$ \\
\hline $\mathrm{C} 12$ & $\mathrm{C} 11$ & $\mathrm{C} 1$ & $123.55(13)$ & $\mathrm{C} 23$ & $\mathrm{C} 24$ & $\mathrm{C} 25$ & $118.96(14)$ \\
\hline $\mathrm{C} 12$ & C11 & C16 & $117.59(13)$ & $\mathrm{C} 24$ & $\mathrm{C} 25$ & $\mathrm{C} 26$ & $120.77(14)$ \\
\hline $\mathrm{C} 16$ & C11 & $\mathrm{C} 1$ & $118.80(12)$ & $\mathrm{C} 25$ & $\mathrm{C} 26$ & $\mathrm{C} 21$ & $120.91(14)$ \\
\hline $\mathrm{C} 13$ & $\mathrm{C} 12$ & $\mathrm{C} 11$ & $121.18(13)$ & $\mathrm{C} 32$ & $\mathrm{C} 31$ & $\mathrm{C} 1$ & $114.48(12)$ \\
\hline C14 & C13 & $\mathrm{C} 12$ & $120.16(13)$ & & & & \\
\hline
\end{tabular}

Table S17. Hydrogen Bonds for 4a.
D H A d(D-H)/Å
$\mathbf{d}(\mathbf{H}-\mathbf{A}) / \AA \quad \mathbf{d}(\mathbf{D}-\mathbf{A}) / \AA$
D-H-A/ ${ }^{\circ}$
$\mathrm{O} 2 \mathrm{H} 2 \mathrm{O} 1^{1}$
0.84
$1.912 .7309(14)$
164.4

13/2-X,1-Y,1/2+Z

Table S18. Torsion Angles for 4a.
$\begin{array}{llll}A & \text { B } & \text { C } & \text { D }\end{array}$
Angle $/^{\circ}$
A B C D Angle ${ }^{\circ}$
O1 $\quad$ C2 23 C4
$8.6(2)$
C13C14C15C16
$-2.6(2)$
O2 C14C15C16 177.09(13) C14C15C16C11
$0.4(2)$
C1 C2 C3 C4 -167.19(13) C16C11C12C13
$-2.0(2)$
C1 C11C12C13 175.09(13) C21C1 C2 O1 15.13(19)
C1 C11C16C15-175.29(13) C21C1 C2 C3 -169.08(12)
C1 C21C22C23-175.75(13) C21C1 C11C12 122.18(14)
C1 C21C26C25 175.17(14) C21C1 C11C16 -60.81(16)
C2 C1 C11C12-117.96(14) C21C1 C31C32 62.53(15) 


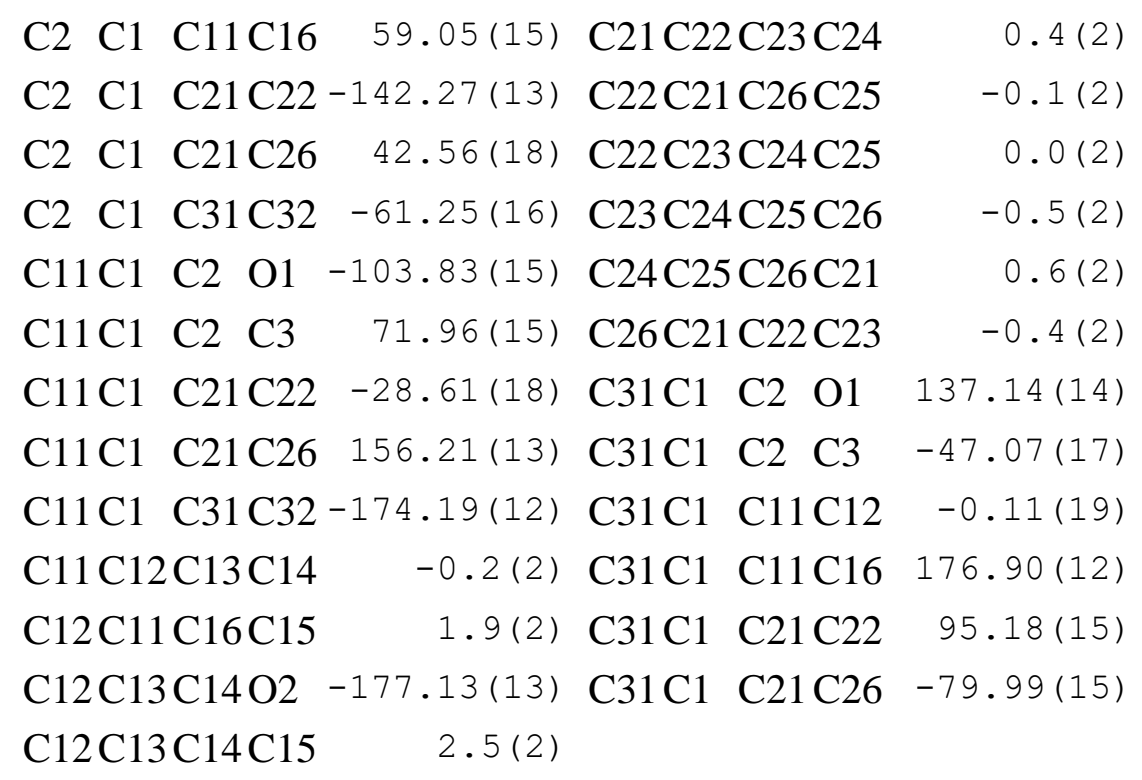

Table S19. Hydrogen Atom Coordinates $\left(\AA \times 10^{4}\right)$ and Isotropic Displacement Parameters $\left(\AA^{2} \times 10^{3}\right)$ for 4 a.

\begin{tabular}{|c|c|c|c|c|}
\hline Atom & $x$ & $y$ & $z$ & $\mathbf{U}(\mathbf{e q})$ \\
\hline $\mathrm{H} 2$ & 9675 & 6025 & 4154 & \\
\hline $\mathrm{H} 3 \mathrm{~A}$ & 4089 & 2796 & 1446 & \\
\hline H3B & 5765 & 3369 & 1897 & \\
\hline $\mathrm{H} 4 \mathrm{~A}$ & 6874 & 2071 & 982 & \\
\hline $\mathrm{H} 4 \mathrm{~B}$ & 7390 & 3180 & 552 & \\
\hline $\mathrm{H} 4 \mathrm{C}$ & 5739 & 2515 & 169 & \\
\hline H12 & 4239 & 4848 & 3442 & \\
\hline H13 & 6784 & 5332 & 4242 & \\
\hline H15 & 8960 & 6610 & 2047 & \\
\hline H16 & 6428 & 6124 & 1255 & \\
\hline $\mathrm{H} 22$ & 3223 & 7052 & 2166 & \\
\hline $\mathrm{H} 23$ & 1660 & 8469 & 1564 & \\
\hline $\mathrm{H} 24$ & 17 & 8261 & 266 & \\
\hline $\mathrm{H} 25$ & -46 & 6616 & -419 & \\
\hline H26 & 1541 & 5203 & 166 & \\
\hline H31A & 2719 & 3827 & 2531 & \\
\hline H31B & 1542 & 4868 & 2656 & \\
\hline $\mathrm{H} 32 \mathrm{~A}$ & -115 & 3429 & 2026 & \\
\hline H32B & 1116 & 3526 & 1168 & \\
\hline $\mathrm{H} 32 \mathrm{C}$ & -194 & 4471 & 1438 & \\
\hline
\end{tabular}


(1) (a) McMurry, J. E.; Felming, M. P. New Method for the Reductive Coupling of Carbonyls to Olefins. Synthesis of .beta.-Carotene. J. Am. Chem. Soc. 1974, 96, 4708-4909. (b) Seo, J. W.; Comninos, J. S.; Chi, D. Y.; Kim, D. W.; Carlson, K. E.; Katzenellenbogen, J. A. Fluorine-Substituted Cyclofenil Derivatives as Estrogen Receptor Ligands: Synthesis and Structure-Affinity Relationship Study of Potential Positron Emission Tomography Agents for Imaging Estrogen Receptors in Breast Cancer. J. Med. Chem. 2006, 49, 2496-2511. (c) Seo, J. W.; Kim, H. J.; Lee, B. S.; Katzenellenbogen, J. A.; Chi, D. Y. Convenient One-Pot Synthesis of 2,2-Bis-(4-hydroxyphenyl)-cyclopentanone. J. Org. Chem. 2008, 73, 715-718. (d) Zhu, H.; Huang, L.; Xu, X.; Shen, Y.-M. Practical Synthesis of FEt-penta-cyclofenil and Its Derivatives for Potential PET Imaging. Synth. Commun. 2010, 40, 3322-3331.

(2) Ahmed, M. S. M.; Mori, A. Sonogashira Coupling with Aqueous Ammonia Directed to the Synthesis of Azotolane Derivatives. Tetrahedron 2004, 60, 9977-9982.

(3) Monks, B. M.; Cook, S. P. Palladium-Catalyzed Alkyne Insertion/Suzuki Reaction of Alkyl Iodides. J. Am. Chem. Soc. 2012, 134, 15297-15300.

(4) Sato, K.; Kuriwaki, J.; Takahashi, K.; Saito, Y.; Oka, J.; Otani, Y.; Sha, Y.; Nakazawa, K.; Sekino, Y.; Ohwada, T. Discovery of a Tamoxifen-Related Compound that Suppresses Glial L-Glutamate Transport Activity without Interaction with Estrogen Receptors. ACS Chem. Neurosci. 2012, 3, 105-113.

(5) Andjouh, S.; Blache, Y. Click-based Synthesis of Bromotyrosine Alkaloid Analogs as Potential Anti-biofilm Leads for SAR Studies. Bioorg. Med. Chem. Lett. 2015, 25, 5762-5766.

(6) Catalyst C1: Kötzner, L.; Webber, M. J. Martinez, A.; De Fusco, C.; List, B. Asymmetric Catalysis on the Nanoscale: The Organocatalytic Approach to Helicenes. Angew. Chem., Int. Ed. 2014, 53, 5202-5205. 
(7) Catalyst A3: Kang, Q.; Zhao, Z.-A.; You, S.-L. Highly Enantioselective Friedel-Crafts Reaction of Indoles with Imines by a Chiral Phosphoric Acid. J. Am. Chem. Soc. 2007, 129, 1484-1485.

(8) Catalyst D: Rueping, M.; Maji, M. S.; Küçük, H. B.; Atodiresei, I. Asymmetric Brønsted Acid Catalyzed Cycloadditions-Efficient Enantioselective Synthesis of Pyrazolidines, Pyrazolines, and 1,3-Diamines from N-Acyl Hyrazones and Alkenes. Angew. Chem., Int. Ed. 2012, 51, 12864-12868.

(9) Catalyst A5: Sun, F.-L.; Zheng, X.-J.; Gu, Q.; He, Q.-L.; You, S.-L. Enantioselective Synthesis of Unsymmetrical Triarylmethanes by Chiral Brønsted Acids. Eur. J. Org. Chem. 2010, 47-50.

(10) Catalyst E: Zhuang, M.; Du, H. Asymmetric Rearrangement of Racemic Epoxides Catalyzed by Chiral Brønsted Acids. Org. Biomol. Chem. 2013, 11, 1460-1462.

(11) Molander, G. A.; Brown, A. R. Suzuki-Miyaura Cross-Coupling Reactions of Potassium Vinyltrifluoroborate with Aryl and Heteroaryl Electrophiles. J. Org. Chem. 2006, 71, 9681-9686.

(12) Itoh, T.; Mase, T. A General Palladium-Catalyzed Coupling of Aryl Bromides/Triflates and Thiols. Org. Lett. 2004, 6, 4587-4590.

(13) Xiao, Y.; Min Q.; Xu, C.; Wang, R.; Zhang, X. Nickel-Catalyzed Difluoroalkylation of (Hetero)Arylborons with Unactivated 1-Bromo-1,1-difluoroalkanes. Angew. Chem., Int. Ed. 2016, 55, 5837-5841.

(14) Peterson, G. A.; Kunng, F.-A.; McCallum, J. S.; Wulffe, W. D. Palladium Catalyzed Reduction of Aryl Triflates - Utilization in the Synthesis of Angelicin, Olivin and Chromomycinone from Phenolsproduced in the Benzannulation Reaction of Chromium Carbenecomplexes. Tetrahedron Lett. 1987, 28, 1381-1384.

(15) (a) Nicolaou, K. C.; Montagnon, T.; Baran, P. S.; Zhong, Y.-L. Iodine(V) Reagents in Organic Synthesis. Part 4. o-Iodoxybenzoic Acid as a 
Chemospecific Tool for Single Electron Transfer-Based Oxidation Processes. J. Am. Chem. Soc. 2002, 124, 2245-2258. (b) Nakajima, R.; Ogino, T.; Yokoshima, S.; Fukuyama, T. Total Synthesis of (-)-Mersicarpine. J. Am. Chem. Soc. 2010, 132, 1236-1237.

(16) Carreño, M. C.; González-López, M.; Latorre, A.; Urbano, A. General Synthesis of 8-Aryl-2-tetralones. J. Org. Chem. 2006, 71, 4956-4964.

(17) Chen, M.; Cai, X.; Chen, Z.; Shi, L.; Zhou, Y. Facile Construction of Three Contiguous Stereogenic Centers via Dynamic Kinetic Resolution in Asymmetric Transfer Hydrogenation of Quinolones. Chem. Commun. 2014, 50, 12526-12529. 


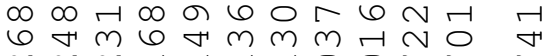

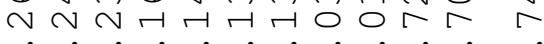

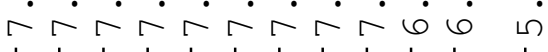

$\longrightarrow$
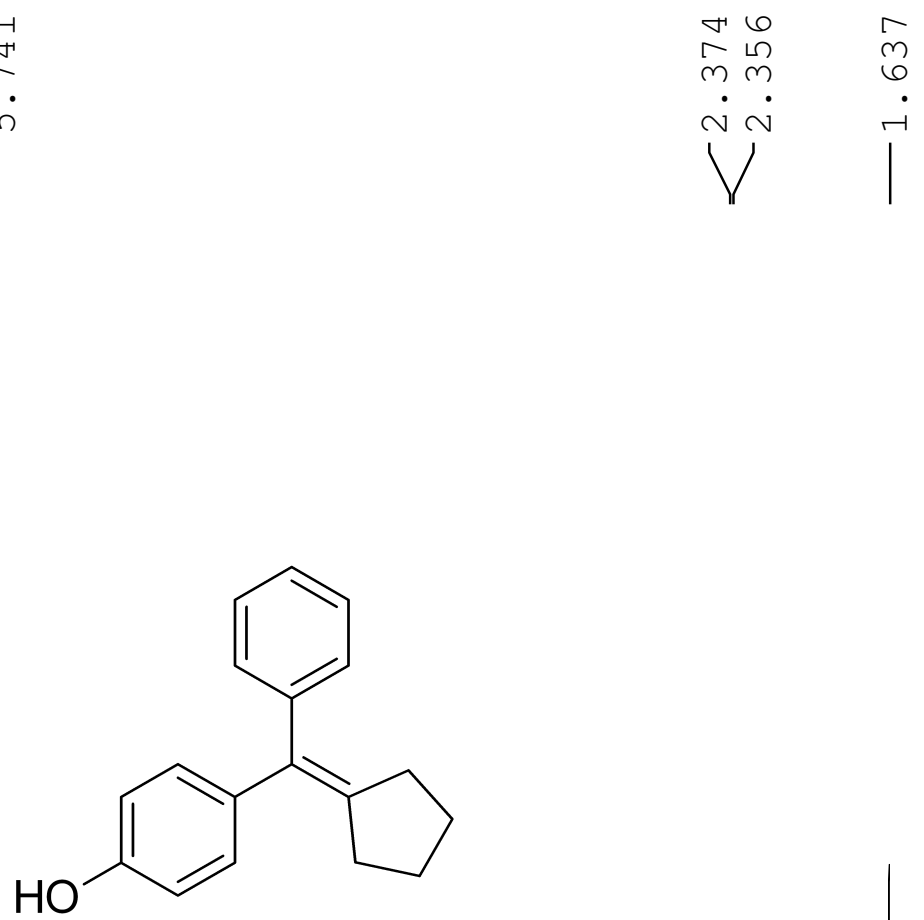

S1a

4

3

\section{2}

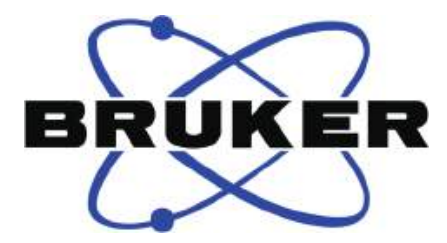

Current Data Parameters NAME

mdk $9171 \mathrm{H}$

PROCNO

F2 - Acquisition Parameters

Date__ 20190530

20.57

INSTRUM spect

$5 \mathrm{~mm}$ PABBO BB/

PULPROG $\quad$ zg30

TD $\quad 65536$

OLVENT

NS

DS

IDRES $0.122266 \mathrm{~Hz}$

AQ

DW

$\mathrm{DE}$

TE

TD 0

12.56

62.400 usec

6.50 usec

$$
1
$$

$======$ CHANNEL $\mathrm{fl}========$

SFO1 $\quad 400.1324710 \mathrm{MHz}$

NuC1 14.50

14.50 usec

PLW1 $11.99499989 \mathrm{~W}$

F2 - Processing parameters

SI 65536

SF $\quad 400.1300592 \mathrm{MHz}$

SSB

$0.30 \mathrm{~Hz}$

1.00 


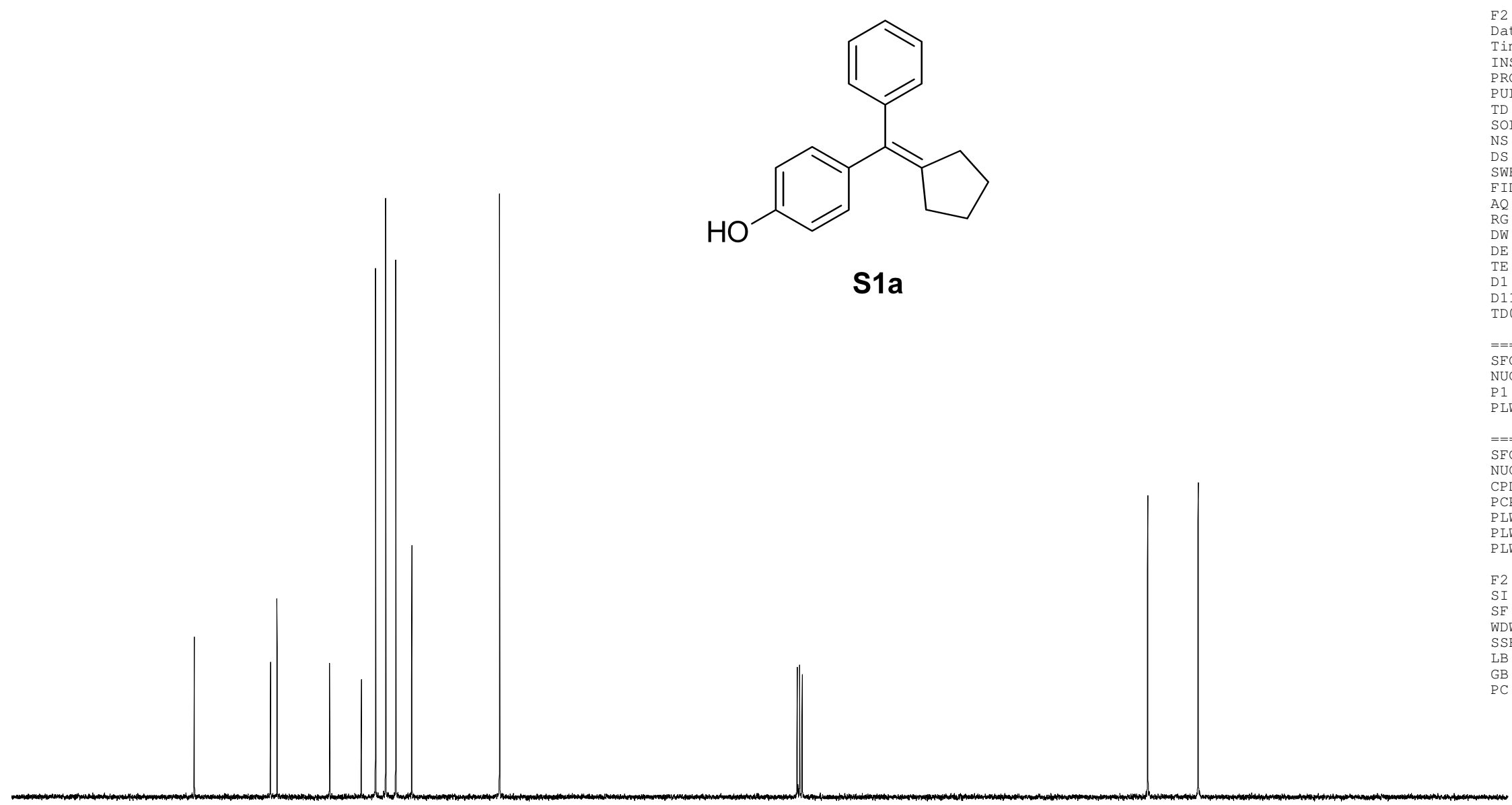

Current Data Parameters Current
NAME
EXPNO
PROCNO

1

2 - Acquisition Parameters

20190530

Time

ULROG

SOLVEN

DS

$0.366798 \mathrm{~Hz}$
$1.3631488 \mathrm{sec}$
196.92

196.92
20.800 usec
6.50

6.50 usec

$2.00000000 \mathrm{sec}$

$\begin{array}{ll}2.00000000 & \mathrm{sec} \\ \text { DDO } & 0.0300001\end{array}$

CHANNEL $\mathrm{f} 1========$
$100.6228298 \mathrm{MHz}$

$\mathrm{NUC1}$
$\mathrm{P} 1$

$93 \mathrm{C}$

$46.98899841 \mathrm{~W}$

$z======$ CHANNEL $f 2 \quad=======$
SFO2
$400.1316005 \mathrm{MHz}$

$1 \mathrm{H}$
CPDC2
waltz16

$11.99499989 \mathrm{~W}$

$1.34293999 \mathrm{~W}$
$\mathrm{PLW12}$

$\begin{array}{rr}\text { F2 } & \text { Processing parameters } \\ \text { SI } & 32768 \\ \mathrm{SF} & 100.6127920 \mathrm{MHz}\end{array}$

WDW $\quad 100.6127920 \mathrm{MHz}$

$1.00 \mathrm{~Hz}$

$\begin{array}{lllllllllllllllllll}170 & 160 & 150 & 140 & 130 & 120 & 110 & 100 & 90 & 80 & \begin{array}{l}70 \\ \mathrm{~S}-102\end{array} & 60 & 50 & 40 & 30 & 20 & 10 & 0 & \mathrm{ppm}\end{array}$



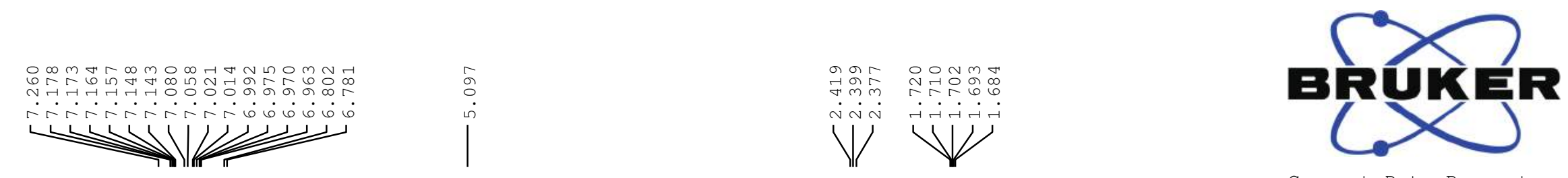
Current Data Parameters
NAME

EXPNO

F2 - Acquisition Parameters

Date_ 20190124

Time $\quad 19.48$

spect
PROBHD $5 \mathrm{~mm} \mathrm{PABBO}$ BB-

$\begin{array}{lr}\text { PROBHD } & 5 \mathrm{~mm} \text { PABBO } \mathrm{BB}- \\ \text { PULPROG } & \mathrm{zg} 30\end{array}$

TD

SOLVENT

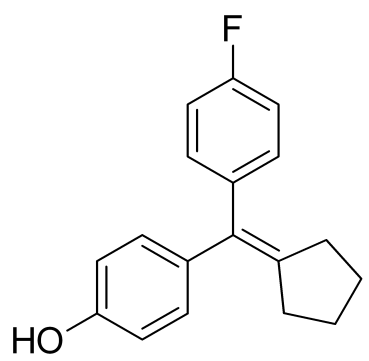

$\begin{array}{lr}\text { DS } & 2 \\ \text { SWH } & 8223.685 \mathrm{~Hz}\end{array}$

FIDRES $\quad 0.125483 \mathrm{~Hz}$

$\mathrm{AQ} \quad 3.9845889 \mathrm{sec}$

RG

DW

DE

TE

D1

60.800 usec

6.00 usec

$294.9 \mathrm{~K}$

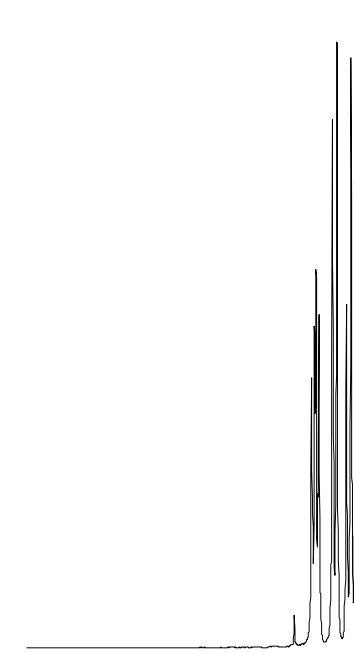

S1b

$\begin{array}{lr}=======\text { CHANNEL } \mathrm{fl}======= \\ \text { NUC1 } & 1 \mathrm{H} \\ \text { P1 } & 15.80 \text { useC } \\ \text { PL1 } & -1.00 \mathrm{~dB}\end{array}$

PL1W

$12.17476940 \mathrm{~W}$

SFOI $400.1324710 \mathrm{MHz}$

F2 - Processing parameter

$\begin{array}{lr}\text { SI } & 32768 \\ \text { SF } & 400.1300090 \mathrm{MHz}\end{array}$

WDW

SSB

SSB

LB
PC

EM

$0.30 \mathrm{~Hz}$

1.00

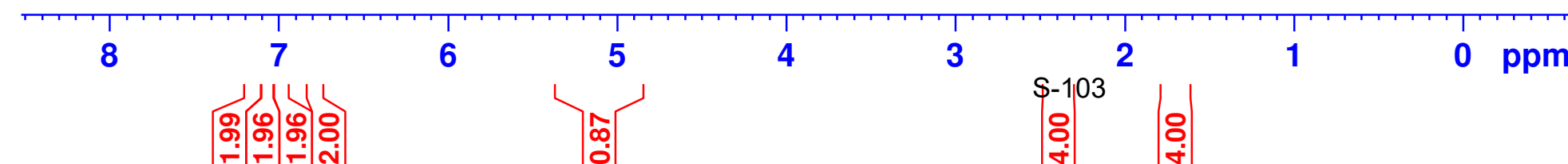




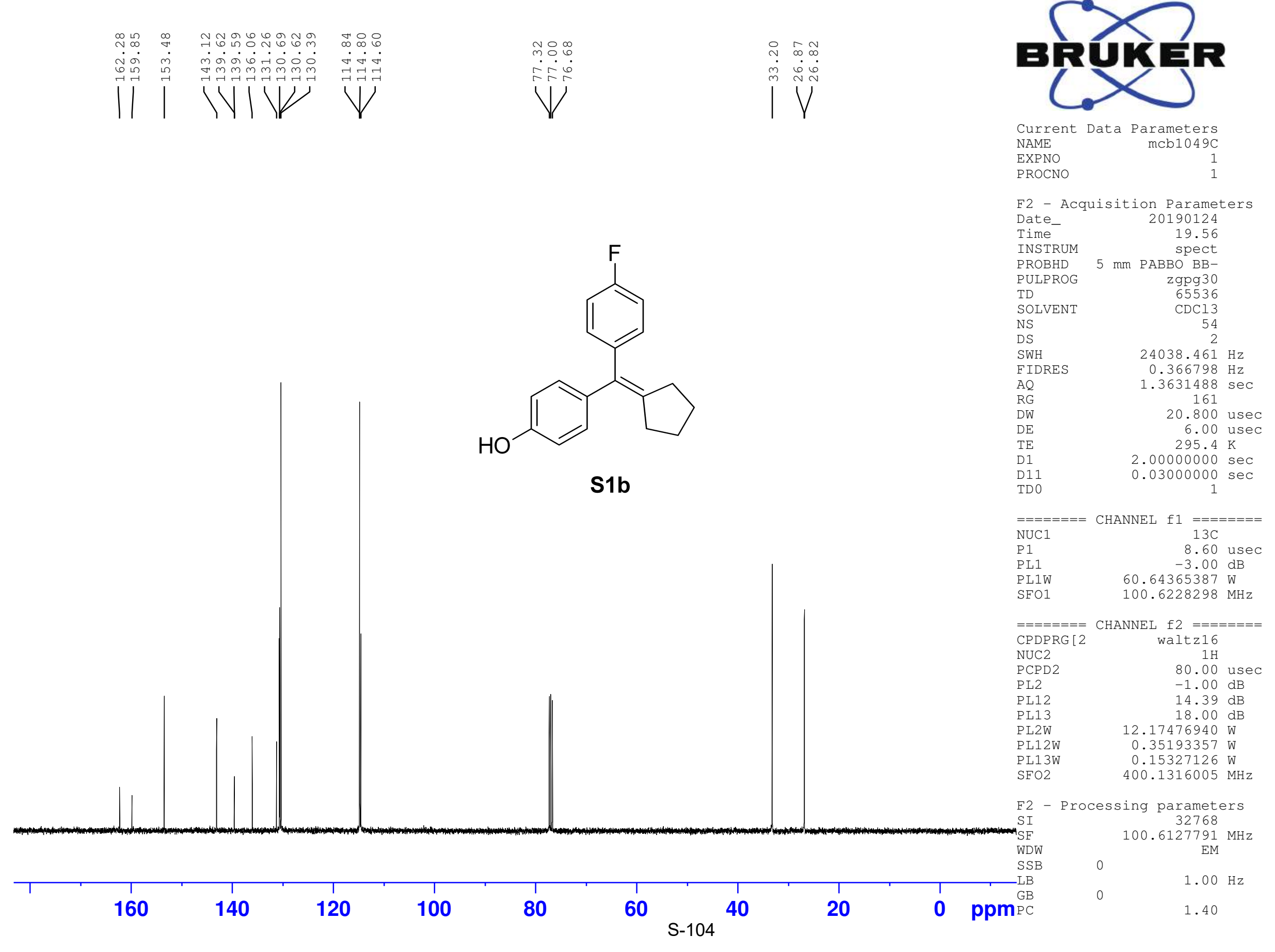




\section{equiren}

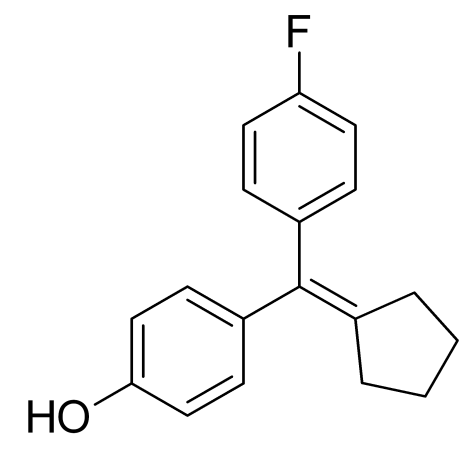

S1b

Current Data Parameters

NAME mcb1049F-decoup

PROCNO

$\begin{array}{ll}\text { F2 - Acquisition Parameters } \\ \text { Date_ } & 20190530 \\ \text { Time- } & 20.52\end{array}$

Time

$\begin{array}{lr}\text { INSTRUM } & \text { spect } \\ \text { PROBHD } & 5 \mathrm{~mm} \text { PABBO BB/ } \\ \text { PULPROG } & \mathrm{zgpg} 30\end{array}$

SOLVENT

NS
DS

$\begin{array}{ll}\text { SWH } & 93750.000 \mathrm{~Hz}\end{array}$

$\begin{array}{ll}90 \quad & 1.430511 \mathrm{~Hz} \\ \mathrm{AQ} & 0.3495253 \mathrm{sec}\end{array}$

AQ
RG

DW

D 11
TD0

$2.00000000 \mathrm{sec}$ $0.03000000 \mathrm{sec}$

$z== \pm===$ CHANNEL $f 1 \quad=======$
SFO1 $376.4607162 \mathrm{MHz}$

NUC1 $19 \mathrm{~F}$

P1 14.70 usec
PLW1 $\quad 15.99600029 \mathrm{~W}$

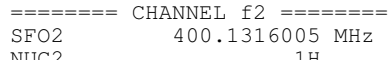

$\begin{array}{lr}\text { NUC2 } & 1 \mathrm{H} \\ \text { CPDPRG [2 } & \text { waltz16 }\end{array}$

$\begin{array}{lr}\text { CPDPRG [2 } & \text { waltz16 } \\ \text { PCPD2 } & 90.00 \text { usec }\end{array}$

$\begin{array}{lr}\text { PLW2 } & 11.99499989 \mathrm{~W} \\ \text { PLW12 } & 0.34213999 \mathrm{~W}\end{array}$

PLW13 0.2713001 W

F2 - Processing parameters
SI

$\begin{array}{ll}\text { SI } & 32768 \\ \text { SF } & 376.4983660 \mathrm{MH} z\end{array}$

WDW

$\mathrm{EM}$
$1.00 \mathrm{~Hz}$

\begin{tabular}{|c|c|c|c|c|c|c|c|c|c|c|c|}
\hline 20 & $\begin{array}{l}1 \\
0\end{array}$ & $\begin{array}{r}1 \\
-20\end{array}$ & -40 & $\begin{array}{r}1 \\
-60\end{array}$ & -80 & $\begin{array}{c}1 \\
-100\end{array}$ & $\begin{array}{l}1 \\
-120\end{array}$ & $\begin{array}{l}140 \\
-140\end{array}$ & $\begin{array}{c}1 \\
-160\end{array}$ & $\begin{array}{c}1 \\
-180\end{array}$ & -200 \\
\hline 20 & 0 & & & -00 & -00 & & S-105 & $=140$ & -100 & -100 & $-\angle 00$ \\
\hline
\end{tabular}




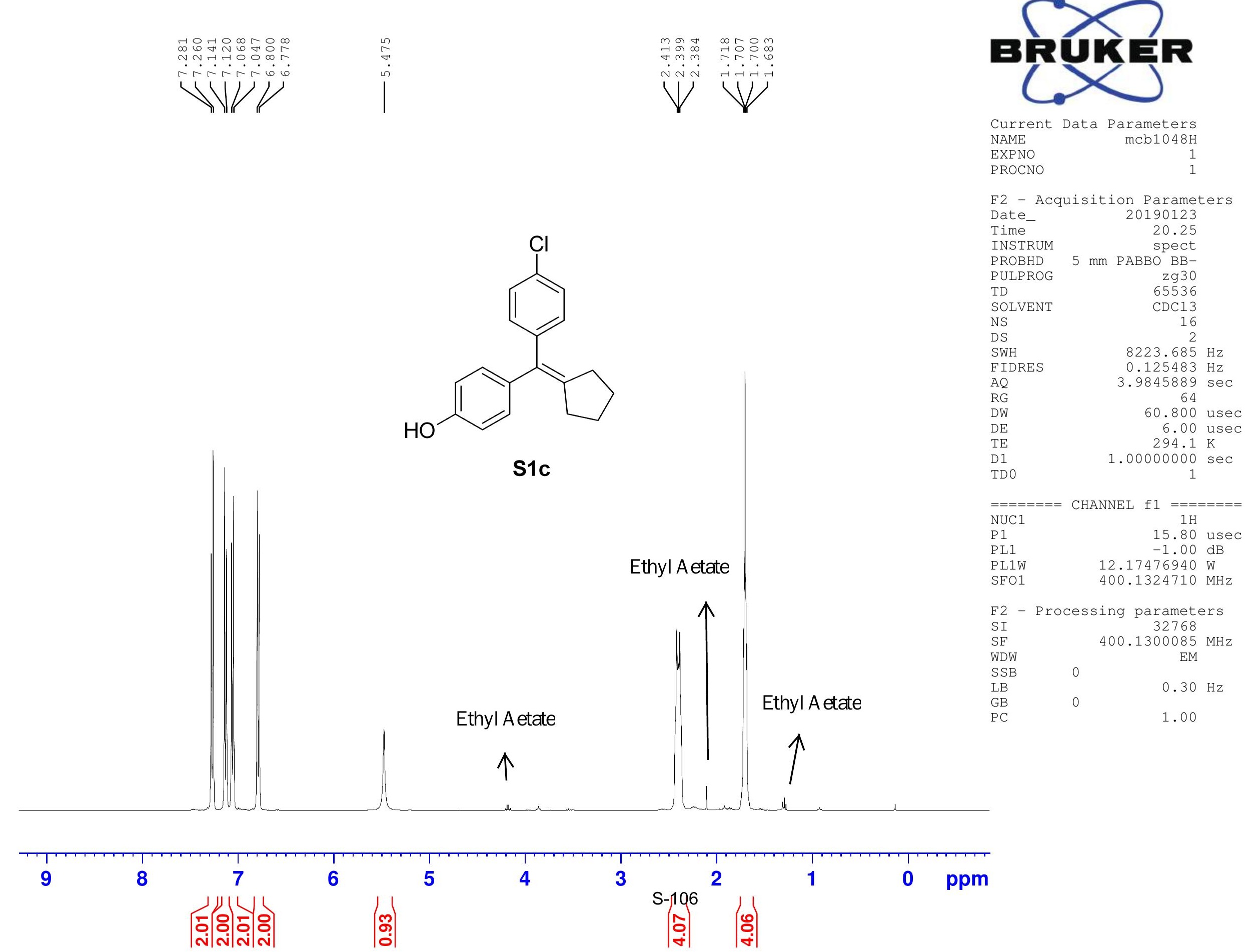


Current Data Parameters

NAME

EXPNO

PROCNO

mcb1048c

F2 - Acquisition Parameters

Date__ 20190123

Time $\quad 20.32$

PROBHD $5 \mathrm{~mm}$ PABBO BB-

PULPROG zgog30

TD $\quad 65536$

SOLVENT CDCl3

NS

SWH $24038.461 \mathrm{~Hz}$

FIDRES $\quad 0.366798 \mathrm{~Hz}$

AQ $\quad 1.3631488 \mathrm{sec}$

RG

2050
20.800 usec

$\begin{array}{lr}\text { DW } & 20.800 \text { usec } \\ \text { DE } & 6.00 \text { usec }\end{array}$

TE $\quad 294.7 \mathrm{~K}$

$\begin{array}{ll}\text { D1 } & 2.00000000 \mathrm{sec} \\ \text { D11 } & 0.03000000 \mathrm{sec}\end{array}$

D11 $\quad 0.03000000 \mathrm{sec}$

$=======$ CHANNEL $\mathrm{f}]$

$=======$ CHANNEL fl $\begin{aligned} & ======== \\ & 13 \mathrm{C}\end{aligned}$
NUC1

PL1
PL1W

SFO1

$-3.00 \mathrm{~dB}$

$60.64365387 \mathrm{~W}$

$100.6228298 \mathrm{MHz}$

waltz16

PCPD2

PL2

PL12

PI $2 \mathrm{~W}$

PL12W

PL13W

$1 \mathrm{H}$
80.00 usec

$\begin{array}{ll}80.00 & \text { use } \\ -1.00 \mathrm{~dB} & \end{array}$

$14.39 \mathrm{~dB}$

$12.17476940 \mathrm{~W}$

$0.15327126 \mathrm{~W}$

$400.1316005 \mathrm{MHz}$

2 - Processing parameters

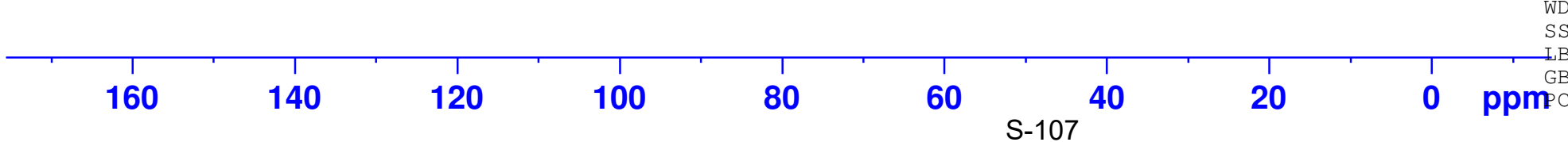

$1.00 \mathrm{~Hz}$

1.40 

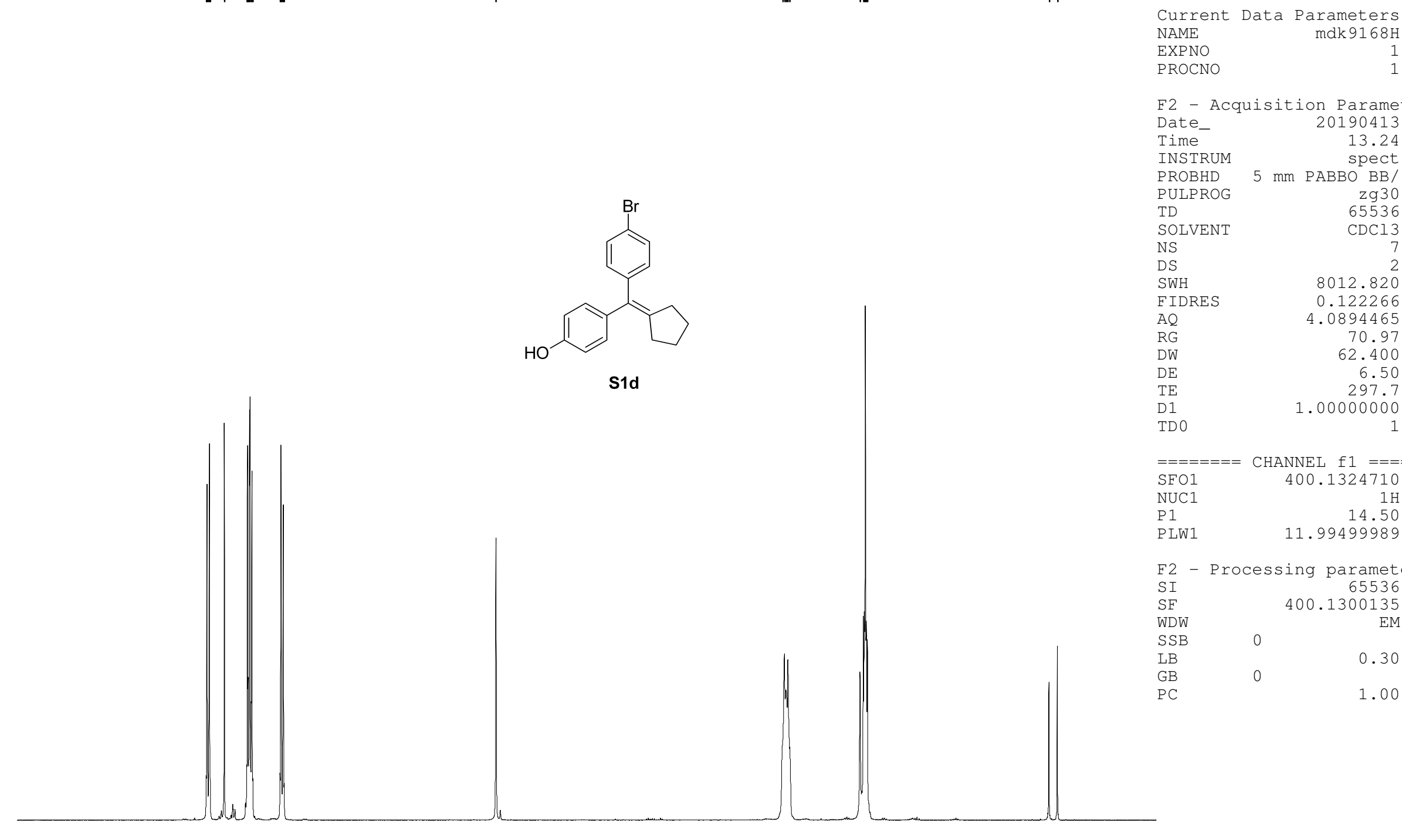

EXPNO

F2 - Acquisition Parameters

Date_ 20190413

Time $\quad 13.24$

SPeCt
PROBHD $5 \mathrm{~mm} \mathrm{PABBO} \mathrm{BB} /$

$\begin{array}{lr}\text { PROBHD } & 5 \mathrm{~mm} \text { PABBO BB/ } \\ \text { PULPROG } & \mathrm{zg} 30\end{array}$

TD

NS

DS

SWH

FIDRES

$\mathrm{AQ}$

AQ
RG
DW
DE

DE

D1

$\mathrm{SFO}$

NUC

CHANNEL $\mathrm{fl}========$

$400.132471 \mathrm{H}$

14.50 usec
PIW1

F2 - Processing parameters

SI 65536

SF $\quad 400.1300135 \mathrm{MHz}$

WDW

SSB

LB

EM

$0.30 \mathrm{~Hz}$

1.00

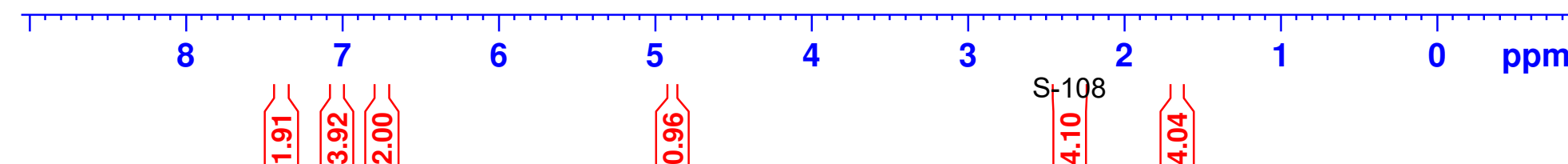




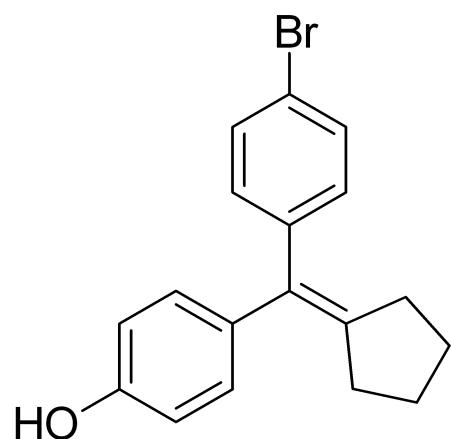

S1d
BR UKKER 1

Current Data Parameters NAME EXPNO

PROCNO

F2 - Acquisition Parameters

Date_ 20190413

Time $\quad 13.26$

5 mm PABBO

PROBHD $5 \mathrm{~mm}$ PABBO BB/

PULPROG zgpg30

TD 65536

SOLVENT

NS

DS

SWH

FIDRES

$\mathrm{AQ}$

RG

DW

TE

D1 11

TDO

$\mathrm{CDCl} 3$

$24038.461 \mathrm{~Hz}$

$0.366798 \mathrm{~Hz}$

$1.3631488 \mathrm{sec}$ 196.92

20.800 usec 6.50 usec $298.4 \mathrm{~K}$

$2.00000000 \mathrm{sec}$ $0.03000000 \mathrm{sec}$

\section{$==$}

PLW1

100.6228298 $\mathrm{MHz}$

$13 \mathrm{C}$

46.98899841

$\mathrm{SFO} 2$

CPDPRG [ 2

PCPD2

PLW2

PLW2

PLW12

HANNEL f2 $========$

$400.1316005 \mathrm{MHz}$

waltz16

90.00 usec

$11.99499989 \mathrm{~W}$

$0.34213999 \mathrm{~W}$

0.27713001

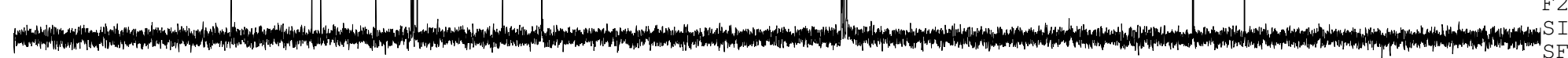

- Processing parameters$$
T
$$

32768
100.6127729

$1.00 \mathrm{~Hz}$

1.40 


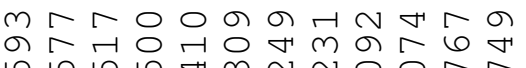

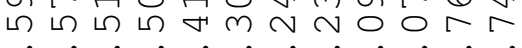

rararararab

$\longrightarrow$

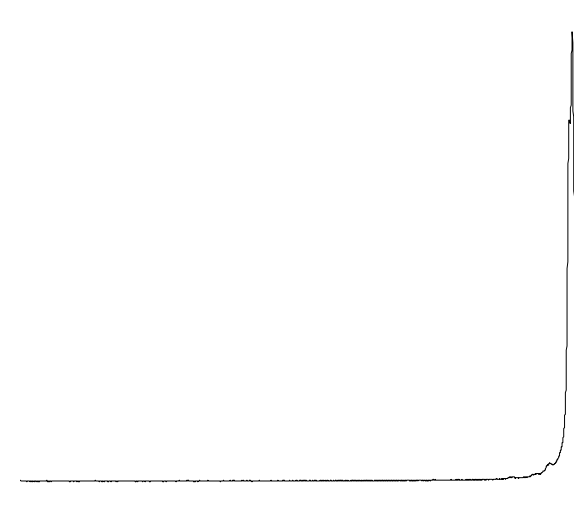

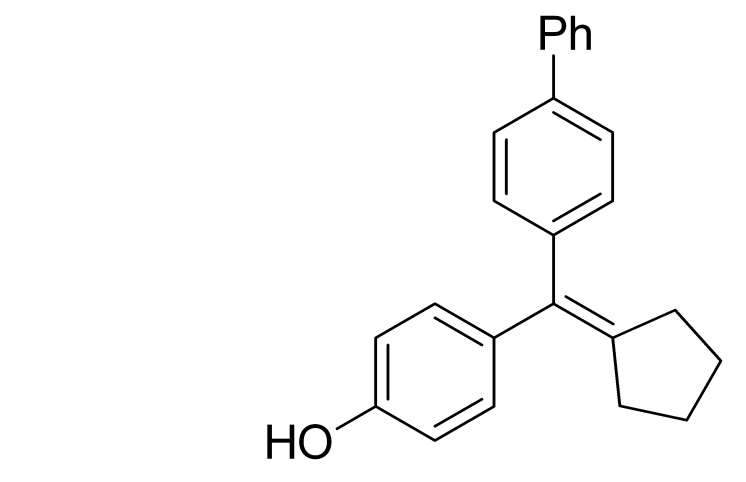

\section{S1e}

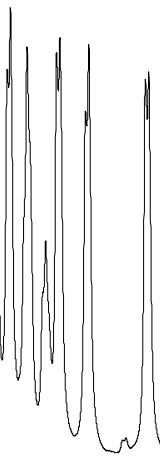

\begin{abstract}
7
\end{abstract}

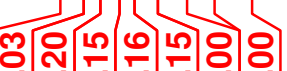

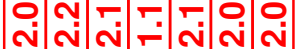

6

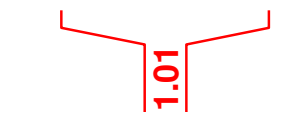

4

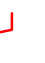

\section{Current Data Parameters NAME \\ EXPNO
PROCNO \\ mdk $9066 \mathrm{H}$}

F2 - Acquisition Parameters

Date__ 20190318

Time 13.31

spect

PULPROG $5 \mathrm{~mm}$ PABBO
zgB 30

$\begin{array}{lr}\text { TD } & \text { zg30 } \\ \text { TD } & 65536\end{array}$

$\begin{array}{ll}\text { TD } & 65536 \\ \text { SOLVENT } & \text { CDCl3 }\end{array}$

DS

$\begin{array}{ll}\text { DS } & 2 \mathrm{~Hz} \\ \text { SWH } & 8012.820 \mathrm{~Hz}\end{array}$

IDRES $\quad 0.122266 \mathrm{~Hz}$

$\mathrm{AQ} \quad 4.0894465 \mathrm{sec}$

RG

DW

$\mathrm{DE}$ 6.50 us
$295.9 \mathrm{~K}$

$1.00000000 \mathrm{sec}$

$========$ CHANNEL $\mathrm{f} 1 \mathrm{l}========$ SFO1 $400.1324710 \mathrm{MHz}$ NUC1 1

P1 14.50 usec PLW1 $11.99499989 \mathrm{~W}$

F2 - Processing parameters

SI 65536

SF $\quad 400.1300269 \mathrm{MHz}$

WDW EM

$0.30 \mathrm{~Hz}$

GB
PC

1.00 


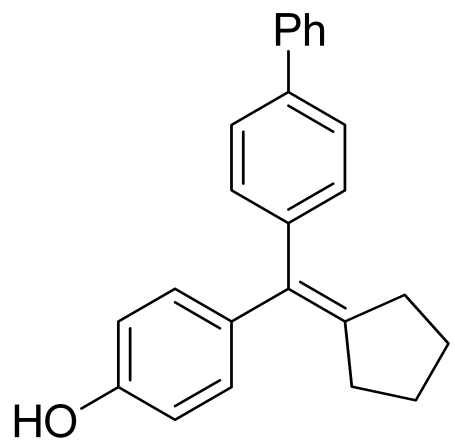

S1e

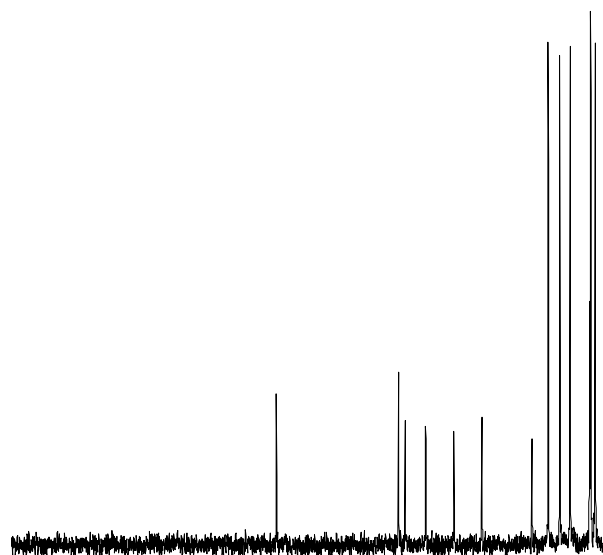

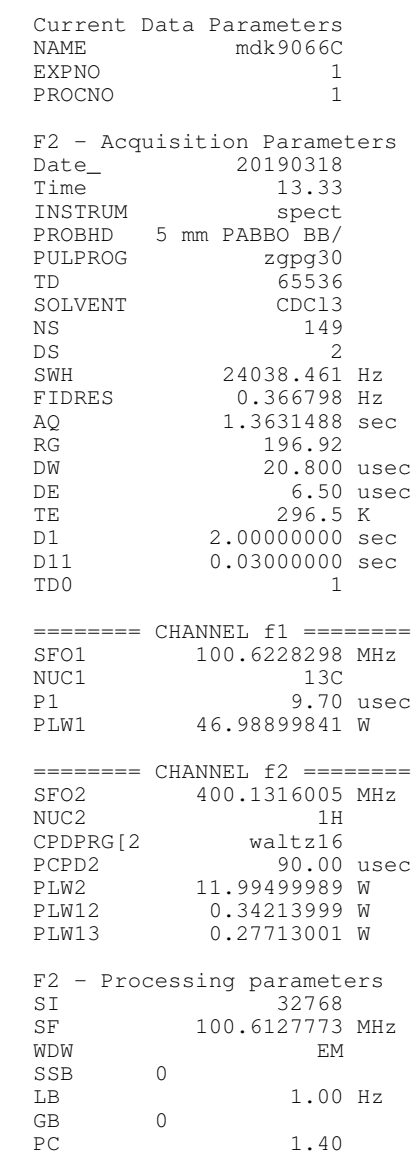

$\begin{array}{lllllllllllllllllll}170 & 160 & 150 & 140 & 130 & 120 & 110 & 100 & 90 & 80 & 70 & 60 & 50 & 40 & 30 & 20 & 10 & 0 & \text { ppm }\end{array}$



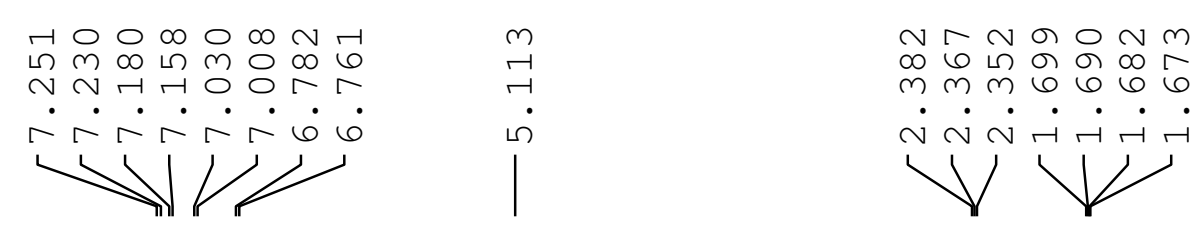

\section{BQ (}

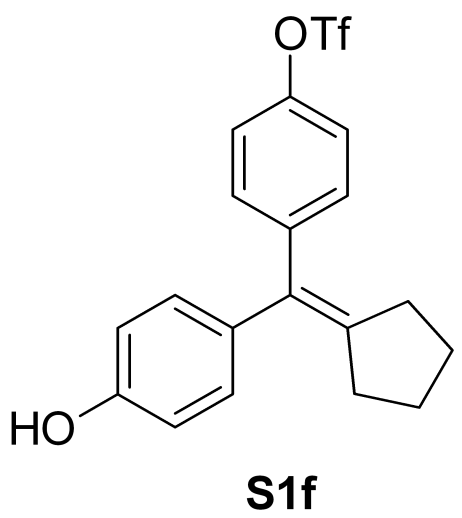

NAME

EXPNO

mdk9100H

ROCNO

F2 - Acquisition Parameters

Date_ 20190323

Time 13.19

$5 \mathrm{mpect}$

TIBOC

zg30
65536

TD $\quad 65536$

SOLVENT

NS

DS

$8012.820 \mathrm{~Hz}$
0.082266

IDRES $\quad 0.122266 \mathrm{~Hz}$

AQ $\quad 4.0894465 \mathrm{sec}$

RG

DW

$\mathrm{DE}$ $6.50 \mathrm{us}$
$295.8 \mathrm{~K}$

TD 0

$====$

$\mathrm{SFO1}$

$\mathrm{NUC} 1$
$\mathrm{P} 1$

CHANNEL $\mathrm{f} 1========$

$400.1324710 \mathrm{MHz}$

PLW1

14.50 usec

SI $\quad 65536$

SF $\quad 400.1300137 \mathrm{MHz}$

WSB

EM

$0.30 \mathrm{~Hz}$

GB

1.00

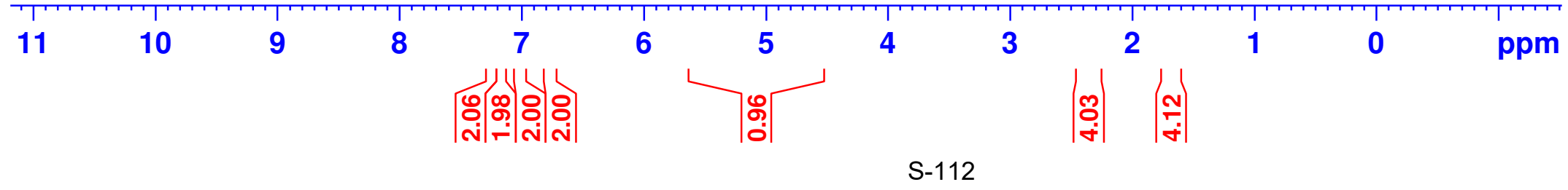



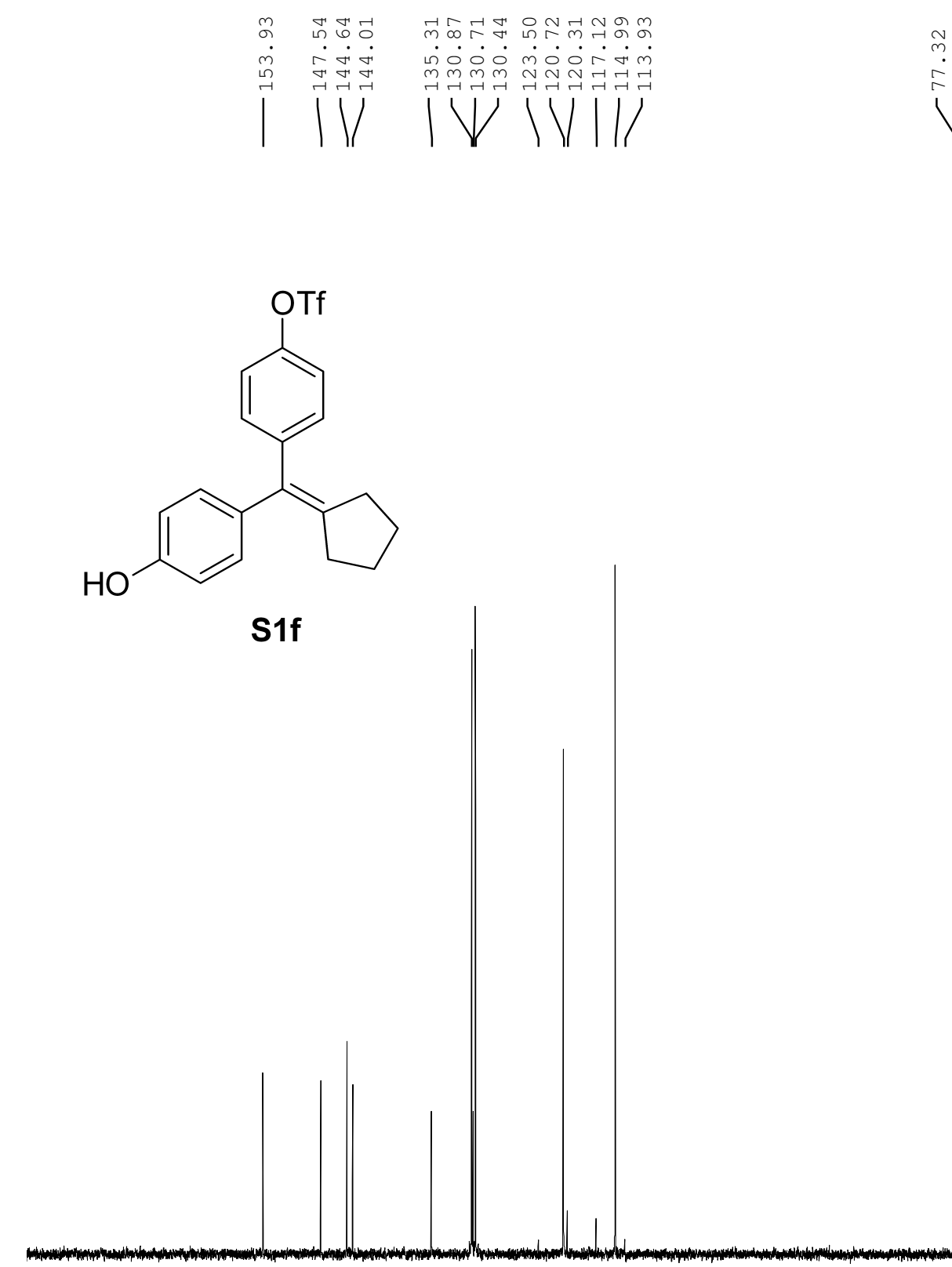

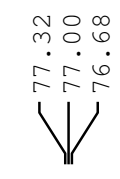

vi

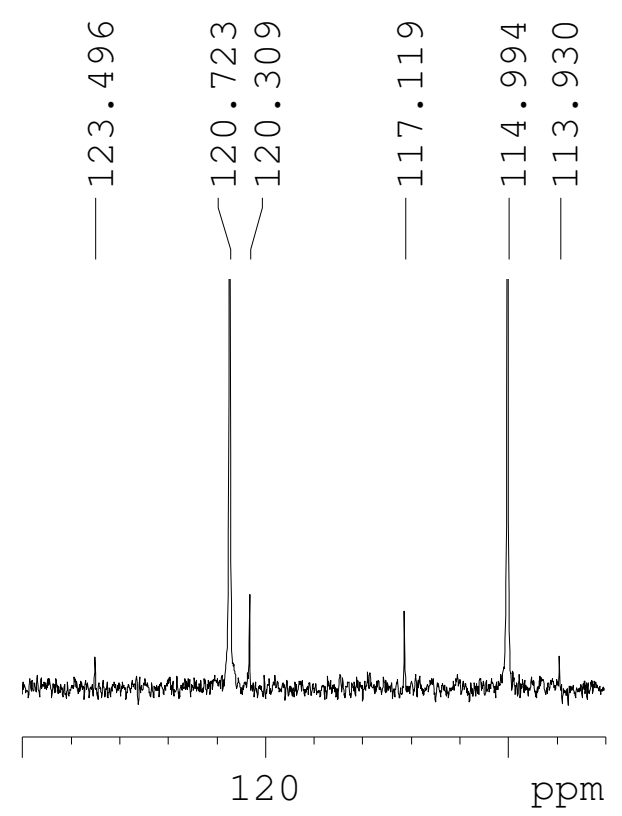

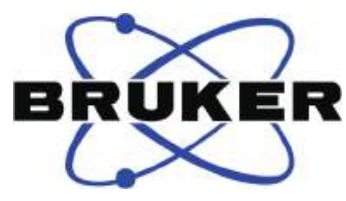

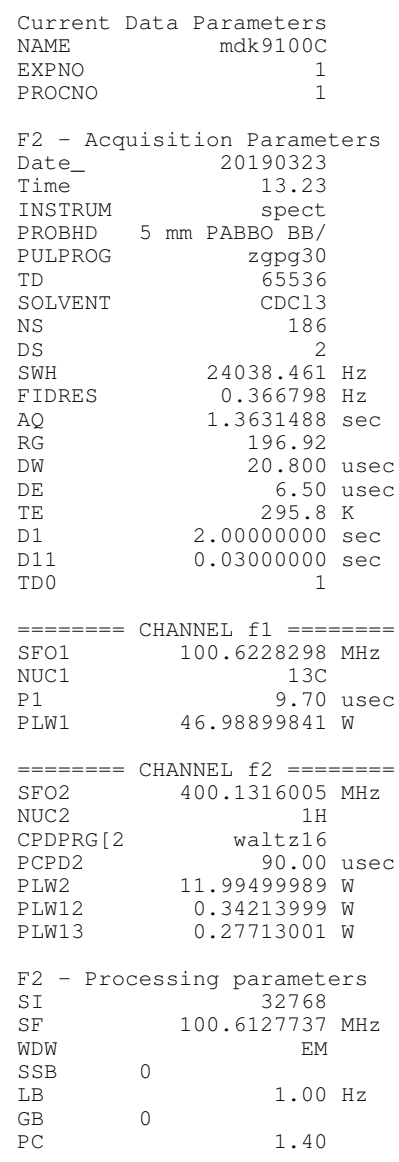




\section{expererer}

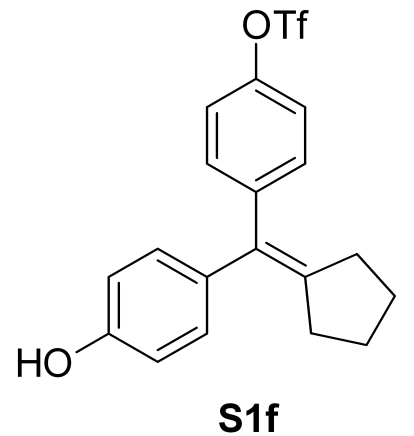

Current Data Parameters
NAME mdk9100F-decoup

EXPNO

$\begin{array}{ll}\text { F2 - Acquisition Parameters } \\ \text { Date } & 20190323 \\ \text { Time } & 13.22\end{array}$

Time

spect
PROBHD $5 \mathrm{~mm}$ PABBO BB/

PULPRO

SOLVENT

NSS
DS

$\begin{array}{ll}\text { SWH } & 93750.000 \mathrm{~Hz}\end{array}$

$\begin{array}{ll}\text { AQ } & 1.430511 \mathrm{~Hz} \\ \text { RG } & 0.3495253 \mathrm{sec}\end{array}$

RG

TE

D1
D11

196.92
5.333 usec

6.50 usec
$295.9 \mathrm{~K}$

$2.00000000 \mathrm{sec}$
$0.03000000 \mathrm{sec}$

TDO

$.0300000 \mathrm{sec}$

$z======$ CHANNEL $\mathrm{f} 1 \quad=======$
SFO1
$376.4607162 \mathrm{MHz}$

NUC1 $\quad 376.460719 \mathrm{~F}$

P1 14.70 usec
PLW1 $\quad 15.99600029 \mathrm{~W}$

$=======$ CHANNEL $f 2 \quad=======$
SFO2
NUC2
CO0.1316005 MHz

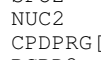

PLW2

waltz16
90.00 usec

PLW12

$.99499989 \mathrm{~W}$
$.34213999 \mathrm{~W}$

F2 - Processing parameters

$\begin{array}{lr}\text { SF } & 376.4983660 \mathrm{MHz} \\ \text { WDW } & \text { EM }\end{array}$

$\mathrm{GB}$
$\mathrm{PC}$

$1.00 \mathrm{~Hz}$

\begin{tabular}{|c|c|c|c|c|c|c|c|c|c|c|c|}
\hline 20 & $\begin{array}{l}1 \\
0\end{array}$ & $\begin{array}{r}1 \\
-20\end{array}$ & -40 & $\begin{array}{r}1 \\
-60\end{array}$ & -80 & $\begin{array}{c}1 \\
-100\end{array}$ & $\begin{array}{l}1 \\
-120\end{array}$ & $\begin{array}{l}140 \\
-140\end{array}$ & $\begin{array}{c}1 \\
-160\end{array}$ & $\begin{array}{c}1 \\
-180\end{array}$ & -200 \\
\hline 20 & 0 & & & & -00 & & S-114 & $=140$ & -100 & -100 & $-\angle 00$ \\
\hline
\end{tabular}




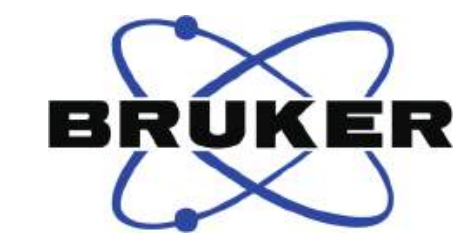

Current Data Parameters

NAME

mdk $9102 \mathrm{H}$

PROCNO

F2 - Acquisition Parameters

Date_ 20190323

$\begin{array}{lr}\text { Time } & 16.14\end{array}$

INSTRUM spect

ROBHD

PULPROG $\quad$ gg30

TD $\quad 65536$

SOLVENT

NS

DS

FIDRES

FIDR
$\mathrm{AQ}$

AQ

$\mathrm{DW}$

$\mathrm{DE}$

TE

S1g

TD 0

$===$

$\mathrm{SFO1}$

$\mathrm{NUC}$
$\mathrm{P} 1$

P1
PLW1

11.99499989 W

F2 - Processing parameters

SI 65536

SF $\quad 400.1300124 \mathrm{MH}$

WDW

SSB

LB

GB
PC

$0.30 \mathrm{~Hz}$

1.00

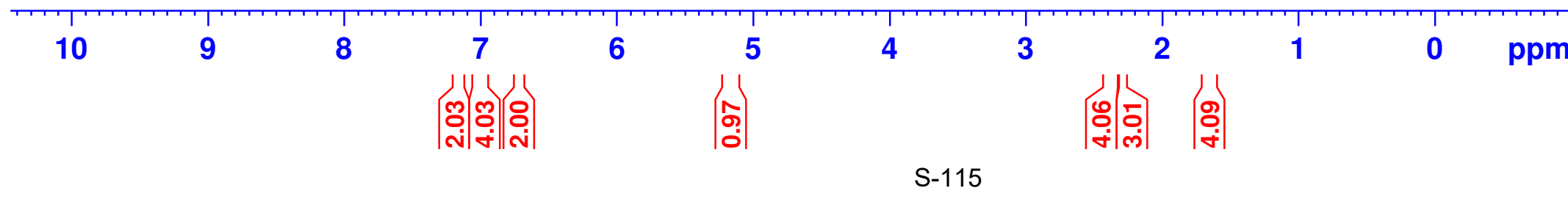




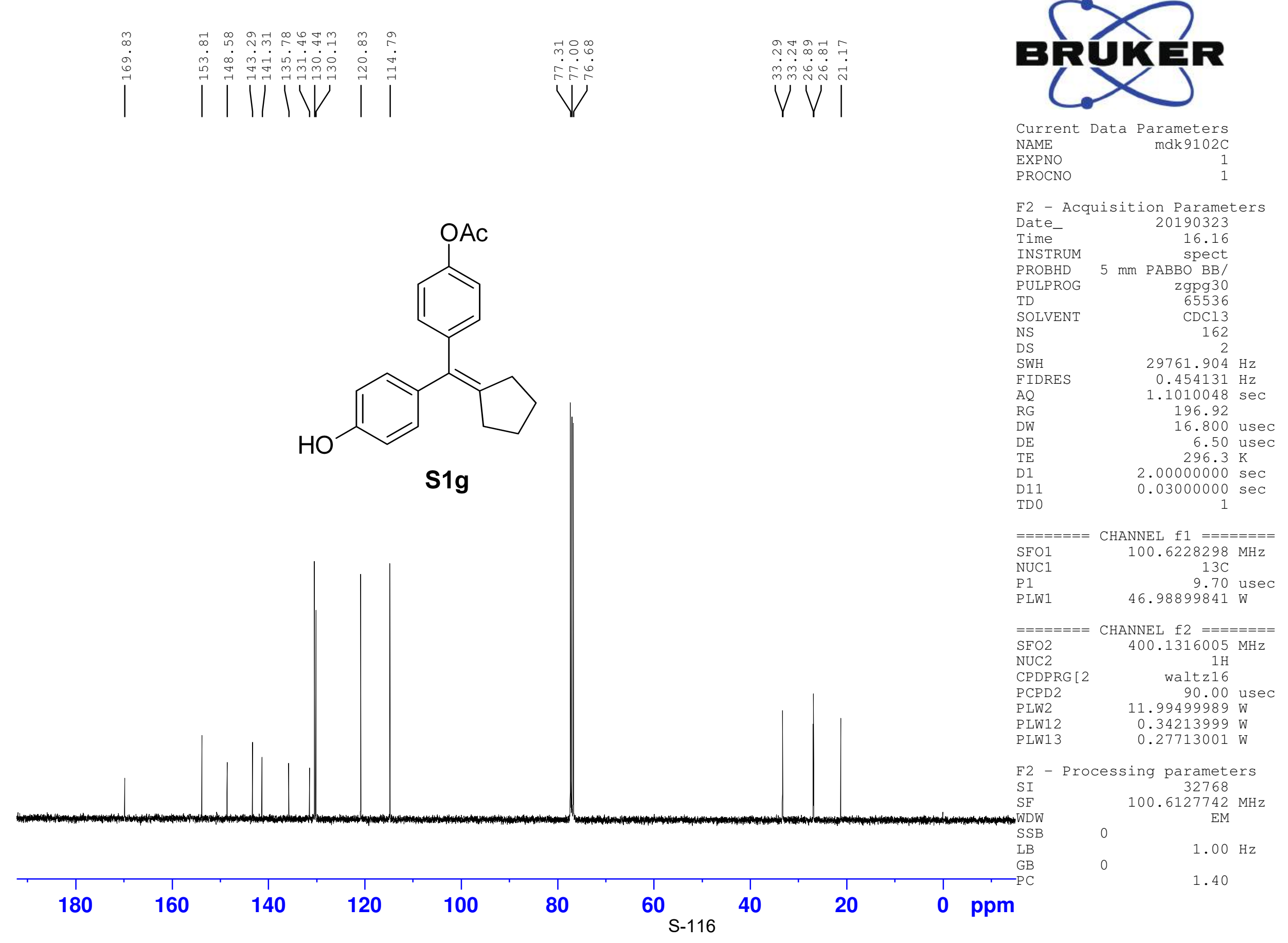




\section{Be $(1>$}

Current Data Parameters NAME

$\operatorname{mdk} 9170 \mathrm{H}$

$$
\begin{aligned}
& \text { EXPNO } \\
& \text { PROCNO }
\end{aligned}
$$

1
1
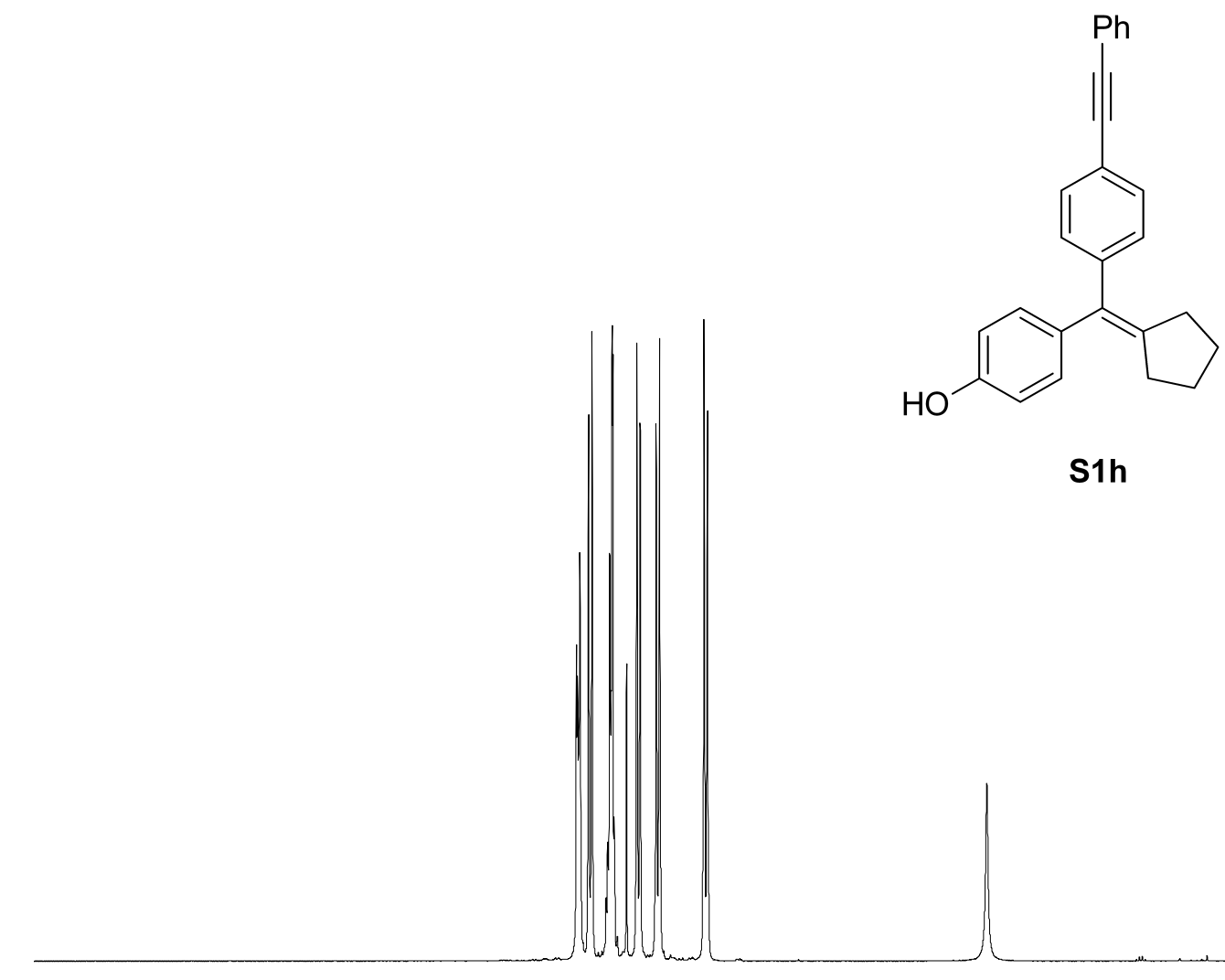

S1h

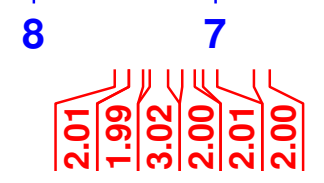

6

$\begin{aligned} & 5 \\ & \mid \\ & 0 \\ & 0\end{aligned} \mid$

4

3

$\left|\begin{array}{l}\mathscr{\gamma} \\ \hdashline \\ \dot{\gamma}\end{array}\right|$

2

$\left|\begin{array}{l}1 \\ 2 \\ 8 \\ 8\end{array}\right|$

F2 - Acquisition Parameters Date__ 20190416 Time 13.47 spect
S $5 \mathrm{~mm}$ PABBO
PUB
zULPRO
65536 TD $\quad 65536$ $\begin{array}{lr}\text { SOLVENT } & \text { CDC13 } \\ \text { NS } & 16\end{array}$ DS

$8012.820 \mathrm{~Hz}$
SWH $0.122266 \mathrm{~Hz}$ $\begin{array}{cr}\text { AQ } & 4.0894465 \\ \text { RG } & 49.32\end{array}$ $\begin{array}{lr}\text { RG } & 49.32 \\ \text { DW } & 62.400 \text { usec }\end{array}$ DE $\quad 6.50$ usec $\begin{array}{lr}T E & 297.6 \mathrm{~K} \\ \mathrm{D} 1 & 1.00000000 \mathrm{sec}\end{array}$ $\begin{array}{lr}\text { D1 } & 1.00000000 \\ \text { TD } & 1\end{array}$

$=======$ CHANNEL $\mathrm{fl} \quad========$ SFO1 $400.1324710 \mathrm{MHz}$ NUC1 $1 \mathrm{H}$ P1 14.50 usec PLW1 $11.99499989 \mathrm{~W}$

F2 - Processing parameters SI 65536 SF $\quad 400.1300246 \mathrm{MHz}$ WDW EM

$0.30 \mathrm{~Hz}$

GB

1.00 


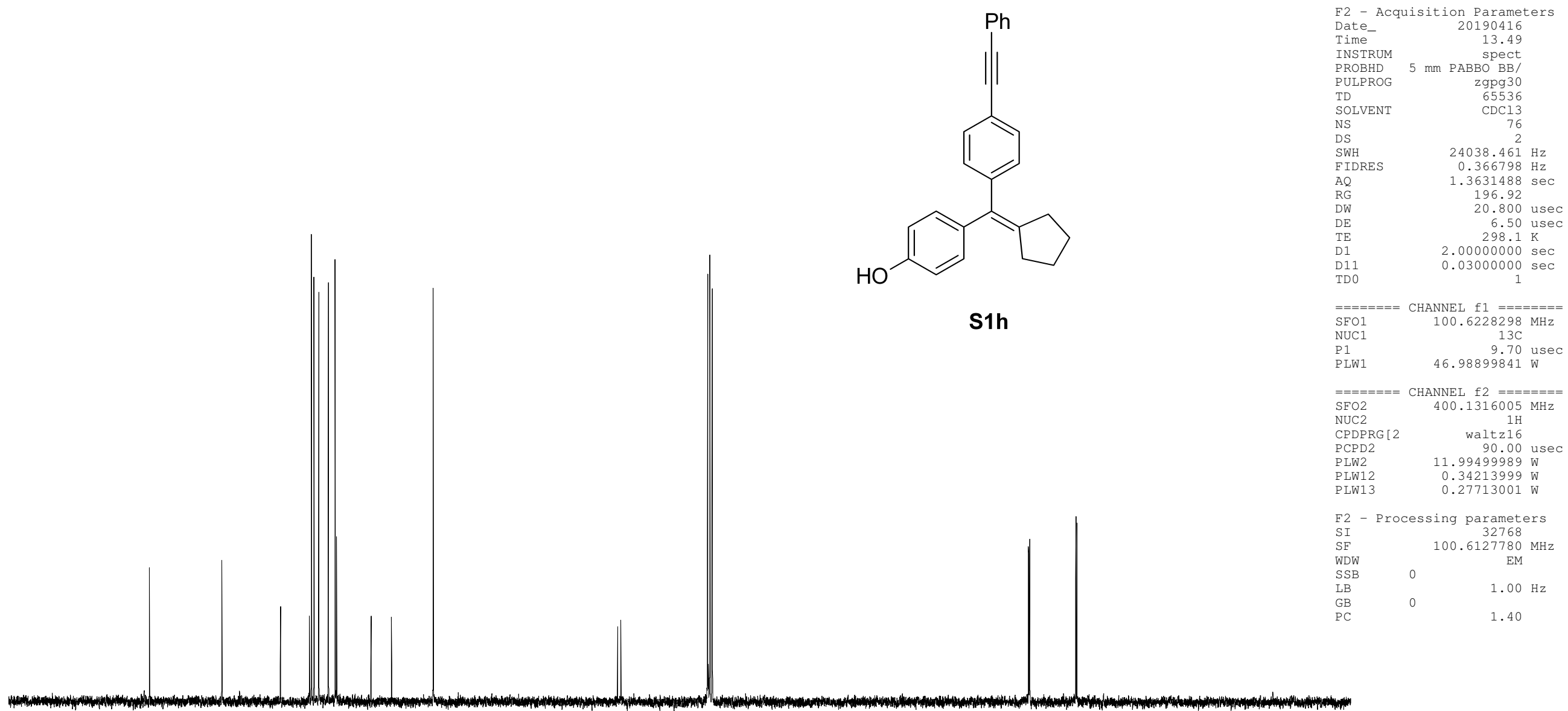

$\begin{array}{lr}\text { Current } & \text { Data } \\ \text { NAME } & \text { mameters } \\ \text { EXPNO } & \text { mdk9170C } \\ \text { PROCNO } & 1\end{array}$ 


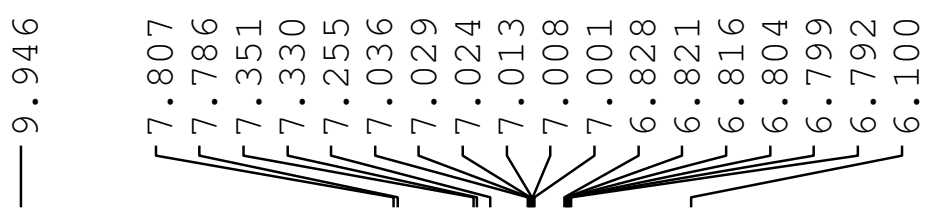

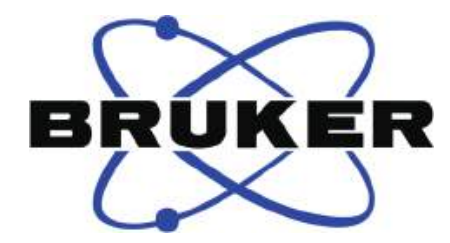

Current Data Parameters NAME

EXPNO

$\operatorname{mdk} 9173 \mathrm{H}$<smiles>O=Cc1ccc(C(=C2CCCC2)c2ccc(O)cc2)cc1</smiles>

S1i

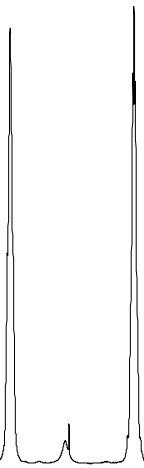

F2 - Acquisition Parameters Date_ 20190424

$\begin{array}{lr}\text { Time } & 13.23\end{array}$

INSTRUM spect

ROBHD

zg30

TD 65536

SOLVENT

NS

DS

FIDRES

AQ

RG

$\mathrm{DW}$
$\mathrm{DE}$
$\mathrm{TE}$

TE

TD 0

$====$
SFO1
NUC1

$\mathrm{NUC1}$
$\mathrm{P} 1$

PLW1

16
16

$8012.820 \mathrm{~Hz}$

$0.122266 \mathrm{~Hz}$

$4.0894465 \mathrm{sec}$ 27.78

62.400 usec 6.50 usec

$1.00000000 \mathrm{sec}$

CHANNEI $f 1=======$ $400.1324710 \mathrm{MHz}$ 14.50 $11.99499989 \mathrm{~W}$

F2 - Processing parameters SI 65536 SF $\quad 400.1300120 \mathrm{MHz}$ WDW

SSB EM

$0.30 \mathrm{~Hz}$

1.00

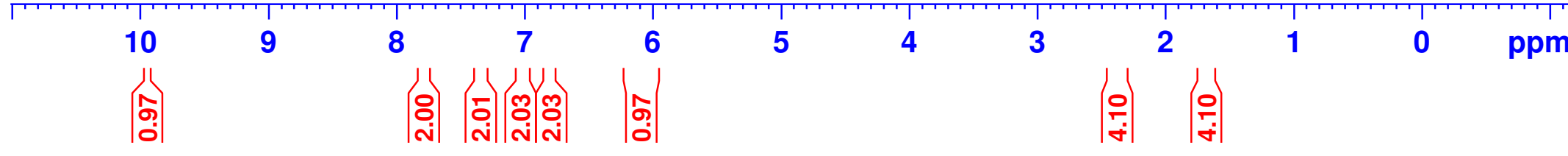




\section{apopien}<smiles>O=Cc1ccc(C(=C2CCCC2)c2ccc(O)cc2)cc1</smiles>

S1i 


\section{ar KXen $(1)$}

Current Data Parameters NAME

PROCNO

mak9032

F2 - Acquisition Parameters

Date__ 20190305

$\begin{array}{lr}\text { Time } & 19.10\end{array}$

INSTRUM spect

PULPROG

$\mathrm{Zg} 30$
65536

TD $\quad 65536$

SOLVENT

NS

DS

FIDRES

$\mathrm{AQ}$

RG

$\mathrm{DW}$
$\mathrm{DE}$
$\mathrm{TE}$

D1
TD 0

S1j

$===$

$\mathrm{SFO1}$

NUC

P1
PLW1

F2 - Processing parameters

SI $\quad 65536$

$\begin{array}{ll}\text { SF } & 400.1300172 \mathrm{MHz}\end{array}$

SSB

LB

GB

PC

$0.30 \mathrm{~Hz}$

1.00

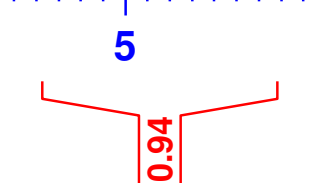

4

ำต

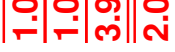

3

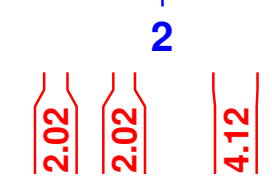




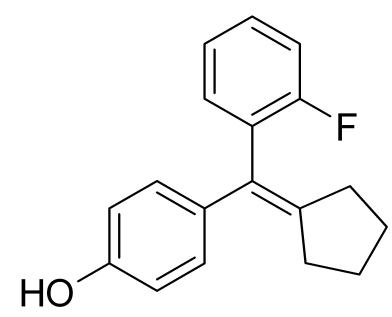

S1j

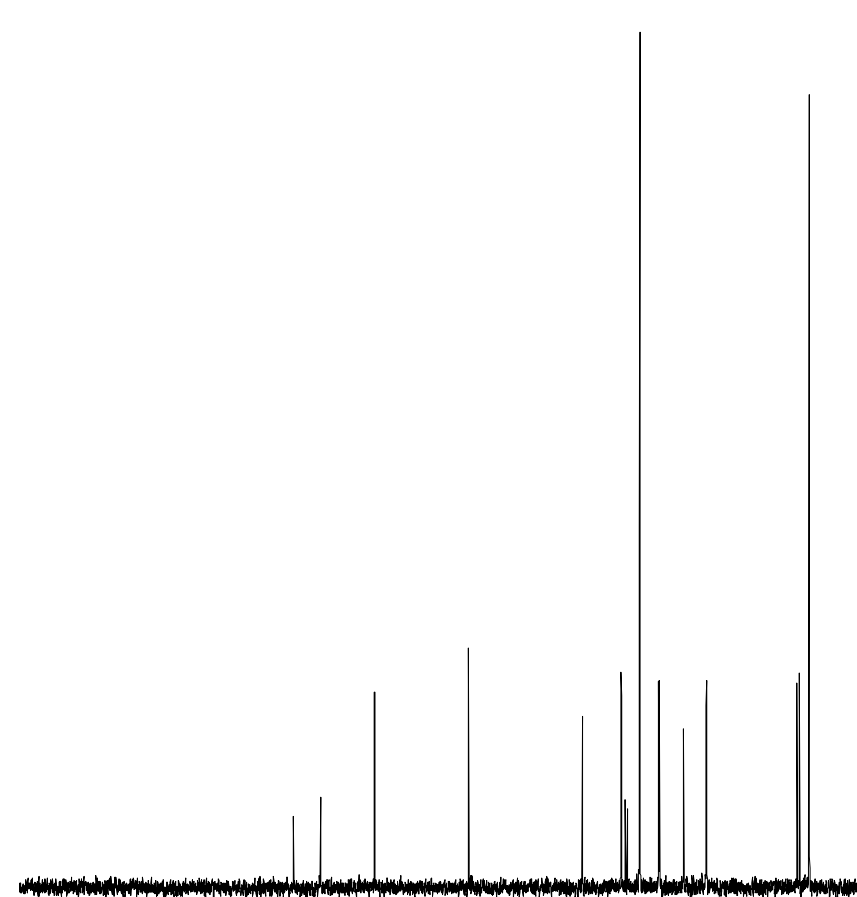

Current Data Parameters
mdk9032C EXPNO
PROCNO

F2 - Acquisition Parameters

Date

$\begin{array}{lr}\text { Time } & 19.14 \\ \text { INSTRUM } & \text { spect } \\ \text { PROBHD } & 5 \mathrm{~mm} \text { PABBO BB/ }\end{array}$

PULPRO

TD SOLVENT

NS
DS

SWH

$A Q$
$R G$

$R G$
$D W$
$D E$
$T E$
$D 1$

TE
D1
D11

D11

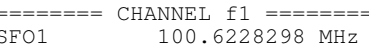

SFO1 $\quad 100.6228298 \mathrm{MHz}$

P1

6.98899841 WT

$\begin{array}{lc}======== & \text { CHANNEL } \mathrm{f} 2 \quad======= \\ \text { SFO2 } & 400.1316005 \mathrm{MHz} \\ \text { NUC2 } & 1 \mathrm{H} \\ \text { CPDPRG } & \text { waltz16 } \\ \text { PCPD2 } & 90.00 \text { usec } \\ \text { PLW2 } & 11.99499989 \mathrm{~W} \\ \text { PLW12 } & 0.34213999 \mathrm{~W} \\ \text { PLW13 } & 0.27713001\end{array}$

$\begin{array}{lr}\text { PLW2 } & 11.99499989 \mathrm{~W} \\ \text { PLW2 } & 0.34213999 \mathrm{~W}\end{array}$

F2 - Processing parameters

F2 - Processing parameters

$\begin{array}{lc}\text { SF } & 100.6127744 \text { MHZ } \\ \text { WDW } & \text { EM }\end{array}$

GB 


\section{equiren}<smiles>Oc1ccc(C(=C2CCCC2)c2ccccc2F)cc1</smiles>

Current Data Parameters
NAME mdk9032F-decoup EROCNO

F2 - Acquisition Parameters
Date- 0190305

Date_ 20190305

TimsTRUM

spect
PROBHD $5 \mathrm{~mm}$ PABBO BB/

PULPRO

SOLVEN

NS

$\begin{array}{ll}2 & 2 \\ \text { SWH } & 93750.000 \mathrm{~Hz}\end{array}$

$\begin{array}{ll}\text { AQ } & 1.430511 \mathrm{~Hz} \\ \mathrm{BG} & 0.3495253 \mathrm{sec}\end{array}$

196.92
5.333

5.333 usec
6.50 usec

$.00000000 \mathrm{sec}$

$0.03000000 \mathrm{sec}$

TDO

$z======$ CHANNEL $f 1=======$
SFO1
$376.4607162 \mathrm{MHz}$

NUC1 $19 \mathrm{~F}$

P1 14.70 usec
PLW1

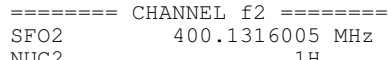

NUC2 $1 \mathrm{H}$

$\begin{array}{lr}\text { CPDPRG [2 } & \text { waltz16 } \\ \text { PCPD2 } & 90.00 \text { usec }\end{array}$

$\begin{array}{lr}\text { PLW2 } & 11.99499989 \mathrm{~W} \\ \text { PLW12 } & 0.34213999 \mathrm{~W}\end{array}$

P.W13

F2 - Processing parameters
SI
32768

$\begin{array}{lc}\text { SI } & 32768 \\ \text { SF } & 376.4983660 \\ \text { WDW } & \text { MHz }\end{array}$

WDW

GB

$1.00 \mathrm{~Hz}$

\begin{tabular}{|c|c|c|c|c|c|c|c|c|c|c|c|}
\hline 20 & 0 & $\begin{array}{c}1 \\
-20\end{array}$ & $\begin{array}{c}1 \\
-40\end{array}$ & $\begin{array}{r}1 \\
-60\end{array}$ & $\begin{array}{l}1 \\
-80\end{array}$ & $\begin{array}{r}1 \\
-100\end{array}$ & $\begin{array}{r}1 \\
-120\end{array}$ & $\begin{array}{l}\text { 1 } \\
-140\end{array}$ & $\begin{array}{r}1 \\
-160\end{array}$ & $\begin{array}{r}1 \\
-180\end{array}$ & $\begin{array}{l}1 \\
-200\end{array}$ \\
\hline 20 & 0 & & & -00 & & & $5-123$ & -140 & -100 & -100 & $-\angle O 0$ \\
\hline
\end{tabular}



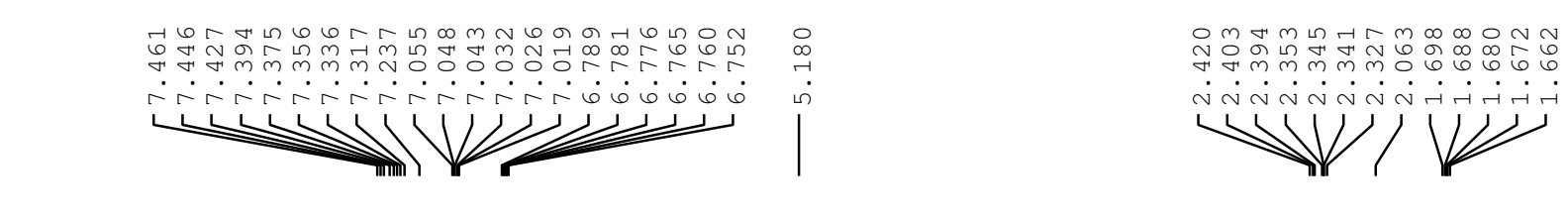

BR UKKER $(>)$

Current Data Parameters NAME EXPNO

ROCNO

F2 - Acquisition Parameters

Date__ 20190306

$\begin{array}{ll}\text { Time } & 22.19 \\ \text { INSTRUM } & \text { spect }\end{array}$

spect
PROBHD $5 \mathrm{~mm} \mathrm{PABBO} \mathrm{BB} /$

PROBHD $5 \mathrm{~mm}$ PABBO $\mathrm{BB} /$
PULPROG
$\mathrm{zg} 30$

$\begin{array}{lr}\text { PULPROG } & \text { zg30 } \\ \text { TD } & 65536\end{array}$<smiles>Oc1ccc(C(=C2CCCC2)c2cccc(C(F)(F)F)c2)cc1</smiles>

NS

DS

SWH
FIDRES

AQ

$\mathrm{AQ}$
$\mathrm{RG}$

DW

$\mathrm{DE}$

TE

TD0

$$
\begin{array}{r}
\mathrm{CDC} 13 \\
16
\end{array}
$$

$8012.820 \mathrm{~Hz}$

$0.122266 \mathrm{~Hz}$

$4.0894465 \mathrm{sec}$

$$
62.400 \text { usec }
$$

6.50 usec

$296.4 \mathrm{~K}$

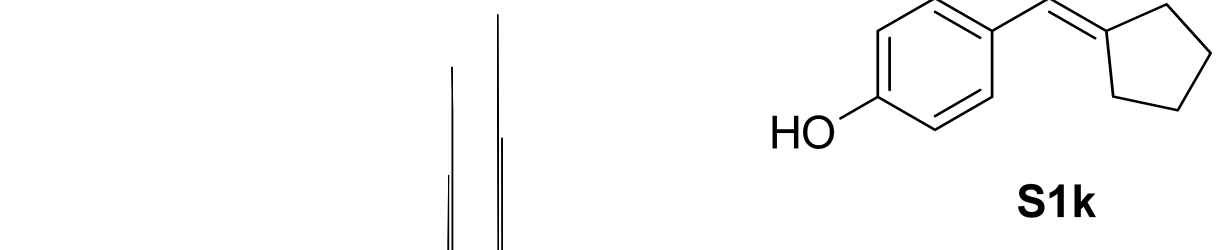

Ethyl A cetate$$
===
$$

$1.00000000 \mathrm{sec}$

$====$
SFO1
NUC1

$\mathrm{NUC1}$

1

$1 \mathrm{H}$

PLW1 $11.99499989 \mathrm{~W}$

F2 - Processing parameters

$\begin{array}{lc}\text { SI } & 65536 \\ \text { SF } & 400.1300193 \mathrm{MHz}\end{array}$

WDW

SSB

GB

0

$30 \mathrm{~Hz}$

Ethyl A cetate

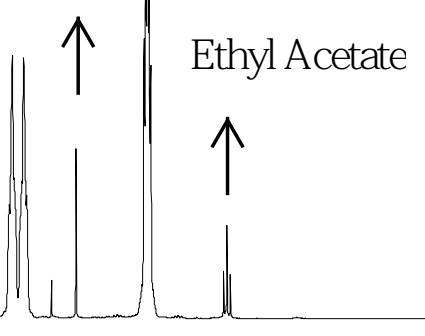

1.00

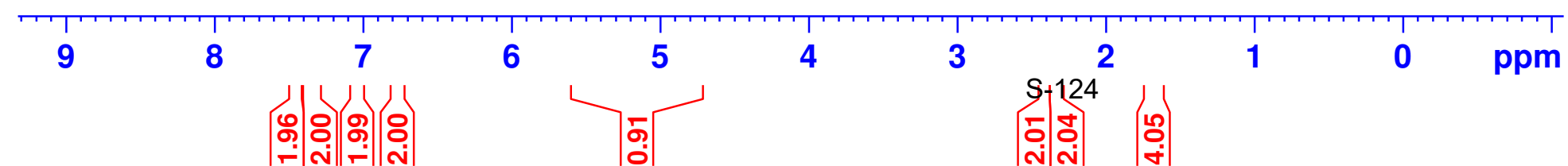




\section{eprieren}

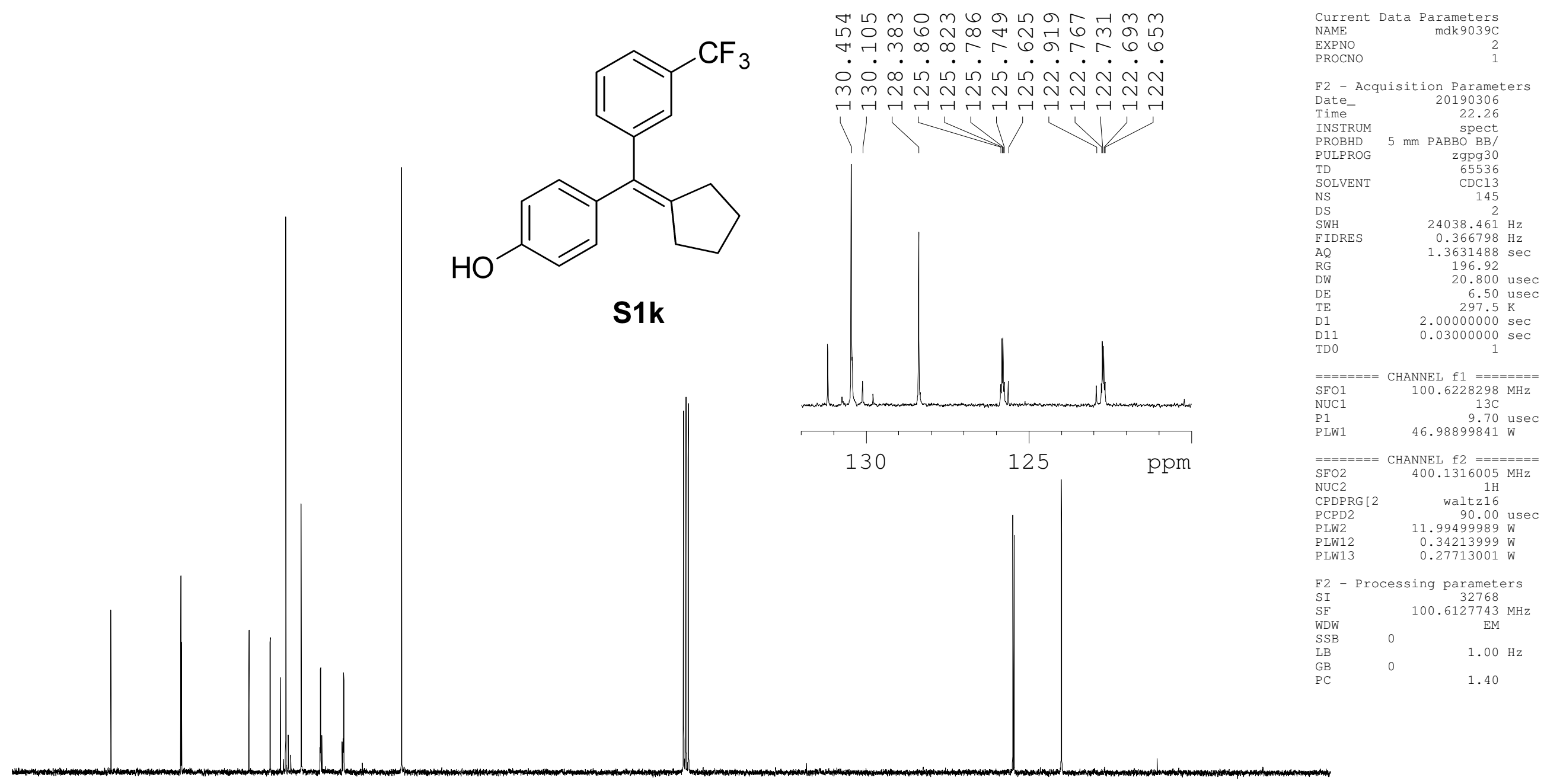
160150
$140 \quad 130$
$\begin{array}{lll}120 & 110 \quad 100\end{array}$
90
$80 \quad 70$
$70 \quad 60$
50
$\begin{array}{lll}40 & 30 & 20\end{array}$
10
ppm 


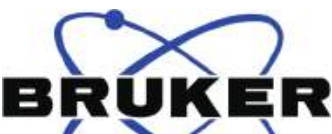

Current Data Parameters
NAME mdk9039F-decoup

PROCNO

F2 - Acquisition Parameters
Date_ 20190306

Date_ 20190306

Time

spect
PROBHD $5 \mathrm{~mm}$ PABBO BB/

PULPRO

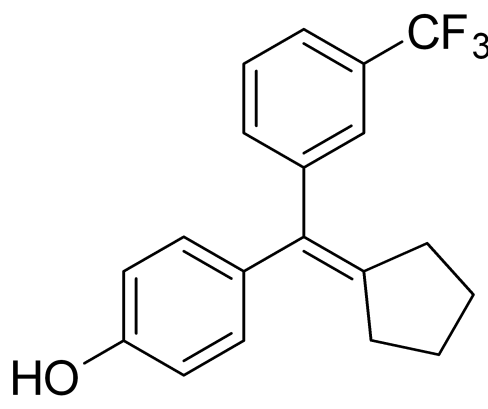

SOIVEN

NS
DS

$\begin{array}{ll}2 & 2 \\ \text { SWH } & 93750.000 \mathrm{~Hz}\end{array}$

$\begin{array}{ll}\text { AQ } & 1.430511 \mathrm{~Hz} \\ \mathrm{RG} & 0.3495253 \mathrm{sec}\end{array}$

196.92
5.333
$6.50 \mathrm{usec}$

6.50 usec
$295.5 \mathrm{~K}$

$\begin{array}{lr}\text { D1 } & 2.00000000 \mathrm{sec} \\ \text { D11 } & 0.03000000 \mathrm{sec} \\ \text { TD0 } & 1\end{array}$

$z======$ CHANNEL $f 1 \quad=======$
SFO1 $\quad 376.4607162 \mathrm{MHz}$

NEC1 $\quad 19 \mathrm{~F}$

S1k

$5.99600029 \mathrm{~W}$

$=======8$ CHANNEL $\mathrm{f} 2 \quad========$
SFO2
S00.1316005 MHz

$\begin{array}{lr}\text { NUC2 } & 1 \mathrm{H} \\ \text { CPDPRG } 2 & \text { waltz16 }\end{array}$

$\begin{array}{lr}\text { PCPD2 } & 90.00 \text { usec }\end{array}$

$\begin{array}{lr}\text { PLW2 } & 11.99499989 \mathrm{~W} \\ \text { PLW12 } & 0.34213999 \mathrm{~W}\end{array}$

PIn13

F2 - Processing parameters
SI

$\begin{array}{ll}\text { SF } & 32768 \\ \text { SF } & \end{array}$

$1.00 \mathrm{~Hz}$

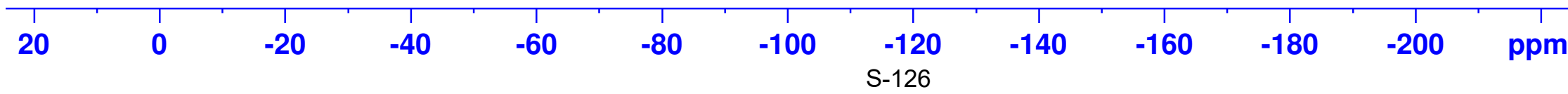




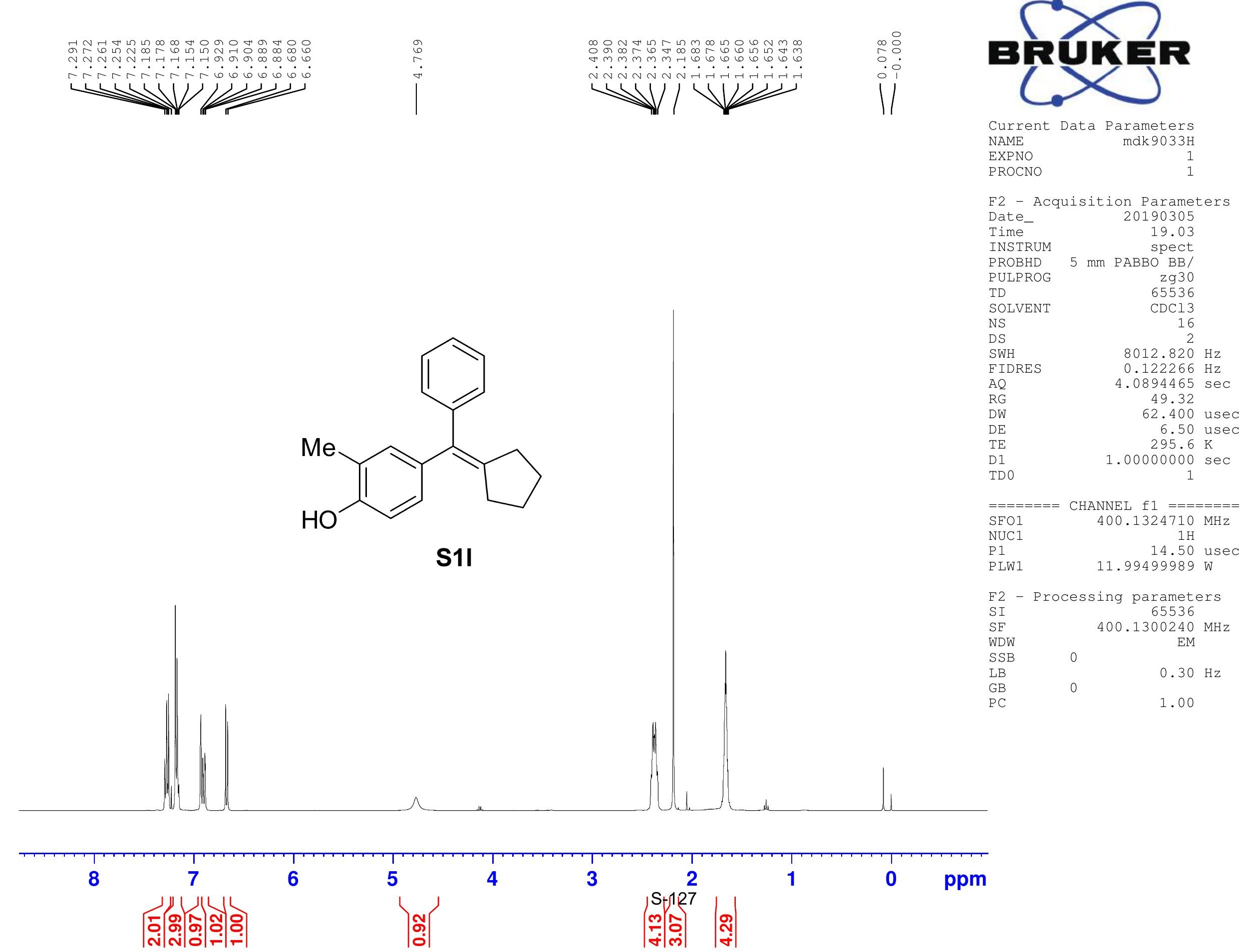




\section{BRUKER}<smiles>Cc1cc(C(=C2CCCC2)c2ccccc2)ccc1O</smiles>
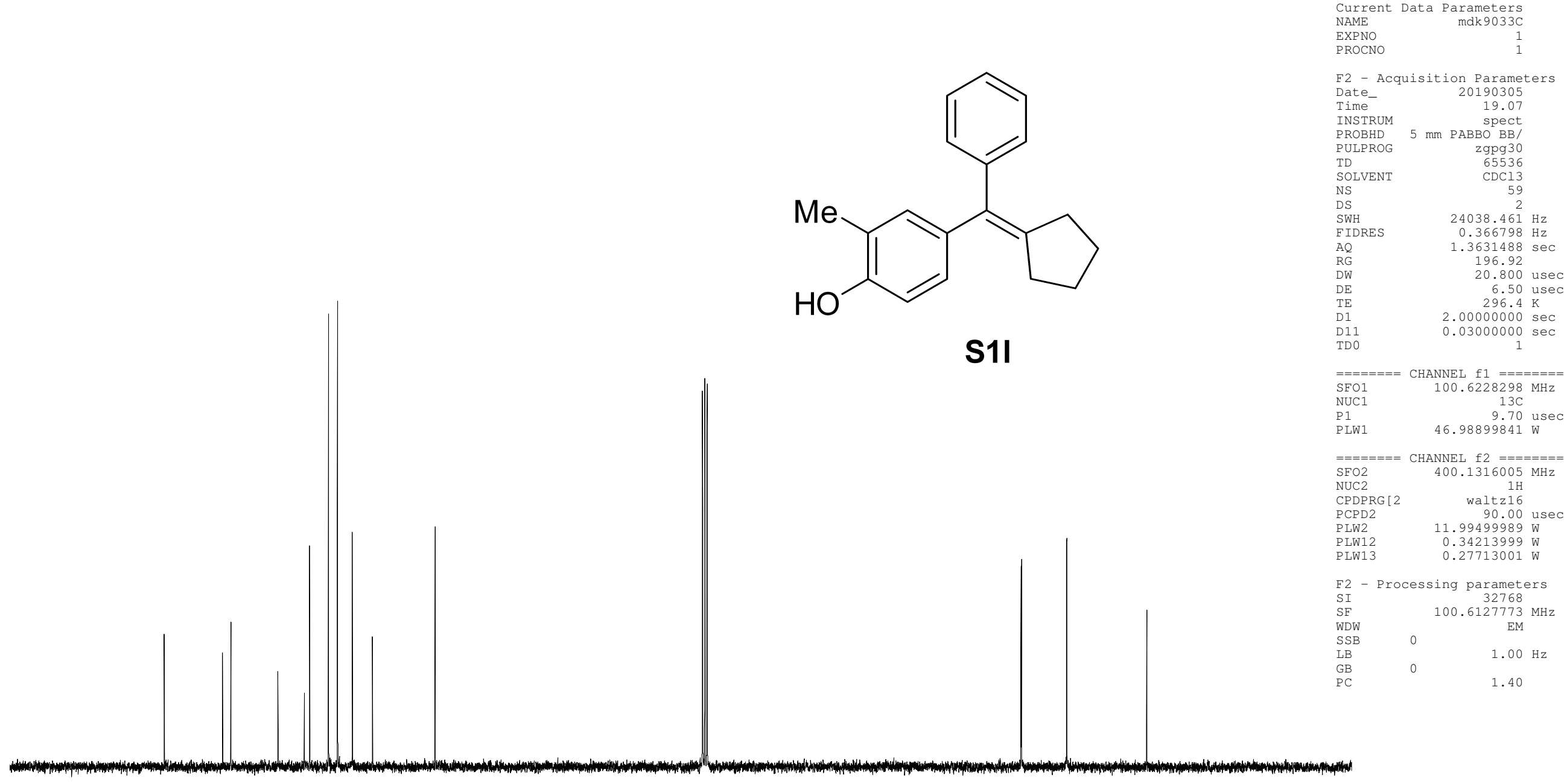

\section{S1I}


BR UKER $C<$

Current Data Parameters NAME EXPNO

F2 - Acquisition Parameters

Date_ 20190306

Time $\quad 9.44$

INSTRUM spect

PROBHD $5 \mathrm{~mm}$ PABBO BB-

PULPROG $\quad \mathrm{zg} 30$

TD $\quad 65536$

SOLVENT CDCl3

NS

$\begin{array}{lr}\text { DS } & 2 \\ \text { SWH } & 8223.685 \mathrm{~Hz}\end{array}$

FIDRES $\quad 0.125483 \mathrm{~Hz}$

$\mathrm{AQ} \quad 3.9845889 \mathrm{sec}$

RG

DW

$\mathrm{DE}$

TE

TDO

60.800 usec

6.00 usec

$295.2 \mathrm{~K}$

$1.00000000 \mathrm{sec}$

$========$ CHANNEL $\mathrm{f} 1$

NUC1

P1

PL1

PL1W

$\mathrm{f} 1=$

\section{S1m}

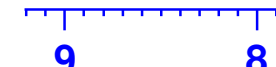

8

7

$\sin 12$ مุت

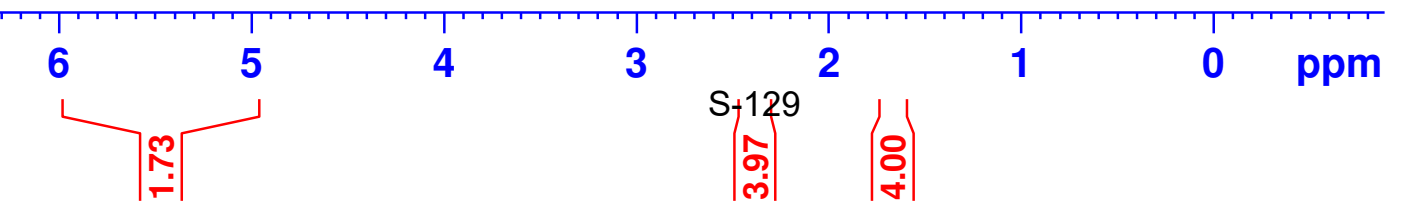




\section{BRUKER $(3)$}
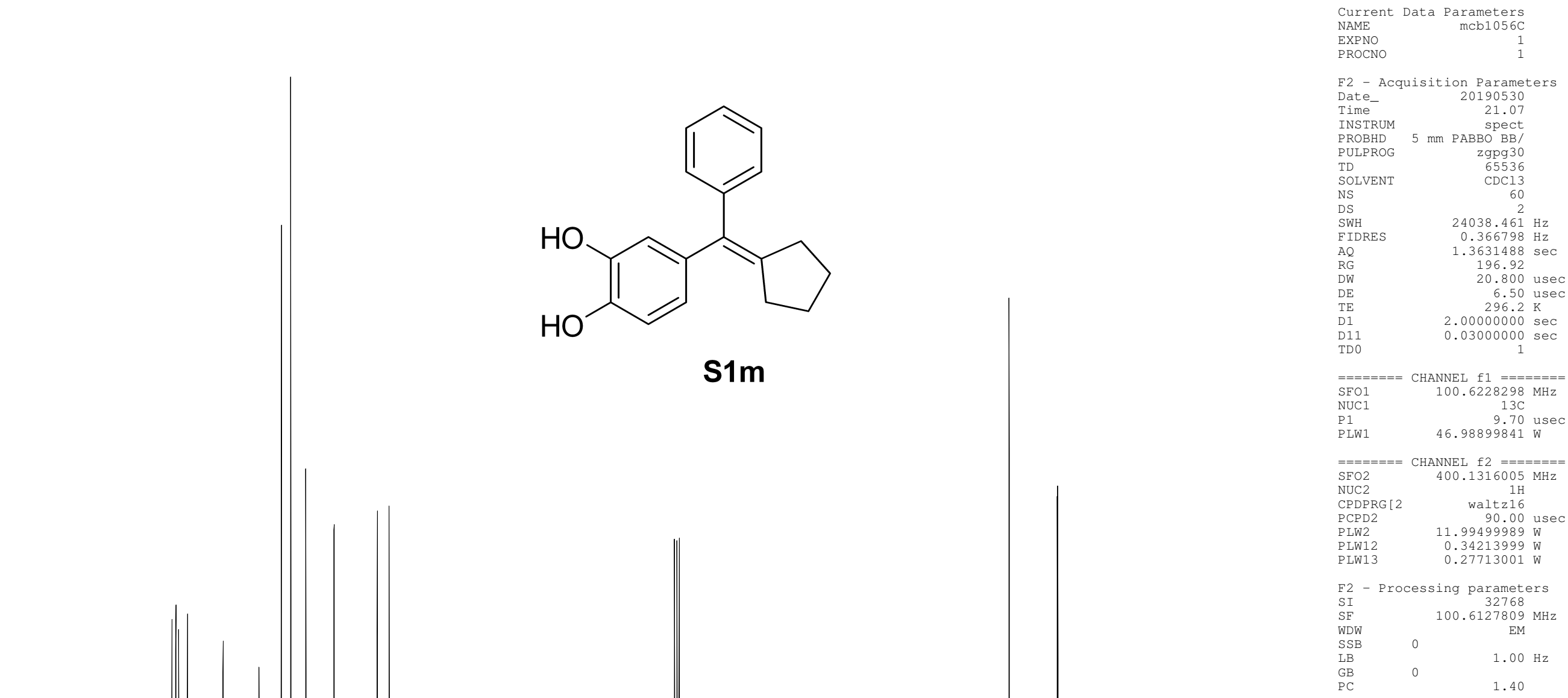

$\begin{array}{llllllllllllllllll}160 & 150 & 140 & 130 & 120 & 110 & 100 & 90 & 80 & 70 & 60 & 50 & 40 & 30 & 20 & 10 & 0 & \text { ppm }\end{array}$



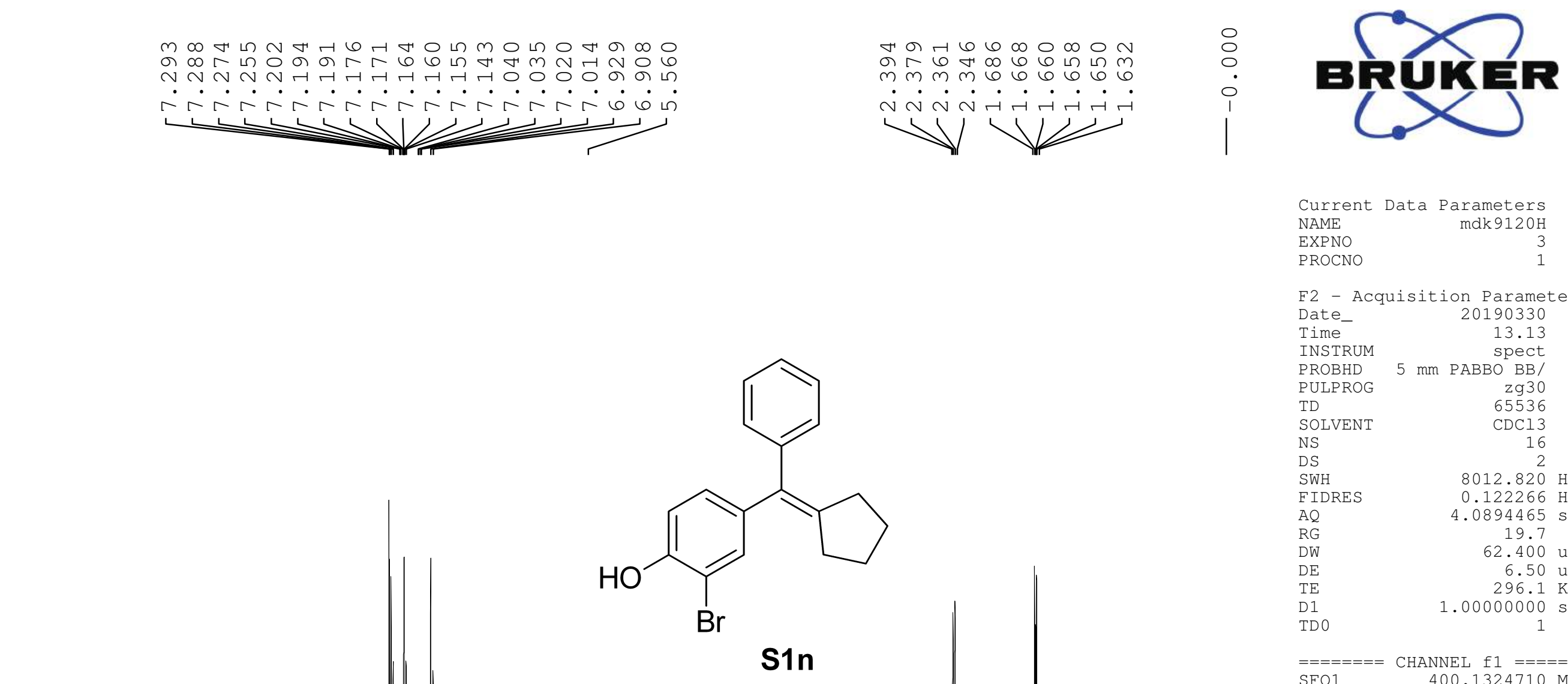

F2 - Acquisition Parameters

Date___ 20190330

Time 13.13

$5 \mathrm{~mm}$ spect

zg30

$\begin{array}{ll}\text { TD } & 65536 \\ \text { SOLVENT } & \text { CDC13 }\end{array}$

NS

SWH

FIDRES

$\mathrm{AQ}$

RG

DW

DE

D1
TD 0

$====$

$\mathrm{SFO}$

NUC1

16
16

$8012.820 \mathrm{~Hz}$ $0.122266 \mathrm{~Hz}$ $4.0894465 \mathrm{sec}$ 19.7

62.400 usec 6.50 usec $1.00000000 \mathrm{sec}$

CHANNEL $\mathrm{f} 1$ $400.1324710 \mathrm{MHz}$ LW1 11.50 us

F2 - Processing paraneters

I Processing parameter.

SF $\quad 400.1300331 \mathrm{MH}$

$\begin{array}{lr}\text { SF } & 400.1300331 \\ \text { WDW } & \text { EM }\end{array}$
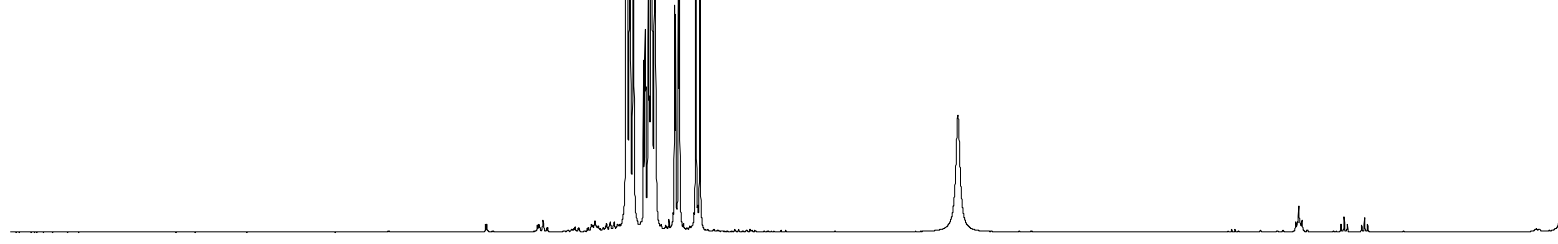

SSB

$0.30 \mathrm{~Hz}$

GB

1.00

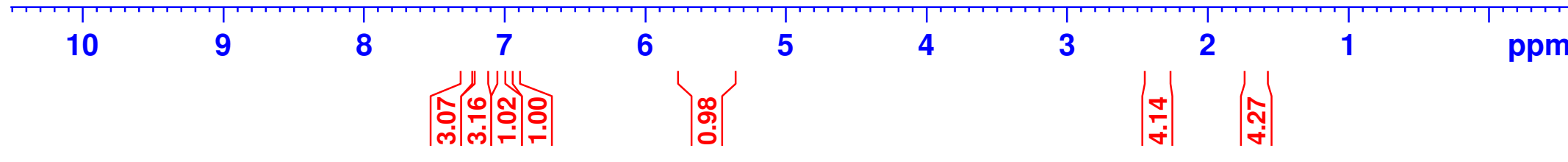



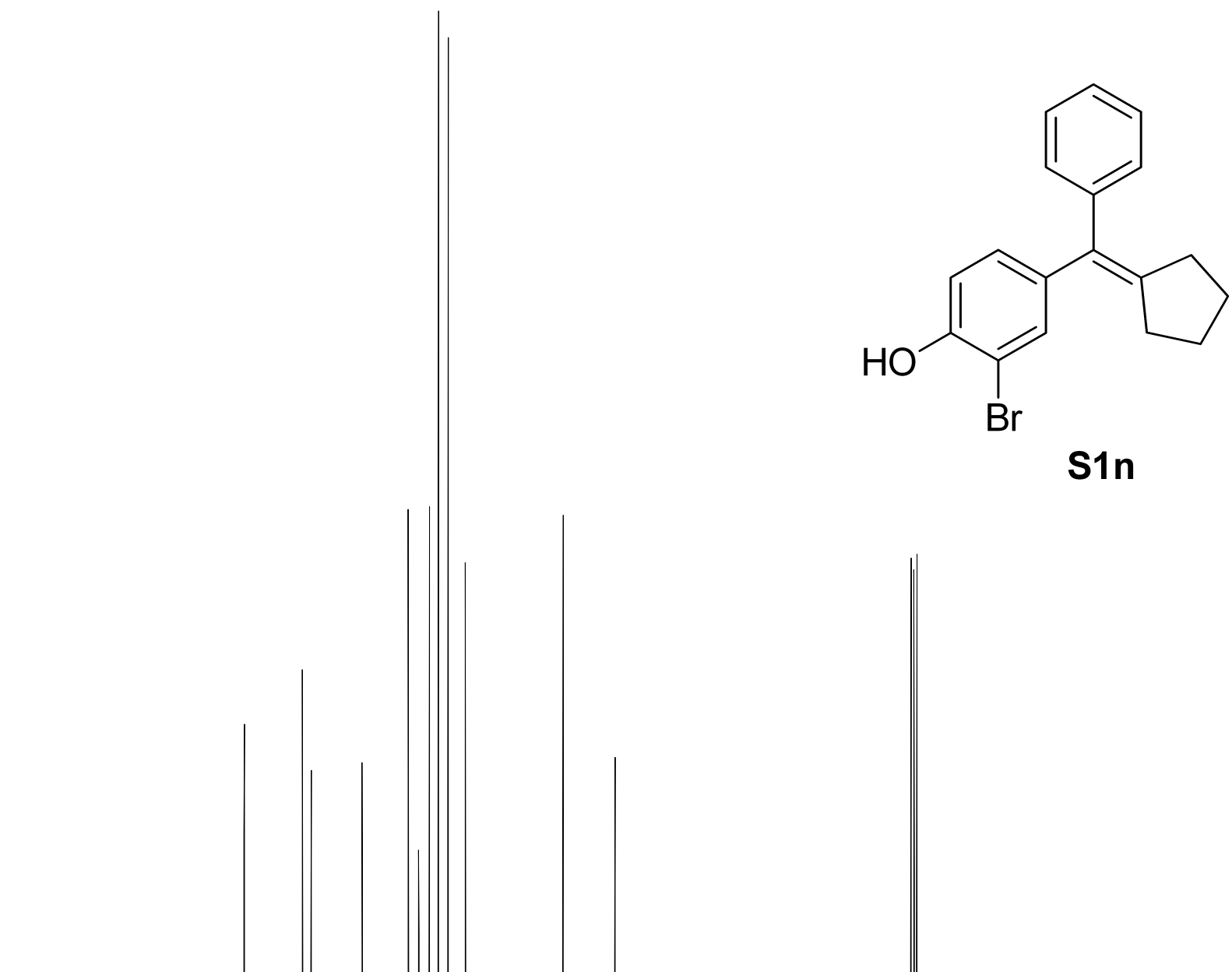

\section{S1n}

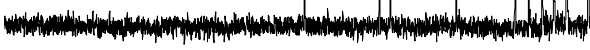

$\begin{array}{lllllllllllllllllll}170 & 160 & 150 & 140 & 130 & 120 & 110 & 100 & 90 & 80 & 70 & 60 & 50 & 40 & 30 & 20 & 10 & 0 & \mathrm{spm}\end{array}$

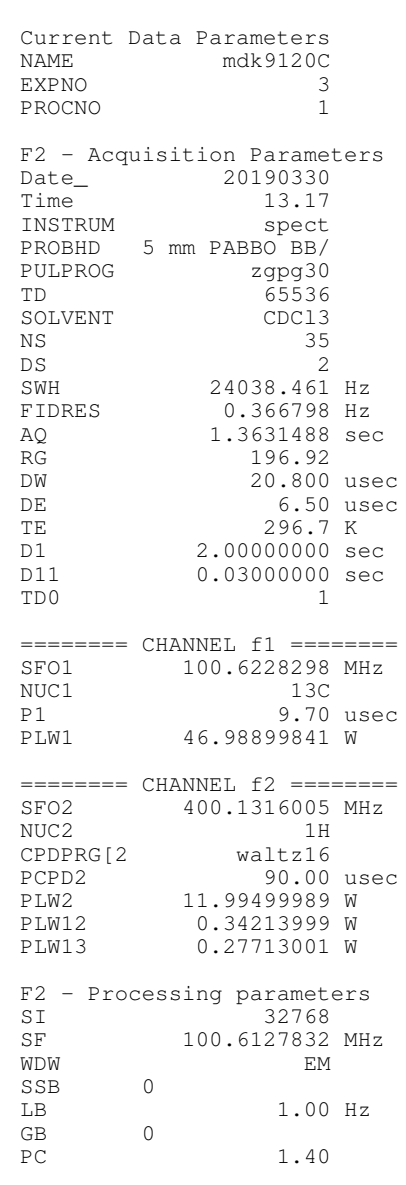




\section{=

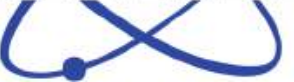

Current Data Parameters NAME

mdk9118H

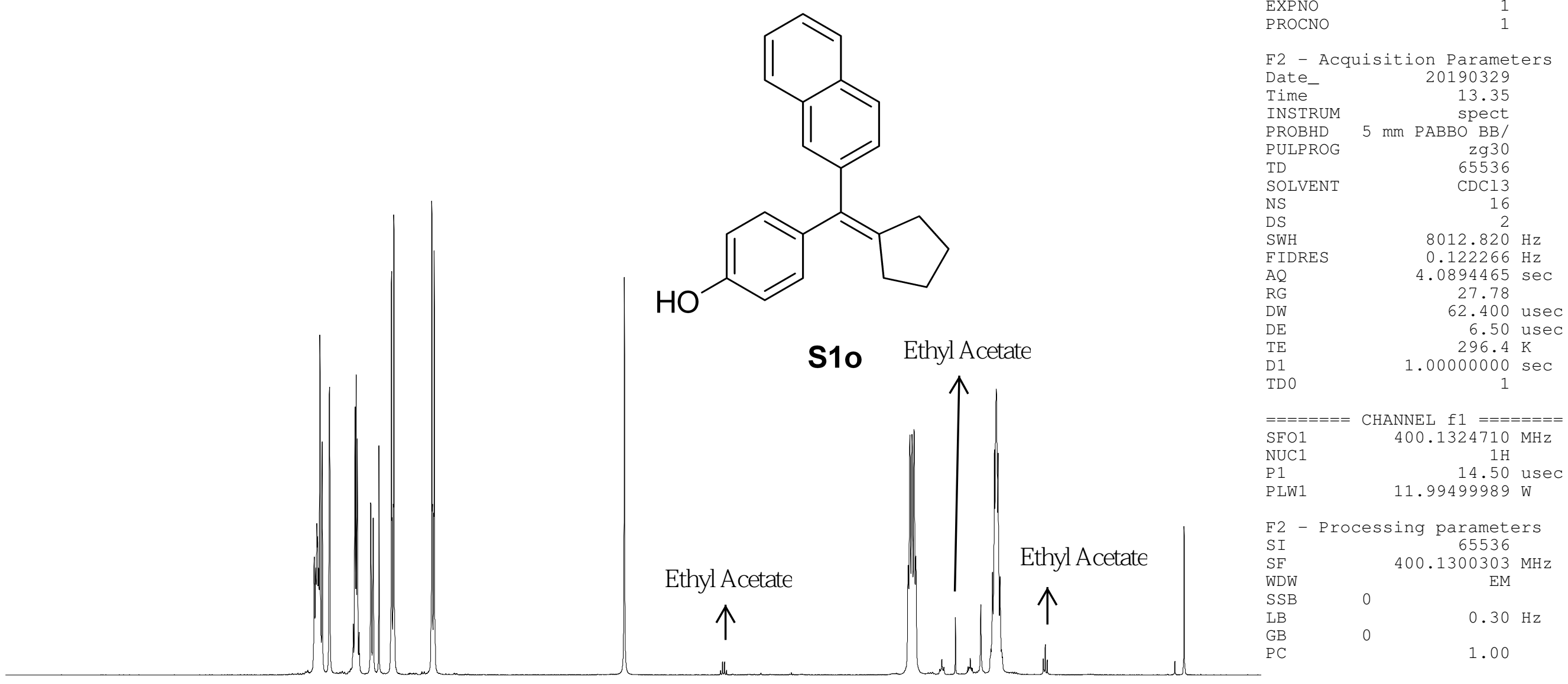

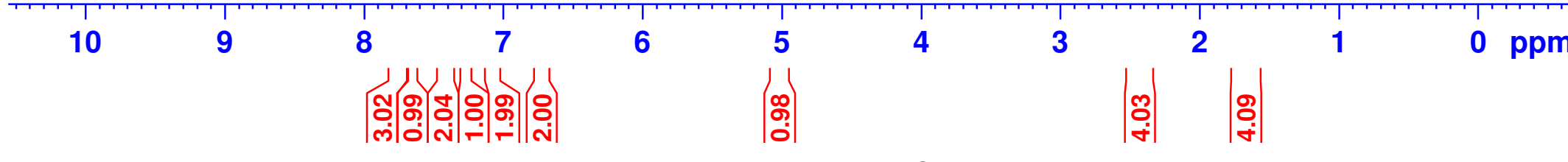



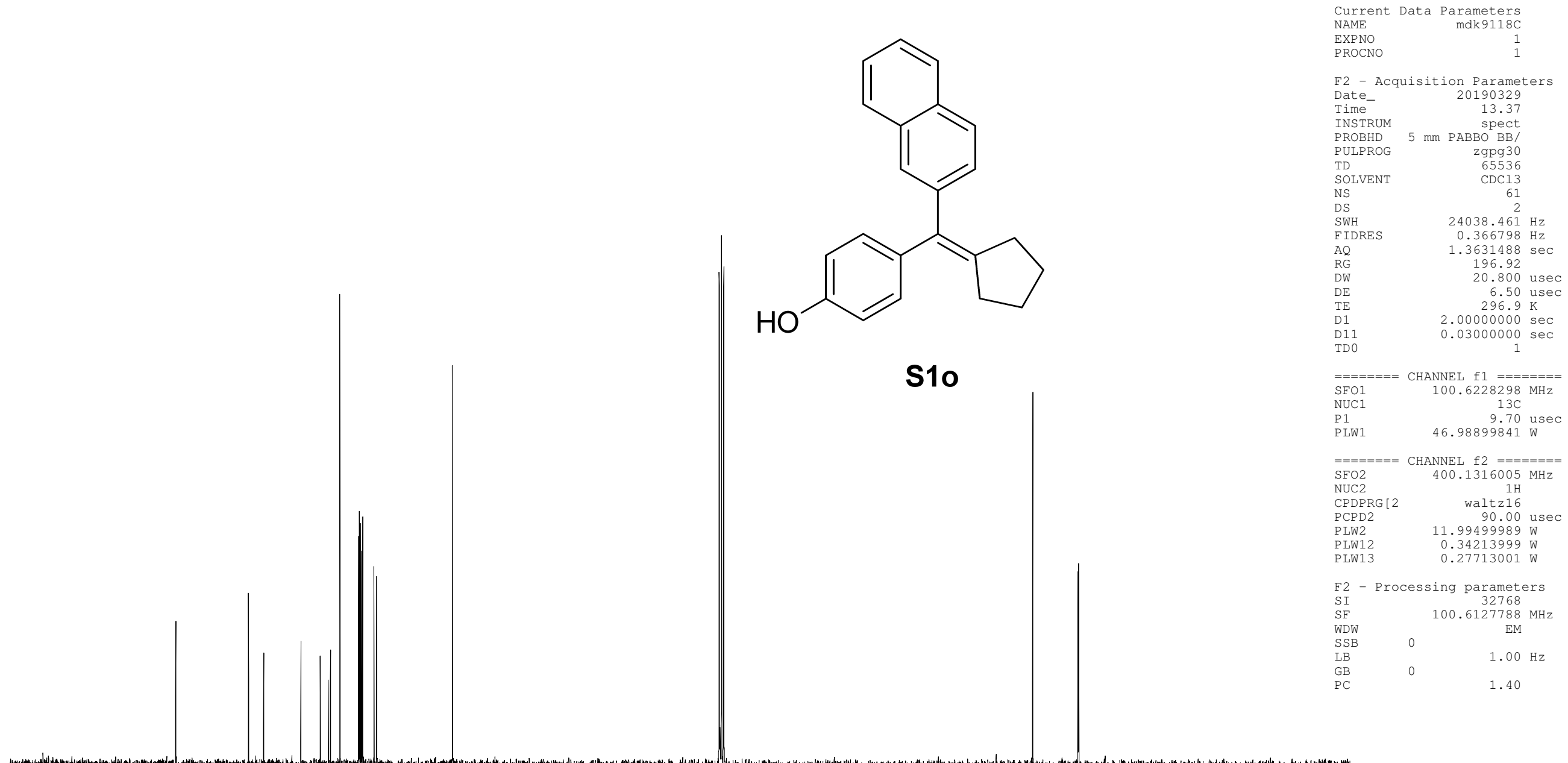

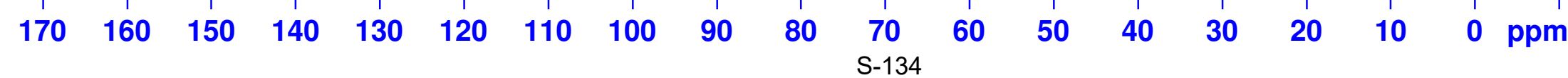




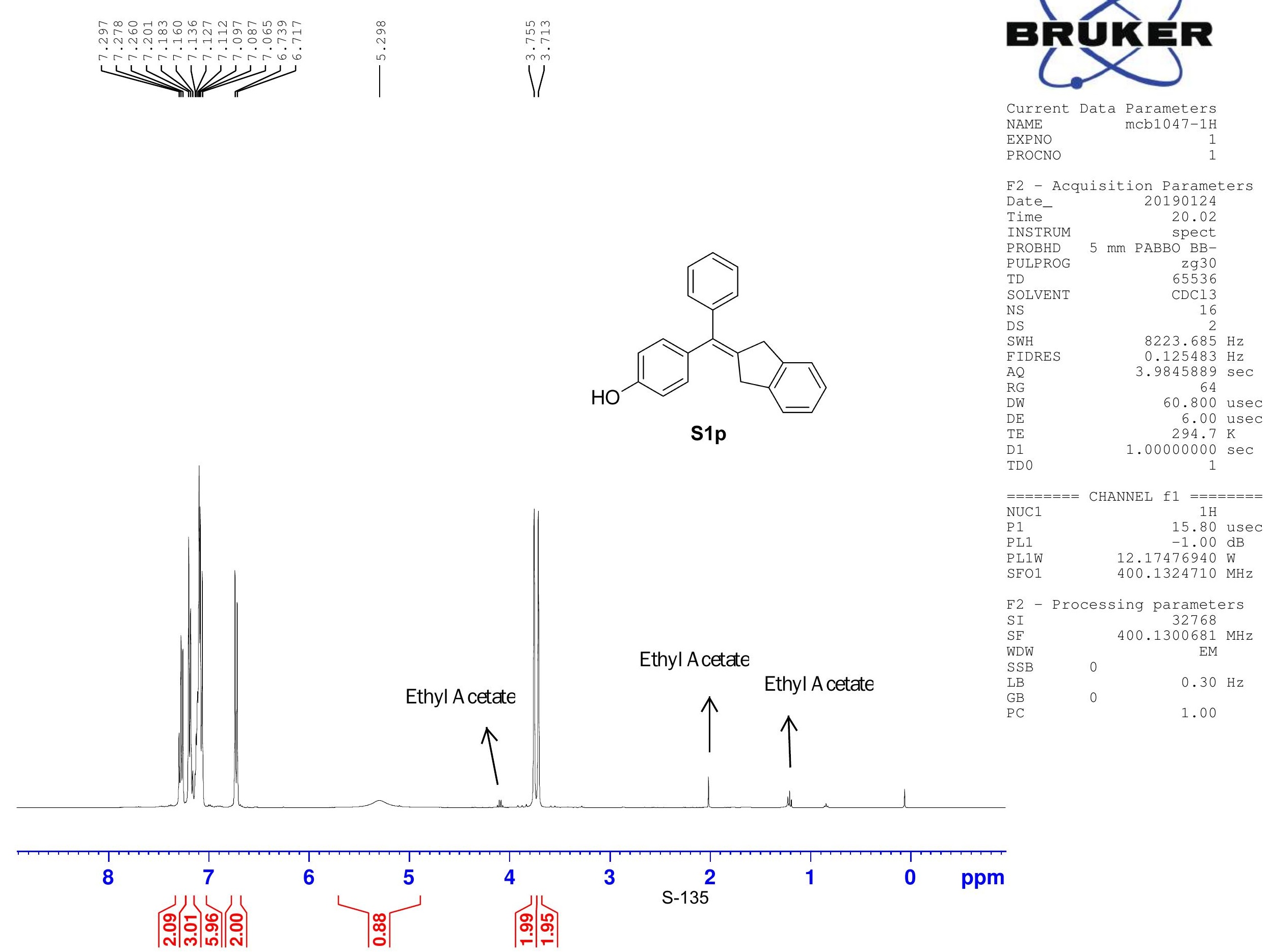




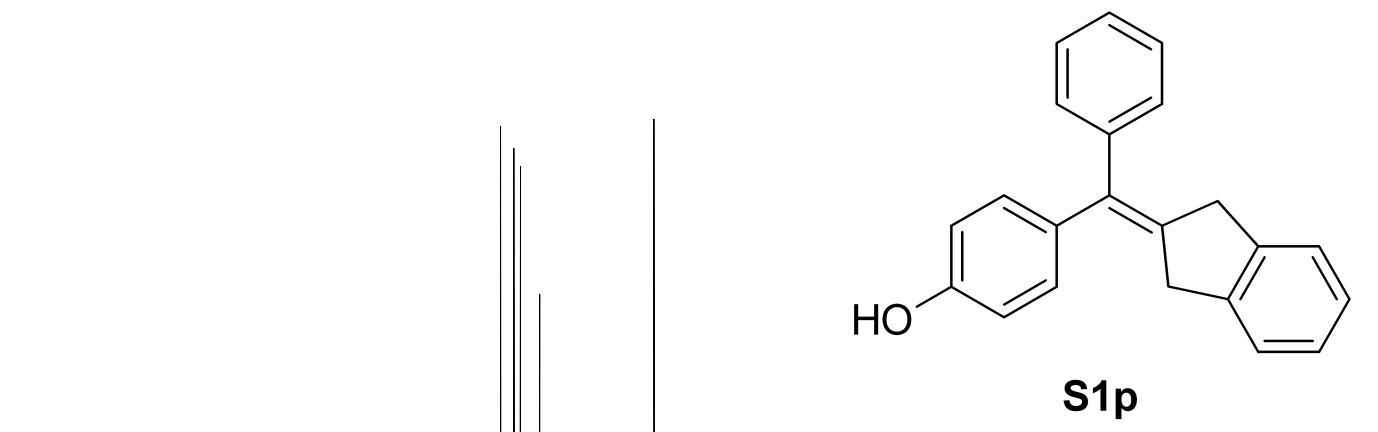

\section{$C$}

Current Data Parameters

NAME

EXPNO

PROCNO

mcb1047-1c

F2 - Acquisition Parameters

Date_ 20190124

Time 20.09

PROBHD $5 \mathrm{~mm}$ PABBO BB-

PULPROG zgpg30

TD 65536

SOLVENT CDCl3

NS

SWH $\quad 24038.461 \mathrm{~Hz}$

FIDRES $\quad 0.366798 \mathrm{~Hz}$

$\mathrm{AQ} \quad 1.3631488 \mathrm{sec}$

RG

20.800 use

DE $\quad 6.00$ usec

TE $\quad 295.3 \mathrm{~K}$

$\begin{array}{ll}\text { D1 } & 2.00000000 \mathrm{sec} \\ \text { D11 } & 0.03000000 \mathrm{sec}\end{array}$

TD 0

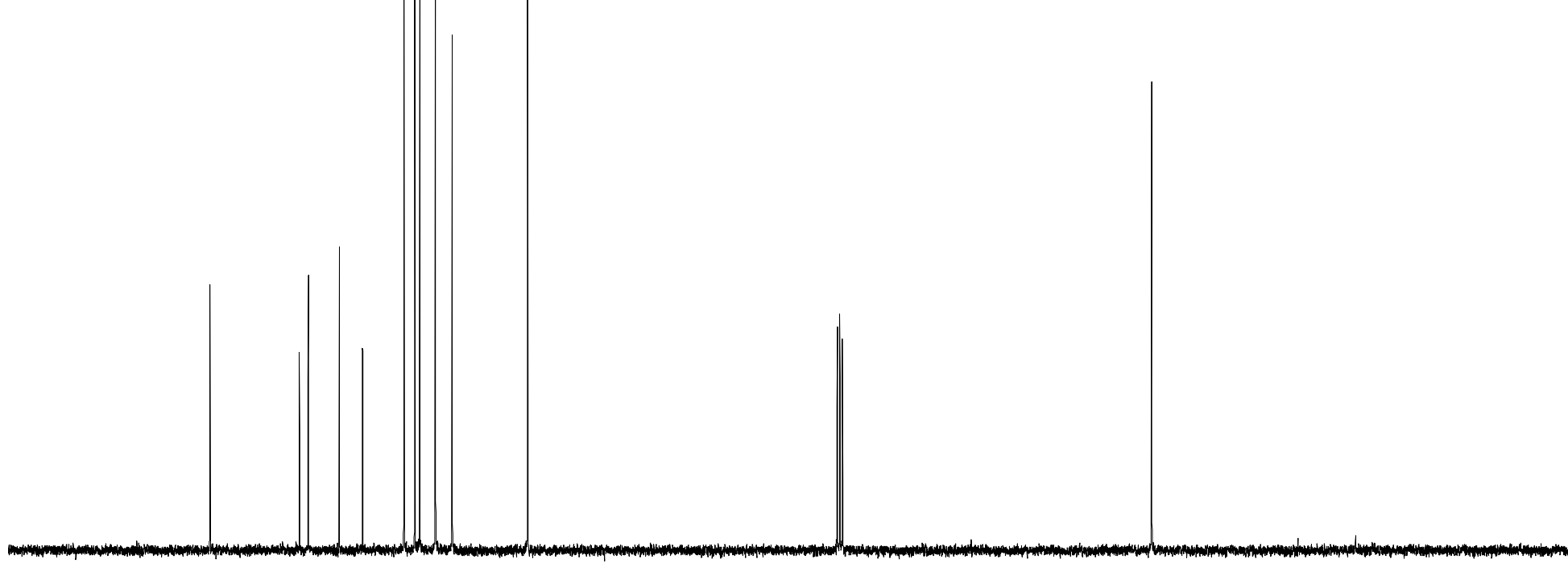

$=====$ CHANNEL $\mathrm{f} 1$

$\mathrm{P} 1$

PL1

PL1

$\mathrm{SFO1}$

$13 \mathrm{C}$

$-3.00 \mathrm{~dB}$
$60.64365387 \mathrm{~W}$

$60.64365387 \mathrm{~W}$

CPDPRG [2 CHANN

TUC2 [2 CHaltzl6

PCPD2

PL2

PL12

PI $2 \mathrm{~W}$

PL12W

PL13W

80.00 usec

$-1.00 \mathrm{~dB}$

$14.39 \mathrm{~dB}$

$0.35193357 \mathrm{~W}$

$0.15327126 \mathrm{~W}$

$-400.1316005 \mathrm{MHz}$

2 - Processing parameters

32768

$100.6127872 \mathrm{MHz}$

EM

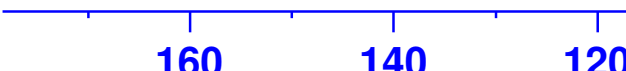

100

80

60

40

0 ppm

$1.00 \mathrm{~Hz}$

1.40 


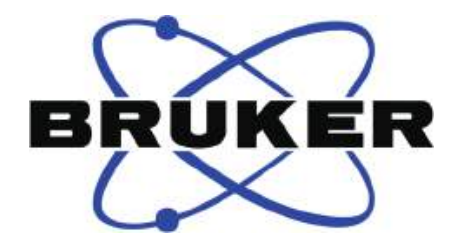

Current Data Parameters NAME

EXPNO

$\operatorname{mdk} 9021 \mathrm{H}$

PROCNO

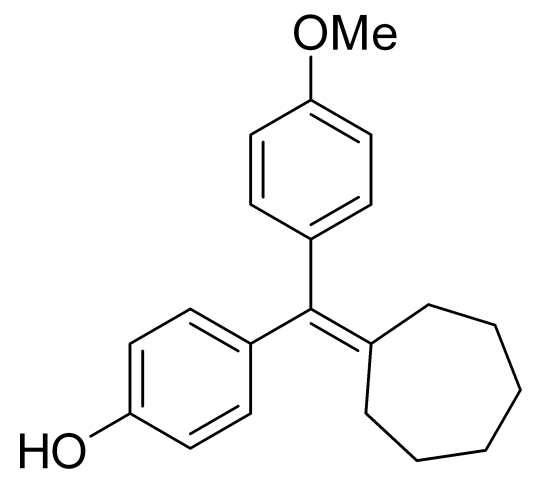

S1q

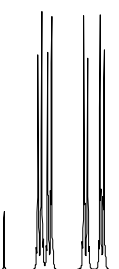

F2 - Acquisition Parameters

Date_

20190301

Time 19.50

$5 \mathrm{~mm}$ spect

PULPROG $\quad$ zg30

$\begin{array}{lr}\text { TD } & \text { Zg30 } \\ & 65536\end{array}$

SOLVENT

NS

DS

SWH

FIDRE
AQ
RG

RG

$\mathrm{DW}$

$\mathrm{DE}$

TE

TD 0

$===$

$\mathrm{SFO1}$

$\mathrm{NUC1}$
$\mathrm{P} 1$

P 1
PLW1

$\mathrm{CDCl}$

$8012.820 \mathrm{~Hz}$

$8012.820 \mathrm{~Hz}$

$4.0894465 \mathrm{sec}$

49.32
62.400

62.400 usec

6.50 usec

$1.00000000 \mathrm{sec}$

CHANNET $\mathrm{f1}$ $400.1324710 \mathrm{MHz}$

$1 \mathrm{H}$

0 usec

$11.99499989 \mathrm{~W}$

F2 - Processing parameters

SI 65536

SF $\quad 400.1300163 \mathrm{MHz}$

WSB

EM

$0.30 \mathrm{~Hz}$

1.00
10

9

\begin{abstract}
8
\end{abstract}

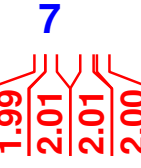

6

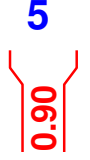

4

金)

S-137
3

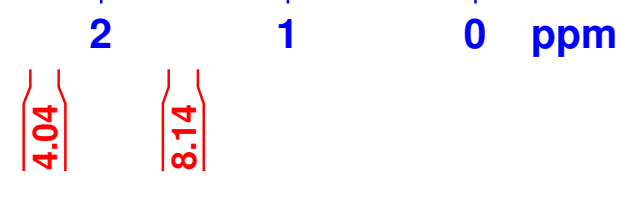




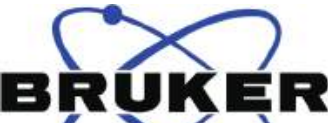

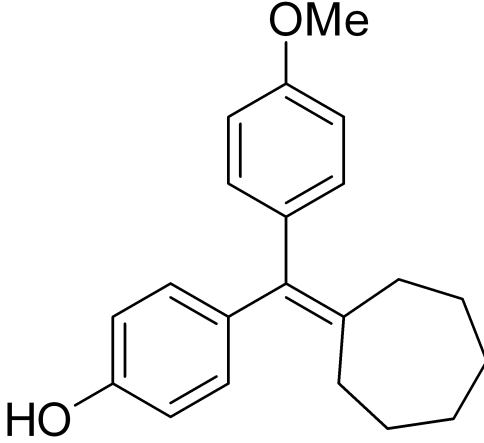

s1q

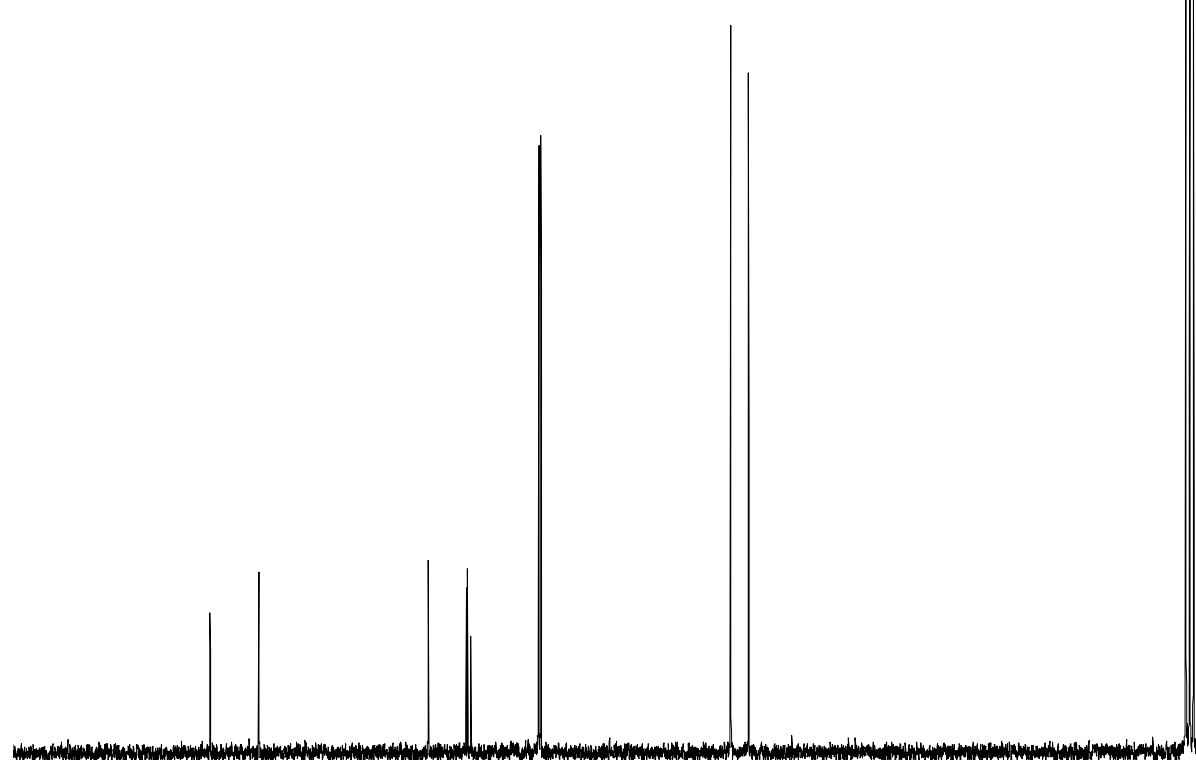

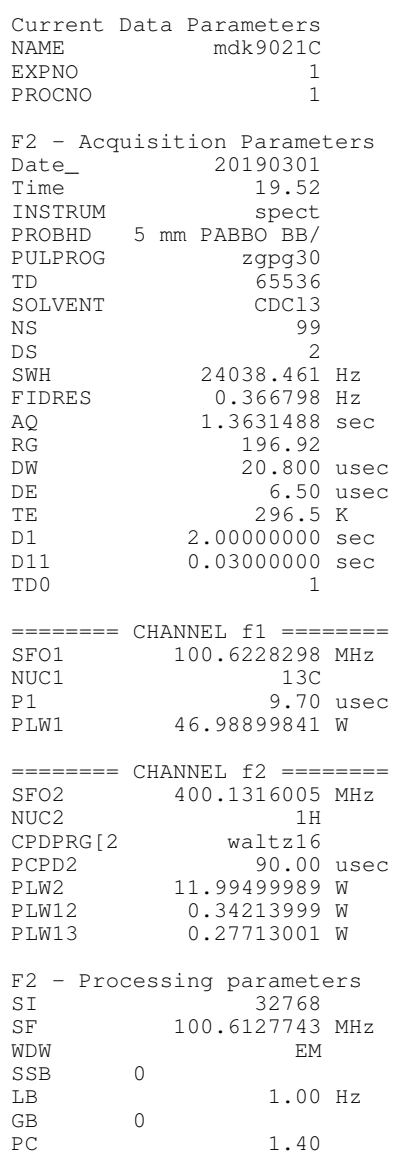

$\begin{array}{llllllllllllllllll}170 & 160 & 150 & 140 & 130 & 120 & 110 & 100 & 90 & 80 & 70 & 60 & 50 & 40 & 30 & 20 & 10 & \mathrm{ppm}\end{array}$




\section{sporep

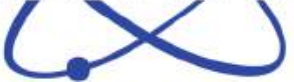

Current Data Parameters

NAME

$\operatorname{mak} 8109 \mathrm{H}$

PROCNO

F2 - Acquisition Parameters

Date_ 20190715

Time $\quad 13.22$

$5 \mathrm{~mm}$ spect

PROBHD

$\mathrm{zg} 30$
65536

TD $\quad 65536$

SOLVENT

NS

DS

FIDRES

FIDR
AQ

RG

$\mathrm{DW}$

$\mathrm{DE}$

$\mathrm{TE}$
$\mathrm{D} 1$

TD 0

$===$

$\mathrm{SFO1}$

$\mathrm{NUC}$
$\mathrm{P} 1$

$\mathrm{CDC} 13$
16
2

$8012.820 \mathrm{~Hz}$

$8012.820 \mathrm{~Hz}$

$4.0894465 \mathrm{~Hz}$

14.29

62.400 usec

6.50 usec

$1.00000000 \mathrm{sec}$ 1

CHANNEL $\mathrm{fl}========$ $400.1324710 \mathrm{MHz}$

14.50

usec

PLW1 $11.99499989 \mathrm{~W}$

F2 - Processing parameters

SI 65536

SF $400.1300560 \mathrm{MH}$

NDW EM

SSB

$0.30 \mathrm{~Hz}$

1.00 

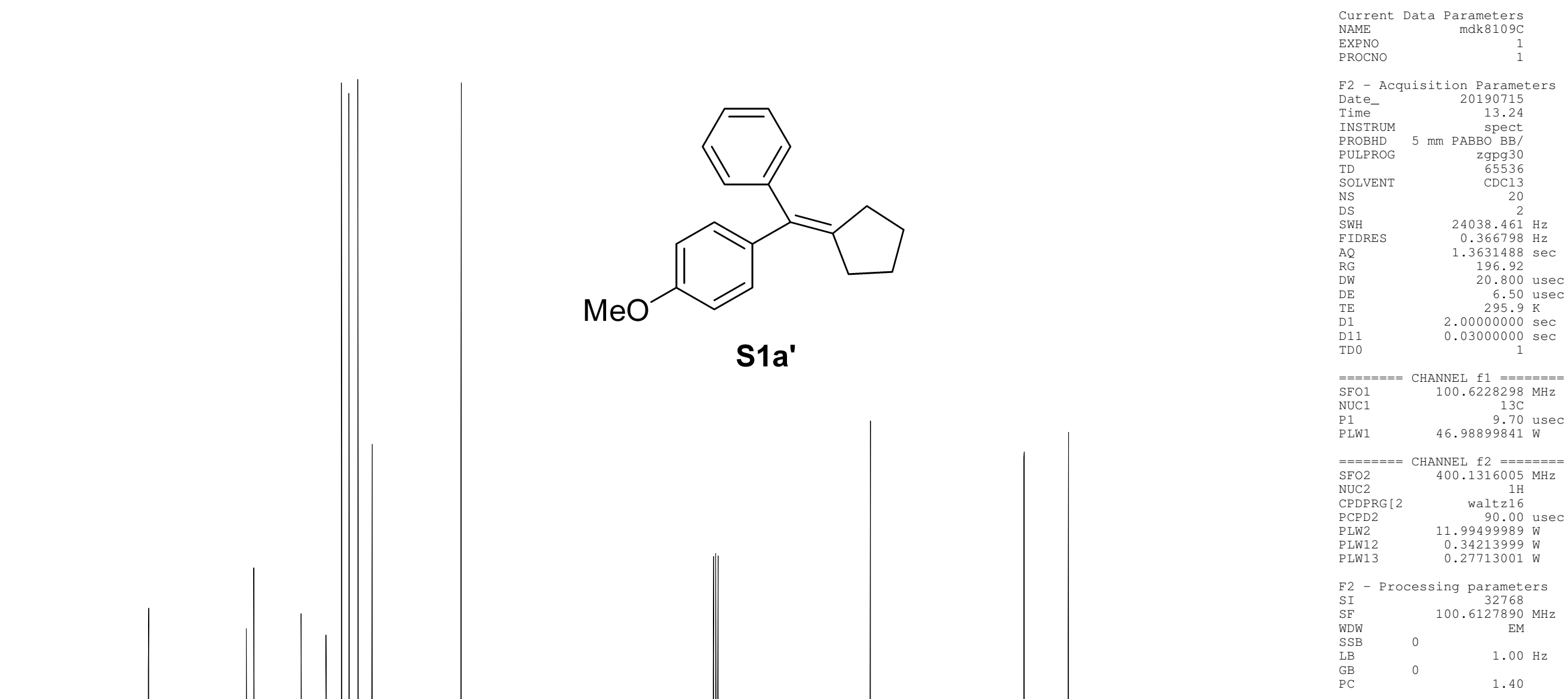

$\begin{array}{llllllllllllllllllll}170 & 160 & 150 & 140 & 130 & 120 & 110 & 100 & 90 & 80 & \begin{array}{l}70 \\ \mathrm{~S}-140\end{array} & 60 & 50 & 40 & 30 & 20 & 10 & 0 & \mathrm{ppm}\end{array}$


00 ஏ

$\operatorname{s} n$ h 4 H

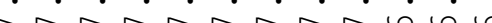

$\leadsto$

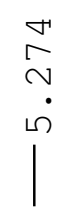

S3a

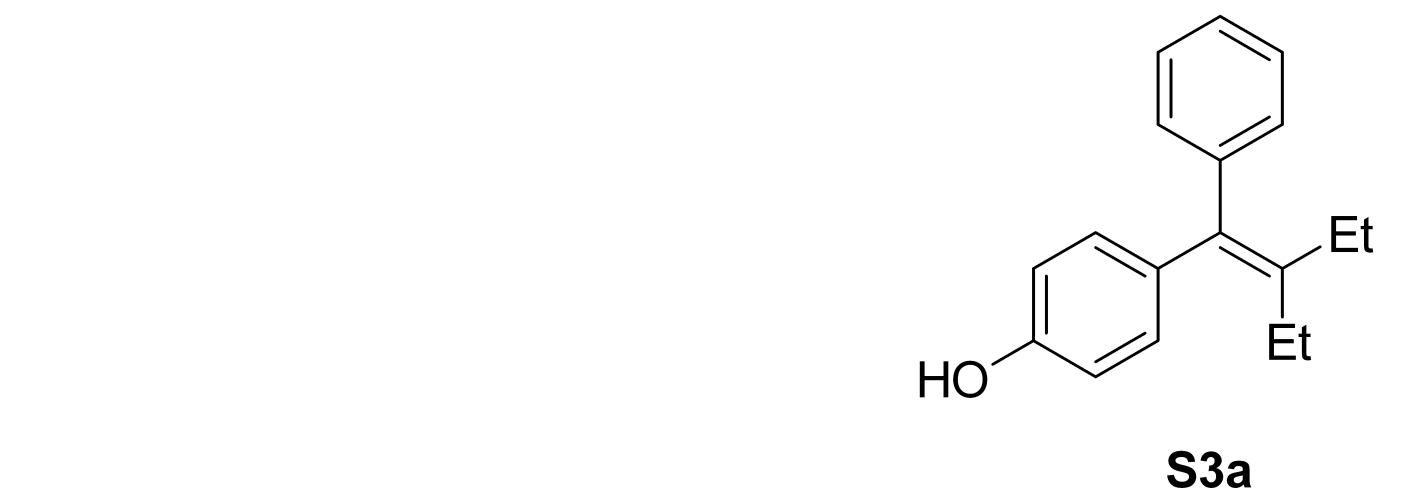

mレ न $\dot{\sim} \dot{\sim} \dot{\sim} \dot{\sim} \dot{H} \dot{H} \dot{0} \dot{0}$

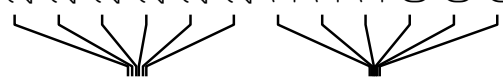

$\stackrel{\circ}{\circ}$

\section{(n)}

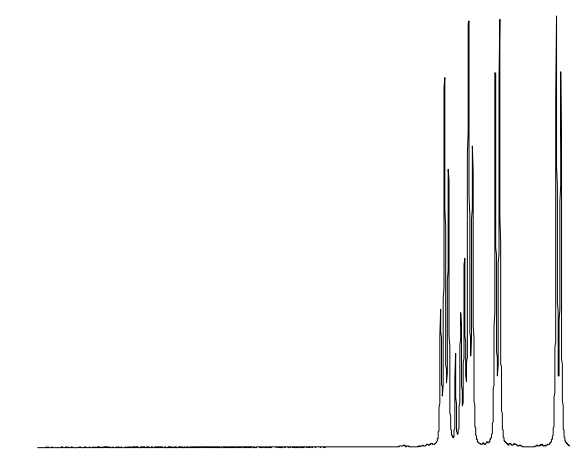

$5 \quad 4$
8

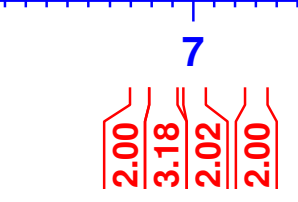

6

$\left|\begin{array}{l}\text { ğ } \\ 0\end{array}\right|$

.
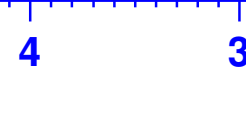

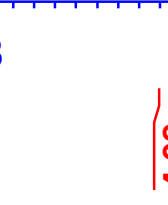

\section{ex $(1)$}

Current Data Parameters NAME

EXPNO
PROCNO

$\mathrm{mdk} 8082 \mathrm{H}$

F2 - Acquisition Parameters

Date__ 20190531

$\begin{array}{lr}\text { Time } & 10.19\end{array}$

INSTRUM spect

$5 \mathrm{~mm}$ PABBO

Zg30
TD

$\begin{array}{ll}\text { TD } & 65536 \\ \text { SOLVENT } & \text { CDCl3 }\end{array}$

TS

NS

DS

$8012.820 \mathrm{~Hz}$
0.0892265

$80122266 \mathrm{~Hz}$
IDRES

$\mathrm{AQ}$

RG
DW
DE
TE

$295.1 \mathrm{~K}$

TD $\quad 1.00000000 \mathrm{sec}$

$======-$ CHANNTL f1 SFO1 $400.1324710 \mathrm{MHz}$ UC1 $1 \mathrm{H}$

P1 14.50 usec

PLW1 11.99499989 W

F2 - Processing parameters

SI 65536

SF $\quad 400.1300356 \mathrm{MHz}$

WDW EM

$0.30 \mathrm{~Hz}$

1.00 

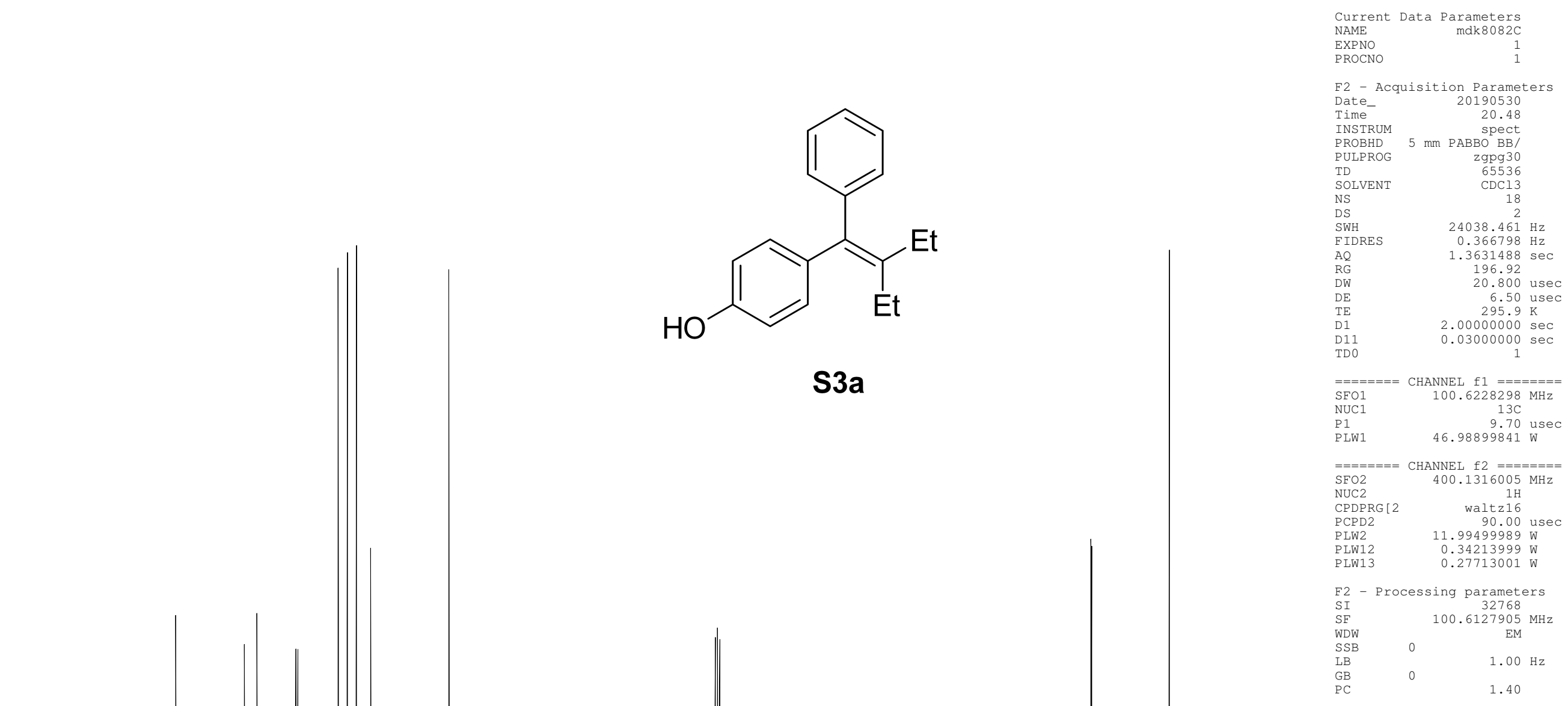

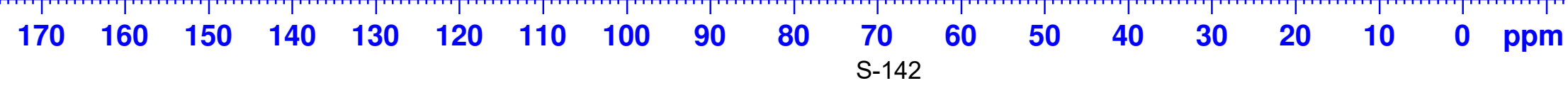




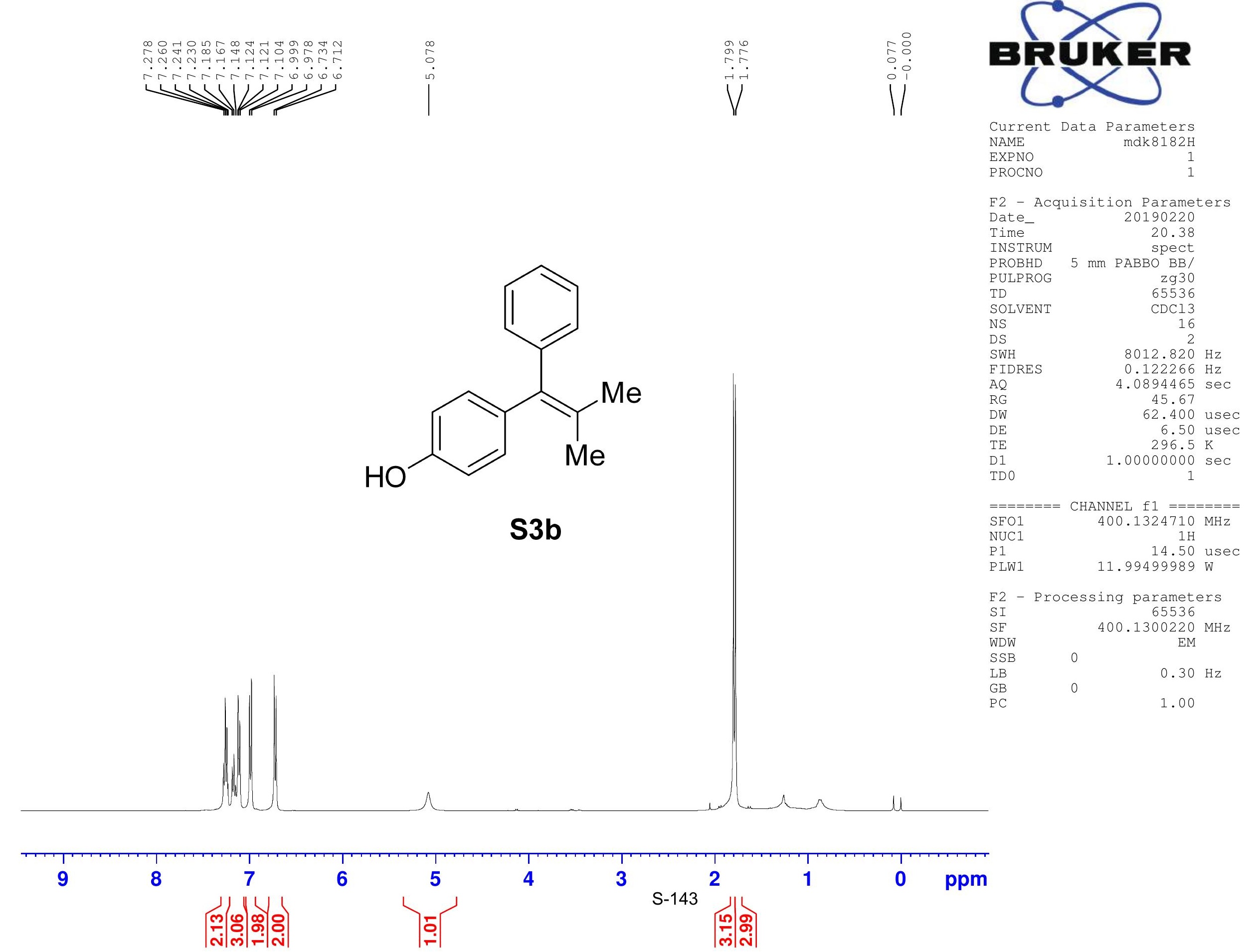




\section{BRUKER}
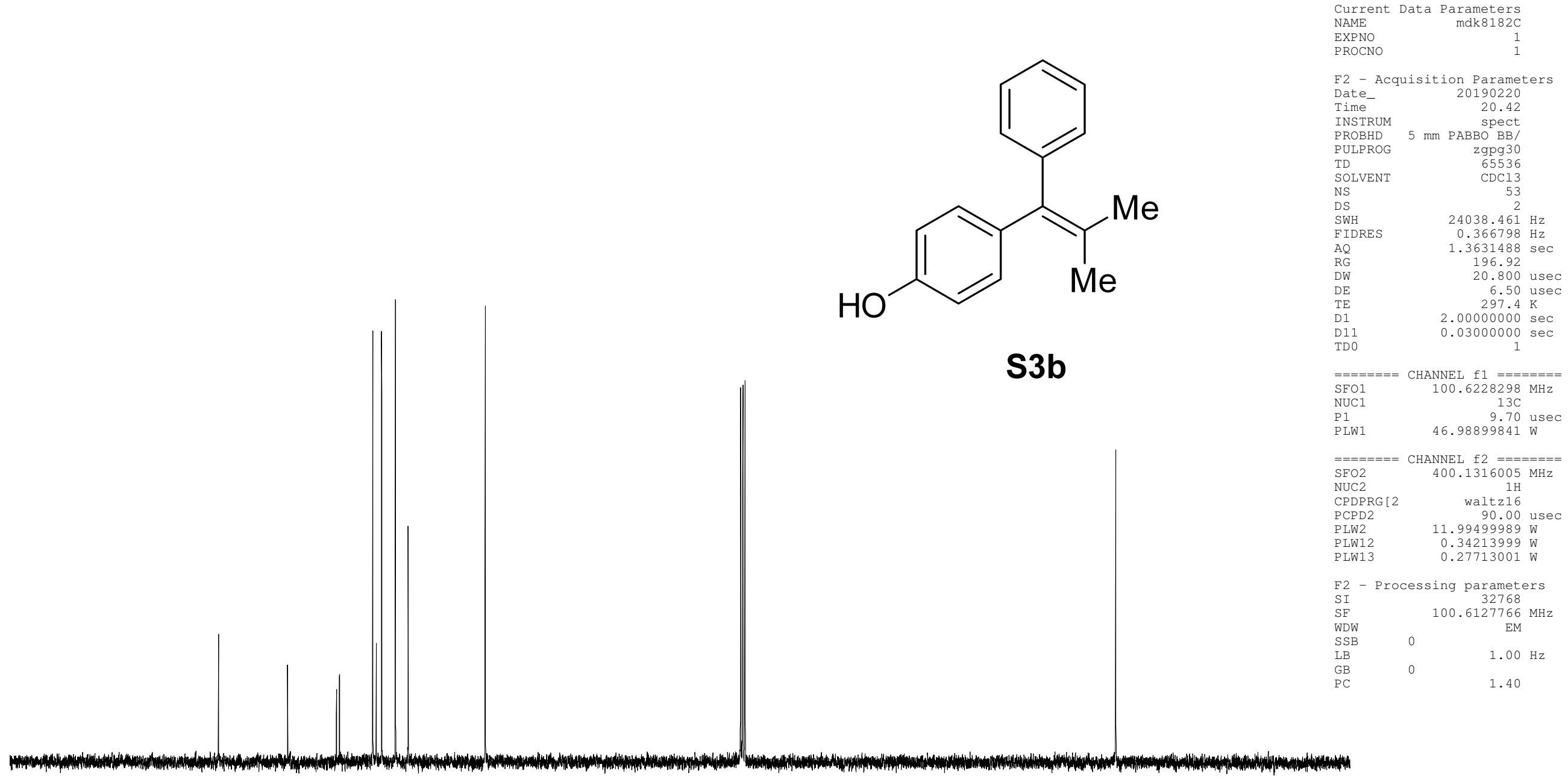

$\begin{array}{rrrrrrrrrrrrrrrrrrrrr}180 & 170 & 160 & 150 & 140 & 130 & 120 & 110 & 100 & 90 & 80 & 70 & 60 & 50 & 40 & 30 & 20 & 10 & 0 & \mathrm{ppm}\end{array}$




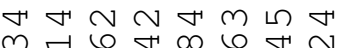

N N O G ब

r

3

ก

ก

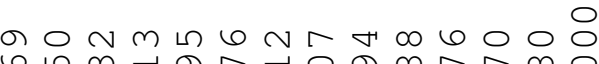
नН Н

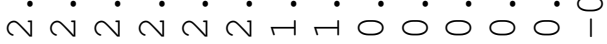

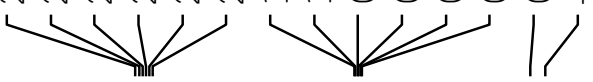<smiles>CCC(CC)=C(c1ccc(O)cc1)c1ccc(Cl)cc1</smiles>

S3c

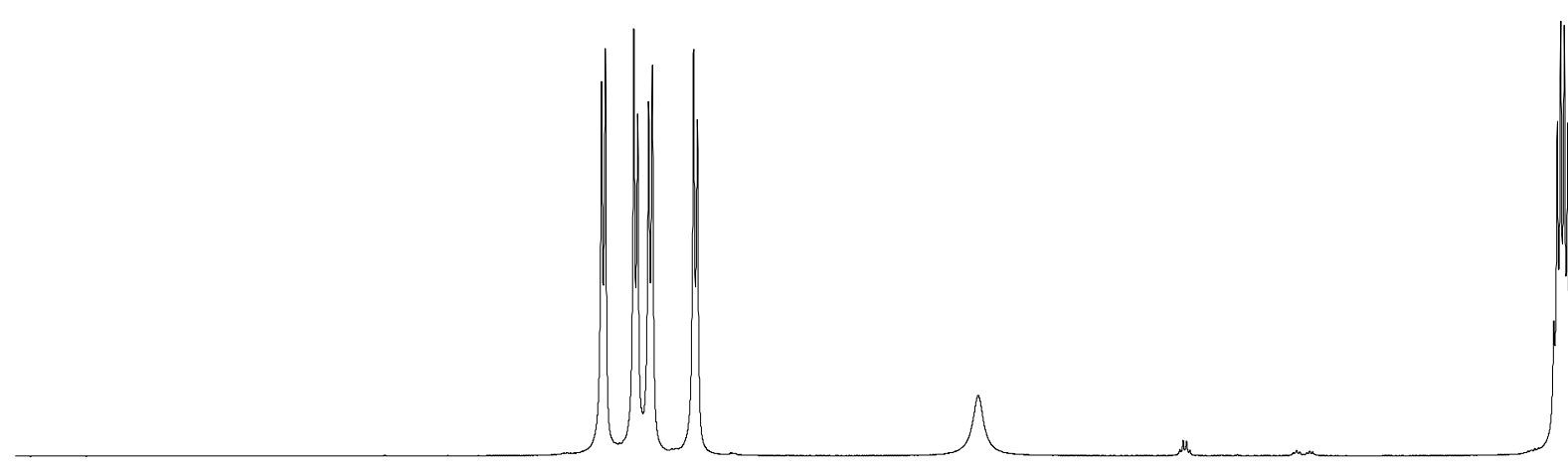

\section{BR KRER $(1)$}

Current Data Parameters NAME

EXPNO

mdk $9072 \mathrm{H}$

F2 - Acquisition Parameter

Date_ 20190318

Time $\quad 13.43$

$5 \mathrm{~mm}$ spect

PUIPROG

$\mathrm{zg} 30$
65536

SD

NS

SWH

$\begin{array}{ll}\text { SWH } & 8012.820 \mathrm{~Hz} \\ \text { FIDRES } & 0.122266 \mathrm{~Hz}\end{array}$

$4.0894465 \mathrm{~Hz}$

RG

DW

$\mathrm{DE}$

$\mathrm{TE}$
$\mathrm{D} 1$

TD 0

$====$

$\mathrm{SFO1}$

$\mathrm{NUC}$
$\mathrm{P} 1$

P1
PLW1

$4.0894465 \mathrm{sec}$
45.67

62.400 usec

6.50 usec

$1.00000000 \mathrm{sec}$

CHANNET $11=-==-=$ $400.1324710 \mathrm{MHz}$ 14.50 usec $11.99499989 \mathrm{~W}$

F2 - Processing parameters

SI 65536

SF $\quad 400.1300209 \mathrm{MHz}$

WDW EM

SSB

$0.30 \mathrm{~Hz}$

C

1.00

\begin{tabular}{|c|c|c|}
\hline 10 & 9 & 8 \\
\hline
\end{tabular}

6 ᄂ 5

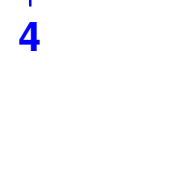

3

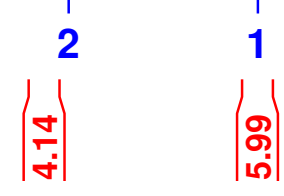

0 ppm 


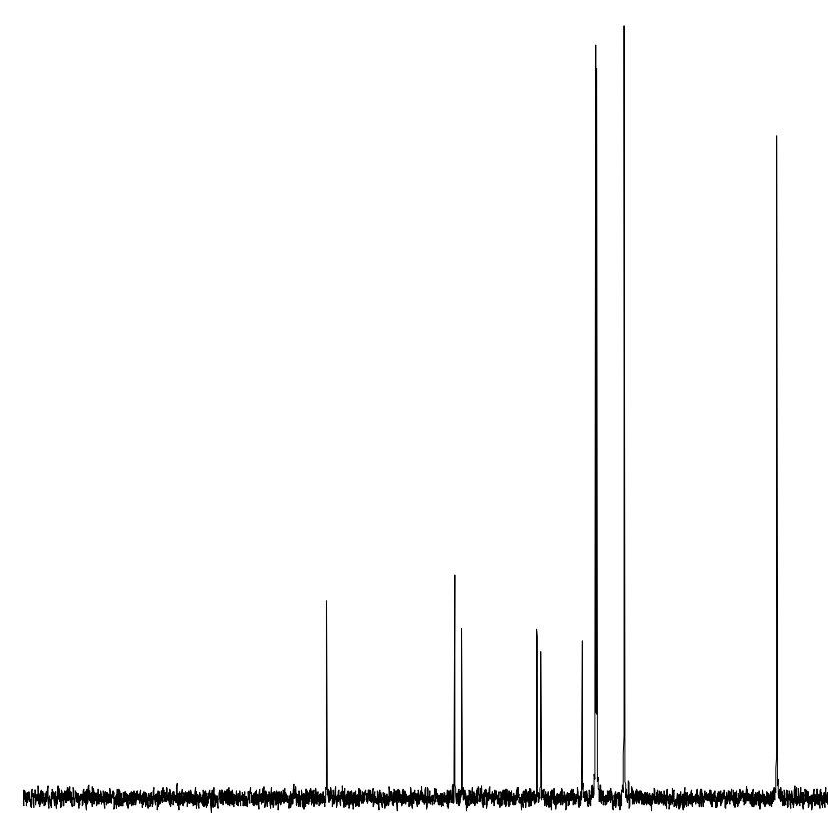<smiles>CCC(CC)=C(c1ccc(O)cc1)c1ccc(Cl)cc1</smiles>

S3c

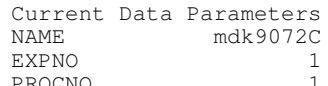
F2 - Acquisition Parameters
Date_ $\begin{array}{lr}\text { Time } & 13.46 \\ \text { INSTRUM } & \text { spect } \\ \text { PROBHD } & 5 \mathrm{~mm} \text { PABBO BB/ }\end{array}$ PULPRO TD
SOLVENT
NS SOL
NS
DS DS
SWH
FIDRE FIDR
AQ
RG AQ
RG
DW $\mathrm{RG}$
$\mathrm{DW}$
$\mathrm{DE}$
$\mathrm{TE}$

TE
D1
D11
TD0

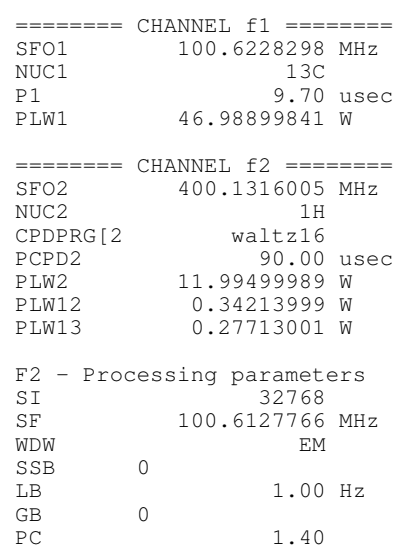




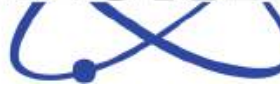

Current Data Parameters NAME

EXPNO

PROCNO

F2 - Acquisition Parameters

Date_ 20190327

Time 21.00

spect
PROBHD $5 \mathrm{~mm} \mathrm{PABBO} \mathrm{BB} /$

$\begin{array}{lr}\text { PROBHD } & 5 \mathrm{~mm} \text { PABBO BB/ } \\ \text { PULPROG } & \mathrm{zg} 30\end{array}$

TD 65536

SOLVENT CDC13

NS

DS

SWH $\quad 8012.820 \mathrm{~Hz}$

FIDRES $\quad 0.122266 \mathrm{~Hz}$

$\mathrm{AQ} \quad 4.0894465 \mathrm{sec}$

RG $\quad 45.67$

DW $\quad 62.400$ usec

$295.9 \mathrm{~K}$

$1.00000000 \mathrm{sec}$

S3d

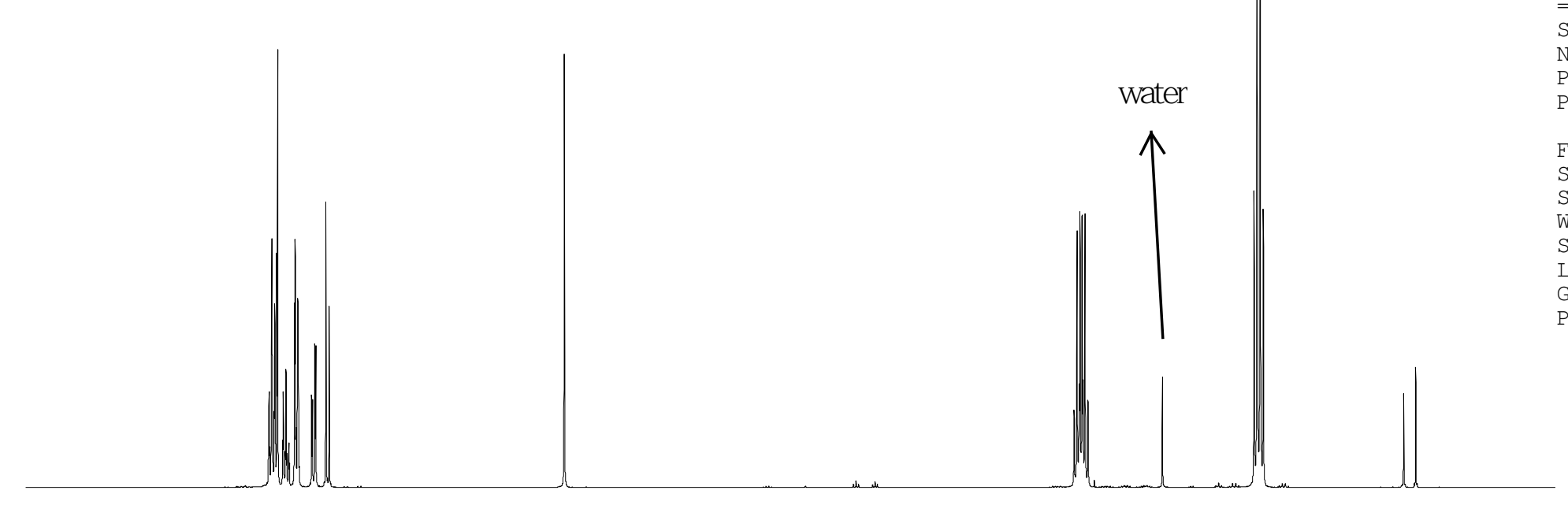

$======$ CHANNEL fl $========$ SFO1 $400.1324710 \mathrm{MHz}$ NUC1 $1 \mathrm{H}$

14.50 usec

F2 - Processing parameters

SI 65536

SF $\quad 400.1300197 \mathrm{MHz}$

WDW

SSB

GB

$0.30 \mathrm{~Hz}$

1.00

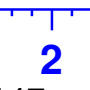

1 
Current Data Parameters

F2 - Acquisition Parameters

Date__ 20190327

Time 21.03

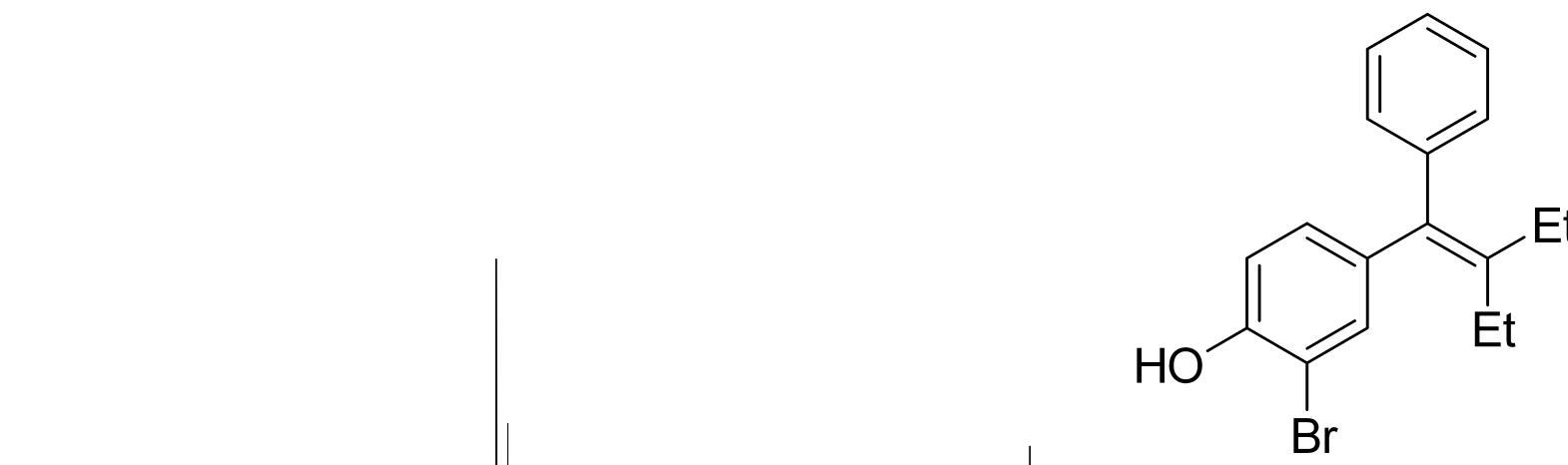

INOBHD

PULPROG Zgpg30

TD 65536

SOLVENT

NS

DS

SWH $\quad 24038.461 \mathrm{~Hz}$

FIDRES $\quad 0.366798 \mathrm{~Hz}$

$\mathrm{AQ} \quad 1.3631488 \mathrm{sec}$

RG $\quad 196.92$

DW 20.800 usec

$\begin{array}{lr}\text { DE } & 6.50 \text { usec } \\ \text { TE } & 296.5 \mathrm{~K}\end{array}$

D1 $2.00000000 \mathrm{sec}$

D11 $0.03000000 \mathrm{sec}$

S3d

CHANNEL $\mathrm{f} 1 \mathrm{1}=======$

$\mathrm{SFO1}$

NUC

P1

$100.6228298 \mathrm{MHz}$

PLW1 9.70 usec

$=======$ CHANNEL $\mathrm{f} 2========$

SFO2 $400.1316005 \mathrm{MHz}$

NUC 2

CPDPRG [2

PCPD2

PLW2

PLW12

waltzl6

r 90.00 usec

$11.99499989 \mathrm{~W}$

$0.34213999 \mathrm{~W}$

0.27713001

F2 - Processing parameters

SI Processing parameters

$\begin{array}{lr}\text { SI } & 32768 \\ \text { SF } & 100.6127751 \mathrm{MHz}\end{array}$

$100.6127751 \mathrm{MHz}$
$\mathrm{EM}$

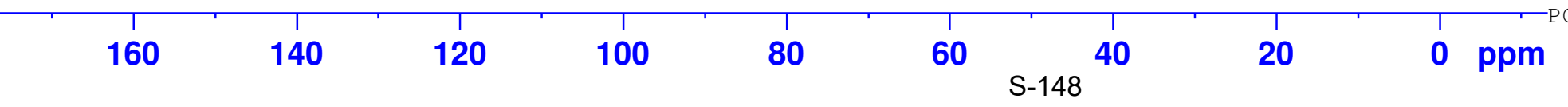

0 


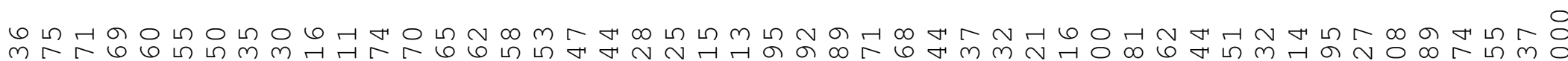

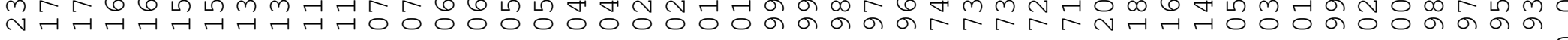

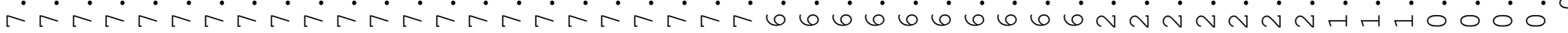<smiles>CCC(CC)=C(c1ccc(O)cc1)c1ccccc1F</smiles>

S3e

\section{sp $(1)$}

Current Data Parameters NAME

EXPNO

$\operatorname{mdk} 11059 \mathrm{H}$

PROCNO

F2 - Acquisition Parameters

Date_ 20190811

Time $\quad 13.42$

$5 \mathrm{~mm}$ spect

PUI PROG

$\mathrm{zg} 30$
65536

TD 65536

NS

SWH

FIDRES

$\mathrm{AQ}$

RG

DW

$\mathrm{DE}$

D1
TD 0

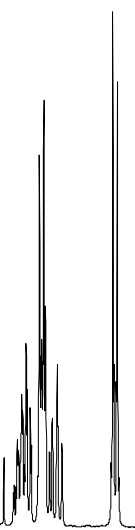

$\mathrm{SFO}$

NUC1

P 1

PLW1

16
2

$8012.820 \mathrm{~Hz}$

$0.122266 \mathrm{~Hz}$

$4.0894465 \mathrm{sec}$ 27.78

62.400 usec

6.50 use

$1.00000000 \mathrm{sec}$

$$
1
$$

CHANNEL $\mathrm{f} 1========$ $400.1324710 \mathrm{MHz}$

14.50

use

Processing parameters

SI $\quad 65536$

SF $\quad 400.1300192 \mathrm{MHz}$

SSB

SSB

$\mathrm{EM}$

$0.30 \mathrm{~Hz}$

1.00

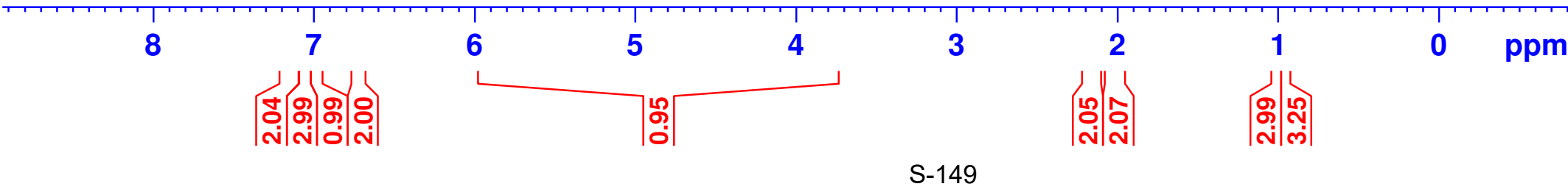



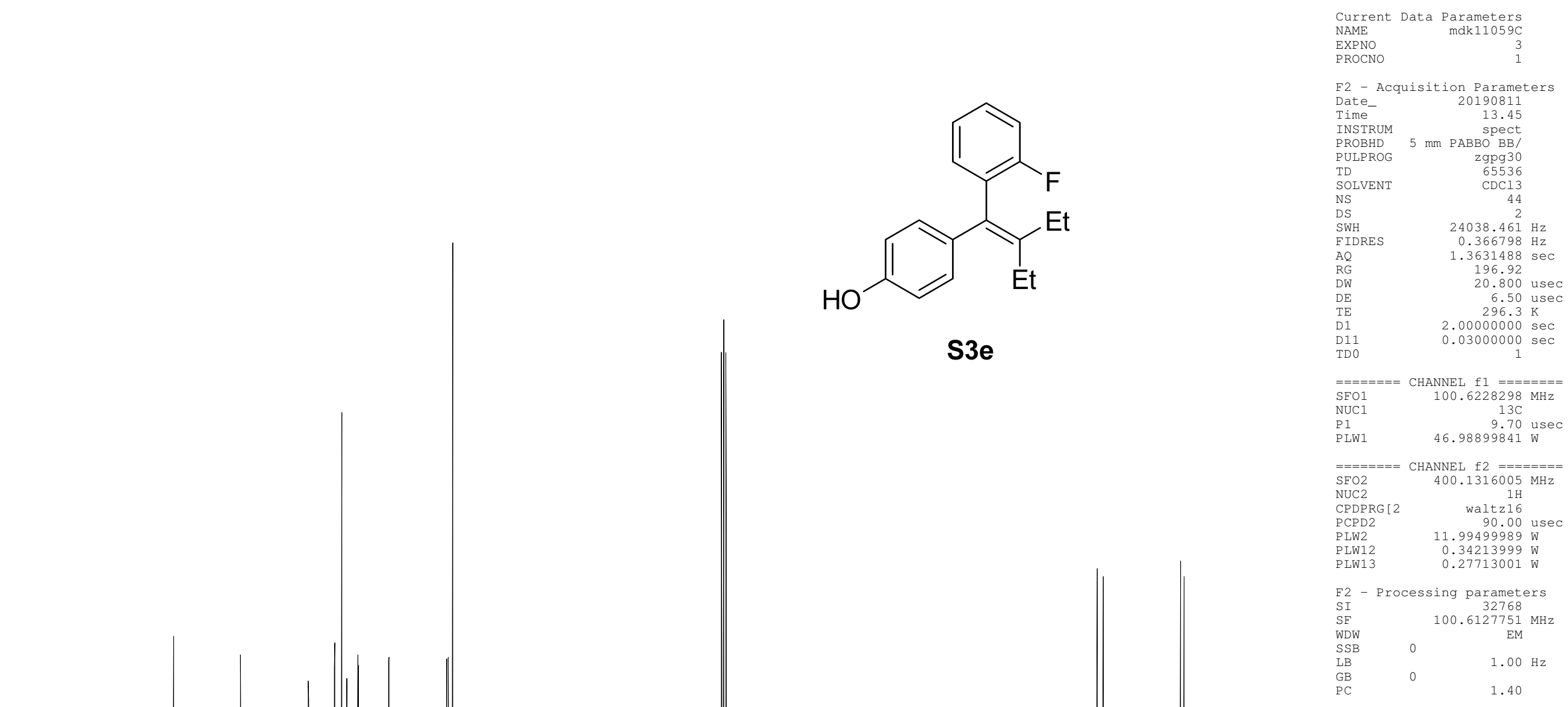

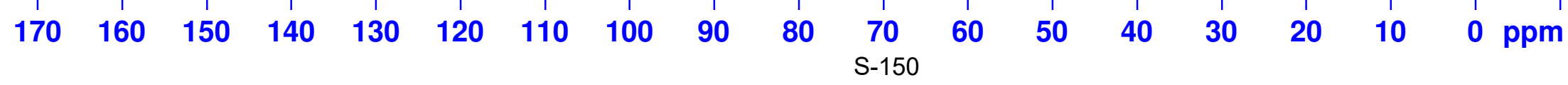




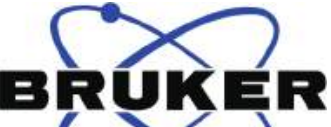

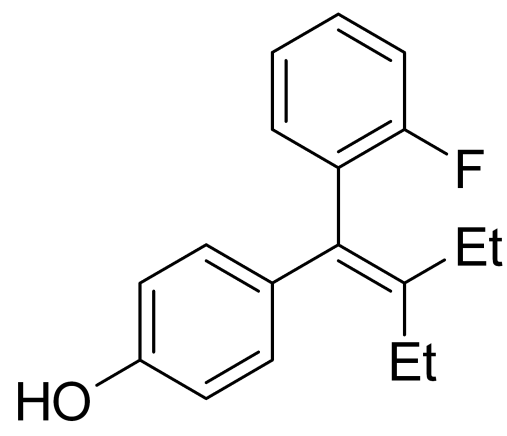

NAME mdk11059F-decoup

PROCNO

F2 - Acquisition Parameters
Date_ 20190810

Date__ 20190810

Time

spect
PROBHD $5 \mathrm{~mm}$ PABBO BB/

PULPRO

SOLVEN

NS
DS

$\begin{array}{ll}2 \\ \text { SWH } & 93750.000 \mathrm{~Hz}\end{array}$

$\begin{array}{ll}A Q & 1.430511 \mathrm{~Hz} \\ \mathrm{BQ} & 0.3495253 \mathrm{sec}\end{array}$

DW

$\mathrm{DE}$

D1

196.92
5.333
6.53

5.333 usec
6.50 usec

$2.00000000 \mathrm{sec}$

TD 0

$0.03000000 \mathrm{sec}$

S3e

\begin{tabular}{llll}
\hline & & & \\
20 & 0 & -20 & -40
\end{tabular}

$-60$

$-80$

$-100$

$-120$

$-140$

$-160$

$-180$

$-200$

ppm 


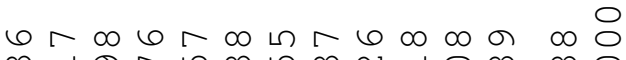
N

\section{sporen ,}

Current Data Parameters

NAME

$\operatorname{mok} 11052 \mathrm{H}$

PROCNO

F2 - Acquisition Parameter

Date__ 20190810

Time $\quad 15.20$

$5 \mathrm{~mm}$ saBBOect

zgB 30
65536

TD $\quad 65536$

SOLVENT

NS

SWH

FIDRES

FIDR
$\mathrm{AQ}$

RG

$\mathrm{DW}$
$\mathrm{DE}$
$\mathrm{TE}$

D1

S3f

Et

TD 0

$====$

$\mathrm{SFO1}$

NUC1

P1
PLW1

16
16

$8012.820 \mathrm{~Hz}$

$0.122266 \mathrm{~Hz}$

$4.0894465 \mathrm{sec}$ 34.77

62.400 usec 6.50 usec

$1.00000000 \mathrm{sec}$ 1

CHANNEL $\mathrm{fl}========$ $400.1324710 \mathrm{MHz}$ 14.50 sec

F2 - Processing parameters SI $\quad 65536$ $\begin{array}{lll}\text { SF } & 400.1300406 \mathrm{MHz}\end{array}$ WDW

$0.30 \mathrm{~Hz}$

1.00

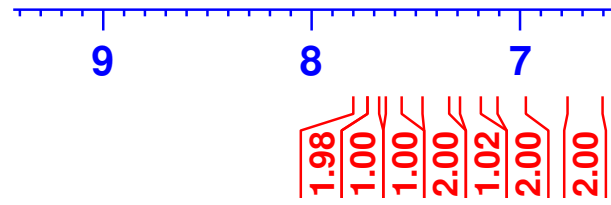

6

5

4

3

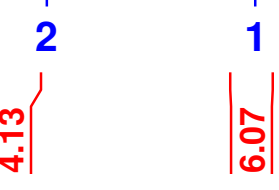

0 ppm 


\section{aponeren}

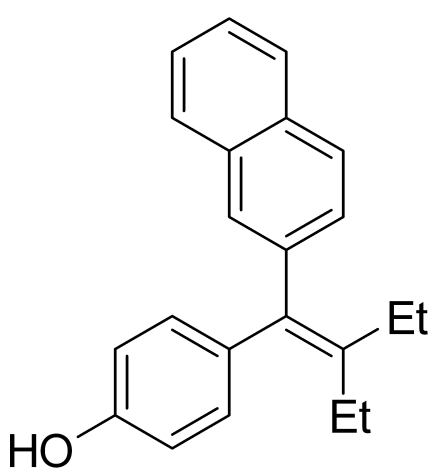

S3f $\begin{array}{lr}\text { Current } & \text { Data Parameters } \\ \text { NAME } & \text { mdk11052C } \\ \text { EXPNO } & 2 \\ \text { PROCNO } & 1\end{array}$

F2 - Acquisition Parameters
20190810

Date_ 20190810

$\begin{array}{lr}\text { Time } & 15.23 \\ \text { INSTRUM } & \text { spect } \\ \text { PROBHD } & 5 \mathrm{~mm} \text { PABBO BB/ }\end{array}$

PULPROC

SOLVEN

DS
SWH

$24038.461 \mathrm{~Hz}$

$\mathrm{AQ}$
$\mathrm{RG}$

DW
DE

D1
D11

$1.3631488 \mathrm{sec}$

196.92
20.800 usec

6.50 usec

$2.00000000 \mathrm{sec}$
$0.03000000 \mathrm{sec}$

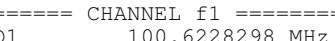

$\mathrm{SE}$
$\mathrm{NU} 1$
$\mathrm{P} 1$

$13 \mathrm{C}$
9.70 usec

$46.98899841 \mathrm{~W}$

$=======$ CHANNEL $\mathrm{f} 2 \quad======= \pm$
SFO2
SOO.1316005 MHz

$\begin{array}{lr}\text { NUC2 } & 1 \mathrm{H} \\ \text { CPDPRG [2 } & \text { waltz16 } \\ \text { PCPD2 } & 90.00\end{array}$

PLW2 11.99499989 W

PLW12 $\quad 0.34213999 \mathrm{~W}$

$\begin{array}{lr}\text { F2 } & \text { - Processing parameters } \\ \text { SI } & 32768 \\ \text { SF } & 100.6127817 \mathrm{MHz}\end{array}$

$\begin{array}{lr}\text { SE } & 100.6127817 \\ \text { WDW } & \text { EM }\end{array}$

$\begin{array}{lll}\mathrm{SSB} & 0 & 1.00 \mathrm{~Hz} \\ \mathrm{IB} & & \end{array}$ 
の N N N

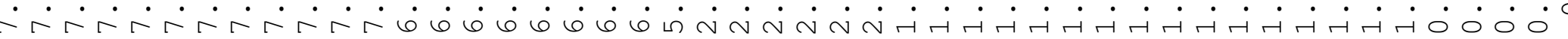

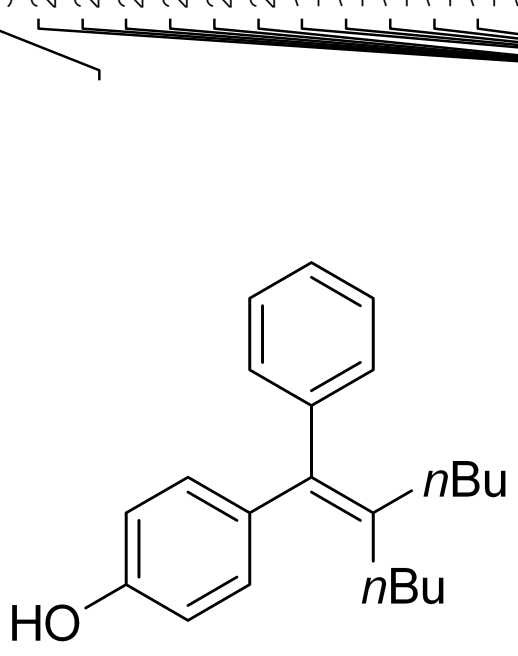

S3g
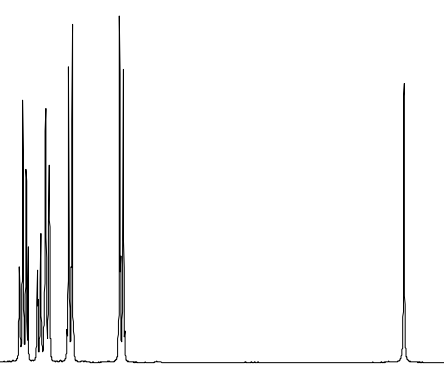

8

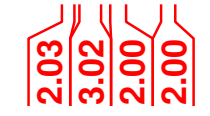

6

ro

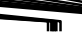

$1 /$

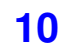
9

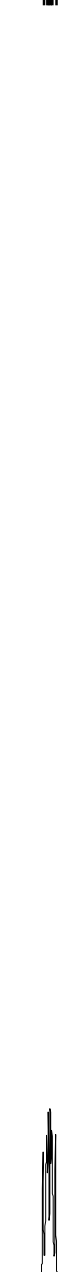

\section{किति $C$}

Current Data Parameters NAME

PROCNO

$\operatorname{mdk} 9131 \mathrm{H}$

F2 - Acquisition Parameters Date__ 20190401

$\begin{array}{lr}\text { Time } & 20.01\end{array}$

INSTRUM spect

BB/

$\begin{array}{lr}\text { PULPROG } & \text { zg30 } \\ \text { TD } & 65536\end{array}$

$\begin{array}{ll}\text { TD } & 65536 \\ \text { SOLVENT } & \text { CDC13 } \\ \text { NS } & 16\end{array}$

NS
DS
SWH

SWH

FIDRES $\quad 0.122266 \mathrm{~Hz}$

$\mathrm{AQ}$

RG

$\mathrm{DW}$
$\mathrm{DE}$

$\mathrm{DE}$

D 1
TD 0

$====$

$\mathrm{SFO1}$

$\mathrm{NUC1}$
$\mathrm{P} 1$

P 1
PLW1 17.38

62.400 usec
6.50 usec $297.0 \mathrm{~K}$

$1.00000000 \mathrm{sec}$

CHANNEL f1 $========$ $400.1324710 \mathrm{MHz}$

4.50 usec

Processing parameters

SI $\quad 65536$

SF $\quad 400.1300294 \mathrm{MHz}$

SSB

$\mathrm{EM}$

$0.30 \mathrm{~Hz}$

1.00 


\section{experen

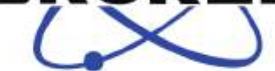

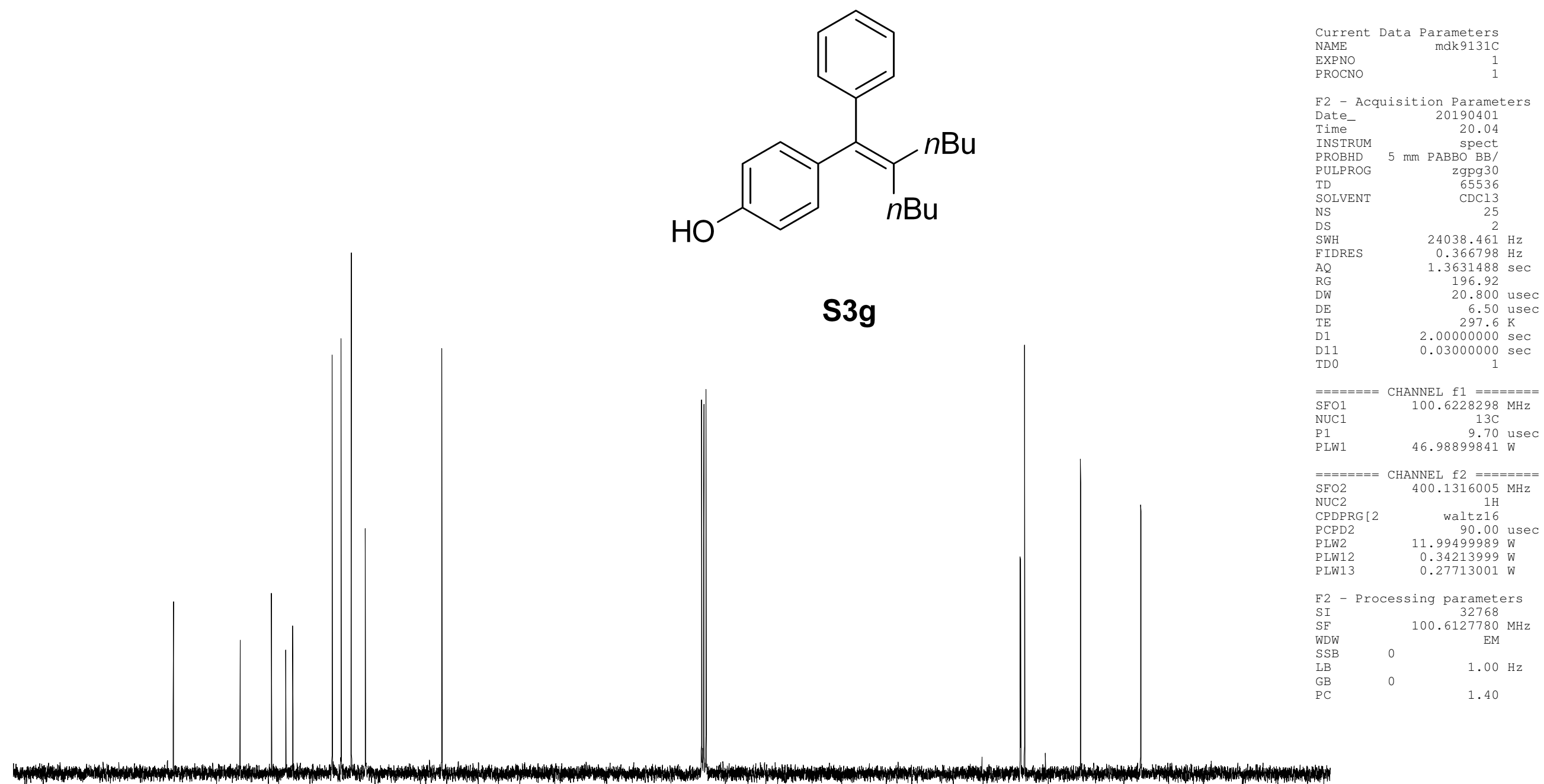

S3g

$\begin{array}{lllllllllllllllllll}170 & 160 & 150 & 140 & 130 & 120 & 110 & 100 & 90 & 80 & \begin{array}{l}70 \\ \mathrm{~S}-155\end{array} & 60 & 50 & 40 & 30 & 20 & 10 & 0 & \mathrm{ppm}\end{array}$




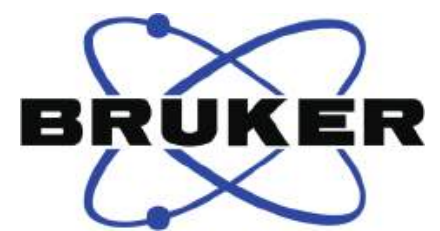

Current Data Parameters NAME

EXPNO

$\operatorname{mdk} 11085 \mathrm{H}$

PROCNO
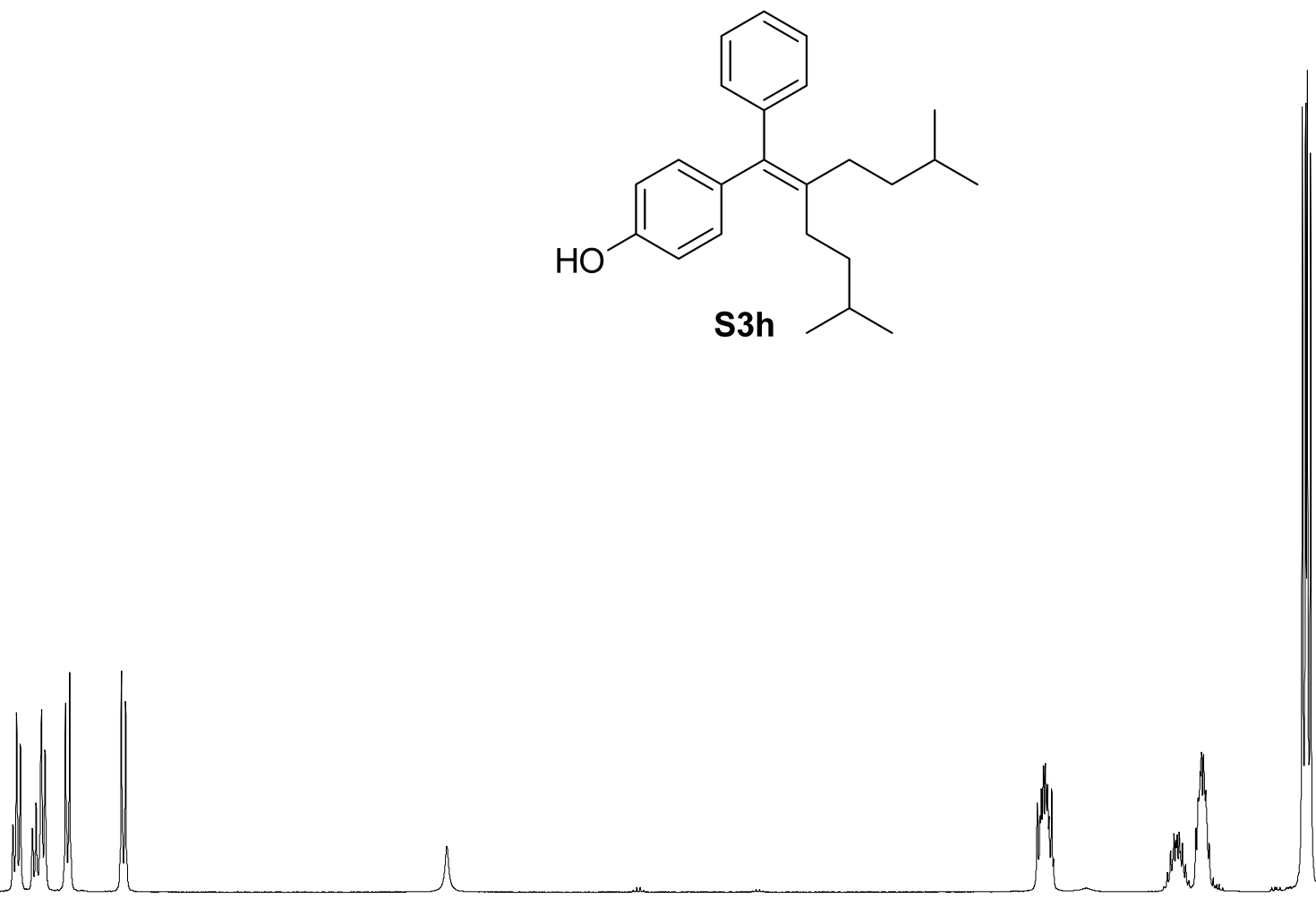

3

œ.

4

5
$\infty$
$\infty$
$\infty$

4

ஸे

6

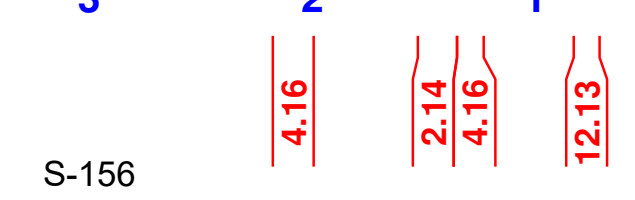




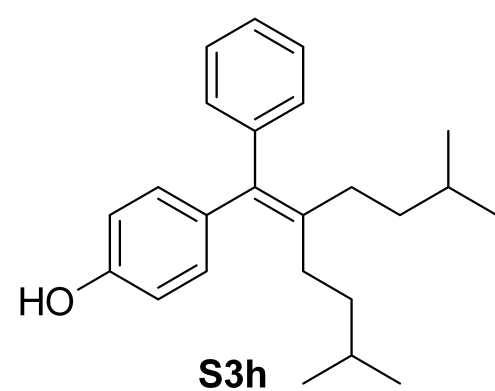

$\begin{array}{lr}\text { Current } & \text { Data } \\ \text { NAME } & \text { mameters } \\ \text { EXPNO } & 11085 \mathrm{C} \\ \text { PXOCNO } & 1\end{array}$

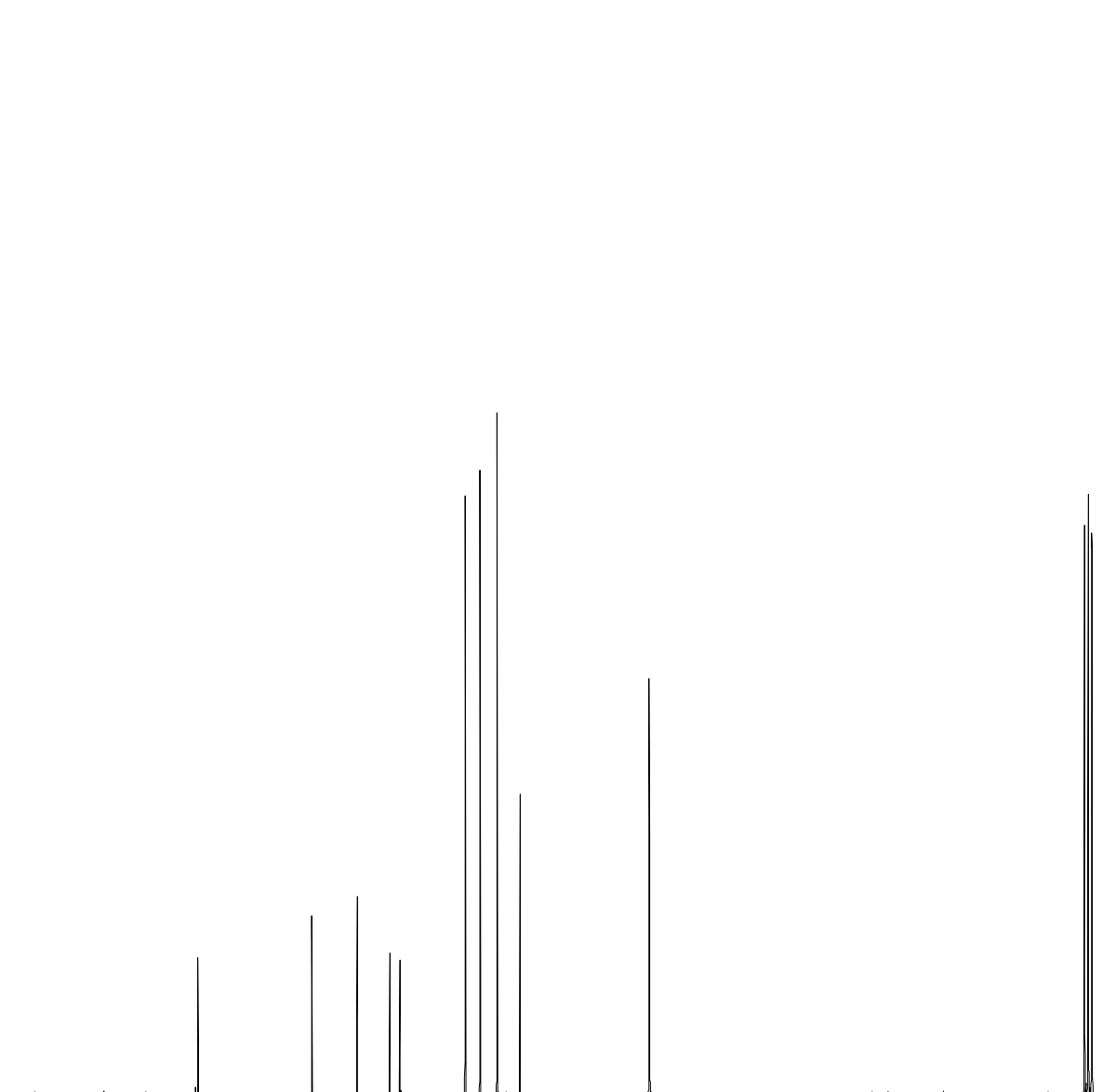

F2 - Acquisition Parameters

Date_ 20190814

$\begin{array}{lr}\text { Time } & 21.00 \\ \text { INSTRUM } & \text { spect } \\ \text { PROBHD } & 5 \mathrm{~mm} \text { PABBO BB/ }\end{array}$

PULPROG

SOLVENT

NS
DS

SWH $\quad 24038.461 \mathrm{~Hz}$

$\begin{array}{ll}\text { AQ } & 0.366798 \mathrm{~Hz} \\ \text { RG } & 1.3631488 \mathrm{sec}\end{array}$

AQ
RG
DW

$\begin{array}{lr}1 & 20.800 \text { usec } \\ \text { DE } & 6.50 \text { usec } \\ \text { TE } & 296.9 \text { K }\end{array}$

TE

D1

$2.00000000 \mathrm{sec}$

$=======$ CHANNEL $\mathrm{fl}=======$

$\begin{array}{ll}\text { SFO1 } & 100.6228298 \mathrm{MHz}\end{array}$

P1 9.70 usec

$6.98899841 \mathrm{~W}$

$=======$ CHANNEL $\mathrm{f} 2 \quad======= \pm$
$\mathrm{SFO2}$
$400.1316005 \mathrm{MHz}$

$\begin{array}{lr}\text { NUC2 } & 1 \mathrm{H} \\ \text { CPDPRG [2 waltz16 } & 90.00\end{array}$

PLW2 $11.99499989 \mathrm{~W}$

PLW12 $\quad 0.34213999$ W

F2 - Processing parameters

$\begin{array}{lr}\text { FI } & \text { Processing parameters } \\ \text { SF } & 100.6127758 \\ \text { SFHz }\end{array}$

$\begin{array}{lc}\text { SF } & 100.6127759 \text { MHz } \\ \text { WDW } & \text { EM }\end{array}$

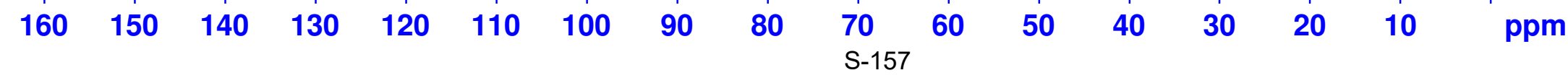




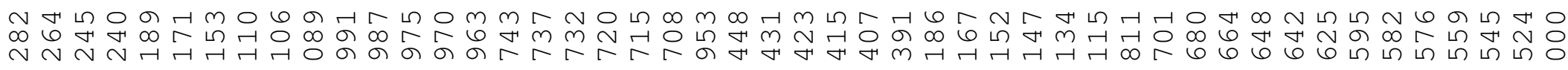

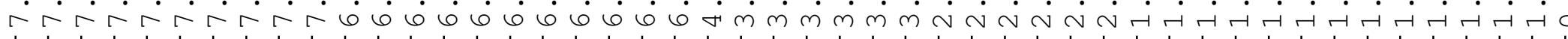
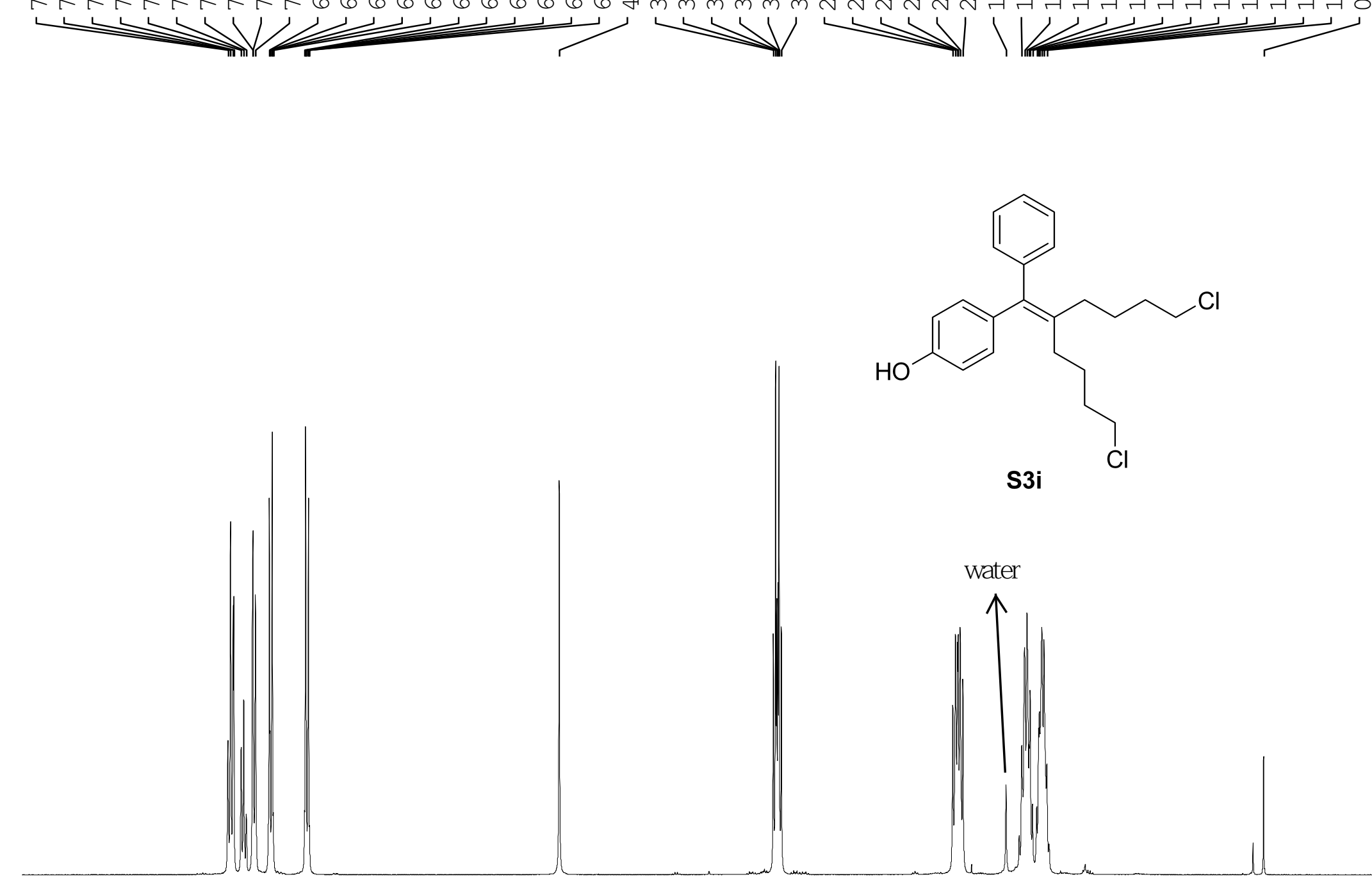

NAME

EXPNO

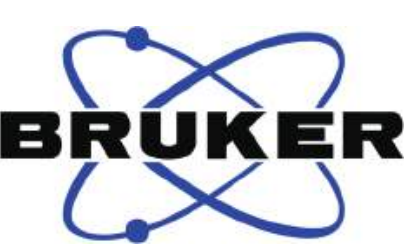

F2 -

Acquisition Parameters

Date

20190430

Time

INSTRUM

PULPROG

$5 \mathrm{~mm} \mathrm{PABBO} \mathrm{BB} /$

SOLVENT

NS

DS

SWH

FID

$\mathrm{AQ}$
$\mathrm{RG}$

RG

DW

DE

D1
TD 0

water

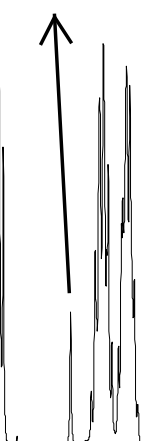

$====$
$\mathrm{SFO} 1$

NUC

P1

PLW1

$\mathrm{zg} 30$
65536
$\mathrm{CDC} 13$

16
2

$8012.820 \mathrm{~Hz}$

$0.122266 \mathrm{~Hz}$

$4.0894465 \mathrm{sec}$

27.78

62.400 usec

6.50 usec

$1.00000000 \mathrm{sec}$

$$
1
$$

CHANNEL $\mathrm{fl}=======$

$400.1324710 \mathrm{MHz}$

14.50

usec

- Processing parameter

SI

ing paramet

$400.1300183 \mathrm{MHz}$

SSB

EM

$0.30 \mathrm{~Hz}$

1.00

8 7

성혀잉ㅇㅇ

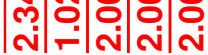

6

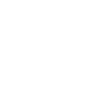

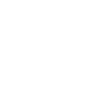

5
$\left|\begin{array}{l}\infty \\ 0 \\ 0\end{array}\right|$

4

|

3

बू.

S-158

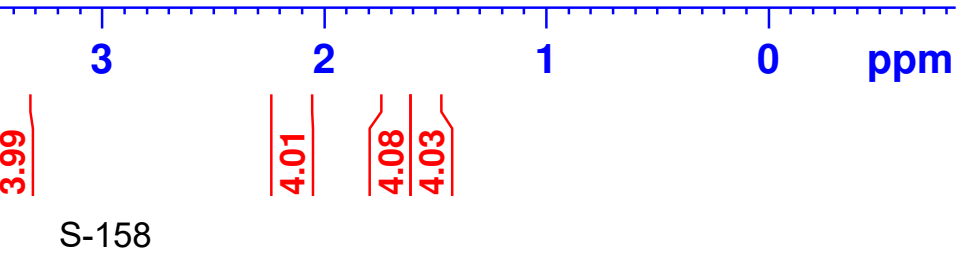



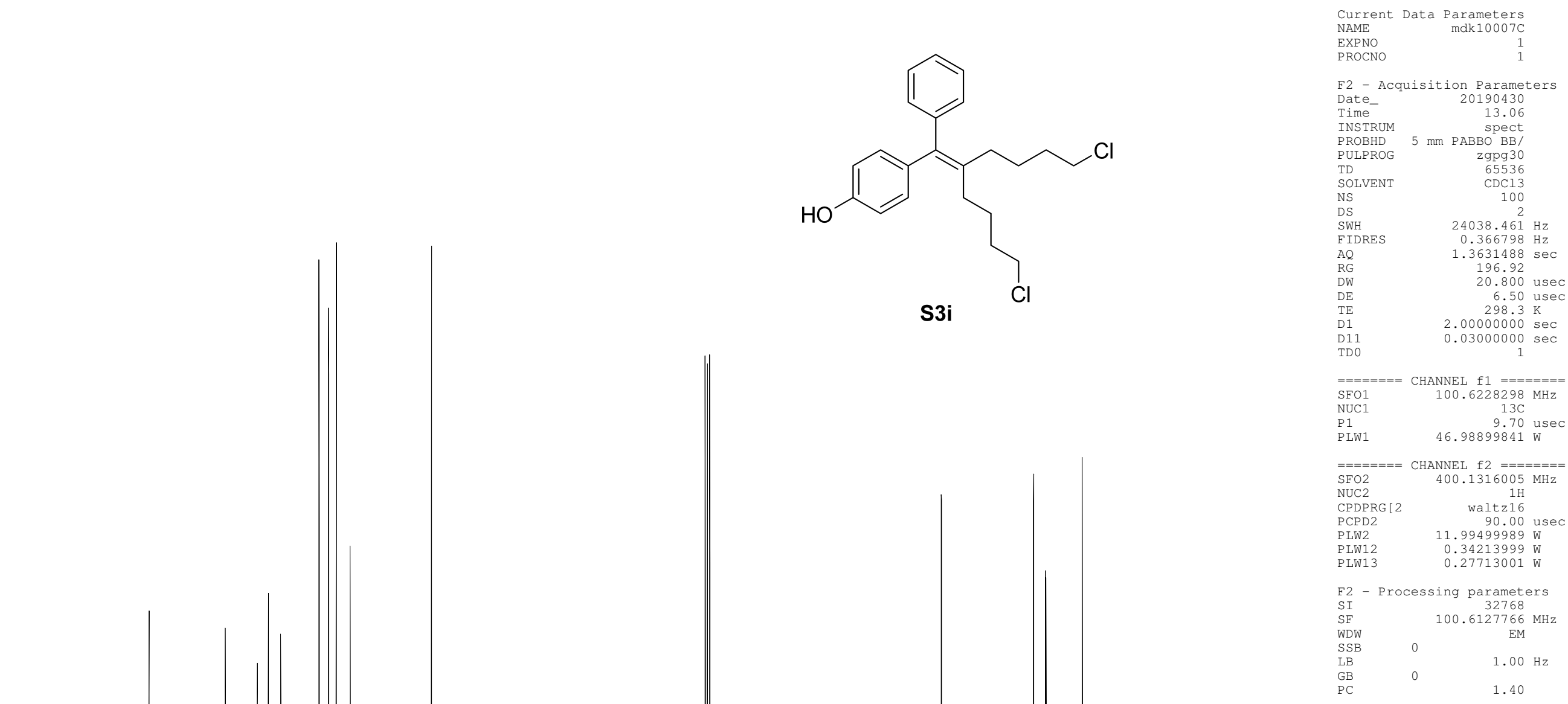

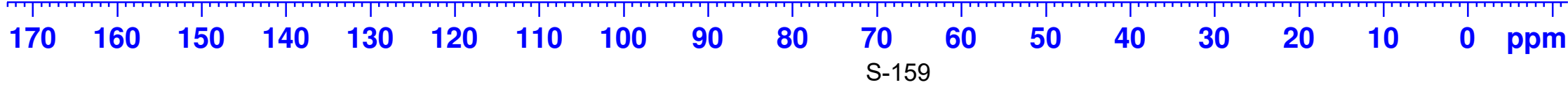




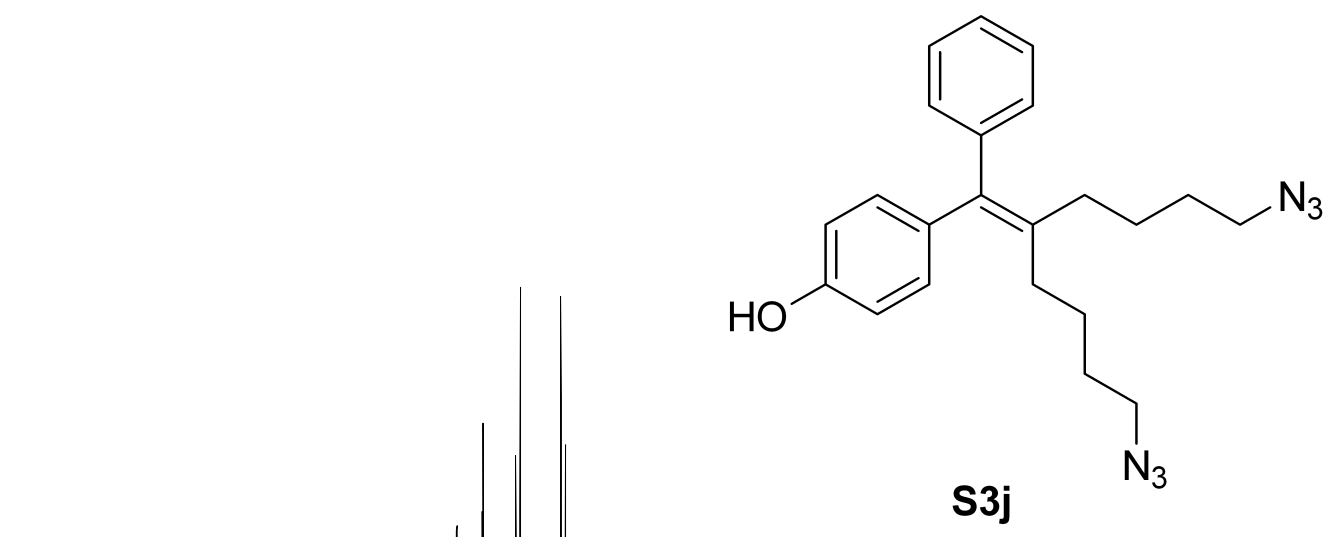

S3j

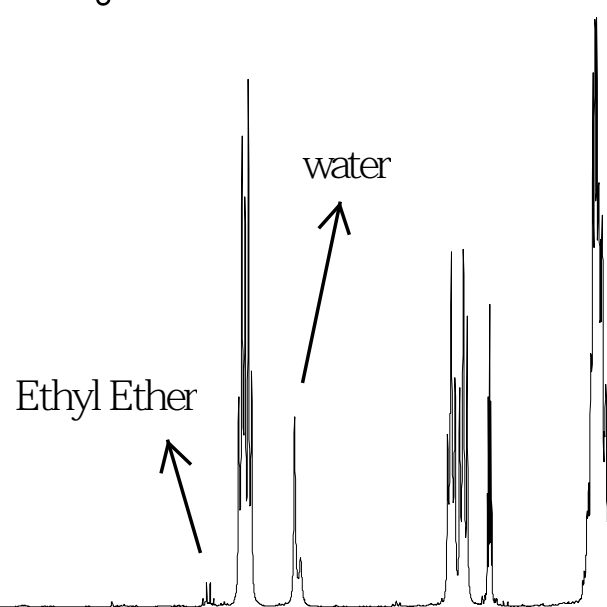

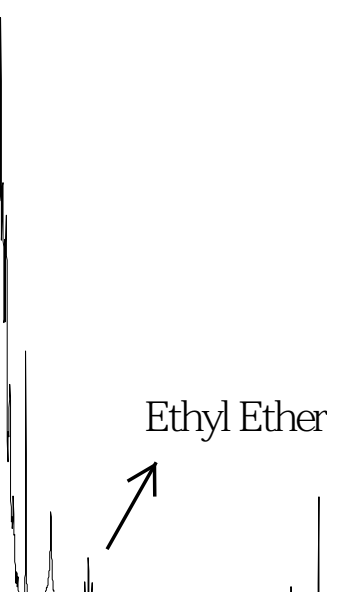

BP UKRER $(1>$

Current Data Parameters NAME

EXPNO

mak $11079 \mathrm{H}$

PROCNO

F2 - Acquisition Parameters

Date__ 20190813

Time $\quad 21.30$

spect

PULPROG

$\begin{array}{lr}\text { TD } & \text { Zg30 } \\ & 65536\end{array}$

SOLVENT Acetone

TS

DS

SWH

FIDRES

FIDR
AQ
RG

$\mathrm{DW}$

$\mathrm{DE}$

TE

TD 0

$====$
SFO1
NUC1

$\mathrm{N} 1$

Acetone
16

$8012.820 \mathrm{~Hz}$

$0.122266 \mathrm{~Hz}$

$4.0894465 \mathrm{sec}$

27.78

62.400 usec

6.50 usec

$1.00000000 \mathrm{sec}$

CHANNEI $\mathrm{f} 1 \mathrm{=}=======$

$400.1324710 \mathrm{MHz}$

(14.50

50 usec

PLW1 11.99499989 W

F2 - Processing parameters

SI 65536

SF $400.1299909 \mathrm{MH}$

EM

$0.30 \mathrm{~Hz}$

1.00

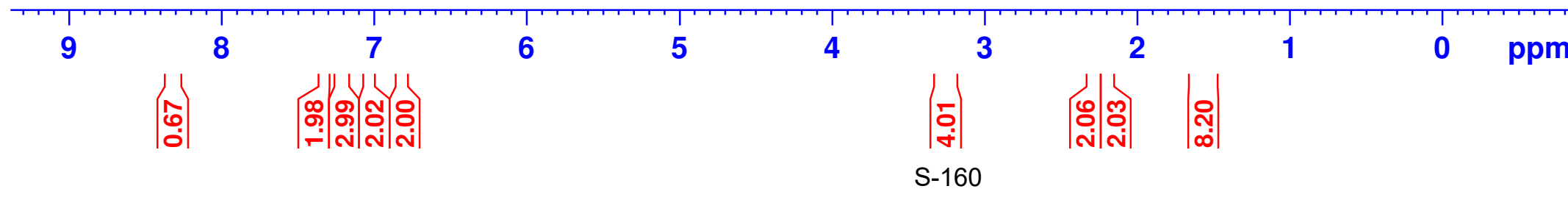




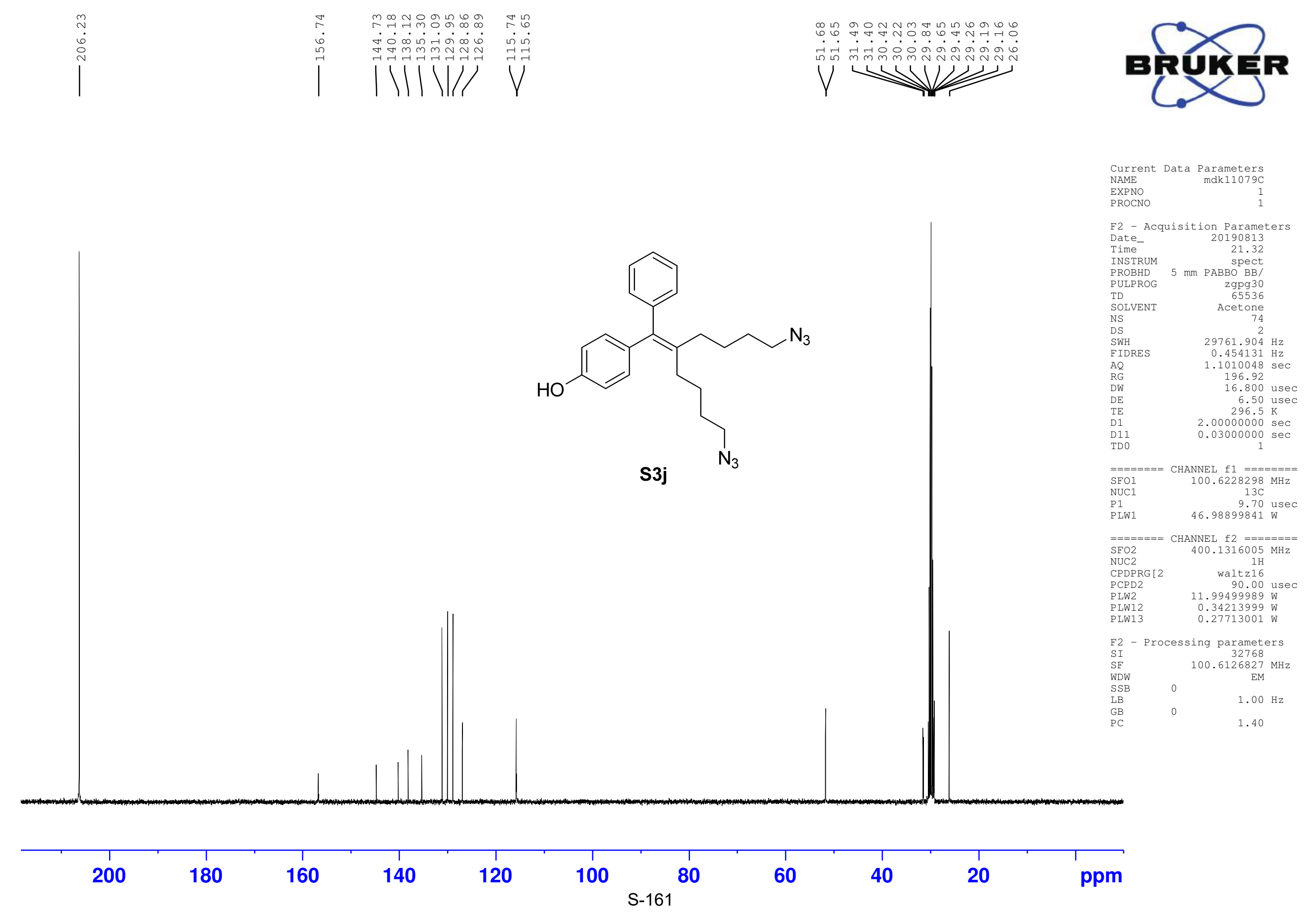


<smiles>CCC(CC)=C(c1ccccc1)c1ccc(OC)cc1</smiles>

$\mathrm{MeO}$

\section{S3a'}
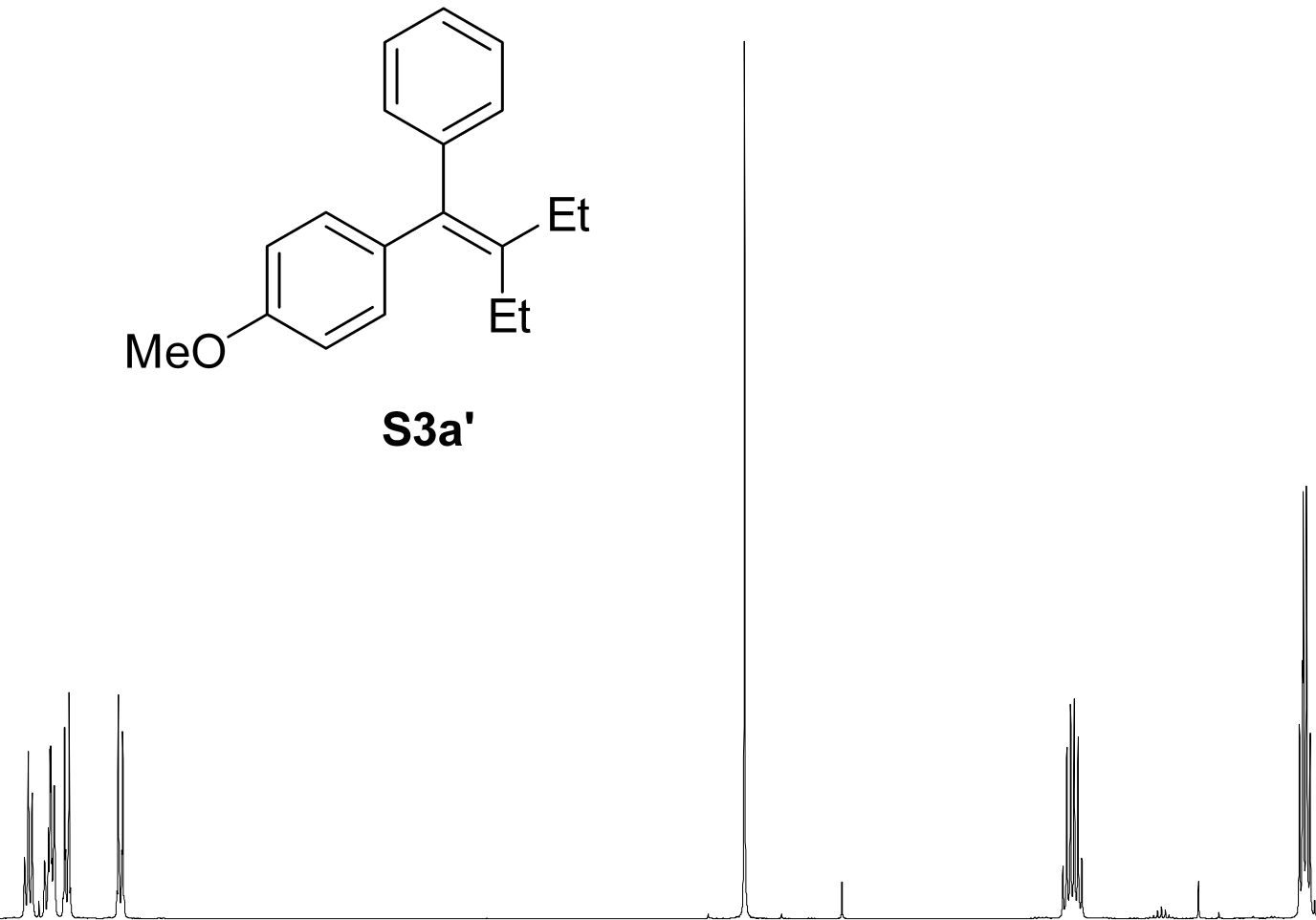

\section{sR $(1)$}
Current Data Parameters NAME
PROCNO
mdk $11053 \mathrm{H}$

F2 - Acquisition Parameters

Date_ 20190810

$\begin{array}{lr}\text { Time } & 10.26\end{array}$

INSTRUM spect

ROBHD

Zg30
PULPROG

TD 65536

$\begin{array}{lr}\text { SOLVENT } & \text { CDCl3 } \\ \text { NS } & 16\end{array}$

NS

DSH

$\quad 8012.820 \mathrm{~Hz}$

$0.122266 \mathrm{~Hz}$

$\begin{array}{lr}\text { AQ } & 4.0894465 \\ \text { RG } & 31.55\end{array}$

$\begin{array}{lr}\text { RG } & 31.55 \\ \text { DW } & 62.400 \text { usec }\end{array}$

6.50
$\mathrm{DE}$

D1 $1.00000000 \mathrm{sec}$

TDO

$====$

$\mathrm{SFO1}$

$\mathrm{NUC1}$
$\mathrm{P} 1$

$1.00000000 \mathrm{sec}$
1

CHANNEL $\mathrm{f} 1 \mathrm{l}========$ $400.1324710 \mathrm{MHz}$ $\begin{array}{r}14.50 \\ \hline\end{array}$ usec PLW1 11.99499989 W

F2 - Processing parameters SI 65536 $\begin{array}{lc}\text { SF } & 400.1300342 \mathrm{MHz} \\ \text { WDW } & \mathrm{EM}\end{array}$

SSB

$0.30 \mathrm{~Hz}$

1.00 


\section{experen}<smiles>CCC(=Cc1ccccc1)c1ccc(OC)cc1</smiles>

$$
\begin{aligned}
& \begin{array}{lr}
\text { Current } & \text { Data } \text { Parameters } \\
\text { NAME } & \text { mdk11053C } \\
\text { EXPNO } & 1 \\
\text { PROCNO } & 1
\end{array} \\
& \text { F2 - Acquisition Parameters } \\
& \text { Date } \quad 20190810 \\
& \begin{array}{lr}
\text { Time } & 10.29 \\
\text { INSTRUM } & \text { spect } \\
\text { PROBHD } & 5 \mathrm{~mm} \text { PABBO BB/ }
\end{array} \\
& \text { PULPROC } \\
& \begin{array}{l}
\text { TD } \\
\text { SOLVENT }
\end{array} \\
& \begin{array}{l}
\text { NS } \\
\text { DS } \\
\text { SWH }
\end{array} \\
& \begin{array}{lr}
\text { SWH } & 24038.461 \mathrm{~Hz} \\
\text { FIDRES } & 0.366798 \mathrm{~Hz}
\end{array} \\
& \begin{array}{l}
\mathrm{AQ} \\
\mathrm{RG}
\end{array} \\
& \begin{array}{l}
\mathrm{DW} \\
\mathrm{DE} \\
\mathrm{TE}
\end{array} \\
& \begin{array}{l}
\text { TE } \\
\text { D1 } \\
\text { D11 }
\end{array} \\
& 1.3631488 \mathrm{sec} \\
& 20.800 \text { usec } \\
& 6.50 \text { usec } \\
& \begin{array}{l}
2.00000000 \mathrm{sec} \\
0.03000000 \mathrm{sec}
\end{array} \\
& \text { TD } 0 \\
& \begin{array}{l}
\text { fHANNEL } \mathrm{fl}== \pm=== \pm \\
\mathrm{SFO1} \quad 100.6228298 \mathrm{MHz}
\end{array} \\
& \begin{array}{l}
\text { NUC1 } \\
\text { P1 } \\
\text { PLW1 }
\end{array} \\
& \begin{array}{r}
13 \mathrm{C} \text { usec } \\
9.70 \text { use }
\end{array} \\
& 46.98899841 \mathrm{~W}
\end{aligned}
$$

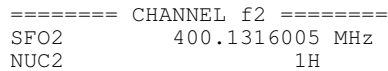

$$
\begin{aligned}
& \begin{array}{lr}
\text { NUC2 } & 1 \mathrm{H} \\
\text { CPDPRG [2 } & \text { waltz16 } \\
\text { PCPD2 } & 90.00 \text { usec }
\end{array} \\
& \begin{array}{lr}
\text { PLW2 } & 11.99499989 \mathrm{~W} \\
\text { PLW12 } & 0.34213999 \mathrm{~W}
\end{array} \\
& \text { PLW13 } \quad 0.27713001 \mathrm{~W} \\
& \begin{array}{c}
\text { F2 - Processing parameters } \\
\text { SI }
\end{array} \\
& \begin{array}{ll}
\text { SF } & 100.6127788 \mathrm{MHz}
\end{array} \\
& \text { GB } \\
& 1.00 \mathrm{~Hz}
\end{aligned}
$$




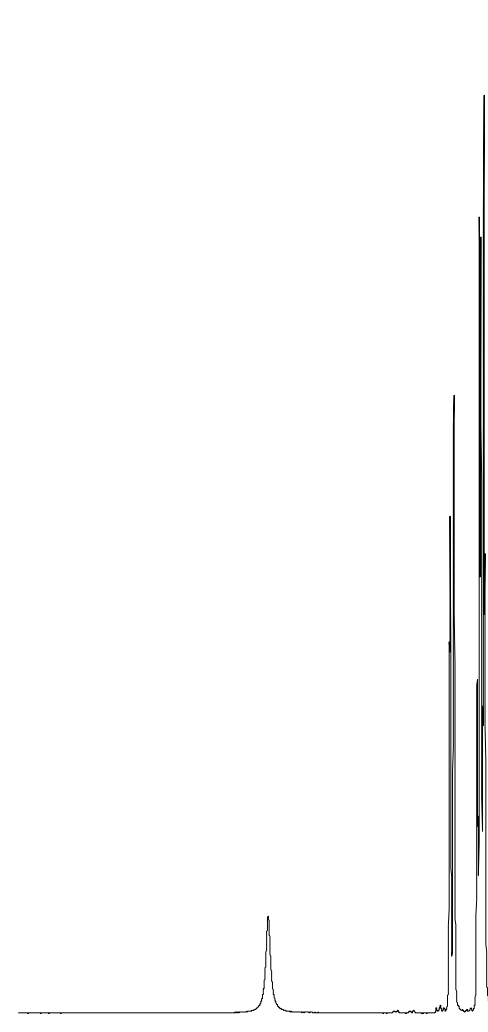

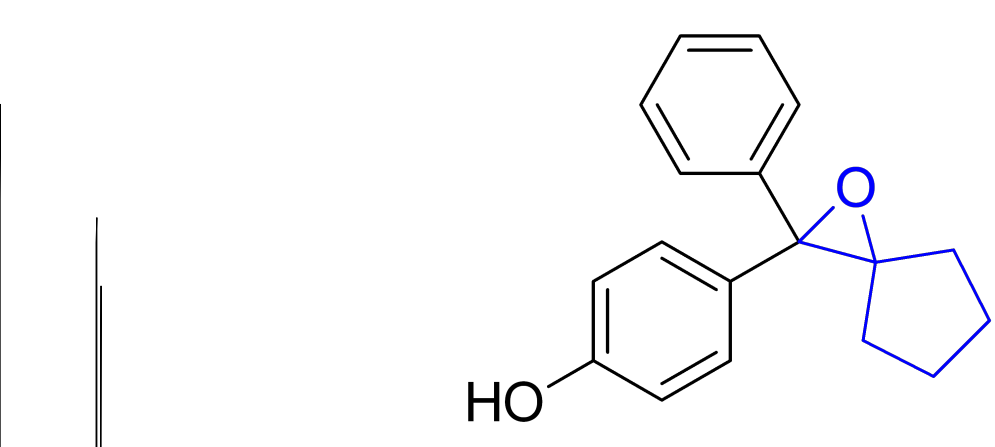

$1 \mathrm{a}$
9

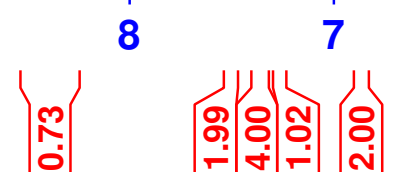

6

5

5

4

4

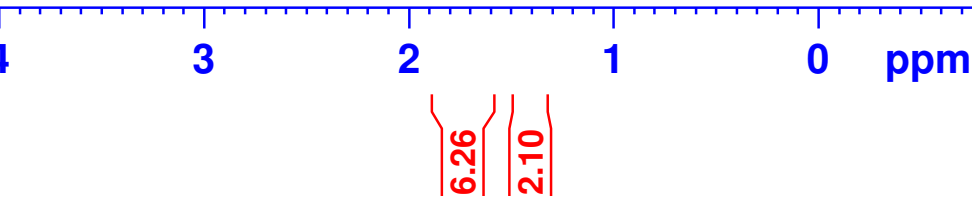

\section{Current Data Parameters NAME \\ PROCNO \\ $\operatorname{mdk} 9163 \mathrm{H}$}

F2 - Acquisition Parameters

Date_ 20190531

$\begin{array}{lr}\text { Time- } & 2019.37\end{array}$

SPect

PULPROG $5 \mathrm{~mm}$ PABBO BB/
zg30

$\begin{array}{lr}\text { TD } & \text { Zg30 } \\ & 65536\end{array}$

SOLVENT Acetone

DS

SWH

FIDRES

AQ

RG

DW

DE

D1
TD 0

$======$

$\mathrm{SFO1}$

$\mathrm{NUC1}$
$\mathrm{P} 1$

16
16

$8012.820 \mathrm{~Hz}$

$0.122266 \mathrm{~Hz}$

$4.0894465 \mathrm{sec}$

34.77

62.400 usec

6.50 usec

$1.00000000 \mathrm{sec}$

$$
1
$$

CHANNEL $\mathrm{fl}========$ $400.1324710 \mathrm{MHz}$ P1 14.50 usec PLW1 $11.99499989 \mathrm{~W}$

F2 - Processing parameters SI 65536 SF $\quad 400.1299912 \mathrm{MHz}$ WDW 


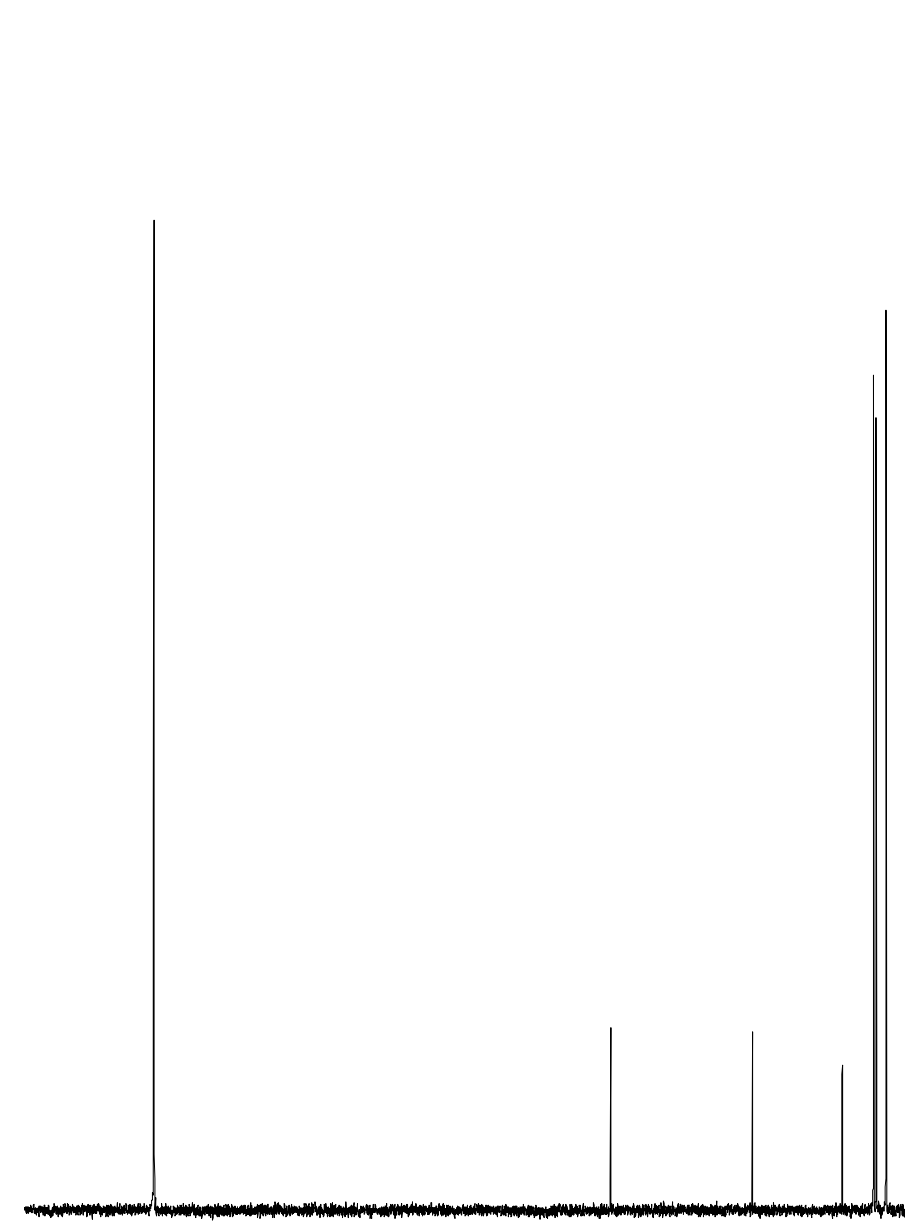

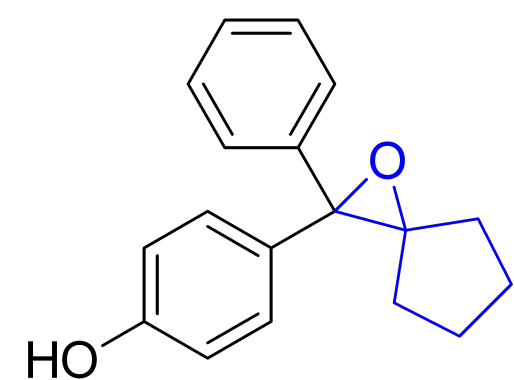

$1 a$

$\begin{array}{lc}\text { F2 - Acquisition Parameters } \\ \text { Date_ } \\ \text { Time } & 20190531\end{array}$

Time
INSTRUM

spect
PROBHD $5 \mathrm{~mm}$ PABBO BB/

PULPROG

TD
SOLVENT

NS
DS
SWH
FIDRES

FIDRES
AQ
RG
DW
DE

$\mathrm{DE}$
$\mathrm{TE}$
$\mathrm{D} 1$

TE
D1
D11
TD0

$=== \pm===$ CHANNEL $\mathrm{f} 1 \quad=======$
SFO1
$100.6228298 \mathrm{MHz}$

NUC1
P1

zgpg30
65536

41
2

$0.366798 \mathrm{~Hz}$

196.92

20.800 usec

6.50 usec

$2.00000000 \mathrm{sec}$
$0.03000000 \mathrm{sec}$

PLW1

. 9889980

$= \pm== \pm===$ CHANNEL $f 2 \quad======= \pm$
SFO2
$400.1316005 \mathrm{MHz}$

$\begin{array}{lr}\text { NUC2 } & 1 \mathrm{H} \\ \text { CPDPRG [2 } & \text { waltz16 }\end{array}$

$11.99499989 \mathrm{WI}$

PLW12
PLW13

$\begin{array}{lr}\text { F2 } & \text { - Processing parameters } \\ \text { SI } & 32768 \\ \text { SF } & 100.6126878 \mathrm{MHz}\end{array}$

SF

$00.6126878 \mathrm{MHz}$
$\mathrm{EM}$ 


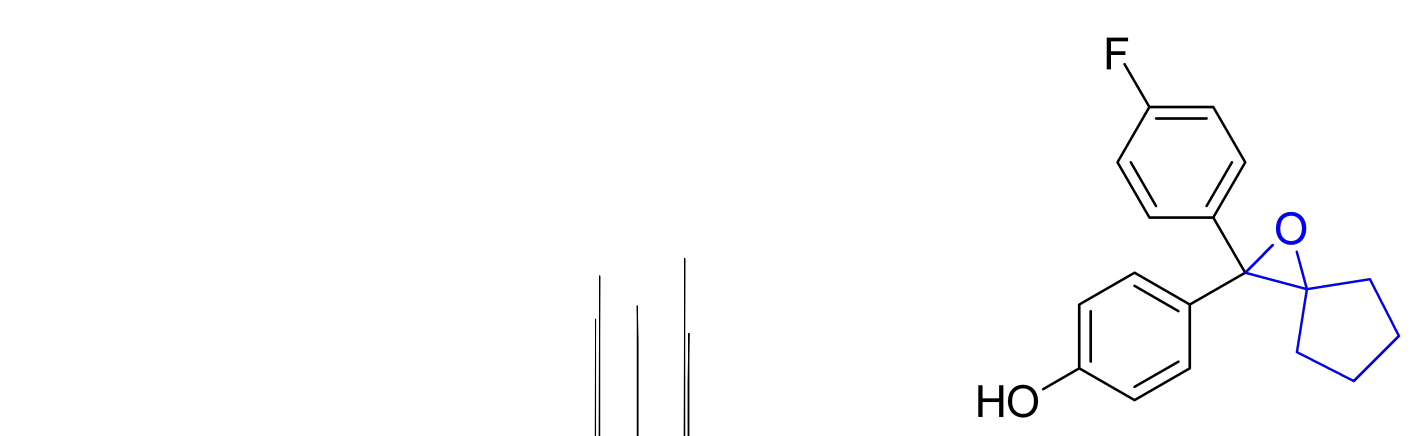

$1 \mathrm{~b}$

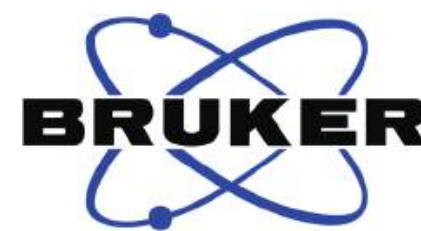

Current Data Parameters NAME

PROCNO

$\operatorname{mak} 8158 \mathrm{H}$

F2 - Acquisition Parameter

Date__ 20190225

Time 13.39

spect

$5 \mathrm{~mm}$ PABBO
PULP
$\mathrm{zg} 30$

$\begin{array}{lr}\mathrm{Zg} 30 \\ \mathrm{TD} & 65536\end{array}$

SOLVENT Acetone

S

DS

SWH

FIDRES

$\mathrm{AQ}$

RG

DW

$\mathrm{DE}$

D1
TD 0

$====$

$\mathrm{SFO1}$

$\mathrm{NUC1}$
$\mathrm{P} 1$

P1
PLW1

Acetone

$8012.820 \mathrm{~Hz}$

$8012.820 \mathrm{~Hz}$
$0.122266 \mathrm{~Hz}$

$4.0894465 \mathrm{~Hz}$

39.46

62.400 usec 6.50 usec

$1.00000000 \mathrm{sec}$ 1

CHANNEL $\mathrm{f} 1========$ $400.1324710 \mathrm{MHz}$ usec

F2 - Processing parameters SI $\quad 65536$ SF $\quad 400.1299910 \mathrm{MHz}$ WSB

SB

GB PC

$0.30 \mathrm{~Hz}$

1.00
10

\begin{abstract}
9
\end{abstract}

$\left|\begin{array}{l}\text { ‘ } \\ 0\end{array}\right|$

8

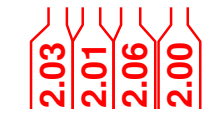

$7 \quad 6$

6

5

4

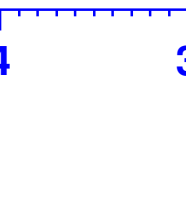

S-166

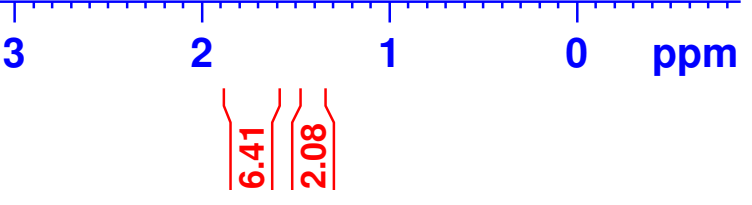




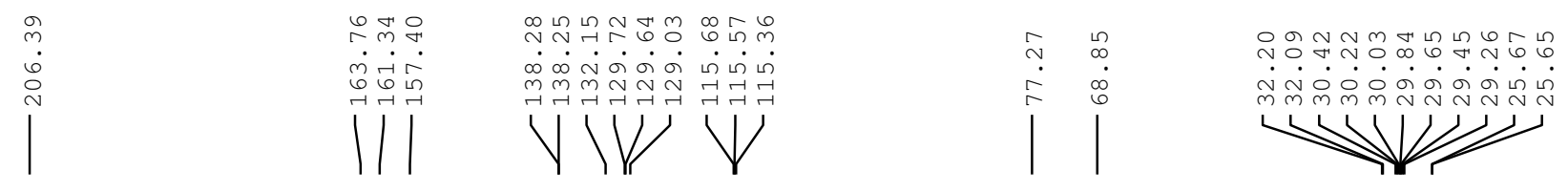

BR

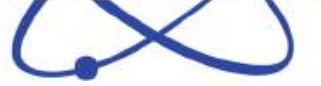

Current Data Parameters NAME

EXPNO

$\operatorname{mdk} 8158 \mathrm{c}$

PROCNO

F2 - Acquisition Parameters

Date__ 20190225

Time $\quad 13.42$

INSTRUM 5 mPECt

PROBHD $5 \mathrm{~mm}$ PABBO BB/

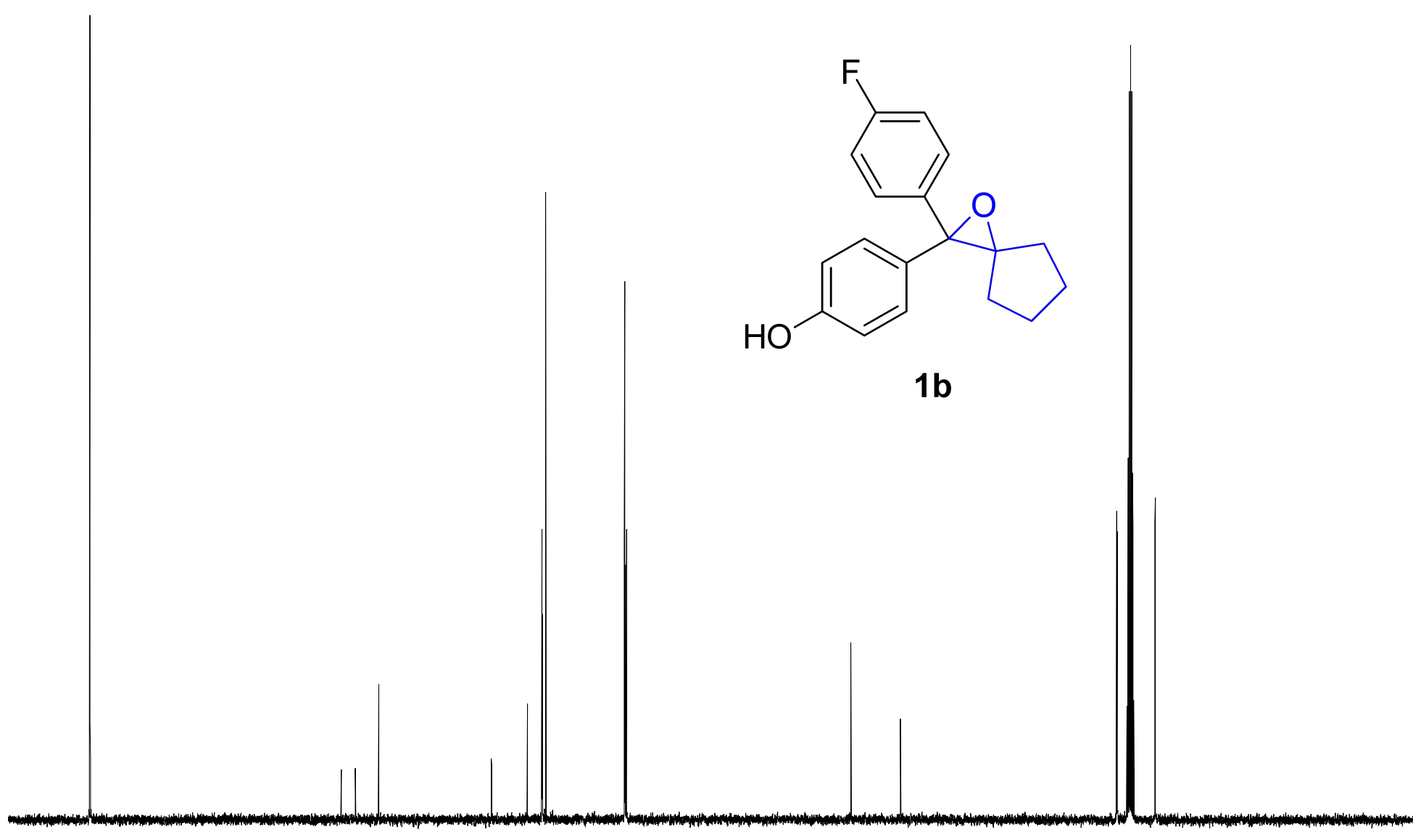

PULPROG

TD

zgpg 30
65536

SOLVENT

NS

DS

FIDRES $\quad 0.366798 \mathrm{~Hz}$

AQ $\quad 1.3631488 \mathrm{sec}$

RG $\quad 196.92$

DW 20.800 usec

$\begin{array}{ll}\text { DE } & 6.50 \text { usec } \\ \text { DE } & 295.8 \mathrm{~K}\end{array}$

$2.00000000 \mathrm{sec}$

D11 $0.03000000 \mathrm{sec}$

TD0

$==$ CHANNEL $f 1=======$

$100.6228298 \mathrm{MHz}$

9.730

PLW1 $\quad 46.98899841 \mathrm{~W}$

$=======$ CHANNEL $\mathrm{f} 2$ $========$

$\mathrm{SFO} 2$

$400.1316005 \mathrm{MHz}$

$1 \mathrm{H}$

CPDPRG [2 waltzl6

PCPD2 290.00 usec

PLW2 11.99499989 W

PIW12 $\quad 0.34213999$

PLW13 0.27713001

F2 - Processing parameters

SI 32768

$\begin{array}{lr}\text { SI } & 32768 \\ \text { SF } & 100.6126860 \mathrm{MHz}\end{array}$

WDW

$100.6126860 \mathrm{MHz}$
$\mathrm{EM}$

$1.00 \mathrm{~Hz}$

1.40

200

180

160

140

120

100

80

60

40

20

0 ppm 


\section{equiren}

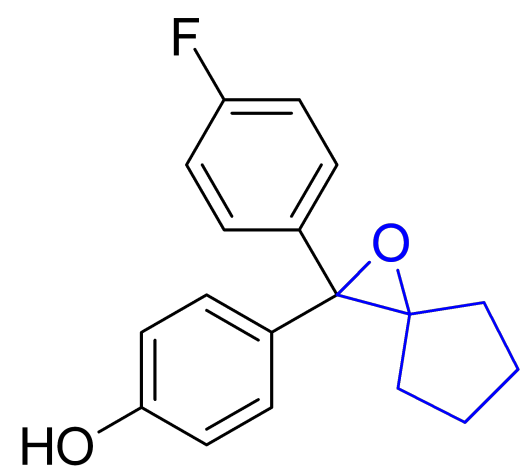

$1 b$
Current Data Parameters

NAME mdk8158F-decoup

EXPNO

F2 - Acquisition Parameters
Date_ 20190225

Date
Time

Time
INSTRUM

PULPROG

TD

NS
DS

SWH

$\mathrm{A} Q$
$\mathrm{RG}$

DW

TE
D1
D11

D11
TD 0

$========$ CHANNEL $\mathrm{f} 1 \mathrm{1}========$

SFO1 $\quad 376.4607162 \mathrm{MHz}$

P1 14.70 usec

$=======$
CHANNEL $f 2 \quad=======$
SFO2
NUC2

NUC2 $1 \mathrm{1H}$

$\begin{array}{lr}\text { PLW2 } & 11.99499989 \mathrm{~W} \\ \text { PLW12 } & 0.34213999 \mathrm{~W}\end{array}$

$\begin{array}{ll}\text { PLW12 } & 0.34213999 \text { W } \\ \text { PLW13 } & 0.27713001 \mathrm{~W}\end{array}$

F2 - Processing parameters
SI

$\begin{array}{lc}\text { SF } & 376.4983660 \mathrm{MHz} \\ \text { WDW } & \text { EM }\end{array}$

GB

\begin{tabular}{|c|c|c|c|c|c|c|c|c|c|c|c|}
\hline 20 & 0 & $\begin{array}{c}1 \\
-20\end{array}$ & $\begin{array}{c}1 \\
-40\end{array}$ & $\begin{array}{r}1 \\
-60\end{array}$ & $\begin{array}{l}1 \\
-80\end{array}$ & $\begin{array}{r}1 \\
-100\end{array}$ & $\begin{array}{r}1 \\
-120\end{array}$ & $\begin{array}{l}\text { 1 } \\
-140\end{array}$ & $\begin{array}{r}1 \\
-160\end{array}$ & $\begin{array}{r}1 \\
-180\end{array}$ & $\begin{array}{l}1 \\
-200\end{array}$ \\
\hline 20 & 0 & & & -00 & -00 & -100 & $5-168$ & -140 & -100 & -100 & $-\angle O 0$ \\
\hline
\end{tabular}


BP UKRER (x)

Current Data Parameters NAME mdk8155H-acetone EXPNO

PROCNO

F2 - Acquisition Parameters Date__ 20190627 $\begin{array}{lr}\text { Time- } & 15.09\end{array}$ INSTRUM spect PROBHD $5 \mathrm{~mm}$ PABBO BB/ PULPROG zg30 TD $\begin{array}{lr}\text { SOLVENT } & \text { Acetone } \\ \text { NS } & 16 \\ \text { DS } & 2\end{array}$ DWH $\mathrm{HO}^{-}$

$1 \mathrm{c}$

\section{water}

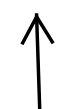

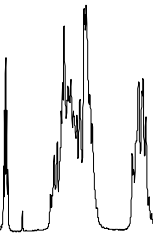
FIDRES F IDRE
$\mathrm{AQ}$
$\mathrm{RG}$ RG DW DE $\mathrm{TE}$
$\mathrm{D} 1$

$8012.820 \mathrm{~Hz}$ $8012.820 \mathrm{~Hz}$
$0.122266 \mathrm{~Hz}$ $4.0894465 \mathrm{sec}$ 27.78 62.400 usec 6.50 usec $1.00000000 \mathrm{~K}$ TD 0 $====$
$\mathrm{SFO1}$ $\mathrm{SFO} 1$
$\mathrm{NUC1}$ P1 P1
PLW1 1

CHANNEL f1 $========$ $400.1324710 \mathrm{MHz}$ 14.50 use $11.99499989 \mathrm{~W}$ $\begin{array}{lcc}\text { F2 } & \text { Processing parameters } \\ \text { SI } & 65536 \\ \text { SF } & 400.1299911 \mathrm{MHz} \\ \text { WDW } & 0 & \text { EM } \\ \text { SSB } & 0 & 0.30 \mathrm{~Hz} \\ \text { LB } & 0 & \\ \text { GB } & 0 & 1.00 \\ \text { PC } & & \end{array}$

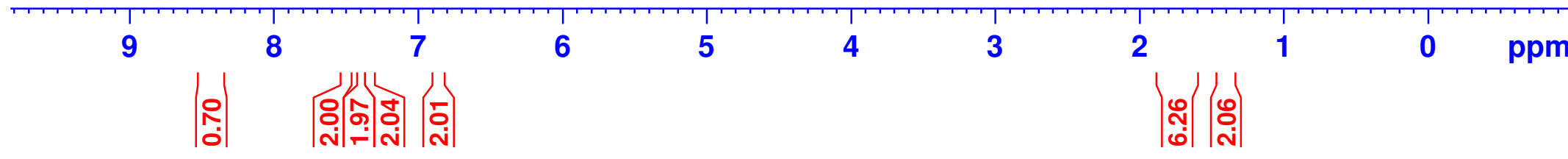




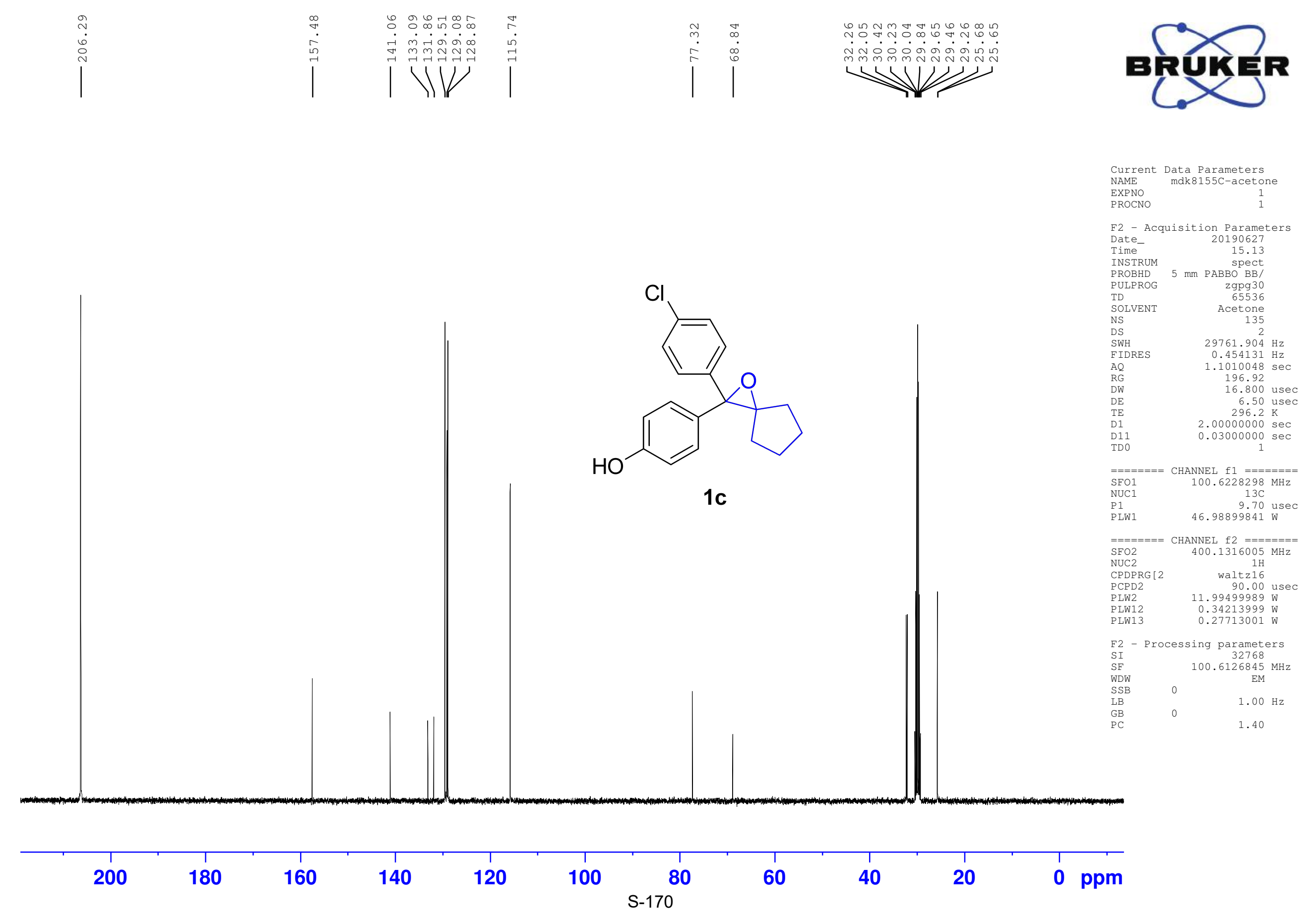




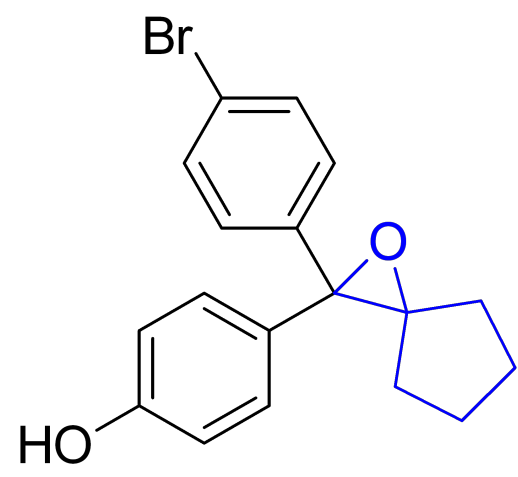

$1 d$
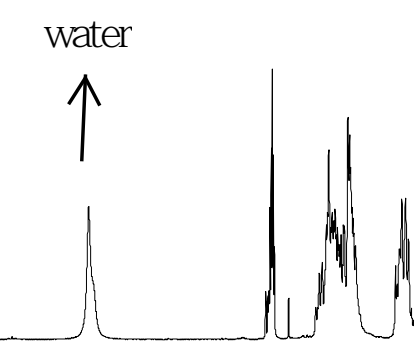

Curre
NAME
EXPNO

PROCNO

mdk9015H

F2 - Acquisition Parameters

Date_ 20190228

Time 13.47

$5 \mathrm{mmect}$

UITROG

$\begin{array}{lr}\text { TD } & \text { zg30 } \\ \text { TD } & 65536\end{array}$

SOLVENT Acetone

ST

DS

SWH

FIDRES

$\mathrm{AQ}$

RG

DW

$\mathrm{DE}$

D1

TD 0

$===$
$\mathrm{SFO} 1$

$\mathrm{SFO1}$

$\mathrm{NUC}$

P1
P LW1

Acetone 16

$8012.820 \mathrm{~Hz}$

$8012.820 \mathrm{~Hz}$

$4.0894465 \mathrm{~Hz}$

49.32
62.400

62.400 usec

6.50 usec

$1.00000000 \mathrm{sec}$

CHANNEI $\mathrm{f} 1=======$ $400.1324710 \mathrm{MHz}$

4.50 use

T2 - Pro

- Processing parameters

SI 65536

SF $\quad 400.1299911 \mathrm{MHz}$

SSB

EM

$0.30 \mathrm{~Hz}$

1.00

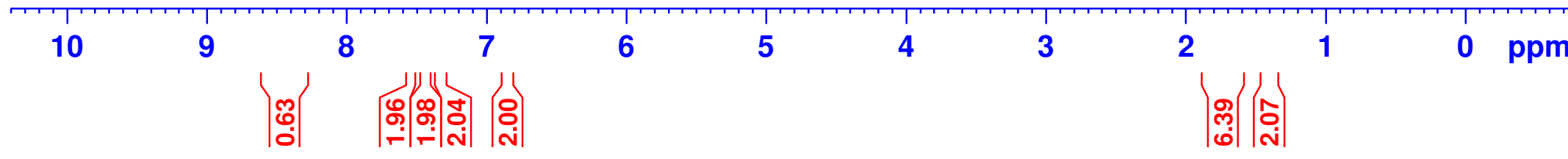




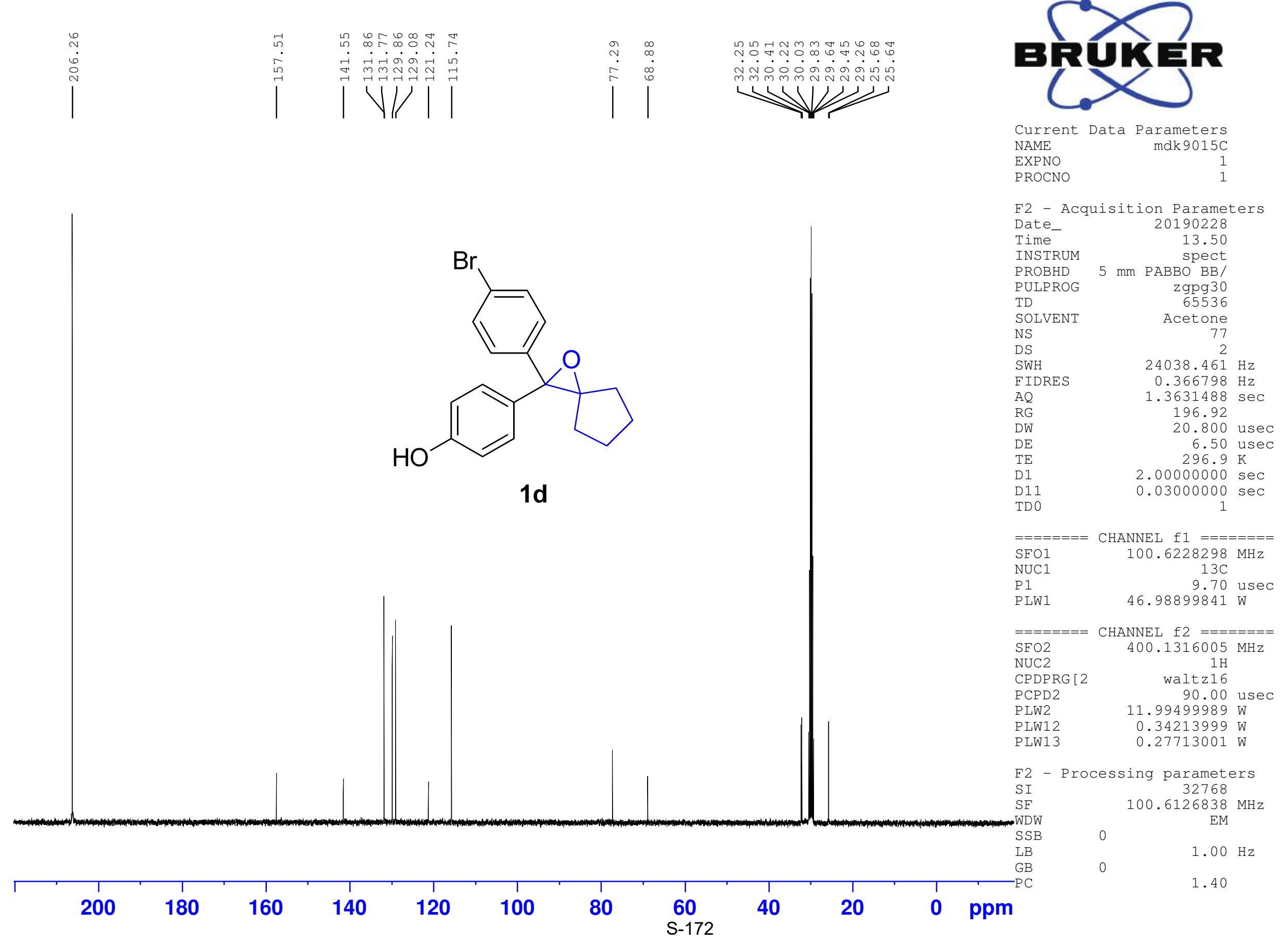




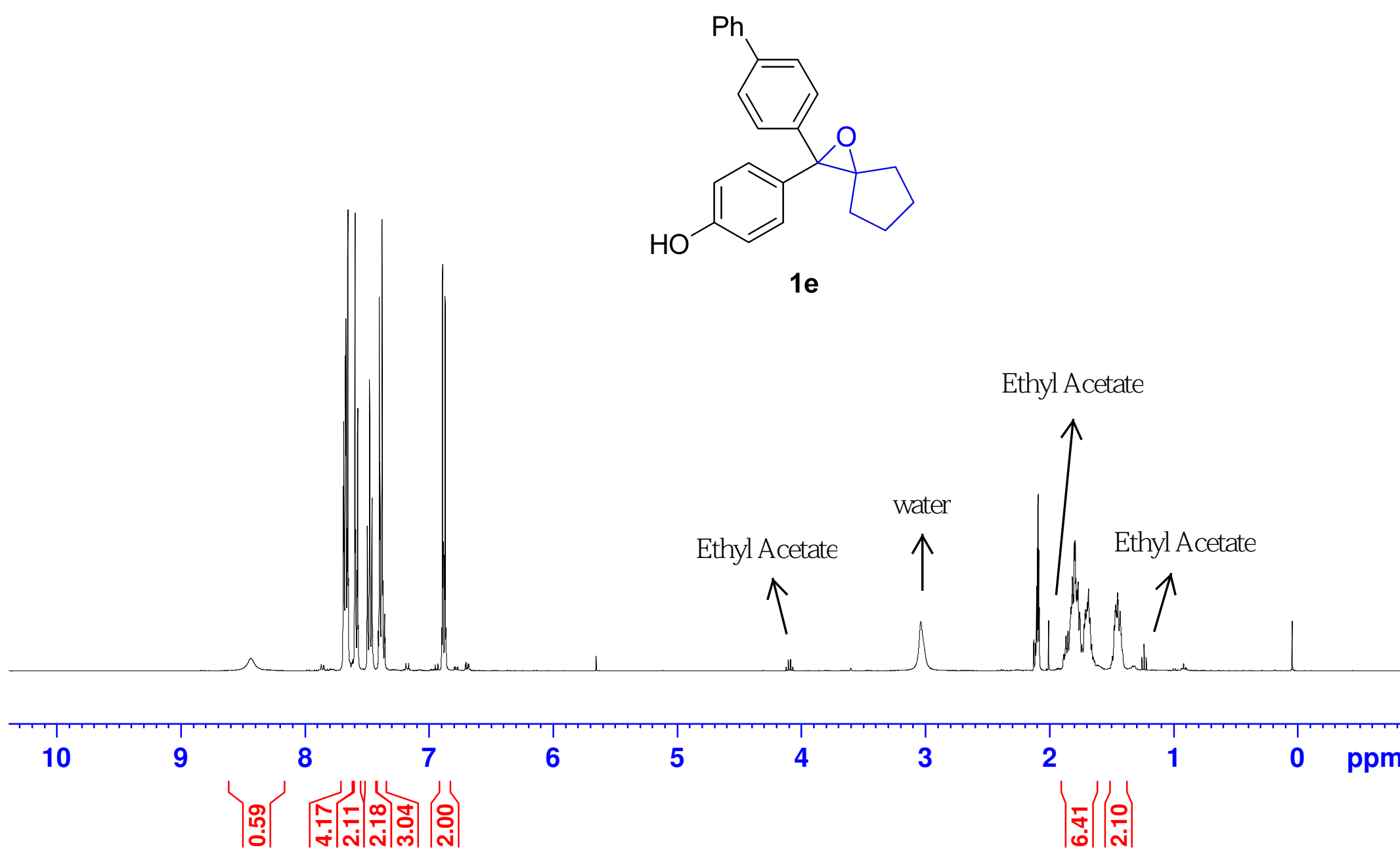

\section{eperen

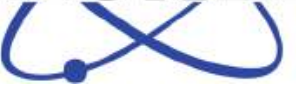

$\begin{array}{lr}\text { Current Data Parameters } \\ \text { NAME } & \text { mak } 9080 \mathrm{H}\end{array}$ EXPNO

1

F2 - Acquisition Parameters Date_ 20190319

$\begin{array}{lr}\text { Time } & 13.09\end{array}$

SPect

PULPROG $5 \mathrm{~mm}$ PABBO $\mathrm{zg} 30$

$\begin{array}{lr}\text { TD } & \text { Zg30 } \\ & 65536\end{array}$

SOLVENT Acetone

NS

SWH

FIDRES

$\mathrm{AQ}$

RG

DW

TE

TD 0

$===$

$\mathrm{SFO1}$

UUC

P 1
PLW1

16
16

$8012.820 \mathrm{~Hz}$

$0.122266 \mathrm{~Hz}$

$4.0894465 \mathrm{sec}$

31.55

62.400 usec

6.50 usec

$1.00000000 \mathrm{sec}$

1

$$
1
$$

CHANNEL $\mathrm{f} 1 \mathrm{l}========$

$400.1324710 \mathrm{MHz}$

LW1 14.50 usec

2 Processing parameters

SI $\quad 65536$

SF $\quad 400.1299910 \mathrm{MH}$

WSW

EM

$0.30 \mathrm{~Hz}$

1.00 

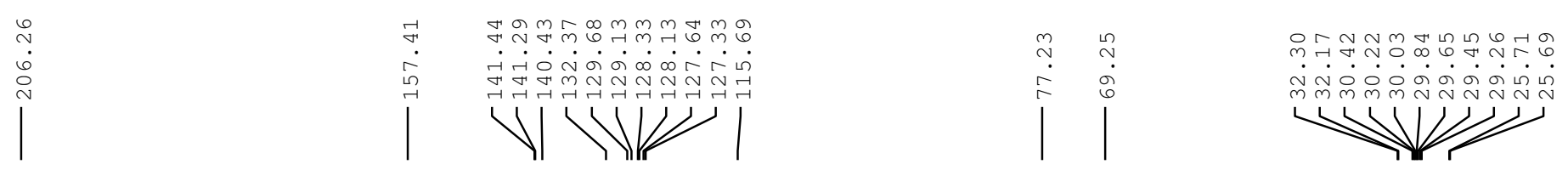

\section{equirien}
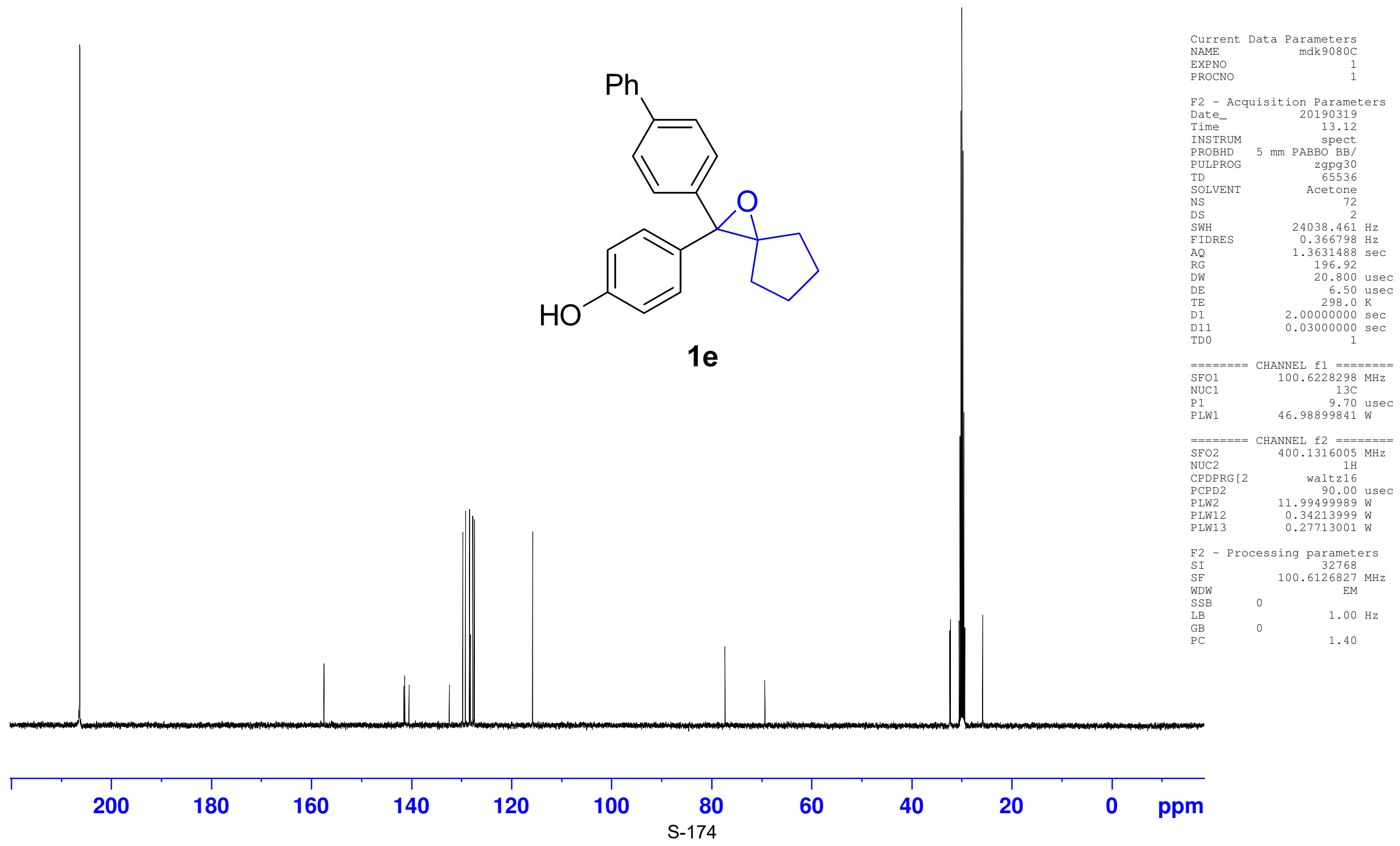


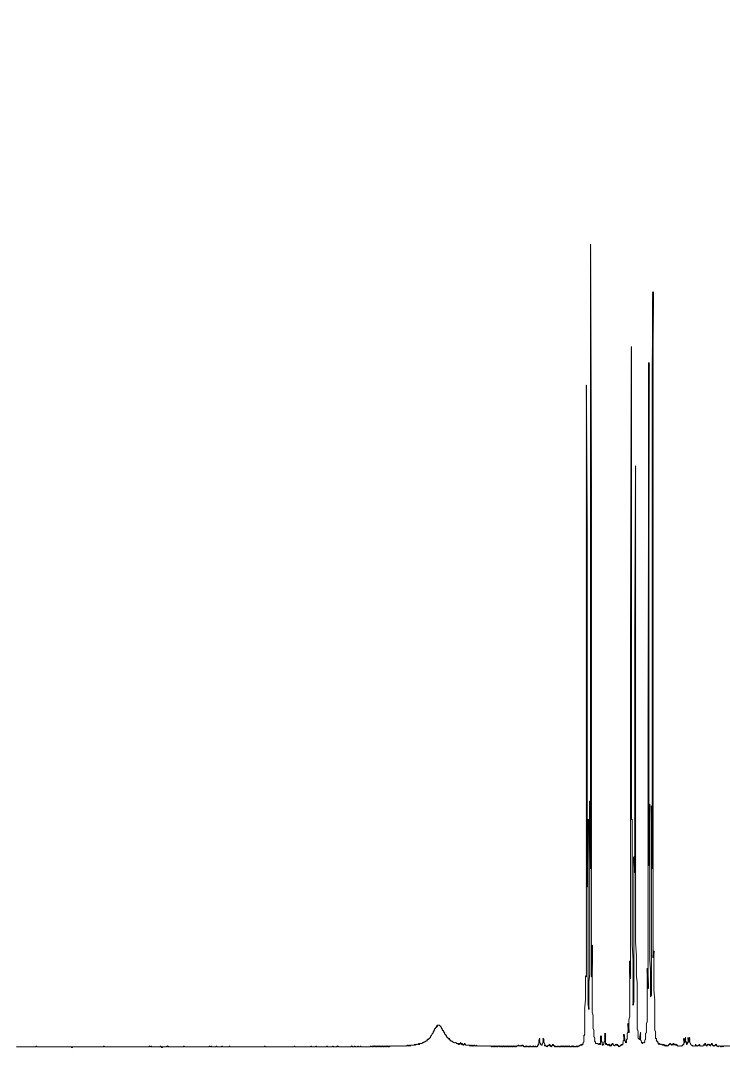

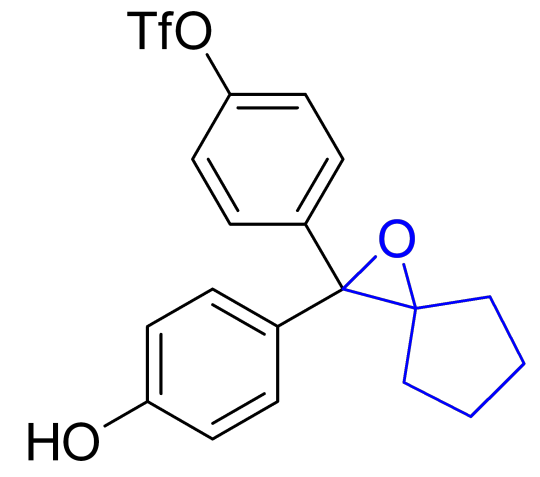

$1 f$
10

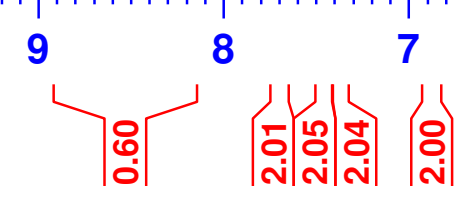

인
6

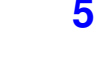

5

s-1

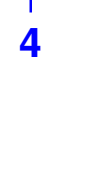

4
Current Data Parameters NAME

EXPNO

mdk $9101 \mathrm{H}$

F2 - Acquisition Parameters

Date__ 20190323

Time $\quad 12.58$

spect
INSTRUM

UIPOG

$\begin{array}{lr}\text { TD } & \text { Zg30 } \\ & 65536\end{array}$

SOLVENT Acetone

$\begin{array}{lr}\text { NS } & \text { Acetone } \\ \text { DS } & 16\end{array}$

DS

$8012.820 \mathrm{~Hz}$

IDRES $\quad 0.122266 \mathrm{~Hz}$

$\mathrm{AQ}$

RG

DW

TE

D1
TD 0

$4.0894465 \mathrm{sec}$ 54.81

62.400 usec

6.50 use

$1.00000000 \mathrm{sec}$

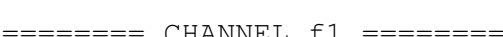

SEO1

SFO1

P1

CHANNEL $\mathrm{f} 1====$ $400.1324710 \mathrm{MHz}$

PLW

14.50 usec

$11.99499989 \mathrm{~W}$

Processing parameters

SI 65536

SF $\quad 400.1299911 \mathrm{MHz}$

SSB

EM

$0.30 \mathrm{~Hz}$

1.00 


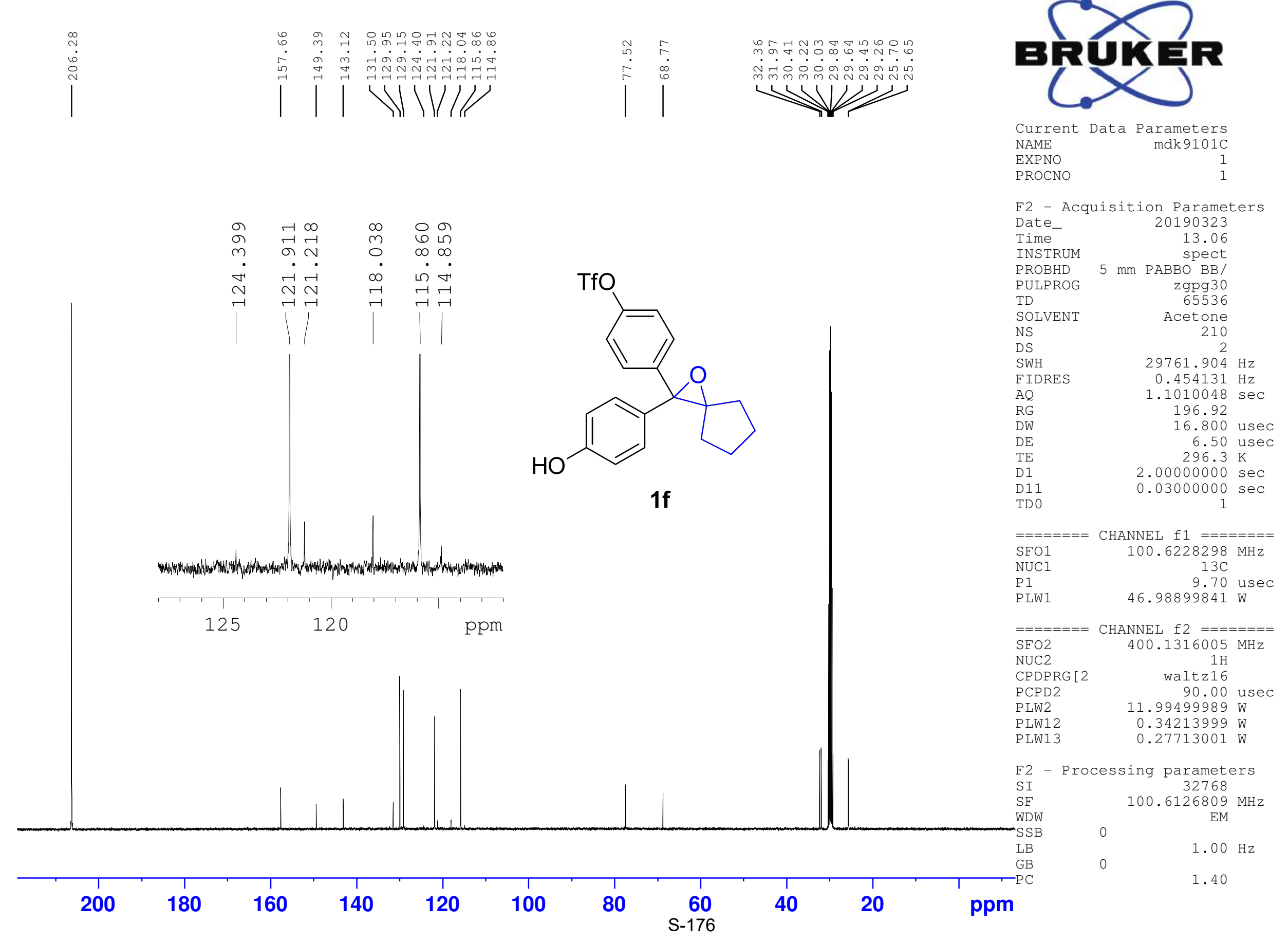




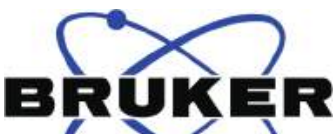
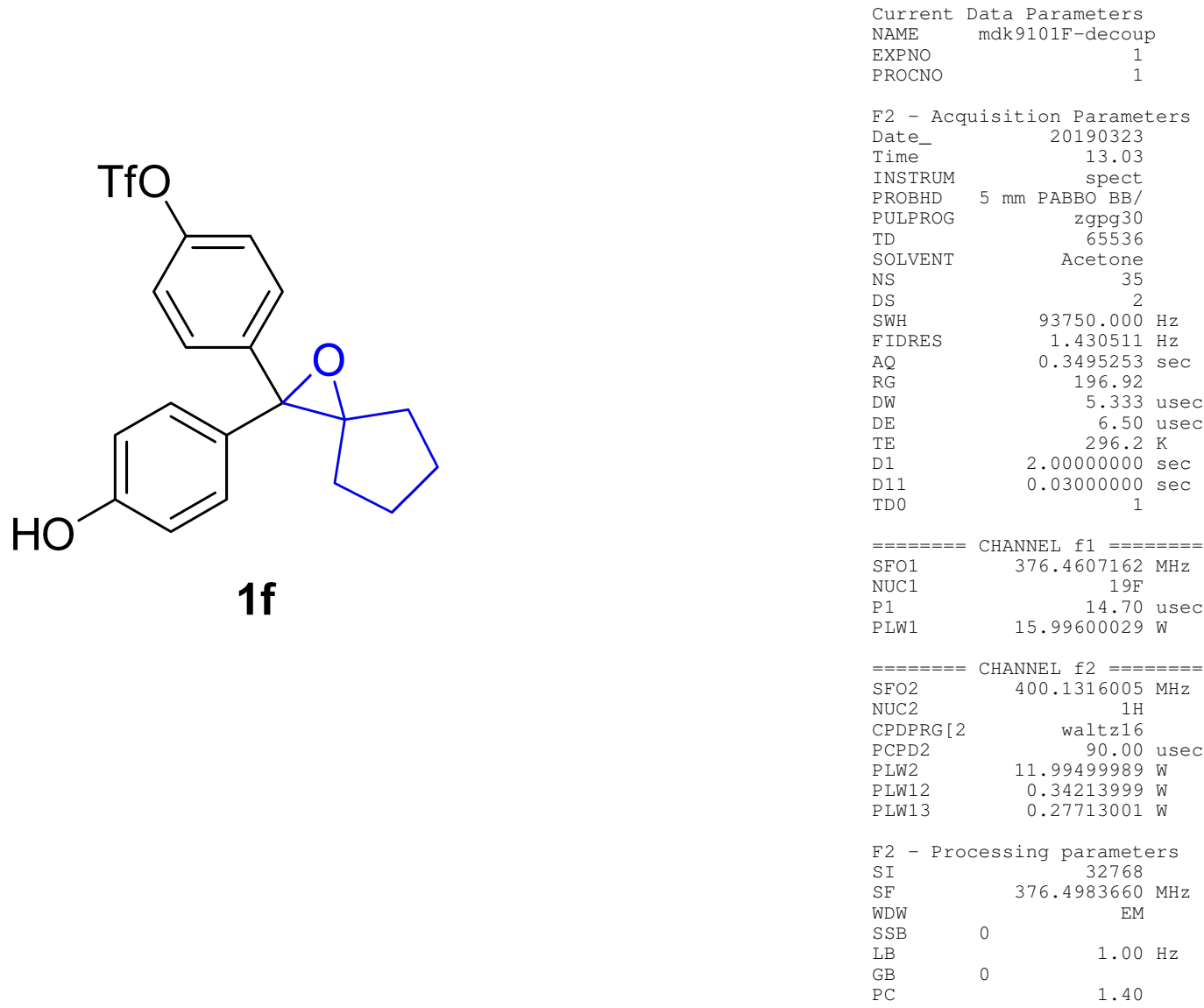

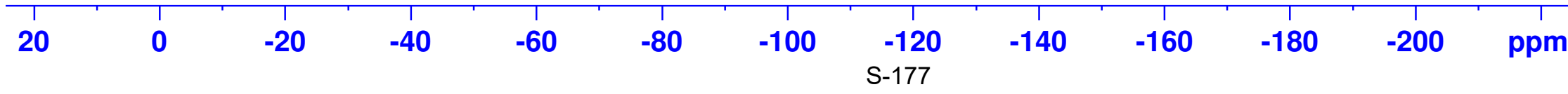


F2 - Acquisition Parameters

Date__ 20190325

Time $\quad 13.01$

spect
PROBHD $5 \mathrm{~mm}$ PABBO BB/

$\begin{array}{rr}\text { PROBHD } 5 \mathrm{~mm} \mathrm{PABBO} \mathrm{BB} / \\ \text { PULPROG } & \mathrm{zg} 30\end{array}$

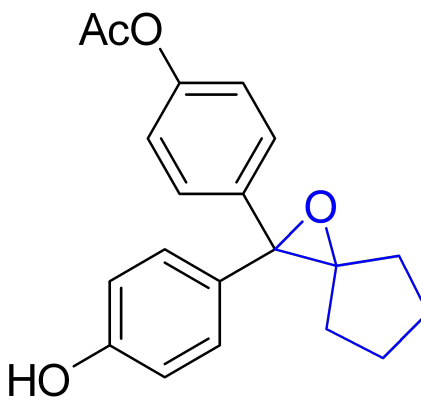

19

TD

SOLVENT

NS

DS

SWH
FIDRES

AO

AQ

DW

$\mathrm{DE}$

TE

TDO

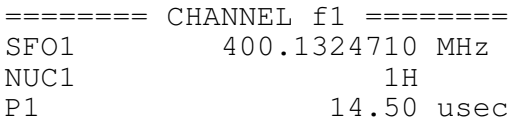

P1 14.50 usec

PLW1 $11.99499989 \mathrm{~W}$

F2 - Processing parameter

SI 65536

SF $\quad 400.1299910 \mathrm{MHz}$

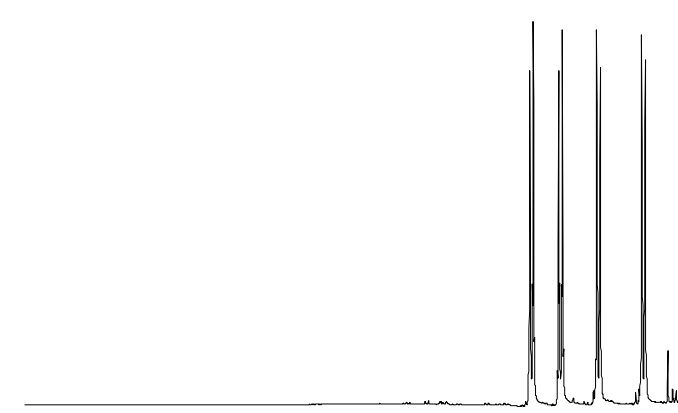

WDW

$S S B$

400.1299910
EM

GB

$0.30 \mathrm{~Hz}$

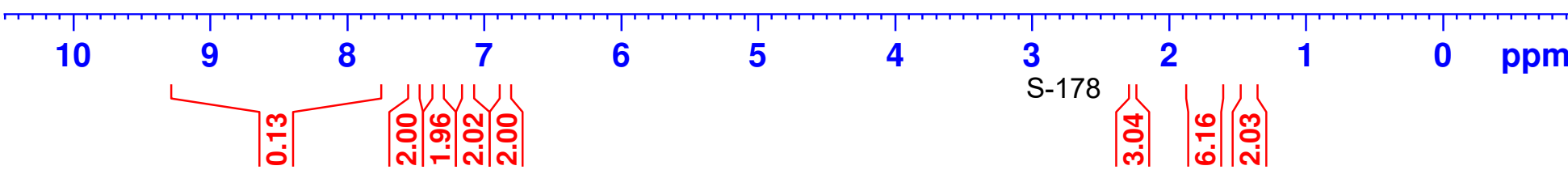




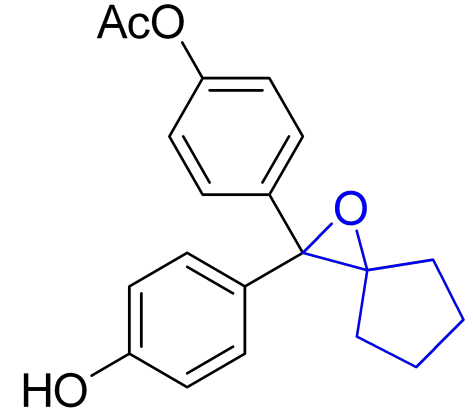

$1 \mathrm{~g}$ $(\times)$

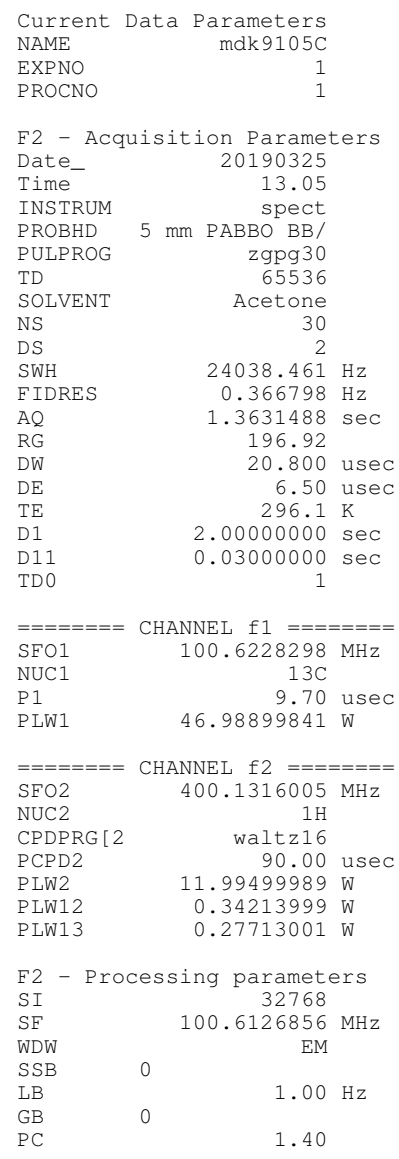

\begin{tabular}{|rrrrrrrrrrrrr}
\hline 200 & 180 & 160 & 140 & 120 & 100 & 80 & 60 & 40 & 20 & 0 & ppm
\end{tabular}



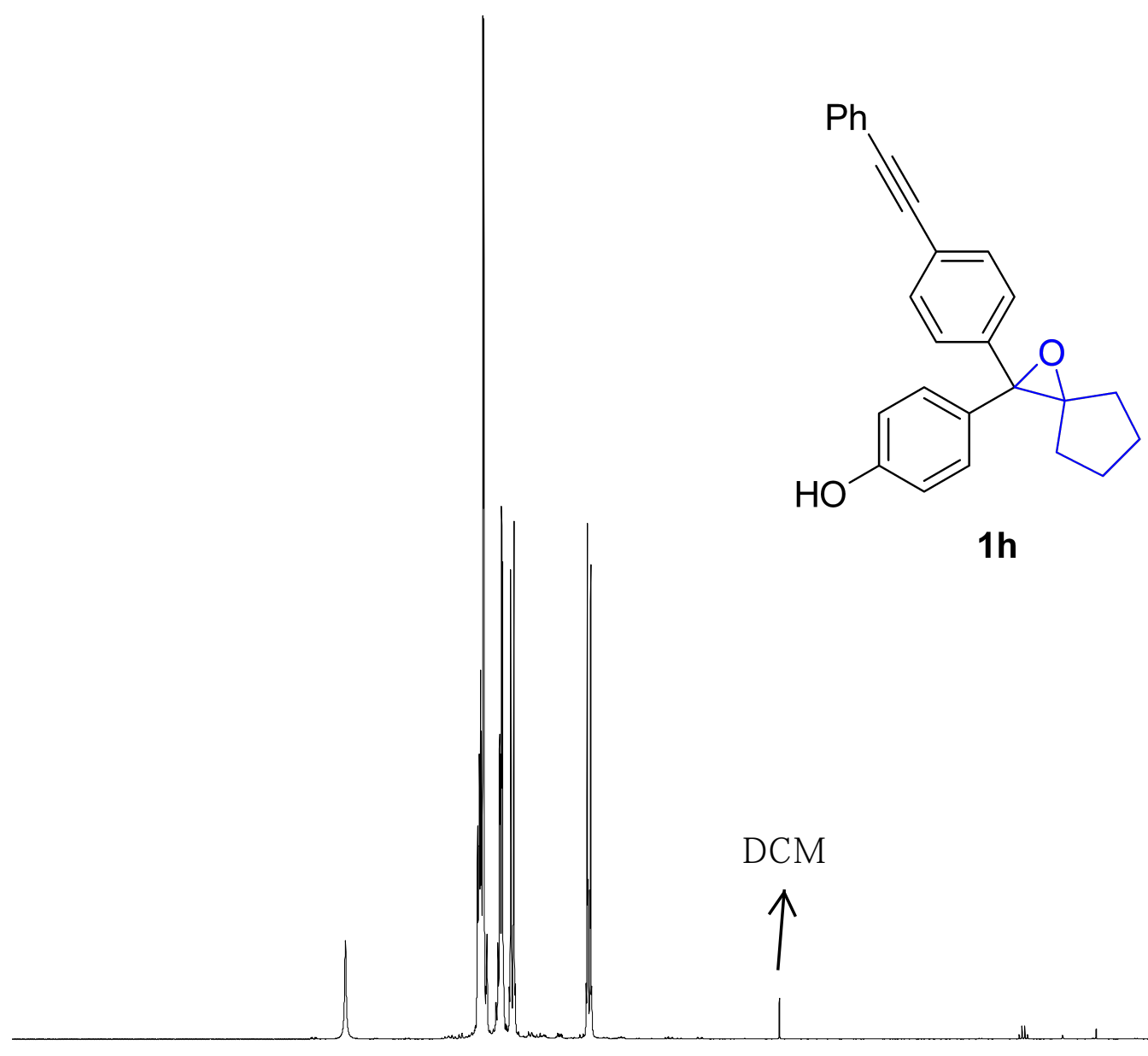

NAME

EXPNO

arameters

PROCNO

1

F2 - Acquisition Parameters

Date_ $\quad 20190416$

$\begin{array}{ll}\text { Time } & 13.37\end{array}$

$5 \mathrm{~mm}$ spect

PULPROG $\quad$ zg30

$\begin{array}{lr}\text { TD } & \text { Zg30 } \\ & 65536\end{array}$

SOLVENT Acetone

NS

DS

SWH

FIDRES

AQ

RG

$\mathrm{DW}$
$\mathrm{DE}$
$\mathrm{TE}$

TE

16
16

$8012.820 \mathrm{~Hz}$

$0.122266 \mathrm{~Hz}$

$4.0894465 \mathrm{sec}$ 49.32

62.400 usec 6.50 usec

$1.00000000 \mathrm{sec}$

TD 0

$$
1
$$

SFO1

NUC

CHANNEL f $1========$

water

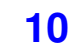

10

9

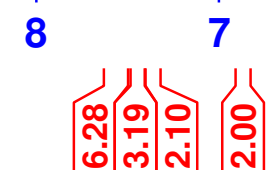

6

5

4

3

2

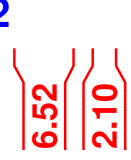

100 ppm 


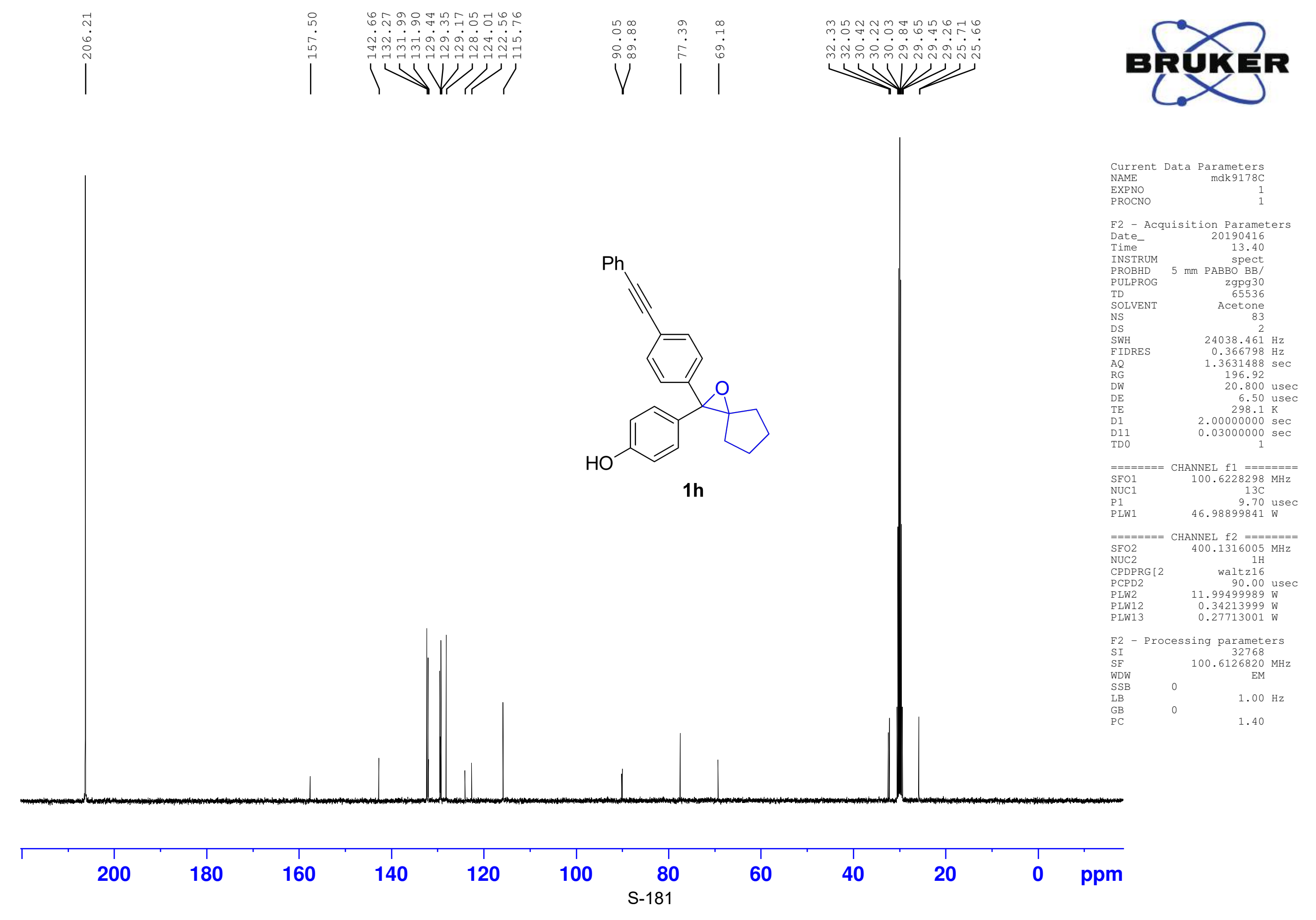




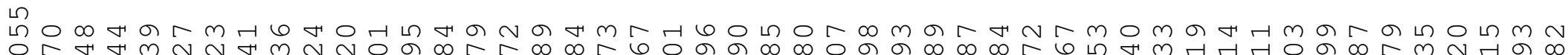

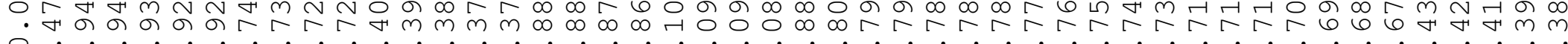

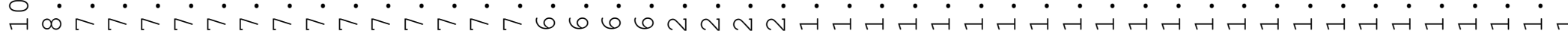

Current Data Parameters NAME mdk $9190 \mathrm{H}$
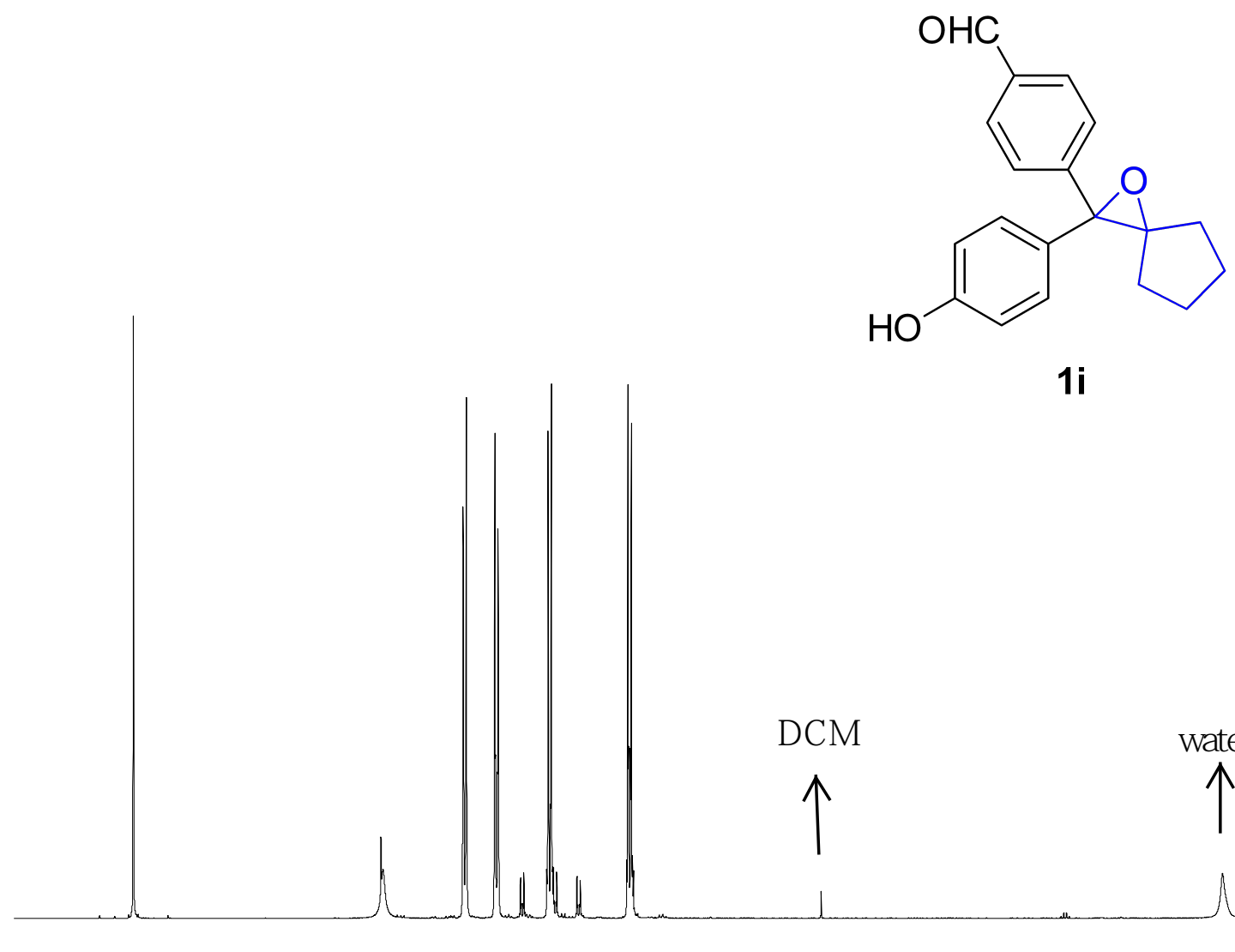

PROCNO

1
1

F2 - Acquisition Parameters Date_ $\quad 20190424$

Time 13.16

$5 \mathrm{~mm} \mathrm{spect}$

PULPROG $\quad$ zg30

$\begin{array}{lr}\text { TD } & \text { 2930 } \\ & 65536\end{array}$

SOLVENT Acetone

NS

DS

SWH

FIDRES

AQ

RG

DW

$\mathrm{DE}$

D1
TD 0

$====$

$\mathrm{SFO1}$

NUC

P1
PLW1

16
2
$8012.820 \mathrm{~Hz}$

$4.0894465 \mathrm{sec}$

27.78

62.400 usec 6.50 usec

$1.00000000 \mathrm{sec}$

CHANNEL $\mathrm{fl}========$ $400.1324710 \mathrm{MHz}$ LW1 $\quad 14.50$ usec

SI Processing parameters

SI 65536

$\begin{array}{lc}\text { SF } & 400.1299910 \mathrm{MHz} \\ \text { WDW } & \text { EM }\end{array}$

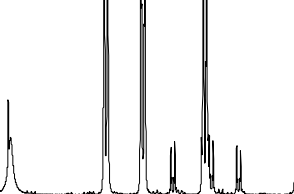

DCM

$\uparrow$ water

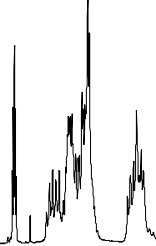

$0.30 \mathrm{~Hz}$

1.00

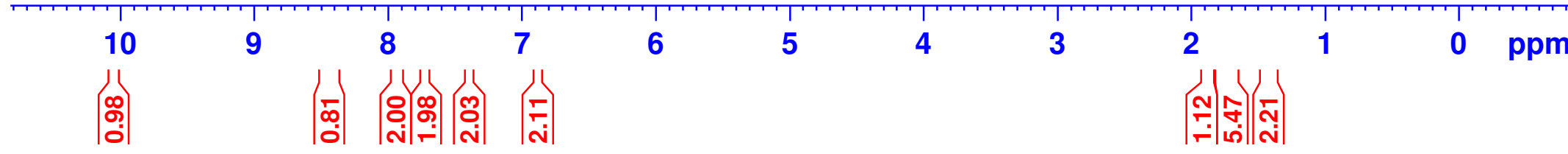




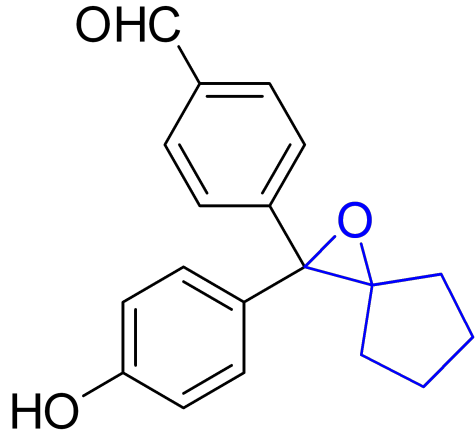

$1 \mathrm{i}$

Current Data Parameters EXPNO

mdk9190C
1

F2 - Acquisition Parameter

Date_ 20190424

Time

PROBHD

PULPROG

TD

NS

SWH
FIDRES

$\mathrm{A} Q$
$\mathrm{RG}$
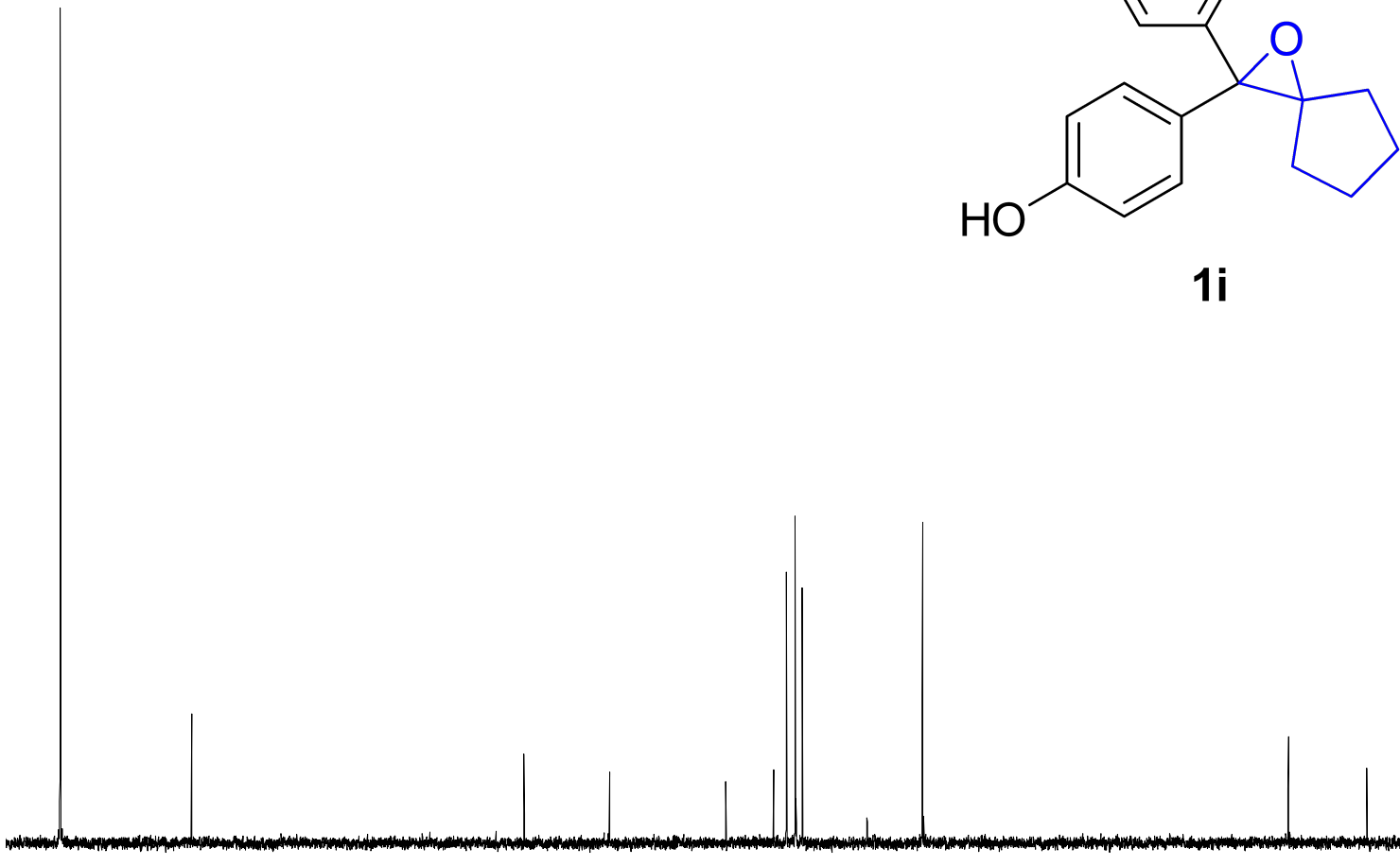


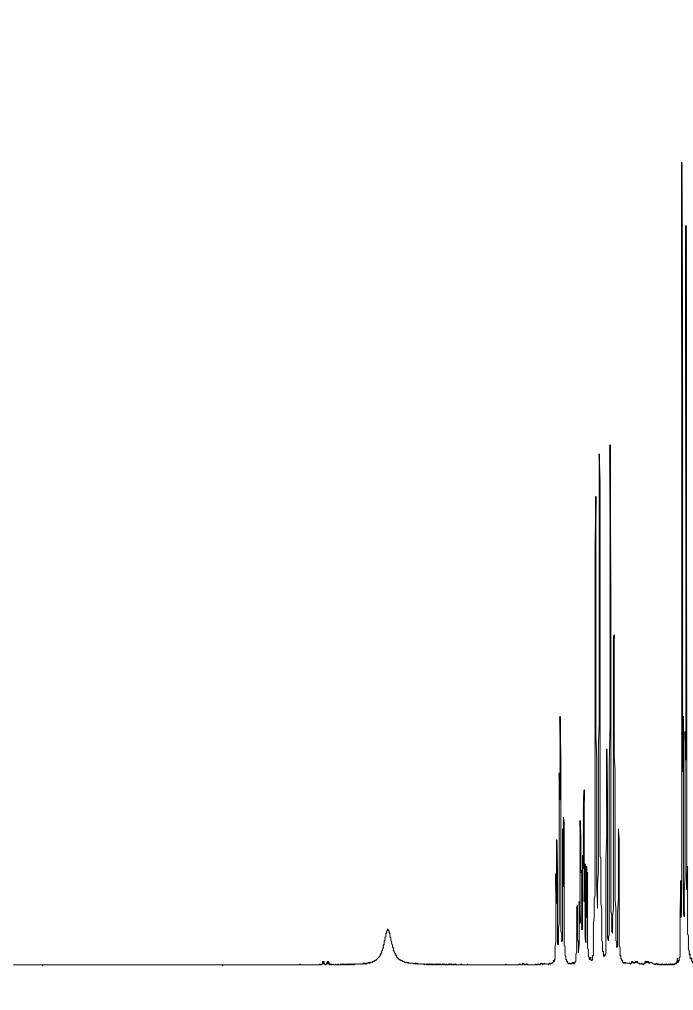

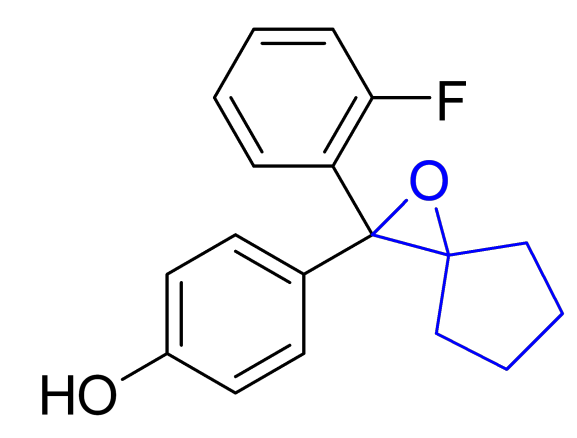

$1 \mathrm{j}$

Current Data Parameters NAME

EXPNO

mdk $9035 \mathrm{H}$

PROCNO

F2 - Acquisition Parameters

Date_

20190306

Time

PROBHD

PULPROG

SOLVENT

NS

DS

SWH

$\mathrm{AQ}$

RG

$\mathrm{DW}$
$\mathrm{DE}$
$\mathrm{TE}$

D1
TD 0

$===$

$\mathrm{SFO1}$

$\mathrm{NUC1}$
$\mathrm{P} 1$

19.27
spect

$5 \mathrm{~mm}$ РАВBO BB/

$\mathrm{zg} 30$
65536

Acetone

16
2

$8012.820 \mathrm{~Hz}$

$4.089465 \mathrm{~Hz}$

49.32

62.400 usec

6.50 usec

$1.00000000 \mathrm{sec}$

CHANNEI $\mathrm{f1}=$ $400.1324710 \mathrm{MHz}$

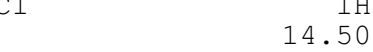

TI Processing parameters

SI 65536

$\begin{array}{lc}\text { SF } & 400.1299911 \mathrm{MHz} \\ \text { WDW } & \text { EM }\end{array}$

water

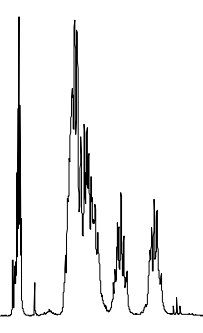

$0.30 \mathrm{~Hz}$

LB

PC

1.00

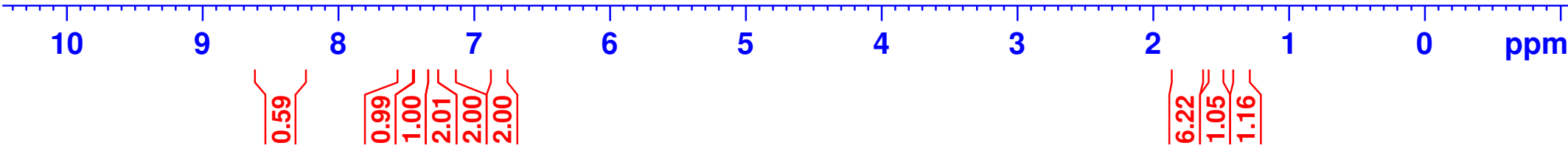



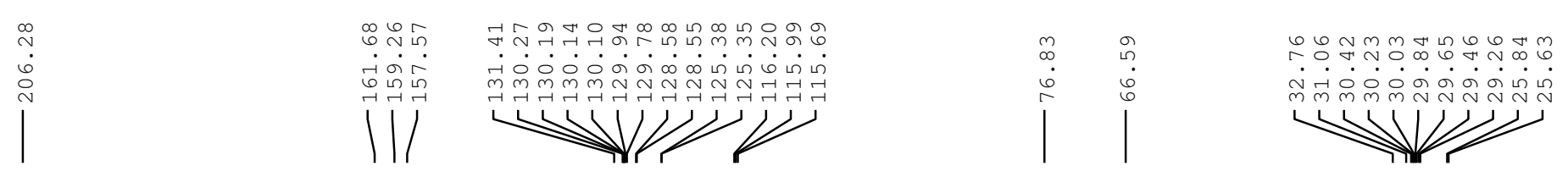

eferieng

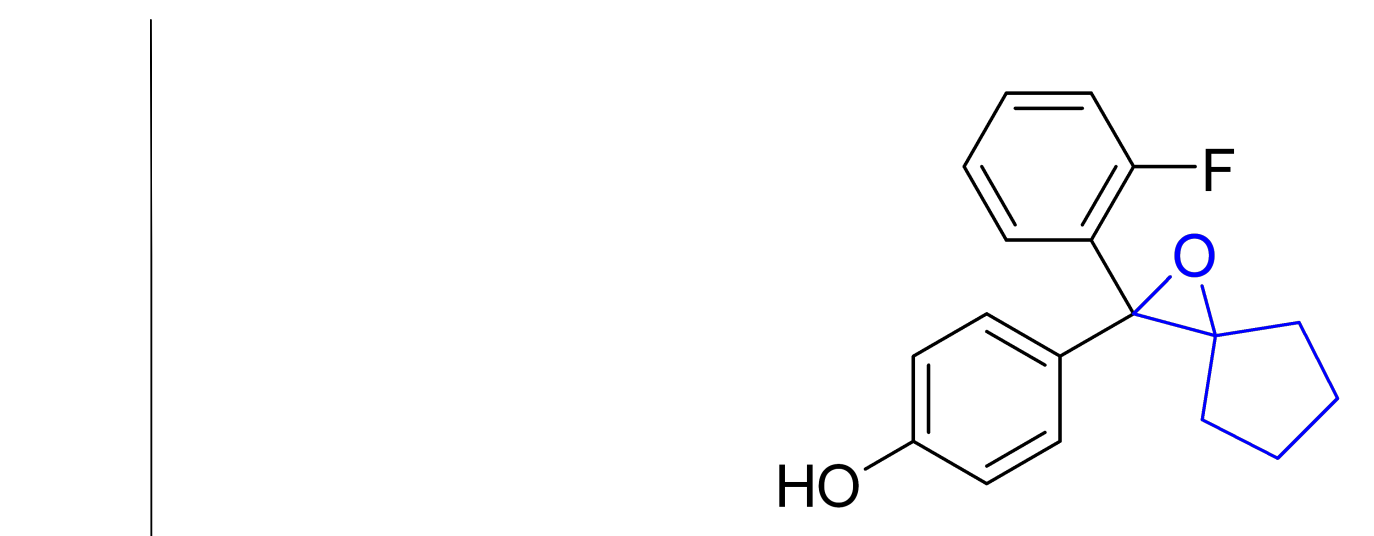

1j

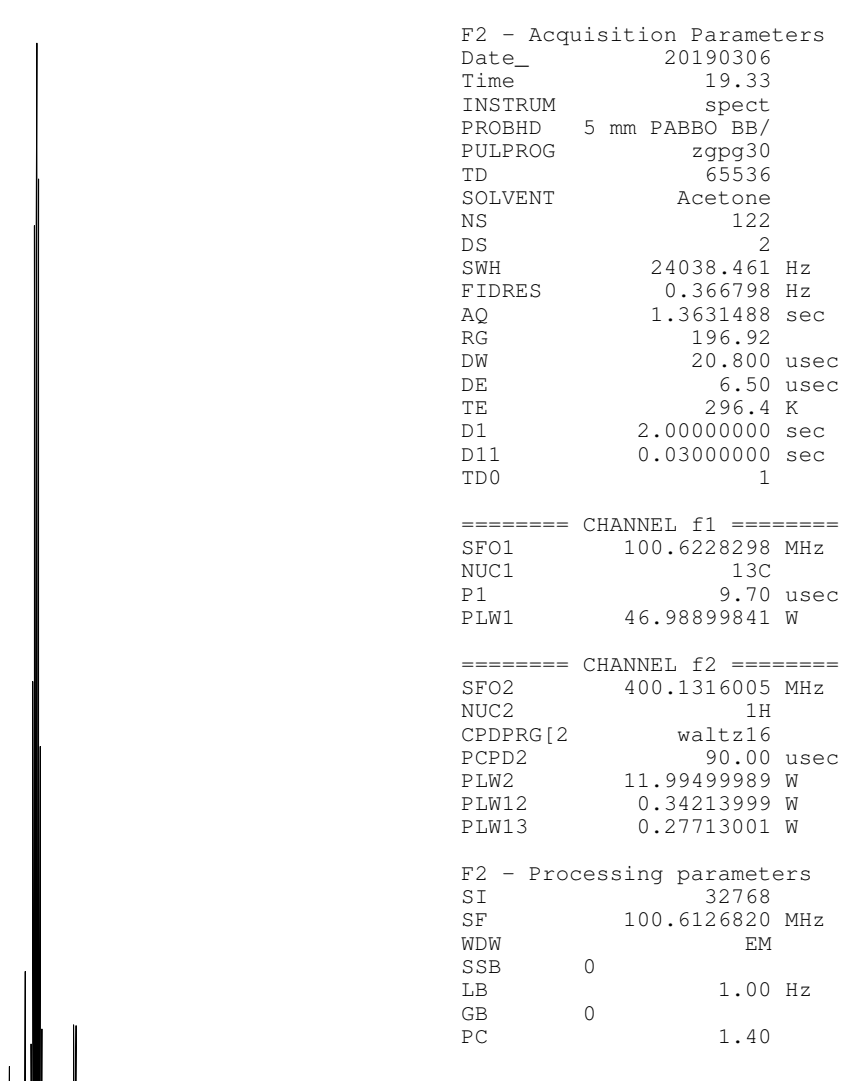

\begin{tabular}{|rrrrrrrrrrrr}
\hline 200 & 180 & 160 & 140 & 120 & 100 & 80 & 60 & 40 & 20 & 0 & ppm
\end{tabular}




\section{expiren}

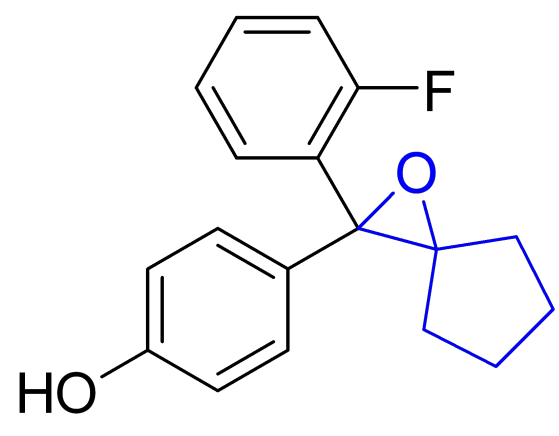

1j

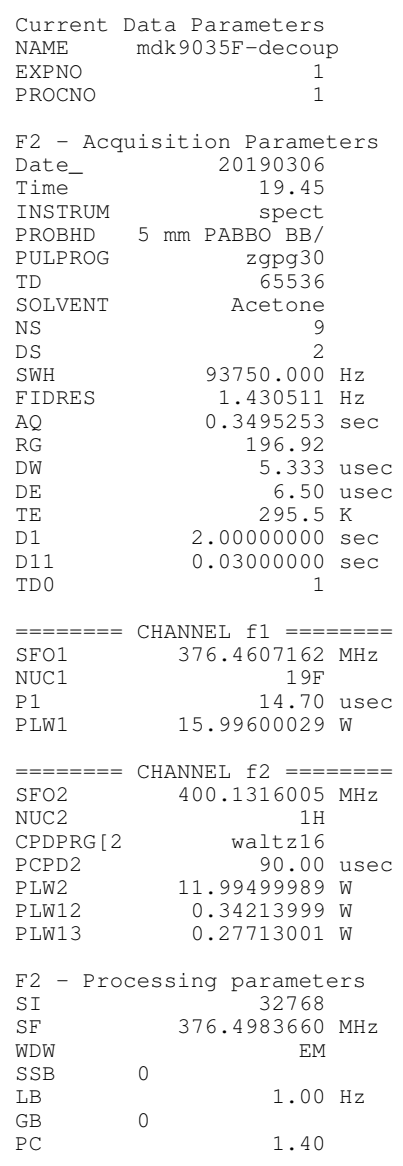

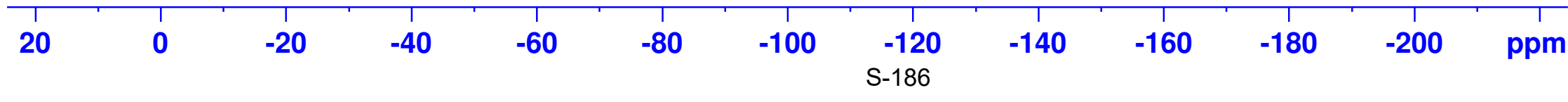




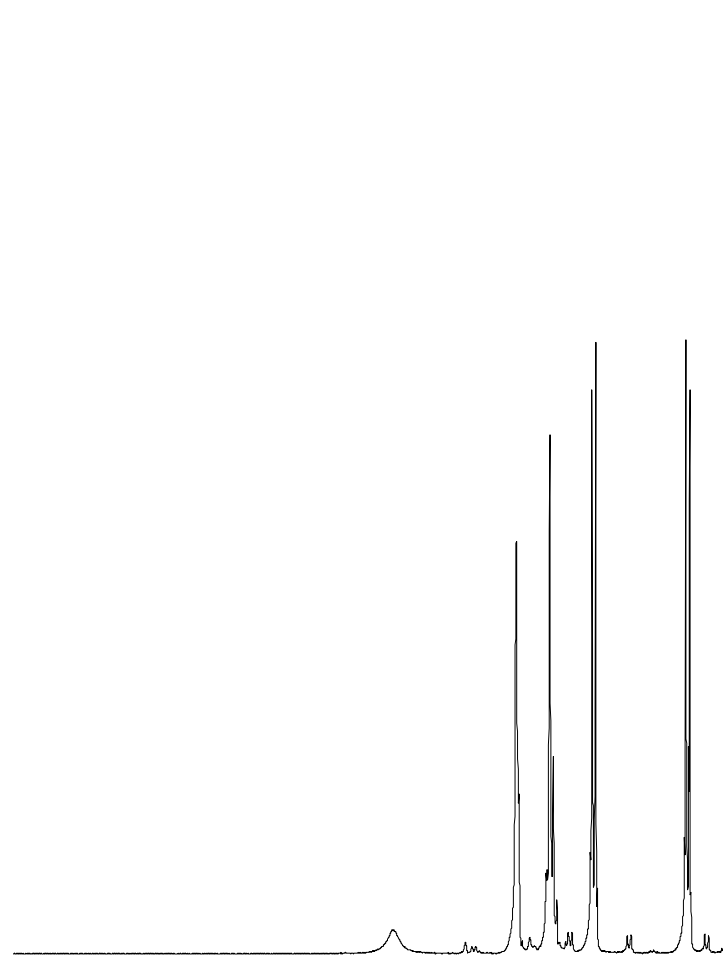

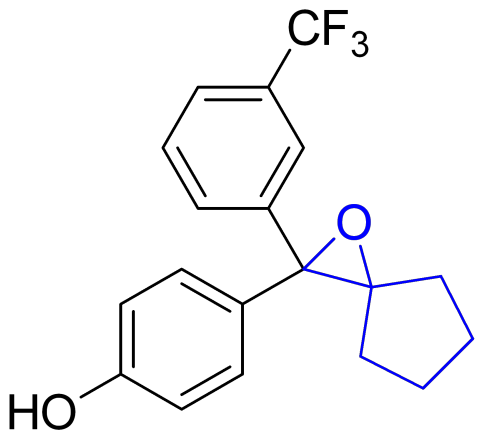

$1 \mathrm{k}$

\section{eperer ,}

Current Data Parameters NAME

PROCNO

$\operatorname{mak} 9045 \mathrm{H}$

F2 - Acquisition Parameters

Date_ 20190309

$\begin{array}{lr}\text { Time } & 20.16\end{array}$

INSTRUM spect

ROBHD $5 \mathrm{~mm}$ PABBC

Zg30
TD

SOLVENT Acetone

TS

DS

SWH

FIDRES

$\mathrm{AQ}$

RG

DW

$\mathrm{DE}$

D1
TD 0

$====$

$\mathrm{SFO1}$

NUC1

PLW1

16
Acetone

$8012.820 \mathrm{~Hz}$

$8012.820 \mathrm{~Hz}$

$4.0894465 \mathrm{sec}$

49.32

62.400 usec 6.50 usec

$1.00000000 \mathrm{sec}$ 1

CHANNEL $\mathrm{f} 1 \mathrm{l}========$ $400.1324710 \mathrm{MHz}$ 14.50 $11.99499989 \mathrm{~W}$

F2 - Processing parameters

SI 65536

SF $\quad 400.1299912 \mathrm{MHz}$

WDW

SSB

EM

$0.30 \mathrm{~Hz}$

1.00 


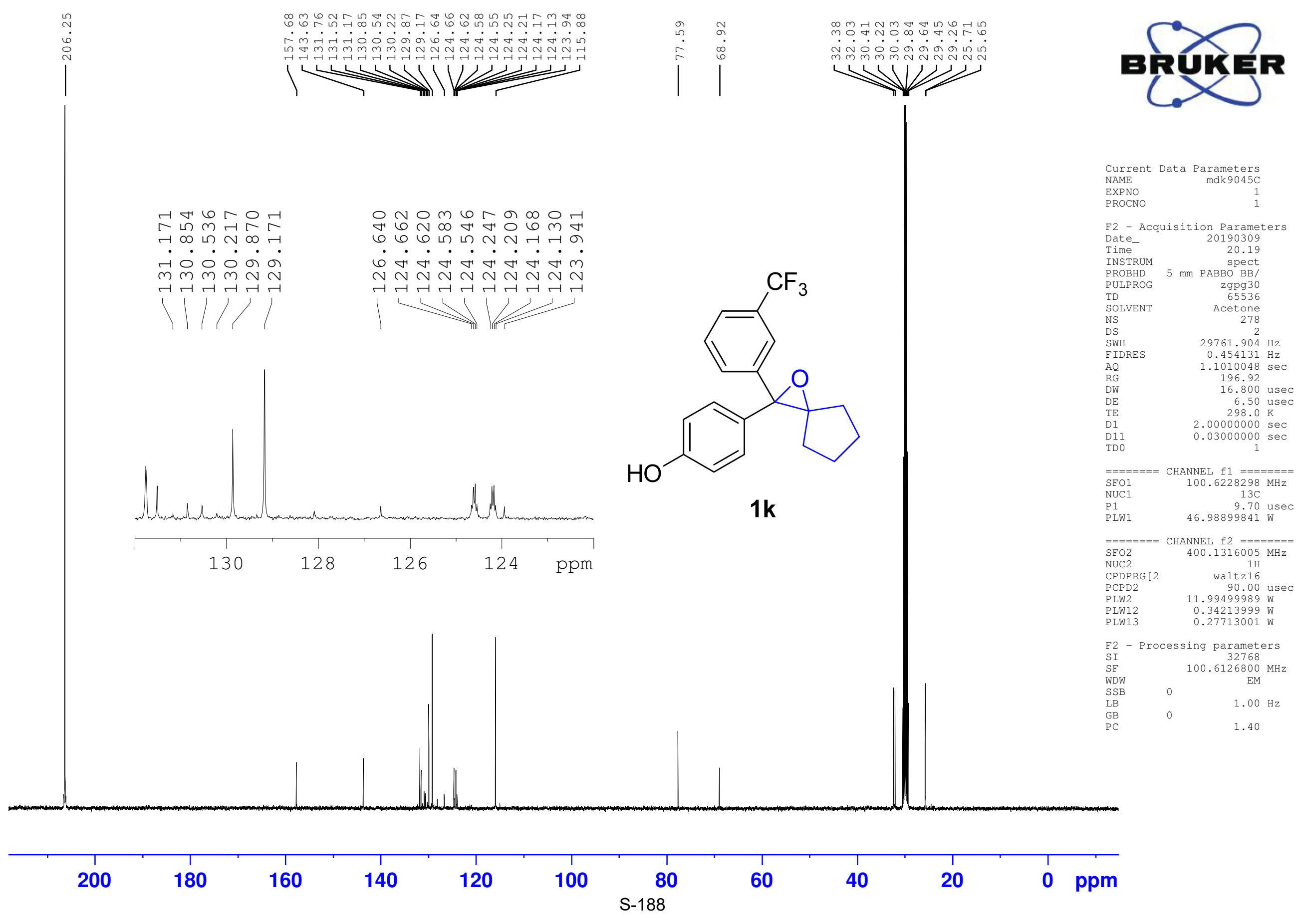




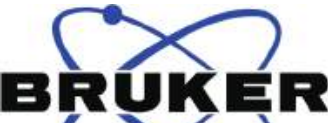
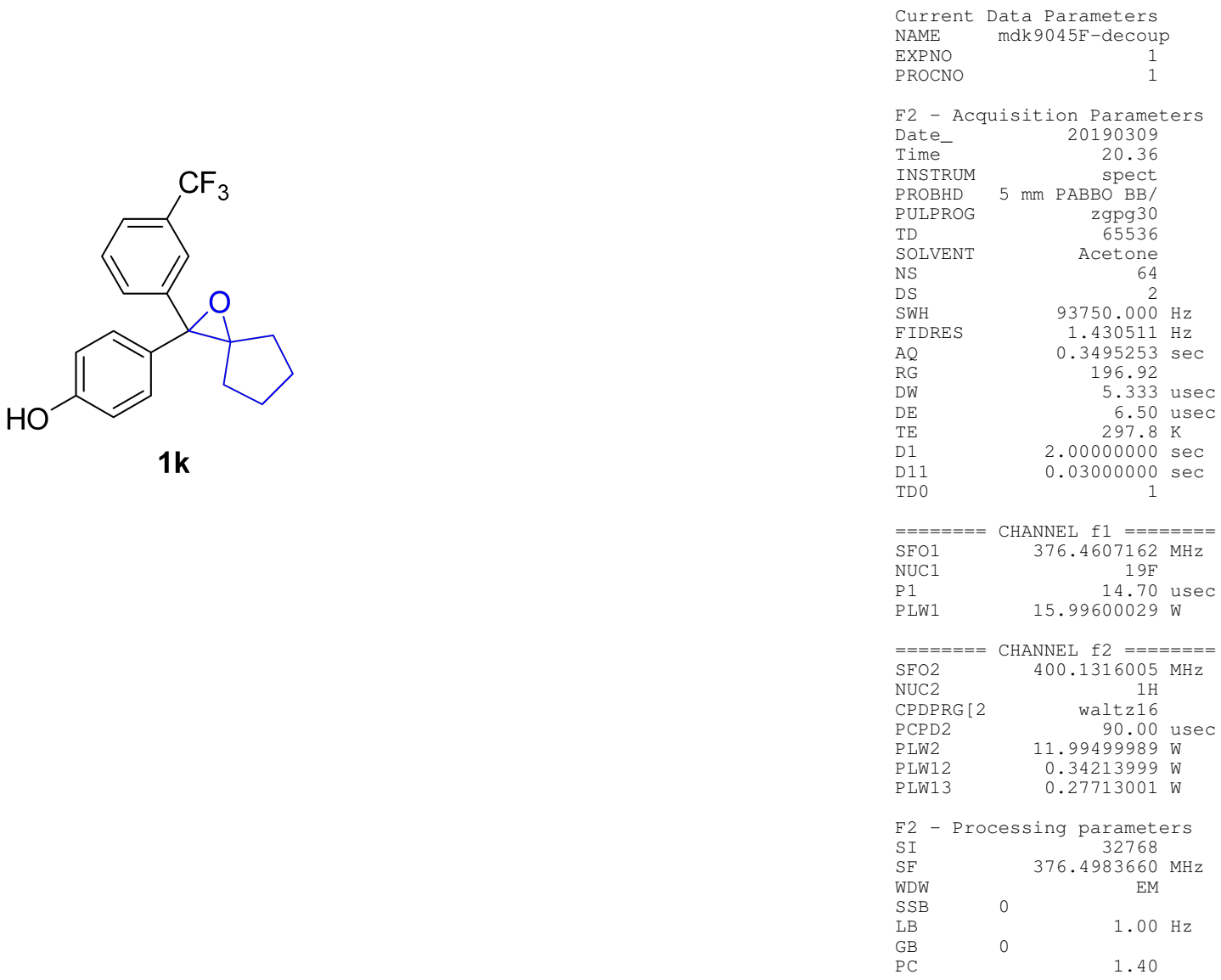

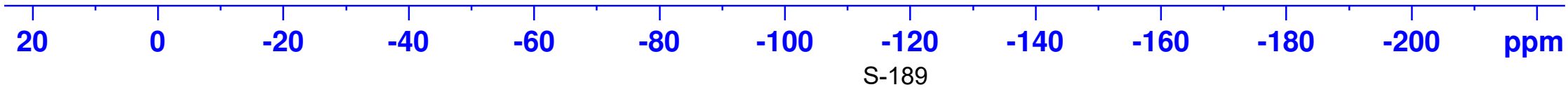




\section{sporen

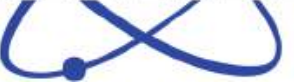

Current Data Parameters NAME

EXPNO

mdk $9038 \mathrm{H}$

PROCNO

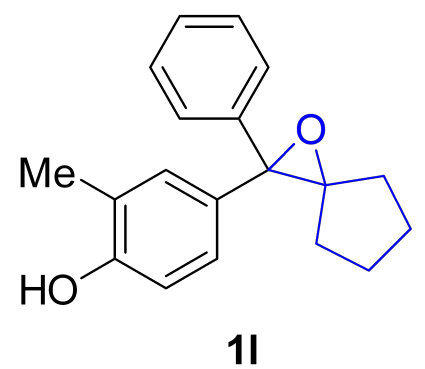

11
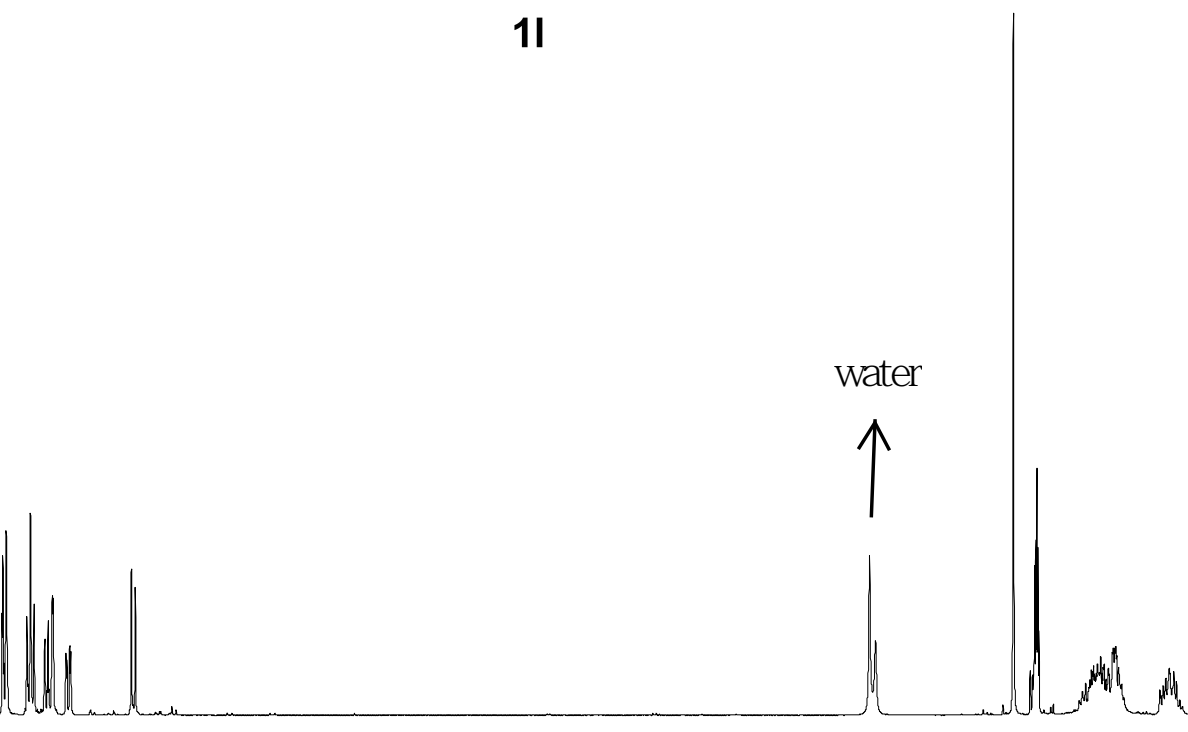

\section{2}

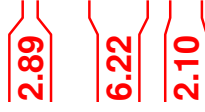

F2 - Acquisition Parameters

Date__ 20190307

Time 13.33

$5 \mathrm{~mm}$ spect

$5 \mathrm{~mm} \mathrm{PABB}$

zg30
65536

SOLVENT Acetone

TS

DS

SWH

FIDRES

$\mathrm{AQ}$

$\mathrm{RG}$

DW

$\mathrm{DE}$

D1
TD 0

$====$

$\mathrm{SFO} 1$

$\mathrm{NUC1}$
$\mathrm{P} 1$

Acetone
16

$8012.820 \mathrm{~Hz}$

$4.0894465 \mathrm{~Hz}$

70.97

62.400 usec

6.50 usec

$1.00000000 \mathrm{~K}$

$$
1
$$

CHANNEL $\mathrm{f} 1========$ $400.1324710 \mathrm{MHz}$

$1 \mathrm{H}$

usec

PLW1 $11.99499989 \mathrm{~W}$

F2 - Processing parameters

SI 65536

SF $\quad 400.1299910 \mathrm{MHz}$

SSB

EM

$0.30 \mathrm{~Hz}$

1.00

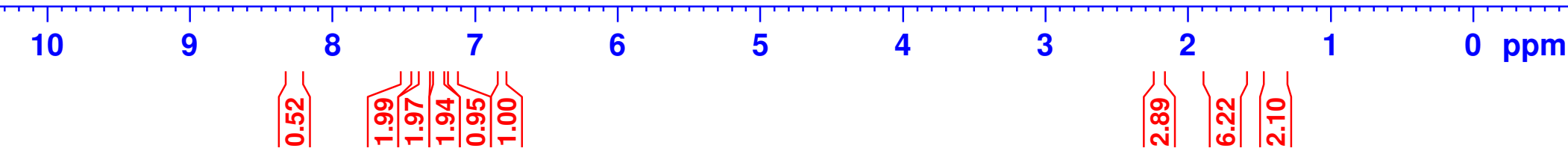




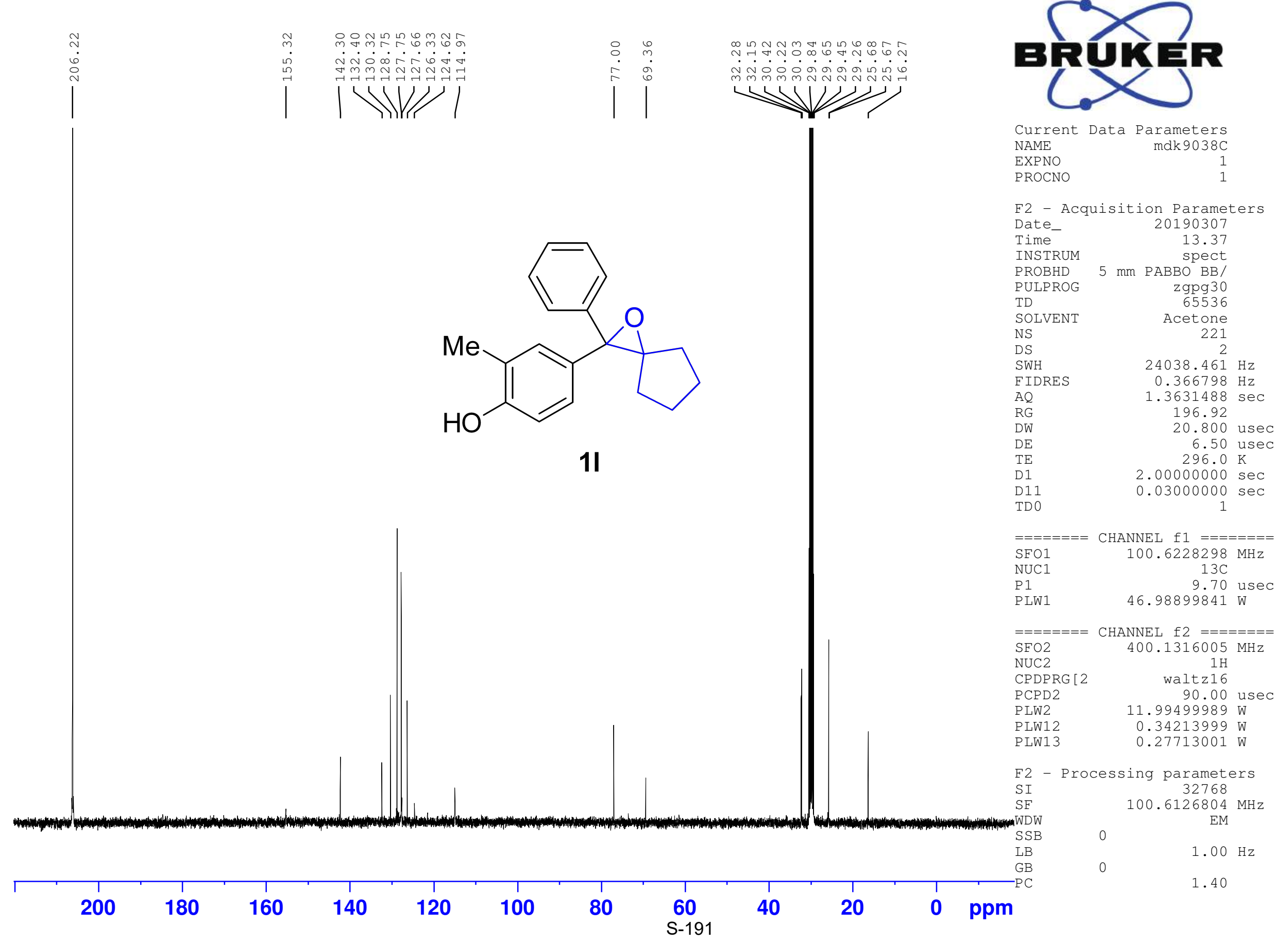




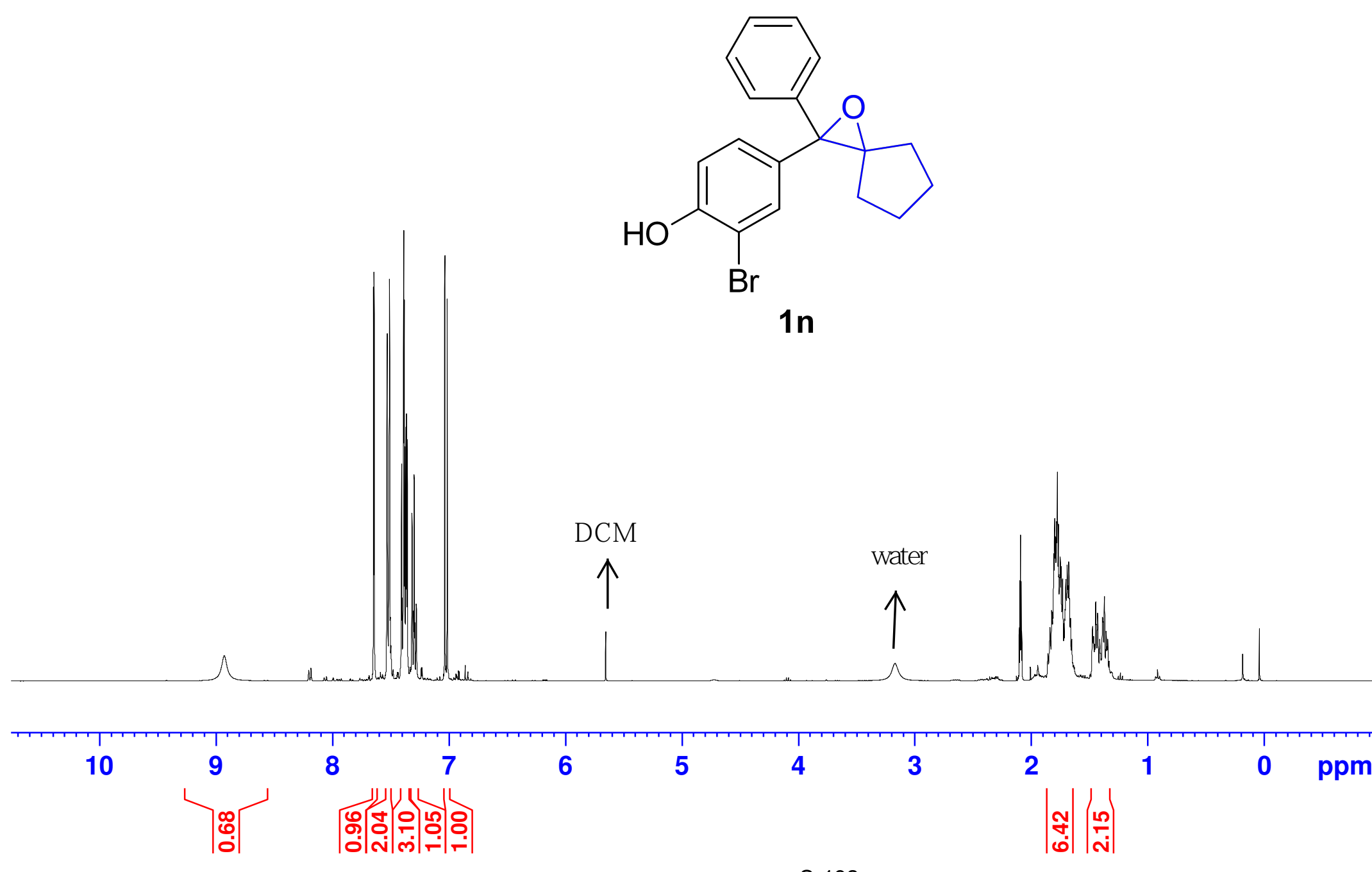

\section{sporen ,}

Current Data Parameters
NAME
mak $9124 \mathrm{H}$

EXPNO

1

F2 - Acquisition Parameters

Date_ 20190330

$\begin{array}{lr}\text { Time } & 12.46\end{array}$

spect

PULPROG zg30

$\begin{array}{lr} & \text { Zg30 } \\ \text { TD } & 65536\end{array}$

SOLVENT Acetone

NS

DS

FID

$\mathrm{AQ}$

RG

DE

TE

TD 0

$=====$
SFO1
NUC1

$\mathrm{P} 1$

16
16

$8012.820 \mathrm{~Hz}$

$0.122266 \mathrm{~Hz}$

$4.0894465 \mathrm{sec}$ 49.32

62.400 usec 6.50 usec

$1.00000000 \mathrm{sec}$

1

$$
1 \text { sec }
$$

PLW1 $400.1324710 \mathrm{MHz}$

$11.99499989 \mathrm{~W}$

F2 - Processing parameters

SI 65536

400.1299911 MH

WDW

$\mathrm{EM}$

$0.30 \mathrm{~Hz}$

GB

$\mathrm{PC}$

1.00 


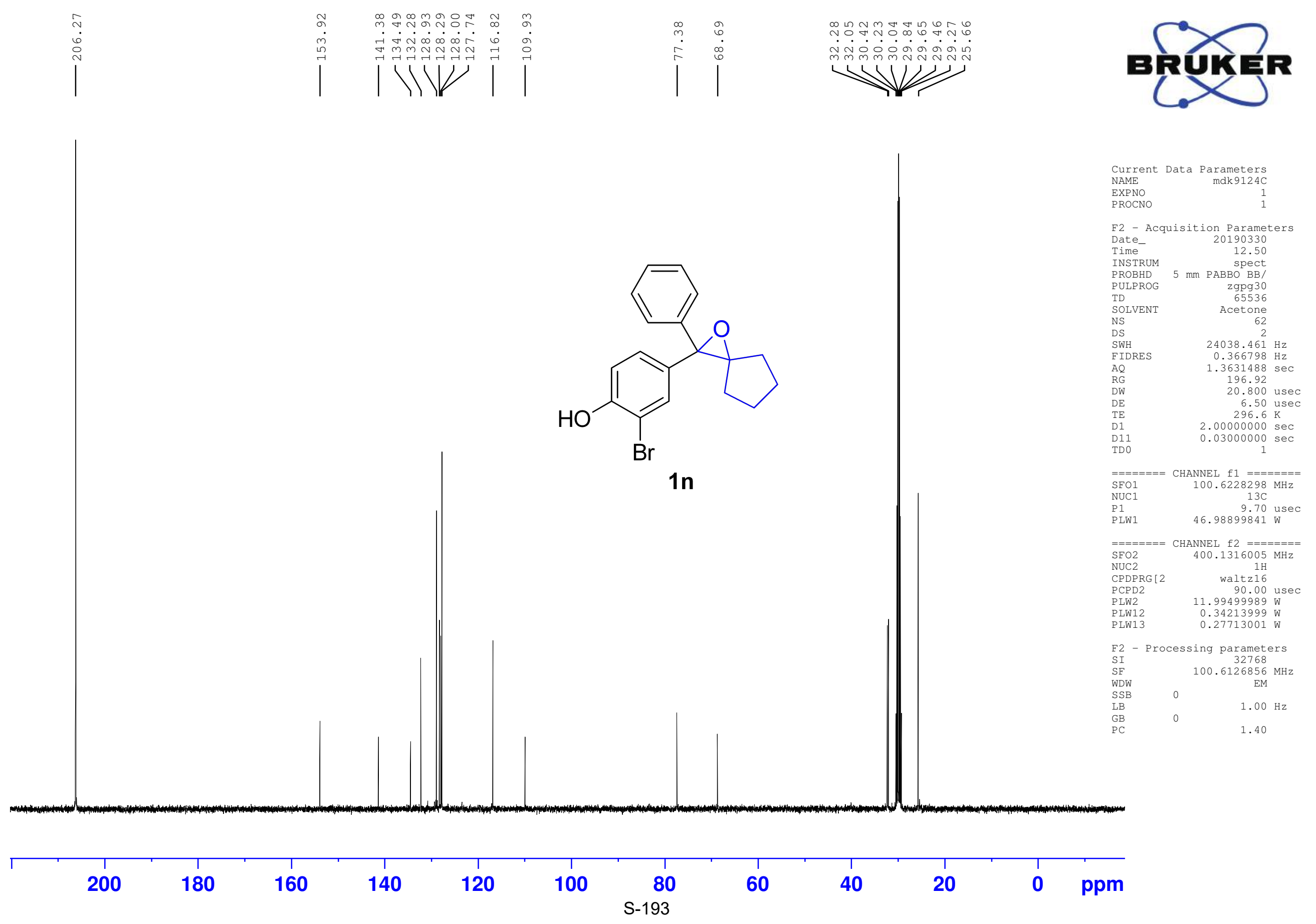




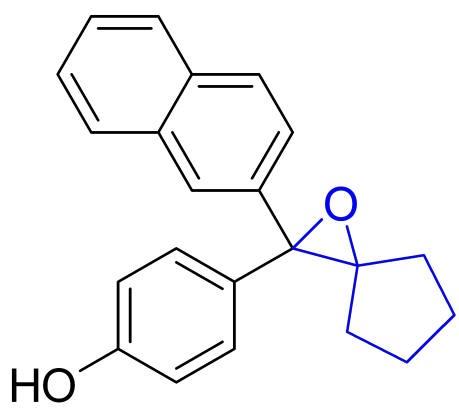

10

Current Data Parameters NAME

EXPNO

$\operatorname{mdk} 9129 \mathrm{H}$

PROCNO

F2 - Acquisition Parameters

Date__ 20190330

Time

20.34

INSTRUM

PROBHD

$5 \mathrm{~mm} \mathrm{PABBO} \mathrm{BB} /$

PUIPROG

zg30
65536

$\begin{array}{lr}65536 \\ \text { SOLVENT } & \text { Acetone }\end{array}$

NS

SWH

FIDRES

FIDR
$\mathrm{AQ}$
$\mathrm{RG}$

AQ

$\mathrm{DW}$

$\mathrm{DE}$

$\mathrm{TE}$
$\mathrm{D} 1$

TD 0

Acetone
16

$8012.820 \mathrm{~Hz}$

$4.0894465 \mathrm{~Hz}$

25.32

62.400 usec 6.50 usec

$1.00000000 \mathrm{sec}$

$===$

$\mathrm{SFO1}$

NUC1

CHANNEL $\mathrm{f} 1========$

$\mathrm{P} 1$

$400.1324710 \mathrm{MHz}$

PLW1

14.50

SI 65536

SF $\quad 400.1299910 \mathrm{MHz}$

WSB

$\mathrm{EM}$

$0.30 \mathrm{~Hz}$

GB

PC

1.00

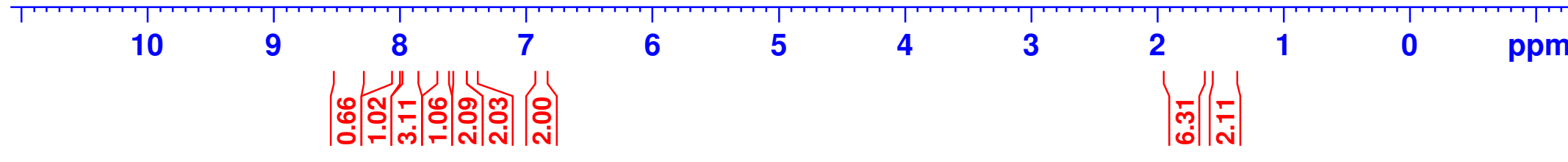




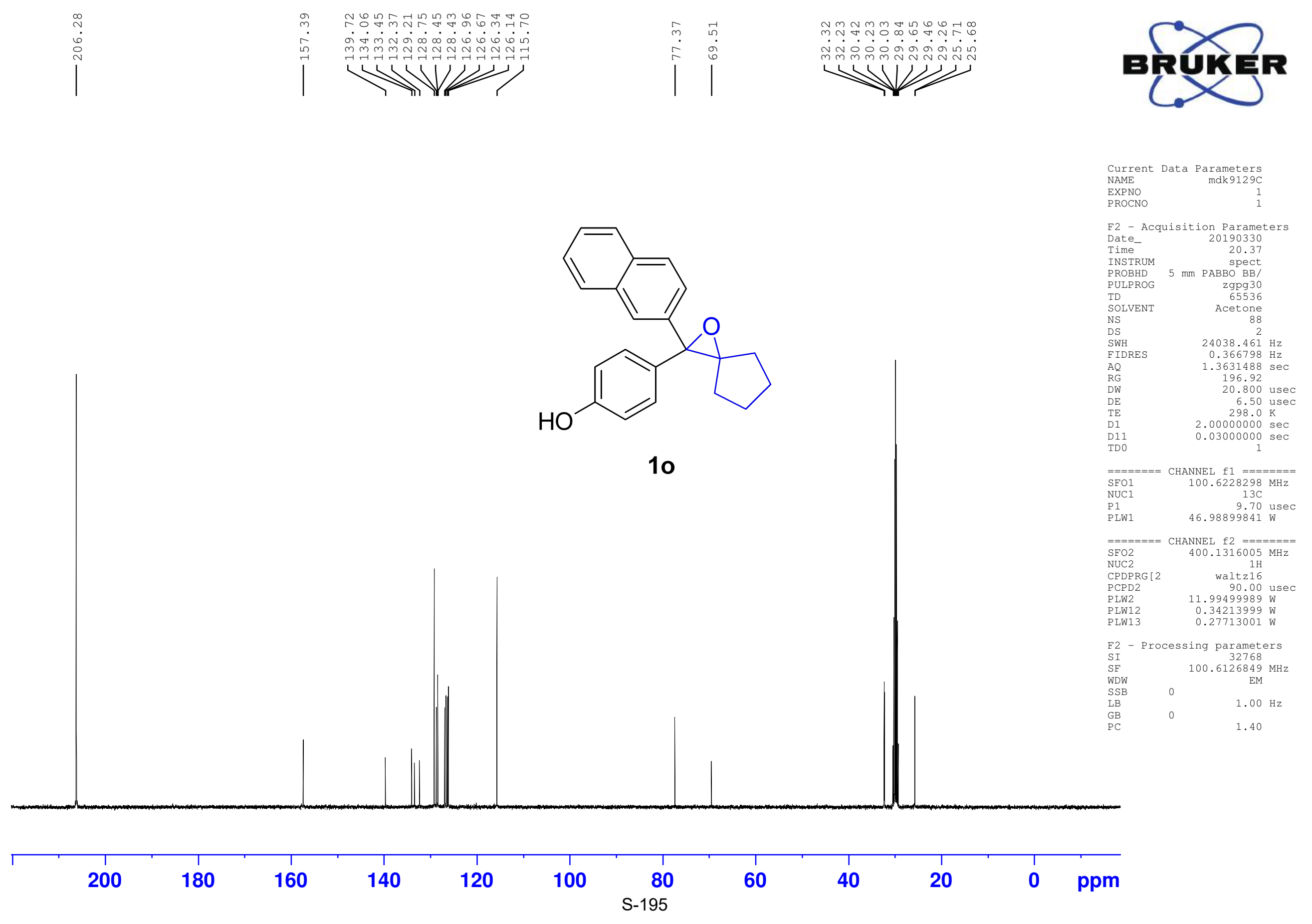




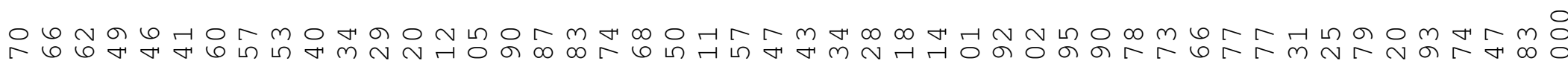

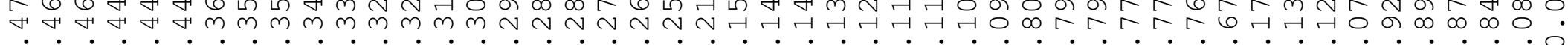

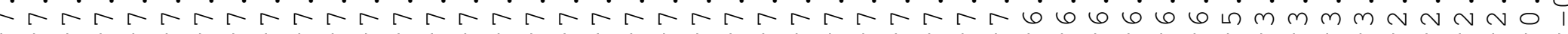
iा1 111

\section{spen

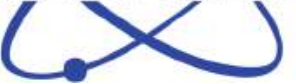
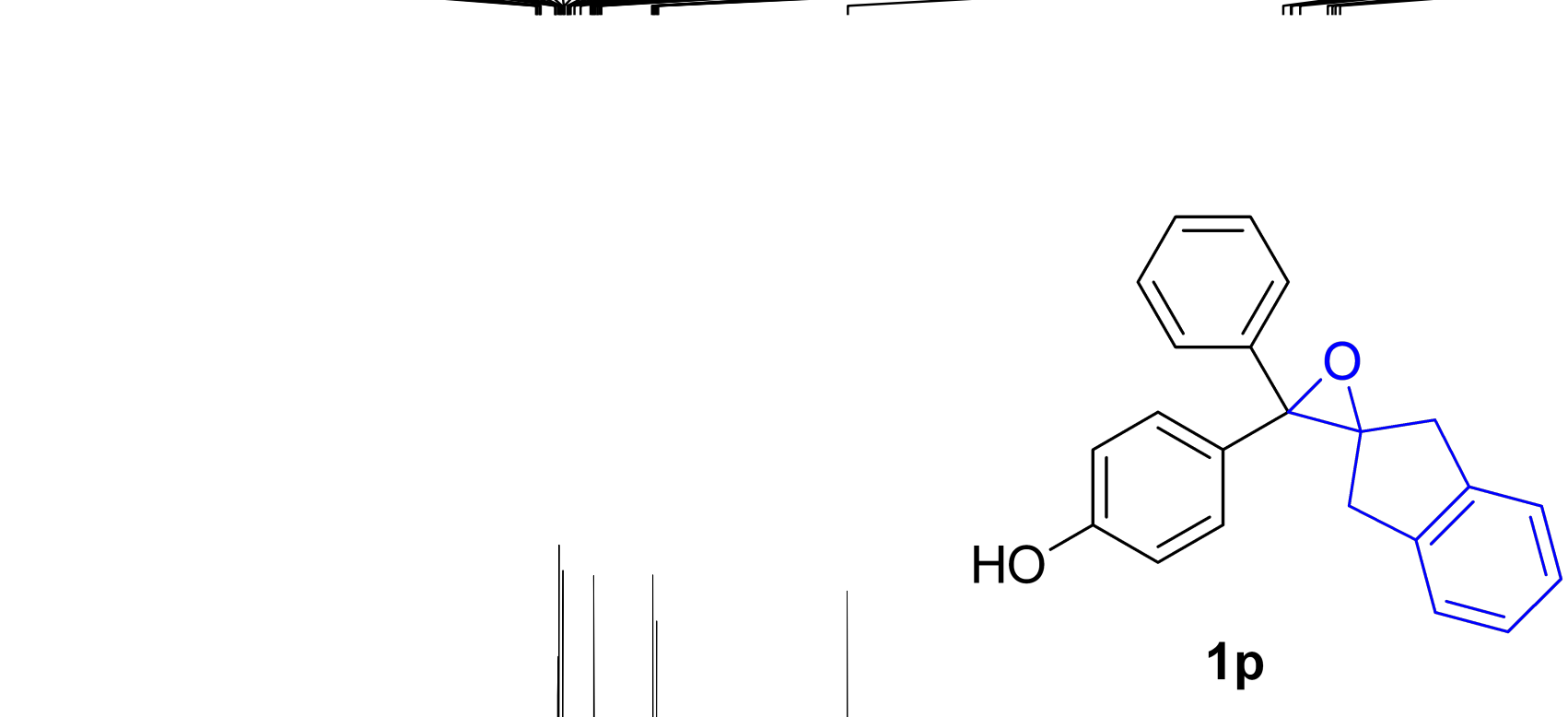

Current Data Parameters NAME

$\operatorname{mdk} 8164 \mathrm{H}$

PROCNO

1

F2 - Acquisition Parameters

Date_ 20190213

Time 14.00

$5 \mathrm{~mm}$ spect

PRTOG

$\mathrm{zg} 30$
65536

TD $\quad 65536$

SOLVENT

NS

SWH

FIDRES

AQ

RG

DW

DE

D1
TD 0

$====$

$\mathrm{SFO1}$

NUC

P 1
PLW1

$\mathrm{CDCl} 3$
16
2

$8012.820 \mathrm{~Hz}$

$0.122266 \mathrm{~Hz}$

$4.0894465 \mathrm{sec}$ 27.78

62.400 usec 6.50 usec $1.00000000 \mathrm{sec}$

CHANNEL $\mathrm{fl}========$ $400.1324710 \mathrm{MHz}$ LW1 $\quad 14.50$ us

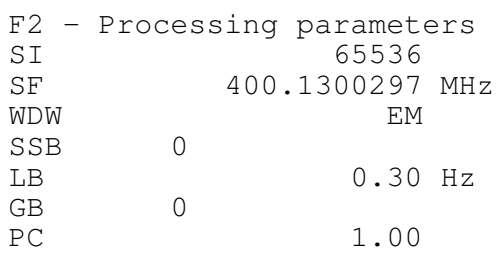

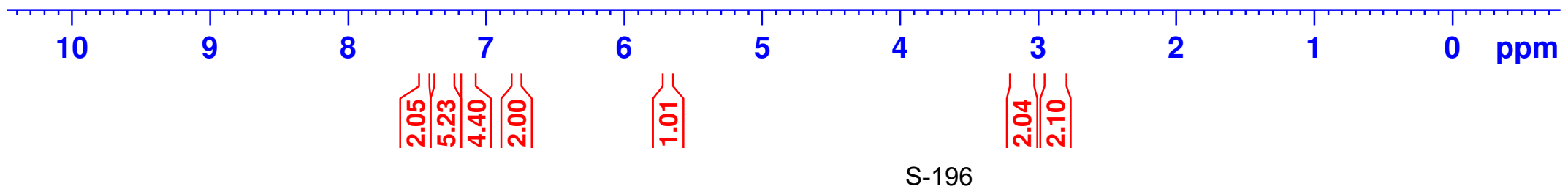




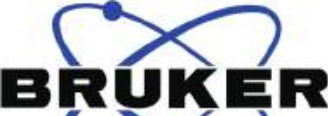 $\mathrm{CO}$}
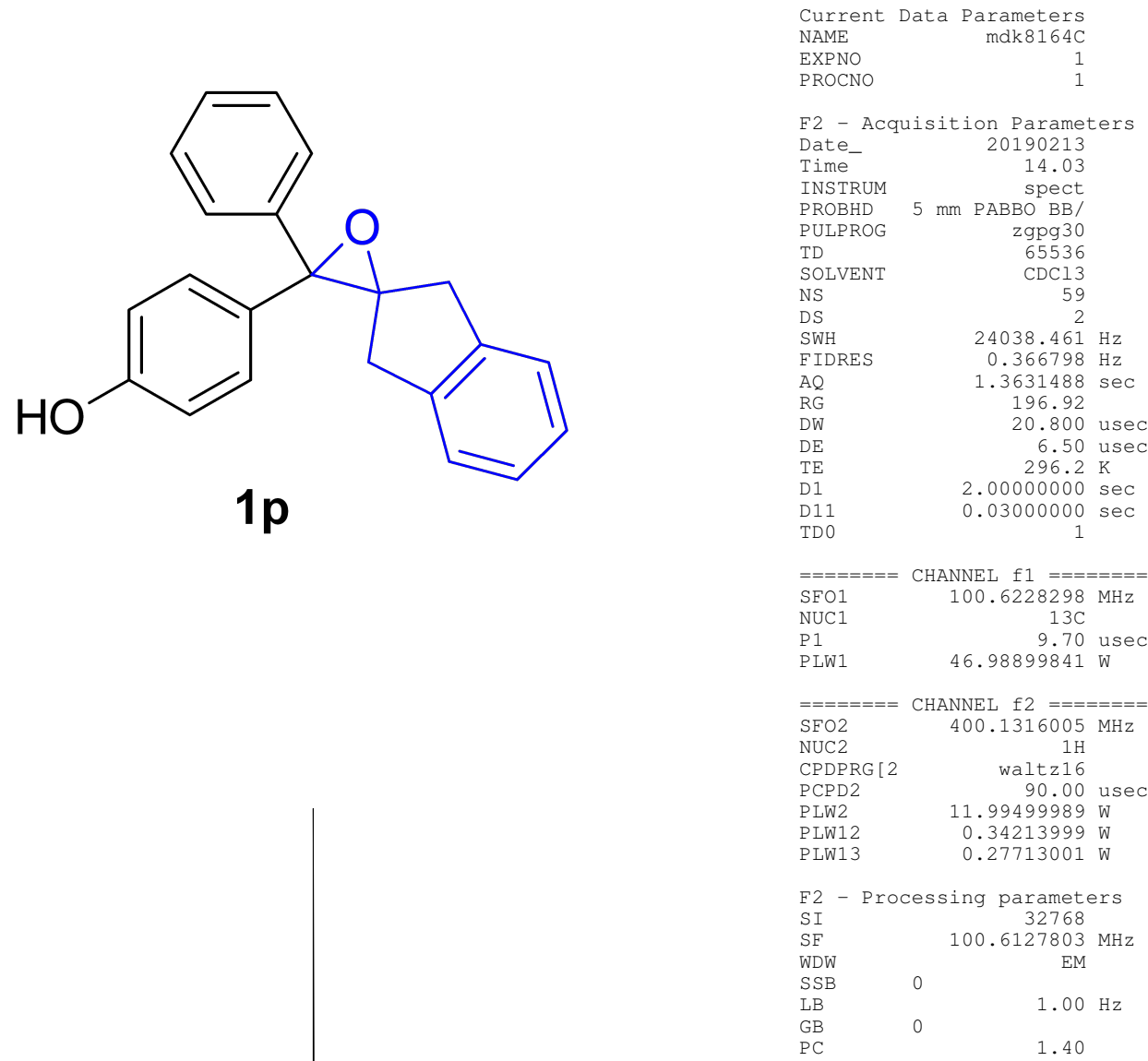

14mom

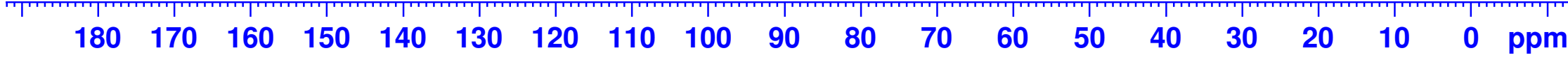




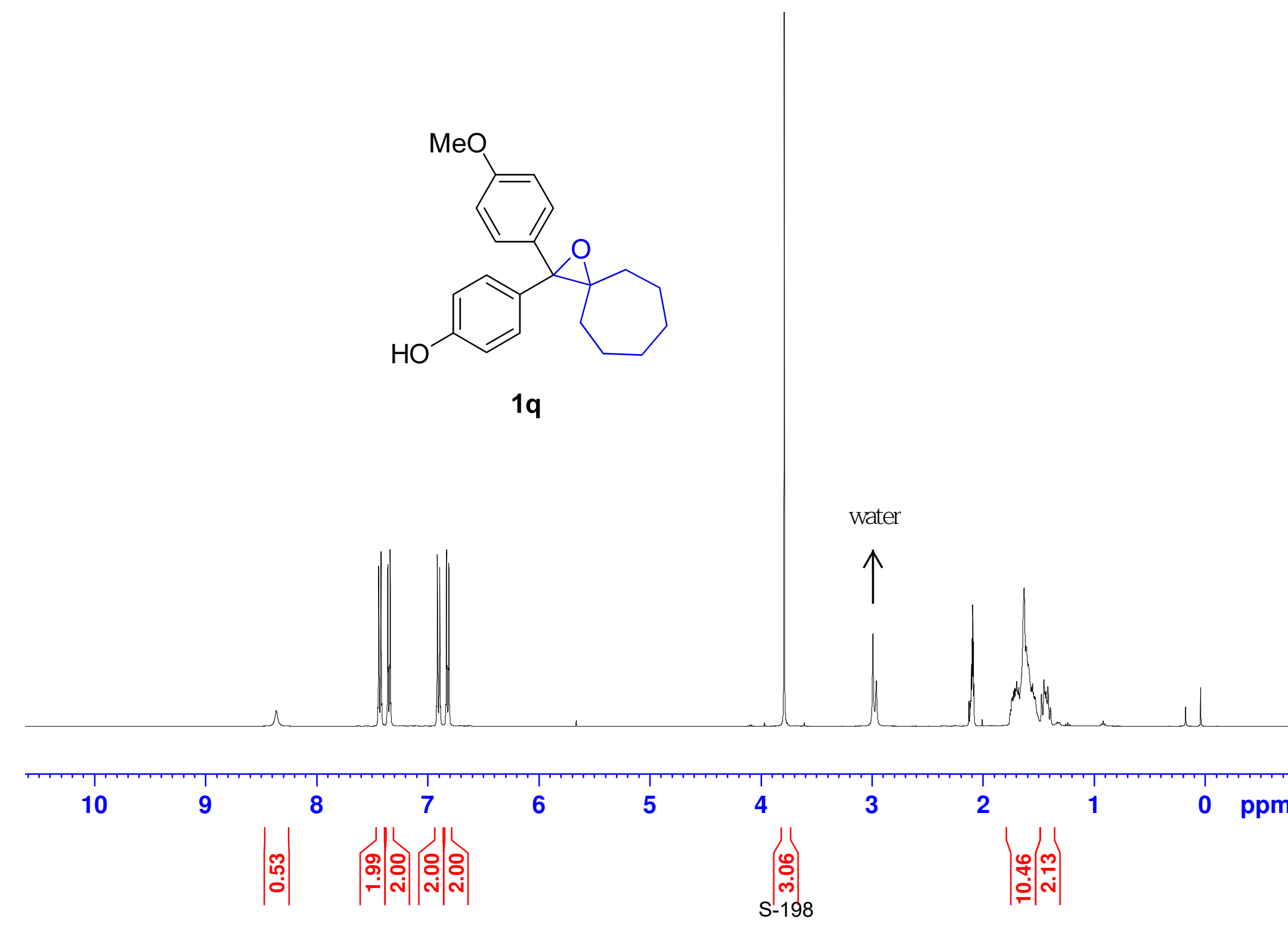

19 water

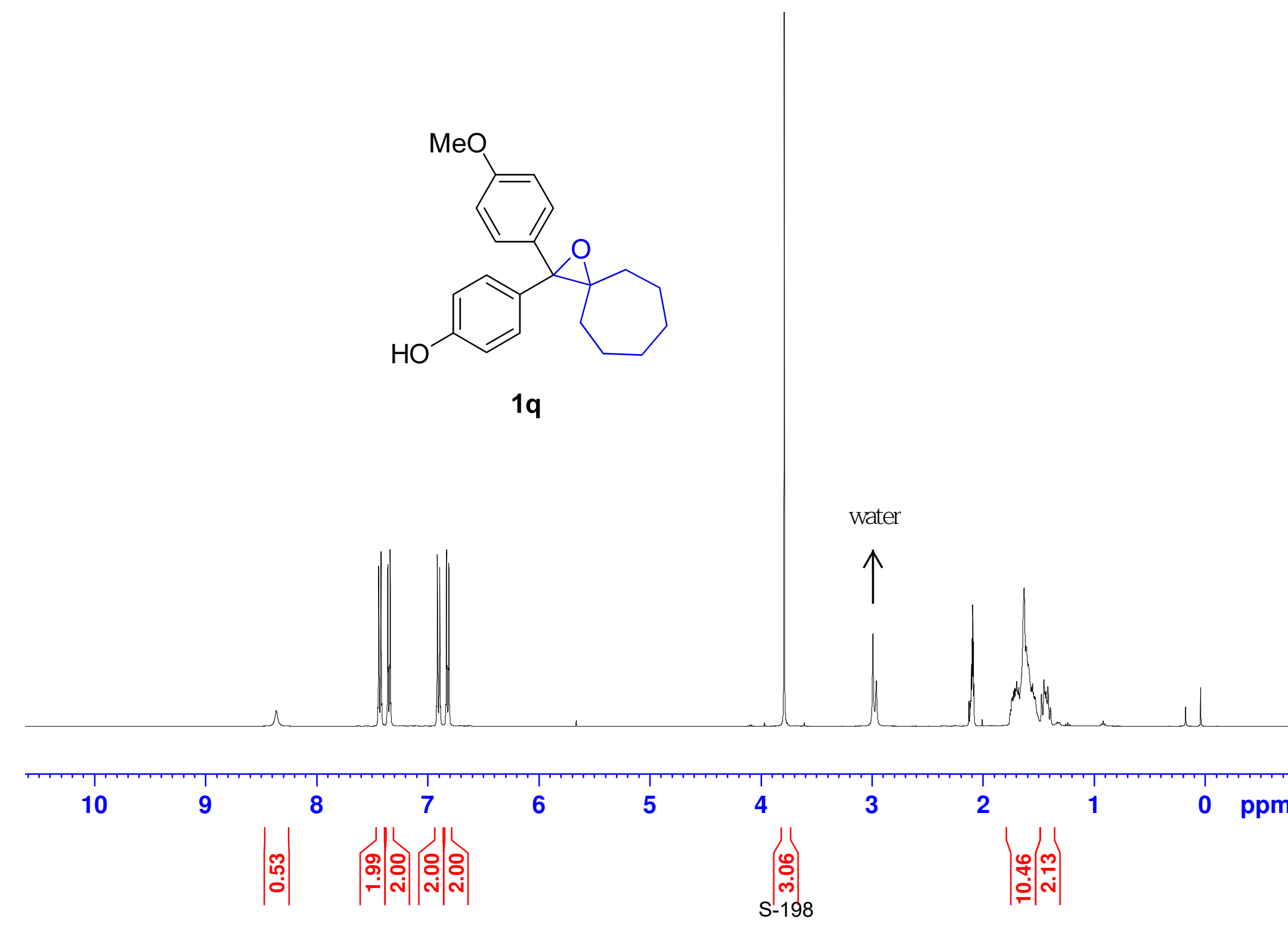

In

(1)
Current Data Parameters NAME

ROCNO

$\operatorname{mdk} 9025 \mathrm{H}$

2 - Acquisition Parameters

Date_ 20190304

Time $\quad 13.07$

$5 \mathrm{mpect}$

zg 30
65536

\begin{tabular}{l}
25536 \\
\hline
\end{tabular}

LVENT

SS

SWH

FIDRES

$\mathrm{AQ}$

RG

DE

TE

TD 0

$===$

$\mathrm{SFO1}$

P 1

Acetone
16

$8012.820 \mathrm{~Hz}$

$8012.820 \mathrm{~Hz}$
$0.122266 \mathrm{~Hz}$

$4.0894465 \mathrm{~Hz}$

54.81

62.400 usec 6.50 usec

$1.00000000 \mathrm{sec}$

CHANNEL $\mathrm{f} 1 \mathrm{l}========$ $400.1324710 \mathrm{MHz}$

F2 - Processing parameters SI 65536 SF $400.1299911 \mathrm{MHz}$ EM

$0.30 \mathrm{~Hz}$

1.00 


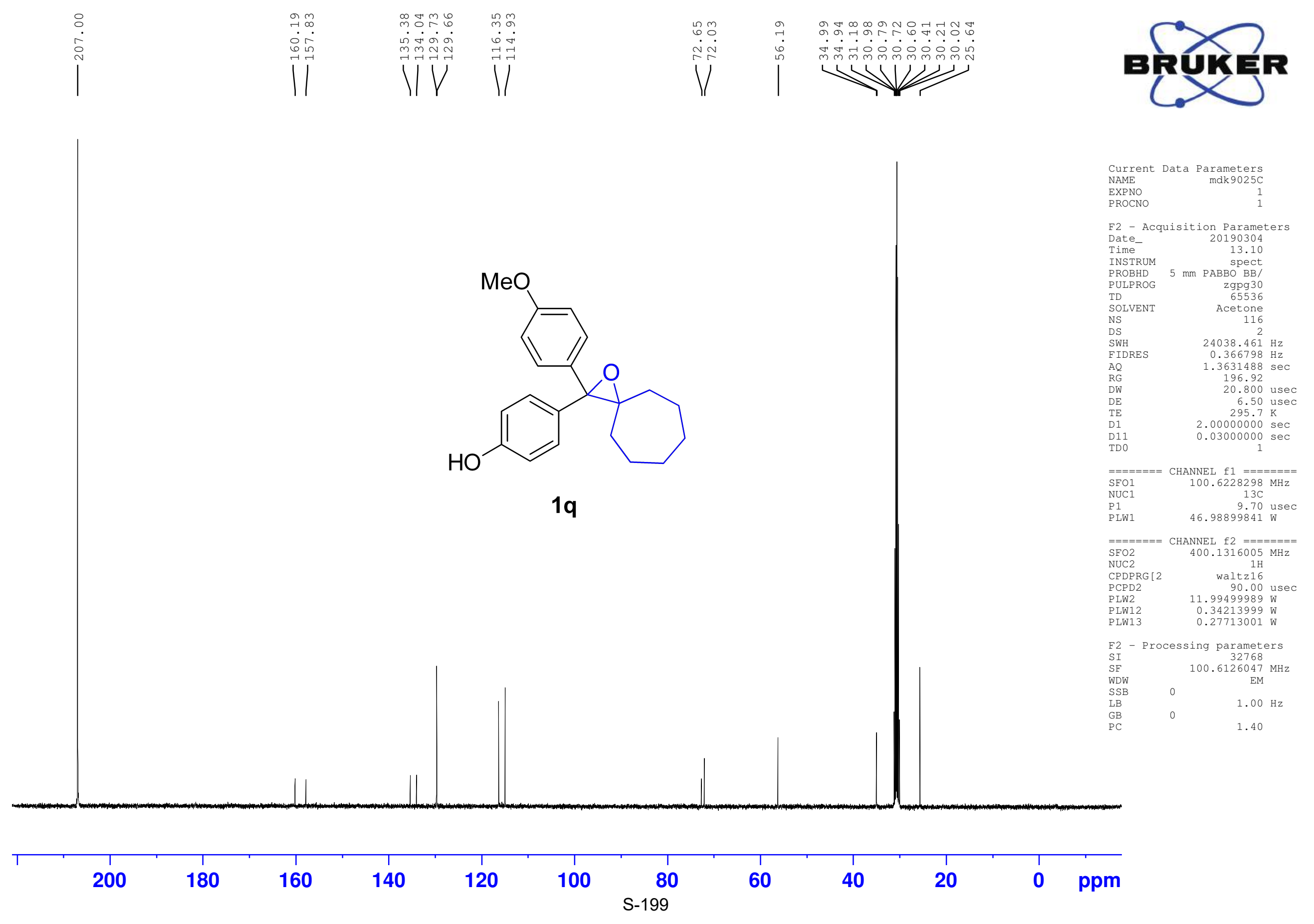




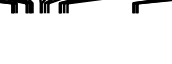

$1 a^{\prime}$

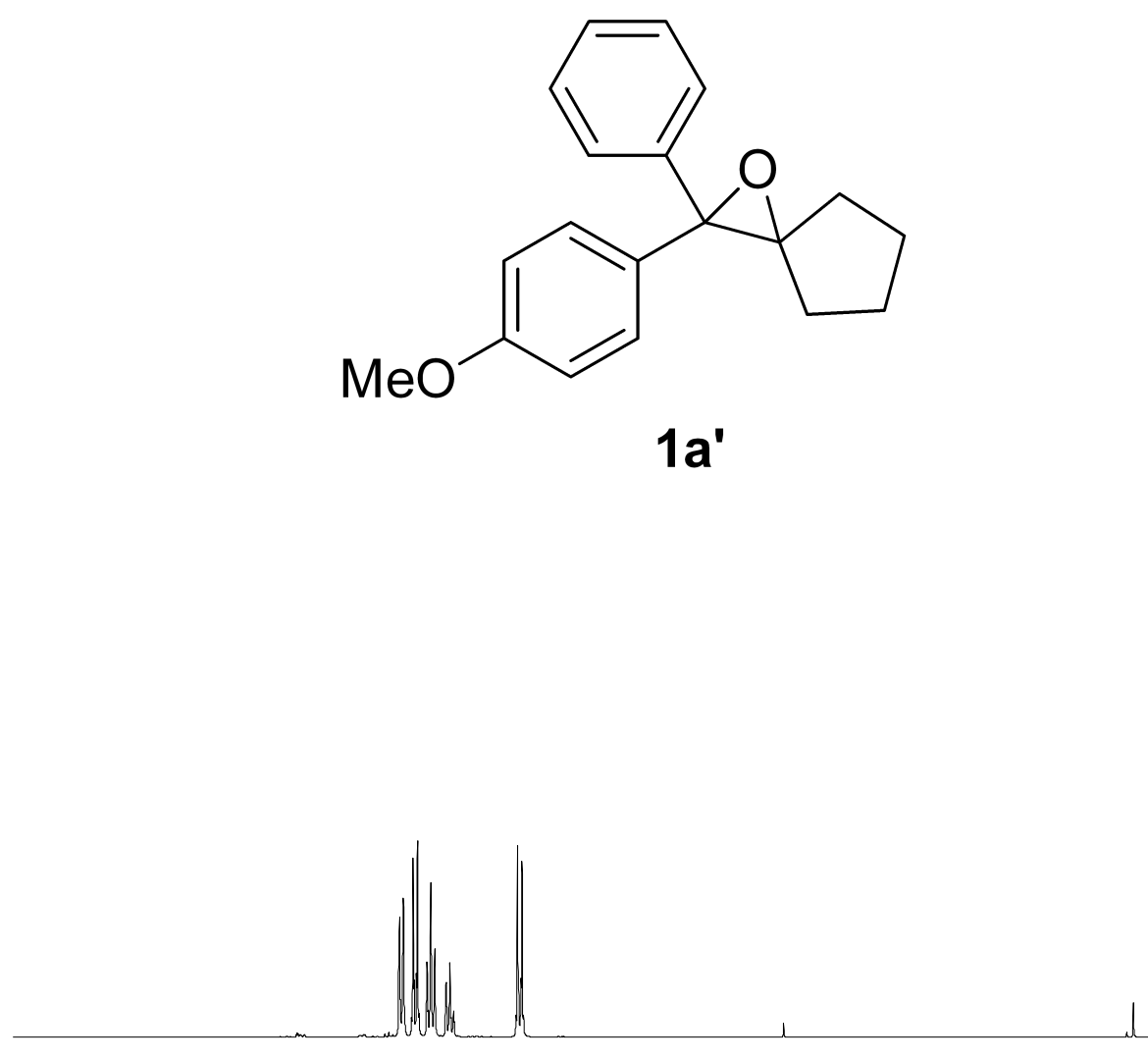

water

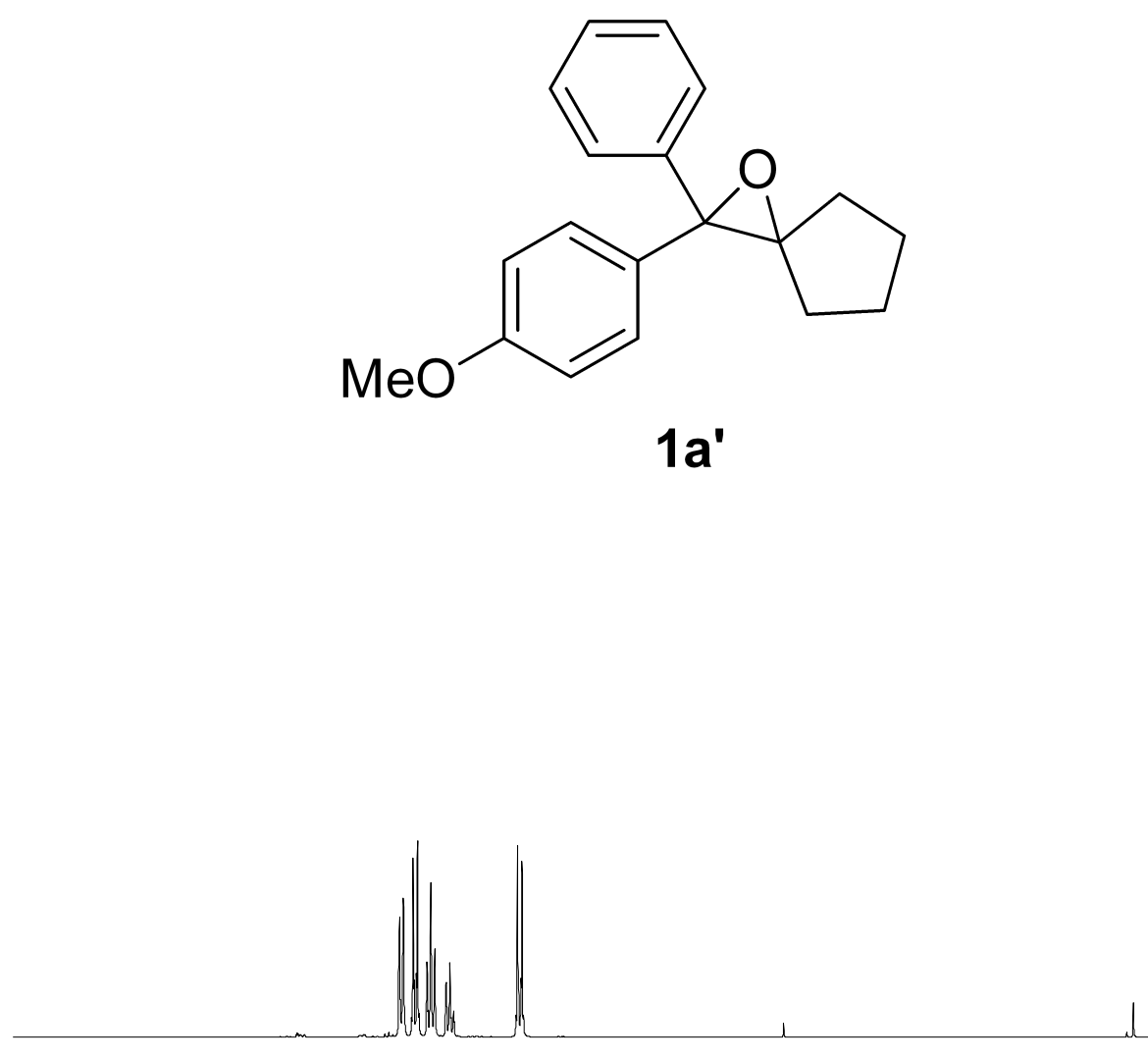

( 11 $\begin{array}{lr}\text { Current Data Parameters } \\ \text { NAME } & \text { mdk10166H } \\ \text { EXPNO } & 1 \\ \text { PROCNO } & 1\end{array}$

F2 - Acquisition Parameters Date_ $\quad 20190715$

$\begin{array}{lr}\text { Time } & 2019.09\end{array}$

INSTRUM spect

PROBHD $5 \mathrm{~mm}$ PABBO BB/

$\begin{array}{lr}\text { PULPROG } & \text { zg30 } \\ \text { TD } & 65536\end{array}$

SOLVENT Acetone

NS

NS

DS

FIDRES

$\mathrm{AQ}$

RG

DW

DE

D 1
TD 0

$===$

$\mathrm{SFO1}$

$\mathrm{NUC1}$
$\mathrm{P} 1$

P 1
PLW1

Acetone
16

$8012.820 \mathrm{~Hz}$

$8012.820 \mathrm{~Hz}$

$4.0894465 \mathrm{sec}$

34.77

62.400 usec

6.50 usec

$1.00000000 \mathrm{sec}$

CHANNEL $\mathrm{f1}========$

$400.1324710 \mathrm{MHz}$

14.50 use

$11.99499989 \mathrm{~W}$

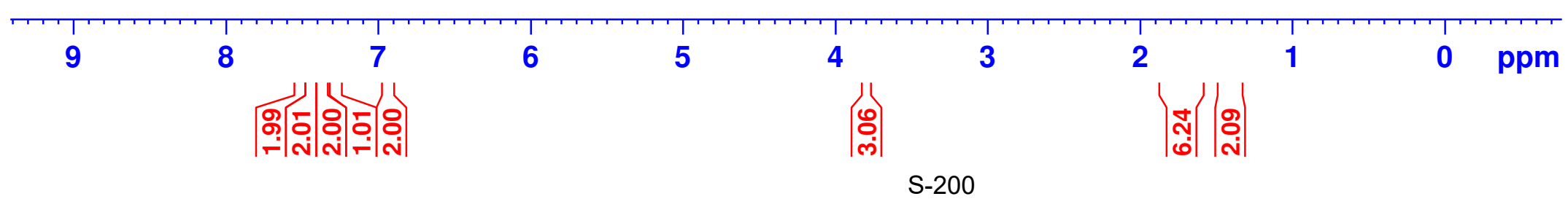




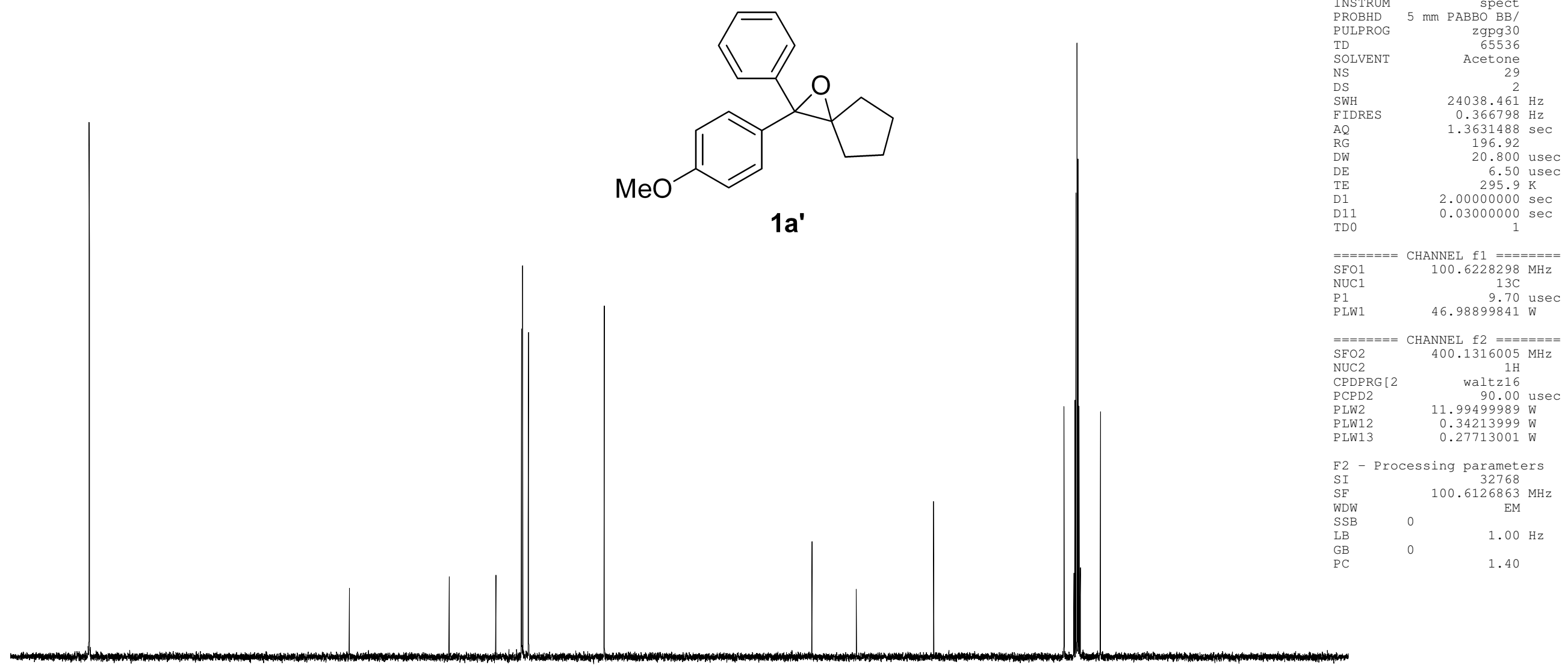

\begin{tabular}{|rrrrrrrrrrrrr}
\hline 200 & 180 & 160 & 140 & 120 & 100 & 80 & 60 & 40 & 20 & 0 & ppm
\end{tabular}




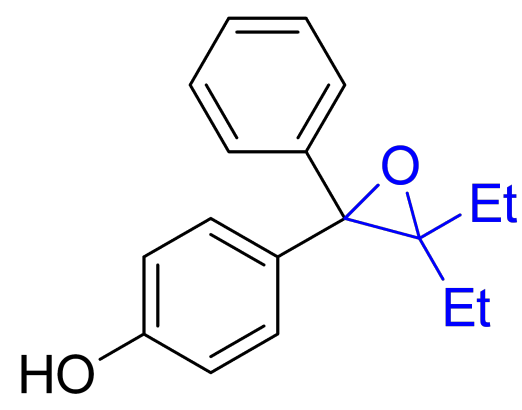

$3 a$

\section{Ethyl A cetate}

Ethyl A cetate

Ethyl A cetate

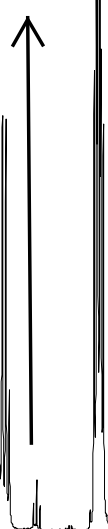

\section{BR UKER}

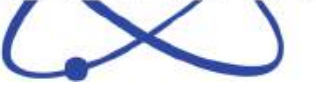

Current Data Parameters NAME EXPNO

PROCNO

F2 - Acquisition Parameters

Date_ 20190125

Time $\quad 10.32$

spect
PROBHD $5 \mathrm{~mm} \mathrm{PABBO} \mathrm{BB} /$

PROBHD $5 \mathrm{~mm}$ PABBO $\mathrm{BB} /$
PULPROG

$\begin{array}{lr}\text { PULPROG } & \text { zg30 } \\ \text { TD } & 65536\end{array}$

$\begin{array}{ll}\text { TD } & 65536 \\ \text { SOLVENT } & \text { CDC13 }\end{array}$

NS

DS

SWH

FIDRES

$\mathrm{AQ}$

RG

DW

DE

D1

IDO

$8012.820 \mathrm{~Hz}$

$0.122266 \mathrm{~Hz}$

$4.0894465 \mathrm{sec}$$$
62.400 \text { use }
$$

6.50 use

$295.6 \mathrm{~K}$

$1.00000000 \mathrm{sec}$

SFO1 $\quad 400.1324710 \mathrm{MHz}$

NUC1 14.50

P1 14.50 usec

PLW1 11.99499989 W

F2 - Processing parameters

SI 65536

SF $\quad 400.1300174 \mathrm{MHz}$

WDW

SSB

GB

EM

EM

$0.30 \mathrm{~Hz}$

1.00

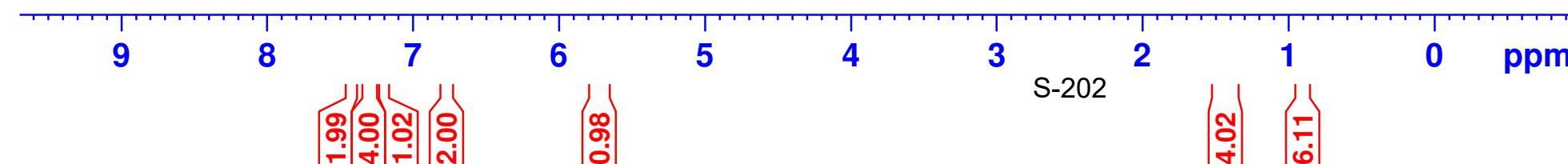




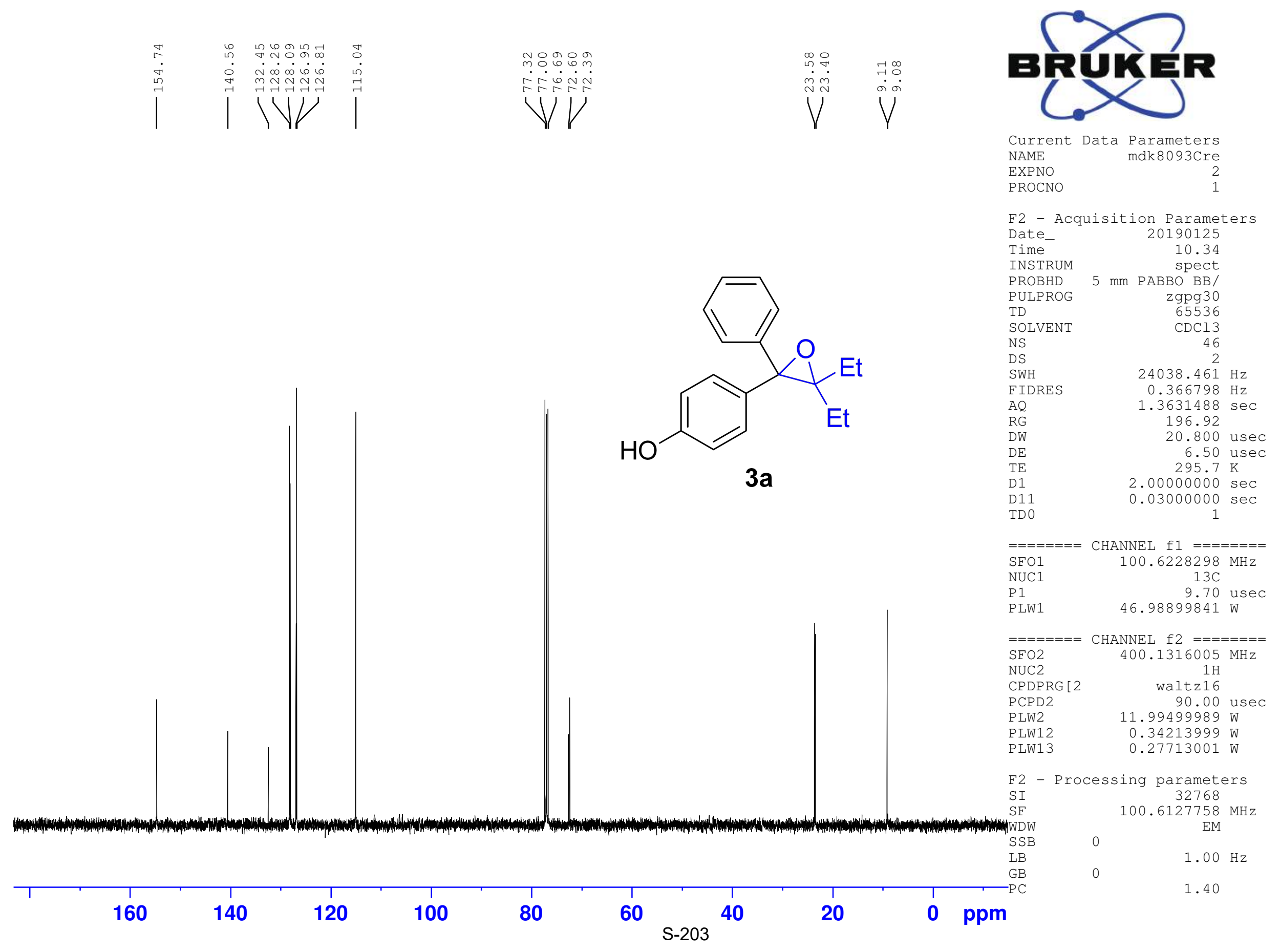




\section{BR $(1)$}

Current Data Parameters NAME

$\operatorname{mdk} 8189 \mathrm{H}$

EXPNO

F2 - Acquisition Parameters

Date__ 20190223

Time 21.59

$5 \mathrm{~mm}$ spect

PULPROG $5 \mathrm{~mm}$ PABBO
zg30

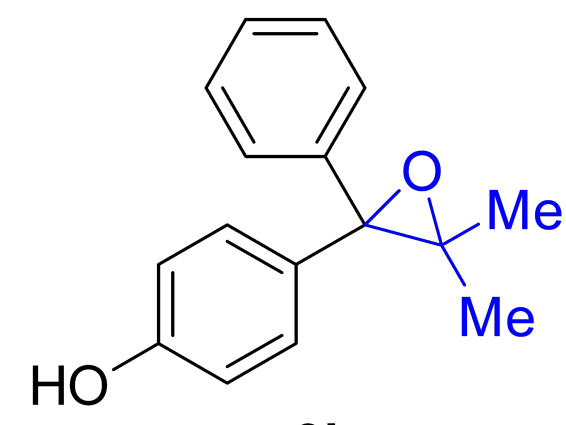

$3 b$
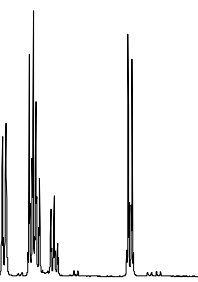

TD

SOLVEN

DS

SWH

FIDRES

$\mathrm{AQ}$

RG

DW

$\mathrm{DE}$

D1
TD 0

Acetone

16
2

$8012.820 \mathrm{~Hz}$

$0.122266 \mathrm{~Hz}$

$4.0894465 \mathrm{sec}$

54.81

62.400 usec 6.50 usec

$1.00000000 \mathrm{~K}$

$$
1
$$

$=======$ CHANNEL f $1========$

$\mathrm{SFO} 1$

NUC1
P1

$400.1324710 \mathrm{MHz}$ 400.1324710
$1 \mathrm{H}$

PLW1

4.50 usec

$11.99499989 \mathrm{~W}$

$\begin{array}{lcc}\text { F2 } & \text { - Processing parameters } \\ \text { SI } & 65536 \\ \text { SF } & 400.1299911 \mathrm{MHz} \\ \text { WDW } & 0 & \text { EM } \\ \text { SSB } & 0 & \\ \text { LB } & 0 & 0.30 \mathrm{~Hz} \\ \text { GB } & 0 & 1.00 \\ \text { PC } & & \end{array}$

6

\begin{abstract}
5
\end{abstract}
4

3

2

1

0 ppm

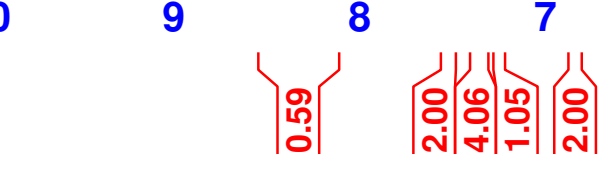

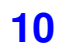




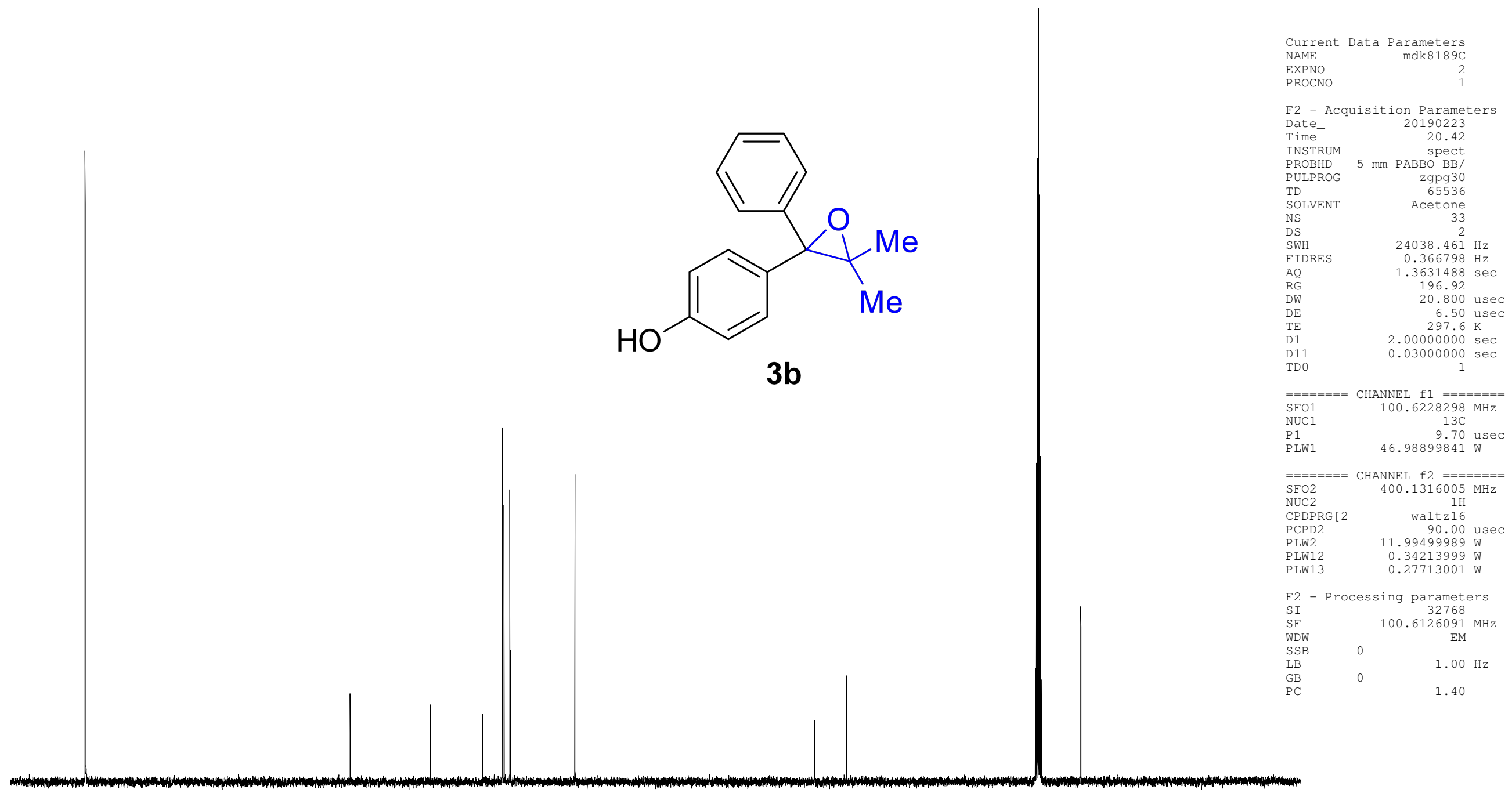

\begin{tabular}{|c|c|c|c|c|c|c|c|c|c|c|}
\hline 200 & 180 & 160 & 140 & 120 & 100 & $\begin{array}{c}1 \\
80 \\
205\end{array}$ & 60 & 40 & 20 & 0 \\
\hline
\end{tabular}




\section{sporen $(1>$}

Current Data Parameters NAME

PROCNO

$\operatorname{mdk} 9079 \mathrm{H}$

F2 - Acquisition Parameters

Date_ 20190318

$\begin{array}{lr}\text { Time } & 13.19\end{array}$

INSTRUM spect

PUIPROG

zg30
65536

SOLVENT

$\begin{array}{lr}\text { SOLVENT } & \text { Acetone } \\ \text { NS } & 16 \\ \text { DS } & 2\end{array}$

SWH

$8012.820 \mathrm{~Hz}$

IDRES $\quad 0.122266 \mathrm{~Hz}$

$\mathrm{AQ} \quad 4.0894465 \mathrm{sec}$

RG

DW

$\mathrm{DE}$

TE

$3 c$

$\mathrm{SFO}$

NUC

P 1
PLW1

27.78

62.400 usec

6.50 usec

$1.00000000 \mathrm{sec}$

CHANNET $112=0$ $400.1324710 \mathrm{MHz}$ $1 \mathrm{H}$

50 usec

F2 - Processing parameters SI $\quad 65536$ $\begin{array}{lc}\text { SF } & 400.1299911 \mathrm{MHz} \\ \text { WDW } & \text { EM }\end{array}$

SSB

$0.30 \mathrm{~Hz}$

GB

PC

1.00

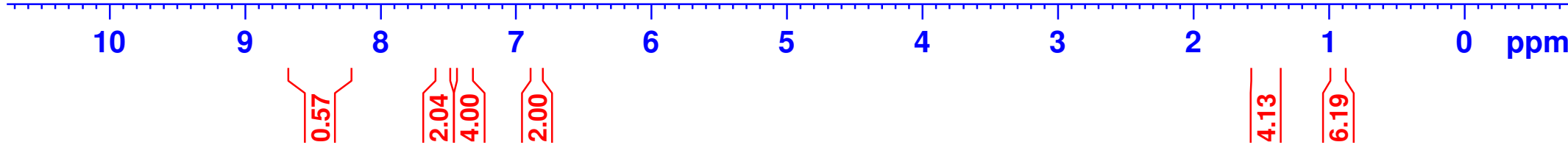




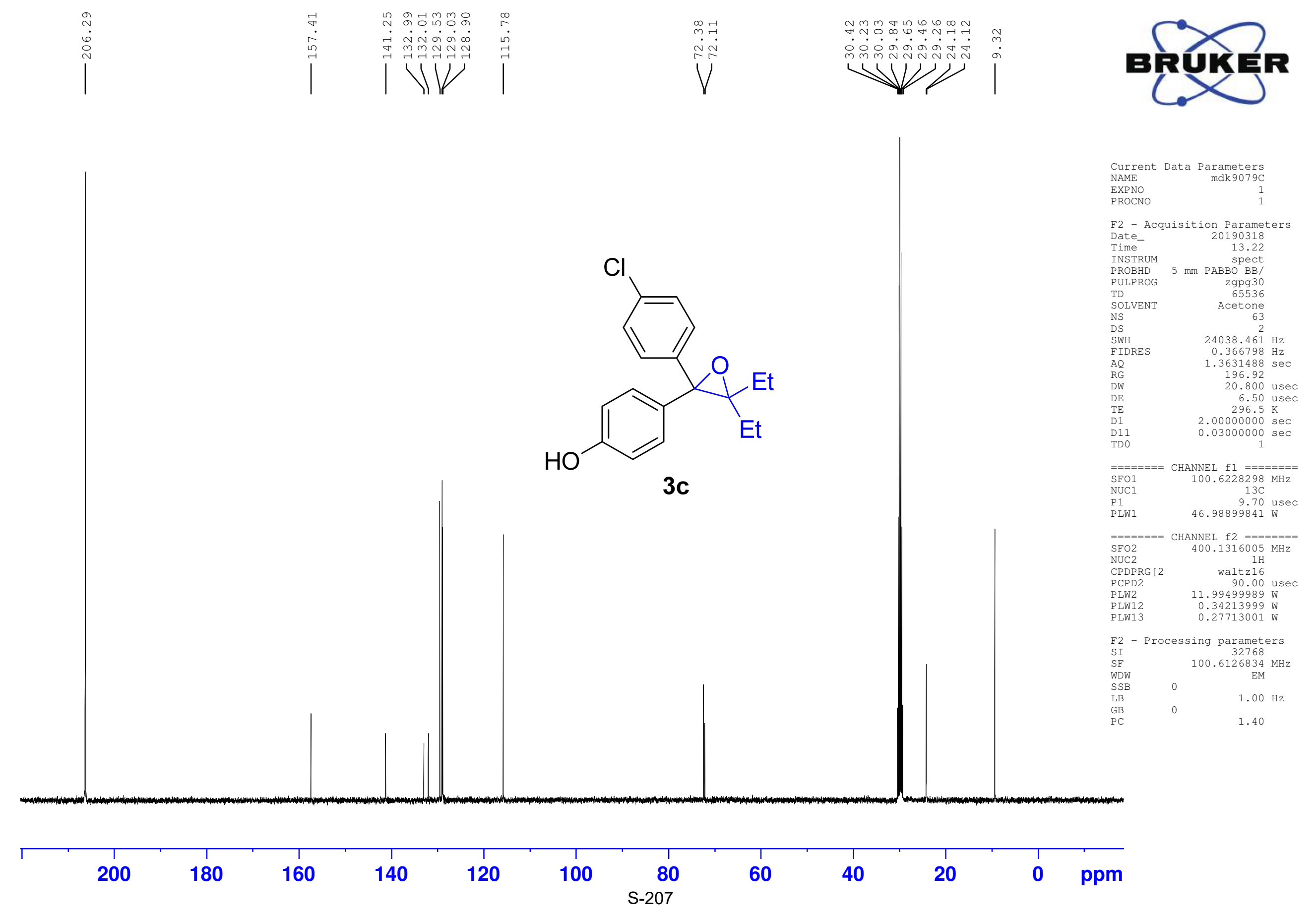




\section{eperer

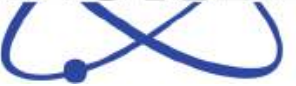

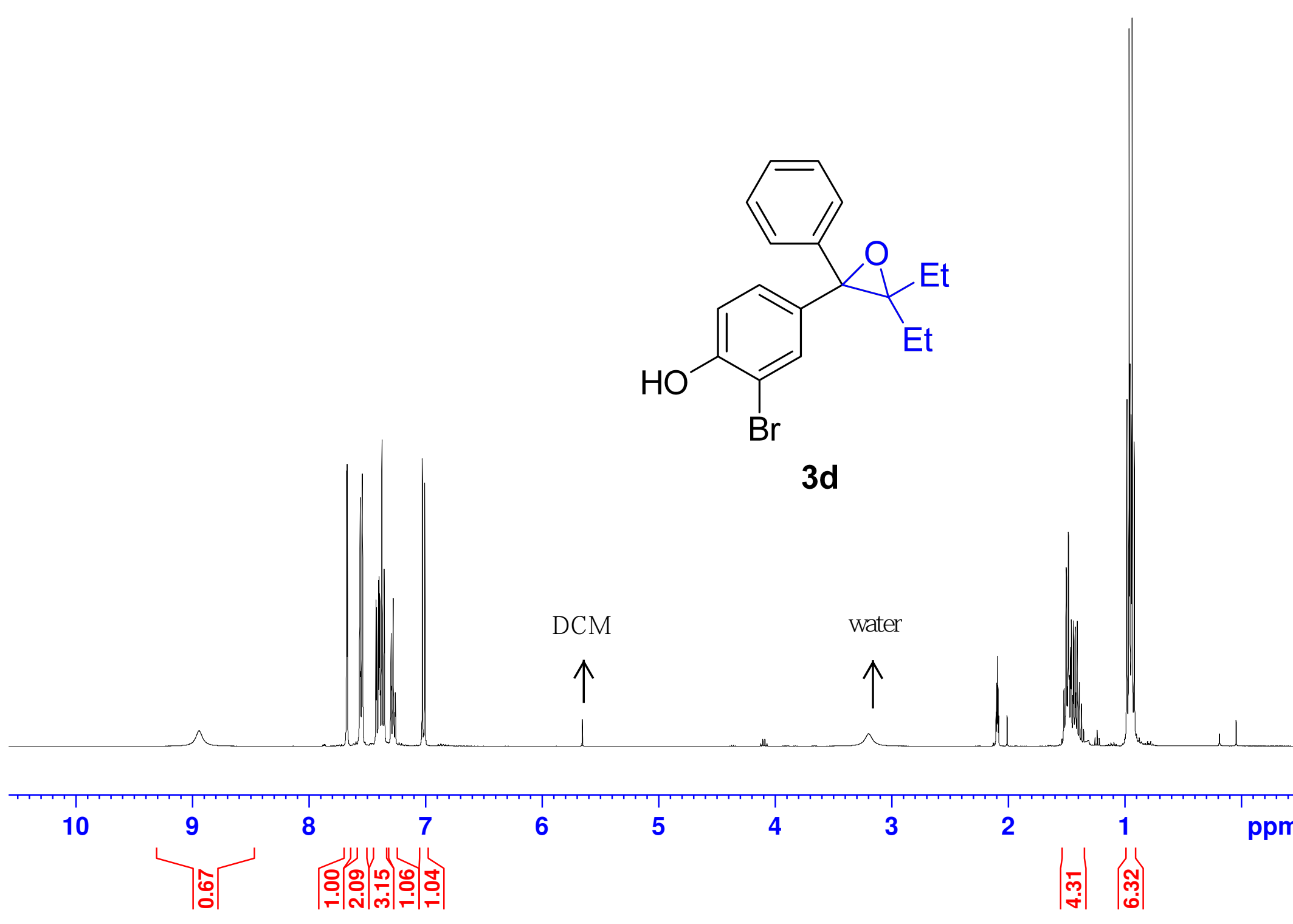

Current Data Parameters NAME

EXPNO
PROCNO

mdk $9123 \mathrm{H}$

ROCNO

2 - Acquisition Parameters

Date_ 20190330

Time $\quad 12.55$

$5 \mathrm{~mm}$ spect

$\begin{array}{lr}\text { PULPROG } & \text { zg30 } \\ \text { TD } & 65536\end{array}$

SOLVENT Acetone

DS

SWH

$8012.820 \mathrm{~Hz}$ $4.0894465 \mathrm{~Hz}$ 


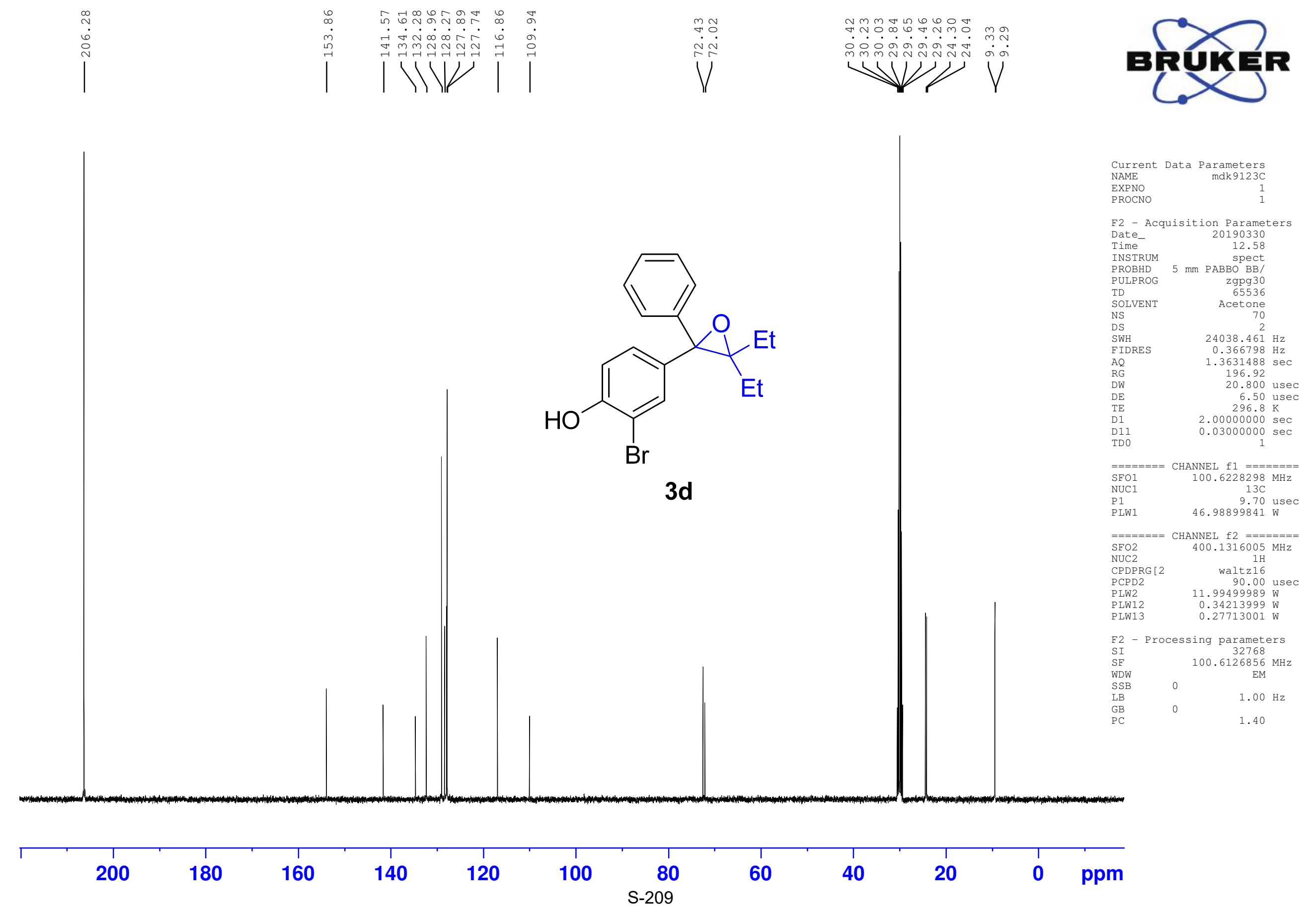




\section{sR KKen $(1)$}

Current Data Parameters
NAME EXPNO

PROCNO

1

F2 - Acquisition Parameters Date__ 20190811 $\begin{array}{lr}\text { Time- } & 13.18\end{array}$ spect
INSTRUM $5 \mathrm{~mm}$ PABBO ULPROG $\begin{array}{lr}\text { TD } & \text { Zg30 } \\ & 65536\end{array}$ SOLVENT Acetone NS 16 SWH AO $\quad 4.0894465 \mathrm{~Hz}$ RG DW $\mathrm{DE}$ TE TD 0

$====$ $\mathrm{SFO1}$ NUC1 P 1
PLW1 $4.0894465 \mathrm{sec}$ 62.67 62.400 usec 6.50 usec $1.00000000 \mathrm{sec}$ $3 e$
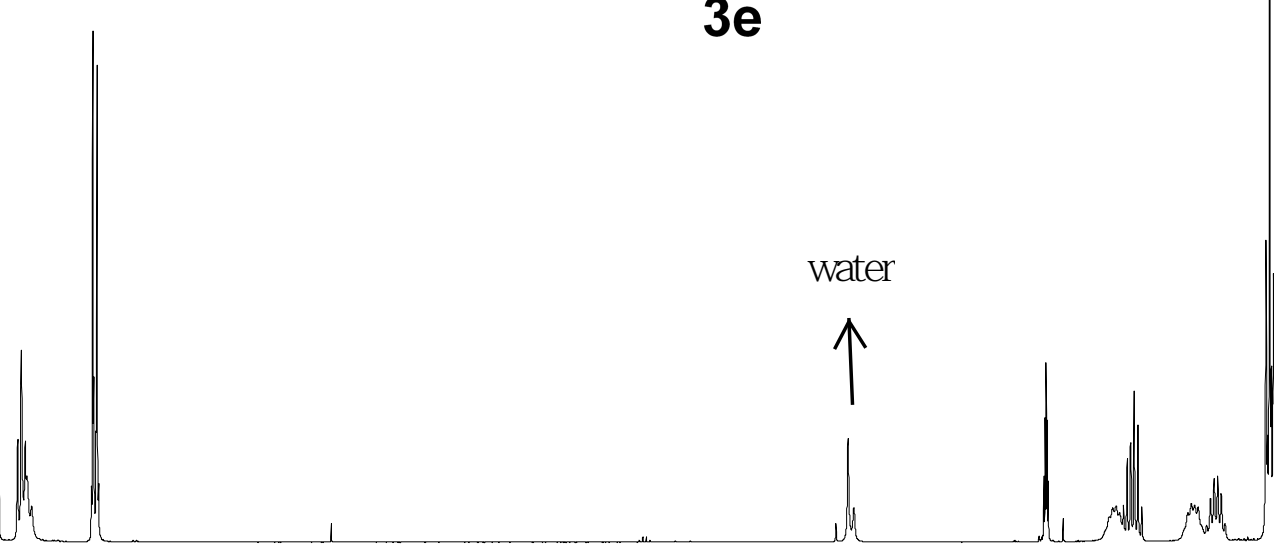

PC

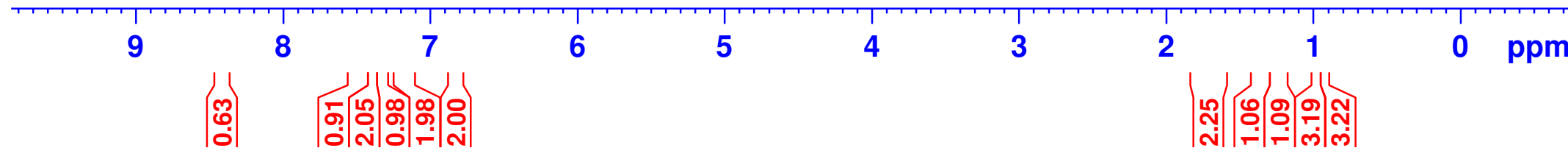



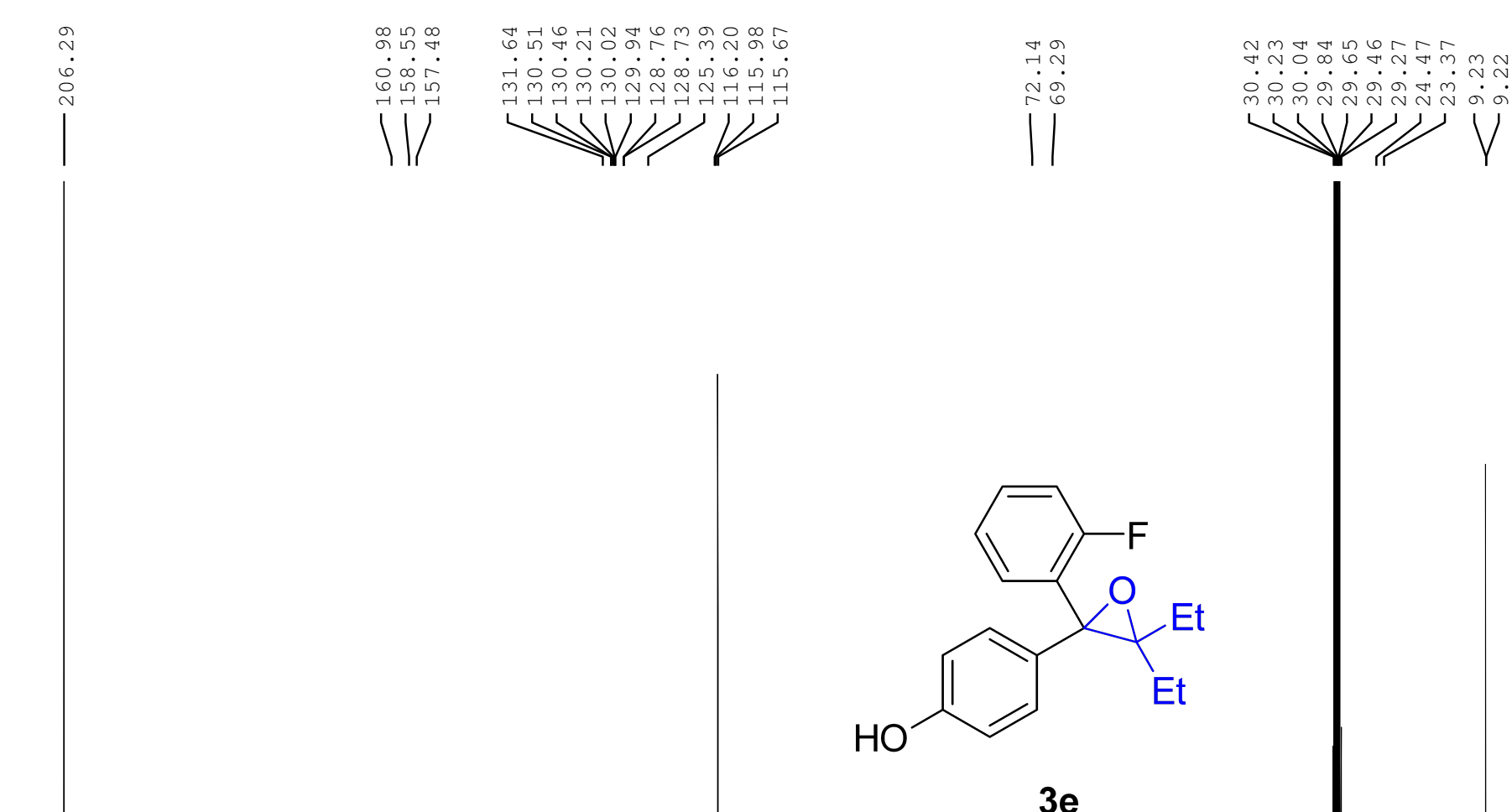

BR UKKER

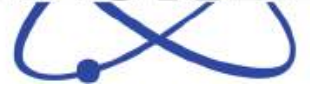

Current Data Parameters NAME

PROCNO

mdk $11064 \mathrm{C}$

F2 - Acquisition Parameters

Date_ 20190811

Time $\quad 17.03$

INSTRUM 5 SPECt

PROBHD $5 \mathrm{~mm}$ PABBO BB/

PULPROG zgpg30

TD

SOLVENT Acetone

NS

DS

SWH

FIDRES

$\mathrm{AQ}$

RG

DW

$\mathrm{DW}$
$\mathrm{DE}$
$\mathrm{TE}$
$\mathrm{D} 1$

D11

$3 e$

$===$

$\mathrm{SFO}$

NUC1

Acetone
3480

$24038.461 \mathrm{~Hz}$

$0.366798 \mathrm{~Hz}$

$1.3631488 \mathrm{sec}$ 196.92

20.800 usec

6.50 usec

$295.9 \mathrm{~K}$

$2.00000000 \mathrm{sec}$

$0.03000000 \mathrm{sec}$

$100.6228298 \mathrm{MHz}$

PLW1 $\quad 46.98899841 \mathrm{~W}$

$\mathrm{SFO} 2$

NOL $12=======$

NUC2

$400.1316005 \mathrm{MHz}$

CPDPRG [2 waltz16

PCPD2 90.00 usec

PLW2 11.99499989

PLW12 0.27713001

F2 - Processing parameters

F2 - Processing parameters

$\begin{array}{lr}\text { SI } & 32768 \\ \text { SF } & 100.6126827 \mathrm{MHz}\end{array}$ WDW

$100.6126827 \mathrm{MHz}$
$\mathrm{EM}$

$1.00 \mathrm{~Hz}$

1.40

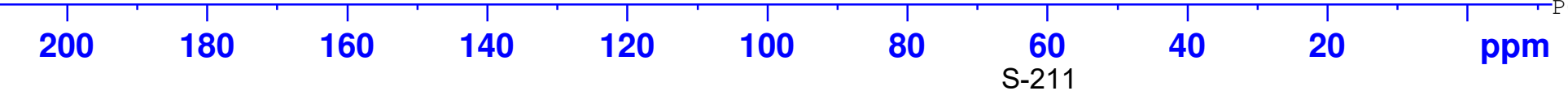




\section{apiren}

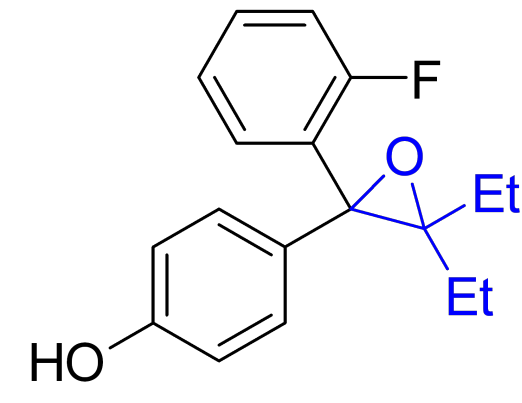

$3 e$
Current Data Parameters NAME mdk11064F-decoup PROCNO F2 - Acquisition Parameters
Date_ 20190811 Time PROBHD $5 \mathrm{~mm}$ PABBO BB/

PULPROC

TD SOLVENT

NS
DS

SWH
FIDRE

$\mathrm{AQ}$
$\mathrm{RG}$

DW

TE
D1
D11

D11
TD 0

$=======$ CHANNEL f 11 = $=======$ SFO1 $376.4607162 \mathrm{MHz}$ P1

14.70 use $15.99600029 \mathrm{~W}$

$=======$ CHANNEL $f 2 \quad======= \pm$
SFO2
$400.1316005 \mathrm{MHz}$ NUC2
CPDPRG waltz16

waltz16
90.00 usec $1.99499989 \mathrm{~W}$
$0.34213999 \mathrm{~W}$
$0.2713001 \mathrm{~W}$

PLW12
PLW13

F2 - Processing parameters

SI Processing parameters

$\begin{array}{ll}\text { SF } & 376.4983660 \mathrm{MHz}\end{array}$

GB

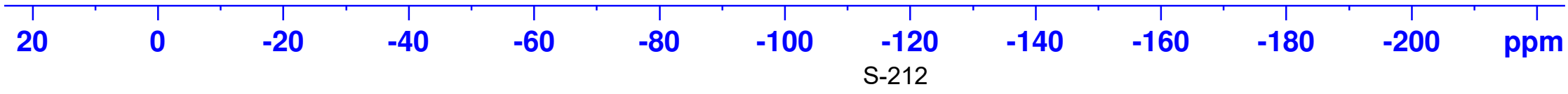




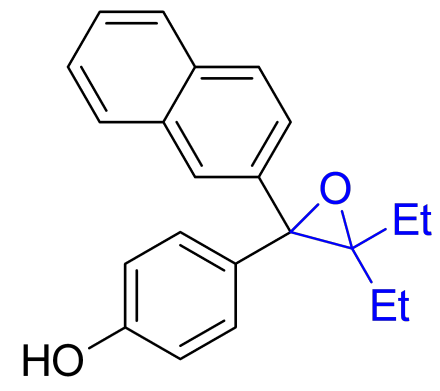

$3 \mathbf{f}$ $\begin{array}{lr}\text { Current } & \text { Data } \\ \text { NAME } & \text { mameters } \\ \text { EXPNO } & 1 \\ \text { PROCNO } & 1\end{array}$

F2 - Acquisition Parameters Date_ 20190810

Time $\quad 14.04$

INSTRUM spect

PROBHD $5 \mathrm{~mm}$ PABBO BB/

PULPROG zg30

TD 65536

SOLVENT Acetone

NS

DS

FIDRES

$8012.820 \mathrm{~Hz}$ AO $\quad 4.0894465 \mathrm{~Hz}$

RG

DW

$\mathrm{DE}$

$\mathrm{TE}$

TD 0

$====$

$\mathrm{SFO1}$

$\mathrm{NUC1}$
$\mathrm{P} 1$

P 1
PLW1

4.0894465 45.67

62.400 usec 6.50 use

$1.00000000 \mathrm{sec}$

$$
1
$$

CHANNEL $\mathrm{f} 1========$ $400.1324710 \mathrm{MHz}$ 1324710
$1 \mathrm{H}$

14.50 usec $11.99499989 \mathrm{~W}$

$\begin{array}{lcc}\text { F2 } & \text { Processing parameters } \\ \text { SI } & 65536 \\ \text { SF } & 400.1299909 \mathrm{MHz} \\ \text { WDW } & & \text { EM } \\ \text { SSB } & 0 & 0.30 \mathrm{~Hz} \\ \text { LB } & 0 & \\ \text { GB } & 0 & 1.00 \\ \text { PC } & & \end{array}$

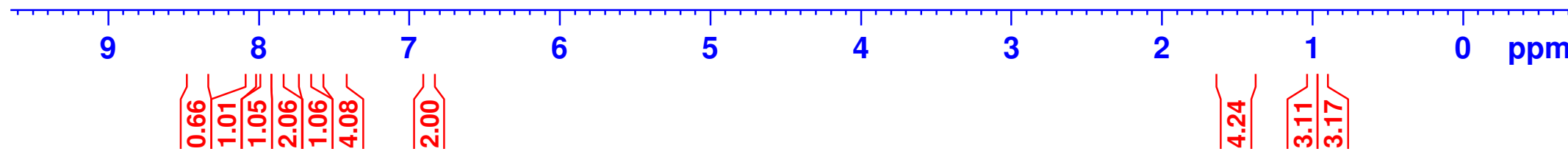



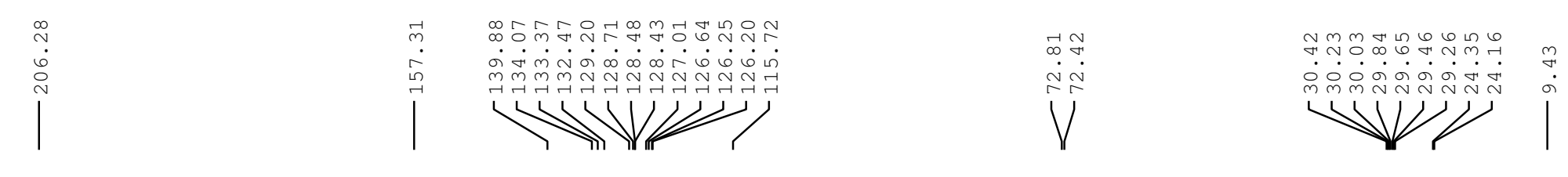

arienger

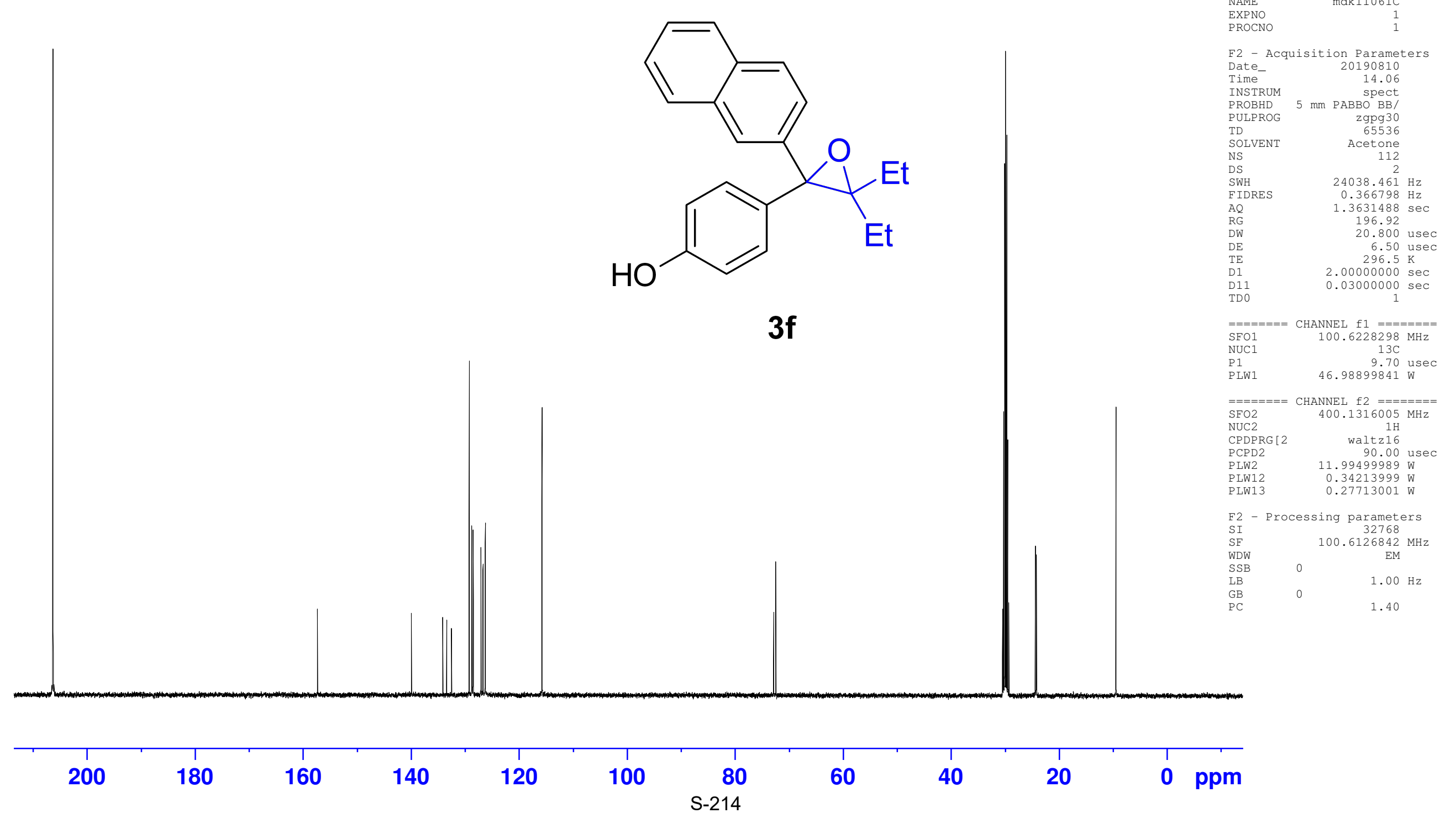




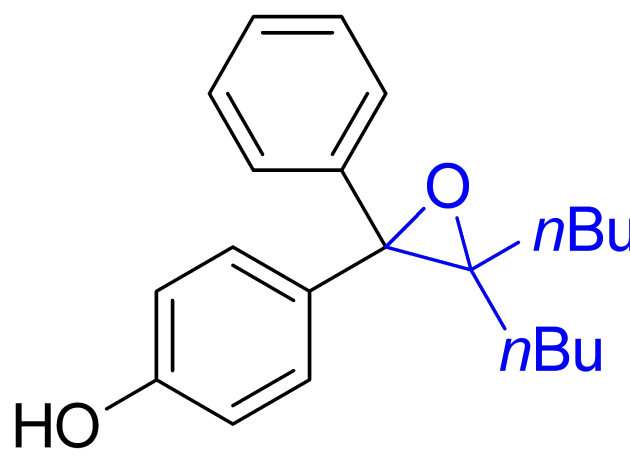

$3 \mathbf{g}$
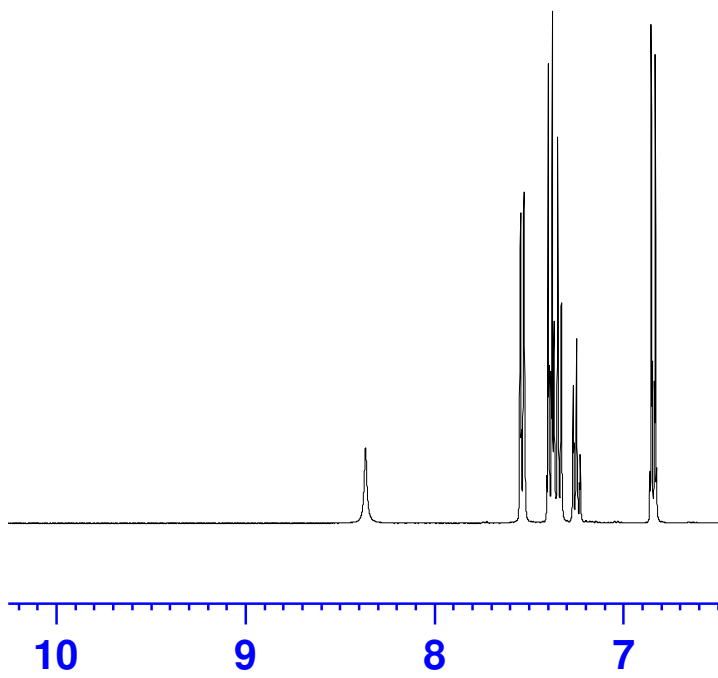

9

8

7

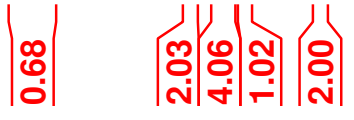

6

5

Ethyl Ether

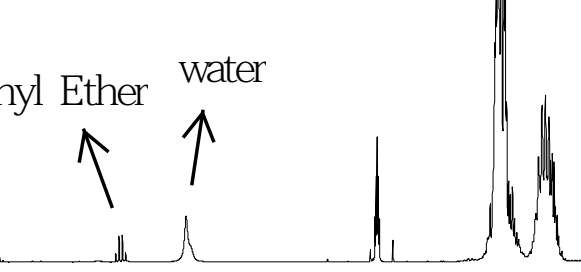

$\begin{array}{lr}\text { Current } & \text { Data } \\ \text { NAME } & \text { mameters } \\ \text { EXPNO } & 1 \\ \text { PROCNO } & 1\end{array}$

F2 - Acquisition Parameters Date__ 20190404

$\begin{array}{lr}\text { Time } & 13.04\end{array}$

INSTRUM spect

PROBHD $5 \mathrm{~mm}$ PABBO

$\begin{array}{lr}\text { TD } & \text { TDO } \\ \text { TD } & 65536\end{array}$

SOLVENT Acetone

$\begin{array}{lr}\text { NS } & \text { Acetone } \\ \text { NS } & 16\end{array}$

DS

$\begin{array}{ll} & 8012.820 \mathrm{~Hz}\end{array}$

FIDRES $\quad 0.122266 \mathrm{~Hz}$

AQ

RG

$\mathrm{DW}$
$\mathrm{DE}$
$\mathrm{TE}$

D1
TD 0

$====$

SFO1

$\mathrm{NUC1}$
$\mathrm{P} 1$

$4.0894465 \mathrm{sec}$
31.55

62.400 usec

6.50 usec

$1.00000000 \mathrm{sec}$

CHANNEL f1 $========$

$400.1324710 \mathrm{MHz}$

$1 \mathrm{H}$

14.50 usec

PLW1 $11.99499989 \mathrm{~W}$

F2 - Processing parameters

SI 65536

$\begin{array}{lc}\text { SF } & 400.1299910 \mathrm{MHz} \\ \text { WDW } & \text { EM }\end{array}$

SSB

$0.30 \mathrm{~Hz}$

GB

1.00 


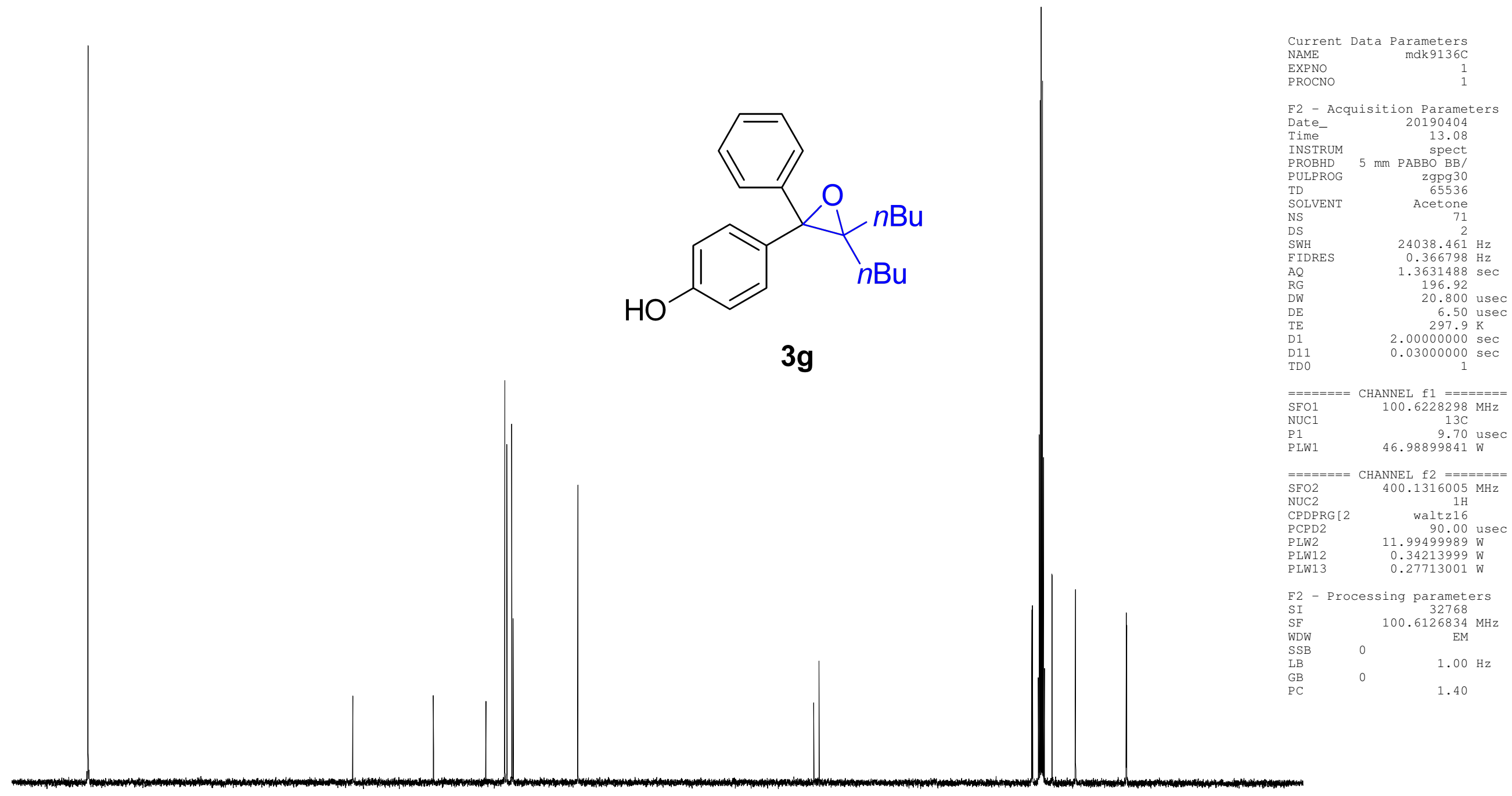

\begin{tabular}{|rrrrrrrrrrrrr}
\hline 200 & 180 & 160 & 140 & 120 & 100 & 80 & 60 & 40 & 20 & 0 & ppm
\end{tabular}




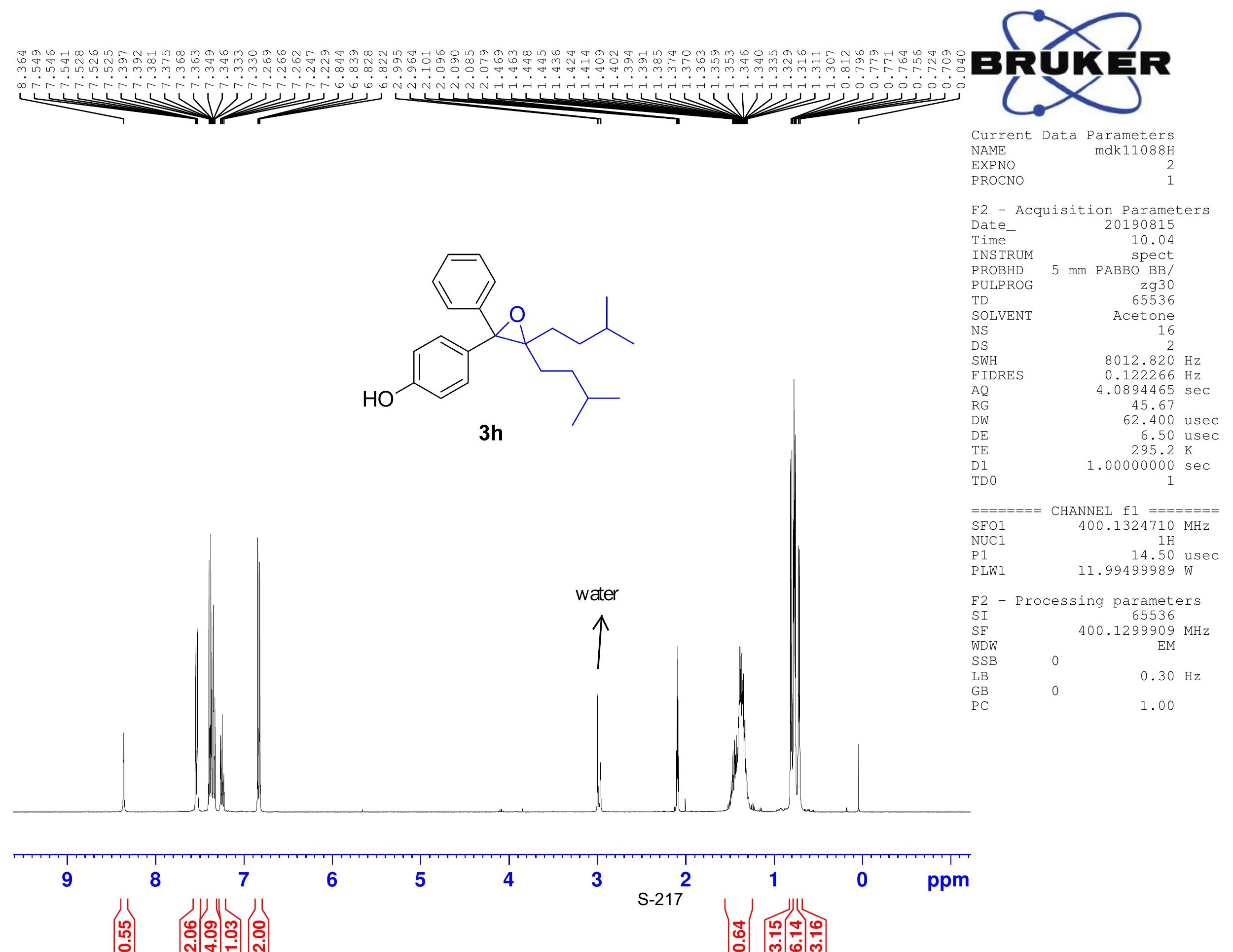




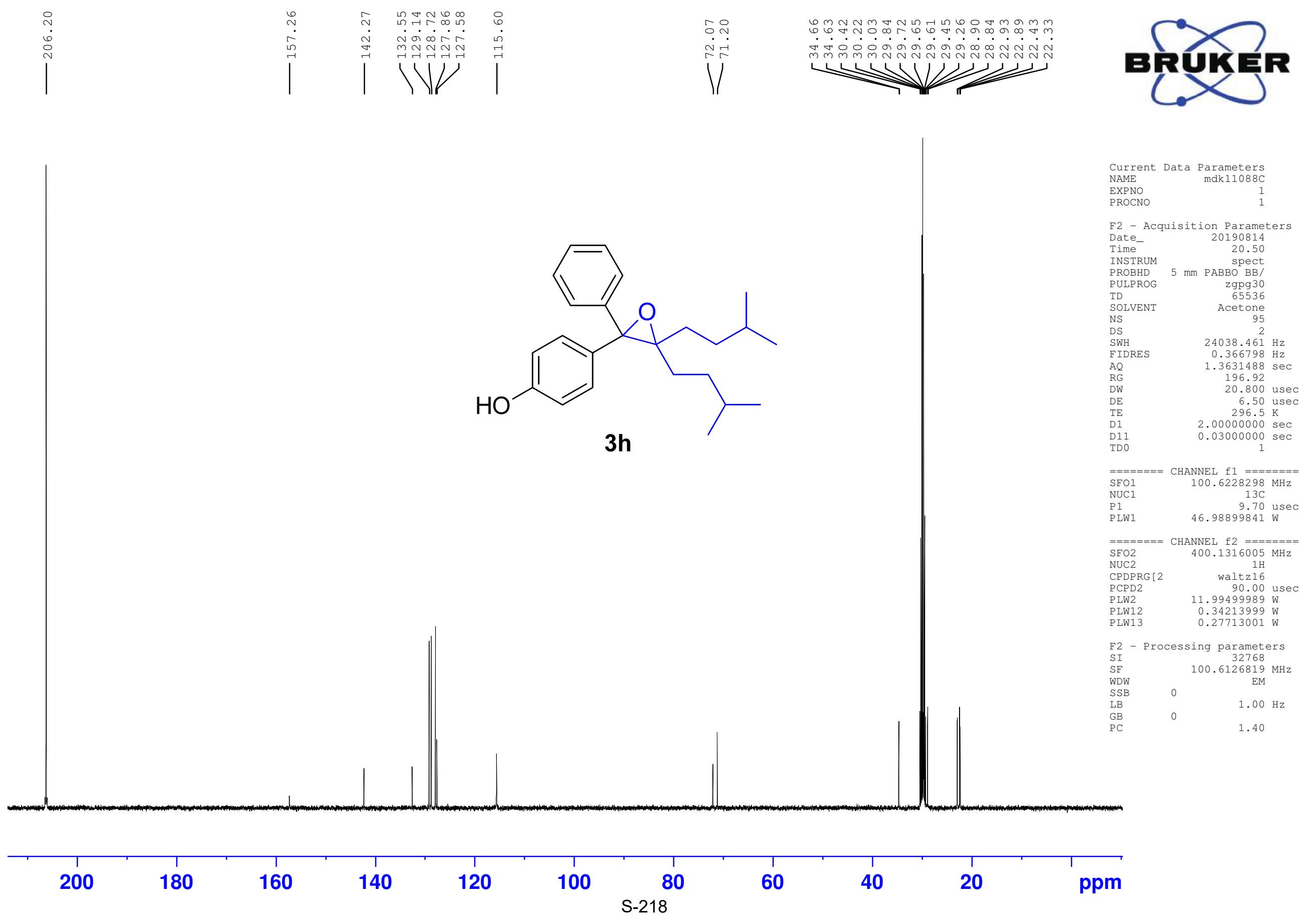




\section{en $(1>$}

Current Data Parameters NAME

PROCNO

$\operatorname{mdk} 10017 \mathrm{H}$

F2 - Acquisition Parameters

Date_ 20190501

Time $\quad 13.26$

spect

PUIPROG

Zg30
65536

SOLVENT Acetone

NS

SWH

FIDRES

AQ

RG

DW

$\mathrm{DE}$

D1
TD 0

$====$

$\mathrm{SFO1}$

NUC

P1
PLW1

Acetone
16

$8012.820 \mathrm{~Hz}$

$8012.820 \mathrm{~Hz}$
$0.122266 \mathrm{~Hz}$

$4.0894465 \mathrm{sec}$

39.46

62.400 usec

6.50 usec

$1.00000000 \mathrm{sec}$

$$
1
$$

CHANNEL $\mathrm{f} 1 \mathrm{l}========$ $400.1324710 \mathrm{MHz}$ 14.50 sec $11.99499989 \mathrm{~W}$

F2 - Processing parameters SI 65536

$\begin{array}{lc}\text { SF } & 400.1299911 \mathrm{MHz} \\ \text { WDW } & \text { EM }\end{array}$

SSB

$0.30 \mathrm{~Hz}$

1.00

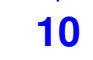

10

9

9

|
5

6

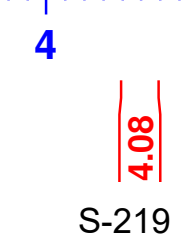

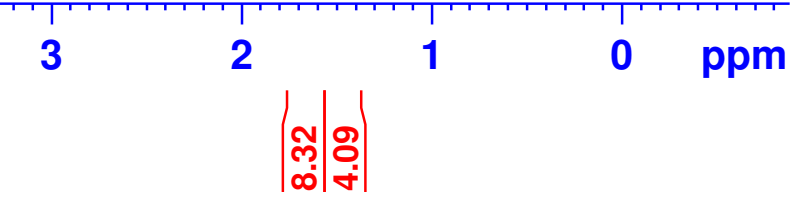



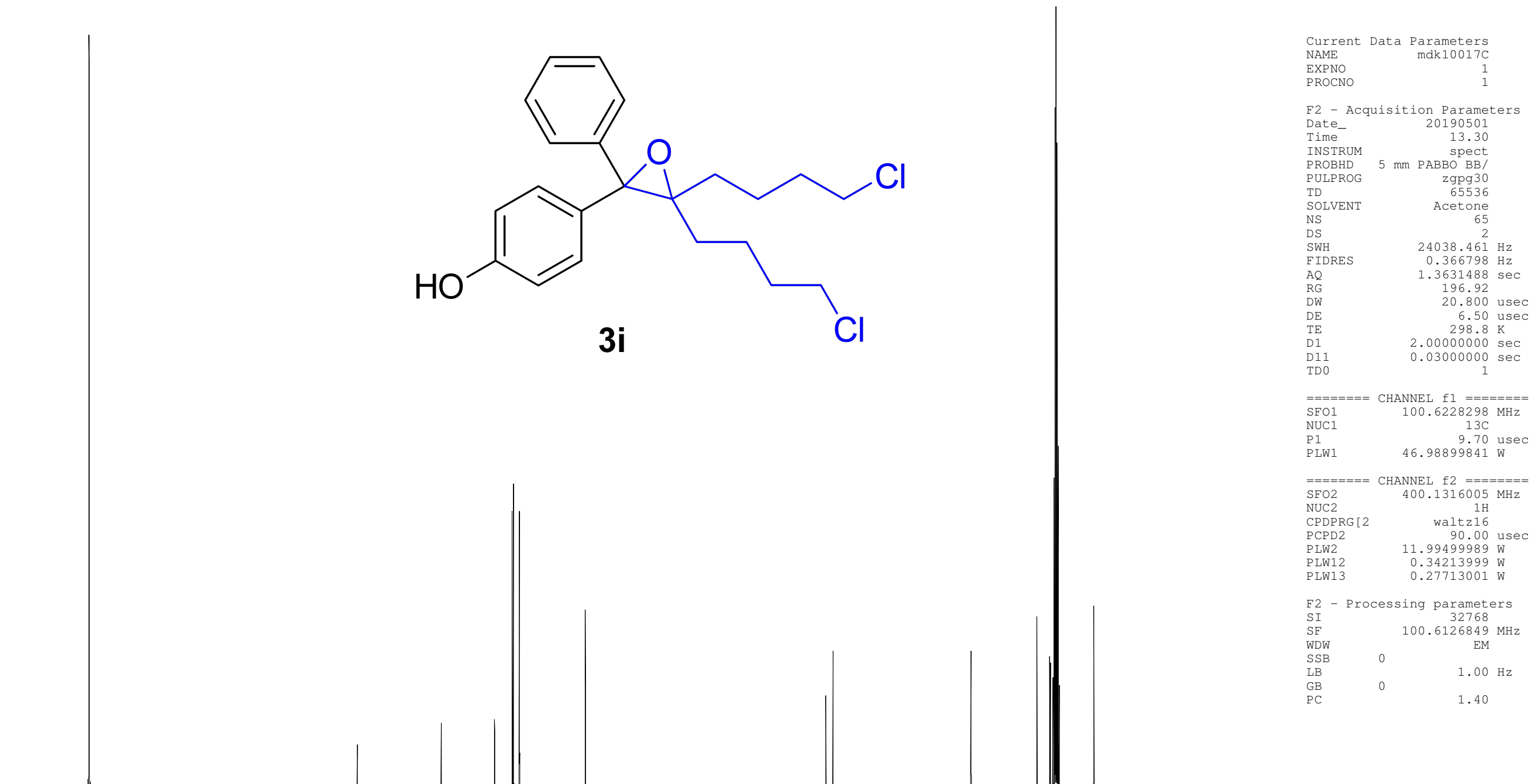

\begin{tabular}{|rrrrrrrrrrrrr}
\hline 200 & 180 & 160 & 140 & 120 & 100 & 80 & 60 & 40 & 20 & 0 & ppm
\end{tabular}




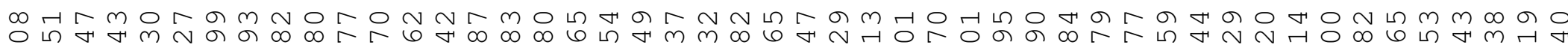
F

\section{sperer ,}

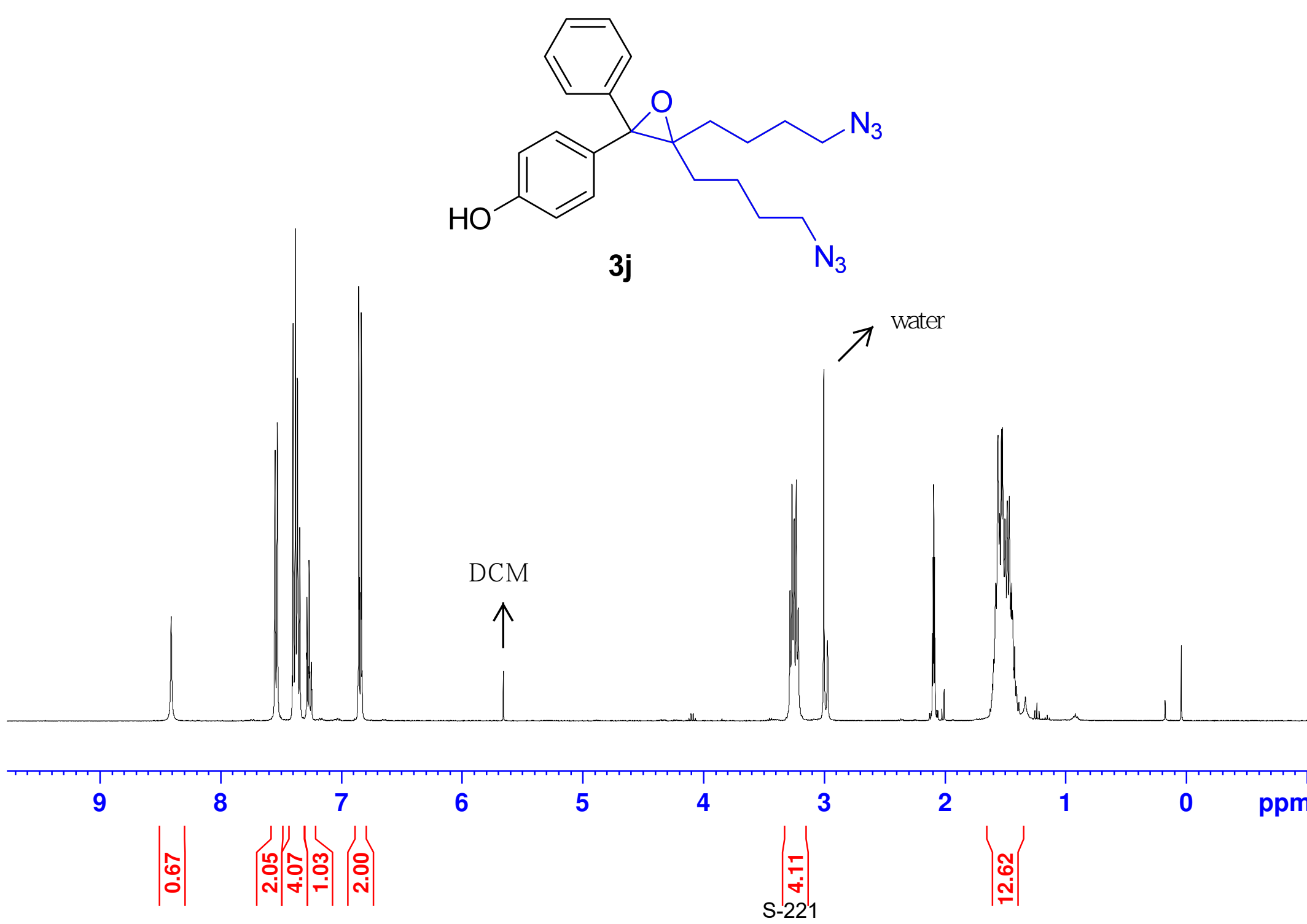

Current Data Parameters
NAME EXPNO

PROCNO

1

F2 - Acquisition Parameters

Date_ 20190814

Time 10.02

spect

PULPROG $\quad$ zg30

TD 65536

Acetone

NS

SWH

FIDRES

$A Q$

$\mathrm{RG}$

DE

$\mathrm{TE}$

D1
TD 0

$===$

$\mathrm{SFO1}$

$\mathrm{NUC}$

16
2

$8012.820 \mathrm{~Hz}$

$0.122266 \mathrm{~Hz}$

$4.0894465 \mathrm{sec}$ 31.55

62.400 usec 6.50 use $1.00000000 \mathrm{sec}$

$1 \mathrm{sec}$
400.1324710 MHz $400.1324710 \mathrm{MHz}$ 14.50 usec LW1 $11.99499989 \mathrm{~W}$

F2 - Processing parameters I 65536 400.1299909 MHz WDW EM

SSB

$0.30 \mathrm{~Hz}$

$\mathrm{PC}$

1.00 


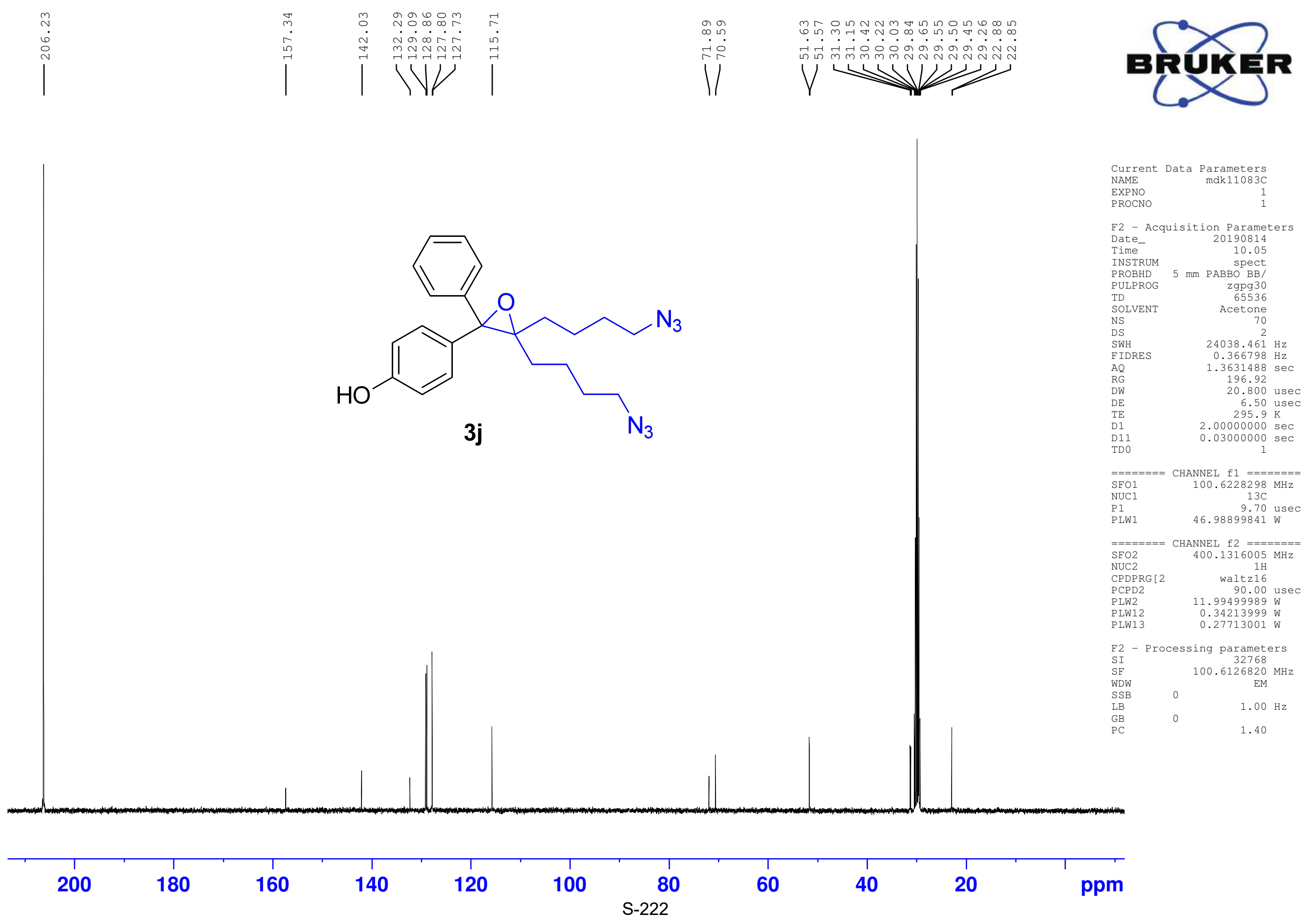




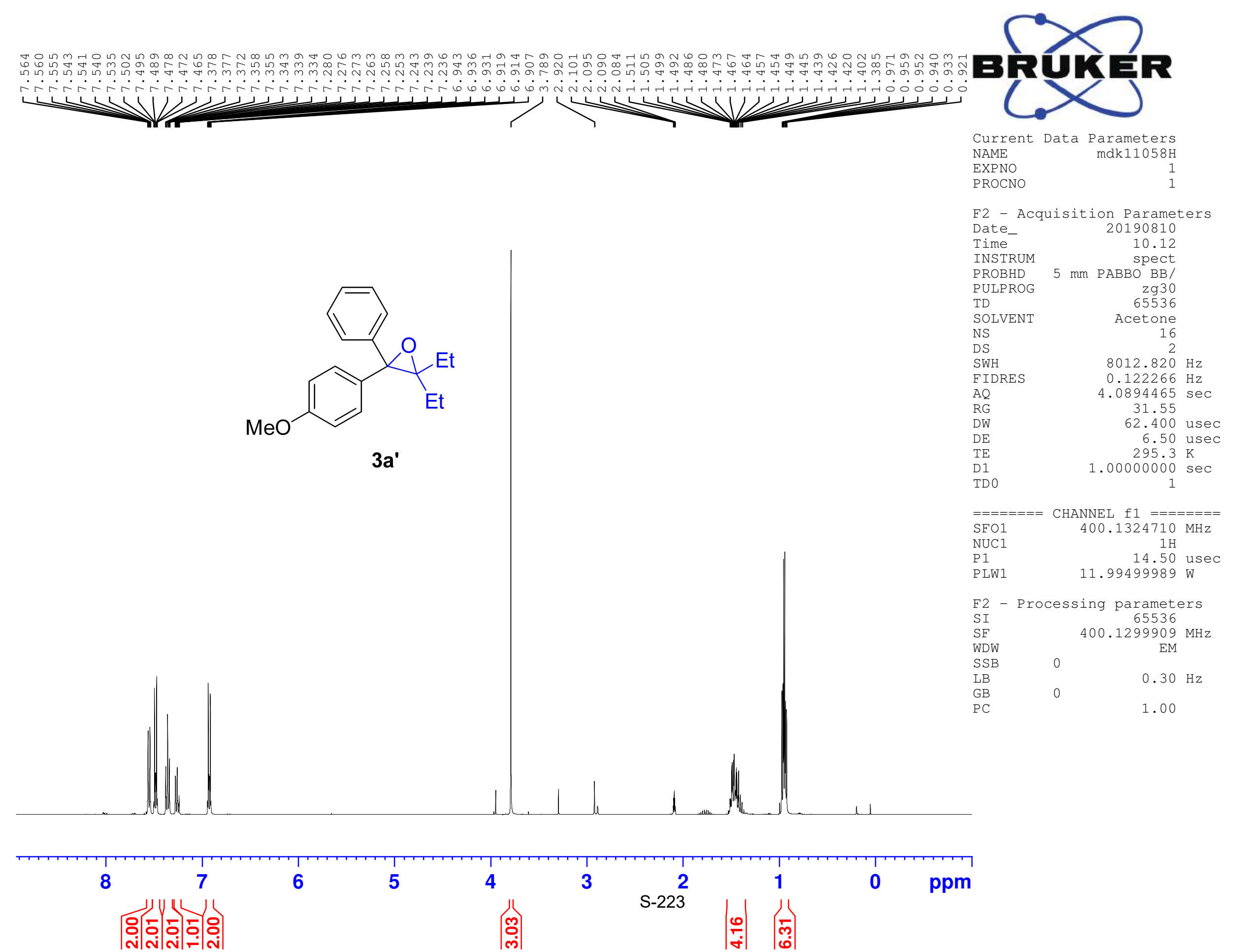




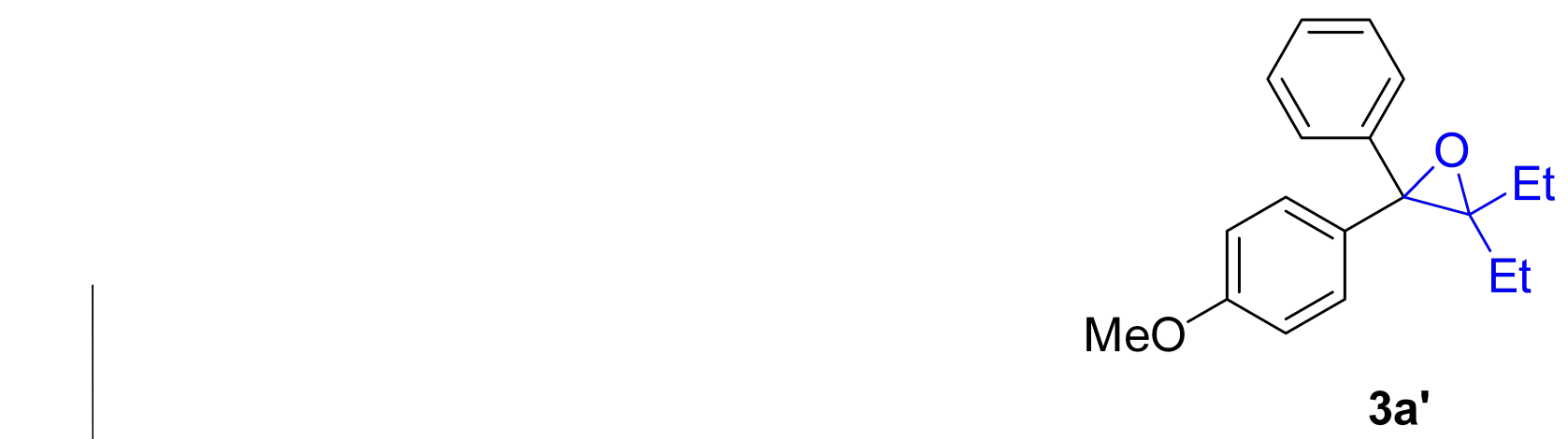

Current Data Parameters EXPNO

mak11058C
1

F2 - Acquisition Parameters
Date_ 20190810

Time-

INSTRUM

PULPRO

TD SOLVENT

NS
DS
SWH

AQ
RG
DW

DE
TE
$D 1$

D1
D1

D11
TD 0

$=== \pm===$ CHANNEL $f 1 \quad========$
SFO1
$100.6228298 \mathrm{MHz}$

$\mathrm{SFO} 1$
$\mathrm{NUC1}$
$\mathrm{P} 1$ spect
zgpg 30

$\begin{array}{r}25 \\ 2 \\ \hline\end{array}$

$0.366798 \mathrm{~Hz}$

20.800 usec

6.50 usec

$2.00000000 \mathrm{sec}$

$913 \mathrm{C}$

$=======$ CHANNEL $f 2 \quad=======$
SFO2
NUC2

$\begin{array}{lr}\text { NUC2 } & 1 \mathrm{H} \\ \text { CPDPRG [2 } & \text { waltz16 }\end{array}$

PLW2 $11.99499989 \mathrm{~W}$

$\begin{array}{ll}\text { PLW12 } & 0.34213999 \mathrm{~W}\end{array}$

$\begin{array}{lr}\text { F2 - Processing parameters } \\ \text { SI } & 32768 \\ \text { SF } & 100.6126856 \mathrm{MHz}\end{array}$

SF

$00.6126856 \mathrm{MHz}$
$\mathrm{EM}$

\begin{tabular}{rrrrrrrrrrrrr}
\hline 200 & 180 & 160 & 140 & 120 & 100 & 80 & 60 & 40 & 20 & 0 ppm
\end{tabular}


のோำ $m m \sim N \sim N \sim \sim 000 \infty \pi \infty$

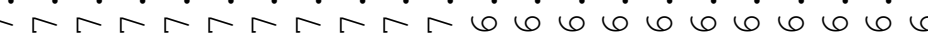
$\longrightarrow+1,1$

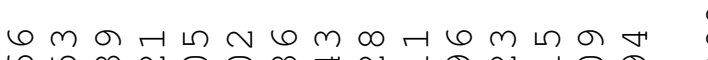

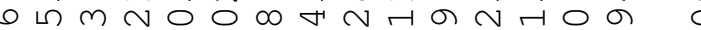

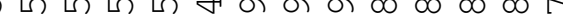

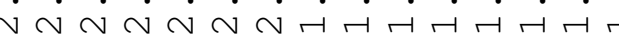

\section{BR (}

Current Data Parameters NAME

PROCNO

mdk $8139 \mathrm{H}$

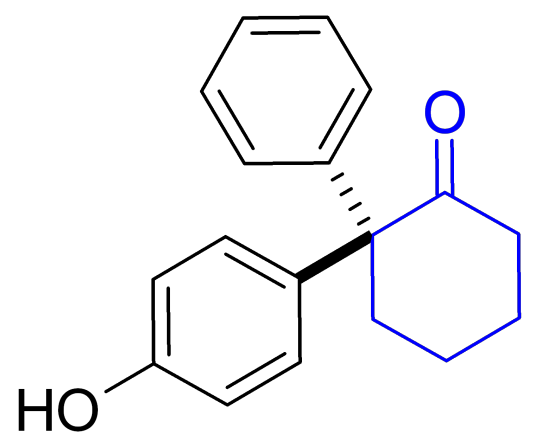

$2 a$

F2 - Acquisition Parameters

Date_ 20190122

Time $\quad 13.41$

$5 \mathrm{~mm}$ spect

PULPROG $\quad$ zg30

$\begin{array}{lr}\text { TD } & \text { Z930 } \\ \text { TD } & 65536\end{array}$

SOLVENT

NS

DS

SWH $4.0894465 \mathrm{~Hz}$ RG

DW

$\mathrm{DE}$

$\mathrm{TE}$
$\mathrm{D} 1$

$.0894465 \mathrm{sec}$
27.78

$1.00000000 \mathrm{sec}$

TD 0 62.400 usec 6.50 usec

$=======$ CHANNEL $f 1$ $\mathrm{SEO}$

$\mathrm{NUC} 1$
$\mathrm{P} 1$

HANNEL $\mathrm{f} 1=$

F2 - Processing parameters SI $\quad 65536$ SF $\quad 400.1300186 \mathrm{MHz}$ WSB

LB EM

$0.30 \mathrm{~Hz}$

1.00

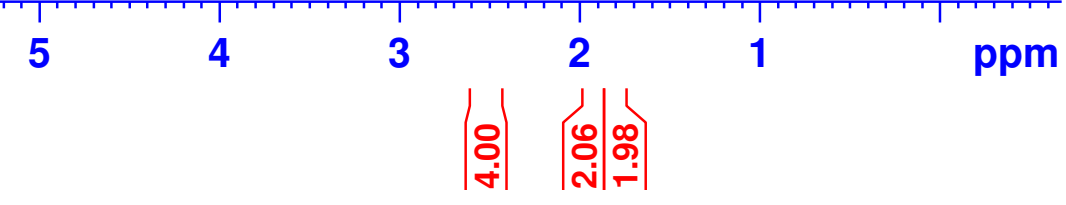

S-225 

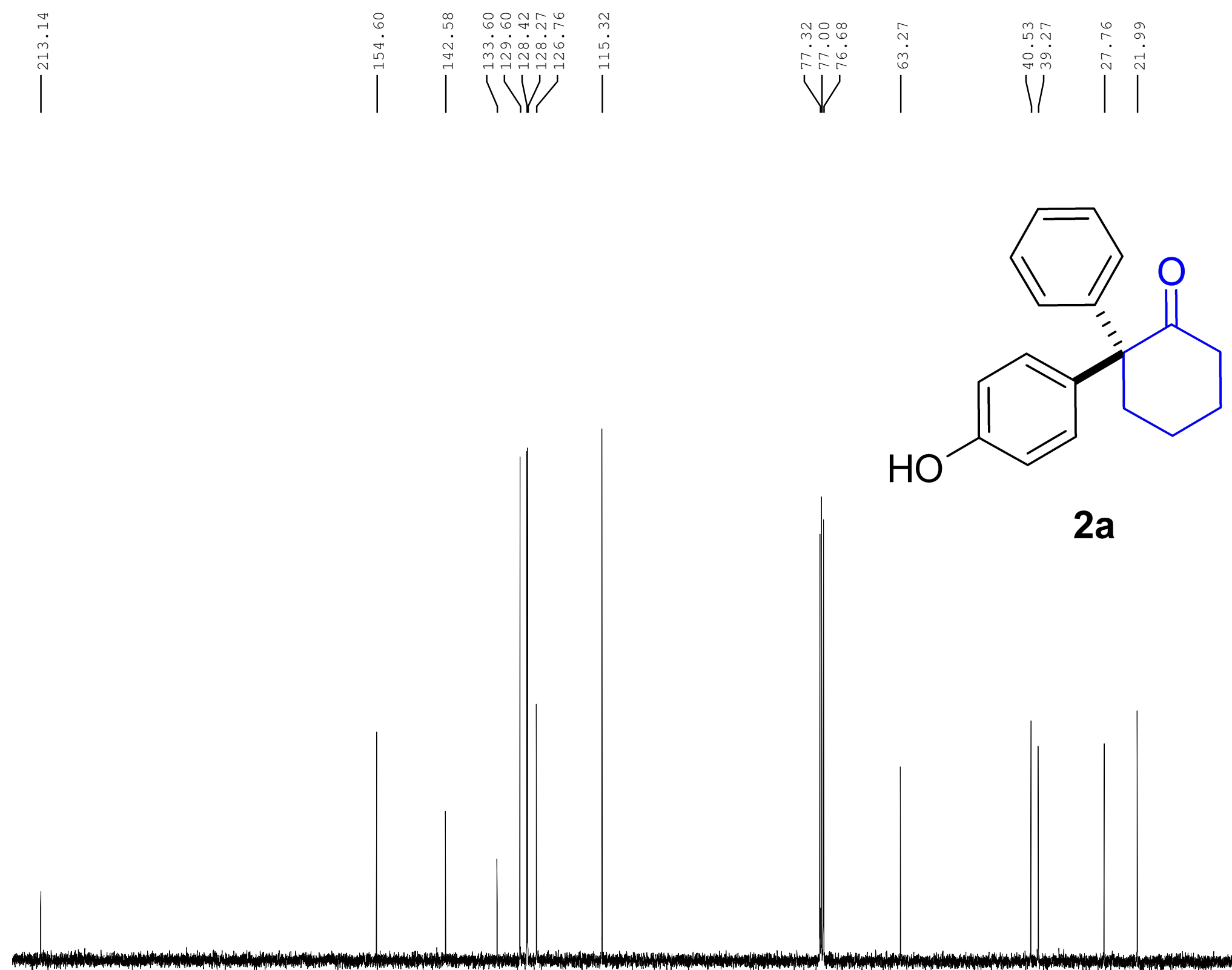

$2 a$

Current Data Parameters
NAME EXPNO

F2 - Acquisition Parameters

Date

INIMe

PROBHD
PULPROG

TD
SOLVEN

NS
DS
SWH

$2 \mathrm{H}$
24038.461 Hz

$\mathrm{AQ}$
$\mathrm{RG}$

RG
DW
DE
TE

196.92 usec
6.800 usec
295.2 K

$\begin{array}{ll}\text { D1 } & 2.00000000 \mathrm{sec} \\ \text { D11 } & 0.03000000 \mathrm{sec} \\ \text { TD0 } & \end{array}$

$=======$ CHANNEL $\mathrm{fl}=======$
SFO1

$\mathrm{SE}$
$\mathrm{NU} 1$
$\mathrm{P} 1$

1

$\begin{array}{ll}======= & \text { CHANNEL } \mathrm{f} 2 \quad======= \\ \text { SFO2 } & 400.1316005 \mathrm{MHz}\end{array}$

NUC2 2
CPDPR
PCPD2

$1 \mathrm{H}$

PLW2 $11.99499989 \mathrm{~W}$

PLW12 11.34213999 W

$\begin{array}{lr}\text { F2 } & \text { - Processing parameters } \\ \text { SI } & 32768 \\ \text { SF } & 100.6127803 \mathrm{MHz}\end{array}$

${ }_{\mathrm{EM}}^{\mathrm{MH}}$

$1.00 \mathrm{~Hz}$

\begin{tabular}{rrrrrrrrrrrrrr}
\hline 200 & 180 & 160 & 140 & 120 & 100 & 80 & 60 & 40 & 20 & 0 & ppm
\end{tabular}




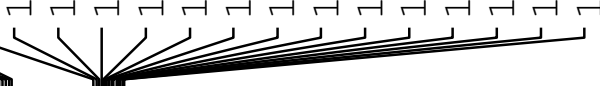

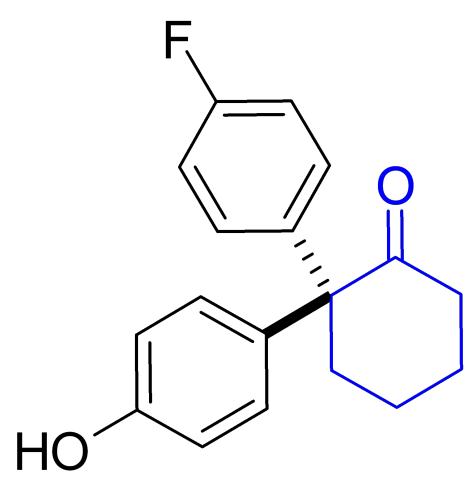

2b

\section{sporer 2}

Current Data Parameters NAME

EXPNO

mdk $8160 \mathrm{H}$

F2 - Acquisition Parameters

Date_ 20190131

Time 21.11

$5 \mathrm{~mm} P A B B O \mathrm{BB} /$

PULPROG $\quad$ zg30

$\begin{array}{lr}\text { TD } & \text { Zg30 } \\ & 65536\end{array}$

$\begin{array}{ll}\text { SOLVENT } & 65536 \\ \text { CDCl3 }\end{array}$

DS

SWH

FIDRES

$\mathrm{AQ}$

RG

DW

$\mathrm{DE}$

D1
TD 0

$====$

$\mathrm{SFO1}$

$\mathrm{NUC1}$
$\mathrm{P} 1$

PLW1

2
$8012.820 \mathrm{~Hz}$

$4.0894465 \mathrm{sec}$

31.55

62.400 usec

6.50 usec

$1.00000000 \mathrm{~K}$$$
1
$$

CHANNEL $\mathrm{f} 1========$ $400.1324710 \mathrm{MHz}$

14.50

usec

$11.99499989 \mathrm{~W}$

F2 - Processing parameters

SI 65536

SF $\quad 400.1300130 \mathrm{MHz}$

WDW

$S S B$

$\mathrm{EM}$

$0.30 \mathrm{~Hz}$

1.00

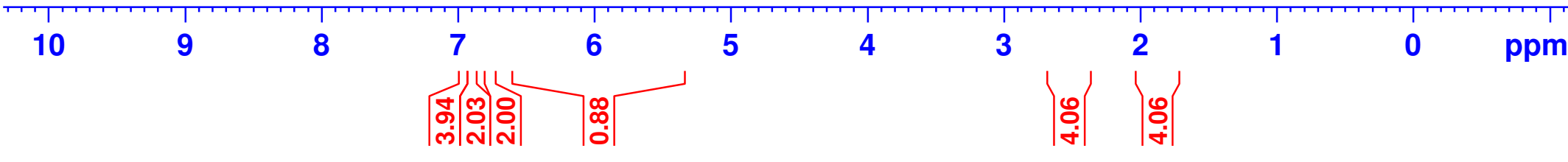


<smiles>O=C1CCCC[C@@]1(c1ccc(O)cc1)c1ccc(F)cc1</smiles>

$2 b$ 


\section{equiren}

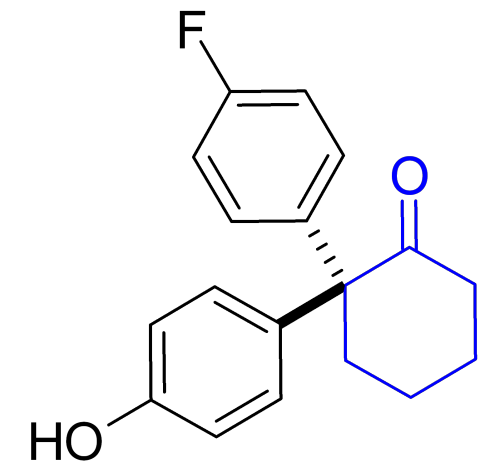

2b

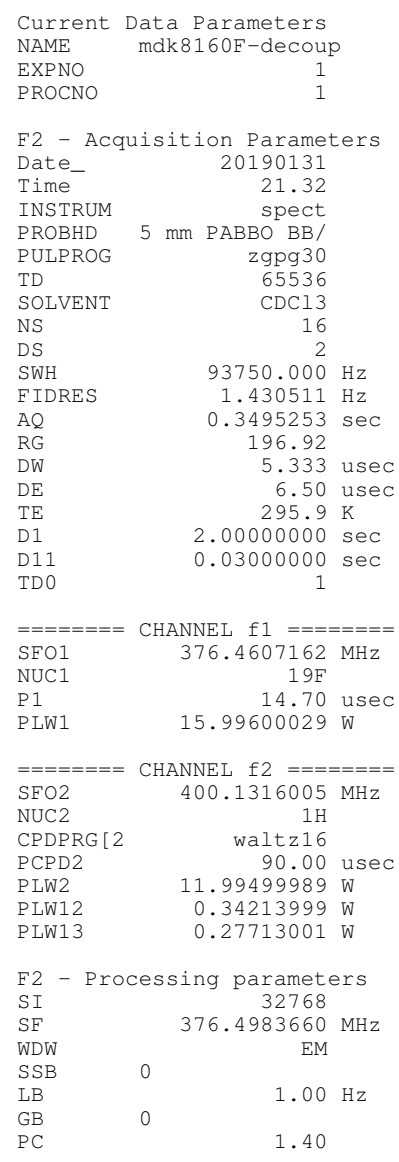

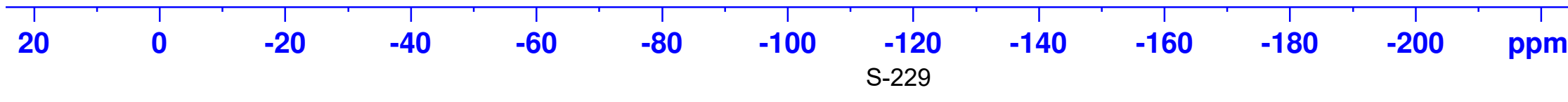



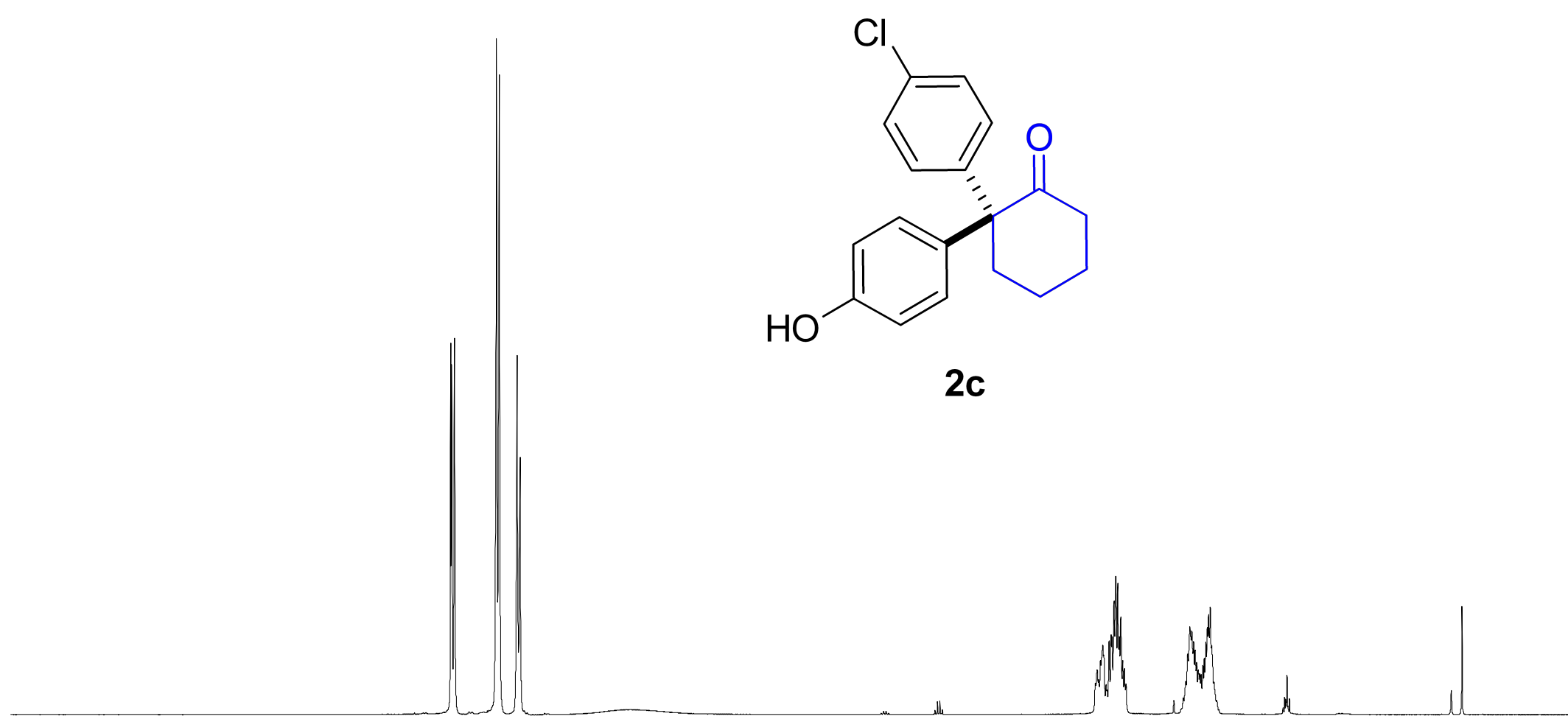

\section{sporen

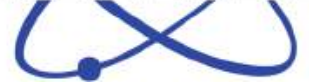

$\begin{array}{lr}\text { Current } & \text { Data } \\ \text { NAME } & \text { mameters } \\ \text { EXPNO } & 2 \\ \text { PROCNO } & 1\end{array}$

F2 - Acquisition Parameters

Date__ 20190130

$\begin{array}{ll}\text { Time } & 20.54\end{array}$

$\begin{array}{lr}\text { PULPROG } & \text { zg30 } \\ \text { TD } & 65536\end{array}$

$5 \mathrm{~mm}$ PABBO BB/

TD $\quad 65536$

DS

SWH

FIDRES

$\mathrm{AQ}$

RG

DW

DE

D1
TD 0

$=====$
SFO1
NUC1

$\mathrm{N} 1$

16
2

$8012.820 \mathrm{~Hz}$

$0.122266 \mathrm{~Hz}$

$4.0894465 \mathrm{sec}$ 34.77

62.400 usec 6.50 usec

$1.00000000 \mathrm{sec}$

CHANNEI $\mathrm{f} 1 \mathrm{=}=======$ $400.1324710 \mathrm{MHz}$

(2)

I Processing parameters

SI $\quad 400.1300124 \mathrm{MHz}$

WDW EM

$\mathrm{SSB}$

$0.30 \mathrm{~Hz}$

GB

1.00

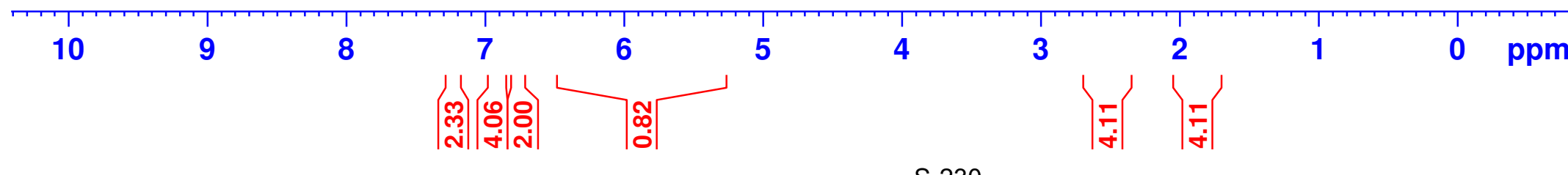


<smiles>O=C1CCCC[C@]1(c1ccc(O)cc1)c1ccc(Cl)cc1</smiles>

2c

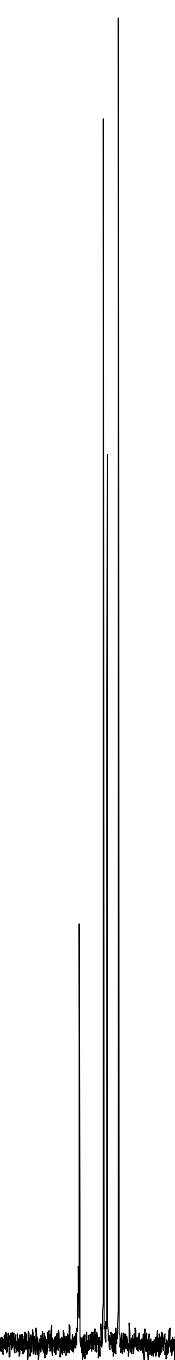

\begin{tabular}{rrrrrrrrrrrrrr}
\hline & 220 & 200 & 180 & 160 & 140 & 120 & 100 & 80 & 60 & 40 & 20 & 0 & ppm
\end{tabular}




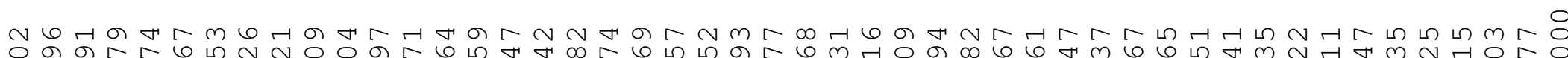

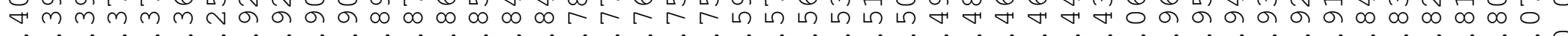

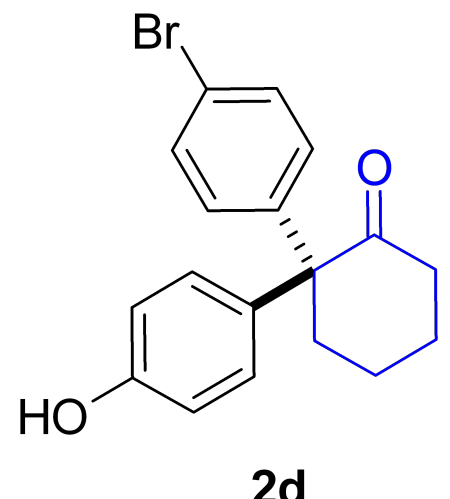

Ethyl A cetate

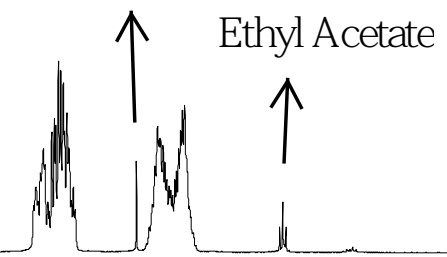

Ethyl A cetate

$\uparrow$ sporer ,

Current Data Parameters NAME

PROCNO

$\mathrm{mdk} 9018 \mathrm{H}$

F2 - Acquisition Parameters Date_ 20190227

$$
\begin{array}{lr}
\text { Date_- } & 20190227 \\
\text { Time } & 19.53 \\
\text { INSTRUM } & \text { spect }
\end{array}
$$

SPect

BB/

PULPROG

SD

SOLV
NS
DS

DS

SWH

$\mathrm{AQ}$

RG

$\mathrm{DW}$
$\mathrm{DE}$
$\mathrm{TE}$

D 10

$====$

$\mathrm{SFO1}$

$\mathrm{NUC1}$
$\mathrm{P} 1$

PLW1

zg30
65536
$\operatorname{CDC} 13$

$\mathrm{CDC} 13$
16

$8012.820 \mathrm{~Hz}$

$8012.820 \mathrm{~Hz}$
$0.122266 \mathrm{~Hz}$

$4.0894465 \mathrm{sz}$

49.32

62.400 usec 6.50 usec

$1.00000000 \mathrm{sec}$

CHANNEL $\mathrm{f} 1========$ $400.1324710 \mathrm{MHz}$

14.50

4.50 usec

Processing parameters

SI 65536

$\begin{array}{ll}\text { SF } & 400.1300129 \mathrm{MHz}\end{array}$ 
<smiles>O=C1CCCC[C@]1(c1ccc(O)cc1)c1ccc(Br)cc1</smiles>

$2 d$

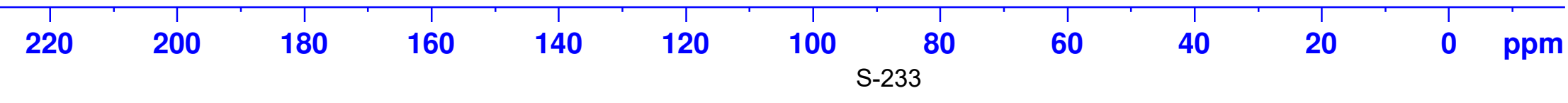

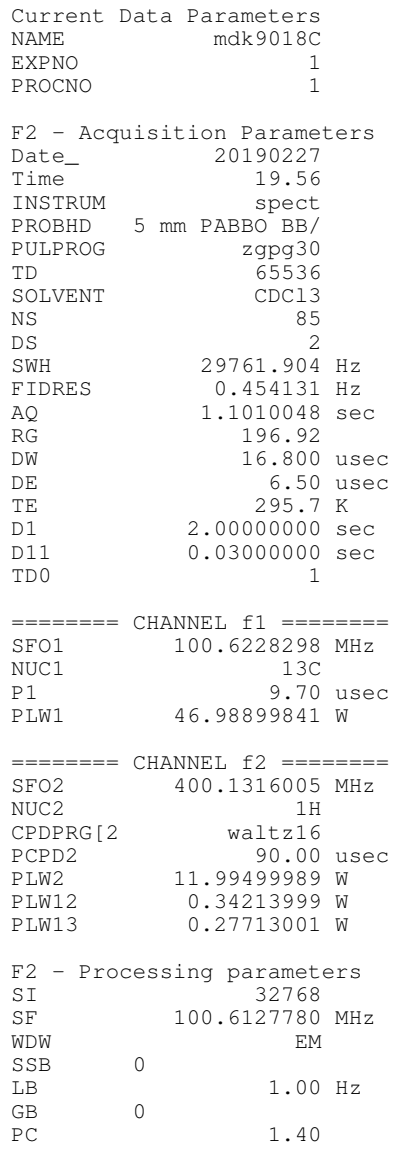




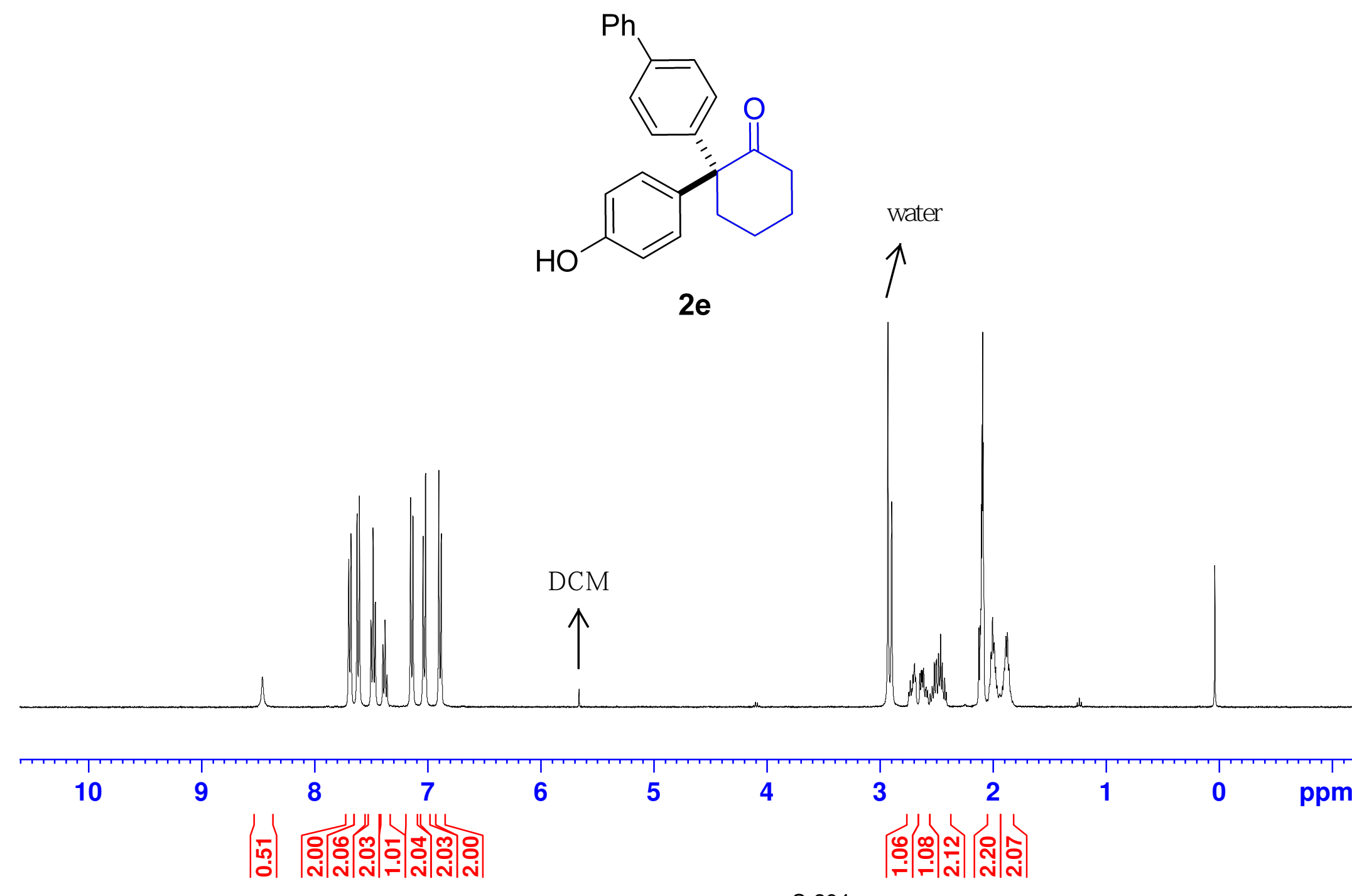

Current Data Parameters NAME

PROCNO

$\mathrm{mdk} 9085 \mathrm{H}$

F2 - Acquisition Parameters

Date_ 20190319

$\begin{array}{lr}\text { Time } & 20.06\end{array}$

spect

PULPROG $\quad$ zg30

$\begin{array}{lr}\text { TD } & \mathrm{Zg} 30 \\ & 65536\end{array}$

SOLVENT Acetone

NS

DS

FIDRES

$\mathrm{AQ}$

$\mathrm{RG}$

DE

TE

TD 0

$===$

$\mathrm{SFO1}$

NUC

16
16

$8012.820 \mathrm{~Hz}$

$0.122266 \mathrm{~Hz}$

$4.0894465 \mathrm{sec}$ 103.52

62.400 usec 6.50 use

$1.00000000 \mathrm{sec}$

CHANNEL $\mathrm{fl}========$ $400.1324710 \mathrm{MHz}$

$2-10$

- Processing parameters

SF $\quad 65536$

S00.1299911 MH

SSB

EM

$0.30 \mathrm{~Hz}$

1.00 


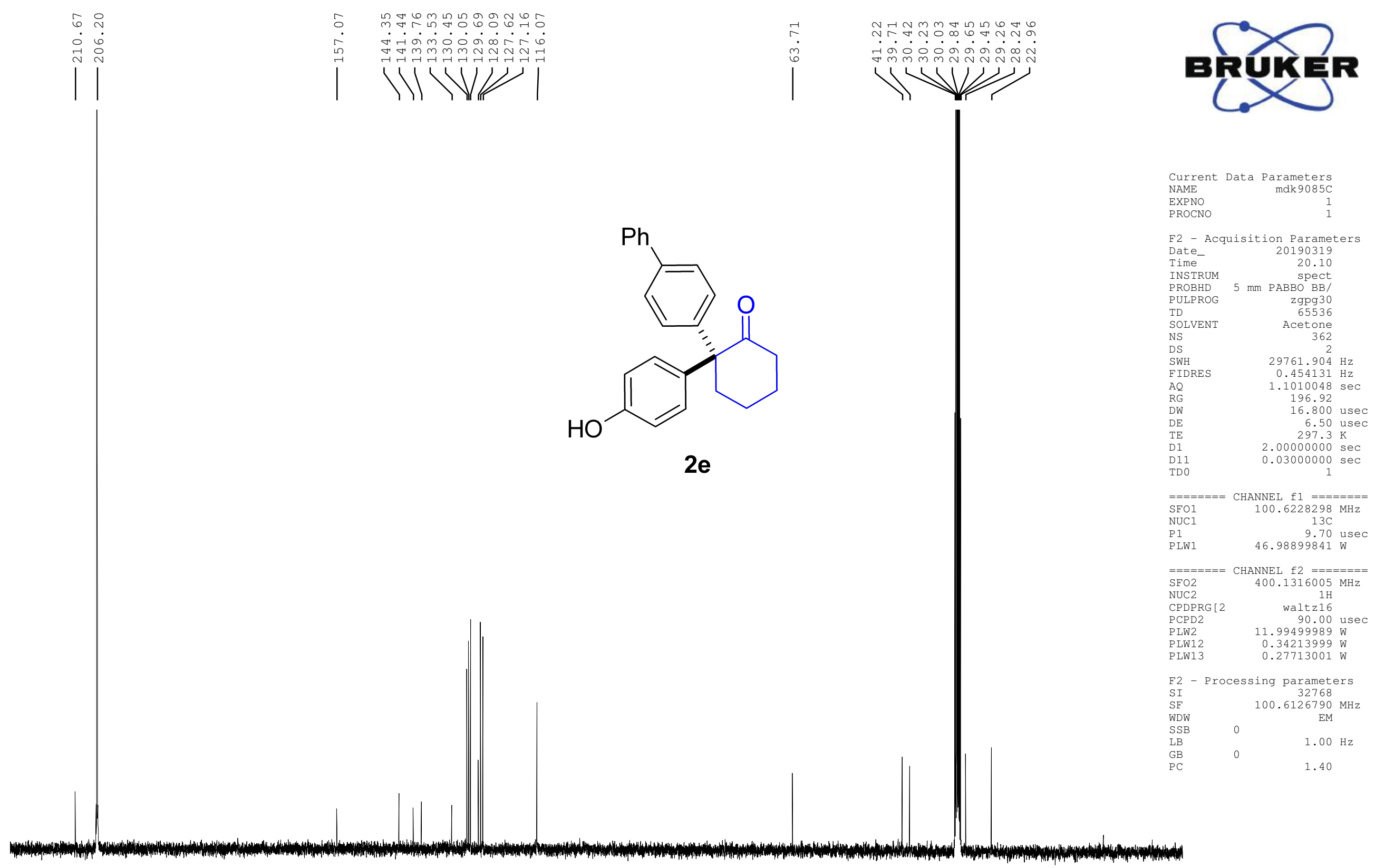

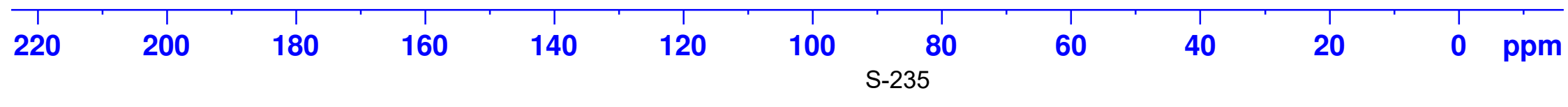




\section{aponen

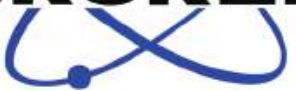

Current Data Parameters NAME

PROCNO

$\operatorname{mdk} 9103 \mathrm{H}$

F2 - Acquisition Parameters Date_ 20190325

$\begin{array}{lr}\text { Time } & 13.15\end{array}$

INSTRUM spect

$\quad \begin{aligned} \text { zg30 } \\ 65536\end{aligned}$

TD $\quad 65536$

NS

Ethyl A cetate

$\uparrow$ Ethyl A cetate
SWH

FIDRES

$\mathrm{AQ}$

RG

DW

$\mathrm{DE}$

TE

TD 0

$$
\begin{aligned}
& ========\text { CHANNEL } \mathrm{fl}======== \\
& \mathrm{SFO1} \\
& \text { NUC1 } \\
& \text { P } 1 \\
& \text { PLW1 }
\end{aligned}
$$

$8012.820 \mathrm{~Hz}$ $0.122266 \mathrm{~Hz}$ $0894465 \mathrm{sec}$ 49.32 .400 usec 6.50 usec .00000000 sec

$\begin{array}{lcc}\text { F2 } & \text { - Processing parameters } \\ \text { SI } & 65536 \\ \text { SF } & 400.1300104 \mathrm{MHz} \\ \text { WDW } & 0 & \text { EM } \\ \text { SSB } & 0 & \\ \text { LB } & 0 & 0.30 \mathrm{~Hz} \\ \text { GB } & 0 & 1.00 \\ \text { PC } & & \end{array}$
Ethyl A cetate

$\uparrow$

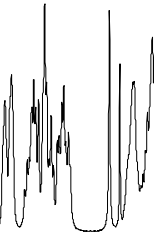

9

8

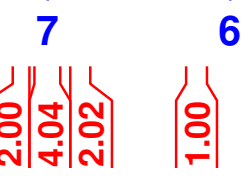

5
4 


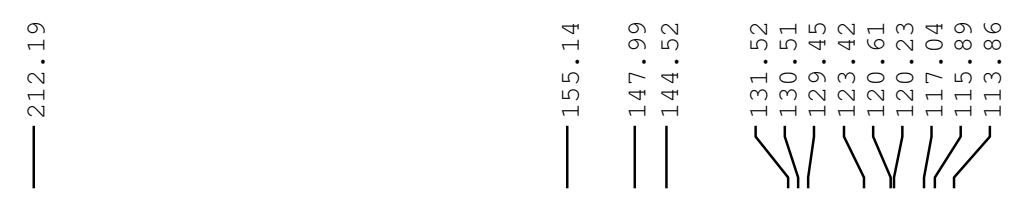

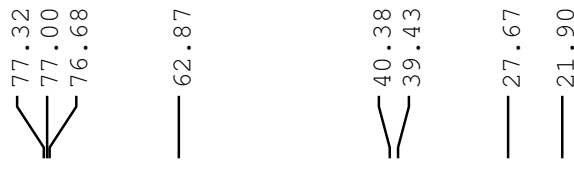

enciser
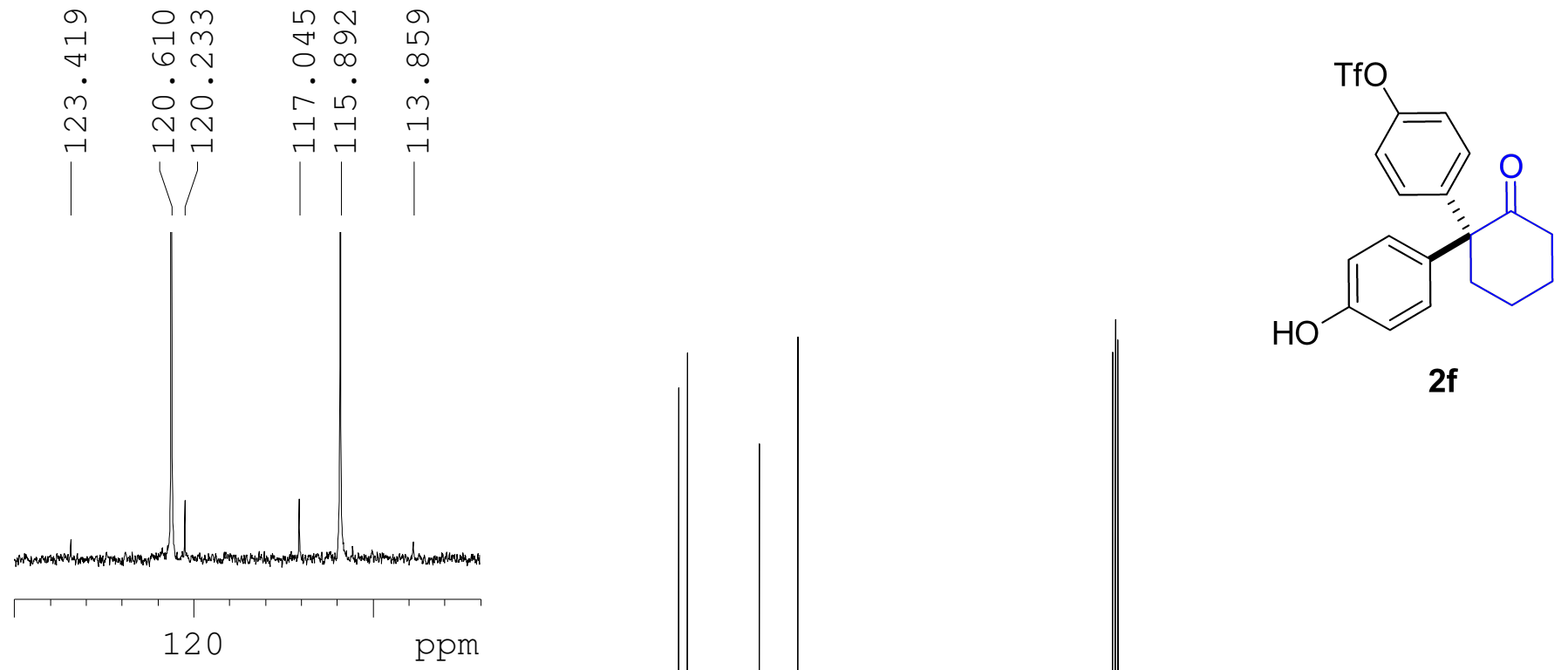

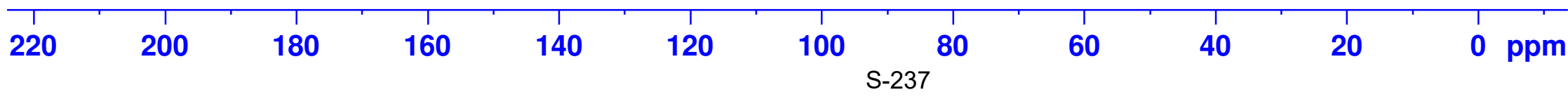




\section{expienten}

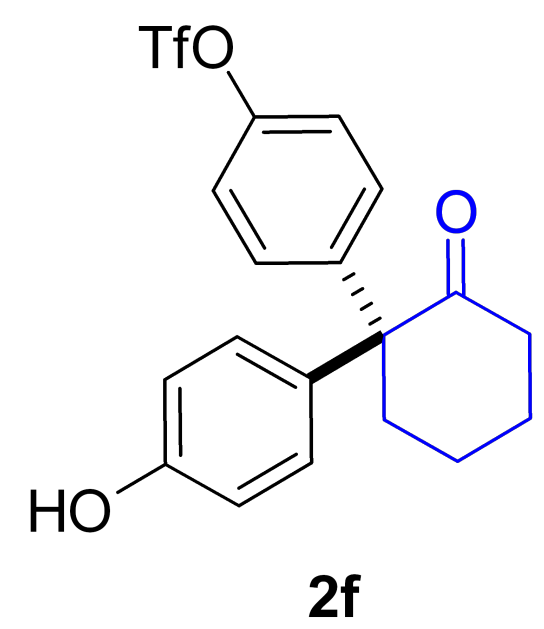

NAME mdk9103F-decoup

PROCNO

$\begin{array}{ll}\text { F2 - Acquisition Parameters } \\ \text { Date_ } & 20190325 \\ \text { Time } & 13.27\end{array}$

Time
INSTRUM

spect
PROBHD $5 \mathrm{~mm}$ PABBO BB/

PULPROG

SOLVEN

DS

$\mathrm{AQ}$
$\mathrm{RG}$

DE

D 11

11
DO

$0.3495253 \mathrm{sec}$

196.92

5.333 usec
6.50 usec

$.00000000 \mathrm{sec}$

$0.03000000 \mathrm{sec}$

$z======$ CHANNEL $f 1 \quad=======$
SFO1 $376.4607162 \mathrm{MHz}$

NUC1
$\mathrm{P} 1$

$6.4607162 \mathrm{MHz}$
$19 \mathrm{~F}$

$5.99600029 \mathrm{~W}$

$=======$ CHANNEL $\mathrm{f} 2 \quad======= \pm$
SFO2
SOO.1316005 MHz

NUC2
CPDPRG

waltz16

PLW2

. $99499989 \mathrm{~W}$

PLW13

$0.27713001 \mathrm{~W}$

F2 - Processing parameters
SI

$\begin{array}{lr}\text { SF } & 376.4983660 \mathrm{MH} \\ \text { WDW } & \mathrm{EM}\end{array}$

$1.00 \mathrm{~Hz}$

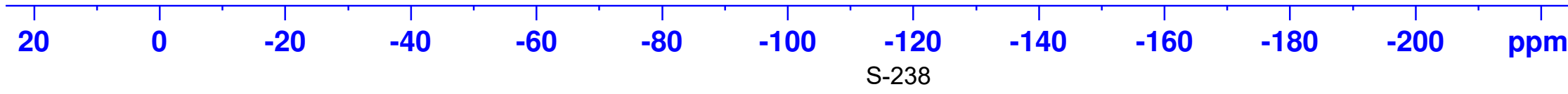


ค ศ - -1666666 $\longrightarrow$

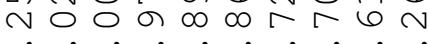

$m$ m

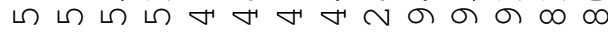

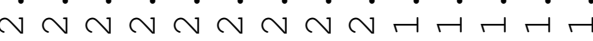
$\longrightarrow$

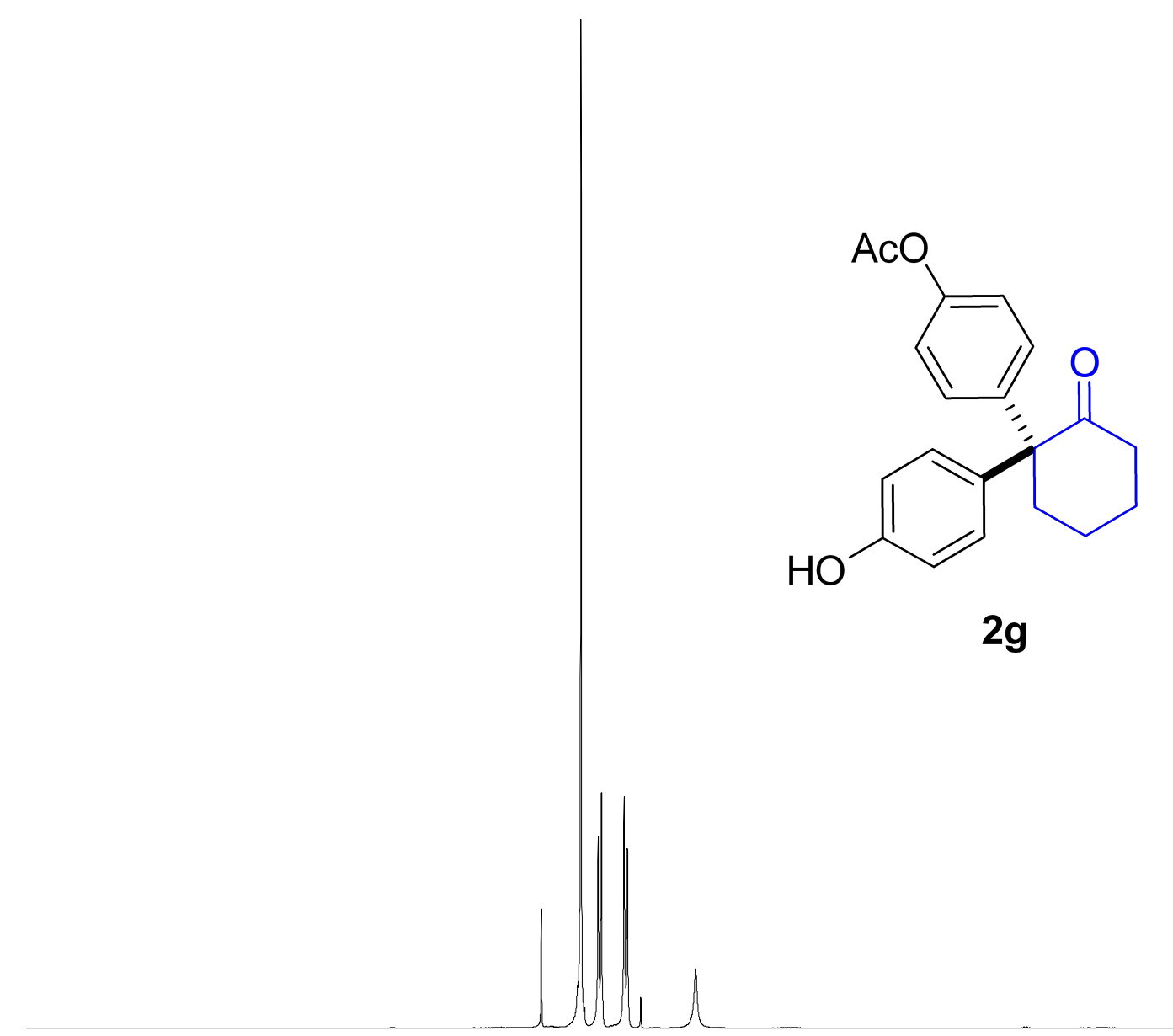

\section{er ,}

Current Data Parameters NAME

EXPNO

mdk $9107 \mathrm{H}$

PROCNO

F2 - Acquisition Parameters

Date__ 20190325

Time $\quad 15.51$

spect

$\mathrm{zg} 30$
PULPROG

TD $\quad 65536$

SOLVENT

NS

DS

FIDRES

$8012.820 \mathrm{~Hz}$
$4.0894465 \mathrm{sec}$

DW

$\mathrm{DE}$ 6.50 us
$295.3 \mathrm{~K}$

$1.00000000 \mathrm{sec}$ TD 01.0000000

$=======$ CHANNEL f1 $========$

SFO1 $\quad 400.1324710 \mathrm{MHz}$ STC1

$\mathrm{NUC1}$
$\mathrm{P} 1$

14.50 usec $11.99499989 \mathrm{~W}$

F2 - Processing parameters

SI 65536

SF $\quad 400.1300111 \mathrm{MHz}$

WDW

SSB

EM

$0.30 \mathrm{~Hz}$

C

1.00

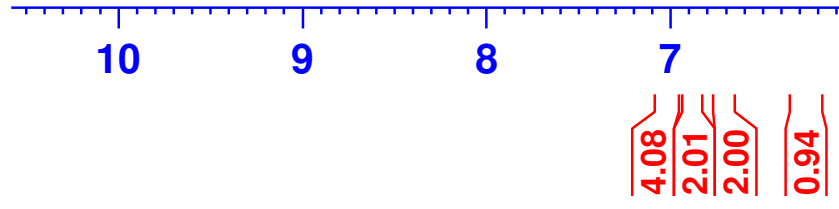

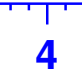

3

2

1

o ppm

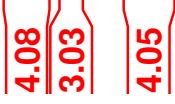




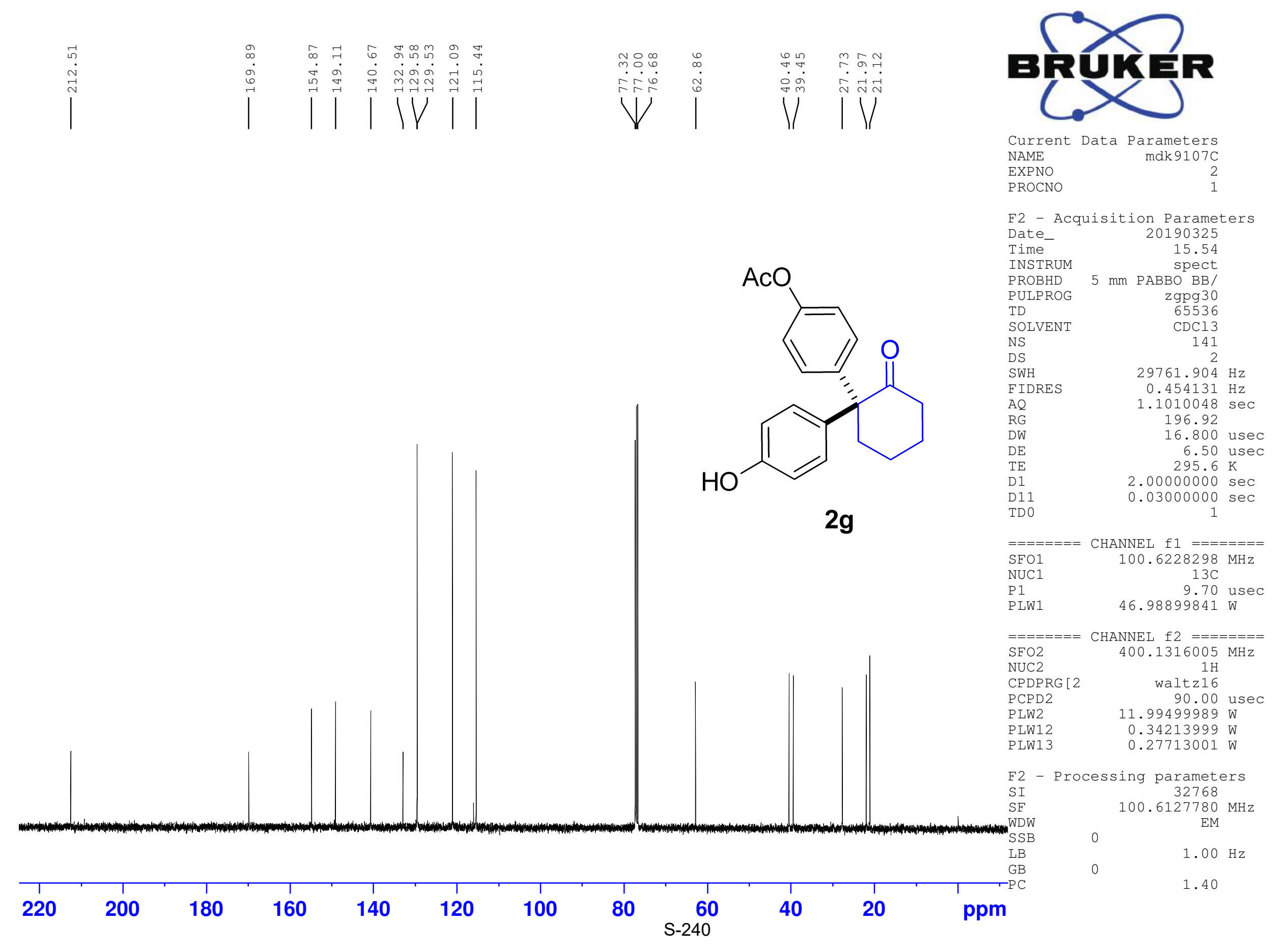



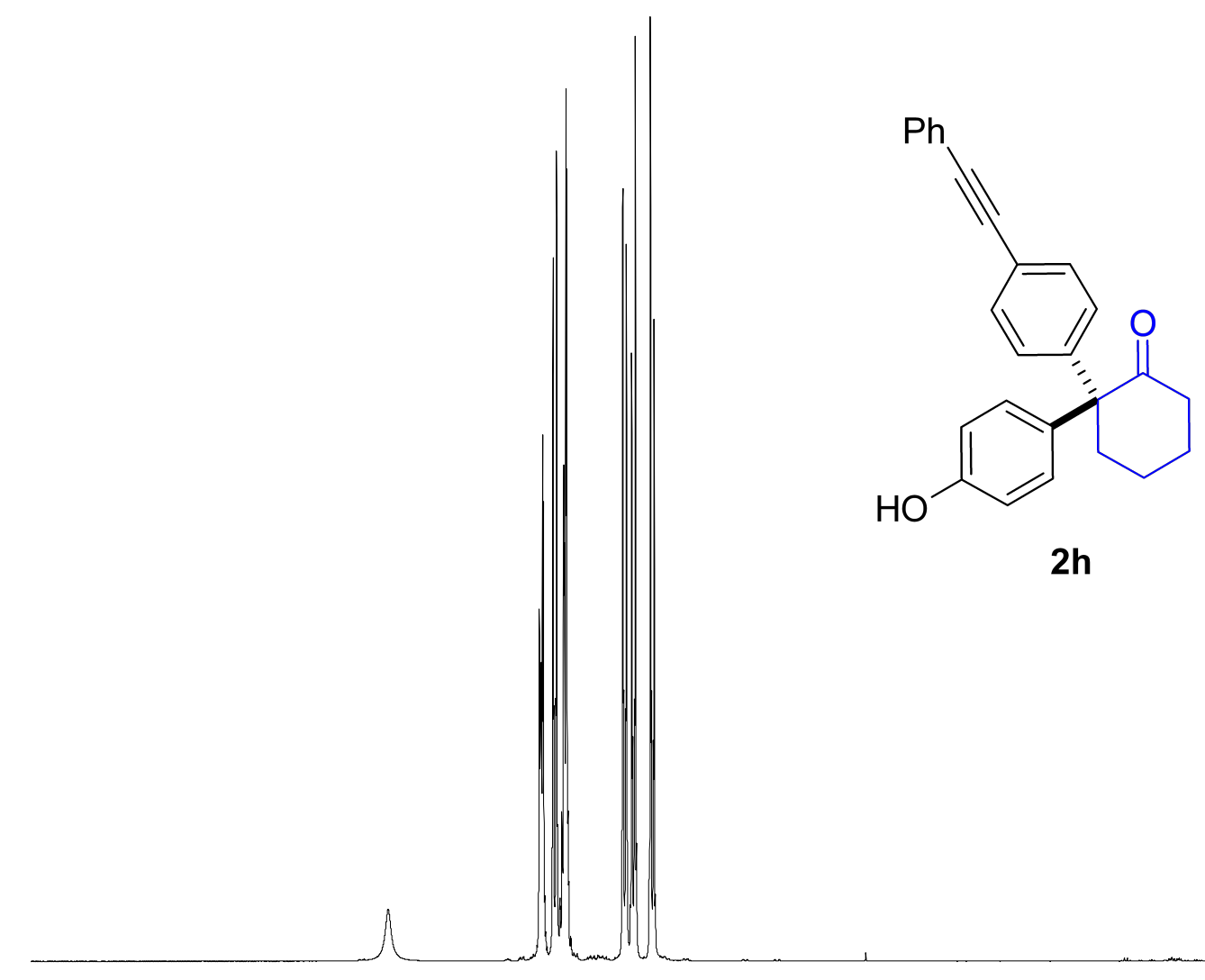

$$
\begin{aligned}
& \text { Current Data Parameters } \\
& \text { NAME } \\
& \text { PROCNO } \\
& \begin{array}{r}
\text { mak } 9179 \mathrm{H} \\
1
\end{array}
\end{aligned}
$$

water

Processing parameters

SI 65536

SF $\quad 400.1299911 \mathrm{MHz}$

SSB

EM

$0.30 \mathrm{~Hz}$

1.00

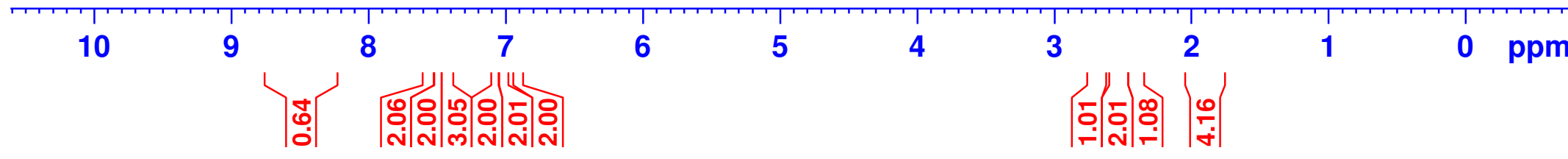




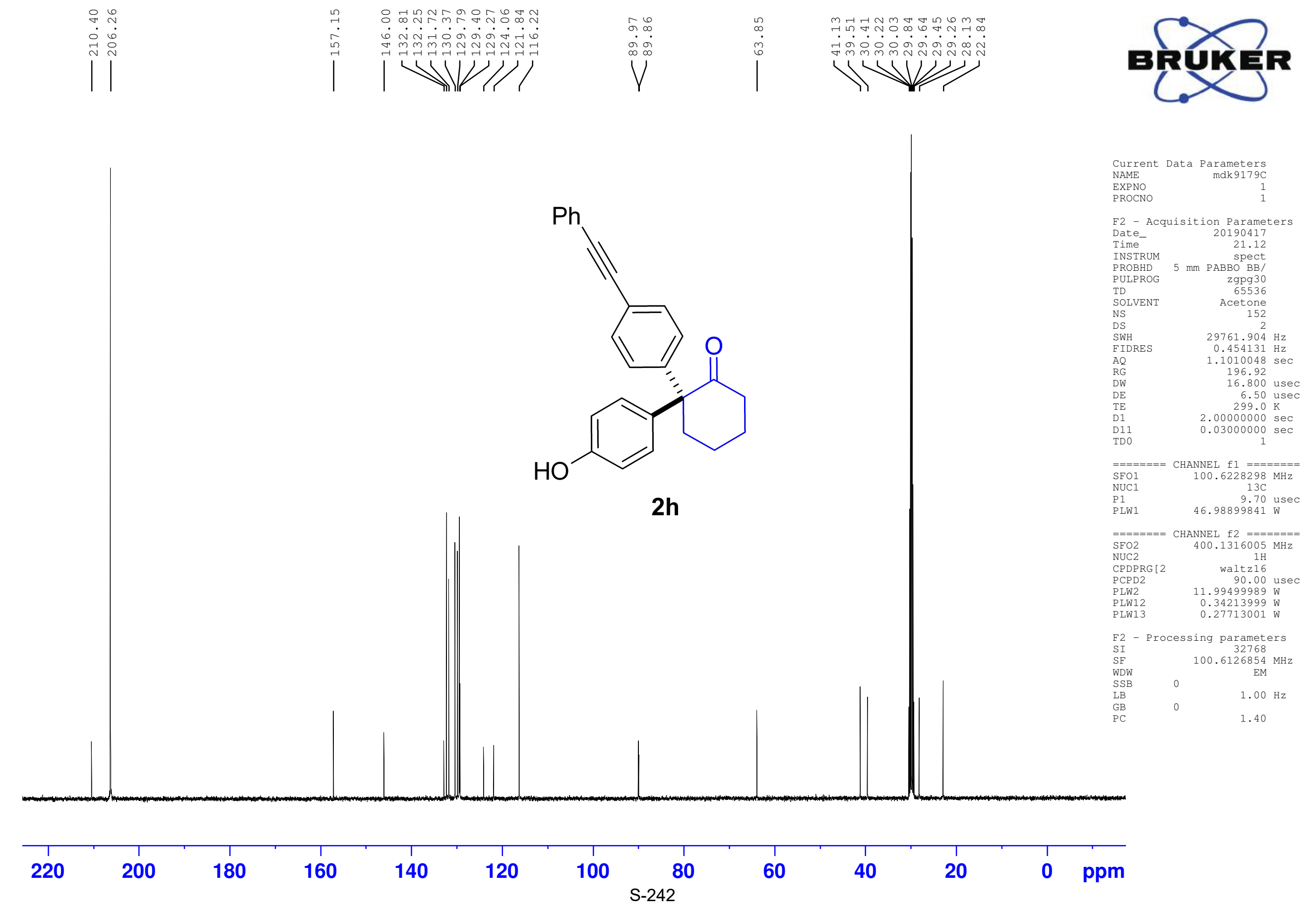


为,

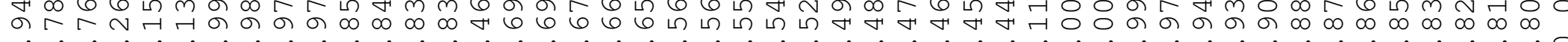

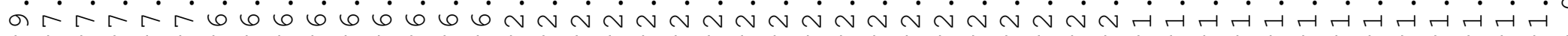
(1)

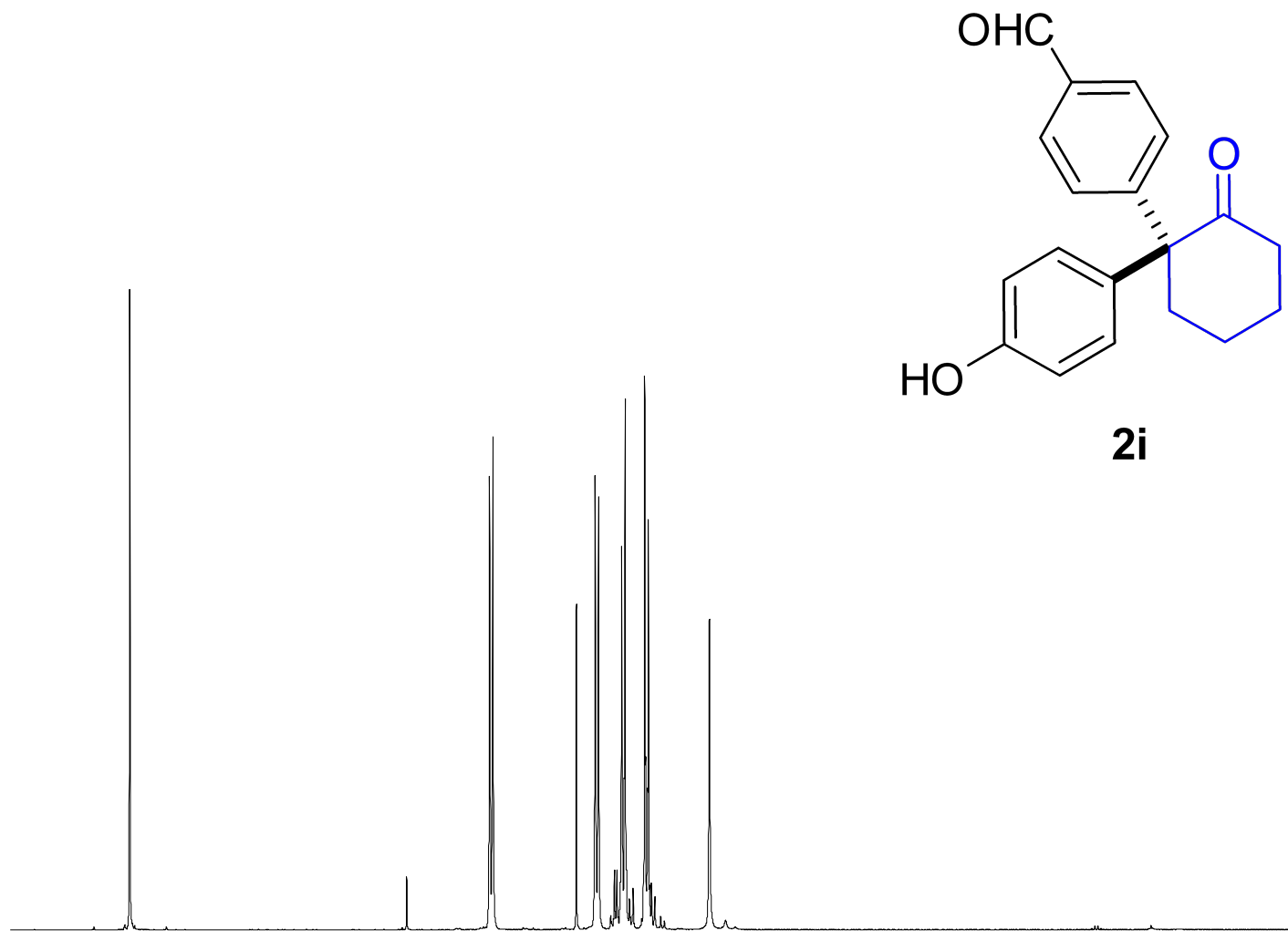

\section{SR KXer

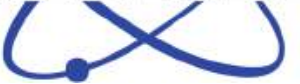

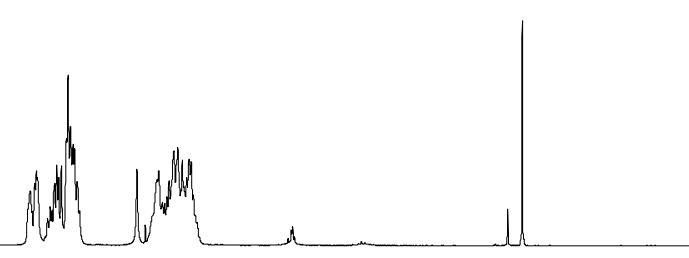
Current Data Parameters NAME
PROCNO
$\operatorname{mdk} 9191 \mathrm{H}$

F2 - Acquisition Parameters

Date_ 20190425

Time $\quad 16.21$

spect

PULPROG $\quad$ zg30

$\begin{array}{lr}\text { TD } & \text { Zg30 } \\ & 65536\end{array}$

$\begin{array}{lr}\text { SOLVENT } & 65536 \\ \text { NS } & 16\end{array}$

DS

SWH

FIDRES

$\mathrm{AQ}$

RG

DW

DE

D1
TD 0

$====$

$\mathrm{SFO1}$

NUC

P 1

16
2
$8012.820 \mathrm{~Hz}$

$8012.820 \mathrm{~Hz}$

$0.122266 \mathrm{~Hz}$

45.67

62.400 usec

6.50 usec

$1.00000000 \mathrm{sec}$

CHANNEL $\mathrm{f} 1 \mathrm{l}========$

$400.1324710 \mathrm{MHz}$

PL1 $\quad 11.50$ usec

Processing parameter

$\begin{array}{lr}\text { SI } & 65536 \\ \text { SF } & 400.1300085 \\ \text { WH }\end{array}$

WDW EM

SSB

$0.30 \mathrm{~Hz}$

BB

1.00

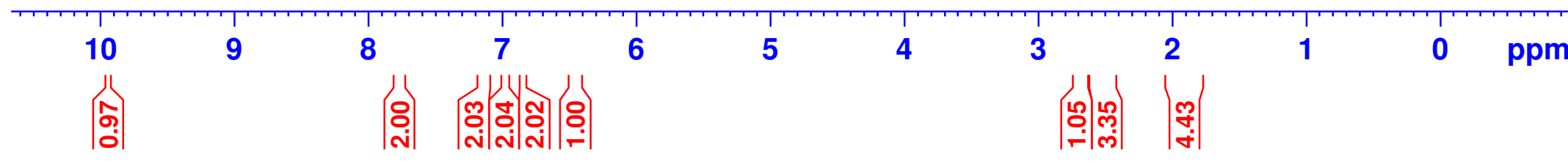




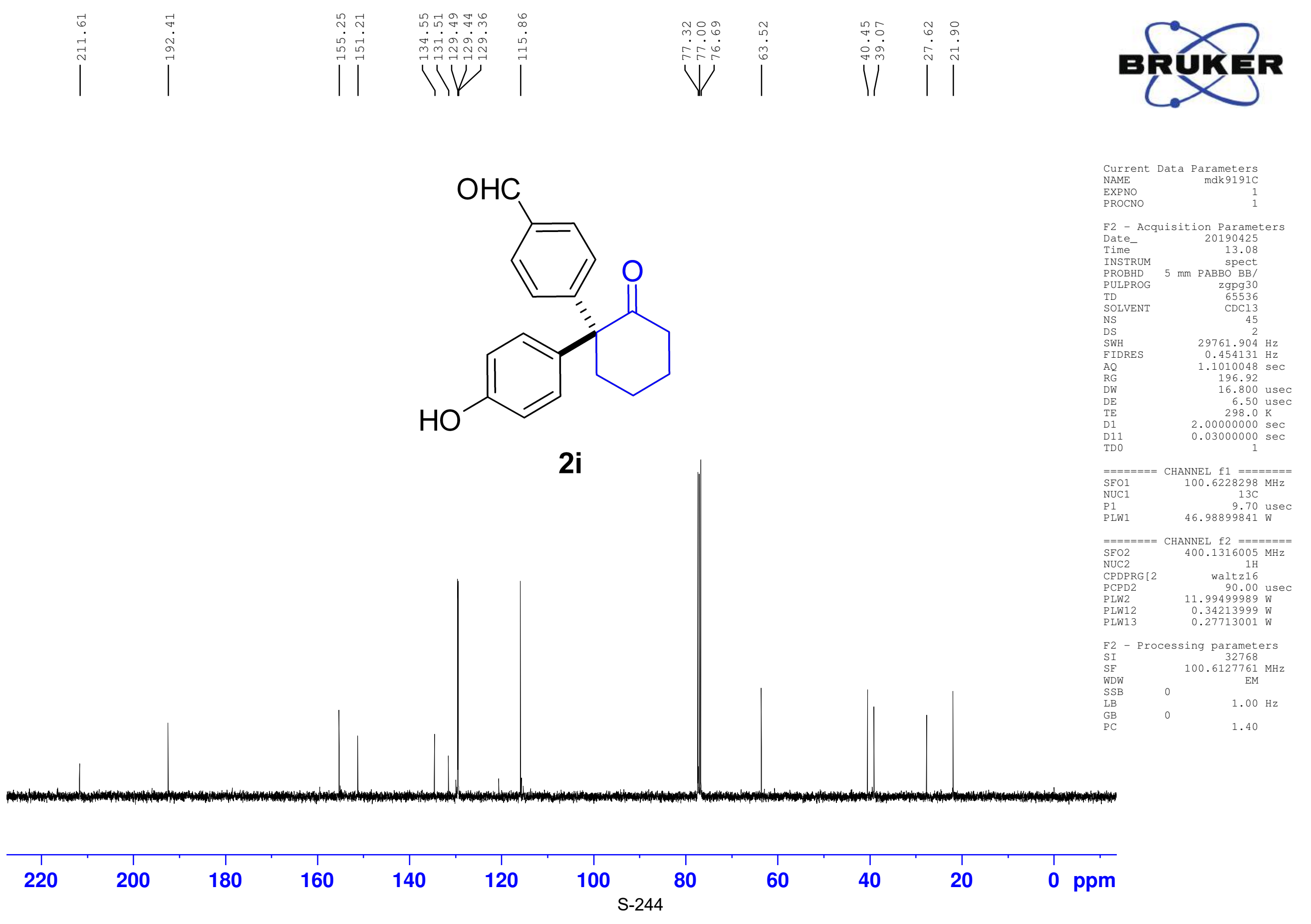




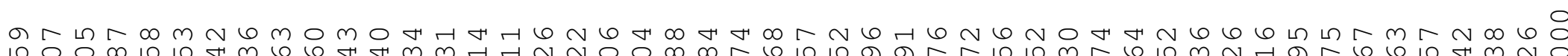

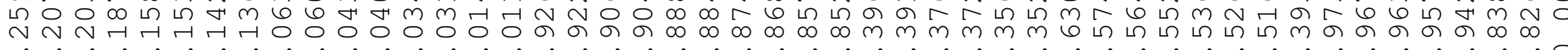
-

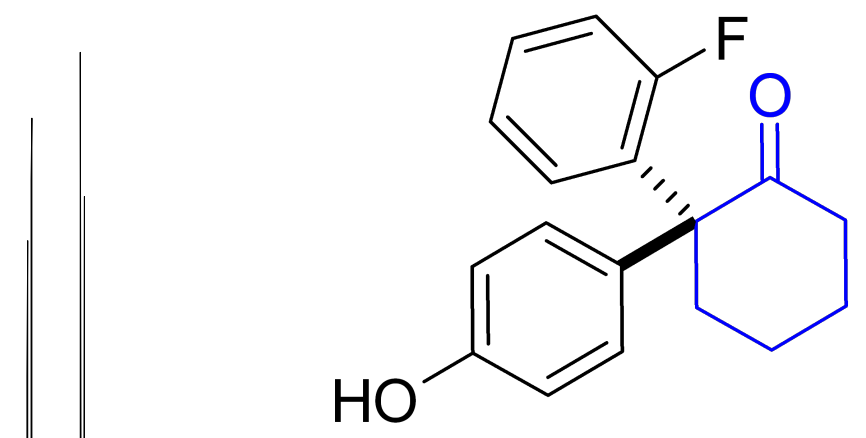

2j

Ethyl A cetate

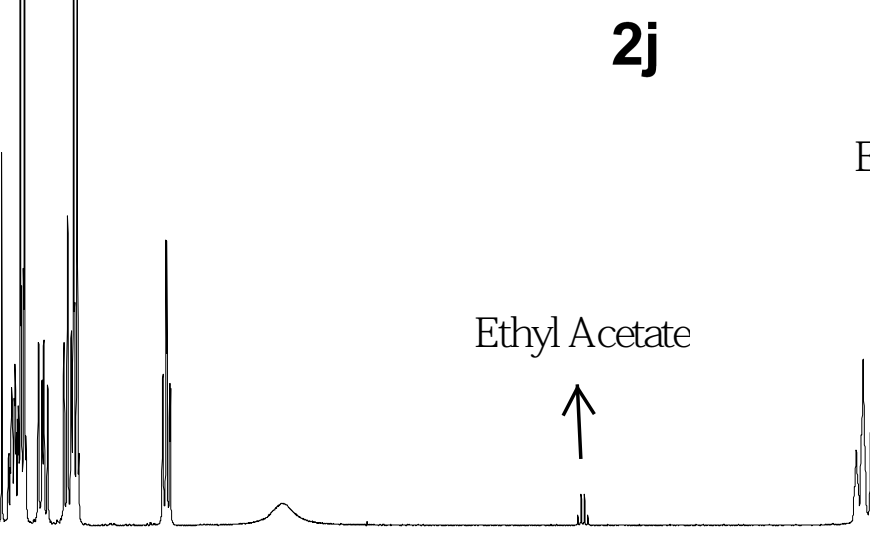

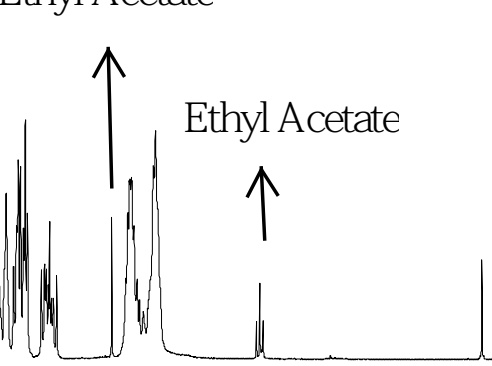

BP UKRER $(1<)$

Current Data Parameters NAME

PROCNO

$\operatorname{mdk} 9037 \mathrm{H}$

F2 - Acquisition Parameters Date_ 20190306 $\begin{array}{lr}\text { Time- } & 19.39\end{array}$ INSTRUM spect UIPR $\mathrm{Zg} 30$
65536 TD $\quad 65536$

NS

DWH

FIDRES

$\mathrm{AQ}$

RG

DW

TE

D1
TD 0

\section{$===$
$\mathrm{SFO} 1$}

$\mathrm{SFO1}$

$\mathrm{NUC}$

P1
PLW1

$\mathrm{CDC} 13$
16
2

$8012.820 \mathrm{~Hz}$

$0.122266 \mathrm{~Hz}$

$4.0894465 \mathrm{sec}$ 88.84

62.400 usec 6.50 usec

$1.00000000 \mathrm{sec}$ 1

CHANNEL $\mathrm{f} 1 \mathrm{l}========$ $400.1324710 \mathrm{MHz}$ 14.50 usec

F2 - Processing parameters SI 65536 SF $\quad 400.1300107 \mathrm{MHz}$ WDW SSB EM $0.30 \mathrm{~Hz}$ 1.00
10

9

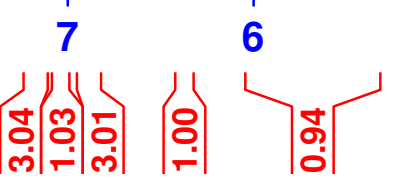

5

4

3

2

|它| 


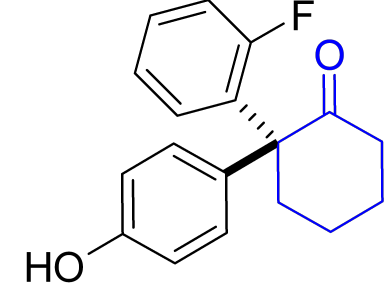

2j

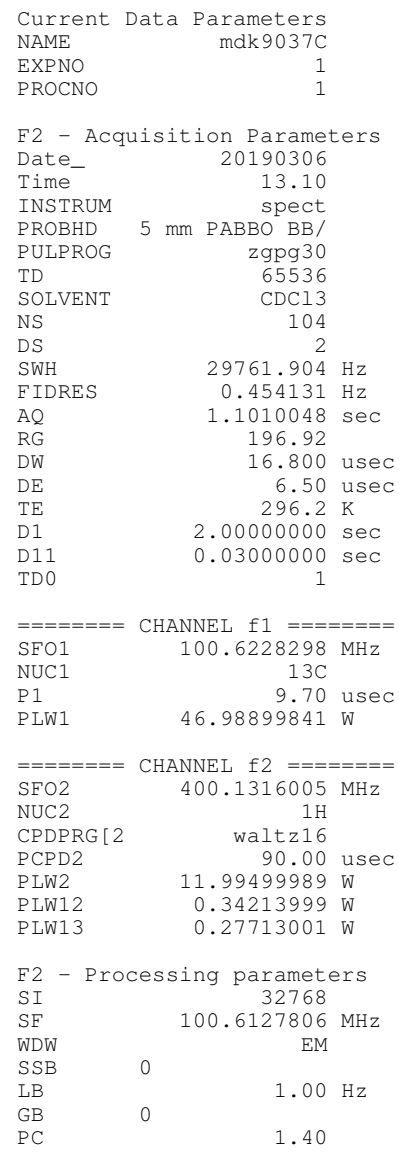




\section{equiren}

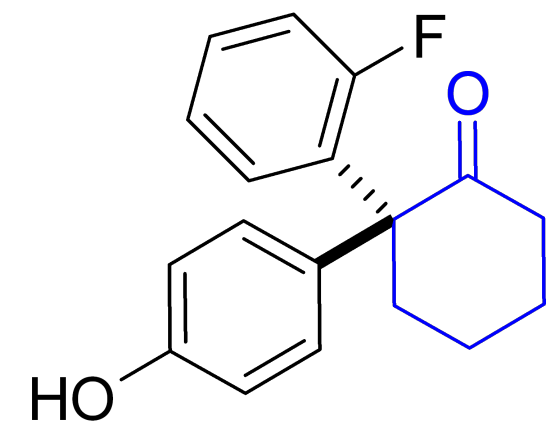

2j

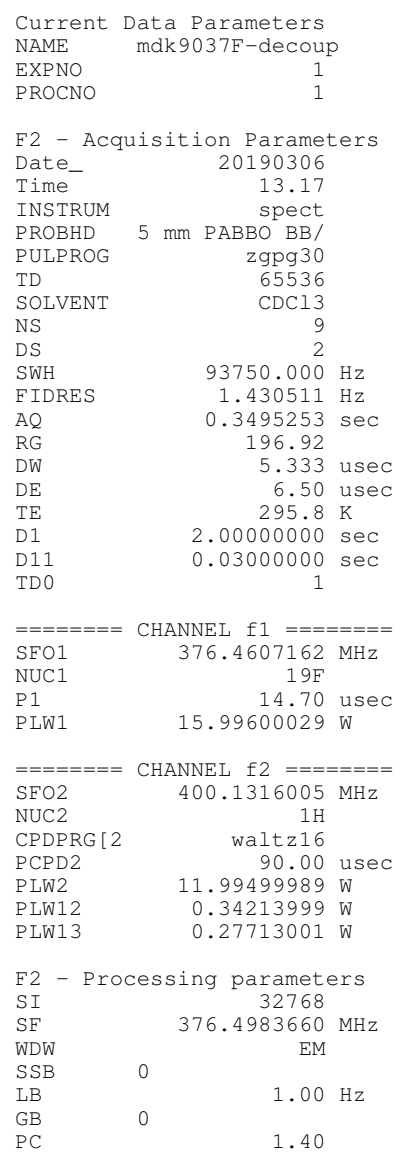

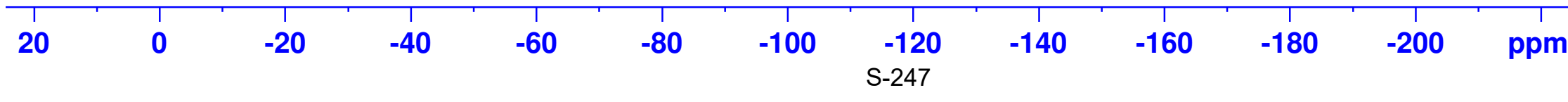




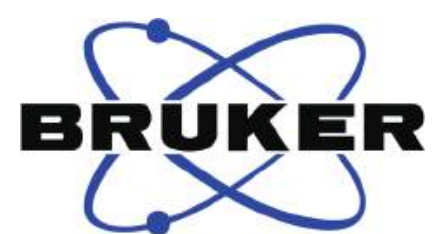

Current Data Parameters NAME

EXPNO

mdk $9048 \mathrm{H}$

PROCNO

F2 - Acquisition Parameter

Date

20190308

Time<smiles>O=C1CCCC[C@]1(c1ccc(O)cc1)c1cccc(C(F)(F)F)c1</smiles>

$2 k$
INSTRUM

PULPROG

SOLVENT

NS

DS

AQ

RG

DW

TE

D1
TD 0

$5 \mathrm{spect}$

zg30

Acetc 16
2

$8012.820 \mathrm{~Hz}$ $0.122266 \mathrm{~Hz}$ $4.0894465 \mathrm{sec}$ 34.77

62.400 usec 6.50 usec 1.000000 $====$ $\mathrm{SFO1}$ NUC $\mathrm{P} 1$ PLW1

CHANNEL $\mathrm{f} 1 \mathrm{l}========$ $400.1324710 \mathrm{MHz}$ 4.50 usec $11.99499989 \mathrm{~W}$

F2 - Processing parameters

water

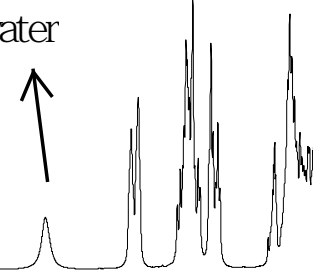

65536

$\mathrm{EM}$
$\mathrm{MHz}$

$0.30 \mathrm{~Hz}$

1.00

\section{0}

9 $\left|\begin{array}{l}1 \\ 0 \\ 0 \\ 0\end{array}\right|$ 7

6

5

4

3

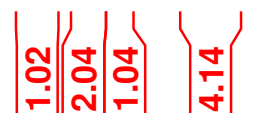




\section{aperken}

인

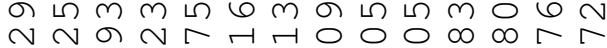

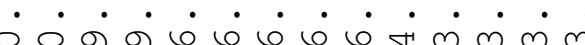

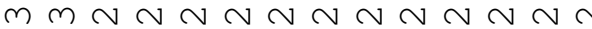

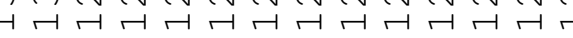
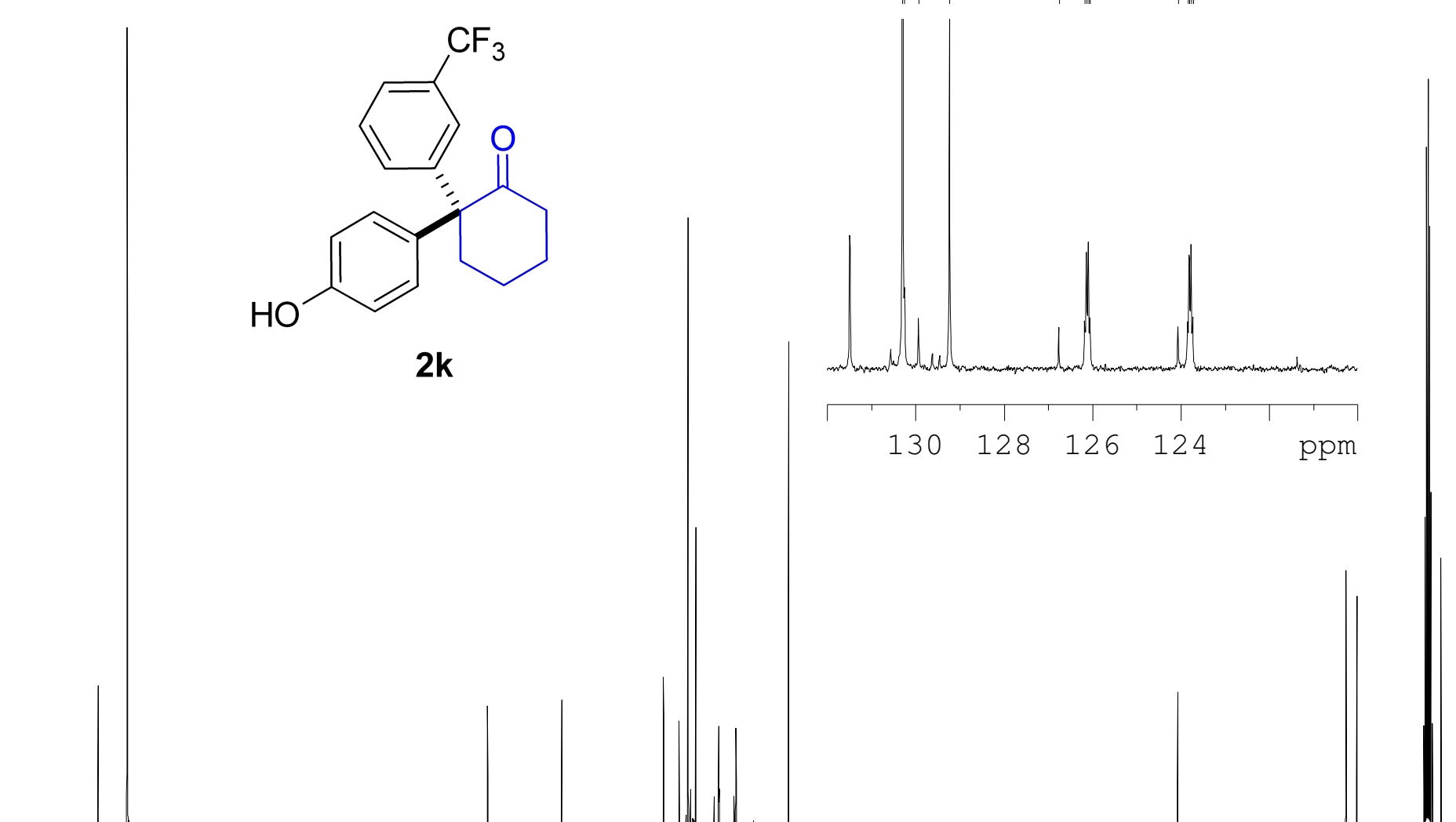

Current Data Parameters EXPNO

mdk9048C
1
1

F2 - Acquisition Parameters
Date_ 20190308

Time
INSTRUM

spect
PROBHD $5 \mathrm{~mm}$ PABBO BB/

TDLPROG
TD

SOLVENT

NSS
DS

SWH
FIDRES

$\mathrm{AQ}$
$\mathrm{RG}$

DW
DE

TE
D1
D11

D11
TD 0

zgpg30
65536

$29761.904 \mathrm{~Hz}$

1.1010048 $\mathrm{Hz}$

196.92
16.800

16.800 usec
6.50 usec

$2.00000000 \mathrm{sec}$
$0.03000000 \mathrm{sec}$

$\begin{aligned} & \text { SFO1 }=\text { CHANNEL } f 1 \quad====== \pm= \\ & 100.6228298 \mathrm{MHz}\end{aligned}$

NUC1

PLT1

$46.98899841 \mathrm{~W}$

$========$ CHANNEL $f 2 \quad======= \pm$
SFO2 $400.1316005 \mathrm{MHz}$

$\begin{array}{lr}\text { NUC2 } & 1 \mathrm{H} \\ \text { CPDPRG [2 } & \text { waltz16 } \\ \text { PCPD2 } & 90.00\end{array}$

11.99499989

$\begin{array}{ll}\text { PLW12 } & 0.34213999 \mathrm{~W} \\ \mathrm{PLW13} & 0.27713901 \mathrm{~W}\end{array}$

$\begin{array}{lr}\text { F2 } & \text { - Processing parameters } \\ \text { SI } & 32768 \\ \text { SF } & 100.6126845 \mathrm{MHz}\end{array}$

SF

$00.6126845 \mathrm{MHz}$
$\mathrm{EM}$

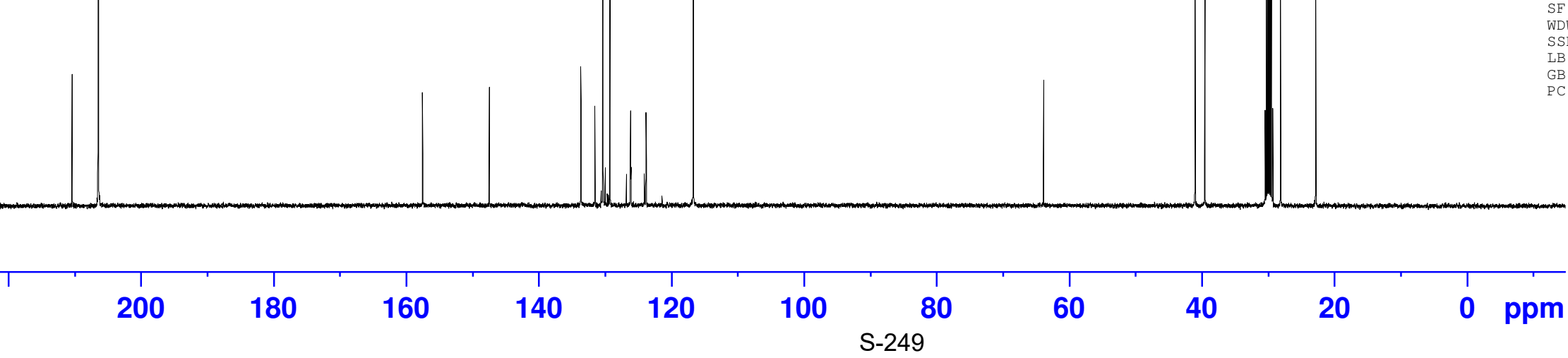




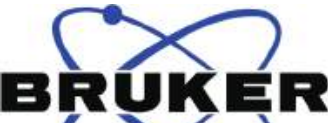 $\mathrm{C}>$}
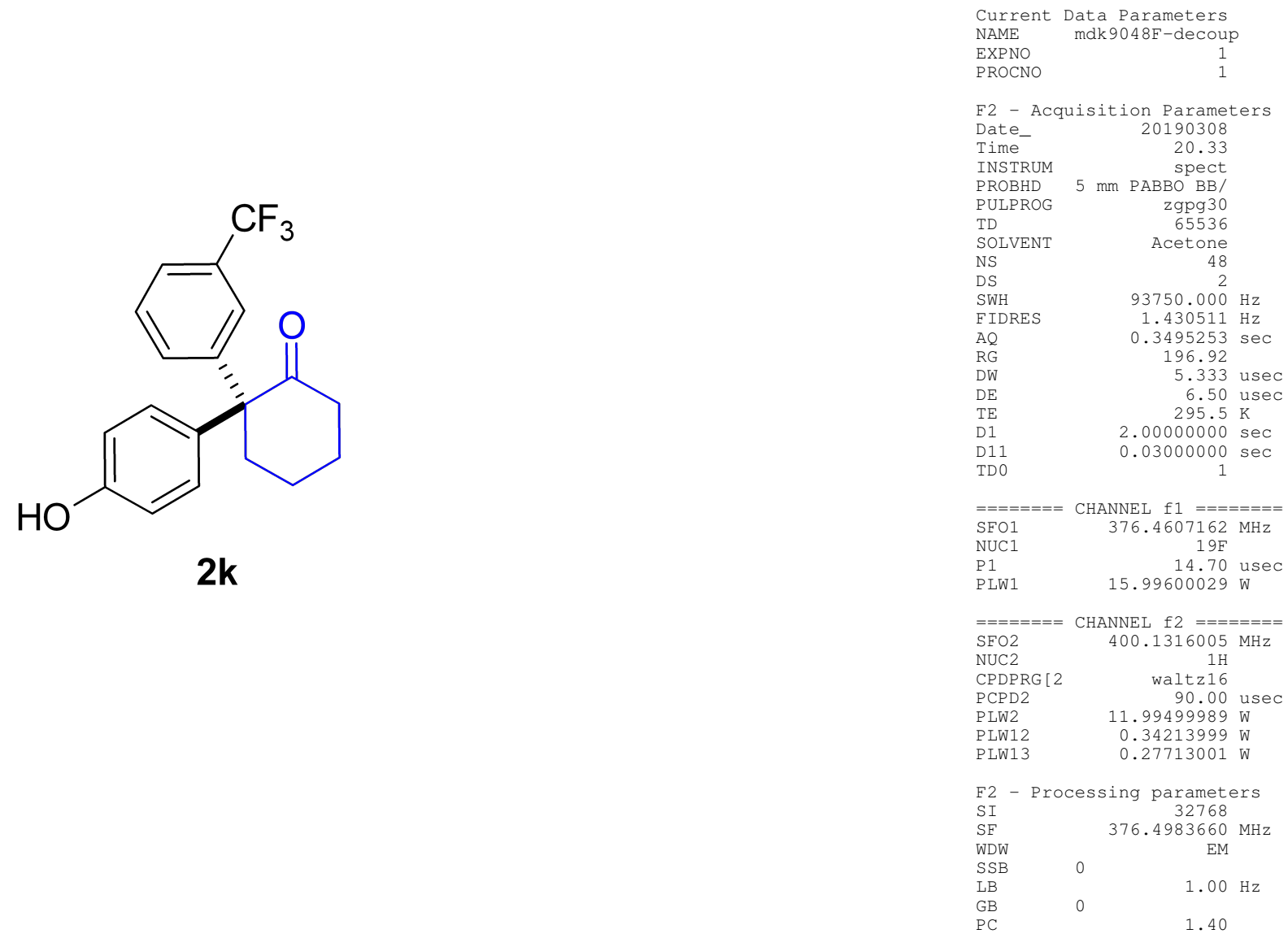

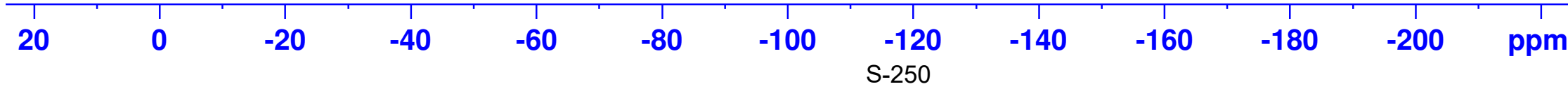




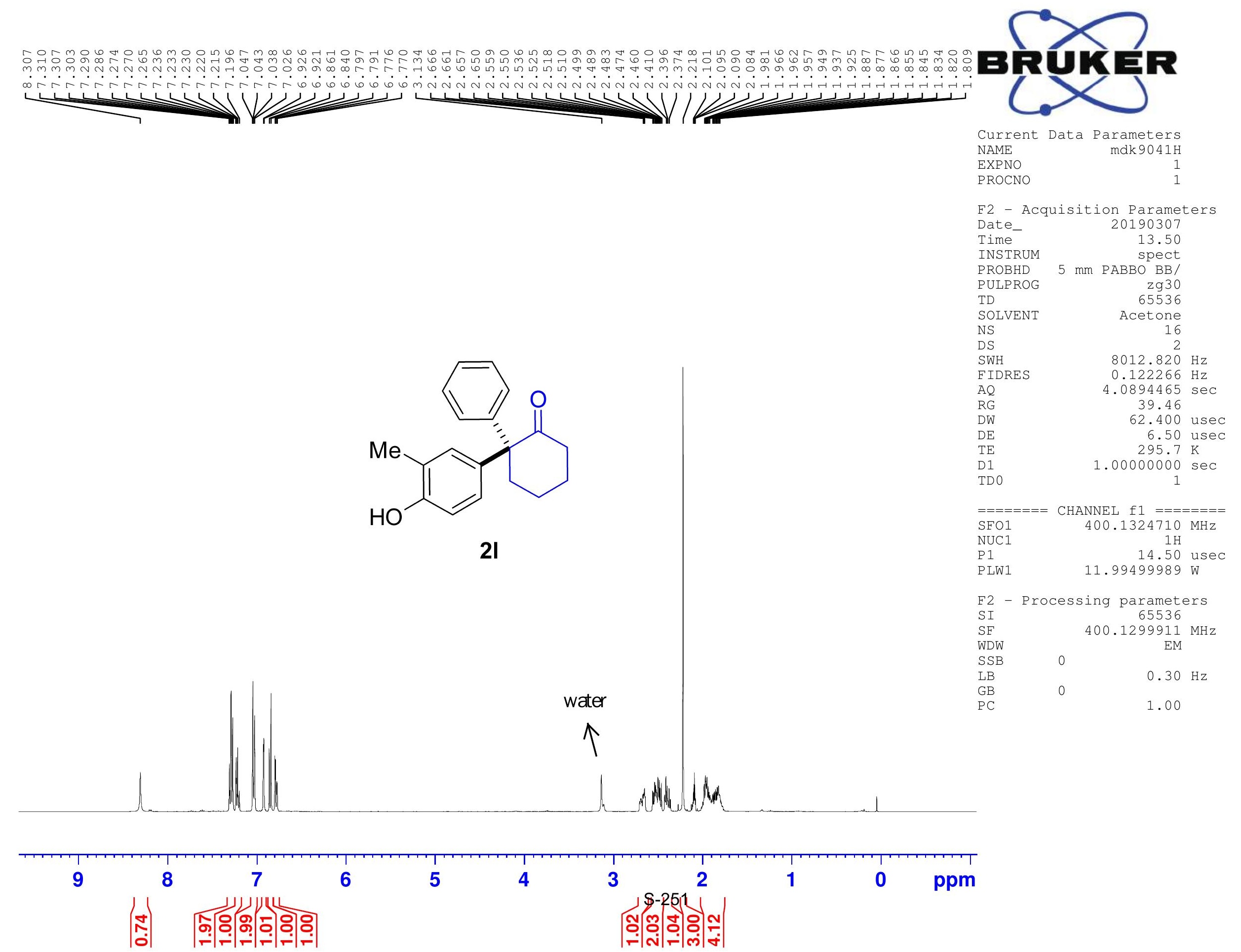




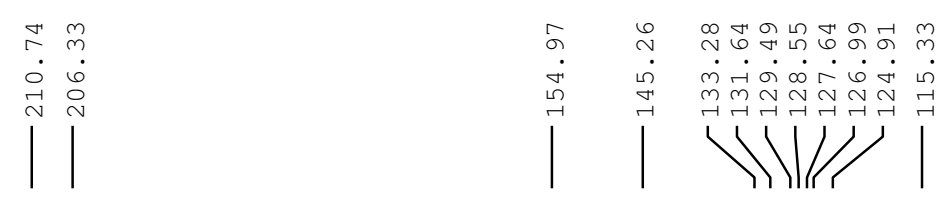

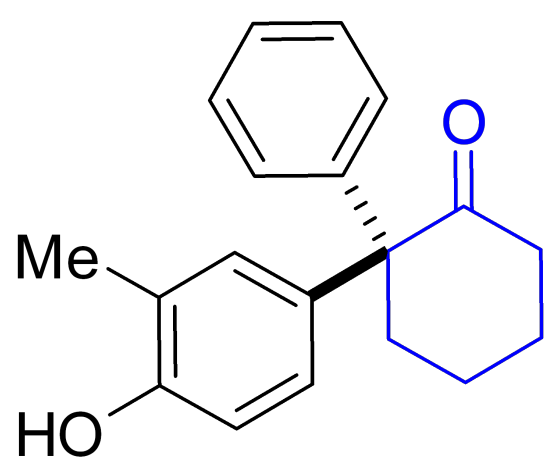

2I

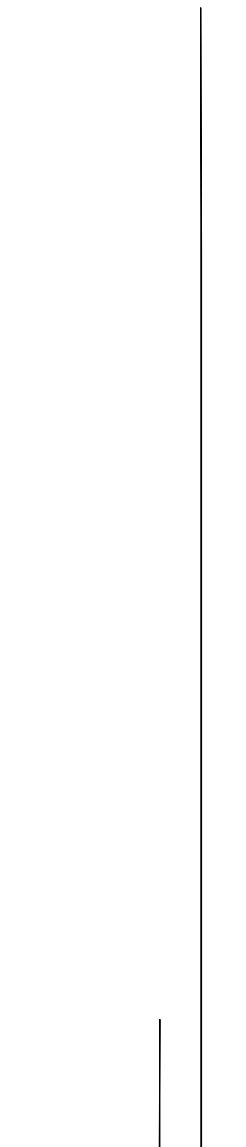

Current Data Parameters EXPNO

mdk $9041 \mathrm{C}$
1

F2 - Acquisition Parameters

Date_ 20190307

Time

spect
PROBHD $5 \mathrm{~mm}$ PABBO BB/

TD

SOLVEN

zgpg30
65536

STR

RQ

TE

D1
D11

D11

$0.454131 \mathrm{~Hz}$

16.800 usec

6.50 usec

$2.00000000 \mathrm{sec}$
$0.03000000 \mathrm{sec}$

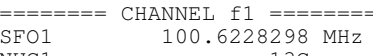

NEO1

$6.98899841 \mathrm{~W}$

$=======$ CHANNEL $\mathrm{f} 2 \quad======= \pm$
$\mathrm{SFO2}$
$400.1316005 \mathrm{MHz}$

$\begin{array}{lr}\text { NUC2 } & 1 \mathrm{H} \\ \text { CPDPRG [2 } & \text { waltz16 } \\ \text { PCPD2 } & 90.00\end{array}$

$\begin{array}{lr}\text { PLW2 } & 11.99499989 \mathrm{~W} \\ \text { PLW12 } & 0.34213999 \mathrm{~W}\end{array}$

PLW13 0.27713001 W

$\begin{array}{lr}\text { F2 } & \text { - Processing parameters } \\ \text { SI } & 32768 \\ \text { SF } & 100.6126855 \mathrm{MHz}\end{array}$

SF
WDW
SSB

${ }_{\mathrm{EM}}^{\mathrm{M}}$

$1.00 \mathrm{~Hz}$

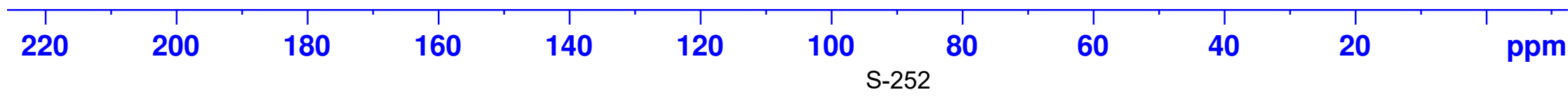




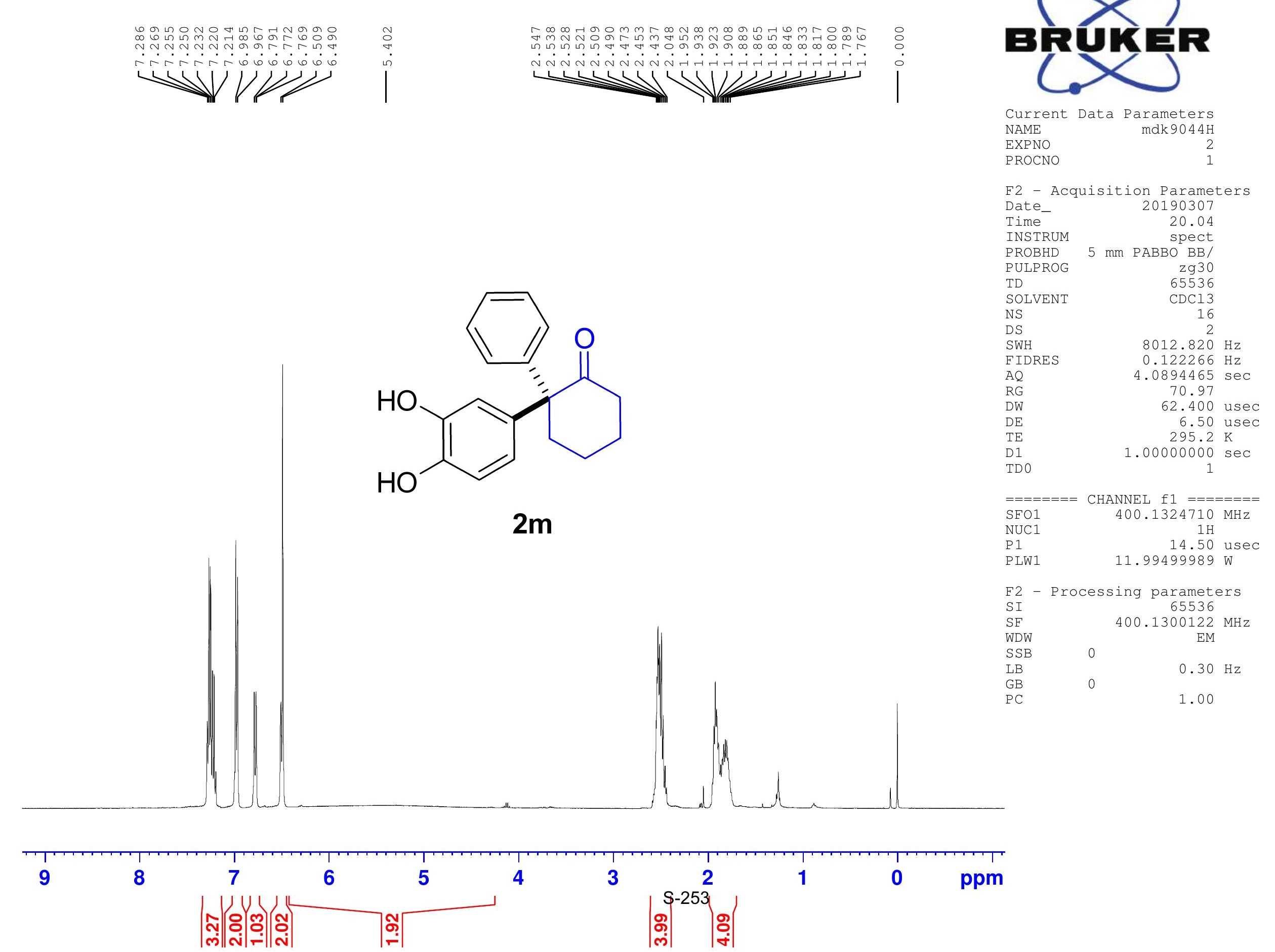




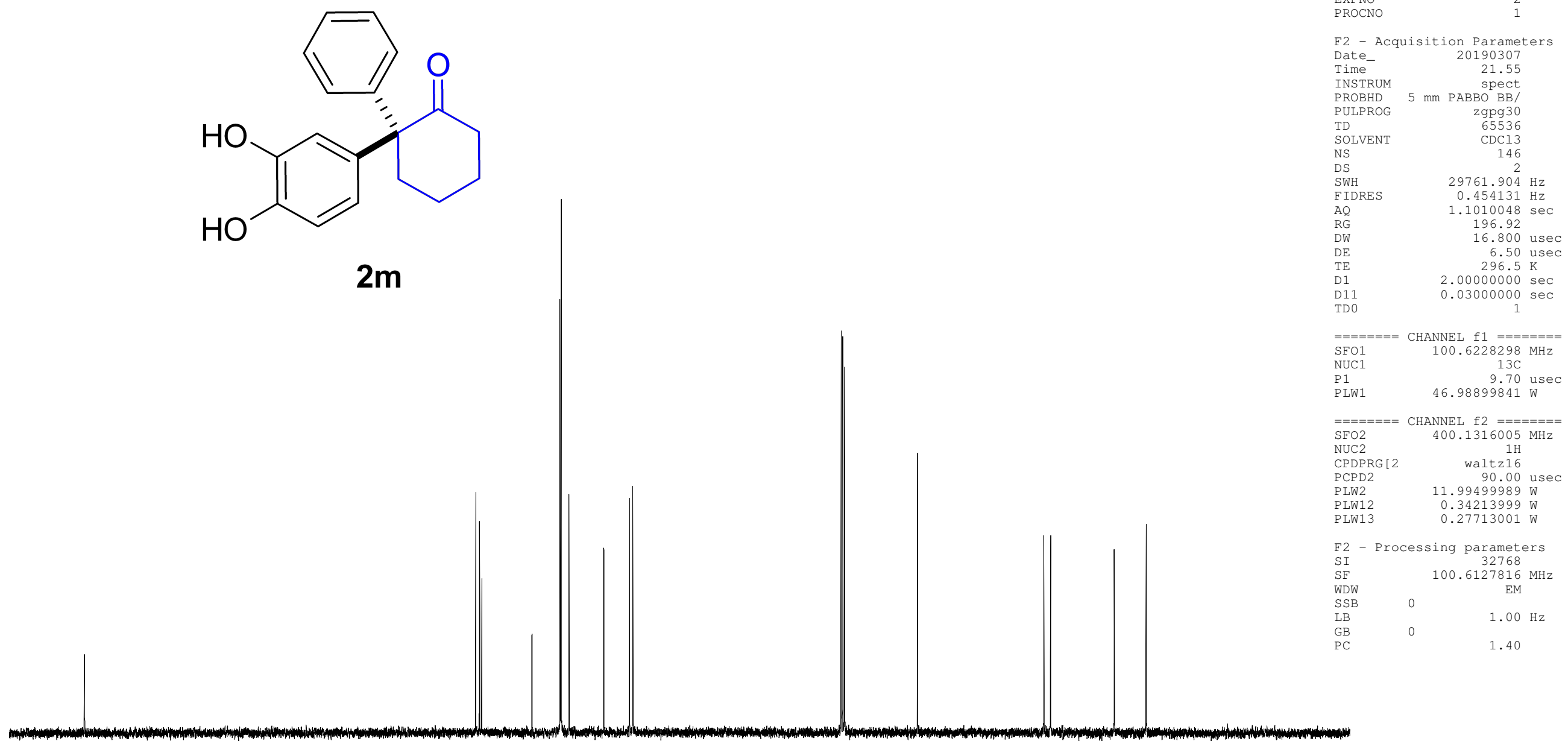

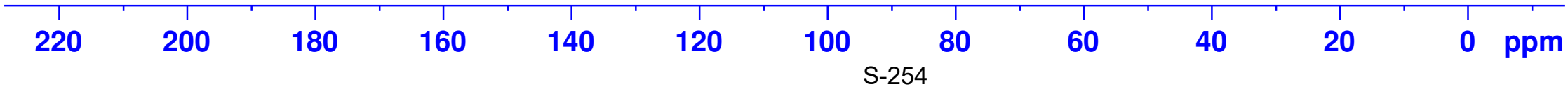


m

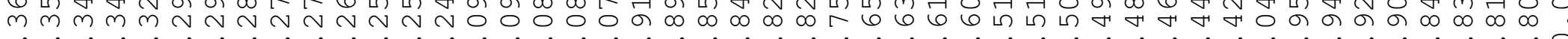

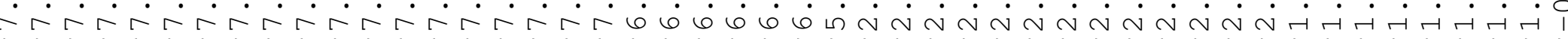
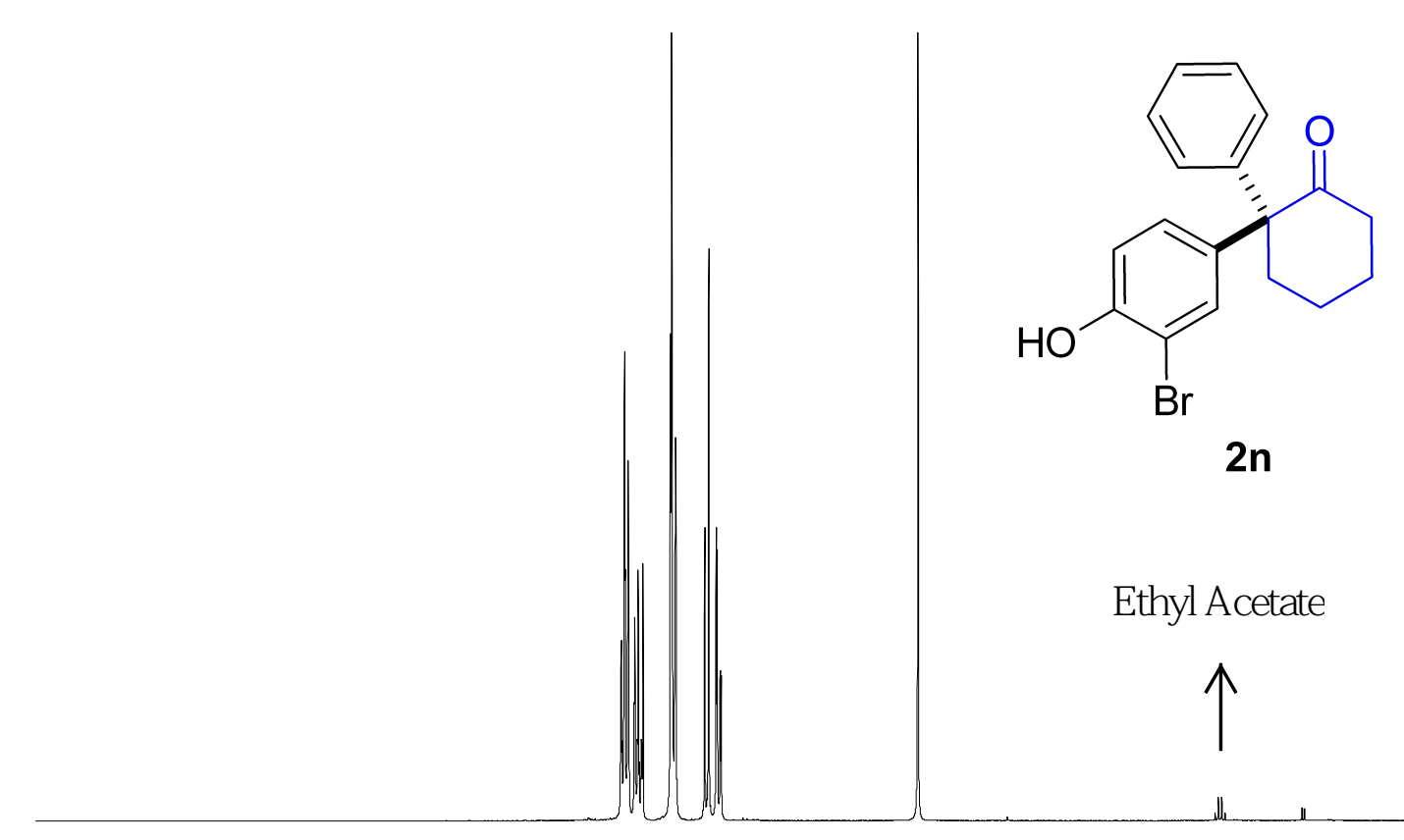

10 9

8

6

Ethyl A cetate

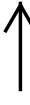

$\uparrow$

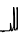

5

4

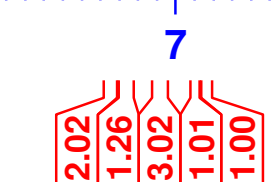
|ิ

\section{Ethyl A cetate}

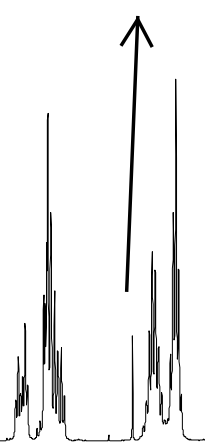

\section{si} ,

Current Data Parameters NAME

PROCNO

mak9125H

F2 - Acquisition Parameters

Date_

20190330

Time

INSTRUM

PROBHD

SOLVENT

NS
DS
SWH

SWH

IDRES

$\mathrm{AQ}$

RG

$\mathrm{DW}$
$\mathrm{DE}$

$\mathrm{DE}$
$\mathrm{TE}$
$\mathrm{D} 1$

DD 0

$====$
SFO1

$\mathrm{SFO} 1$
NUC1

$\mathrm{NUC1}$
$\mathrm{P} 1$

PLW1

13.05
spect

$5 \mathrm{~mm}$ PABBO BB/

$\mathrm{zg} 30$
65536

$\mathrm{CDCl} 3$

$8012.820 \mathrm{~Hz}$

$8012.820 \mathrm{~Hz}$

$4.0894465 \mathrm{sec}$

49.32

62.400 usec

6.50 usec

$1.00000000 \mathrm{sec}$

CHANNEL $\mathrm{f} 1========$

$400.1324710 \mathrm{MHz}$ 14.50

$11.99499989 \mathrm{~W}$

Ethyl A cetate

3

2

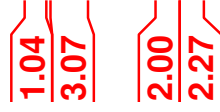


<smiles>O=C1CCCC[C@@]1(c1ccccc1)c1ccc(O)c(Br)c1</smiles>

$2 n$

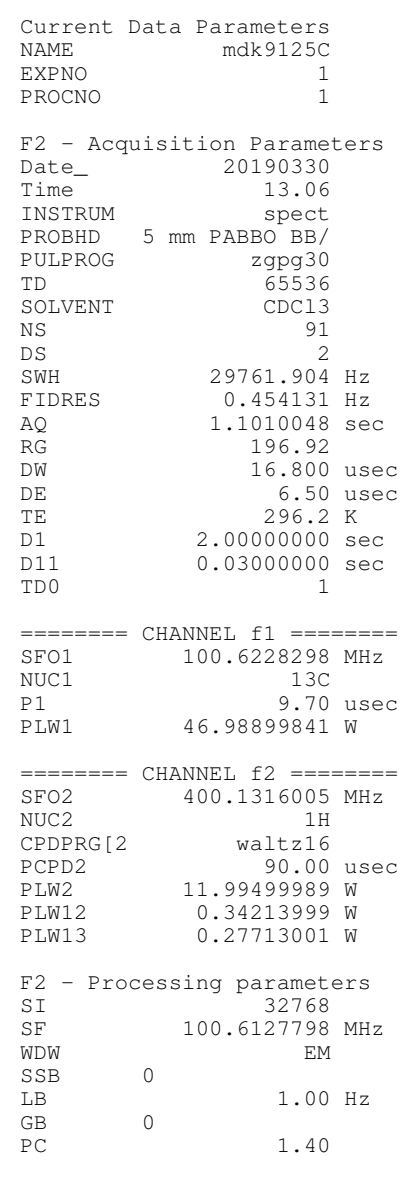




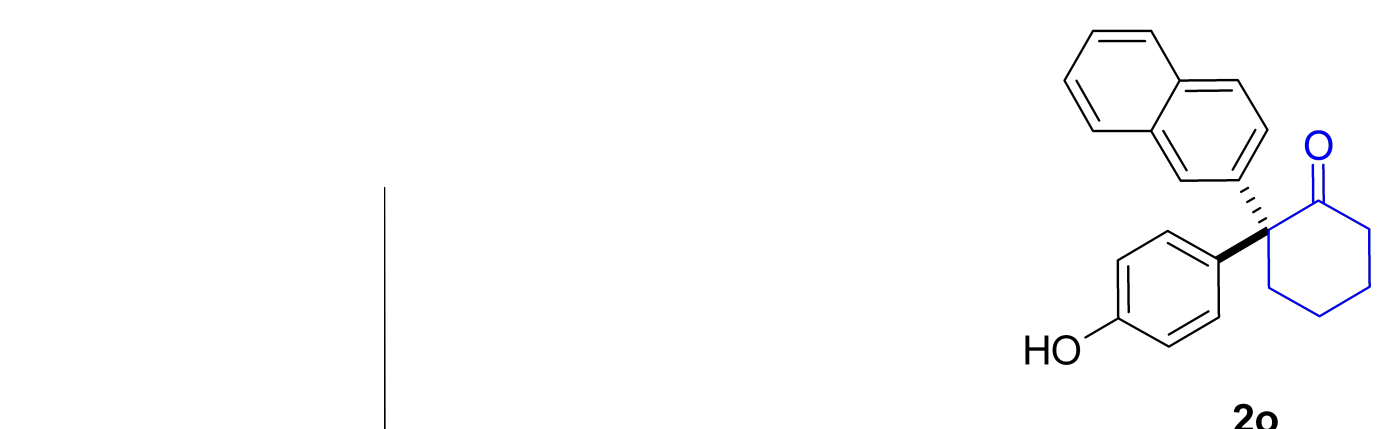

\section{BR UKER

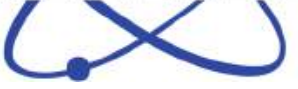

Current Data Parameters NAME

PROCNO

mdk $9130 \mathrm{H}$

F2 - Acquisition Parameters

Date__ 20190330

$\begin{array}{ll}\text { Time } & 20.55 \\ \text { INSTRUM } & \text { spect }\end{array}$

SPeCt
PROBHD $5 \mathrm{~mm} \mathrm{PABBO} \mathrm{BB} /$

PROBHD $5 \mathrm{~mm}$ PABBO BB/
PULPROG
zg30

$\begin{array}{lr}\text { PULPROG } & \text { zg30 } \\ \text { TD } & 65536\end{array}$

$\begin{array}{ll}\text { TD } & 65536 \\ \text { SOLVENT } & \text { CDCl3 }\end{array}$

SOLVENT
NS

DS

SWH

FIDRES $\quad 8012.820 \mathrm{~Hz}$

$0.122266 \mathrm{~Hz}$

AQ $4.0894465 \mathrm{sec}$

RG

DW

$\mathrm{DE}$

TE

TD 0

$$
\begin{array}{r}
82.92 \\
62.400 \text { usec }
\end{array}
$$

62.400 usec

$\begin{aligned} & 6.50 \text { usec } \\ & 297.8 \mathrm{~K}\end{aligned}$

20

$===$
$\mathrm{SFO1}$

P1

$1.00000000 \mathrm{sec}$
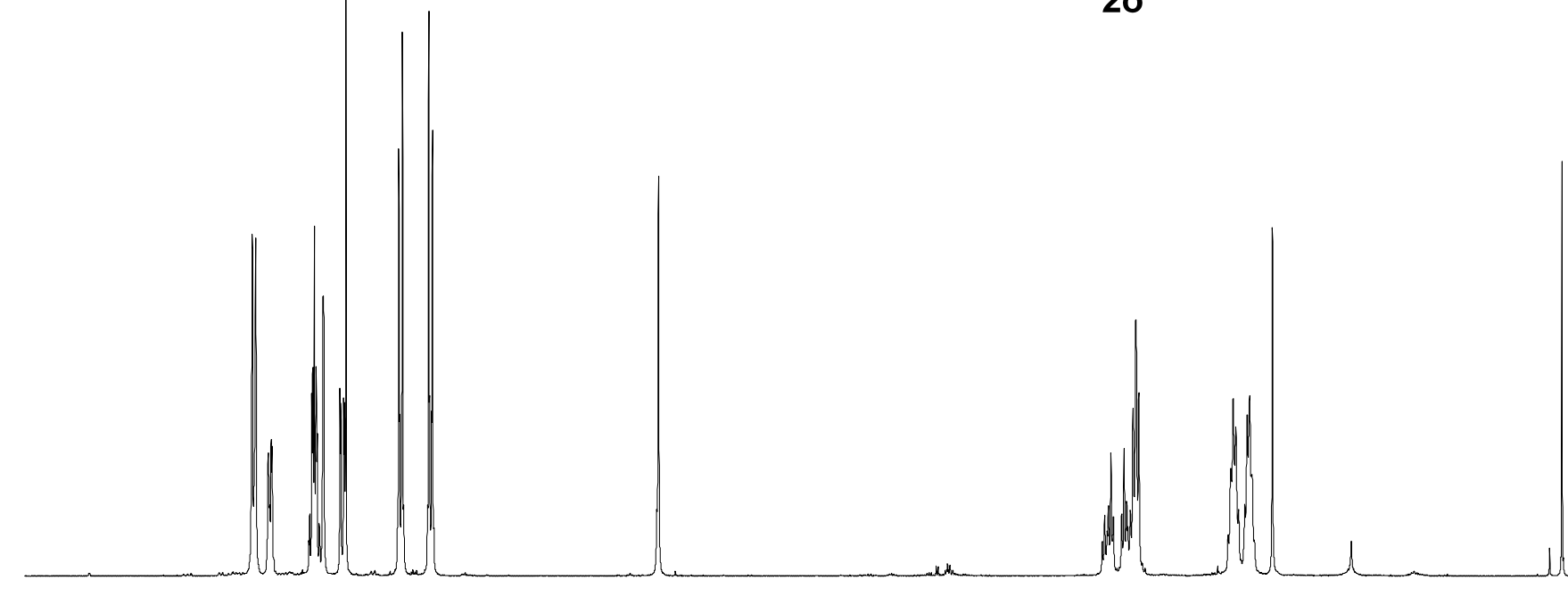

14.50 usec

F2 - Processing parameters

SI 65536

SF $\quad 400.1300154 \mathrm{MHz}$

WDW

$\mathrm{SSB}$

GB

$0.30 \mathrm{~Hz}$

1.00

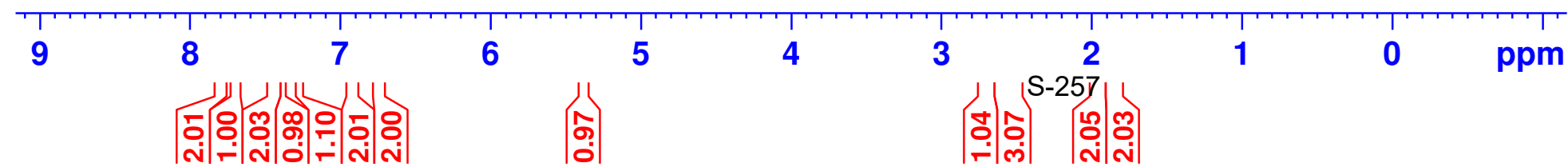




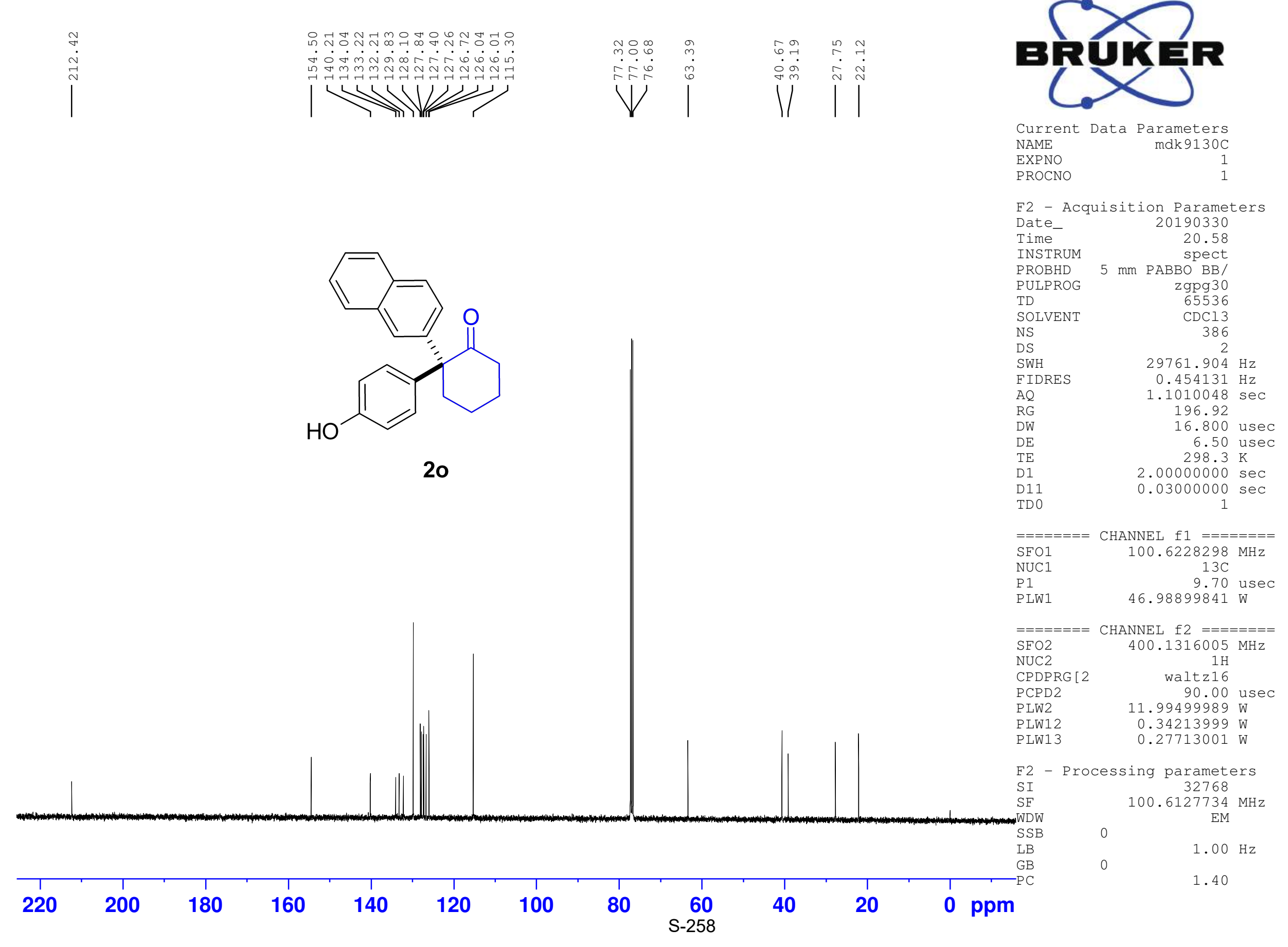


<smiles>O=C1Cc2ccccc2CC1(c1ccccc1)c1ccc(O)cc1</smiles>

$2 p$

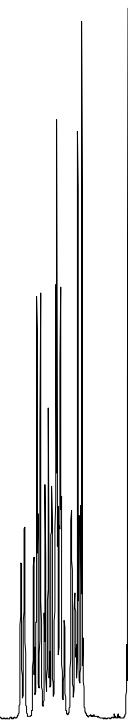

\section{thyl A cetate}

\section{Ethyl A cetate}
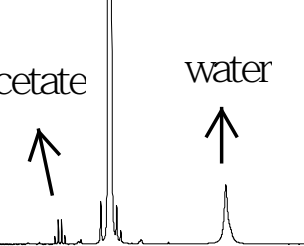

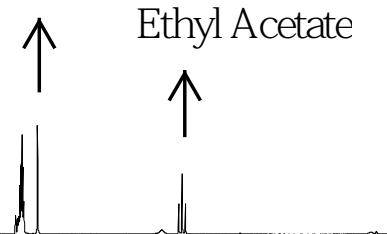

$\begin{array}{lr}\text { Current } & \text { Data } \\ \text { NAME } & \text { mameters } \\ \text { EXPNO } & 1 \\ \text { PROCNO } & 1\end{array}$

F2 - Acquisition Parameters

Date__ 20190216

Time $\quad 13.16$

$5 \mathrm{~mm}$ spect

PULPROG

$\begin{array}{lr}\text { TD } & \text { Zg30 } \\ & 65536\end{array}$

SOLVENT Acetone

NS

NS

DS

FIDRES

AQ

RG

DW

DE

D1 0

$===$

$\mathrm{SFO1}$

$\mathrm{NUC1}$
$\mathrm{P} 1$

Acetone
16

$8012.820 \mathrm{~Hz}$

$0.122266 \mathrm{~Hz}$

$4.0894465 \mathrm{sec}$ 34.77

62.400 usec 6.50 usec $1.00000000 \mathrm{sec}$ $400.1324710 \mathrm{MHz}$

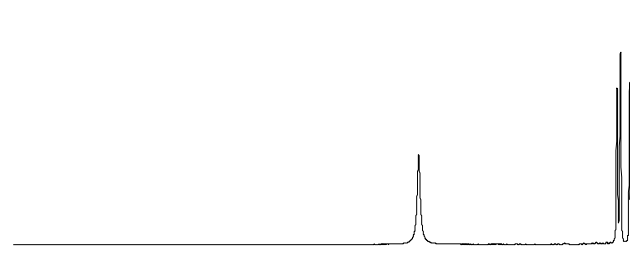

$\begin{array}{lcc}\text { F2 } & \text { - Processing parameters } \\ \text { SI } & 65536 \\ \text { SF } & 400.1299911 \mathrm{MHz} \\ \text { WDW } & 0 & \text { EM } \\ \text { SSB } & 0 & \\ \text { LB } & 0 & 0.30 \mathrm{~Hz} \\ \text { GB } & 0 & 1.00 \\ \text { PC } & & \end{array}$

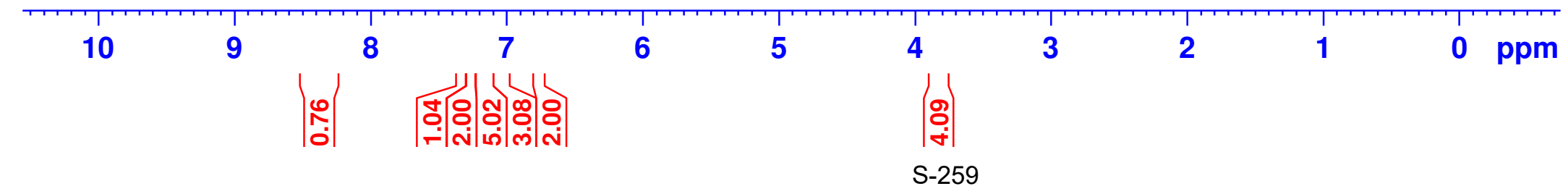




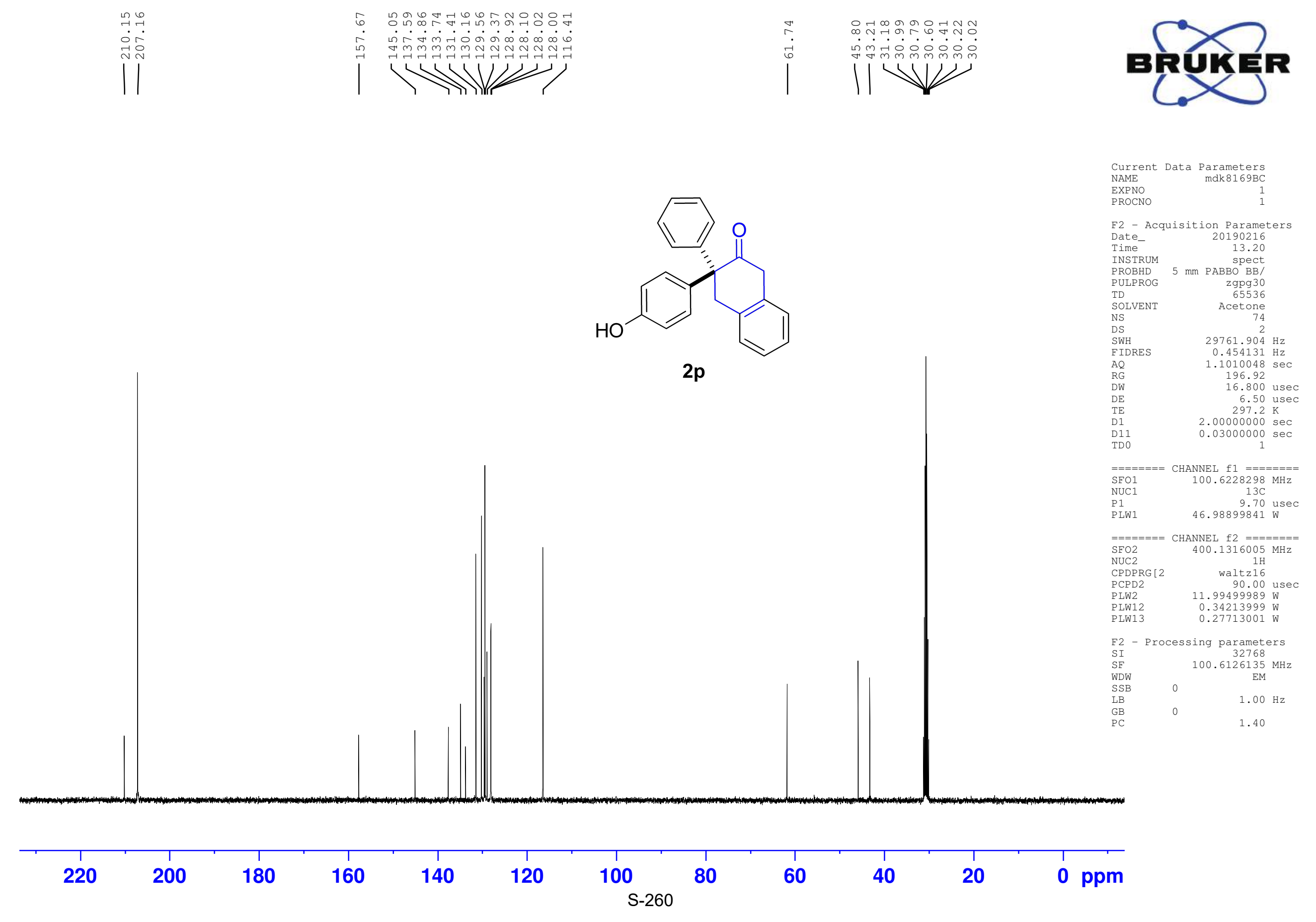




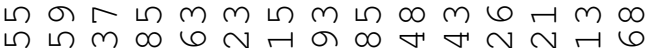

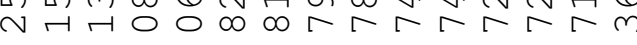

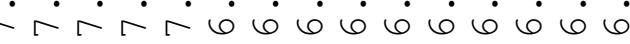

$\ldots$

$\rightarrow$ तो

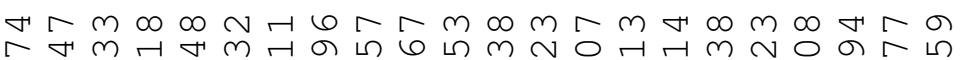
$\kappa$ เ

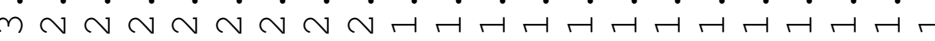
N v N v N

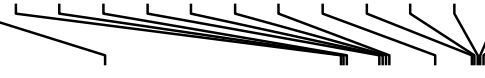

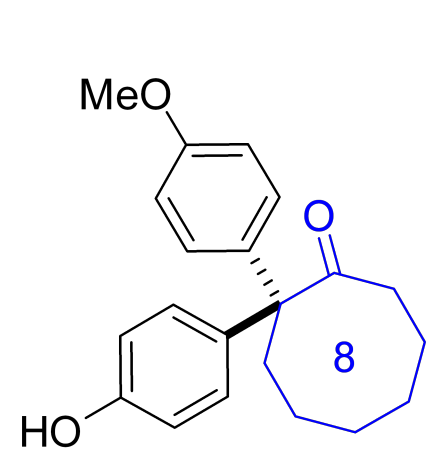

2q

\section{se $(1)$}

Current Data Parameters NAME

PROCNO

$\operatorname{mdk} 9027 \mathrm{H}$

F2 - Acquisition Parameters Date__ 20190304

$\begin{array}{lr}\text { Time } & 13.18\end{array}$

INSTRUM spect

zg30
65536

TD 65536

NS

DS

FIDRES

$\mathrm{AQ}$

RG

DW

$\mathrm{DE}$

D1
TD 0

$===$

$\mathrm{SFO1}$

NUC

P1
PLW1

Ethyl A cetate

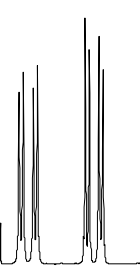

Ethyl A cetate

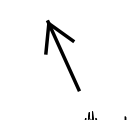

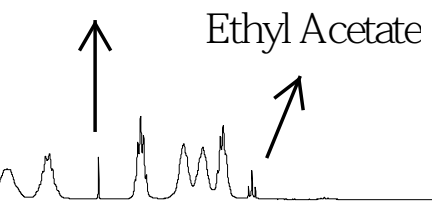

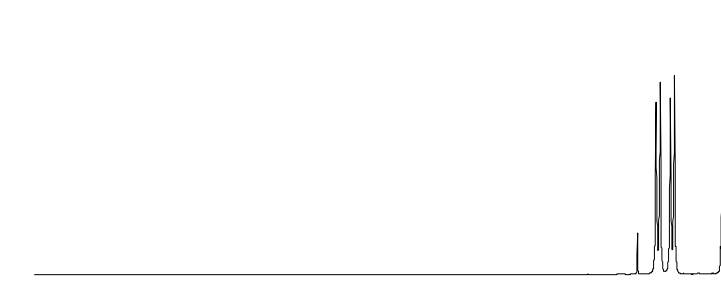

8

8

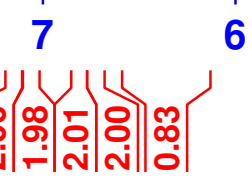

5

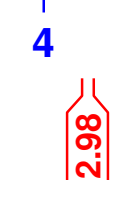

3

2

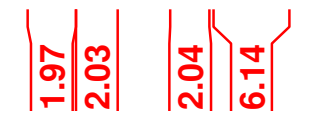




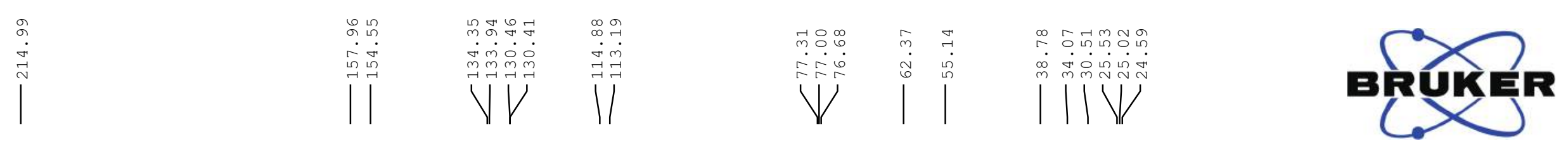

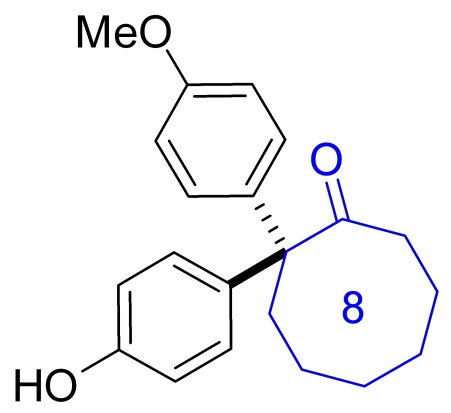

$2 q$

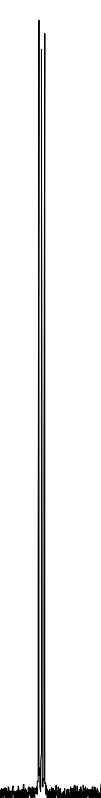

Current Data Parameters
NAME

EXPNO
PROCNO

F2 - Acquisition Parameter

Date ${ }_{\text {Tim }} \quad 20190304$

Time
INSTRUM

PROBHD
PULPROC

TD SOLVENT

NS

SWH

$\mathrm{AQ}$
$\mathrm{RG}$

$\mathrm{RG}$
$\mathrm{DW}$
$\mathrm{DE}$

TE
D1
D11

D11
TD0

$=======$ CHANNEL $\mathrm{f} 1 \quad========$
SFO1

SFO1
NUC1
P1
PLW1

$13 \mathrm{C}$
$9.70 \mathrm{usec}$

$46.98899841 \mathrm{~W}$

$\begin{array}{ll}======= & \text { CHANNEL } \quad \text { f2 }======== \\ \text { SFO2 } & 400.1316005 \mathrm{MHz}\end{array}$

$\begin{array}{lr}\text { NUC2 } & 1 \mathrm{H} \\ \text { CPDPRG [2 } & \text { waltz16 } \\ \text { PCPD2 } & 90.00\end{array}$

11.99499989

$\begin{array}{ll}\text { PLW12 } & 0.34213999 \mathrm{~W} \\ \text { PLW13 } & 0.2771301\end{array}$

F2 - Processing parameters

$\begin{array}{lr}\text { F2 } & \text { - Processing parameters } \\ \text { SI } & 32768 \\ \text { SF } & 100.6127779 \mathrm{MHz}\end{array}$

WE

GB

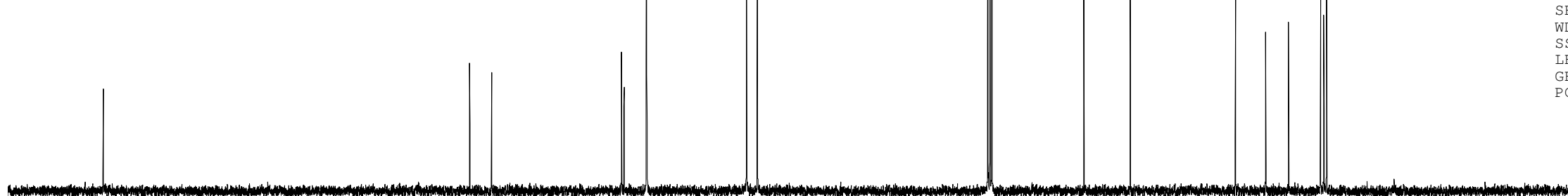

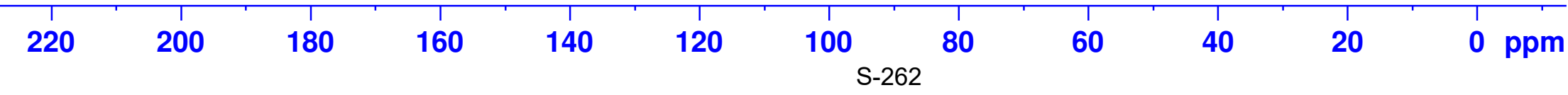




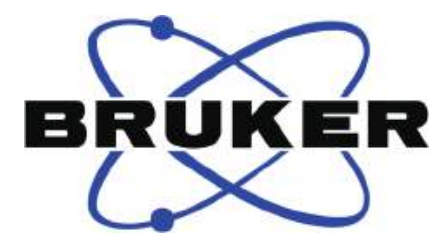

Current Data Parameters NAME

EXPNO

$\operatorname{mdk} 10167 \mathrm{H}$

PROCNO

F2 - Acquisition Parameters

Date_ 20190715

$\begin{array}{lr}\text { Time } & 2013.16\end{array}$

INSTRUM spect

PROBHD

PULPROG $\quad$ zg30

TD $\quad 65536$

SOLVENT

NS

DS

FIDRES

$2 a^{\prime}$

$\mathrm{AQ}$

RG

DW

$\mathrm{DE}$

D 10

$====$

$\mathrm{SFO1}$

$\mathrm{NUC}$
$\mathrm{P} 1$

P1
PLW1

$\mathrm{CDC} 13$
16
2

$8012.820 \mathrm{~Hz}$

$0.122266 \mathrm{~Hz}$

$4.0894465 \mathrm{sec}$ 19.7

62.400 usec 6.50 usec $1.00000000 \mathrm{sec}$ 1

CHANNEL $\mathrm{fl}========$ $400.1324710 \mathrm{MHz}$ sec SI $\quad 65536$ SF $\quad 400.1300258 \mathrm{MHz}$ WDW EM

$0.30 \mathrm{~Hz}$

1.00

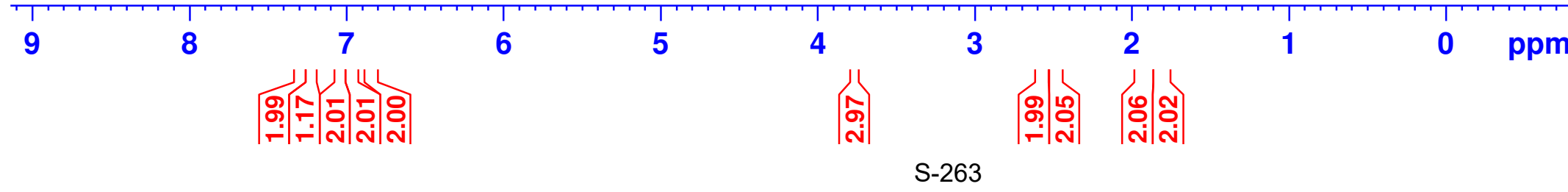




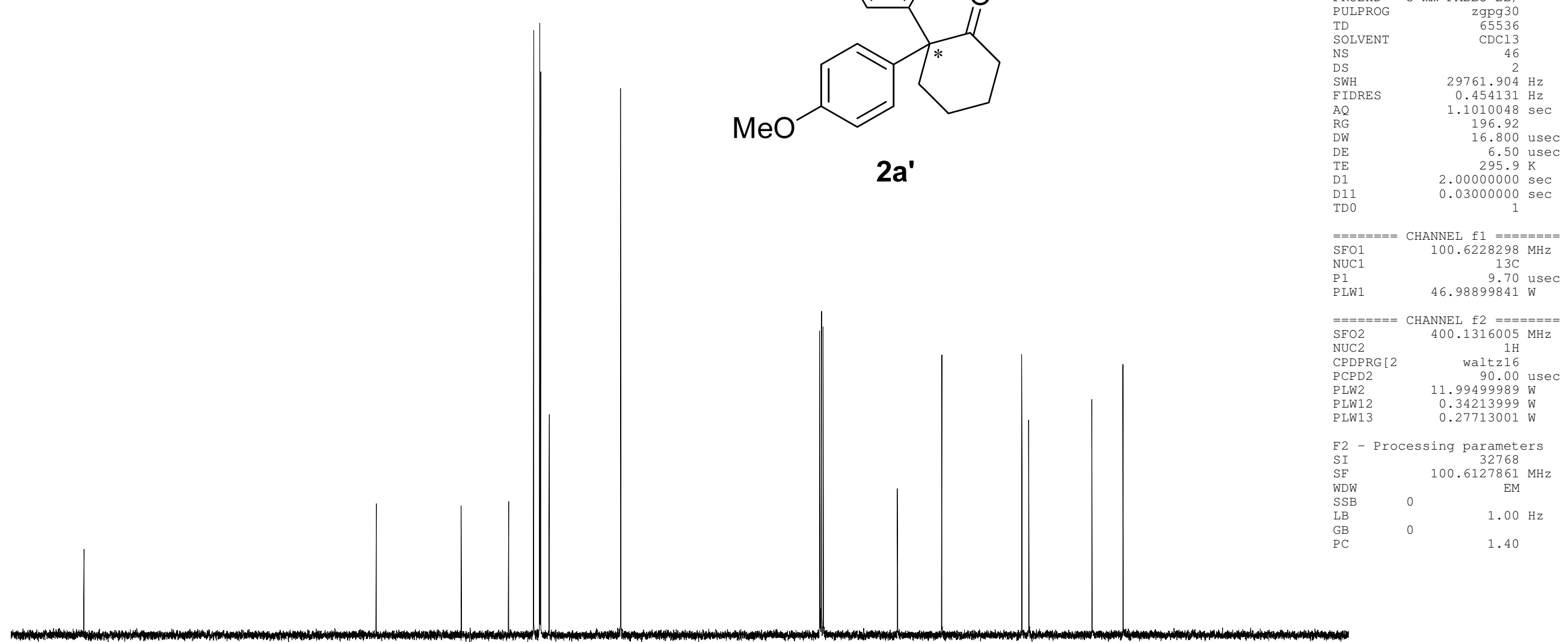

\begin{tabular}{lrrrrrrrrrrrrr}
\hline 220 & 200 & 180 & 160 & 140 & 120 & 100 & 80 & 60 & 40 & 20 & 0 & ppm
\end{tabular}




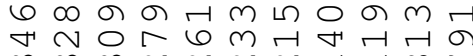
$m m m \sim \sim \sim \sim \sim 冂 卄$.

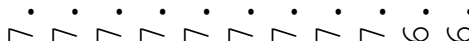

$\longrightarrow$

\section{sporen

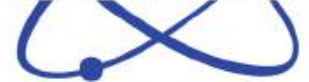

Current Data Parameters NAME $\operatorname{mdk} 8151 \mathrm{H}$

EXPNO

F2 - Acquisition Parameters<smiles>CCC(=O)[C@@](CC)(c1ccccc1)c1ccc(O)cc1</smiles>

$4 a$
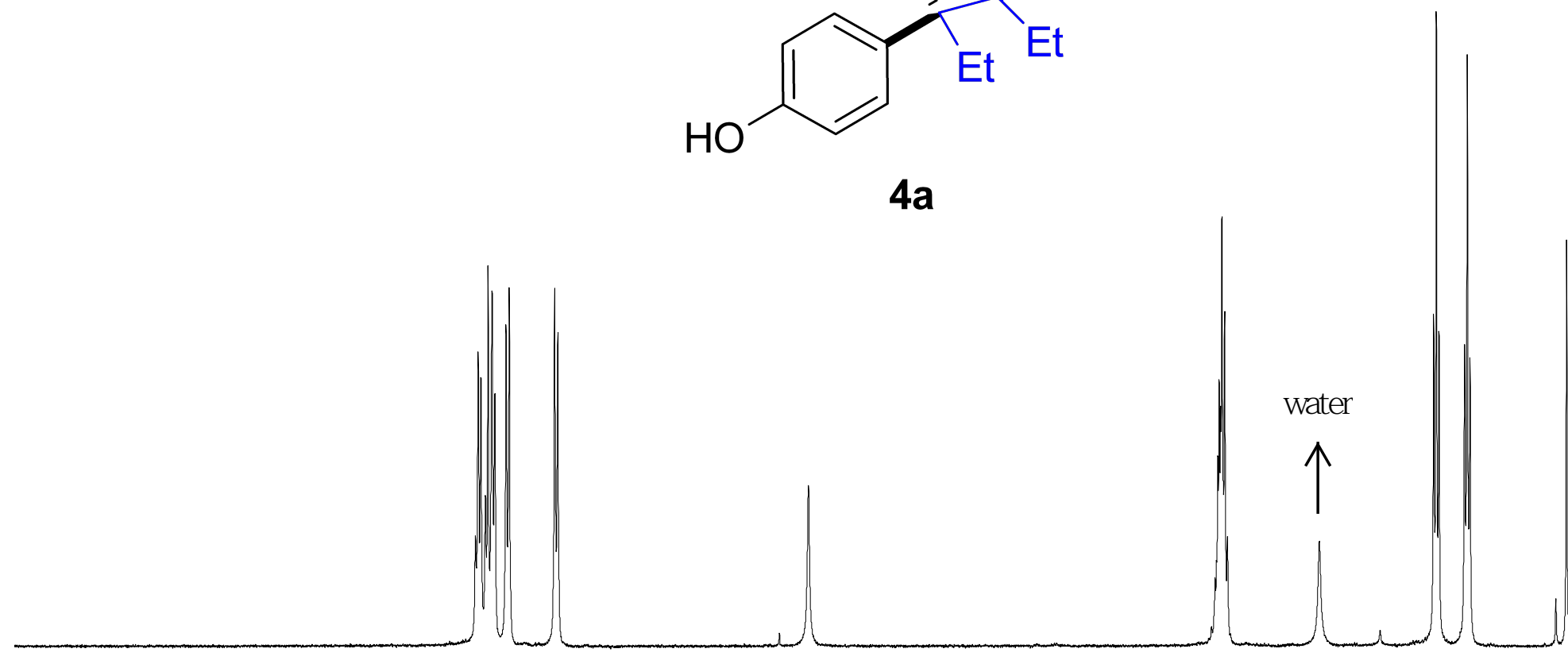

0190129

$\begin{array}{lr}\text { Time } & 2013.12\end{array}$

INSTRUM spect

PROBHD $5 \mathrm{~mm}$

PULPROG zg30

TD 65536

SOLVENT

NS

SWH

FIDRES

AQ

RG

$\mathrm{DW}$

$\mathrm{DE}$

TE

TD 0

$===$

$\mathrm{SFO1}$

$\mathrm{NUC1}$
$\mathrm{P} 1$

PLW1

$\mathrm{CDC} 13$
16
2

$8012.820 \mathrm{~Hz}$

$0.122266 \mathrm{~Hz}$

$4.0894465 \mathrm{sec}$ 112.31

62.400 usec 6.50 usec
$295.3 \mathrm{~K}$ $1.00000000 \mathrm{sec}$

CHANNEI $f 1=======$ $400.1324710 \mathrm{MHz}$ 14.50 usec

F2 - Processing parameter SI 65536 SF $400.1300095 \mathrm{MH}$ WDW

SSB EM

$0.30 \mathrm{~Hz}$

1.00

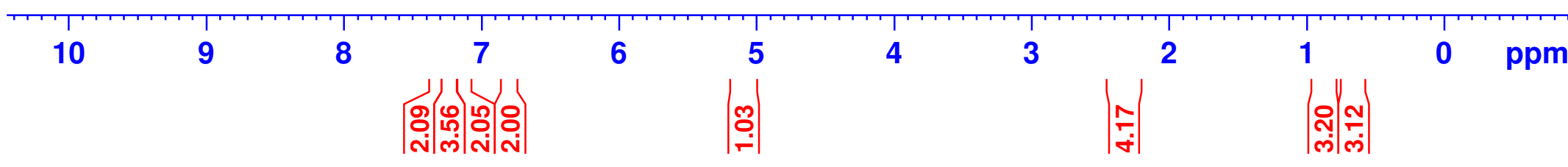




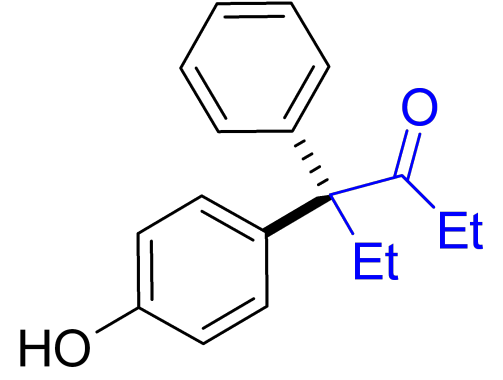

$4 a$

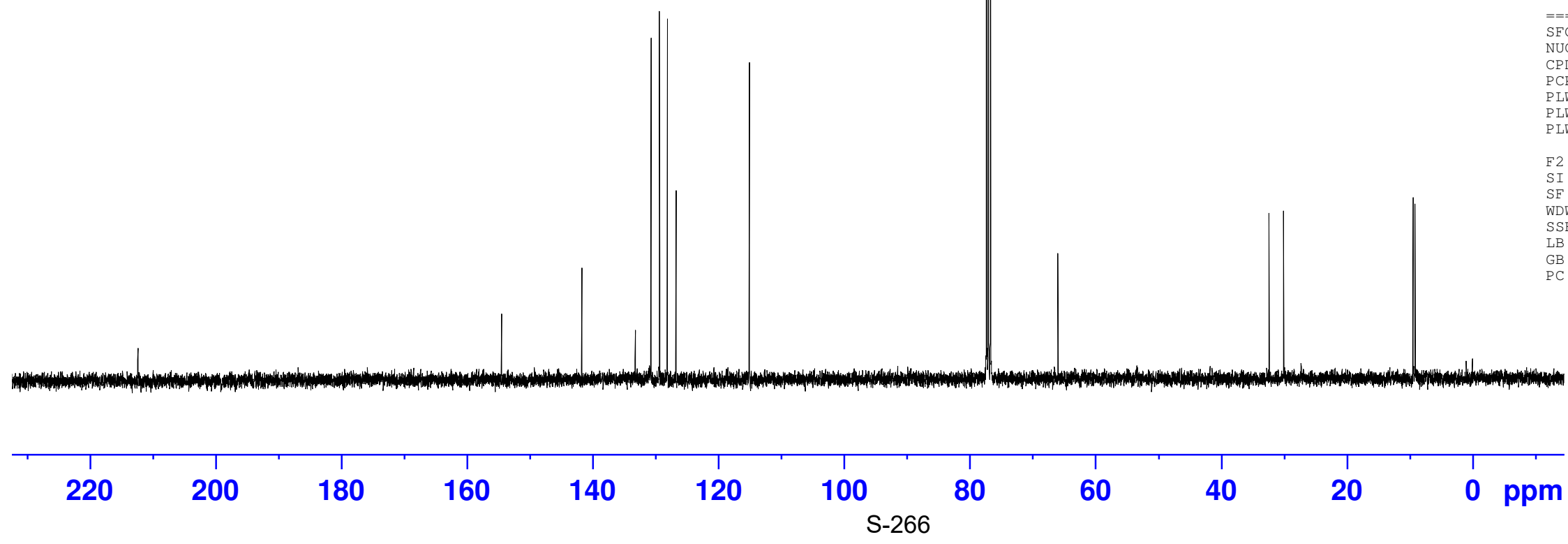

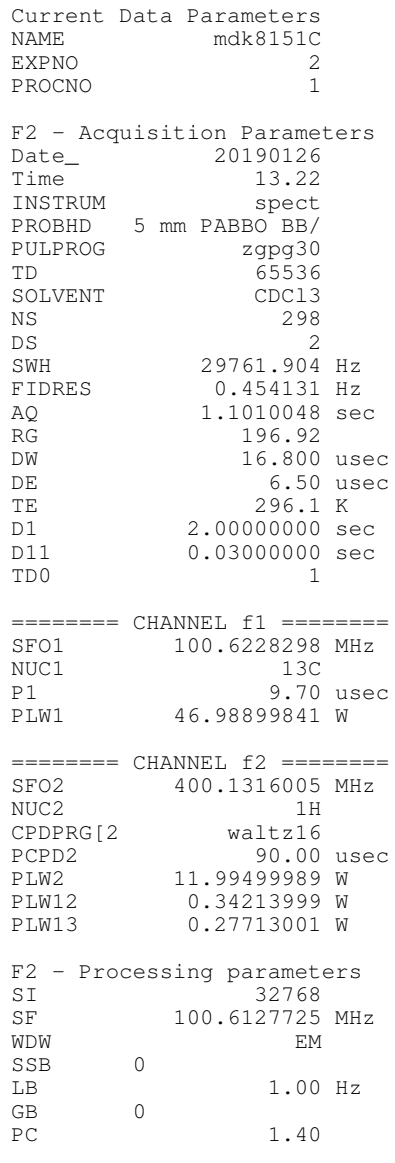




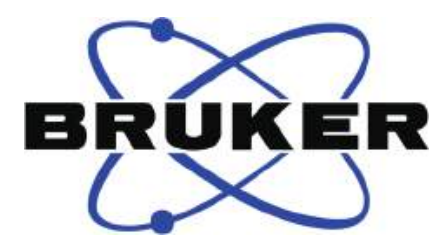

Current

EXPNO

PROCNO

F2 - Acquisition Parameters

Date__ 20190223

13.14

INSTRUM

PROBHD

$5 \mathrm{~mm}$ PABBO BB/

UTROB

TD

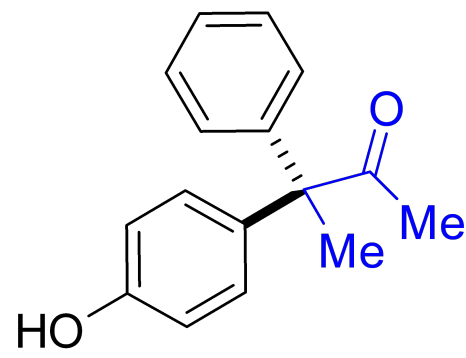

4b

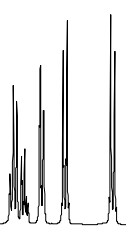

7

6

5

4

3

2

1

o ppm

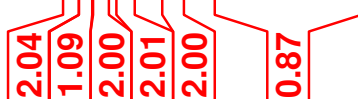

(ి) 
<smiles>CC(=O)C(C)(c1ccccc1)c1ccc(O)cc1</smiles>

$4 b$

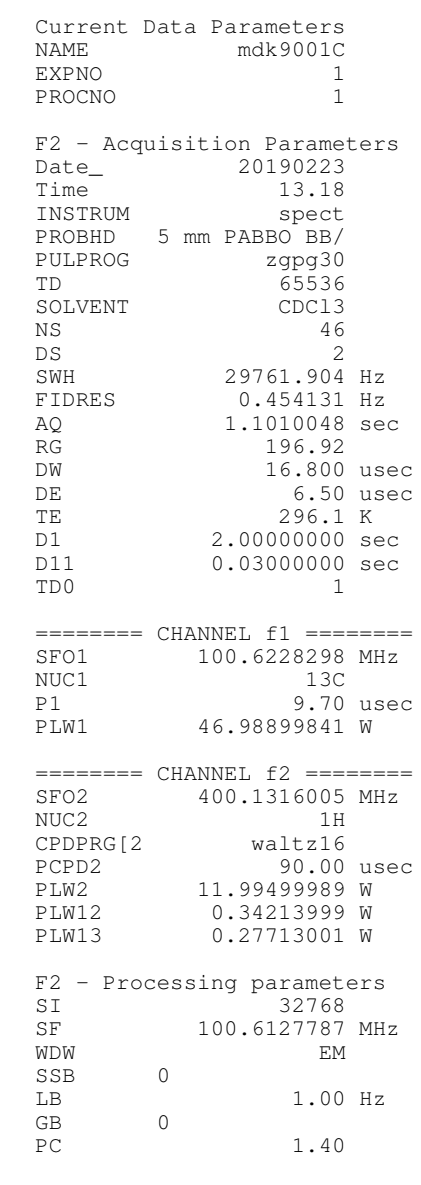




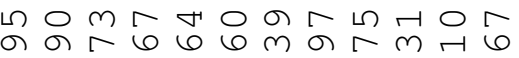

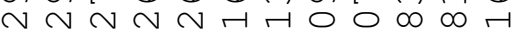

$\operatorname{rar} r \dot{r} \times \dot{0} 6$

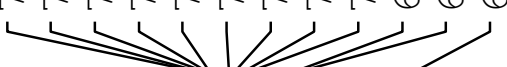

办

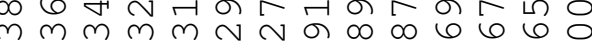
$\sim \sim \sim \sim \sim \sim \sim 0000000$ 3
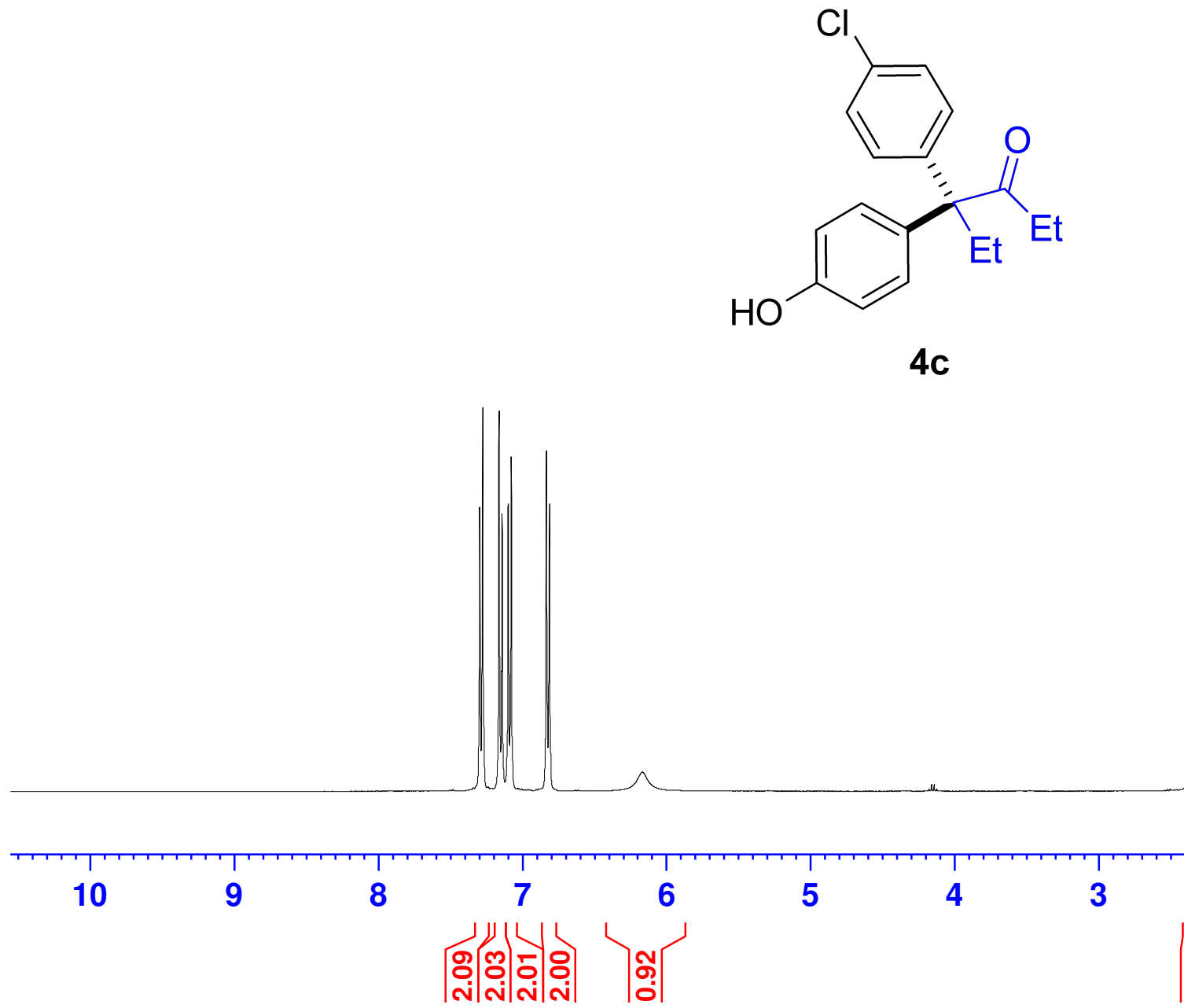

3

\section{sporen $(1)$}

$\begin{array}{lr}\text { Current Data Parameters } \\ \text { NAME } & \text { mak9082 } \mathrm{H}\end{array}$

EXPNO

2

PROCNO

F2 - Acquisition Parameters

Date_ 20190319

$\begin{array}{ll}\text { Time } & 21.30\end{array}$

$5 \mathrm{~mm}$ spect

PULPROG $\quad$ zg30

$\begin{array}{lr}\text { TD } & \text { Zg30 } \\ & 65536\end{array}$

SOLVENT CDC13

DS

SWH

FIDRES

$\mathrm{AQ}$

RG

DW

$\mathrm{DE}$

D1
TD 0

$====$

$\mathrm{SFO1}$

NUC

P1
PLW1

16
2
$8012.820 \mathrm{~Hz}$

$4.0894465 \mathrm{sec}$ 45.67

62.400 usec 6.50 usec

$1.00000000 \mathrm{sec}$

CHANNET 61 = $==2=$ $400.1324710 \mathrm{MHz}$

use $11.99499989 \mathrm{~W}$

F2 - Processing parameters

SI 65536

$\begin{array}{ll}\text { SF } & 400.1300088 \mathrm{MHz}\end{array}$

WSB

EM

$0.30 \mathrm{~Hz}$

1.00

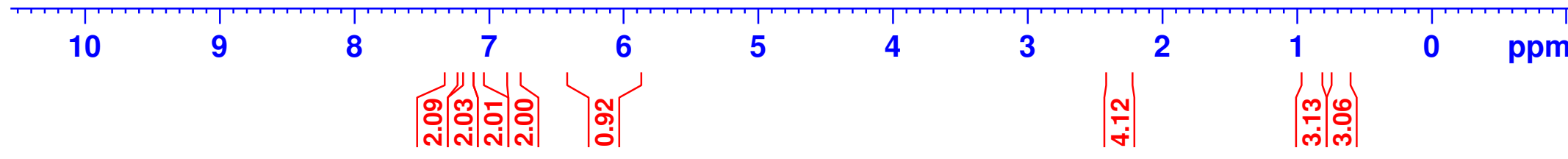




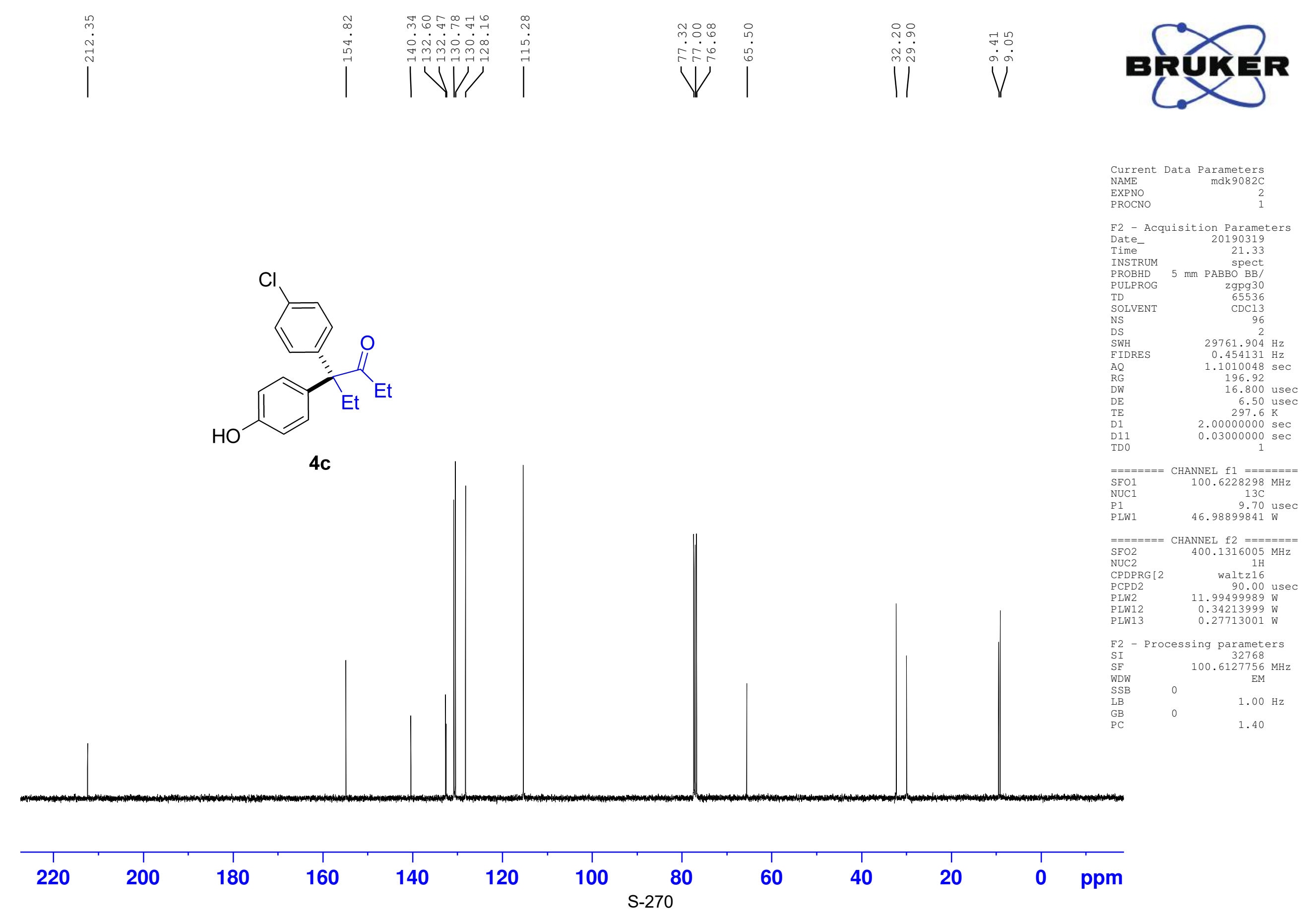




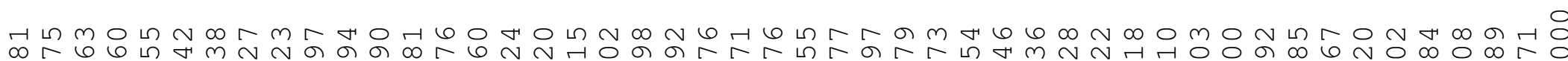

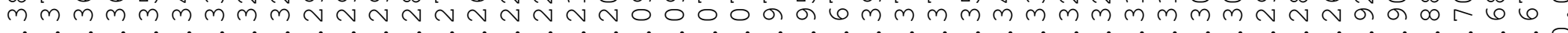

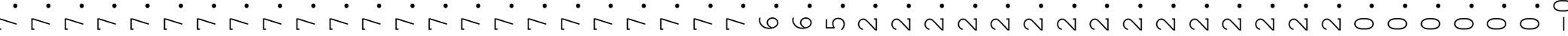

\section{sp ,}

-<smiles>CCC(=O)c1ccccc1-c1ccccc1</smiles>
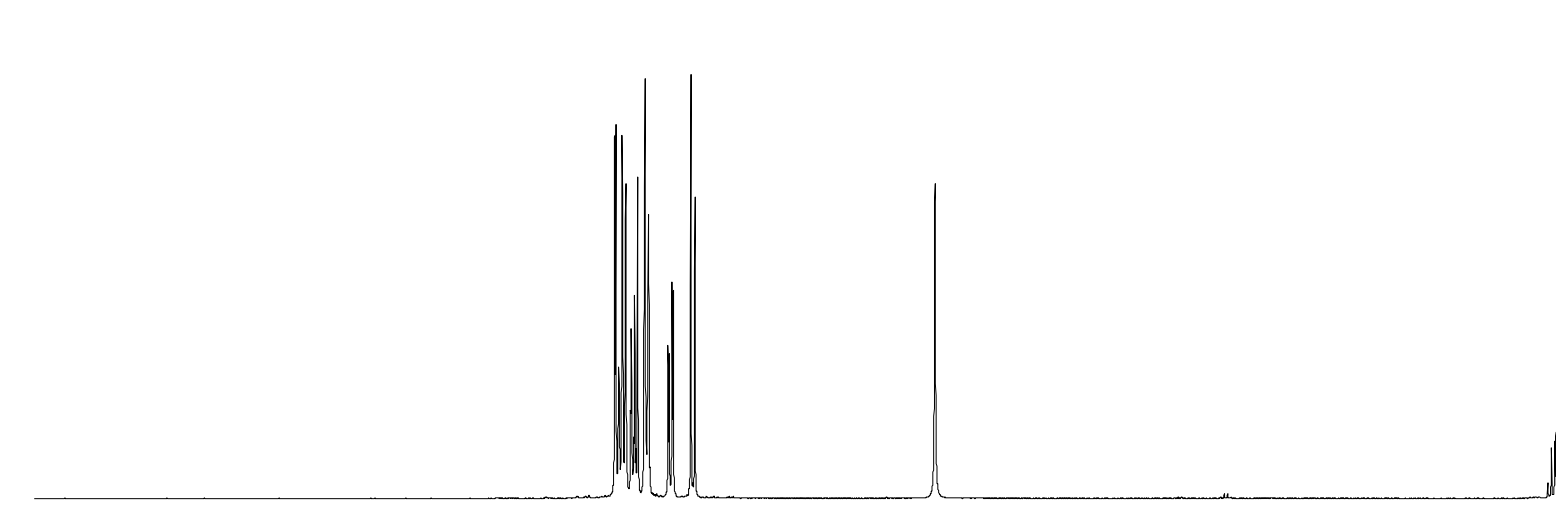

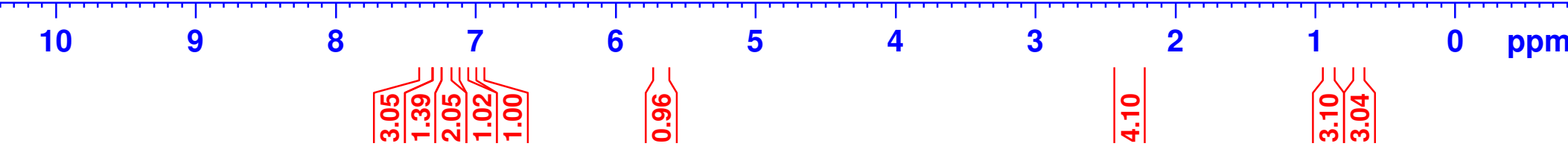

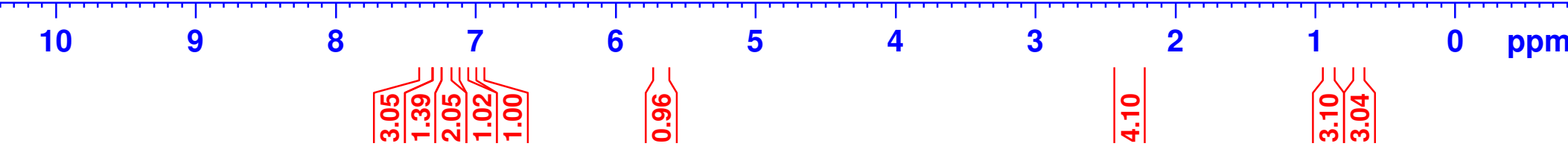

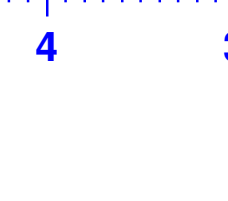

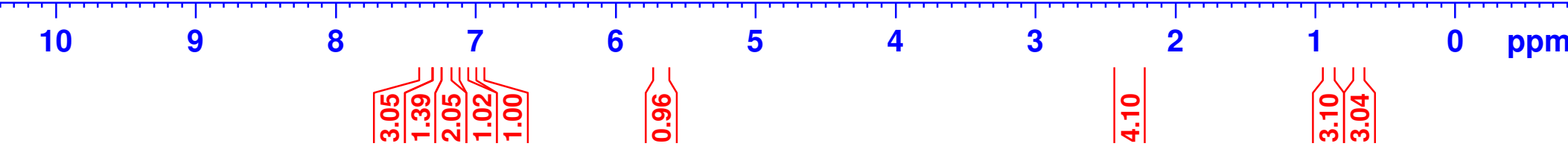

S-271

Current Data Parameters
NAME
mdk $9127 \mathrm{H}$ EXPNO

PROCNO

1

F2 - Acquisition Parameters Date_ 20190330

$\begin{array}{lr}\text { Time- } & 20.45\end{array}$

INSTRUM spect

PROBHD

$\begin{array}{lr}\text { TD } & \text { zg30 } \\ \text { TD } & 65536\end{array}$

$\begin{array}{ll}\text { TD } & 65536 \\ \text { SOLVENT } & \text { CDC13 }\end{array}$

NS
DS
SWH

$\begin{array}{ll}\text { SWH } & 8012.820 \mathrm{~Hz} \\ \text { FIDRES } & 0.122266 \mathrm{~Hz}\end{array}$ AQ $\quad 4.0894465 \mathrm{~Hz}$ RG

DW

$\mathrm{DE}$

$\mathrm{TE}$
$\mathrm{D} 1$

TDO

$===$

$\mathrm{SFO1}$

$\mathrm{NUC1}$
$\mathrm{P} 1$

P 1
PLW1

$4.0894465 \mathrm{sec}$ 627.78

62.400 usec
6.50 usec $297.7 \mathrm{~K}$

$1.00000000 \mathrm{sec}$

CHANNET 61 = $=0=0$ $400.1324710 \mathrm{MHz}$ $1 \mathrm{H}$ 14.50 usec $11.99499989 \mathrm{~W}$

F2 - Processing parameters SI 65536 SF $\quad 400.1300102 \mathrm{MHz}$ SSB EM $0.30 \mathrm{~Hz}$ 1.00

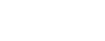




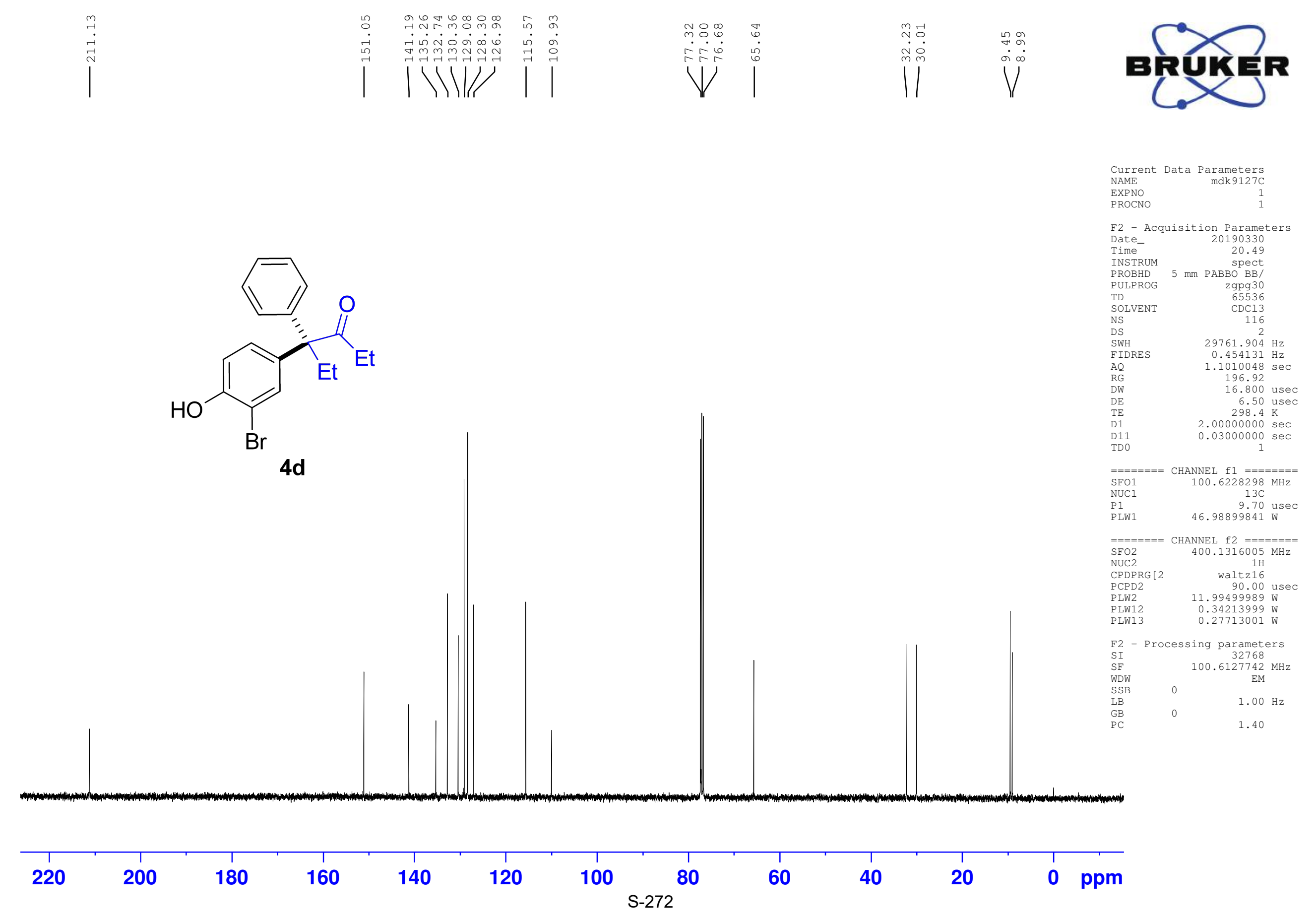




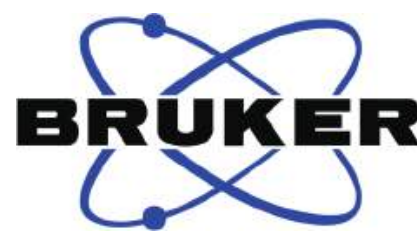

Current Data Parameters NAME

PROCNO

mdk11070H

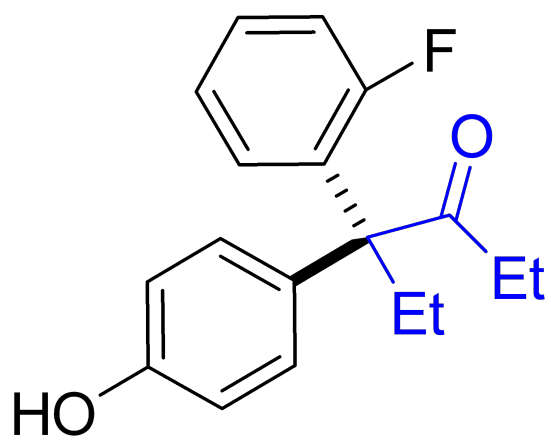

$4 e$

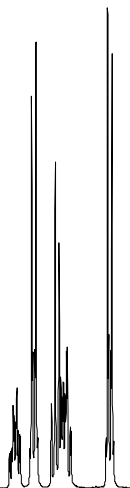

water

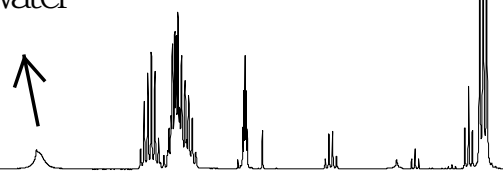

F2 - Acquisition Parameters

Date_ 20190811

Time 16.49

$5 \mathrm{~mm}$ spect

PULPROG

zg30
65536

TD

SOLVENT

NS

SWH

FIDRES

$\mathrm{AQ}$

RG

DW

$\mathrm{DE}$

D1
TD 0

$====$

$\mathrm{SFO1}$

$\mathrm{NUC}$
$\mathrm{P} 1$

Acetone

16
2

$8012.820 \mathrm{~Hz}$

$0.122266 \mathrm{~Hz}$

$4.0894465 \mathrm{sec}$

45.67

$1.00000000 \mathrm{sec}$

CHANNEL $\mathrm{f} 1 \mathrm{l}========$

$400.1324710 \mathrm{MHz}$

11 14.50

PLW1 $11.99499989 \mathrm{~W}$

F2 - Processing parameters

SI 65536

SF $\quad 400.1299910 \mathrm{MHz}$

EM

1.00

62.400 usec

6.50 usec

$0.30 \mathrm{~Hz}$

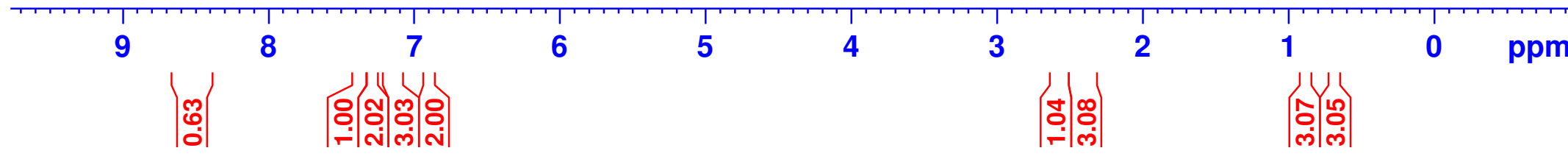



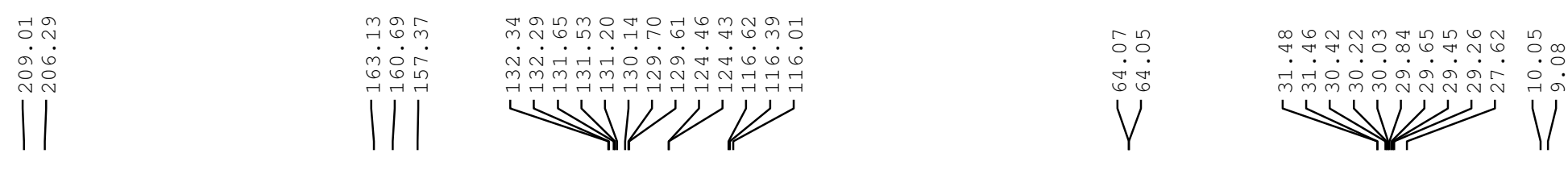

eferieng

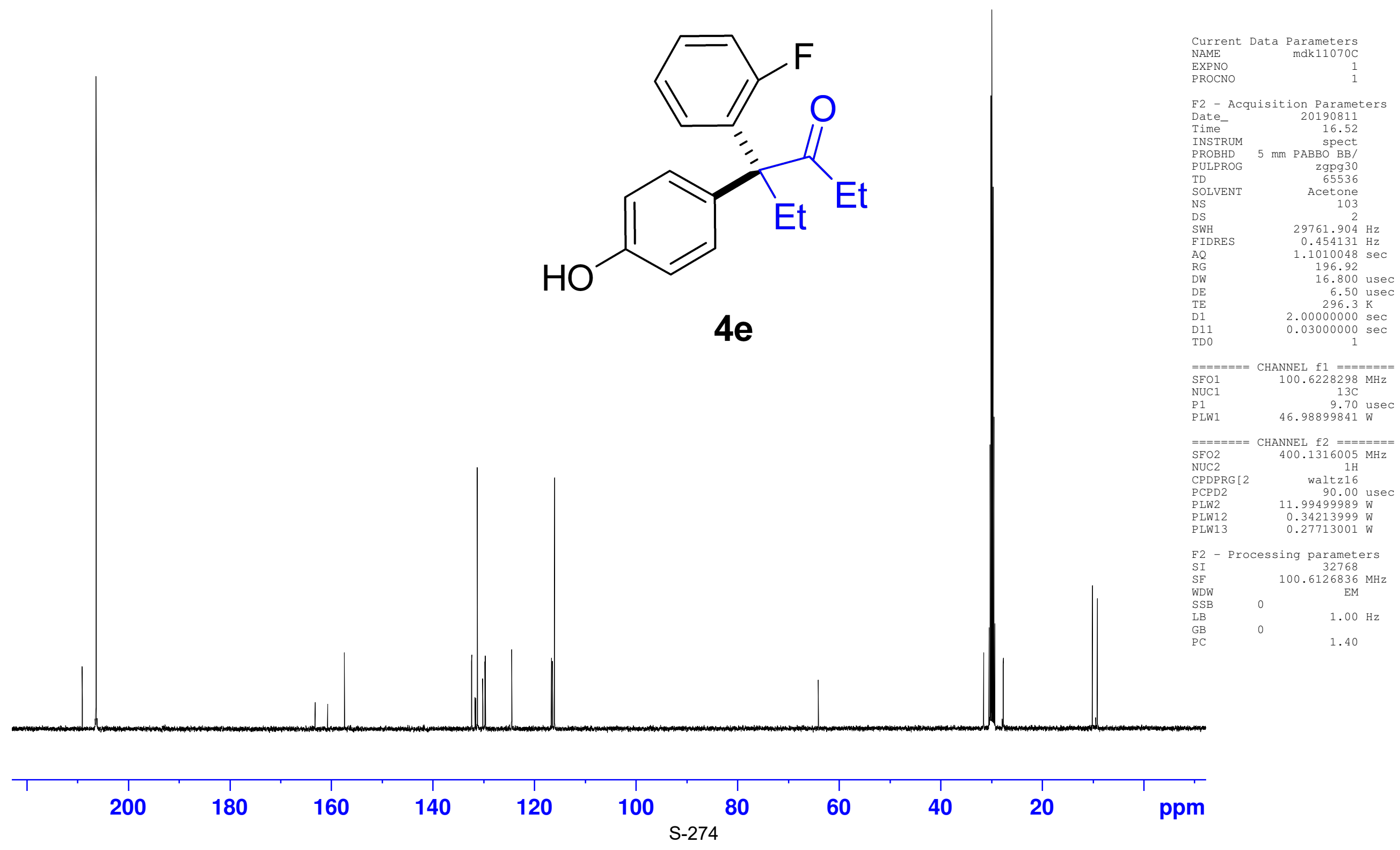




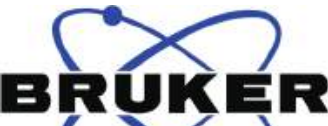 (2)}
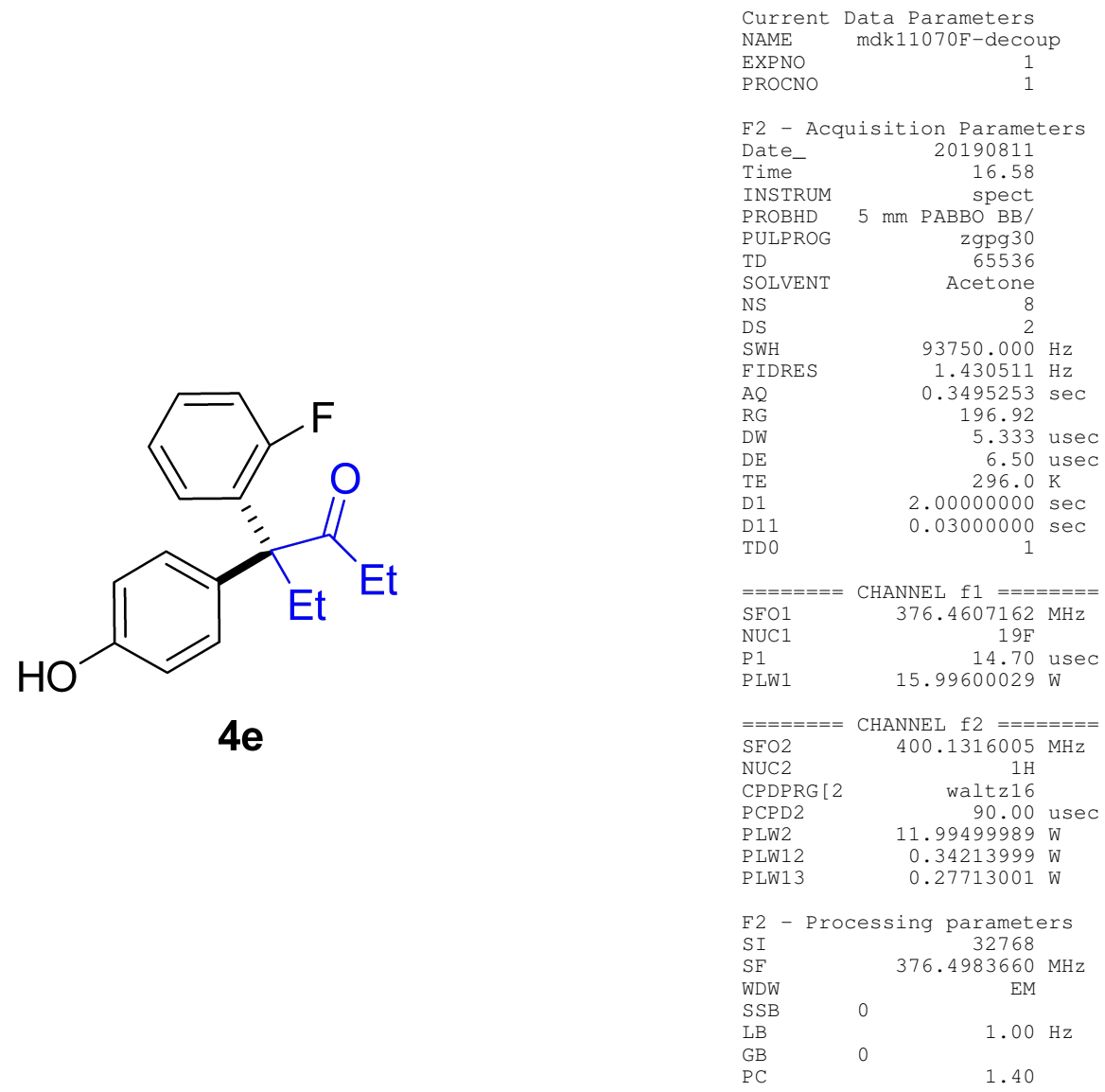

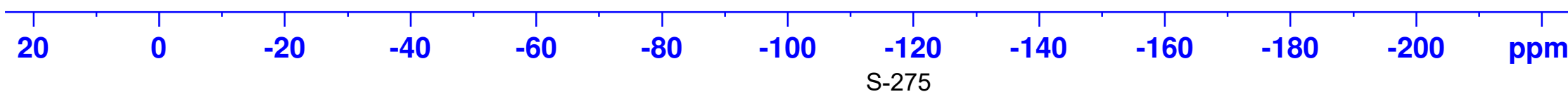




\section{sporen $(1)$}

$\begin{array}{lr}\text { Current Data } & \text { Parameters } \\ \text { NAME } & \text { mdk11065H } \\ \text { EXPNO } & 2 \\ \text { PROCNO } & 1\end{array}$<smiles>CCC(=O)[C@@](CC)(c1ccc(O)cc1)c1ccc2ccccc2c1</smiles>

$4 f$

ROCNO

F2 - Acquisition Parameters

Date__ 20190811

Time 15.12

spect

PULPROG

zg30
65536

SOLVENT Acetone

NS

SWH

FIDRES

$\mathrm{AQ}$

RG

16
16

$8012.820 \mathrm{~Hz}$

$0.122266 \mathrm{~Hz}$

$4.0894465 \mathrm{sec}$ 49.32
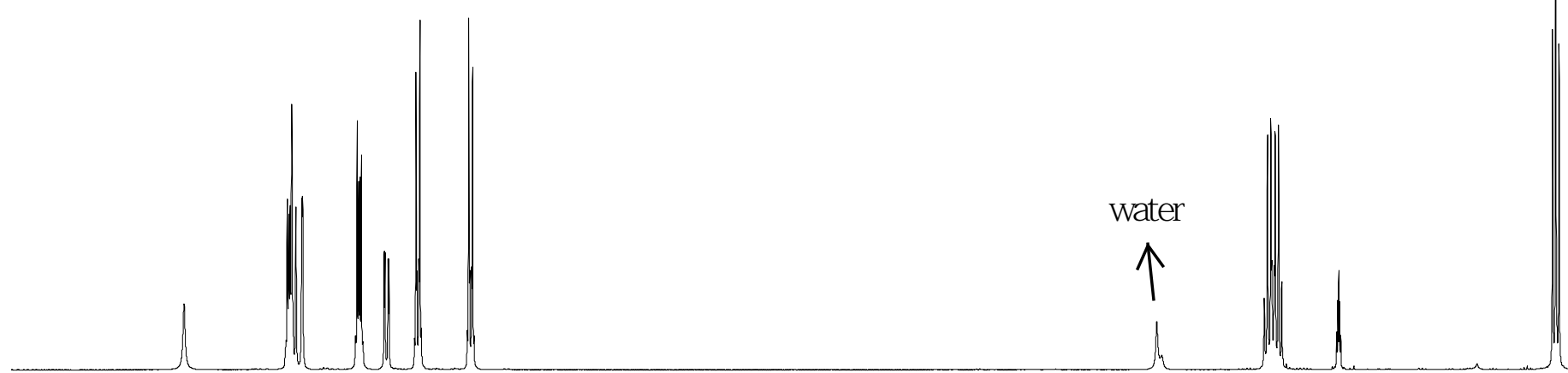

$\mathrm{DW}$
$\mathrm{DE}$
$\mathrm{TE}$

D1
TD 0

$====$

$\mathrm{SFO1}$

$\mathrm{NUC}$
$\mathrm{P} 1$

62.400 usec 6.50 usec

$1.00000000 \mathrm{sec}$

$$
1 \text { sec }
$$
$400.1324710 \mathrm{MHz}$ 14.50 usec PLW1 $11.99499989 \mathrm{~W}$

F2 - Processing parameters SI 65536 SF $400.1299909 \mathrm{MH}$ WDW EM

$0.30 \mathrm{~Hz}$

1.00

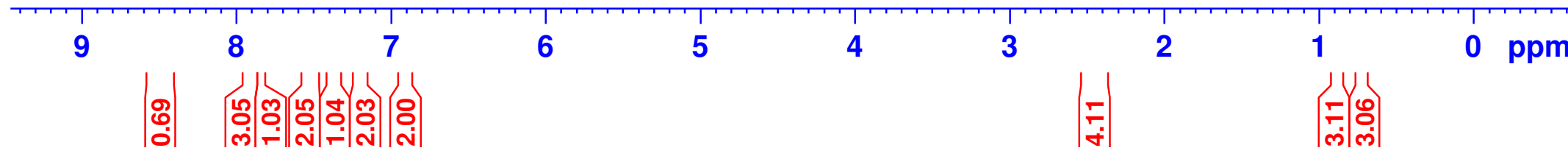



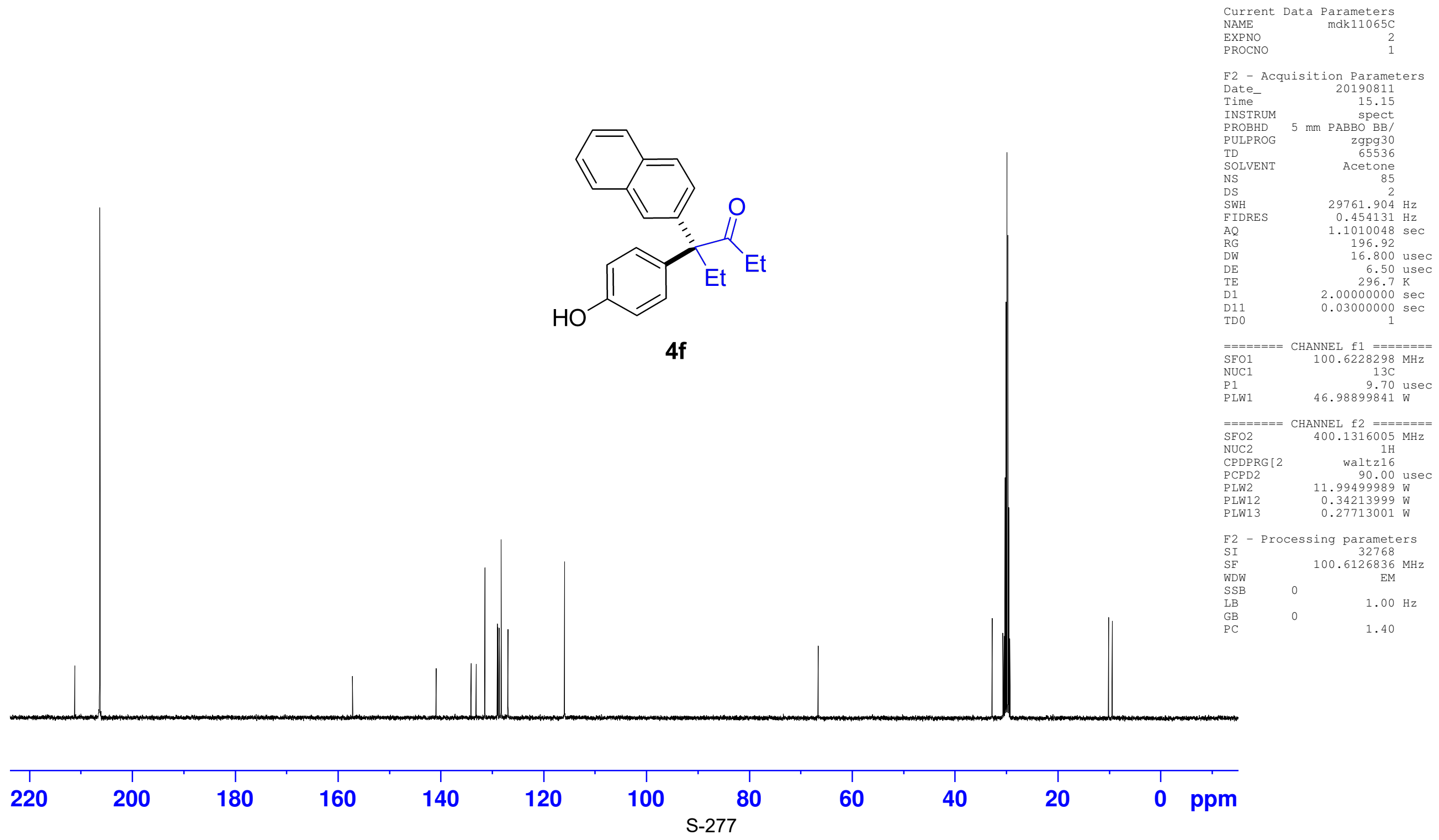


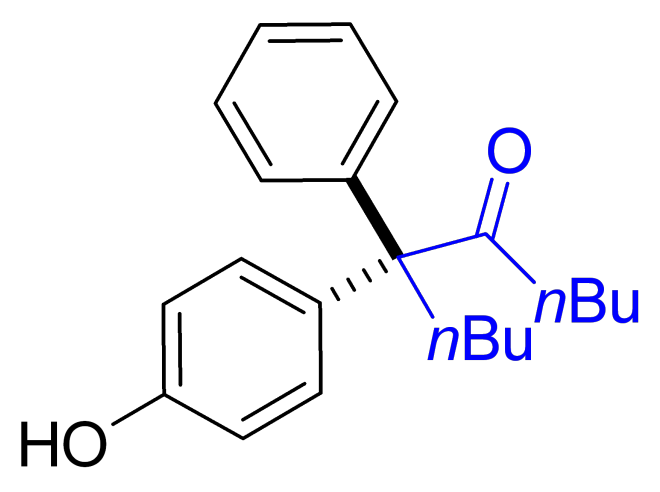

$4 \mathrm{~g}$

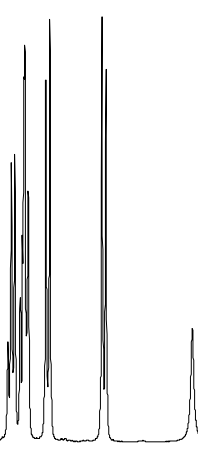

10

9

8

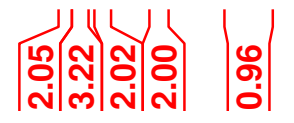

5

4

3

|ำ

ஸิ|Nิ|

2

Current Data Parameters NAME

EXPNO
PROCNO

1

F2 - Acquisition Parameters Date_ 20190413 $\begin{array}{lr}\text { Time } & 13.30\end{array}$ INSTRUM spect ROBHD $\mathrm{zg} 30$
65536 TD 65536 $\begin{array}{lr}\text { NS } & 16\end{array}$ DS

SWH $\quad 8012.820 \mathrm{~Hz}$ FIDRES $\quad 0.122266 \mathrm{~Hz}$ AQ $\quad 4.0894465 \mathrm{sec}$ RG
DW
DE
TE DE 6.50 usec
$297.7 \mathrm{~K}$ $1.00000000 \mathrm{sec}$ 


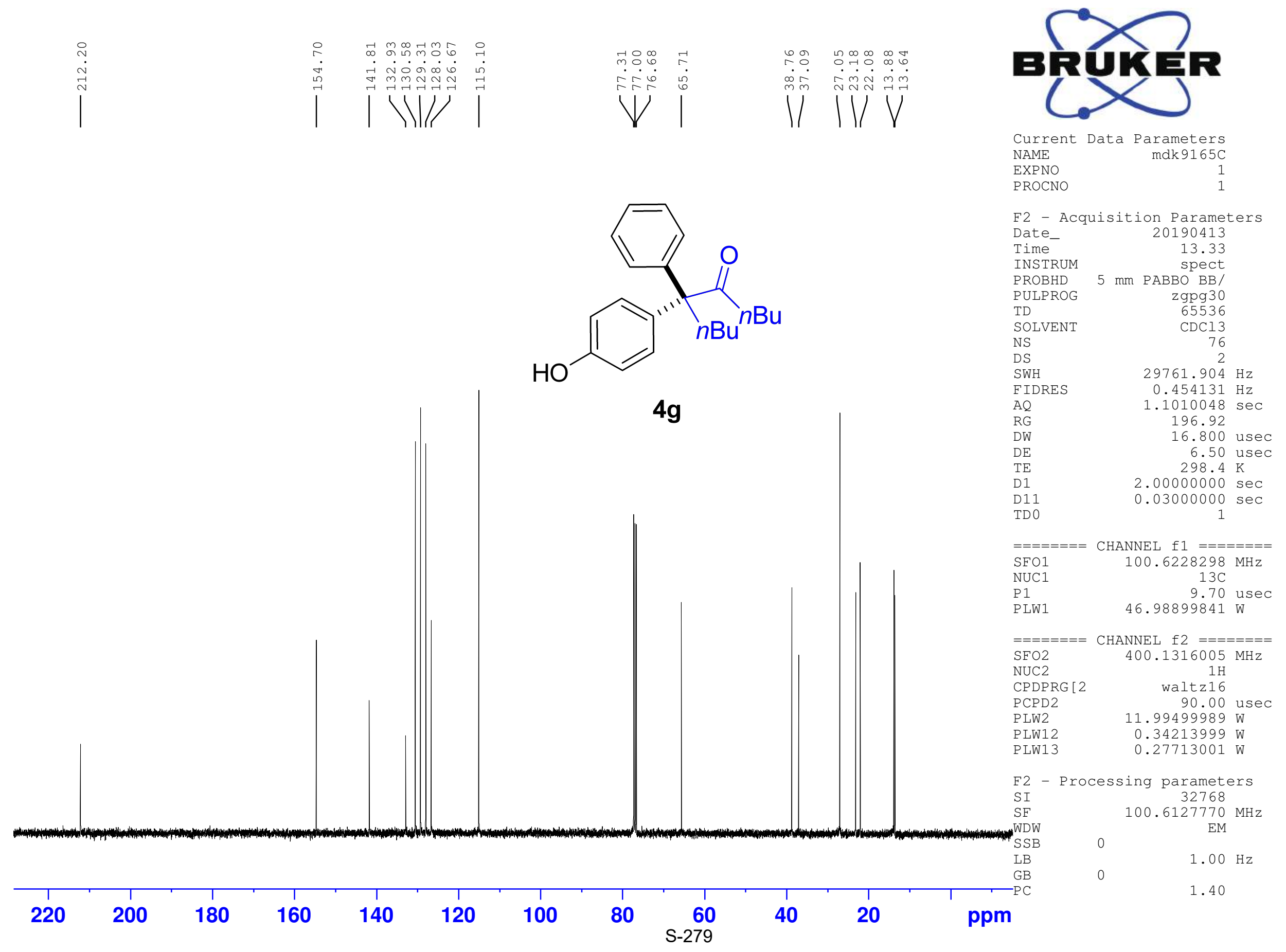



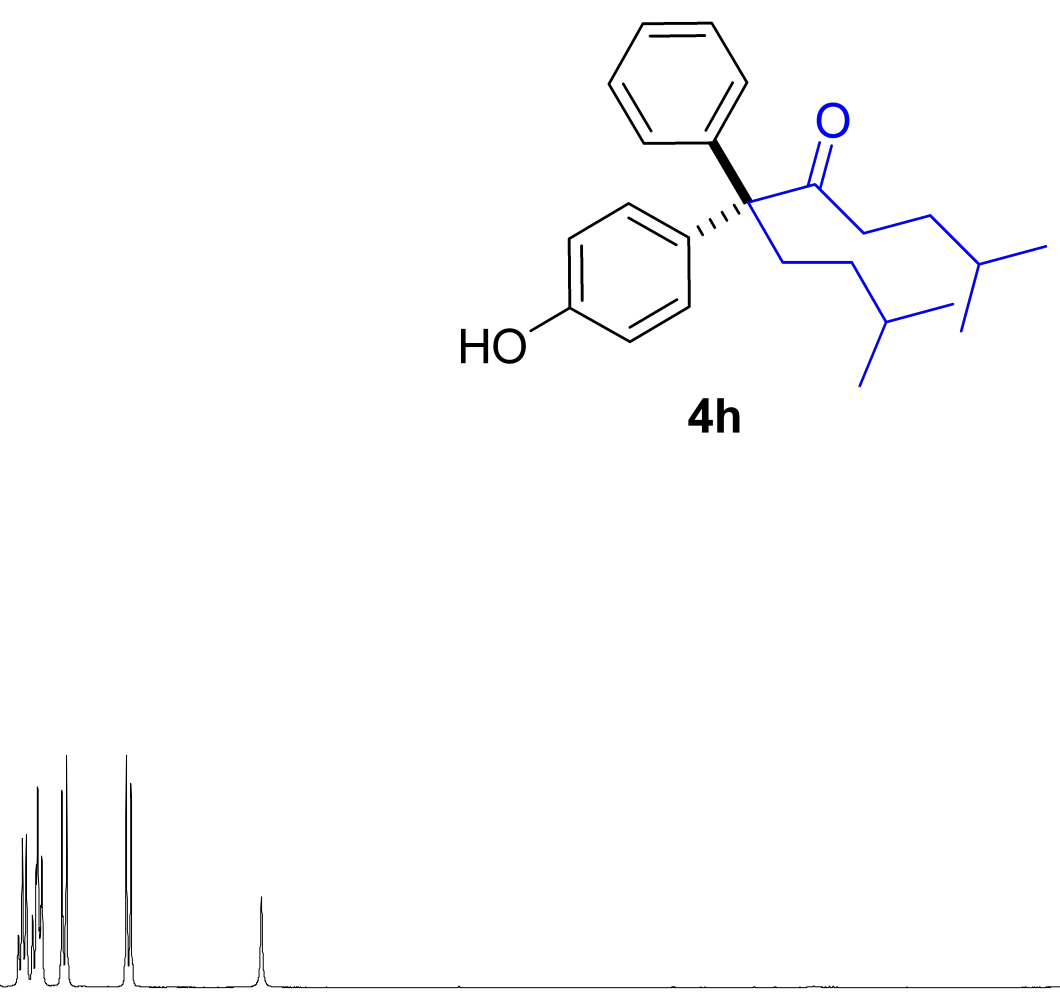

$4 h$
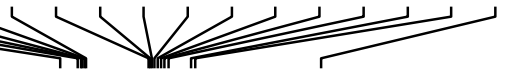

\section{eperer

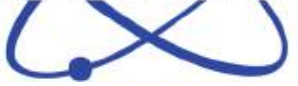
Current Data Parameters NAME $\operatorname{mdk} 11089 \mathrm{H}$
PROCNO

F2 - Acquisition Parameters

Date__ 20190815

$\begin{array}{lr}\text { Time- } & 19.17\end{array}$

spect

PUI PROG

$\mathrm{zg} 30$
$\quad 6536$

TD $\quad 65536$

SOLVENT

NS

SWH

FIDRES

$\mathrm{AQ}$

RG

DW

DE

D1
TD 0

$====$

$\mathrm{SFO1}$

$\mathrm{NUC}$
$\mathrm{P} 1$

16
2

\section{$8012.820 \mathrm{~Hz}$}

$0.122266 \mathrm{~Hz}$

$4.0894465 \mathrm{sec}$

62.40

62.400 usec

6.50 use

$1.00000000 \mathrm{sec}$

1

$1 \mathrm{sec}$

$400.1324710 \mathrm{MHz}$

$11.99499989 \mathrm{~W}$
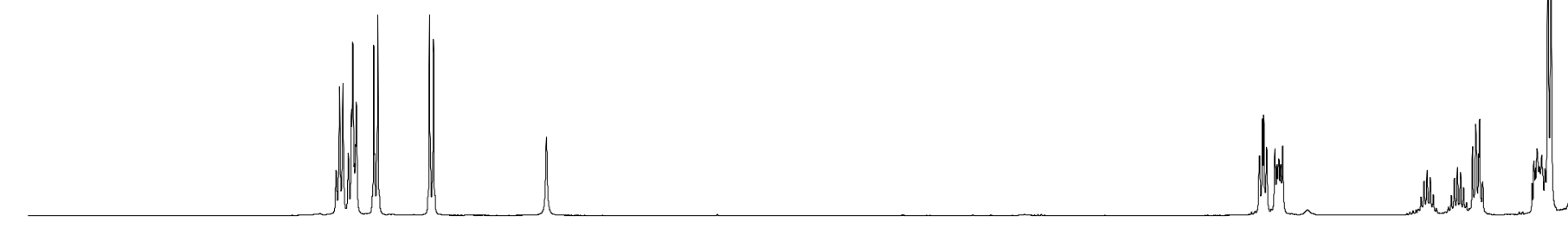

$\begin{array}{lcc}\text { F2 - Processing parameters } \\ \text { SI } & 65536 \\ \text { SF } & 400.1300120 \mathrm{MHz} \\ \text { WDW } & 0 & \text { EM } \\ \text { SSB } & 0 & 0.30 \mathrm{~Hz} \\ \text { LB } & 0 & \\ \text { GB } & 0 & 1.00\end{array}$

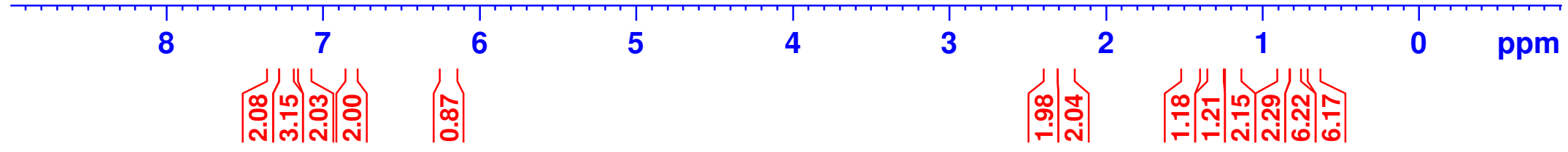




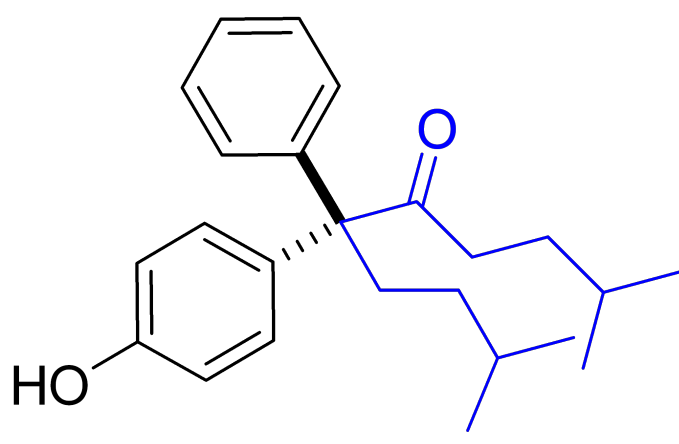

4h
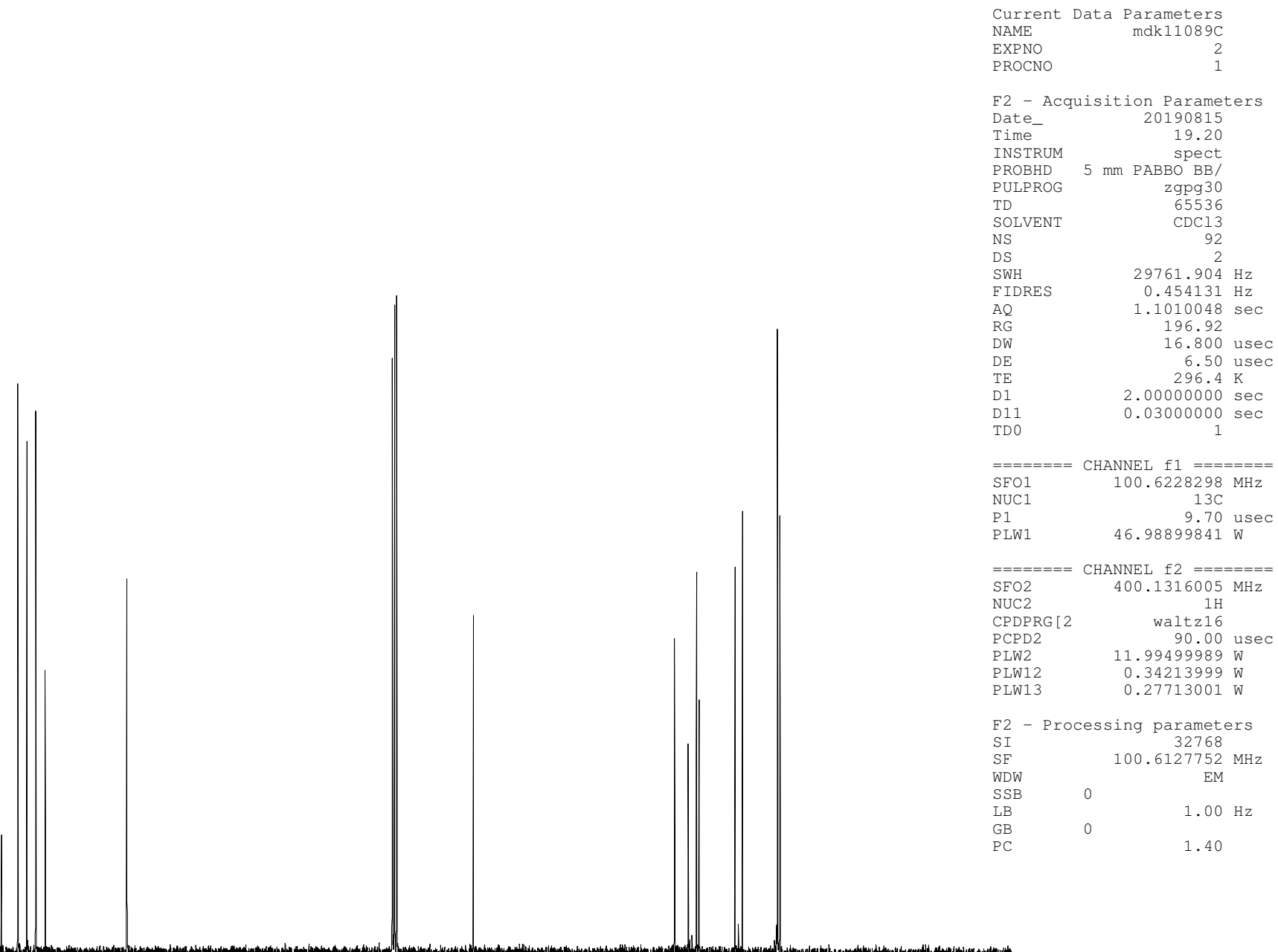


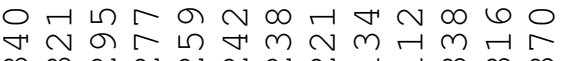
$m m \sim \sim \sim \sim \sim \sim \sim \infty \infty \infty$ arrararab I

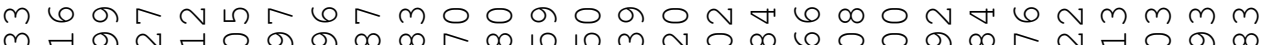

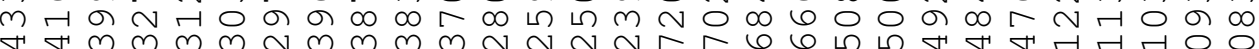

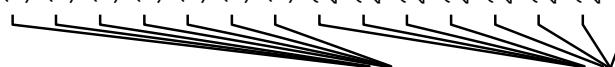
$m m m m m$. . . . . . . . . . . . .

\section{epengen $(1)$}

Current Data Parameters NAME

PROCNO

$\operatorname{mdk} 10019 \mathrm{H}$

F2 - Acquisition Parameters

Date_ 20190510

Time 13.44

$5 \mathrm{~mm}$ spect

PUI PROG

$\mathrm{Zg} 30$
65536

TD $\quad 65536$

NS

SWH

FIDRES

$\mathrm{AQ}$

$4 \mathbf{i}$

Cl

$\mathrm{RG}$

DW

$\mathrm{DE}$

D1

TD 0

$====$
SFO1
NUC1

$\mathrm{NUC}$

P1
PLW1

16
2

$8012.820 \mathrm{~Hz}$

$0.122266 \mathrm{~Hz}$

$4.0894465 \mathrm{sec}$ 31.55

62.400 usec 6.50 usec

$1.00000000 \mathrm{sec}$

CHANNET $f 1=======$ $400.1324710 \mathrm{MHz}$ 14.50 sec

F2 - Processing parameters SI $\quad 65536$ SF $\quad 400.1300105 \mathrm{MHz}$

$\mathrm{LB}$
$\mathrm{GB}$ PC

$0.30 \mathrm{~Hz}$

1.00

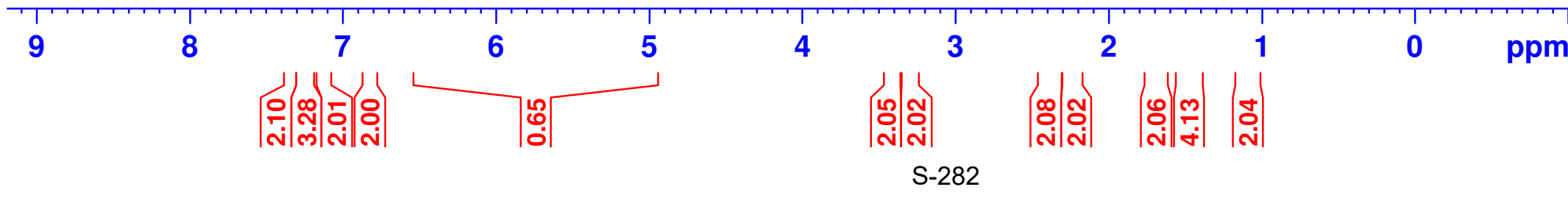




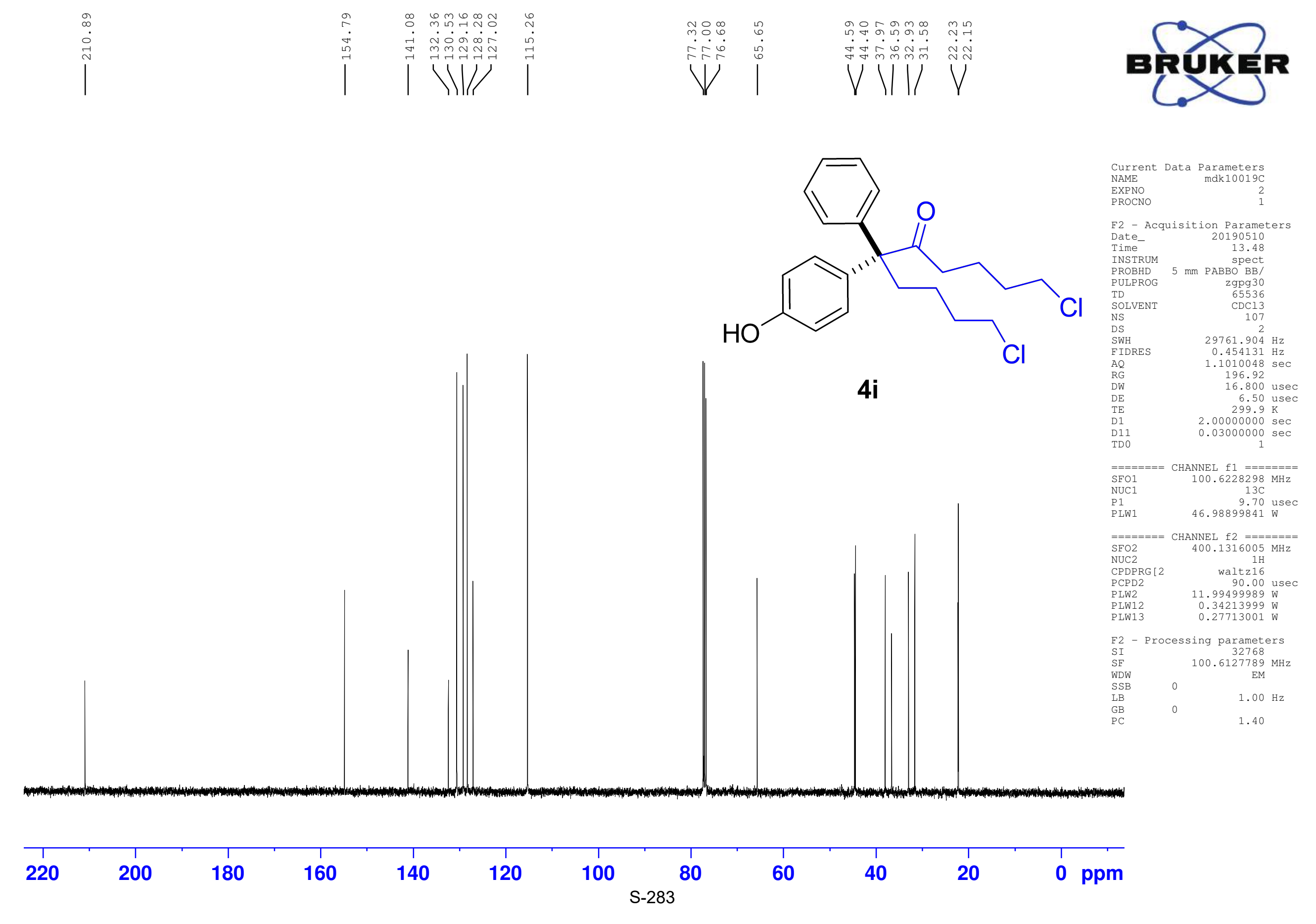



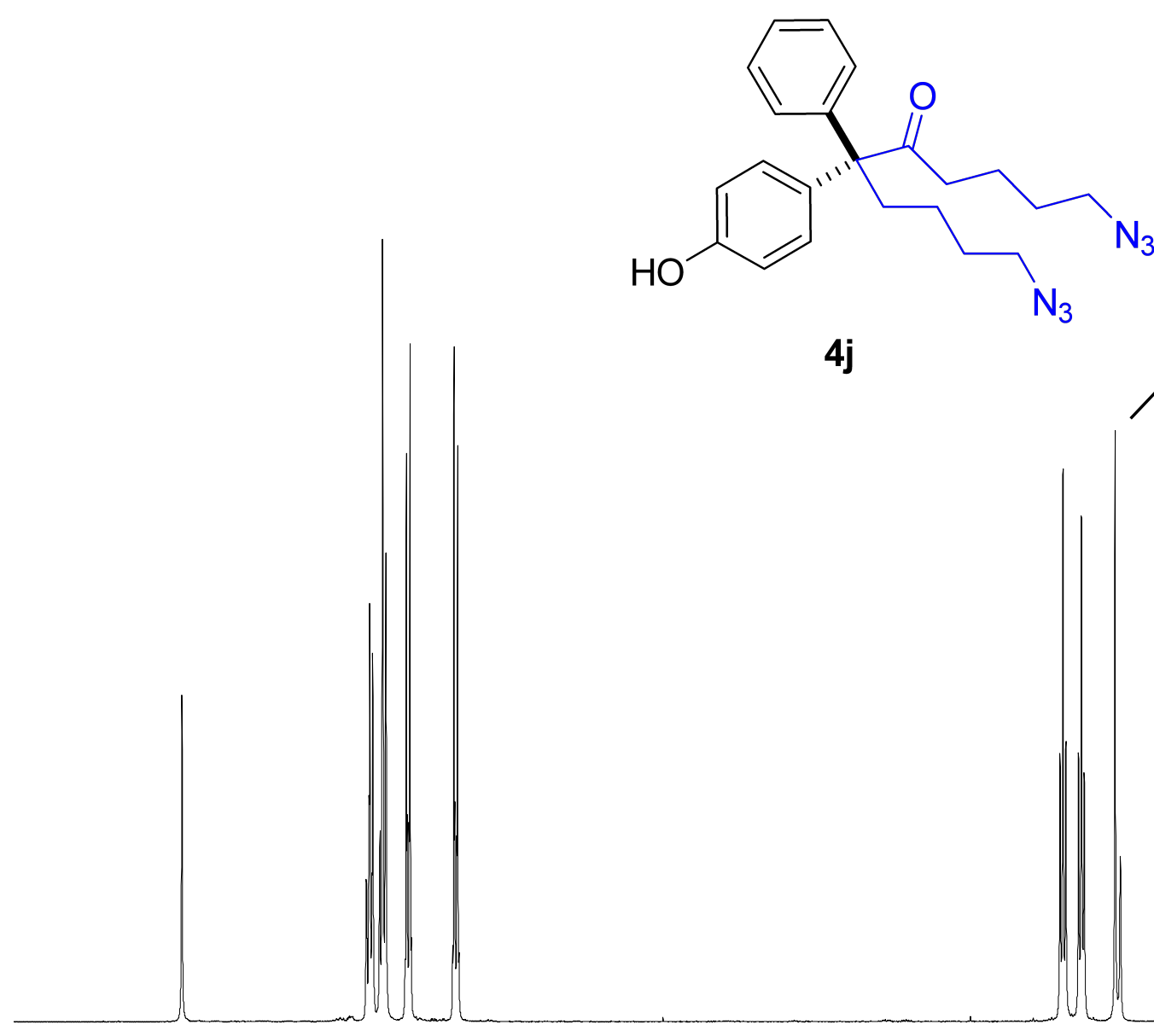

4j

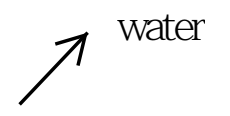

\section{siner

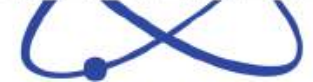

$\begin{array}{lr}\text { Current Data } & \text { Parameters } \\ \text { NAME } & \text { mdk11086 } \\ \text { EXPNO } & 1 \\ \text { PROCNO } & 1\end{array}$

F2 - Acquisition Parameters

Date__ 20190815

Time 14.56

spect

PULPROG

$\begin{array}{lr}\text { TD } & \text { Zg30 } \\ \text { TD } & 65536\end{array}$

SOLVENT Acetone

DS

SWH

FIDRES

$\mathrm{AQ}$

RG

DW

$\mathrm{DE}$

D1
TD 0

$===$

$\mathrm{SFO1}$

NUC

P 1
PLW1

16
16

$8012.820 \mathrm{~Hz}$

$0.122266 \mathrm{~Hz}$

$4.0894465 \mathrm{sec}$

54.81

62.400 usec

6.50 usec

$1.00000000 \mathrm{sec}$

CHANNEL $\mathrm{f} 1========$

$400.1324710 \mathrm{MHz}$

LW1 $\quad 14.50$ usec

F2 - Processing paraneters

SI Processing parameters

SF

WDW

SSB

$400.1299910 \mathrm{MHz}$
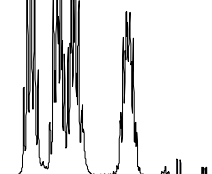

EM

$0.30 \mathrm{~Hz}$

1.00

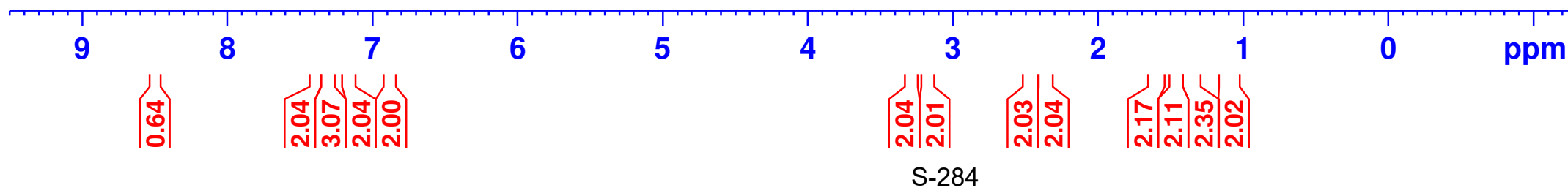




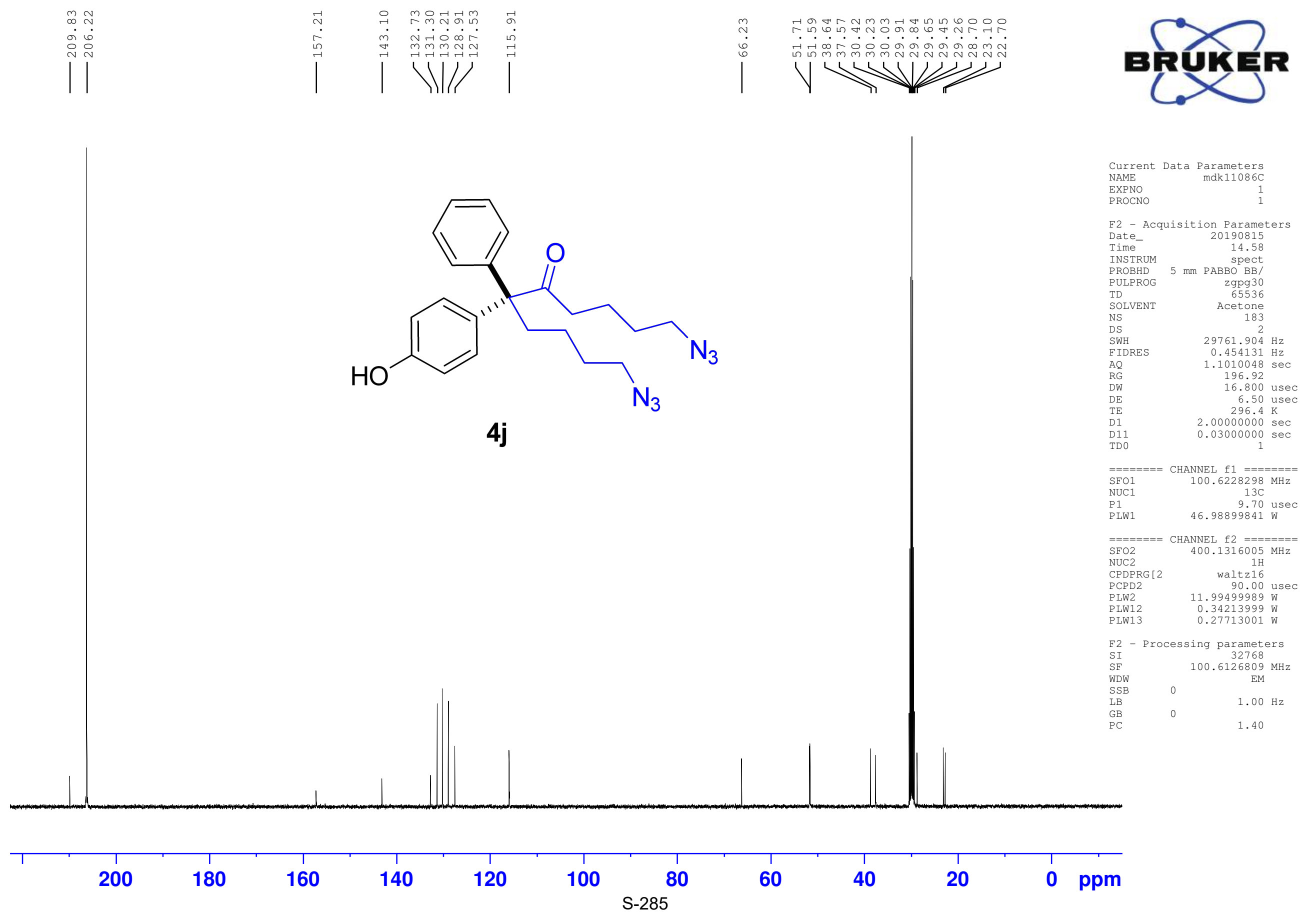




\section{a

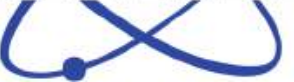

$\begin{array}{lr}\text { Current Data } & \text { Parameters } \\ \text { NAME } & \text { mdk11091H } \\ \text { EXPNO } & 1 \\ \text { PROCNO } & 1\end{array}$

F2 - Acquisition Parameters

Date_ 20190815

Time $\quad 19.26$

$5 \mathrm{~mm}$ spect

$\mathrm{zg} 30$
65536

$\begin{array}{ll}\text { TD } & 65536 \\ \text { SOLVENT } & \text { CDC13 }\end{array}$

NS

DS

FIDRES

$\mathrm{AQ}$

RG

DW

$\mathrm{DE}$

D1
TD 0

$====$

$\mathrm{SFO1}$

NUC

P1
PLW1

16
2

$8012.820 \mathrm{~Hz}$

$8012.820 \mathrm{~Hz}$

$4.0894465 \mathrm{~Hz}$

45.67

62.400 usec 6.50 usec

$1.00000000 \mathrm{sec}$ 1

CHANNEL $\mathrm{fl}========$ $400.1324710 \mathrm{MHz}$ $1 \mathrm{H}$
14.50 sec

F2 - Processing parameters SI 65536 SF $\quad 400.1300115 \mathrm{MHz}$ WDW

SSB EM

$0.30 \mathrm{~Hz}$

1.00
8 7 สำㅇㅇㅇ잉

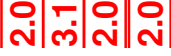

$6 \quad 5$

5

$\left|\begin{array}{l}0 \\ 0 \\ \text { m. }\end{array}\right|$
().
3

3

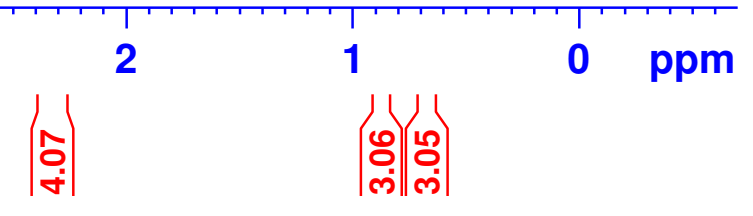




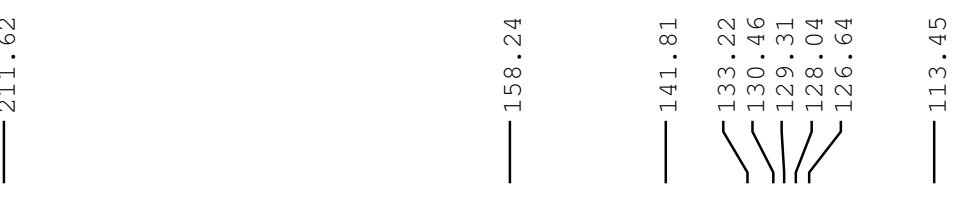

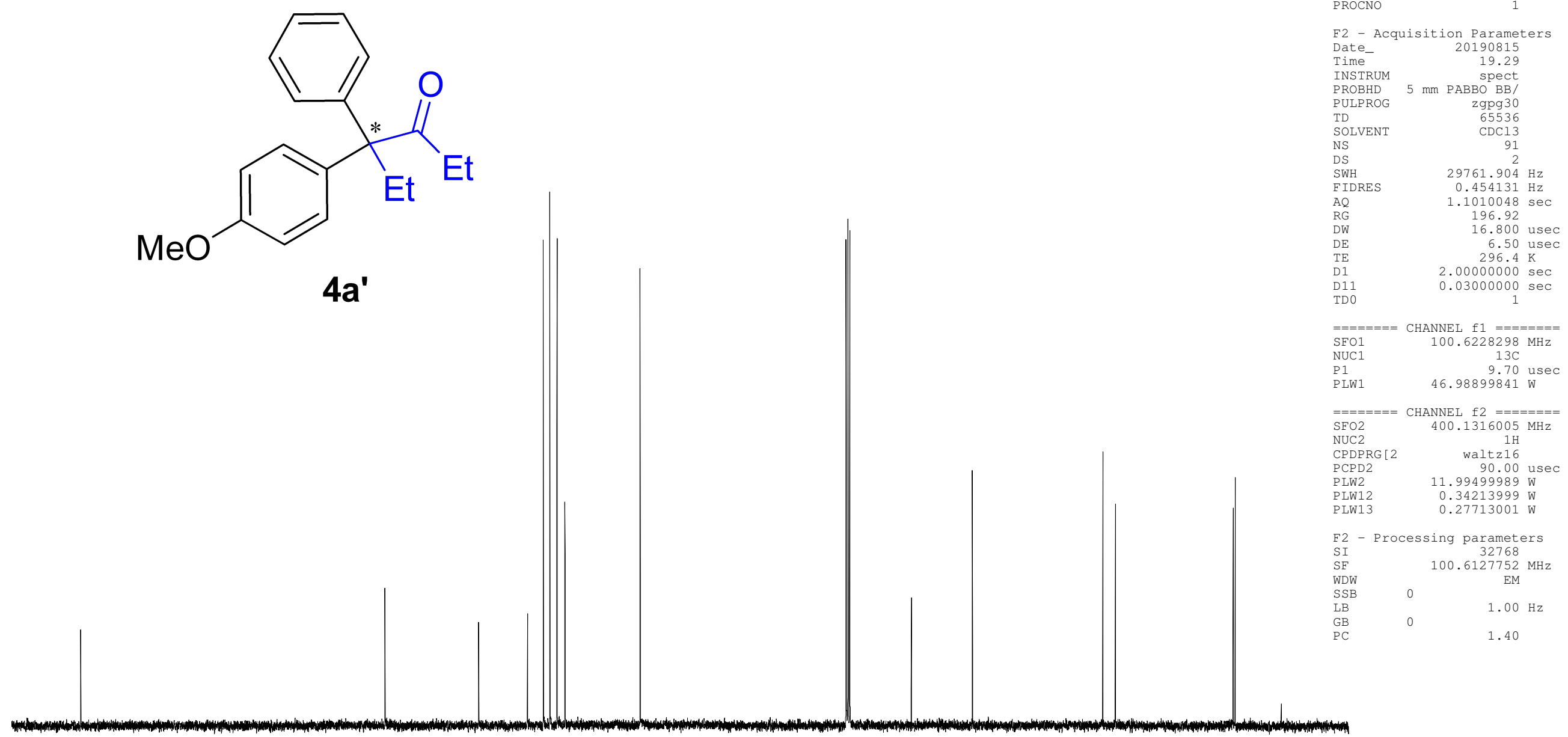

Current Data Parameters
NAME EXPNO
PROCNO

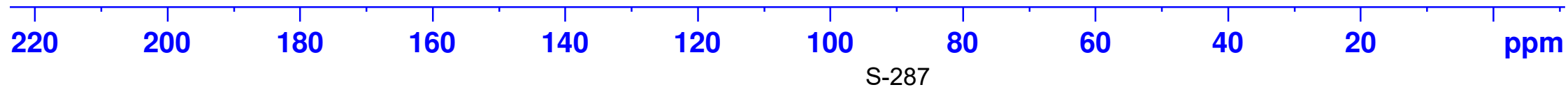



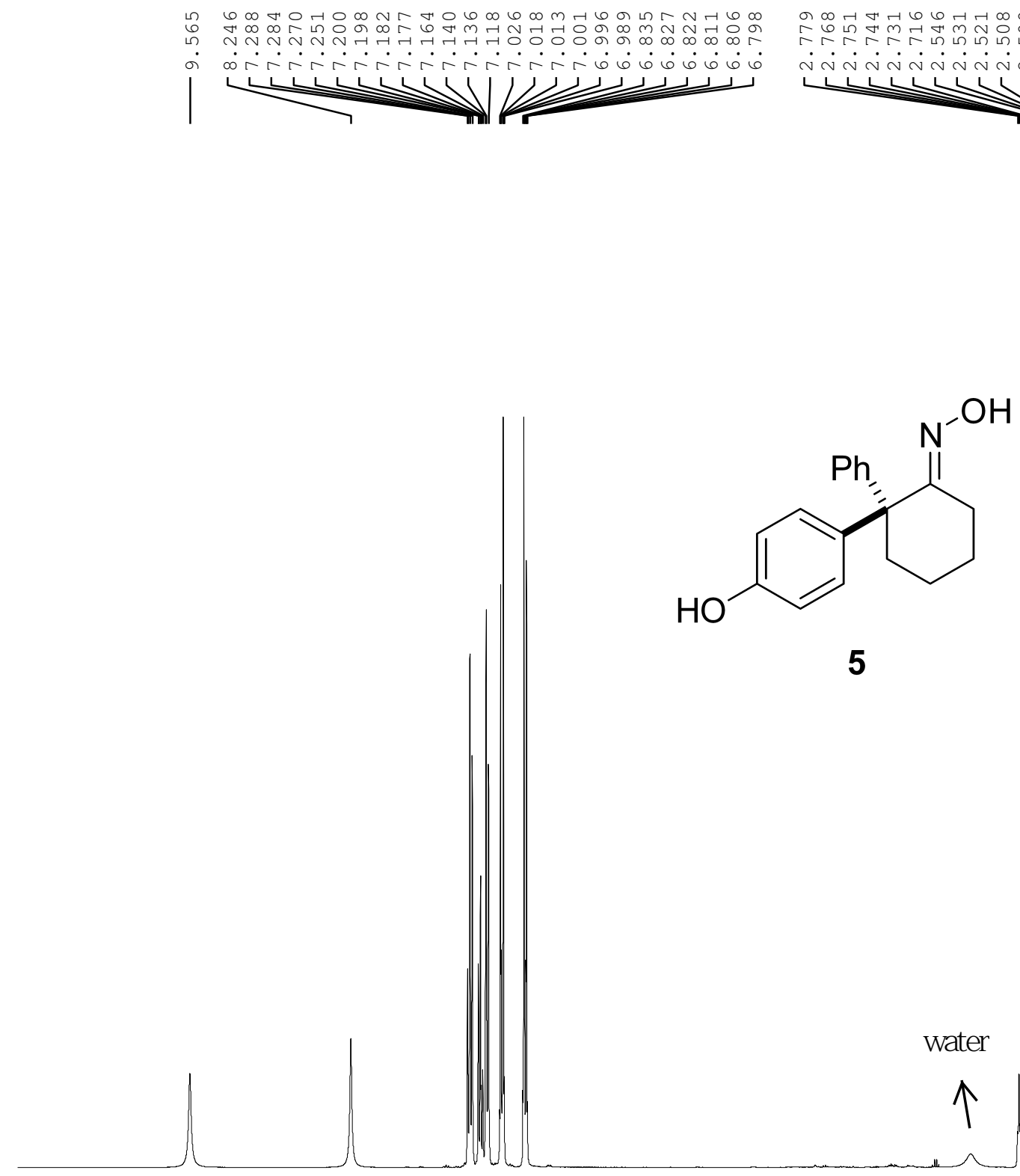

\section{BR UKE

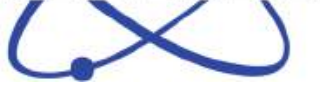

Current Data Parameters NAME mak10028H EXPNO

ROCNO

F2 - Acquisition Parameters

Date_ 20190510

Time $\quad 13.30$

SPeCt
PROBHD $5 \mathrm{~mm} \mathrm{PABBO} \mathrm{BB} /$

PROBHD $5 \mathrm{~mm} \mathrm{PABBO}$ BB/
PULPROG

$\begin{array}{lr}\text { PULPROG } & \text { zg30 } \\ \text { TD } & 65536\end{array}$

SOLVENT

NS

DS
SWH

SWH $\quad 8012.820 \mathrm{~Hz}$

FIDRES $\quad 0.122266 \mathrm{~Hz}$

$\mathrm{AQ} \quad 4.0894465 \mathrm{sec}$

RG $\quad 34.77$

$\begin{array}{lr}\text { DW } & 62.400 \text { usec } \\ \text { DE } & 6.50 \text { usec }\end{array}$

TE $\quad 300.5 \mathrm{~K}$

D1 $\quad 1.00000000 \mathrm{sec}$

$\begin{array}{lr}======= & \text { CHANNEL } \mathrm{fl}======= \\ \text { SFO1 } & 400.1324710 \mathrm{MHz} \\ \text { NUC1 } & 1 \mathrm{H} \\ \text { P1 } & 14.50 \mathrm{usec}\end{array}$

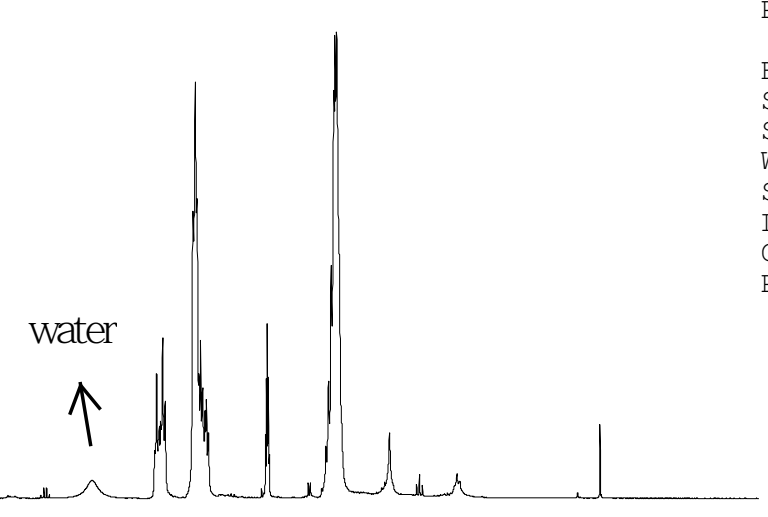

PLW1 11.99499989 .W

F2 - Processing parameters

SI 65536

SF $\quad 400.1299912 \mathrm{MHz}$

WDW

$0.30 \mathrm{~Hz}$

GB

1.00

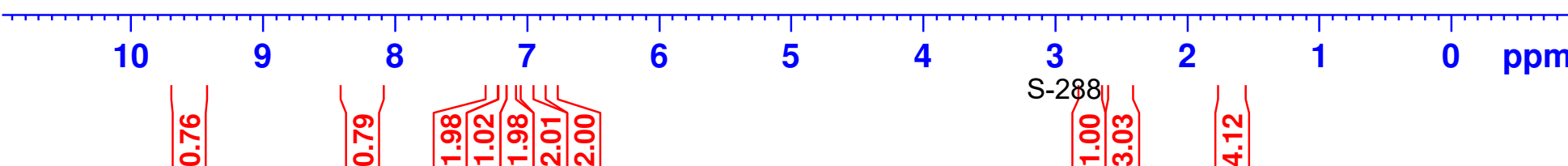




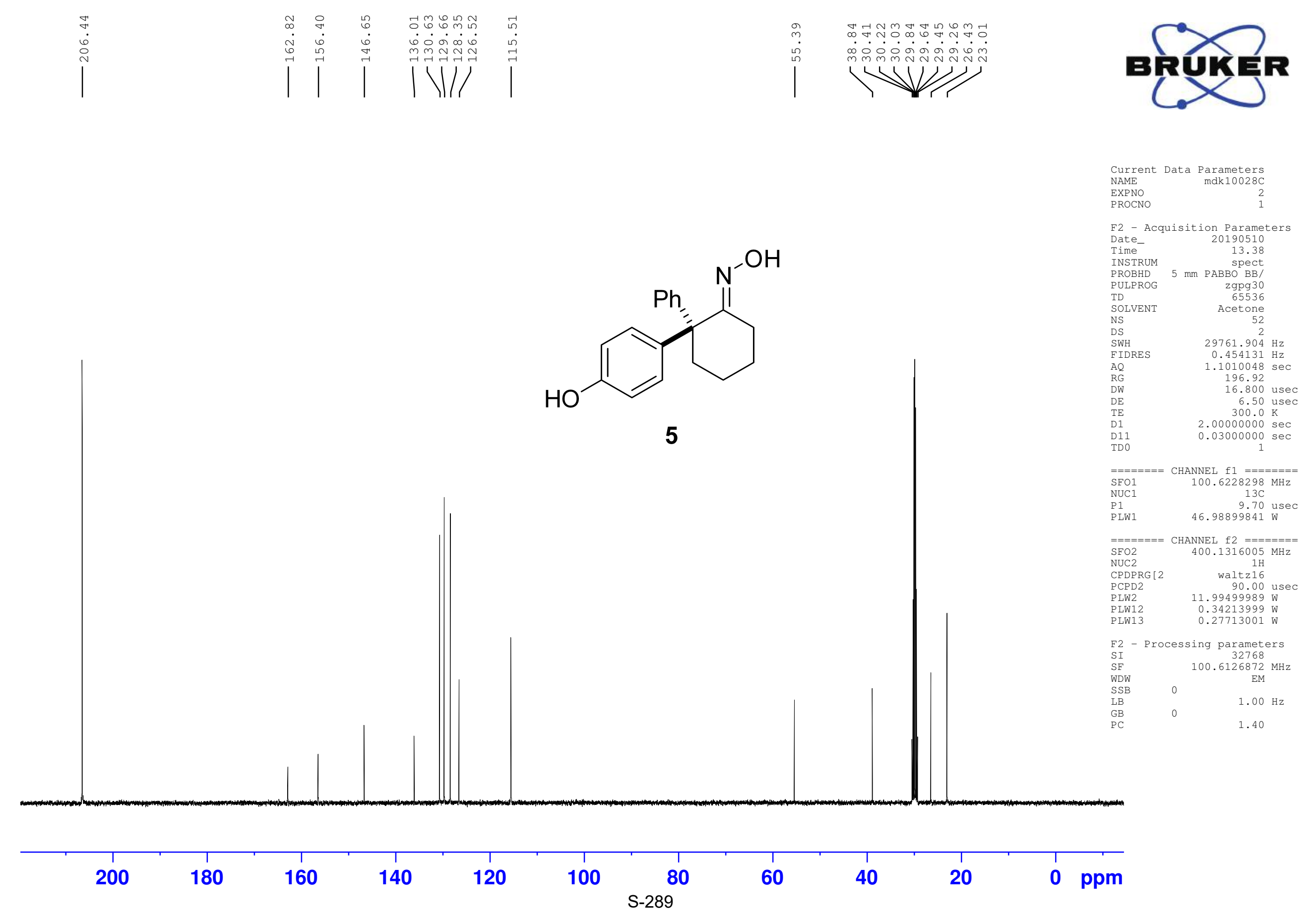


.
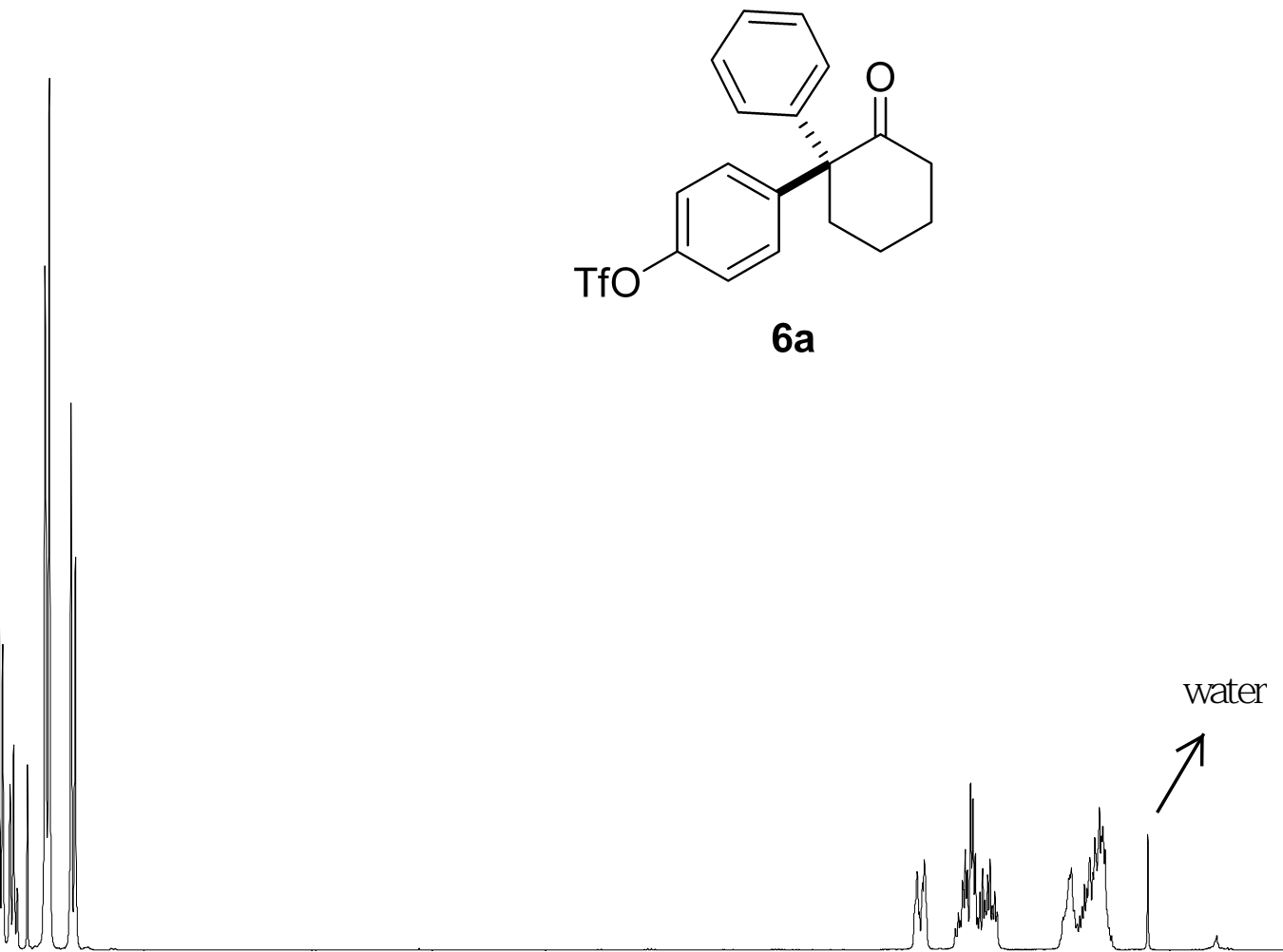

5

4

3

2

ㅇํㅇㅇำ

6

|웜| 음

일.

1

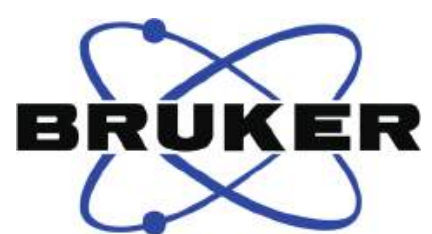

Current Data Parameters NAME

PROCNO

$\operatorname{mdk} 10046 \mathrm{H}$

F2 - Acquisition Parameters

Date__ 20190521

Time 14.10

spect

PULPROG $\quad$ zg30

TD 65536

SOLVENT CDCl3

NS

DS

$80122266 \mathrm{~Hz}$
IDRES

AQ

DW

$\mathrm{DE}$

$\mathrm{TE}$

TD 0

$====$

NUC1

$\mathrm{NUC1}$
$\mathrm{P} 1$

$.0894465 \mathrm{sec}$
27.78

62.400 usec

6.50 usec

$1.00000000 \mathrm{~K}$$$
1
$$

CHANNEL $\mathrm{f} 1========$ $400.1324710 \mathrm{MHz}$ 14.50 $11.99499989 \mathrm{~W}$

$\begin{array}{lcc}\text { F2 } & \text { Processing parameters } \\ \text { SI } & 65536 \\ \text { SF } & 400.1300134 \mathrm{MHz} \\ \text { WDW } & 0 & \text { EM } \\ \text { SSB } & 0 & 0.30 \mathrm{~Hz} \\ \text { LB } & 0 & 1.00 \\ \text { GB } & 0 & \end{array}$ 


\section{BR UKE}

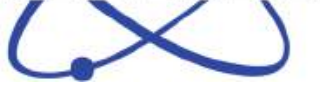

Current Data Parameters NAME

EXPNO

$\operatorname{mdk} 10046 \mathrm{C}$

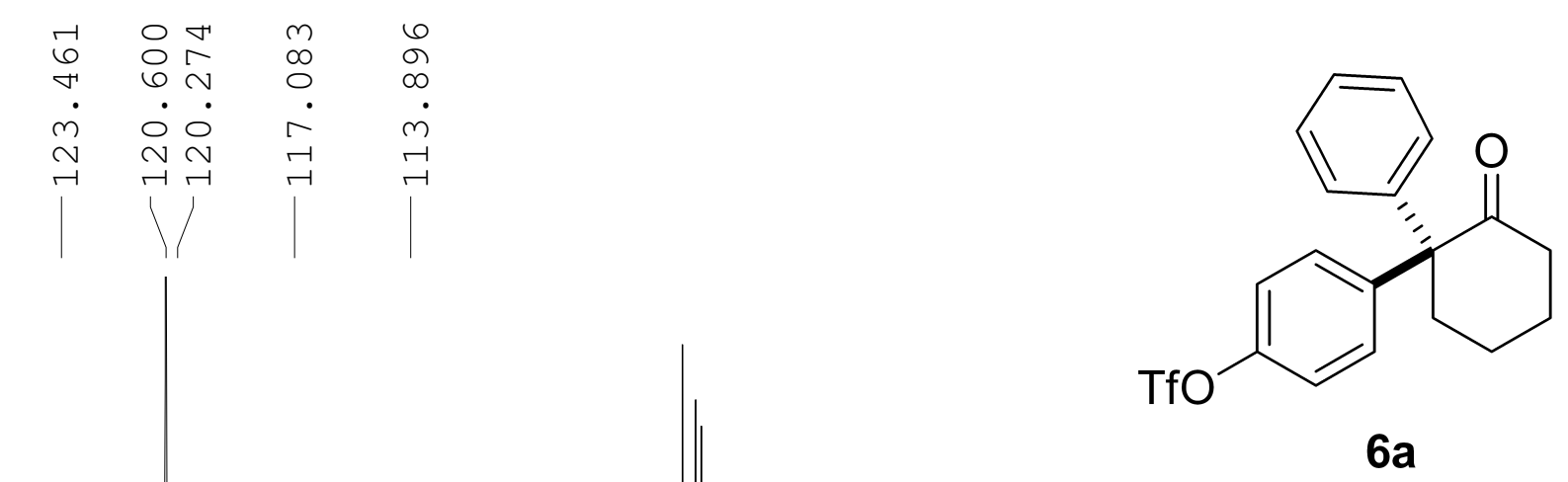

F2 - Acquisition Parameters

Date_ 20190521

Time $\quad 14.13$

INSIRUM 5 SPECt

PROBHD $5 \mathrm{~mm}$ PABBO BB/

PULPROG zgpg30

TD 65536

SOLVENT CDCl3

NS

DS

SWH $\quad 29761.904 \mathrm{~Hz}$

FIDRES $\quad 0.454131 \mathrm{~Hz}$

$\mathrm{AQ} \quad 1.1010048 \mathrm{sec}$

RG $\quad 196.92$

$\begin{array}{lr}\mathrm{DW} & 16.800 \text { usec } \\ \mathrm{DE} & 6.50 \mathrm{usec}\end{array}$

TE $\quad 298.1 \mathrm{~K}$

D1 $2.00000000 \mathrm{sec}$

D11 $0.03000000 \mathrm{sec}$
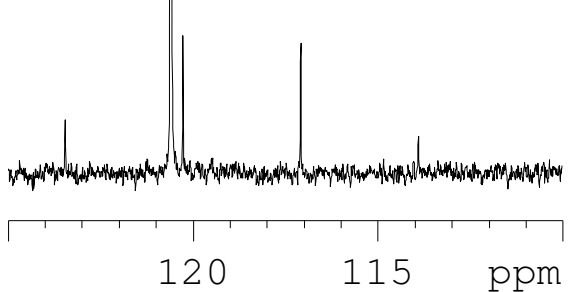

pm

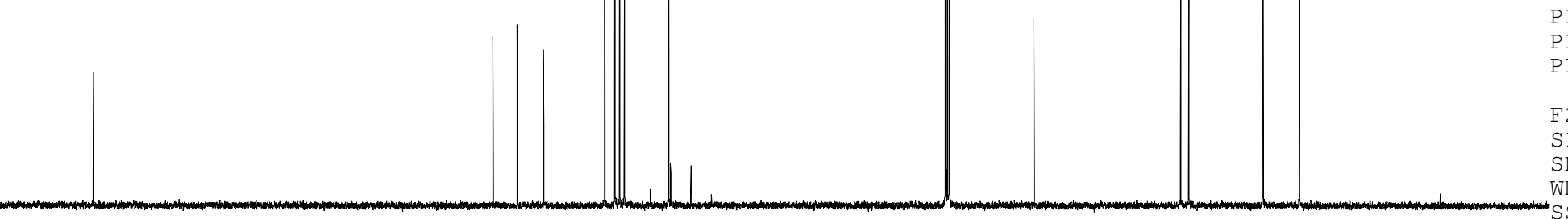

$=======0$ CHANNEL f $1 \quad========$

$100.6228298 \mathrm{MHz}$

NUC1 $13 \mathrm{C}$

P1 9.70 usec

PLW1 $46.98899841 \mathrm{~W}$

$=======$ CHANNEL $\mathrm{f} 2 \mathrm{2}=======$

$\mathrm{SFO} 2$

$1 \mathrm{H}$

waltz16

PCPD2

$\mathrm{PLW} 12$

$11.99499989 \mathrm{~W}$

$11.99499989 \mathrm{~W}$
$0.34213999 \mathrm{~W}$

F2 - Processing parameters

$\begin{array}{lr}\text { SI } & 32768 \\ \text { SF } & 100.6127743 \mathrm{MHz}\end{array}$ WDW

$100.6127743 \mathrm{MHz}$
EM

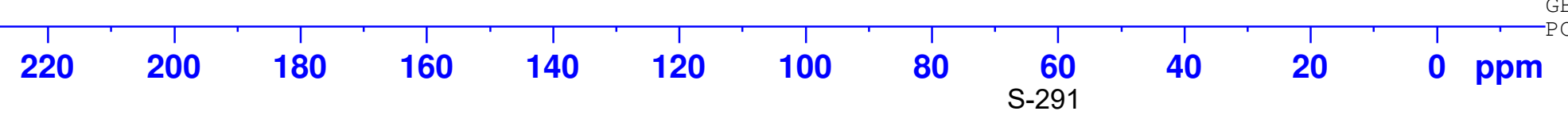

$1.00 \mathrm{~Hz}$

1.40 


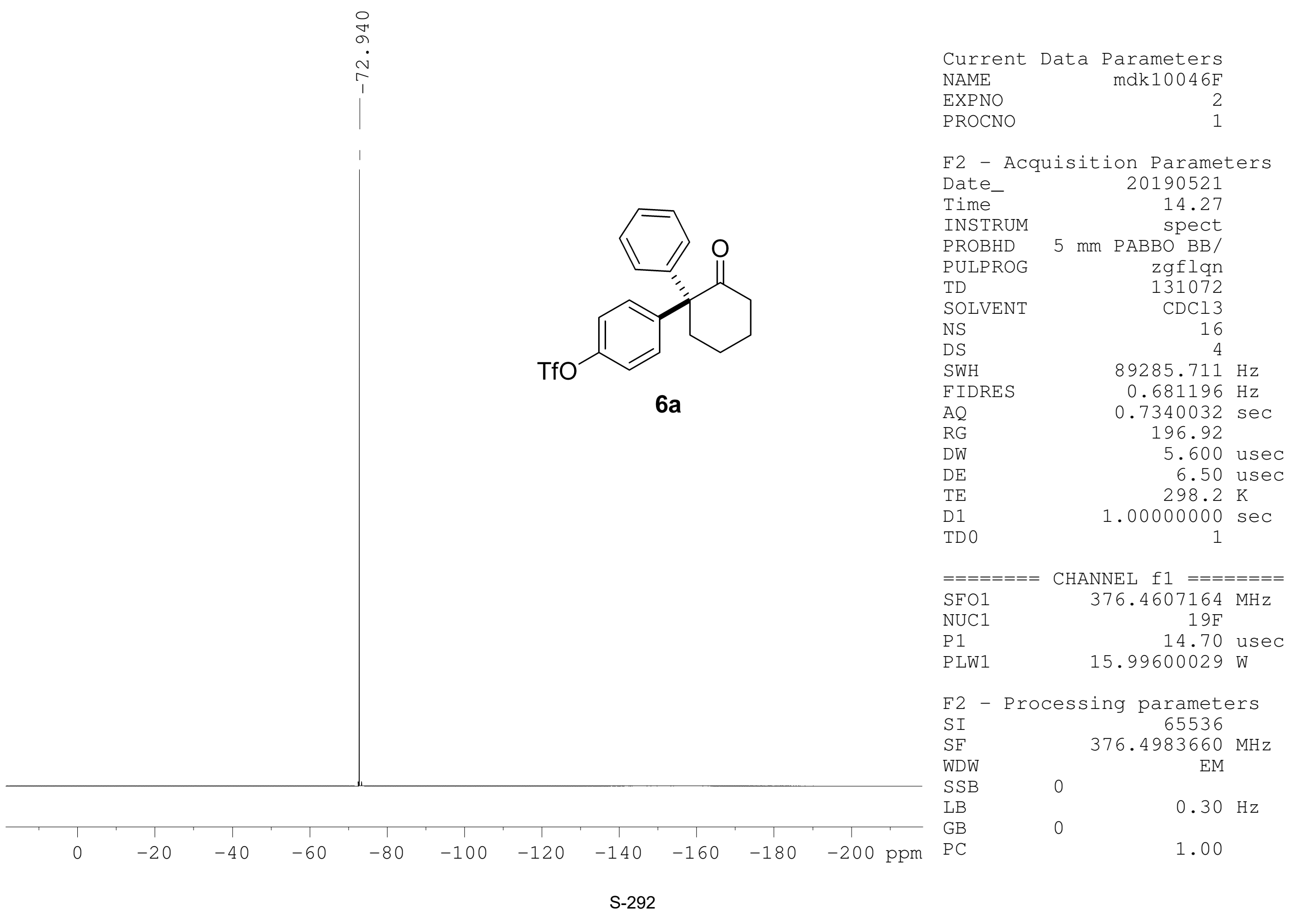




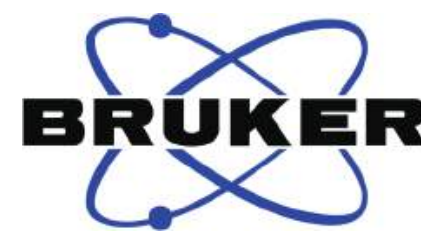

Curren

NAME

PROCNO

arameters

$\operatorname{mdk} 10110 \mathrm{H}$

F2 - Acquisition Parameters

Date__ 20190615

Time 13.08<smiles>CCOc1ccc([C@]2(c3ccc(Cl)cc3)CCCCC2=O)cc1</smiles>

$\begin{array}{lr}\text { PULPROG } & \text { zg30 } \\ & 65536\end{array}$

$5 \mathrm{~mm}$ PABBO $\mathrm{BB} /$

TD $\quad 65536$

DS

SWH

FIDRES

FIDR
AQ

AQ

$\mathrm{DW}$

DE

TE

TD 0

$===$

$\mathrm{SFO1}$

$\mathrm{NUC1}$
$\mathrm{P} 1$

P 1
PLW1

CHANNEL $\mathrm{f} 1==$ $400.1324710 \mathrm{MHz}$ 14.50 $11.99499989 \mathrm{~W}$ 


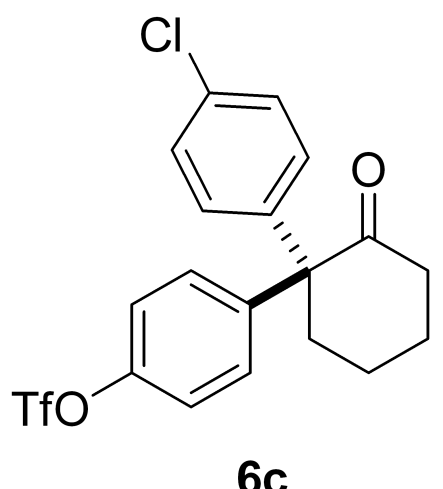

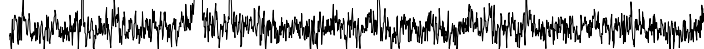
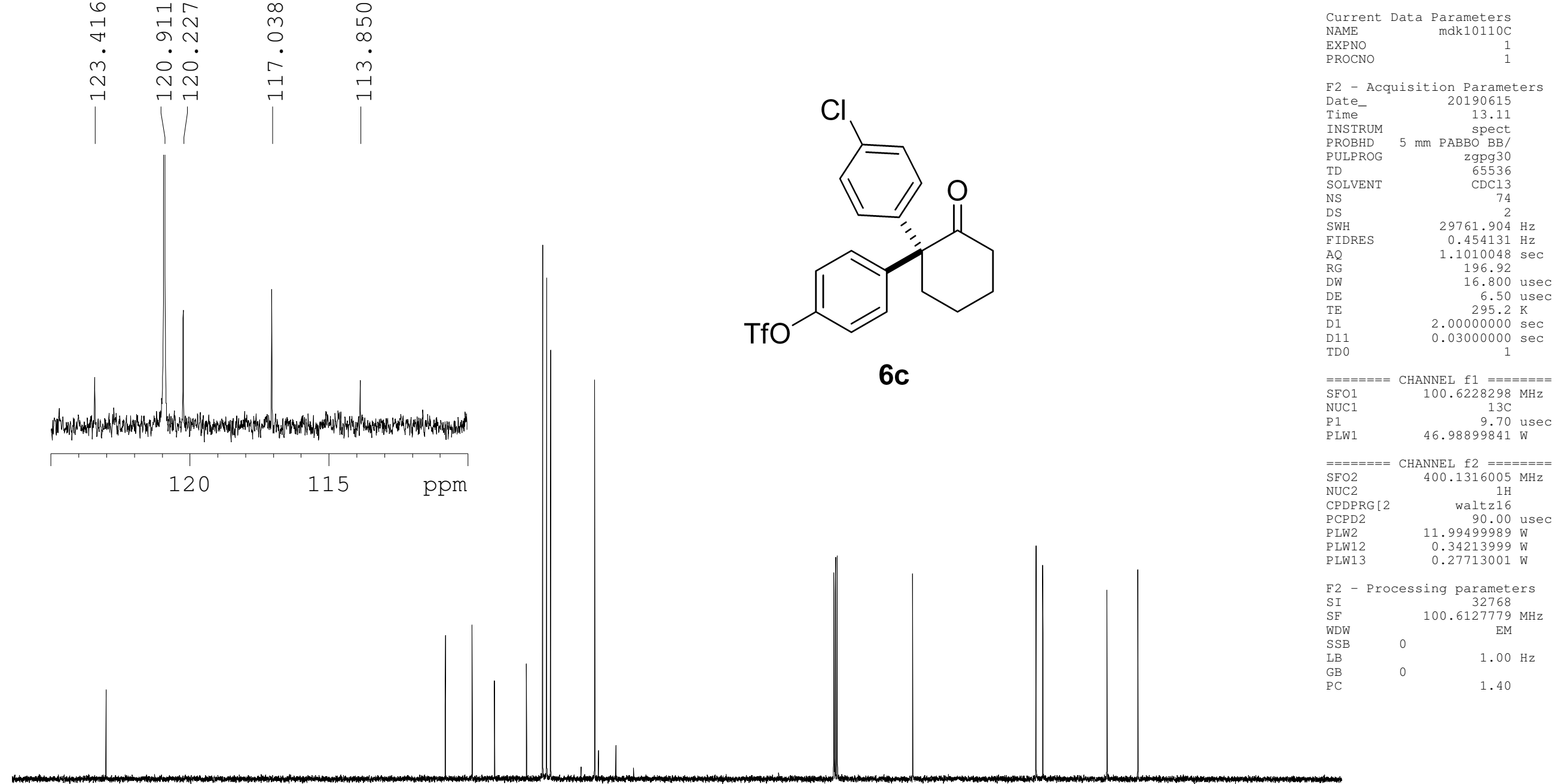

\begin{tabular}{|c|c|c|c|c|c|c|c|c|c|c|c|}
\hline \multirow{2}{*}{220} & 1 & 1 & $T$ & $T$ & 1 & 1 & 1 & $T$ & 1 & 1 & 1 \\
\hline & 200 & 180 & 160 & 140 & 120 & 100 & 80 & 60 & 40 & 20 & 0 \\
\hline
\end{tabular}




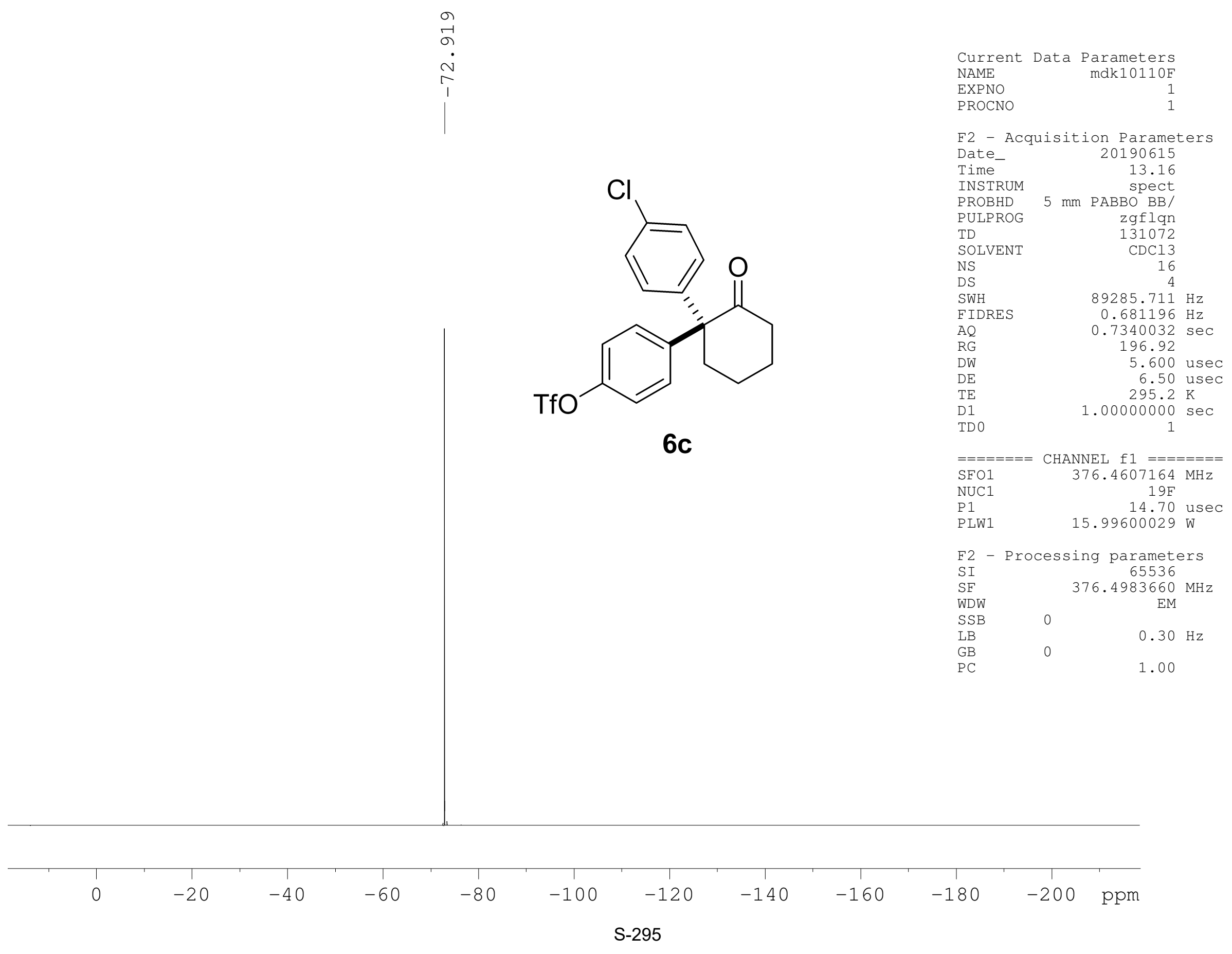




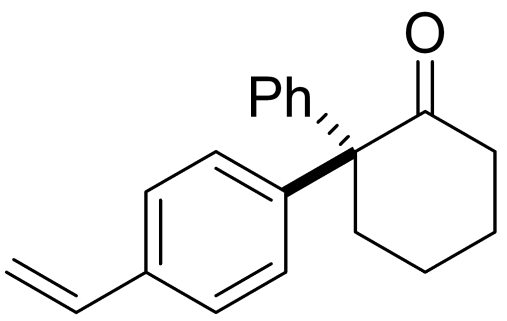

7

\section{$C$}

Current Data Parameters

NAME

mak10061H

EXPNO

F2 - Acquisition Parameters

Date__ 20190521

$\begin{array}{ll}\text { Time } & 19.33 \\ \text { INSTRUM } & \text { spect }\end{array}$

PROBHD $5 \mathrm{~mm} \mathrm{PABBO}$ BB/

PROBHD $5 \mathrm{~mm} \mathrm{PABBO}$ BB/
PULPROG

PULPROG

TD

SOLVENT

DS

SWH

FIDRES

$\mathrm{AQ}$
$\mathrm{RG}$
$\mathrm{DW}$

DW

TE

D 1

TDO
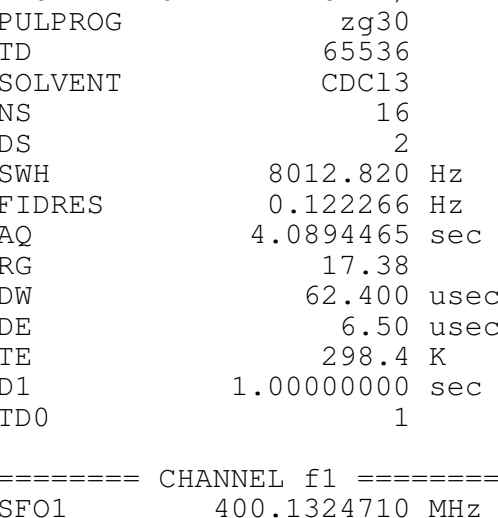

P1

NEL $f 1=======$

14.50 usec

$11.99499989 \mathrm{~W}$

F2 - Processing parameter

SI $\quad 400.1300316$ MHz

WDW

EM

LB

$0.30 \mathrm{~Hz}$

1.00

\section{6} ㄱํ웡요

$\left|\begin{array}{l|l|}0 \\ 0\end{array}\right|$

3

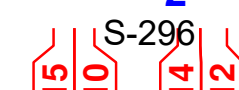

ลุ: 


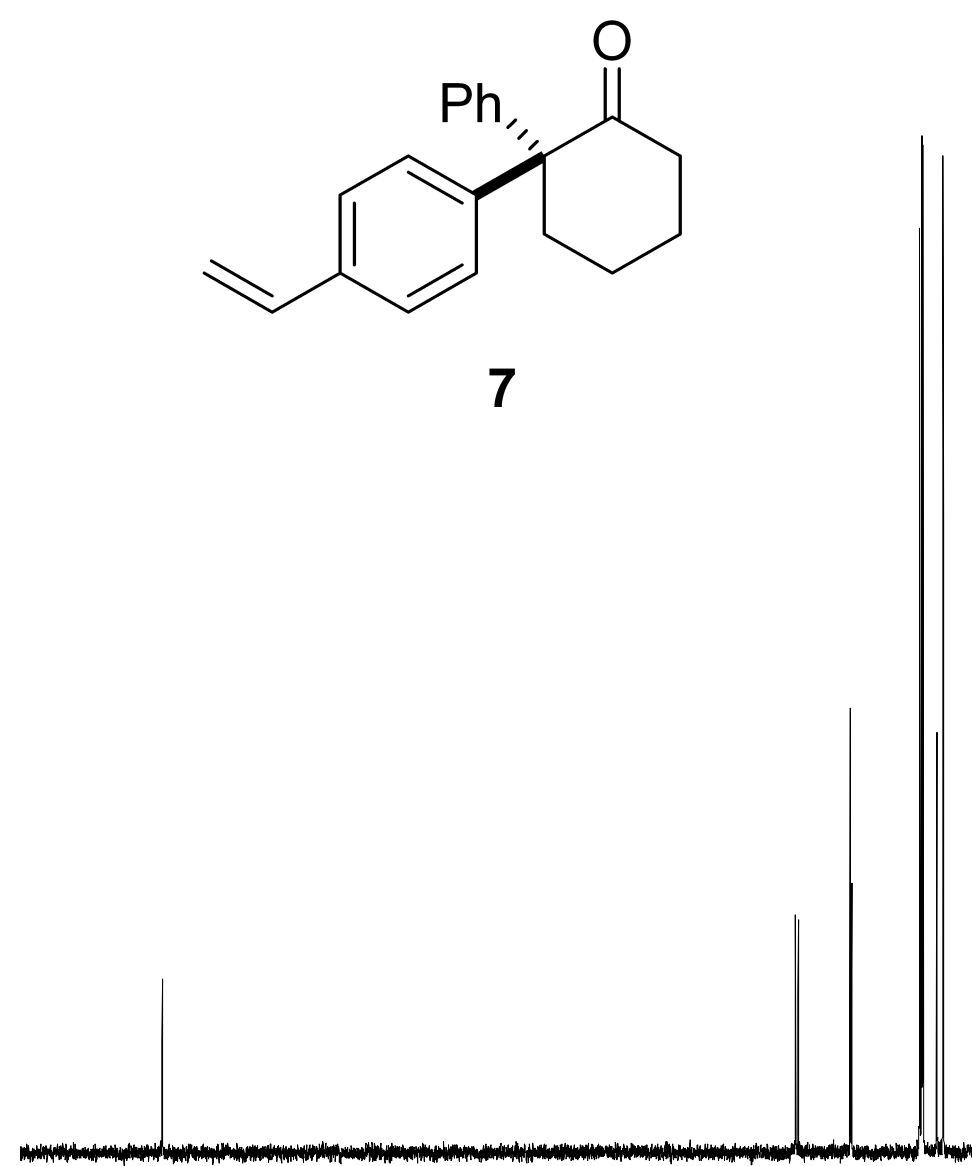

$\begin{array}{lr}\text { Current Data } & \text { Parameters } \\ \text { NAME } & \text { mdk10061C } \\ \text { EXPNO } & 1\end{array}$ EXPNO
PROCNO

F2 - Acquisition Parameters

Date

Time
INSTRUM

spect
PROBHD $5 \mathrm{~mm}$ PABBO BB/

PULPRO

SOLVENT

NS
DS
SWH

SWH
FIDRES

FIDRES
RG
DW
DE

$\mathrm{DE}$
$\mathrm{TE}$
$\mathrm{D} 1$

D1
DD0

$=== \pm===$ CHANNEL $\mathrm{f} 1 \quad======= \pm$
SFO1

SFO1
NUC1
P1

$13 \mathrm{C}$
$9.70 \mathrm{usec}$

PLW1

$46.98899841 \mathrm{~W}$

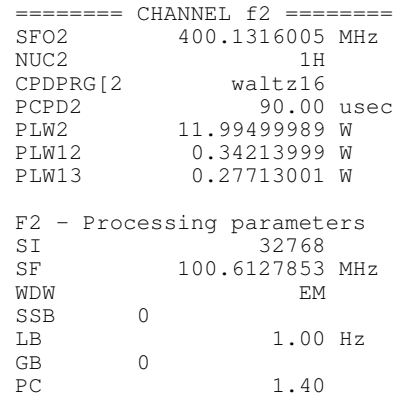

\begin{tabular}{lllllllllllllll}
\hline 220 & 200 & 180 & 160 & 140 & 120 & 100 & 80 & 60 & 40 & 20 & 0 & ppm
\end{tabular}



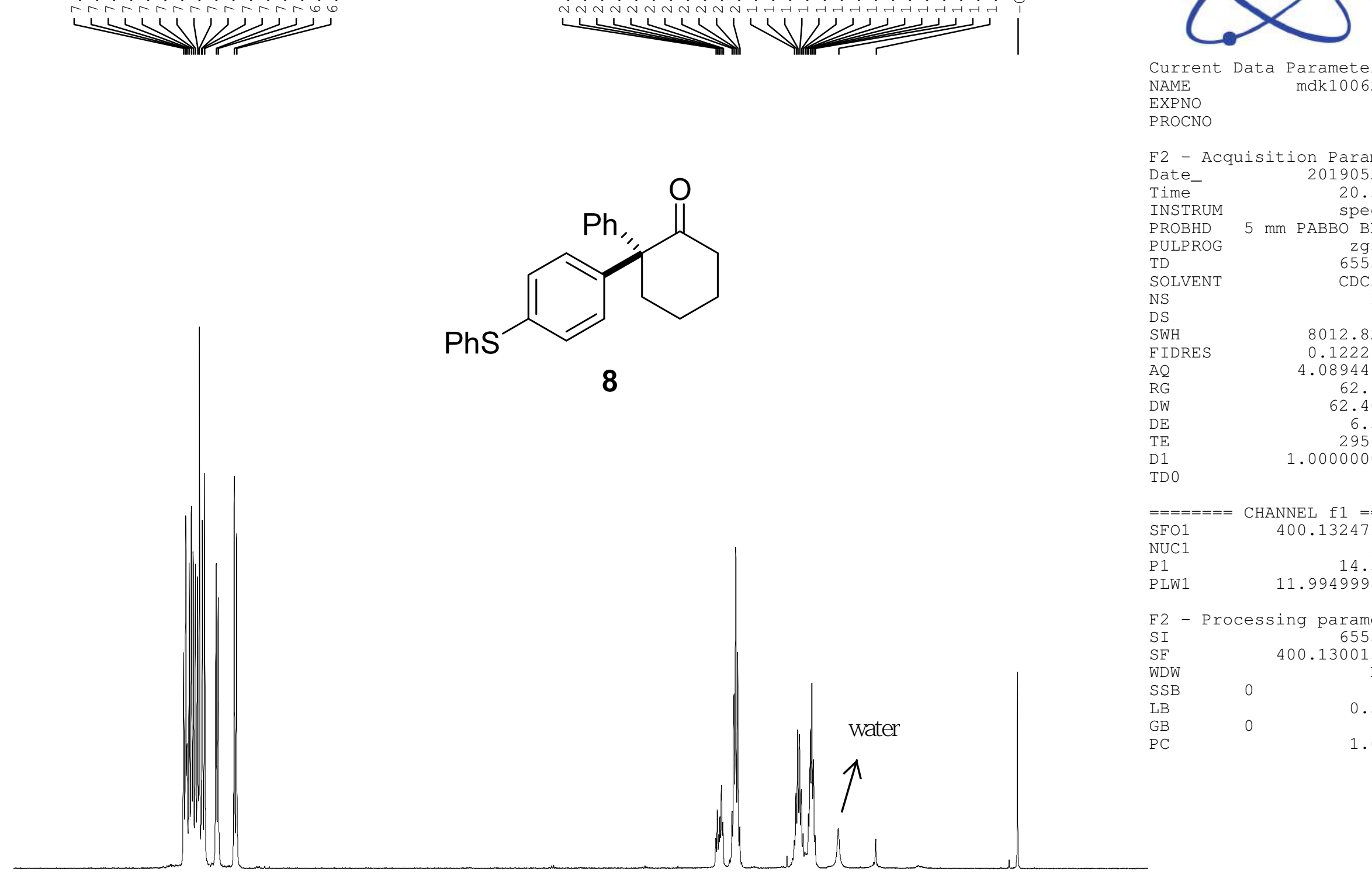

Current Data Parameters NAME

EXPNO

$\operatorname{mdk} 10062 \mathrm{H}$

F2 - Acquisition Parameters

Date__ 20190523

Time 20.39

SPeCt
PROBHD $5 \mathrm{~mm} \mathrm{PABBO} \mathrm{BB} /$

$\begin{array}{lr}\text { PROBHD } & 5 \mathrm{~mm} \text { PABBO BB/ } \\ \text { PULPROG } & \mathrm{zg} 30\end{array}$

TD

SOLVENT

NS

DS

SWH

FIDRES

$\mathrm{AQ}$

RG

DW

TE

TDO

$$
\mathrm{CDCl} 3
$$

$8012.820 \mathrm{~Hz}$

$0.122266 \mathrm{~Hz}$

$4.0894465 \mathrm{sec}$ 62.93

62.400 usec

6.50 usec

$295.8 \mathrm{~K}$

$1.00000000 \mathrm{sec}$

$\mathrm{SFO}$

NUC1

P1

CHANNEL f1 $========$

14.50 usec

F2 - Processing parameter

SI 65536

SF $\quad 400.1300151 \mathrm{MHz}$

WDW

$\mathrm{SSB}$

GB

$0.30 \mathrm{~Hz}$

water

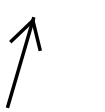

1.00

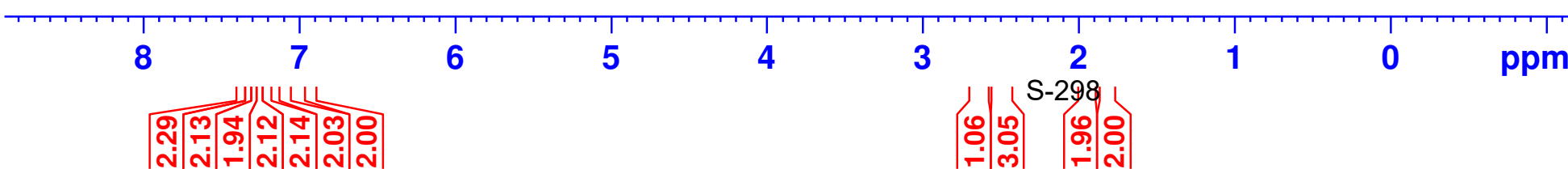




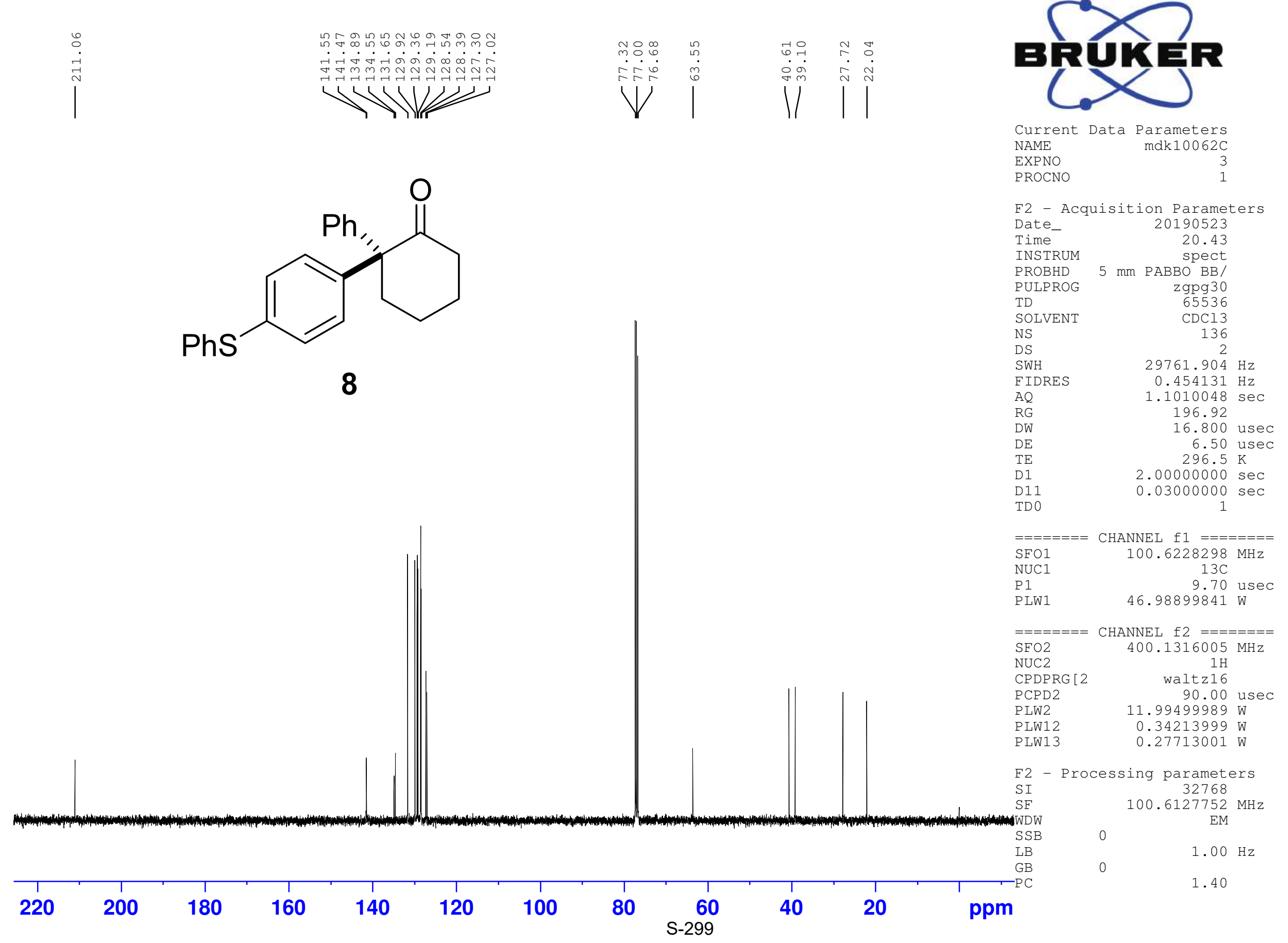




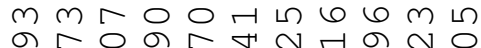

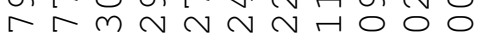

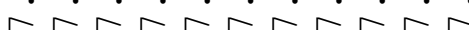
$\rightarrow$
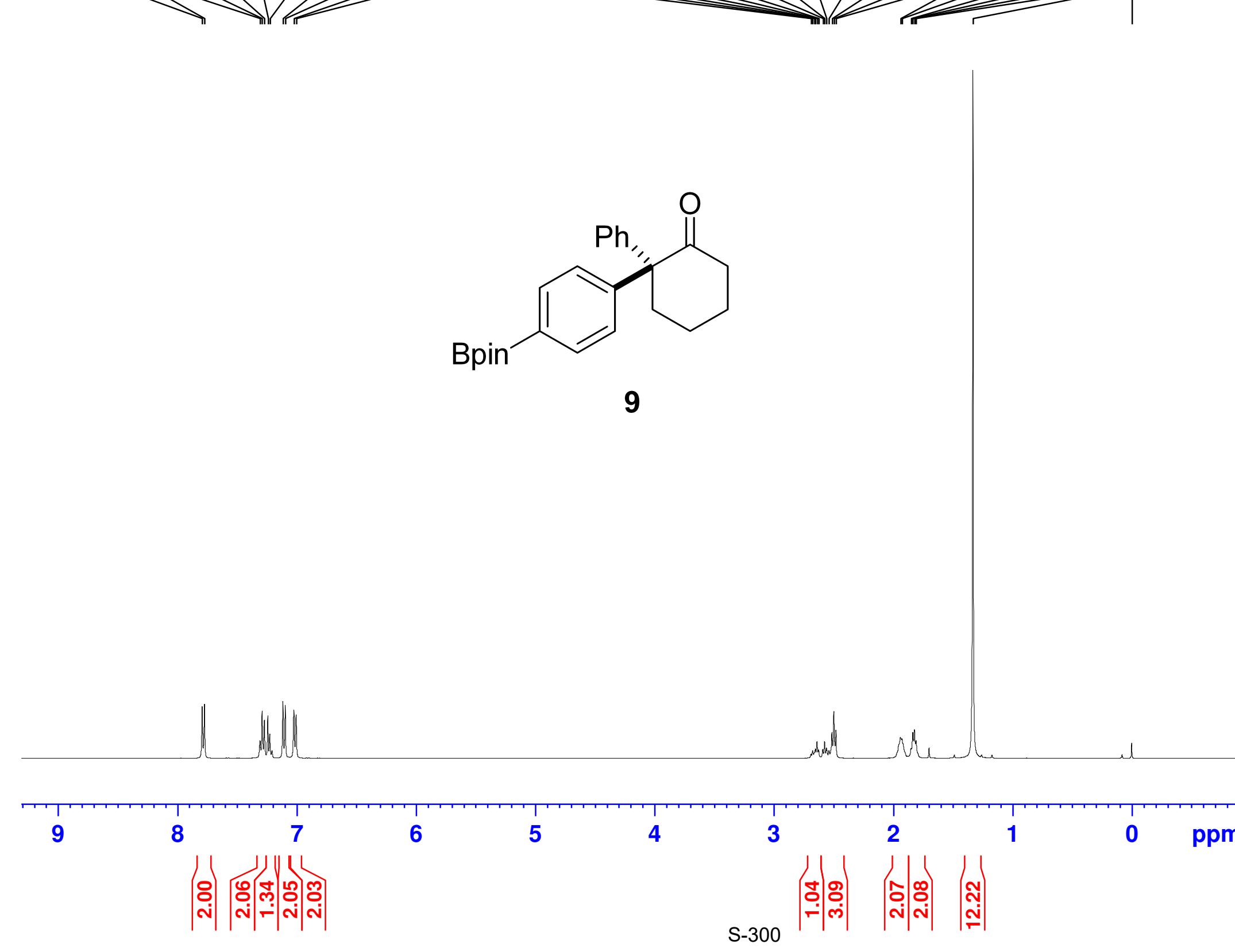

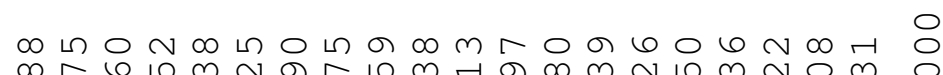

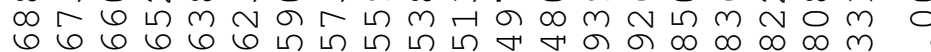

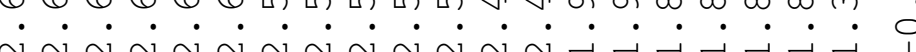
$\ldots$

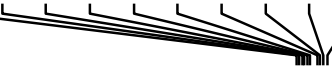

\section{sR $(1)$}

Current Data Parameters NAME

PROCNO

mdk $10063 \mathrm{H}$

F2 - Acquisition Parameters

Date__ 20190522

Time 20.02

$5 \mathrm{~mm}$ spect

PULPROG $\quad$ zg30

$\begin{array}{lr}\text { TD } & \text { Z930 } \\ \text { TD } & 65536\end{array}$

SOLVENT

SS

SWH

FIDRES

$\mathrm{AQ}$

RG

DE

TE

TD 0

$===$

$\mathrm{SFO}$

$\mathrm{N} 1$

PLW1

$\mathrm{CDCl} 3$
16

$8012.820 \mathrm{~Hz}$

$8012.820 \mathrm{~Hz}$

$4.0894465 \mathrm{sec}$

34.77

62.400 usec

6.50 usec

$1.00000000 \mathrm{sec}$

f1 $====2=5$

$400.1324710 \mathrm{MHz}$ $1 \mathrm{H}$

$11.99499989 \mathrm{~W}$

F2 - Processing parameters

I 65536

F $\quad 400.1300178 \mathrm{MHz}$

SB $\quad 0$

SSB

$0.30 \mathrm{~Hz}$

1.00 


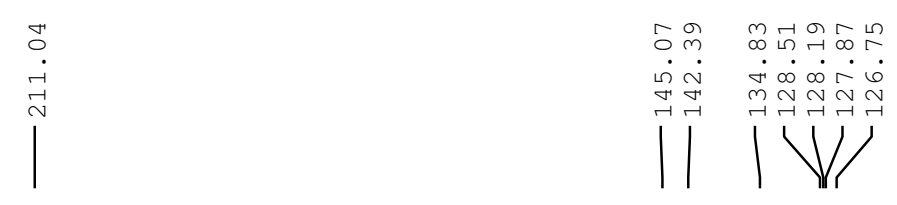

\section{expiren}

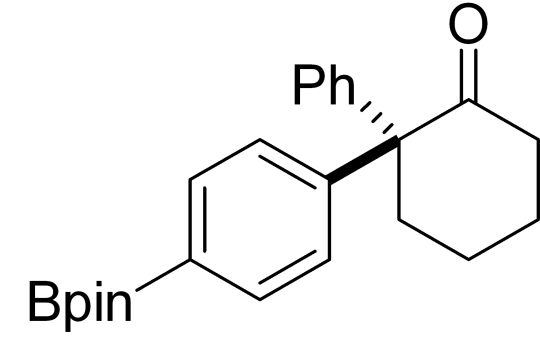

9

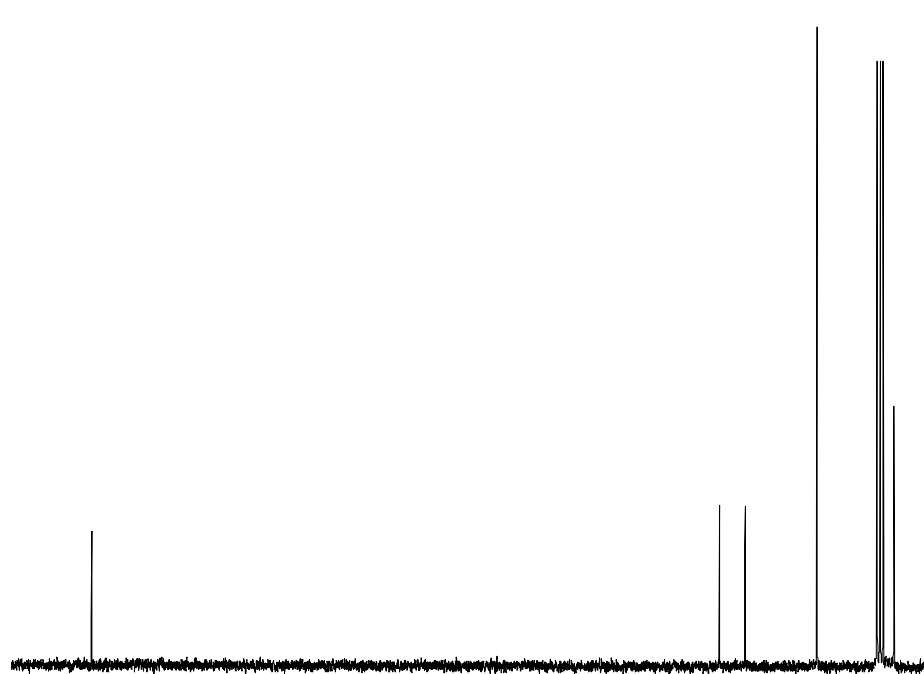

Current Data Parameters EXPNO F2 - Acquisition Parameters
Date 20190522 Time spect
PROBHD $5 \mathrm{~mm}$ PABBO BB/ PULPRO TD SOLVEN
NS
DS
SWH
FIDRES $\mathrm{AQ}$
$\mathrm{RG}$ DW TE
D1
D11

D11
TD0

$=======$ CHANNEL f1 $======= \pm$ SEO1 $\quad 100.6228298 \mathrm{MHz}$ $13 \mathrm{C}$
$9.70 \mathrm{usec}$ $6.98899841 \mathrm{~W}$ $== \pm== \pm=$ CHANNEL $\mathrm{f} 2 \quad====== \pm=$
$\mathrm{SFO2} \quad 400.1316005 \mathrm{MHz}$ $\begin{array}{lr}\text { NUC2 } & 1 \mathrm{H} \\ \text { CDDPRG [2 } & \text { waltz16 }\end{array}$ PCPD2
PIW2 90.00 use $\begin{array}{lr}\text { PLW2 } & 11.99499989 \mathrm{~W} \\ \text { PLW12 } & 0.34213999 \mathrm{~W}\end{array}$ Processing F2 - Processing parameters
SI $\begin{array}{ll}\text { SI } & 32768 \\ \text { SF } & 100.6127788 \mathrm{MHz}\end{array}$ SF SS

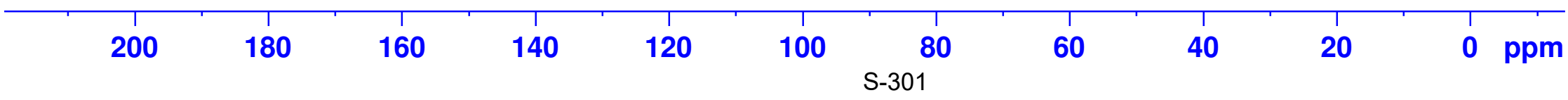




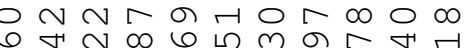
$m m m \sim N \sim 0$ व -

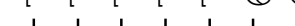
$\longrightarrow$ $m$ m $\infty$ m mmm

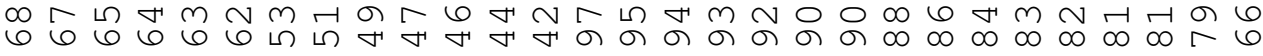

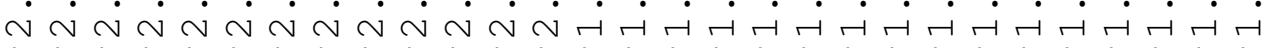

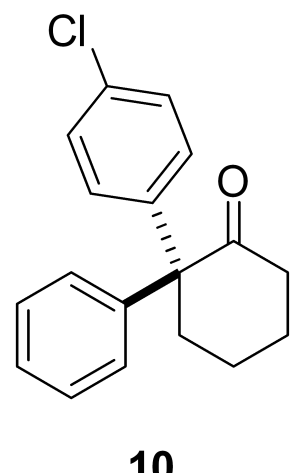

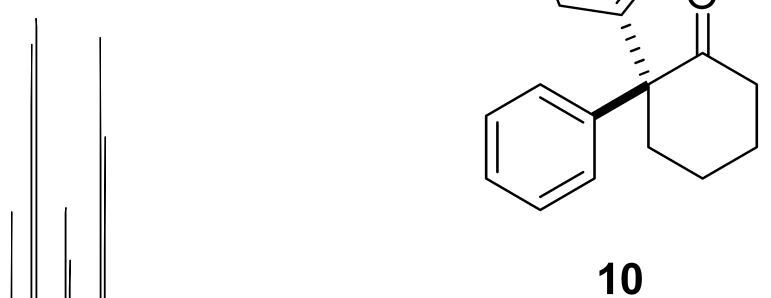

\section{8}

6

7

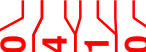

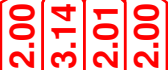

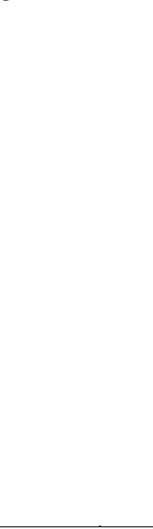

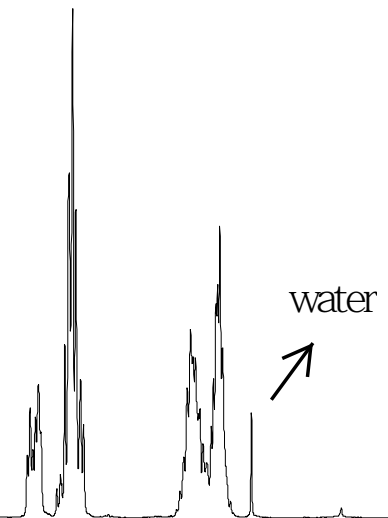

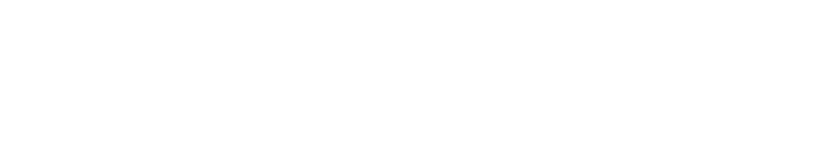

Current Data Parameters NAME

PROCNO

$\operatorname{mdk} 10116 \mathrm{H}$

F2 - Acquisition Parameters Date__ 20190615

$\begin{array}{lr}\text { Time- } & 13.24 \\ \text { INSTRUM } & \end{array}$

INSTRUM spect

PROBHD $5 \mathrm{~mm}$ PABB

PULPROG $\quad$ zg30

TD $\quad 65536$

$\begin{array}{lr}\text { SOLVENT } & \text { CDC13 } \\ \text { NS } & 16 \\ \text { DS } & 2\end{array}$

DS

FIDRES

AQ

RG

DW

DE

D1
TD 0

$====$

$\mathrm{SFO1}$

$\mathrm{NUC1}$
$\mathrm{P} 1$

$8012.820 \mathrm{~Hz}$

$8012.820 \mathrm{~Hz}$

$4.0894465 \mathrm{sec}$

31.55

62.400 usec

6.50 usec

$1.00000000 \mathrm{sec}$

CHANNEL $\mathrm{f} 1========$ $400.1324710 \mathrm{MHz}$

14.50

4.50 usec

PLW1 11.99499989 W

F2 - Processing parameters

SI 65536

SF $\quad 400.1300235 \mathrm{MHz}$

WDW

SSB

EM

$0.30 \mathrm{~Hz}$

1.00 


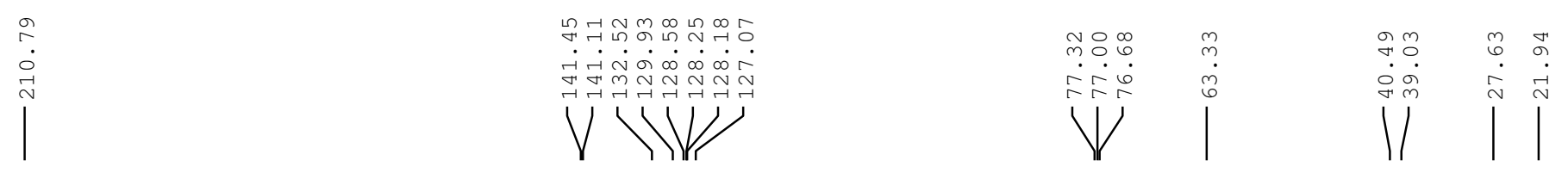

말

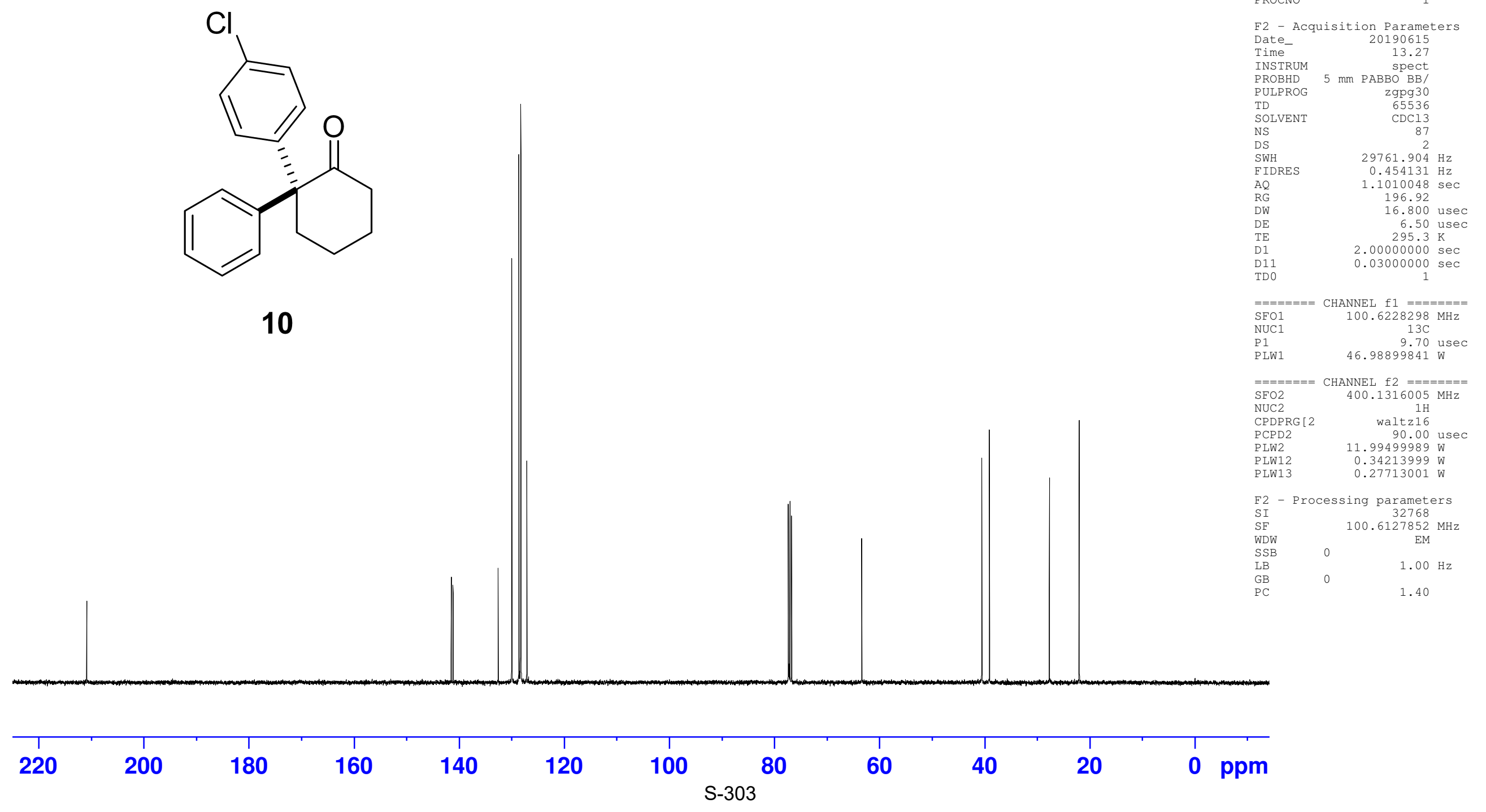




\section{Bg $(1)$}

Current Data Parameters NAME

$\operatorname{mdk} 10127 \mathrm{H}$

EXPNO

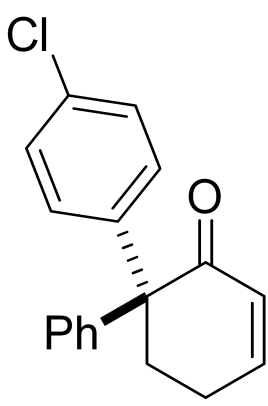

11

F2 - Acquisition Parameters

Date_ 20190620

$\begin{array}{lr}\text { Time } & 20.22\end{array}$

INSTRUM spect

PUTPROG

$\mathrm{zg} 30$
65536

$\begin{array}{ll}\text { TD } & 65536 \\ \text { SOLVENT } & \text { CDC13 }\end{array}$

NS

SWH

FIDRES

$\mathrm{AQ}$

RG

$\mathrm{DW}$

16
16

$8012.820 \mathrm{~Hz}$

$0.122266 \mathrm{~Hz}$

$4.0894465 \mathrm{sec}$ 45.67

$\mathrm{DE}$

TE

62.400 usec 6.50 usec $295.1 \mathrm{~K}$

$400.1324710 \mathrm{MHz}$

F2 - Processing parameters

SI $\quad 65536$

SF $\quad 400.1300182 \mathrm{MH}$

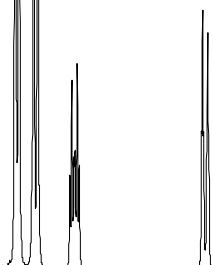

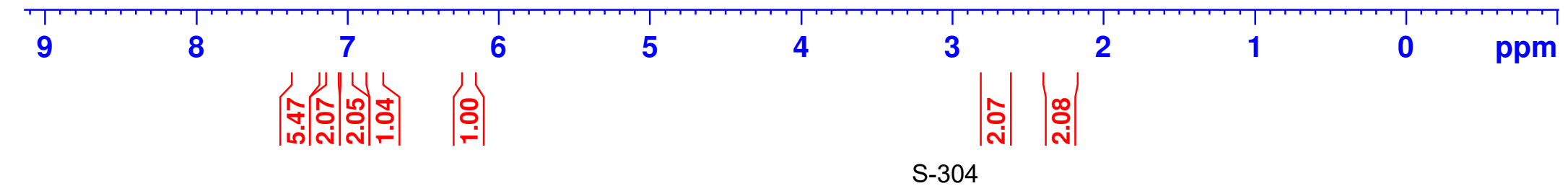



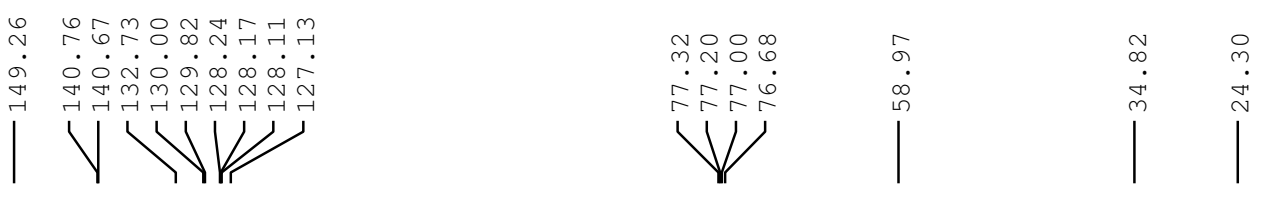

arienger

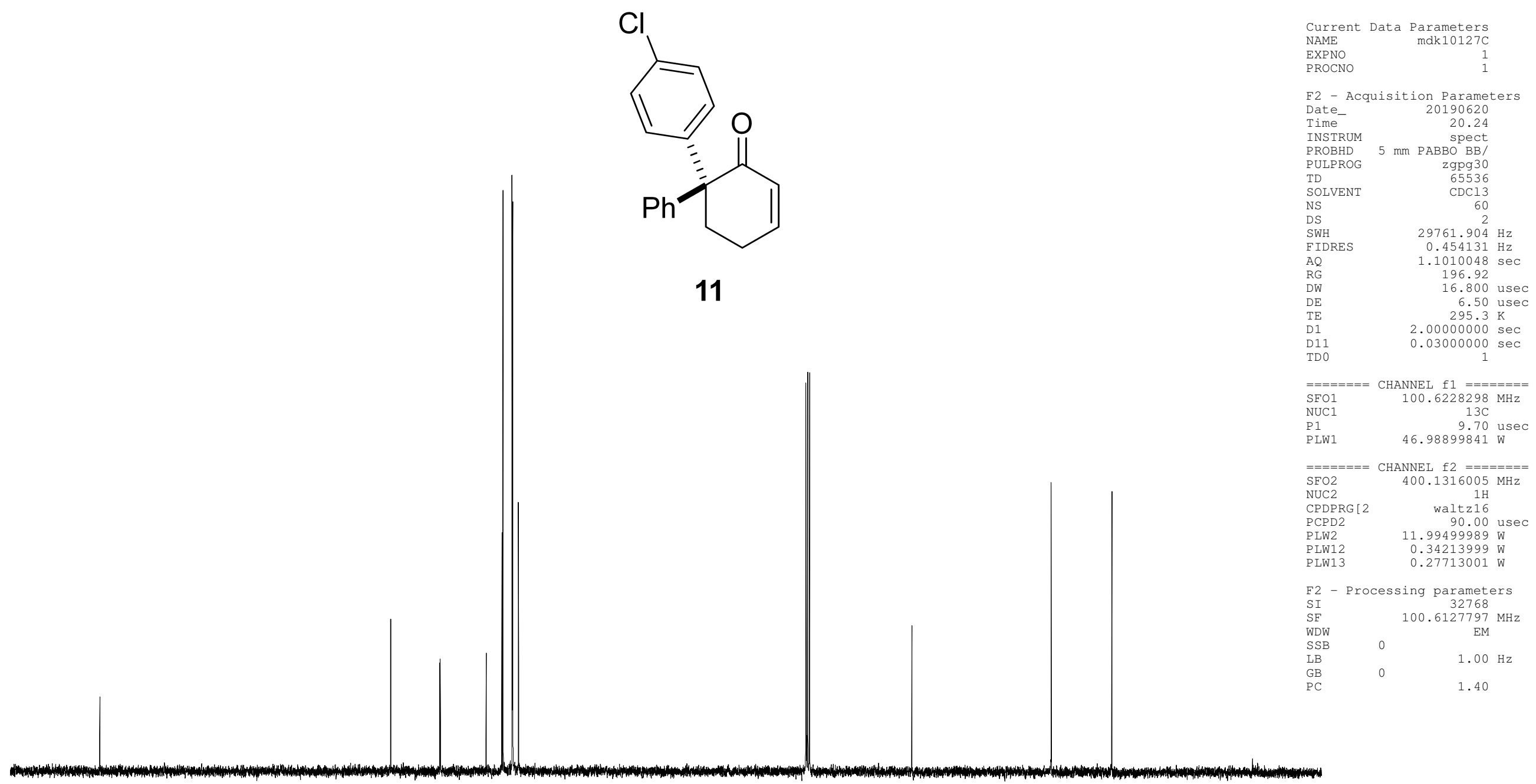

\begin{tabular}{|c|c|c|c|c|c|c|c|c|c|c|}
\hline 1 & 1 & 1 & 1 & 1 & 1 & 1 & 1 & 1 & 1 & \\
\hline 200 & 180 & 160 & 140 & 120 & 100 & & 60 & 40 & 20 & ppm \\
\hline
\end{tabular}


의 $\ln$ 다 ๙ $\dot{\forall} \dot{\sim} \dot{\sim} \dot{\sim} \dot{\sim} \dot{\sim} \dot{\sim} \dot{\sim} \dot{\sim} \dot{\sim} \dot{\sim} \dot{\sim} \dot{\sim} \dot{\sim} \dot{\sim} \dot{\sim} \dot{\sim}$

$\underbrace{+}$

12

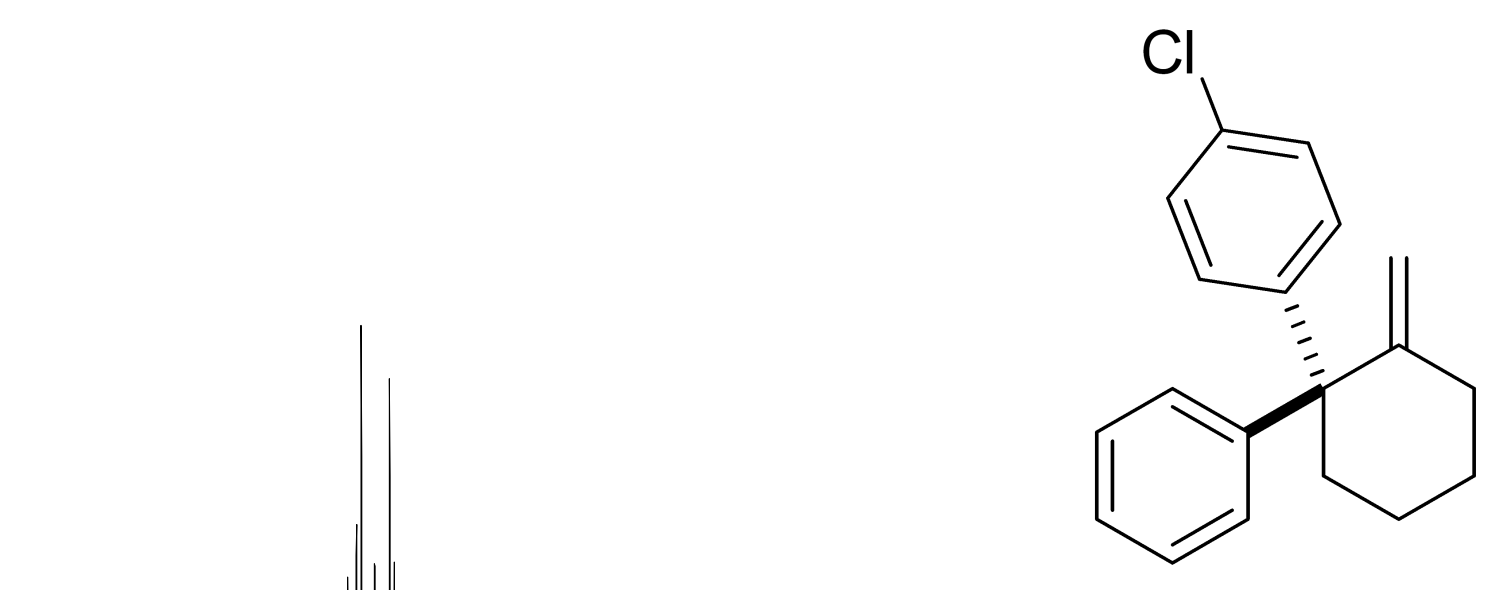

\section{Bg $(1)$}

Current Data Parameters NAME

PROCNO

$\operatorname{mdk} 10118 \mathrm{H}$

F2 - Acquisition Parameters

Date_ 20190617

Time $\quad 20.15$

$5 \mathrm{~mm}$ PABBO $\mathrm{BB} /$

PULPROG $\quad$ zg30

$\begin{array}{lr}\text { TD } & \text { zg30 } \\ & 65536\end{array}$

SOLVENT

NS

DS

SWH

$\mathrm{AQ}$

RG

$\mathrm{DW}$
$\mathrm{DE}$
$\mathrm{TE}$

D1
TD 0

$====$

$\mathrm{SFO1}$

$\mathrm{NUC1}$
$\mathrm{P} 1$

PLW1

$\mathrm{CDCl}$
16

$8012.820 \mathrm{~Hz}$

$0.122266 \mathrm{~Hz}$

$4.0894465 \mathrm{sec}$ 31.55

62.400 usec 6.50 usec

$1.00000000 \mathrm{sec}$ 1

CHANNEL $\mathrm{f} 1========$ $400.1324710 \mathrm{MHz}$ 14.50 $11.99499989 \mathrm{~W}$

22 - Processing parameters

SI Processing parameter

SF $\quad 400.1300326 \mathrm{MHz}$

WDW

$0.30 \mathrm{~Hz}$

1.00

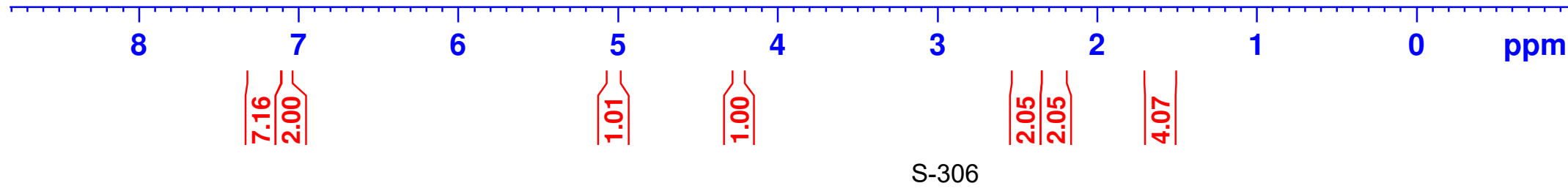


12
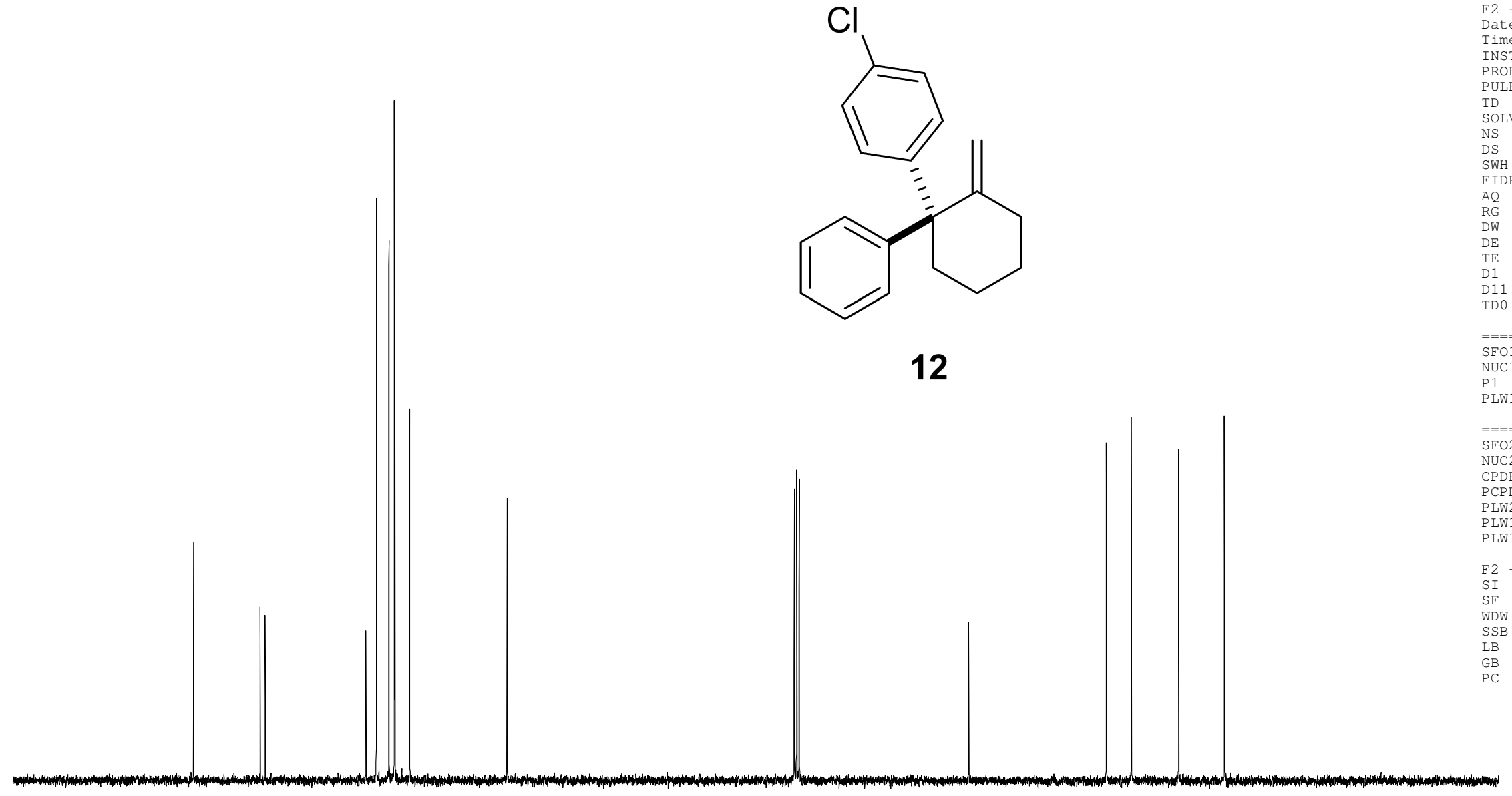

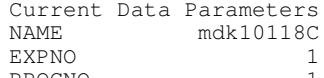

F2 - Acquisition Parameter

$\begin{array}{lr}\text { Time } & 20.18 \\ \text { INSTRUM } & \text { spect } \\ \text { PROBHD } & 5 \mathrm{~mm} \text { PABBO BB/ }\end{array}$

PROBHD

TD

SOLVE
NS
DS

SWH
FIDRES

$\mathrm{AQ}$
$\mathrm{RG}$

DW
DE

D1
D11

D11
TD 0

$=======$ CHANNEL $\mathrm{fI}========$

SFO1 $\quad 100.6228298 \mathrm{MHz}$

$13 \mathrm{C}$ usec
9.70 use

$6.98899841 \mathrm{~W}$

$\begin{array}{ll}z====== & \text { CHANNEL } f 2 \quad======= \\ \text { SFO2 } & 400.1316005 \mathrm{MHz}\end{array}$

$\begin{array}{lr}\text { NUC2 } & 1 \mathrm{H} \\ \text { CPDPRG [2 } & \text { waltz16 } \\ \text { PCPD2 } & 90.00\end{array}$

$11.99499989 \mathrm{~W}$

$0.34213999 \mathrm{~W}$
$\mathrm{~W}$

$\begin{array}{lr}\text { F2 - Processing parameters } \\ \mathrm{SI} \\ \mathrm{SF} & 32768 \\ \mathrm{SF} & 100.6127789 \mathrm{MHz}\end{array}$

WDW 


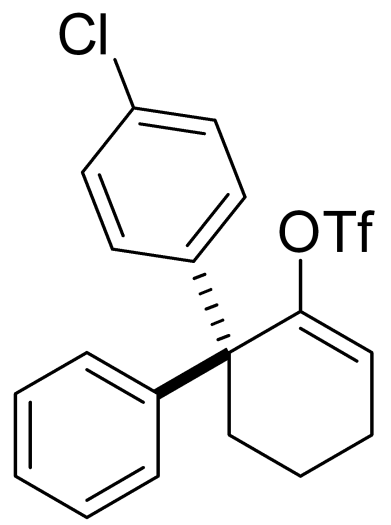

13

\section{BR $(1)$}

Current Data Parameters NAME

PROCNO

mdk $10119 \mathrm{H}$

F2 - Acquisition Parameters

Date_ 20190617

Time $\quad 20.22$

$5 \mathrm{~mm}$ spect

PULPROG $\quad$ zg30

$\begin{array}{lr}\text { TD } & \text { Zg30 } \\ & 65536\end{array}$

$\begin{array}{ll}\text { SOLVENT } & \text { CDC13 }\end{array}$

DS

SWH

FIDRES

FIDR
AQ

RG

$\mathrm{DW}$
$\mathrm{DE}$
$\mathrm{TE}$

D1
TD 0

$===$

$\mathrm{SFO}$

$\mathrm{NUC1}$
$\mathrm{P} 1$

16
2

$8012.820 \mathrm{~Hz}$

$0.122266 \mathrm{~Hz}$

$4.0894465 \mathrm{sec}$ 27.78

62.400 usec 6.50 usec

$1.00000000 \mathrm{sec}$ 1

CHANNEL $\mathrm{f} 1========$ $400.1324710 \mathrm{MHz}$ 14 PLW1 $11.99499989 \mathrm{~W}$

- Processing parameter

SF $\quad 65536$

SFW $\quad 400.1300179 \mathrm{MHz}$

SSB

EM

$0.30 \mathrm{~Hz}$

1.00

\begin{tabular}{ll}
7 & 6 \\
8 & \\
\hline &
\end{tabular}

5

4

3

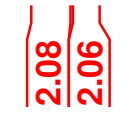

2

1

0 ppm 


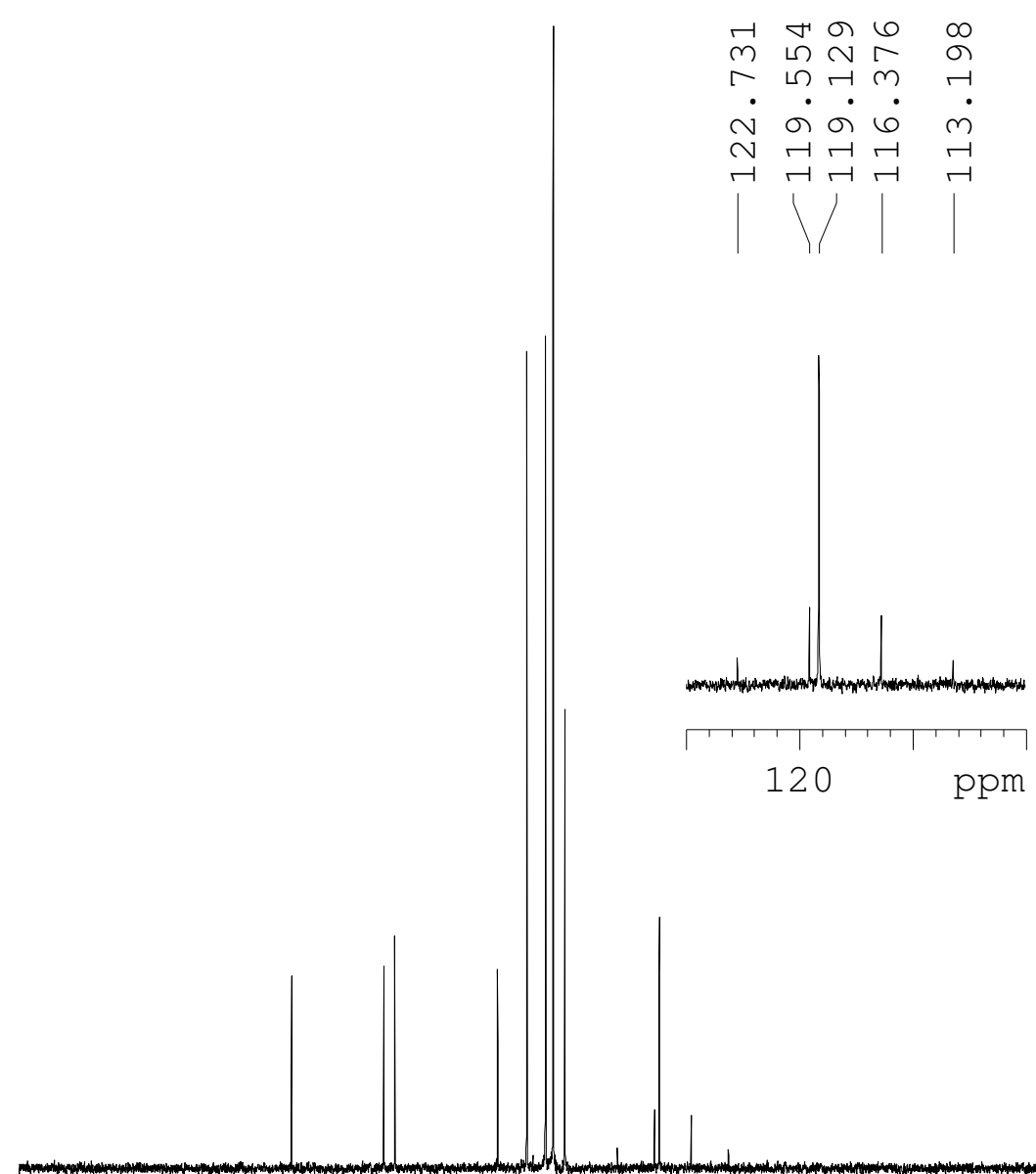

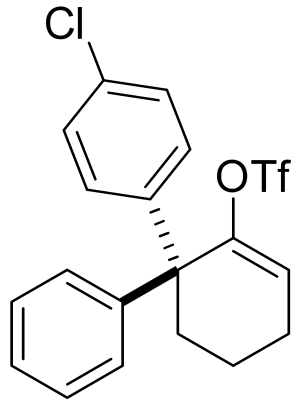

13

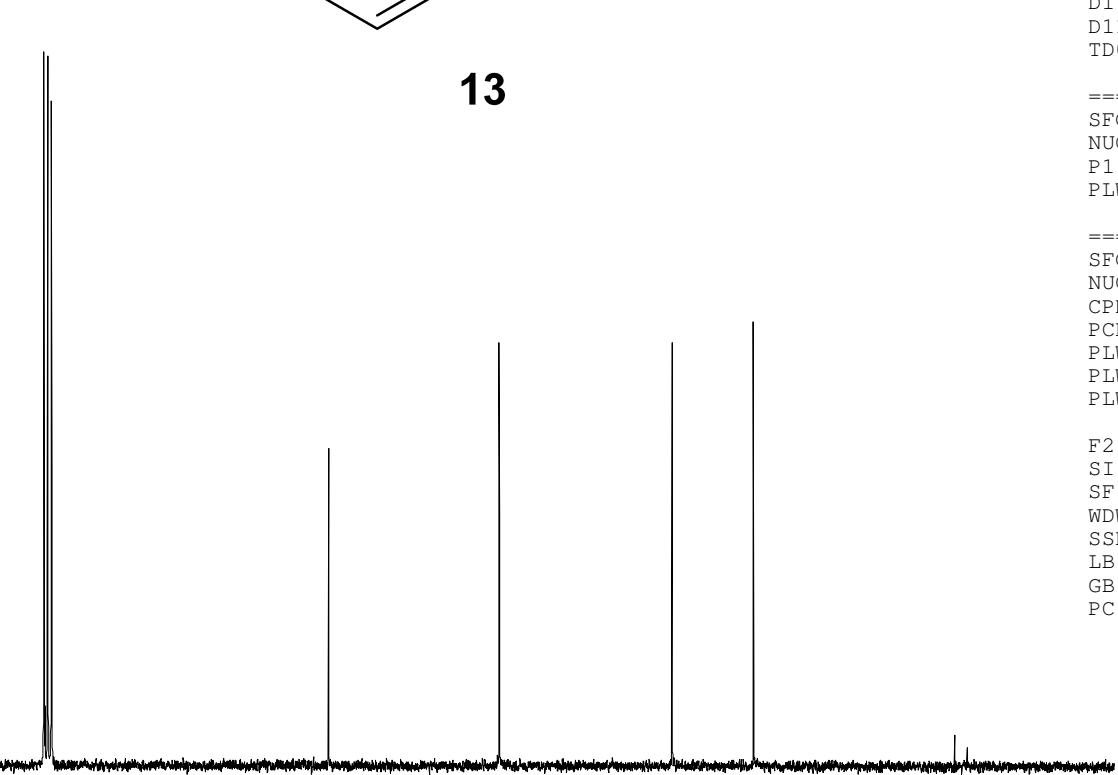

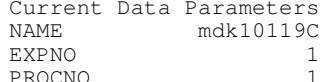
F2 - Acquisition Parameters
Date_

Time spect
PROBHD $5 \mathrm{~mm}$ PABBO BB/

TD

SOLVEN

NS

SWH
FIDRES

$\mathrm{AQ}$
$\mathrm{RG}$

DW
DE

D 11

DD 11

100. $f 1========$

NUC

$913 \mathrm{C}$

$46.98899841 \mathrm{~W}$

$y=====$ CHANNEL f2 $=======$
SFO2 $400.1316005 \mathrm{MHz}$

$\begin{array}{lr}\text { NUC2 } & 1 \mathrm{H} \\ \text { CPDPRG [2 } & 90.00\end{array}$

$11.99499989 \mathrm{~W}$

$0.27713001 \mathrm{~W}$

$\begin{array}{lr}\text { F2 - Processing parameters } \\ \text { SI } \\ \text { SF } & 32768 \\ \mathrm{SF} & 100.6127743 \mathrm{MHz}\end{array}$

100.6127743
WDW

$1.00 \mathrm{~Hz}$ 


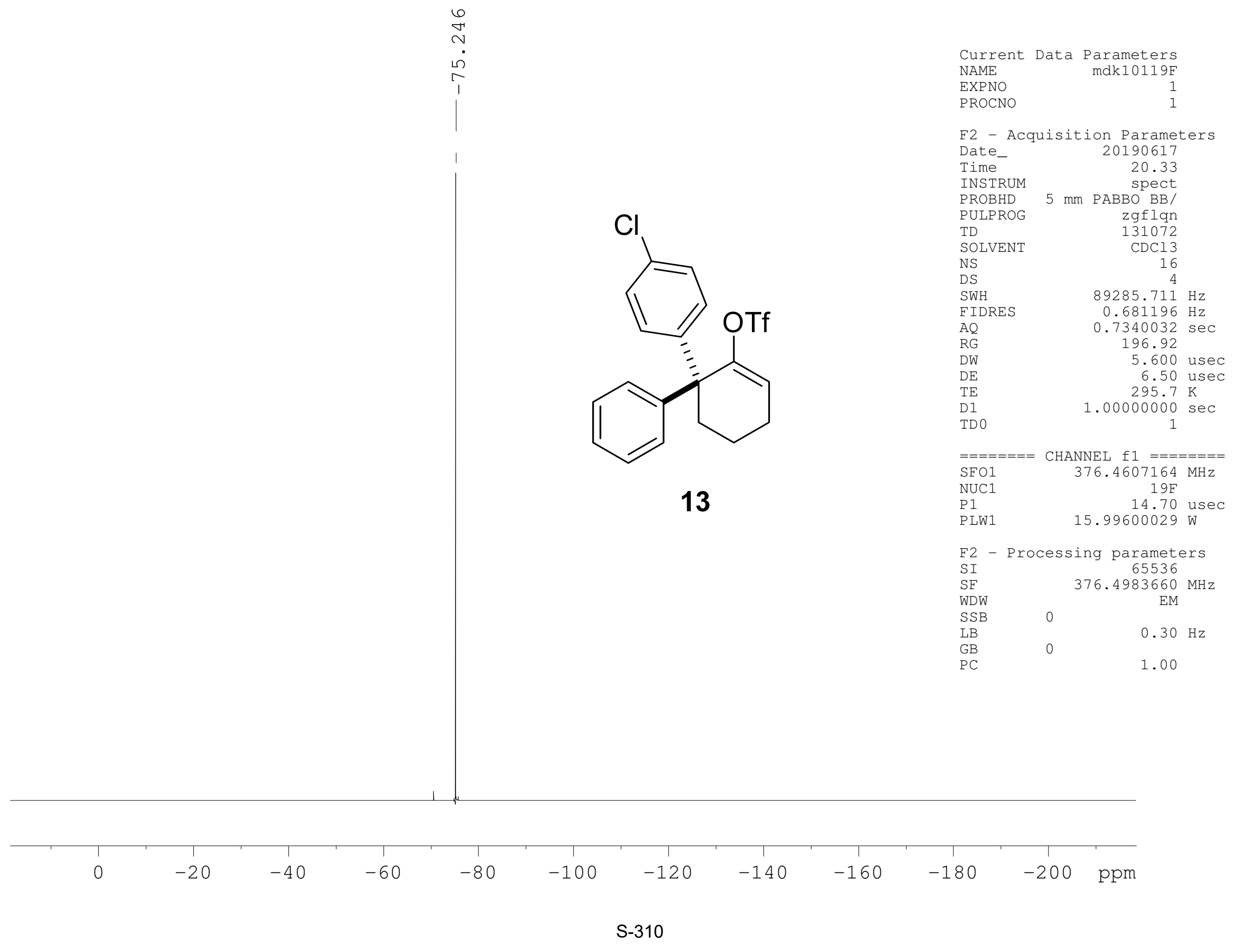




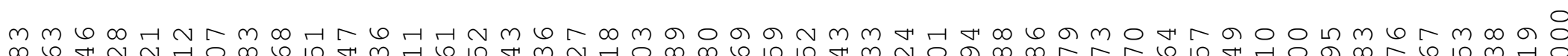
ㅅN N N

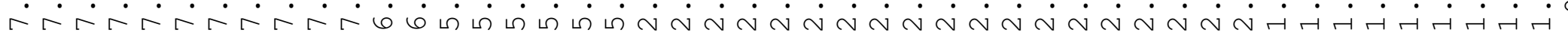
$+1$

\section{sperer

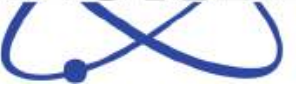

Current Data Parameters NAME $\operatorname{mak} 10126 \mathrm{H}$

PROCNO

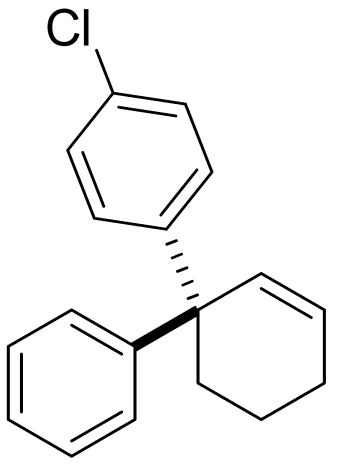

F2 - Acquisition Parameters Date 20190619 $\begin{array}{lr}\text { Time- } & 19.50\end{array}$ INSTRUM spect PROBHD
PULPROG TD 65536 SOLVENT NS DS

$\begin{array}{ll}\text { SWH } & 8012.820 \mathrm{~Hz} \\ \text { IDRES } & 0.122266 \mathrm{~Hz}\end{array}$ $\mathrm{AO} \quad 4.0894465 \mathrm{~Hz}$ DW $\mathrm{DE}$ 14 62.67 62.400 usec
6.50 usec $294.9 \mathrm{~K}$ $1.00000000 \mathrm{sec}$ $=======$ CHANNEL f1 $=======$ SFO1 NUC1 $\mathrm{NUC1}$ $400.1324710 \mathrm{MHz}$ PLW1 14.50 usec
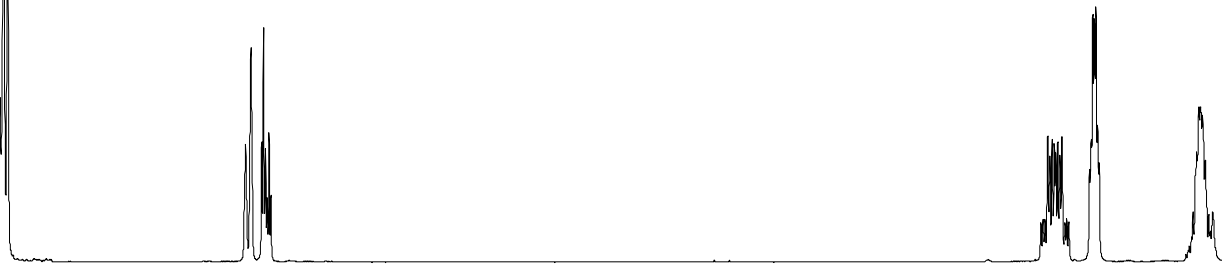

$\begin{array}{lcc}\text { F2 } & \text { Processing parameters } \\ \text { SI } & 65536 \\ \text { SF } & 400.1300260 \mathrm{MHz} \\ \text { WDW } & 0 & \text { EM } \\ \text { SSB } & 0 & \\ \text { LB } & 0 & 0.30 \mathrm{~Hz} \\ \text { GB } & 0 & 1.00 \\ \text { PC } & & \end{array}$

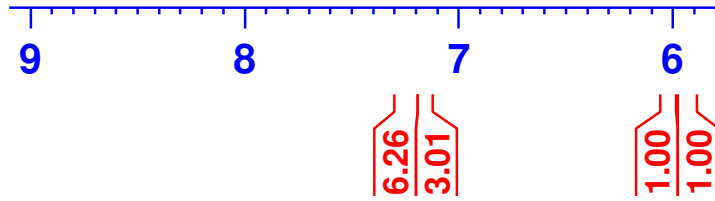

5

4

3

2

1

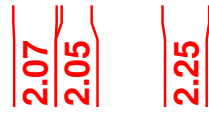




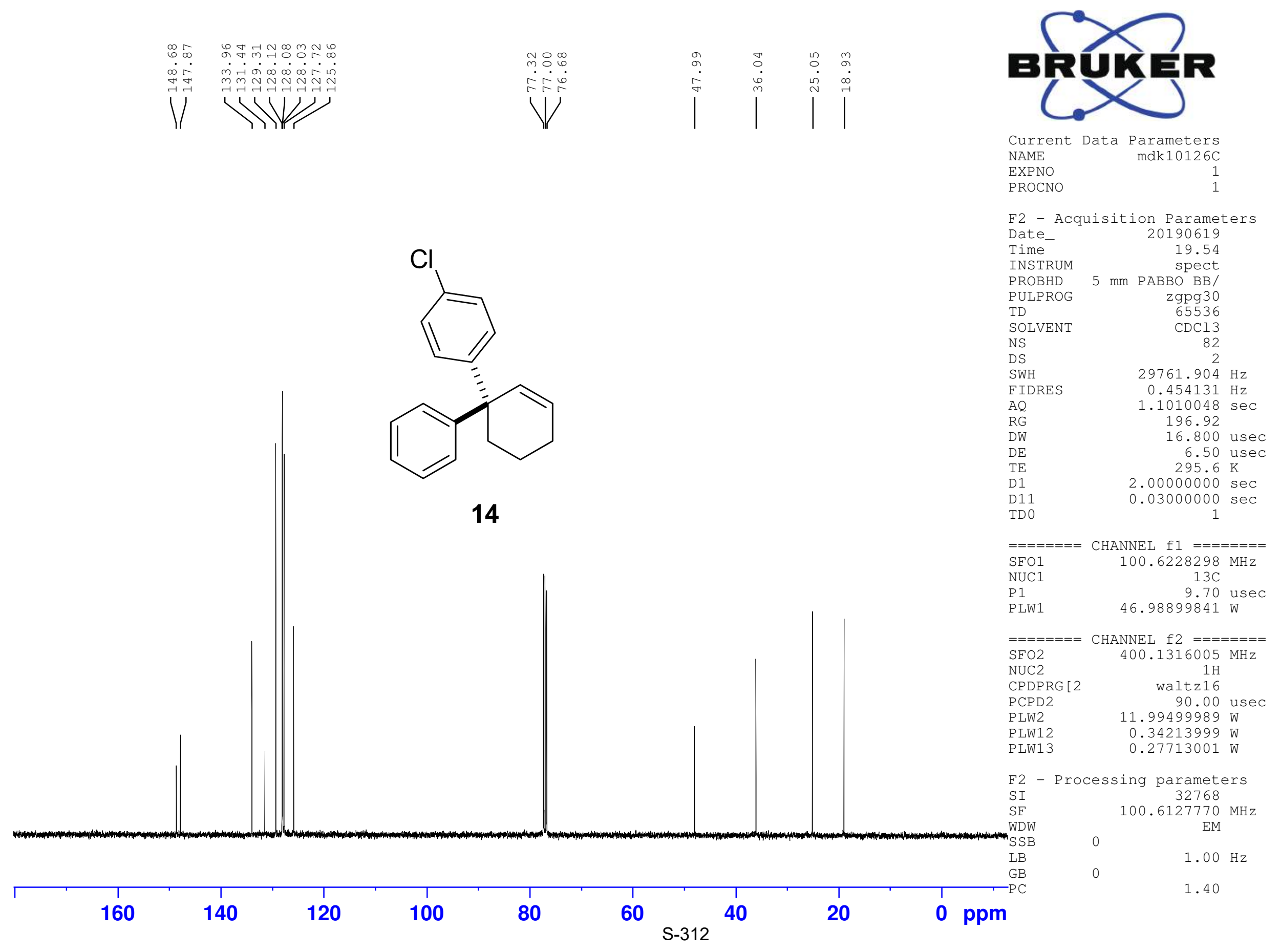




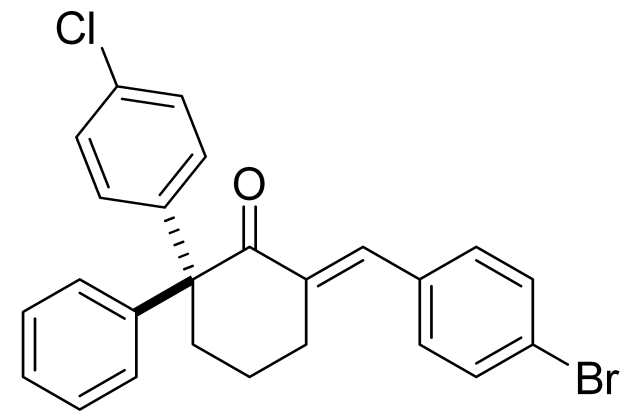

15

\section{Ethyl Acetate}
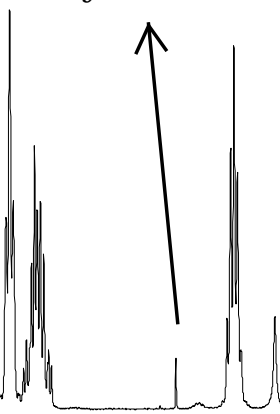

water

$\uparrow_{\text {Ethyl Acetate }}$

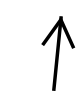

5
4
3

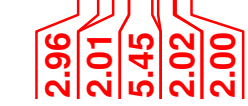

6 


\section{=RUस}

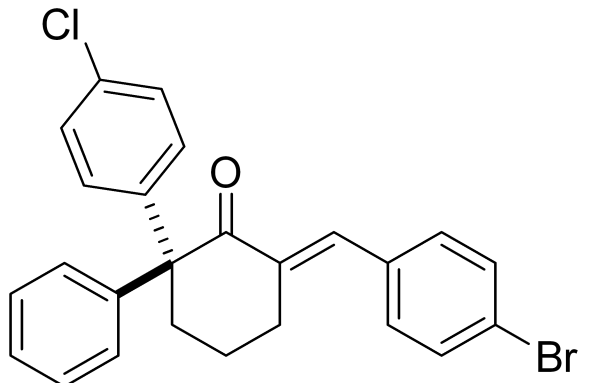

15

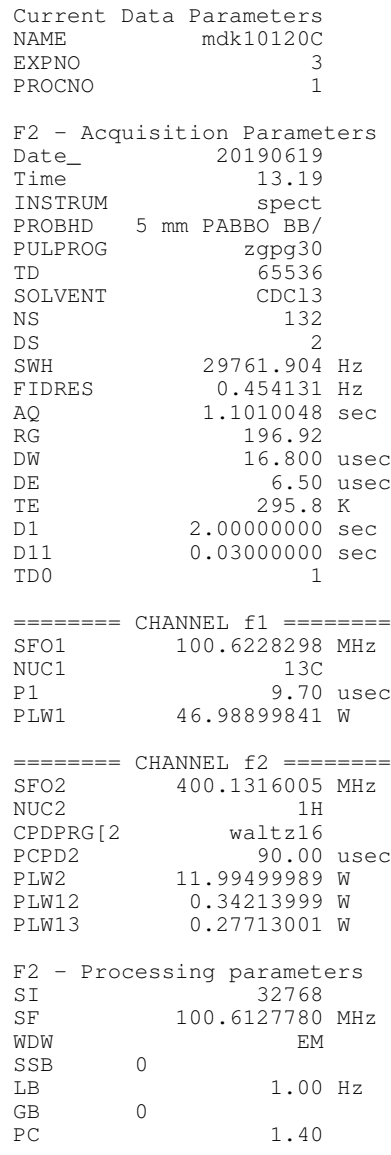

\begin{tabular}{rrrrrrrrrrrrr}
\hline 200 & 180 & 160 & 140 & 120 & 100 & 80 & 60 & 40 & 20 & 0 & ppm
\end{tabular}




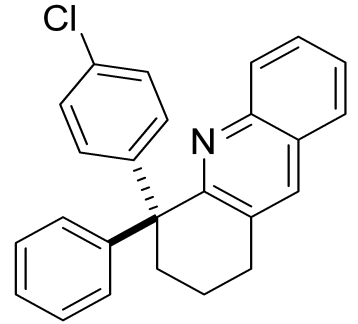

16

\section{$C$}

Current Data Parameters NAME mdk10117H

PROCNO

F2 - Acquisition Parameters

Date__ 20190617

Time $\quad 20.03$

INSTRUM 5 spect

PROBHD $5 \mathrm{~mm}$ PABBO BB/

PULPROG $\quad \mathrm{zg} 30$

TD $\quad 65536$

SOLVENT CDC13

N.S

DS

SWH $\quad 8012.820 \mathrm{~Hz}$

FIDRES $\quad 0.122266 \mathrm{~Hz}$

$\mathrm{AQ} \quad 4.0894465 \mathrm{sec}$

RG

DW

$\mathrm{DE}$

D1

TDO

62.400 use 6.50 usec $295.1 \mathrm{~K}$

$1.00000000 \mathrm{sec}$

$\begin{array}{lr}======= & \text { CHANNEL } 11======= \\ \text { SFO1 } & 400.1324710 \mathrm{MHz} \\ \text { NUC1 } & 1 \mathrm{H} \\ \text { P1 } & 14.50 \text { usec }\end{array}$

PLW1 11.99499989 W

F2 - Processing parameters

SI 65536

SF $\quad 400.1300309 \mathrm{MHz}$

WDW

$S S B$

GB
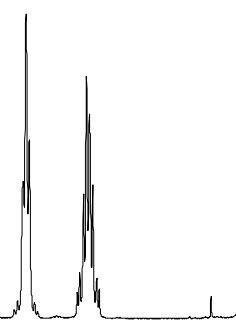

$0.30 \mathrm{~Hz}$

1.00

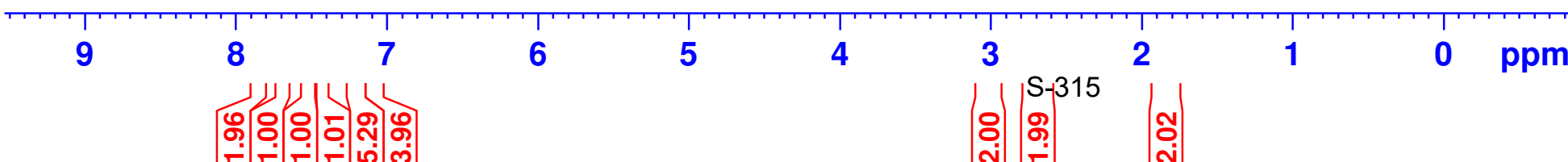




\section{BR}

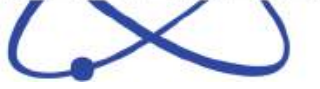

Current Data Parameters

NAME

mdk10117C

EXPNO

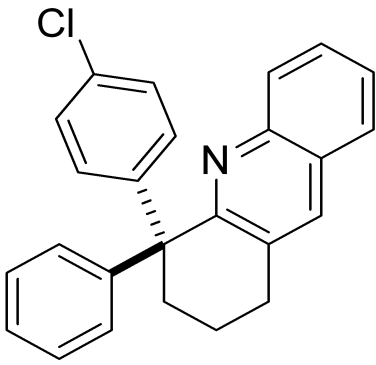

PROCNO

F2 - Acquisition Parameters

Date__ 20190617

Time $\quad 20.06$

INSTRUM 5 mpect

PROBHD $5 \mathrm{~mm}$ PABBO BB/

PULPROG zgpg30

TD 65536

SOLVENT CDC13

NS

$\begin{array}{lr}\text { DS } & 2 \\ \text { SWH } & 29761.904 \mathrm{~Hz}\end{array}$

FIDRES $\quad 0.454131 \mathrm{~Hz}$

$\mathrm{AQ} \quad 1.1010048 \mathrm{sec}$

16

$$
196.92
$$

DW $\quad 16.800$ usec

DE $\quad 6.50$ use

TE $\quad 295.8 \mathrm{~K}$

D1 $2.00000000 \mathrm{sec}$

TDO $\quad 0.03000000$

$=======$ CHANNEL $\mathrm{fl}========$
$\mathrm{SFO1}$

NUC1 100.622829

P1 $\quad 9.70$ usec

PLW1 46.98899841 W

$=======$ CHANNEL f $2=======$

$\mathrm{SFO} 2$

$1 \mathrm{H}$
$\mathrm{NUC2}$

waltz16

$\begin{array}{ll}\text { PCPD2 } & 90.00 \text { us }\end{array}$

PLW2 11.99499989 W

PLW12 $\quad 0.37713999$

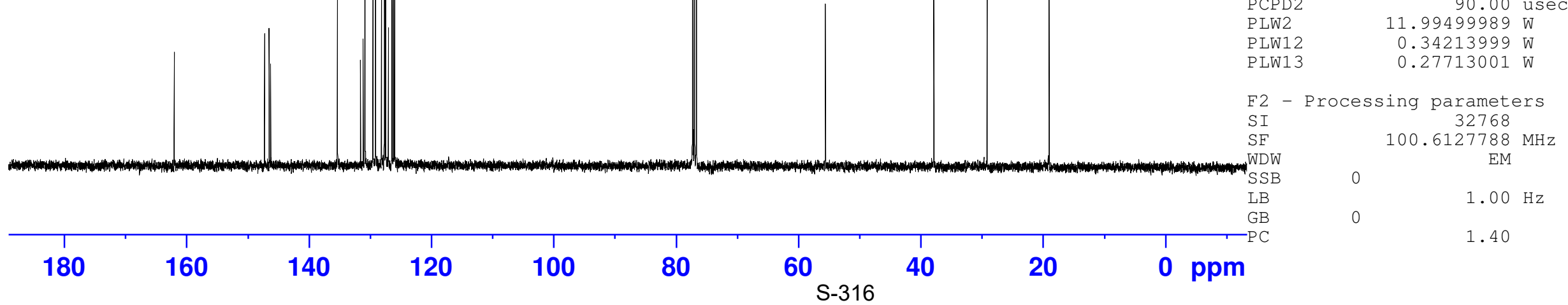


Area Percent Report

\begin{tabular}{|c|c|c|}
\hline $\begin{array}{l}\text { Acq. Operator } \\
\text { Acq. Instrument }\end{array}$ & Instrument 1 & Seq. Line : 4 \\
\hline Injection Date & 1/22/2019 $11: 24: 29$ AM & $\begin{array}{c}\text { Location : vial } 88 \\
\text { Inj }: \quad 1\end{array}$ \\
\hline
\end{tabular}

Different Inj Volume from Sequence! Actual Inj Volume : $5.000 \mu$

Acq. Method : C: \CHEM32\1\DATA \QDY 2019-01-22 10-38-43\AD-10-20.M

Last changed : 11/28/2014 10:15:17 AM

Analysis Method : C: \CHEM32\1\METHODS \OD-01-10-0.3.M

Last changed : 5/29/2019 9:25:17 PM

Additional Info: Peak

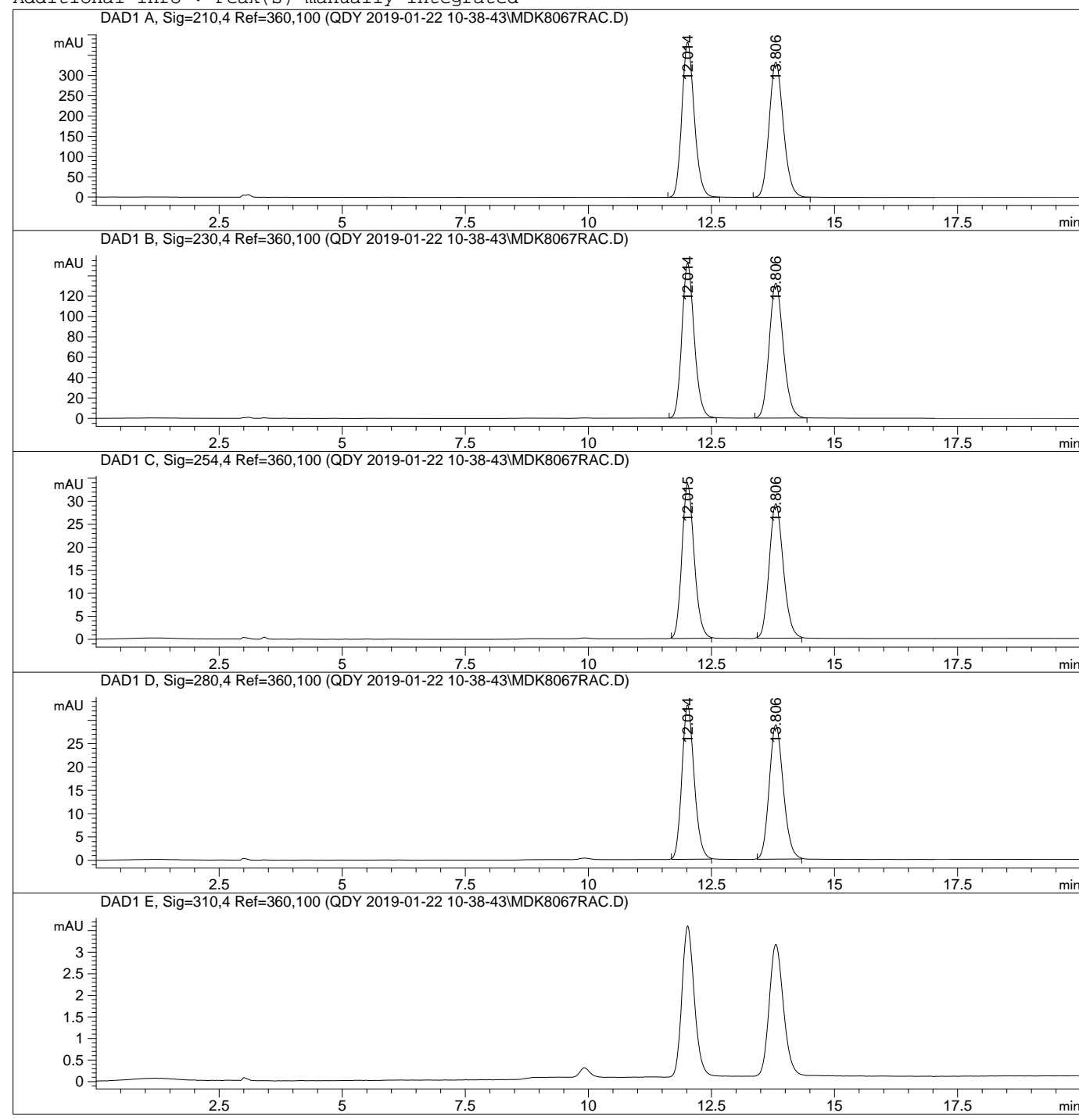

$\begin{array}{lll} & & \\ \text { Sorted By } & : & \text { Signal } \\ \text { Multiplier } & \vdots & 1.0000 \\ \text { Dilution } & : & 1.0000\end{array}$

Use Multiplier \& Dilution Factor with ISTDS

Signal 1: DAD1 A, Sig=210, 4 Ref $=360,100$

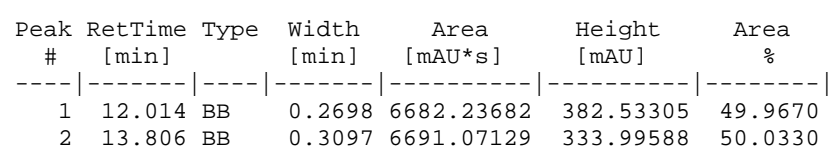

Totals :

$1.33733 \mathrm{e} 4 \quad 716.52893$

Signal 2: DAD1 B, Sig $=230,4$ Ref $=360,100$

\begin{tabular}{cccccc}
$\begin{array}{c}\text { Peak RetTime Type } \\
\text { \# }\end{array}\left[\begin{array}{c}\text { Width } \\
{[\mathrm{min}]}\end{array}\right.$ & $\begin{array}{c}\text { Area } \\
{[\mathrm{mAU}}\end{array}$ & $\begin{array}{l}\text { Height } \\
{[\mathrm{mAU}]}\end{array}$ & $\begin{array}{c}\text { Area } \\
\%\end{array}$ \\
\hdashline-12.014 & $\mathrm{BB}$ & 0.2687 & 2642.23193 & 152.05118 & 50.0072 \\
\hline 2 & $13.806 \mathrm{BB}$ & 0.3086 & 2641.47583 & 132.50363 & 49.9928
\end{tabular}

Totals :

$5283.70776 \quad 284.55481$

Signal 3: DAD1 C, Sig $=254,4$ Ref $=360,100$

\begin{tabular}{|c|c|c|c|c|c|}
\hline $\begin{array}{c}\text { Peak } \\
\#\end{array}$ & $\begin{array}{l}\text { RetTime Type } \\
\text { [min] }\end{array}$ & $\begin{array}{l}\text { Width } \\
\text { [min] }\end{array}$ & $\begin{array}{c}\text { Area } \\
{\left[\mathrm{mAU} U^{\star} \mathrm{s}\right]}\end{array}$ & $\begin{array}{l}\text { Height } \\
{[\mathrm{mAU}]}\end{array}$ & $\begin{array}{c}\text { Area } \\
\%\end{array}$ \\
\hline 1 & $12.015 \mathrm{BB}$ & 0.2685 & 581.44623 & 33.50232 & 49.9912 \\
\hline 2 & $13.806 \mathrm{BB}$ & 0.3083 & 581.65192 & 29.20953 & 50.0088 \\
\hline
\end{tabular}

Totals :

$1163.09814 \quad 62.71185$

Signal 4: DAD1 D, Sig=280, 4 Ref $=360,100$

Peak RetTime Type width Area Height Area

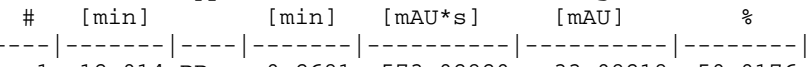

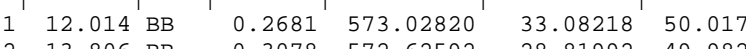

Totals : $\quad 1145.65411 \quad 61.90210$

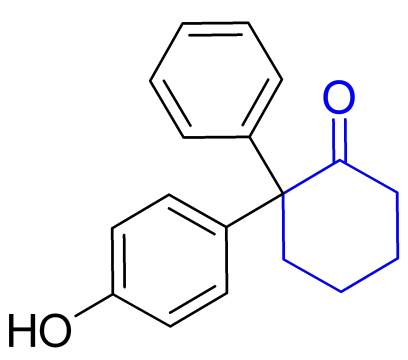

Signal 5: DAD1 E, Sig $=310,4$ Ref $=360,100$

racemic 


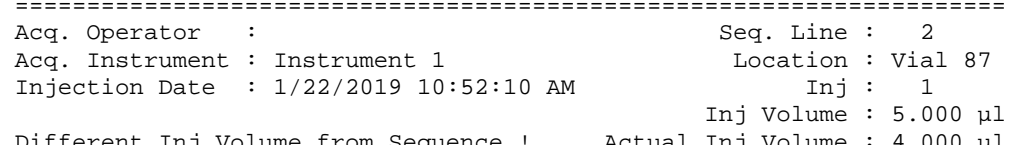

Injection Date : 1/22/2019 10:52:10 AM Inj Volume $\quad \begin{gathered}1 \\ \end{gathered}$ Acq. Method : C: \CHEM32\1\DATA Last changed : 1/22/2019 10:51:17 AM

Analysis Method : C: \CHEM32\1\METHODS\OD-01-10-0.3.M

changed : 5/29/2019 9:25:17 PM

Additional Info: Peak(s) manually integrated

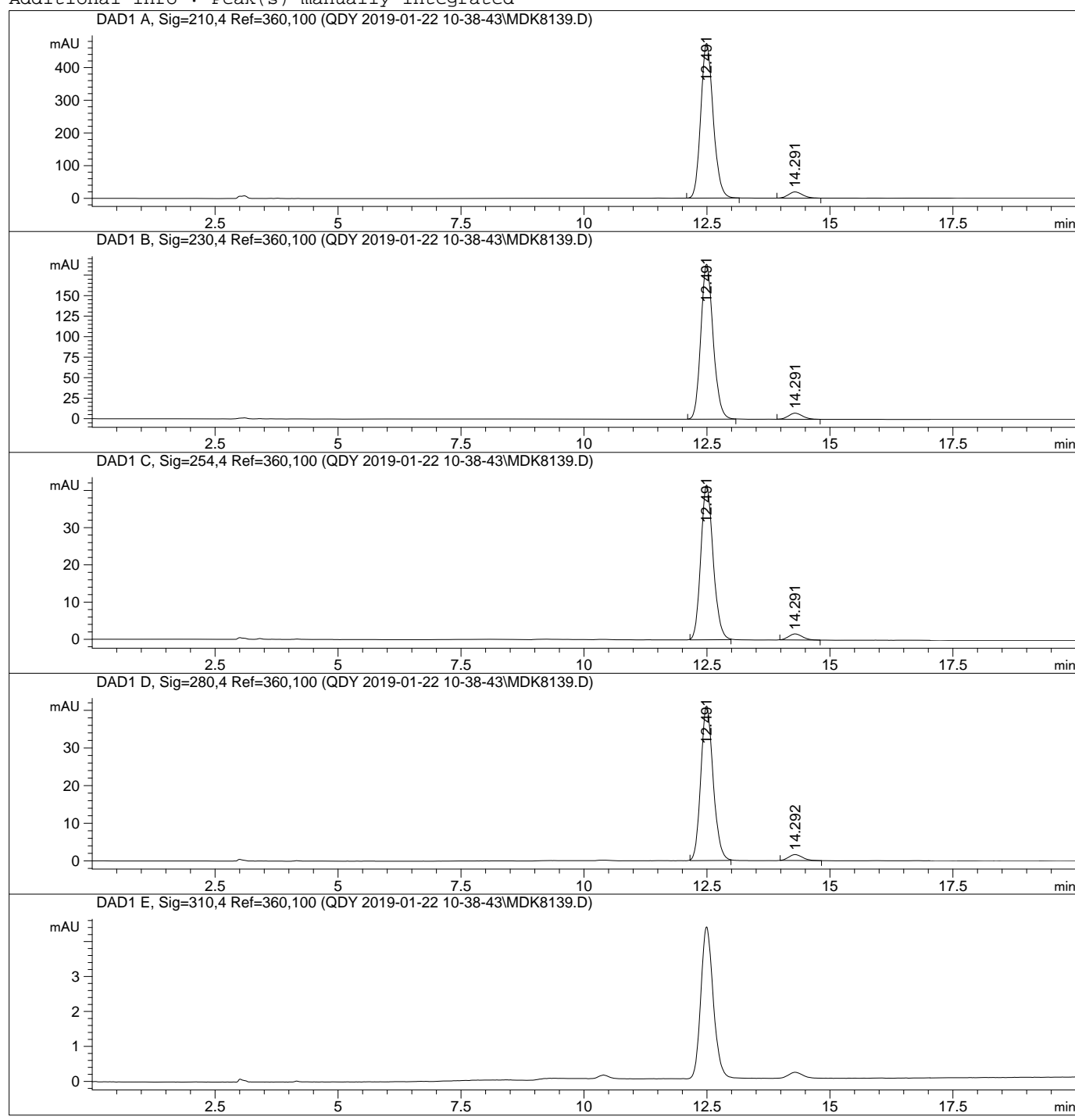

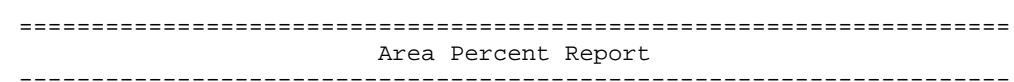

Sorted By

\section{Signal}

Multiplier

1.0000

Use Multiplier \& Dilution Factor with ISTDs

Signal 1: DAD1 A, Sig=210, 4 Ref $=360,100$

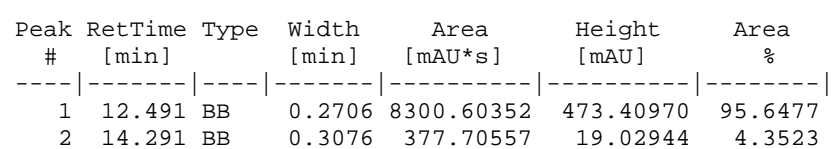

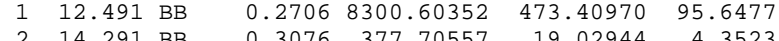

Totals :

$8678.30908 \quad 492.43914$

Signal 2: DAD1 B, Sig $=230,4$ Ref $=360,100$

\begin{tabular}{|c|c|c|c|c|c|}
\hline $\begin{array}{c}\text { Peak } \\
\#\end{array}$ & $\begin{array}{l}\text { RetTime Type } \\
\text { [min] }\end{array}$ & $\begin{array}{l}\text { Width } \\
\text { [min] }\end{array}$ & $\begin{array}{c}\text { Area } \\
{\left[\mathrm{mAU} U^{*} \mathrm{~s}\right]}\end{array}$ & $\begin{array}{l}\text { Height } \\
{[\mathrm{mAU}]}\end{array}$ & $\begin{array}{c}\text { Area } \\
\%\end{array}$ \\
\hline 1 & 12. $491 \mathrm{BB}$ & $\begin{array}{l}0.2672 \\
0.3070\end{array}$ & $\begin{array}{r}3289.61328 \\
148.11389\end{array}$ & $\begin{array}{r}188.89381 \\
7.48055\end{array}$ & $\begin{array}{r}95.6915 \\
4.3885\end{array}$ \\
\hline
\end{tabular}

Totals :

$3437.72717 \quad 196.37436$

Signal 3: DAD1 C, Sig $=254,4$ Ref $=360,100$

\begin{tabular}{|c|c|c|c|c|c|}
\hline $\begin{array}{c}\text { eak } \\
\#\end{array}$ & $\begin{array}{l}\text { RetTime Type } \\
\text { [min] }\end{array}$ & $\begin{array}{l}\text { Width } \\
\text { [min] }\end{array}$ & $\begin{array}{c}\text { Area } \\
{\left[\mathrm{mAU} U^{*} \mathrm{~s}\right]}\end{array}$ & $\begin{array}{l}\text { Height } \\
\text { [mAU] }\end{array}$ & $\begin{array}{c}\text { Area } \\
\%\end{array}$ \\
\hline & & & & & 95.8325 \\
\hline
\end{tabular}

Totals :

$755.10536 \quad 43.19107$

Signal 4: DAD1 D, Sig=280, 4 Ref $=360,100$

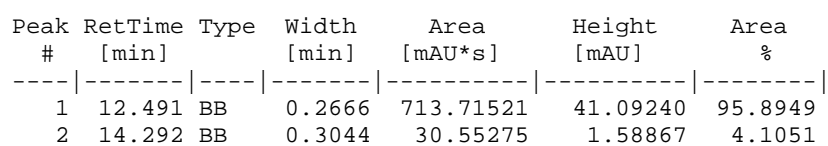

Totals :

$744.26796 \quad 42.68107$

Enantioenriched 
Analysis Method : C: \CHEM32\1\METHODS \OD-01-10-0.3.M

Last changed : 5/29/2019 9:25:17 PM

Additional Info : Peak(s) manually integrated
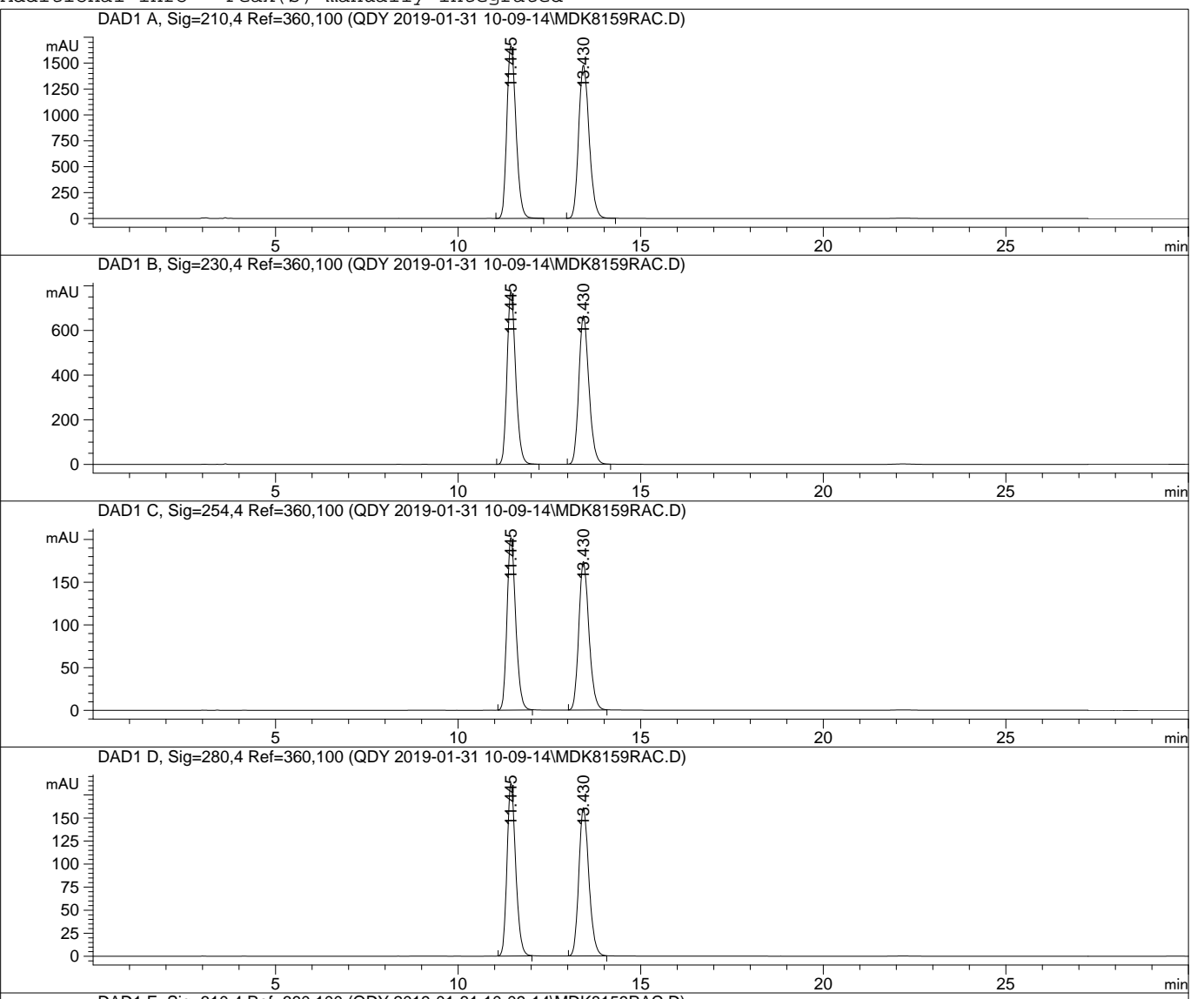

DAD1 E, Sig=310,4 Ref=360, 100 (QDY 2019-01-31 10

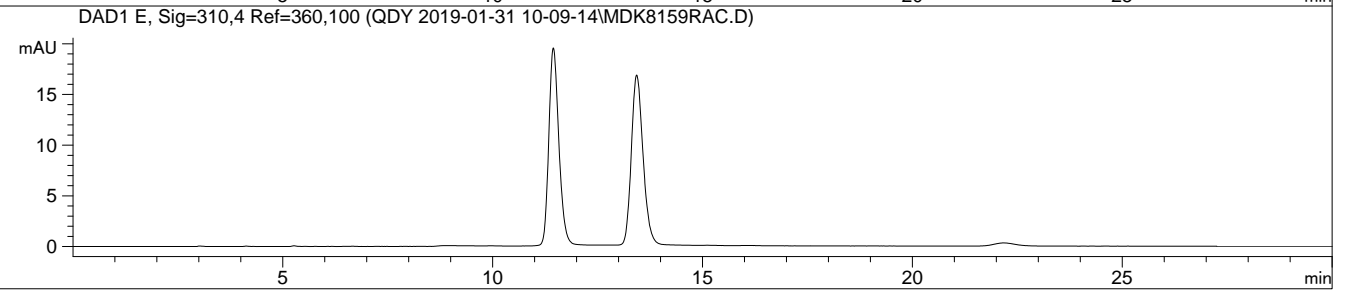

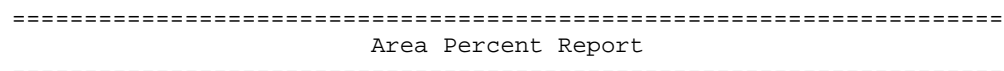

Sorted By

Signal

Multiplier

1.0000

Use Multiplier \& Dilution Factor with ISTDs

Signal 1: DAD1 A, Sig=210, 4 Ref $=360,100$

\begin{tabular}{|c|c|c|c|c|c|}
\hline $\begin{array}{l}\text { Peak } \\
\#\end{array}$ & $\begin{array}{l}\text { RetTime Type } \\
\text { [min] }\end{array}$ & $\begin{array}{l}\text { Width } \\
\text { [min] }\end{array}$ & $\begin{array}{c}\text { Area } \\
{\left[\mathrm{mAU} U^{*} \mathrm{~s}\right]}\end{array}$ & $\begin{array}{l}\text { Height } \\
{[\mathrm{mAU}]}\end{array}$ & $\begin{array}{c}\text { Area } \\
\%\end{array}$ \\
\hline 1 & $11.445 \mathrm{BB}$ & $\begin{array}{l}0.2735 \\
0.3138\end{array}$ & $2.90975 \mathrm{e} 4$ & 1668.00378 & $\begin{array}{l}49.5712 \\
50.4288\end{array}$ \\
\hline
\end{tabular}

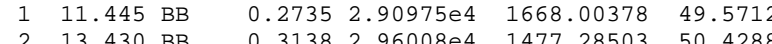

Totals : $\quad 5.86983 \mathrm{e} 4 \quad 3145.28882$

Signal 2: DAD1 B, Sig=230, 4 Ref $=360,100$

\begin{tabular}{|c|c|c|c|c|c|}
\hline $\begin{array}{c}\text { Peak } \\
\#\end{array}$ & $\begin{array}{l}\text { RetTime Type } \\
\text { [min] }\end{array}$ & $\begin{array}{l}\text { Width } \\
\text { [min] }\end{array}$ & $\begin{array}{c}\text { Area } \\
{\left[\mathrm{mAU} U^{*} \mathrm{~s}\right]}\end{array}$ & $\begin{array}{l}\text { Height } \\
{[\mathrm{mAU}]}\end{array}$ & $\begin{array}{c}\text { Area } \\
\%\end{array}$ \\
\hline 1 & $11.445 \mathrm{BB}$ & 0.2550 & 1. $27928 \mathrm{e} 4$ & $\begin{array}{r}773.37354 \\
664.35667\end{array}$ & 49. 9901 \\
\hline
\end{tabular}

Totals :

$2.55907 \mathrm{e} 4 \quad 1437.73029$

Signal 3: DAD1 C, Sig=254, 4 Ref $=360,100$

\begin{tabular}{|c|c|c|c|c|c|}
\hline $\begin{array}{c}\text { Peak } \\
\#\end{array}$ & $\begin{array}{l}\text { RetTime Type } \\
\text { [min] }\end{array}$ & $\begin{array}{l}\text { Width } \\
\text { [min] }\end{array}$ & $\begin{array}{c}\text { Area } \\
{\left[\mathrm{mAU}^{*} \mathrm{~s}\right]}\end{array}$ & $\begin{array}{l}\text { Height } \\
{[\mathrm{mAU}]}\end{array}$ & $\begin{array}{c}\text { Area } \\
\%\end{array}$ \\
\hline 1 & $11.445 \mathrm{BB}$ & $\odot .2553$ & 3346.96191 & 202.01096 & 49.9378 \\
\hline 2 & $13.430 \mathrm{BB}$ & 0.2973 & 3355.30029 & 173.72388 & 50.0622 \\
\hline
\end{tabular}

Signal 4: DAD1 D, Sig $=280,4$ Ref $=360,100$

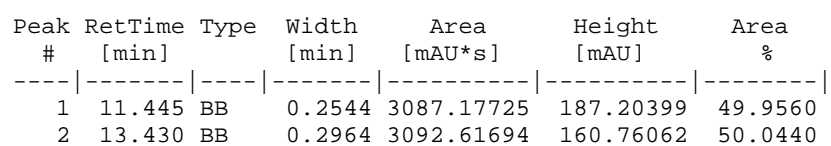

Totals :

$6179.79419 \quad 347.96461$

Signal 5: DAD1 E, Sig=310, 4 Ref $=360,100$

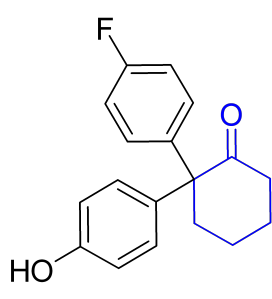

racemic

*** End of Report *** 


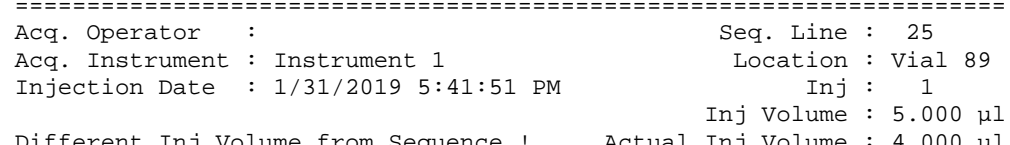

Different Inj Volume from Sequence ! Actual Inj Volume : $5.000 \mu \mathrm{\mu}$

Acq. Method : C: \CHEM32\1\DATA

Last changed : 1/31/2019 5:40:57 PM

Analysis Method : C: \CHEM32\1\METHODS \OD-01-10-0.3.M

Last changed : 5/29/2019 9:25:17 PM

$\frac{\text { Additional Info : Peak (s) manually integrated }}{\text { DAD1 A, Sig=210,4 Ref }=360,100 \text { (QDY 2019-01-31 10-09-14MDK8160. D) }}$
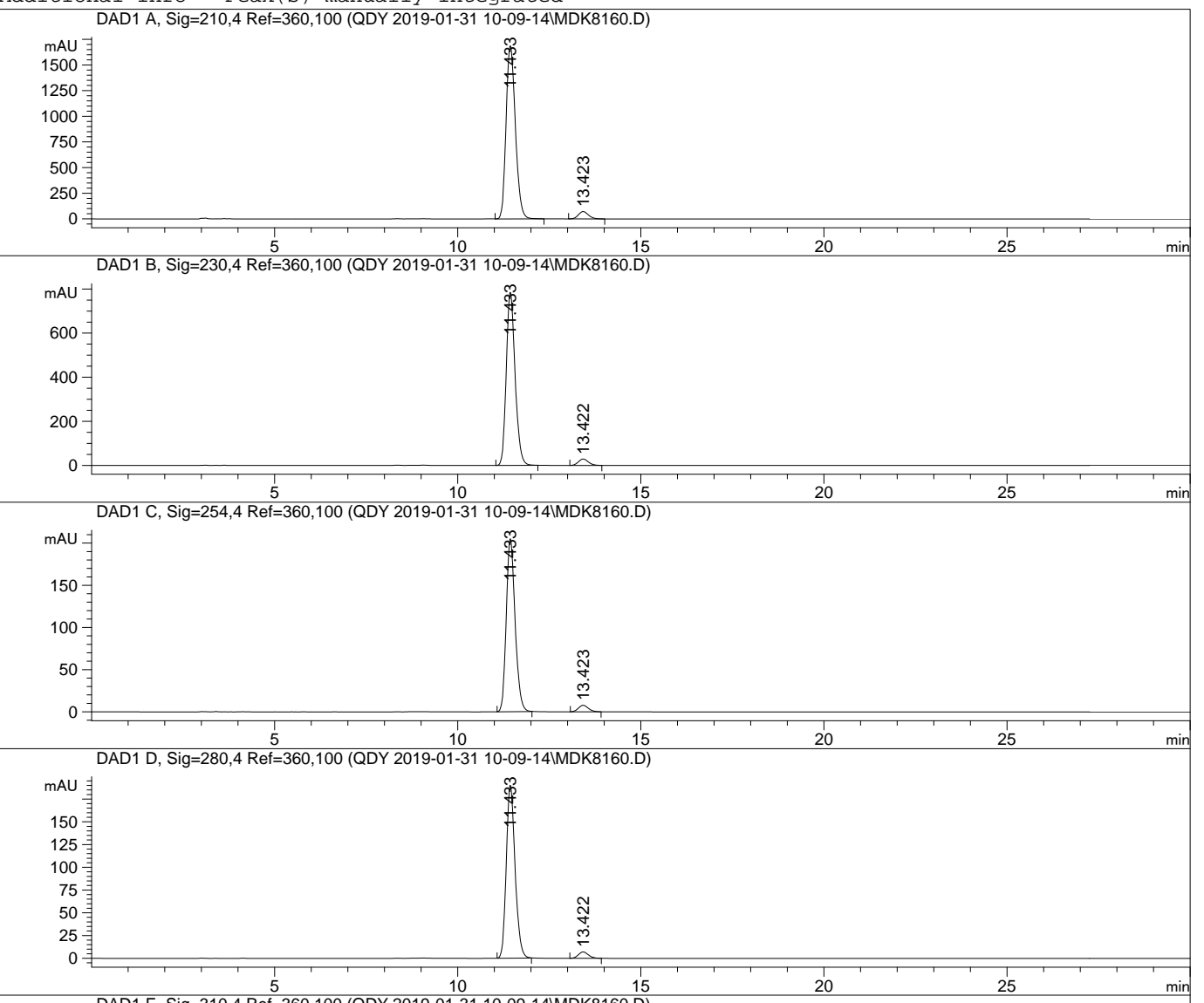

$\frac{1}{\text { DAD1 E, Sig }=310,4 \text { Ref }=360,100} \frac{10}{\text { (QDY 2019-01-31 }} \frac{1}{10-09-14 \text { M MDK8160.D) }}$

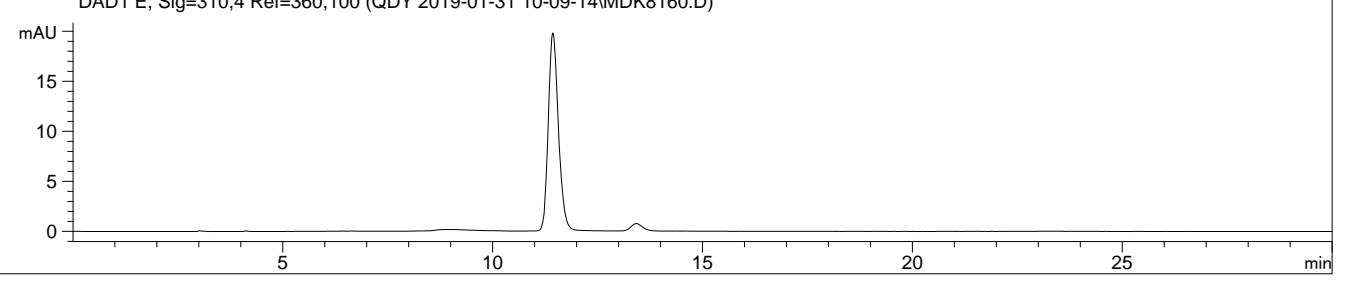

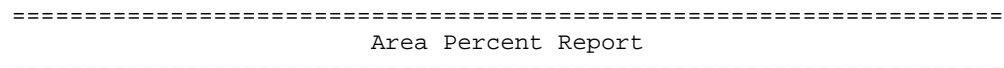

Sorted By

Signal

Multiplier

1.0000

Use Multiplier \& Dilution Factor with ISTDs

Signal 1: DAD1 A, Sig=210, 4 Ref $=360,100$

\begin{tabular}{|c|c|c|c|c|c|}
\hline $\begin{array}{c}\text { Peak } \\
\#\end{array}$ & $\begin{array}{l}\text { RetTime Type } \\
\text { [min] }\end{array}$ & $\begin{array}{l}\text { Width } \\
\text { [min] }\end{array}$ & $\begin{array}{c}\text { Area } \\
{\left[\mathrm{mAU} U^{*} \mathrm{~s}\right]}\end{array}$ & $\begin{array}{l}\text { Height } \\
{[\mathrm{mAU}]}\end{array}$ & $\begin{array}{c}\text { Area } \\
\%\end{array}$ \\
\hline 1 & $11.433 \mathrm{BB}$ & 0.2761 & $2.98042 \mathrm{e} 4$ & 1687.18335 & $\begin{array}{r}95.5607 \\
4.4393\end{array}$ \\
\hline
\end{tabular}

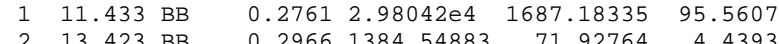

Totals : $\quad 3.11888 \mathrm{e} 4 \quad 1759.11098$

Signal 2: DAD1 B, Sig $=230,4$ Ref $=360,100$

\begin{tabular}{|c|c|c|c|c|c|}
\hline$\underset{\#}{\text { eak }}$ & $\begin{array}{l}\text { RetTime Type } \\
\text { [min] }\end{array}$ & $\begin{array}{l}\text { Width } \\
\text { [min] }\end{array}$ & $\begin{array}{c}\text { Area } \\
{\left[\mathrm{mAU}^{*} \mathrm{~s}\right]}\end{array}$ & $\begin{array}{l}\text { Height } \\
{[\mathrm{mAU}]}\end{array}$ & $\begin{array}{c}\text { Area } \\
\%\end{array}$ \\
\hline & $3 \mathrm{~B}$ & 0.295 & $\begin{array}{r}1.31288 \mathrm{e} 4 \\
557.4272\end{array}$ & & \\
\hline
\end{tabular}

Totals :

$1.36862 \mathrm{e} 4 \quad 814.40220$

Signal 3: DAD1 C, Sig=254, 4 Ref $=360,100$

\begin{tabular}{|c|c|c|c|c|c|}
\hline $\begin{array}{c}\text { Peak } \\
\#\end{array}$ & $\begin{array}{l}\text { RetTime Type } \\
{[\text { min] }}\end{array}$ & $\begin{array}{l}\text { Width } \\
\text { [min] }\end{array}$ & $\begin{array}{c}\text { Area } \\
{\left[\mathrm{mAU} U^{*} \mathrm{~s}\right]}\end{array}$ & $\begin{array}{l}\text { Height } \\
{[\mathrm{mAU}]}\end{array}$ & $\begin{array}{c}\text { Area } \\
\%\end{array}$ \\
\hline 1 & $11.433 \mathrm{BB}$ & 0.2574 & 3434.78662 & 205.07155 & 95.8801 \\
\hline 2 & $13.423 \mathrm{BB}$ & 0.2947 & 147.58890 & 7.73210 & 4.1199 \\
\hline
\end{tabular}

Signal 4: DAD1 D, Sig=280, 4 Ref $=360,100$

\begin{tabular}{|c|c|c|c|c|c|}
\hline $\begin{array}{c}\text { Peak } \\
\#\end{array}$ & $\begin{array}{l}\text { RetTime Type } \\
\text { [min] }\end{array}$ & $\begin{array}{l}\text { Width } \\
\text { [min] }\end{array}$ & $\begin{array}{c}\text { Area } \\
{\left[\mathrm{mAU}^{\star} \mathrm{s}\right]}\end{array}$ & $\begin{array}{l}\text { Height } \\
{[\mathrm{mAU}]}\end{array}$ & $\begin{array}{c}\text { Area } \\
\%\end{array}$ \\
\hline 1 & $\begin{array}{l}11.433 \mathrm{BB} \\
13.422 \mathrm{BB}\end{array}$ & $\begin{array}{l}0.2565 \\
0.2965\end{array}$ & $\begin{array}{r}3169.35620 \\
134.37640\end{array}$ & $\begin{array}{r}190.09380 \\
7.04481\end{array}$ & $\begin{array}{r}95.9326 \\
4.0674\end{array}$ \\
\hline
\end{tabular}

Totals : $3303.73260 \quad 197.13861$

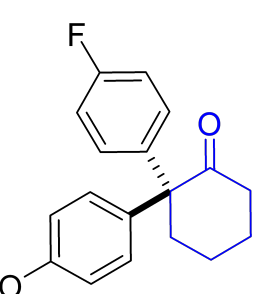

$2 b$

Signal 5: DAD1 E, Sig $=310,4$ Ref $=360,100$

*** End of Report *** 


\begin{tabular}{|c|c|c|}
\hline $\begin{array}{l}\text { Acq. Operator } \\
\text { Acq. Instrument } \\
\text { Injection Date }\end{array}$ & $\begin{array}{l}\text { Instrument } 1 \\
1 / 30 / 20191: 41: 42 \text { PM }\end{array}$ & $\begin{array}{r}\text { Seq. Line } \\
\text { Location } \\
\text { Inj }\end{array}$ \\
\hline
\end{tabular}

Injection Date $\quad \begin{array}{cc}\text { Inj } & 1 \\ & \text { Inj Volume }: 5.000 \mu \mathrm{\mu l}\end{array}$

Acq. Method : C: \CHEM32\1\DATA QQDY 2019-01-30 13-28-12\AD-10-20.M

Last changed $: 1 / 30 / 20191: 40: 50$ PM

Analysis Method : C: \CHEM32\1\METHODS\OD-01-10-0.3.M

Last changed : 5/29/2019 9:25:17 PM

Additional Info: Peak(s) manually integrated
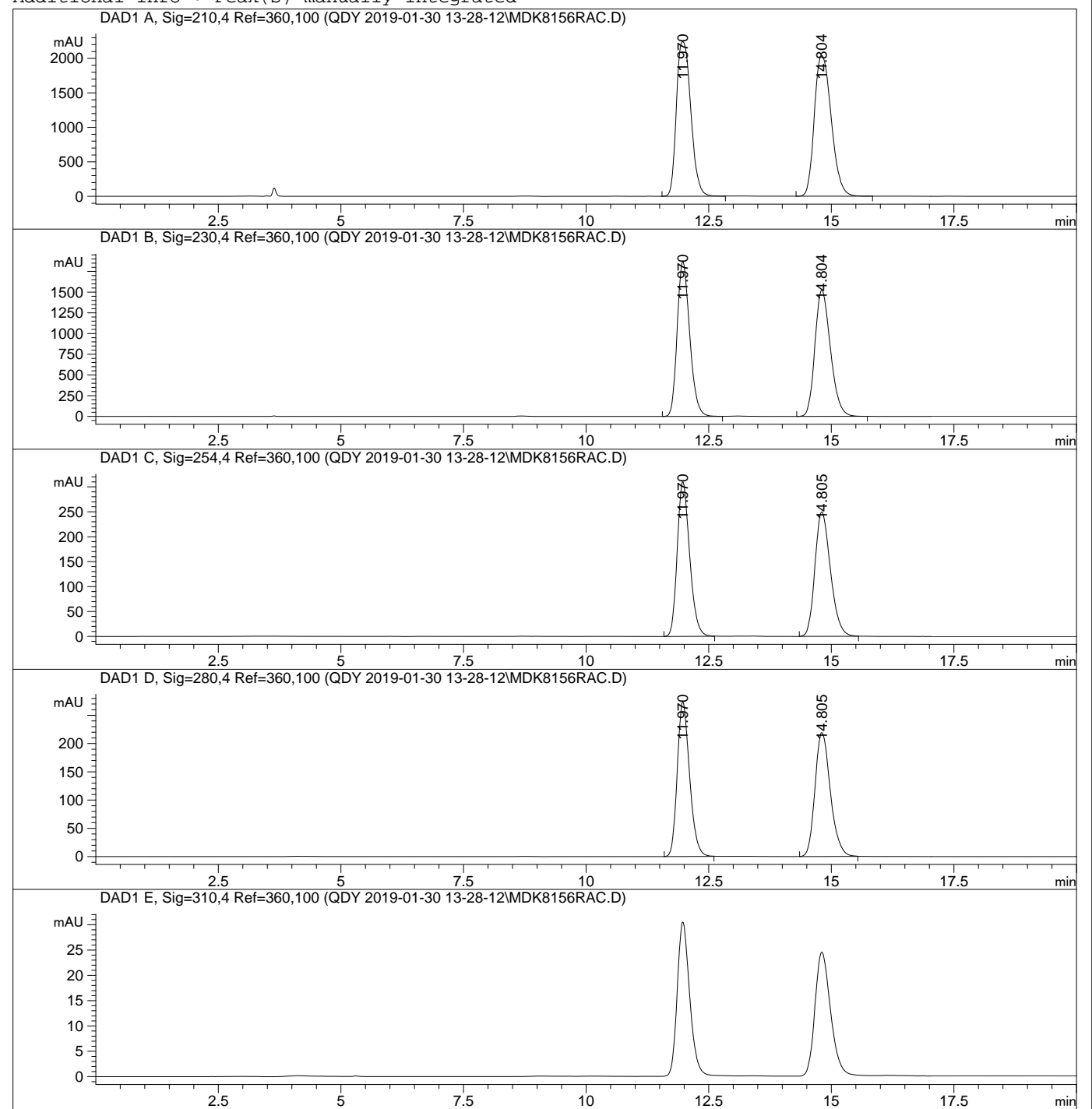

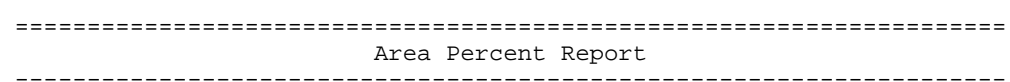

Sorted By
Multiplier

Signal

1.0000

Use Multiplier \& Dilution Factor with ISTDs

Signal 1: DAD1 A, Sig=210, 4 Ref $=360,100$

\begin{tabular}{cccccc}
$\begin{array}{c}\text { Peak RetTime Type } \\
\text { \# }\end{array}\left[\begin{array}{c}\text { Width } \\
{[\mathrm{min}]}\end{array}\right.$ & $\begin{array}{c}\text { Area } \\
{[\mathrm{mAU}}\end{array}$ & $\begin{array}{l}\text { Height } \\
{[\mathrm{mAU}]}\end{array}$ & $\begin{array}{c}\text { Area } \\
\%\end{array}$ \\
\hdashline-11.970 & $\mathrm{BB}$ & 0.3218 & $4.57030 \mathrm{e} 4$ & 2242.48511 & 48.3322 \\
\hline 2 & $14.804 \mathrm{BB}$ & 0.3773 & $4.88573 \mathrm{e} 4$ & 2040.67371 & 51.6678
\end{tabular}

$\begin{array}{lllllll}1 & 11.970 & \text { BB } & 0.3218 & 4.57030 \mathrm{e} 4 & 2242.48511 & 48.3322\end{array}$

Totals : $\quad 9.45603 \mathrm{e} 4 \quad 4283.15881$

Signal 2: DAD1 B, Sig=230, 4 Ref $=360,100$

\begin{tabular}{cccccc}
$\begin{array}{c}\text { Peak RetTime Type } \\
\#\end{array}\left[\begin{array}{c}\text { Width } \\
{[\mathrm{min}]}\end{array}\right.$ & $\begin{array}{c}\text { Area } \\
{[\mathrm{mAU}}\end{array}$ & $\begin{array}{l}\text { Height } \\
{[\mathrm{mAU}]}\end{array}$ & $\begin{array}{c}\text { Area } \\
\%\end{array}$ \\
\hdashline-11.970 & $\mathrm{BB}$ & 0.2753 & $3.31938 \mathrm{e} 4$ & 1867.73608 & 49.7577 \\
\hline 2 & $14.804 \mathrm{BB}$ & 0.3406 & $3.35171 \mathrm{e} 4$ & 1523.88025 & 50.2423
\end{tabular}

Totals :

$6.67109 \mathrm{e} 4 \quad 3391.61633$

Signal 3: DAD1 C, Sig $=254,4$ Ref $=360,100$

\begin{tabular}{|c|c|c|c|c|c|}
\hline $\begin{array}{c}\text { Peak } \\
\#\end{array}$ & $\begin{array}{l}\text { RetTime Type } \\
\text { [min] }\end{array}$ & $\begin{array}{l}\text { Width } \\
\text { [min] }\end{array}$ & $\begin{array}{c}\text { Area } \\
{\left[\mathrm{mAU}^{\star} \mathrm{s}\right]}\end{array}$ & $\begin{array}{l}\text { Height } \\
{[\mathrm{mAU}]}\end{array}$ & $\begin{array}{c}\text { Area } \\
\%\end{array}$ \\
\hline 1 & $\begin{array}{l}11.970 \mathrm{BB} \\
14.805 \mathrm{BB}\end{array}$ & $\begin{array}{l}0.2712 \\
0.3383\end{array}$ & 5450.22412 & 309.94727 & $\begin{array}{l}49.8428 \\
50.1572\end{array}$ \\
\hline
\end{tabular}

Totals :

$1.09348 \mathrm{e} 4 \quad 559.56456$

Signal 4: DAD1 D, Sig=280, 4 Ref $=360,100$

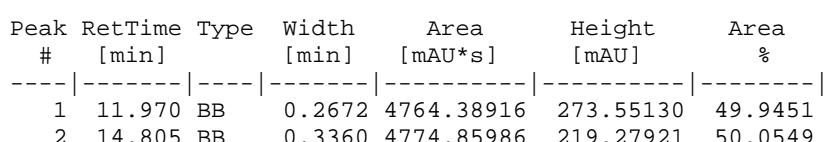

Totals :

$9539.24902 \quad 492.83051$

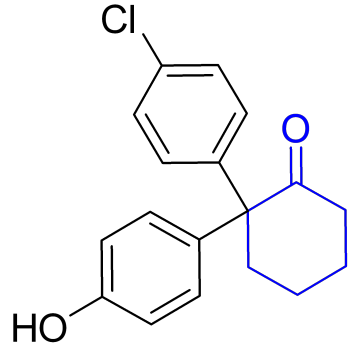

Signal 5: DAD1 E, Sig=310, 4 Ref $=360,100$

$* * *$ End of Report *** 


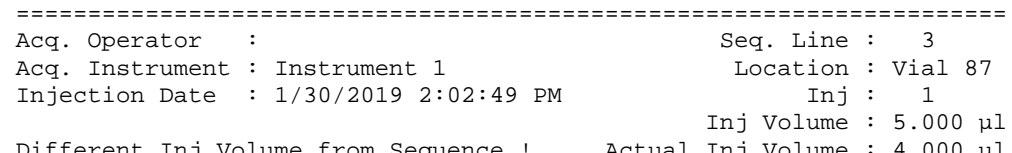

Different Inj Volume from Sequence ! Actual Inj Volume : $5.000 \mu \mathrm{\mu}$ Acq. Method : C: \CHEM32\1\DATA Last changed : $1 / 30 / 20191: 40: 50$ PM

Analysis Method : C: \CHEM32\1\METHODS\OD-01-10-0.3.M

: $5 / 29 / 2019$ 9:25:17 PM

Additional Info : Peak(s) manually integrated
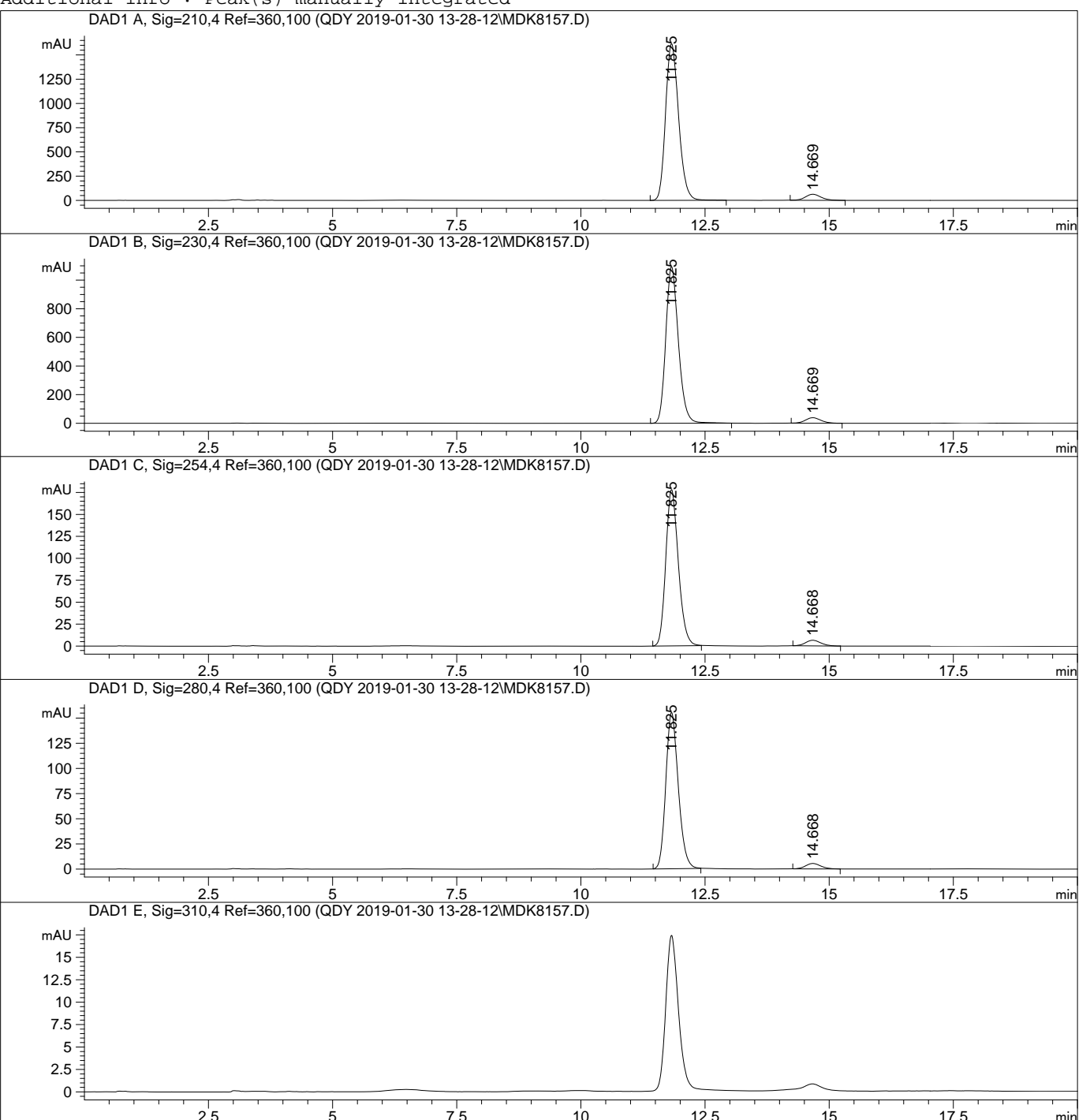

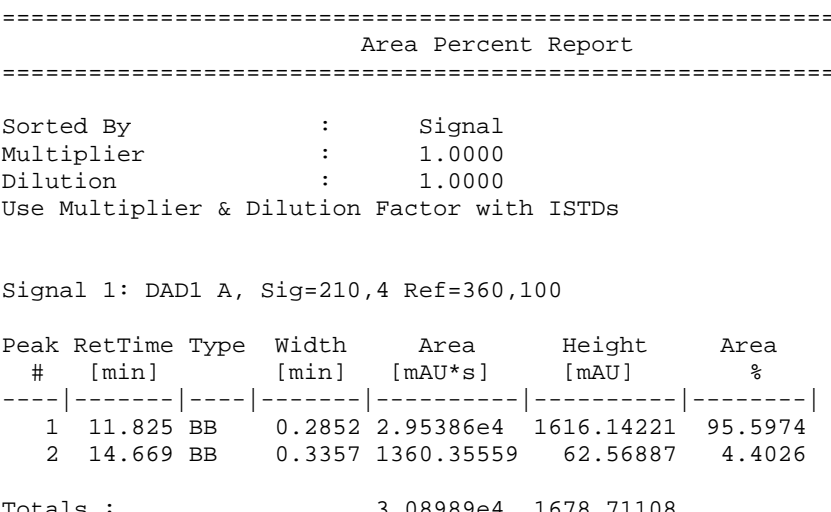

Totals :

3. $08989 \mathrm{e} 4 \quad 1678.71108$

Signal 2: DAD1 B, Sig $=230,4$ Ref $=360,100$

\begin{tabular}{|c|c|c|c|c|c|}
\hline $\begin{array}{c}\text { Peak } \\
\text { \# }\end{array}$ & $\begin{array}{l}\text { RetTime Type } \\
\text { [min] }\end{array}$ & $\begin{array}{l}\text { Width } \\
\text { [min] }\end{array}$ & $\begin{array}{c}\text { Area } \\
{\left[\mathrm{mAU}^{\star} \mathrm{s}\right]}\end{array}$ & $\begin{array}{l}\text { Height } \\
{[\mathrm{mAU}]}\end{array}$ & $\begin{array}{c}\text { Area } \\
\%\end{array}$ \\
\hline & $\begin{array}{l}11.825 \mathrm{BB} \\
14.669 \mathrm{BB}\end{array}$ & $\begin{array}{l}0.2728 \\
0.3352\end{array}$ & $\begin{array}{c}1.93980 \mathrm{e} 4 \\
837.4356\end{array}$ & $\begin{array}{r}1094.51636 \\
38.58882\end{array}$ & $\begin{array}{r}95.8615 \\
4.1385\end{array}$ \\
\hline
\end{tabular}

Totals :

$2.02354 \mathrm{e} 4 \quad 1133.10518$

Signal 3: DAD1 C, Sig $=254,4$ Ref $=360,100$

\begin{tabular}{|c|c|c|c|c|c|c|}
\hline $\begin{array}{c}\text { Peak } \\
\#\end{array}$ & $\begin{array}{l}\text { RetTime } \\
{[\text { min }]}\end{array}$ & Type & $\begin{array}{l}\text { Width } \\
\text { [min] }\end{array}$ & $\begin{array}{c}\text { Area } \\
{\left[\mathrm{mAU}^{\star} \mathrm{s}\right]}\end{array}$ & $\begin{array}{l}\text { Height } \\
{[\mathrm{mAU}]}\end{array}$ & $\begin{array}{c}\text { Area } \\
\%\end{array}$ \\
\hline 1 & 11.825 & $B B$ & 0.2714 & 3137.05786 & 178.14732 & 95.7560 \\
\hline 2 & 14.668 & $B B$ & $\odot .3352$ & 139.03633 & 6.45818 & 4.2440 \\
\hline
\end{tabular}

Signal 4: DAD1 D, Sig=280, 4 Ref $=360,100$

\begin{tabular}{|c|c|c|c|c|c|}
\hline $\begin{array}{c}\text { Peak } \\
\#\end{array}$ & $\begin{array}{l}\text { RetTime Type } \\
\text { [min] }\end{array}$ & $\begin{array}{l}\text { Width } \\
\text { [min] }\end{array}$ & $\begin{array}{c}\text { Area } \\
{\left[\mathrm{mAU} U^{*} \mathrm{~s}\right]}\end{array}$ & $\begin{array}{l}\text { Height } \\
{[\mathrm{mAU}]}\end{array}$ & $\begin{array}{c}\text { Area } \\
\%\end{array}$ \\
\hline 1 & $11.825 \mathrm{BB}$ & 0.2 & 2714.91138 & 155.3 & 399 \\
\hline 2 & $14.668 \mathrm{BB}$ & 0.3303 & 114.89316 & 5.39916 & 4.0601 \\
\hline
\end{tabular}

Totals : $2829.80453 \quad 160.79685$

Signal 5: DAD1 E, Sig $=310,4$ Ref $=360,100$

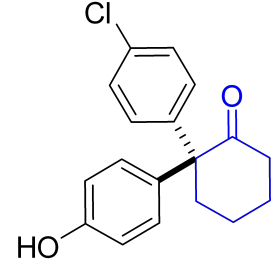

2c

Enantioenriched

*** End of Report *** 


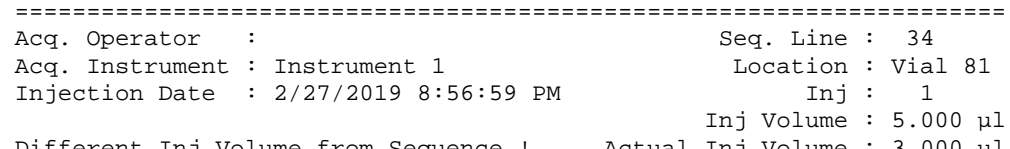

Different Inj Volume from Sequence ! Actual Inj Volume : $5.000 \mu \mathrm{\mu}$

Acq. Method : C: \CHEM32\1\DATA\QDY 2019-02-27 10-32-22\AD-10-30.M

Last changed : 11/30/2015 12:57:24 PM

Analysis Method : C: \CHEM32\1\METHODS \OD-02-10.M

Last changed : 7/4/2019 9:46:26 PM

Additional Info (modified after loading)
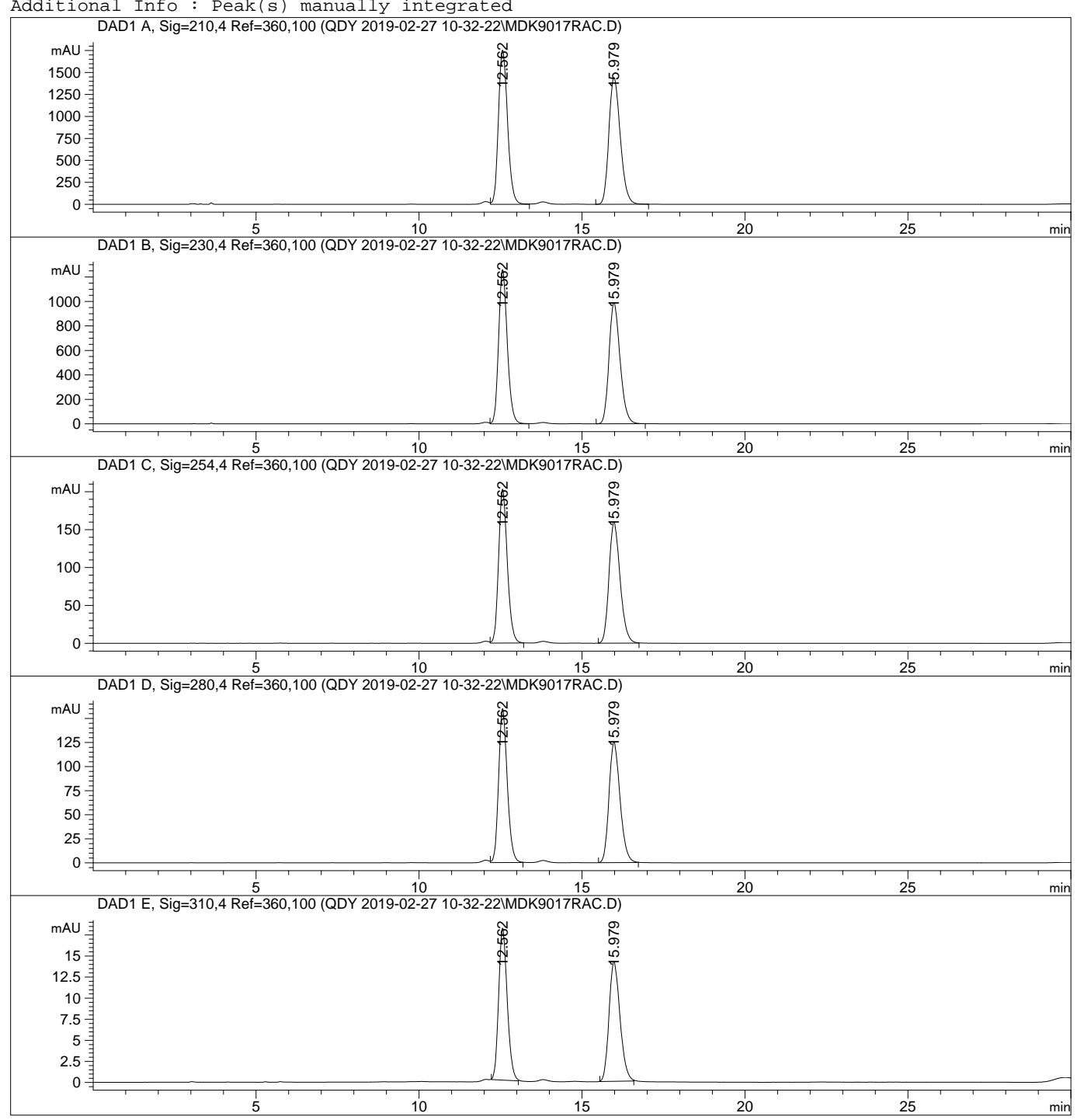

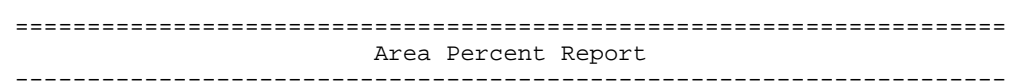

Sorted By
Multiplier

\section{Signal}

1.0000

Use Multiplier \& Dilution Factor with ISTDs

Signal 1: DAD1 A, Sig=210, 4 Ref $=360,100$

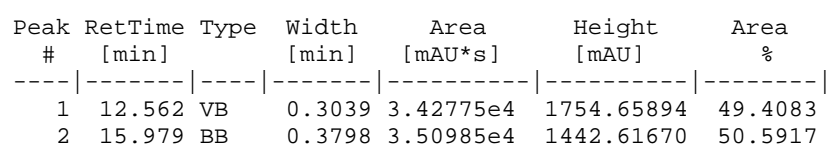

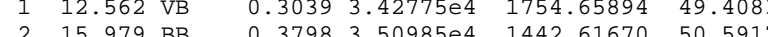

Totals :

$6.93760 \mathrm{e} 4 \quad 3197.27563$

Signal 2: DAD1 B, Sig=230, 4 Ref $=360,100$

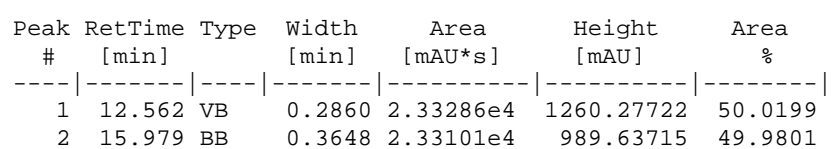

Totals :

4.66387e4 2249.91437

Signal 3: DAD1 C, Sig $=254,4$ Ref $=360,100$

\begin{tabular}{|c|c|c|c|c|c|}
\hline $\begin{array}{c}\text { Peak } \\
\#\end{array}$ & $\begin{array}{l}\text { RetTime Type } \\
\text { [min] }\end{array}$ & $\begin{array}{l}\text { Width } \\
\text { [min] }\end{array}$ & $\begin{array}{c}\text { Area } \\
{\left[\mathrm{mAU}^{\star} \mathrm{s}\right]}\end{array}$ & $\begin{array}{l}\text { Height } \\
{[\mathrm{mAU}]}\end{array}$ & $\begin{array}{c}\text { Area } \\
\%\end{array}$ \\
\hline 1 & $12.562 \mathrm{VB}$ & 0.2867 & 3773.07446 & 203.15865 & 49.9263 \\
\hline 2 & $15.979 \mathrm{BB}$ & 0.3662 & 3784.21265 & 159.85712 & 50.0737 \\
\hline
\end{tabular}

Totals :

$7557.28711 \quad 363.01576$

Signal 4: DAD1 D, Sig=280, 4 Ref $=360,100$

\begin{tabular}{|c|c|c|c|c|c|}
\hline eak & $\begin{array}{c}\text { RetTime } T \\
{[\text { [min] }}\end{array}$ & $\begin{array}{l}\text { Width } \\
\text { [min] }\end{array}$ & $\begin{array}{c}\text { Area } \\
{\left[\mathrm{mAU}^{*} \mathrm{~s}\right]}\end{array}$ & $\begin{array}{l}\text { Height } \\
\text { [mAU] }\end{array}$ & $\begin{array}{c}\text { Area } \\
\%\end{array}$ \\
\hline & $.00 \angle \mathrm{VD}$ & & 50 & & \\
\hline
\end{tabular}

Totals :

$5893.62744 \quad 285.22494$

Signal 5: DAD1 E, Sig=310, 4 Ref $=360,100$

\begin{tabular}{|c|c|c|c|c|c|}
\hline eak & $\begin{array}{l}\text { RetTime Type } \\
\text { [min] }\end{array}$ & $\begin{array}{l}\text { Width } \\
\text { [min] }\end{array}$ & $\begin{array}{c}\text { Area } \\
{\left[\mathrm{mAU} U^{*} \mathrm{~s}\right]}\end{array}$ & $\begin{array}{l}\text { Height } \\
{[\mathrm{mAU}]}\end{array}$ & $\begin{array}{c}\text { Area } \\
\%\end{array}$ \\
\hline & $2 \mathrm{BE}$ & .2830 & $\begin{array}{l}329.19049 \\
332.11105\end{array}$ & $\begin{array}{l}18.02747 \\
14.19235\end{array}$ & \\
\hline
\end{tabular}

Totals :

$661.30154 \quad 32.21982$ 


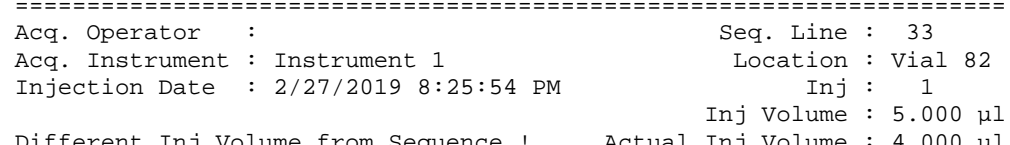

Different Inj Volume from Sequence! Actual Inj Volume : $5.000 \mu \mathrm{\mu}$

Acq. Method : C: \CHEM32\1\DATA QQDY 2019-02-27 10-32-22\AD-10-30.M

Last changed : 11/30/2015 12:57:24 PM

Analysis Method : C: \CHEM32\1\METHODS \OD-01-10-0.3.M

Last changed : 5/29/2019 9:25:17 PM

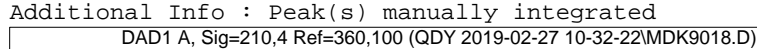

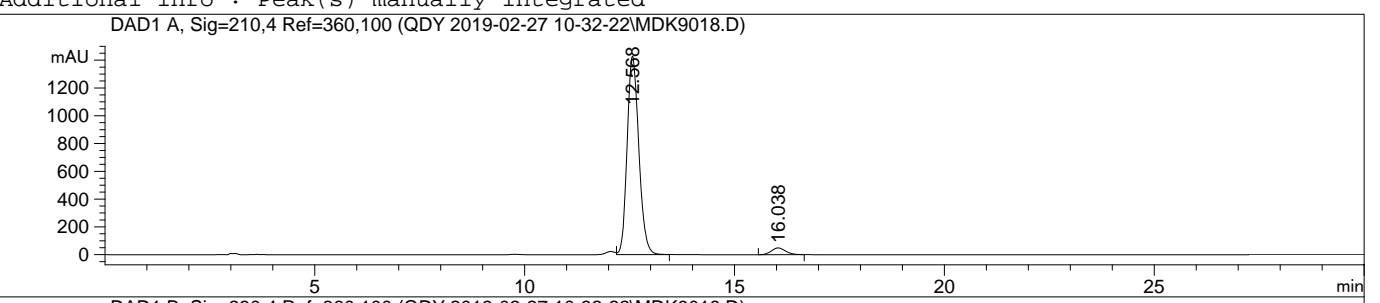

DAD1 B, Sig=230,4 Ref=360, 100 (QDY 2019-02-27 10-32-221MDK9018.D)

20

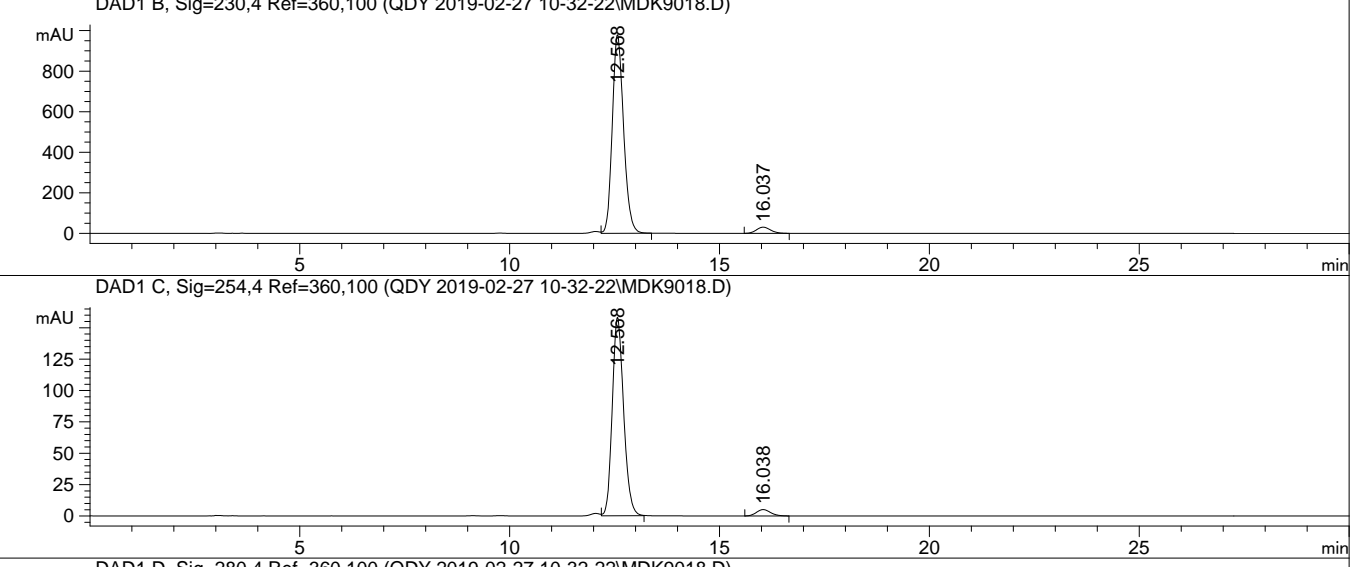

DAD1 D, Sig=280,4 Ref=360, 100 (QDY 2019-02-27 10-32-221MDK9018.D)

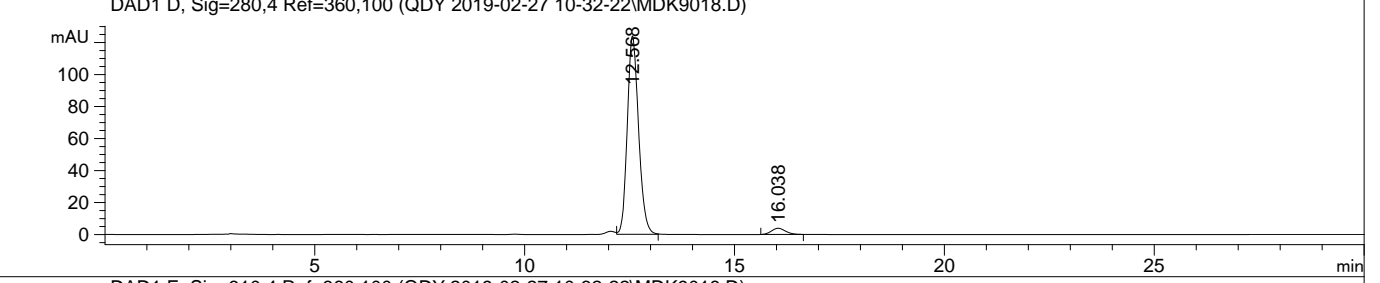

$\begin{array}{ccr}5 & 10 & 15 \\ \text { DAD1 E, Sig=310,4 Reff=360, } 100 \text { (QDY 2019-02-27 } & 10-32-22 \text { MDLK9018.D) }\end{array}$

$\mathrm{mAU}$ 手

12奏

等

2 $\min$

(1)

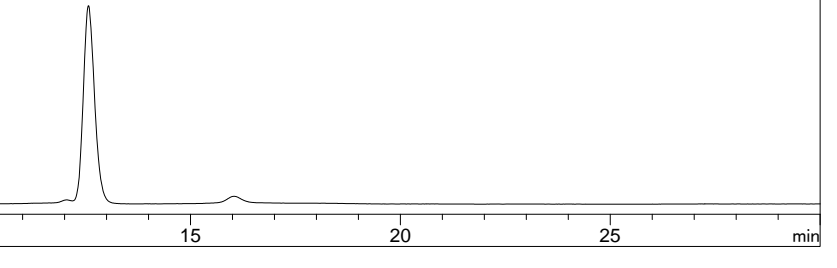

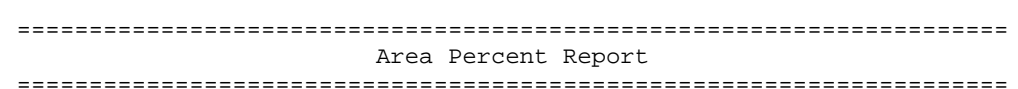

Sorted By
Multiplier

\section{Signal}

1.0000

Use Multiplier \& Dilution Factor with ISTDS

Signal 1: DAD1 A, Sig=210, 4 Ref $=360,100$

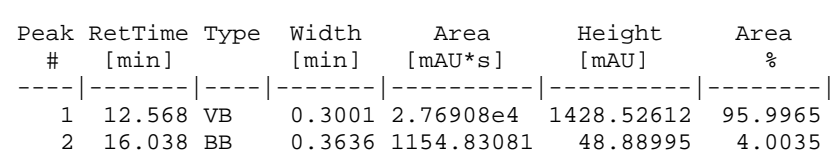

Totals :

$2.88456 \mathrm{e} 4 \quad 1477.41608$

Signal 2: DAD1 B, Sig $=230,4$ Ref $=360,100$

\begin{tabular}{|c|c|c|c|c|c|}
\hline $\begin{array}{c}\text { Peak } \\
\#\end{array}$ & $\begin{array}{l}\text { RetTime Type } \\
\text { [min] }\end{array}$ & $\begin{array}{l}\text { Width } \\
\text { [min] }\end{array}$ & $\begin{array}{c}\text { Area } \\
{\left[\mathrm{mAU}^{\star} \mathrm{s}\right]}\end{array}$ & $\begin{array}{l}\text { Height } \\
{[\mathrm{mAU}]}\end{array}$ & $\begin{array}{c}\text { Area } \\
\%\end{array}$ \\
\hline $\begin{array}{l}1 \\
2\end{array}$ & $\begin{array}{l}12.568 \mathrm{VB} \\
16.037 \mathrm{BB}\end{array}$ & $\begin{array}{l}0.2890 \\
0.3649\end{array}$ & $\begin{array}{c}1.83723 \mathrm{e} 4 \\
724.36249\end{array}$ & $\begin{array}{r}978.83838 \\
30.74253\end{array}$ & $\begin{array}{r}96.2069 \\
3.7931\end{array}$ \\
\hline
\end{tabular}

Totals :

$1.90967 \mathrm{e} 4 \quad 1009.58090$

Signal 3: DAD1 C, Sig=254, 4 Ref $=360,100$

\begin{tabular}{cccccc}
$\begin{array}{c}\text { Peak RetTime Type } \\
\text { \# }\end{array}\left[\begin{array}{c}\text { Width } \\
{[\mathrm{min}]}\end{array}\right.$ & $\begin{array}{c}\text { Area } \\
{[\mathrm{mAU}}\end{array}$ & $\begin{array}{l}\text { Height } \\
{[\mathrm{mAU}]}\end{array}$ & $\begin{array}{c}\text { Area } \\
\%\end{array}$ \\
\hdashline & $12.568 \mathrm{VB}$ & 0.2902 & 2983.38306 & 158.08659 & 96.1441 \\
\hline 2 & $16.038 \mathrm{BB}$ & 0.3657 & 119.64977 & 5.09970 & 3.8559
\end{tabular}

Totals :

$3103.03283 \quad 163.18630$

Signal 4: DAD1 D, Sig=280, 4 Ref $=360,100$

Peak RetTime Type Width Area Height Area

\# [min] 1 [min] [mAU*s] [mAU] $\%$ \%

$\begin{array}{llllll}1 & 12.568 \text { VB } & 0.2887 & 2322.63501 & 123.89622 & 96.2601\end{array}$

$\begin{array}{rrrr} & \\ 12.038 \text { BB } & 0.3613 & 90.23863 & 3.88078 \\ \text { Totals : } & 2412.87364 & 127.77700\end{array}$

(l)

2d

Enantioenriched

Signal 5: DAD1 E, Sig=310, 4 Ref $=360,100$

*** End of Report *** 


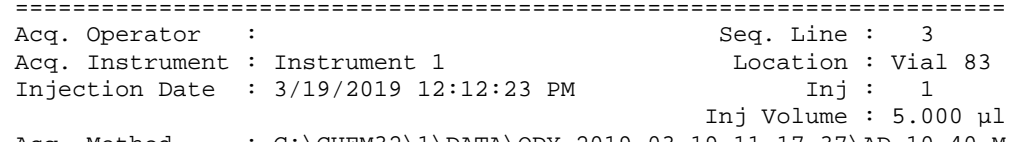

Injection Date Inj volume : $5.000 \mu \mathrm{\mu l}$

Acq. Method changed $3 / 19 / 2019$ 11:30:23 AM

Analysis Method : C: \CHEM32\1\METHODS \OD-01-10-0.3.M

Last changed : 5/29/2019 9:25:17 PM

Additional Info: : Peak(s) manually int
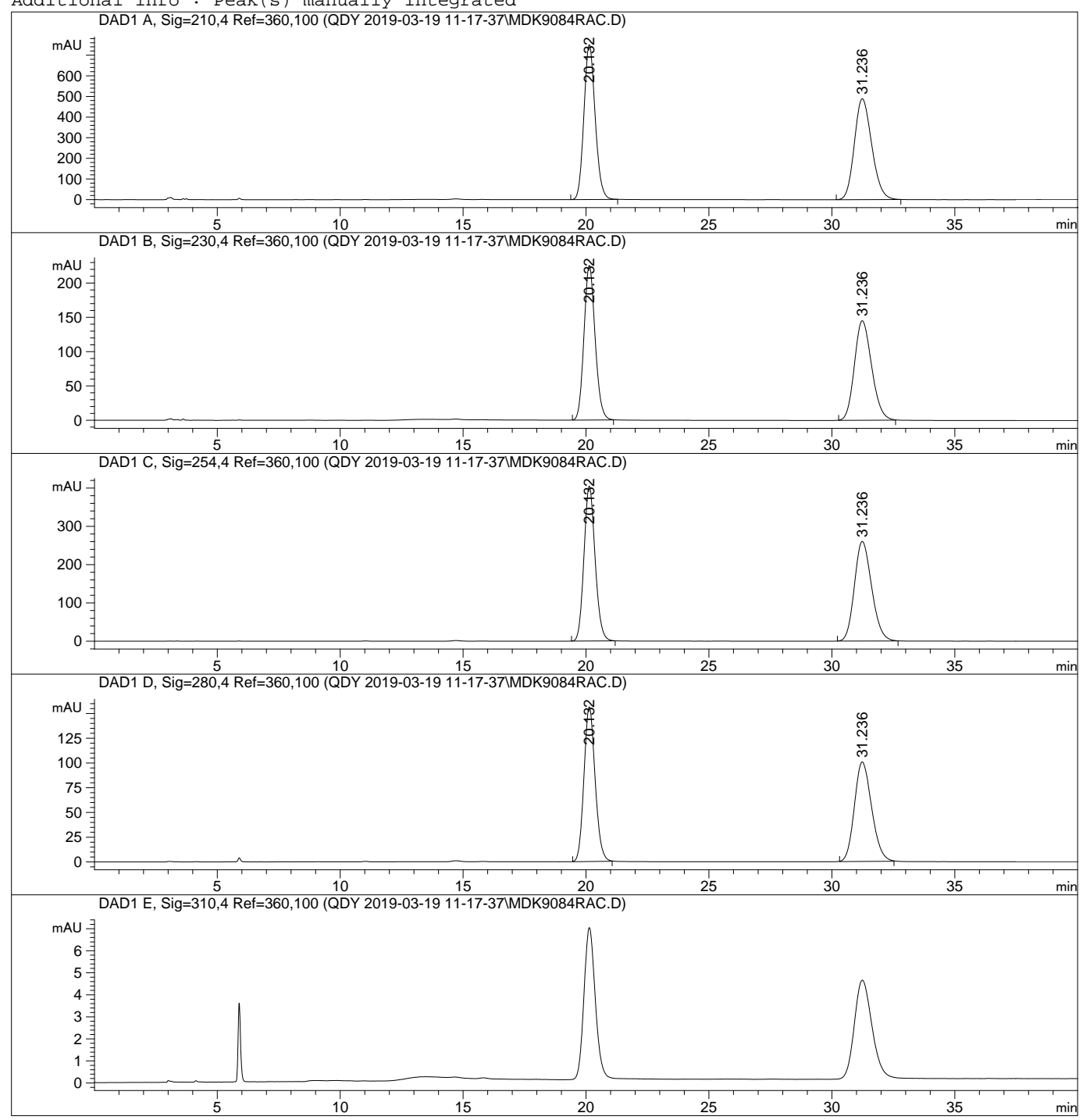

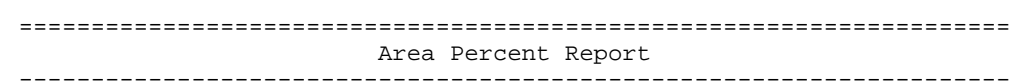

Sorted By

Signal

Multiplier

1.0000

Use Multiplier \& Dilution Factor with ISTDs

Signal 1: DAD1 A, Sig=210, 4 Ref $=360,100$

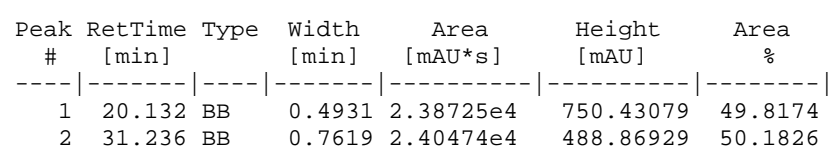

Totals :

$4.79199 \mathrm{e} 4 \quad 1239.30008$

Signal 2: DAD1 B, Sig=230, 4 Ref $=360,100$

Peak RetTime Type Width Area Height Area \# [min] $[$ [min] [mAU*s] [mAU] $\%$ \%

$\begin{array}{lllllll}1 & 20.132 & \text { BB } & 0.4868 & 7088.27979 & 225.44545 & 50.0700\end{array}$

$\begin{array}{llllll}1 & 30.132 \mathrm{BB} & 0.4868 & & & \end{array}$

Totals :

$1.41567 \mathrm{e} 4 \quad 370.48312$

Signal 3: DAD1 C, Sig $=254,4$ Ref $=360,100$

\begin{tabular}{cccccc}
$\begin{array}{c}\text { Peak RetTime Type } \\
\#\end{array}\left[\begin{array}{c}\text { Width } \\
{[\mathrm{min}]}\end{array}\right.$ & $\begin{array}{c}\text { Area } \\
{[\mathrm{min}]}\end{array}$ & $\begin{array}{l}\text { Height } \\
{[\mathrm{mAU}]}\end{array}$ & $\begin{array}{c}\text { Area } \\
\%\end{array}$ \\
\hdashline-1 & $20.132 \mathrm{BB}$ & 0.4870 & $1.27229 \mathrm{e}$ & 404.49435 & 50.0475 \\
\hline 2 & $31.236 \mathrm{BB}$ & 0.7535 & $1.26987 \mathrm{e} 4$ & 260.15036 & 49.9525
\end{tabular}

Totals :

$2.54216 \mathrm{e} 4 \quad 664.64471$

Signal 4: DAD1 D, Sig $=280,4$ Ref $=360,100$

Peak RetTime Type Width Area Height Area

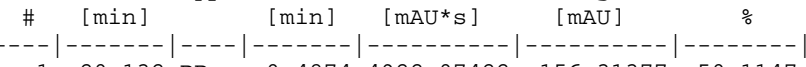

$\begin{array}{llllll}1 & 20.132 \text { BB } & 0.4874 & 4922.07422 & 156.31377 & 50.1147\end{array}$

$\begin{array}{rlrrr}2 & 31.236 \text { BB } & 0.7565 & 4899.55078 & 100.55277 \\ \text { tals : } & 9821.62500 & 256.86654\end{array}$

Signal 5: DAD1 E, Sig=310, 4 Ref $=360,100$

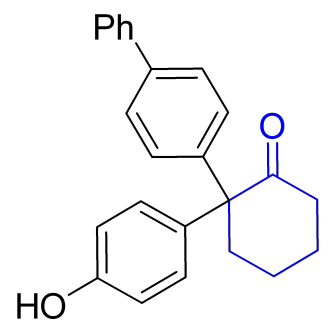

racemic

*** End of Report *** 
Analysis Method : C: \CHEM32\1\METHODS \OD-01-10-0.3.M

Last changed : 5/29/2019 9:25:17 PM

Additional Info : Peak(s) manually integrated
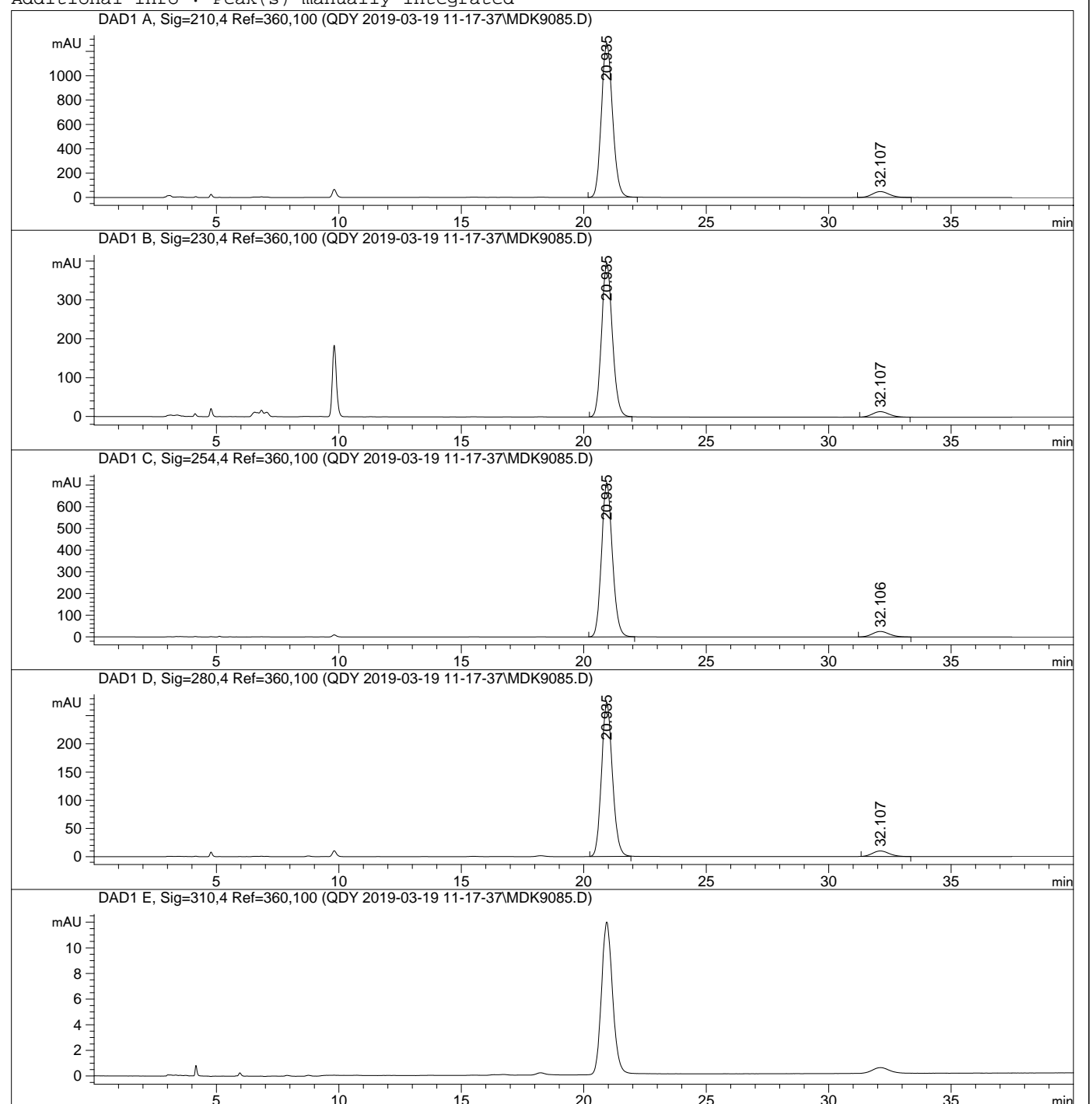

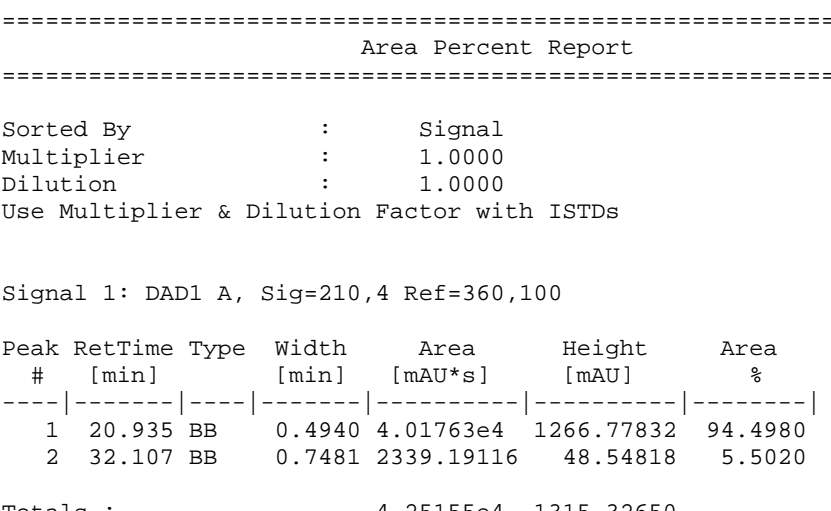

Totals :

$4.25155 \mathrm{e} 4 \quad 1315.32650$

Signal 2: DAD1 B, Sig $=230,4$ Ref $=360,100$

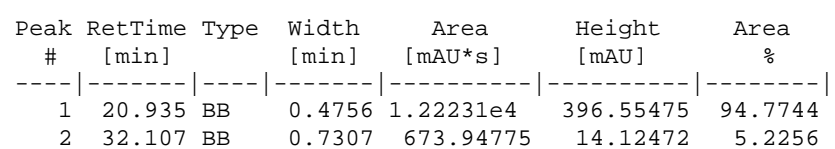

Totals :

$1.28971 \mathrm{e} 4 \quad 410.67947$

Signal 3: DAD1 C, Sig $=254,4$ Ref $=360,100$

\begin{tabular}{|c|c|c|c|c|c|}
\hline $\begin{array}{c}\text { Peak } \\
\#\end{array}$ & $\begin{array}{l}\text { RetTime Type } \\
\text { [min] }\end{array}$ & $\begin{array}{l}\text { Width } \\
\text { [min] }\end{array}$ & $\begin{array}{c}\text { Area } \\
{\left[\mathrm{mAU} U^{*} \mathrm{~s}\right]}\end{array}$ & $\begin{array}{l}\text { Height } \\
{[\mathrm{mAU}]}\end{array}$ & $\begin{array}{c}\text { Area } \\
\%\end{array}$ \\
\hline 1 & $20.935 \mathrm{BB}$ & 0.4759 & $2.19555 \mathrm{e} 4$ & 711.79822 & 94.7340 \\
\hline 2 & $32.106 \mathrm{BB}$ & 0.7418 & 1220.44336 & 25.43350 & 5.2660 \\
\hline
\end{tabular}

Totals :

$2.31759 \mathrm{e} 4 \quad 737.23172$

Signal 4: DAD1 D, Sig=280, 4 Ref $=360,100$

Peak RetTime Type width Area Height Area

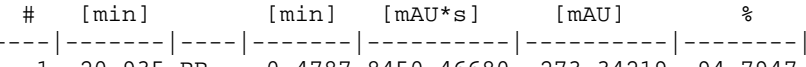

$\begin{array}{llllll}1 & 20.935 \text { BB } & 0.4787 & 8450.46680 & 273.34219 & 94.7947\end{array}$

Totals :

$8914.49033 \quad 283.13052$

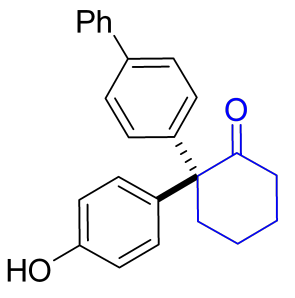

$2 \mathrm{e}$

Enantioenriched

Signal 5: DAD1 E, Sig=310, 4 Ref $=360,100$

*** End of Report *** 
Area Percent Report

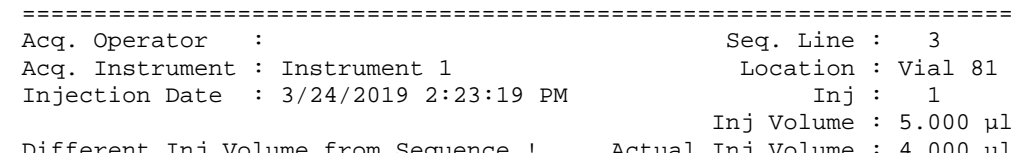

Different Inj Volume from Sequence ! Actual Inj Volume : $5.000 \mu \mathrm{\mu}$

Acq. Method : C: \CHEM32\1\DATA

Last changed : 3/24/2019 1:51:22 PM

Analysis Method : C: \CHEM32\1\METHODS \OD-01-10-0.3.M

Last changed: 5/29/2019 9:25:17 PM

Additional Info : Peak(s) manually integrated
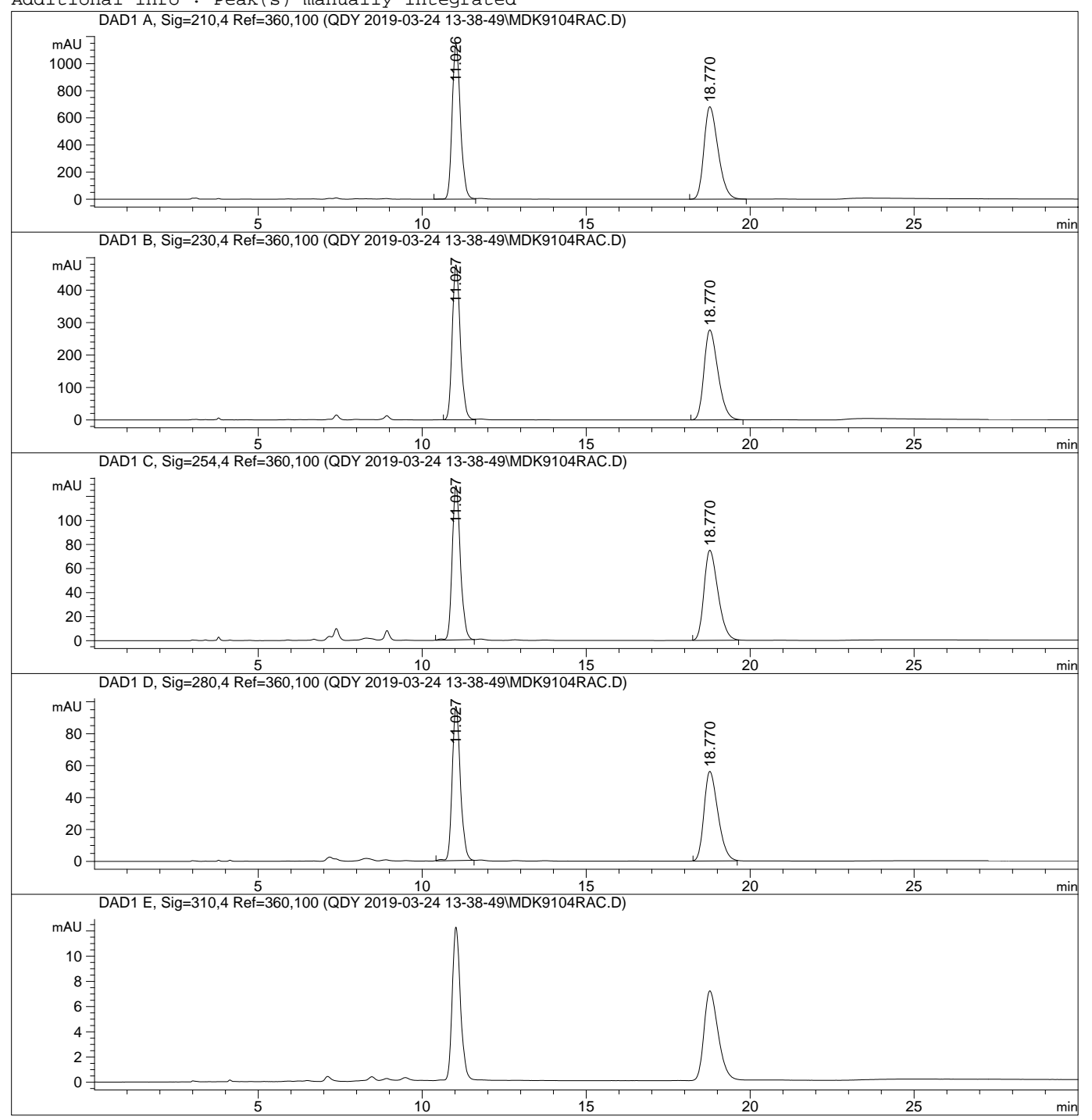

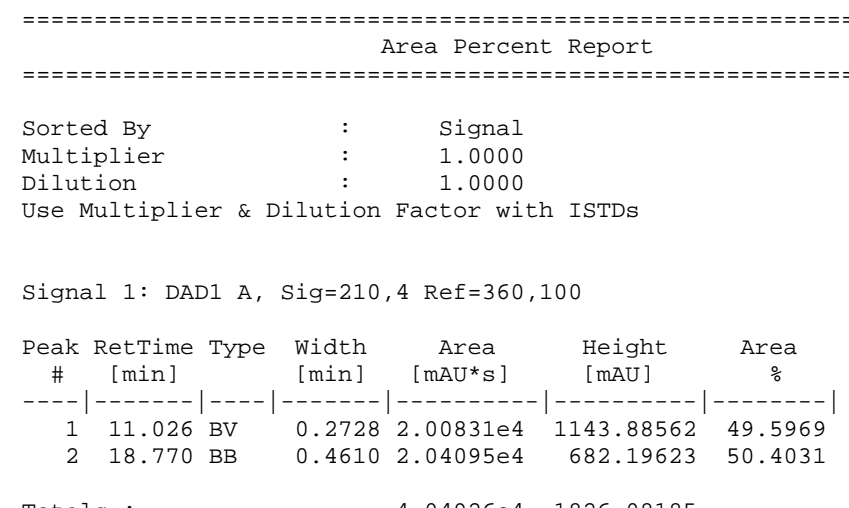

Totals :

4. $04926 \mathrm{e} 4 \quad 1826.08185$

Signal 2: DAD1 B, Sig=230, 4 Ref $=360,100$

\begin{tabular}{|c|c|c|c|c|c|}
\hline $\begin{array}{c}\text { Peak } \\
\#\end{array}$ & $\begin{array}{l}\text { RetTime Type } \\
\text { [min] }\end{array}$ & $\begin{array}{l}\text { Width } \\
\text { [min] }\end{array}$ & $\begin{array}{c}\text { Area } \\
{\left[\mathrm{mAU} U^{*} \mathrm{~s}\right]}\end{array}$ & $\begin{array}{l}\text { Height } \\
{[\mathrm{mAU}]}\end{array}$ & $\begin{array}{c}\text { Area } \\
\%\end{array}$ \\
\hline 1 & $11.027 \mathrm{BB}$ & 0.2667 & 8217.59863 & 477.61136 & 49.9853 \\
\hline
\end{tabular}

Totals :

$1.64400 \mathrm{e} 4 \quad 754.88110$

Signal 3: DAD1 C, Sig $=254,4$ Ref $=360,100$

\begin{tabular}{|c|c|c|c|c|c|}
\hline $\begin{array}{c}\text { Peak } \\
\#\end{array}$ & $\begin{array}{l}\text { RetTime Type } \\
{[\text { min] }}\end{array}$ & $\begin{array}{l}\text { Width } \\
\text { [min] }\end{array}$ & $\begin{array}{c}\text { Area } \\
{\left[\mathrm{mAU} U^{*} \mathrm{~s}\right]}\end{array}$ & $\begin{array}{l}\text { Height } \\
{[\mathrm{mAU}]}\end{array}$ & $\begin{array}{c}\text { Area } \\
\%\end{array}$ \\
\hline 1 & 11. $027 \mathrm{BB}$ & 0.2685 & 2226.79346 & 128.31960 & 382 \\
\hline 2 & $18.770 \mathrm{BB}$ & 0.4581 & 2223.39160 & 74.94193 & 49.9618 \\
\hline
\end{tabular}

Totals :

$4450.18506 \quad 203.26153$

Signal 4: DAD1 D, Sig $=280,4$ Ref $=360,100$

\begin{tabular}{|c|c|c|c|c|c|}
\hline eak & $\begin{array}{l}\text { RetTime Type } \\
\text { [min] }\end{array}$ & $\begin{array}{l}\text { Width } \\
\text { [min] }\end{array}$ & $\begin{array}{c}\text { Area } \\
{\left[\mathrm{mAU} U^{*} \mathrm{~s}\right]}\end{array}$ & $\begin{array}{l}\text { Height } \\
{[\mathrm{mAU}]}\end{array}$ & $\begin{array}{c}\text { Area } \\
\%\end{array}$ \\
\hline & 770 & 45 & $\begin{array}{l}1672.75769 \\
1658.90527\end{array}$ & 96.61972 & \\
\hline
\end{tabular}

Totals :

$3331.66296 \quad 152.75715$

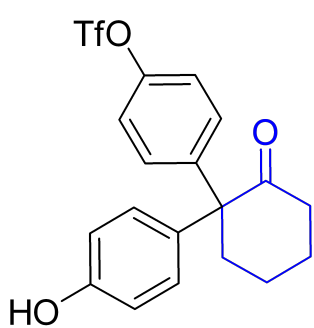

racemic

Signal 5: DAD1 E, Sig $=310,4$ Ref $=360,100$

$* * *$ End of Report *** 


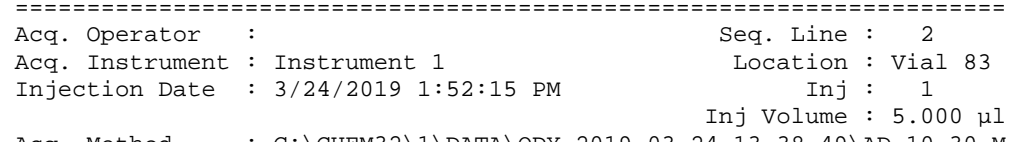

Inj volume : $5.000 \mu \mathrm{I}$

Acq. Method $\quad$ : $3 / 24 / 2019$ 1:51:22 PM

Analysis Method : C: \CHEM32\1\METHODS \OD-01-10-0.3.M

Last changed : 5/29/2019 9:25:17 PM

Additional Info: Peak(s) nonualiy i
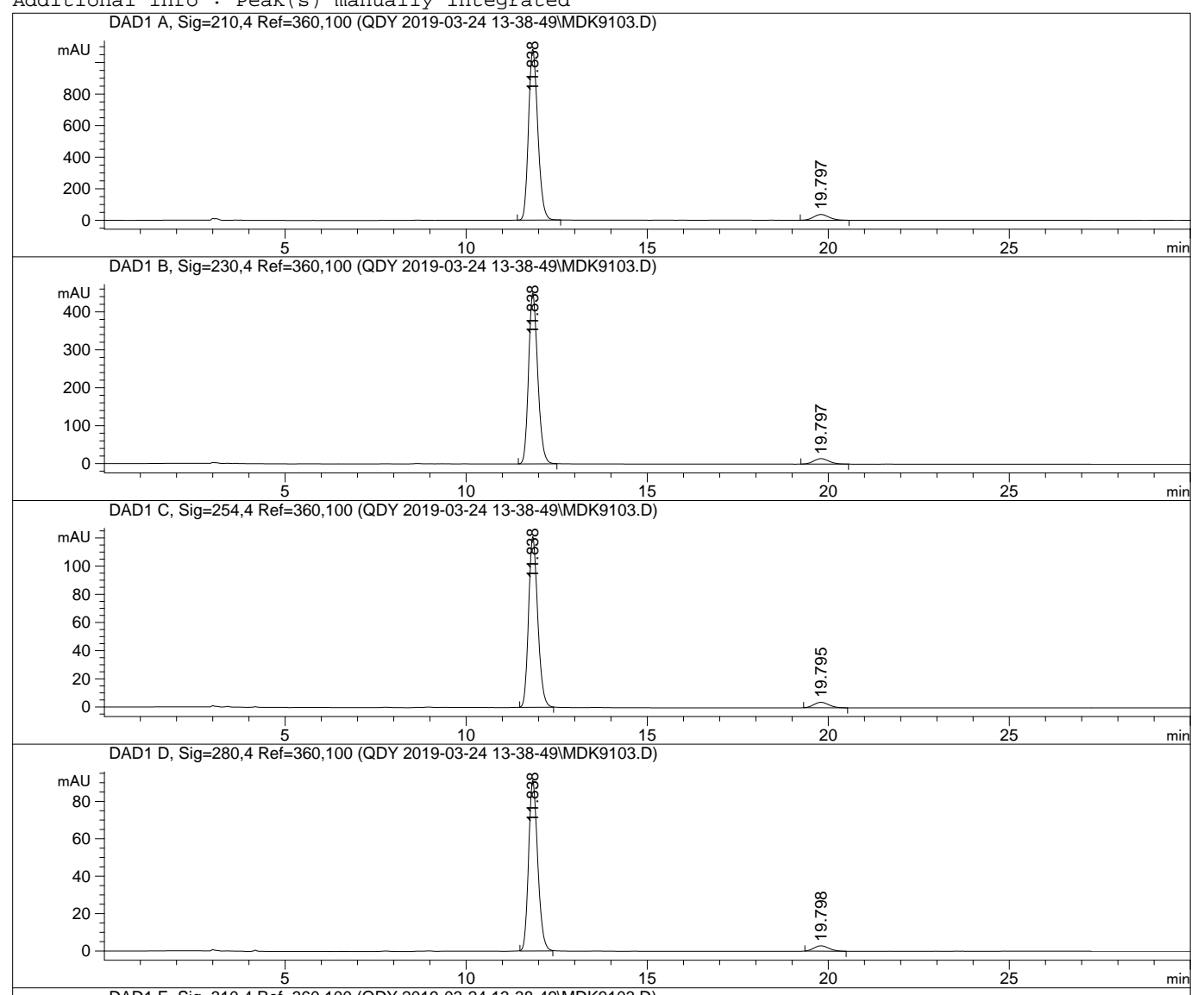

$\begin{array}{crr}5 & 10 & 15 \\ \text { DAD1 E, Sig=310,4 Ref=360,100 (QDY 2019-03-24 } & \text { 13-38-49IMDK9103.D) }\end{array}$

$$
\begin{array}{r}
\text { mAU } \\
10 \\
8 \\
6 \\
6 \\
4 \\
4 \\
2 \\
2
\end{array}
$$

Area Percent Report

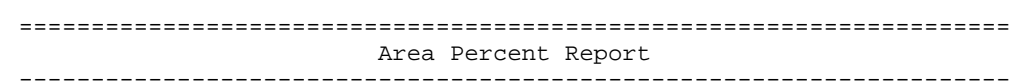

\section{Sorted By}

Signal

Multiplie

1.0000

Use Multiplier \& Dilution Factor with ISTDs

\begin{tabular}{|c|c|c|c|c|c|}
\hline $\begin{array}{c}\text { Peak } \\
\#\end{array}$ & $\begin{array}{l}\text { RetTime Type } \\
{[\mathrm{min}]}\end{array}$ & $\begin{array}{l}\text { Width } \\
\text { [min] }\end{array}$ & $\begin{array}{c}\text { Area } \\
{\left[\mathrm{mAU} U^{*} \mathrm{~s}\right]}\end{array}$ & $\begin{array}{l}\text { Height } \\
{[\mathrm{mAU}]}\end{array}$ & $\begin{array}{c}\text { Area } \\
\%\end{array}$ \\
\hline $\begin{array}{l}1 \\
2\end{array}$ & $\begin{array}{l}11.838 \text { BВ } \\
19.797 \mathrm{BB}\end{array}$ & $\begin{array}{l}0.2763 \\
0.4579\end{array}$ & $\begin{array}{l}1.93353 \mathrm{e} 4 \\
1076.33728\end{array}$ & $\begin{array}{r}1082.84961 \\
36.50950\end{array}$ & $\begin{array}{r}94.7269 \\
5.2731\end{array}$ \\
\hline
\end{tabular}

Signal 1: DAD1 A, Sig=210, 4 Ref $=360,100$

Totals :

2.04117e4 1119.35911

\begin{tabular}{|c|c|c|c|c|c|}
\hline $\begin{array}{c}\text { Peak } \\
\#\end{array}$ & $\begin{array}{l}\text { RetTime Type } \\
{[\text { min] }}\end{array}$ & $\begin{array}{l}\text { Width } \\
\text { [min] }\end{array}$ & $\begin{array}{c}\text { Area } \\
{\left[\mathrm{mAU} U^{\star} \mathrm{s}\right]}\end{array}$ & $\begin{array}{l}\text { Height } \\
{[\mathrm{mAU}]}\end{array}$ & $\begin{array}{c}\text { Area } \\
\%\end{array}$ \\
\hline $\begin{array}{l}1 \\
2\end{array}$ & $\begin{array}{l}11.838 \text { BB } \\
19.797 \mathrm{BB}\end{array}$ & $\begin{array}{l}0.2707 \\
0.4536\end{array}$ & $\begin{array}{r}7902.23926 \\
423.74338\end{array}$ & $\begin{array}{r}450.40689 \\
14.47032\end{array}$ & $\begin{array}{r}94.9106 \\
5.0894\end{array}$ \\
\hline
\end{tabular}

Signal 2: DAD1 B, Sig $=230,4$ Ref $=360,100$

Totals :

$8325.98264 \quad 464.87721$

Signal 3: DAD1 C, Sig $=254,4$ Ref $=360,100$

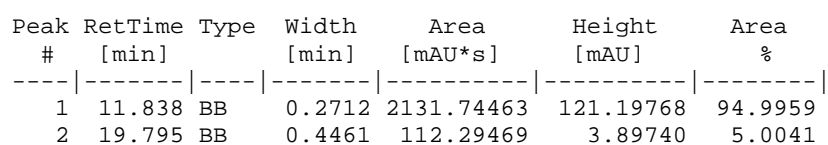

Totals :

$2244.03932 \quad 125.09508$

Signal 4: DAD1 D, Sig=280, 4 Ref $=360,100$

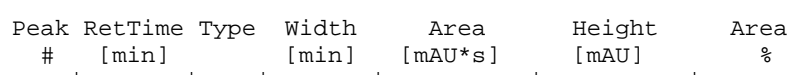

111.838 BB $0.27051599 .78430 \quad 01.25175 \quad 05.1448$

$\begin{array}{lrrrrr}11.838 & \text { BB } & 0.2705 & 159.78430 & 91.25175 & 95.1448 \\ 2 & 19.798 & 0.4360 & 81.63613 & 2.86855 & 4.8552\end{array}$

Totals :

$1681.42043 \quad 94.12030$

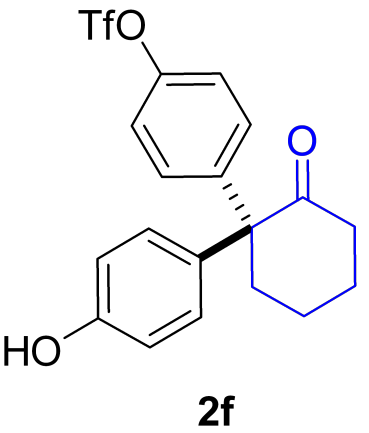

Enantioenriched

Signal 5: DAD1 E, Sig $=310,4$ Ref $=360,100$

*** End of Report *** 
Area Percent Report

\begin{tabular}{|c|c|c|}
\hline Acq. Operator & & Seq. Line : \\
\hline Acq. Instrument & Instrument 1 & Location \\
\hline Injection Date & $3 / 24 / 2019$ 4:07:54 PM & 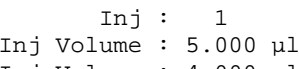 \\
\hline
\end{tabular}

Injection Date $\quad$ Inj Volume $: 5.000 \mu \mathrm{\mu l}$

Acq. Method : C: \CHEM32\1\DATA Q QDY 2019-03-24 13-38-49\AD-10-60.M

Last changed : 3/24/2019 4:07:03 PM

Analysis Method : C: \CHEM32\1\METHODS\OD-01-10-0.3.M

Last changed: 5/29/2019 9:25:17 PM

Additional Info : Peak(s) manually integrated
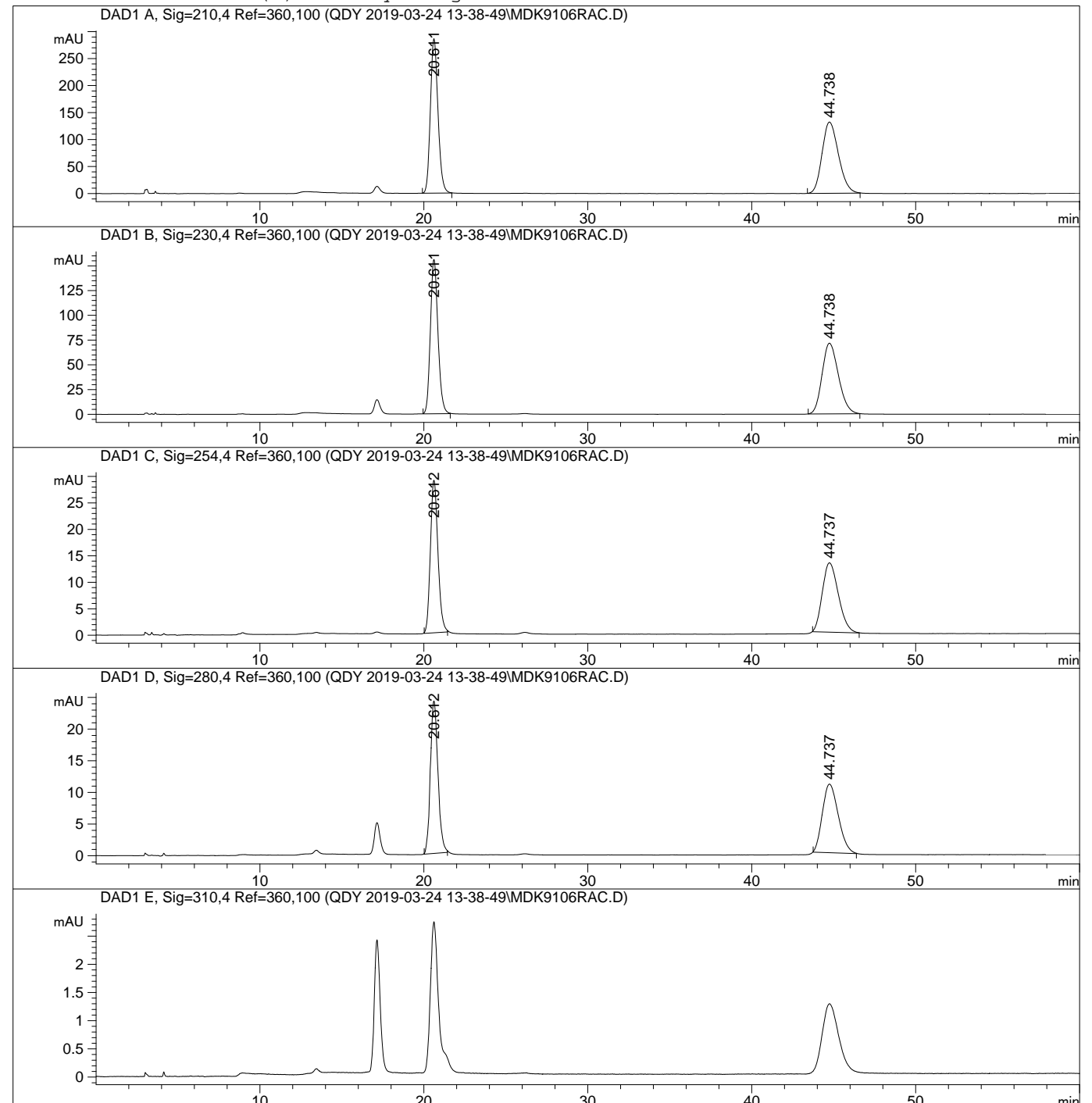

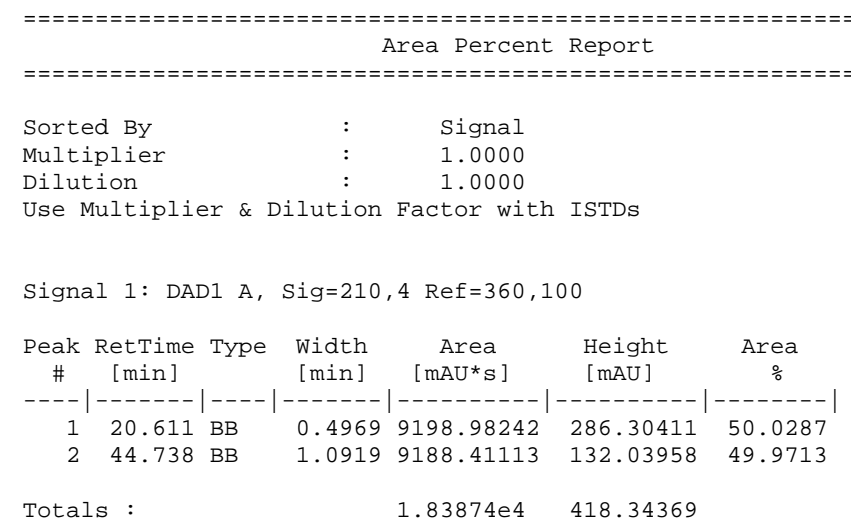

Signal 2: DAD1 B, Sig=230, 4 Ref $=360,100$

\begin{tabular}{|c|c|c|c|c|c|}
\hline $\begin{array}{c}\text { Peak } \\
\#\end{array}$ & $\begin{array}{l}\text { RetTime Type } \\
{[\text { min] }}\end{array}$ & $\begin{array}{l}\text { Width } \\
\text { [min] }\end{array}$ & $\begin{array}{c}\text { Area } \\
{\left[\mathrm{mAU} U^{\star} \mathrm{s}\right]}\end{array}$ & $\begin{array}{l}\text { Height } \\
{[\mathrm{mAU}]}\end{array}$ & $\begin{array}{c}\text { Area } \\
\%\end{array}$ \\
\hline $\begin{array}{l}1 \\
2\end{array}$ & $\begin{array}{l}20.611 \text { BB } \\
44.738 \text { BB }\end{array}$ & $\begin{array}{l}0.4927 \\
1.0869\end{array}$ & $\begin{array}{l}4979.70898 \\
4952.65625\end{array}$ & $\begin{array}{r}155.86249 \\
71.43053\end{array}$ & $\begin{array}{l}50.1362 \\
49.8638\end{array}$ \\
\hline
\end{tabular}

Totals :

$9932.36523 \quad 227.29302$

Signal 3: DAD1 C, Sig $=254,4$ Ref $=360,100$

\begin{tabular}{|c|c|c|c|c|c|}
\hline $\begin{array}{c}\text { Peak } \\
\#\end{array}$ & $\begin{array}{l}\text { RetTime Type } \\
\text { [min] }\end{array}$ & $\begin{array}{l}\text { Width } \\
\text { [min] }\end{array}$ & $\begin{array}{c}\text { Area } \\
{\left[\mathrm{mAU} U^{\star} \mathrm{s}\right]}\end{array}$ & $\begin{array}{l}\text { Height } \\
{[\mathrm{mAU}]}\end{array}$ & $\begin{array}{c}\text { Area } \\
\%\end{array}$ \\
\hline 1 & $20.612 \mathrm{BB}$ & 0.4913 & 917.73438 & 28.84000 & 50.7171 \\
\hline 2 & $44.737 \mathrm{BB}$ & 1.0663 & 891.78168 & 13.09884 & 49.2829 \\
\hline
\end{tabular}

Totals :

$1809.51605 \quad 41.93884$

Signal 4: DAD1 D, Sig=280, 4 Ref $=360,100$

\begin{tabular}{|c|c|c|c|c|c|}
\hline \# & $\begin{array}{l}\text { RetTime Type } \\
\text { [min] }\end{array}$ & $\begin{array}{l}\text { Width } \\
\text { [min] }\end{array}$ & $\begin{array}{c}\text { Area } \\
{\left[\mathrm{mAU} U^{*} \mathrm{~s}\right]}\end{array}$ & $\begin{array}{l}\text { Height } \\
{[\mathrm{mAU}]}\end{array}$ & $\begin{array}{c}\text { Area } \\
\%\end{array}$ \\
\hline & 37 & & $\begin{array}{l}764.45172 \\
730.49231\end{array}$ & $\begin{array}{l}24.07485 \\
10.84075\end{array}$ & $\begin{array}{l}51.1358 \\
48.8642\end{array}$ \\
\hline
\end{tabular}

Totals :

$1494.94403 \quad 34.91560$

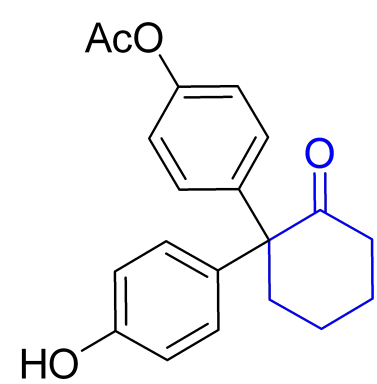

Signal 5: DAD1 E, Sig=310, 4 Ref $=360,100$

racemic 
Area Percent Report

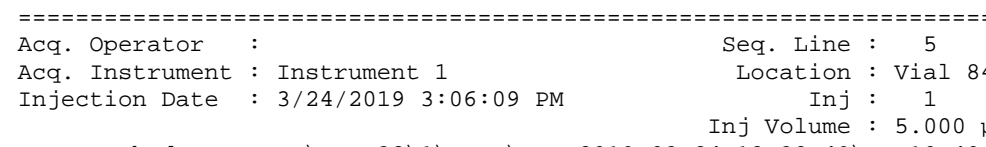

Injection Date Inj Volume $: 5.000 \mu \mathrm{\mu l}$

Last changed $\quad 3 / 24 / 2019$ 3:38:14 PM

Analysis Method : C: \CHEM32\1\METHODS\OD-01-10-0.3.M

Last changed : 5/29/2019 9:25:17 PM

Additional Info : Peak(s) manually integrated
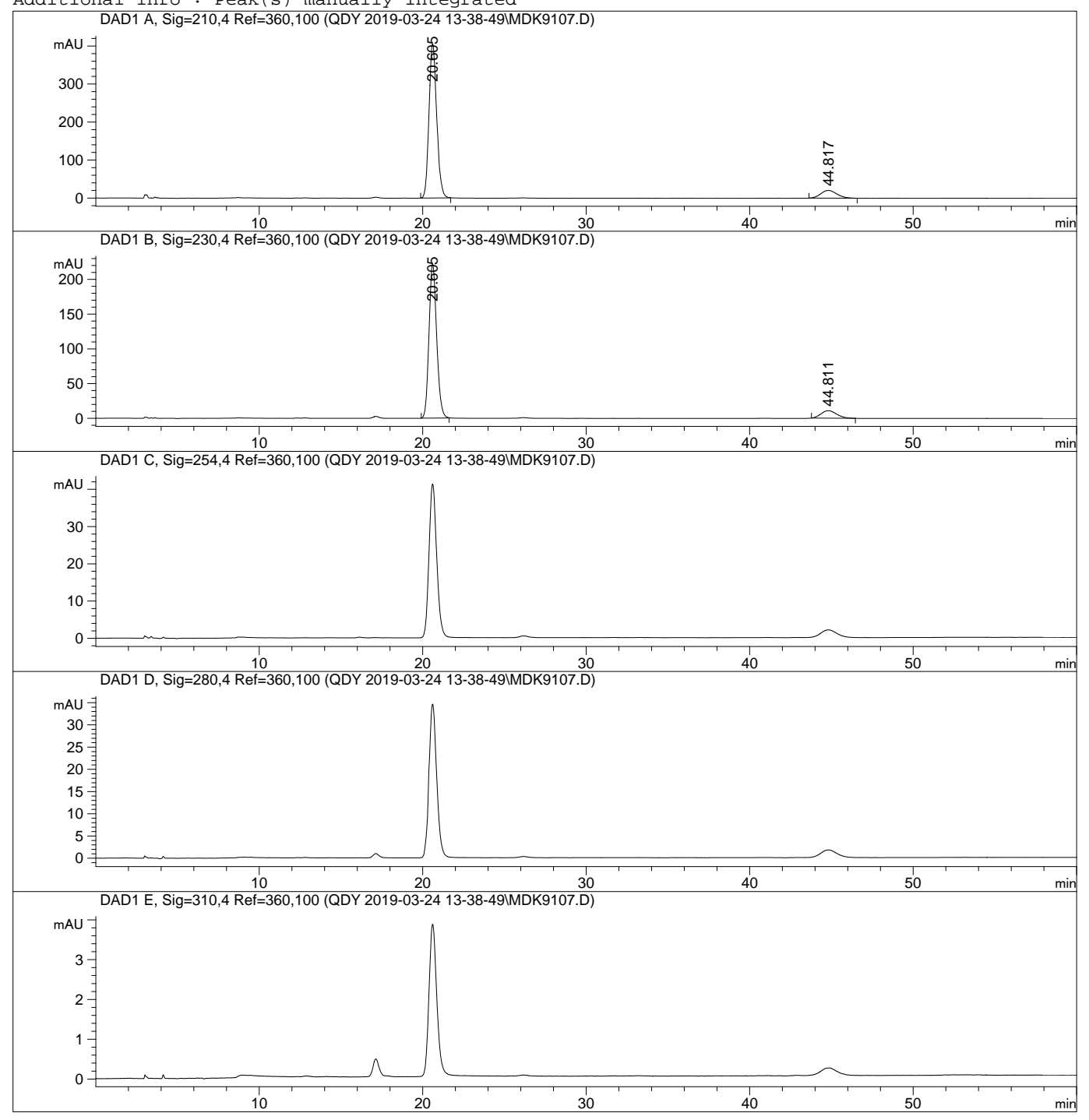

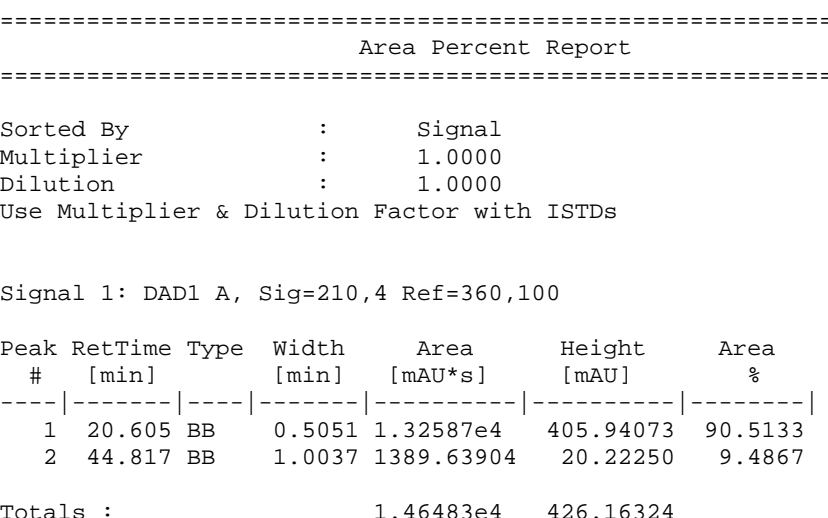

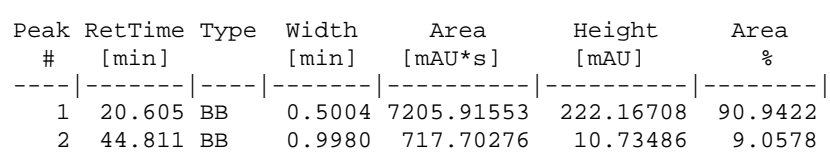

Totals :

$7923.61829 \quad 232.90195$

Signal 3: DAD1 C, Sig $=254,4$ Ref $=360,100$

Signal 4: DAD1 D, Sig $=280,4$ Ref $=360,100$

Signal 5: DAD1 E, Sig=310, 4 Ref $=360,100$

$* \star *$ End of Report ***

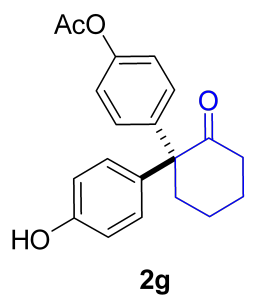

Enantioenriched 


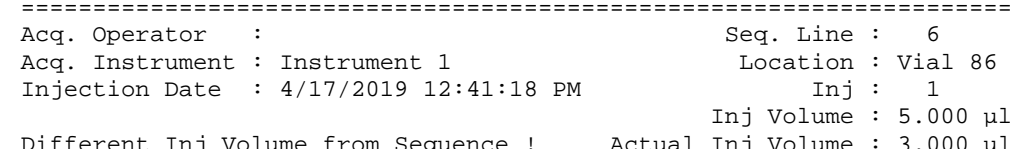

Different Inj Volume from Sequence! Actual Inj Volume : $5.000 \mu \mathrm{I}$ Acq. Method : C: \CHEM32\1\DATA \QDY 2019-04-17 10-42-20\AD-10-40.M Last changed : 4/17/2019 11:59:16 AM

Analysis Method : C: \CHEM32\1\METHODS \OD-01-10-0.3.M

: $5 / 29 / 20199: 25: 17$ PM

Additional Info : Peak(s) manually integrated

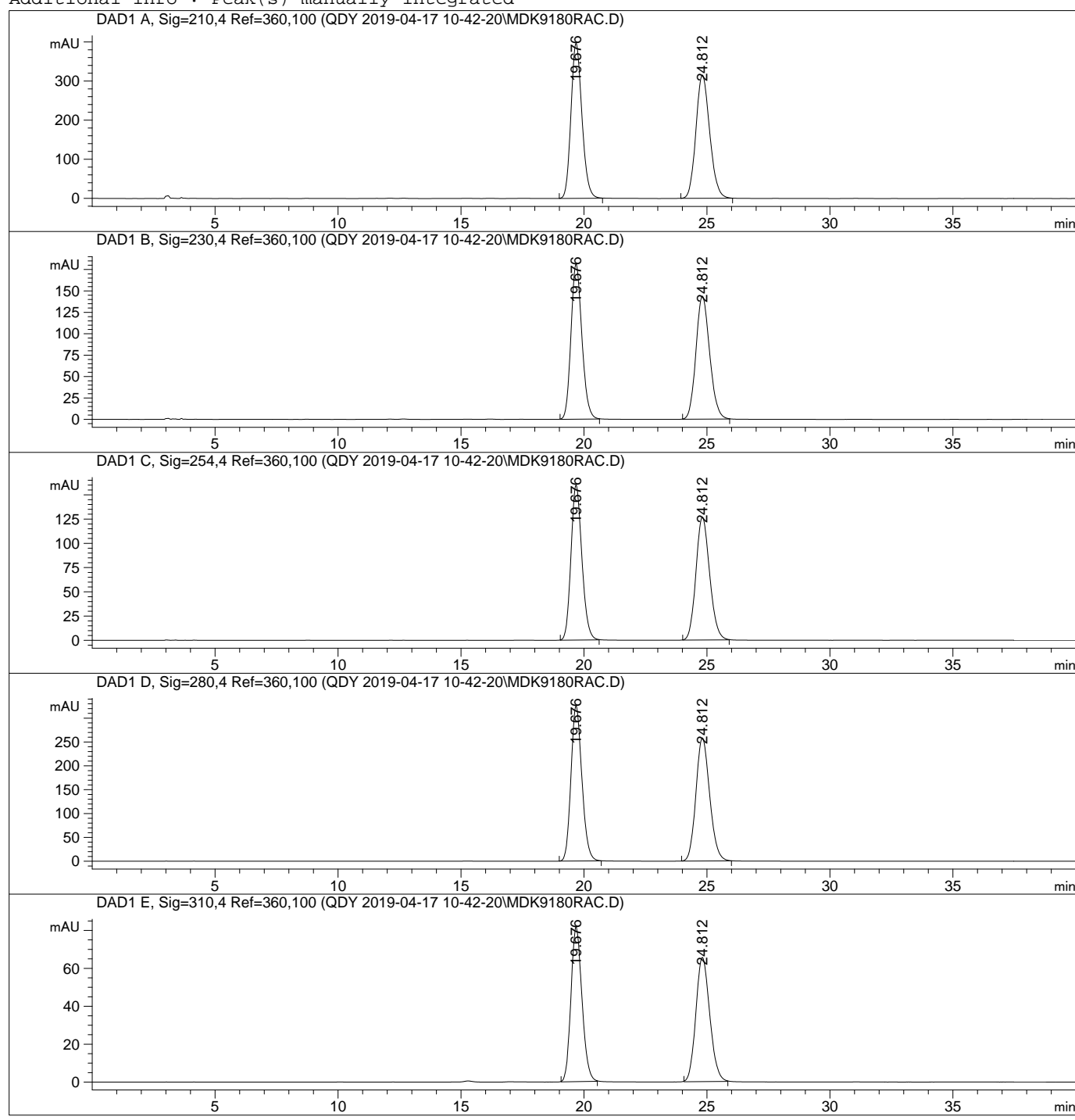

Area Percent Report

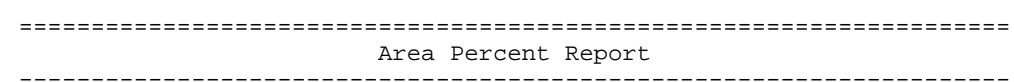

Sorted By

\section{Signal}

Multiplier
Dilution

1.0000

Use Multiplier \& Dilution Factor with ISTDs

Signal 1: DAD1 A, Sig=210, 4 Ref $=360,100$

\begin{tabular}{|c|c|c|c|c|c|}
\hline $\begin{array}{l}\text { Peak } \\
\#\end{array}$ & $\begin{array}{l}\text { RetTime Type } \\
\text { [min] }\end{array}$ & $\begin{array}{l}\text { Width } \\
\text { [min] }\end{array}$ & $\begin{array}{c}\text { Area } \\
{\left[\mathrm{mAU} U^{*} \mathrm{~s}\right]}\end{array}$ & $\begin{array}{l}\text { Height } \\
{[\mathrm{mAU}]}\end{array}$ & $\begin{array}{c}\text { Area } \\
\%\end{array}$ \\
\hline 1 & $19.676 \mathrm{BB}$ & 0.4793 & 1.22732e 4 & 396.33746 & 49.9577 \\
\hline
\end{tabular}

Totals :

$2.45672 \mathrm{e} 4 \quad 711.79718$

Signal 2: DAD1 B, Sig=230, 4 Ref $=360,100$

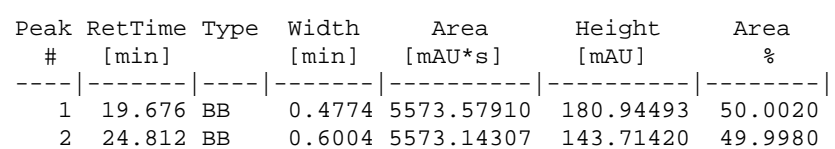

Totals :

$1.11467 \mathrm{e} 4 \quad 324.65913$

Signal 3: DAD1 C, Sig $=254,4$ Ref $=360,100$

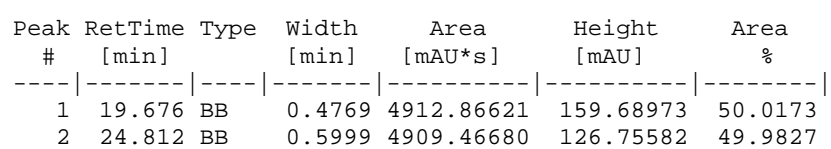

Totals :

$9822.33301 \quad 286.44555$

Signal 4: DAD1 D, Sig=280, 4 Ref $=360,100$

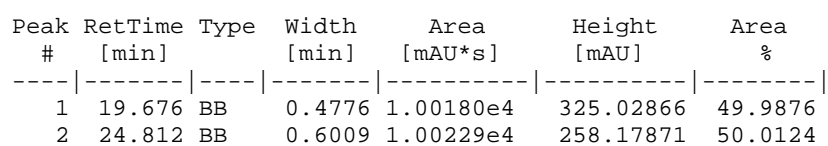

Totals :

$2.00409 \mathrm{e} 4 \quad 583.20737$

Signal 5: DAD1 E, Sig $=310,4$ Ref $=360,100$

\begin{tabular}{|c|c|c|c|c|c|}
\hline $\begin{array}{c}\text { eak } \\
\#\end{array}$ & $\begin{array}{c}\text { RetTime T } \\
{[\mathrm{min}]}\end{array}$ & $\begin{array}{l}\text { Width } \\
\text { [min] }\end{array}$ & $\begin{array}{c}\text { Area } \\
{\left[\mathrm{mAU} U^{*} \mathrm{~s}\right]}\end{array}$ & $\begin{array}{l}\text { Height } \\
{[\mathrm{mAU}]}\end{array}$ & $\begin{array}{c}\text { Area } \\
\%\end{array}$ \\
\hline & .676 & .486 & $\begin{array}{l}2547.68750 \\
2565.66113\end{array}$ & $\begin{array}{l}81.62657 \\
65.49895\end{array}$ & $\begin{array}{l}49.8242 \\
50.1758\end{array}$ \\
\hline
\end{tabular}

Totals : $\quad 5113.34863 \quad 147.12552$

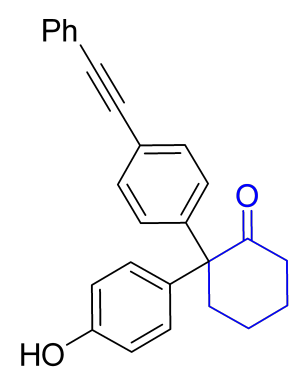

racemic 


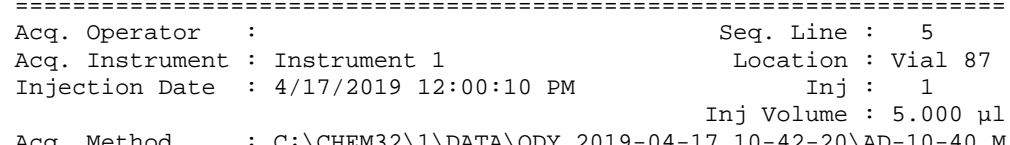

Inj Volume $: 5.000 \mu \mathrm{I}$
C.

Last changed $\quad 4 / 17 / 2019$ 11:59:16 AM

Analysis Method : C: \CHEM32\1\METHODS \OD-01-10-0.3.M

Last changed : 5/29/2019 9:25:17 PM

Additional Info: Peak(s) manually integr
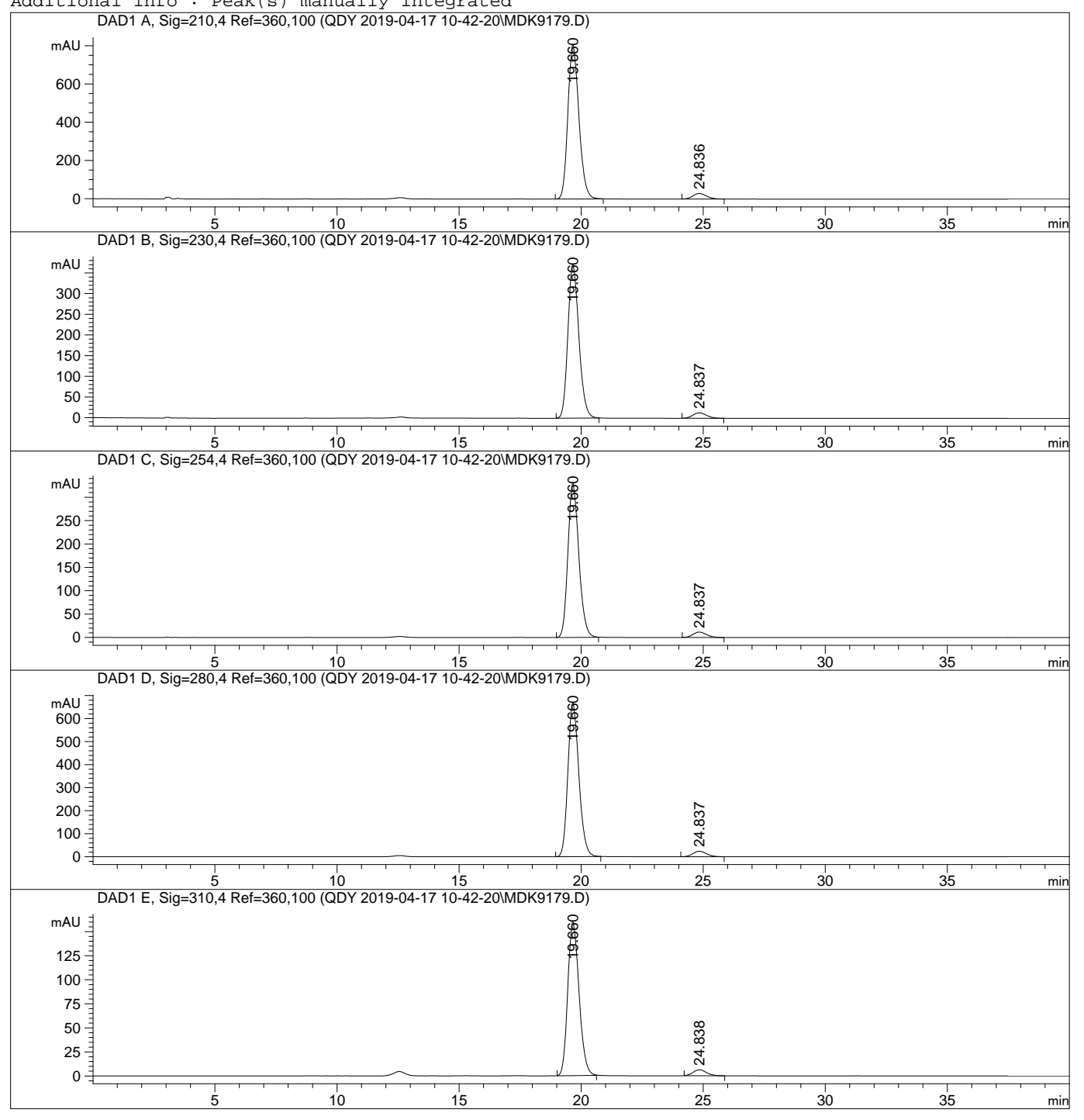

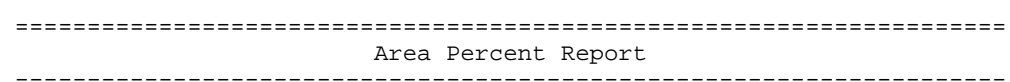

Sorted By

\section{Signal}

Multiplie
Dilution

1.0000

Use Multiplier \& Dilution Factor with ISTDs

Signal 1: DAD1 A, Sig=210, 4 Ref $=360,100$

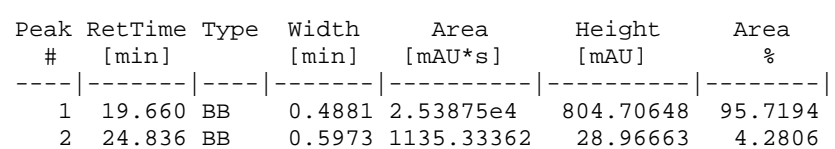

Totals :

$2.65229 \mathrm{e} 4 \quad 833.67311$

Signal 2: DAD1 B, Sig=230, 4 Ref $=360,100$

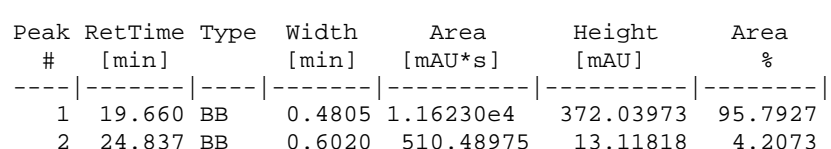

$\begin{array}{llllll}1 & 19.660 \mathrm{BB} & 0.4805 & 1.16230 \mathrm{e} 4 & 372.03973 & 95.7927\end{array}$

Totals : $\quad 1.21335 \mathrm{e} 4 \quad 385.15792$

Signal 3: DAD1 C, Sig $=254,4$ Ref $=360,100$

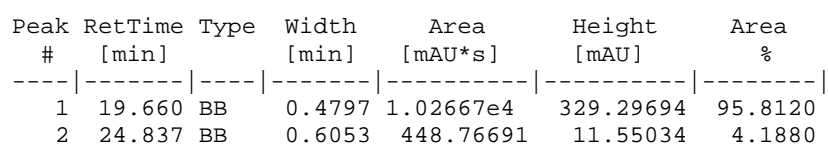

Totals :

$1.07155 \mathrm{e} 4 \quad 340.84728$

Signal 4: DAD1 D, Sig=280, 4 Ref $=360,100$

\begin{tabular}{|c|c|c|c|c|c|}
\hline eak & $\begin{array}{l}\text { RetTime Type } \\
\text { [min] }\end{array}$ & $\begin{array}{l}\text { Width } \\
\text { [min] }\end{array}$ & $\begin{array}{c}\text { Area } \\
{\left[\mathrm{mAU} U^{*} \mathrm{~s}\right]}\end{array}$ & $\begin{array}{l}\text { Height } \\
{[\mathrm{mAU}]}\end{array}$ & $\begin{array}{c}\text { Area } \\
\%\end{array}$ \\
\hline & 24 & & & & \\
\hline
\end{tabular}

Totals :

$2.18132 \mathrm{e} 4 \quad 692.26295$

Signal 5: DAD1 E, Sig=310, 4 Ref $=360,100$

Peak RetTime Type Width Area Height Area

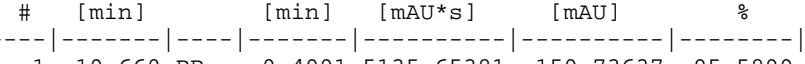

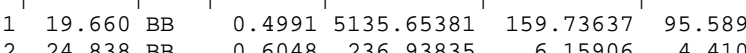

Totals : $\quad 5372.59216 \quad 165.89543$

$-332$

Instrument 1 5/30/2019 8:10:31 PM

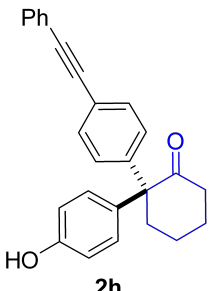




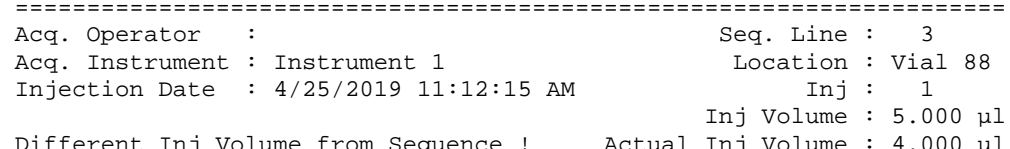

Injection Date Inj Volume : $5.000 \mu \mathrm{\mu l}$ Acq. Method : C: \CHEM32\1\DATA \QDY 2019-04-25 10-27-22\AD-15-30.M Last changed $\quad$ 4/25/2019 10:40:13 AM

Analysis Method : C: \CHEM32\1\METHODS \OD-01-10-0.3.M

Additional Info : Peak(s) manually integrated
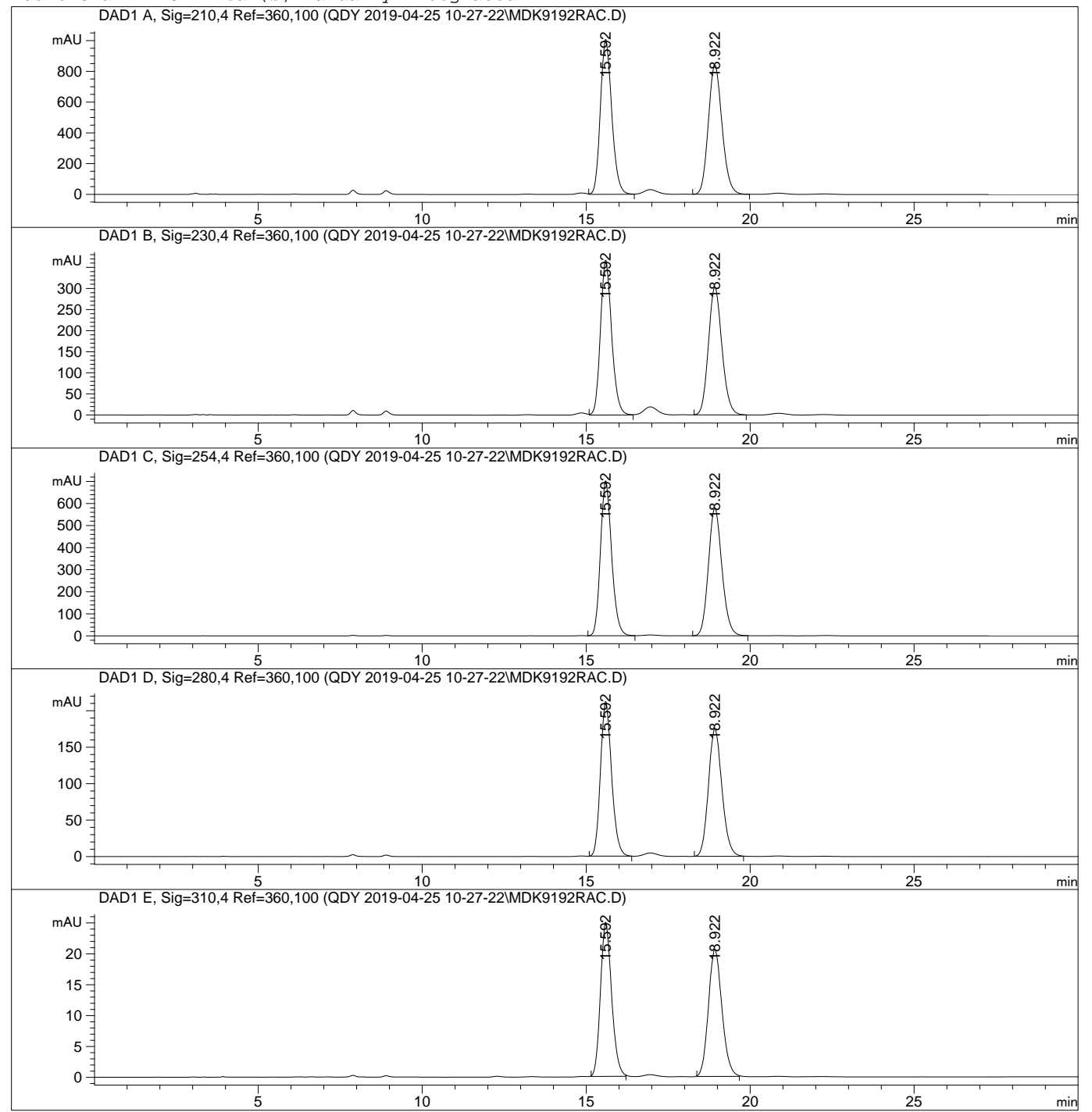

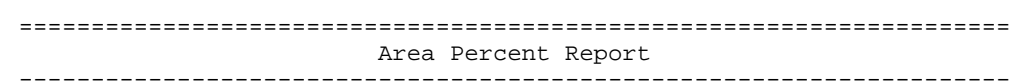

Sorted By

\section{Signal}

Multiplier

1.0000

Use Multiplier \& Dilution Factor with ISTDs

Signal 1: DAD1 A, Sig=210, 4 Ref $=360,100$

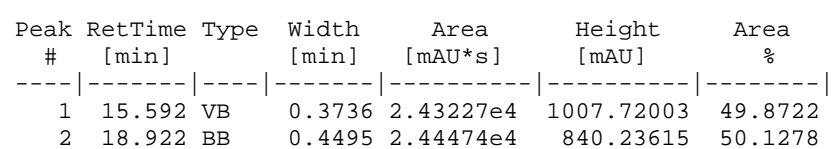

$\begin{array}{llllll}1 & 15.592 \mathrm{VB} & 0.3736 & 2.43227 \mathrm{e} 4 & 1007.72003 & 49.8722\end{array}$

Totals :

$4.87700 \mathrm{e} 4 \quad 1847.95618$

Signal 2: DAD1 B, Sig=230, 4 Ref $=360,100$

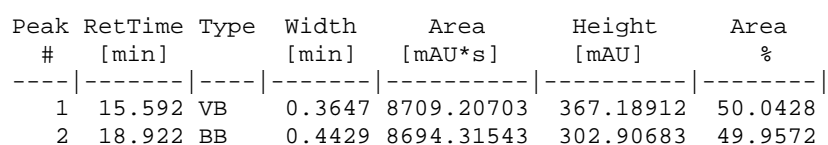

Totals :

$1.74035 \mathrm{e} 4 \quad 670.09595$

Signal 3: DAD1 C, Sig $=254,4$ Ref $=360,100$

\begin{tabular}{|c|c|c|c|c|c|}
\hline $\begin{array}{c}\text { Peak } \\
\#\end{array}$ & $\begin{array}{l}\text { RetTime Type } \\
\text { [min] }\end{array}$ & $\begin{array}{l}\text { Width } \\
\text { [min] }\end{array}$ & $\begin{array}{c}\text { Area } \\
{\left[\mathrm{mAU} U^{\star} \mathrm{s}\right]}\end{array}$ & $\begin{array}{l}\text { Height } \\
{[\mathrm{mAU}]}\end{array}$ & $\begin{array}{c}\text { Area } \\
\%\end{array}$ \\
\hline $\begin{array}{l}1 \\
2\end{array}$ & $\begin{array}{l}15.592 \mathrm{BB} \\
18.922 \mathrm{BB}\end{array}$ & $\begin{array}{l}0.3643 \\
0.4454\end{array}$ & $\begin{array}{l}1.66272 \mathrm{e} 4 \\
1.66338 \mathrm{e} 4\end{array}$ & $\begin{array}{l}702.12903 \\
578.67206\end{array}$ & $\begin{array}{l}49.9902 \\
50.0098\end{array}$ \\
\hline
\end{tabular}

Totals :

$3.32610 \mathrm{e} 4 \quad 1280.80109$

Signal 4: DAD1 D, Sig=280, 4 Ref $=360,100$

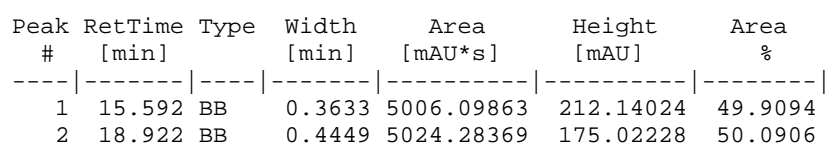

Totals :

$1.00304 \mathrm{e} 4 \quad 387.16252$

Signal 5: DAD1 E, Sig $=310,4$ Ref $=360,100$

\begin{tabular}{|c|c|c|c|c|c|}
\hline $\begin{array}{c}\text { eak } \\
\#\end{array}$ & $\begin{array}{l}\text { RetTime Type } \\
\text { [min] }\end{array}$ & $\begin{array}{l}\text { Width } \\
\text { [min] }\end{array}$ & $\begin{array}{c}\text { Area } \\
{\left[\mathrm{mAU} U^{*} \mathrm{~s}\right]}\end{array}$ & $\begin{array}{l}\text { Height } \\
{[\mathrm{mAU}]}\end{array}$ & $\begin{array}{c}\text { Area } \\
\%\end{array}$ \\
\hline & 0 & $\begin{array}{l}0.3628 \\
0.4438\end{array}$ & $\begin{array}{l}587.82098 \\
588.66876\end{array}$ & $\begin{array}{l}24.95882 \\
20.57427\end{array}$ & $\begin{array}{l}49.9640 \\
50.0360\end{array}$ \\
\hline
\end{tabular}

Totals : $\quad 1176.48975 \quad 45.53310$ 


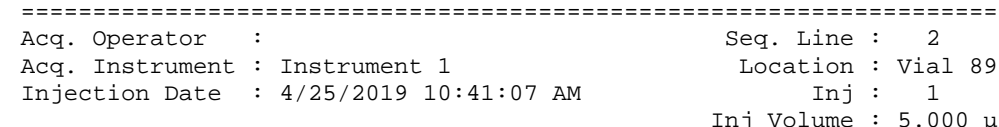

Injection Date Inj volume $: 5.000 \mu \mathrm{\mu}$ Acq. Method
Last changed $4 / 25 / 2019$ 10:40:13 AM

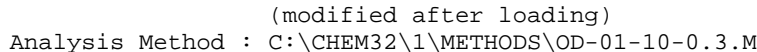

Last changed : 5/29/2019 9:25:17 PM

Additional Info:
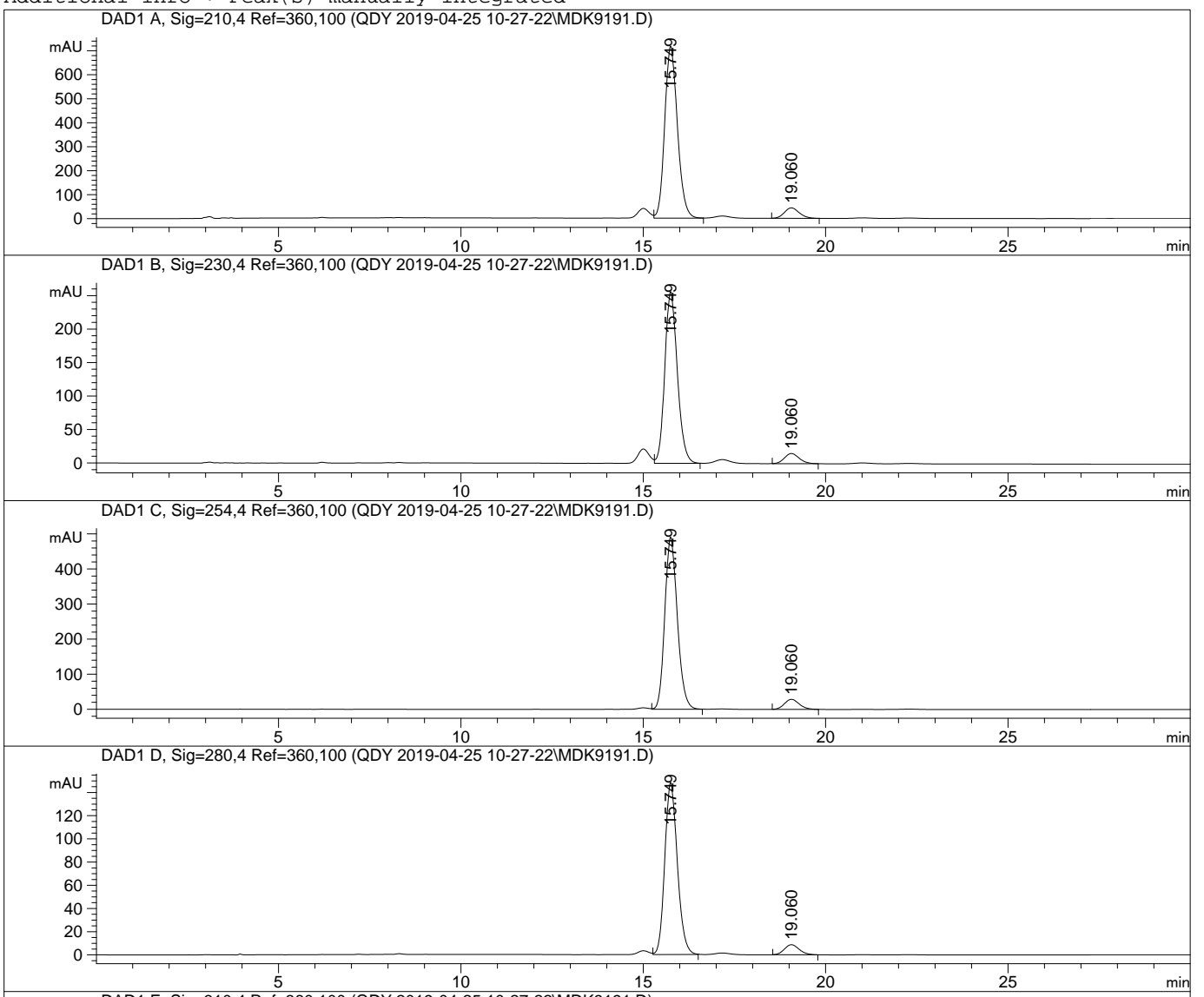

DAD1 E, Sig=310,4 Reft=360,100 (QDY 2019-04-25 10-27-22IMDK9191.D)

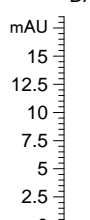

2.5 彗

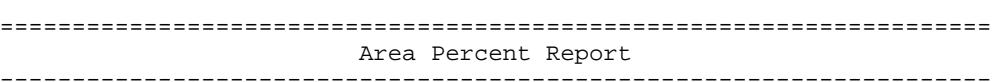

Sorted By

Signal

Multiplier

1.0000

Use Multiplier \& Dilution Factor with ISTDs

Signal 1: DAD1 A, Sig=210, 4 Ref $=360,100$

\begin{tabular}{cccccc}
$\begin{array}{c}\text { Peak RetTime Type } \\
\text { \# }\end{array}\left[\begin{array}{c}\text { Width } \\
{[\mathrm{min}]}\end{array}\right.$ & $\begin{array}{c}\text { Area } \\
{[\mathrm{mAU}}\end{array}$ & $\begin{array}{l}\text { Height } \\
{[\mathrm{mAU}]}\end{array}$ & $\begin{array}{c}\text { Area } \\
\%\end{array}$ \\
\hdashline$-15.749 \mathrm{VB}$ & 0.3753 & $1.74097 \mathrm{e} 4$ & 717.04938 & 93.3069 \\
\hline 2 & $19.060 \mathrm{BB}$ & 0.4407 & 1248.84058 & 43.78655 & 6.6931
\end{tabular}

Totals :

$1.86585 \mathrm{e} 4 \quad 760.83593$

Signal 2: DAD1 B, Sig=230, 4 Ref $=360,100$

\begin{tabular}{cccccc}
$\begin{array}{c}\text { Peak RetTime Type } \\
\#\end{array}\left[\begin{array}{c}\text { Width } \\
{[\mathrm{min}]}\end{array}\right.$ & $\begin{array}{c}\text { Area } \\
{[\mathrm{mAU}}\end{array}$ & $\begin{array}{l}\text { Height } \\
{[\mathrm{mAU}]}\end{array}$ & $\begin{array}{c}\text { Area } \\
\%\end{array}$ \\
\hdashline & $15.749 \mathrm{VB}$ & 0.3698 & 6152.39551 & 256.50116 & 93.4484 \\
\hline 2 & $19.060 \mathrm{BB}$ & 0.4368 & 431.34009 & 15.21080 & 6.5516
\end{tabular}

Totals :

$6583.73560 \quad 271.71196$

Signal 3: DAD1 C, Sig $=254,4$ Ref $=360,100$

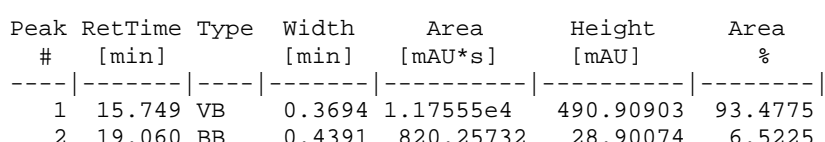

Totals : $\quad 1.25757 \mathrm{e} 4 \quad 519.80977$

Signal 4: DAD1 D, Sig=280, 4 Ref $=360,100$

Peak RetTime Type Width Area Height Area

\# [min] $[$ [min] [mAU*s] [mAU] $1 \%$

$\begin{array}{rrrrrr}1 & 15.749 \mathrm{VB} & 0.3713 & 3550.69824 & 148.31688 & 93.4974 \\ 2 & 19.060 \mathrm{BB} & 0.4338 & 246.94508 & 8.73579 & 6.5026\end{array}$

Totals :

$3797.64333 \quad 157.05267$

$\mathrm{HO}$

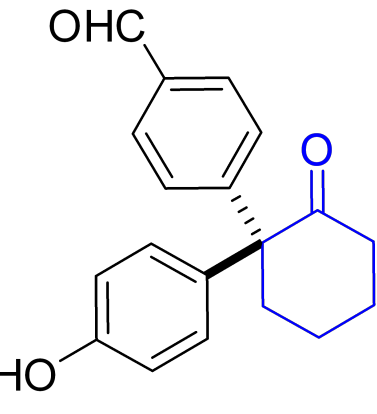

2i

Enantioenriched

Signal 5: DAD1 E, Sig $=310,4$ Ref $=360,100$

*** End of Report *** 
Area Percent Report

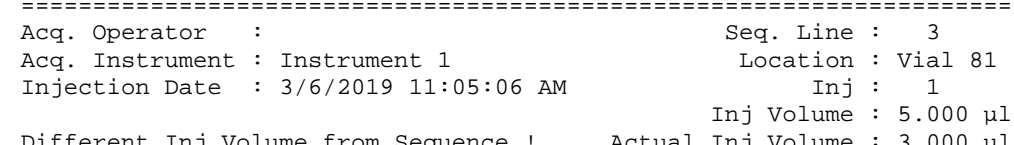

Different Inj volume from Sequence ! Actual Inj Volume : $5.000 \mu \mathrm{\mu}$

Acq. Method : C: \CHEM32\1\DATA IQDY 2019-03-06 10-25-26\AD-10-25.M

Last changed : 3/6/2019 10:38:08 AM

Analysis Method : C: \CHEM32\1\METHODS\OD-01-10-0.3.M

Last changed : 5/29/2019 9:25:17 PM

Additional Info : Peak(s) manually integrated
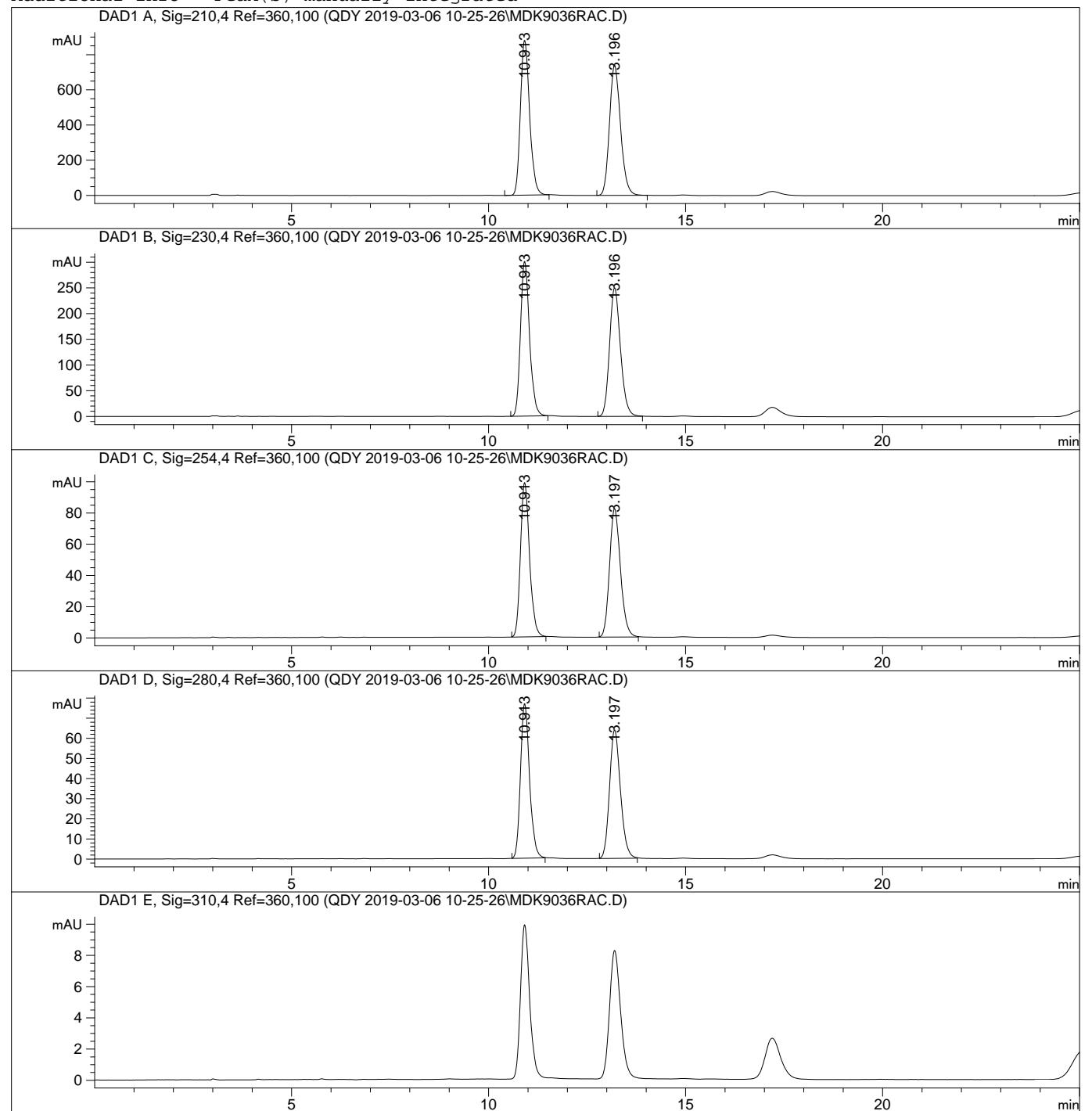

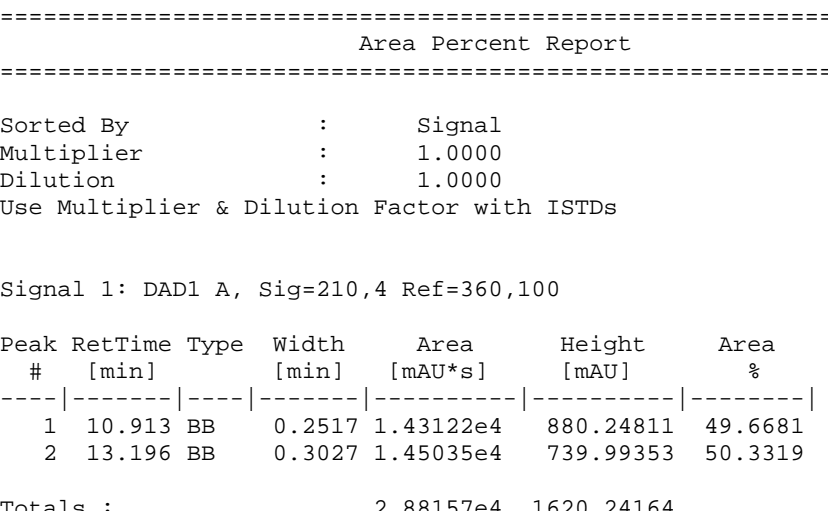

Signal 2: DAD1 B, Sig=230, 4 Ref $=360,100$

\begin{tabular}{|c|c|c|c|c|c|}
\hline $\begin{array}{l}\text { Peak } \\
\#\end{array}$ & $\begin{array}{l}\text { RetTime Type } \\
{[\text { min] }}\end{array}$ & $\begin{array}{l}\text { Width } \\
\text { [min] }\end{array}$ & $\begin{array}{c}\text { Area } \\
{\left[\mathrm{mAU} U^{*} \mathrm{~s}\right]}\end{array}$ & $\begin{array}{l}\text { Height } \\
{[\mathrm{mAU}]}\end{array}$ & $\begin{array}{c}\text { Area } \\
\%\end{array}$ \\
\hline 1 & $10.913 \mathrm{BB}$ & 0.246 & 4808.7 & 300. & 956 \\
\hline 2 & $13.196 \mathrm{BB}$ & 0.2972 & 4828.87256 & 250.12534 & 50.1044 \\
\hline
\end{tabular}

Totals :

$9637.62793 \quad 551.08960$

Signal 3: DAD1 C, Sig $=254,4$ Ref $=360,100$

\begin{tabular}{|c|c|c|c|c|c|}
\hline $\begin{array}{c}\text { Peak } \\
\#\end{array}$ & $\begin{array}{l}\text { RetTime Type } \\
\text { [min] }\end{array}$ & $\begin{array}{l}\text { Width } \\
\text { [min] }\end{array}$ & $\begin{array}{c}\text { Area } \\
{\left[\mathrm{mAU}^{*} \mathrm{~s}\right]}\end{array}$ & $\begin{array}{l}\text { Height } \\
{[\mathrm{mAU}]}\end{array}$ & $\begin{array}{c}\text { Area } \\
\%\end{array}$ \\
\hline 1 & $10.913 \mathrm{BB}$ & 0.2469 & 1583.37817 & 98.81934 & 49.9078 \\
\hline 2 & $13.197 \mathrm{BB}$ & 0.2975 & 1589.23010 & 82.23269 & 50.0922 \\
\hline
\end{tabular}

Totals :

$3172.60828 \quad 181.05203$

Signal 4: DAD1 D, Sig=280, 4 Ref $=360,100$

\begin{tabular}{|c|c|c|c|c|c|}
\hline $\begin{array}{c}\text { Peak } \\
\#\end{array}$ & $\begin{array}{l}\text { RetTime Type } \\
\text { [min] }\end{array}$ & $\begin{array}{l}\text { Width } \\
\text { [min] }\end{array}$ & $\begin{array}{c}\text { Area } \\
{\left[\mathrm{mAU} U^{*} \mathrm{~s}\right]}\end{array}$ & $\begin{array}{l}\text { Height } \\
{[\mathrm{mAU}]}\end{array}$ & $\begin{array}{c}\text { Area } \\
\%\end{array}$ \\
\hline & $10.913 \mathrm{BB}$ & & 1227.84399 & 76.84303 & 49.9315 \\
\hline 2 & $13.197 \mathrm{BB}$ & 0.2969 & 1231.21472 & 63.87856 & 50.0685 \\
\hline
\end{tabular}

Totals :

$2459.05872 \quad 140.72159$

Signal 5: DAD1 E, Sig $=310,4$ Ref $=360,100$

$\mathrm{HO}$

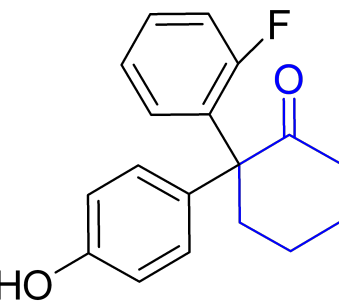

racemic

$\star \star *$ End of Report *** 
Area Percent Report

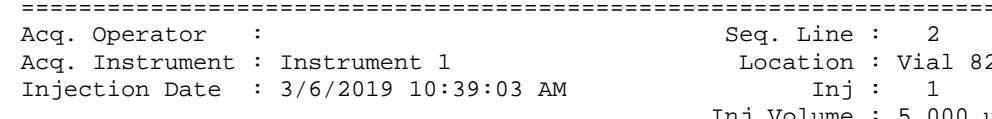

Injection Inj Volume : $5.000 \mu \mathrm{\mu l}$

$\begin{array}{ll}\text { Acq. Method } & \text { C: } \backslash \text { CHEM32 } 10: 38: 08 \text { AM } \\ \text { Last changed } & 3 / 6 / 2019 \text { 10:38:08 A }\end{array}$

Analysis Method : C: \CHEM32\1\METHODS\OD-01-10-0.3.M

Last changed : 5/29/2019 9:25:17 PM

Additional Info : Peak(s) manually integrated
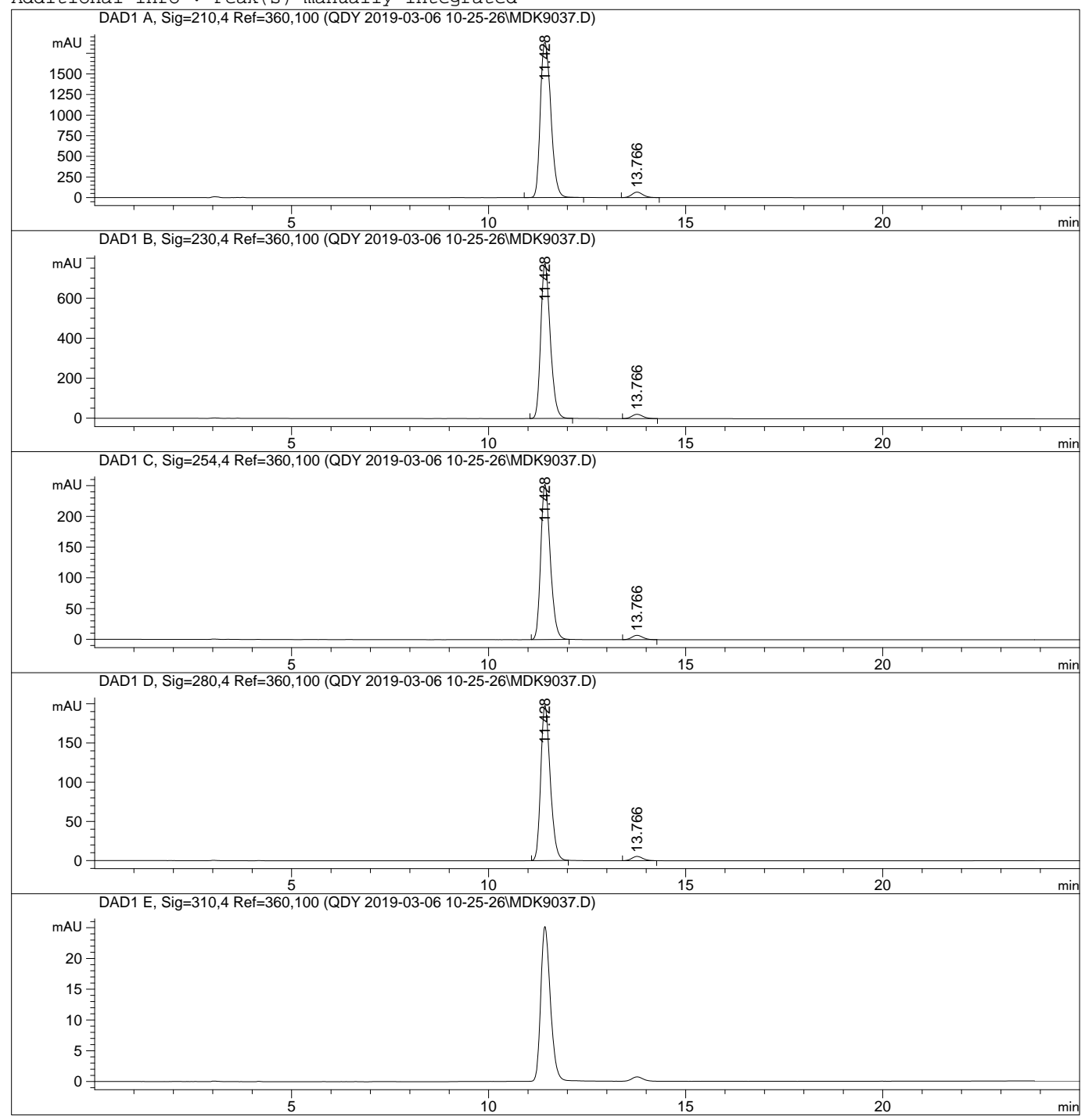

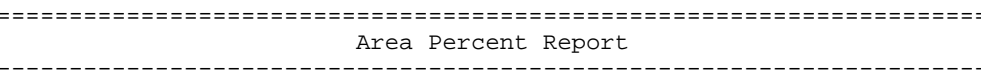

Sorted By

Signal

Multiplier
Dilution

1.0000

Use Multiplier \& Dilution Factor with ISTDs

Signal 1: DAD1 A, Sig=210, 4 Ref $=360,100$

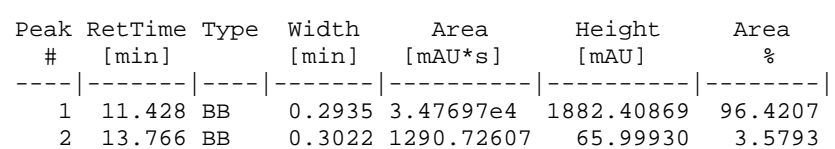

Totals :

$3.60604 \mathrm{e} 4 \quad 1948.40799$

Signal 2: DAD1 B, Sig $=230,4$ Ref $=360,100$

\begin{tabular}{cccccc}
$\begin{array}{c}\text { Peak RetTime Type } \\
\text { \# }\end{array}\left[\begin{array}{c}\text { Width } \\
{[\mathrm{min}]}\end{array}\right.$ & $\begin{array}{c}\text { Area } \\
{[\mathrm{mAU}}\end{array}$ & $\begin{array}{l}\text { Height } \\
{[\mathrm{mAU}]}\end{array}$ & $\begin{array}{c}\text { Area } \\
\%\end{array}$ \\
\hdashline-11.428 & $\mathrm{BB}$ & 0.2588 & $1.29543 \mathrm{e} 4$ & 775.64429 & 96.8828 \\
\hline 2 & $13.766 \mathrm{BB}$ & 0.3012 & 416.80280 & 21.40347 & 3.1172
\end{tabular}

Totals :

$1.33711 \mathrm{e} 4 \quad 797.04776$

Signal 3: DAD1 C, Sig $=254,4$ Ref $=360,100$

\begin{tabular}{|c|c|c|c|c|c|}
\hline$\underset{\#}{e a k}$ & $\begin{array}{l}\text { RetTime Type } \\
\text { [min] }\end{array}$ & $\begin{array}{l}\text { Width } \\
\text { [min] }\end{array}$ & $\begin{array}{c}\text { Area } \\
{\left[\mathrm{mAU} U^{*} \mathrm{~s}\right]}\end{array}$ & $\begin{array}{l}\text { Height } \\
{[\mathrm{mAU}]}\end{array}$ & $\begin{array}{c}\text { Area } \\
\%\end{array}$ \\
\hline & $8 \mathrm{~B}$ & 0.2595 & 4230.1460 & 252.40833 & \\
\hline
\end{tabular}

Totals :

$4367.77913 \quad 259.49959$

Signal 4: DAD1 D, Sig=280, 4 Ref $=360,100$

Peak RetTime Type Width Area Height Area

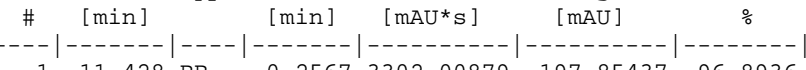

$\begin{array}{llllll}1 & 11.428 \mathrm{BB} & 0.2567 & 3302.00879 & 197.85437 & 96.8936\end{array}$

Totals : $\quad 3407.87244 \quad 203.32283$

$\mathrm{HO}$

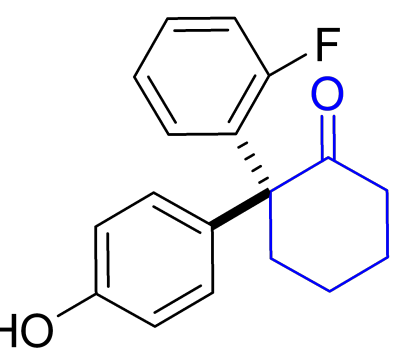

2j

Signal 5: DAD1 E, Sig $=310,4$ Ref $=360,100$

Enantioenriched

$* * *$ End of Report *** 


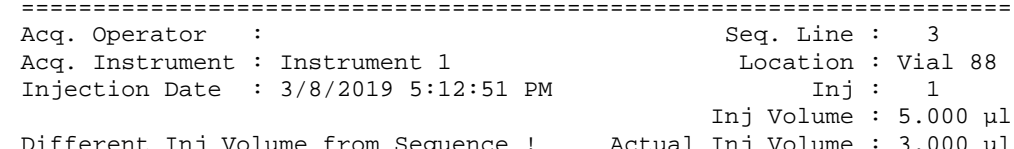

Injection Date $\quad \begin{aligned} \text { Inj } & 1 \\ & \text { Inj Volume }: 5.000 \mu 1\end{aligned}$

Acq. Method : C: \CHEM32\1\DATA QQDY 2019-03-08 16-18-03\AD-10-40.M

Last changed : 3/8/2019 4:30:52 PM

Analysis Method : C: \CHEM32\1\METHODS\OD-01-10-0.3.M

Adet : $5 / 29 / 2019$ 9:25:17 PM

Additional Info : Peak(s) manialily
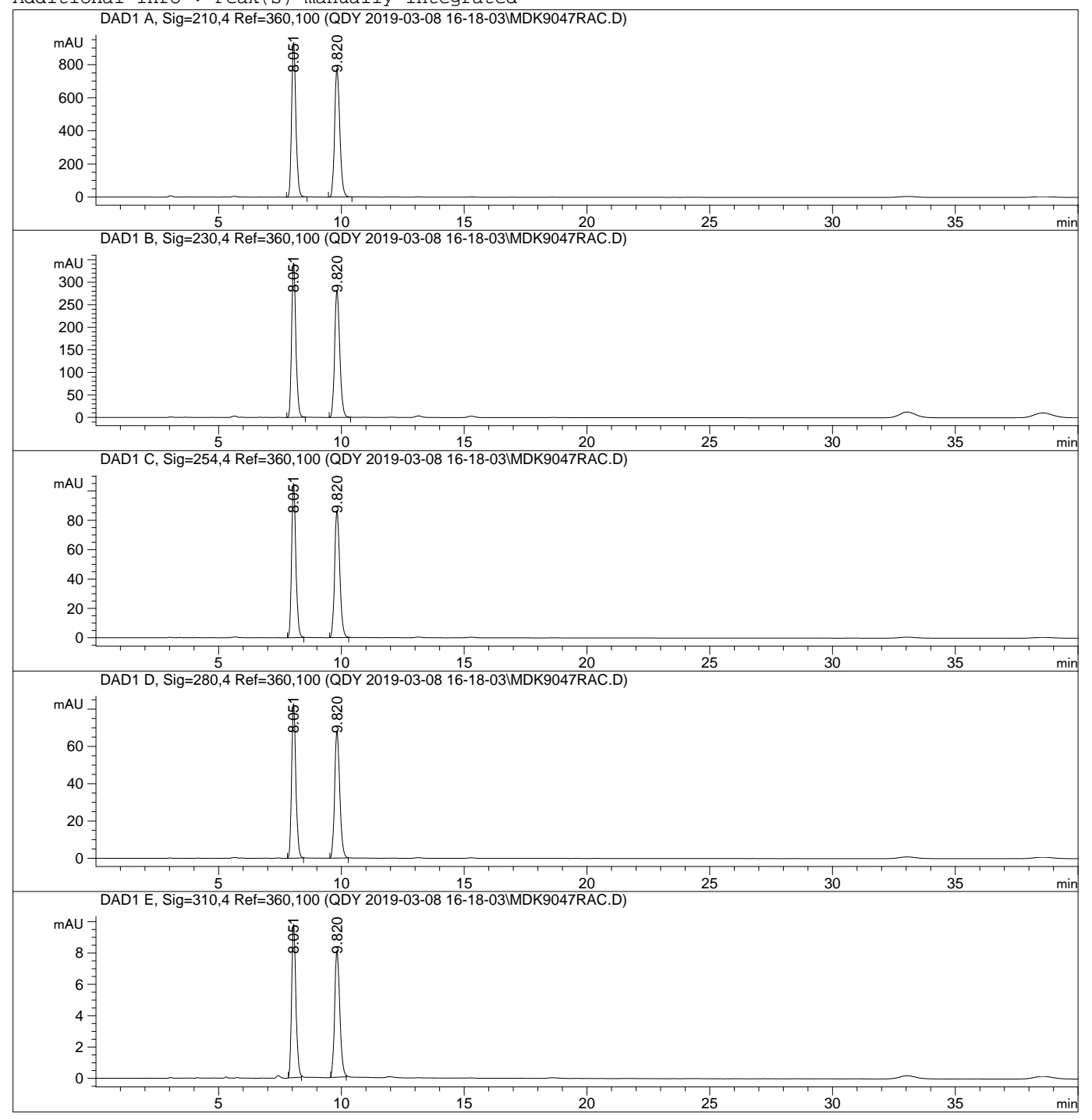

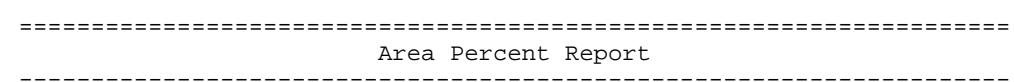

\section{Sorted By}

Signal

Multiplier
Dilution

1.0000

Use Multiplier \& Dilution Factor with ISTDs

Signal 1: DAD1 A, Sig=210, 4 Ref $=360,100$

\begin{tabular}{|c|c|c|c|c|c|}
\hline $\begin{array}{c}\text { Peak } \\
\#\end{array}$ & $\begin{array}{l}\text { RetTime Type } \\
{[\mathrm{min}]}\end{array}$ & $\begin{array}{l}\text { Width } \\
\text { [min] }\end{array}$ & $\begin{array}{c}\text { Area } \\
{\left[\mathrm{mAU} U^{*} \mathrm{~s}\right]}\end{array}$ & $\begin{array}{l}\text { Height } \\
{[\mathrm{mAU}]}\end{array}$ & $\begin{array}{c}\text { Area } \\
\%\end{array}$ \\
\hline $\begin{array}{l}1 \\
2\end{array}$ & $\begin{array}{l}8.051 \mathrm{BB} \\
9.820 \mathrm{BB}\end{array}$ & $\begin{array}{l}0.1868 \\
0.2267\end{array}$ & $\begin{array}{l}1.12736 \mathrm{e} 4 \\
1.13593 \mathrm{e} 4\end{array}$ & $\begin{array}{l}933.92279 \\
776.47992\end{array}$ & $\begin{array}{l}49.8105 \\
50.1895\end{array}$ \\
\hline
\end{tabular}

Totals :

$2.26329 \mathrm{e} 4 \quad 1710.40271$

Signal 2: DAD1 B, Sig=230, 4 Ref $=360,100$

\begin{tabular}{|c|c|c|c|c|c|}
\hline $\begin{array}{c}\text { Peak } \\
\#\end{array}$ & $\begin{array}{l}\text { RetTime Type } \\
{[\text { min] }}\end{array}$ & $\begin{array}{l}\text { Width } \\
\text { [min] }\end{array}$ & $\begin{array}{c}\text { Area } \\
{\left[\mathrm{mAU} U^{\star} \mathrm{s}\right]}\end{array}$ & $\begin{array}{l}\text { Height } \\
{[\mathrm{mAU}]}\end{array}$ & $\begin{array}{c}\text { Area } \\
\%\end{array}$ \\
\hline $\begin{array}{l}1 \\
2\end{array}$ & $\begin{array}{l}8.051 \mathrm{BB} \\
9.820 \mathrm{BB}\end{array}$ & $\begin{array}{l}0.1827 \\
0.2228\end{array}$ & $\begin{array}{l}4076.86035 \\
4085.37085\end{array}$ & $\begin{array}{l}342.90671 \\
282.41019\end{array}$ & $\begin{array}{l}49.9479 \\
50.0521\end{array}$ \\
\hline
\end{tabular}

Totals :

$8162.23120 \quad 625.31689$

Signal 3: DAD1 C, Sig $=254,4$ Ref $=360,100$

\begin{tabular}{|c|c|c|c|c|c|}
\hline eak & $\begin{array}{l}\text { RetTime Type } \\
\text { [min] }\end{array}$ & $\begin{array}{l}\text { Width } \\
\text { [min] }\end{array}$ & $\begin{array}{c}\text { Area } \\
{\left[\mathrm{mAU}^{*} \mathrm{~s}\right]}\end{array}$ & $\begin{array}{l}\text { Height } \\
{[\mathrm{mAU}]}\end{array}$ & $\begin{array}{c}\text { Area } \\
\%\end{array}$ \\
\hline & $.051 \mathrm{BE}$ & 020 & 1252.68311 & & \\
\hline
\end{tabular}

Totals :

$2507.86304 \quad 191.68414$

Signal 4: DAD1 D, Sig=280, 4 Ref $=360,100$

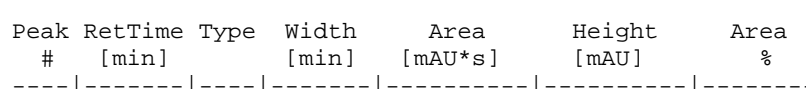

10.051 BB $0.1827 \quad 985.01123 \quad 82.83432 \quad 090591$

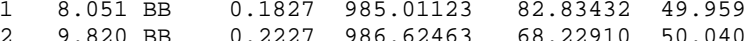

Totals :

$1971.63586 \quad 151.06342$

Signal 5: DAD1 E, Sig=310, 4 Ref $=360,100$

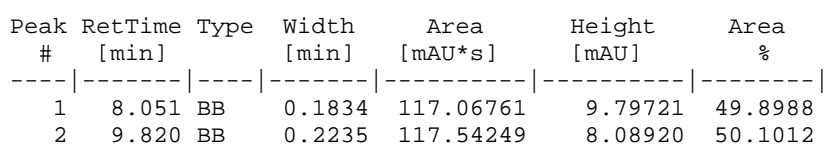

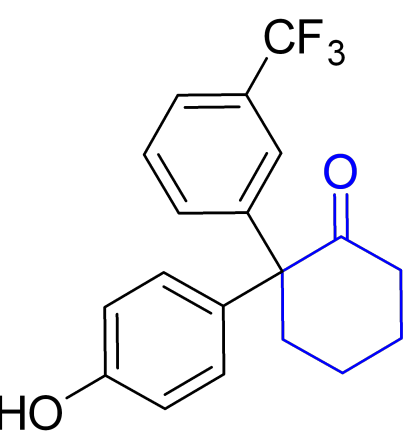

racemic 
Analysis Method : C: \CHEM32\1\METHODS \OD-01-10-0.3.M

Last changed : 5/29/2019 9:25:17 PM

$\frac{\text { Additional Info : Peak (s) manually integrated }}{\text { DAD1 A, Sig=210,4 Ref }=360,100 \text { (QDY 2019-03-08 16-18-031MDK9048.D) }}$
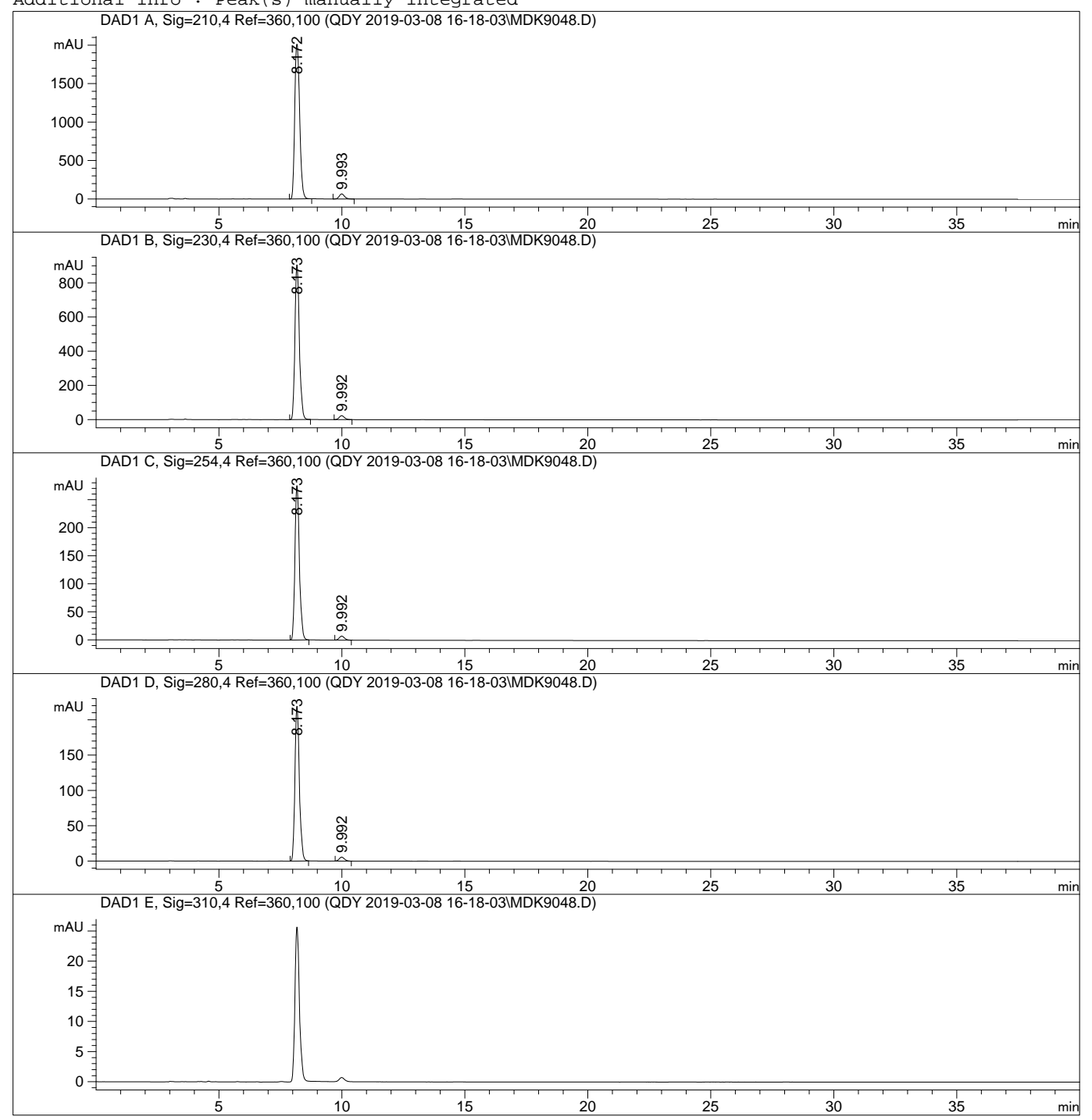

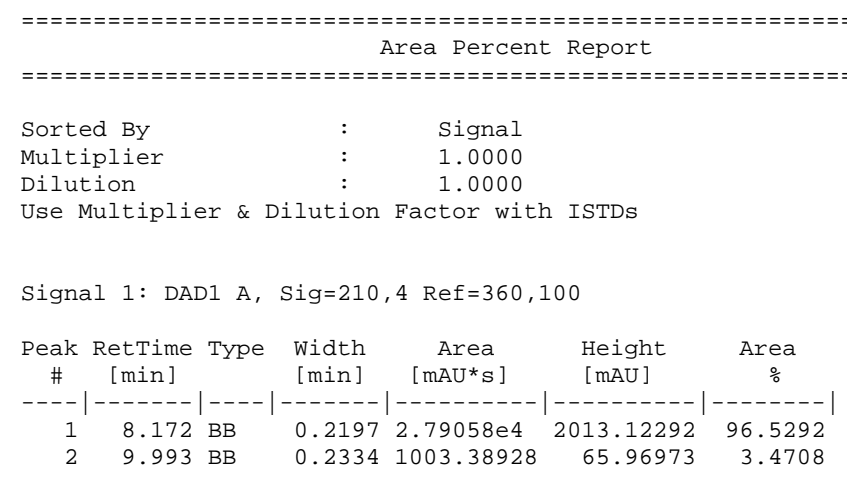

Totals :

$2.89092 \mathrm{e} 4 \quad 2079.09265$

Signal 2: DAD1 B, Sig=230, 4 Ref $=360,100$

\begin{tabular}{|c|c|c|c|c|c|}
\hline $\begin{array}{c}\text { Peak } \\
\#\end{array}$ & $\begin{array}{l}\text { RetTime Type } \\
\text { [min] }\end{array}$ & $\begin{array}{l}\text { Width } \\
\text { [min] }\end{array}$ & $\begin{array}{c}\text { Area } \\
{\left[\mathrm{mAU} U^{*} \mathrm{~s}\right]}\end{array}$ & $\begin{array}{l}\text { Height } \\
{[\mathrm{mAU}]}\end{array}$ & $\begin{array}{c}\text { Area } \\
\%\end{array}$ \\
\hline 1 & $8.173 \mathrm{BB}$ & 0.1913 & $\begin{array}{c}1.12801 \mathrm{e} 4 \\
\end{array}$ & 905.52655 & $\begin{array}{r}96.9385 \\
3.0615\end{array}$ \\
\hline
\end{tabular}

Totals :

$1.16363 \mathrm{e} 4 \quad 928.86514$

Signal 3: DAD1 C, Sig $=254,4$ Ref $=360,100$

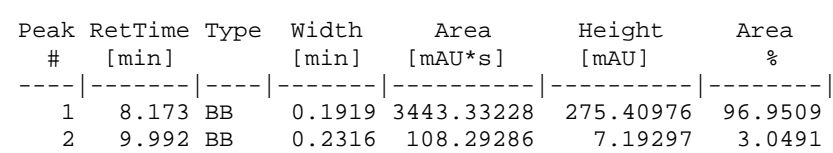

Totals :

$3551.62514 \quad 282.60273$

Signal 4: DAD1 D, Sig $=280,4$ Ref $=360,100$

Peak RetTime Type Width Area Height Area

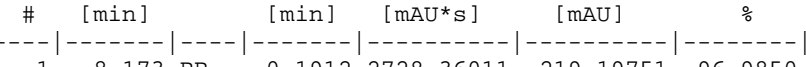

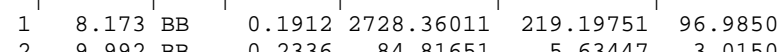

Totals :

$2813.17662 \quad 224.83198$

Signal 5: DAD1 E, Sig=310, 4 Ref $=360,100$

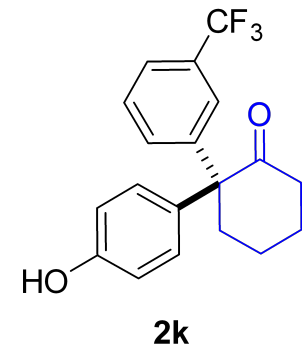

Enantioenriched

$\star \star *$ End of Report *** 


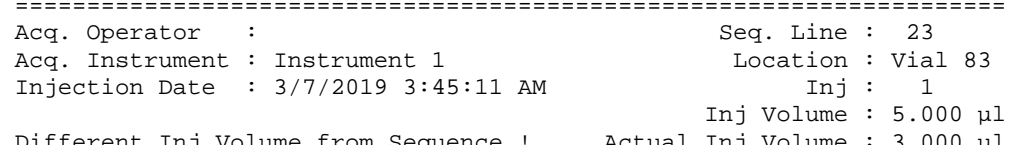

Injection Date $\quad$ Inj Volume $: 5.000 \mu \mathrm{\mu l}$ Acq. Method : C: \CHEM32\1\DATA Q QDY 2019-03-06 20-24-29\AD-10-20.M Last changed : 3/7/2019 3:23:14 AM

Analysis Method : C: \CHEM32\1\METHODS\OD-๑1-10-0.3.M

: $5 / 29 / 20199: 25: 17$ PM

Additional Info : Peak(s) manually integrated
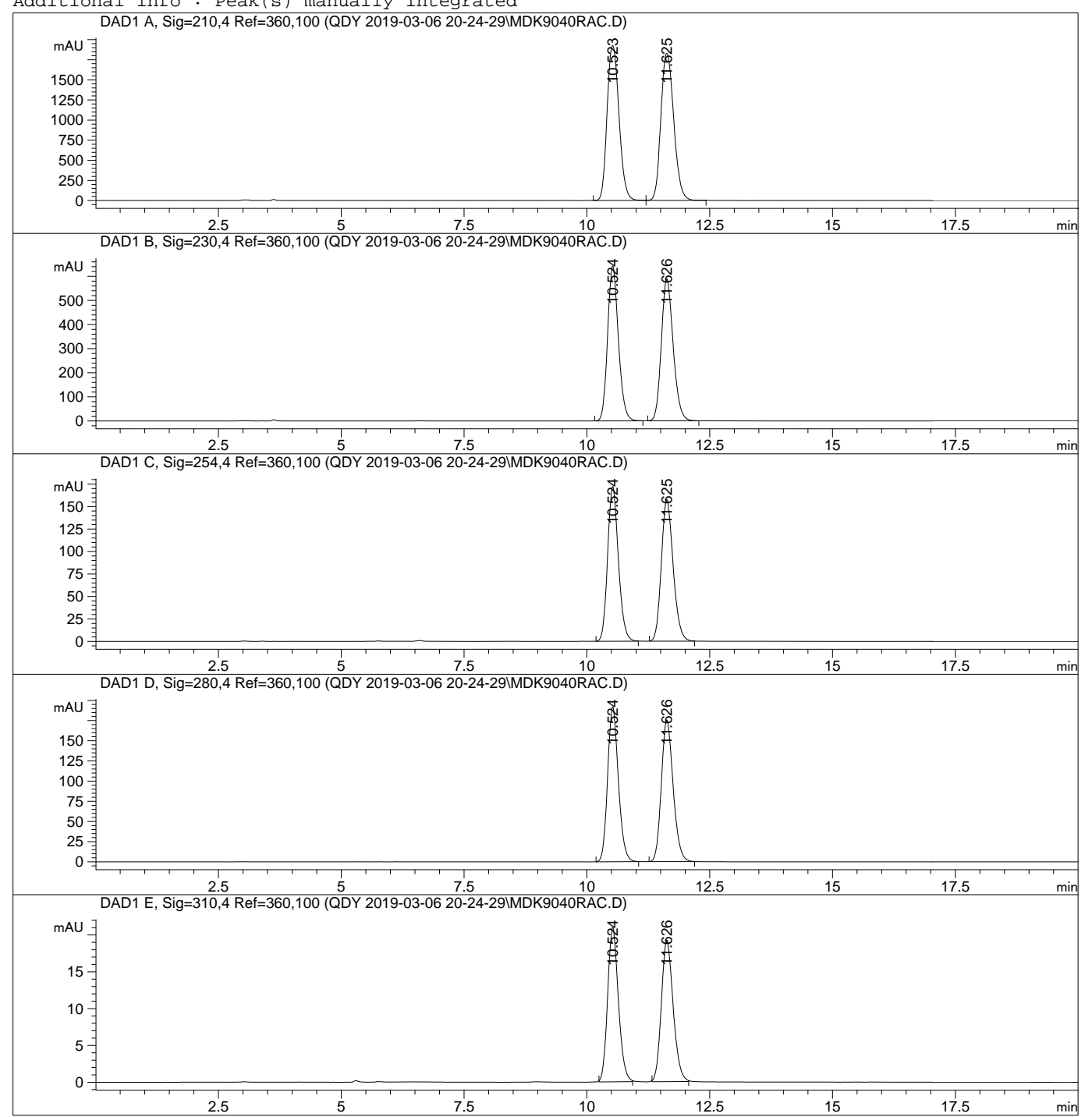

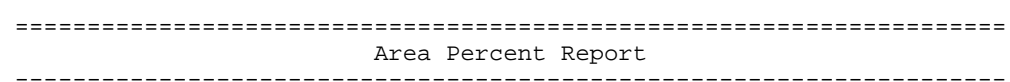

Sorted By

\section{Signal}

Multiplier
Dilution

1.0000

Use Multiplier \& Dilution Factor with ISTDs

Signal 1: DAD1 A, Sig=210, 4 Ref $=360,100$

\begin{tabular}{|c|c|c|c|c|c|}
\hline $\begin{array}{l}\text { Peak } \\
\#\end{array}$ & $\begin{array}{l}\text { RetTime Type } \\
\text { [min] }\end{array}$ & $\begin{array}{l}\text { Width } \\
\text { [min] }\end{array}$ & $\begin{array}{c}\text { Area } \\
{\left[\mathrm{mAU} U^{*} \mathrm{~s}\right]}\end{array}$ & $\begin{array}{l}\text { Height } \\
{[\mathrm{mAU}]}\end{array}$ & $\begin{array}{c}\text { Area } \\
\%\end{array}$ \\
\hline 1 & 10.523 BV & 0.2667 & $3.24554 \mathrm{e} 4$ & 1925.08972 & $\begin{array}{l}49.4048 \\
50\end{array}$ \\
\hline
\end{tabular}

Totals :

$6.56929 \mathrm{e} 4 \quad 3753.06567$

Signal 2: DAD1 B, Sig=230, 4 Ref $=360,100$

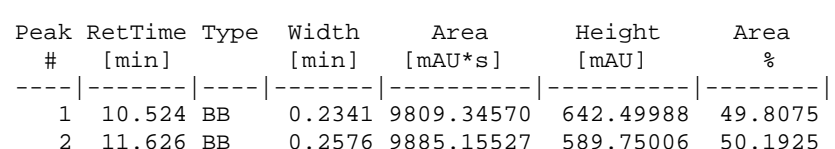

Totals :

$1.96945 \mathrm{e} 4 \quad 1232.24994$

Signal 3: DAD1 C, Sig $=254,4$ Ref $=360,100$

\begin{tabular}{cccccc}
$\begin{array}{c}\text { Peak RetTime Type } \\
\text { \# }\end{array}\left[\begin{array}{c}\text { Width } \\
{[\mathrm{min}]}\end{array}\right.$ & $\begin{array}{c}\text { Area } \\
{[\mathrm{mAU}}\end{array}$ & $\begin{array}{l}\text { Height } \\
{[\mathrm{mAU}]}\end{array}$ & $\begin{array}{c}\text { Area } \\
\%\end{array}$ \\
\hdashline-10.524 & $\mathrm{BB}$ & 0.2339 & 2625.75464 & 172.16739 & 49.8874 \\
\hline 2 & $11.625 \mathrm{BB}$ & 0.2572 & 2637.60400 & 157.63029 & 50.1126
\end{tabular}

Totals :

$5263.35864 \quad 329.79768$

Signal 4: DAD1 D, Sig=280, 4 Ref $=360,100$

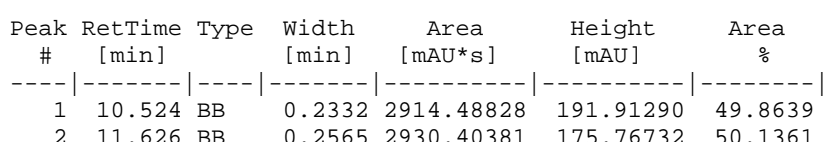

Totals :

$5844.89209 \quad 367.68022$

Signal 5: DAD1 E, Sig $=310,4$ Ref $=360,100$

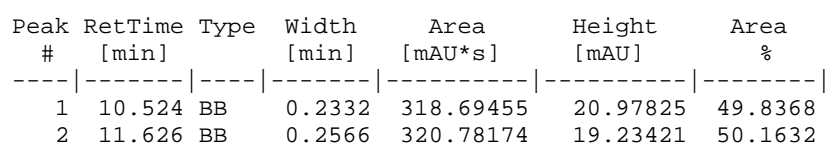

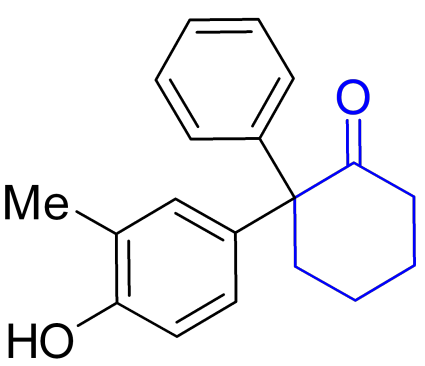

racemic

$\begin{array}{lll}\text { Totals : } & 639.47629 & 40.21247\end{array}$ 


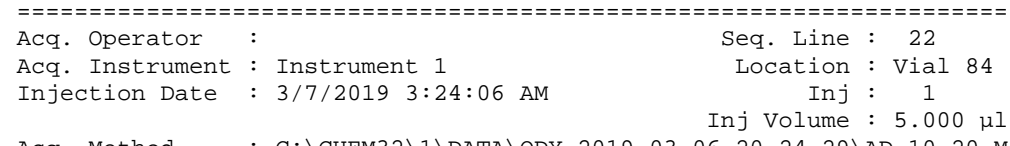

Inj volume : $5.000 \mu \mathrm{I}$ $\begin{array}{ll}\text { Acq. Method } & \text { C: }: \text { CHEM32 } 1 \text { DATA } \\ \text { Last changed } & \text { 3/7/2019 3:23:14 AM }\end{array}$

Analysis Method : C: $\backslash$ CHEM32\1\METHODS\OD-01-10-0.3.M

Last changed : 5/29/2019 9:25:17 PM

Additional Info: Peak(s) manually i
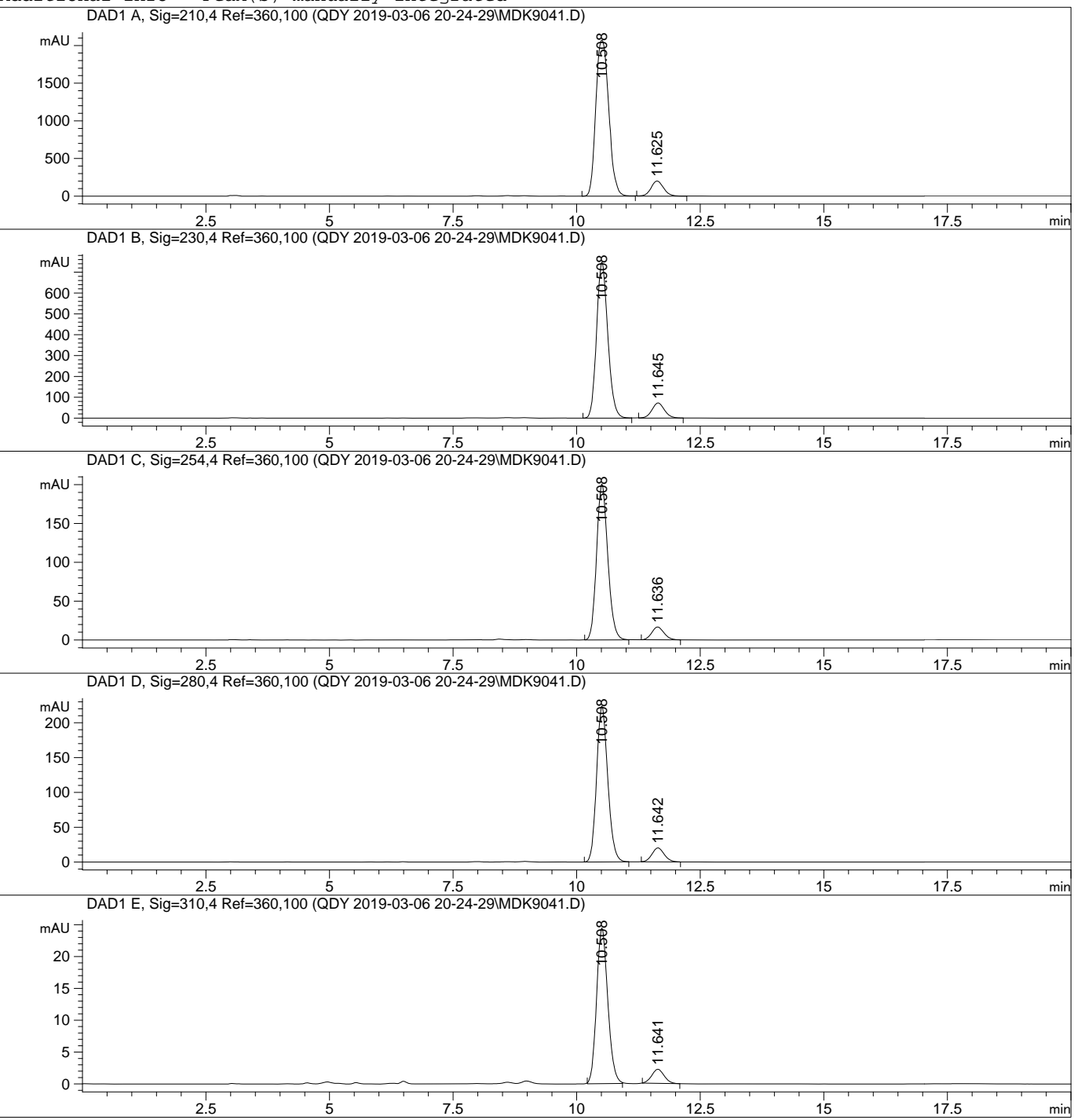

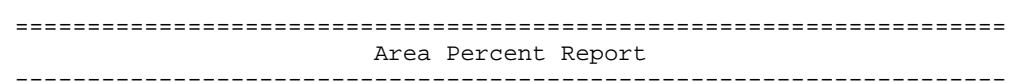

Sorted By

\section{Signal}

Multiplier
Dilution

1.0000

Use Multiplier \& Dilution Factor with ISTDs

Signal 1: DAD1 A, Sig=210, 4 Ref $=360,100$

\begin{tabular}{cccccc}
$\begin{array}{c}\text { Peak RetTime Type } \\
\text { \# }\end{array}\left[\begin{array}{c}\text { Width } \\
{[\mathrm{min}]}\end{array}\right.$ & $\begin{array}{c}\text { Area } \\
{[\mathrm{mAU}}\end{array}$ & $\begin{array}{l}\text { Height } \\
{[\mathrm{mAU}]}\end{array}$ & $\begin{array}{c}\text { Area } \\
\%\end{array}$ \\
\hdashline-10.508 & $\mathrm{BB}$ & 0.2880 & $3.76263 \mathrm{e} 4$ & 2070.63403 & 91.2276 \\
\hline 2 & $11.625 \mathrm{BB}$ & 0.2748 & 3618.10425 & 202.18167 & 8.7724
\end{tabular}

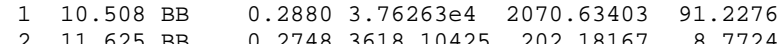

Totals :

$4.12444 \mathrm{e} 4 \quad 2272.81570$

Signal 2: DAD1 B, Sig $=230,4$ Ref $=360,100$

\begin{tabular}{|c|c|c|c|c|c|}
\hline $\begin{array}{c}\text { Peak } \\
\text { \# }\end{array}$ & $\begin{array}{l}\text { RetTime Type } \\
\text { [min] }\end{array}$ & $\begin{array}{l}\text { Width } \\
\text { [min] }\end{array}$ & $\begin{array}{c}\text { Area } \\
{\left[\mathrm{mAU}^{*} \mathrm{~s}\right]}\end{array}$ & $\begin{array}{l}\text { Height } \\
{[\mathrm{mAU}]}\end{array}$ & $\begin{array}{c}\text { Area } \\
\%\end{array}$ \\
\hline & $\begin{array}{l}10.508 \mathrm{BB} \\
11.645 \mathrm{BB}\end{array}$ & $\begin{array}{l}0.2471 \\
0.2726\end{array}$ & $\begin{array}{l}1.18629 \mathrm{e} 4 \\
1283.53638\end{array}$ & $\begin{array}{r}747.79645 \\
72.48436\end{array}$ & $\begin{array}{r}90.2366 \\
9.7634\end{array}$ \\
\hline
\end{tabular}

Totals :

$1.31464 \mathrm{e} 4 \quad 820.28081$

Signal 3: DAD1 C, Sig $=254,4$ Ref $=360,100$

\begin{tabular}{cccccc}
$\begin{array}{c}\text { Peak RetTime Type } \\
\text { \# }\end{array}\left[\begin{array}{c}\text { Width } \\
{[\mathrm{min}]}\end{array}\right.$ & $\begin{array}{c}\text { Area } \\
{[\mathrm{mAU}}\end{array}$ & $\begin{array}{l}\text { Height } \\
{[\mathrm{mAU}]}\end{array}$ & $\begin{array}{c}\text { Area } \\
\%\end{array}$ \\
\hdashline-10.508 & $\mathrm{BB}$ & 0.2448 & 3176.19287 & 200.48097 & 91.7041 \\
\hline 2 & $11.636 \mathrm{BB}$ & 0.2685 & 287.32950 & 16.55358 & 8.2959
\end{tabular}

Totals :

$3463.52237 \quad 217.03455$

Signal 4: DAD1 D, Sig=280, 4 Ref $=360,100$

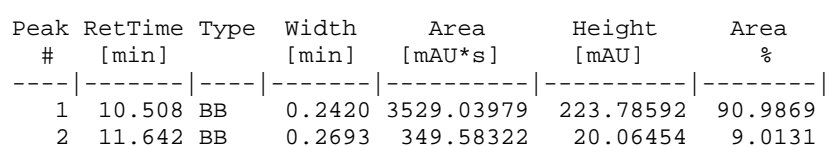

Totals :

$3878.62302 \quad 243.85046$

Signal 5: DAD1 E, Sig $=310,4$ Ref $=360,100$

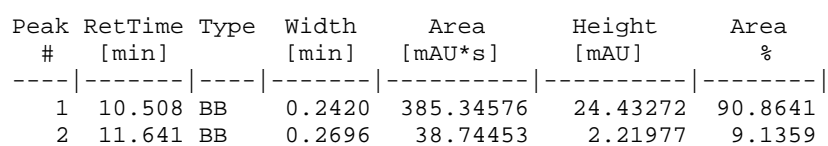

Totals :

$424.09029 \quad 26.65249$

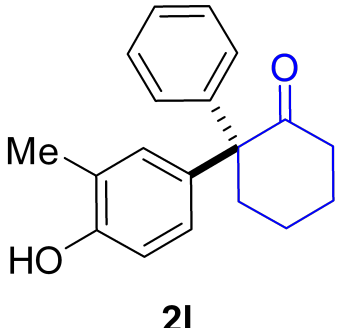

Enantioenriched 
Area Percent Report

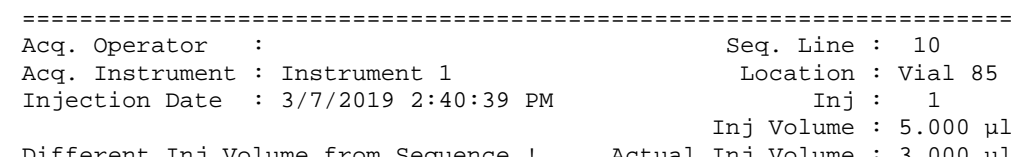

Different Inj Volume from Sequence ! Actual Inj Volume : $5.000 \mu \mathrm{\mu}$ Acq. Method : C: \CHEM32\1\DATA Last changed : 3/7/2019 1:58:43 PM

Analysis Method : C: \CHEM32\1\METHODS\OD-01-10-0.3.M

: $5 / 29 / 2019$ 9:25:17 PM

Additional Info : Peak(s) manually integrated
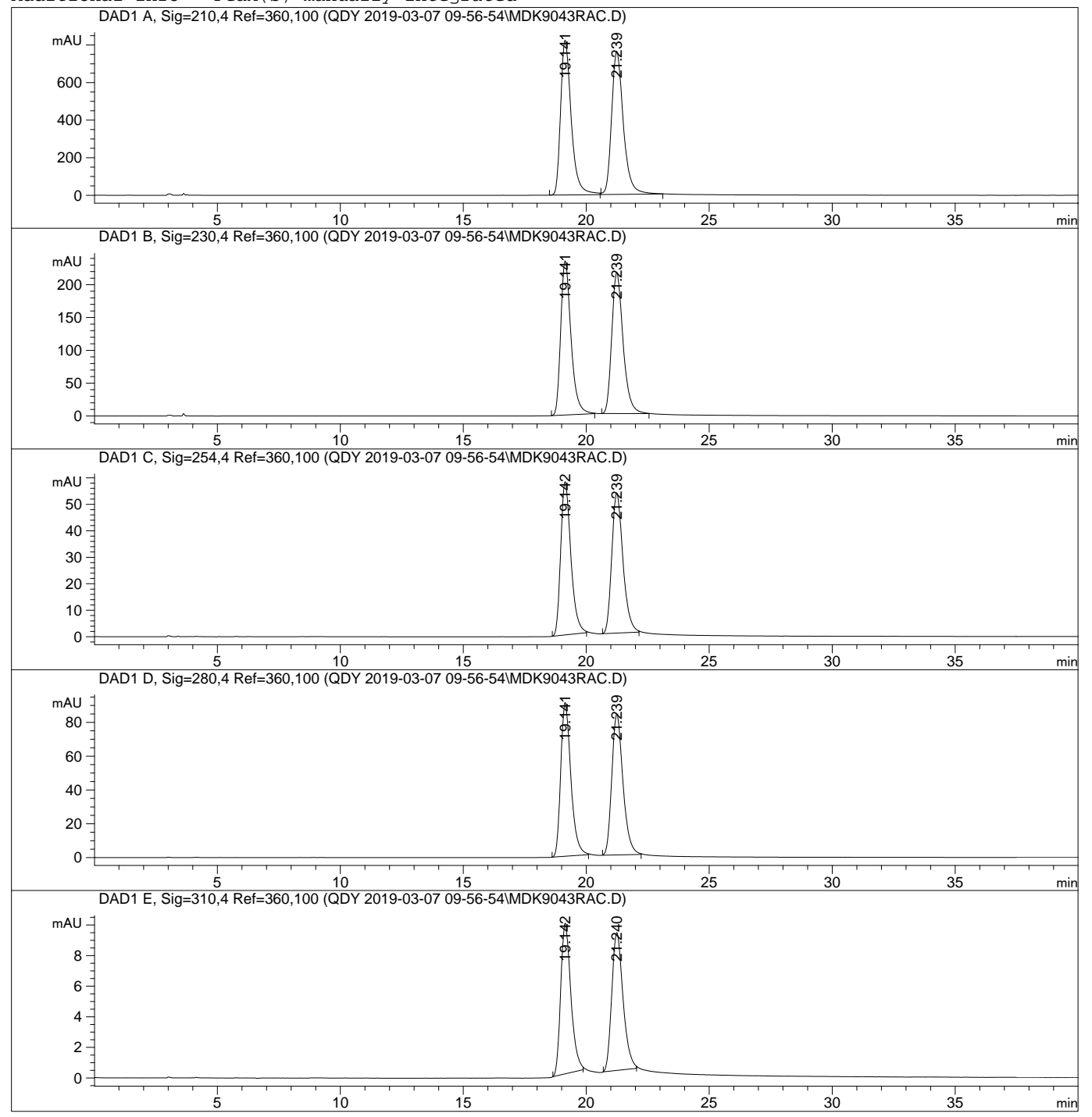

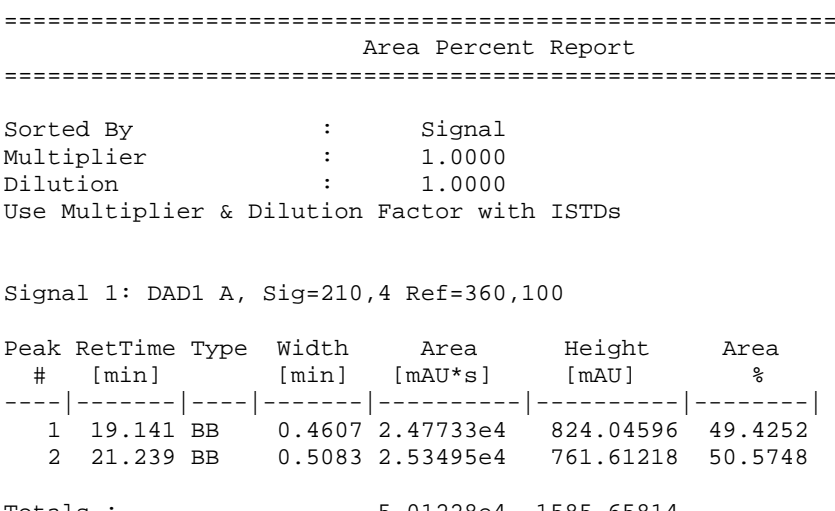

Totals :

$5.01228 \mathrm{e} 4 \quad 1585.65814$

Signal 2: DAD1 B, Sig=230, 4 Ref $=360,100$

\begin{tabular}{|c|c|c|c|c|c|}
\hline $\begin{array}{c}\text { Peak } \\
\#\end{array}$ & $\begin{array}{l}\text { RetTime Type } \\
\text { [min] }\end{array}$ & $\begin{array}{l}\text { Width } \\
\text { [min] }\end{array}$ & $\begin{array}{c}\text { Area } \\
{\left[\mathrm{mAU} U^{*} \mathrm{~s}\right]}\end{array}$ & $\begin{array}{l}\text { Height } \\
{[\mathrm{mAU}]}\end{array}$ & $\begin{array}{c}\text { Area } \\
\%\end{array}$ \\
\hline 1 & $19.141 \mathrm{BB}$ & 0.4457 & 6832.79248 & 234.68440 & $\begin{array}{l}49.7430 \\
50.7578\end{array}$ \\
\hline
\end{tabular}

Totals :

$1.37362 \mathrm{e} 4 \quad 449.65807$

Signal 3: DAD1 C, Sig $=254,4$ Ref $=360,100$

\begin{tabular}{|c|c|c|c|c|c|}
\hline $\begin{array}{c}\text { Peak } \\
\#\end{array}$ & $\begin{array}{l}\text { RetTime Type } \\
\text { [min] }\end{array}$ & $\begin{array}{l}\text { Width } \\
\text { [min] }\end{array}$ & $\begin{array}{c}\text { Area } \\
{\left[\mathrm{mAU}^{*} \mathrm{~s}\right]}\end{array}$ & $\begin{array}{l}\text { Height } \\
{[\mathrm{mAU}]}\end{array}$ & $\begin{array}{c}\text { Area } \\
\%\end{array}$ \\
\hline 1 & $19.142 \mathrm{BB}$ & 0.4398 & 1654.89917 & 57.84290 & 49.7487 \\
\hline 2 & $21.239 \mathrm{BB}$ & 0.4844 & 1671.61670 & 52.93922 & 50.2513 \\
\hline
\end{tabular}

Totals :

$3326.51587 \quad 110.78212$

Signal 4: DAD1 D, Sig=280, 4 Ref $=360,100$

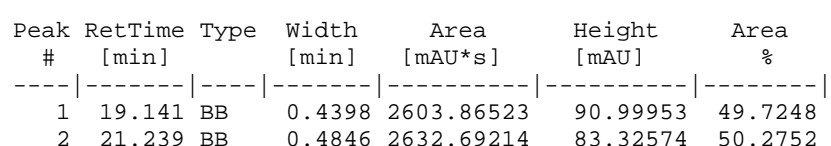

Totals : $5236.55737 \quad 174.32527$

Signal 5: DAD1 E, Sig $=310,4$ Ref $=360,100$

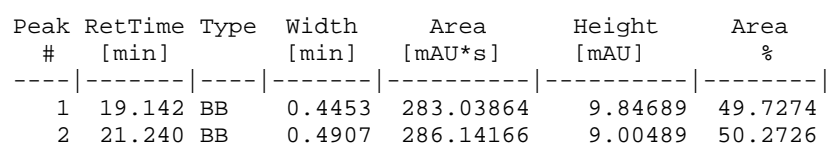

$\begin{array}{lll}\text { Totals : } & 569.18030 & 18.85178\end{array}$ 

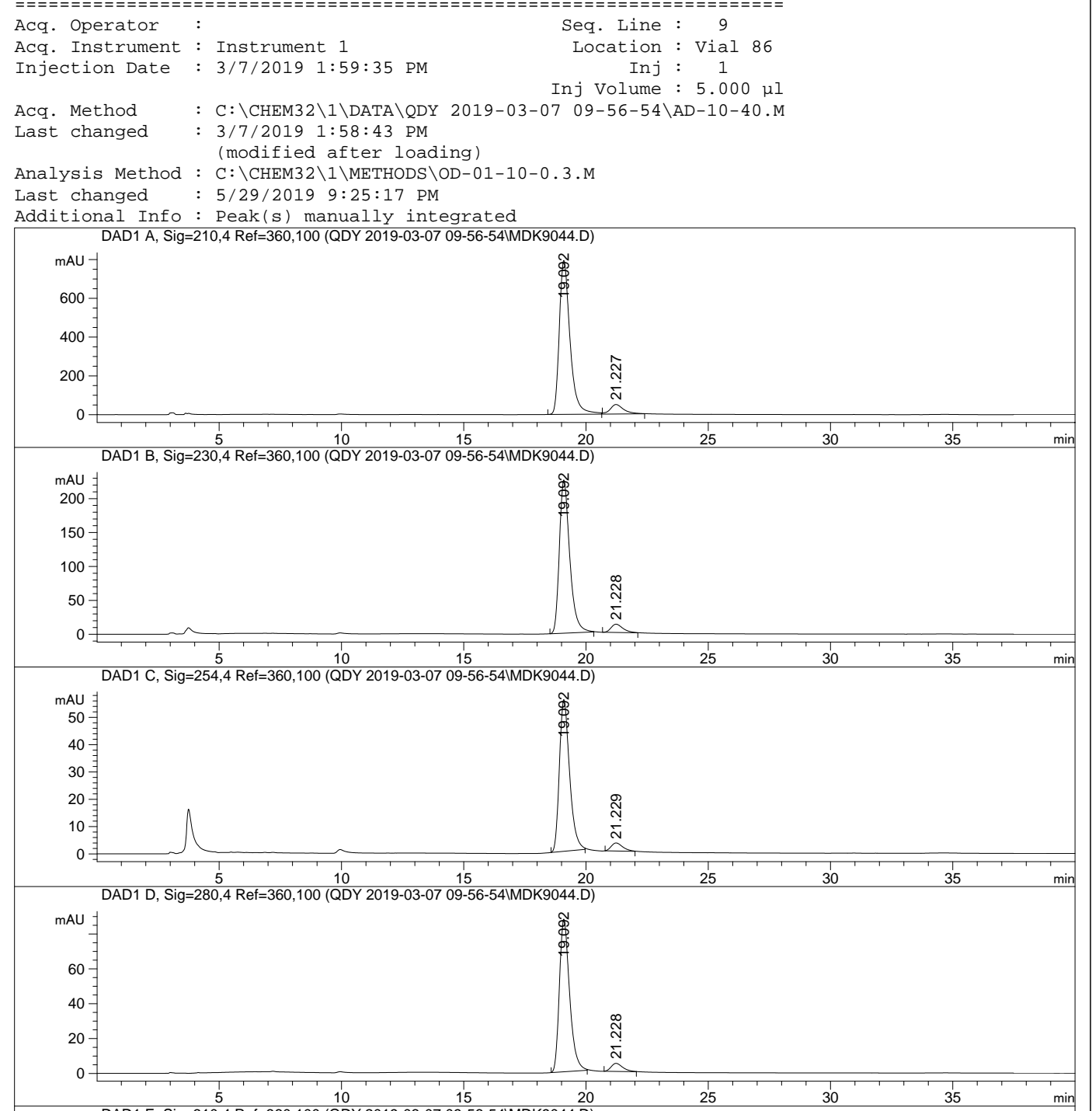

$\begin{array}{cccr}5 & 10 & 15 & 20 \\ \text { DAD1 E, Sig=310,4 Ret=360, } 100(\text { ODY 2019-03-07 09-56-54/MDK9044 D) }\end{array}$

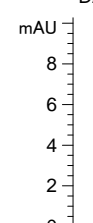

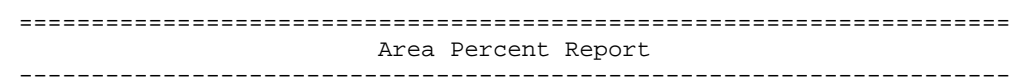

Sorted By

\section{Signal}

Multiplier

1.0000

Use Multiplier \& Dilution Factor with ISTDs

Signal 1: DAD1 A, Sig=210, 4 Ref $=360,100$

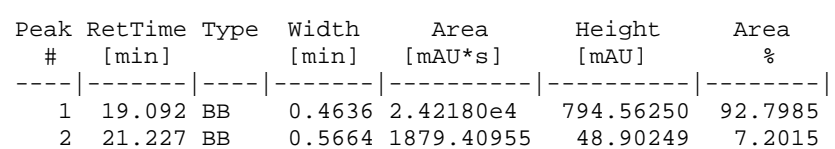

Totals :

$2.60975 \mathrm{e} 4 \quad 843.46499$

Signal 2: DAD1 B, Sig $=230,4$ Ref $=360,100$

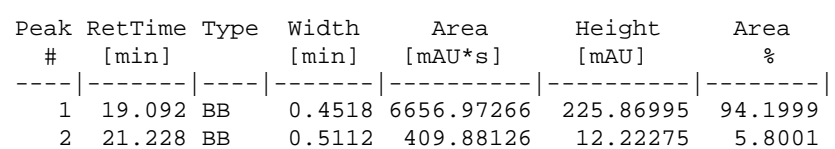

Totals :

$7066.85391 \quad 238.09270$

Signal 3: DAD1 C, Sig $=254,4$ Ref $=360,100$

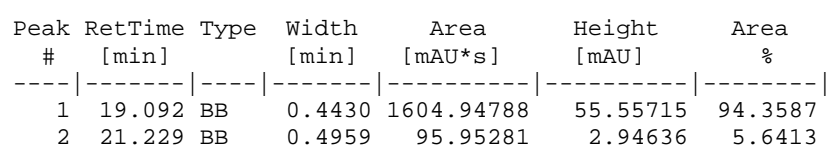

Totals :

$1700.90069 \quad 58.50351$

Signal 4: DAD1 D, Sig=280, 4 Ref $=360,100$

$\begin{array}{ccccc}\text { Peak RetTime Type } & \text { Width } & \text { Area } & \text { Height } & \text { Area } \\ \text { \# }[\mathrm{min}] & {[\mathrm{min}]} & {\left[\mathrm{mAU} \mathrm{U}^{\star} \mathrm{s}\right]} & {[\mathrm{mAU}]} & \%\end{array}$

$\begin{array}{llllllll}1 & 19.092 & \text { BB } & 0.4418 & 2535.20508 & 87.57144 & 94.3794\end{array}$

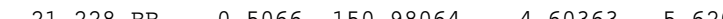

Totals :

$2686.18571 \quad 92.17507$

*** End of Report ***

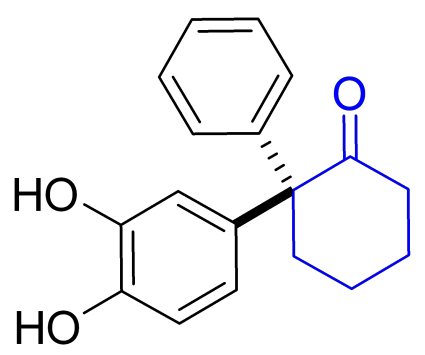

$2 m$

\section{Enantioenriched}


Area Percent Report

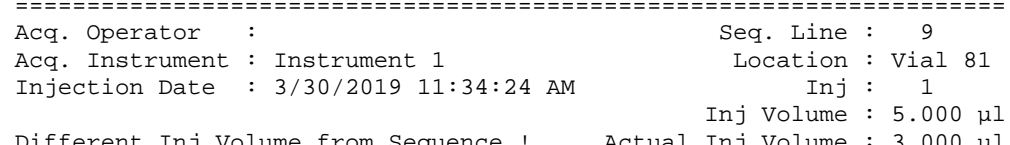

Different Inj volume from Sequence ! Actual Inj Volume : $5.000 \mu \mathrm{\mu}$

Acq. Method : C: \CHEM32\1\DATA

Last changed : $3 / 30 / 201911: 02: 27 \mathrm{AM}$

Analysis Method : C: \CHEM32\1\METHODS\OD-01-10-0.3.M

Last changed : 5/29/2019 9:25:17 PM

Additional Info : Peak(s) manually integrated
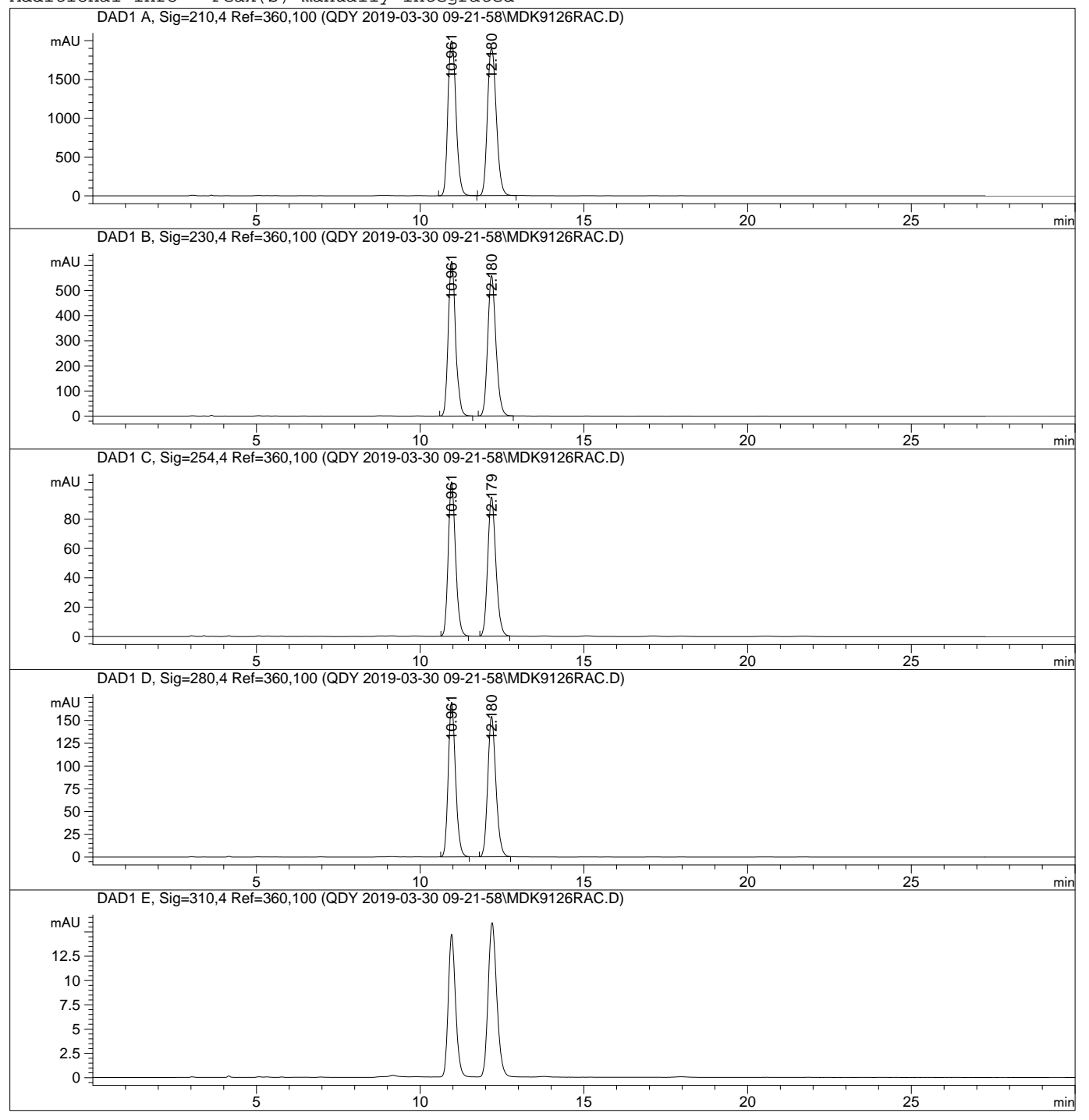

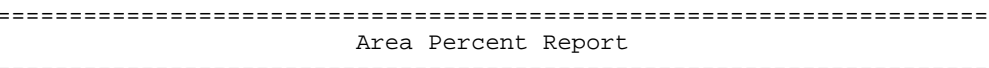

Sorted By

Signal

Multiplier

1.0000

Use Multiplier \& Dilution Factor with ISTDs

Signal 1: DAD1 A, Sig=210, 4 Ref $=360,100$

\begin{tabular}{|c|c|c|c|c|c|}
\hline $\begin{array}{l}\text { Peak } \\
\#\end{array}$ & $\begin{array}{l}\text { RetTime Type } \\
\text { [min] }\end{array}$ & $\begin{array}{l}\text { Width } \\
\text { [min] }\end{array}$ & $\begin{array}{c}\text { Area } \\
{\left[\mathrm{mAU} U^{*} \mathrm{~s}\right]}\end{array}$ & $\begin{array}{l}\text { Height } \\
{[\mathrm{mAU}]}\end{array}$ & $\begin{array}{c}\text { Area } \\
\%\end{array}$ \\
\hline 1 & 10. $961 \mathrm{BB}$ & 0.2717 & 3. $44883 \mathrm{e} 4$ & 1994.91382 & $\begin{array}{l}49.1925 \\
50.8075\end{array}$ \\
\hline
\end{tabular}

Totals :

7. $01089 \mathrm{e} 4 \quad 3888.70850$

Signal 2: DAD1 B, Sig=230, 4 Ref $=360,100$

\begin{tabular}{|c|c|c|c|c|c|}
\hline $\begin{array}{c}\text { Peak } \\
\#\end{array}$ & $\begin{array}{l}\text { RetTime Type } \\
{[\text { min] }}\end{array}$ & $\begin{array}{l}\text { Width } \\
\text { [min] }\end{array}$ & $\begin{array}{c}\text { Area } \\
{\left[\mathrm{mAU} U^{\star} \mathrm{s}\right]}\end{array}$ & $\begin{array}{l}\text { Height } \\
{[\mathrm{mAU}]}\end{array}$ & $\begin{array}{c}\text { Area } \\
\%\end{array}$ \\
\hline & $\begin{array}{l}10.961 \mathrm{BB} \\
12.180 \mathrm{BB}\end{array}$ & $\begin{array}{l}0.2421 \\
0.2669\end{array}$ & $\begin{array}{l}9614.01660 \\
9716.93555\end{array}$ & $\begin{array}{l}615.87665 \\
558.72687\end{array}$ & $\begin{array}{l}49.7338 \\
50.2662\end{array}$ \\
\hline
\end{tabular}

Totals :

$1.93310 \mathrm{e} 4 \quad 1174.60352$

Signal 3: DAD1 C, Sig $=254,4$ Ref $=360,100$

\begin{tabular}{|c|c|c|c|c|c|}
\hline $\begin{array}{c}\text { Peak } \\
\#\end{array}$ & $\begin{array}{l}\text { RetTime Type } \\
\text { [min] }\end{array}$ & $\begin{array}{l}\text { Width } \\
\text { [min] }\end{array}$ & $\begin{array}{c}\text { Area } \\
{\left[\mathrm{mAU}^{*} \mathrm{~s}\right]}\end{array}$ & $\begin{array}{l}\text { Height } \\
{[\mathrm{mAU}]}\end{array}$ & $\begin{array}{c}\text { Area } \\
\%\end{array}$ \\
\hline 1 & $10.961 \mathrm{BB}$ & 0.2392 & 1634.13123 & 105.20583 & 49.9345 \\
\hline 2 & $12.179 \mathrm{BB}$ & 0.2656 & 1638.41626 & 94.81535 & 50.0655 \\
\hline
\end{tabular}

Totals :

$3272.54749 \quad 200.02117$

Signal 4: DAD1 D, Sig=280, 4 Ref $=360,100$

\begin{tabular}{|c|c|c|c|c|c|}
\hline $\begin{array}{c}\text { Peak } \\
\#\end{array}$ & $\begin{array}{l}\text { RetTime Type } \\
\text { [min] }\end{array}$ & $\begin{array}{l}\text { Width } \\
\text { [min] }\end{array}$ & $\begin{array}{c}\text { Area } \\
{\left[\mathrm{mAU} U^{*} \mathrm{~s}\right]}\end{array}$ & $\begin{array}{l}\text { Height } \\
{[\mathrm{mAU}]}\end{array}$ & $\begin{array}{c}\text { Area } \\
\%\end{array}$ \\
\hline 1 & 10.961 BB & & 2624.63745 & 169.82080 & 49.6520 \\
\hline 2 & $12.180 \mathrm{BB}$ & 0.2654 & 2661.42920 & 154.14549 & 50.3480 \\
\hline
\end{tabular}

Totals :

$5286.06665 \quad 323.96629$

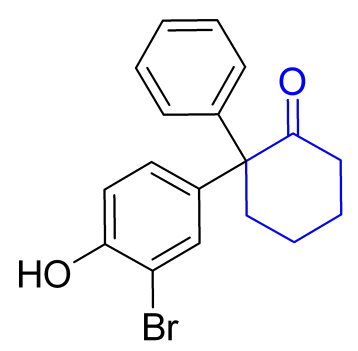

racemic

Signal 5: DAD1 E, Sig $=310,4$ Ref $=360,100$

$\star \star *$ End of Report *** 


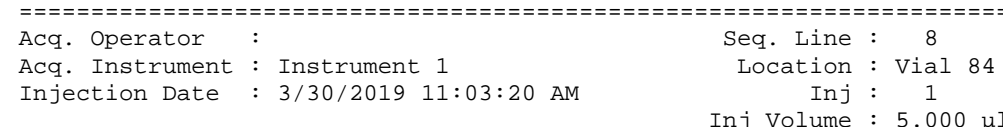

Injection Date Inj volume : $5.000 \mu \mathrm{\mu l}$ $\begin{array}{ll}\text { Acq. Method } & : \text { C: } \backslash \text { CHEM32 } \\ \text { Last changed } & \text { 3/30/2019 11:02:27 AM }\end{array}$

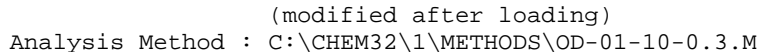

Last changed : 5/29/2019 9:25:17 PM

Additional Info : Peak(s) manually integrated

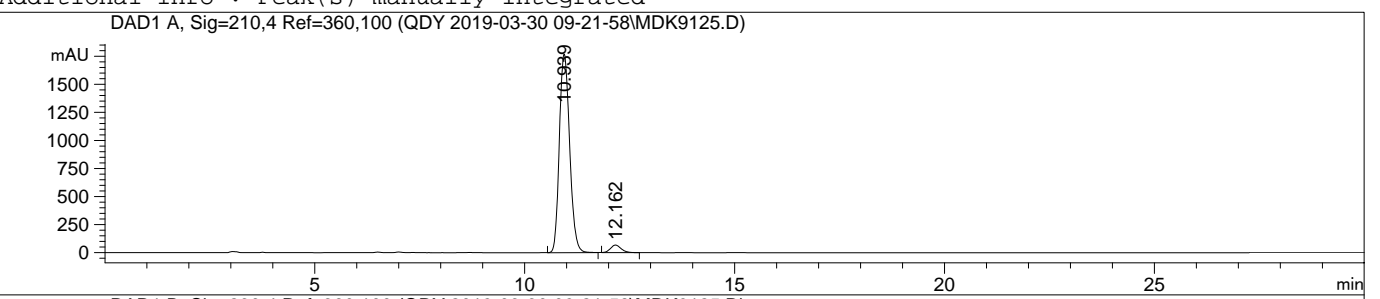

$\begin{array}{ccr}5 & 10 & 15 \\ \text { DAD1 B, Sig=230,4 Ref=360,100 (QDY 2019-03-30 09-21-58/MDK9125.D) }\end{array}$
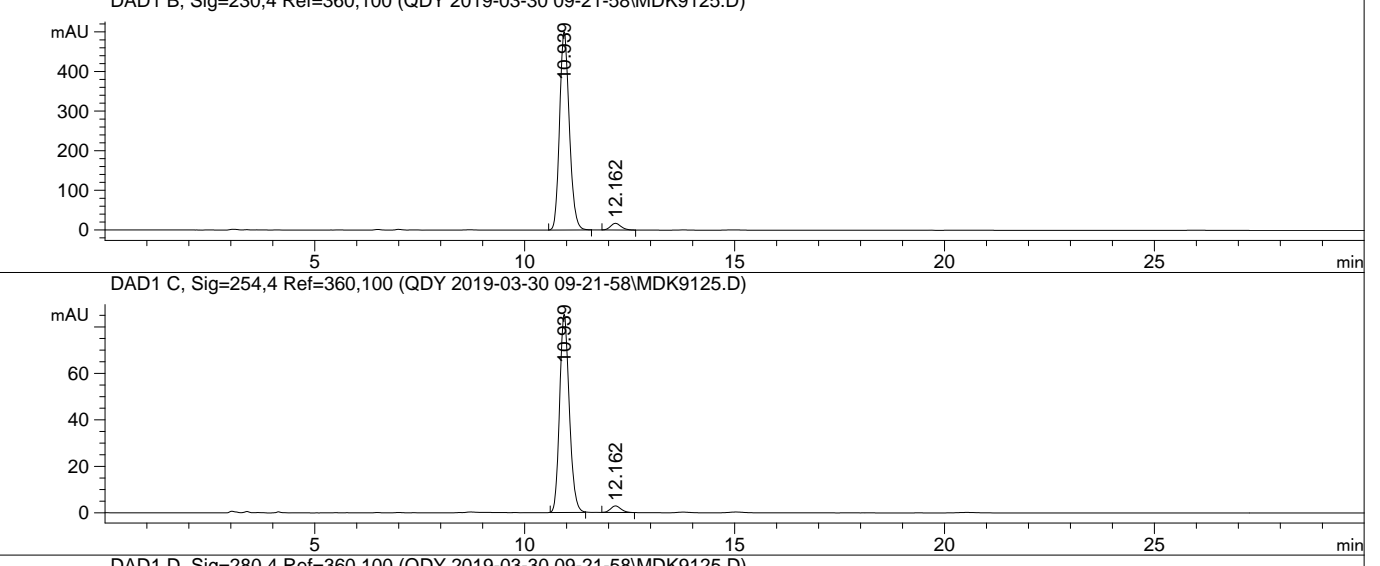

DAD1 D, Sig=280,4 Ref=360,100 (QDY 2019-03-30 09-21-58IMDK9125.D)

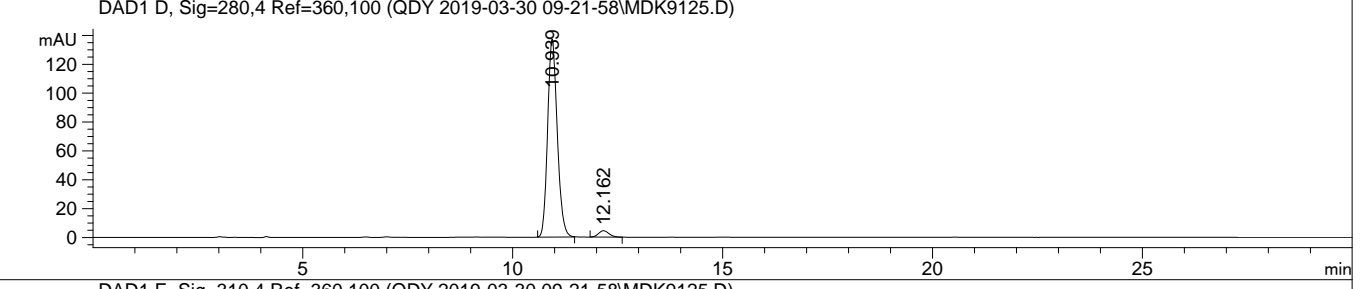

DAD1 E, Sig=310,4 Ref=360,100 (QDY 2019-03-30 09-21-58|MDK9125.D)

$$
\begin{aligned}
& \begin{array}{r}
\mathrm{mAU}- \\
10
\end{array} \\
& 8 \text { 㬰 } \\
& \text { 2. }
\end{aligned}
$$$$
\text { 10刍 }
$$

兽

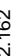

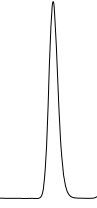

10

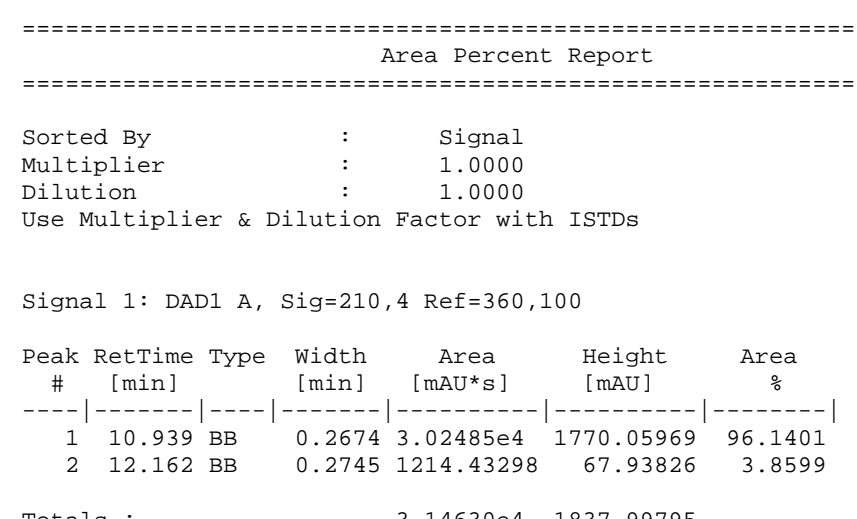

Totals :

\begin{tabular}{|c|c|c|c|c|c|}
\hline $\begin{array}{c}\text { Peak } \\
\#\end{array}$ & $\begin{array}{l}\text { RetTime Type } \\
\text { [min] }\end{array}$ & $\begin{array}{l}\text { Width } \\
\text { [min] }\end{array}$ & $\begin{array}{c}\text { Area } \\
{\left[\mathrm{mAU}^{*} \mathrm{~s}\right]}\end{array}$ & $\begin{array}{l}\text { Height } \\
{[\mathrm{mAU}]}\end{array}$ & $\begin{array}{c}\text { Area } \\
\%\end{array}$ \\
\hline 1 & $10.939 \mathrm{BB}$ & 0.2479 & 8065.69141 & 500.78339 & 96.5036 \\
\hline 2 & $12.162 \mathrm{BB}$ & 0.2688 & 292.22964 & 16.81185 & 3.4964 \\
\hline
\end{tabular}

$3.14630 \mathrm{e} 4 \quad 1837.99795$

Signal 2: DAD1 B, Sig=230, 4 Ref $=360,100$

Totals :

\begin{tabular}{|c|c|c|c|c|c|}
\hline $\begin{array}{c}\text { Peak } \\
\#\end{array}$ & $\begin{array}{l}\text { RetTime Type } \\
{[\mathrm{min}]}\end{array}$ & $\begin{array}{l}\text { Width } \\
\text { [min] }\end{array}$ & $\begin{array}{c}\text { Area } \\
{\left[\mathrm{mAU}^{*} \mathrm{~s}\right]}\end{array}$ & $\begin{array}{l}\text { Height } \\
{[\mathrm{mAU}]}\end{array}$ & $\begin{array}{c}\text { Area } \\
\%\end{array}$ \\
\hline 1 & 10.939 BB & 0.2470 & 1366.94287 & 85.26957 & 96.5278 \\
\hline 2 & $12.162 \mathrm{BB}$ & 0.2702 & 49.17006 & 2.83712 & 3.4722 \\
\hline
\end{tabular}

$8357.92105 \quad 517.59524$

Signal 3: DAD1 C, Sig=254, 4 Ref $=360,100$

Totals :

$1416.11293 \quad 88.10669$

Signal 4: DAD1 D, Sig=280, 4 Ref $=360,100$

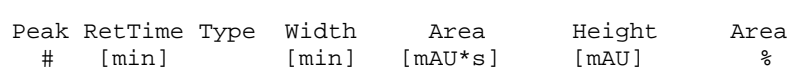

\# [min] $[\mathrm{min}]\left[\mathrm{mAU} \mathrm{U}^{*}\right][\mathrm{mAU}] \quad \%$

$\begin{array}{llllll}1 & 10.939 \mathrm{BB} & 0.2462 & 2191.27246 & 137.30928 & 96.5768\end{array}$

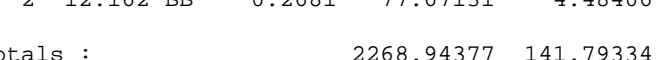

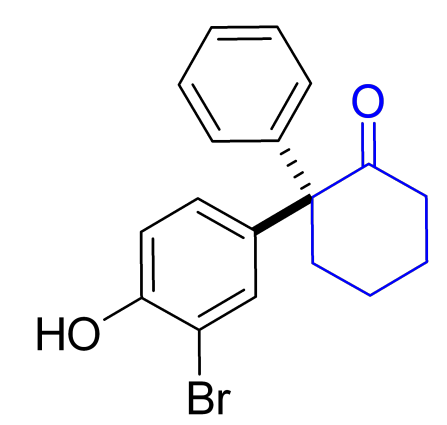

2n

Enantioenriched

Signal 5: DAD1 E, Sig=310, 4 Ref $=360,100$

$\star * *$ End of Report *** 


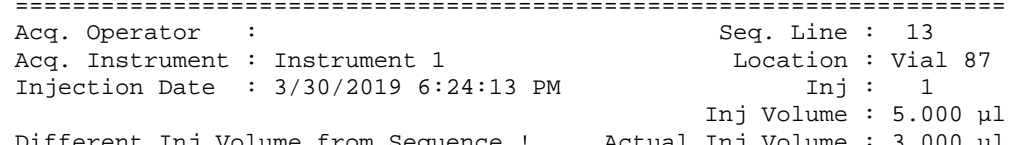

Injection Date $\quad$ Inj Volume $: 5.000 \mu \mathrm{\mu l}$ Acq. Method : C: \CHEM32\1\DATA Q QDY 2019-03-30 14-56-54\AD-10-40.M Last changed : 3/30/2019 5:42:14 PM

Analysis Method : C: \CHEM32\1\METHODS\OD-๑1-10-0.3.M

: $5 / 29 / 2019$ 9:25:17 PM

Additional Info: Peak(s) manually integrated
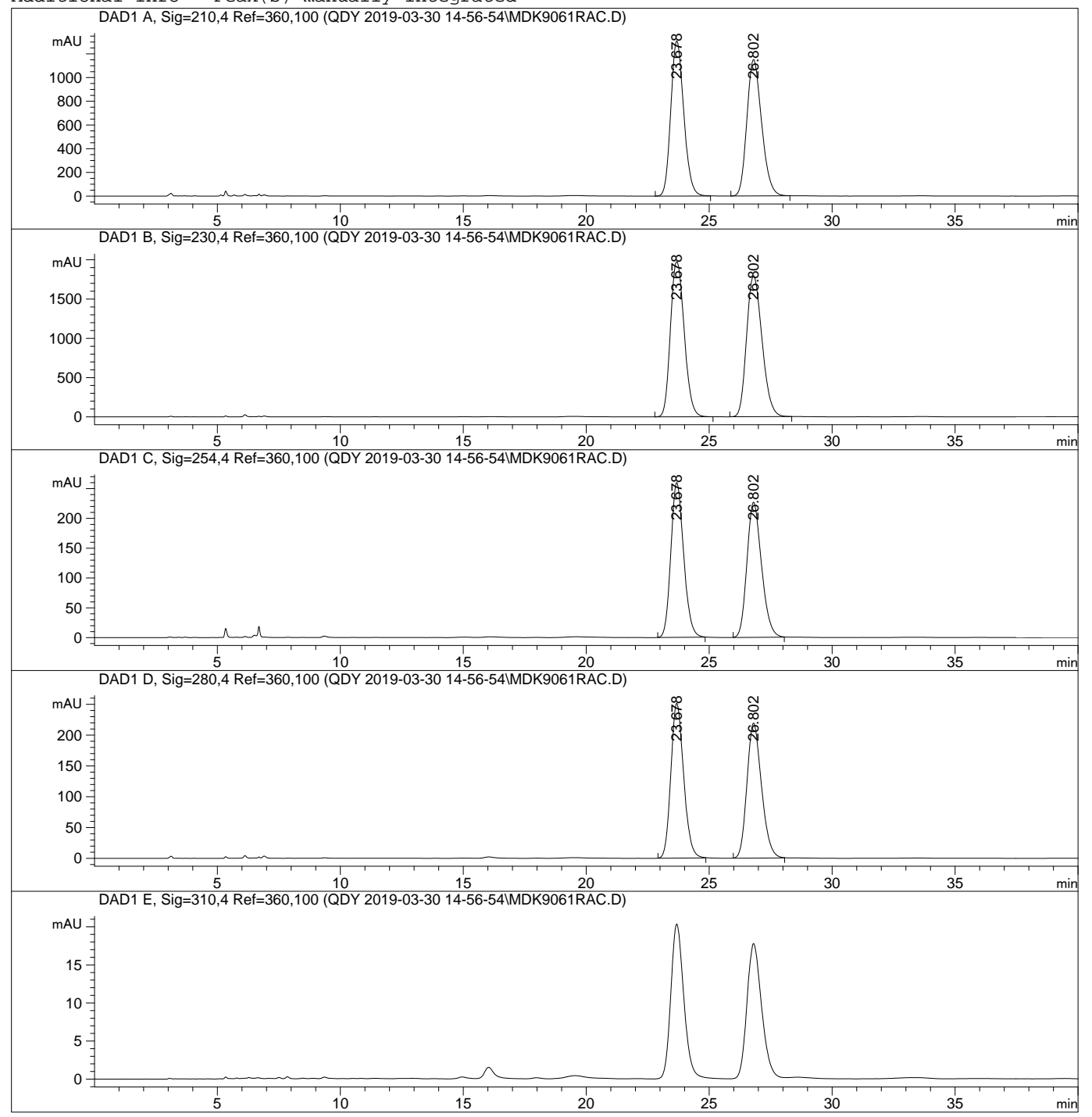

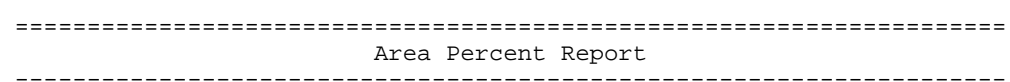

Sorted By

\section{Signal}

Multiplier
Dilution

1.0000

Use Multiplier \& Dilution Factor with ISTDs

Signal 1: DAD1 A, Sig=210, 4 Ref $=360,100$

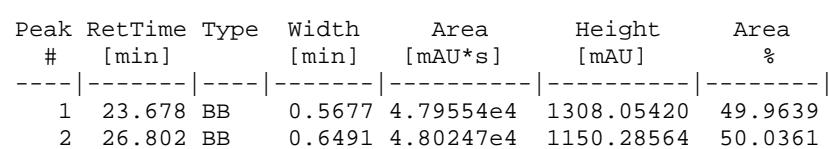

Totals :

$9.59801 \mathrm{e} 4 \quad 2458.33984$

Signal 2: DAD1 B, Sig $=230,4$ Ref $=360,100$

\begin{tabular}{cccccc}
$\begin{array}{c}\text { Peak RetTime Type } \\
\#\end{array}\left[\begin{array}{c}\text { Width } \\
{[\mathrm{min}]}\end{array}\right.$ & $\begin{array}{c}\text { Area } \\
{[\mathrm{mAU}}\end{array}$ & $\begin{array}{l}\text { Height } \\
{[\mathrm{mAU}]}\end{array}$ & $\begin{array}{c}\text { Area } \\
\%\end{array}$ \\
\hdashline-1 & $23.678 \mathrm{BB}$ & 0.6117 & $7.67746 \mathrm{e} 4$ & 1974.00659 & 49.4992 \\
\hline 2 & $26.802 \mathrm{BB}$ & 0.6881 & $7.83282 \mathrm{e} 4$ & 1791.36255 & 50.5008
\end{tabular}

Totals :

$1.55103 \mathrm{e} 5 \quad 3765.36914$

Signal 3: DAD1 C, Sig $=254,4$ Ref $=360,100$

\begin{tabular}{|c|c|c|c|c|c|}
\hline $\begin{array}{c}\text { Peak } \\
\#\end{array}$ & $\begin{array}{l}\text { RetTime Type } \\
{[\text { min] }}\end{array}$ & $\begin{array}{l}\text { Width } \\
\text { [min] }\end{array}$ & $\begin{array}{c}\text { Area } \\
{\left[\mathrm{mAU} U^{*} \mathrm{~s}\right]}\end{array}$ & $\begin{array}{l}\text { Height } \\
{[\mathrm{mAU}]}\end{array}$ & $\begin{array}{c}\text { Area } \\
\%\end{array}$ \\
\hline 1 & $23.678 \mathrm{BB}$ & 0.5573 & 9384.12402 & 259.86374 & 9728 \\
\hline 2 & $26.802 \mathrm{BB}$ & 0.6416 & 9356.83301 & 226.66582 & 49.9272 \\
\hline
\end{tabular}

Signal 4: DAD1 D, Sig $=280,4$ Ref $=360,100$

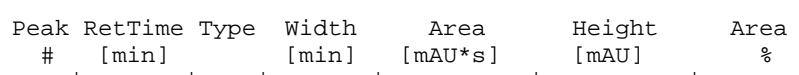

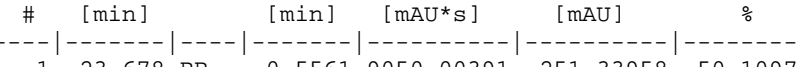

$\begin{array}{llllll}1 & 23.678 \text { BB } & 0.5561 & 9050.00391 & 251.33958 & 50.1097\end{array}$

Totals :

$1.80604 \mathrm{e} 4 \quad 470.38771$

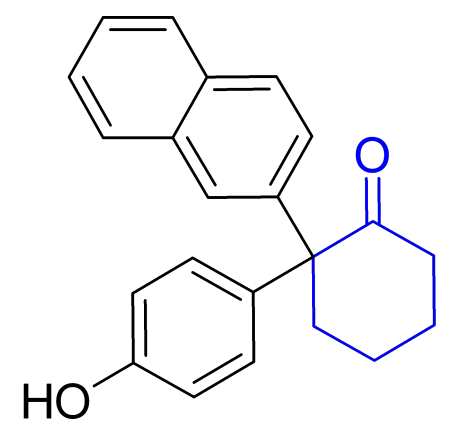

*** End of Report *** racemic 

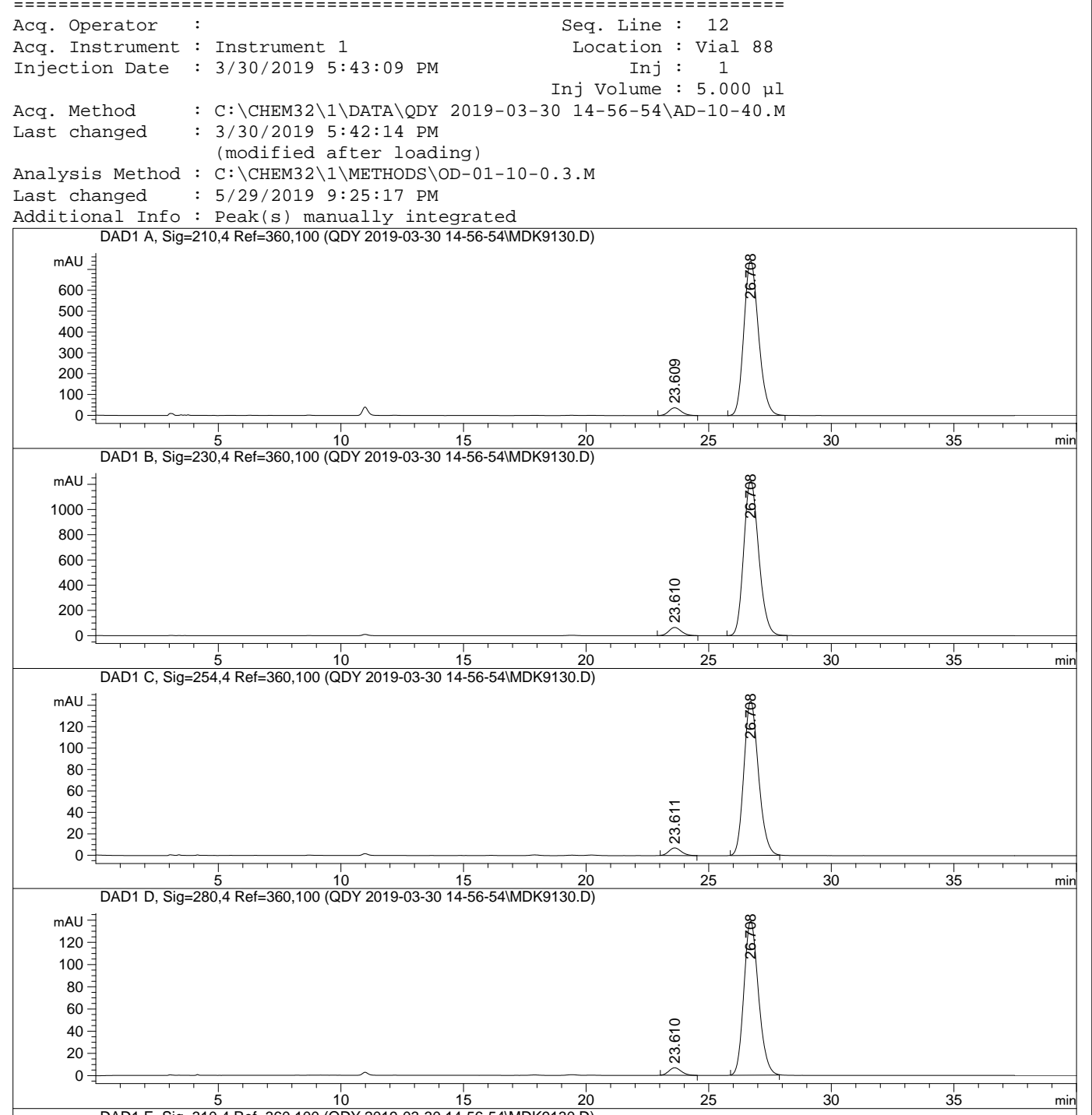

$\begin{array}{crrr}5 & 10 & 15 & 20 \\ \text { DAD1 E, Sig=310,4 Ref=360, } 100 \text { (QDY 2019-03-30 } & 14-56-54 \text { IMDK9130.D) }\end{array}$

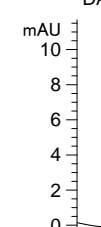

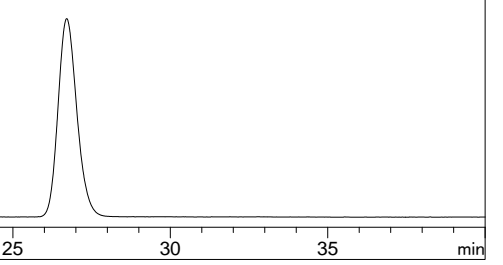

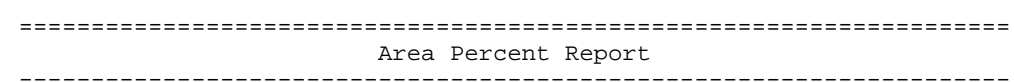

Sorted By

Signal

Multiplier

1.0000

Use Multiplier \& Dilution Factor with ISTDs

Signal 1: DAD1 A, Sig=210, 4 Ref $=360,100$

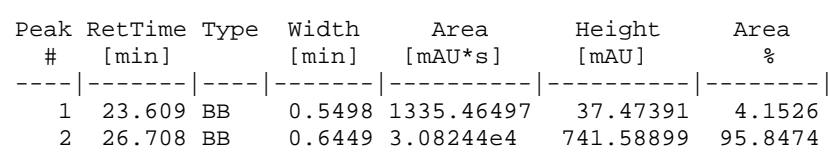

Totals :

$3.21598 \mathrm{e} 4 \quad 779.06290$

Signal 2: DAD1 B, Sig=230, 4 Ref $=360,100$

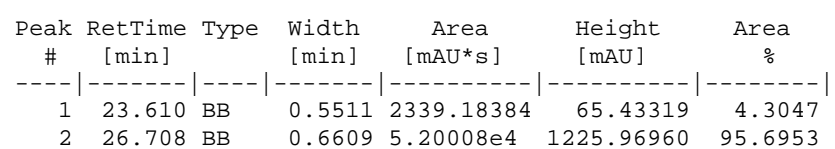

Totals :

$5.43400 \mathrm{e} 4 \quad 1291.40279$

Signal 3: DAD1 C, Sig $=254,4$ Ref $=360,100$

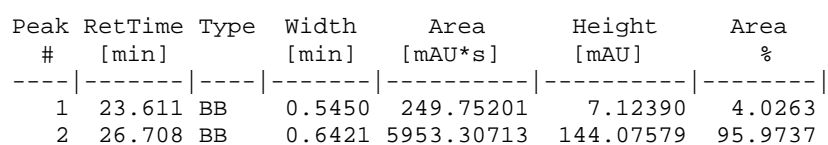
Totals : $6203.05914 \quad 151.19969$

Signal 4: DAD1 D, Sig=280, 4 Ref $=360,100$

Peak RetTime Type Width Area Height Area \# [min] $[$ [min] [mAU*s] [mAU] \%

$\begin{array}{llllll}1 & 23.610 \mathrm{BB} & 0.5467 & 237.26971 & 6.77378 & 3.9866\end{array}$

Totals :

$5951.74188 \quad 145.53439$

$\mathrm{HO}$

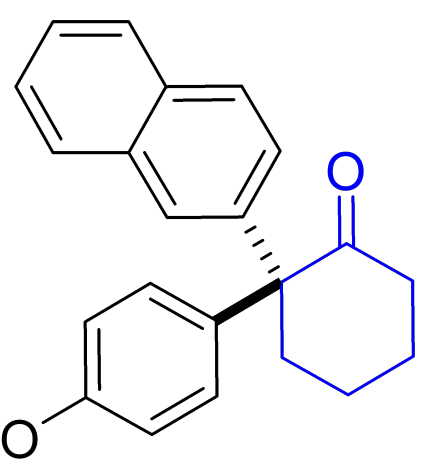

20

Signal 5: DAD1 E, Sig=310, 4 Ref $=360,100$

Enantioenriched 


\section{Sample Name:}
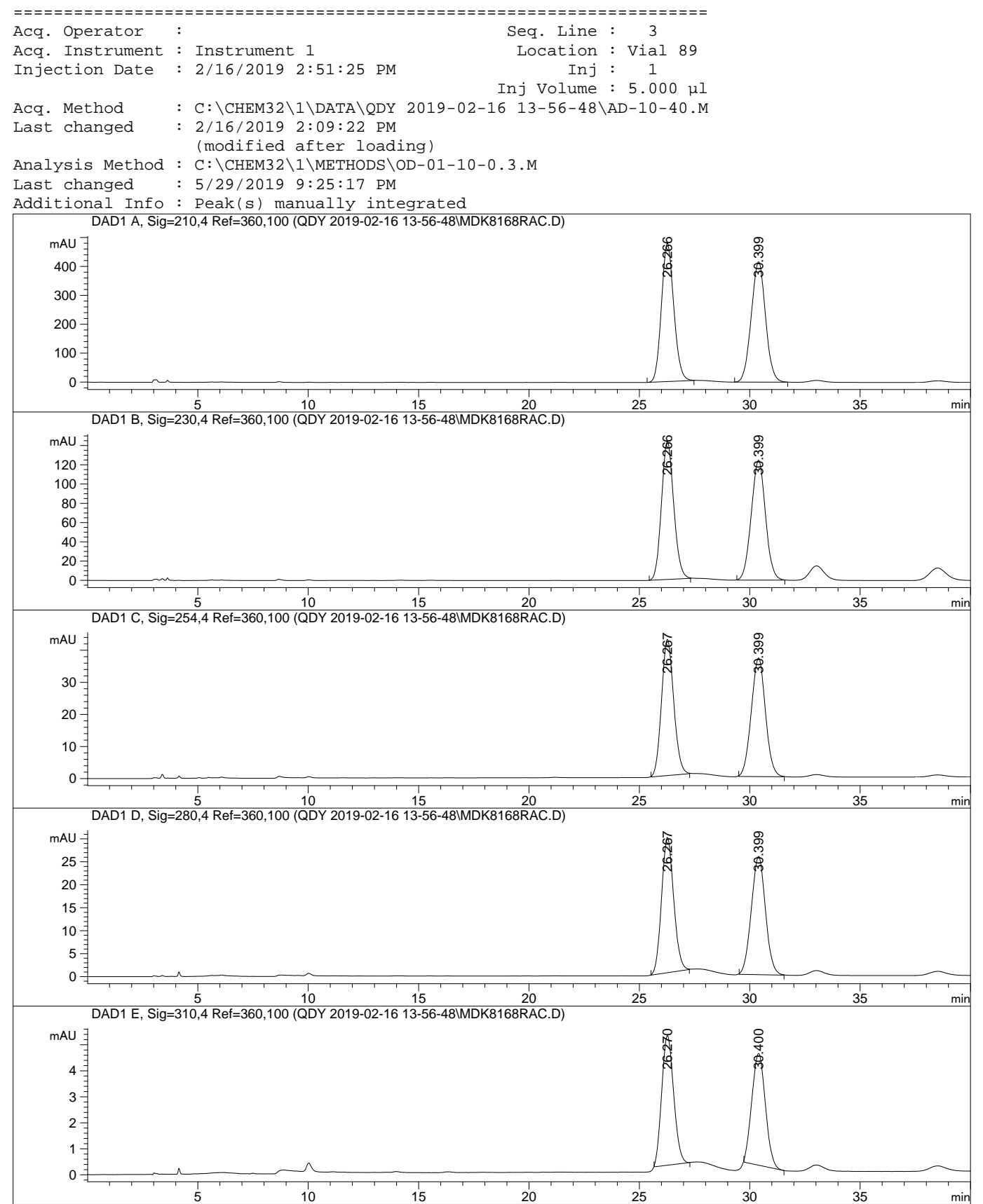

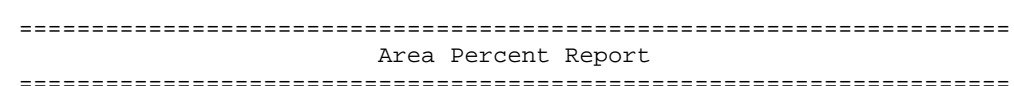

Sorted By

\section{Signal}

Multiplier
Dilution

1.0000

Use Multiplier \& Dilution Factor with ISTDs

Signal 1: DAD1 A, Sig=210, 4 Ref $=360,100$

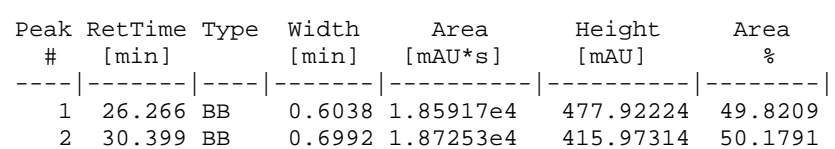

Totals :

$3.73170 \mathrm{e} 4 \quad 893.89539$

Signal 2: DAD1 B, Sig=230, 4 Ref $=360,100$

\begin{tabular}{|c|c|c|c|c|c|}
\hline $\begin{array}{c}\text { eak } \\
\#\end{array}$ & $\begin{array}{l}\text { RetTime Type } \\
\text { [min] }\end{array}$ & $\begin{array}{l}\text { Width } \\
\text { [min] }\end{array}$ & $\begin{array}{c}\text { Area } \\
{\left[\mathrm{mAU}^{\star} \mathrm{s}\right]}\end{array}$ & $\begin{array}{l}\text { Height } \\
\text { [mAU] }\end{array}$ & $\begin{array}{c}\text { Area } \\
\%\end{array}$ \\
\hline & 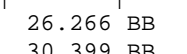 & 6 & 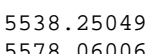 & $\begin{array}{r}43.41974 \\
24.60730\end{array}$ & \\
\hline
\end{tabular}

Totals :

$1.11163 \mathrm{e} 4 \quad 268.02704$

Signal 3: DAD1 C, Sig $=254,4$ Ref $=360,100$

\begin{tabular}{|c|c|c|c|c|c|}
\hline $\begin{array}{l}\text { eak } \\
\#\end{array}$ & $\begin{array}{l}\text { RetTime Type } \\
\text { [min] }\end{array}$ & $\begin{array}{l}\text { Width } \\
\text { [min] }\end{array}$ & $\begin{array}{c}\text { Area } \\
{\left[\mathrm{mAU}^{*} \mathrm{~s}\right]}\end{array}$ & $\begin{array}{l}\text { Height } \\
{[\mathrm{mAU}]}\end{array}$ & $\begin{array}{c}\text { Area } \\
\%\end{array}$ \\
\hline & $\begin{array}{ll}67 \mathrm{~B} \\
99\end{array}$ & & 4903223 & & \\
\hline
\end{tabular}

Totals :

$3279.87683 \quad 79.42656$

Signal 4: DAD1 D, Sig=280, 4 Ref $=360,100$

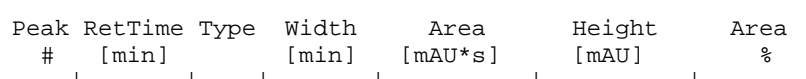

126.267 BB $0.59321123 .67188 \quad 29.44492 \quad 49.5384$

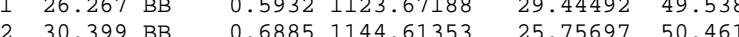

Totals :

$2268.28540 \quad 55.20189$

$\mathrm{HO}$

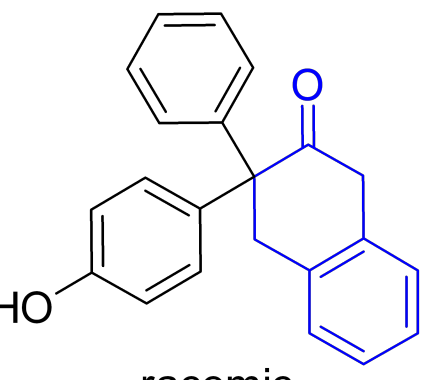

racemic

Signal 5: DAD1 E, Sig $=310,4$ Ref $=360,100$

Peak RetTime Type Width Area Height Area

\# [min] $\left[\begin{array}{l}\text { min] } \\ {\left[\mathrm{mAU} U^{*} \mathrm{~s}\right]} \\ {[\mathrm{mAU}]}\end{array} \%\right.$

$\begin{array}{llllll}1 & 26.270 \text { BB } & 0.5906 & 187.45422 & 4.98597 & 50.6696\end{array}$

$\begin{array}{lll}\text { Totals : } & 369.95412 & 9.27814\end{array}$ 


\section{Data File C: \CHEM32\1\DATA\QDY 2019-02-16 13-56-48\MDK8169-B.D}

Sample Name:
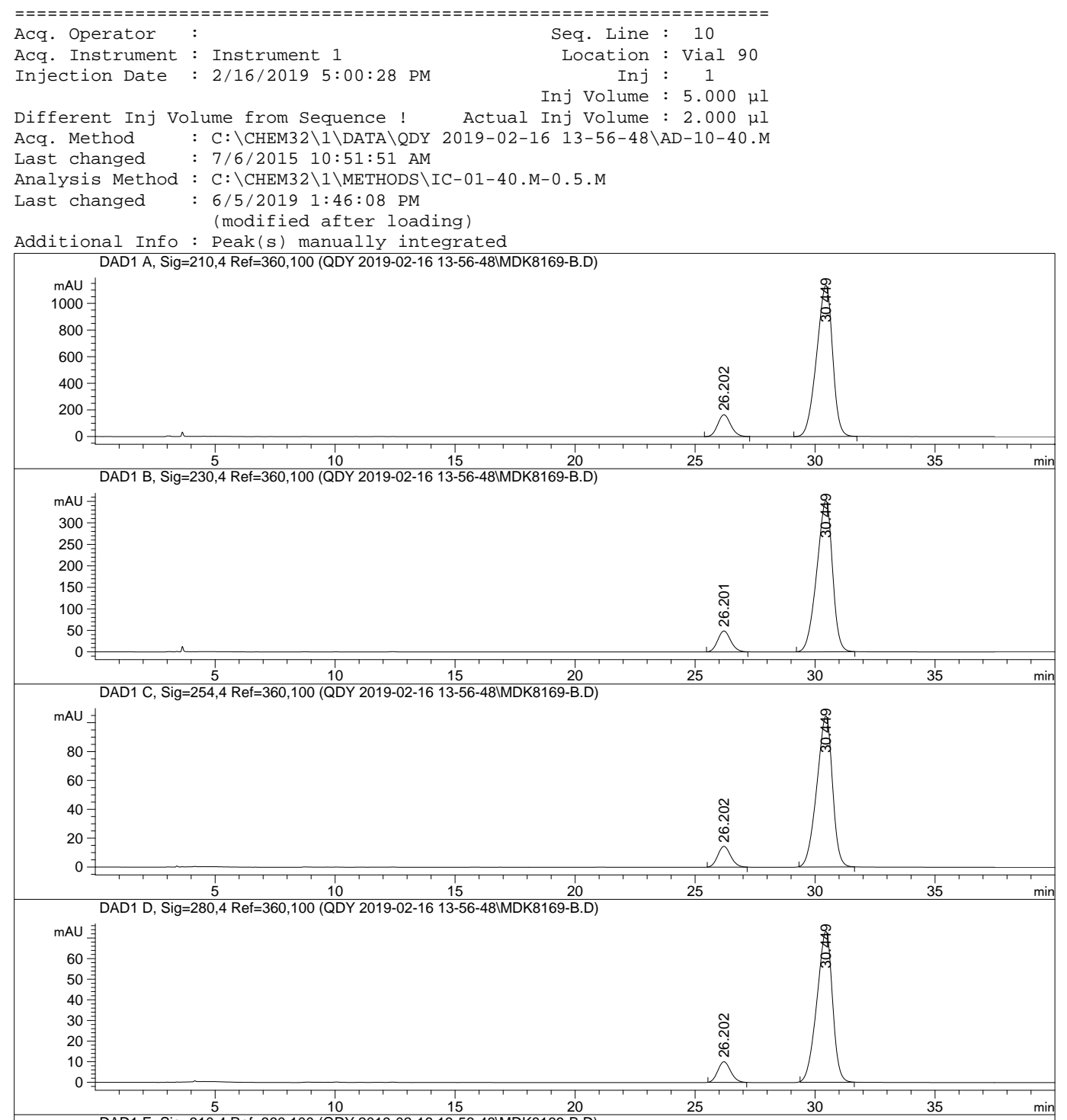

mAU
$10=$
8
6
4
2
0
DAD1 E, Sig $=310,4$ Ref $=360,100$ (QDY 2019-02-16 13-56-48/MDK8169-B.D)

啨

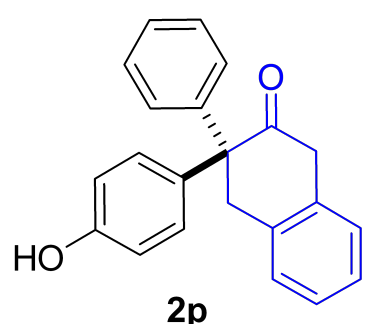

Peak RetTime Type Width Area Height Area \# [min] $[$ [min] [mAU*s] [mAU] $1-\%$ $\begin{array}{llllll}1 & 26.201 \mathrm{BB} & 0.5812 & 1843.58215 & 48.75009 & 10.3801\end{array}$

Totals : $1.77607 \mathrm{e} 4 \quad 400.28097$

Signal 3: DAD1 C, Sig=254, 4 Ref $=360,100$

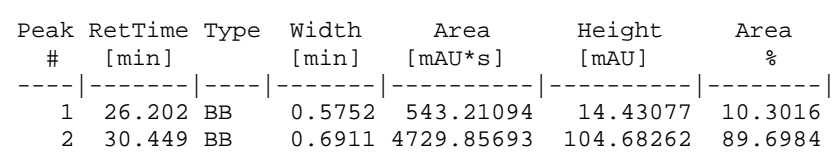
Totals : $5273.06787 \quad 119.11339$

Signal 4: DAD1 D, Sig=280, 4 Ref $=360,100$

Peak RetTime Type Width Area Height Area

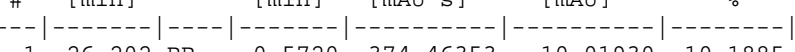

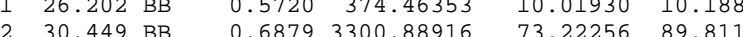
Totals : $3675.35269 \quad 83.24186$

Signal 5: DAD1 E, Sig=310, 4 Ref $=360,100$

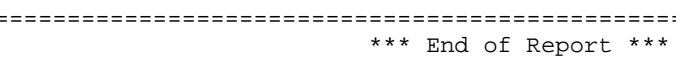

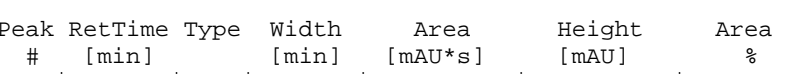 [min] $\left[\begin{array}{l}\text { [min] }\left[\mathrm{mAU}{ }^{\star} \mathrm{S}\right] \\ {[\mathrm{mAU}]}\end{array}\right.$ \% $\begin{array}{llllll}26.202 \text { BB } & 0.5874 & 6227.84131 & 163.87392 & 10.6393\end{array}$

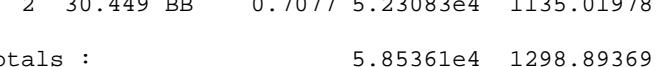




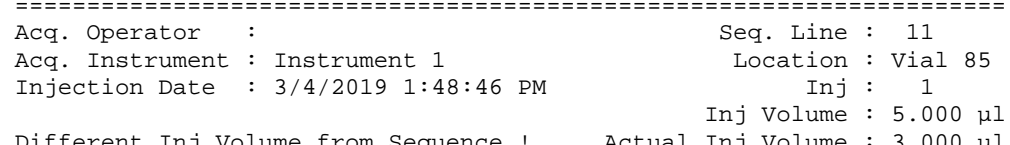

Injection Date $\quad$ Inj Volume $: 5.000 \mu \mathrm{\mu l}$ Acq. Method : C: \CHEM32\1\DATA Last changed : 3/4/2019 1:16:49 PM

Analysis Method : C: \CHEM32\1\METHODS\OD-๑1-10-0.3.M

: $5 / 29 / 20199: 25: 17$ PM

Additional Info: Peak(s) manually integrated
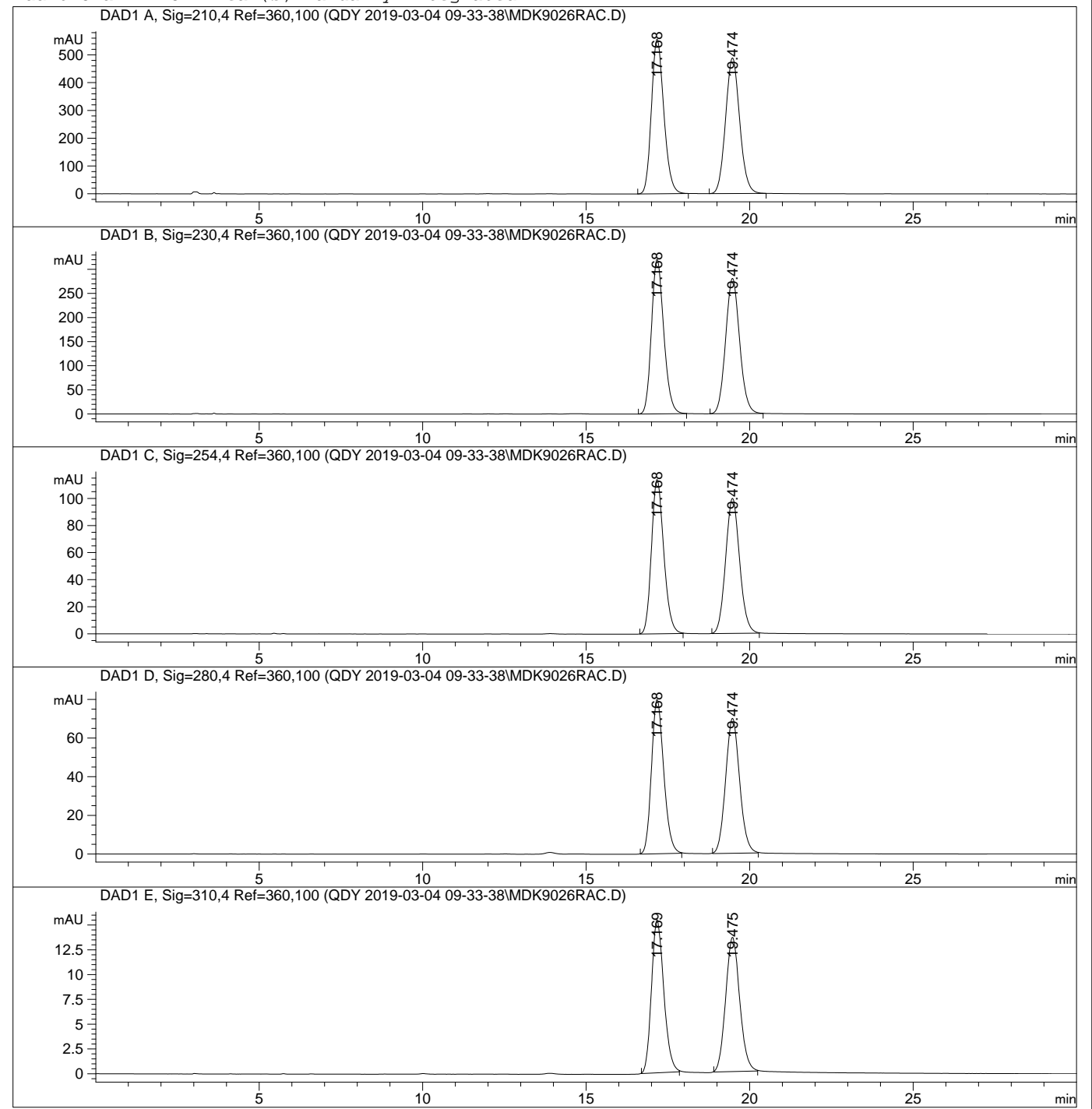

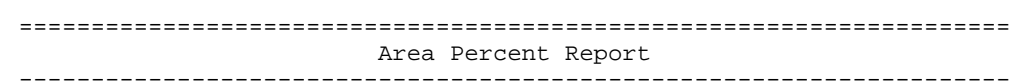

Sorted By

\section{Signal}

Multiplier
Dilution

1.0000

Use Multiplier \& Dilution Factor with ISTDs

Signal 1: DAD1 A, Sig=210, 4 Ref $=360,100$

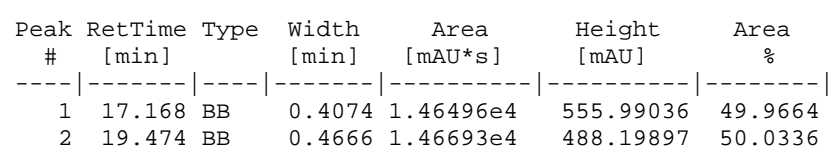

Totals :

$2.93189 \mathrm{e} 4 \quad 1044.18933$

Signal 2: DAD1 B, Sig=230, 4 Ref $=360,100$

\begin{tabular}{|c|c|c|c|c|c|}
\hline $\begin{array}{c}\text { eak } \\
\#\end{array}$ & $\begin{array}{l}\text { RetTime Type } \\
\text { [min] }\end{array}$ & $\begin{array}{l}\text { Width } \\
\text { [min] }\end{array}$ & $\begin{array}{c}\text { Area } \\
{\left[\mathrm{mAU}^{*} \mathrm{~s}\right]}\end{array}$ & $\begin{array}{l}\text { Height } \\
\text { [mAU] }\end{array}$ & $\begin{array}{c}\text { Area } \\
\%\end{array}$ \\
\hline & s & 0.4604 & & & \\
\hline
\end{tabular}

Totals :

$1.67709 \mathrm{e} 4 \quad 601.16757$

Signal 3: DAD1 C, Sig $=254,4$ Ref $=360,100$

\begin{tabular}{|c|c|c|c|c|c|}
\hline $\begin{array}{c}\text { Peak } \\
\#\end{array}$ & $\begin{array}{l}\text { RetTime Type } \\
\text { [min] }\end{array}$ & $\begin{array}{l}\text { Width } \\
\text { [min] }\end{array}$ & $\begin{array}{c}\text { Area } \\
{\left[\mathrm{mAU} U^{*} \mathrm{~s}\right]}\end{array}$ & $\begin{array}{l}\text { Height } \\
{[\mathrm{mAU}]}\end{array}$ & $\begin{array}{c}\text { Area } \\
\%\end{array}$ \\
\hline 1 & $17.168 \mathrm{BB}$ & 0.4029 & 2979.90845 & 114.01297 & 50.0436 \\
\hline 2 & $19.474 \mathrm{BB}$ & $\odot .4597$ & 2974.71436 & 99.81287 & 49.9564 \\
\hline
\end{tabular}

Signal 4: DAD1 D, Sig $=280,4$ Ref $=360,100$

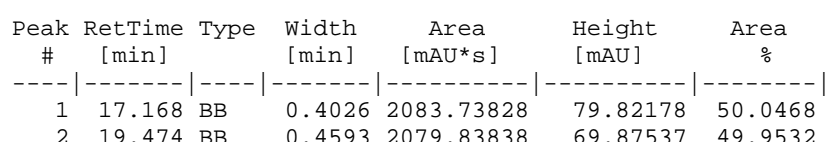

Totals :

$4163.57666 \quad 149.69714$

Signal 5: DAD1 E, Sig=310, 4 Ref $=360,100$

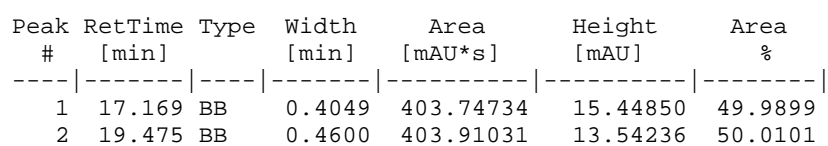

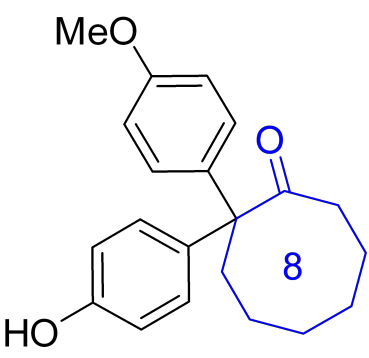

racemic

Totals : $\quad 807.65765 \quad 28.99086$ 


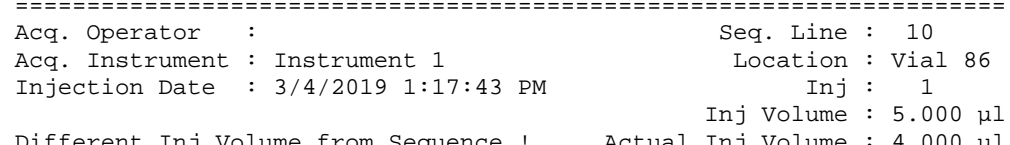

Injection Date $\quad$ Inj Volume $: 5.000 \mu \mathrm{\mu l}$ Acq. Method : C: \CHEM32\1\DATA\QDY 2019-03-04 09-33-38\AD-10-30.M Last changed : 3/4/2019 1:16:49 PM

Analysis Method : C: \CHEM32\1\METHODS\OD-๑1-10-0.3.M

: $5 / 29 / 20199: 25: 17$ PM

Additional Info : Peak(s) manualiy integra

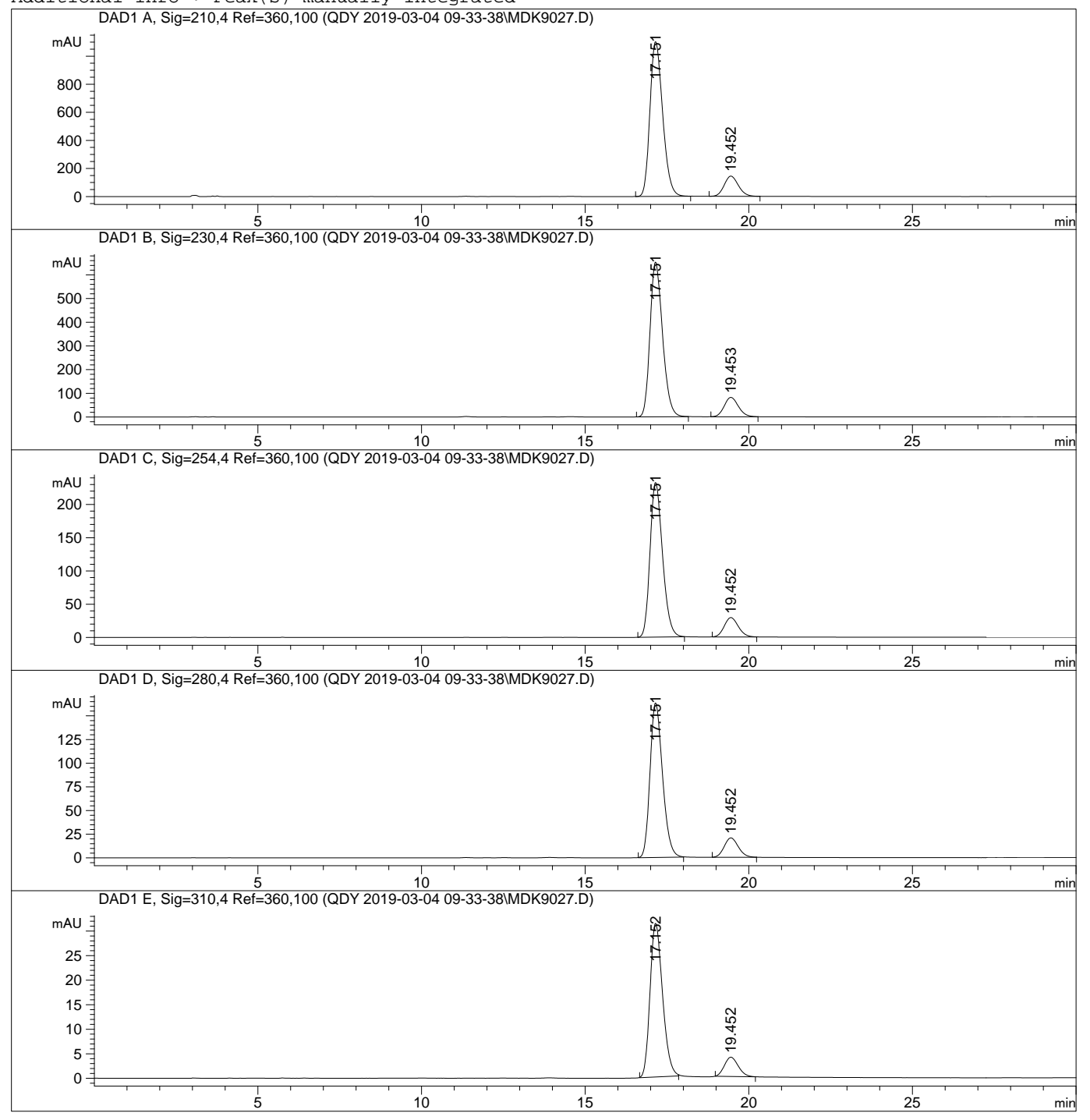

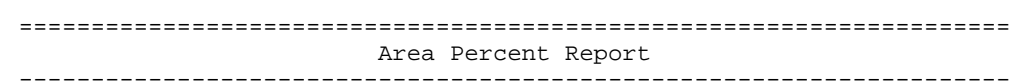

Sorted By

Signal

Multiplier

1.0000

Use Multiplier \& Dilution Factor with ISTDs

Signal 1: DAD1 A, Sig=210, 4 Ref $=360,100$

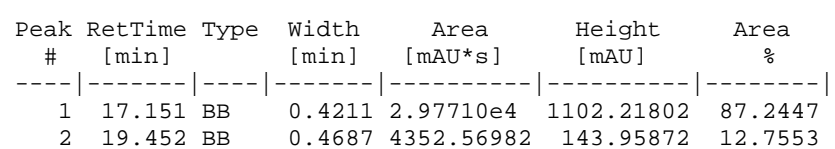

Totals :

$3.41235 \mathrm{e} 4 \quad 1246.17674$

Signal 2: DAD1 B, Sig=230, 4 Ref $=360,100$

Peak RetTime Type Width Area Height Area \# [min] $[$ [min] [mAU*s] [mAU] $\%$

$\begin{array}{lllllll}1 & 17.151 & \text { BB } & 0.4119 & 1.73462 \mathrm{e} 4 & 653.07092 & 87.5555\end{array}$

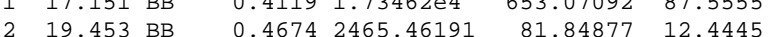

Totals :

$1.98117 \mathrm{e} 4 \quad 734.91969$

Signal 3: DAD1 C, Sig $=254,4$ Ref $=360,100$

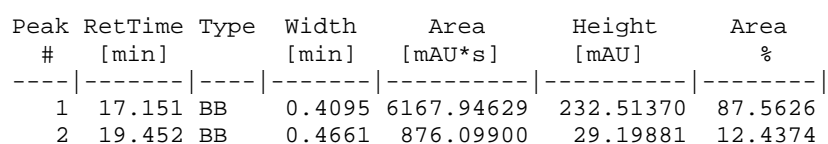

Totals :

$7044.04529 \quad 261.71251$

Signal 4: DAD1 D, Sig=280, 4 Ref $=360,100$

Peak RetTime Type Width Area Height Area

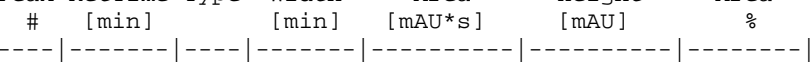

$\begin{array}{lllllll}1 & 17.151 & \text { BB } & 0.4091 & 4322.34131 & 163.14525 & 87.5425\end{array}$

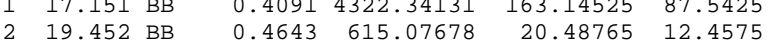

Totals :

$4937.41809 \quad 183.63289$

Signal 5: DAD1 E, Sig $=310,4$ Ref $=360,100$

Peak RetTime Type Width Area Height Area

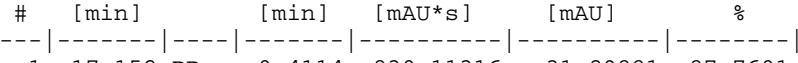

$\begin{array}{llllll}1 & 17.152 \mathrm{BB} & 0.4114 & 830.11316 & 31.29881 & 87.7601\end{array}$

Totals : $\quad 945.88899 \quad 35.21952$

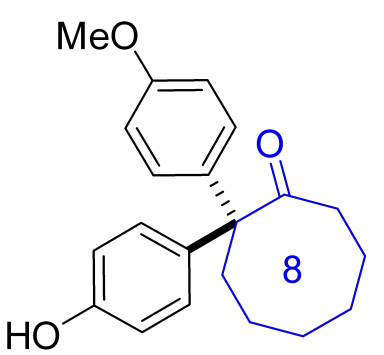

$2 q$

Enantioenriched 


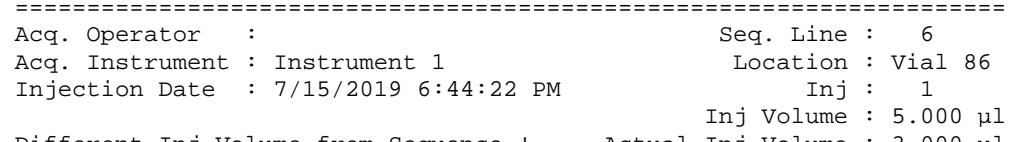

Different Inj Volume from Sequence! Actual Inj Volume : $5.000 \mu \mathrm{I}$

Acq. Method : C: \CHEM32\1\DATA\QDY 2019-07-15 17-27-34\AD-05-20.M

$\begin{array}{l:l}\text { Last changed } & \text { 10/14/2015 } 3: 51: 32 \text { PM } \\ \text { Analysis Method } & \text { C: } \backslash \text { CHEM32\1 } 1 \text { METHODS } \backslash \text { OD - } 02-10 . M\end{array}$

Last changed : 7/4/2019 9:46:26 PM

(modified after loading)

$\frac{\text { Additional Info : Peak (s) manually integrated }}{\text { DAD1 A, Sig=210,4 Ref } 360,100 \text { (QDY 2019-07-15 17-27-3412A-RAC.D) }}$
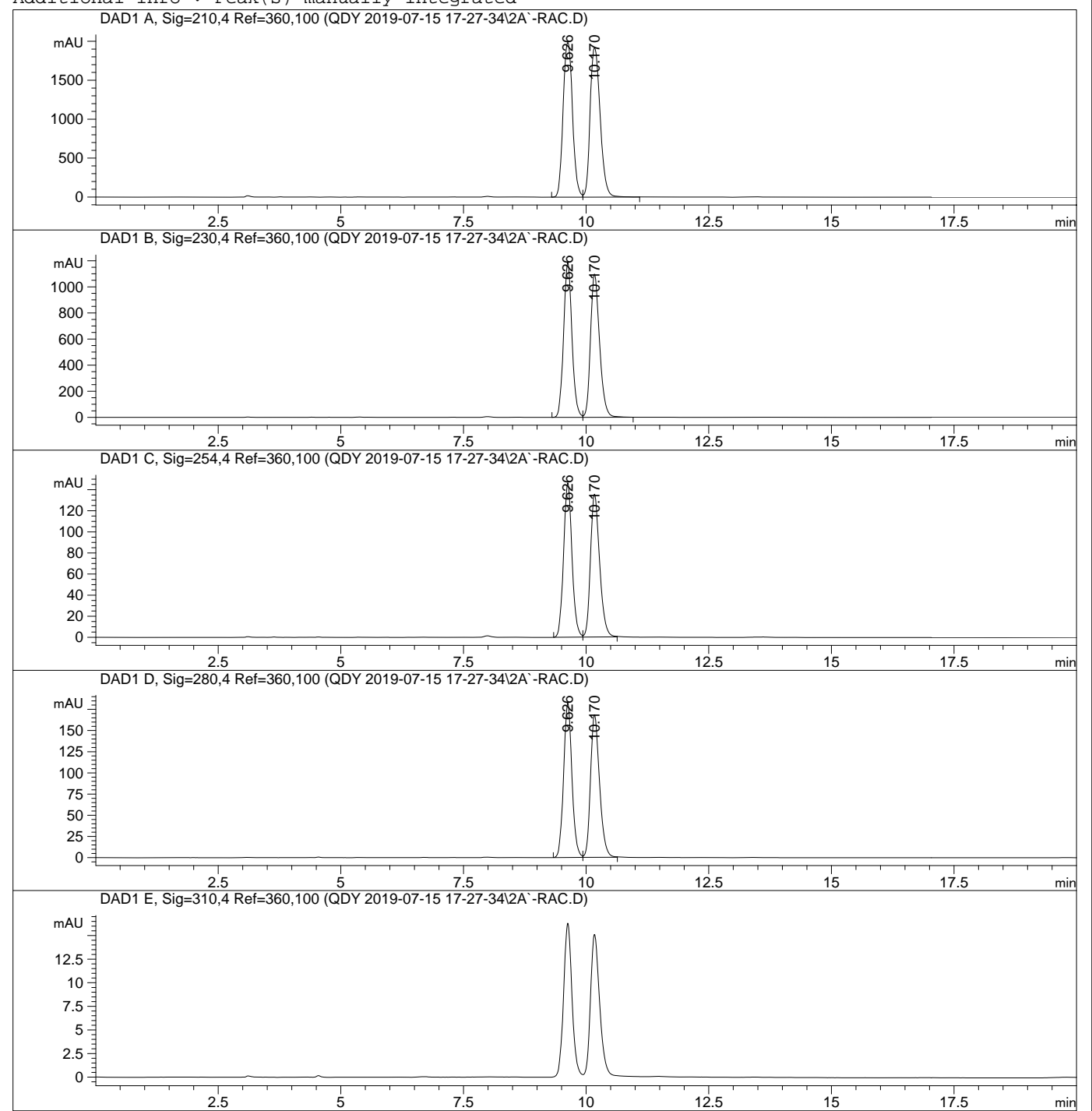

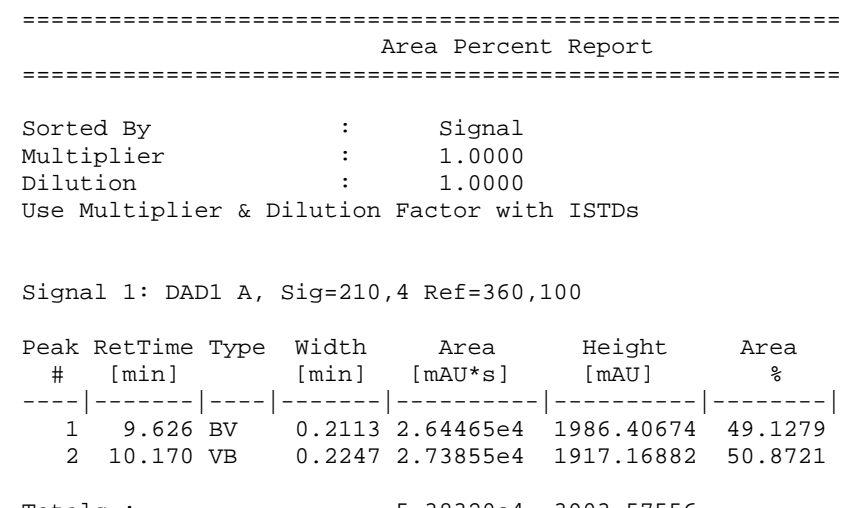

Totals :

$5.38320 \mathrm{e} 4 \quad 3903.57556$

Signal 2: DAD1 B, Sig=230, 4 Ref $=360,100$

\begin{tabular}{cccccc}
$\begin{array}{c}\text { Peak } \\
\#\end{array}$ RetTime Type & $\begin{array}{c}\text { Width } \\
{[\mathrm{min}]}\end{array}$ & $\begin{array}{c}\text { Area } \\
{[\mathrm{mAU}}\end{array}$ & $\begin{array}{l}\text { Height } \\
{[\mathrm{mAU}]}\end{array}$ & $\begin{array}{c}\text { Area } \\
\%\end{array}$ \\
\hdashline-1 & $9.626 \mathrm{BV}$ & 0.1817 & $1.39968 \mathrm{e} 4$ & 1185.89734 & 49.7217 \\
\hline 2 & 10.170 VB & 0.1964 & $1.41535 \mathrm{e} 4$ & 1097.94836 & 50.2783
\end{tabular}

Totals :

$2.81503 \mathrm{e} 4 \quad 2283.84570$

Signal 3: DAD1 C, Sig $=254,4$ Ref $=360,100$

\begin{tabular}{cccccc}
$\begin{array}{c}\text { Peak RetTime Type } \\
\text { \# }\end{array}\left[\begin{array}{c}\text { Width } \\
{[\mathrm{min}]}\end{array}\right.$ & $\begin{array}{c}\text { Area } \\
{[\mathrm{mAU}}\end{array}$ & $\begin{array}{l}\text { Height } \\
{[\mathrm{mAU}]}\end{array}$ & $\begin{array}{c}\text { Area } \\
\%\end{array}$ \\
\hdashline-1 & $9.626 \mathrm{BV}$ & 0.1827 & 1741.13721 & 146.45526 & 49.8319 \\
\hline 2 & 10.170 VB & 0.1969 & 1752.88513 & 135.51564 & 50.1681
\end{tabular}

Totals :

$3494.02234 \quad 281.97090$

Signal 4: DAD1 D, Sig=280, 4 Ref $=360,100$

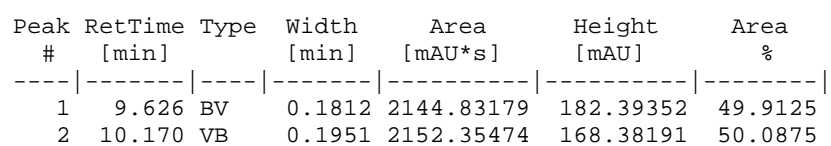

Totals :

$4297.18652 \quad 350.77544$

Signal 5: DAD1 E, Sig=310, 4 Ref $=360,100$

*** End of Report *** 


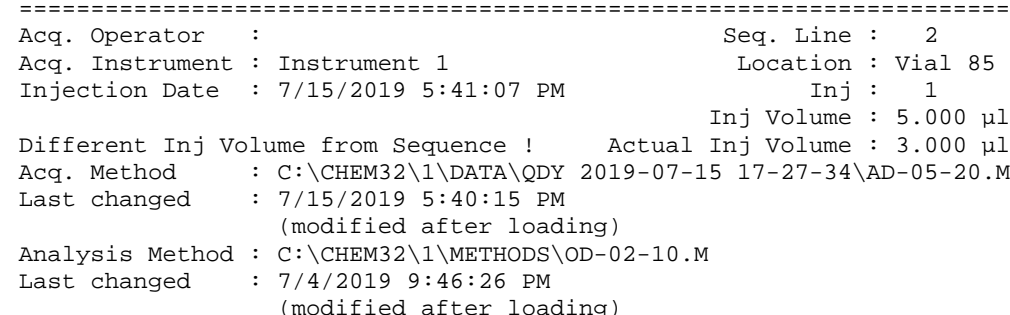

2.5

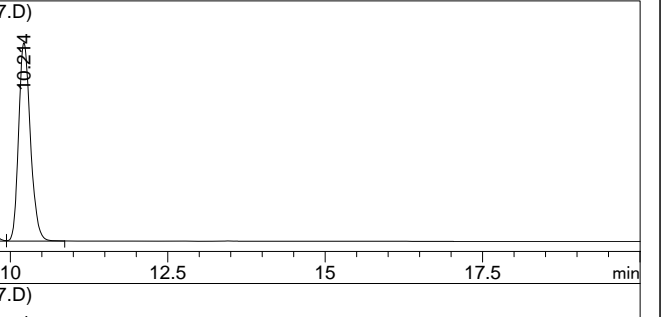

$\stackrel{+}{\stackrel{0}{*}}$

.

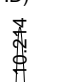

$\overbrace{\infty}^{\infty}$

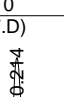

4
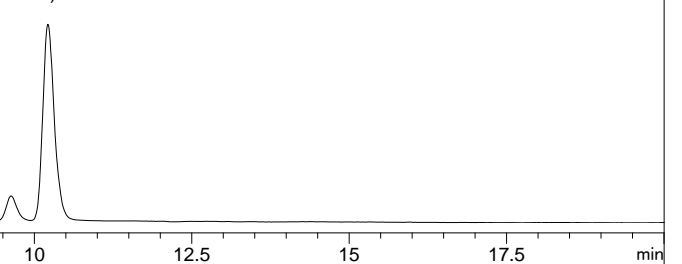

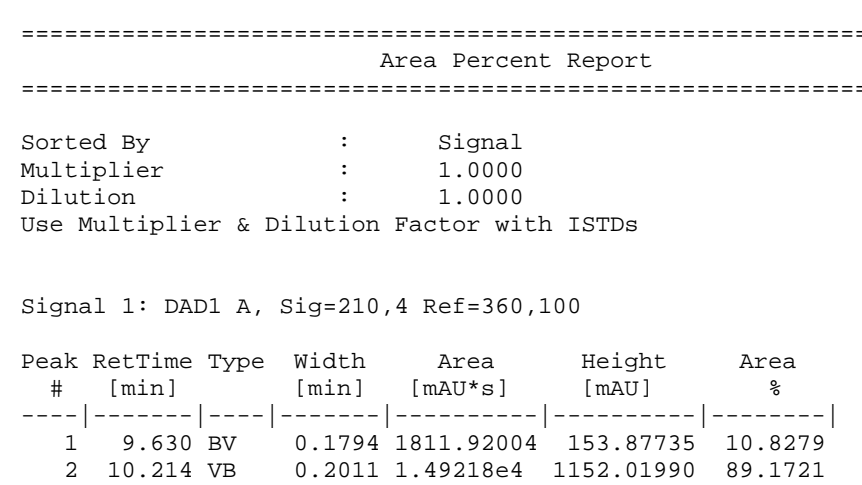

Totals :

$1.67337 \mathrm{e} 4 \quad 1305.89725$

Signal 2: DAD1 B, Sig=230, 4 Ref $=360,100$

\begin{tabular}{|c|c|c|c|c|c|}
\hline $\begin{array}{c}\text { Peak } \\
\#\end{array}$ & $\begin{array}{l}\text { RetTime Type } \\
\text { [min] }\end{array}$ & $\begin{array}{l}\text { Width } \\
\text { [min] }\end{array}$ & $\begin{array}{c}\text { Area } \\
{\left[\mathrm{mAU} U^{*} \mathrm{~s}\right]}\end{array}$ & $\begin{array}{l}\text { Height } \\
{[\mathrm{mAU}]}\end{array}$ & $\begin{array}{c}\text { Area } \\
\%\end{array}$ \\
\hline 1 & $9.630 \mathrm{BV}$ & 0.1789 & 824.33954 & 70.26449 & 10.4547 \\
\hline 2 & $10.214 \mathrm{VB}$ & 0.1934 & 7060.51953 & 558.70325 & 89.5453 \\
\hline
\end{tabular}

Totals :

$7884.85907 \quad 628.96774$

Signal 3: DAD1 C, Sig=254, 4 Ref $=360,100$

\begin{tabular}{|c|c|c|c|c|c|}
\hline $\begin{array}{c}\text { Peak } \\
\#\end{array}$ & $\begin{array}{l}\text { RetTime Type } \\
\text { [min] }\end{array}$ & $\begin{array}{l}\text { Width } \\
{[\mathrm{min}]}\end{array}$ & $\begin{array}{c}\text { Area } \\
{\left[\mathrm{mAU} U^{\star} \mathrm{s}\right]}\end{array}$ & $\begin{array}{l}\text { Height } \\
{[\mathrm{mAU}]}\end{array}$ & $\begin{array}{c}\text { Area } \\
\%\end{array}$ \\
\hline 1 & $9.630 \mathrm{BB}$ & 0.1788 & 104.85526 & 8.94379 & 10.5953 \\
\hline 2 & $10.214 \mathrm{BB}$ & 0.1965 & 884.78589 & 69.53242 & 89.4047 \\
\hline
\end{tabular}

Signal 4: DAD1 D, Sig=280, 4 Ref $=360,100$

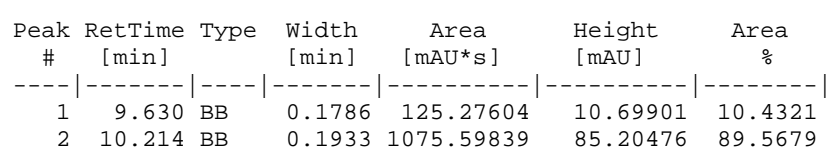
Totals : $1200.87443 \quad 95.90377$

$\mathrm{MeO}$<smiles>COc1ccc(C2(c3ccccc3)CCCCC2=O)cc1</smiles>

Enantioenriched

Signal 5: DAD1 E, Sig=310, 4 Ref $=360,100$

*** End of Report *** 


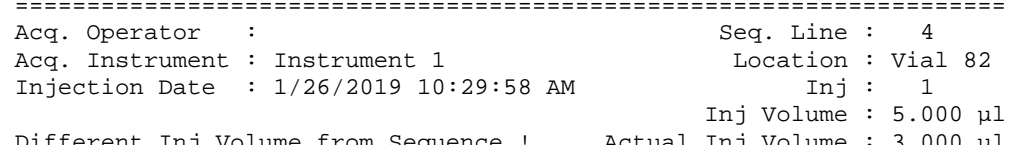

Injection Date Inj Volume : $5.000 \mu \mathrm{\mu l}$ Acq. Method : C: \CHEM32\1\DATA QQDY 2019-01-26 09-34-19\AS-05-20.M Last changed : 1/26/2019 10:08:01 AM

Analysis Method : C: \CHEM32\1\METHODS\OD-01-10-0.3.M

Last changed : 5/29/2019 9:25:17 PM

Additional Info : Peak(s) manually integrated

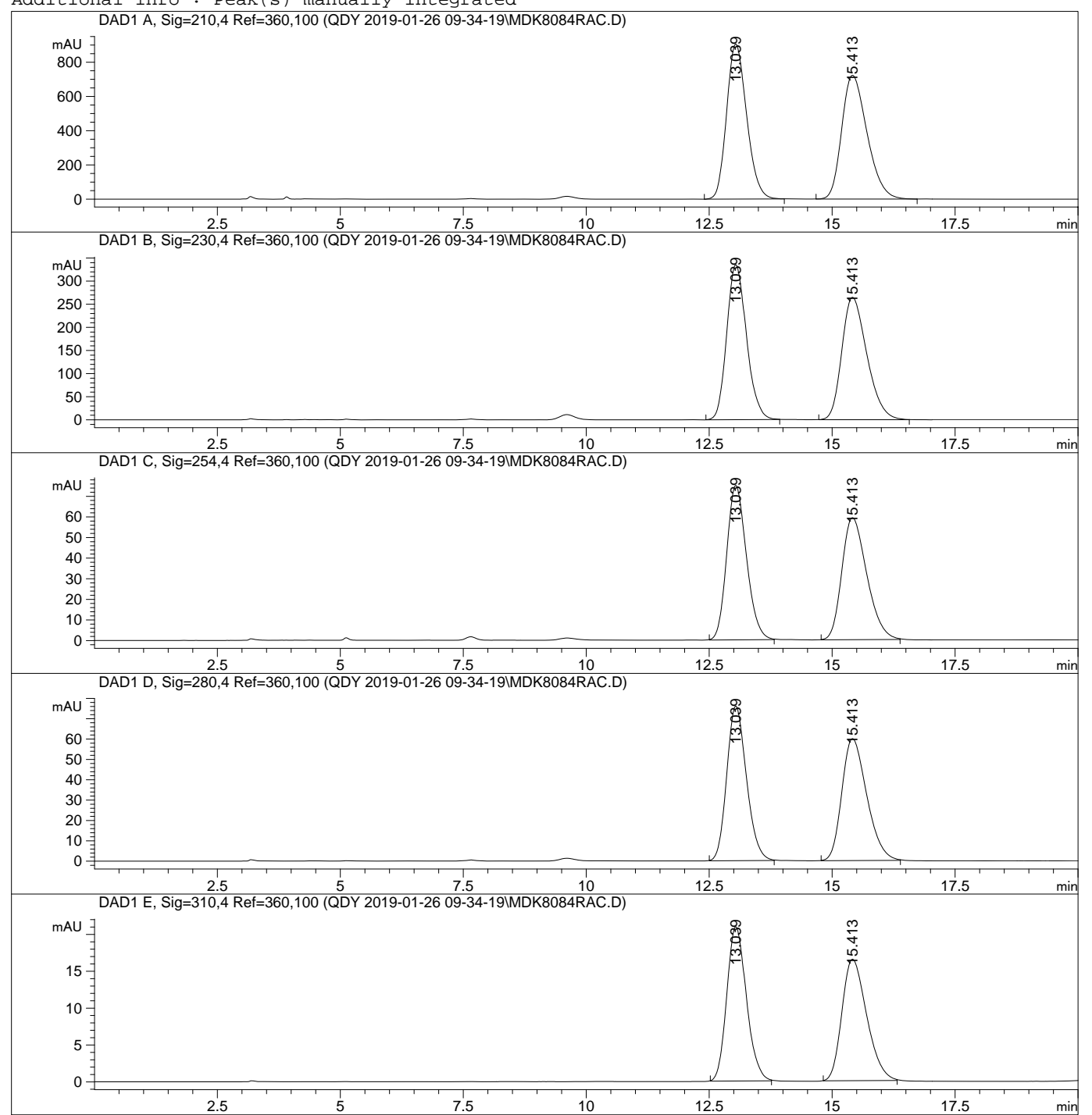

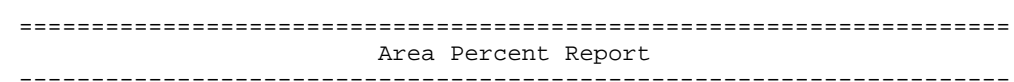

Sorted By

\section{Signal}

Multiplier
Dilution

1.0000

Use Multiplier \& Dilution Factor with ISTDs

Signal 1: DAD1 A, Sig=210, 4 Ref $=360,100$

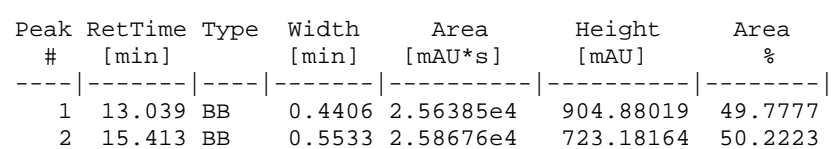

Totals :

$5.15061 \mathrm{e} 4 \quad 1628.06183$

Signal 2: DAD1 B, Sig=230, 4 Ref $=360,100$

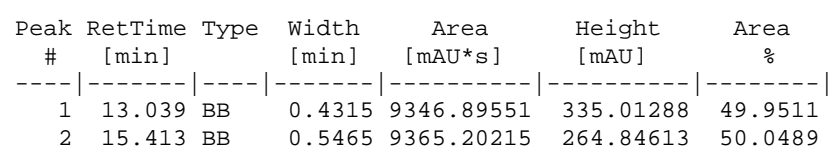

Totals :

$1.87121 \mathrm{e} 4 \quad 599.85901$

Signal 3: DAD1 C, Sig $=254,4$ Ref $=360,100$

\begin{tabular}{|c|c|c|c|c|c|}
\hline $\begin{array}{c}\text { eak } \\
\#\end{array}$ & $\begin{array}{l}\text { RetTime Type } \\
\text { [min] }\end{array}$ & $\begin{array}{l}\text { Width } \\
\text { [min] }\end{array}$ & $\begin{array}{c}\text { Area } \\
{\left[\mathrm{mAU}^{\star} \mathrm{s}\right]}\end{array}$ & $\begin{array}{l}\text { Height } \\
{[\mathrm{mAU}]}\end{array}$ & $\begin{array}{c}\text { Area } \\
\%\end{array}$ \\
\hline & 15 & & 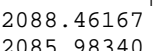 & & \\
\hline
\end{tabular}

Totals :

$4174.44507 \quad 134.15686$

Signal 4: DAD1 D, Sig=280, 4 Ref $=360,100$

\begin{tabular}{|c|c|c|c|c|c|}
\hline eak & $\begin{array}{l}\text { RetTime Type } \\
\text { [min] }\end{array}$ & $\begin{array}{l}\text { Width } \\
\text { [min] }\end{array}$ & $\begin{array}{c}\text { Area } \\
{\left[\mathrm{mAU}^{*} \mathrm{~s}\right]}\end{array}$ & $\begin{array}{l}\text { Height } \\
{[\mathrm{mAU}]}\end{array}$ & $\begin{array}{c}\text { Area } \\
\%\end{array}$ \\
\hline & 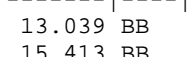 & & & & \\
\hline
\end{tabular}

Totals :

$4220.73633 \quad 135.88544$

\section{Signal 5: DAD1 E, Sig $=310,4$ Ref $=360,100$}

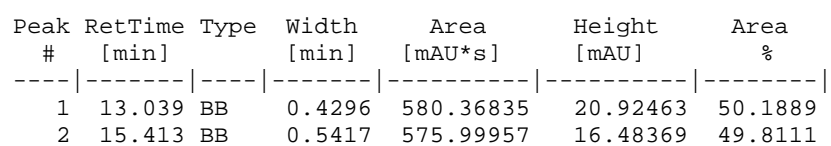

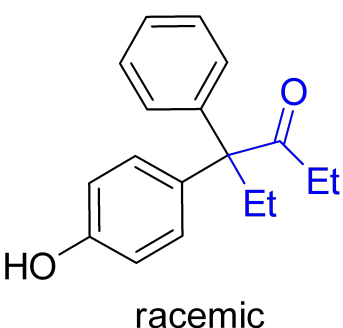

Totals : 


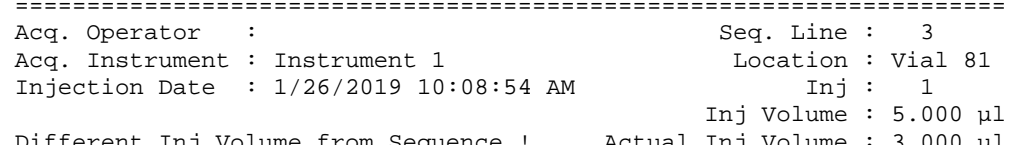

Injection Dater Inj Volume $: 5.000 \mu \mathrm{\mu l}$

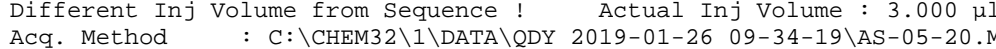
Last changed : 1/26/2019 10:08:01 AM

Analysis Method : C: \CHEM32\1\METHODS \OD-01-10-0.3.M

Last changed : 5/29/2019 9:25:17 PM
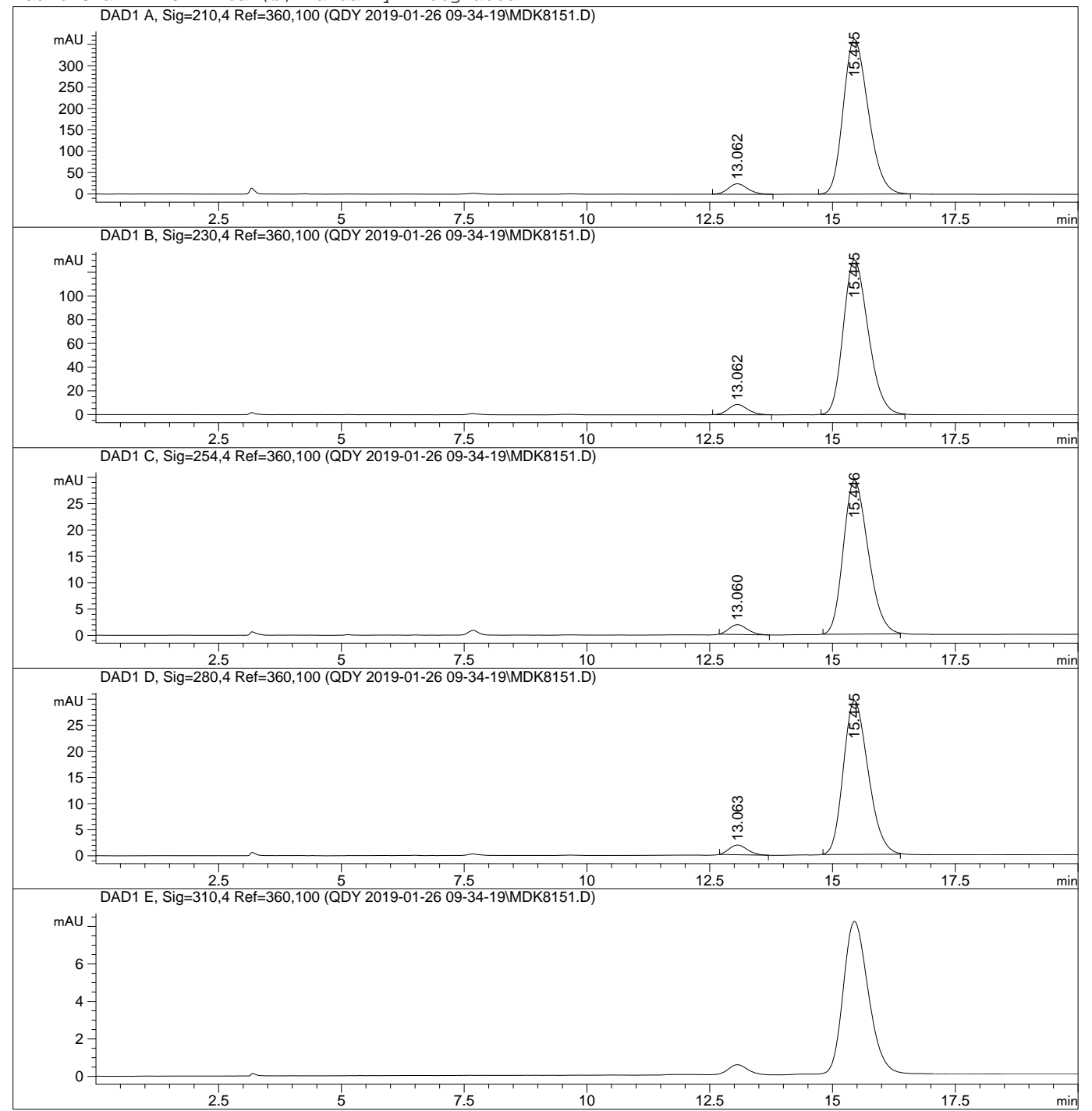

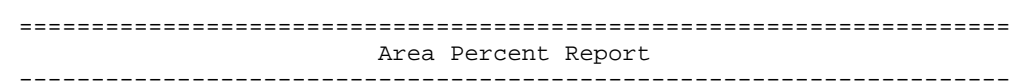

Sorted By

\section{Signal}

Multiplie
Dilution

1.0000

Use Multiplier \& Dilution Factor with ISTDs

Signal 1: DAD1 A, Sig=210, 4 Ref $=360,100$

\begin{tabular}{|c|c|c|c|c|c|}
\hline $\begin{array}{c}\text { Peak } \\
\#\end{array}$ & $\begin{array}{l}\text { RetTime Type } \\
{[\mathrm{min}]}\end{array}$ & $\begin{array}{l}\text { Width } \\
\text { [min] }\end{array}$ & $\begin{array}{c}\text { Area } \\
{\left[\mathrm{mAU} U^{*} \mathrm{~s}\right]}\end{array}$ & $\begin{array}{l}\text { Height } \\
{[\mathrm{mAU}]}\end{array}$ & $\begin{array}{c}\text { Area } \\
\%\end{array}$ \\
\hline $\begin{array}{l}1 \\
2\end{array}$ & $\begin{array}{l}13.062 \mathrm{BB} \\
15.445 \mathrm{BB}\end{array}$ & $\begin{array}{l}0.4215 \\
0.5514\end{array}$ & $\begin{array}{c}673.72339 \\
1.28299 \mathrm{e} 4\end{array}$ & $\begin{array}{r}24.60467 \\
362.10245\end{array}$ & $\begin{array}{r}4.9892 \\
95.0108\end{array}$ \\
\hline
\end{tabular}

Totals :

$1.35036 \mathrm{e} 4 \quad 386.70712$

Signal 2: DAD1 B, Sig=230, 4 Ref $=360,100$

\begin{tabular}{|c|c|c|c|c|c|}
\hline $\begin{array}{c}\text { Peak } \\
\#\end{array}$ & $\begin{array}{l}\text { RetTime Type } \\
{[\text { min] }}\end{array}$ & $\begin{array}{l}\text { Width } \\
\text { [min] }\end{array}$ & $\begin{array}{c}\text { Area } \\
{\left[\mathrm{mAU} U^{\star} \mathrm{s}\right]}\end{array}$ & $\begin{array}{l}\text { Height } \\
{[\mathrm{mAU}]}\end{array}$ & $\begin{array}{c}\text { Area } \\
\%\end{array}$ \\
\hline $\begin{array}{l}1 \\
2\end{array}$ & $\begin{array}{l}13.062 \mathrm{BB} \\
15.445 \mathrm{BB}\end{array}$ & $\begin{array}{l}0.4196 \\
0.5471\end{array}$ & $\begin{array}{r}236.95398 \\
4595.33691\end{array}$ & $\begin{array}{r}8.70294 \\
130.40134\end{array}$ & $\begin{array}{r}4.9036 \\
95.0964\end{array}$ \\
\hline
\end{tabular}

Totals :

$4832.29089 \quad 139.10428$

Signal 3: DAD1 C, Sig $=254,4$ Ref $=360,100$

\begin{tabular}{|c|c|c|c|c|c|}
\hline $\begin{array}{c}\text { Peak } \\
\#\end{array}$ & $\begin{array}{l}\text { RetTime Type } \\
{[\text { min] }}\end{array}$ & $\begin{array}{l}\text { Width } \\
\text { [min] }\end{array}$ & $\begin{array}{c}\text { Area } \\
{\left[\mathrm{mAU} U^{*} \mathrm{~s}\right]}\end{array}$ & $\begin{array}{l}\text { Height } \\
{[\mathrm{mAU}]}\end{array}$ & $\begin{array}{c}\text { Area } \\
\%\end{array}$ \\
\hline 1 & $13.060 \mathrm{BB}$ & 0. & 48.18580 & 1.86005 & 4909 \\
\hline 2 & $15.446 \mathrm{BB}$ & $\odot .5497$ & 1024.77722 & 29.17820 & 95.5091 \\
\hline
\end{tabular}

Signal 4: DAD1 D, Sig $=280,4$ Ref $=360,100$

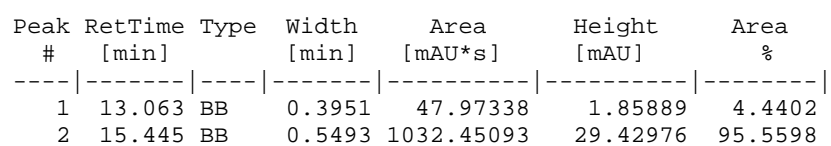

Totals :

$1080.42431 \quad 31.28865$

$\mathrm{HO}$

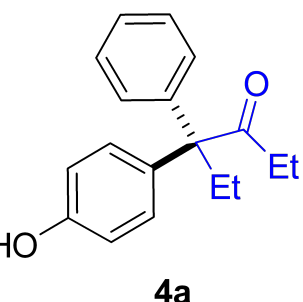

Enantioenriched

Signal 5: DAD1 E, Sig=310, 4 Ref $=360,100$

*** End of Report *** 


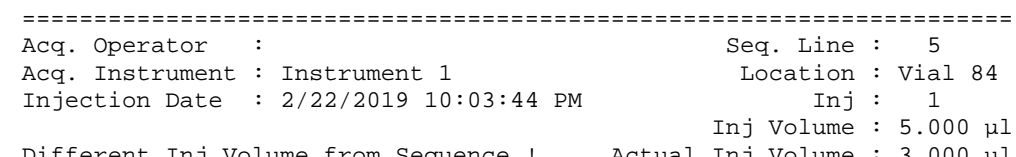

Different Inj volume from Sequence ! Actual Inj Volume : $5.000 \mu \mathrm{\mu}$

Acq. Method : C: \CHEM32\1\DATA\QDY 2019-02-22 20-41-16\AS-10-30.M

Last changed : 2/22/2019 9:31:25 PM

Analysis Method : C: \CHEM32\1\METHODS\OD-01-10-0.3.M

Last changed : 5/29/2019 9:25:17 PM

Additional Info : Peak(s) manually integrated
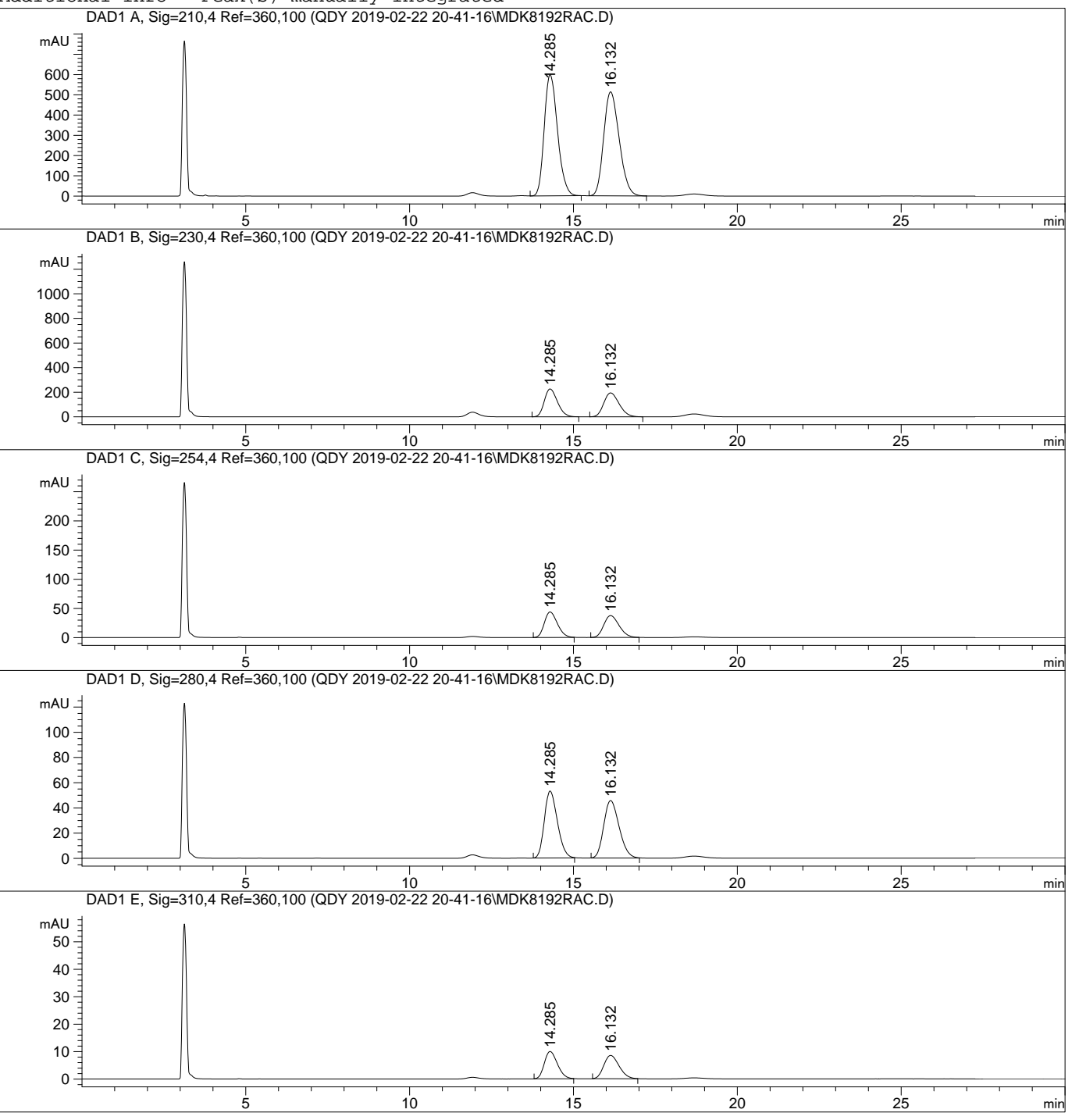

Area Percent Report

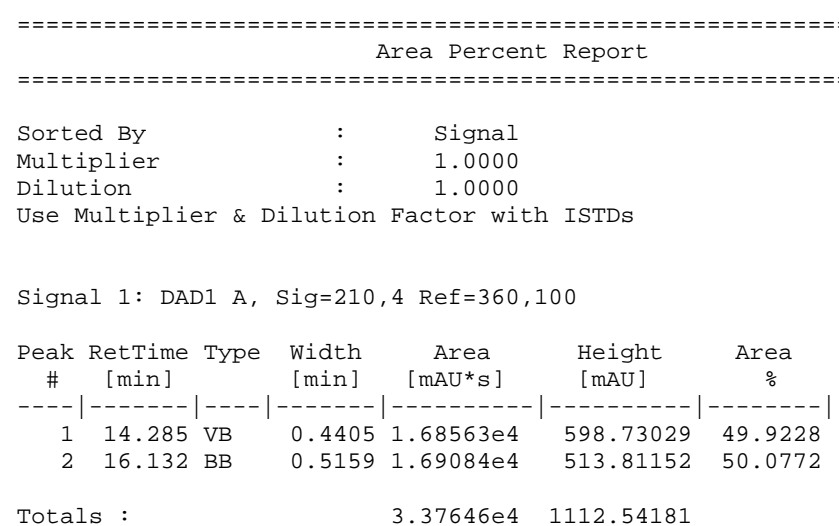

Signal 2: DAD1 B, Sig $=230,4$ Ref $=360,100$

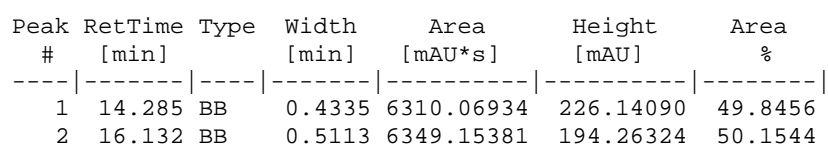

Totals : $1.26592 \mathrm{e} 4 \quad 420.40414$

Signal 3: DAD1 C, Sig $=254,4$ Ref $=360,100$

\begin{tabular}{|c|c|c|c|c|c|}
\hline $\begin{array}{c}\text { Peak } \\
\#\end{array}$ & $\begin{array}{l}\text { RetTime Type } \\
\text { [min] }\end{array}$ & $\begin{array}{l}\text { Width } \\
\text { [min] }\end{array}$ & $\begin{array}{c}\text { Area } \\
{\left[\mathrm{mAU} U^{*} \mathrm{~s}\right]}\end{array}$ & $\begin{array}{l}\text { Height } \\
{[\mathrm{mAU}]}\end{array}$ & $\begin{array}{c}\text { Area } \\
\%\end{array}$ \\
\hline 1 & $14.285 \mathrm{BB}$ & $\odot .4330$ & 1217.22314 & 43.69048 & 49.8751 \\
\hline 2 & $16.132 \mathrm{BB}$ & $\odot .5106$ & 1223.31860 & 37.49690 & 50.1249 \\
\hline
\end{tabular}

Signal 4: DAD1 D, Sig $=280,4$ Ref $=360,100$

\begin{tabular}{|c|c|c|c|c|c|}
\hline $\begin{array}{c}\text { Peak } \\
\#\end{array}$ & $\begin{array}{l}\text { RetTime Type } \\
\text { [min] }\end{array}$ & $\begin{array}{l}\text { Width } \\
\text { [min] }\end{array}$ & $\begin{array}{c}\text { Area } \\
{\left[\mathrm{mAU} U^{*} \mathrm{~s}\right]}\end{array}$ & $\begin{array}{l}\text { Height } \\
{[\mathrm{mAU}]}\end{array}$ & $\begin{array}{c}\text { Area } \\
\%\end{array}$ \\
\hline 1 & $14.285 \mathrm{BB}$ & 0.4323 & 1477.58081 & 53.14955 & 49.8468 \\
\hline 2 & $16.132 \mathrm{BB}$ & 0.5121 & 1486.66443 & 45.62757 & 50.1532 \\
\hline
\end{tabular}

Totals : $2964.24524 \quad 98.77711$

Signal 5: DAD1 E, Sig $=310,4$ Ref $=360,100$

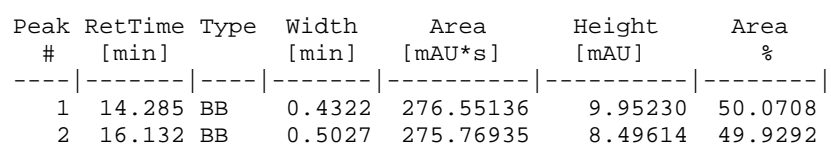

$\begin{array}{lll}\text { Totals : } & 552.32071 & 18.44844\end{array}$

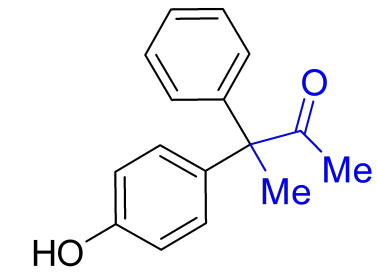

racemic 


\section{Data File C: \CHEM32\1\DATA\QDY 2019-02-22 20-41-16\MDK-9001.D}

Sample Name
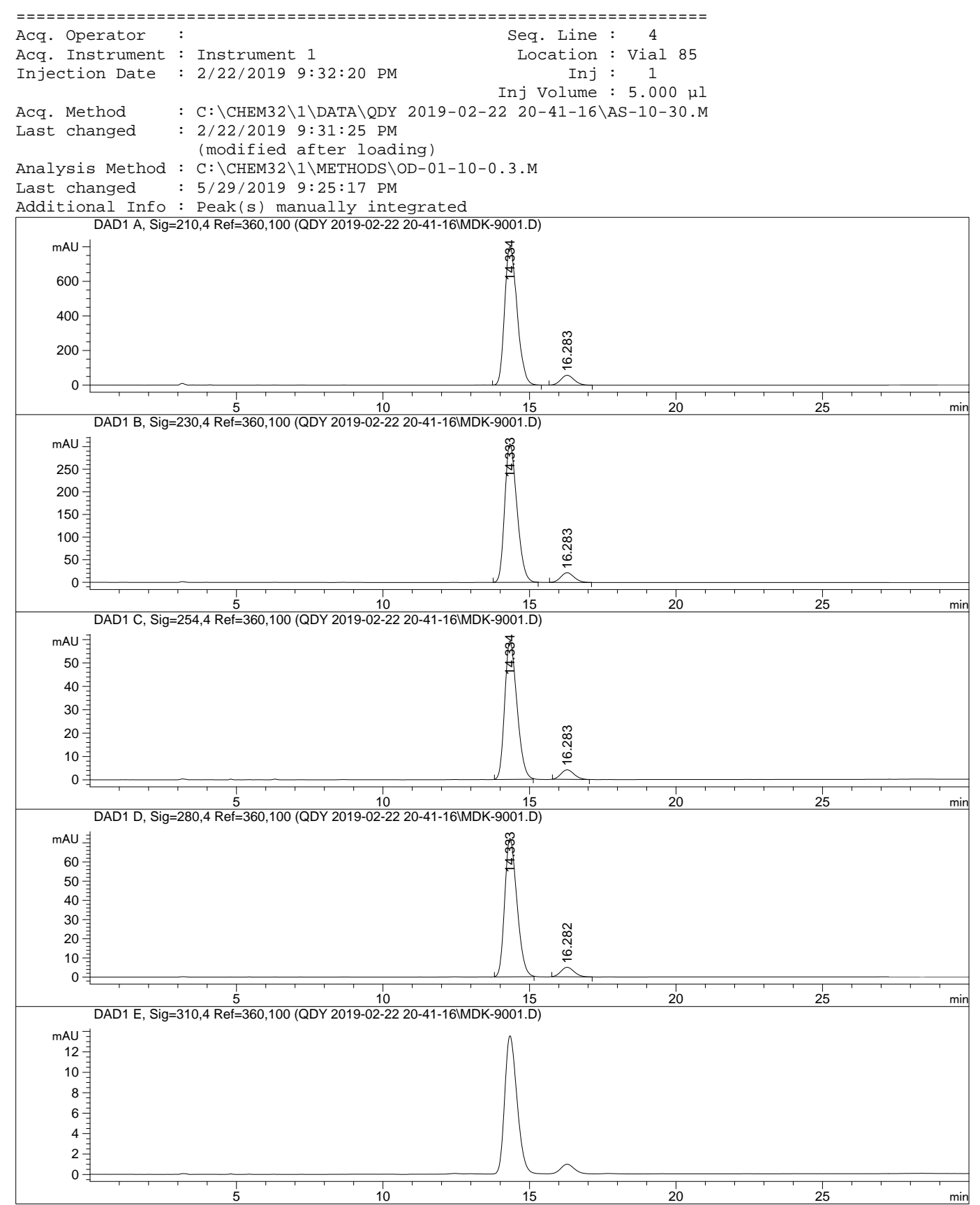

File C: \CHEM32\IDATA \QDY 2019-02-22 20-41-16\MDK-9001.D Sample Name:

Area Percent Report

$\begin{array}{lll}\text { Sorted By } & : & \text { Signal } \\ \text { Multiplier } & \vdots & 1.0000 \\ \text { Dilution } & : & 1.0000\end{array}$

Use Multiplier \& Dilution Factor with ISTDs

Signal 1: DAD1 A, Sig=210, 4 Ref $=360,100$

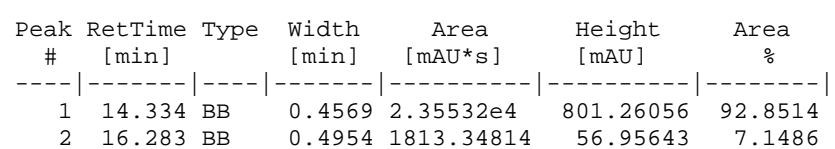

Totals :

$2.53665 \mathrm{e} 4 \quad 858.21699$

Signal 2: DAD1 B, Sig=230, 4 Ref $=360,100$

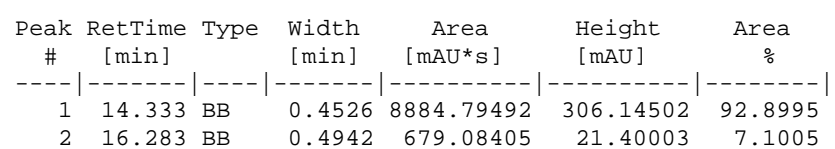

Totals :

$9563.87897 \quad 327.54505$

Signal 3: DAD1 C, Sig=254, 4 Ref $=360,100$

\begin{tabular}{cccccc}
$\begin{array}{c}\text { Peak RetTime Type } \\
\text { \# }\end{array}\left[\begin{array}{c}\text { Width } \\
{[\mathrm{min}]}\end{array}\right.$ & $\begin{array}{c}\text { Area } \\
{[\mathrm{mAU}}\end{array}$ & $\begin{array}{l}\text { Height } \\
{[\mathrm{mAU}]}\end{array}$ & $\begin{array}{c}\text { Area } \\
\%\end{array}$ \\
\hdashline$-14.334 \mathrm{BB}$ & 0.4519 & 1712.74365 & 59.14365 & 93.1277 \\
\hline 2 & $16.283 \mathrm{BB}$ & 0.4762 & 126.38988 & 4.07134 & 6.8723
\end{tabular}

Totals :

$1839.13354 \quad 63.21499$

Signal 4: DAD1 D, Sig=280, 4 Ref $=360,100$

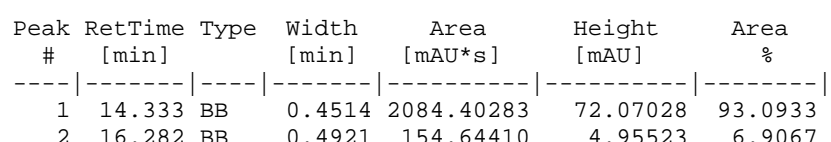

Totals :

$2239.04694 \quad 77.02551$

$\mathrm{HO}$

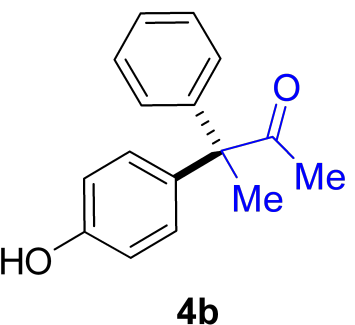

Enantioenriched

Signal 5: DAD1 E, Sig=310, 4 Ref $=360,100$

*** End of Report *** 


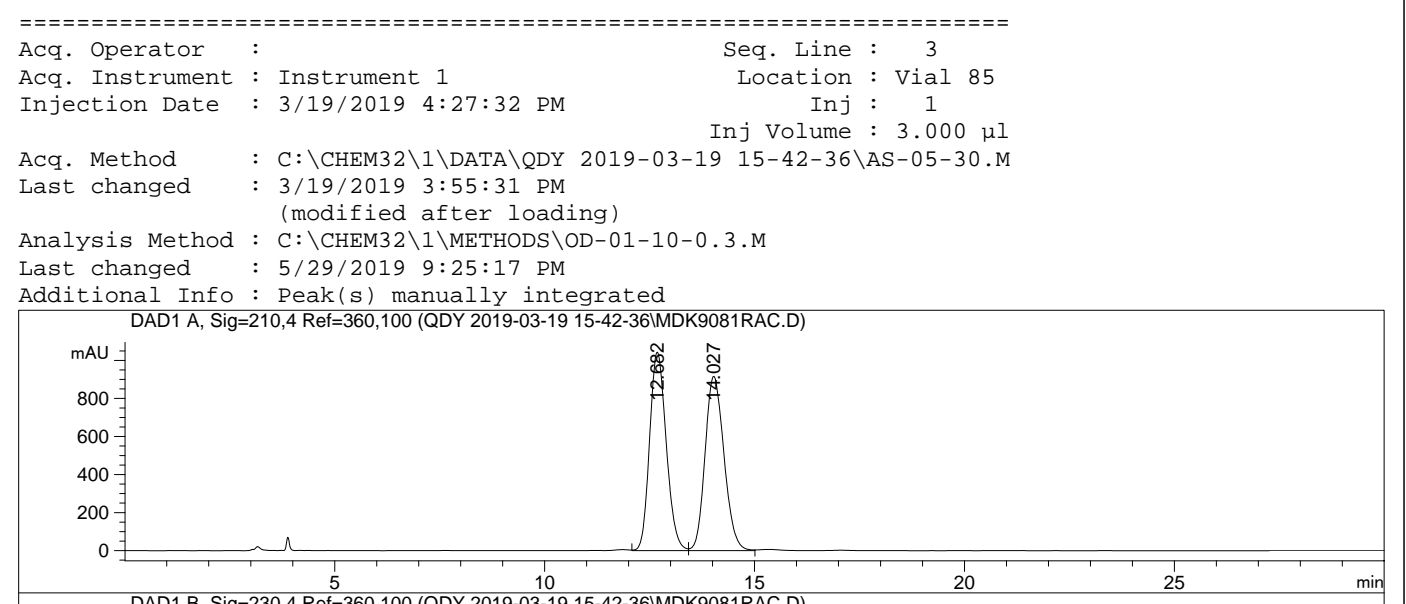
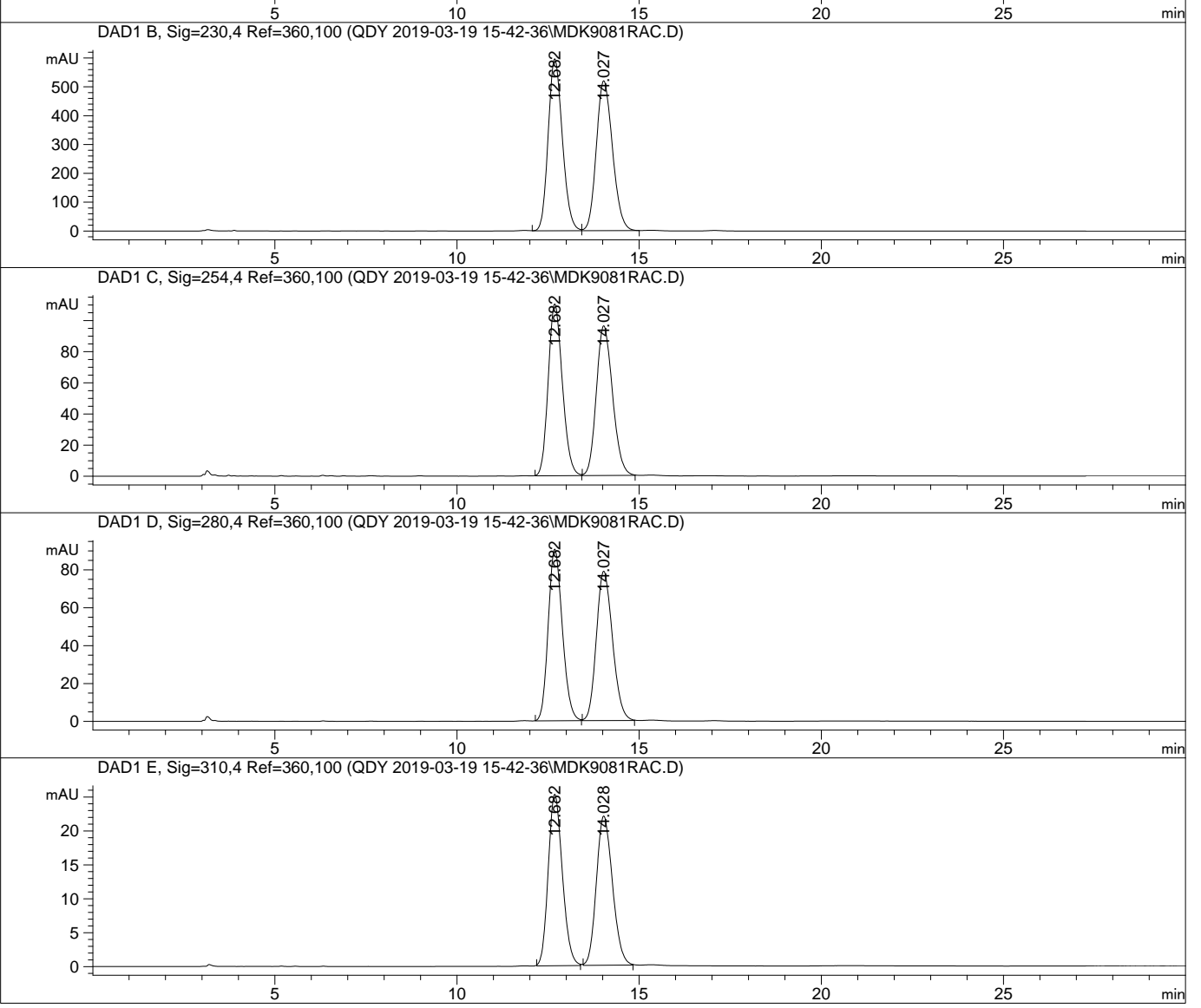

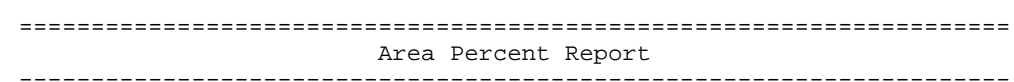

\section{Sorted By}

\section{Signal}

Multiplier
Dilution

1.0000

Use Multiplier \& Dilution Factor with ISTDs

Signal 1: DAD1 A, Sig=210, 4 Ref $=360,100$

\begin{tabular}{|c|c|c|c|c|c|}
\hline $\begin{array}{c}\text { Peak } \\
\#\end{array}$ & $\begin{array}{l}\text { RetTime Type } \\
\text { [min] }\end{array}$ & $\begin{array}{l}\text { Width } \\
\text { [min] }\end{array}$ & $\begin{array}{c}\text { Area } \\
{\left[\mathrm{mAU} U^{*} \mathrm{~s}\right]}\end{array}$ & $\begin{array}{l}\text { Height } \\
{[\mathrm{mAU}]}\end{array}$ & $\begin{array}{c}\text { Area } \\
\%\end{array}$ \\
\hline 1 & $12.682 \mathrm{VV}$ & & $2.87183 e 4$ & 1042.78491 & 50.0145 \\
\hline 2 & $14.027 \mathrm{VB}$ & 0.4898 & $2.87017 \mathrm{e} 4$ & 915.46344 & 49.9855 \\
\hline
\end{tabular}

Totals :

$5.74201 \mathrm{e} 4 \quad 1958.24835$

Signal 2: DAD1 B, Sig $=230,4$ Ref $=360,100$

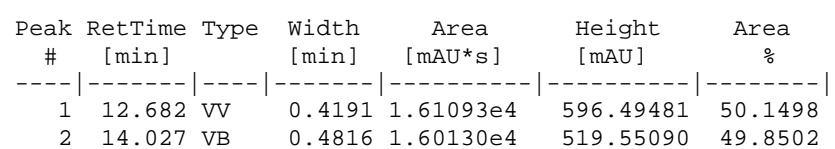

Totals :

3. 21222e4 1116.04572

Signal 3: DAD1 C, Sig $=254,4$ Ref $=360,100$

\begin{tabular}{|c|c|c|c|c|c|}
\hline $\begin{array}{c}\text { Peak } \\
\#\end{array}$ & $\begin{array}{l}\text { RetTime Type } \\
\text { [min] }\end{array}$ & $\begin{array}{l}\text { Width } \\
\text { [min] }\end{array}$ & $\begin{array}{c}\text { Area } \\
{\left[\mathrm{mAU} U^{*} \mathrm{~s}\right]}\end{array}$ & $\begin{array}{l}\text { Height } \\
{[\mathrm{mAU}]}\end{array}$ & $\begin{array}{c}\text { Area } \\
\%\end{array}$ \\
\hline 1 & $12.682 \mathrm{BB}$ & 0.4210 & 2978.91870 & 110.31512 & 50.0952 \\
\hline
\end{tabular}

Totals :

5946.51050206 .42516

Signal 4: DAD1 D, Sig=280, 4 Ref $=360,100$

$\begin{array}{ccccc}\text { Peak RetTime Type } & \text { Width } & \text { Area } & \text { Height } & \text { Area } \\ \text { \# }[\mathrm{min}] & {[\mathrm{min}]} & {\left[\mathrm{mAU} \mathrm{U}^{\star} \mathrm{s}\right]} & {[\mathrm{mAU}]} & \%\end{array}$ $112.682 \mathrm{BB} \quad 0.42022440 .59546 \quad 90.6204550 .1612$ $\begin{array}{lllllll}12.682 & \mathrm{BB} & 0.4202 & 2440.59546 & 90.62045 & 50.1612\end{array}$

Totals : $4865.49976 \quad 169.48018$

Signal 5: DAD1 E, Sig $=310,4$ Ref $=360,100$

Peak RetTime Type Width Area Height Area

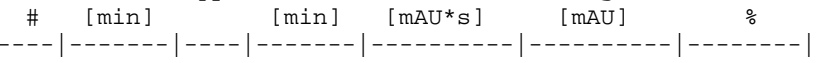

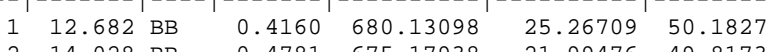
Totals : $\quad 1355.31036 \quad 47.26186$ 


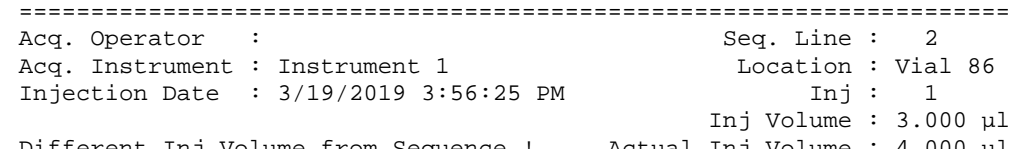

Different Inj Volume from Sequence ! Actual Inj Volume : $3.000 \mu \mathrm{\mu}$

Acq. Method : C: \CHEM32\1\DATA\QDY 2019-03-19 15-42-36\AS-05-30.M

Last changed : 3/19/2019 3:55:31 PM

Analysis Method : C: \CHEM32\1\METHODS\OD-01-10-0.3.M

A : 5/29/2019 9:25:17 PM

Additional Info : Peak(s) manually integrated
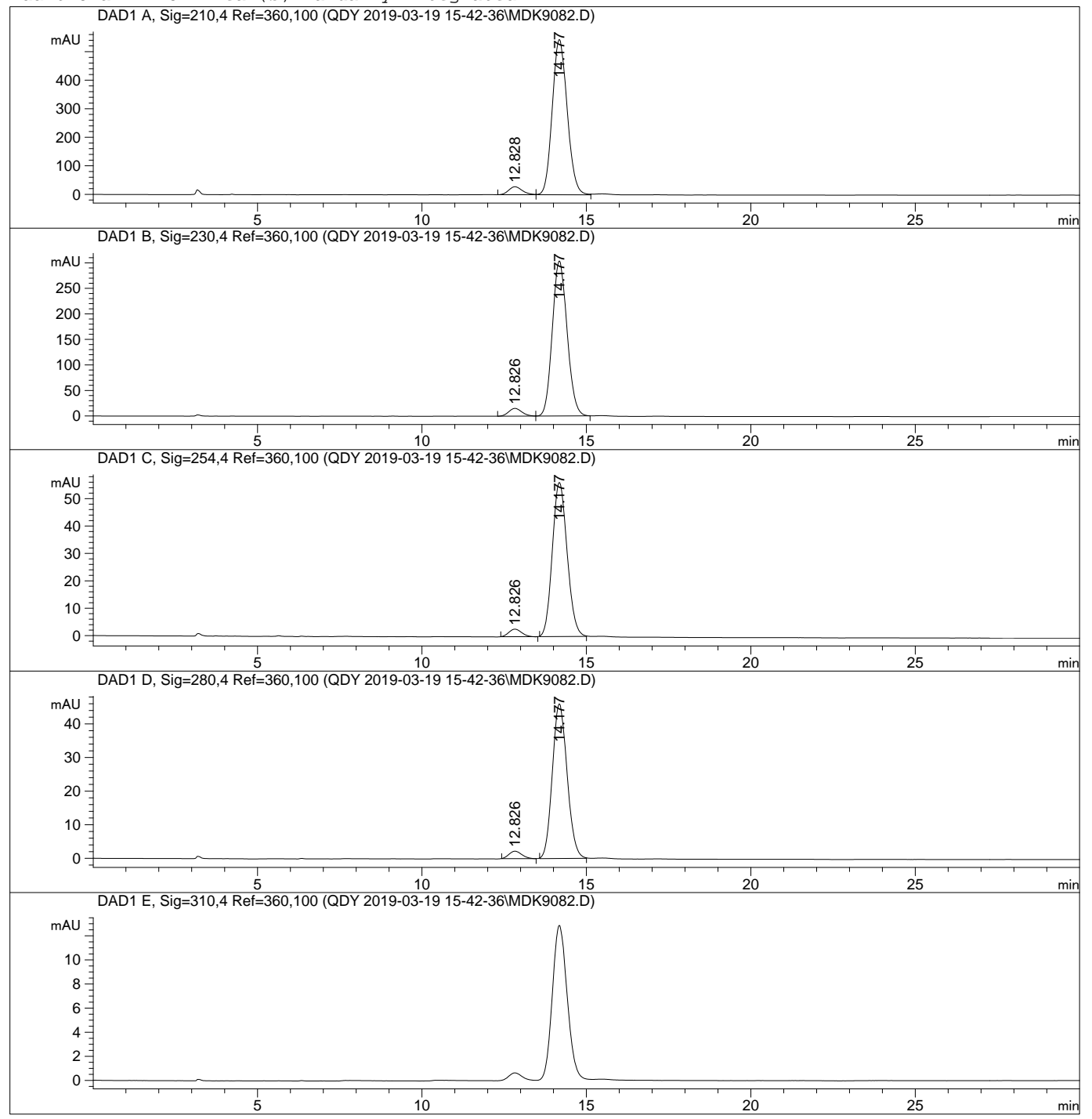

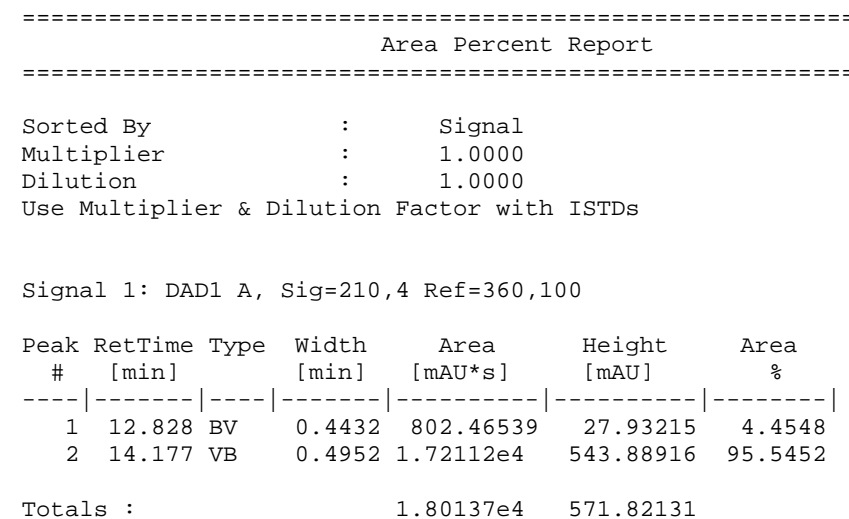

Signal 2: DAD1 B, Sig=230, 4 Ref $=360,100$

\begin{tabular}{|c|c|c|c|c|c|}
\hline $\begin{array}{c}\text { Peak } \\
\#\end{array}$ & $\begin{array}{l}\text { RetTime Type } \\
{[\mathrm{min}]}\end{array}$ & $\begin{array}{l}\text { Width } \\
\text { [min] }\end{array}$ & $\begin{array}{c}\text { Area } \\
{\left[\mathrm{mAU} U^{\star} \mathrm{s}\right]}\end{array}$ & $\begin{array}{l}\text { Height } \\
{[\mathrm{mAU}]}\end{array}$ & $\begin{array}{c}\text { Area } \\
\%\end{array}$ \\
\hline 1 & $12.826 \mathrm{BV}$ & 0.4324 & 418.49191 & 15.14580 & 4.2214 \\
\hline 2 & $14.177 \mathrm{VB}$ & 0.4870 & 9495.08691 & 303.51028 & 95.7786 \\
\hline
\end{tabular}

Totals :

$9913.57883 \quad 318.65608$

Signal 3: DAD1 C, Sig $=254,4$ Ref $=360,100$

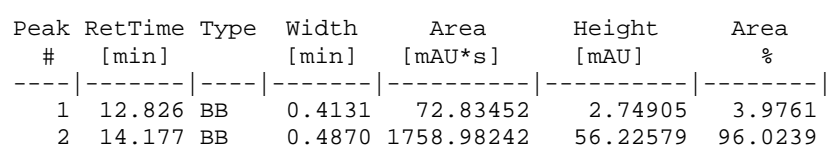

Totals :

$1831.81694 \quad 58.97484$

Signal 4: DAD1 D, Sig=280, 4 Ref $=360,100$

Peak RetTime Type width Area Height Area

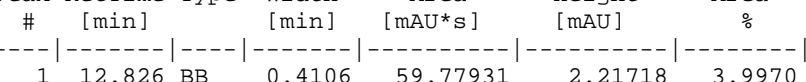

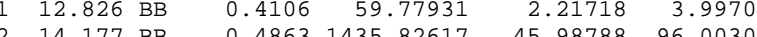

Totals :

$1495.60548 \quad 48.20506$

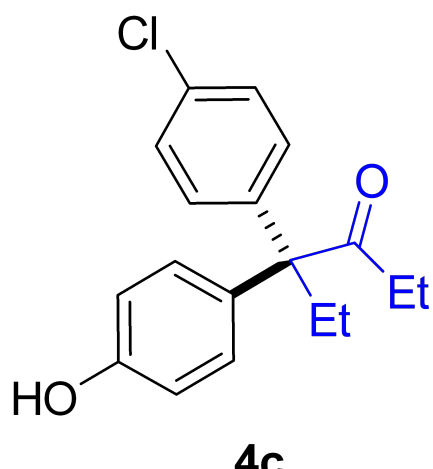

Signal 5: DAD1 E, Sig $=310,4$ Ref $=360,100$

Enantioenriched

$* * *$ End of Report *** 
Area Percent Report

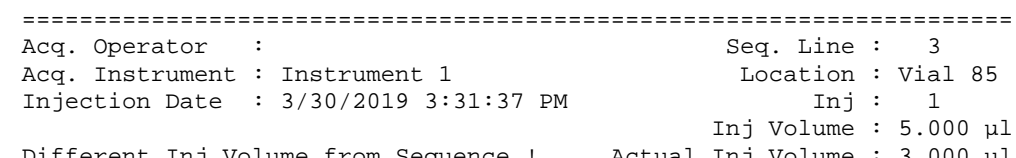

Different Inj Volume from Sequence ! Actual Inj Volume : $5.000 \mu \mathrm{\mu}$

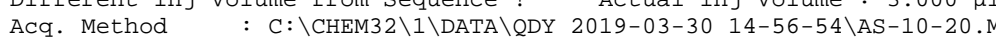

Last changed : 3/30/2019 3:09:06 PM

Analysis Method : C: \CHEM32\1\METHODS\OD-01-10-0.3.M

Last changed : 5/29/2019 9:25:17 PM

Additional Info : Peak(s) manually integrated
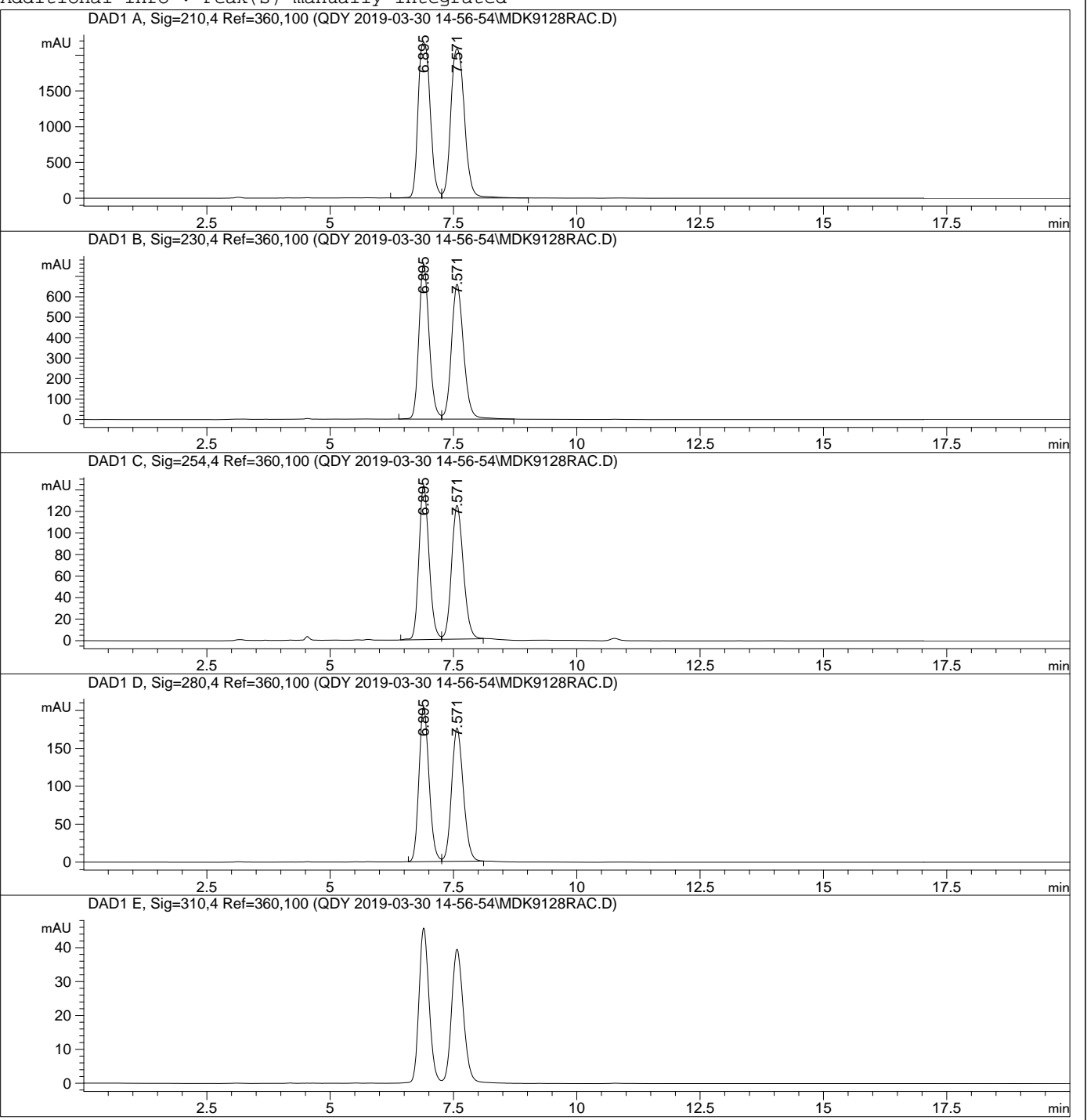

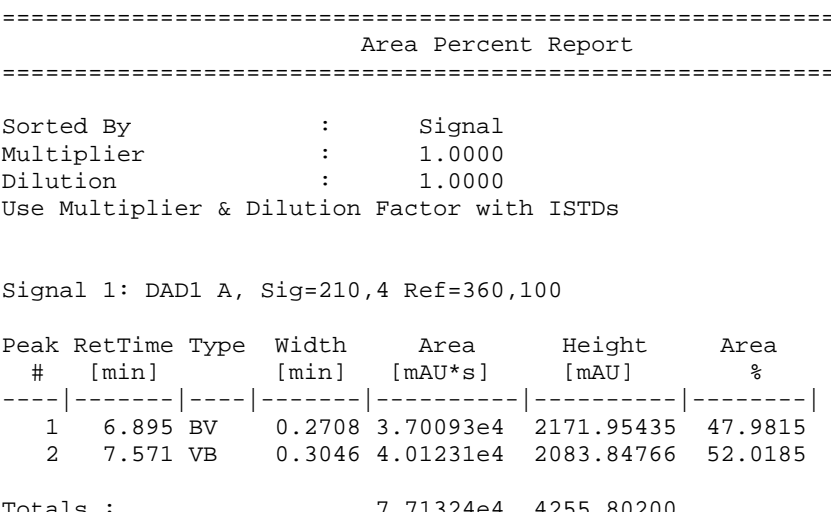

Totals :

$7.71324 \mathrm{e} 4 \quad 4255.80200$

Signal 2: DAD1 B, Sig $=230,4$ Ref $=360,100$

\begin{tabular}{|c|c|c|c|c|c|}
\hline $\begin{array}{c}\text { Peak } \\
\#\end{array}$ & $\begin{array}{l}\text { RetTime Type } \\
\text { [min] }\end{array}$ & $\begin{array}{l}\text { Width } \\
\text { [min] }\end{array}$ & $\begin{array}{c}\text { Area } \\
{\left[\mathrm{mAU} U^{*} \mathrm{~s}\right]}\end{array}$ & $\begin{array}{l}\text { Height } \\
{[\mathrm{mAU}]}\end{array}$ & $\begin{array}{c}\text { Area } \\
\%\end{array}$ \\
\hline 1 & $6.895 \mathrm{BV}$ & 0.2244 & 1. $09455 \mathrm{e} 4$ & 758.50519 & 49.1928 \\
\hline
\end{tabular}

Totals :

$2.22502 \mathrm{e} 4 \quad 1417.96515$

Signal 3: DAD1 C, Sig $=254,4$ Ref $=360,100$

\begin{tabular}{|c|c|c|c|c|c|}
\hline $\begin{array}{c}\text { Peak } \\
\#\end{array}$ & $\begin{array}{l}\text { RetTime Type } \\
\text { [min] }\end{array}$ & $\begin{array}{l}\text { Width } \\
\text { [min] }\end{array}$ & $\begin{array}{c}\text { Area } \\
{\left[\mathrm{mAU}^{*} \mathrm{~s}\right]}\end{array}$ & $\begin{array}{l}\text { Height } \\
{[\mathrm{mAU}]}\end{array}$ & $\begin{array}{c}\text { Area } \\
\%\end{array}$ \\
\hline 1 & $6.895 \mathrm{BV}$ & 0.2224 & 2044.74756 & 143.33133 & 49.7200 \\
\hline 2 & $7.571 \mathrm{VB}$ & 0.2582 & 2067.77930 & 124.25388 & 50.2800 \\
\hline
\end{tabular}

Totals :

$4112.52686 \quad 267.58521$

Signal 4: DAD1 D, Sig=280, 4 Ref $=360,100$

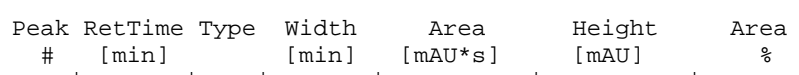

$10.895 \mathrm{BV} \quad 0.21952900 .17090 \quad 204.49521 \quad 49.9685$

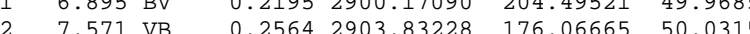

Totals :

$5804.00317 \quad 380.56186$

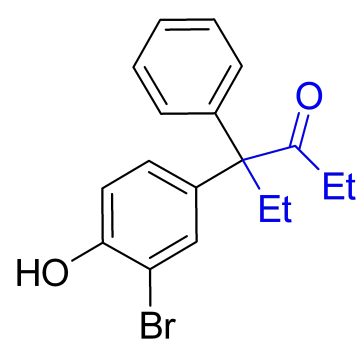

racemic

Signal 5: DAD1 E, Sig=310, 4 Ref $=360,100$

*** End of Report *** 


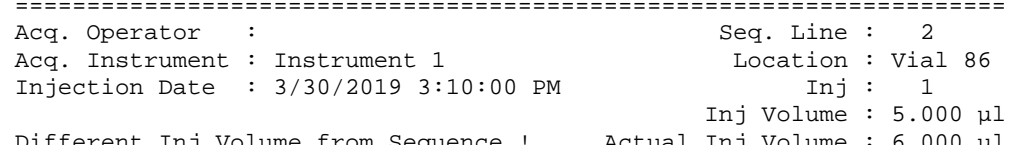

Injection Date Inj Volume $: 5.000 \mu \mathrm{\mu l}$

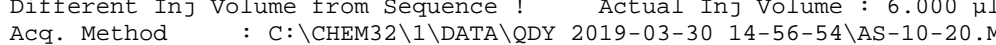

Last changed : 3/30/2019 3:09:06 PM

Analysis Method : C: \CHEM32\1\METHODS\OD-01-10-0.3.M

Last changed : 5/29/2019 9:25:17 PM

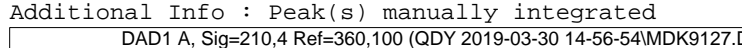
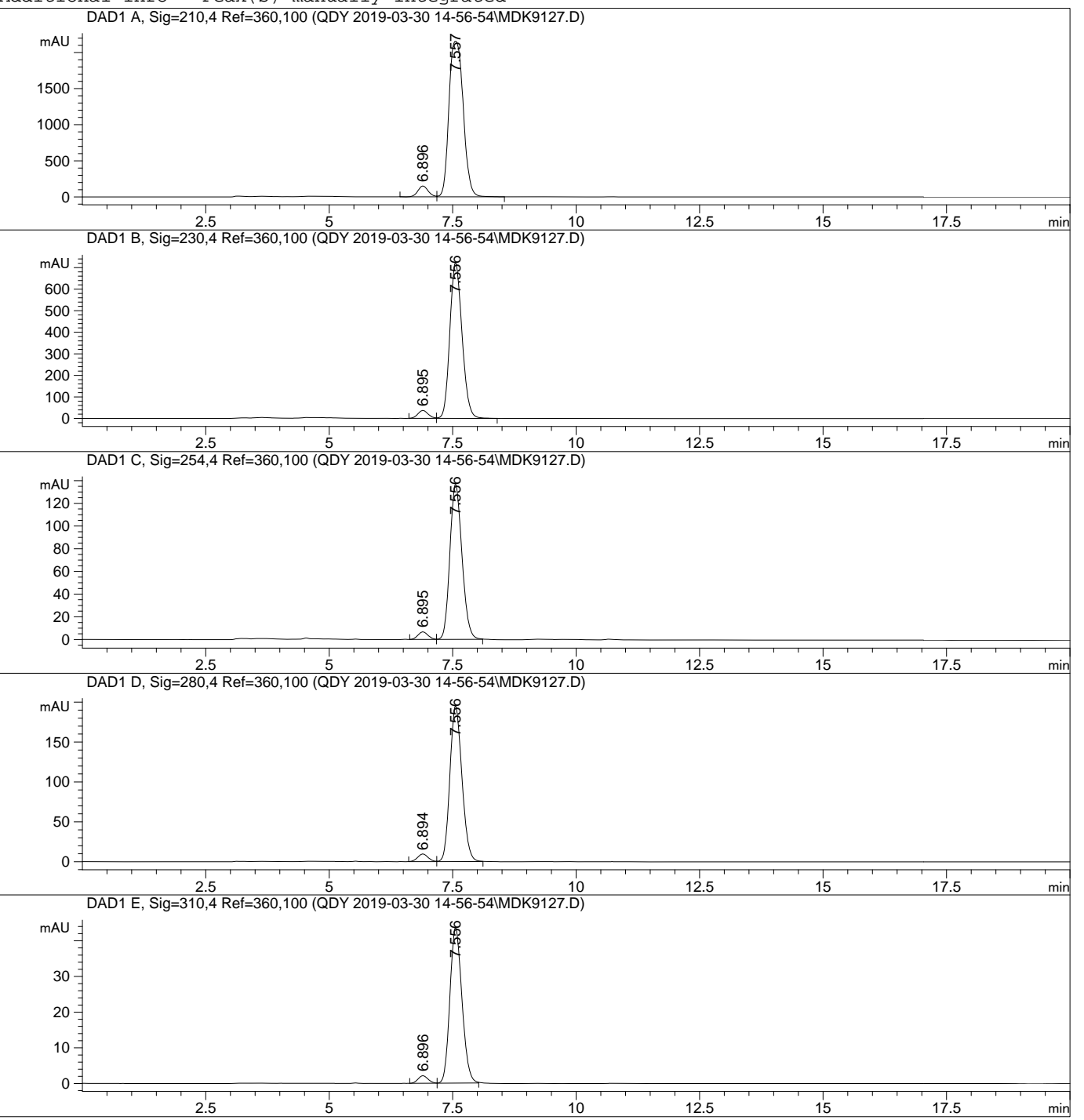

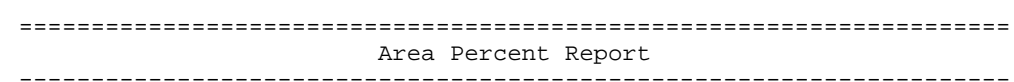

Sorted By
Multiplier

\section{Signal}

1.0000

Use Multiplier \& Dilution Factor with ISTDs

Signal 1: DAD1 A, Sig=210, 4 Ref $=360,100$

\begin{tabular}{|c|c|c|c|c|c|}
\hline eak & $\begin{array}{l}\text { RetTime Type } \\
\text { [min] }\end{array}$ & $\begin{array}{l}\text { Width } \\
\text { [min] }\end{array}$ & $\begin{array}{c}\text { Area } \\
{\left[\mathrm{mAU}{ }^{\star} \mathrm{s}\right]}\end{array}$ & $\begin{array}{l}\text { Height } \\
{[\mathrm{mAU}]}\end{array}$ & $\begin{array}{c}\text { Area } \\
\%\end{array}$ \\
\hline & 5 & & i. 6104 & & \\
\hline
\end{tabular}

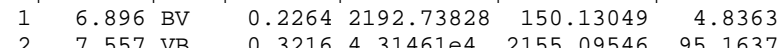

Totals : $\quad 4.53388 \mathrm{e} 4 \quad 2305.22595$

Signal 2: DAD1 B, Sig=230, 4 Ref $=360,100$

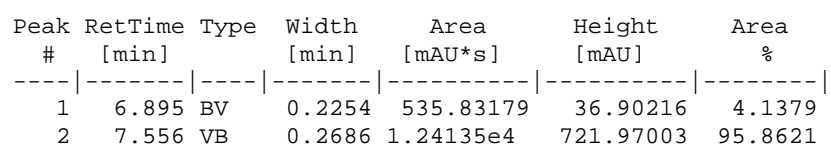

Totals :

$1.29494 \mathrm{e} 4 \quad 758.87220$

Signal 3: DAD1 C, Sig=254, 4 Ref $=360,100$

\begin{tabular}{|c|c|c|c|c|c|}
\hline $\begin{array}{c}\text { Peak } \\
\#\end{array}$ & $\begin{array}{l}\text { RetTime Type } \\
\text { [min] }\end{array}$ & $\begin{array}{l}\text { Width } \\
\text { [min] }\end{array}$ & $\begin{array}{c}\text { Area } \\
{\left[\mathrm{mAU}^{*} \mathrm{~s}\right]}\end{array}$ & $\begin{array}{l}\text { Height } \\
{[\mathrm{mAU}]}\end{array}$ & $\begin{array}{c}\text { Area } \\
\%\end{array}$ \\
\hline 1 & $6.895 \mathrm{BV}$ & 0.2181 & 93.81573 & 6.66835 & 3.8746 \\
\hline 2 & 7.556 VB & 0.2668 & 2327.50586 & 136.56580 & 96.1254 \\
\hline
\end{tabular}

Totals :

$2421.32159 \quad 143.23415$

Signal 4: DAD1 D, Sig=280, 4 Ref $=360,100$

\begin{tabular}{|c|c|c|c|c|c|}
\hline eak & $\begin{array}{l}\text { RetTime Type } \\
\text { [min] }\end{array}$ & $\begin{array}{l}\text { Width } \\
\text { [min] }\end{array}$ & $\begin{array}{c}\text { Area } \\
{\left[\mathrm{mAU} U^{*} \mathrm{~s}\right]}\end{array}$ & $\begin{array}{l}\text { Height } \\
{[\mathrm{mAU}]}\end{array}$ & $\begin{array}{c}\text { Area } \\
\%\end{array}$ \\
\hline & 94 & & & & \\
\hline
\end{tabular}

Totals :

$3444.72769 \quad 204.47962$

Signal 5: DAD1 E, Sig=310, 4 Ref $=360,100$

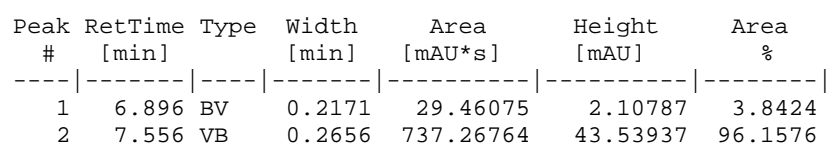

Totals : $\quad 766.72839 \quad 45.64725$

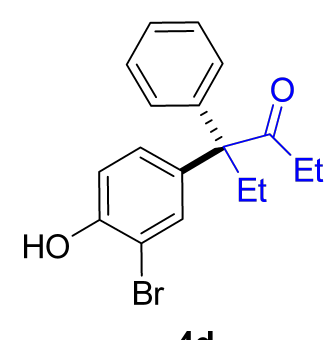

Enantioenriched 


\begin{tabular}{|c|c|c|}
\hline Acq. Oper & & Seq. Line : \\
\hline & Instrument 1 & Location : \\
\hline Injection Date & 8/11/2019 4:31:57 PM & 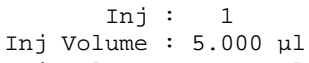 \\
\hline
\end{tabular}

Injection Date : 8/11/2019 4:31:57 PM $\quad$ Inj $\quad \begin{gathered}1 \\ \end{gathered}$

Acq. Method : C: \CHEM32\1\DATA :QDY 2019-08-11 15-57-17\OD-05-20.M

Last changed : 8/11/2019 4:10:02 PM

Analysis Method : C: \CHEM32\1\METHODS \OD-01-10.M

Last changed : 8/15/2019 4:47:06 PM

Additional Info: Peak(s) manually integrated
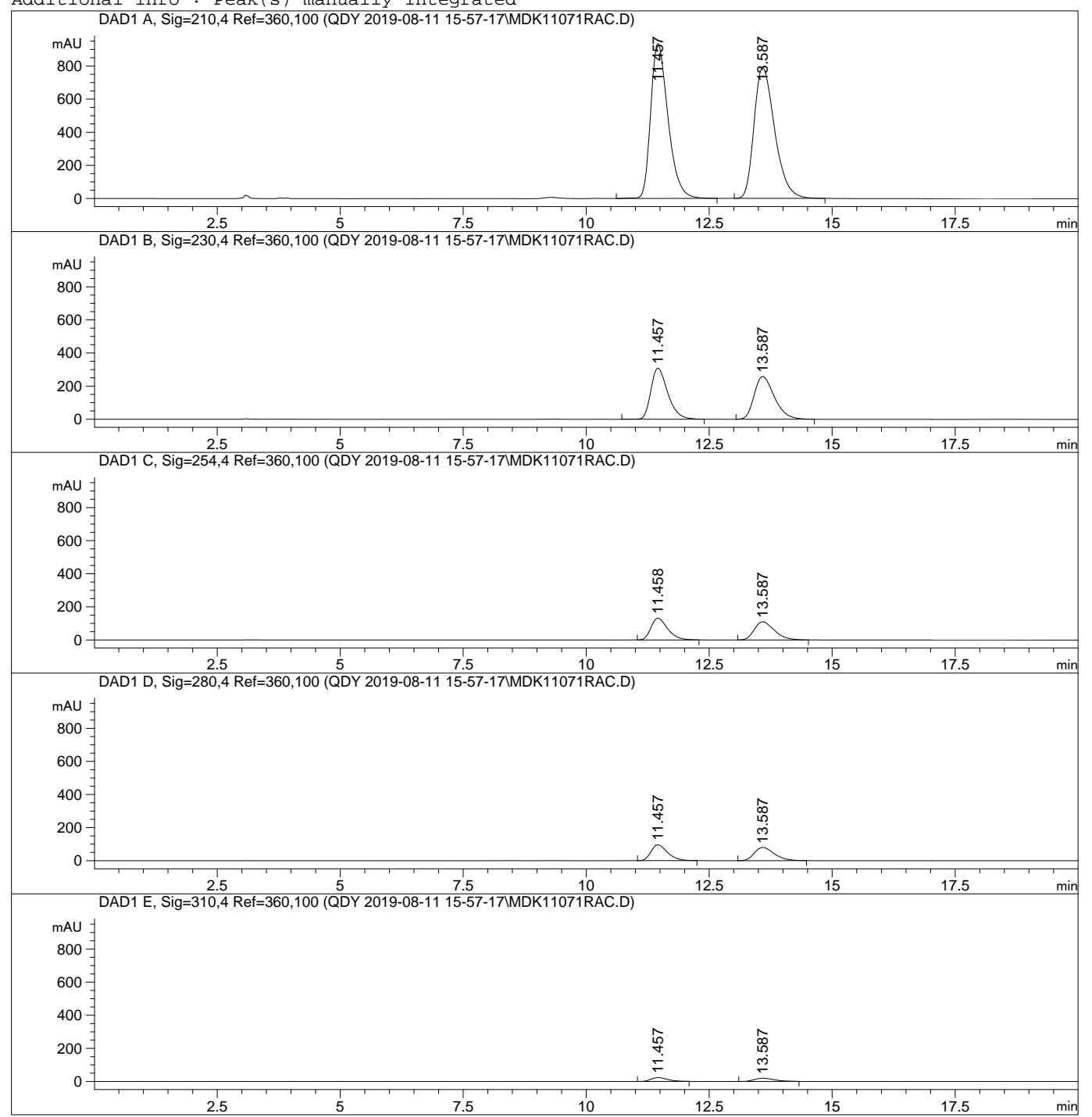

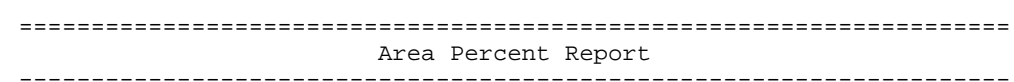

Sorted By
Multiplier

\section{Signal}

1.0000

Use Multiplier \& Dilution Factor with ISTDs

Signal 1: DAD1 A, Sig=210, 4 Ref $=360,100$

\begin{tabular}{|c|c|c|c|c|c|}
\hline $\begin{array}{l}\text { Peak } \\
\#\end{array}$ & $\begin{array}{l}\text { RetTime Type } \\
\text { [min] }\end{array}$ & $\begin{array}{l}\text { Width } \\
\text { [min] }\end{array}$ & $\begin{array}{c}\text { Area } \\
{\left[\mathrm{mAU} U^{*} \mathrm{~s}\right]}\end{array}$ & $\begin{array}{l}\text { Height } \\
{[\mathrm{mAU}]}\end{array}$ & $\begin{array}{c}\text { Area } \\
\%\end{array}$ \\
\hline 1 & $11.457 \mathrm{BB}$ & $\begin{array}{l}0.3784 \\
0.4520\end{array}$ & 2. $29675 \mathrm{e} 4$ & 935.65527 & 49.8972 \\
\hline
\end{tabular}

Totals :

4.60296e4 1726.98547

Signal 2: DAD1 B, Sig=230, 4 Ref $=360,100$

\begin{tabular}{|c|c|c|c|c|c|}
\hline $\begin{array}{c}\text { Peak } \\
\#\end{array}$ & $\begin{array}{l}\text { RetTime Type } \\
\text { [min] }\end{array}$ & $\begin{array}{l}\text { Width } \\
\text { [min] }\end{array}$ & $\begin{array}{c}\text { Area } \\
{\left[\mathrm{mAU} U^{*} \mathrm{~s}\right]}\end{array}$ & $\begin{array}{l}\text { Height } \\
{[\mathrm{mAU}]}\end{array}$ & $\begin{array}{c}\text { Area } \\
\%\end{array}$ \\
\hline 1 & $11.457 \mathrm{BB}$ & 0.3643 & 7374.27100 & $\begin{array}{l}309.13165 \\
258.71088\end{array}$ & $\begin{array}{l}49.9564 \\
58.9436\end{array}$ \\
\hline
\end{tabular}

Totals :

$1.47614 \mathrm{e} 4 \quad 567.84253$

Signal 3: DAD1 C, Sig $=254,4$ Ref $=360,100$

\begin{tabular}{|c|c|c|c|c|c|}
\hline $\begin{array}{c}\text { Peak } \\
\#\end{array}$ & $\begin{array}{l}\text { RetTime Type } \\
\text { [min] }\end{array}$ & $\begin{array}{l}\text { Width } \\
\text { [min] }\end{array}$ & $\begin{array}{c}\text { Area } \\
{\left[\mathrm{mAU}^{\star} \mathrm{s}\right]}\end{array}$ & $\begin{array}{l}\text { Height } \\
{[\mathrm{mAU}]}\end{array}$ & $\begin{array}{c}\text { Area } \\
\%\end{array}$ \\
\hline 1 & $11.458 \mathrm{BB}$ & 0.3626 & 3113.73535 & 131.31824 & 49.8359 \\
\hline 2 & $13.587 \mathrm{BB}$ & 0.4428 & 3134.23901 & 109.88017 & 50.1641 \\
\hline
\end{tabular}

Totals :

$6247.97437 \quad 241.19840$

Signal 4: DAD1 D, Sig=280, 4 Ref $=360,100$

\begin{tabular}{|c|c|c|c|c|c|c|}
\hline $\begin{array}{l}\text { eak } \\
\#\end{array}$ & $\begin{array}{c}\text { RetTime } \\
{[\text { min }]}\end{array}$ & & $\begin{array}{l}\text { Width } \\
\text { [min] }\end{array}$ & $\begin{array}{c}\text { Area } \\
{\left[\mathrm{mAU} U^{*} \mathrm{~s}\right]}\end{array}$ & $\begin{array}{l}\text { Height } \\
{[\mathrm{mAU}]}\end{array}$ & $\begin{array}{c}\text { Area } \\
\%\end{array}$ \\
\hline & & & 4378 & $\begin{array}{l}2261.45923 \\
2276.08325\end{array}$ & $\begin{array}{l}95.67754 \\
80.03597\end{array}$ & $\begin{array}{l}49.8389 \\
50.1611\end{array}$ \\
\hline
\end{tabular}

Totals :

$4537.54248 \quad 175.71352$

Signal 5: DAD1 E, Sig=310, 4 Ref $=360,100$

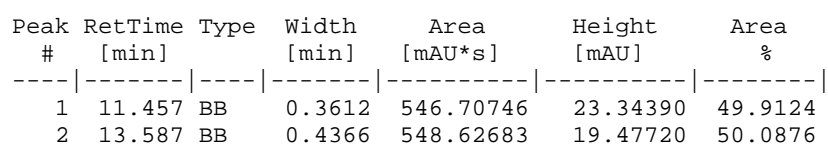

Totals :

$1095.33429 \quad 42.82110$

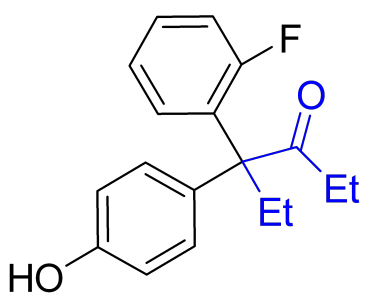

Racemic 


\section{Data File C: \CHEM32\1\DATA\QDY 2019-08-11 15-57-17\MDK11070.D}

Sample Name
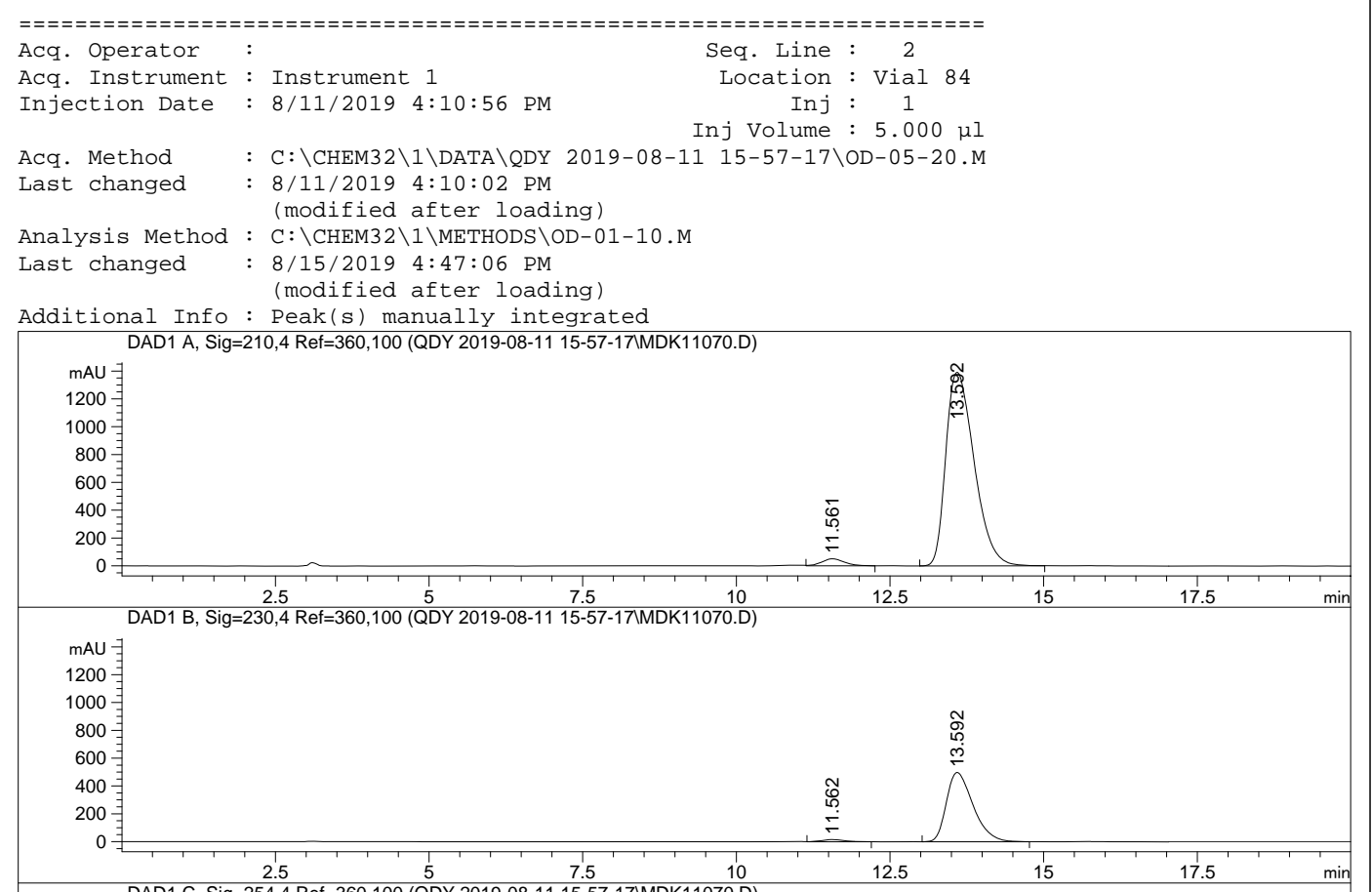

D1 C, Sig=254, 4 Ref=360, 100 (QDY 2019-08-11 15-57-17MDDK11070.D)
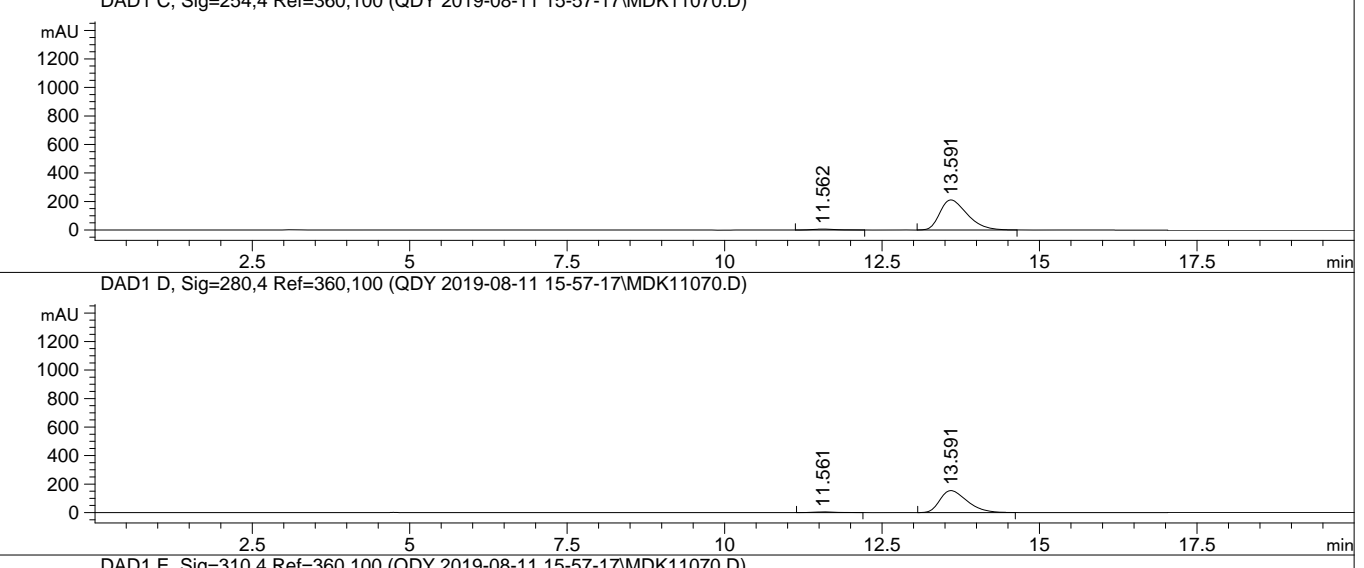

mAU 7

200

800 犊

400 韦

200

$0=\frac{1,1}{1}, 1$ 


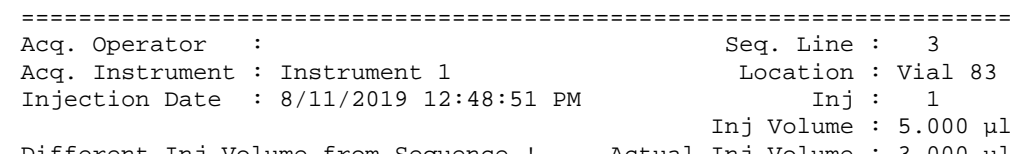

Different Inj volume from Sequence! Actual Inj Volume : $5.000 \mu \mathrm{I}$

Acq. Method : C: \CHEM32\1\DATA\QDY 2019-08-11 12-14-47\IC-05-20.M

Last changed: 6/25/2018 1:35:20 AM

Analysis Method : C: \CHEM32\1\METHODS\OD-01-10.M

Last changed : 8/15/2019 4:47:06 PM

Additional Info: Peak(s) manually integrated
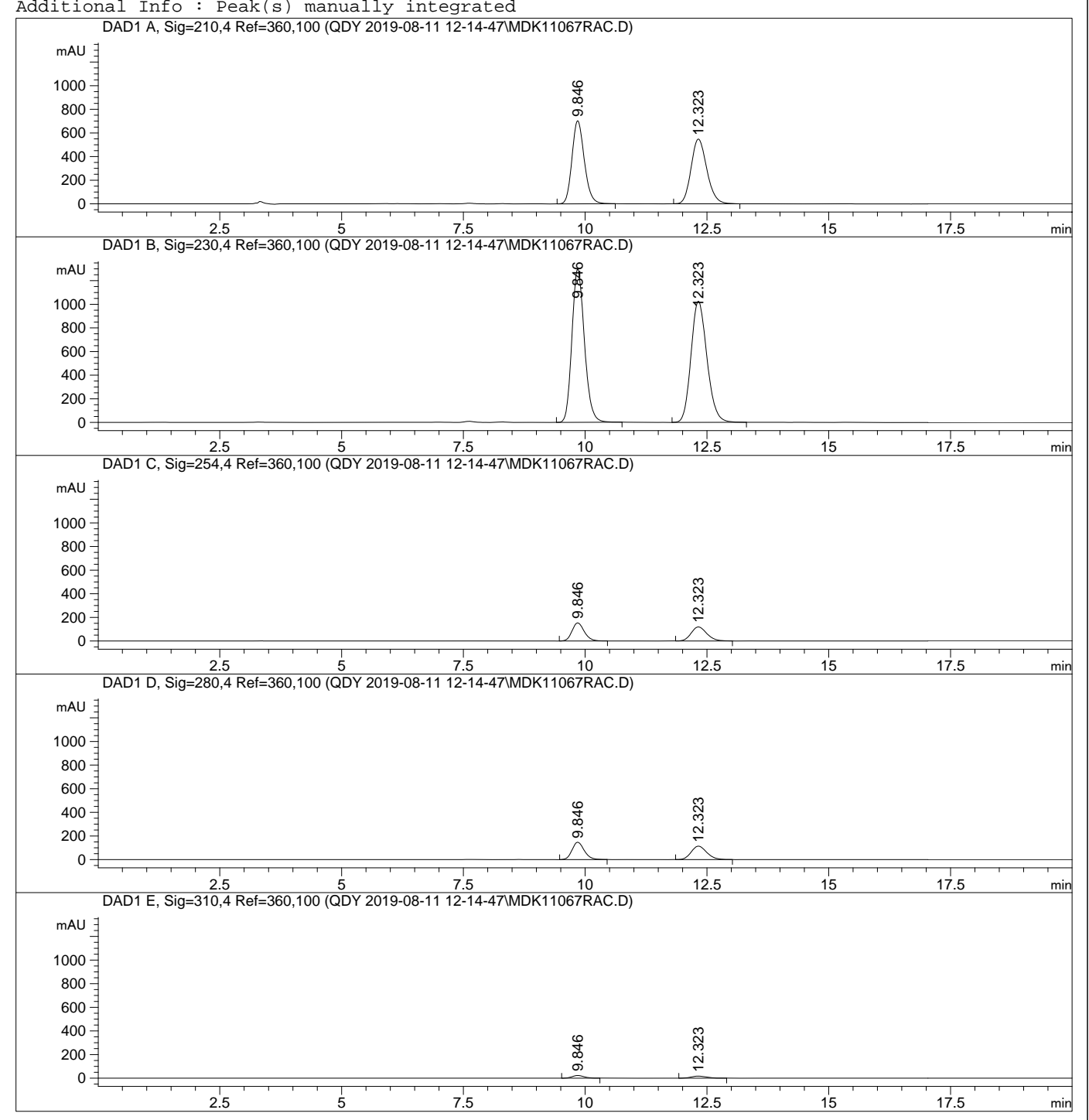

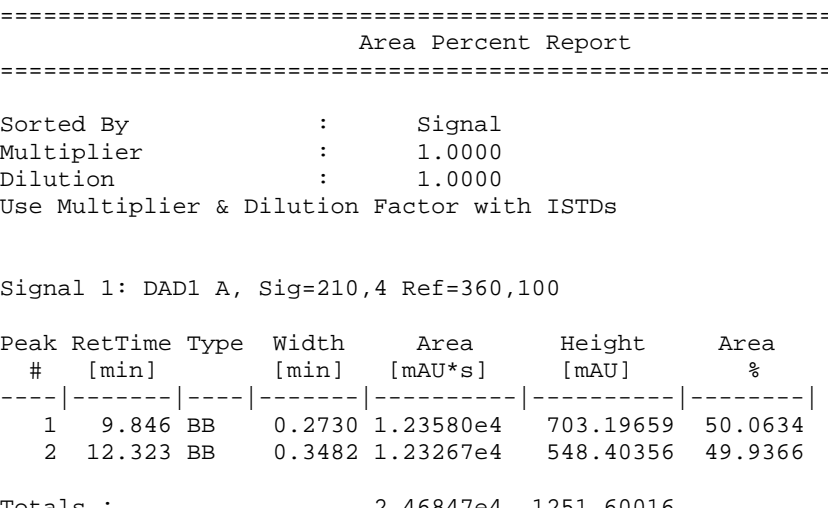

Totals :

$2.46847 \mathrm{e} 4 \quad 1251.60016$

Signal 2: DAD1 B, Sig $=230,4$ Ref $=360,100$

\begin{tabular}{cccccc}
$\begin{array}{c}\text { Peak RetTime Type } \\
\text { \# }\end{array}\left[\begin{array}{c}\text { Width } \\
{[\mathrm{min}]}\end{array}\right.$ & $\begin{array}{c}\text { Area } \\
{[\mathrm{mAU}}\end{array}$ & $\begin{array}{l}\text { Height } \\
{[\mathrm{mAU}]}\end{array}$ & $\begin{array}{c}\text { Area } \\
\%\end{array}$ \\
\hdashline-1 & $9.846 \mathrm{BB}$ & 0.2765 & $2.31728 \mathrm{e} 4$ & 1296.37817 & 49.8061 \\
\hline 2 & $12.323 \mathrm{BB}$ & 0.3536 & $2.33533 \mathrm{e} 4$ & 1025.98914 & 50.1939
\end{tabular}

Totals :

$4.65261 \mathrm{e} 4 \quad 2322.36731$

Signal 3: DAD1 C, Sig $=254,4$ Ref $=360,100$

\begin{tabular}{|c|c|c|c|c|c|}
\hline $\begin{array}{c}\text { Peak } \\
\#\end{array}$ & $\begin{array}{l}\text { RetTime Type } \\
\text { [min] }\end{array}$ & $\begin{array}{l}\text { Width } \\
\text { [min] }\end{array}$ & $\begin{array}{c}\text { Area } \\
{\left[\mathrm{mAU}^{\star} \mathrm{s}\right]}\end{array}$ & $\begin{array}{l}\text { Height } \\
{[\mathrm{mAU}]}\end{array}$ & $\begin{array}{c}\text { Area } \\
\%\end{array}$ \\
\hline$\frac{1}{2}$ & $\begin{array}{r}9.846 \mathrm{BB} \\
12.323 \mathrm{BB}\end{array}$ & $\begin{array}{l}0.2696 \\
0.3472\end{array}$ & $\begin{array}{l}2673.24609 \\
266.47388\end{array}$ & 153.17670 & 50.0634 \\
\hline
\end{tabular}

Totals :

$5339.71997 \quad 272.26922$

Signal 4: DAD1 D, Sig=280, 4 Ref $=360,100$ $\begin{array}{ccccc}\text { Peak RetTime Type Width } & \text { Area } & \text { Height } & \text { Area } \\ \# \quad[\mathrm{~min}] & {[\mathrm{min}]} & {\left[\mathrm{mAU}^{\star} \mathrm{s}\right]} & {[\mathrm{mAU}]} & \%\end{array}$ 10.846 BB 0.26892554 .46460146 .8471250 .0846 $\begin{array}{lllllllll}12.323 & B B & 0.3465 & 2545.83154 & 113.99933 & 49.0154\end{array}$ Totals : $5100.29614 \quad 260.84645$

Signal 5: DAD1 E, Sig $=310,4$ Ref $=360,100$

Peak RetTime Type width Area [ Heigh [

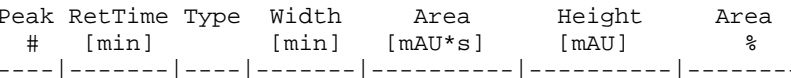
$\begin{array}{lllllll}1 & 9.846 & \text { BB } & 0.2678 & 387.76407 & 22.41559 & 50.0610\end{array}$ $\begin{array}{lrllll}2 & 12.323 \text { BB } & 0.3433 & 386.81940 & 17.40712 & 49.9390\end{array}$<smiles>[101In]</smiles>

$\begin{array}{lll}\text { Totals : } & 774.58347 & 39.82271\end{array}$ 


\section{Data File C: \CHEM32\1\DATA\QDY 2019-08-11 12-14-47\MDK11065.D}

Sample Name
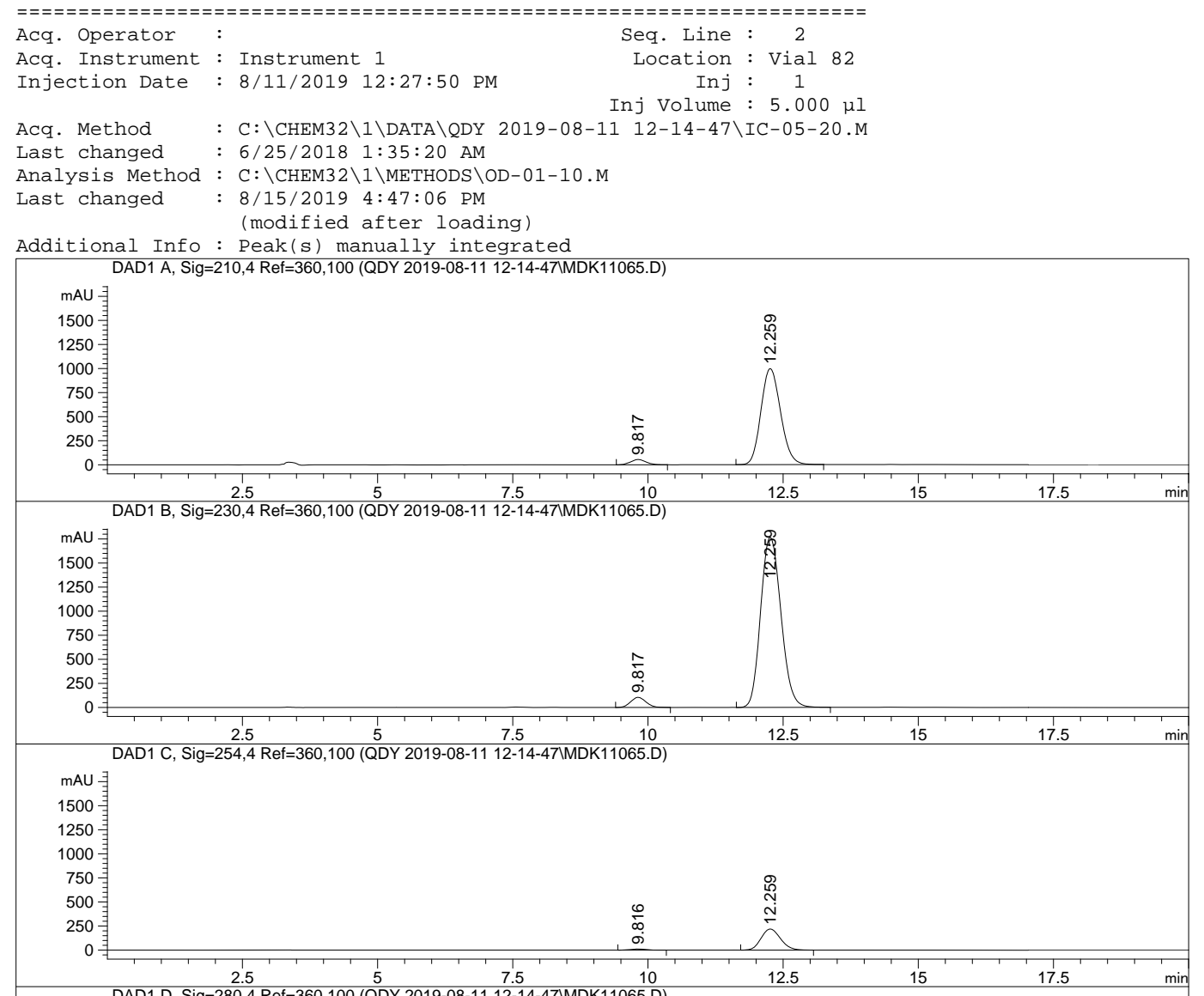

DAD1 D, Sig=280,4 Ref=360,100 (QDY 2019-08-11 $1.52-54-47$ MDK11065.D)

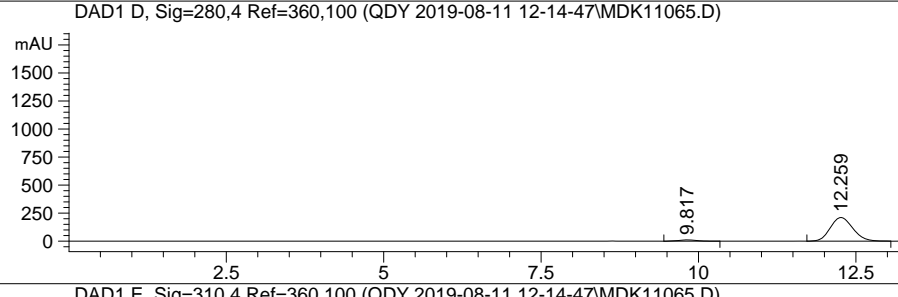

mAU 青

1500 事

1250

500

250 青

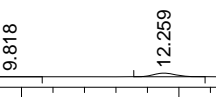

A File C: \CHEM32\1\DATA \QDY 2019-08-11 12-14-47\MDK11065.D

Area Percent Report

\section{Sorted By
Multiplier}

\section{Signal}

1.0000

Use Multiplier \& Dilution Factor with ISTDs

Signal 1: DAD1 A, Sig=210, 4 Ref $=360,100$

\begin{tabular}{cccccc}
$\begin{array}{c}\text { Peak RetTime Type } \\
\text { \# }\end{array}\left[\begin{array}{c}\text { Width } \\
{[\mathrm{min}]}\end{array}\right.$ & $\begin{array}{c}\text { Area } \\
{[\mathrm{mAU}}\end{array}$ & $\begin{array}{c}\text { Height } \\
{[\mathrm{mAU}]}\end{array}$ & $\begin{array}{c}\text { Area } \\
\%\end{array}$ \\
\hdashline-1 & $9.817 \mathrm{BB}$ & 0.3069 & 1079.42041 & 55.00963 & 4.1135 \\
\hline 2 & $12.259 \mathrm{BB}$ & 0.3964 & $2.51614 \mathrm{e} 4$ & 997.03577 & 95.8865
\end{tabular}

Totals :

$2.62409 \mathrm{e} 4 \quad 1052.04540$

Signal 2: DAD1 B, Sig=230, 4 Ref $=360,100$

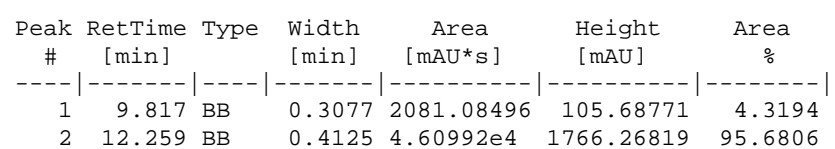

Totals :

$4.81802 \mathrm{e} 4 \quad 1871.95590$

Signal 3: DAD1 C, Sig $=254,4$ Ref $=360,100$

\begin{tabular}{|c|c|c|c|c|c|}
\hline $\begin{array}{c}\text { Peak } \\
\#\end{array}$ & $\begin{array}{l}\text { RetTime Type } \\
\text { [min] }\end{array}$ & $\begin{array}{l}\text { Width } \\
\text { [min] }\end{array}$ & $\begin{array}{c}\text { Area } \\
{\left[\mathrm{mAU} U^{*} \mathrm{~s}\right]}\end{array}$ & $\begin{array}{l}\text { Height } \\
{[\mathrm{mAU}]}\end{array}$ & $\begin{array}{c}\text { Area } \\
\%\end{array}$ \\
\hline 1 & $9.816 \mathrm{BB}$ & 0.3068 & 232.54973 & 11.85949 & 4.068 \\
\hline 2 & $12.259 \mathrm{BB}$ & 0.3895 & 5484.05078 & 219.46227 & 95.9320 \\
\hline
\end{tabular}

Totals :

$5716.60051 \quad 231.32176$

Signal 4: DAD1 D, Sig=280, 4 Ref $=360,100$

\begin{tabular}{|c|c|c|c|c|c|}
\hline $\begin{array}{c}\text { eak } \\
\#\end{array}$ & $\begin{array}{l}\text { RetTime Type } \\
\text { [min] }\end{array}$ & $\begin{array}{l}\text { Width } \\
\text { [min] }\end{array}$ & $\begin{array}{c}\text { Area } \\
{\left[\mathrm{mAU}^{\star} \mathrm{s}\right]}\end{array}$ & $\begin{array}{l}\text { Height } \\
{[\mathrm{mAU}]}\end{array}$ & $\begin{array}{c}\text { Area } \\
\%\end{array}$ \\
\hline & 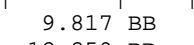 & & . & 10.88199 & \\
\hline
\end{tabular}

Totals :

$5471.65207 \quad 222.12691$

Signal 5: DAD1 E, Sig=310, 4 Ref $=360,100$

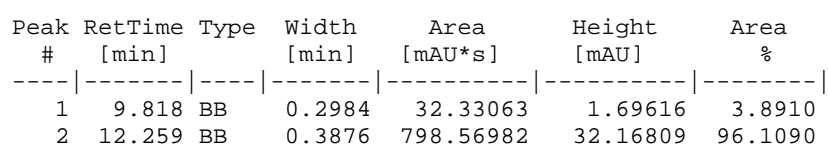

Totals : $\quad 830.90046 \quad 33.86425$

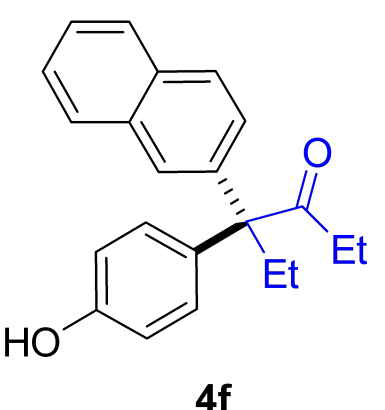

Enantioenriched 


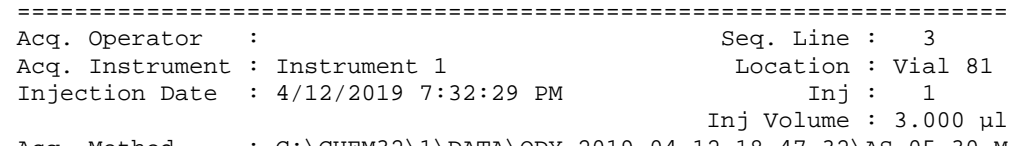

Injection Date Inj volume : $3.000 \mu \mathrm{\mu}$

Acq. Method $:$ C:
Last changed $4 / 12 / 2019$ 7:00:28 PM

Analysis Method : C: \CHEM32\1\METHODS \OD-01-10-0.3.M

Last changed : 5/29/2019 9:25:17 PM

Additional Info: Peak(s) manually integrated

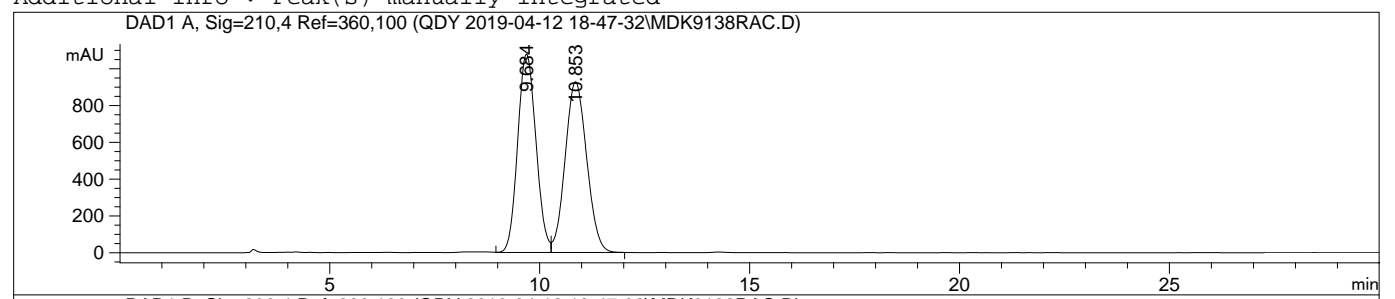

DAD1 B, Sig=230,4 Ref=360,100 (QDY 2019-04-12 18 -47-32IMDK9138RAC.D)

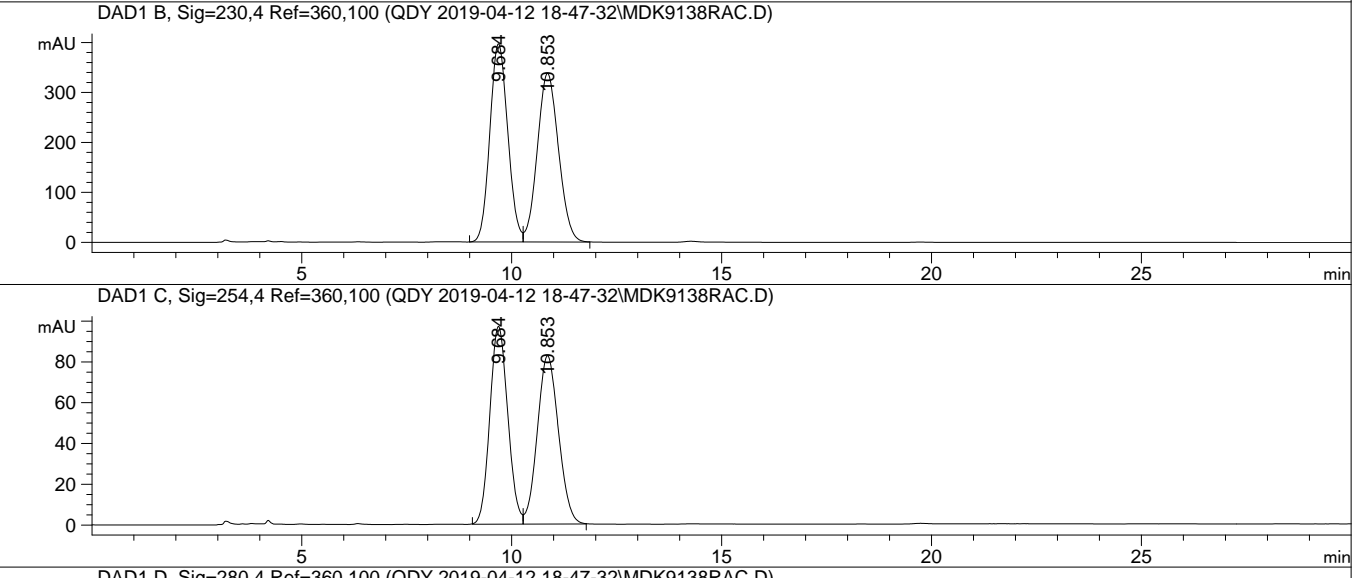

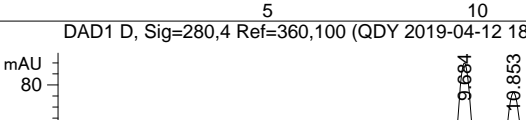

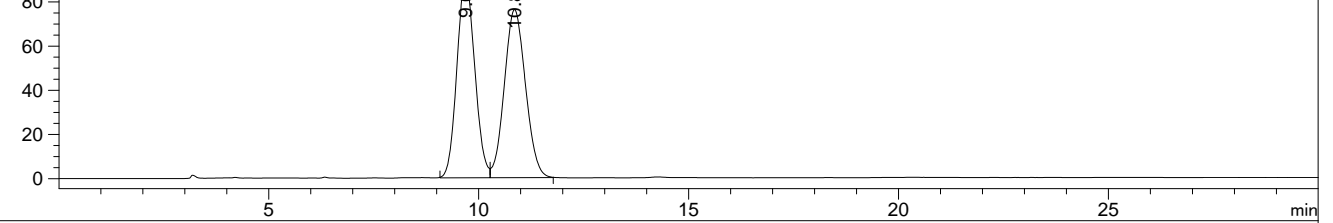

DAD1 E, Sig=310,4 Ref=360,100 (QDY 2019-04-12 18 -47-32IMDK9138RAC.D)

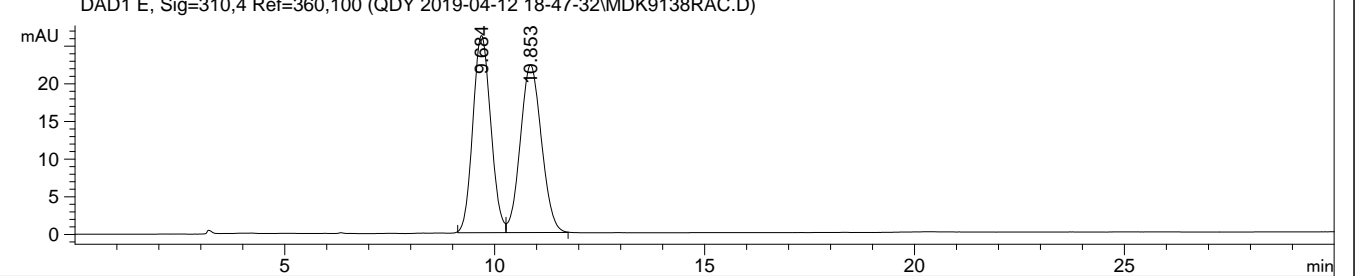

Area Percent Report

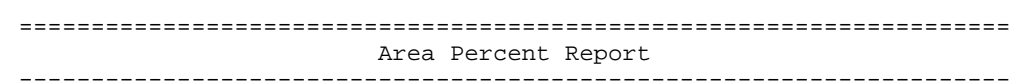

Sorted By

Signal

Multiplier

1.0000

Use Multiplier \& Dilution Factor with ISTDs

Signal 1: DAD1 A, Sig=210, 4 Ref $=360,100$

\begin{tabular}{cccccc}
$\begin{array}{c}\text { Peak RetTime Type } \\
\text { \# }\end{array}\left[\begin{array}{c}\text { Width } \\
{[\mathrm{min}]}\end{array}\right.$ & $\begin{array}{c}\text { Area } \\
{[\mathrm{mAU}}\end{array}$ & $\begin{array}{l}\text { Height } \\
{[\mathrm{mAU}]}\end{array}$ & $\begin{array}{c}\text { Area } \\
\%\end{array}$ \\
\hdashline-1 & $9.684 \mathrm{VV}$ & 0.4775 & $3.26503 \mathrm{e} 4$ & 1077.68079 & 49.6717 \\
\hline 2 & $10.853 \mathrm{VB}$ & 0.5559 & $3.30819 \mathrm{e} 4$ & 928.01483 & 50.3283
\end{tabular}

Totals :

$6.57322 \mathrm{e} 4 \quad 2005.69562$

Signal 2: DAD1 B, Sig=230, 4 Ref $=360,100$

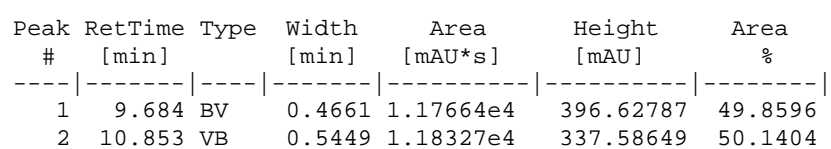

Totals :

$2.35991 \mathrm{e} 4 \quad 734.21436$

Signal 3: DAD1 C, Sig $=254,4$ Ref $=360,100$

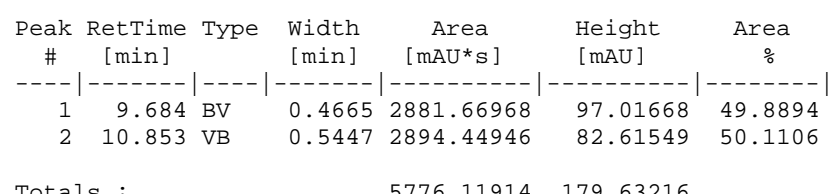

Signal 4: DAD1 D, Sig=280, 4 Ref $=360,100$

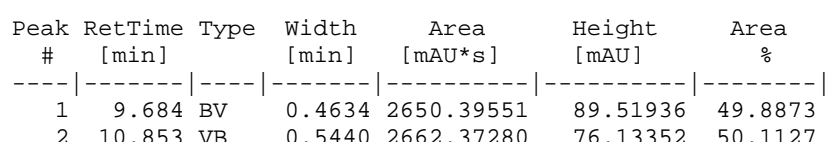

Totals :

$5312.76831 \quad 165.65288$

Signal 5: DAD1 E, Sig=310, 4 Ref $=360,100$

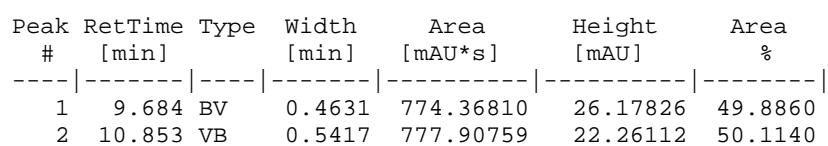

Totals : $\quad 1552.27570 \quad 48.43939$

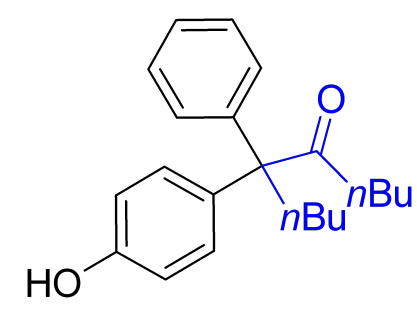

racemic 


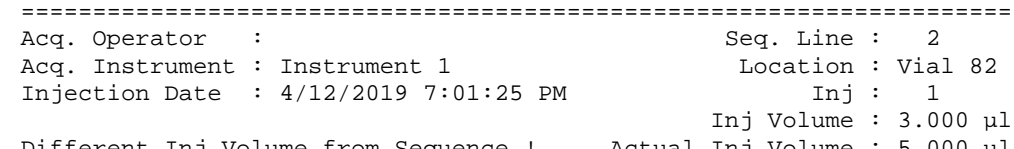

Different Inj Volume from Sequence ! Actual Inj Volume : $3.000 \mu \mathrm{\mu}$

Acq. Method : C: \CHEM32\1\DATA\QDY 2019-04-12 18-47-32\AS-05-30.M

Last changed : 4/12/2019 7:00:28 PM

Analysis Method : C: \CHEM32\1\METHODS\OD-01-10-0.3.M

Last changed : 5/29/2019 9:25:17 PM

$\frac{\text { Additional Info : Peak (s) manually integrated }}{\text { DAD1 A, Sig=210,4 Ref }=360,100 \text { (QDY 2019-04-12 18-47-321MDK9165-D) }}$
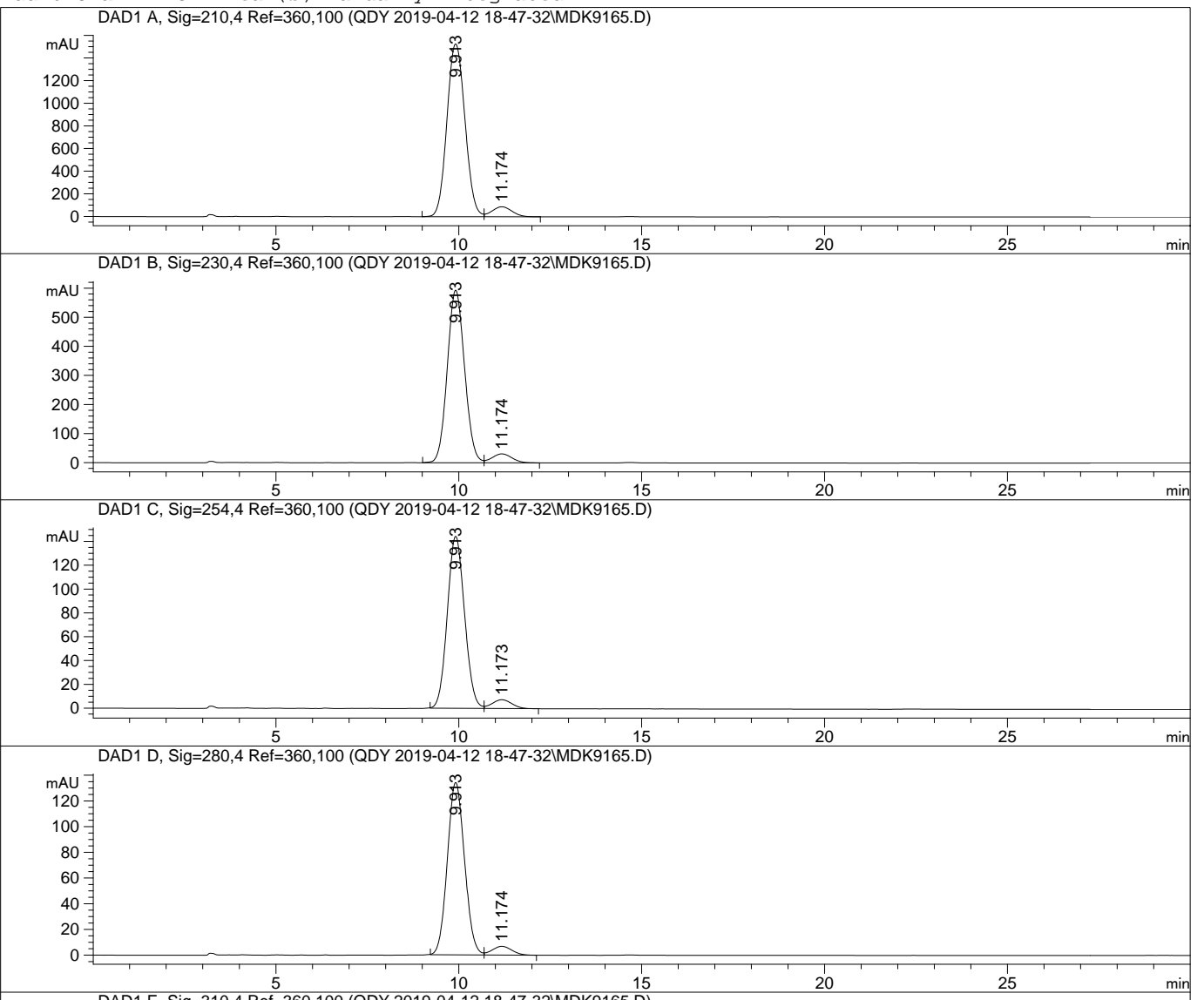

DAD1 E, Sig=310,4 Ref=360,100 (QDY 2019-04-12 18 -47-321MDK9165 D)

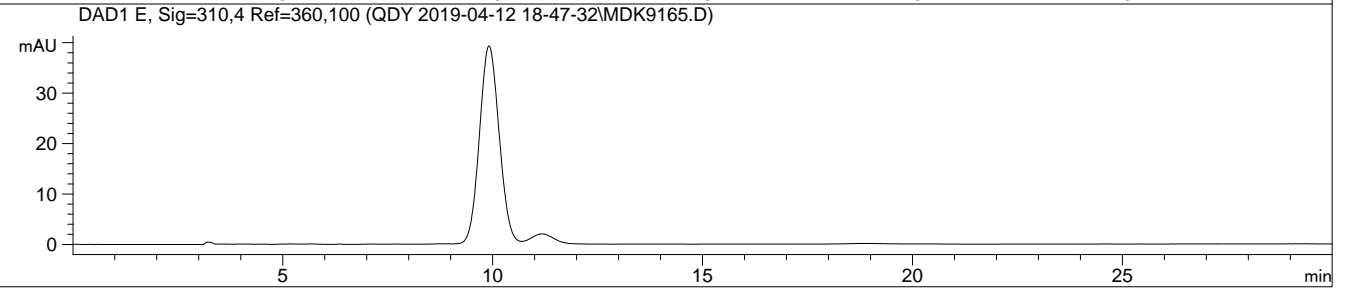

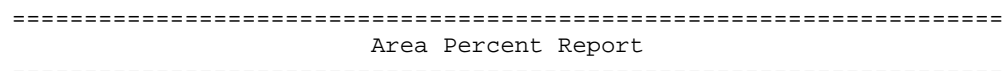

Sorted By

\section{Signal}

Dilution

1.0000

Use Multiplier \& Dilution Factor with ISTDs

Signal 1: DAD1 A, Sig=210, 4 Ref $=360,100$

\begin{tabular}{cccccc}
$\begin{array}{c}\text { Peak RetTime Type } \\
\text { \# }\end{array}\left[\begin{array}{c}\text { Width } \\
{[\mathrm{min}]}\end{array}\right.$ & $\begin{array}{c}\text { Area } \\
{[\mathrm{mAU}}\end{array}$ & $\begin{array}{l}\text { Height } \\
{[\mathrm{mAU}]}\end{array}$ & $\begin{array}{c}\text { Area } \\
\%\end{array}$ \\
\hdashline-1 & $9.913 \mathrm{BV}$ & 0.5414 & $5.22561 \mathrm{e} 4$ & 1526.45264 & 93.9807 \\
\hline 2 & $11.174 \mathrm{VB}$ & 0.5921 & 3346.92676 & 88.32896 & 6.0193
\end{tabular}

Totals :

$5.56030 \mathrm{e} 4 \quad 1614.78160$

Signal 2: DAD1 B, Sig $=230,4$ Ref $=360,100$

\begin{tabular}{cccccc}
$\begin{array}{c}\text { Peak RetTime Type } \\
\text { \# }\end{array}\left[\begin{array}{c}\text { Width } \\
{[\mathrm{min}]}\end{array}\right.$ & $\begin{array}{c}\text { Area } \\
{[\mathrm{mAU}}\end{array}$ & $\begin{array}{l}\text { Height } \\
{[\mathrm{mAU}]}\end{array}$ & $\begin{array}{c}\text { Area } \\
\%\end{array}$ \\
\hdashline-1 & $9.913 \mathrm{BV}$ & 0.5107 & $1.94715 \mathrm{e} 4$ & 593.66528 & 94.3866 \\
2 & $11.174 \mathrm{VB}$ & 0.5920 & 1158.01965 & 30.70811 & 5.6134
\end{tabular}

Totals :

$2.06295 \mathrm{e} 4 \quad 624.37340$

Signal 3: DAD1 C, Sig $=254,4$ Ref $=360,100$

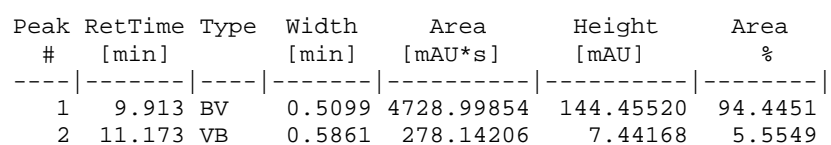

Totals :

$5007.14059 \quad 151.89688$

Signal 4: DAD1 D, Sig=280, 4 Ref $=360,100$

Peak RetTime Type Width Area Height Area

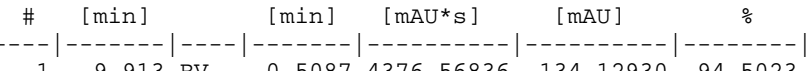

$\begin{array}{llllll}9.913 & \text { BV } & 0.5087 & 4376.56836 & 134.12930 & 94.5023\end{array}$

Totals :

$4631.17429 \quad 140.93648$

Signal 5: DAD1 E, Sig=310, 4 Ref $=360,100$

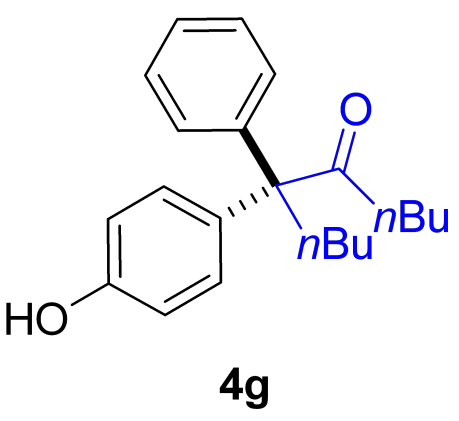

Enantioenriched

$* * *$ End of Report *** 

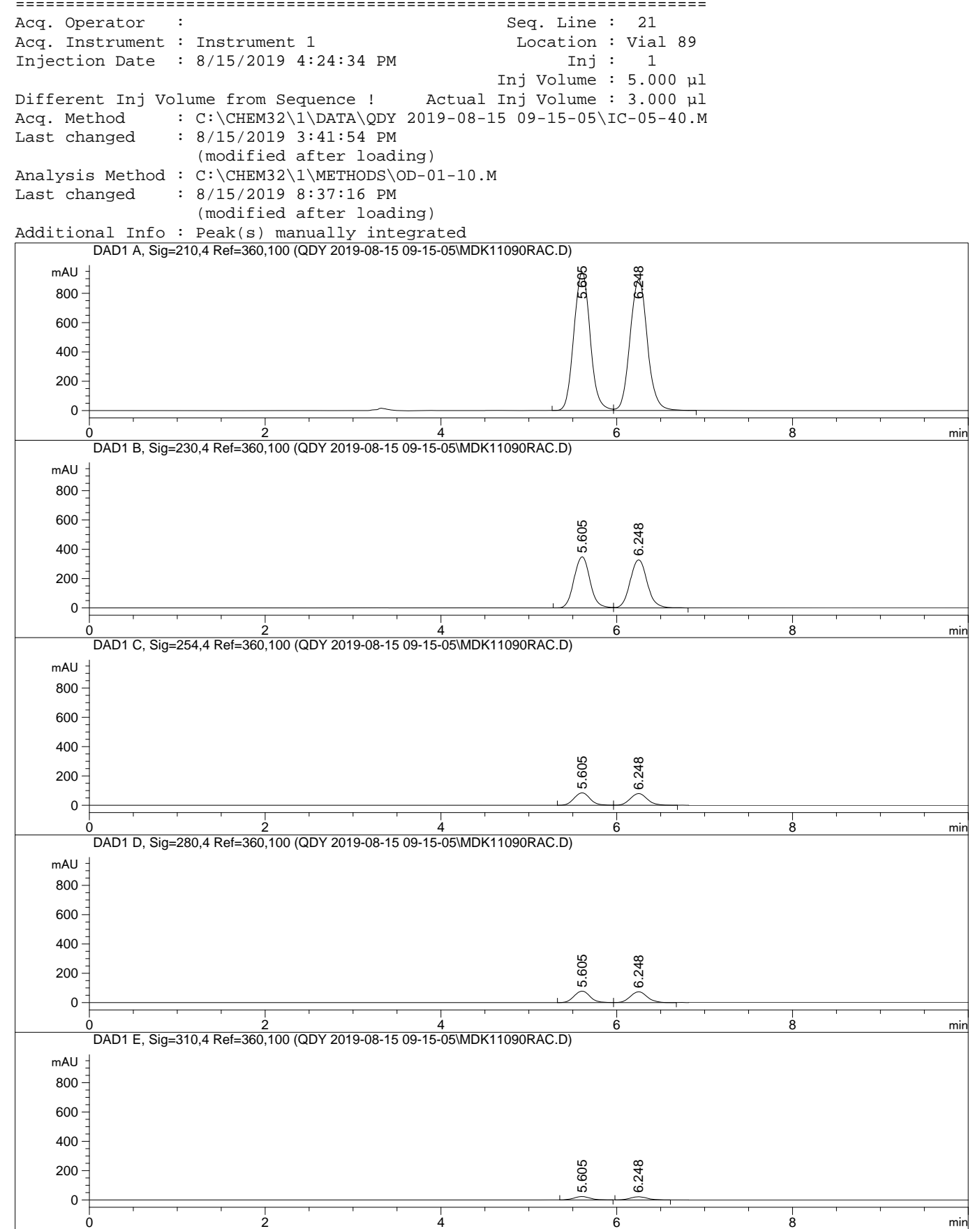

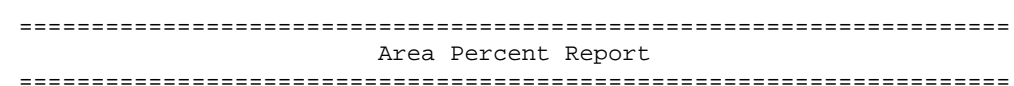

Sorted By
Multiplier

\section{Signal}

1.0000

Use Multiplier \& Dilution Factor with ISTDs

Signal 1: DAD1 A, Sig=210, 4 Ref $=360,100$

\begin{tabular}{cccccc}
$\begin{array}{c}\text { Peak RetTime Type } \\
\#\end{array}\left[\begin{array}{c}\text { Width } \\
{[\mathrm{min}]}\end{array}\right.$ & $\begin{array}{c}\text { Area } \\
{[\mathrm{mAU}}\end{array}$ & $\begin{array}{l}\text { Height } \\
{[\mathrm{mAU}]}\end{array}$ & $\begin{array}{c}\text { Area } \\
\%\end{array}$ \\
\hdashline-1 & $5.605 \mathrm{BV}$ & 0.2037 & $1.21076 \mathrm{e}$ & 943.54852 & 49.7825 \\
\hline 2 & $6.248 \mathrm{VB}$ & 0.2155 & $1.22135 \mathrm{e} 4$ & 893.37189 & 50.2175
\end{tabular}

Totals :

$2.43211 \mathrm{e} 4 \quad 1836.92041$

Signal 2: DAD1 B, Sig=230, 4 Ref $=360,100$

\begin{tabular}{cccccc}
$\begin{array}{c}\text { Peak RetTime Type } \\
\text { \# }\end{array}\left[\begin{array}{c}\text { Width } \\
{[\mathrm{min}]}\end{array}\right.$ & $\begin{array}{c}\text { Area } \\
{[\mathrm{mAU}}\end{array}$ & $\begin{array}{l}\text { Height } \\
{[\mathrm{mAU}]}\end{array}$ & $\begin{array}{c}\text { Area } \\
\%\end{array}$ \\
\hdashline 1 & $5.605 \mathrm{BV}$ & 0.1991 & 4392.48486 & 348.39810 & 49.8930 \\
2 & 6.248 VB & 0.2107 & 4411.32520 & 328.36343 & 50.1070
\end{tabular}

Totals :

$8803.81006 \quad 676.76154$

Signal 3: DAD1 C, Sig $=254,4$ Ref $=360,100$

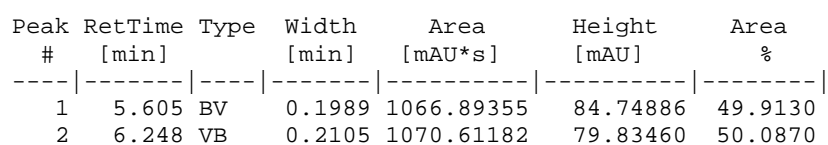

Totals :

$2137.50537 \quad 164.58347$

Signal 4: DAD1 D, Sig=280, 4 Ref $=360,100$

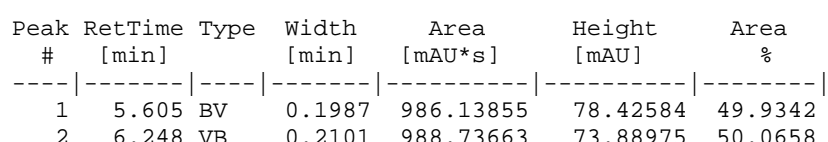

Totals :

$1974.87518 \quad 152.31559$

Signal 5: DAD1 E, Sig $=310,4$ Ref $=360,100$

Peak RetTime Type Width Area Height Area

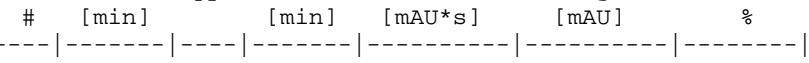

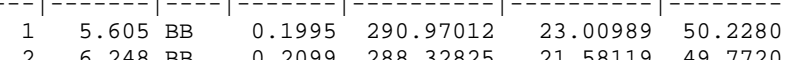
Totals : $\quad 579.29837 \quad 44.59108$

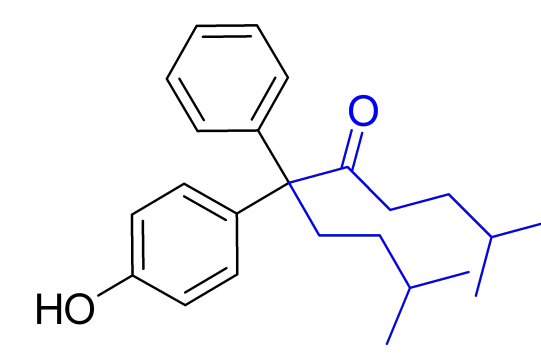

Racemic 


\section{Data File C: \CHEM32\1\DATA\QDY 2019-08-15 09-15-05\MDK11089.D}

Sample Name:
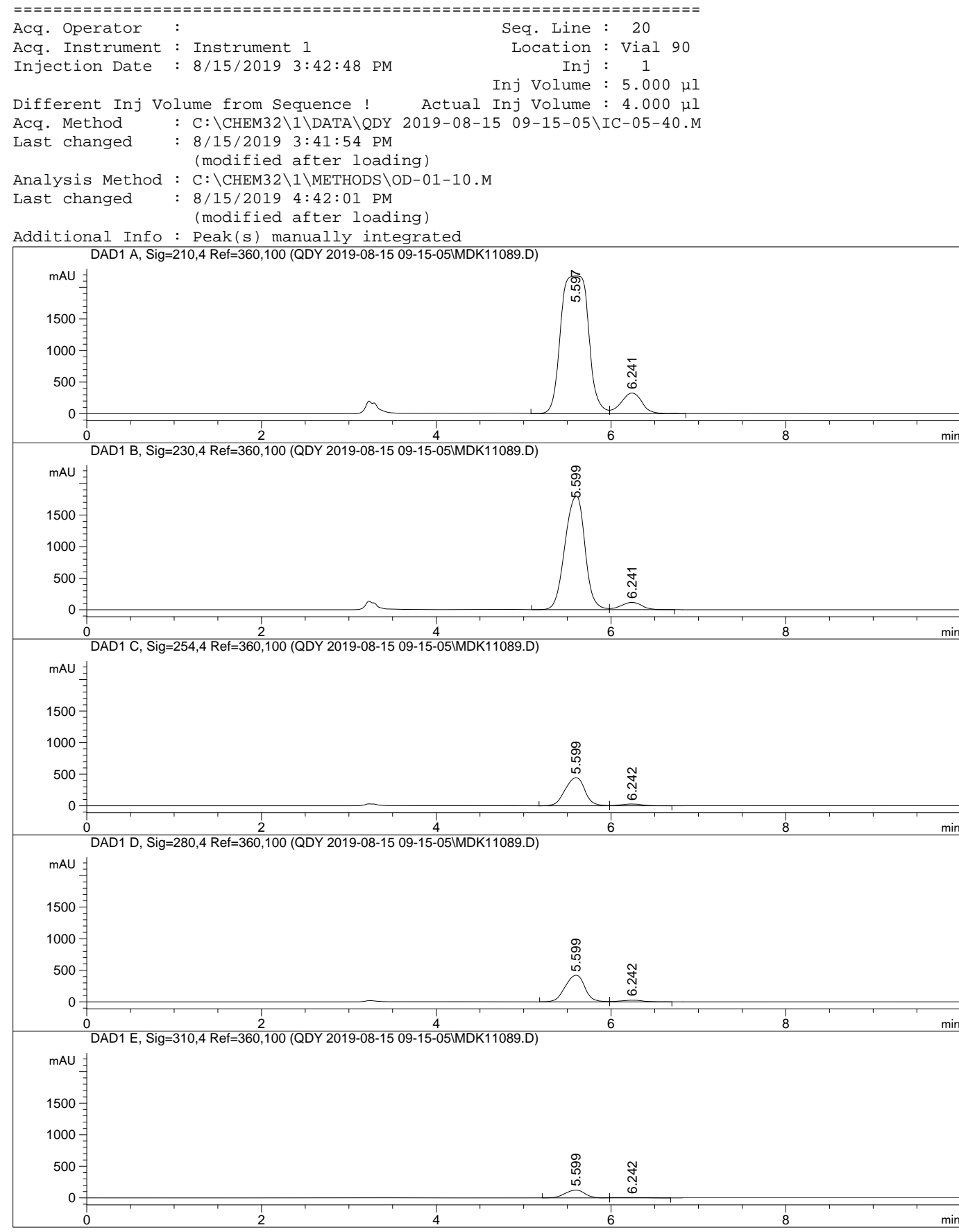

Data File C: \CHEM32\1\DATA\QDY 2019-08-15 09-15-05\MDK11089.D Sample Name

Area Percent Report

$\begin{array}{lcr}\text { Sorted By } & : & \text { Signal } \\ \text { Multiplier } & \vdots & 1.0000 \\ \text { Dilution } & \vdots & 1.0000 \\ \text { Use Multiplier \& Dilution } & \text { Factor with }\end{array}$

Signal 1: DAD1 A, Sig=210, 4 Ref $=360,100$

\begin{tabular}{cccccc}
$\begin{array}{c}\text { Peak RetTime Type } \\
\#\end{array}\left[\begin{array}{c}\text { Width } \\
{[\mathrm{min}]}\end{array}\right.$ & $\begin{array}{c}\text { Area } \\
{[\mathrm{mAU}}\end{array}$ & $\begin{array}{c}\text { Height } \\
{[\mathrm{mAU}]}\end{array}$ & $\begin{array}{c}\text { Area } \\
\%\end{array}$ \\
\hdashline-1 & $5.597 \mathrm{VV}$ & 0.3545 & $4.79588 \mathrm{e} 4$ & 2181.65356 & 89.7470 \\
\hline 2 & $6.241 \mathrm{VB}$ & 0.2637 & 5478.96338 & 326.69977 & 10.2530
\end{tabular}

Totals :

$5.34378 \mathrm{e} 4 \quad 2508.35333$

Signal 2: DAD1 B, Sig=230, 4 Ref $=360,100$

\begin{tabular}{cccccc}
$\begin{array}{c}\text { Peak RetTime Type } \\
\#\end{array}\left[\begin{array}{c}\text { Width } \\
{[\mathrm{min}]}\end{array}\right.$ & $\begin{array}{c}\text { Area } \\
{[\mathrm{mAU}}\end{array}$ & $\begin{array}{l}\text { Height } \\
{[\mathrm{mAU}]}\end{array}$ & $\begin{array}{c}\text { Area } \\
\%\end{array}$ \\
\hdashline-1 & $5.599 \mathrm{VV}$ & 0.2550 & $2.85504 \mathrm{e} 4$ & 1800.67358 & 93.7569 \\
2 & $6.241 \mathrm{VB}$ & 0.2601 & 1901.11230 & 115.45100 & 6.2431
\end{tabular}

Totals :

$3.04515 \mathrm{e} 4 \quad 1916.12458$

Signal 3: DAD1 C, Sig=254, 4 Ref $=360,100$

$$
\begin{aligned}
& \text { Peak RetTime Type Width Area Height Area }
\end{aligned}
$$

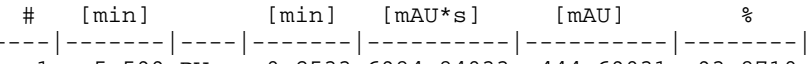

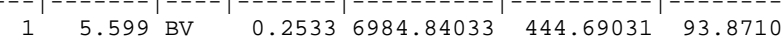

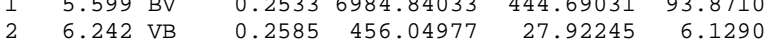$$
\text { Totals : } \quad 7440.89011 \quad 472.61276
$$

Signal 4: DAD1 D, Sig $=280,4$ Ref $=360,100$

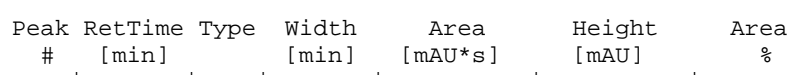

\# [min] $[$ [min] [mAU*s] [mAU] 1 \%

$\begin{array}{llllll}1 & 5.599 \text { BV } & 0.2500 & 6561.51660 & 420.55557 & 93.9538\end{array}$

Totals :

$6983.76965 \quad 446.36930$

$\mathrm{HO}$

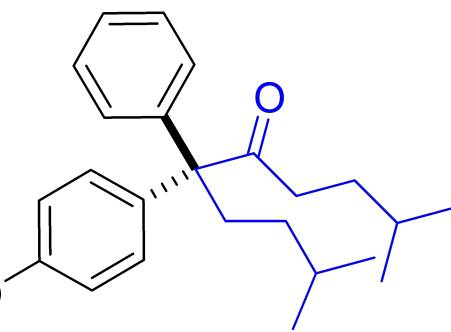

$4 \mathrm{~h}$

Signal 5: DAD1 E, Sig $=310,4$ Ref $=360,100$

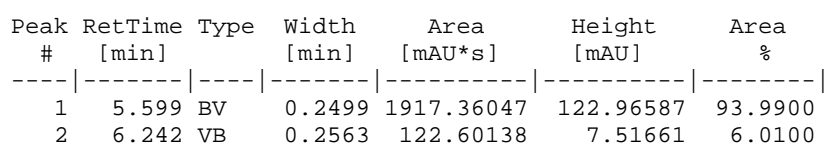

$\begin{array}{lll}\text { Totals : } & 2039.96185 & 130.48249\end{array}$

\section{Enantioenriched}




\section{Data File C: \CHEM32\1\DATA\QDY 2019-05-01 09-18-04\MDK10020-RAC.D}

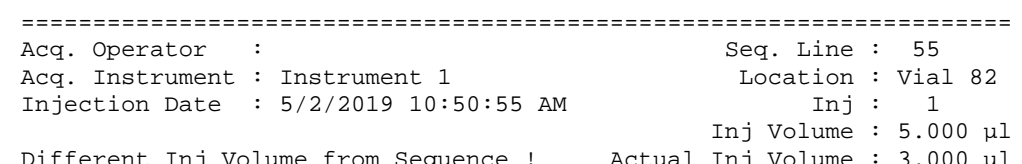

Injection Date $\quad$ Inj Volume $: 5.000 \mu \mathrm{\mu l}$ Acq. Method : C:ICHEM32\1 \DATA\QDY 2019-05-01 09-18-04\AS-10-40.M Last changed : 5/2/2019 10:50: 03 AM

Analysis Method: C: \CHEM32\1\METHODS\OD-01-10-0.3.M

Last changed : 5/29/2019 9:25:17 PM

Additional Info: : Peak(s) manually integrated

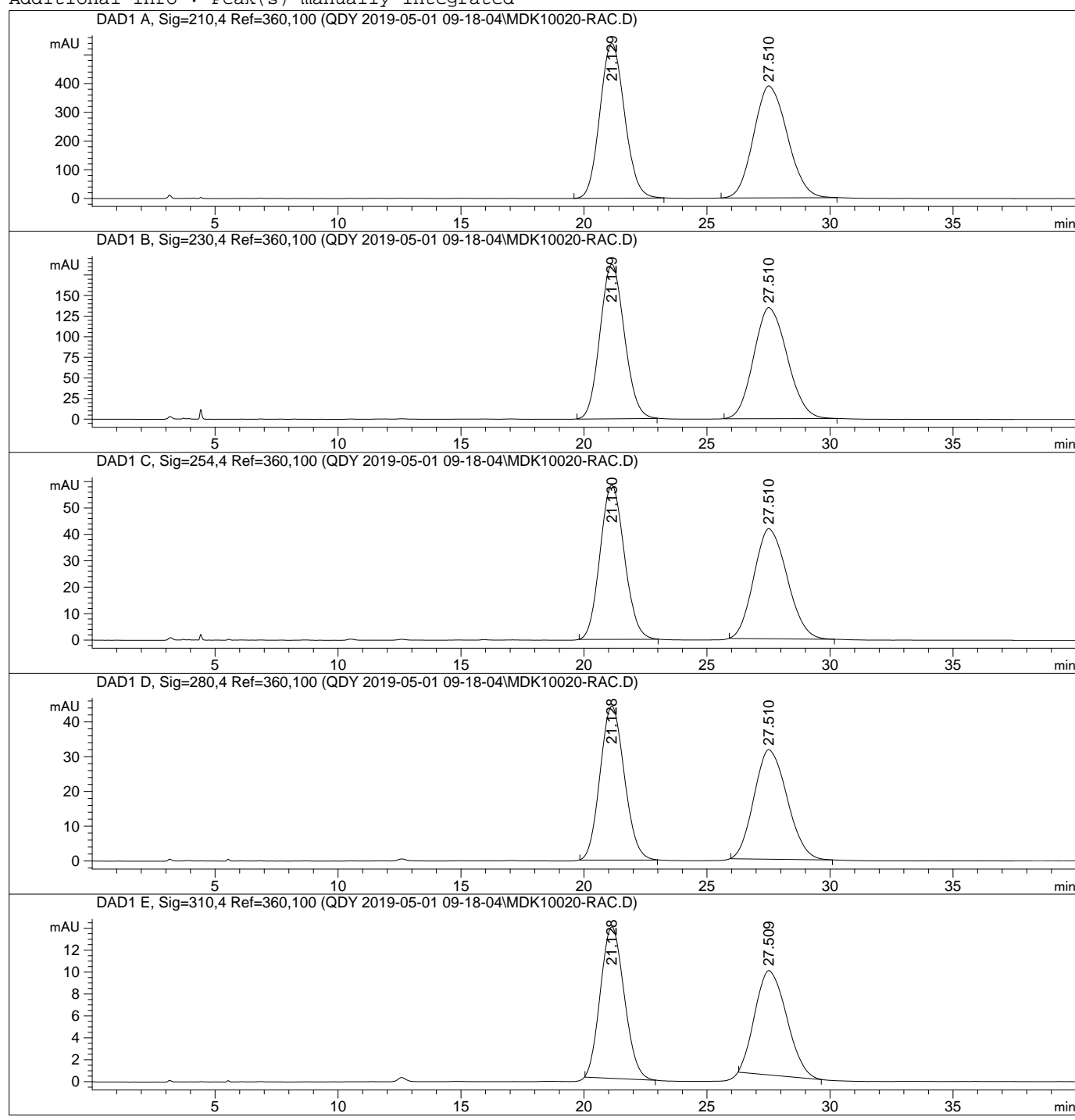

Data File C:\CHEM32\1\DATA\QDY 2019-05-01 09-18-04\MDK10020-RAC.D Sample Name

Area Percent Report

$\begin{array}{lcc}\text { Sorted By } & : & \text { Signal } \\ \text { Multiplier } & \vdots & 1.0000 \\ \text { Dilution } & \vdots & 1.0000 \\ \text { Use Multiplier } & \text { D Dilution } & \text { Factor with }\end{array}$

Signal 1: DAD1 A, Sig=210, 4 Ref $=360,100$

\begin{tabular}{cccccc}
$\begin{array}{c}\text { Peak RetTime Type } \\
\#\end{array}\left[\begin{array}{c}\text { Width } \\
{[\mathrm{min}]}\end{array}\right.$ & $\begin{array}{c}\text { Area } \\
{[\mathrm{mAn}]}\end{array}$ & $\begin{array}{l}\text { Height } \\
{[\mathrm{mAU}]}\end{array}$ & $\begin{array}{c}\text { Area } \\
\%\end{array}$ \\
\hdashline-1 & $21.129 \mathrm{BB}$ & 1.0892 & $3.74760 \mathrm{e} 4$ & 538.99854 & 50.1914 \\
\hline 2 & $27.510 \mathrm{BB}$ & 1.5294 & $3.71902 \mathrm{e} 4$ & 389.69217 & 49.8086
\end{tabular}

Totals :

$7.46663 \mathrm{e} 4 \quad 928.69070$

Signal 2: DAD1 B, Sig=230, 4 Ref $=360,100$

\begin{tabular}{|c|c|c|c|c|c|}
\hline $\begin{array}{c}\text { Peak } \\
\#\end{array}$ & $\begin{array}{l}\text { RetTime Type } \\
\text { [min] }\end{array}$ & $\begin{array}{l}\text { Width } \\
\text { [min] }\end{array}$ & $\begin{array}{c}\text { Area } \\
{\left[\mathrm{mAU} U^{*} \mathrm{~s}\right]}\end{array}$ & $\begin{array}{l}\text { Height } \\
{[\mathrm{mAU}]}\end{array}$ & $\begin{array}{c}\text { Area } \\
\%\end{array}$ \\
\hline 1 & $21.129 \mathrm{BB}$ & $\begin{array}{l}1.0790 \\
1.5228\end{array}$ & $\begin{array}{l}1.29112 \mathrm{e} 4 \\
1.97952 \mathrm{e}\end{array}$ & $\begin{array}{l}187.58882 \\
134.87300\end{array}$ & $\begin{array}{l}50.2258 \\
49.7742\end{array}$ \\
\hline
\end{tabular}

Totals :

$2.57064 \mathrm{e} 4 \quad 322.46182$

Signal 3: DAD1 C, Sig $=254,4$ Ref $=360,100$

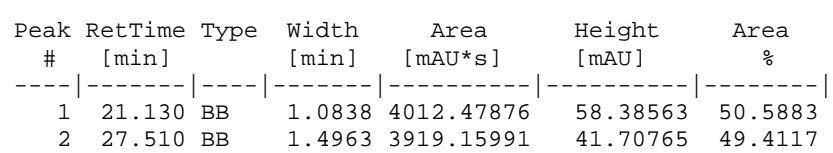

Totals :

$7931.63867 \quad 100.09328$

Signal 4: DAD1 D, Sig $=280,4$ Ref $=360,100$

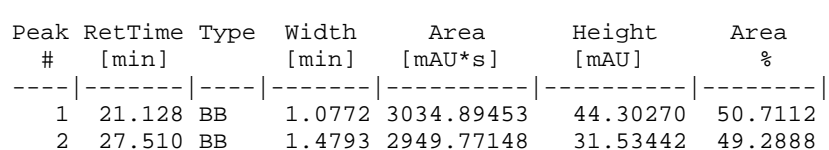

Totals :

$5984.66602 \quad 75.83713$

Signal 5: DAD1 E, Sig $=310,4$ Ref $=360,100$

Peak RetTime Type Width Area Height Area \# [min] $[$ [min] [mAU*s] [mAU] $\%$

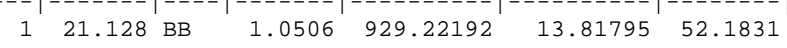

Totals : $\quad 1780.69659 \quad 23.34602$

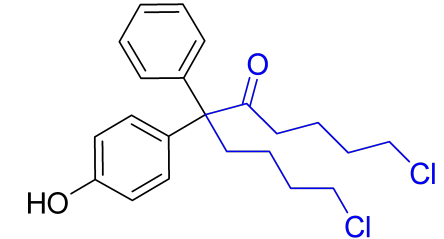

racemic 


\section{Data File C: \CHEM32\1\DATA\QDY 2019-05-01 09-18-04\MDK10019.D}

Sample Name:

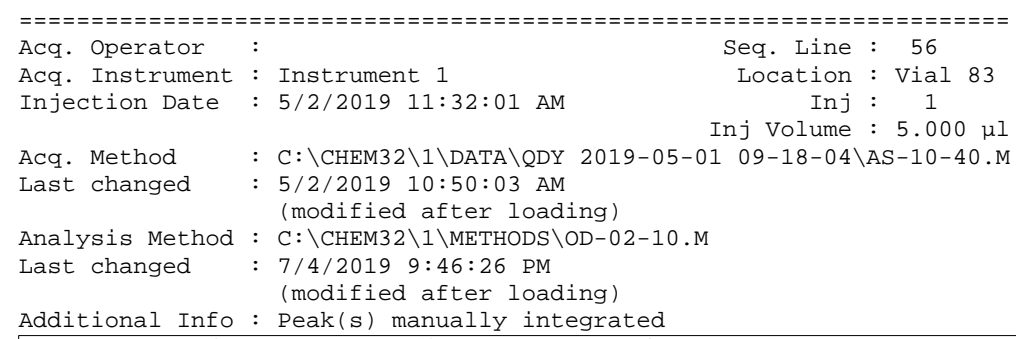

Analysis Method : C: \CHEM32\1\METHODS \OD-02-10.M

Last changed : 7/4/2019 9:46:26 PM

Additional Info: Peak(s) manually integrated
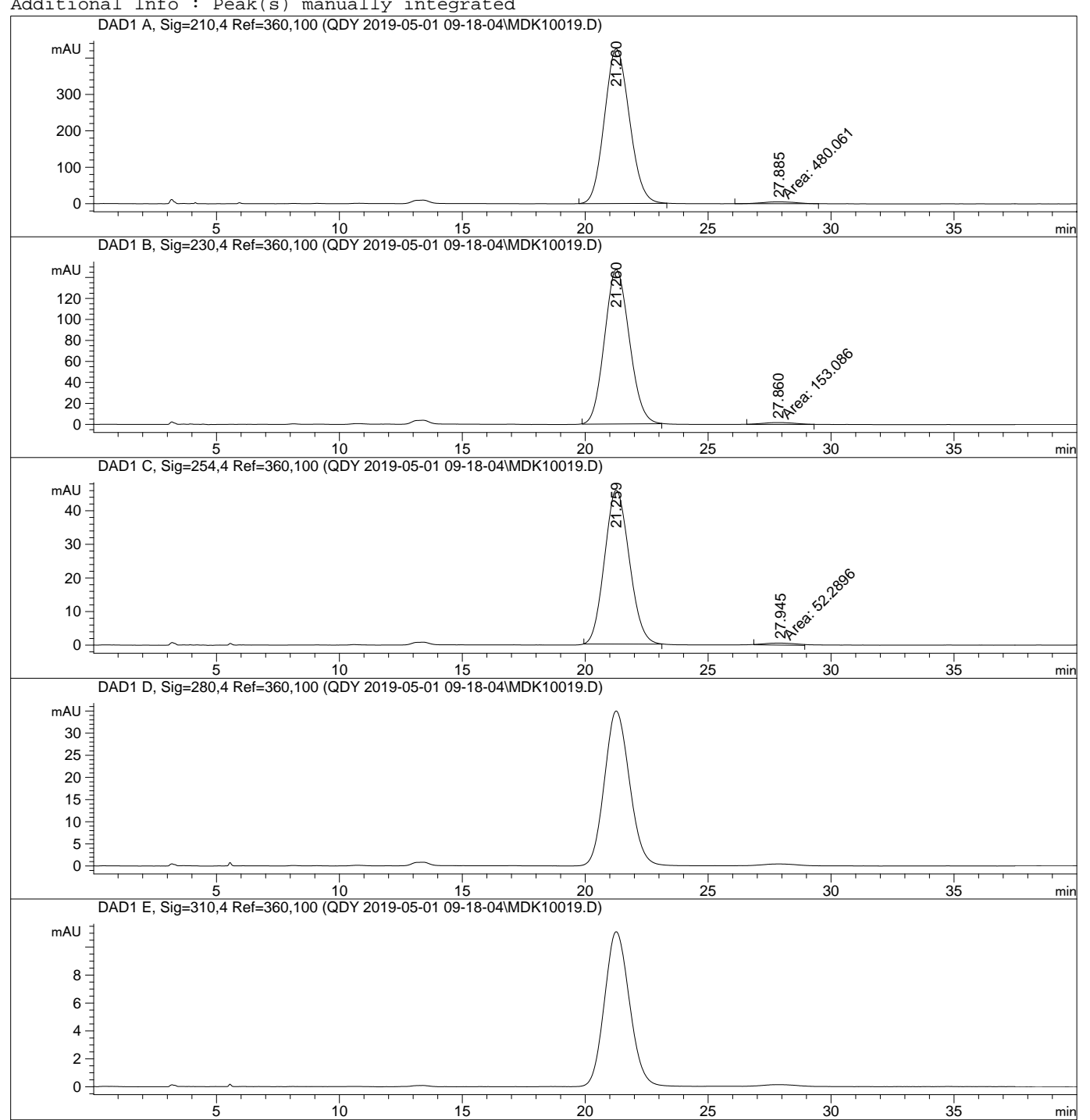

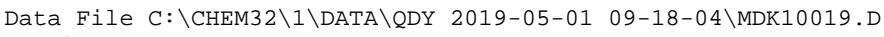
Sample Name:

Area Percent Report

$\begin{array}{lll} & & \\ \text { Sorted By } & : & \text { Signal } \\ \text { Multiplier } & \vdots & 1.0000 \\ \text { Dilution } & \vdots & 1.0000\end{array}$

Use Multiplier \& Dilution Factor with ISTDs

Signal 1: DAD1 A, Sig=210, 4 Ref $=360,100$

\begin{tabular}{|c|c|c|c|c|c|}
\hline $\begin{array}{c}\text { Peak } \\
\#\end{array}$ & $\begin{array}{l}\text { RetTime Type } \\
{[\mathrm{min}]}\end{array}$ & $\begin{array}{l}\text { Width } \\
\text { [min] }\end{array}$ & $\begin{array}{c}\text { Area } \\
{\left[\mathrm{mAU} U^{*} \mathrm{~s}\right]}\end{array}$ & $\begin{array}{l}\text { Height } \\
{[\mathrm{mAU}]}\end{array}$ & $\begin{array}{c}\text { Area } \\
\%\end{array}$ \\
\hline $\begin{array}{l}1 \\
2\end{array}$ & $\begin{array}{l}21.260 \mathrm{BB} \\
27.885 \mathrm{MM}\end{array}$ & $\begin{array}{l}1.0978 \\
1.5654\end{array}$ & $\begin{array}{c}3.00097 \mathrm{e} 4 \\
480.06116\end{array}$ & $\begin{array}{r}425.00214 \\
5.11105\end{array}$ & $\begin{array}{r}98.4255 \\
1.5745\end{array}$ \\
\hline
\end{tabular}

Totals :

3. $04898 \mathrm{e} 4 \quad 430.11319$

Signal 2: DAD1 B, Sig=230, 4 Ref $=360,100$

\begin{tabular}{cccccc}
$\begin{array}{c}\text { Peak RetTime Type } \\
\#\end{array}\left[\begin{array}{c}\text { Width } \\
{[\mathrm{min}]}\end{array}\right.$ & $\begin{array}{c}\text { Area } \\
{[\mathrm{mAU}}\end{array}$ & $\begin{array}{l}\text { Height } \\
{[\mathrm{mAU}]}\end{array}$ & $\begin{array}{c}\text { Area } \\
\%\end{array}$ \\
\hdashline-1 & $21.260 \mathrm{BB}$ & 1.0911 & $1.03070 \mathrm{e} 4$ & 147.15715 & 98.5365 \\
\hline 2 & $27.860 \mathrm{MM}$ & 1.4899 & 153.08630 & 1.71247 & 1.4635
\end{tabular}

Totals :

$1.04600 \mathrm{e} 4 \quad 148.86962$

Signal 3: DAD1 C, Sig $=254,4$ Ref $=360,100$

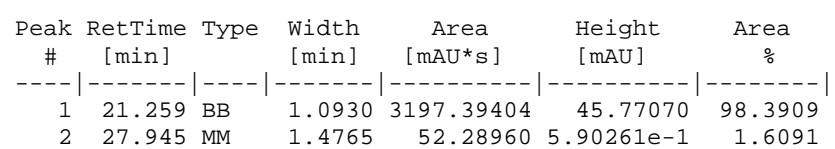

Totals :

$3249.68364 \quad 46.36096$

Signal 4: DAD1 D, Sig=280, 4 Ref $=360,100$

Signal 5: DAD1 E, Sig $=310,4$ Ref $=360,100$

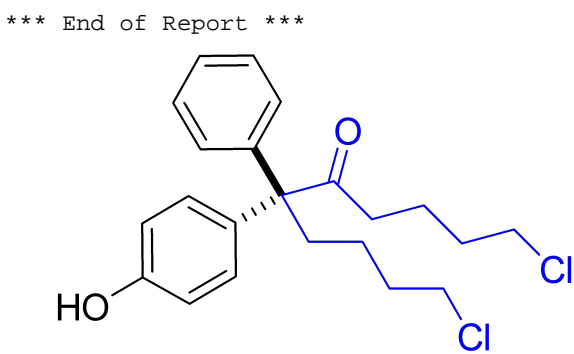

$4 \mathbf{i}$

Enantioenriched 


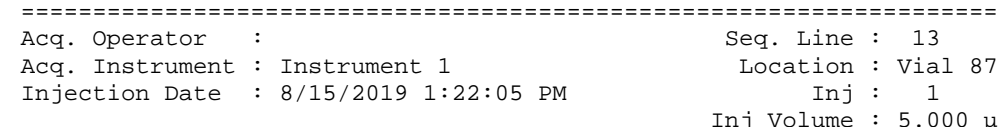

Injection Inj volume : $5.000 \mu \mathrm{I}$

Acq. Method : 8/15/2019 12:40:06 PM

Analysis Method : C: $:$ CHEM32\1\METHODS \OD-01-10.M

Last changed : 8/15/2019 4:38:57 PM

Additional Info : Poak(s) manually integrated
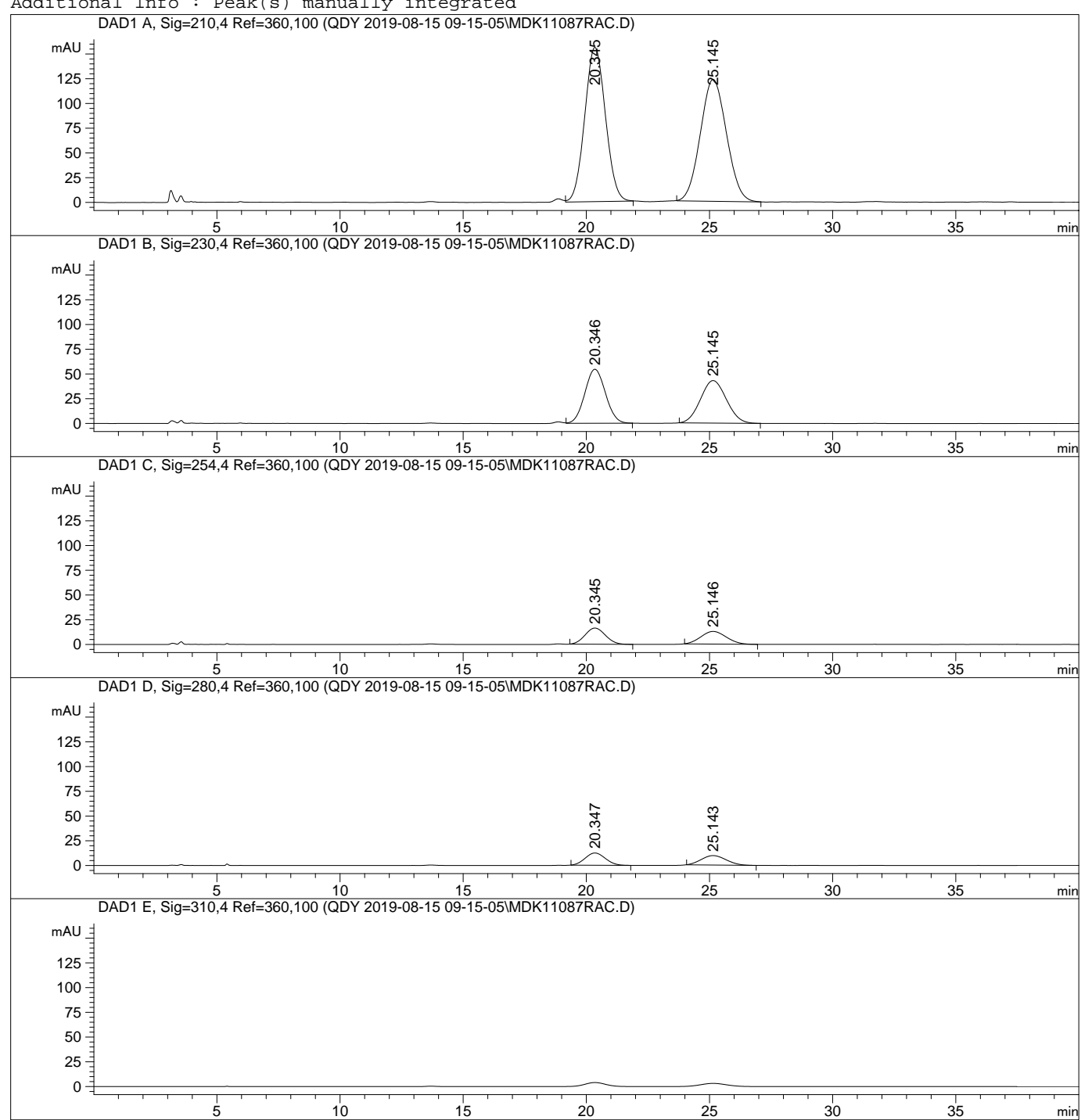

Area Percent Report

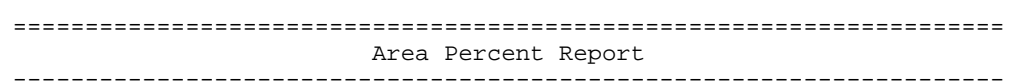

Sorted By

Signal

Multiplier

1.0000

Use Multiplier \& Dilution Factor with ISTDs

Signal 1: DAD1 A, Sig=210, 4 Ref $=360,100$

\begin{tabular}{cccccc}
$\begin{array}{c}\text { Peak RetTime Type } \\
\text { \# }\end{array}\left[\begin{array}{c}\text { Width } \\
{[\mathrm{min}]}\end{array}\right.$ & $\begin{array}{c}\text { Area } \\
{[\mathrm{mAU}}\end{array}$ & $\begin{array}{l}\text { Height } \\
{[\mathrm{mAU}]}\end{array}$ & $\begin{array}{c}\text { Area } \\
\%\end{array}$ \\
\hdashline-1 & $20.345 \mathrm{VB}$ & 0.9083 & 9121.34082 & 155.95680 & 50.1068 \\
\hline 2 & $25.145 \mathrm{BB}$ & 1.1355 & 9082.47363 & 123.28167 & 49.8932
\end{tabular}

Totals :

$1.82038 \mathrm{e} 4 \quad 279.23847$

Signal 2: DAD1 B, Sig=230, 4 Ref $=360,100$

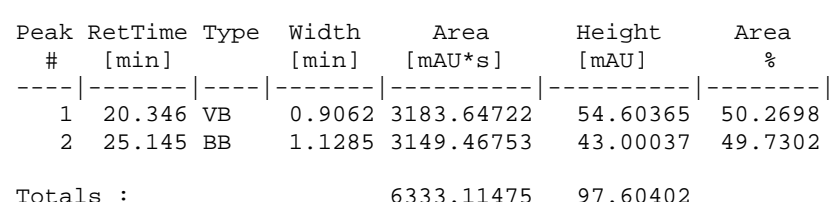

Totals :

$6333.11475 \quad 97.60402$

Signal 3: DAD1 C, Sig $=254,4$ Ref $=360,100$

\begin{tabular}{|c|c|c|c|c|c|}
\hline $\begin{array}{c}\text { Peak } \\
\#\end{array}$ & $\begin{array}{l}\text { RetTime Type } \\
\text { [min] }\end{array}$ & $\begin{array}{l}\text { Width } \\
{[\mathrm{min}]}\end{array}$ & $\begin{array}{c}\text { Area } \\
{\left[\mathrm{mAU} U^{*} \mathrm{~s}\right]}\end{array}$ & $\begin{array}{l}\text { Height } \\
{[\mathrm{mAU}]}\end{array}$ & $\begin{array}{c}\text { Area } \\
\%\end{array}$ \\
\hline 1 & $20.345 \mathrm{BB}$ & 0.8996 & 937.21857 & 16.32848 & 50.6023 \\
\hline 2 & $25.146 \mathrm{BB}$ & 1.0592 & 914.90814 & 12.77999 & 49.3977 \\
\hline
\end{tabular}

Signal 4: DAD1 D, Sig $=280,4$ Ref $=360,100$

Peak RetTime Type Width Area Height Area

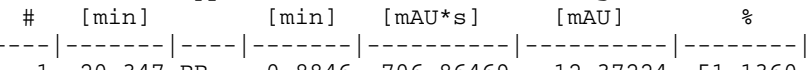

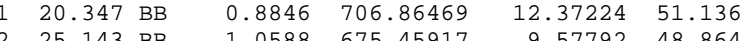

Totals : 1382.32385

21.95017

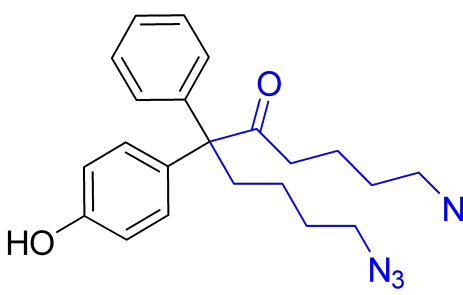

Racemic

Signal 5: DAD1 E, Sig $=310,4$ Ref $=360,100$ 

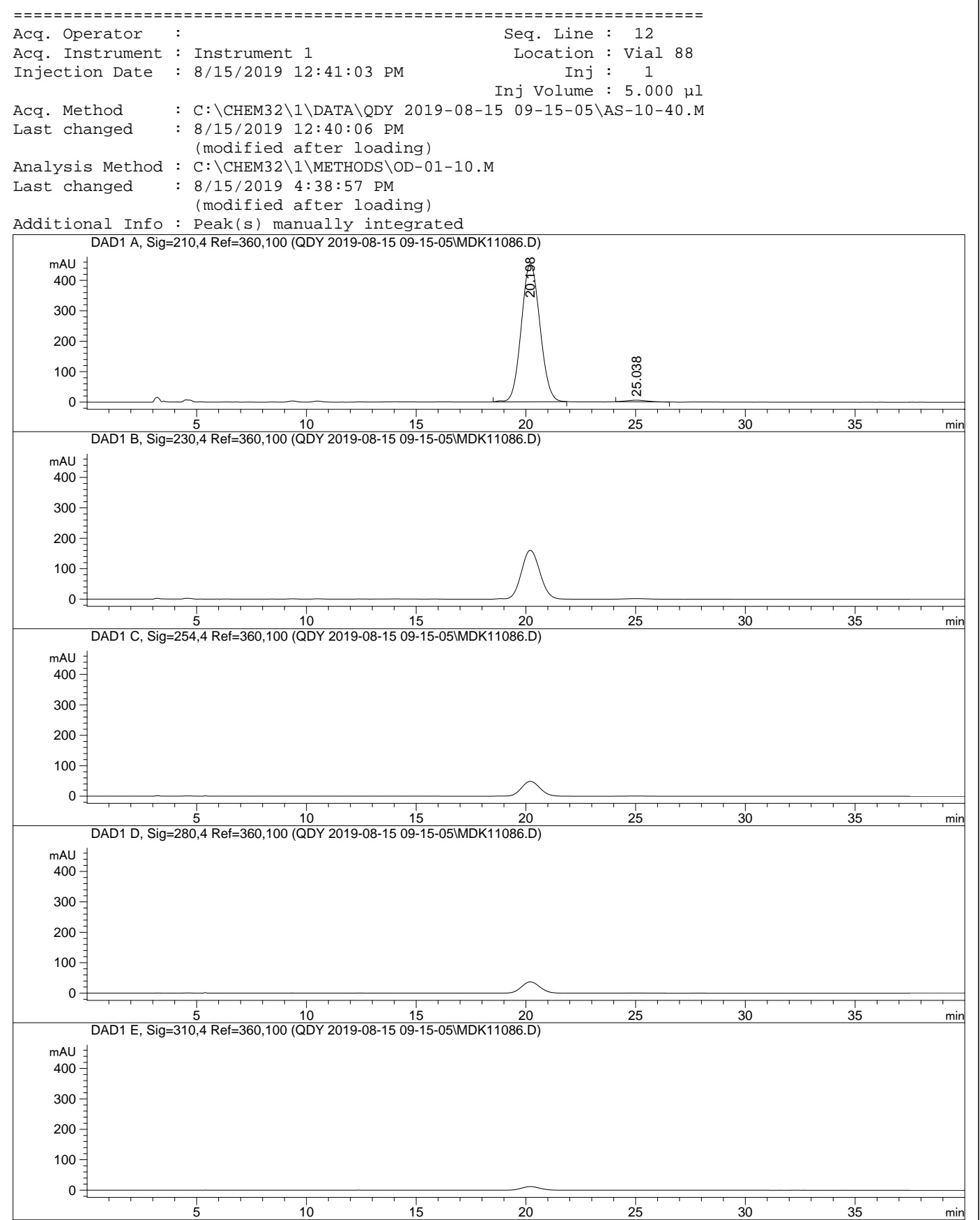

$\begin{array}{lll}\text { Sorted By } & : & \text { Signal } \\ \text { Multiplier } & : & 1.0000\end{array}$

Dilution

Use Multiplier \& Dilution Factor with ISTDs

Signal 1: DAD1 A, Sig=210, 4 Ref $=360,100$

\begin{tabular}{|c|c|c|c|c|c|}
\hline $\begin{array}{c}\text { Peak } \\
\#\end{array}$ & $\begin{array}{l}\text { RetTime Type } \\
{[\mathrm{min}]}\end{array}$ & $\begin{array}{l}\text { Width } \\
\text { [min] }\end{array}$ & $\begin{array}{c}\text { Area } \\
{\left[\mathrm{mAU} U^{*} \mathrm{~s}\right]}\end{array}$ & $\begin{array}{l}\text { Height } \\
{[\mathrm{mAU}]}\end{array}$ & $\begin{array}{c}\text { Area } \\
\%\end{array}$ \\
\hline $\begin{array}{l}1 \\
2\end{array}$ & $\begin{array}{l}20.198 \text { BВ } \\
25.038 \text { BВ }\end{array}$ & $\begin{array}{l}0.9176 \\
0.7704\end{array}$ & $\begin{array}{c}2.67148 \mathrm{e} 4 \\
349.36710\end{array}$ & $\begin{array}{r}453.28952 \\
5.41739\end{array}$ & $\begin{array}{r}98.7091 \\
1.2909\end{array}$ \\
\hline
\end{tabular}

Totals :

$2.70642 \mathrm{e} 4 \quad 458.70691$

Signal 2: DAD1 B, Sig=230, 4 Ref $=360,100$

Signal 3: DAD1 C, Sig $=254,4$ Ref $=360,100$

Signal 4: DAD1 D, Sig $=280,4$ Ref $=360,100$

Signal 5: DAD1 E, Sig=310, 4 Ref $=360,100$

$* * *$ End of Report ***
**

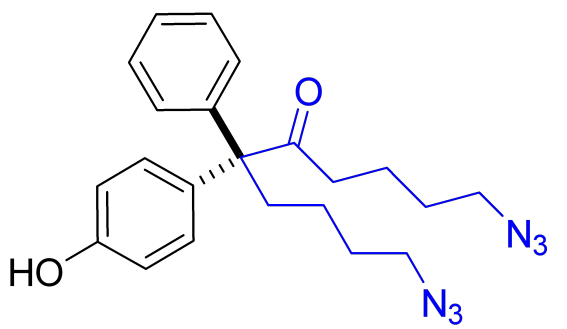

$4 j$

Enantioenriched 


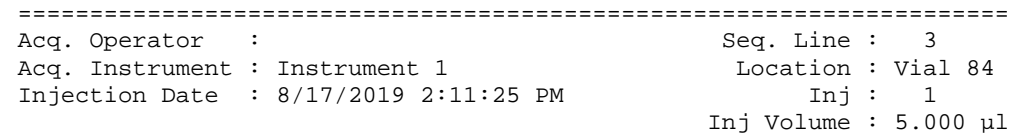

Different Inj volume from Sequence ! Actual Inj Volume : $5.000 \mu \mathrm{\mu}$ Acq. Method : C: \CHEM32\1\DATA QQDY 2019-08-17 13-37-12\AD-01-20.M Last changed : 8/17/2019 1:49:28 PM

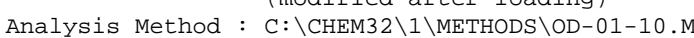

Last changed : 8/15/2019 8:37:16 PM

Additional Info: Peak(s) manually integrated

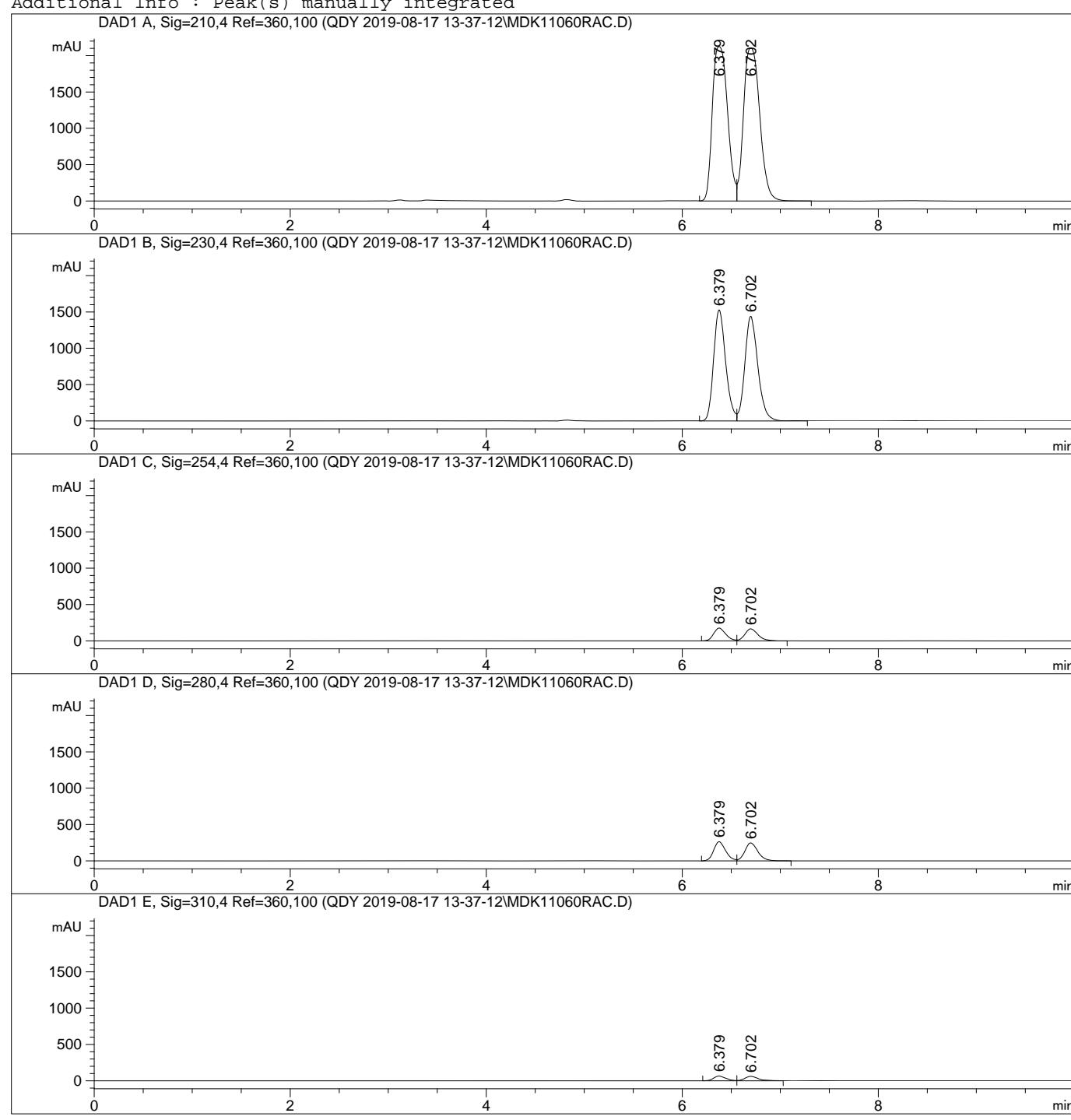

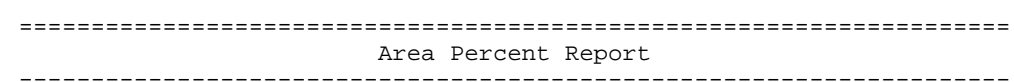

Sorted By
Multiplier

\section{Signal}

1.0000

Use Multiplier \& Dilution Factor with ISTDs

Signal 1: DAD1 A, Sig=210, 4 Ref $=360,100$

\begin{tabular}{cccccc}
$\begin{array}{c}\text { Peak RetTime Type } \\
\#\end{array}\left[\begin{array}{c}\text { Width } \\
{[\mathrm{min}]}\end{array}\right.$ & $\begin{array}{c}\text { Area } \\
{[\mathrm{mAU}}\end{array}$ & $\begin{array}{l}\text { Height } \\
{[\mathrm{mAU}]}\end{array}$ & $\begin{array}{c}\text { Area } \\
\%\end{array}$ \\
\hdashline-1 & $6.379 \mathrm{VV}$ & 0.1708 & $2.27526 \mathrm{e} 4$ & 2126.87061 & 48.4449 \\
\hline 2 & $6.702 \mathrm{VB}$ & 0.1820 & $2.42133 \mathrm{e} 4$ & 2107.60913 & 51.5551
\end{tabular}

$\begin{array}{llllll}1 & 6.302 \mathrm{VB} & 0.1820 & 2.42133 \mathrm{e} & 2126.87061 & 48.4449\end{array}$

Totals : $\quad 4.69659 \mathrm{e} 4 \quad 4234.47974$

Signal 2: DAD1 B, Sig=230, 4 Ref $=360,100$

\begin{tabular}{cccccc}
$\begin{array}{c}\text { Peak RetTime Type } \\
\#\end{array}\left[\begin{array}{c}\text { Width } \\
{[\mathrm{min}]}\end{array}\right.$ & $\begin{array}{c}\text { Area } \\
{[\mathrm{mAU}}\end{array}$ & $\begin{array}{l}\text { Height } \\
{[\mathrm{mAU}]}\end{array}$ & $\begin{array}{c}\text { Area } \\
\%\end{array}$ \\
\hdashline-1 & $6.379 \mathrm{VV}$ & 0.1313 & $1.30184 \mathrm{e} 4$ & 1529.38806 & 49.3747 \\
2 & $6.702 \mathrm{VB}$ & 0.1421 & $1.33482 \mathrm{e} 4$ & 1440.70532 & 50.6253
\end{tabular}

$\begin{array}{llllll}1 & 6.379 \mathrm{VV} & 0.1313 & 1.30184 \mathrm{e} 4 & 1529.38806 & 49.3747 \\ 2 & 6.702 \mathrm{VB} & 0.1421 & 1.33482 \mathrm{e} 4 & 1440.70532 & 50.6253\end{array}$

Totals : $\quad 2.63666 \mathrm{e} 4 \quad 2970.09338$

Signal 3: DAD1 C, Sig=254, 4 Ref $=360,100$

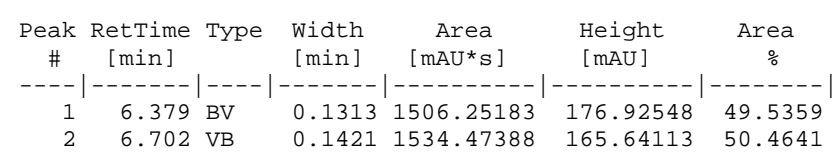

Totals : $\quad 3040.72571 \quad 342.56660$

Signal 4: DAD1 D, Sig=280, 4 Ref $=360,100$

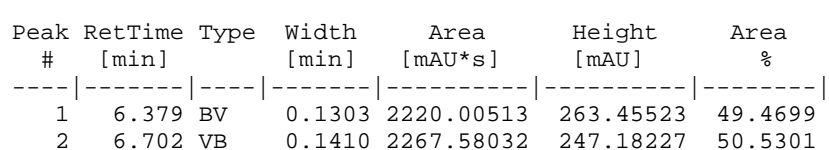

Totals :

$4487.58545 \quad 510.63750$

Signal 5: DAD1 E, Sig=310, 4 Ref $=360,100$

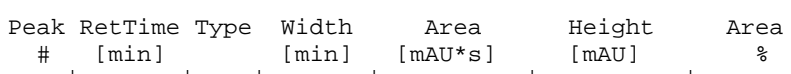

10.379 BV $0.1301551 .93579 \quad 65.6227049 .5141$

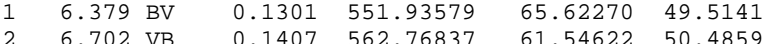

Totals : $\quad 1114.70416 \quad 127.16892$

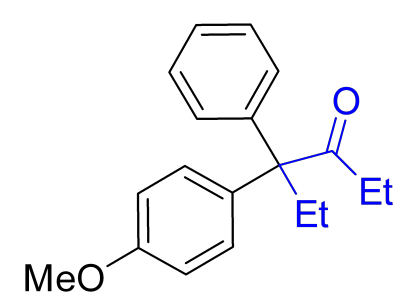

Racemic 


\section{Data File C: \CHEM32\1\DATA\QDY 2019-08-17 13-37-12\MDK11091.D}

Sample Name

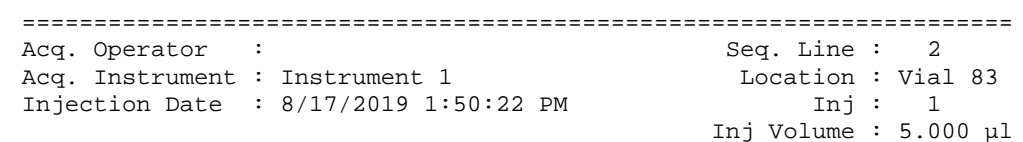

Injection Date : 8/17/2019 1:50:22 PM Inj Volume : 1

Acq. Method : C: \CHEM32\1\DATA\QDY 2019-08-17 13-37-12\AD-01-20.M

Last changed : 8/17/2019 1:49:28 PM

Analysis Method : C: $:$ CHEM32\1\METHODS\OD-01-10.M

Last changed : 8/15/2019 8:37:16 PM

Additional Info:
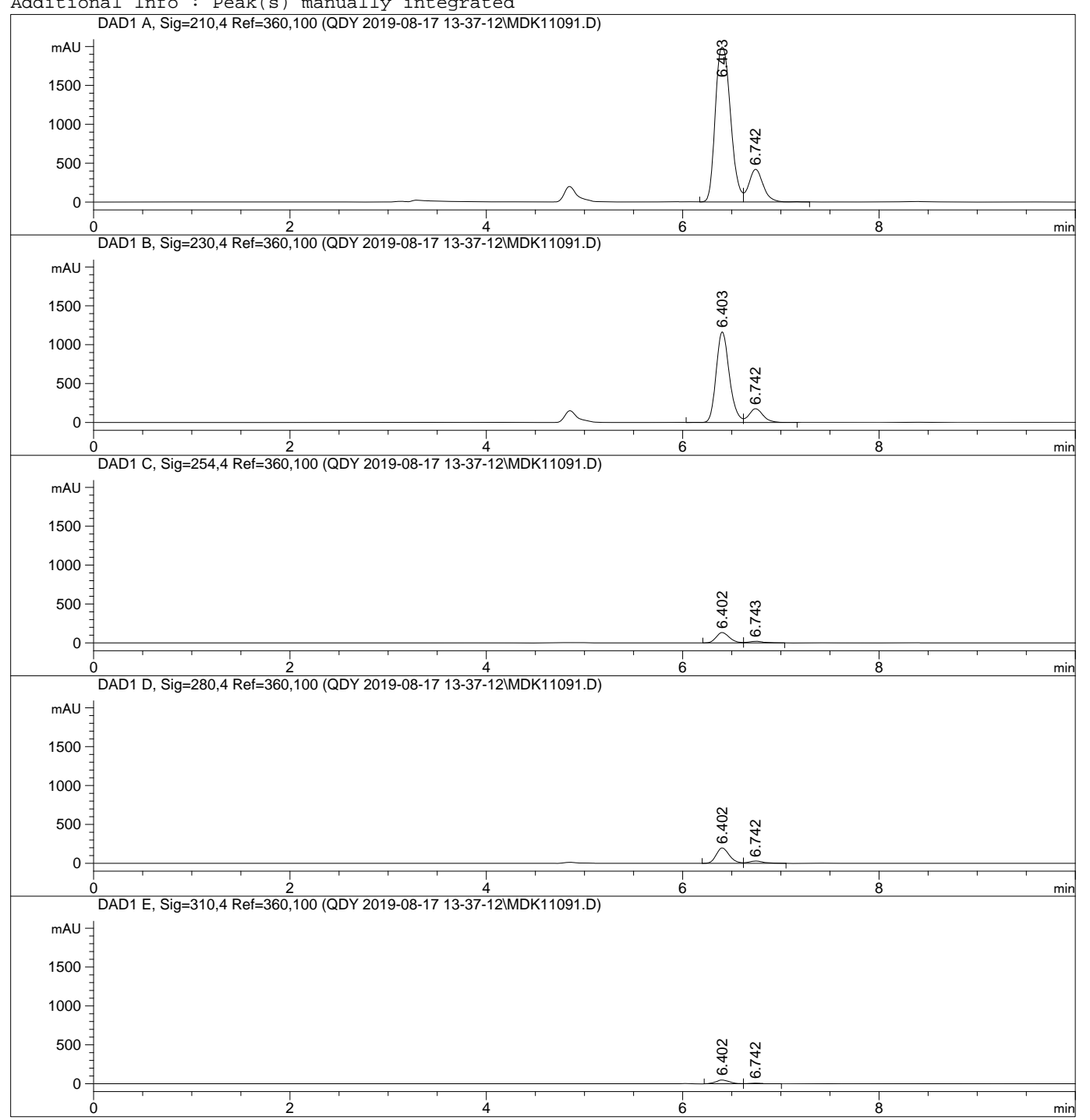

S-374
Area Percent Report

$\begin{array}{lll}\text { Sorted By } & : & \text { Signal } \\ \text { Multiplier } & : & 1.0000 \\ \text { Dilution } & : & 1.0000\end{array}$

Use Multiplier \& Dilution Factor with ISTDs

Signal 1: DAD1 A, Sig=210, 4 Ref $=360,100$

\begin{tabular}{cccccc}
$\begin{array}{c}\text { Peak RetTime Type } \\
\#\end{array}\left[\begin{array}{c}\text { Width } \\
{[\mathrm{min}]}\end{array}\right.$ & $\begin{array}{c}\text { Area } \\
{[\mathrm{mAU}}\end{array}$ & $\begin{array}{l}\text { Height } \\
{[\mathrm{mAU}]}\end{array}$ & $\begin{array}{c}\text { Area } \\
\%\end{array}$ \\
\hdashline-1 & $6.403 \mathrm{VV}$ & 0.1718 & $2.14645 \mathrm{e} 4$ & 1990.62854 & 83.4668 \\
\hline 2 & $6.742 \mathrm{VB}$ & 0.1541 & 4251.70605 & 419.90948 & 16.5332
\end{tabular}

$\begin{array}{lllllll}1 & 6.403 & \text { VV } & 0.1718 & 2.14645 \mathrm{e} 4 & 1990.62854 & 83.4668 \\ 2 & 6.742 \text { VB } & 0.1541 & 4251.70605 & 419.90948 & 16.5332\end{array}$

$\begin{array}{lll}\text { Totals : } & 2.57162 \mathrm{e} 4 & 2410.53802\end{array}$

Signal 2: DAD1 B, Sig=230, 4 Ref $=360,100$

\begin{tabular}{|c|c|c|c|c|c|}
\hline $\begin{array}{c}\text { Peak } \\
\text { \# }\end{array}$ & $\begin{array}{l}\text { RetTime Type } \\
\text { [min] }\end{array}$ & $\begin{array}{l}\text { Width } \\
\text { [min] }\end{array}$ & $\begin{array}{c}\text { Area } \\
{\left[\mathrm{mAU}^{*} \mathrm{~s}\right]}\end{array}$ & $\begin{array}{l}\text { Height } \\
{[\mathrm{mAU}]}\end{array}$ & $\begin{array}{c}\text { Area } \\
\%\end{array}$ \\
\hline $\begin{array}{l}1 \\
2\end{array}$ & $\begin{array}{l}6.403 \mathrm{VV} \\
6.742 \mathrm{VB}\end{array}$ & $\begin{array}{l}0.1449 \\
0.1530\end{array}$ & $\begin{array}{l}1.08717 \mathrm{e} 4 \\
1773.90784\end{array}$ & $\begin{array}{r}1164.76465 \\
176.95950\end{array}$ & $\begin{array}{l}85.9722 \\
14.0278\end{array}$ \\
\hline
\end{tabular}

Totals :

$1.26456 \mathrm{e} 4 \quad 1341.72415$

Signal 3: DAD1 C, Sig=254, 4 Ref $=360,100$

\begin{tabular}{|c|c|c|c|c|c|}
\hline $\begin{array}{c}\text { Peak } \\
\#\end{array}$ & $\begin{array}{l}\text { RetTime Type } \\
{[\text { min] }}\end{array}$ & $\begin{array}{l}\text { Width } \\
\text { [min] }\end{array}$ & $\begin{array}{c}\text { Area } \\
{\left[\mathrm{mAU} U^{\star} \mathrm{s}\right]}\end{array}$ & $\begin{array}{l}\text { Height } \\
{[\mathrm{mAU}]}\end{array}$ & $\begin{array}{c}\text { Area } \\
\%\end{array}$ \\
\hline 1 & $6.402 \mathrm{BV}$ & 0.1453 & 1252.60486 & 133.75671 & 85.8874 \\
\hline 2 & $6.743 \mathrm{VB}$ & 0.1526 & 205.82225 & 20.59250 & 14.1126 \\
\hline
\end{tabular}

Signal 4: DAD1 D, Sig=280, 4 Ref $=360,100$

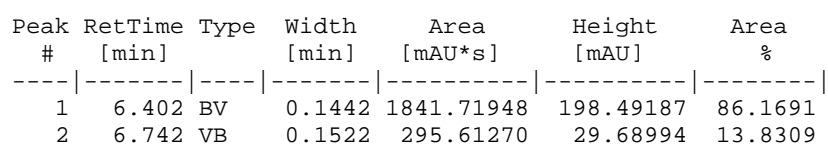
$\begin{array}{lll}\text { Totals : } & 2137.33218 & 228.18181\end{array}$

Signal 5: DAD1 E, Sig=310, 4 Ref $=360,100$

\begin{tabular}{|c|c|c|c|c|c|c|}
\hline $\begin{array}{l}\text { eak } \\
\#\end{array}$ & $\begin{array}{c}\text { RetTime } \\
{[\text { min] }}\end{array}$ & & $\begin{array}{l}\text { Width } \\
\text { [min] }\end{array}$ & $\begin{array}{c}\text { Area } \\
{\left[\mathrm{mAU}^{*} \mathrm{~s}\right]}\end{array}$ & $\begin{array}{l}\text { Height } \\
{[\mathrm{mAU}]}\end{array}$ & $\begin{array}{c}\text { Area } \\
\%\end{array}$ \\
\hline & & & $\begin{array}{l}0.1441 \\
0.1516\end{array}$ & $\begin{array}{r}457.19849 \\
72.81673\end{array}$ & $\begin{array}{r}49.35667 \\
7.35073\end{array}$ & \\
\hline
\end{tabular}

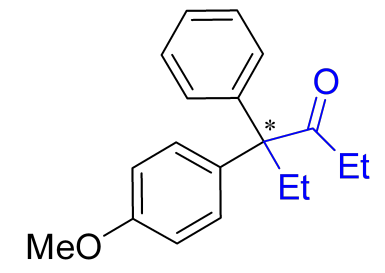

$4 a^{\prime}$

Enantioenriched

Totals : $\quad 530.01522 \quad 56.70740$ 


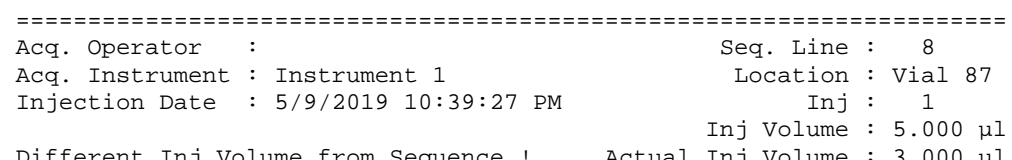

Different Inj Volume from Sequence ! Actual Inj Volume : $5.000 \mu \mathrm{\mu}$

Acq. Method : C: \CHEM32\1\DATA

Last changed : 5/9/2019 10:38:34 PM

Analysis Method : C: $:$ CHEM32\1\METHODS \IC-01-40.M-0.5.M

Last changed : 6/5/2019 1:46:08 PM

Additional Info : (modified after loading)
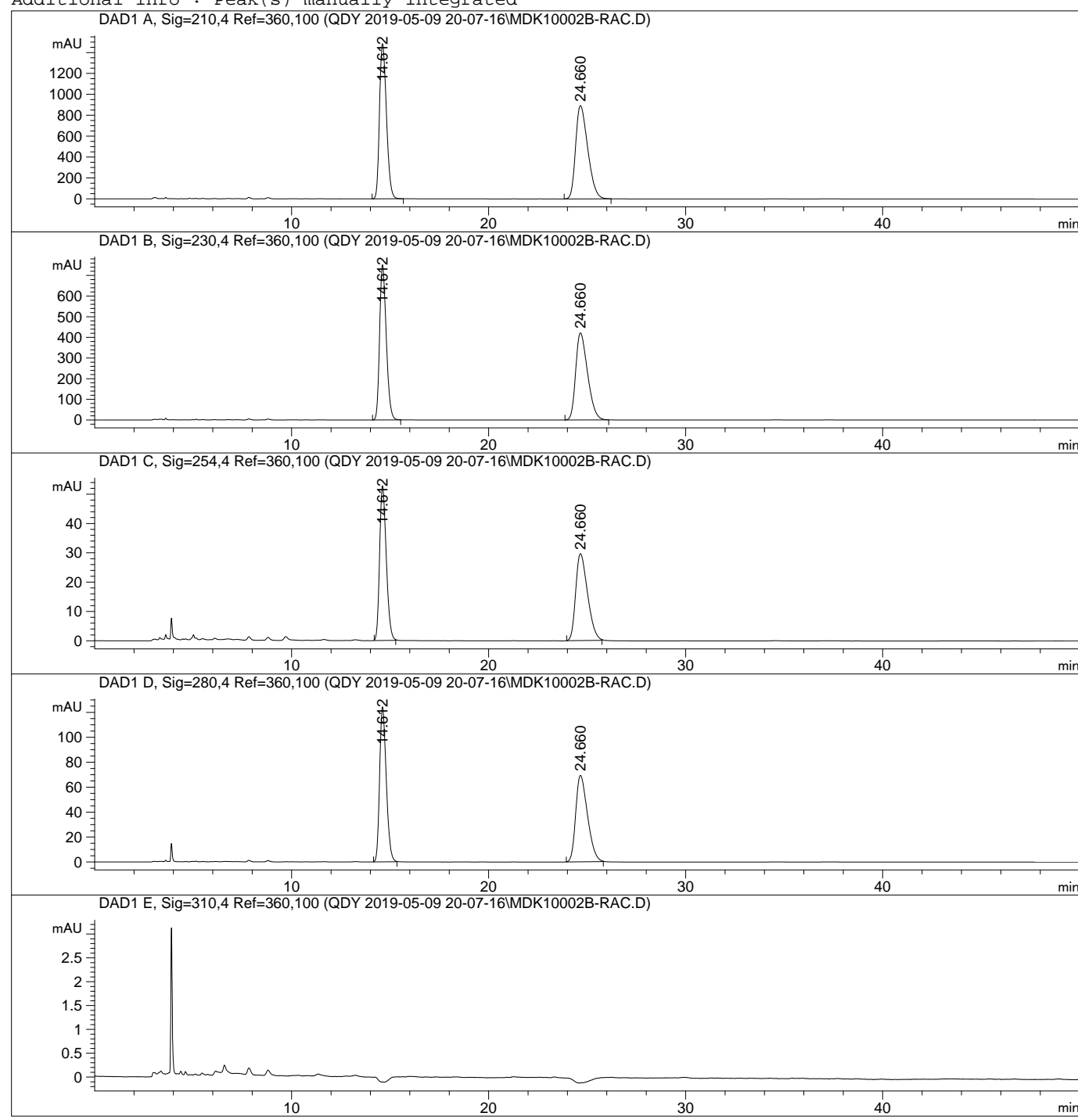

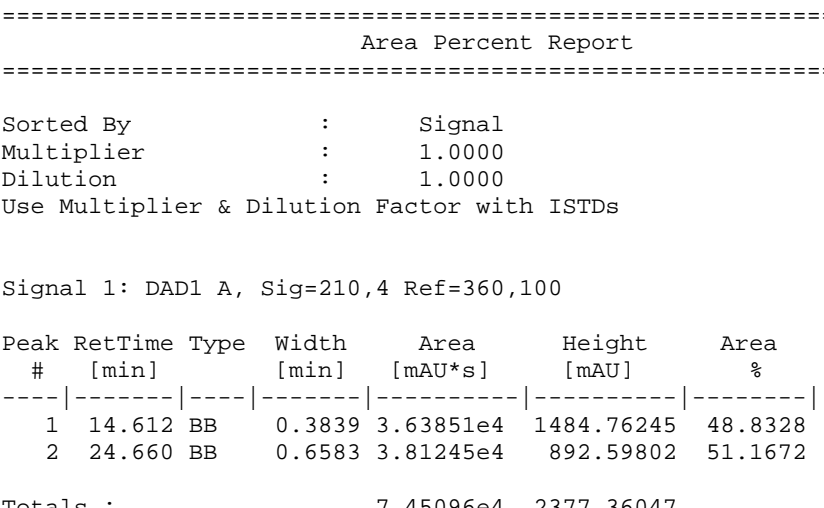

Totals :

$7.45096 \mathrm{e} 4 \quad 2377.36047$

Signal 2: DAD1 B, Sig $=230,4$ Ref $=360,100$

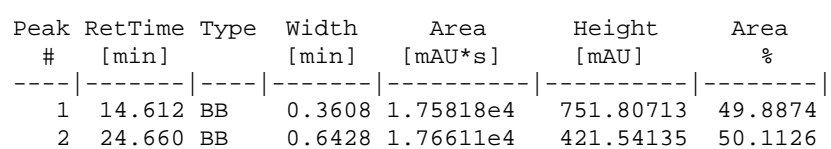

Totals :

$3.52429 \mathrm{e} 4 \quad 1173.34848$

Signal 3: DAD1 C, Sig $=254,4$ Ref $=360,100$

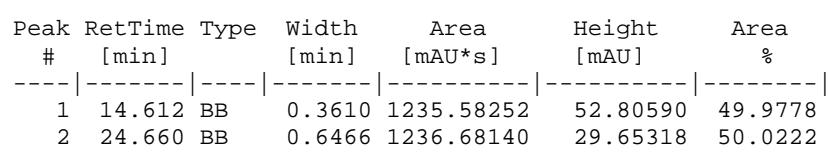

Totals :

$2472.26392 \quad 82.45908$

Signal 4: DAD1 D, Sig=280, 4 Ref $=360,100$

\begin{tabular}{|c|c|c|c|c|c|}
\hline eak & $\begin{array}{c}\text { RetTime } \\
\text { [min] }\end{array}$ & $\begin{array}{l}\text { Width } \\
\text { [min] }\end{array}$ & $\begin{array}{c}\text { Area } \\
{\left[\mathrm{mAU}^{*} \mathrm{~s}\right]}\end{array}$ & $\begin{array}{l}\text { Height } \\
{[\mathrm{mAU}]}\end{array}$ & $\begin{array}{c}\text { Area } \\
\%\end{array}$ \\
\hline & 660 & 641 & $\begin{array}{l}2894.80493 \\
2885.75342\end{array}$ & $\begin{array}{r}124.44032 \\
69.33379\end{array}$ & 50.0783 \\
\hline
\end{tabular}

Totals :

$5780.55835 \quad 193.77411$

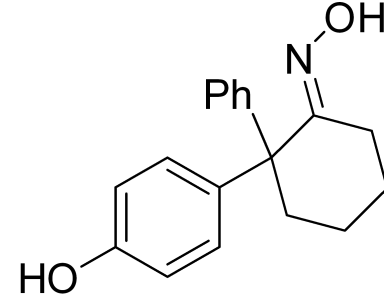

racemic

Signal 5: DAD1 E, Sig=310, 4 Ref $=360,100$

*** End of Report *** 

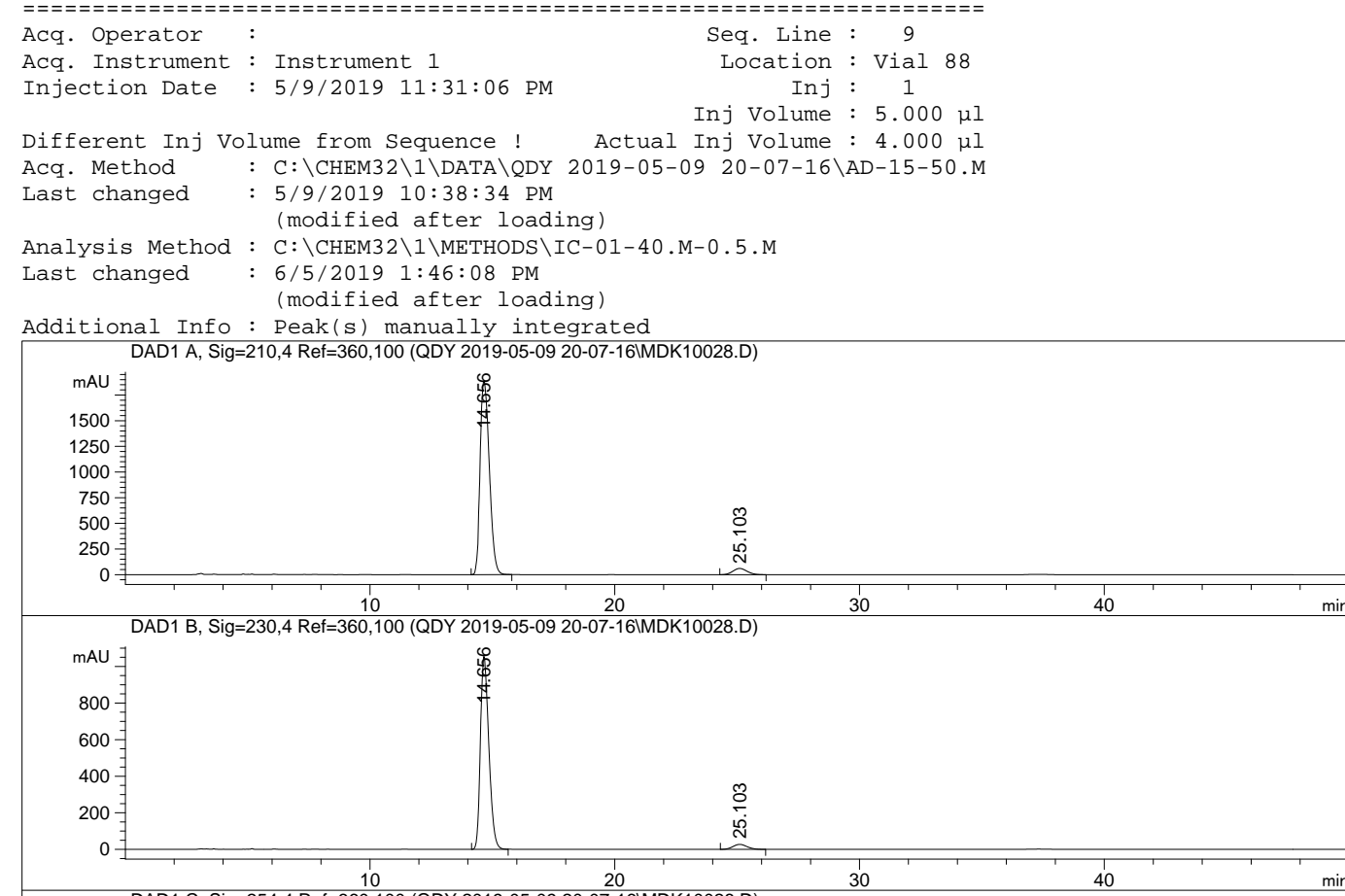

$\frac{10}{10} \frac{20}{20}$
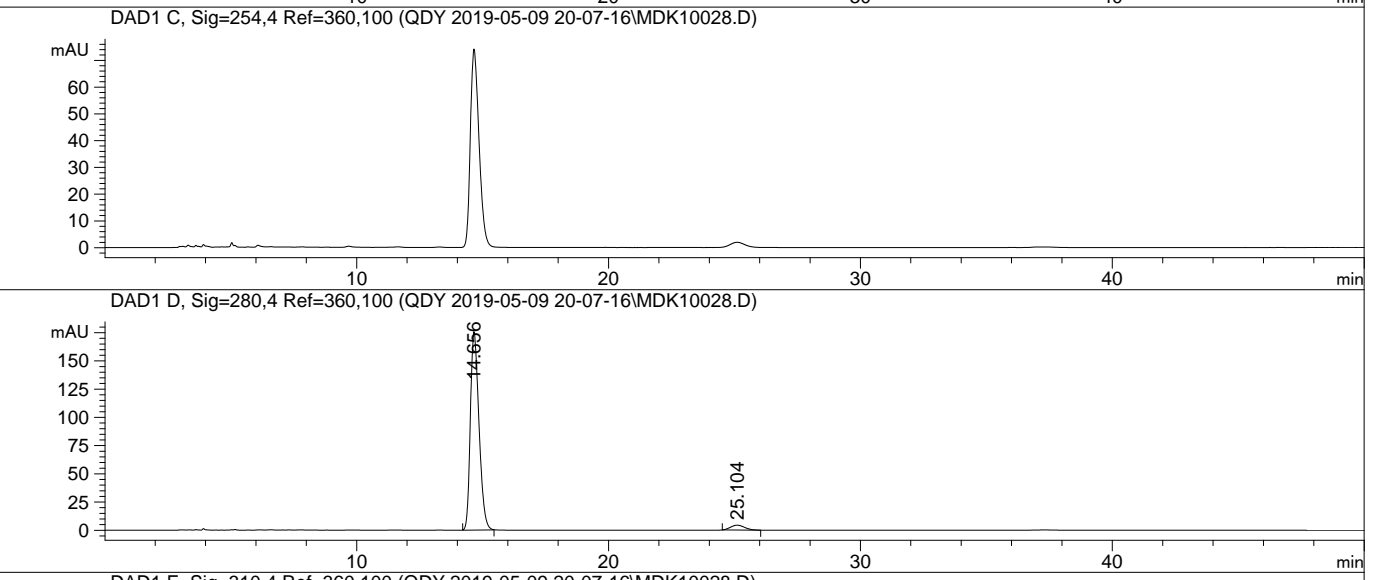

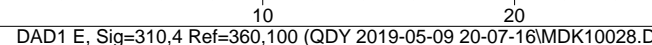

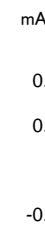

0.2
0.1
0
-0.1
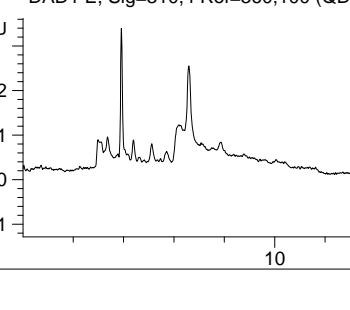

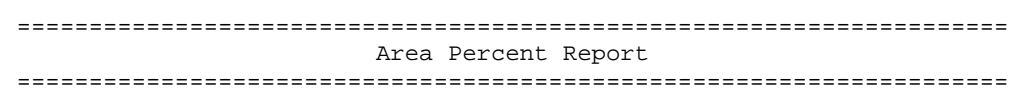

$\begin{array}{lll}\text { Sorted By } & : & \text { Signal } \\ \text { Multiplier } & : & 1.0000\end{array}$

Dilution 1.0000

Use Multiplier \& Dilution Factor with ISTDs

Signal 1: DAD1 A, Sig=210, 4 Ref $=360,100$

\begin{tabular}{|c|c|c|c|c|c|}
\hline $\begin{array}{c}\text { Peak } \\
\#\end{array}$ & $\begin{array}{l}\text { RetTime Type } \\
\text { [min] }\end{array}$ & $\begin{array}{l}\text { Width } \\
\text { [min] }\end{array}$ & $\begin{array}{c}\text { Area } \\
{\left[\mathrm{mAU} U^{*} \mathrm{~s}\right]}\end{array}$ & $\begin{array}{l}\text { Height } \\
{[\mathrm{mAU}]}\end{array}$ & $\begin{array}{c}\text { Area } \\
\%\end{array}$ \\
\hline 1 & $14.656 \mathrm{BB}$ & 0.4189 & $4.94422 \mathrm{e} 4$ & 1879.80652 & $\begin{array}{r}95.2145 \\
4.7855\end{array}$ \\
\hline
\end{tabular}

Totals : $\quad 5.19272 \mathrm{e} 4 \quad 1940.90131$

Signal 2: DAD1 B, Sig=230, 4 Ref $=360,100$

\begin{tabular}{|c|c|c|c|c|c|}
\hline $\begin{array}{c}\text { Peak } \\
\#\end{array}$ & $\begin{array}{l}\text { RetTime Type } \\
{[\text { min] }}\end{array}$ & $\begin{array}{l}\text { Width } \\
\text { [min] }\end{array}$ & $\begin{array}{c}\text { Area } \\
{\left[\mathrm{mAU}^{*} \mathrm{~s}\right]}\end{array}$ & $\begin{array}{l}\text { Height } \\
{[\mathrm{mAU}]}\end{array}$ & $\begin{array}{c}\text { Area } \\
\%\end{array}$ \\
\hline 1 & $14.656 \mathrm{BB}$ & 0.3715 & $2.52671 \mathrm{e} 4$ & $\begin{array}{r}1054.70679 \\
27\end{array}$ & $\begin{array}{r}95.8109 \\
4.1891\end{array}$ \\
\hline
\end{tabular}

Totals :

$2.63718 \mathrm{e} 4 \quad 1082.00021$

Signal 3: DAD1 C, Sig=254, 4 Ref $=360,100$

Signal 4: DAD1 D, Sig $=280,4$ Ref $=360,100$

\begin{tabular}{|c|c|c|c|c|c|}
\hline $\begin{array}{c}\text { Peak } \\
\#\end{array}$ & $\begin{array}{l}\text { RetTime Type } \\
\text { [min] }\end{array}$ & $\begin{array}{l}\text { Width } \\
\text { [min] }\end{array}$ & $\begin{array}{c}\text { Area } \\
{\left[\mathrm{mAU} U^{*} \mathrm{~s}\right]}\end{array}$ & $\begin{array}{l}\text { Height } \\
{[\mathrm{mAU}]}\end{array}$ & $\begin{array}{c}\text { Area } \\
\%\end{array}$ \\
\hline 1 & $14.656 \mathrm{BB}$ & 0.3674 & 4180.29688 & 175.79358 & 96.1205 \\
\hline 2 & $25.104 \mathrm{BB}$ & $\odot .5942$ & 168.72006 & 4.33401 & 3.8795 \\
\hline & & & 0.01 & 180.12759 & \\
\hline
\end{tabular}

Signal 5: DAD1 E, Sig=310, 4 Ref $=360,100$

$* * *$ End of Report ***

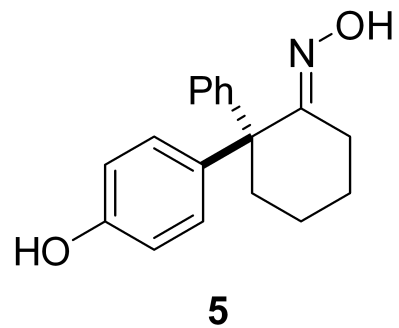

Enantioenriched 


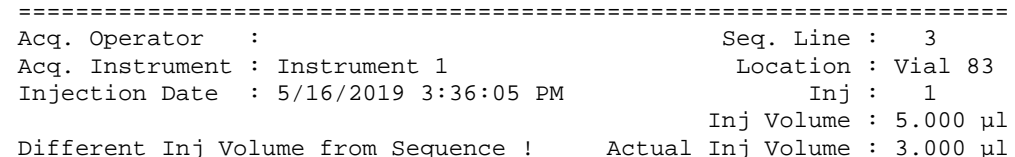

Injection Date $\quad$ Inj $\quad \begin{array}{r}1 \\ \text { Inj Volume }: 5.000 \mu \mathrm{\mu l}\end{array}$

Acq. Method : C: \CHEM32\1\DATA \QDY 2019-05-16 15-01-54\AD-03-20.M

Last changed : 7/2/2016 3:26:02 PM

Analysis Method : C: \CHEM32\1\METHODS \IC-01-40.M-0.5.M

Last changed: 6/5/2019 1:46:08 PM

Additional (modified after loading)

Additional Info : Peak (s) manually integrated
DAD1 A, Sig=210,4 Ref=360,100 (QDY 2019-05-16 15-01-54IMDK10036RAC.D)
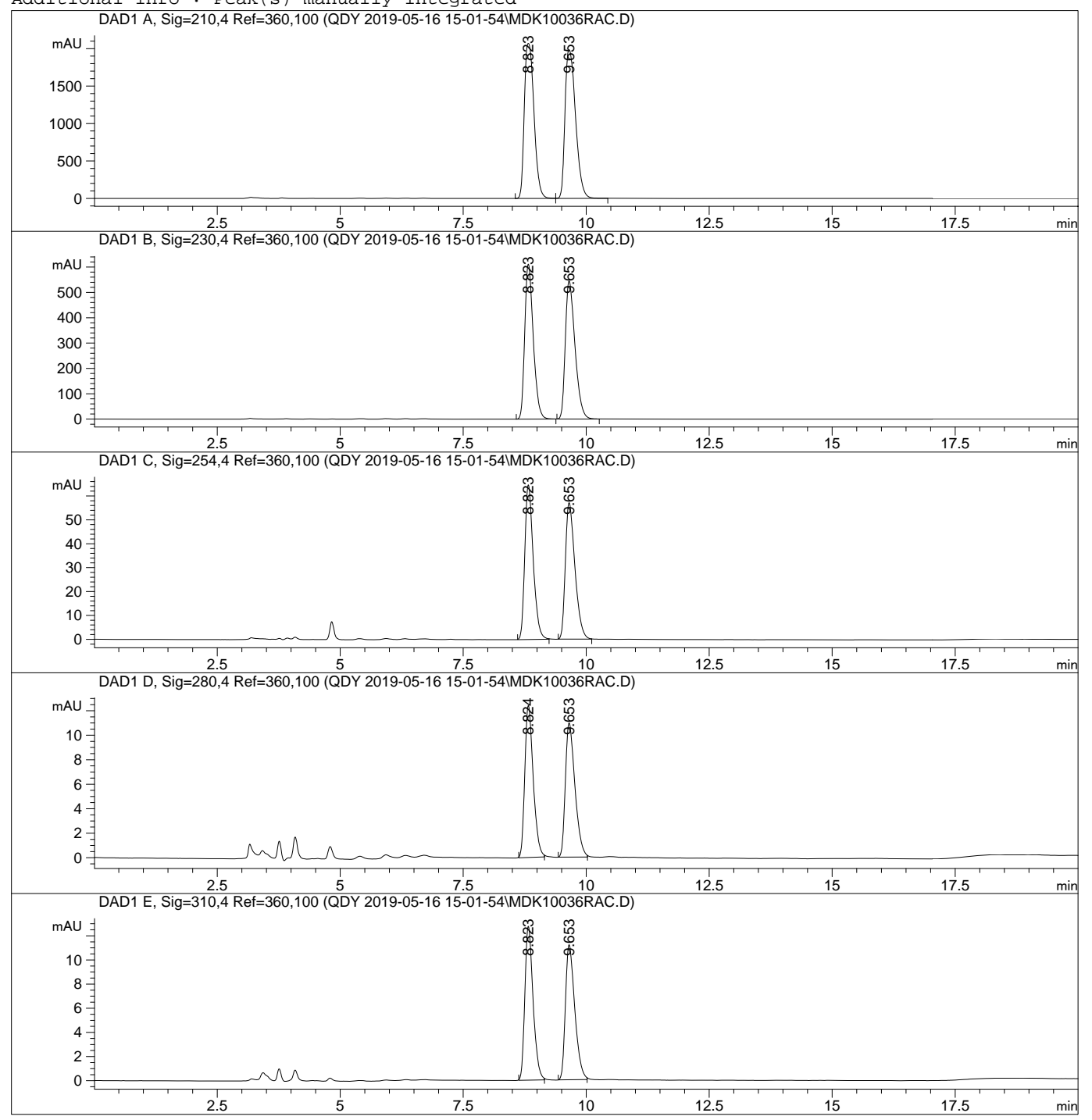

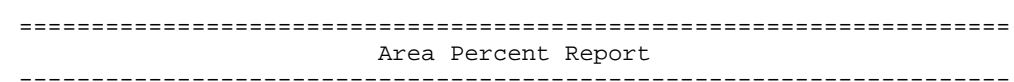

Sorted By

Signal

Multiplier

1.0000

Use Multiplier \& Dilution Factor with ISTDs

Signal 1: DAD1 A, Sig=210, 4 Ref $=360,100$

\begin{tabular}{|c|c|c|c|c|c|}
\hline $\begin{array}{c}\text { Peak } \\
\#\end{array}$ & $\begin{array}{l}\text { RetTime Type } \\
\text { [min] }\end{array}$ & $\begin{array}{l}\text { Width } \\
\text { [min] }\end{array}$ & $\begin{array}{c}\text { Area } \\
{\left[\mathrm{mAU} U^{*} \mathrm{~s}\right]}\end{array}$ & $\begin{array}{l}\text { Height } \\
{[\mathrm{mAU}]}\end{array}$ & $\begin{array}{c}\text { Area } \\
\%\end{array}$ \\
\hline 1 & $8.823 \mathrm{BV}$ & 0.2168 & 2. $81937 \mathrm{e} 4$ & 2071.19482 & 48.7840 \\
\hline
\end{tabular}

Totals : $\quad 5.77930 \mathrm{e} 4 \quad 4056.43591$

Signal 2: DAD1 B, Sig=230, 4 Ref $=360,100$

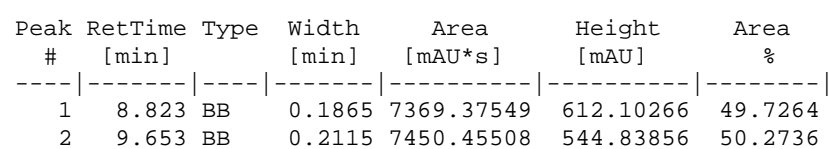

Totals :

$1.48198 \mathrm{e} 4 \quad 1156.94122$

Signal 3: DAD1 C, Sig $=254,4$ Ref $=360,100$

\begin{tabular}{|c|c|c|c|c|c|}
\hline $\begin{array}{c}\text { Peak } \\
\#\end{array}$ & $\begin{array}{l}\text { RetTime Type } \\
{[\text { min] }}\end{array}$ & $\begin{array}{l}\text { Width } \\
\text { [min] }\end{array}$ & $\begin{array}{c}\text { Area } \\
{\left[\mathrm{mAU} U^{\star} \mathrm{s}\right]}\end{array}$ & $\begin{array}{l}\text { Height } \\
{[\mathrm{mAU}]}\end{array}$ & $\begin{array}{c}\text { Area } \\
\%\end{array}$ \\
\hline 1 & $8.823 \mathrm{BB}$ & 0.1807 & 769.99084 & 64.75954 & 6665 \\
\hline 2 & $9.653 \mathrm{BB}$ & 0.2075 & 771.02307 & 57.11434 & 50.0335 \\
\hline
\end{tabular}

Signal 4: DAD1 D, Sig=280, 4 Ref $=360,100$

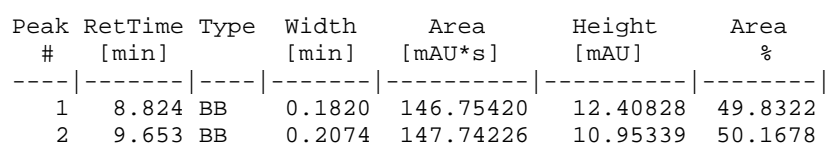

Totals :

$294.49646 \quad 23.36167$

Signal 5: DAD1 E, Sig=310, 4 Ref $=360,100$

\begin{tabular}{cccccc}
$\begin{array}{c}\text { Peak RetTime Type } \\
\# \\
{[\text { [min] }}\end{array}$ & $\begin{array}{c}\text { Width } \\
{[\mathrm{min}]}\end{array}$ & $\begin{array}{c}\text { Area } \\
{[\mathrm{mAU} \text { s }]}\end{array}$ & $\begin{array}{l}\text { Height } \\
{[\mathrm{mAU}]}\end{array}$ & $\begin{array}{c}\text { Area } \\
\%\end{array}$ \\
\hdashline 1 & $8.823 \mathrm{BB}$ & 0.1770 & 147.58678 & 12.75288 & 50.0344 \\
2 & $9.653 \mathrm{BB}$ & 0.2018 & 147.38412 & 11.18110 & 49.9656
\end{tabular}

$\begin{array}{lll}\text { Totals : } & 294.97090 \quad 23.93398\end{array}$
$\mathrm{TfO}$

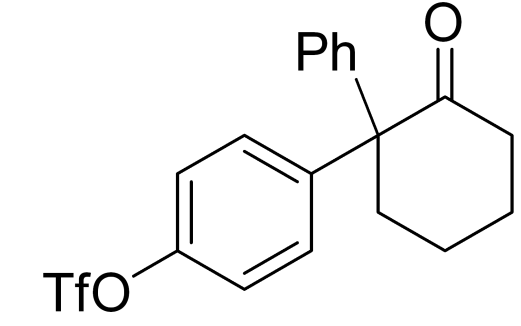

racemic 


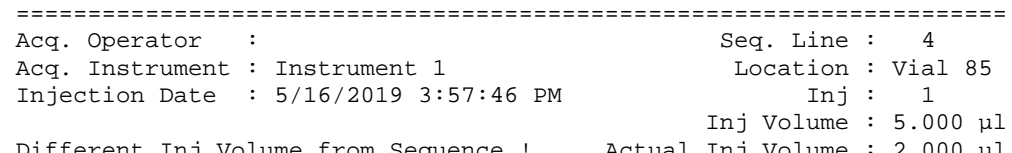

Different Inj volume from Sequence ! Actual Inj Volume : $5.000 \mu \mathrm{\mu}$

Acq. Method : C: \CHEM32\1\DATA\QDY 2019-05-16 15-01-54\AD-03-20.M

Last changed : 7/2/2016 3:26:02 PM

Analysis Method : C: \CHEM32\1\METHODS \IC-01-40.M-0.5.M

Last changed : 6/5/2019 1:46:08 PM

Additional Info (modified after loading)
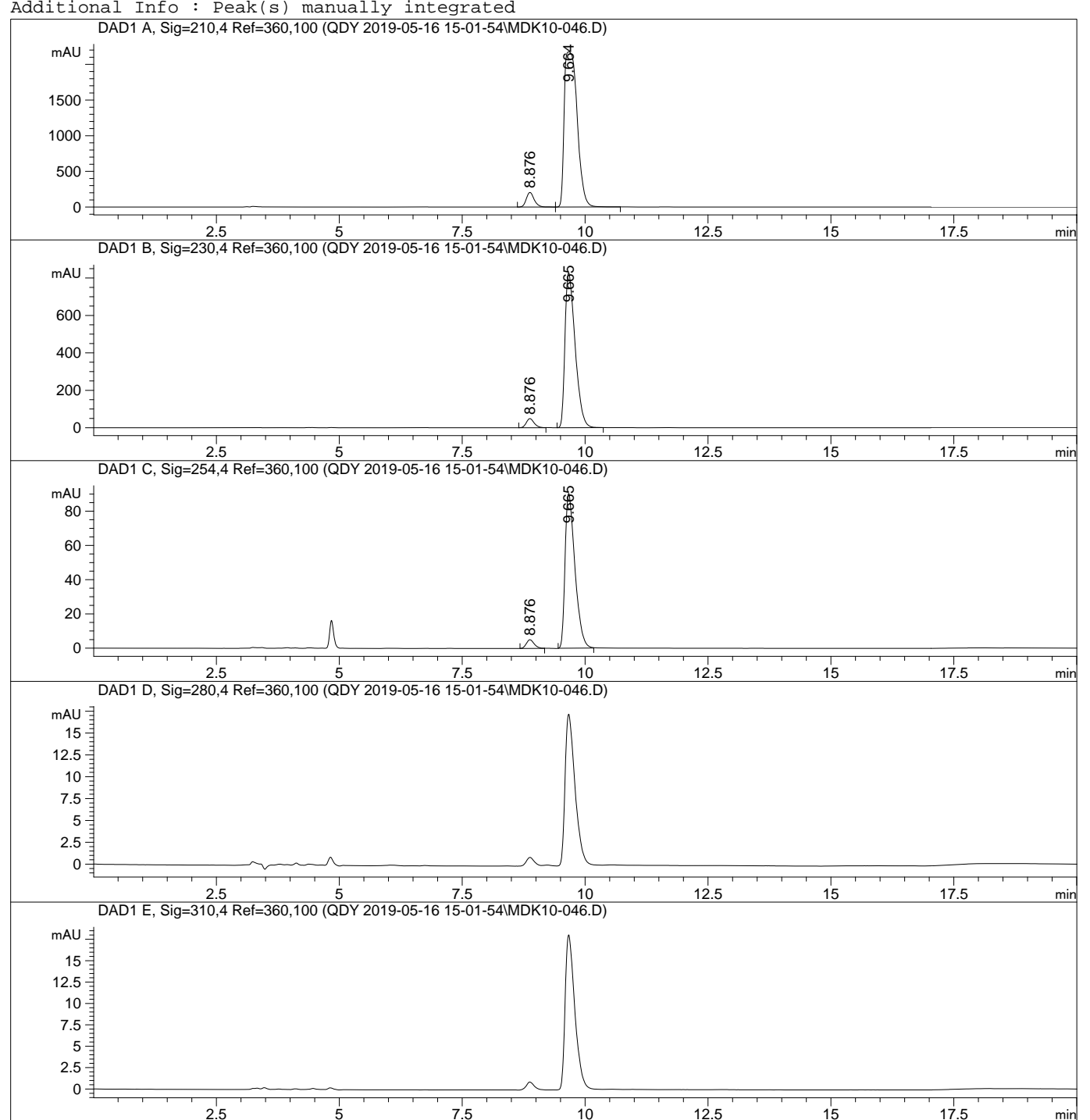

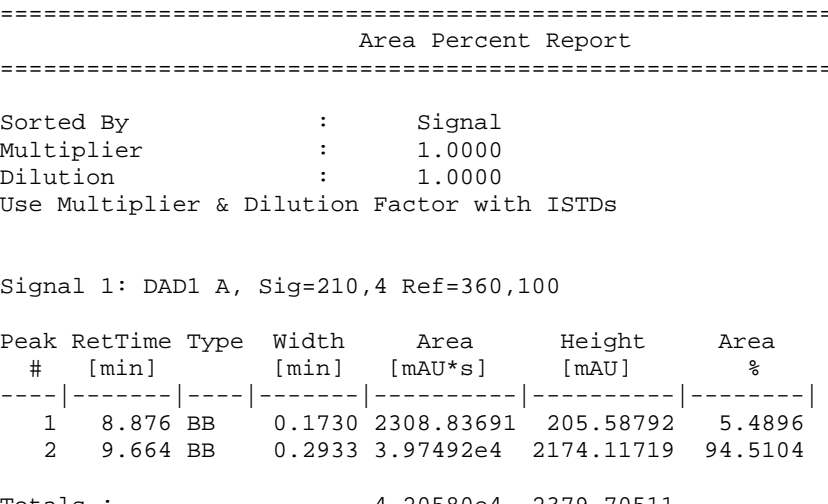

Totals :

$4.20580 \mathrm{e} 4 \quad 2379.70511$

Signal 2: DAD1 B, Sig $=230,4$ Ref $=360,100$

\begin{tabular}{|c|c|c|c|c|c|}
\hline $\begin{array}{c}\text { Peak } \\
\#\end{array}$ & $\begin{array}{l}\text { RetTime Type } \\
\text { [min] }\end{array}$ & $\begin{array}{l}\text { Width } \\
\text { [min] }\end{array}$ & $\begin{array}{c}\text { Area } \\
{\left[\mathrm{mAU} U^{*} \mathrm{~s}\right]}\end{array}$ & $\begin{array}{l}\text { Height } \\
{[\mathrm{mAU}]}\end{array}$ & $\begin{array}{c}\text { Area } \\
\%\end{array}$ \\
\hline 1 & $8.876 \mathrm{BB}$ & 0.1728 & 550.07 & 49.07628 & 4.4348 \\
\hline 2 & $9.665 \mathrm{BB}$ & 0.2209 & $1.18536 \mathrm{e} 4$ & 828.50977 & 95.5652 \\
\hline
\end{tabular}

Totals :

$1.24037 \mathrm{e} 4 \quad 877.58605$

Signal 3: DAD1 C, Sig $=254,4$ Ref $=360,100$

\begin{tabular}{|c|c|c|c|c|c|}
\hline $\begin{array}{c}\text { Peak } \\
\#\end{array}$ & $\begin{array}{l}\text { RetTime Type } \\
{[\mathrm{min}]}\end{array}$ & $\begin{array}{l}\text { Width } \\
\text { [min] }\end{array}$ & $\begin{array}{c}\text { Area } \\
{\left[\mathrm{mAU} U^{*} \mathrm{~s}\right]}\end{array}$ & $\begin{array}{l}\text { Height } \\
{[\mathrm{mAU}]}\end{array}$ & $\begin{array}{c}\text { Area } \\
\%\end{array}$ \\
\hline 1 & $8.876 \mathrm{BB}$ & $\odot .1718$ & 56.58400 & 5.08520 & 4.3055 \\
\hline 2 & $9.665 \mathrm{BB}$ & 0.2122 & 1257.65540 & 90.48111 & 95.6945 \\
\hline Tot & & & & 95.56631 & \\
\hline
\end{tabular}

Signal 4: DAD1 D, Sig $=280,4$ Ref $=360,100$

Signal 5: DAD1 E, Sig $=310,4$ Ref $=360,100$

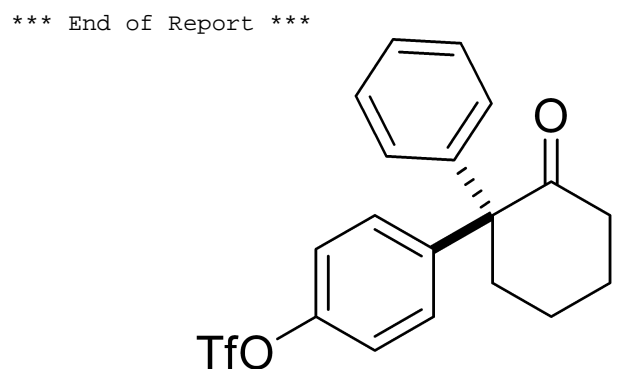

$6 a$

S-378 


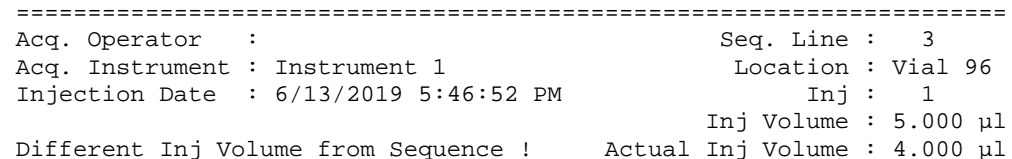

Injection Date $\quad$ Inj Volume $: 5.000 \mu \mathrm{\mu l}$

Acq. Method : C: \CHEM32\1\DATA QDDY 2019-06-13 17-12-47\IC-01-20.M

Last changed : 6/13/2019 5:24:55 PM

Analysis Method : C: \CHEM32\1\METHODS\IC-01-40.M- - .5.M

Last changed : 6/5/2019 1:46:08 PM

Additional Info (modified after loading)
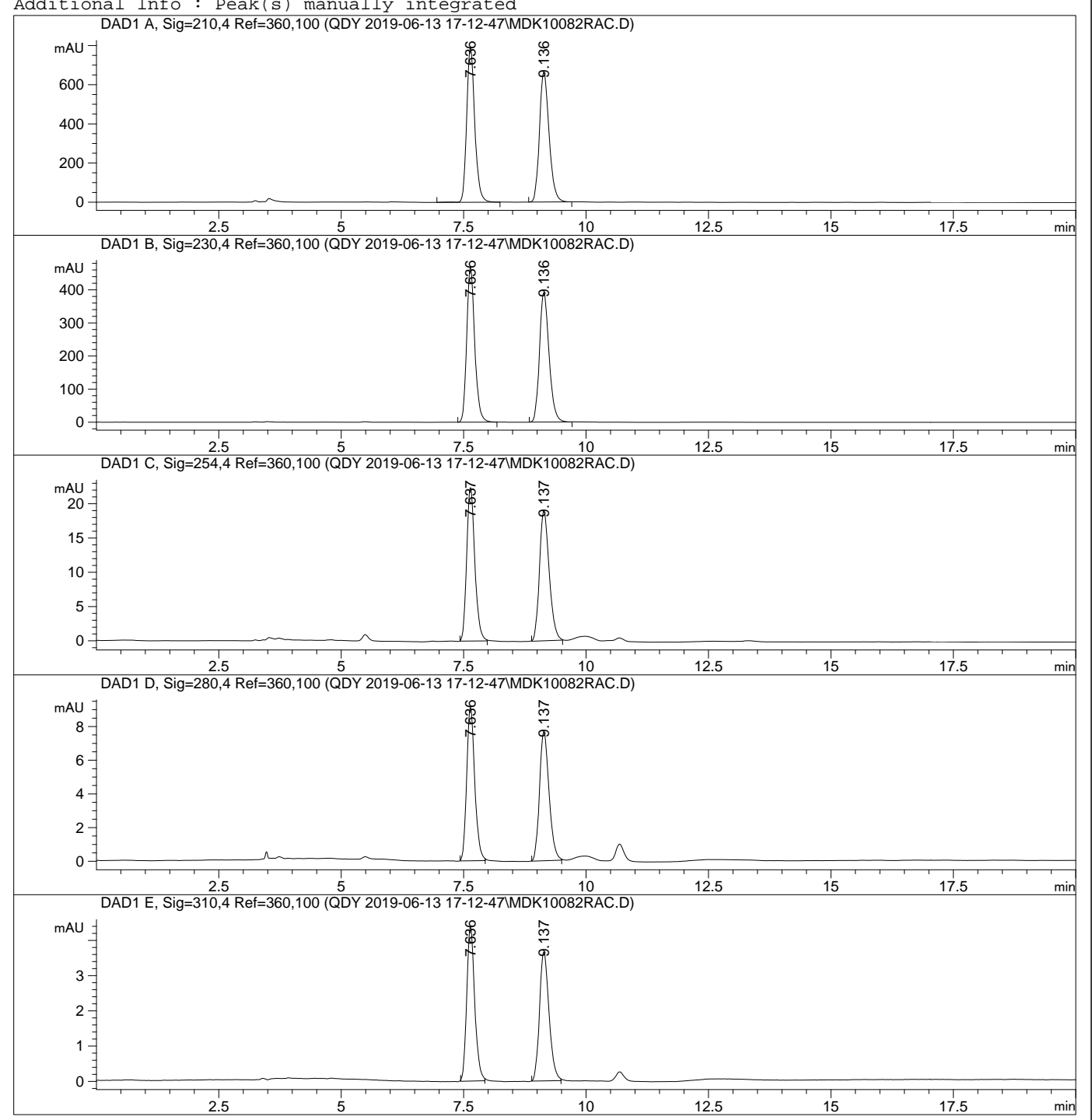

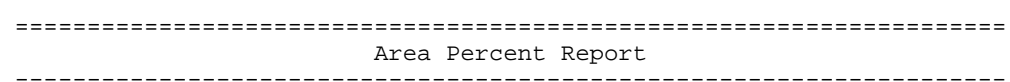

Sorted By

Signal

Multiplier

1.0000

Use Multiplier \& Dilution Factor with ISTDs

Signal 1: DAD1 A, Sig=210, 4 Ref $=360,100$

\begin{tabular}{cccccc}
$\begin{array}{c}\text { Peak RetTime Type } \\
\#\end{array}\left[\begin{array}{c}\text { Width } \\
{[\mathrm{min}]}\end{array}\right.$ & $\begin{array}{c}\text { Area } \\
{[\mathrm{mAU}}\end{array}$ & $\begin{array}{l}\text { Height } \\
{[\mathrm{mAU}]}\end{array}$ & $\begin{array}{c}\text { Area } \\
\%\end{array}$ \\
\hdashline-1 & $7.636 \mathrm{BB}$ & 0.1784 & 9075.38770 & 787.94690 & 50.0948 \\
\hline 2 & $9.136 \mathrm{BB}$ & 0.2111 & 9041.04395 & 662.86804 & 49.9052
\end{tabular}

Totals :

$1.81164 \mathrm{e} 4 \quad 1450.81494$

Signal 2: DAD1 B, Sig=230, 4 Ref $=360,100$

\begin{tabular}{cccccc}
$\begin{array}{c}\text { Peak RetTime Type } \\
\text { \# }\end{array}\left[\begin{array}{c}\text { Width } \\
{[\mathrm{min}]}\end{array}\right.$ & $\begin{array}{c}\text { Area } \\
{[\mathrm{mAU}}\end{array}$ & $\begin{array}{l}\text { Height } \\
{[\mathrm{mAU}]}\end{array}$ & $\begin{array}{c}\text { Area } \\
\%\end{array}$ \\
\hdashline 1 & $7.636 \mathrm{BB}$ & 0.1749 & 5316.97900 & 466.67169 & 50.0266 \\
\hdashline 2 & $9.136 \mathrm{BB}$ & 0.2103 & 5311.32324 & 391.32581 & 49.9734
\end{tabular}

Totals :

$1.06283 \mathrm{e} 4 \quad 857.99750$

Signal 3: DAD1 C, Sig=254, 4 Ref $=360,100$

\begin{tabular}{|c|c|c|c|c|c|}
\hline $\begin{array}{c}\text { Peak } \\
\#\end{array}$ & $\begin{array}{l}\text { RetTime Type } \\
\text { [min] }\end{array}$ & $\begin{array}{l}\text { Width } \\
{[\mathrm{min}]}\end{array}$ & $\begin{array}{c}\text { Area } \\
{\left[\mathrm{mAU} U^{\star} \mathrm{s}\right]}\end{array}$ & $\begin{array}{l}\text { Height } \\
{[\mathrm{mAU}]}\end{array}$ & $\begin{array}{c}\text { Area } \\
\%\end{array}$ \\
\hline 1 & $7.637 \mathrm{BB}$ & 0.1784 & & 0559 & 6621 \\
\hline 2 & $9.137 \mathrm{BB}$ & 0.2132 & 261.68677 & 18.93822 & 50.3379 \\
\hline
\end{tabular}

Totals

$519.86038 \quad 41.34381$

Signal 4: DAD1 D, Sig=280, 4 Ref $=360,100$

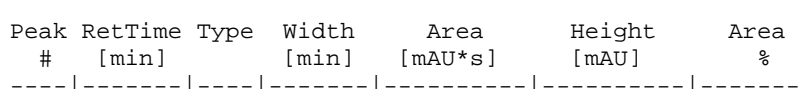
1 7.636 BB $0.1766 \quad 103.51466 \quad 9.1083749 .9719$

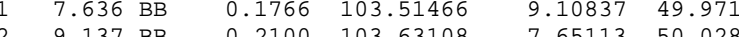
Totals : $207.14574 \quad 16.75949$

Signal 5: DAD1 E, Sig $=310,4$ Ref $=360,100$ $\begin{array}{ccccc}\text { Peak RetTime Type Width } & \text { Area } & \text { Height } & \text { Area } \\ \text { [ }[\mathrm{min}] & {[\mathrm{min}]} & {\left[\mathrm{mAU} \mathrm{U}^{\star} \mathrm{s}\right]} & {[\mathrm{mAU}]} & \%\end{array}$

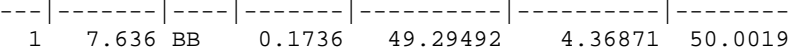

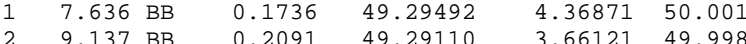

$98.58602 \quad 8.02992$ 


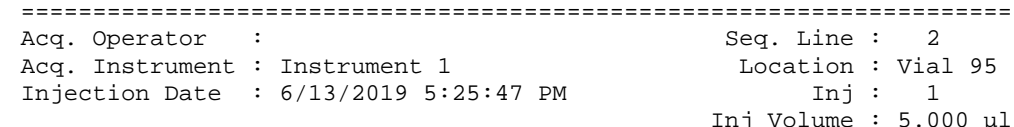

Injection Date Inj volume $\vdots 5.000 \mu \mathrm{l}$

Last changed : 6/13/2019 5:24:55 PM

Analysis Method: C: \CHEM32\1\METHODS\IC-01-40.M- - .5.M

Last changed : 6/5/2019 1:46:08 PM

Additional Info: Peak(s) manually inteng)
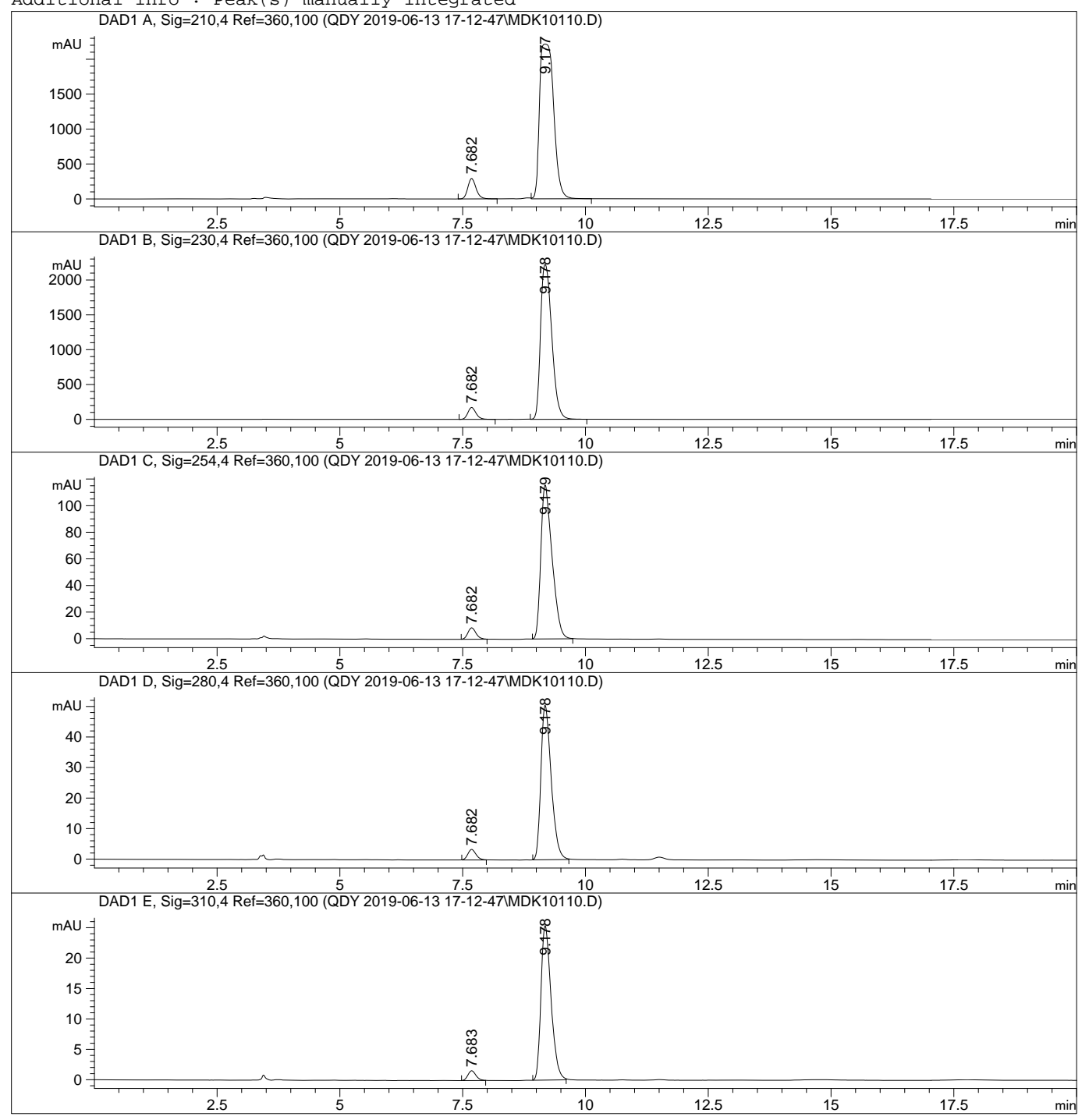

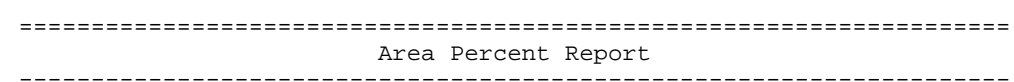

Sorted By

Signal

Multiplier

1.0000

Use Multiplier \& Dilution Factor with ISTDs

Signal 1: DAD1 A, Sig=210, 4 Ref $=360,100$

\begin{tabular}{|c|c|c|c|c|c|}
\hline eak & $\begin{array}{l}\text { RetTime Type } \\
\text { [min] }\end{array}$ & $\begin{array}{l}\text { Width } \\
\text { [min] }\end{array}$ & $\begin{array}{c}\text { Area } \\
{\left[\mathrm{mAU}{ }^{\star} \mathrm{s}\right]}\end{array}$ & $\begin{array}{l}\text { Height } \\
{[\mathrm{mAU}]}\end{array}$ & $\begin{array}{c}\text { Area } \\
\%\end{array}$ \\
\hline & 9 & & & & \\
\hline
\end{tabular}

$\begin{array}{lllllll}1 & 7.682 & \text { BB } & 0.1782 & 3426.79370 & 293.56964 & 7.1751 \\ 2 & 9.177 \text { VB } & 0.3195 & 4.43327 \mathrm{e} 4 & 2215.40601 & 92.8249\end{array}$

Totals : $\quad 4.77595 \mathrm{e} 4 \quad 2508.97565$

Signal 2: DAD1 B, Sig=230, 4 Ref $=360,100$

\begin{tabular}{cccccc}
$\begin{array}{c}\text { Peak RetTime Type } \\
\#\end{array}\left[\begin{array}{c}\text { Width } \\
{[\mathrm{min}]}\end{array}\right.$ & $\begin{array}{c}\text { Area } \\
{[\mathrm{mAU}}\end{array}$ & $\begin{array}{l}\text { Height } \\
{[\mathrm{mAU}]}\end{array}$ & $\begin{array}{c}\text { Area } \\
\%\end{array}$ \\
\hdashline-1 & $7.682 \mathrm{BB}$ & 0.1776 & 1993.10388 & 171.46590 & 5.3600 \\
2 & 9.178 VB & 0.2488 & $3.51917 \mathrm{e} 4$ & 2222.18213 & 94.6400
\end{tabular}

Totals :

$3.71848 \mathrm{e} 4 \quad 2393.64803$

Signal 3: DAD1 C, Sig=254, 4 Ref $=360,100$

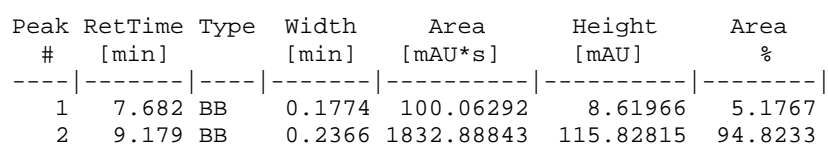

Totals :

$1932.95135 \quad 124.44780$

Signal 4: DAD1 D, Sig=280, 4 Ref $=360,100$

\begin{tabular}{|c|c|c|c|c|c|}
\hline $\begin{array}{c}\text { Peak } \\
\#\end{array}$ & $\begin{array}{l}\text { RetTime Type } \\
\text { [min] }\end{array}$ & $\begin{array}{l}\text { Width } \\
\text { [min] }\end{array}$ & $\begin{array}{c}\text { Area } \\
{\left[\mathrm{mAU} U^{*} \mathrm{~s}\right]}\end{array}$ & $\begin{array}{l}\text { Height } \\
{[\mathrm{mAU}]}\end{array}$ & $\begin{array}{c}\text { Area } \\
\%\end{array}$ \\
\hline 1 & $7.682 \mathrm{BB}$ & 0.1772 & 40.04298 & 3.45654 & 5.1604 \\
\hline 2 & $9.178 \mathrm{BB}$ & 0.2237 & 735.91656 & 50.59505 & 94.8396 \\
\hline
\end{tabular}

Totals :

$775.95955 \quad 54.05159$

Signal 5: DAD1 E, Sig $=310,4$ Ref $=360,100$

\begin{tabular}{|c|c|c|c|c|c|}
\hline eak & $\begin{array}{l}\text { RetTime Type } \\
\text { [min] }\end{array}$ & $\begin{array}{l}\text { Width } \\
\text { [min] }\end{array}$ & $\begin{array}{c}\text { Area } \\
{\left[\mathrm{mAU} U^{*} \mathrm{~s}\right]}\end{array}$ & $\begin{array}{l}\text { Height } \\
{[\mathrm{mAU}]}\end{array}$ & $\begin{array}{c}\text { Area } \\
\%\end{array}$ \\
\hline & $\begin{array}{l}7.683 \\
9.178\end{array}$ & 2020 & $\begin{array}{ll}10.4130 \\
62\end{array}$ & & \\
\hline
\end{tabular}




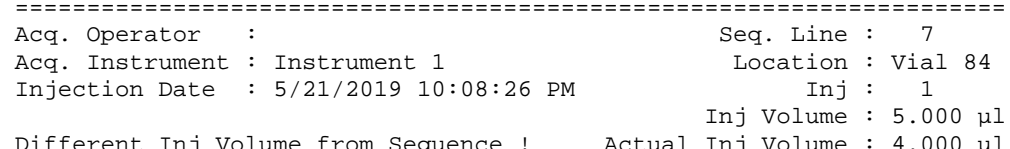

Injection Date Inj Volume : $5.000 \mu \mathrm{\mu l}$ Acq. Method : C: \CHEM32\1\DATA QQDY 2019-05-21 19-08-11\AD-01-20.M Last changed : 5/21/2019 9:46:26 PM

Analysis Method : C: \CHEM32\1\METHODS \IC-01-40.M-0.5.M

Last changed : 6/5/2019 1:46:08 PM

Additional Info: (modified after loading)
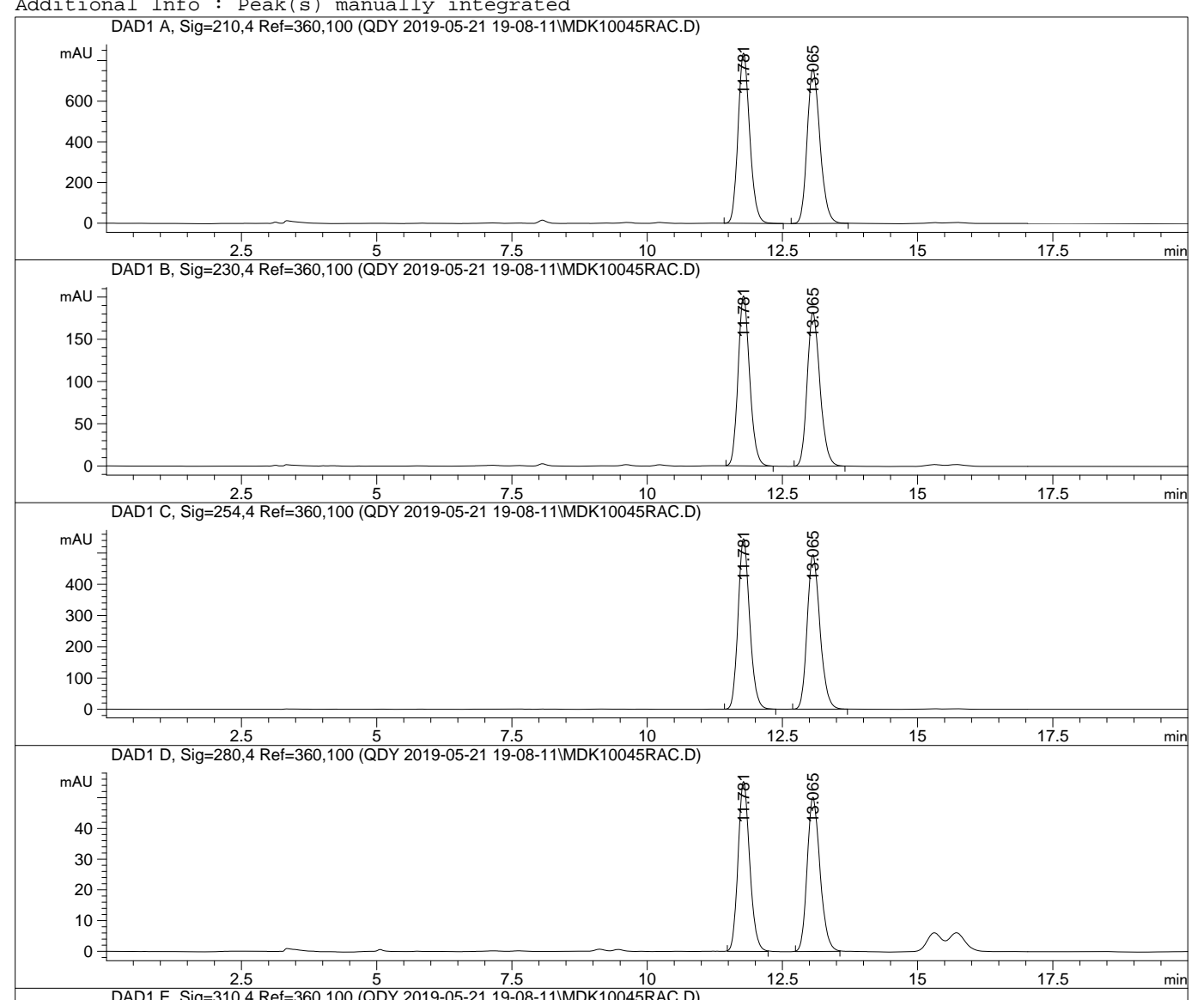

mAU
$5=$
$4=$
$3=$
2
2
1
0

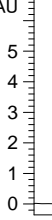

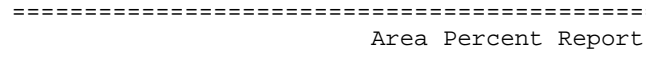

Sorted By

Signal

Multiplie
Dilution

1.0000

Use Multiplier \& Dilution Factor with ISTDs

Signal 1: DAD1 A, Sig=210, 4 Ref $=360,100$

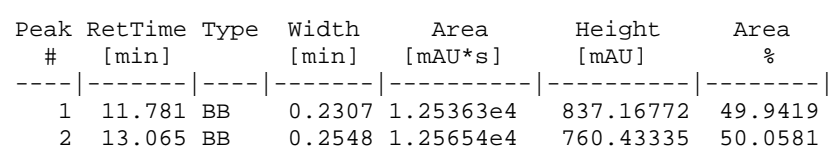

Totals :

$2.51017 \mathrm{e} 4 \quad 1597.60107$

Signal 2: DAD1 B, Sig=230, 4 Ref $=360,100$

\begin{tabular}{cccccc}
$\begin{array}{c}\text { Peak RetTime Type } \\
\#\end{array}\left[\begin{array}{c}\text { Width } \\
{[\mathrm{min}]}\end{array}\right.$ & $\begin{array}{c}\text { Area } \\
{[\mathrm{mAU}}\end{array}$ & $\begin{array}{l}\text { Height } \\
{[\mathrm{mAU}]}\end{array}$ & $\begin{array}{c}\text { Area } \\
\%\end{array}$ \\
\hdashline-11.781 & $\mathrm{BB}$ & 0.2295 & 2994.56470 & 201.36710 & 49.9937 \\
\hline 2 & $13.065 \mathrm{BB}$ & 0.2536 & 2995.31836 & 182.34737 & 50.0063
\end{tabular}

Totals :

$5989.88306 \quad 383.71446$

Signal 3: DAD1 C, Sig=254, 4 Ref $=360,100$

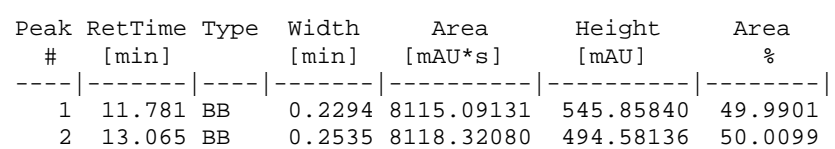

Totals :

$1.62334 \mathrm{e} 4 \quad 1040.43976$

Signal 4: DAD1 D, Sig=280, 4 Ref $=360,100$

Peak RetTime Type Width Area Height Area

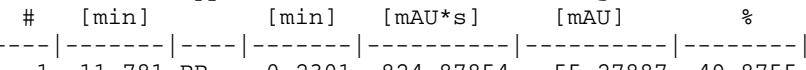

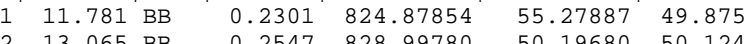

Totals :

$1653.87634 \quad 105.47567$

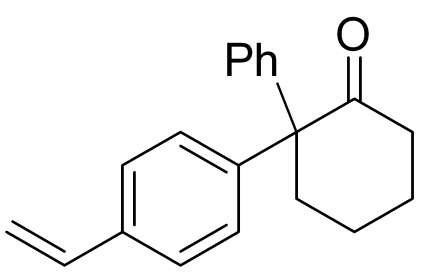

racemic

Signal 5: DAD1 E, Sig=310, 4 Ref $=360,100$

*** End of Report *** 


\section{Data File C: \CHEM32\1\DATA\QDY 2019-05-21 19-08-11\MDK10061.D}

Sample Name
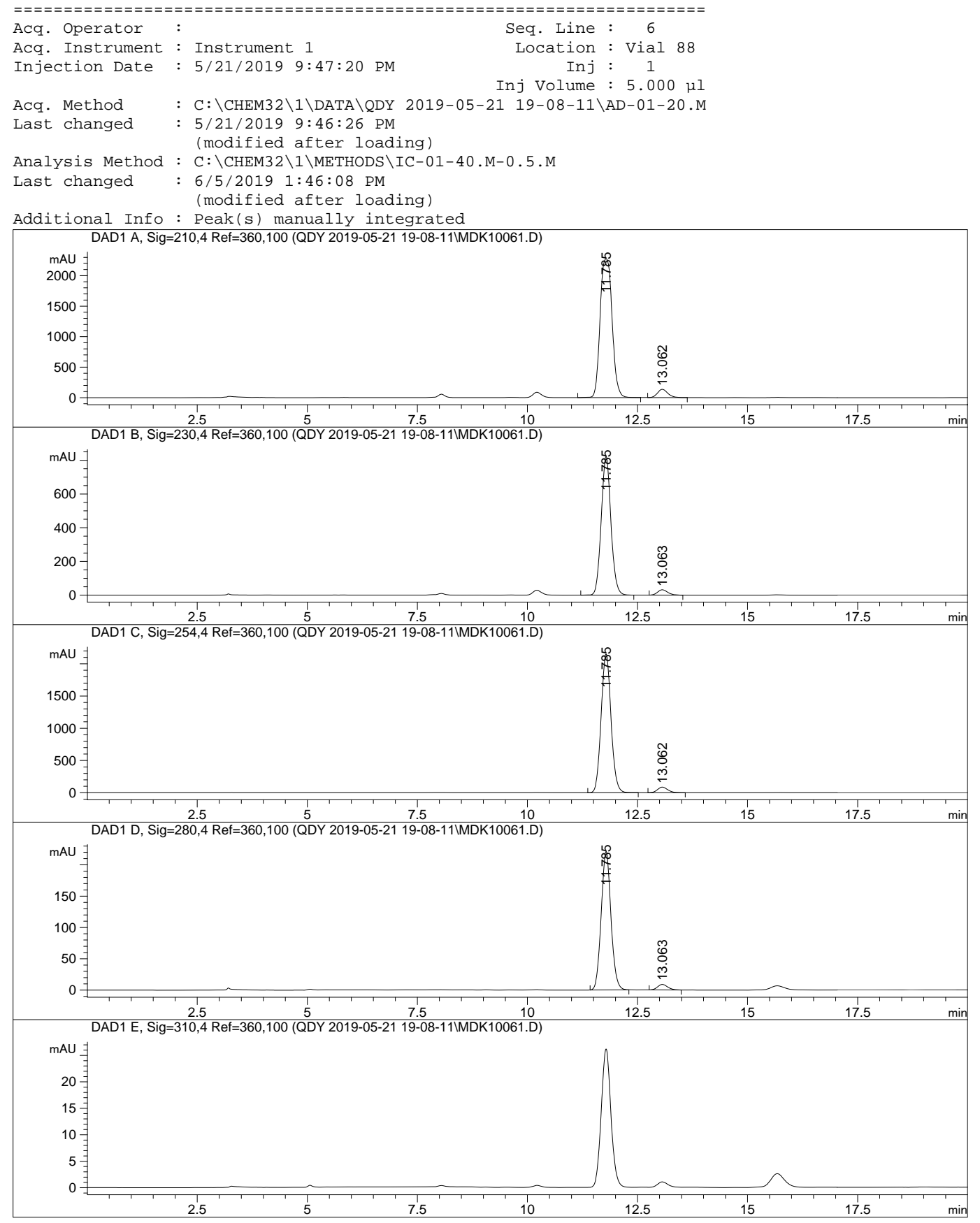

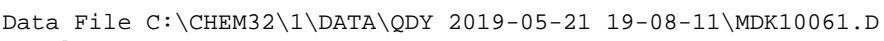
Sample Name

Area Percent Report

$\begin{array}{lll}\text { Sorted By } & : & \text { Signal } \\ \text { Multiplier } & \vdots & 1.0000 \\ \text { Dilution } & : & 1.0000\end{array}$

Use Multiplier \& Dilution Factor with ISTDs

Signal 1: DAD1 A, Sig=210, 4 Ref $=360,100$

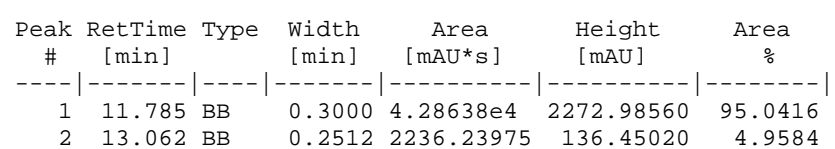

Totals :

$4.51000 \mathrm{e} 4 \quad 2409.43579$

Signal 2: DAD1 B, Sig=230, 4 Ref $=360,100$

\begin{tabular}{cccccc}
$\begin{array}{c}\text { Peak RetTime Type } \\
\#\end{array}\left[\begin{array}{c}\text { Width } \\
{[\mathrm{min}]}\end{array}\right.$ & $\begin{array}{c}\text { Area } \\
{[\mathrm{mAU}}\end{array}$ & $\begin{array}{c}\text { Height } \\
{[\mathrm{mAU}]}\end{array}$ & $\begin{array}{c}\text { Area } \\
\%\end{array}$ \\
\hdashline-11.785 & $\mathrm{BB}$ & 0.2348 & $1.26028 \mathrm{e} 4$ & 822.45331 & 96.0896 \\
\hline 2 & $13.063 \mathrm{BB}$ & 0.2465 & 512.87469 & 32.07772 & 3.9104
\end{tabular}

Totals :

$1.31157 \mathrm{e} 4 \quad 854.53103$

Signal 3: DAD1 C, Sig $=254,4$ Ref $=360,100$

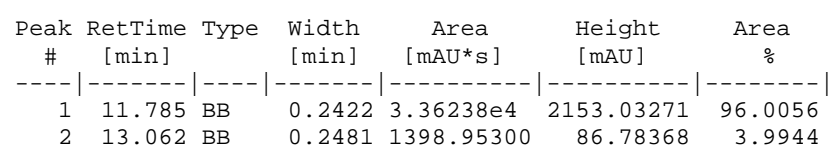

Totals :

$3.50228 \mathrm{e} 4 \quad 2239.81640$

Signal 4: DAD1 D, Sig=280, 4 Ref $=360,100$

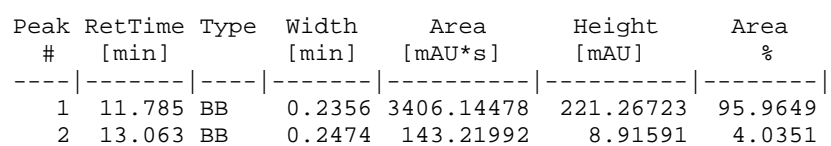

Totals :

$3549.36470 \quad 230.18314$

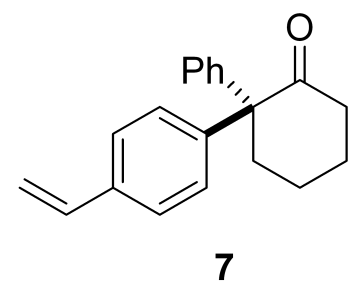

Signal 5: DAD1 E, Sig=310, 4 Ref $=360,100$

Enantioenriched

$* * *$ End of Report *** 


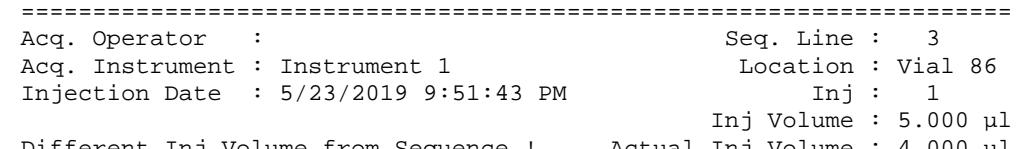

$\begin{array}{ll}\text { Injection Date : } & \text { Inj }: 1 \\ \text { Different Inj Volume from Sequence ! Actual Inj Volume }: 5.000 \mu \mathrm{\mu}\end{array}$

Acq. Method : C: \CHEM32\1\DATA\QDY 2019-05-23 21-06-33\AD-01-30.M

Last changed : 5/23/2019 9:19:34 PM

Analysis Method : C: $\backslash$ CHEM32\1\METHODS \IC-01-40.M- - 0.5.M

Last changed : 6/5/2019 1:46:08 PM

Additional Info: Peak(s) manually integrated
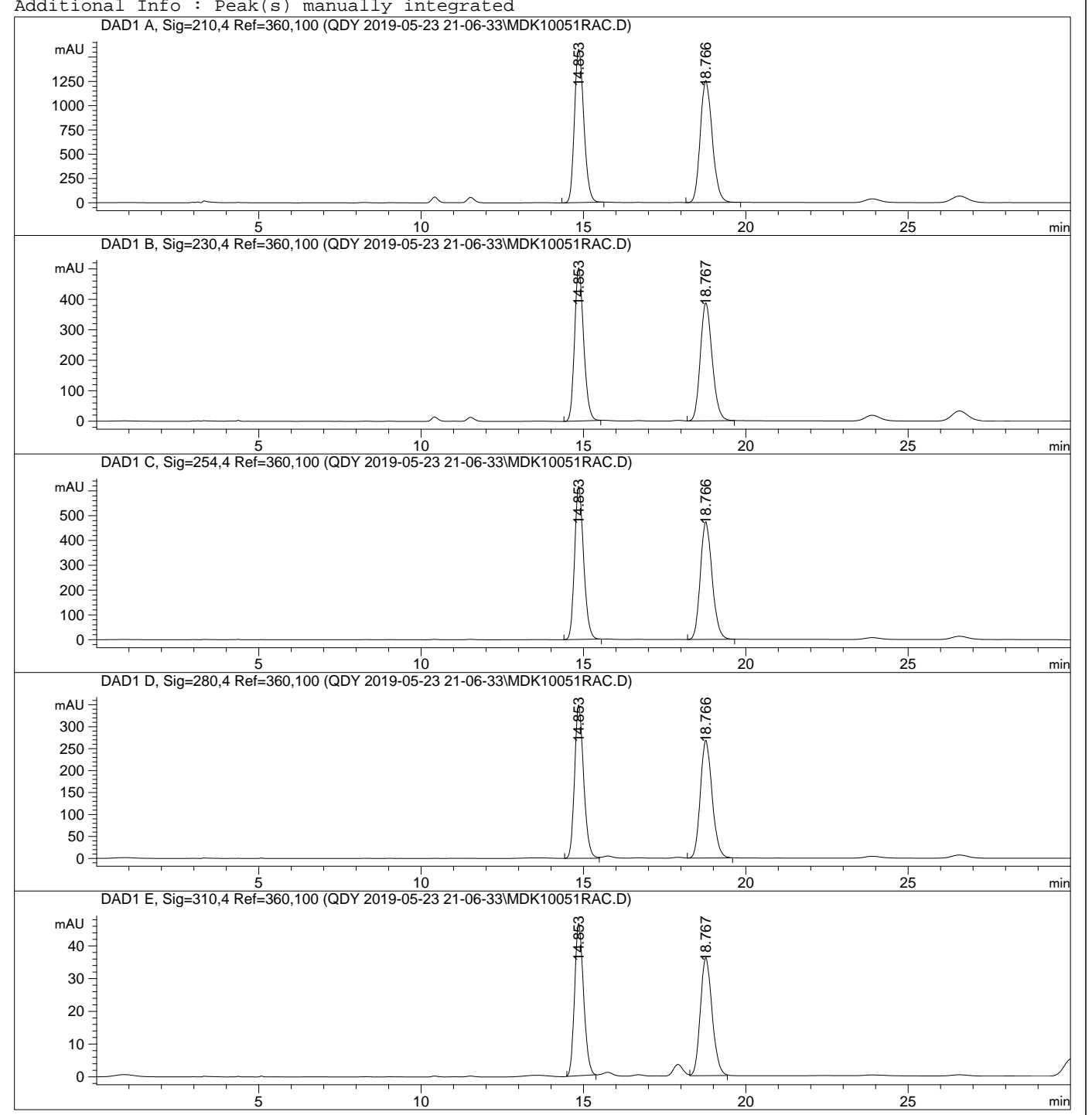

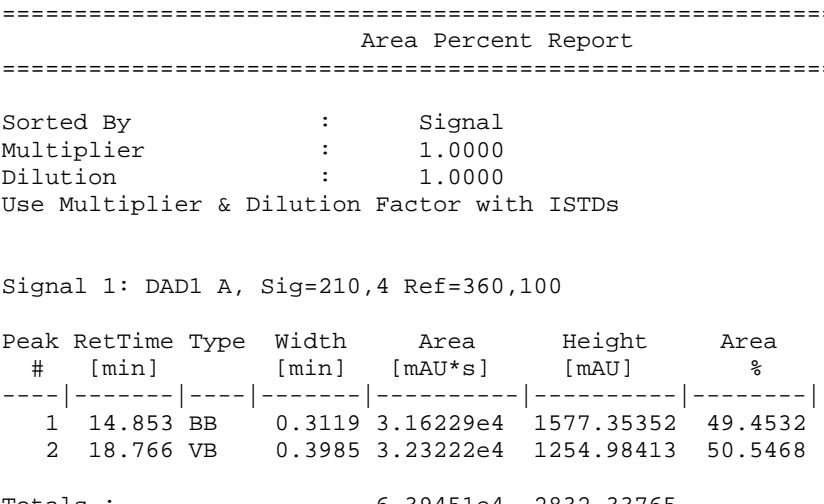

Totals :

$6.39451 \mathrm{e} 4 \quad 2832.33765$

Signal 2: DAD1 B, Sig $=230,4$ Ref $=360,100$

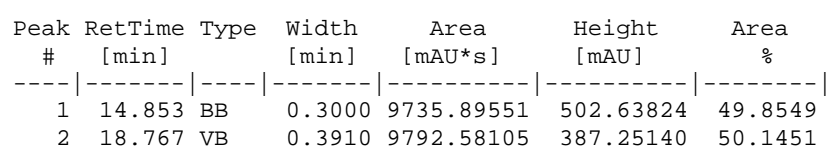

Totals :

$1.95285 \mathrm{e} 4 \quad 889.88965$

Signal 3: DAD1 C, Sig $=254,4$ Ref $=360,100$

\begin{tabular}{|c|c|c|c|c|c|}
\hline $\begin{array}{c}\text { Peak } \\
\#\end{array}$ & $\begin{array}{l}\text { RetTime Type } \\
\text { [min] }\end{array}$ & $\begin{array}{l}\text { Width } \\
\text { [min] }\end{array}$ & $\begin{array}{c}\text { Area } \\
{\left[\mathrm{mAU}^{\star} \mathrm{s}\right]}\end{array}$ & $\begin{array}{l}\text { Height } \\
{[\mathrm{mAU}]}\end{array}$ & $\begin{array}{c}\text { Area } \\
\%\end{array}$ \\
\hline 1 & $14.853 \mathrm{BB}$ & 0.2993 & $1.19155 \mathrm{e} 4$ & 617.12683 & 49.9097 \\
\hline 2 & $18.766 \mathrm{BB}$ & 0.3900 & $1.19587 \mathrm{e} 4$ & 474.47964 & 50.0903 \\
\hline
\end{tabular}

$2.38742 \mathrm{e} 4 \quad 1091.60648$

Signal 4: DAD1 D, Sig=280, 4 Ref $=360,100$

$\begin{array}{ccccc}\text { Peak RetTime Type } & \text { Width } & \text { Area } & \text { Height } & \text { Area } \\ \# \quad[\mathrm{~min}] & {[\mathrm{min}]} & {\left[\mathrm{mAU} U^{\star} \mathrm{s}\right]} & {[\mathrm{mAU}]} & \%\end{array}$

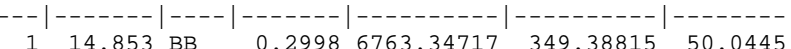

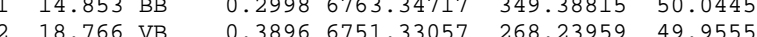

Totals :

$1.35147 \mathrm{e} 4 \quad 617.62775$

$\mathrm{PhS}$

Signal 5: DAD1 E, Sig=310, 4 Ref $=360,100$

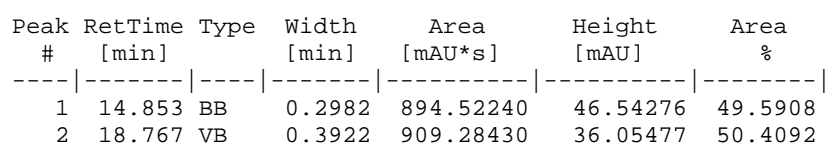

racemic

Totals : $\quad 1803.80670 \quad 82.59753$ 


\begin{tabular}{|c|c|c|}
\hline $\begin{array}{l}\text { Acq. Operator } \\
\text { Acq. Instrument }\end{array}$ & Instrument 1 & $\begin{array}{r}\text { Seq. Line : } \\
\text { Location }\end{array}$ \\
\hline Injection Date & $5 / 23 / 20199: 20: 30 \mathrm{PM}$ & Inj : 1 \\
\hline
\end{tabular}

Injection Date Inj volume $: 5.000 \mu \mathrm{l}$

Acq. Method
Last changed $5 / 23 / 2019$ 9:19:34 PM

Analysis Method : C: : \CHEM32\1\METHODS\IC-01-40.M-0.5.M

Last changed : 6/5/2019 1:46:08 PM

Additional Info: Peak(s) manually integr
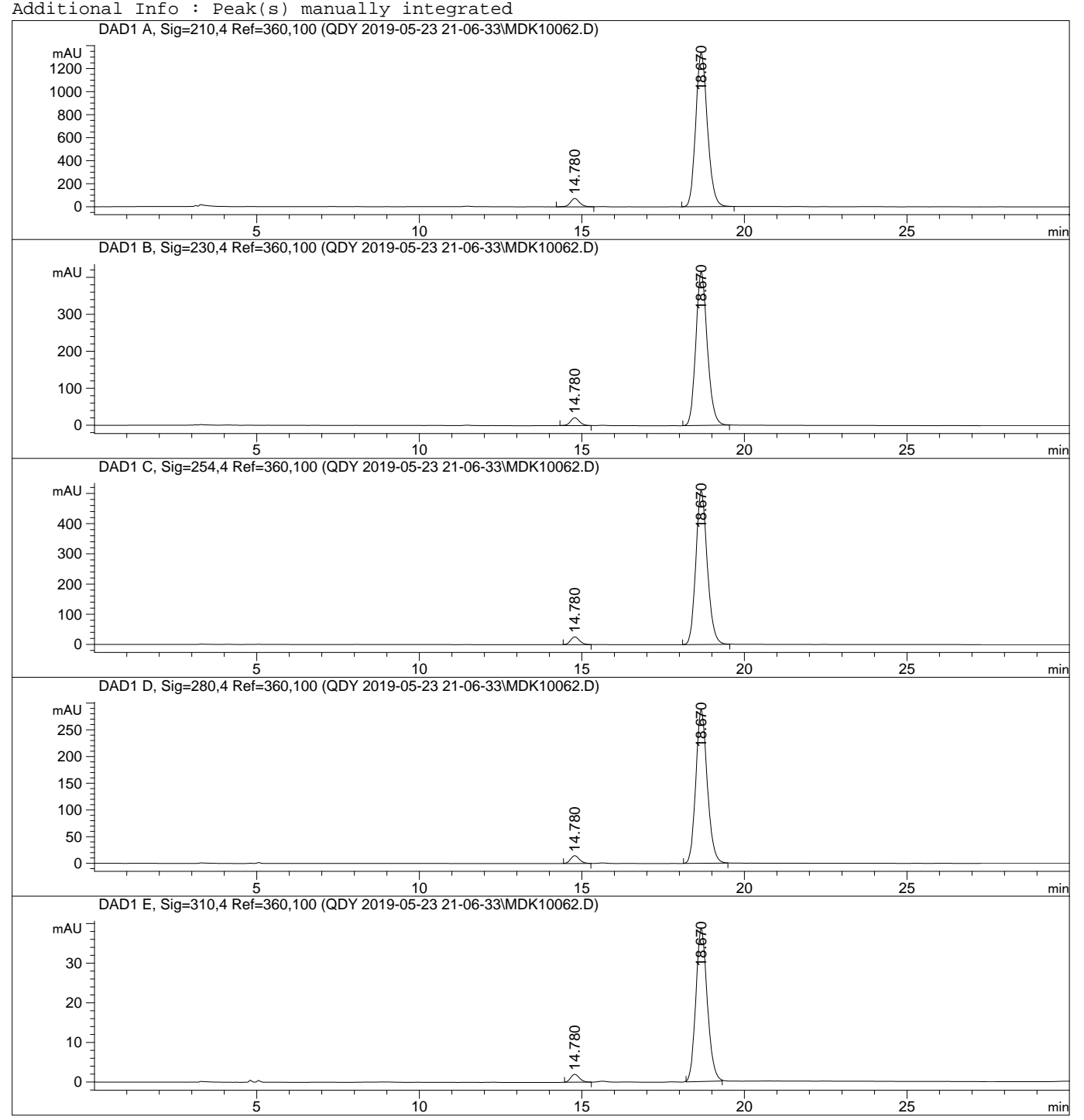

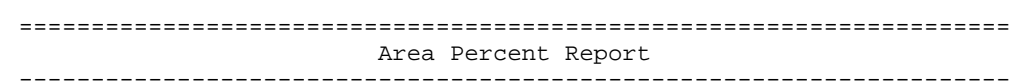

Sorted By

Signal

Multiplier

1.0000

Use Multiplier \& Dilution Factor with ISTDs

Signal 1: DAD1 A, Sig=210, 4 Ref $=360,100$

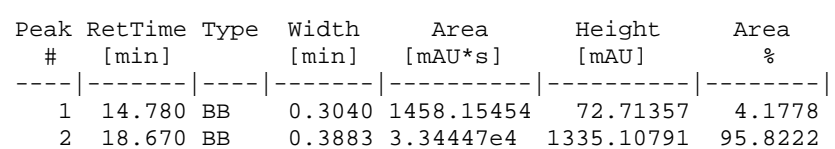

Totals :

$3.49028 \mathrm{e} 4 \quad 1407.82148$

Signal 2: DAD1 B, Sig=230, 4 Ref $=360,100$

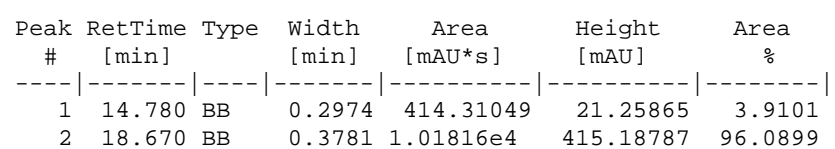

Totals :

$1.05959 \mathrm{e} 4 \quad 436.44652$

Signal 3: DAD1 C, Sig $=254,4$ Ref $=360,100$

\begin{tabular}{cccccc}
$\begin{array}{c}\text { Peak RetTime Type } \\
\text { \# }\end{array}\left[\begin{array}{c}\text { Width } \\
{[\mathrm{min}]}\end{array}\right.$ & $\begin{array}{c}\text { Area } \\
{[\mathrm{mAU}}\end{array}$ & $\begin{array}{c}\text { Height } \\
{[\mathrm{mAU}]}\end{array}$ & $\begin{array}{c}\text { Area } \\
\%\end{array}$ \\
\hdashline-14.780 & $\mathrm{BB}$ & 0.2922 & 492.48422 & 25.85876 & 3.8103 \\
\hline 2 & $18.670 \mathrm{BB}$ & 0.3771 & $1.24326 \mathrm{e} 4$ & 508.77737 & 96.1897
\end{tabular}

Totals :

$1.29251 \mathrm{e} 4 \quad 534.63613$

Signal 4: DAD1 D, Sig=280, 4 Ref $=360,100$

$\begin{array}{ccccc}\text { Peak RetTime Type } & \text { Width } & \text { Area } & \text { Height } & \text { Area } \\ \# \quad[\mathrm{~min}] & {[\mathrm{min}]} & {\left[\mathrm{mAU} U^{\star} \mathrm{s}\right]} & {[\mathrm{mAU}]} & \%\end{array}$

$\begin{array}{llllll}14.780 \mathrm{BB} & 0.2909 & 275.31250 & 14.53987 & 3.7759\end{array}$

$2 \quad 18.670 \mathrm{BB} \quad 0.3767 \quad 7016.00244 \quad 287.55405 \quad 06.2241$

Totals :

$7291.31494 \quad 302.09391$

$\mathrm{PhS}$

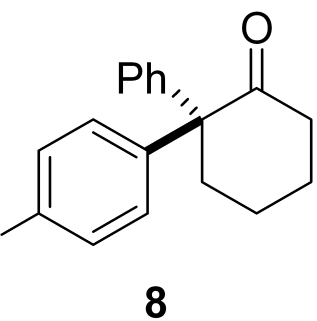

Signal 5: DAD1 E, Sig=310, 4 Ref $=360,100$

\begin{tabular}{|c|c|c|c|c|c|}
\hline $\begin{array}{c}\text { Peak } \\
\text { \# }\end{array}$ & $\begin{array}{l}\text { RetTime Type } \\
\text { [min] }\end{array}$ & $\begin{array}{l}\text { Width } \\
\text { [min] }\end{array}$ & $\begin{array}{c}\text { Area } \\
{\left[\mathrm{mAU}^{\star} \mathrm{s}\right]}\end{array}$ & $\begin{array}{l}\text { Height } \\
{[\mathrm{mAU}]}\end{array}$ & $\begin{array}{c}\text { Area } \\
\%\end{array}$ \\
\hline 1 & $14.780 \mathrm{BB}$ & $\odot .2929$ & 36.69494 & 1.97381 & 3.7465 \\
\hline 2 & $18.670 \mathrm{BB}$ & 0.3770 & 942.76196 & 38.59030 & 96.2535 \\
\hline
\end{tabular}

Enantioenriched 


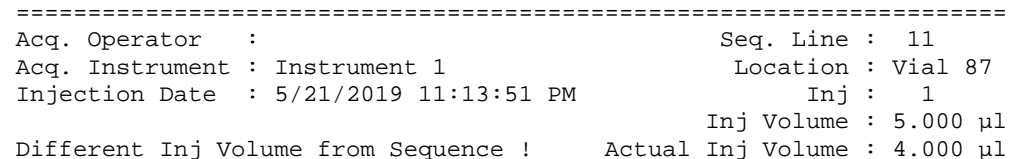

Injection Date $\quad$ Inj Volume $: 5.000 \mu \mathrm{\mu l}$

Acq. Method : C: \CHEM32\1\DATA\QDY 2019-05-21 19-08-11\AD-01-20.M

Last changed : 9/4/2012 9:48:16 AM

Analysis Method : C: $\backslash$ CHEM32\1\METHODS \IC-01-40.M-0.5.M

Last changed : 6/5/2019 1:46:08 PM

Additional Info (modified after loading)
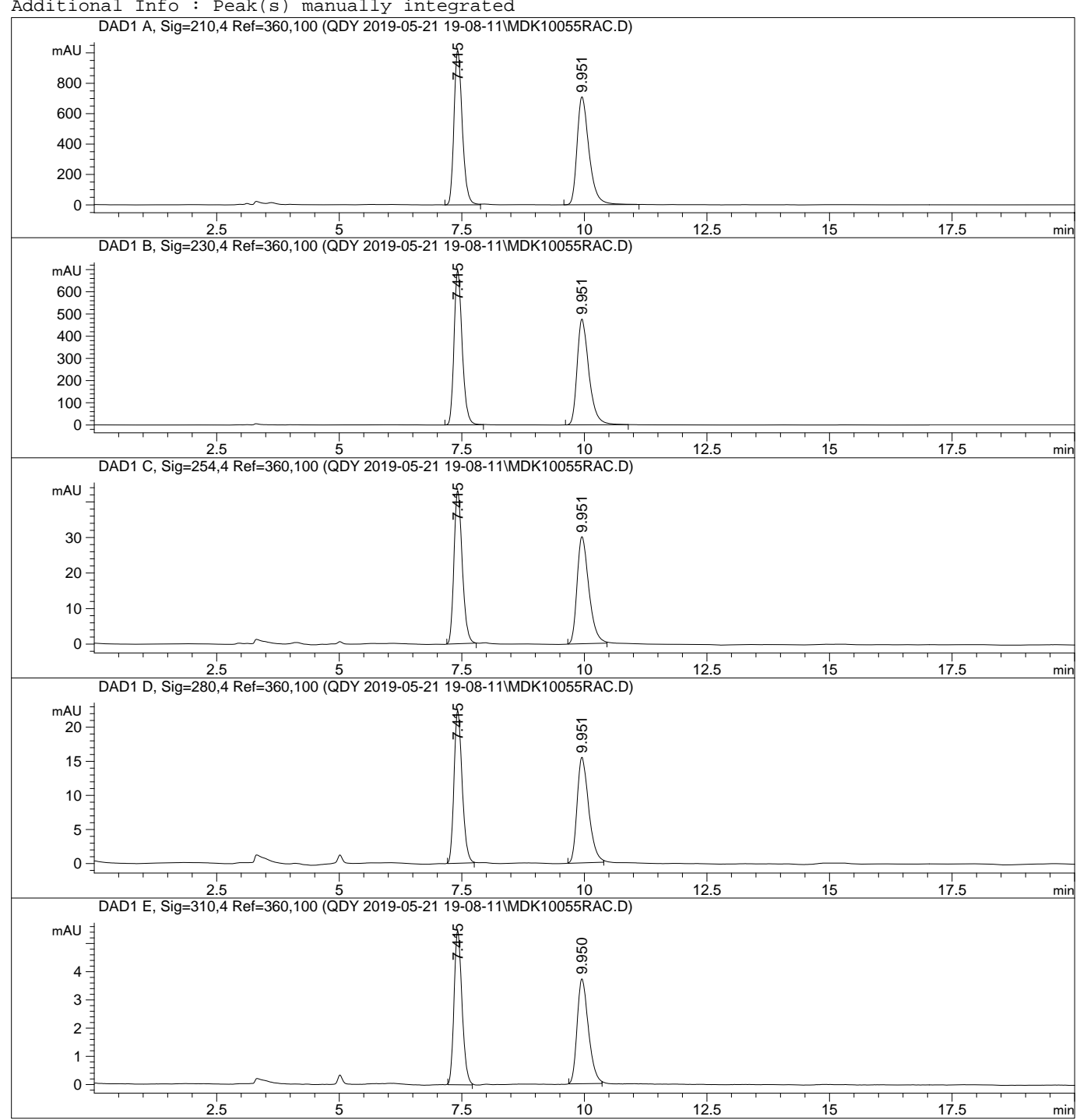

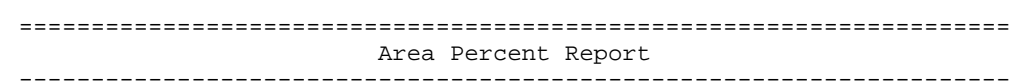

Sorted By
Multiplier

Signal

1.0000

Use Multiplier \& Dilution Factor with ISTDs

Signal 1: DAD1 A, Sig=210, 4 Ref $=360,100$

\begin{tabular}{cccccc}
$\begin{array}{c}\text { Peak RetTime Type } \\
\text { \# }\end{array}\left[\begin{array}{c}\text { Width } \\
{[\mathrm{min}]}\end{array}\right.$ & $\begin{array}{c}\text { Area } \\
{[\mathrm{mAU}}\end{array}$ & $\begin{array}{c}\text { Height } \\
{[\mathrm{mAU}]}\end{array}$ & $\begin{array}{c}\text { Area } \\
\%\end{array}$ \\
\hdashline-1 & $7.415 \mathrm{BV}$ & 0.1803 & $1.18852 \mathrm{e} 4$ & 1017.33466 & 49.5994 \\
\hline 2 & $9.951 \mathrm{BB}$ & 0.2583 & $1.20772 \mathrm{e} 4$ & 710.38940 & 50.4006
\end{tabular}

Totals :

$2.39625 \mathrm{e} 4 \quad 1727.72406$

Signal 2: DAD1 B, Sig=230, 4 Ref $=360,100$

\begin{tabular}{cccccc}
$\begin{array}{c}\text { Peak RetTime Type } \\
\#\end{array}\left[\begin{array}{c}\text { Width } \\
{[\mathrm{min}]}\end{array}\right.$ & $\begin{array}{c}\text { Area } \\
{[\mathrm{mAU}}\end{array}$ & $\begin{array}{l}\text { Height } \\
{[\mathrm{mAU}]}\end{array}$ & $\begin{array}{c}\text { Area } \\
\%\end{array}$ \\
\hdashline-1 & $7.415 \mathrm{BB}$ & 0.1757 & 7984.08740 & 696.47046 & 50.0641 \\
2 & $9.951 \mathrm{BB}$ & 0.2549 & 7963.65430 & 476.79517 & 49.9359
\end{tabular}

Totals :

$1.59477 \mathrm{e} 4 \quad 1173.26563$

Signal 3: DAD1 C, Sig=254, 4 Ref $=360,100$

\begin{tabular}{|c|c|c|c|c|c|}
\hline $\begin{array}{c}\text { Peak } \\
\#\end{array}$ & $\begin{array}{l}\text { RetTime Type } \\
{[\text { min] }}\end{array}$ & $\begin{array}{l}\text { Width } \\
\text { [min] }\end{array}$ & $\begin{array}{c}\text { Area } \\
{\left[\mathrm{mAU} U^{\star} \mathrm{s}\right]}\end{array}$ & $\begin{array}{l}\text { Height } \\
{[\mathrm{mAU}]}\end{array}$ & $\begin{array}{c}\text { Area } \\
\%\end{array}$ \\
\hline 1 & $7.415 \mathrm{BB}$ & 0.180 & 502.85742 & 43.12761 & 344 \\
\hline 2 & $9.951 \mathrm{BB}$ & $\odot .2552$ & 504.17883 & 30.13542 & 50.0656 \\
\hline
\end{tabular}

Signal 4: DAD1 D, Sig $=280,4$ Ref $=360,100$

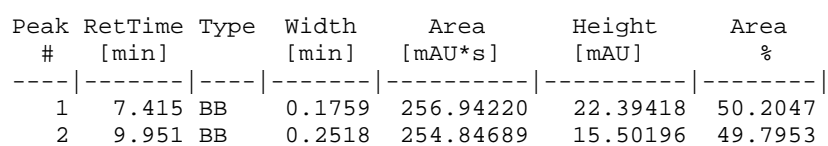

Totals :

$511.78909 \quad 37.89615$

Signal 5: DAD1 E, Sig $=310,4$ Ref $=360,100$

\begin{tabular}{|c|c|c|c|c|c|}
\hline $\begin{array}{l}\text { eak } \\
\#\end{array}$ & $\begin{array}{l}\text { RetTime Type } \\
\text { [min] }\end{array}$ & $\begin{array}{l}\text { Width } \\
\text { [min] }\end{array}$ & $\begin{array}{c}\text { Area } \\
{\left[\mathrm{mAU}^{\star} \mathrm{s}\right]}\end{array}$ & $\begin{array}{l}\text { Height } \\
{[\mathrm{mAU}]}\end{array}$ & $\begin{array}{c}\text { Area } \\
\%\end{array}$ \\
\hline & $\begin{array}{l}.415 \mathrm{BB} \\
.950 \mathrm{BB}\end{array}$ & $\begin{array}{l}.1733 \\
.2466\end{array}$ & $\begin{array}{l}61.46511 \\
59.43413\end{array}$ & & $\begin{array}{l}50.8400 \\
49.1600\end{array}$ \\
\hline
\end{tabular}

Totals :

$120.89924 \quad 9.17777$
Bpin

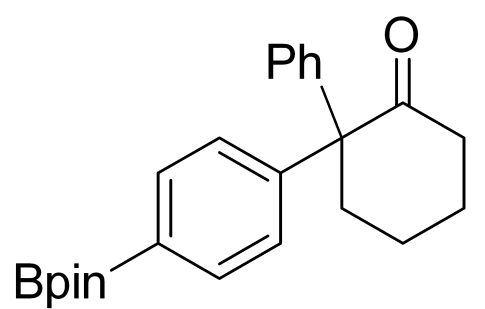

racemic 


\section{Data File C: \CHEM32\1\DATA\QDY 2019-05-21 19-08-11\MDK10063.D}

Sample Name:
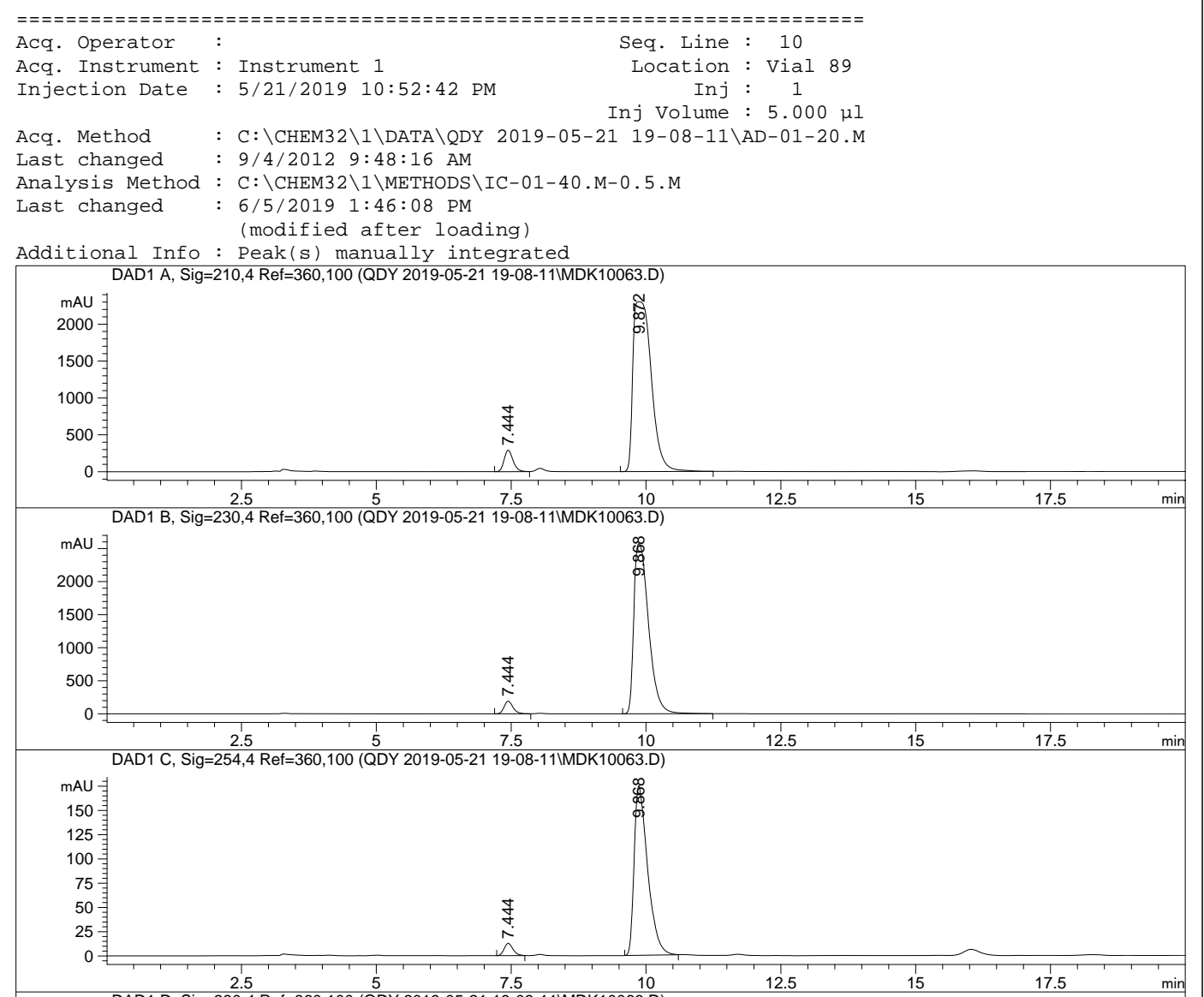

$\begin{array}{cccr}2.5 & 5 & 7.5 & 10 \\ \text { DAD1 D, Sig=280,4 Ref=360,100 (QDY 2019-05-21 } & 19-08-11 \text { IMDK10063.D }\end{array}$
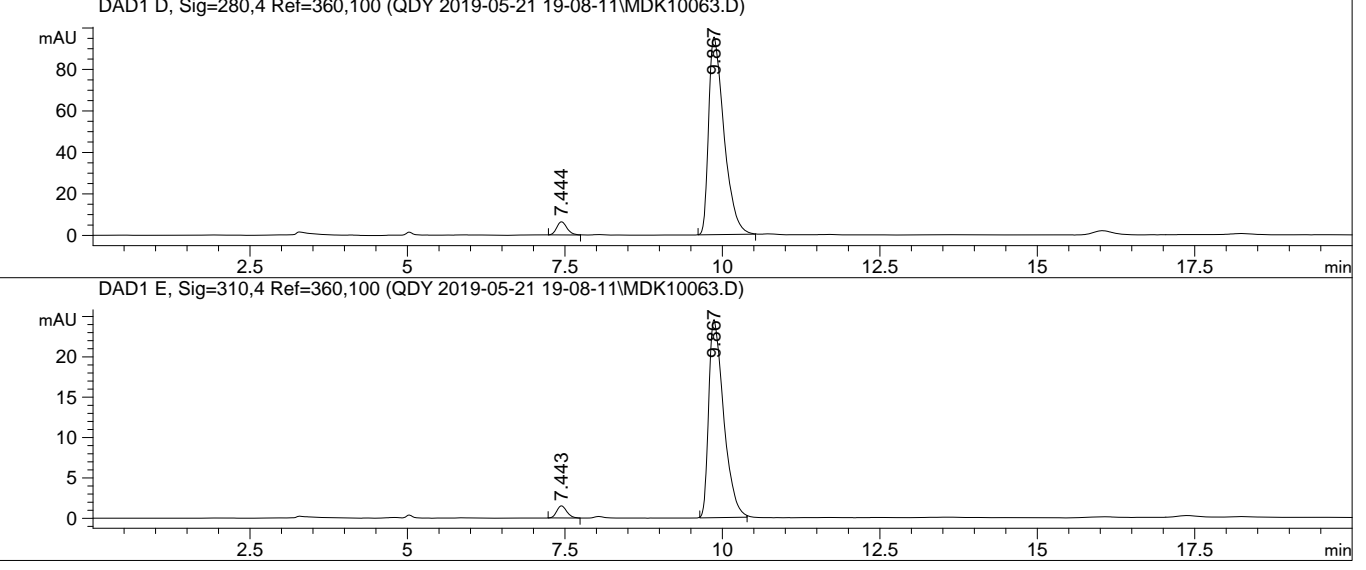

File C: \CHEM32\IDATA \ODY 2019-05-21 19-08-11\MDK10063.D sample Name:

Area Percent Report

$\begin{array}{lll}\text { Sorted By } & : & \text { Signal } \\ \text { Multiplier } & \vdots & 1.0000 \\ \text { Dilution } & : & 1.0000\end{array}$

Use Multiplier \& Dilution Factor with ISTDs

Signal 1: DAD1 A, Sig=210, 4 Ref $=360,100$

\begin{tabular}{cccccc}
$\begin{array}{c}\text { Peak RetTime Type } \\
\#\end{array}\left[\begin{array}{c}\text { Width } \\
{[\mathrm{min}]}\end{array}\right.$ & $\begin{array}{c}\text { Area } \\
{[\mathrm{mAU}}\end{array}$ & $\begin{array}{c}\text { Height } \\
{[\mathrm{mAU}]}\end{array}$ & $\begin{array}{c}\text { Area } \\
\%\end{array}$ \\
\hdashline-1 & $7.444 \mathrm{BV}$ & 0.1788 & 3365.94238 & 291.27136 & 5.8134 \\
\hline 2 & $9.872 \mathrm{BB}$ & 0.3763 & $5.45342 \mathrm{e} 4$ & 2302.44507 & 94.1866
\end{tabular}

Totals :

$5.79002 \mathrm{e} 4 \quad 2593.71643$

Signal 2: DAD1 B, Sig=230, 4 Ref $=360,100$

\begin{tabular}{|c|c|c|c|c|c|}
\hline eak & $\begin{array}{l}\text { RetTime Type } \\
\text { [min] }\end{array}$ & $\begin{array}{l}\text { Width } \\
\text { [min] }\end{array}$ & $\begin{array}{c}\text { Area } \\
{\left[\mathrm{mAU}^{*} \mathrm{~s}\right]}\end{array}$ & $\begin{array}{l}\text { Height } \\
{[\mathrm{mAU}]}\end{array}$ & $\begin{array}{c}\text { Area } \\
\%\end{array}$ \\
\hline & $\begin{array}{ll}7.444 \mathrm{~B} \\
9.868\end{array}$ & 2955 & $8307 \mathrm{e} 4$ & & \\
\hline
\end{tabular}

Totals :

$5.10225 \mathrm{e} 4 \quad 2763.68233$

Signal 3: DAD1 C, Sig=254, 4 Ref $=360,100$

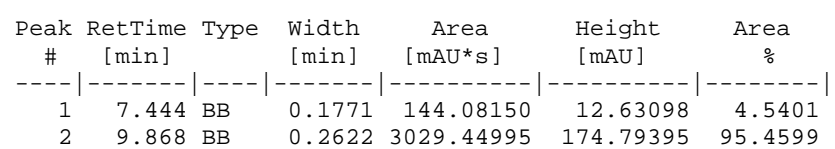

Totals :

$3173.53145 \quad 187.42492$

Signal 4: DAD1 D, Sig=280, 4 Ref $=360,100$

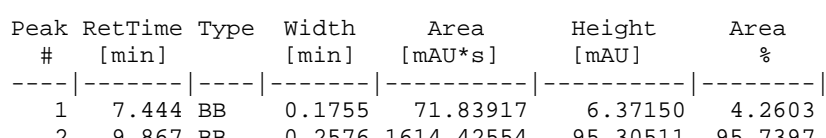

Totals :

$1686.26471 \quad 101.67661$

Signal 5: DAD1 E, Sig $=310,4$ Ref $=360,100$

\begin{tabular}{|c|c|c|c|c|c|c|}
\hline $\begin{array}{l}\text { eak } \\
\#\end{array}$ & $\begin{array}{c}\text { RetTime } \\
{[\text { min] }}\end{array}$ & & $\begin{array}{l}\text { Width } \\
\text { [min] }\end{array}$ & $\begin{array}{c}\text { Area } \\
{\left[\mathrm{mAU}^{*} \mathrm{~s}\right]}\end{array}$ & $\begin{array}{l}\text { Height } \\
{[\mathrm{mAU}]}\end{array}$ & $\begin{array}{c}\text { Area } \\
\%\end{array}$ \\
\hline & & & $\begin{array}{l}0.1735 \\
0.2503\end{array}$ & $\begin{array}{r}16.75410 \\
404.36636\end{array}$ & $\begin{array}{r}1.50934 \\
24.53359\end{array}$ & \\
\hline
\end{tabular}

Totals : $\quad 421.12047 \quad 26.04292$
Bpin

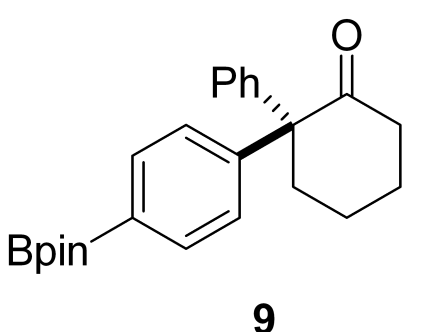

Enantioenriched 


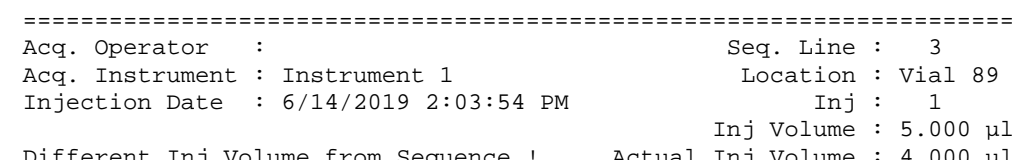

Different Inj Volume from Sequence ! Actual Inj Volume : $5.000 \mu \mathrm{\mu}$

Acq. Method : C: \CHEM32\1\DATA QDY 2019-06-14 13-19-33\AD-01-30.M

Last changed : 6/14/2019 1:31:54 PM

Analysis Method : C: $:$ CHEM32\1\METHODS\IC-01-40.M-0.5.M

Last changed : 6/5/2019 1:46:08 PM

Additional Info (modified after loading)

Additional Info : Peak (s) manually integrated
DAD1 A, Sig=210,4 Ref=360,100 (QDY 2019-06-14 13-19-33!MDK10083RAC.D)

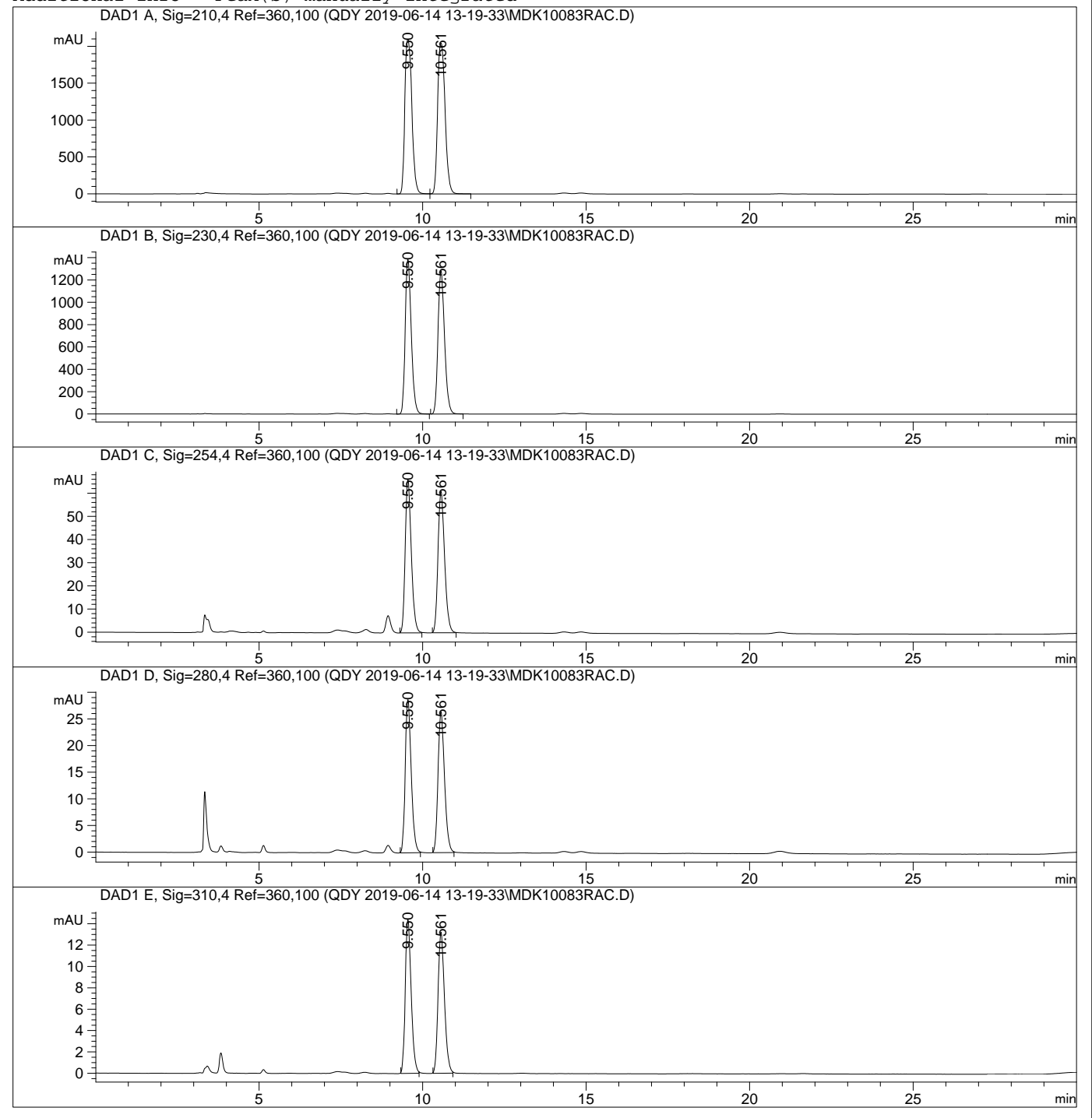




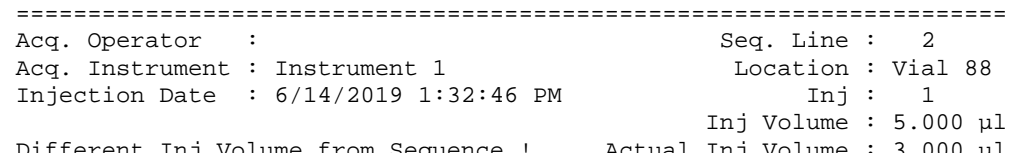

Different Inj Volume from Sequence ! Actual Inj Volume : $5.000 \mu \mathrm{\mu}$

Acq. Method : C: \CHEM32\1\DATA\QDY 2019-06-14 13-19-33\AD-01-30.M

Last changed : 6/14/2019 1:31:54 PM

Analysis Method : C: \CHEM32\1\METHODS\IC-01-40.M-0.5.M

Last changed : 6/5/2019 1:46:08 PM

Additional Info (modified after loading)
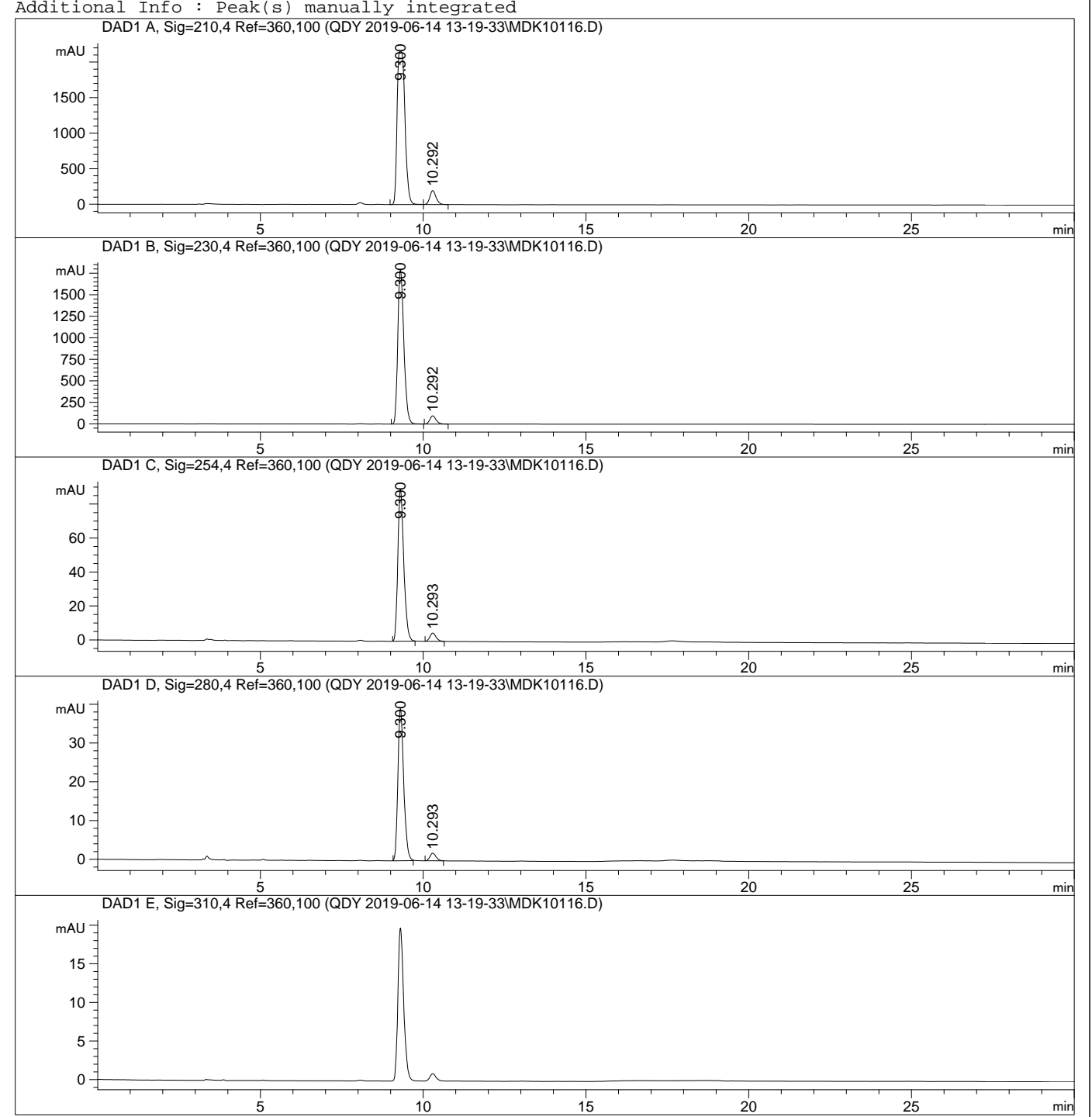

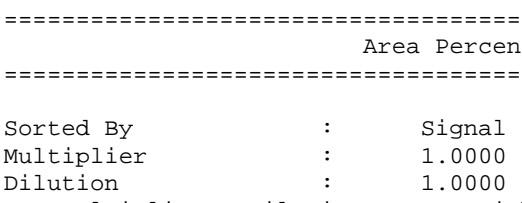

Use Multiplier \& Dilution Factor with ISTDs

Signal 1: DAD1 A, Sig=210, 4 Ref $=360,100$

\begin{tabular}{cccccc}
$\begin{array}{c}\text { Peak RetTime Type } \\
\text { \# }\end{array}\left[\begin{array}{c}\text { Width } \\
{[\mathrm{min}]}\end{array}\right.$ & $\begin{array}{c}\text { Area } \\
{[\mathrm{mAU}}\end{array}$ & $\begin{array}{l}\text { Height } \\
{[\mathrm{mAU}]}\end{array}$ & $\begin{array}{c}\text { Area } \\
\%\end{array}$ \\
\hdashline-1 & $9.300 \mathrm{BV}$ & 0.2652 & $3.57645 \mathrm{e} 4$ & 2159.35132 & 93.2176 \\
\hline 2 & $10.292 \mathrm{VB}$ & 0.2029 & 2602.16919 & 196.03308 & 6.7824
\end{tabular}

Totals :

$3.83667 \mathrm{e} 4 \quad 2355.38440$

Signal 2: DAD1 B, Sig $=230,4$ Ref $=360,100$

\begin{tabular}{|c|c|c|c|c|c|}
\hline $\begin{array}{c}\text { Peak } \\
\#\end{array}$ & $\begin{array}{l}\text { RetTime Type } \\
\text { [min] }\end{array}$ & $\begin{array}{l}\text { Width } \\
\text { [min] }\end{array}$ & $\begin{array}{c}\text { Area } \\
{\left[\mathrm{mAU}^{*} \mathrm{~s}\right]}\end{array}$ & $\begin{array}{l}\text { Height } \\
{[\mathrm{mAU}]}\end{array}$ & $\begin{array}{c}\text { Area } \\
\%\end{array}$ \\
\hline & $\begin{array}{r}9.300 \mathrm{BB} \\
10.292 \mathrm{BB}\end{array}$ & $\begin{array}{l}0.2035 \\
0.2036\end{array}$ & $\begin{array}{l}2.32047 \mathrm{e} 4 \\
1278.53894\end{array}$ & $\begin{array}{r}1787.13477 \\
95.86061\end{array}$ & $\begin{array}{r}94.7779 \\
5.2221\end{array}$ \\
\hline
\end{tabular}

Totals :

$2.44832 \mathrm{e} 4 \quad 1882.99538$

Signal 3: DAD1 C, Sig $=254,4$ Ref $=360,100$

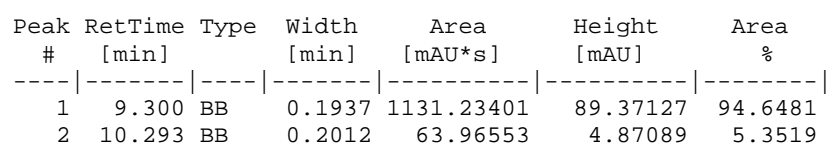

Totals :

$1195.19954 \quad 94.24216$

Signal 4: DAD1 D, Sig $=280,4$ Ref $=360,100$

\begin{tabular}{|c|c|c|c|c|c|}
\hline eak & $\begin{array}{c}\text { RetTime } \\
\text { [min] }\end{array}$ & $\begin{array}{l}\text { Width } \\
\text { [min] }\end{array}$ & $\begin{array}{c}\text { Area } \\
{\left[\mathrm{mAU} U^{*} \mathrm{~s}\right]}\end{array}$ & $\begin{array}{l}\text { Height } \\
{[\mathrm{mAU}]}\end{array}$ & $\begin{array}{c}\text { Area } \\
\%\end{array}$ \\
\hline & $293 \mathrm{~B}$ & $\begin{array}{l}0.1909 \\
0.2002\end{array}$ & $\begin{array}{r}487.30170 \\
25.70511\end{array}$ & $\begin{array}{r}39.23238 \\
1.97048\end{array}$ & \\
\hline
\end{tabular}

Totals :

$513.00681 \quad 41.20286$

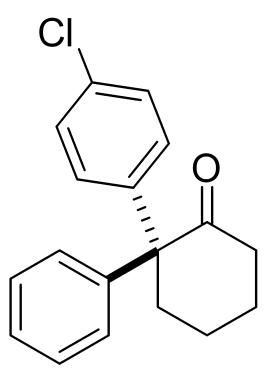

10

Signal 5: DAD1 E, Sig=310, 4 Ref $=360,100$

\section{Enantioenriched}


Area Percent Report

\begin{tabular}{|c|c|c|}
\hline $\begin{array}{l}\text { Acq. Operator } \\
\text { Acq. Instrument } \\
\text { Injection Date }\end{array}$ & $\begin{array}{l}\text { Instrument } 1 \\
6 / 20 / 20198: 54: 04 \mathrm{PM}\end{array}$ & $\begin{array}{r}\text { Seq. Line } \\
\text { Location } \\
\text { Inj }\end{array}$ \\
\hline
\end{tabular}

Different Inj Volume from Sequence ! Actual Inj Volume : $5.000 \mu \mathrm{\mu}$

Acq. Method : C: \CHEM32\1\DATA IQDY 2019-06-20 19-48-07\IC-01-30.M

Last changed : 6/20/2019 8:21:35 PM

Analysis Method : C: \CHEM32\1\METHODS\AS-00-60-0.1.M

: $6 / 19 / 2019$ 8:22:59 PM

Additional Info: Peak(s) manually integrated
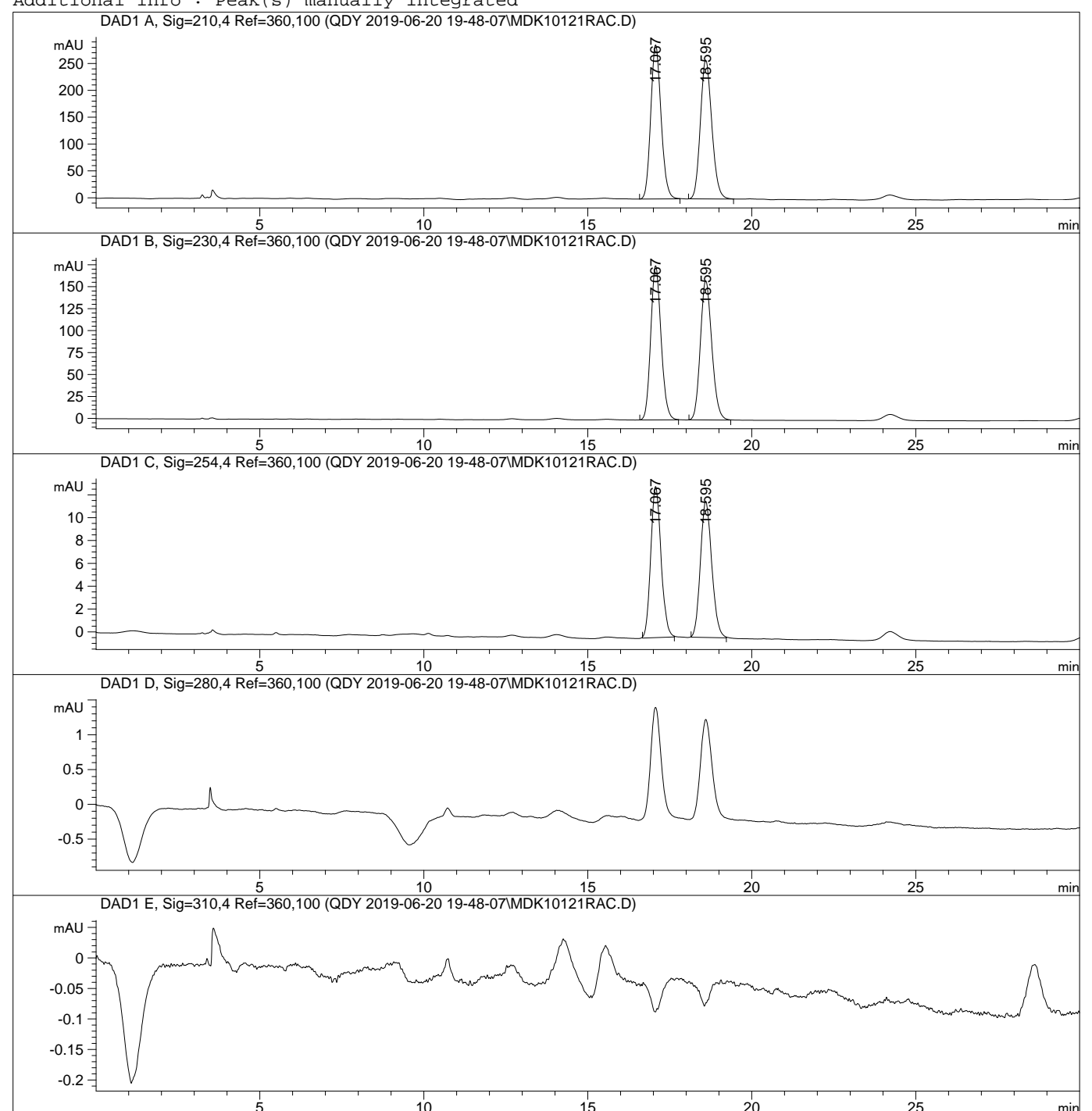

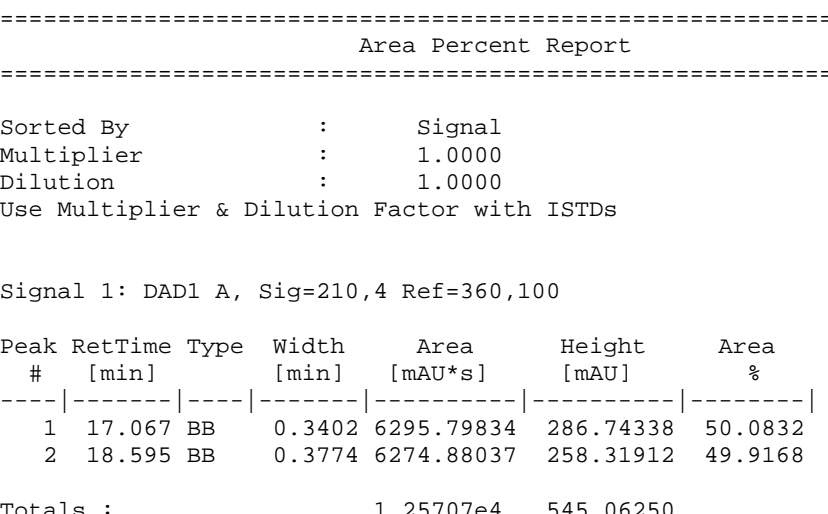

Signal 2: DAD1 B, Sig=230, 4 Ref $=360,100$

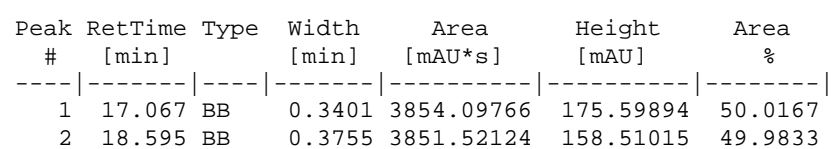

Totals :

$7705.61890 \quad 334.10909$

Signal 3: DAD1 C, Sig=254, 4 Ref $=360,100$

\begin{tabular}{|c|c|c|c|c|c|}
\hline $\begin{array}{c}\text { Peak } \\
\#\end{array}$ & $\begin{array}{l}\text { RetTime Type } \\
\text { [min] }\end{array}$ & $\begin{array}{l}\text { Width } \\
\text { [min] }\end{array}$ & $\begin{array}{c}\text { Area } \\
{\left[\mathrm{mAU} U^{*} \mathrm{~s}\right]}\end{array}$ & $\begin{array}{l}\text { Height } \\
{[\mathrm{mAU}]}\end{array}$ & $\begin{array}{c}\text { Area } \\
\%\end{array}$ \\
\hline 1 & $17.067 \mathrm{BB}$ & 0.3385 & 288.63580 & 13.23017 & \\
\hline 2 & $18.595 \mathrm{BB}$ & 0.3734 & 287.38614 & 11.91654 & 49.8915 \\
\hline & & & 76.02194 & 25.14671 & \\
\hline
\end{tabular}

Signal 4: DAD1 D, Sig=280, 4 Ref $=360,100$

Signal 5: DAD1 E, Sig $=310,4$ Ref $=360,100$

*** End of Report ***

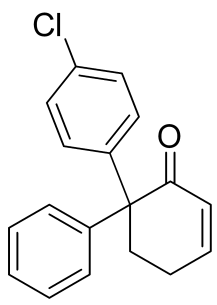

racemic 


\section{Data File C: \CHEM32\1\DATA\QDY 2019-06-20 19-48-๑7\MDK10127.D}

Sample Name

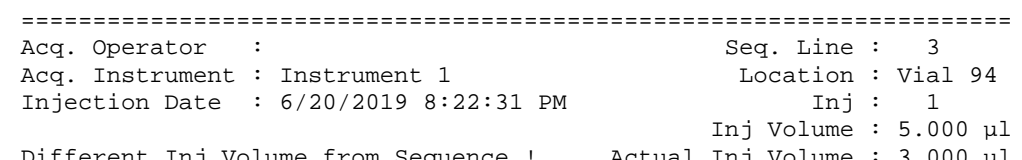

Injection Date $\quad$ Inj Volume $: 5.000 \mu \mathrm{\mu l}$

Acq. Method : C: \CHEM32\1\DATA QQDY 2019-06-20 19-48-07\IC-01-30.M

Last changed : 6/20/2019 8:21:35 PM

Analysis Method : C: \CHEM32\1\METHODS \AS-00-60-0.1.M

Last changed: $6 / 19 / 2019$ 8:22:59 PM

Additional Info: : Peak(s) manually integrated
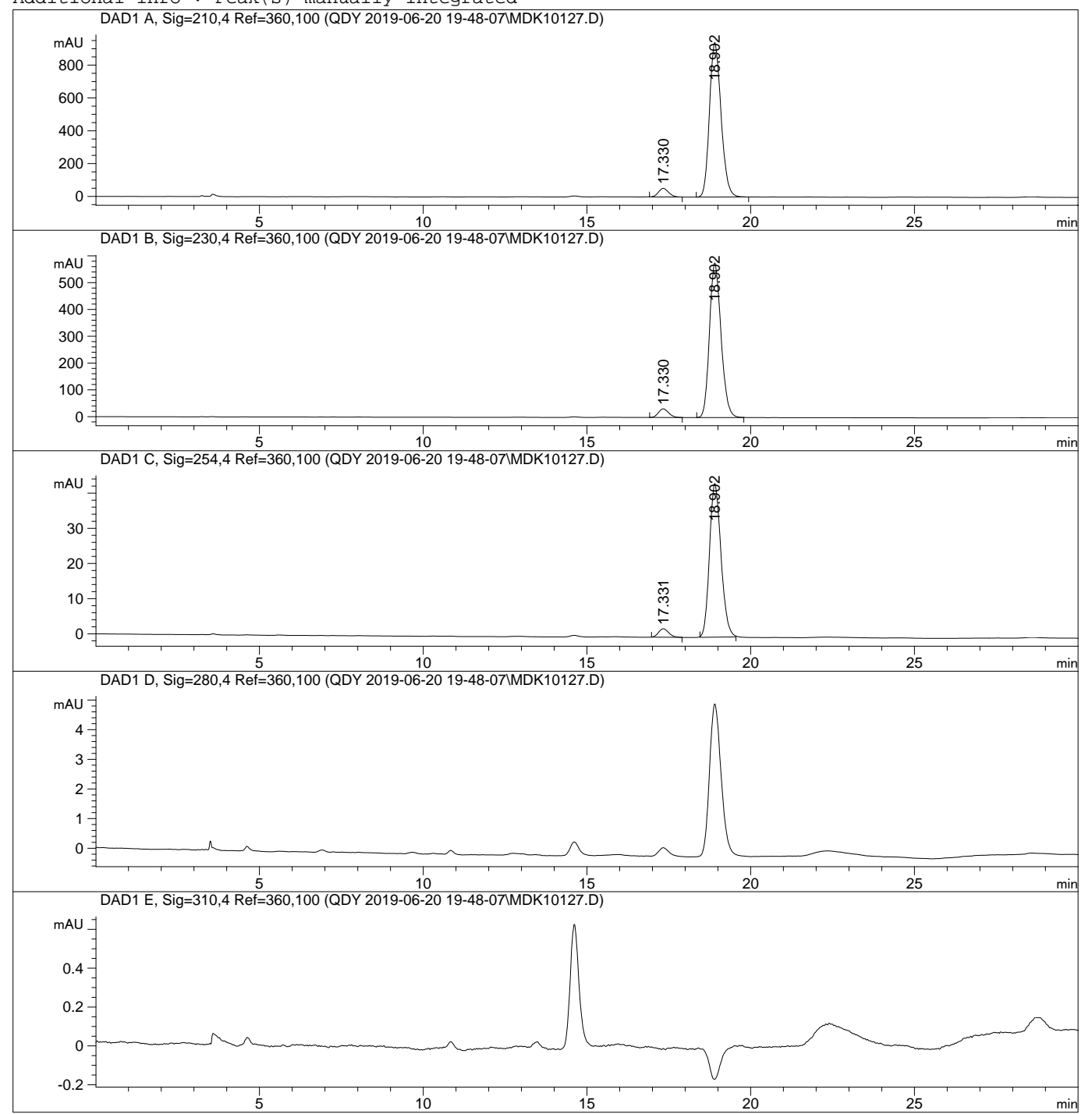

Data File C: \CHEM32\1\DATA \QDY 2019-06-20 19-48-07\MDK10127.D Sample Name

Area Percent Report

$\begin{array}{lcc} & \text { Area Percent } \\ \text { ==================================+ } & \\ \text { Sorted By } & \vdots & \text { Signal } \\ \text { Multiplier } & \vdots & 1.0000 \\ \text { Dilution } & 1.0000\end{array}$

Use Multiplier \& Dilution Factor with ISTDS

Signal 1: DAD1 A, Sig=210, 4 Ref $=360,100$

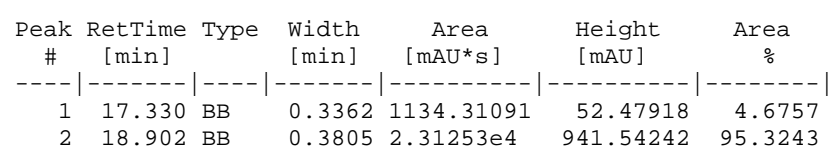

Totals :

$2.42596 \mathrm{e} 4 \quad 994.02160$

Signal 2: DAD1 B, Sig=230, 4 Ref $=360,100$

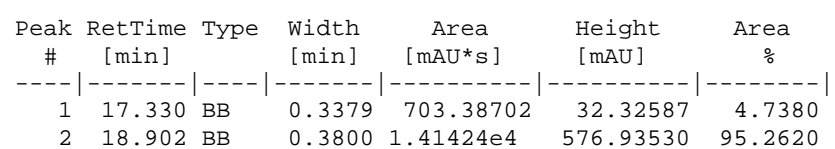

Totals :

$1.48457 \mathrm{e} 4 \quad 609.26118$

Signal 3: DAD1 C, Sig $=254,4$ Ref $=360,100$

\begin{tabular}{|c|c|c|c|c|c|c|}
\hline $\begin{array}{c}\text { Peak } \\
\#\end{array}$ & $\begin{array}{l}\text { RetTime } \\
{[\text { min] }}\end{array}$ & Type & $\begin{array}{l}\text { Width } \\
\text { [min] }\end{array}$ & $\begin{array}{c}\text { Area } \\
{\left[\mathrm{mAU}^{*} \mathrm{~s}\right]}\end{array}$ & $\begin{array}{l}\text { Height } \\
\text { [mAU] }\end{array}$ & $\begin{array}{c}\text { Area } \\
\%\end{array}$ \\
\hline 1 & 17.331 & BB & $\odot .3358$ & 51.58968 & 2.40965 & 4.6258 \\
\hline 2 & 18.902 & BB & 0.3779 & 1063.68201 & 43.70976 & 95.3742 \\
\hline Tot & & & & & 46.119 & \\
\hline
\end{tabular}

Signal 4: DAD1 D, Sig=280, 4 Ref $=360,100$

Signal 5: DAD1 E, Sig $=310,4$ Ref $=360,100$

*** End of Report ***

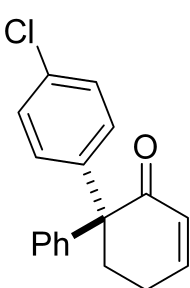

11

Enantioenriched 
Different Inj Volume from Sequence ! Actual Inj Volume : $1.000 \mu \mathrm{l}$

Acq. Method : C: \CHEM32\1\DATA

Last changed $\quad 6 / 18 / 2019$ 4:17:58 PM

Analysis Method : C: \CHEM32\1\METHODS \AD-005-40-0.7.M

Last changed : 6/18/2019 2:26:39 PM

Additional Info : Peak(s) manually integrated
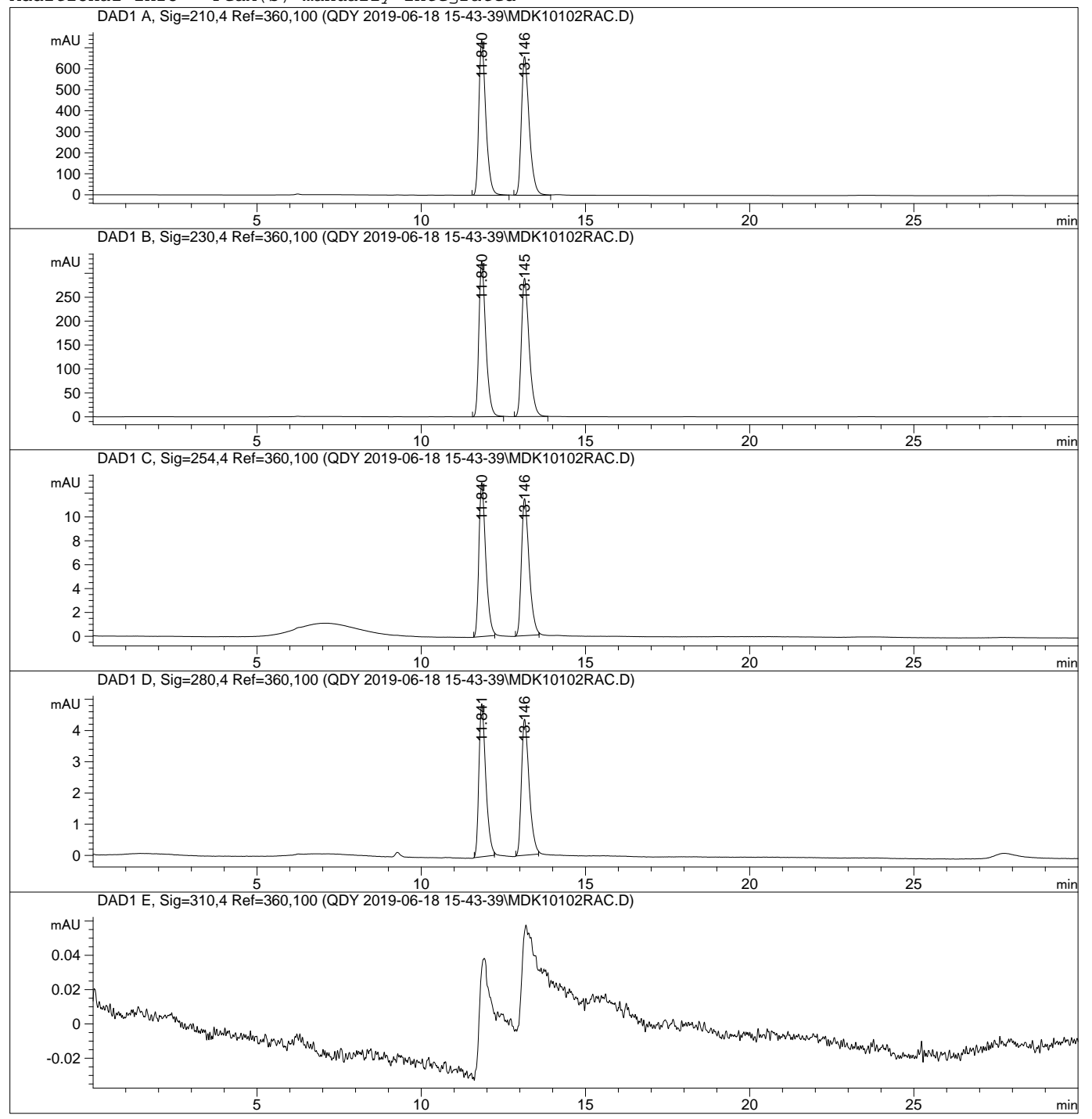

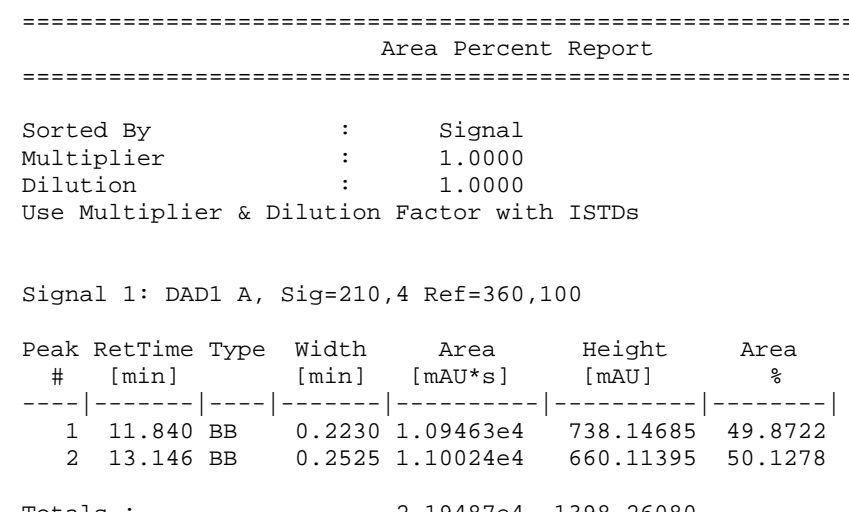

Totals :

$2.19487 \mathrm{e} 4 \quad 1398.26080$

Signal 2: DAD1 B, Sig $=230,4$ Ref $=360,100$

\begin{tabular}{|c|c|c|c|c|c|}
\hline $\begin{array}{c}\text { Peak } \\
\#\end{array}$ & $\begin{array}{l}\text { RetTime Type } \\
\text { [min] }\end{array}$ & $\begin{array}{l}\text { Width } \\
\text { [min] }\end{array}$ & $\begin{array}{c}\text { Area } \\
{\left[\mathrm{mAU}^{*} \mathrm{~s}\right]}\end{array}$ & $\begin{array}{l}\text { Height } \\
{[\mathrm{mAU}]}\end{array}$ & $\begin{array}{c}\text { Area } \\
\%\end{array}$ \\
\hline 1 & $11.840 \mathrm{BB}$ & 0.2208 & 4751.54736 & 324.61374 & $\begin{array}{l}49.9645 \\
50.0355\end{array}$ \\
\hline
\end{tabular}

Totals :

$9509.84277 \quad 613.80283$

Signal 3: DAD1 C, Sig $=254,4$ Ref $=360,100$

\begin{tabular}{|c|c|c|c|c|c|}
\hline $\begin{array}{c}\text { Peak } \\
\#\end{array}$ & $\begin{array}{l}\text { RetTime Type } \\
\text { [min] }\end{array}$ & $\begin{array}{l}\text { Width } \\
\text { [min] }\end{array}$ & $\begin{array}{c}\text { Area } \\
{\left[\mathrm{mAU}^{\star} \mathrm{s}\right]}\end{array}$ & $\begin{array}{l}\text { Height } \\
{[\mathrm{mAU}]}\end{array}$ & $\begin{array}{c}\text { Area } \\
\%\end{array}$ \\
\hline 1 & $11.840 \mathrm{BB}$ & 0.2189 & 186.76347 & 12.90118 & 49.9817 \\
\hline 2 & $13.146 \mathrm{BB}$ & 0.2479 & 186.90045 & 11.48213 & 50.0183 \\
\hline
\end{tabular}

Totals :

$373.66393 \quad 24.38332$

Signal 4: DAD1 D, Sig=280, 4 Ref $=360,100$

\begin{tabular}{|c|c|c|c|c|c|}
\hline $\begin{array}{c}\text { Peak } \\
\#\end{array}$ & $\begin{array}{l}\text { RetTime Type } \\
\text { [min] }\end{array}$ & $\begin{array}{l}\text { Width } \\
\text { [min] }\end{array}$ & $\begin{array}{c}\text { Area } \\
{\left[\mathrm{mAU} U^{*} \mathrm{~s}\right]}\end{array}$ & $\begin{array}{l}\text { Height } \\
{[\mathrm{mAU}]}\end{array}$ & $\begin{array}{c}\text { Area } \\
\%\end{array}$ \\
\hline 1 & $11.841 \mathrm{BB}$ & & 71.03078 & 4.90440 & 50.0245 \\
\hline 2 & $13.146 \mathrm{BB}$ & 0.2479 & 70.96111 & 4.36021 & 49.9755 \\
\hline
\end{tabular}

Totals :

$141.99189 \quad 9.26460$

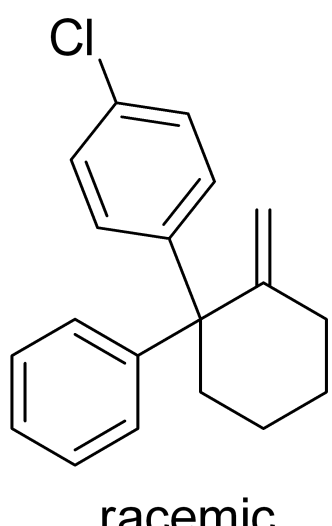

Signal 5: DAD1 E, Sig=310, 4 Ref $=360,100$

*** End of Report *** 
Analysis Method : C: \CHEM32\1 \METHODS \AD-005-40-0.7.M

Last changed : 6/18/2019 2:26:39 PM

Additional Info : Peak(s) manually integrated
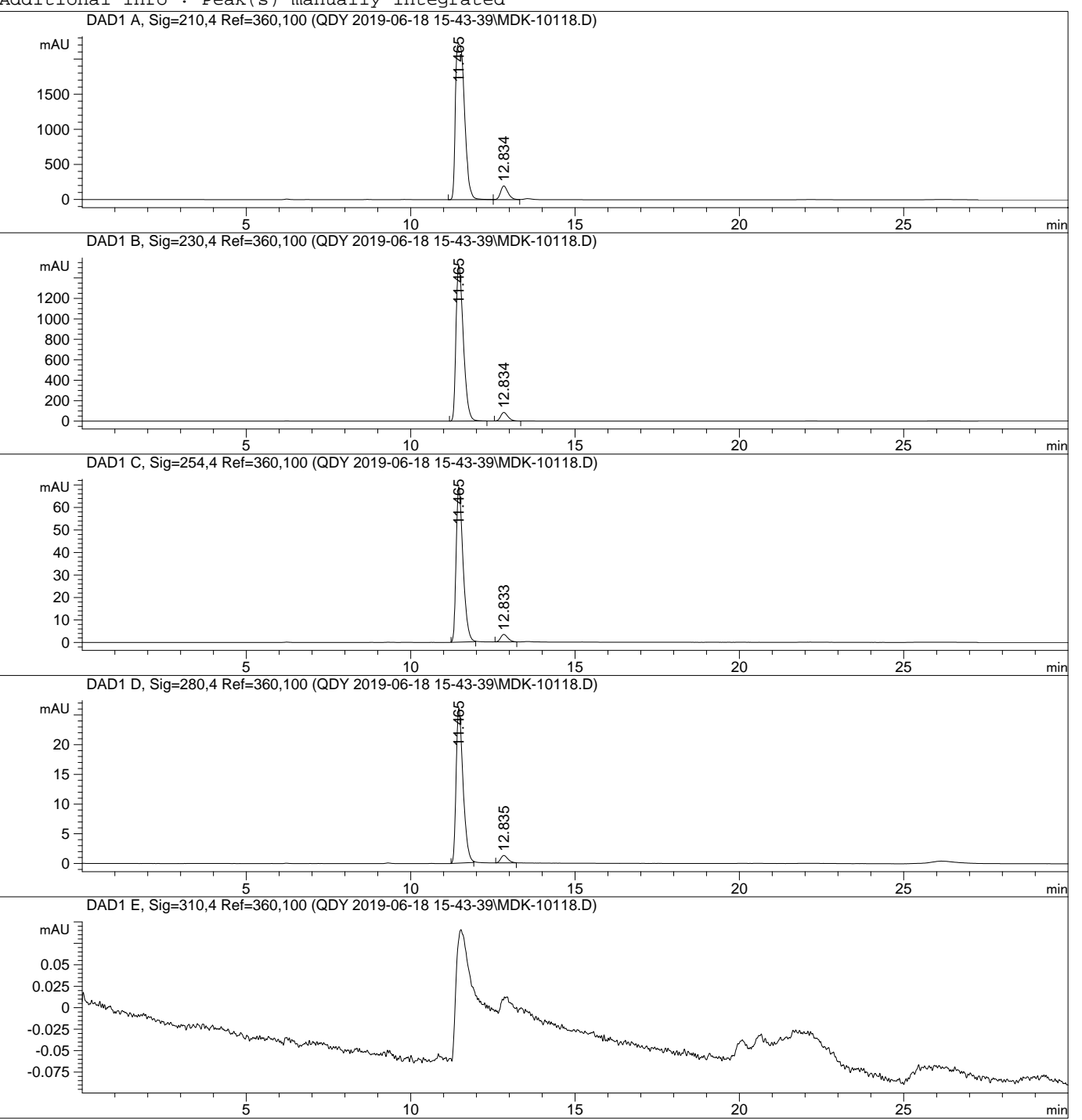

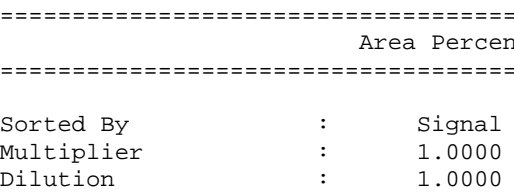

Use Multiplier \& Dilution Factor with ISTDS

Signal 1: DAD1 A, Sig=210, 4 Ref $=360,100$

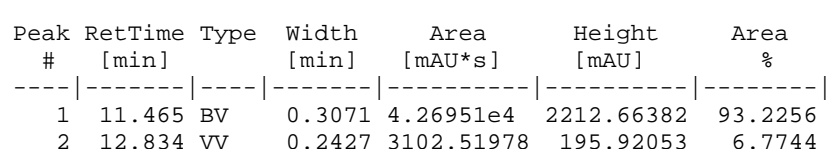

Totals :

$4.57976 \mathrm{e} 4 \quad 2408.58435$

Signal 2: DAD1 B, Sig=230, 4 Ref $=360,100$

\begin{tabular}{|c|c|c|c|c|c|}
\hline $\begin{array}{c}\text { Peak } \\
\#\end{array}$ & $\begin{array}{l}\text { RetTime Type } \\
{[\text { min] }}\end{array}$ & $\begin{array}{l}\text { Width } \\
\text { [min] }\end{array}$ & $\begin{array}{c}\text { Area } \\
{\left[\mathrm{mAU}^{*} \mathrm{~s}\right]}\end{array}$ & $\begin{array}{l}\text { Height } \\
{[\mathrm{mAU}]}\end{array}$ & $\begin{array}{c}\text { Area } \\
\%\end{array}$ \\
\hline & $\begin{array}{l}11.465 \mathrm{BB} \\
12.834 \mathrm{BB}\end{array}$ & $\begin{array}{l}0.2389 \\
0.2381\end{array}$ & $\begin{array}{l}2.35616 \mathrm{e} 4 \\
1293.27832\end{array}$ & $\begin{array}{r}1519.88940 \\
83.75905\end{array}$ & $\begin{array}{r}94.7967 \\
5.2033\end{array}$ \\
\hline
\end{tabular}

Totals :

$2.48549 \mathrm{e} 4 \quad 1603.64845$

Signal 3: DAD1 C, Sig=254, 4 Ref $=360,100$

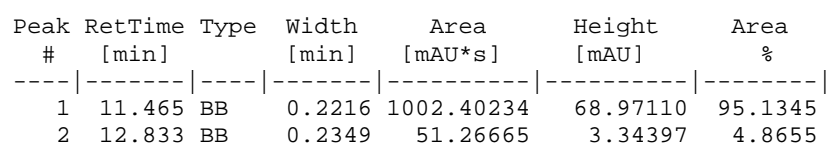

Totals :

$1053.66899 \quad 72.31507$

Signal 4: DAD1 D, Sig=280, 4 Ref $=360,100$

\begin{tabular}{|c|c|c|c|c|c|}
\hline $\begin{array}{c}\text { Peak } \\
\#\end{array}$ & $\begin{array}{l}\text { RetTime Type } \\
\text { [min] }\end{array}$ & $\begin{array}{l}\text { Width } \\
\text { [min] }\end{array}$ & $\begin{array}{c}\text { Area } \\
{\left[\mathrm{mAU} U^{*} \mathrm{~s}\right]}\end{array}$ & $\begin{array}{l}\text { Height } \\
{[\mathrm{mAU}]}\end{array}$ & $\begin{array}{c}\text { Area } \\
\%\end{array}$ \\
\hline 1 & $11.465 \mathrm{BB}$ & & 378.55869 & 26.05864 & 95.2056 \\
\hline 2 & $12.835 \mathrm{BB}$ & 0.2347 & 19.06345 & 1.24473 & 4.7944 \\
\hline
\end{tabular}

Totals :

$397.62213 \quad 27.30337$

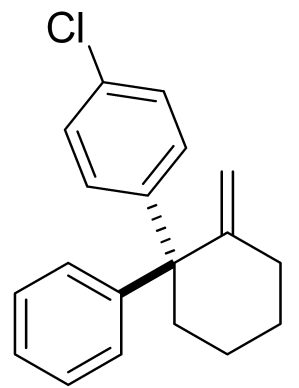

12

Signal 5: DAD1 E, Sig=310, 4 Ref $=360,100$

Enantioenriched

*** End of Report *** 
Analysis Method : C: \CHEM32\1\METHODS \IC-01-40.M- - . .M

Last changed : 6/5/2019 1:46:08 PM

Additional Info : Peak(s) manually integrated
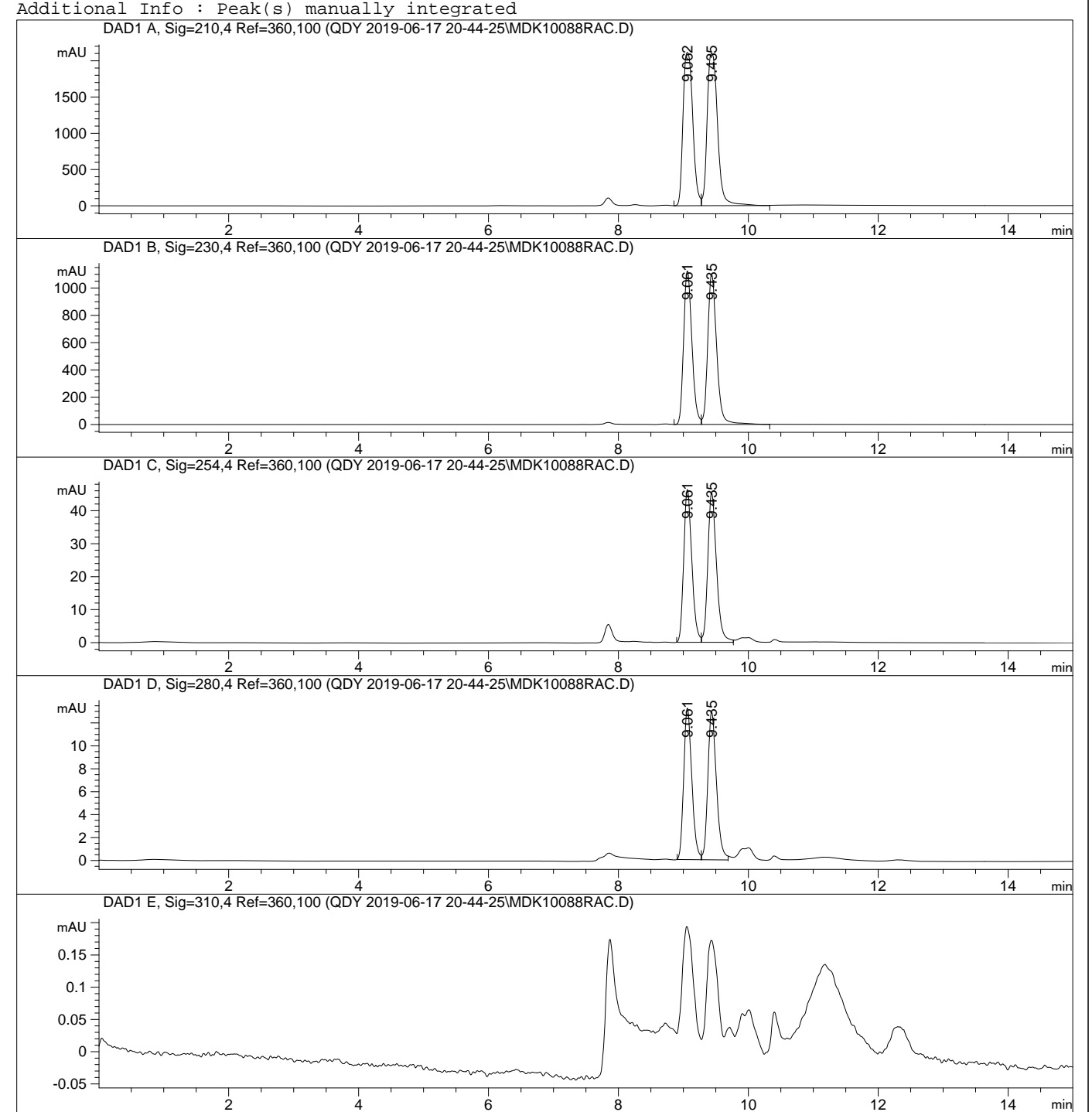

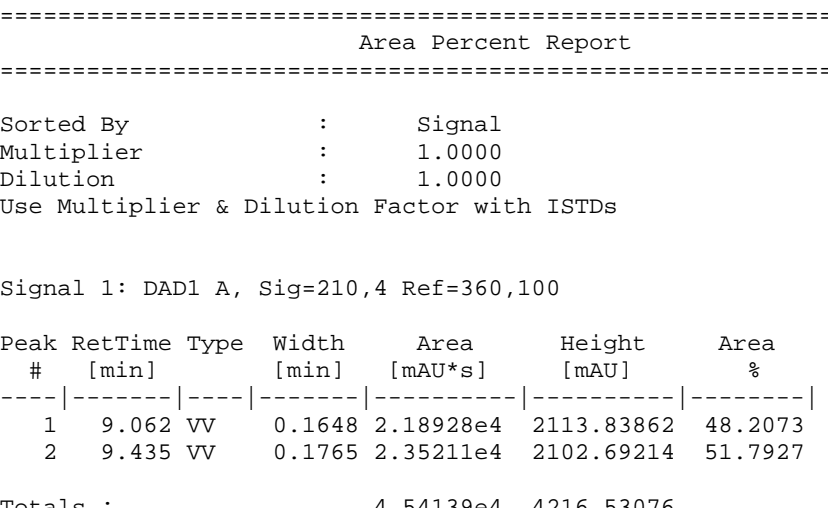

Totals :

$4.54139 \mathrm{e} 4 \quad 4216.53076$

Signal 2: DAD1 B, Sig=230, 4 Ref $=360,100$

\begin{tabular}{cccccc}
$\begin{array}{c}\text { Peak RetTime Type } \\
\text { W }\end{array}[\mathrm{min}]$ & $\begin{array}{c}\text { Width } \\
{[\mathrm{min}]}\end{array}$ & $\begin{array}{c}\text { Area } \\
{[\mathrm{mAU}}\end{array}$ & $\begin{array}{c}\text { Height } \\
{[\mathrm{mAU}]}\end{array}$ & $\begin{array}{c}\text { Area } \\
\%\end{array}$ \\
\hdashline-1 & $9.061 \mathrm{VV}$ & 0.1373 & 9961.98242 & 1124.78137 & 48.5741 \\
2 & $9.435 \mathrm{VB}$ & 0.1479 & $1.05468 \mathrm{e} 4$ & 1099.60571 & 51.4259
\end{tabular}

Totals :

$2.05088 \mathrm{e} 4 \quad 2224.38708$

Signal 3: DAD1 C, Sig $=254,4$ Ref $=360,100$

\begin{tabular}{|c|c|c|c|c|c|}
\hline $\begin{array}{c}\text { Peak } \\
\#\end{array}$ & $\begin{array}{l}\text { RetTime Type } \\
{[\text { min] }}\end{array}$ & $\begin{array}{l}\text { Width } \\
\text { [min] }\end{array}$ & $\begin{array}{c}\text { Area } \\
{\left[\mathrm{mAU} U^{\star} \mathrm{s}\right]}\end{array}$ & $\begin{array}{l}\text { Height } \\
{[\mathrm{mAU}]}\end{array}$ & $\begin{array}{c}\text { Area } \\
\%\end{array}$ \\
\hline 1 & $9.061 \mathrm{BV}$ & 0.1352 & 401.62747 & 46.29647 & 48.8289 \\
\hline 2 & $9.435 \mathrm{VB}$ & 0.1426 & 420.89221 & 45.22142 & 51.1711 \\
\hline
\end{tabular}

Totals :

$822.51968 \quad 91.51789$

Signal 4: DAD1 D, Sig=280, 4 Ref $=360,100$

\begin{tabular}{|c|c|c|c|c|c|}
\hline $\begin{array}{c}\text { Peak } \\
\#\end{array}$ & $\begin{array}{l}\text { RetTime Type } \\
\text { [min] }\end{array}$ & $\begin{array}{l}\text { Width } \\
\text { [min] }\end{array}$ & $\begin{array}{c}\text { Area } \\
{\left[\mathrm{mAU} U^{*} \mathrm{~s}\right]}\end{array}$ & $\begin{array}{l}\text { Height } \\
{[\mathrm{mAU}]}\end{array}$ & $\begin{array}{c}\text { Area } \\
\%\end{array}$ \\
\hline 1 & $9.061 \mathrm{BV}$ & & 114.50626 & 13.22214 & 49.0974 \\
\hline 2 & 9.435 VB & 0.1414 & 118.71625 & 12.89843 & 50.9026 \\
\hline
\end{tabular}

Totals :

$233.22250 \quad 26.12057$

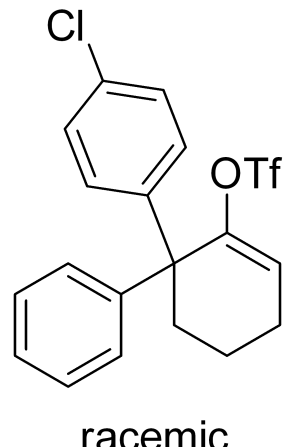

Signal 5: DAD1 E, Sig=310,4 Ref $=360,100$

*** End of Report *** 

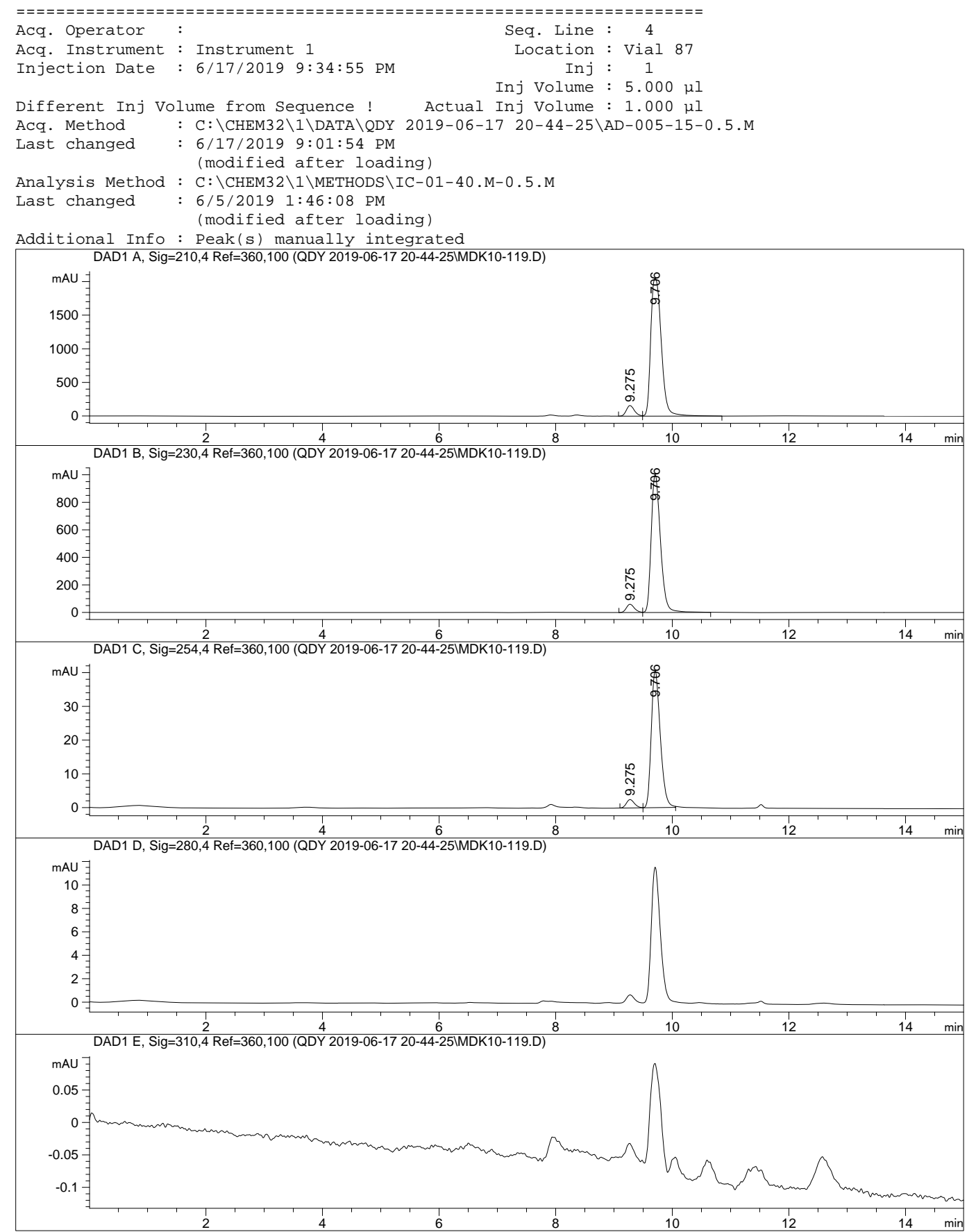

\begin{tabular}{|c|c|c|c|c|c|}
\hline $\begin{array}{l}\text { eak } \\
\text { e }\end{array}$ & $\begin{array}{l}\text { RetTime Type } \\
\text { [min] }\end{array}$ & $\begin{array}{l}\text { Width } \\
{[\mathrm{min}]}\end{array}$ & $\begin{array}{c}\text { Area } \\
{\left[\mathrm{mAU}^{\star} \mathrm{s}\right]}\end{array}$ & $\begin{array}{l}\text { Height } \\
{[\mathrm{mAU}]}\end{array}$ & $\begin{array}{c}\text { Area } \\
\%\end{array}$ \\
\hline 1 & $9.275 \mathrm{BV}$ & 0.1499 & 23.87741 & 2.48841 & 5.2101 \\
\hline 2 & $9.706 \mathrm{VB}$ & 0.1650 & 434.41232 & 40.54437 & 94.7899 \\
\hline
\end{tabular}

Signal 4: DAD1 D, Sig $=280,4$ Ref $=360,100$

Signal 5: DAD1 E, Sig=310, 4 Ref $=360,100$

*** End of Report ***

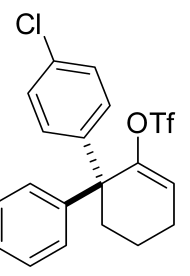

13

Enantioenriched 


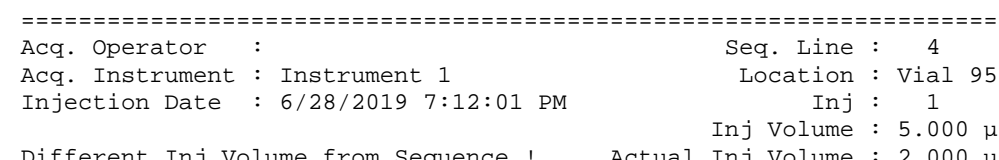

Different Inj Volume from Sequence! Actual Inj Volume : $5.000 \mu$

Acq. Method : C: \CHEM32\1\DATA \QDY 2019-06-28 18-36-36\0J-02-10-0.9.M

Last changed 6/27/2019 4:30:50 PM

Analysis Method: C: \CHEM32\1 \METHODS $\backslash 0 \mathrm{~J}-05-10-1.5 . M$

Last changed : 6/27/2019 7:06:44 PM
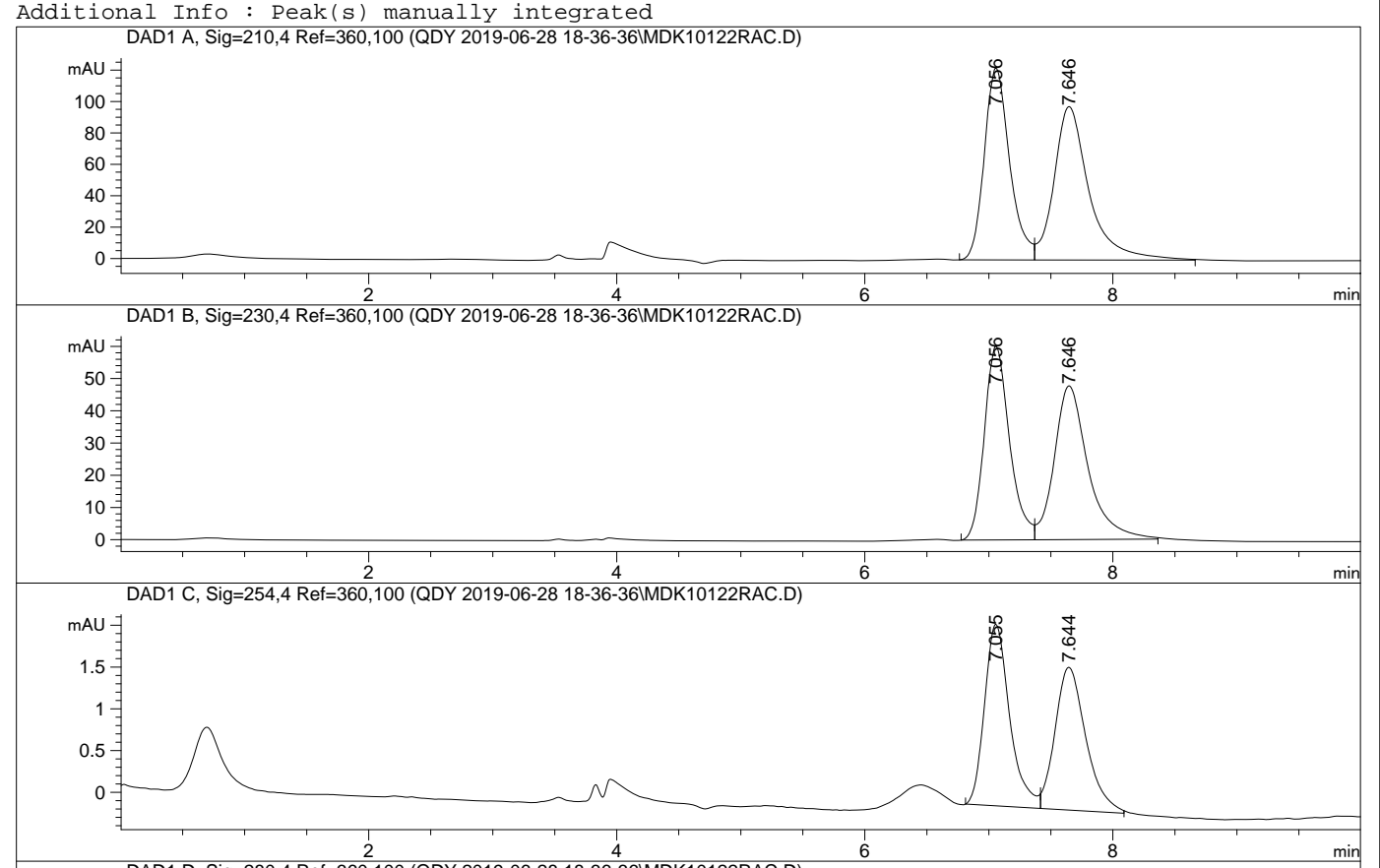

DAD1 D, Sig=280,4 Ref=360,100 (QDY 2019-06-28 $\frac{4}{18-36-36 \text { IMDK10122RAC.D) }}$

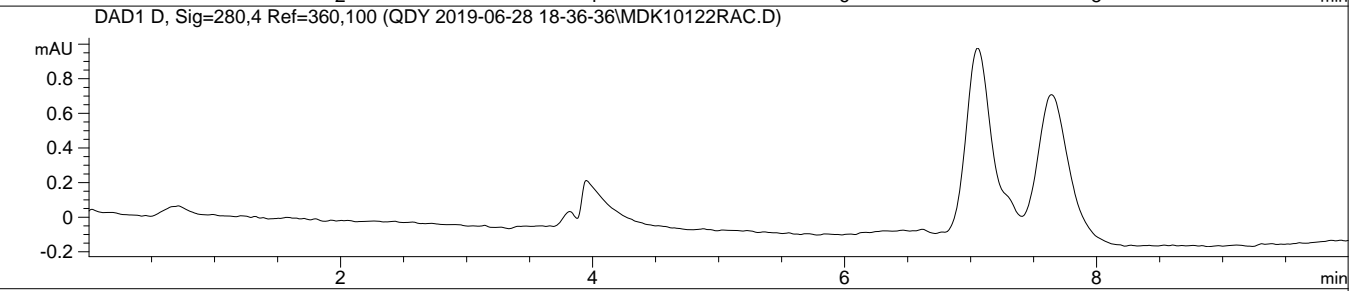

DAD1 E, Sig=310,4 Ref=360,100 (QDY 2019-06-28 18-36-36IMDK10122RACD)

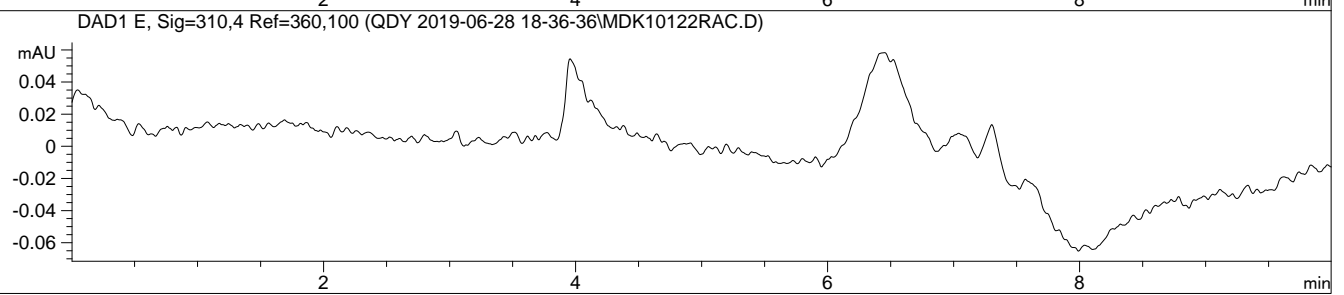

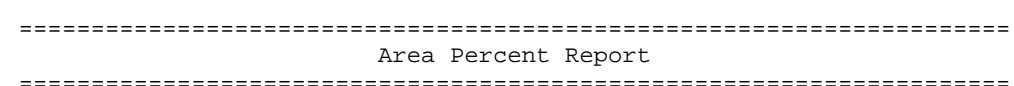

$\begin{array}{lll}\text { Sorted By } & : & \text { Signal } \\ \text { Multiplier } & : & 1.0000\end{array}$

Dilution : 1.0000

Use Multiplier \& Dilution Factor with ISTDs

Signal 1: DAD1 A, Sig=210, 4 Ref $=360,100$

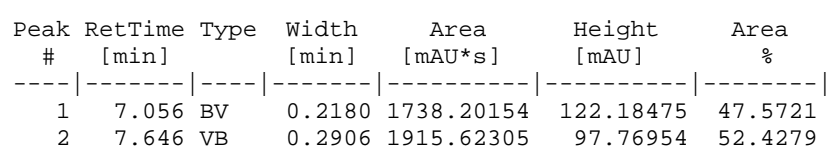

Totals :

$3653.82458 \quad 219.95428$

Signal 2: DAD1 B, Sig=230, 4 Ref $=360,100$

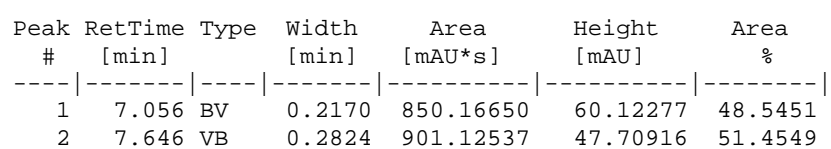

Totals :

$1751.29187 \quad 107.83192$

Signal 3: DAD1 C, Sig=254, 4 Ref $=360,100$

\begin{tabular}{|c|c|c|c|c|c|}
\hline $\begin{array}{c}\text { Peak } \\
\#\end{array}$ & $\begin{array}{l}\text { RetTime Type } \\
\text { [min] }\end{array}$ & $\begin{array}{l}\text { Width } \\
\text { [min] }\end{array}$ & $\begin{array}{c}\text { Area } \\
{\left[\mathrm{mAU} U^{*} \mathrm{~s}\right]}\end{array}$ & $\begin{array}{l}\text { Height } \\
{[\mathrm{mAU}]}\end{array}$ & $\begin{array}{c}\text { Area } \\
\%\end{array}$ \\
\hline 1 & $7.055 \mathrm{BB}$ & $\odot .2129$ & 30.71817 & 2.17384 & 51.0756 \\
\hline 2 & $7.644 \mathrm{BB}$ & $\odot .2608$ & 29.42441 & 1.70960 & 48.9244 \\
\hline
\end{tabular}

Signal 4: DAD1 D, Sig=280, 4 Ref $=360,100$

Signal 5: DAD1 E, Sig $=310,4$ Ref $=360,100$

** End of Report ***

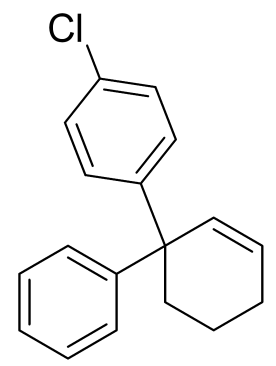

racemic 

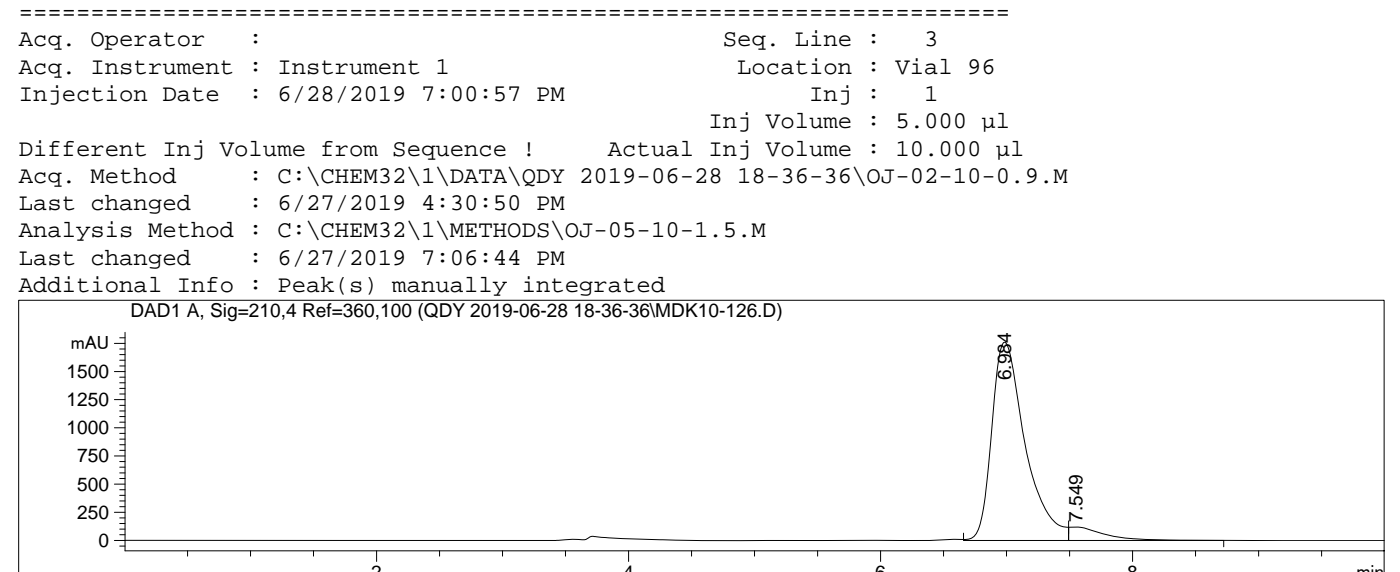

DAD1 B, Sig=230,4 Ref=360, 100 (QDY 2019-06-28 $18-\frac{4}{18-36-36 \text { IMDK10-126.D) }}$

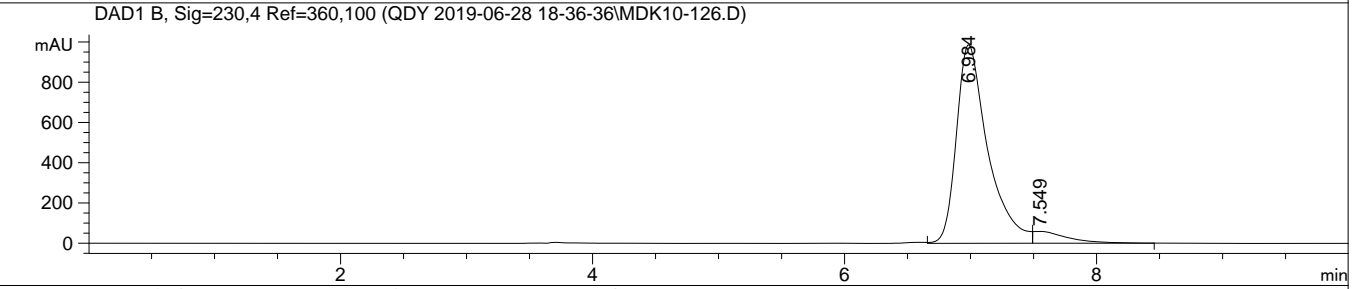

$\frac{2}{\text { DAD1 C, Sig=254,4 Ref }=360,100 \text { (QDY 2019-06-28 }} \frac{4}{18-36-36 \text { IMDK10-126-D) }}$
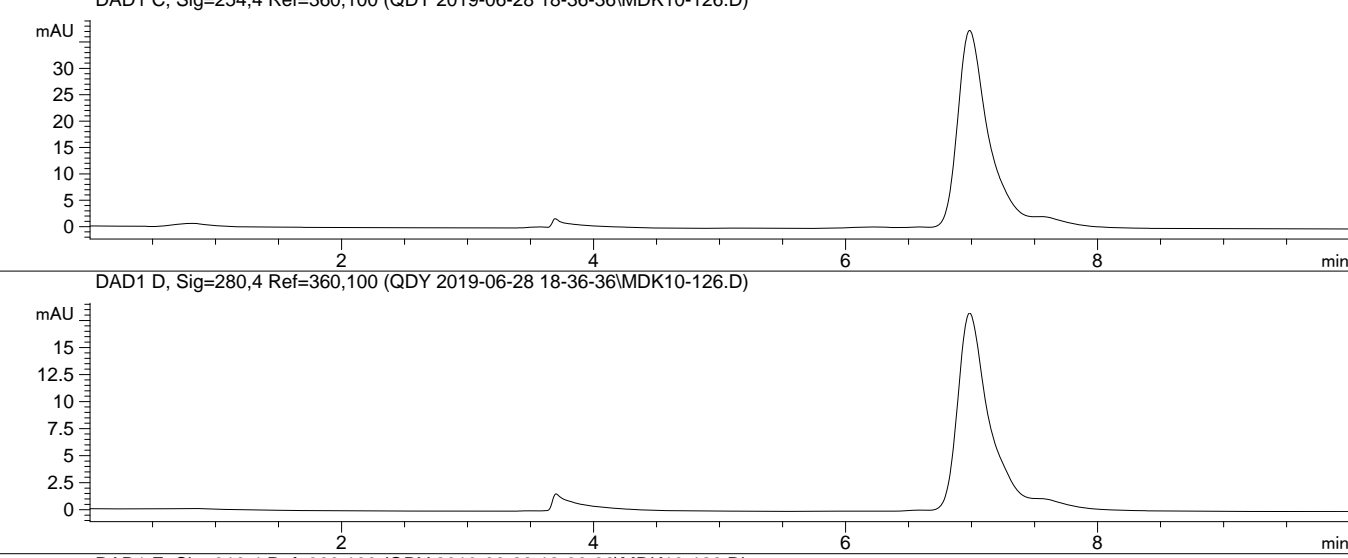

$\frac{2}{D A D 1}$ E, Sig=310,4 Ref=360,100(ODY 2019-06-28 $\frac{4}{18-36-36 \text { IMDK10-126.D) }}$

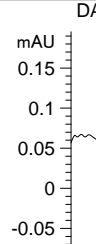

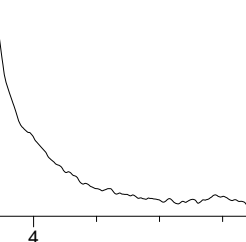

Area Percent Report

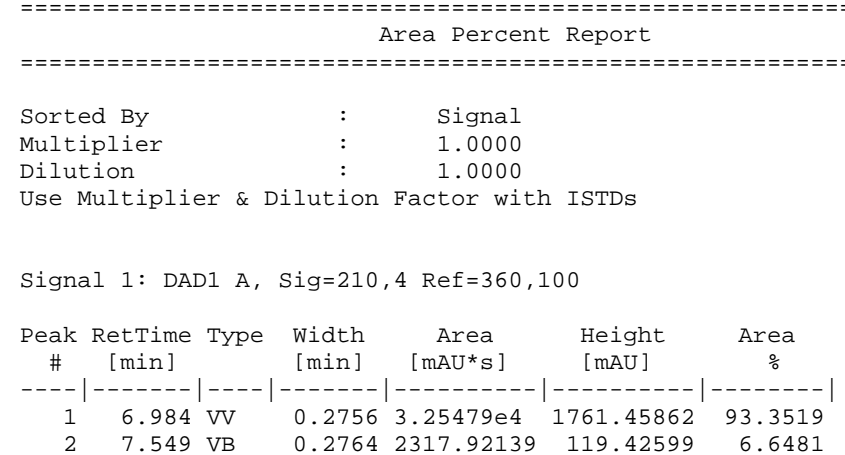

Totals :

$3.48658 \mathrm{e} 4 \quad 1880.88461$

Signal 2: DAD1 B, Sig=230, 4 Ref $=360,100$

\begin{tabular}{cccccc}
$\begin{array}{c}\text { Peak RetTime Type } \\
\#\end{array}\left[\begin{array}{c}\text { Width } \\
{[\mathrm{min}]}\end{array}\right.$ & $\begin{array}{c}\text { Area } \\
{[\mathrm{mAU}}\end{array}$ & $\begin{array}{c}\text { Height } \\
{[\mathrm{mAU}]}\end{array}$ & $\begin{array}{c}\text { Area } \\
\%\end{array}$ \\
\hdashline-1 & $6.984 \mathrm{VV}$ & 0.2557 & $1.70479 \mathrm{e} 4$ & 985.84442 & 93.9134 \\
2 & $7.549 \mathrm{VB}$ & 0.2699 & 1104.87817 & 58.61847 & 6.0866
\end{tabular}

Totals

$1.81528 \mathrm{e} 4 \quad 1044.46289$

Signal 3: DAD1 C, Sig=254, 4 Ref $=360,100$

Signal 4: DAD1 D, Sig $=280,4$ Ref $=360,100$

Signal 5: DAD1 E, Sig $=310,4$ Ref $=360,100$

$* * *$ End of Report ***

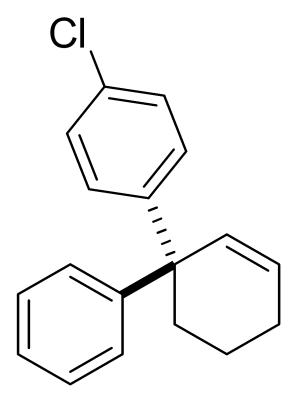

14

Enantioenriched 


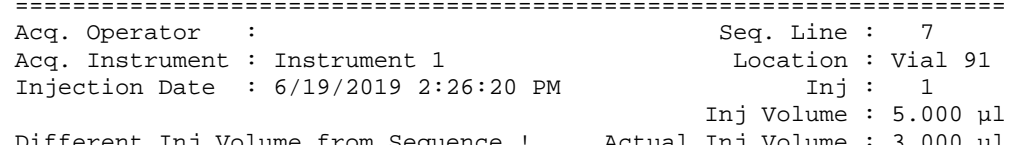

Different Inj volume from sequence ! Actual Inj Volume : $5.000 \mu \mathrm{\mu}$

Acq. Method : C: \CHEM32\1\DATA\QDY 2019-06-19 12-27-08\IC-01-30.M

Last changed : 6/19/2019 1:54:23 PM

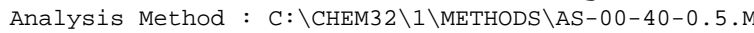

: 6/19/2019 12:24:17 PM

Additional Info : Peak(s) manually integrated
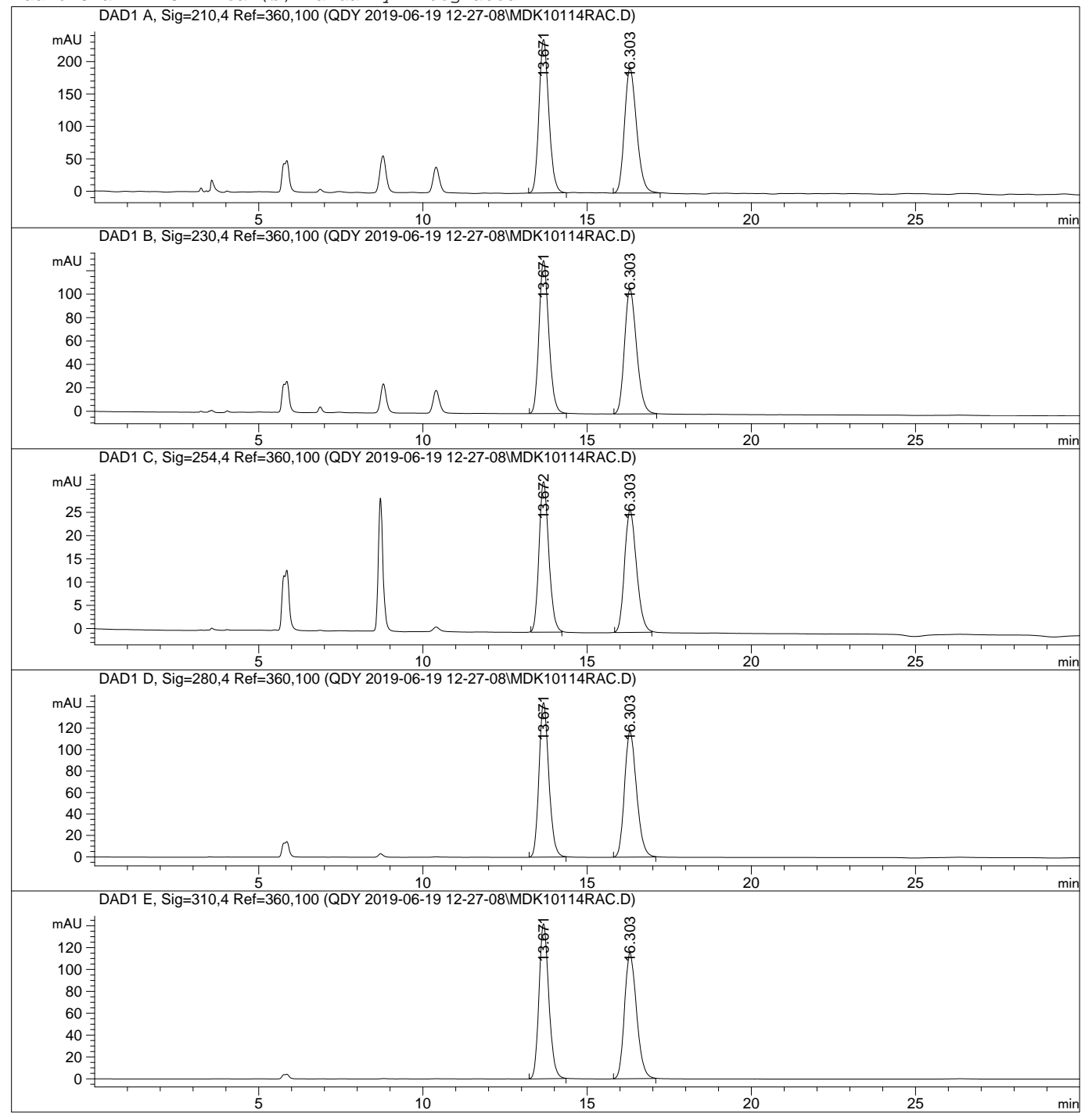

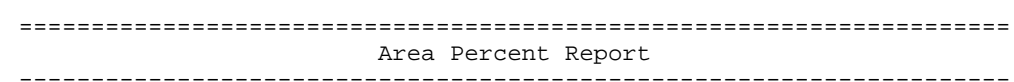

Sorted By
Multiplier

Signal

1.0000

Use Multiplier \& Dilution Factor with ISTDs

Signal 1: DAD1 A, Sig=210, 4 Ref $=360,100$

\begin{tabular}{|c|c|c|c|c|c|}
\hline $\begin{array}{c}\text { Peak } \\
\#\end{array}$ & $\begin{array}{l}\text { RetTime Type } \\
{[\mathrm{min}]}\end{array}$ & $\begin{array}{l}\text { Width } \\
\text { [min] }\end{array}$ & $\begin{array}{c}\text { Area } \\
{\left[\mathrm{mAU} U^{*} \mathrm{~s}\right]}\end{array}$ & $\begin{array}{l}\text { Height } \\
{[\mathrm{mAU}]}\end{array}$ & $\begin{array}{c}\text { Area } \\
\%\end{array}$ \\
\hline $\begin{array}{l}1 \\
2\end{array}$ & $\begin{array}{l}13.671 \mathrm{BB} \\
16.303 \mathrm{BB}\end{array}$ & $\begin{array}{l}0.3215 \\
0.3965\end{array}$ & $\begin{array}{l}4902.57080 \\
4918.88477\end{array}$ & $\begin{array}{l}236.86815 \\
192.24547\end{array}$ & $\begin{array}{l}49.9169 \\
50.0831\end{array}$ \\
\hline
\end{tabular}

Totals :

$9821.45557 \quad 429.11362$

Signal 2: DAD1 B, Sig=230, 4 Ref $=360,100$

\begin{tabular}{|c|c|c|c|c|c|}
\hline $\begin{array}{c}\text { eak } \\
\#\end{array}$ & $\begin{array}{l}\text { RetTime Type } \\
{[\mathrm{min}]}\end{array}$ & $\begin{array}{l}\text { Width } \\
\text { [min] }\end{array}$ & $\begin{array}{c}\text { Area } \\
{\left[\mathrm{mAU} U^{*} \mathrm{~s}\right]}\end{array}$ & $\begin{array}{l}\text { Height } \\
\text { [mAU] }\end{array}$ & $\begin{array}{c}\text { Area } \\
\%\end{array}$ \\
\hline & 12 & 2) 395 & 531 & $\begin{array}{l}30.70554 \\
06.47125\end{array}$ & \\
\hline
\end{tabular}

Totals :

$5423.76953 \quad 237.17679$

Signal 3: DAD1 C, Sig $=254,4$ Ref $=360,100$

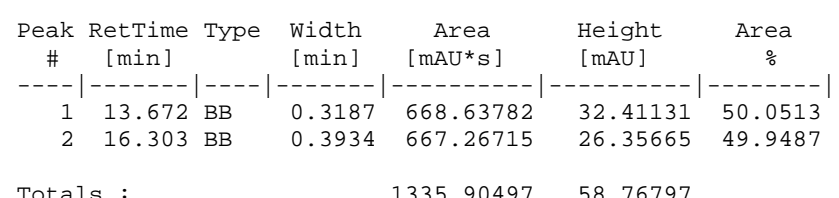

Signal 4: DAD1 D, Sig=280, 4 Ref $=360,100$

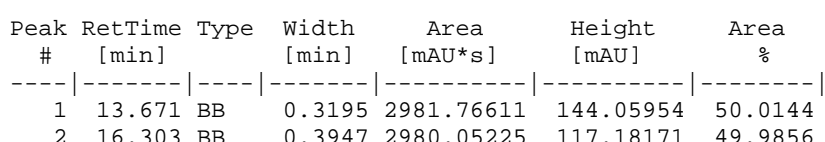

Totals :

$5961.81836 \quad 261.24125$

Signal 5: DAD1 E, Sig=310, 4 Ref $=360,100$

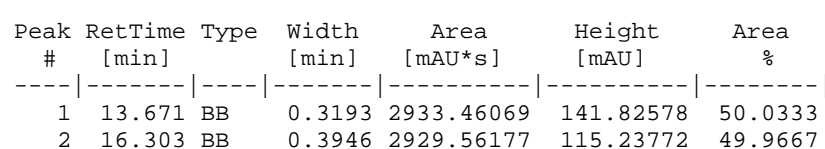

Totals : $\quad 5863.02246 \quad 257.06350$

397

$\begin{array}{lll}\text { Page } 1 \text { of } 3 & \text { Instrument } 1 \text { 6/19/2019 5:02:59 PM }\end{array}$ 
Area Percent Report

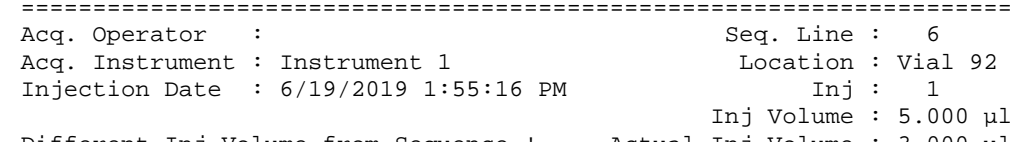

Different Inj volume from Sequence ! Actual Inj Volume : $5.000 \mu \mathrm{\mu}$

Acq. Method : C: \CHEM32\1\DATA\QDY 2019-06-19 12-27-08\IC-01-30.M

Last changed $: 6 / 19 / 20191: 54: 23$ PM

Analysis Method : C: \CHEM32\1\METHODS \AS- $00-40-0.5 . \mathrm{M}$

: 6/19/2019 12:24:17 PM

Additional Info:
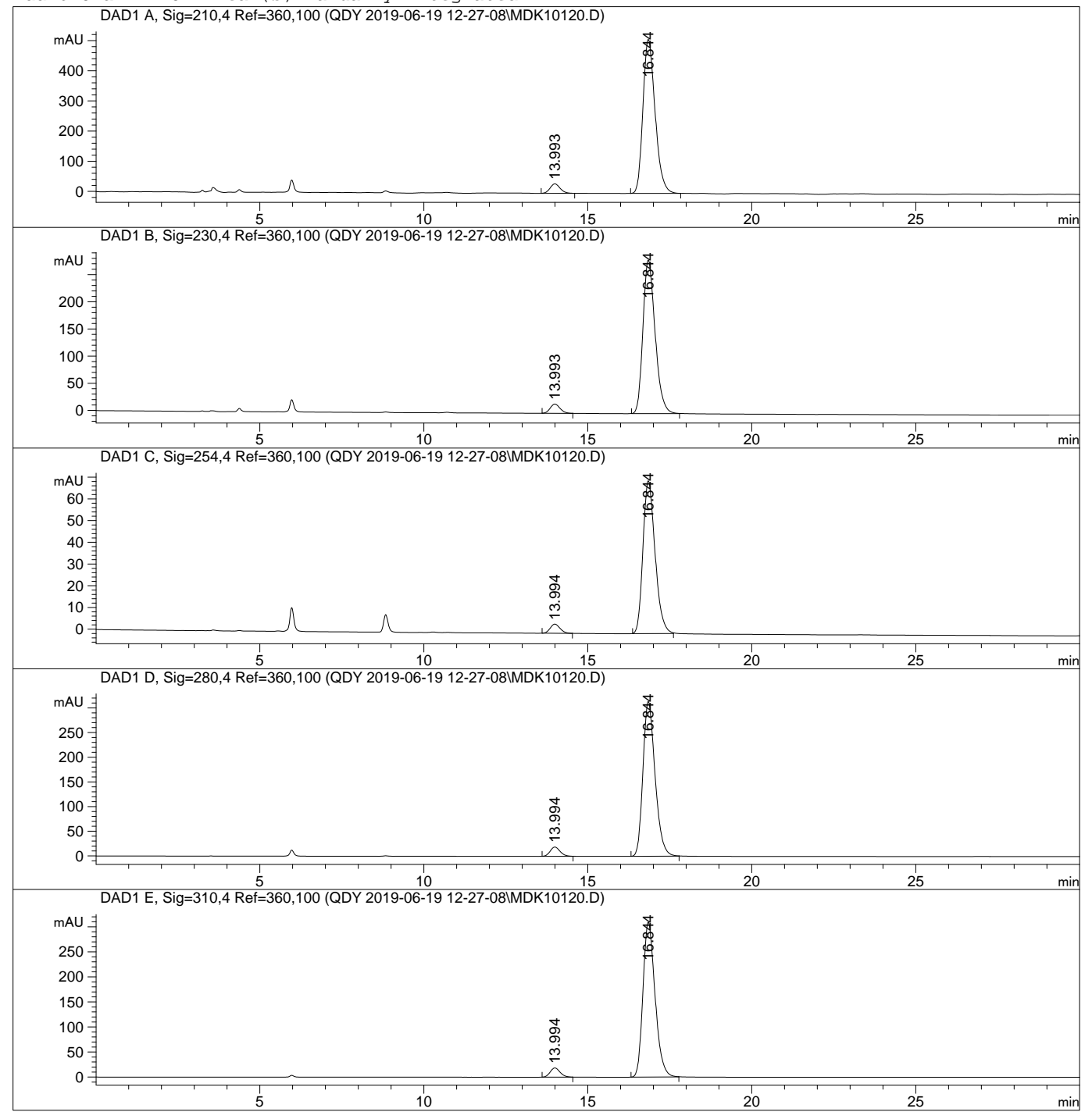

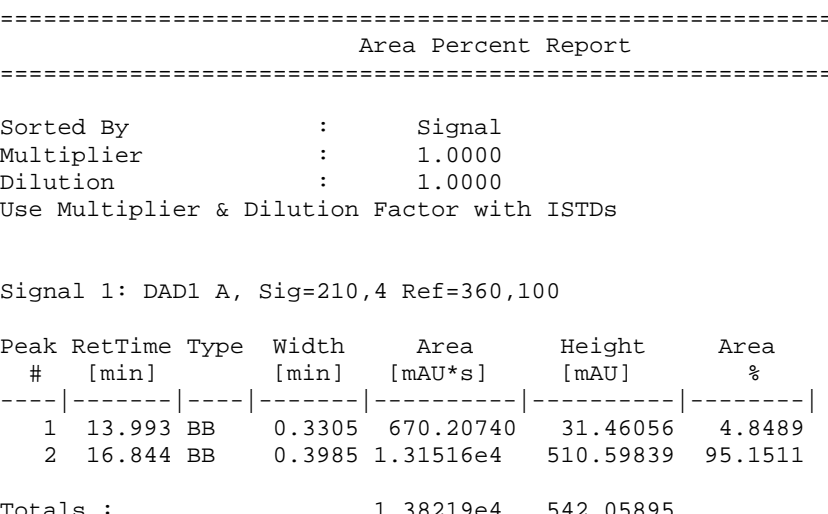

Signal 2: DAD1 B, Sig $=230,4$ Ref $=360,100$
Peak RetTime Type Width Area Height Area

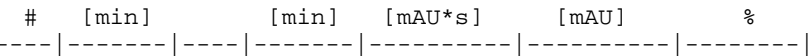

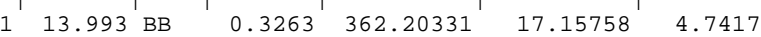

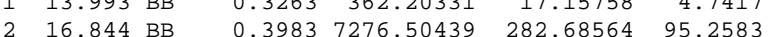

Totals :

$7638.70770 \quad 299.84321$

Signal 3: DAD1 C, Sig $=254,4$ Ref $=360,100$

\begin{tabular}{|c|c|c|c|c|c|}
\hline $\begin{array}{c}\text { Peak } \\
\#\end{array}$ & $\begin{array}{l}\text { RetTime Type } \\
\text { [min] }\end{array}$ & $\begin{array}{l}\text { Width } \\
\text { [min] }\end{array}$ & $\begin{array}{c}\text { Area } \\
{\left[\mathrm{mAU} U^{\star} \mathrm{s}\right]}\end{array}$ & $\begin{array}{l}\text { Height } \\
{[\mathrm{mAU}]}\end{array}$ & $\begin{array}{c}\text { Area } \\
\%\end{array}$ \\
\hline 1 & $13.994 \mathrm{BB}$ & 0.3246 & 88.53929 & 4.22248 & 4.6872 \\
\hline 2 & $16.844 \mathrm{BB}$ & 0.3965 & 1800.41748 & 70.35632 & 95.3128 \\
\hline
\end{tabular}

Signal 4: DAD1 D, Sig $=280,4$ Ref $=360,100$

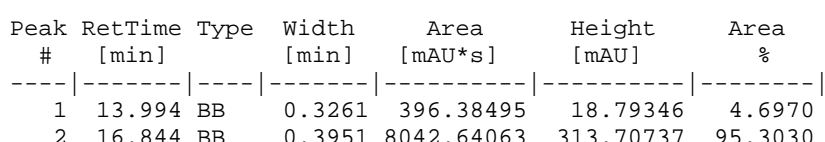

Totals :

$8439.02557 \quad 332.50083$

Signal 5: DAD1 E, Sig $=310,4$ Ref $=360,100$

\begin{tabular}{|c|c|c|c|c|c|c|}
\hline eak & $\begin{array}{c}\text { RetTime } \\
{[\text { min }]}\end{array}$ & & $\begin{array}{l}\text { Width } \\
\text { [min] }\end{array}$ & $\begin{array}{c}\text { Area } \\
{\left[\mathrm{mAU} U^{*} \mathrm{~s}\right]}\end{array}$ & $\begin{array}{l}\text { Height } \\
{[\mathrm{mAU}]}\end{array}$ & $\begin{array}{c}\text { Area } \\
\%\end{array}$ \\
\hline & & & 394 & $\begin{array}{r}389.3227 \\
7920.699\end{array}$ & $\begin{array}{r}18.47845 \\
309.27103\end{array}$ & \\
\hline
\end{tabular}

Totals : $\quad 8310.02246 \quad 327.74947$ 


\begin{tabular}{|c|c|c|}
\hline Acq. Operator & & Seq. Line : \\
\hline Acq. Instrument & Instrument 1 & Location \\
\hline Injection Date & 6/15/2019 4:34:06 PM & 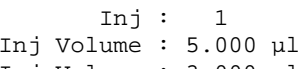 \\
\hline
\end{tabular}

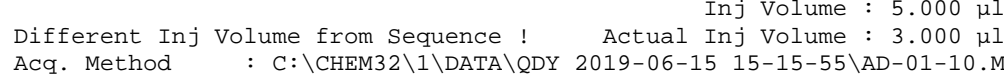

Last changed : 11/2/2015 11:18:23 AM

Analysis Method : C: \CHEM32\1\METHODS \IC-01-40.M-0.5.M

Last changed : 6/5/2019 1:46:08 PM

Additional Info: (modified after loading)
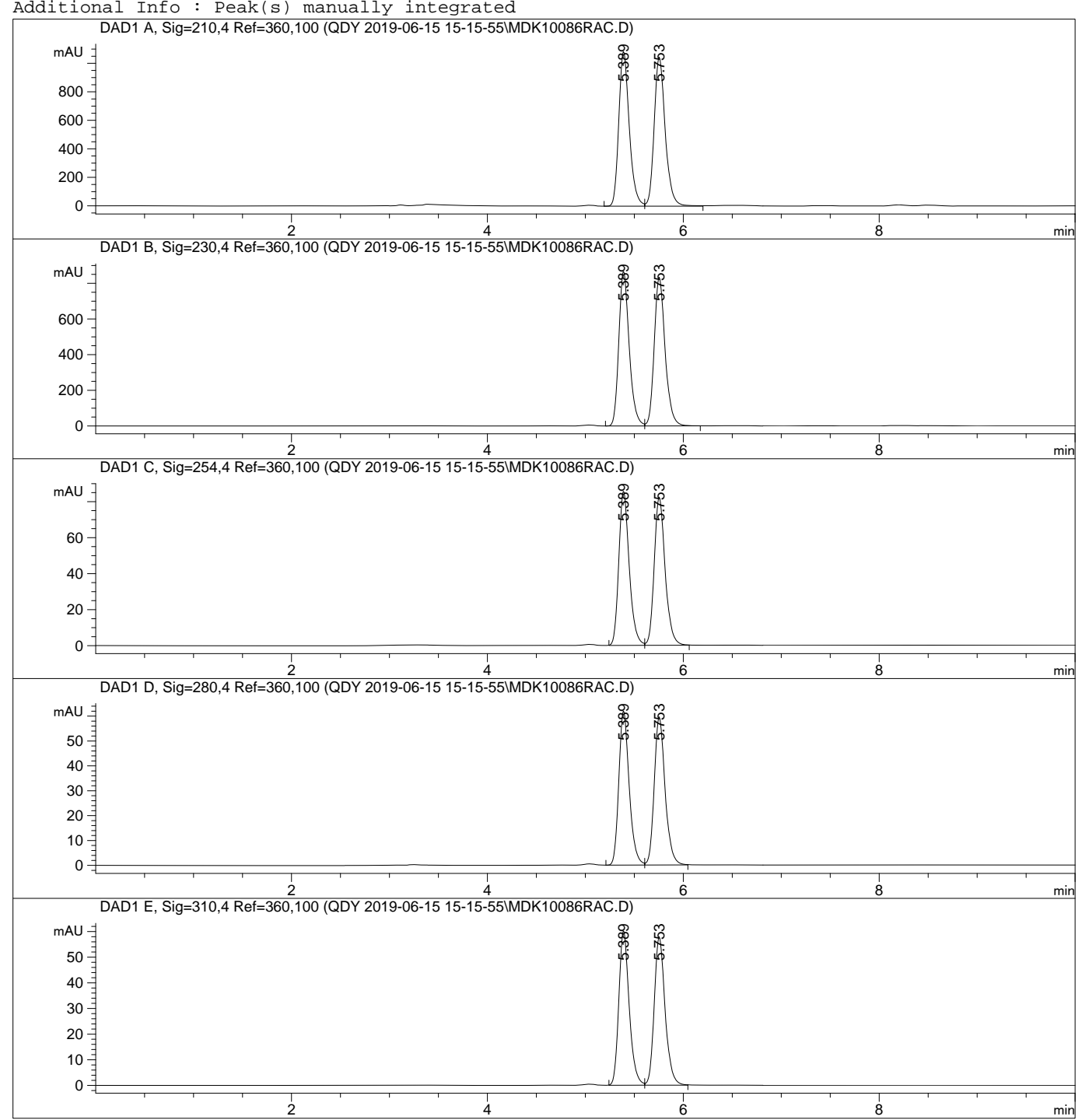

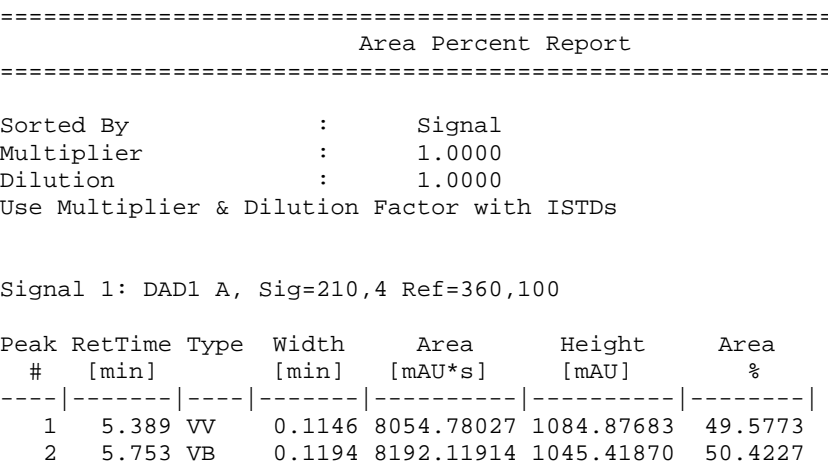

Totals :

$1.62469 \mathrm{e} 4 \quad 2130.29553$

Signal 2: DAD1 B, Sig=230, 4 Ref $=360,100$

\begin{tabular}{cccccc}
$\begin{array}{c}\text { Peak RetTime Type } \\
\#\end{array}\left[\begin{array}{c}\text { Width } \\
{[\mathrm{min}]}\end{array}\right.$ & $\begin{array}{c}\text { Area } \\
{[\mathrm{mAU}}\end{array}$ & $\begin{array}{l}\text { Height } \\
{[\mathrm{mAU}]}\end{array}$ & $\begin{array}{c}\text { Area } \\
\%\end{array}$ \\
\hdashline-1 & $5.389 \mathrm{VV}$ & 0.1118 & 6375.77002 & 866.91235 & 49.8014 \\
2 & $5.753 \mathrm{VB}$ & 0.1180 & 6426.63184 & 833.25488 & 50.1986
\end{tabular}

Totals :

$1.28024 \mathrm{e} 4 \quad 1700.16724$

Signal 3: DAD1 C, Sig $=254,4$ Ref $=360,100$

\begin{tabular}{|c|c|c|c|c|c|}
\hline $\begin{array}{c}\text { Peak } \\
\#\end{array}$ & $\begin{array}{l}\text { RetTime Type } \\
\text { [min] }\end{array}$ & $\begin{array}{l}\text { Width } \\
\text { [min] }\end{array}$ & $\begin{array}{c}\text { Area } \\
{\left[\mathrm{mAU}^{*} \mathrm{~s}\right]}\end{array}$ & $\begin{array}{l}\text { Height } \\
{[\mathrm{mAU}]}\end{array}$ & $\begin{array}{c}\text { Area } \\
\%\end{array}$ \\
\hline 1 & $5.389 \mathrm{BV}$ & 0.1120 & 631.18652 & 85.65644 & 49.8061 \\
\hline 2 & $5.753 \mathrm{VB}$ & 0.1181 & 636.10181 & 82.34420 & 50.1939 \\
\hline
\end{tabular}

Signal 4: DAD1 D, Sig=280, 4 Ref $=360,100$

\begin{tabular}{|c|c|c|c|c|c|}
\hline $\begin{array}{c}\text { Peak } \\
\#\end{array}$ & $\begin{array}{l}\text { RetTime Type } \\
\text { [min] }\end{array}$ & $\begin{array}{l}\text { Width } \\
\text { [min] }\end{array}$ & $\begin{array}{c}\text { Area } \\
{\left[\mathrm{mAU}^{\star} \mathrm{s}\right]}\end{array}$ & $\begin{array}{l}\text { Height } \\
{[\mathrm{mAU}]}\end{array}$ & $\begin{array}{c}\text { Area } \\
\%\end{array}$ \\
\hline 1 & $5.389 \mathrm{VV}$ & & & 5590 & 8224 \\
\hline 2 & 5.753 VB & 0.1177 & 457.55756 & 59.52918 & 50.1776 \\
\hline
\end{tabular}

Totals :

$911.87662 \quad 121.48508$

Signal 5: DAD1 E, Sig $=310,4$ Ref $=360,100$

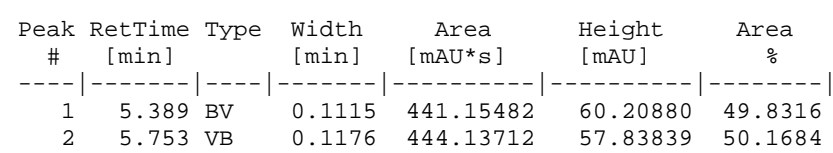

Totals : $\quad 885.29193 \quad 118.04720$ 

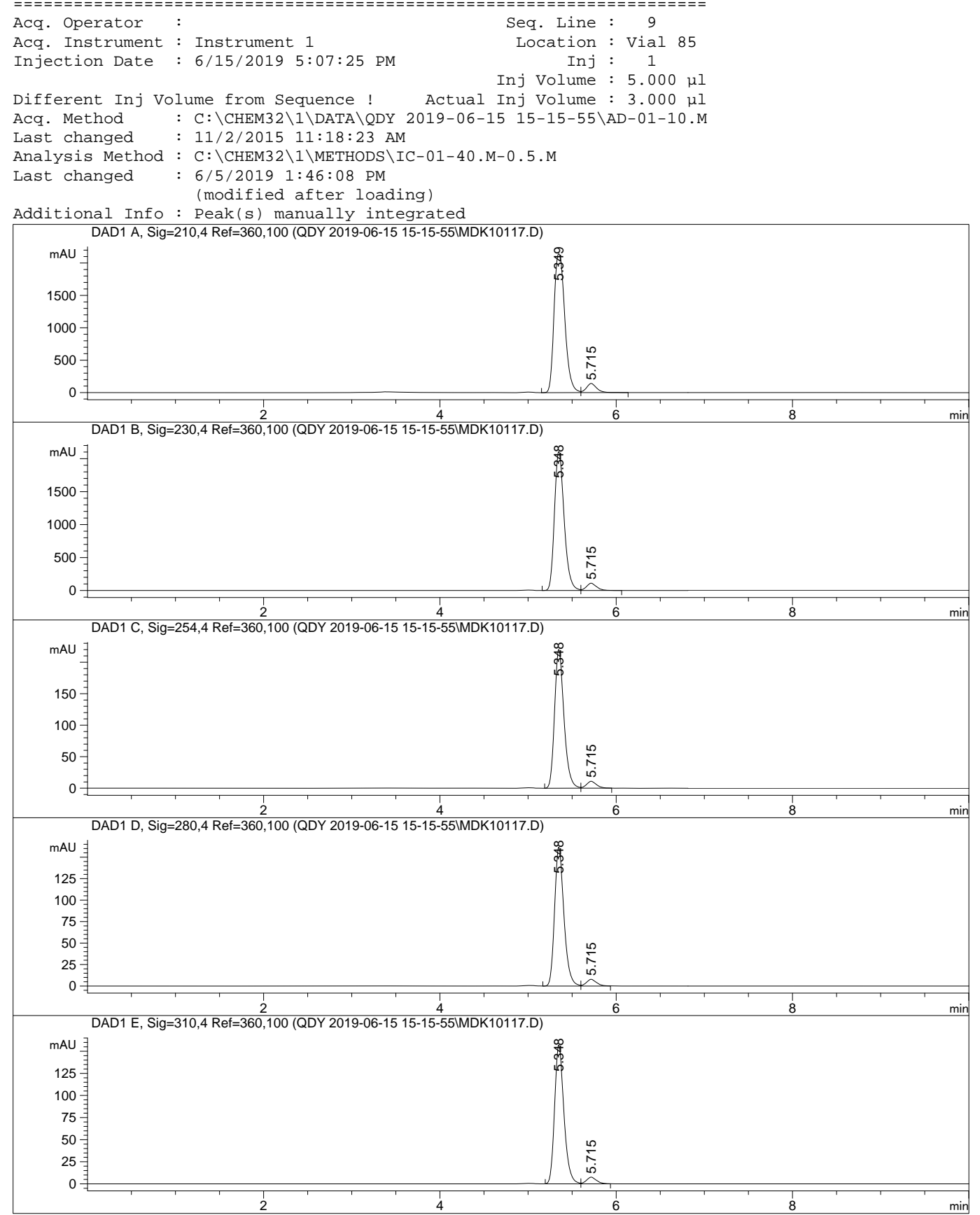

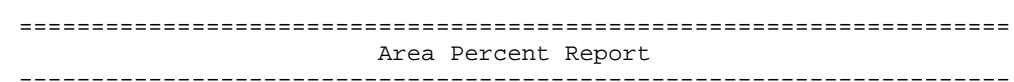

Sorted By

Signal

Multiplier

1.0000

Use Multiplier \& Dilution Factor with ISTDs

Signal 1: DAD1 A, Sig=210, 4 Ref $=360,100$

\begin{tabular}{cccccc}
$\begin{array}{c}\text { Peak RetTime Type } \\
\#\end{array}\left[\begin{array}{c}\text { Width } \\
{[\mathrm{min}]}\end{array}\right.$ & $\begin{array}{c}\text { Area } \\
{[\mathrm{mAU}}\end{array}$ & $\begin{array}{c}\text { Height } \\
{[\mathrm{mAU}]}\end{array}$ & $\begin{array}{c}\text { Area } \\
\%\end{array}$ \\
\hdashline-1 & $5.349 \mathrm{VV}$ & 0.1347 & $1.81441 \mathrm{e} 4$ & 2145.30298 & 94.0925 \\
\hline 2 & $5.715 \mathrm{VB}$ & 0.1200 & 1139.15088 & 141.37256 & 5.9075
\end{tabular}

Totals :

$1.92832 \mathrm{e} 4 \quad 2286.67554$

Signal 2: DAD1 B, Sig=230, 4 Ref $=360,100$

\begin{tabular}{cccccc}
$\begin{array}{c}\text { Peak RetTime Type } \\
\#\end{array}\left[\begin{array}{c}\text { Width } \\
{[\mathrm{min}]}\end{array}\right.$ & $\begin{array}{c}\text { Area } \\
{[\mathrm{mAU}}\end{array}$ & $\begin{array}{c}\text { Height } \\
{[\mathrm{mAU}]}\end{array}$ & $\begin{array}{c}\text { Area } \\
\%\end{array}$ \\
\hdashline-1 & $5.348 \mathrm{VV}$ & 0.1187 & $1.60278 \mathrm{e} 4$ & 2108.77710 & 94.7923 \\
2 & $5.715 \mathrm{VB}$ & 0.1187 & 880.54175 & 110.87341 & 5.2077
\end{tabular}

Totals :

$1.69083 \mathrm{e} 4 \quad 2219.65051$

Signal 3: DAD1 C, Sig $=254,4$ Ref $=360,100$

\begin{tabular}{|c|c|c|c|c|c|}
\hline $\begin{array}{c}\text { Peak } \\
\#\end{array}$ & $\begin{array}{l}\text { RetTime Type } \\
{[\text { min] }}\end{array}$ & $\begin{array}{l}\text { Width } \\
\text { [min] }\end{array}$ & $\begin{array}{c}\text { Area } \\
{\left[\mathrm{mAU} U^{\star} \mathrm{s}\right]}\end{array}$ & $\begin{array}{l}\text { Height } \\
{[\mathrm{mAU}]}\end{array}$ & $\begin{array}{c}\text { Area } \\
\%\end{array}$ \\
\hline 1 & $5.348 \mathrm{BV}$ & 0.1121 & 1627.64734 & 220.55936 & 174 \\
\hline 2 & 5.715 VB & 0.1182 & 87.15585 & 11.03393 & 5.0826 \\
\hline
\end{tabular}

Signal 4: DAD1 D, Sig=280, 4 Ref $=360,100$

Peak RetTime Type width Area Height Area \# [min] $[\mathrm{min}][\mathrm{mAU} * \mathrm{~s}][\mathrm{mAU}] \quad \%$ $\begin{array}{llllll}1 & 5.348 \mathrm{VV} & 0.1115 & 1185.82629 & 161.74858 & 95.0484\end{array}$ $\begin{array}{rrrrrr}1 & 5.348 \text { VB } & 0.1115 & 1185.82629 & 161.74858 & 95.0484 \\ & 5.715 \text { VB } & 0.1178 & 61.77656 & 7.85064 & 4.9516\end{array}$ Totals : $\quad 1247.60286 \quad 169.59922$

Signal 5: DAD1 E, Sig $=310,4$ Ref $=360,100$

Peak RetTime Type Width Area Height Area \# [min] $[\mathrm{min}]\left[\mathrm{mAU}^{*} \mathrm{~s}\right][\mathrm{mAU}] \quad \%$ $\begin{array}{lrrrrr}1 & 5.348 \text { BV } & 0.1115 & 1152.11768 & 157.19804 & 95.0684\end{array}$ Totals : $\quad 1211.88347 \quad 164.81002$

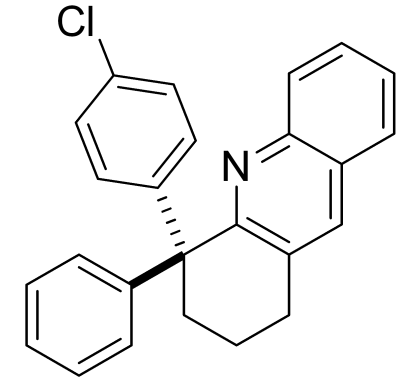

16

Enantioenriched 